\title{
Nondestructive Evaluation of Thick Concrete Using Advanced Signal Processing Techniques
}

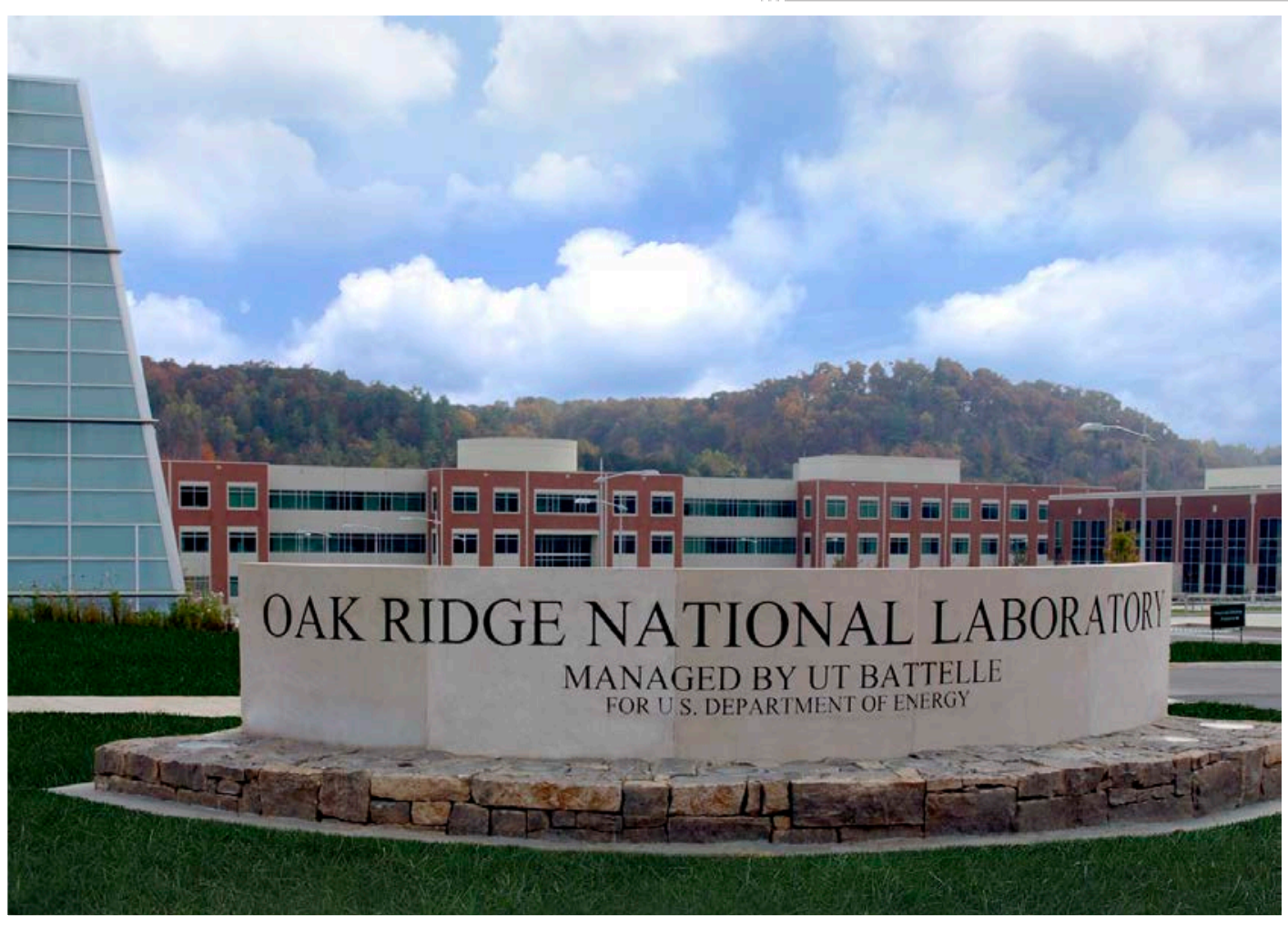

Approved for public release. Distribution is unlimited.

OAK RIDGE NATIONAL LABORATORY MANAGED BY UT-BATTELLE FOR THE US DEPARTMENT OF ENERGY
Dwight Clayton

Alan Barker Hector Santos-Villalobos Austin Albright Kyle Hoegh Lev Khazanovich

September 2015 


\section{DOCUMENT AVAILABILITY}

Reports produced after January 1, 1996, are generally available free via US Department of Energy (DOE) SciTech Connect.

Website http://www.osti.gov/scitech/

Reports produced before January 1, 1996, may be purchased by members of the public from the following source:

National Technical Information Service

5285 Port Royal Road

Springfield, VA 22161

Telephone 703-605-6000 (1-800-553-6847)

TDD 703-487-4639

Fax 703-605-6900

E-mail info@ntis.gov

Website http://www.ntis.gov/help/ordermethods.aspx

Reports are available to DOE employees, DOE contractors, Energy Technology Data Exchange representatives, and International Nuclear Information System representatives from the following source:

Office of Scientific and Technical Information

PO Box 62

Oak Ridge, TN 37831

Telephone 865-576-8401

Fax 865-576-5728

E-mail reports@osti.gov

Website http://www.osti.gov/contact.html

This report was prepared as an account of work sponsored by an agency of the United States Government. Neither the United States Government nor any agency thereof, nor any of their employees, makes any warranty, express or implied, or assumes any legal liability or responsibility for the accuracy, completeness, or usefulness of any information, apparatus, product, or process disclosed, or represents that its use would not infringe privately owned rights. Reference herein to any specific commercial product, process, or service by trade name, trademark, manufacturer, or otherwise, does not necessarily constitute or imply its endorsement, recommendation, or favoring by the United States Government or any agency thereof. The views and opinions of authors expressed herein do not necessarily state or reflect those of the United States Government or any agency thereof. 


\title{
THICK CONCRETE SPECIMEN CONSTRUCTION, TESTING, AND PRELIMINARY ANALYSIS
}

\author{
Dwight Clayton \\ Alan Barker \\ Hector Santos-Villalobos \\ Austin Albright \\ Kyle Hoegh \\ Lev Khazanovich
}

Date Published: September 2015

\author{
Prepared by \\ OAK RIDGE NATIONAL LABORATORY \\ Oak Ridge, Tennessee 37831-6283 \\ managed by \\ UT-BATTELLE, LLC \\ for the \\ US DEPARTMENT OF ENERGY \\ under contract DE-AC05-00OR22725
}





\section{CONTENTS}

\section{Page}

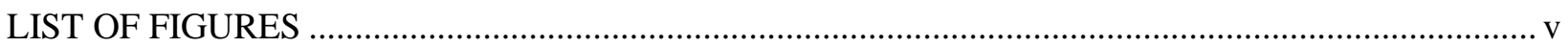

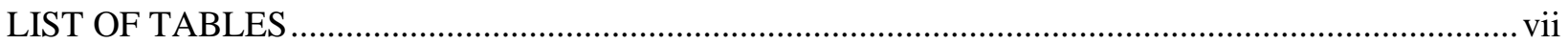

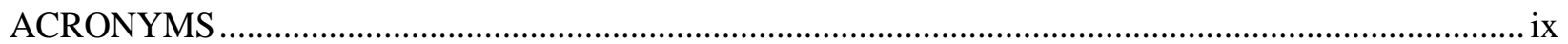

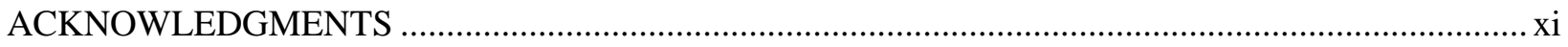

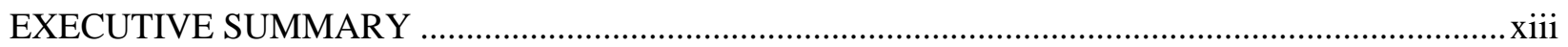

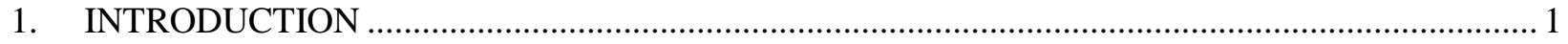

2. NEED FOR SUITABLE CONCRETE SPECIMENS ..................................................................... 3

3. TYPICAL PARAMETERS FOR CONCRETE USED IN NPPs ….................................................. 7

3.1 Typical Containment Wall Design....................................................................................... 7

3.2 Previous Concrete Failures in NPP Containment Structures ................................................. 8

4. DESIGN REQUIREMENTS FOR The REPRESENTATIVE NPP CONCRETE SAMPLE............ 13

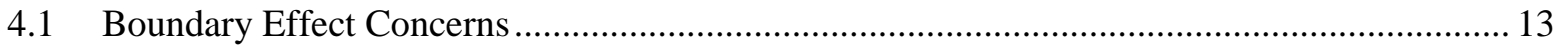

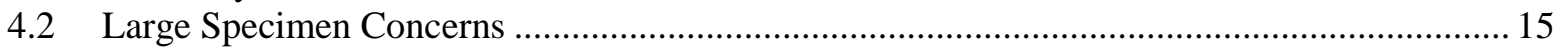

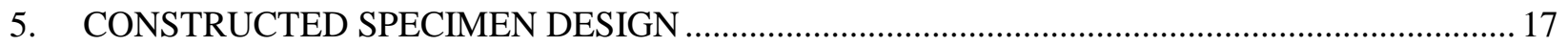

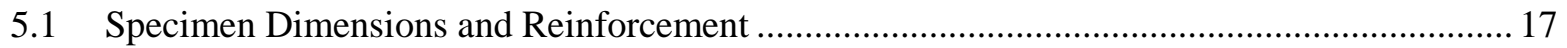

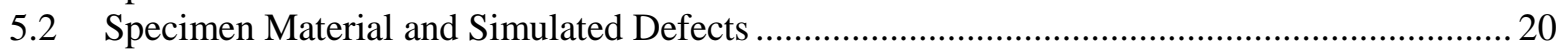

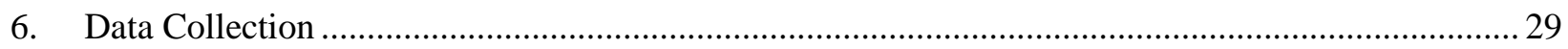

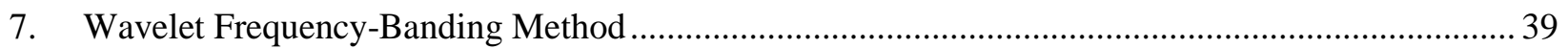

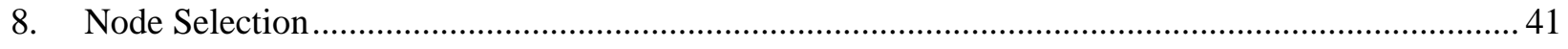

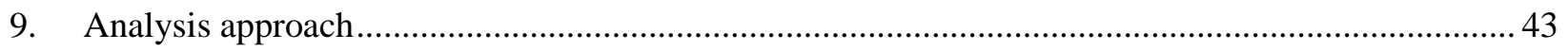

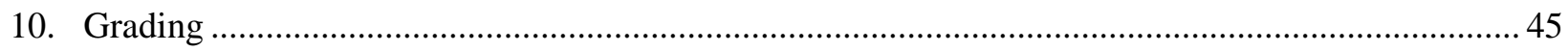

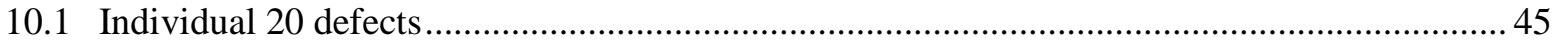

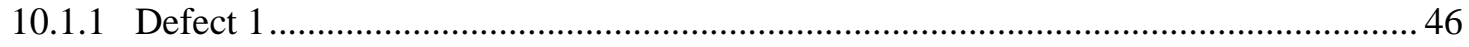

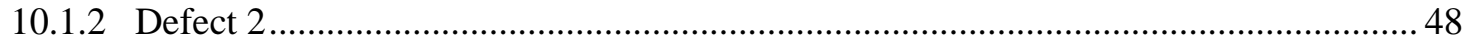

10.1.3 Defect 3

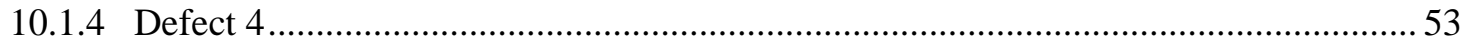

10.1.5 Defect 5

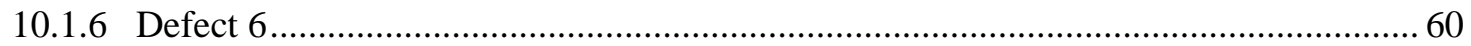

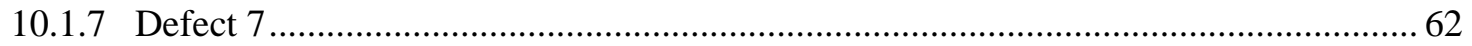

10.1 .8 Defect 8

10.1.9 Defect 9

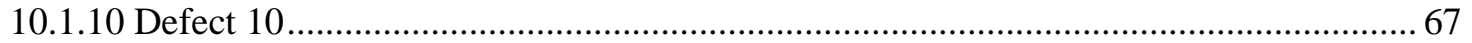

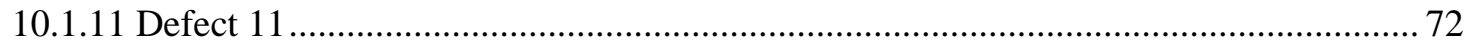

10.1.12 Defect 12

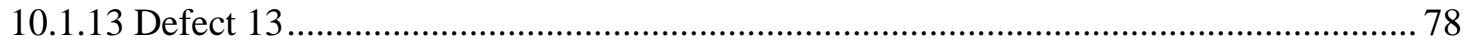

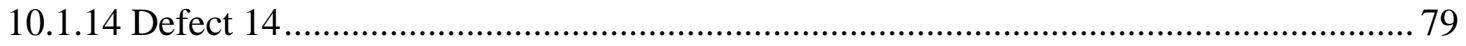

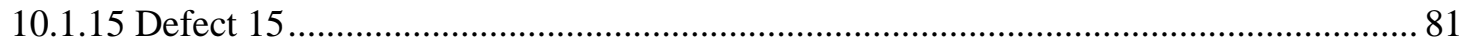

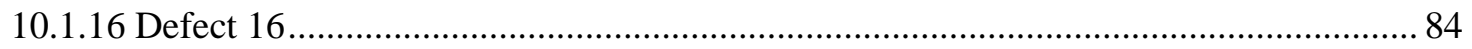

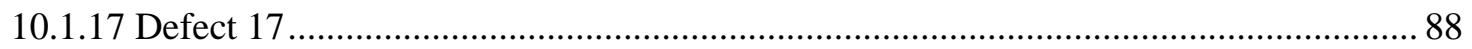

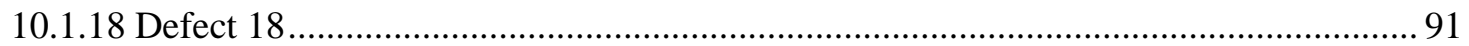

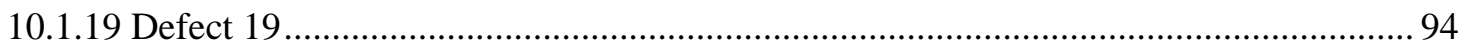

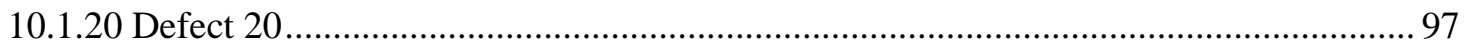




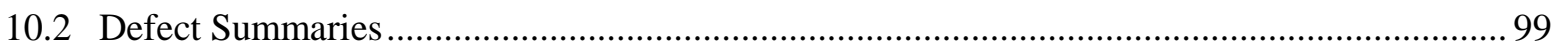

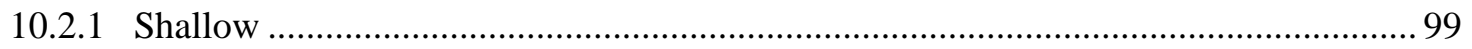

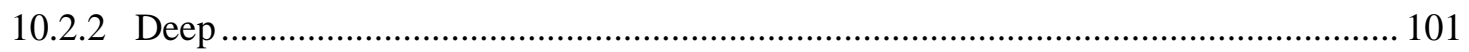

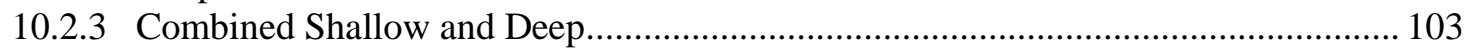

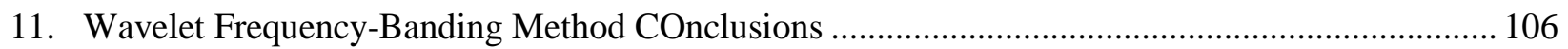

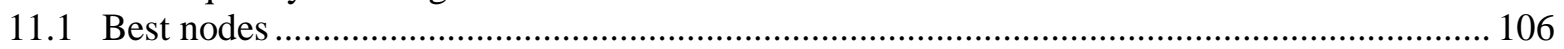

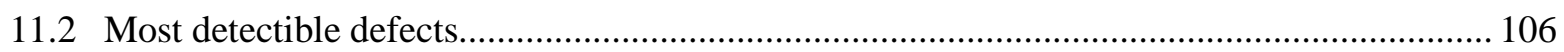

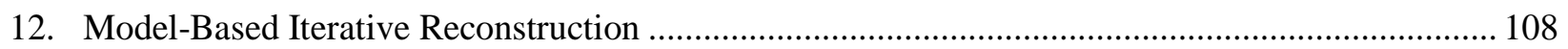

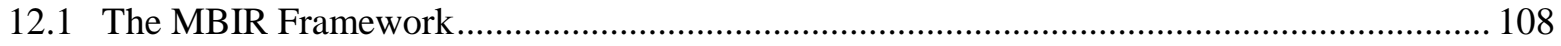

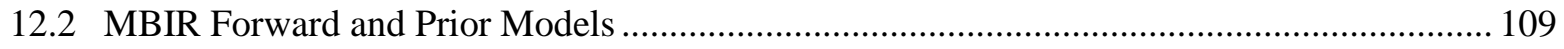

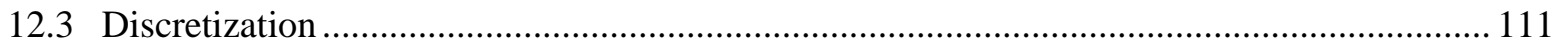

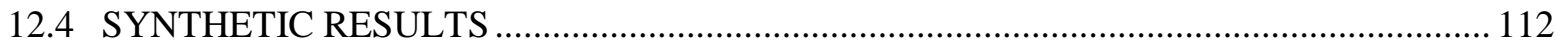

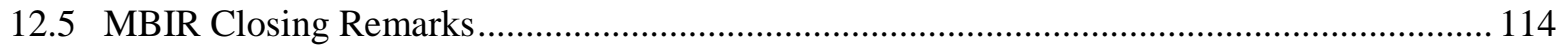

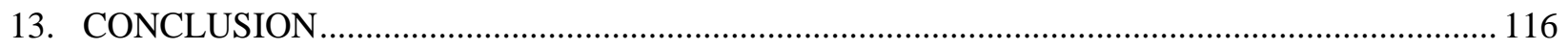

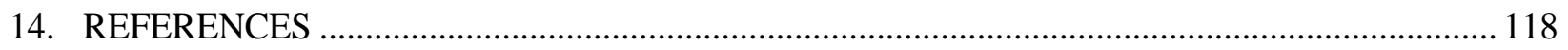

APPENDIX A. ROUGH SIDE DEFECT IMAGES …..................................................................

APPENDIX B. SMOOTH SIDE DEFECT IMAGES ….............................................................. B-1 


\section{LIST OF FIGURES}

Figure $\quad$ Page

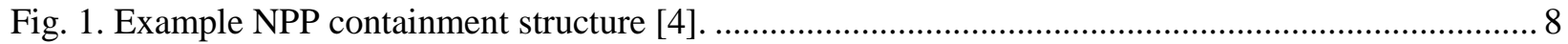

Fig. 2. Delamination crack running from horizontal tendons at approximately $10 \mathrm{in.}$ deep....................... 9

Fig. 3. Davis-Besse Nuclear Power Station [30]............................................................................... 10

Fig. 4. View of the Davis-Besse Nuclear Power Station layout and reinforcement scheme [30]............. 10

Fig. 5. Laminar cracking at reinforcements from an interface cut out by hydrodemolition [30]................ 11

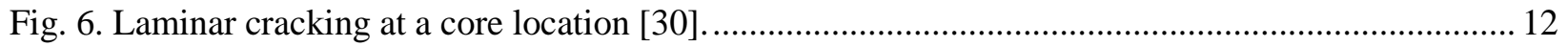

Fig. 7. Beam pattern from a four element ultrasonic array..................................................................... 13

Fig. 8. Picture of MIRA ultrasound scanner with an approximately 1-m-thick reinforced concrete.......... 14

Fig. 9. \#18 reinforcement used to replicate the large diameter mesh used in NPP containment walls....... 17

Fig. 10. Concrete dimensions and reinforcement cross section to represent an NPP containment

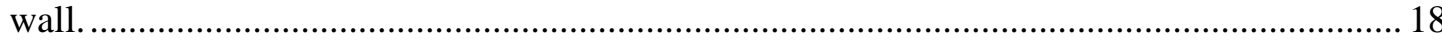

Fig. 11. Length/height view of the proposed concrete dimensions and reinforcement............................. 19

Fig. 12. Length/depth view of the proposed concrete dimensions and reinforcement............................... 19

Fig. 13. Use of SCC to allow for consolidation without affecting simulated defects.............................. 20

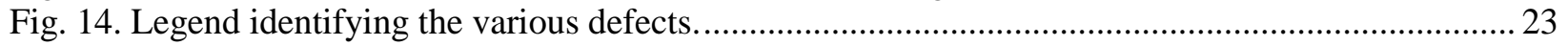

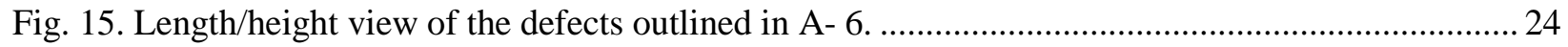

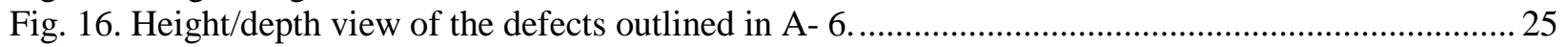

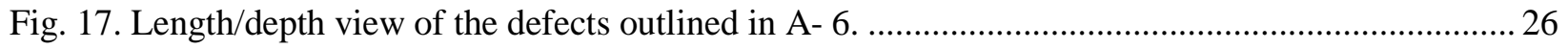

Fig. 18. Example controlled defect simulating a void with dissolving Styrofoam (D12)......................... 26

Fig. 19. Example realistic defect simulating concrete damage with embedded porous concrete. .............. 27

Fig. 20. Length data collection used to create a SAFT-PAN reconstruction............................................ 33

Fig. 21. Height data collection used to create a SAFT-PAN reconstruction. ............................................. 33

Fig. 22. Rough-side measurement procedure for length orientation measurements................................. 34

Fig. 23. Smooth-side measurement procedure for length orientation measurements............................... 35

Fig. 24. Rough-side measurement procedure for height orientation measurements................................. 36

Fig. 25. Smooth-side measurement procedure for height orientation measurements. .............................. 37

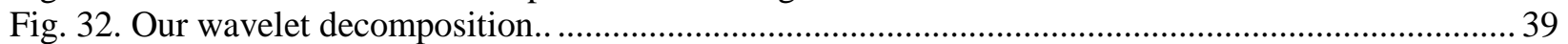

Fig. 33. Defect 1 analysis chart illustrating the normalized score for each node...................................... 46

Fig. 34. Shallow cover improved detectability for defect 1 for node 16 compared to regular SAFT......... 47

Fig. 35. Defect 2 analysis chart illustrating the normalized score for each node....................................... 48

Fig. 36. Shallow cover improved detectability for defect 2 for node 32, horizontal orientation................. 49

Fig. 37. Shallow cover improved detectability for defect 2 for node 32, vertical orientation. ................... 50

Fig. 38. Defect 3 analysis chart illustrating the normalized score for each node..................................... 51

Fig. 39. Shallow cover improved detectability for defect 3 for node 32, vertical orientation. ................... 52

Fig. 40. Defect 4 analysis chart illustrating the normalized score for each node....................................... 53

Fig. 41. Shallow cover improved detectability for defect 4 for node 16, horizontal orientation................ 54

Fig. 42. Shallow cover improved detectability for defect 4 for node 16, vertical orientation \#1............... 55

Fig. 43. Shallow cover improved detectability for defect 4 for node 16, vertical orientation \#2. .............. 56

Fig. 44. Defect 5 analysis chart illustrating the normalized score for each node..................................... 57

Fig. 45. Shallow cover improved detectability for defect 5 for node 66, vertical orientation \#1............... 58

Fig. 46. Shallow cover improved detectability for defect 5 for node 66, vertical orientation \#2. .............. 59

Fig. 47. Defect 6 analysis chart illustrating the normalized score for each node..................................... 60

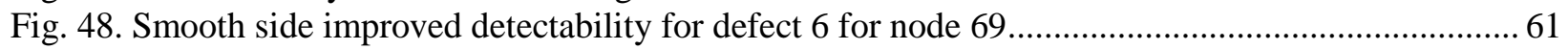

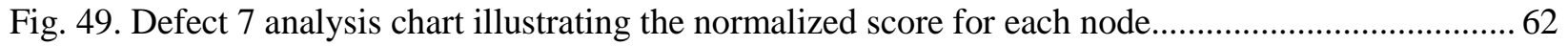

Fig. 50. Defect 8 analysis chart illustrating the normalized score for each node......................................6 62

Fig. 51. Deep cover improved detectability for defect 8 for node 32, horizontal orientation..................... 63

Fig. 52. Deep cover improved detectability for defect 8 for node 66, vertical orientation........................ 64 
Fig. 53. Defect 9 analysis chart illustrating the normalized score for each node..................................... 65

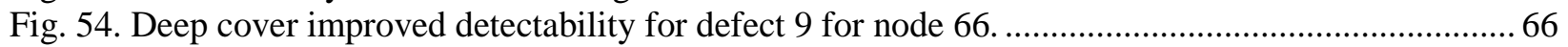

Fig. 55. Defect 10 analysis chart illustrating the normalized score for each node...................................6 67

Fig. 56. Shallow cover improved detectability for defect 10 for node 32 horizontal orientation \#1.......... 68

Fig. 57. Shallow cover improved detectability for defect 10 for node 32, horizontal orientation \#2......... 69

Fig. 58. Deep cover improved detectability for defect 10 for node 34, horizontal orientation \#1.............. 70

Fig. 59. Deep cover improved detectability for defect 10 for node 34, horizontal orientation \#2.............. 71

Fig. 60. Defect 11 analysis chart illustrating the normalized score for each node.................................... 72

Fig. 61. Shallow cover improved detectabilityfor defect 11 for node 18, horizontal orientation............... 73

Fig. 62. Shallow cover improved detectability for defect 11 for node 16, vertical orientation .................. 74

Fig. 63. Deep cover improved detectability for defect 11 for node 18, horizontal orientation................... 75

Fig. 64. Deep cover improved detectability for defect 11 for node 18, vertical orientation...................... 76

Fig. 65. Defect 12 analysis chart illustrating the normalized score for each node...................................... 77

Fig. 66. Defect 13 analysis chart illustrating the normalized score for each node..................................... 78

Fig. 67. Defect 14 analysis chart illustrating the normalized score for each node..................................... 79

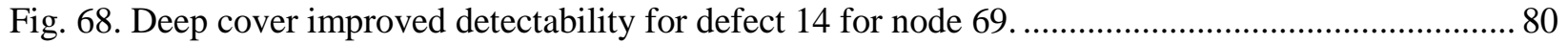

Fig. 69. Defect 15 analysis chart illustrating the normalized score for each node...................................... 81

Fig. 70. Deep cover improved detectability for defect 15 for node 16, horizontal orientation.................... 82

Fig. 71. Deep cover improved detectability for defect 15 for node 16, vertical orientation........................ 83

Fig. 72. Defect 16 analysis chart illustrating the normalized score for each node..................................... 84

Fig. 73. Deep cover improved detectability for defect 6 for node 32, horizontal orientation \#1................ 85

Fig. 74. Deep cover improved detectability for defect 6 for node 32, horizontal orientation \#2................ 86

Fig. 75. Deep cover improved detectability for defect 6 for node 16, vertical orientation......................... 87

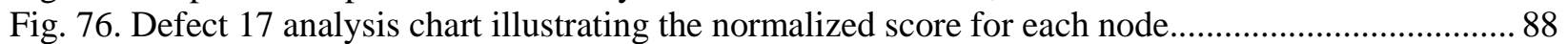

Fig. 77. Shallow cover improved detectability for defect 17 for node 16, horizontal orientation \#1......... 89

Fig. 78. Shallow cover improved detectability for defect 17 for node 16, horizontal orientation \#2 ....... 90

Fig. 79. Defect 18 analysis chart illustrating the normalized score for each node..................................... 91

Fig. 80. Shallow cover improved detectability for defect 18 for node 34, horizontal orientation \#2......... 92

Fig. 81. Shallow cover improved detectability for defect 18 for node 34, horizontal orientation \#2......... 93

Fig. 82. Defect 19 analysis chart illustrating the normalized score for each node.................................... 94

Fig. 83. Shallow cover improved detectability for defect 19 for node 34, horizontal orientation............. 95

Fig. 84. Shallow cover improved detectability for defect 19 for node 34, vertical orientation. ................ 96

Fig. 85. Defect 20 analysis chart illustrating the normalized score for each node....................................97

Fig. 86. Shallow cover improved detectability for defect 20 for node 16 ..............................................9 98

Fig. 87. Best node for shallow side sorted by normalized total across all defects................................... 100

Fig. 88. Most detectible defect for shallow side sorted by normalized total across all nodes. ................. 100

Fig. 89. Best node for deep side sorted by normalized total across all defects....................................... 102

Fig. 90. Most detectible defect for deep side sorted by normalized total across all nodes....................... 102

Fig. 91. Best node for combined shallow and deep sides sorted by normalized total.............................. 104

Fig. 92. Most detectible defect for combined shallow and deep sides sorted by normalized total........... 104

Fig. 93. Illustration of system matrix coefficient amplitudes for two transducers at time 133us............. 110

Fig. 94. Phantom samples used for the synthetic test.. …..................................................................... 113

Fig. 95. Synthetic measured reflections for the phantom in Figure 2 .................................................. 114 


\section{LIST OF TABLES}

Table

Page

Table 1 . Wave type information

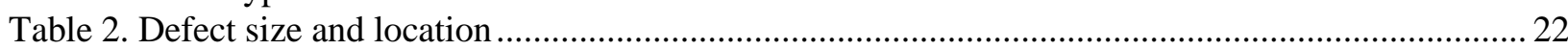

Table 3. Smooth side testing locations (December 16, 2014)............................................................ 31

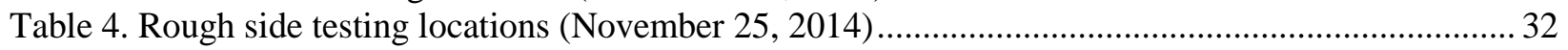

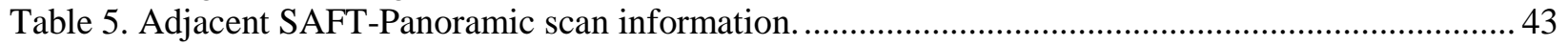

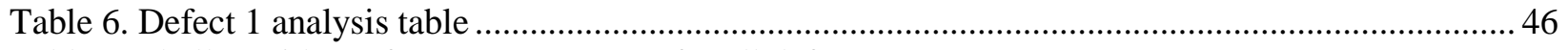

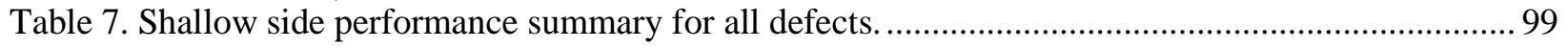

Table 8. Deep side performance summary for all defects....................................................................... 101

Table 9. Combined Shallow and deep side performance summary for all defects.................................. 103 


\section{ACRONYMS}

\begin{tabular}{ll} 
BIM & Born Iterative Method \\
CG & Conjugate Gradient \\
CR3 & Crystal River Nuclear Plant \\
CS & compressed sensing \\
CT & Computed Tomography \\
DPC & dry point contact \\
EFIT & Elastodynamic Finite Integration Technique \\
GD & Gradient Descent \\
GPR & Ground Penetrating Radar \\
HFIR & High Flux Isotope Reactor \\
ICD & Iterative Coordinate Descent \\
IRC & intensity reflectivity coefficients \\
LWR & Light Water Reactor \\
MAP & maximum a posteriori \\
MAST & Multi-Axial Subassemblage Testing \\
MBIR & Model-Based Iterative Reconstruction \\
MLE & maximum likelihood estimation \\
NDE & Nondestructive evaluation \\
NPP & Nuclear power plant \\
NUFFT & Non-uniform Fast Fourier Transform \\
ORNL & Oak Ridge National Laboratory \\
PCC & Portland cement concrete \\
PET & positron emission tomography \\
PSF & point spread function \\
q-GGMRF & q-Generalized Gaussian Markov Random Fields \\
ROI & region of interest \\
SAFT & Synthetic Aperture Frequency Technique \\
SCC & self-consolidating concrete \\
SIRT & simultaneous iterative reconstruction technique \\
TV & Total Variation \\
UCT & ultrasound computed tomography \\
UMN & University of Minnesota \\
UMN-TGL & University of Minnesota - Theodore V. Galambos Structural Engineering Laboratory \\
& \\
\hline
\end{tabular}





\section{ACKNOWLEDGMENTS}

The work is funded by the U.S. Department of Energy's office of Nuclear Energy under the Light Water Reactor Sustainability (LWRS) program. The authors want to thank Mohammed Olama of ORNL for technical consultation and review. The authors also want to acknowledge the assistance of our summer interns, Luke Prince and Joseph Clayton, who helped to make this report possible. Luke and Joseph processed the data and generated many of the result figures. 



\section{EXECUTIVE SUMMARY}

The purpose of the U.S. Department of Energy Office of Nuclear Energy's Light Water Reactor Sustainability (LWRS) Program is to develop technologies and other solutions that can improve the reliability, sustain the safety, and extend the operating lifetimes of nuclear power plants (NPPs) beyond 60 years [1]. Since many important safety structures in an NPP are constructed of concrete, inspection techniques must be developed and tested to evaluate the internal condition. In-service containment structures generally do not allow for the destructive measures necessary to validate the accuracy of these inspection techniques. This creates a need for comparative testing of the various nondestructive evaluation (NDE) measurement techniques on concrete specimens with known material properties, voids, internal microstructure flaws, and reinforcement locations.

Since it is not feasible to build specimens that are to the scale of in-service structures, compact sample specimens must be built while still replicating the NDE needs of real structures. This includes minimizing artifacts caused by boundary effects. Although significant NDE research has been conducted on relatively thin concrete structures to assess pavement, bridge decks, and other infrastructure applications, construction of large reinforced concrete specimens specifically for NDE comparisons is less common [2].Comparative studies have shown various NDE techniques to be successful in identifying the types of internal characteristics of interest. However, these applications are typically conducted to evaluate thin specimens ( $1 \mathrm{ft}$. in thickness), while NPP containment walls are often much thicker (over $3 \mathrm{ft}$. in thickness). Even though previous results for thinner specimens show promise in the ability to nondestructively evaluate internal characteristics, the results need to be validated for thicker and more heavily reinforced structures. Two major NDE challenges associated with thicker structures remain: 1) low signal-to-noise ratio with greater depths due to heterogeneous materials with a dense and complex arrangement of reinforcements, and 2) effects from boundaries at similar distances to the region of interest.

A preliminary report detailed some of the challenges associated with thick reinforced concrete sections and prioritized conceptual designs of specimens that could be fabricated to represent NPP concrete structures for using in NDE evaluation comparisons [3]. As documented in ORNL/TM-2015/72, a specimen thickness of 3 feet 4 inches $(1.016 \mathrm{~m})$ was selected to be representative of a NPP. This thickness is consistent with NPP containment wall specifications. The height and width of the specimen was constricted to 7 feet to accommodate the use of a maximum 20-ton crane while still mitigating boundary effects. NPP concrete is normally embedded with heavily reinforced cross sections using mild steel \#18 bars (2.257 inch diameter, $4.00 \mathrm{in}^{2}$ cross sectional area) and \#8 bars (1.000 inch diameter, 0.79 in $^{2}$ cross sectional area) at 6 to 12 inch spacing. The type and spacing of the reinforcement has a significant effect on shielding evaluation of defects below the level of reinforcements. While elastic wave-based methods are less sensitive to reinforcement than ground penetrating radar (GPR), characterization of defects within more heavily reinforced structures are more difficult than for less heavily reinforced structures. The constructed specimen contained \#18 rebar at 12 in. spacing in both horizontal and vertical orientation. This provides a realistic reinforcement size that also allows for space between reinforcement for semicontrolled evaluation of the effects of concrete depth on defect characterization. With these factors in mind, the defects were designed to give a mix of realistic and controlled defects for assessment of both the necessary measures needed to overcome the challenges with more heavily reinforced concrete structures, while also ensuring the correct type of features for effective NPP evaluation have been included. Information on each of the twenty embedded defects is documented in [4]. 
A linear ultrasonic array with $50 \mathrm{kHz}$ center frequency was used to take scans of the thick concrete specimen. One side is referred to as the "rough", and the other "smooth", due to physical characteristics of the surface after the concrete pour. For each side, scans were taken both horizontally and vertically. This report examines each of the twenty defects using "standard” or full frequency Synthetic Aperture Frequency Technique (SAFT) and "frequency banded" SAFT. The majority of the panoramic reconstructions appear in Appendix A or B.

While standard SAFT techniques are adequate for many defects with shallow concrete cover, some defects that are located under deep concrete cover are not easily identified using the standard SAFT techniques. For many defects, particularly defects under deep cover, the use of frequency banded SAFT improves the detectability over standard SAFT. Improvements include seeing an intensity corresponding to a defect with our technique that is either not visible at all in regular, full frequency content SAFT, or an improvement in clarity or brightness over the SAFT images rendered using the full frequency content. However, further research is needed to understand in what circumstances (depth, defect type, etc.) which wavelet decomposition node is best. Also, further investigation is needed to determine why some "bright spots” are defects, and others are not, i.e. false positives. 


\section{INTRODUCTION}

Extending reactor life of the existing nuclear reactor fleet to 60 years and beyond will likely increase the susceptibility and severity of known forms of degradation, making materials issues a key concern [5][6]. A multitude of concrete-based structures are typically part of a light water reactor (LWR) plant to provide foundation, support, shielding, and containment functions. Concrete has been used in the construction of nuclear power plants (NPPs) because of three primary properties: its inexpensiveness, structural strength, and ability to shield radiation. Examples of concrete structures important to the safety of LWR plants include the containment building, spent fuel pool, and cooling towers. Use of these structures has made concrete's long-term performance crucial for the safe operation of commercial NPPs and creates a need to nondestructively evaluate the current subsurface concrete condition of aging concrete material in NPP containment structures.

The size and complexity of NPP containment structures and heterogeneity of Portland cement concrete (PCC) make characterization of the degradation extent a difficult task. Unlike most metallic materials, reinforced concrete is a composite with a relatively low density matrix; it is a mixture of Portland cement, fine aggregate or sand, aggregate, water, admixtures, and a high density reinforcement (typically 5 percent in NPP containment structures) made up of steel rebar or tendons. NPPs have been typically built with local cement and aggregate fulfilling the design specifications regarding strength, workability, and durability, but as a consequence each plant's concrete composition is unique and complex. In addition, an NPP's concrete structures are often inaccessible, containing large volumes and massively thick concrete structures exposed to different environments (moisture, temperature) and a diversity of degradation mechanisms (high temperatures, radiation exposure, chemical reactions, and other physical mechanisms) at different plant sites, all of which add to the complexity of determining the integrity/quality of the concrete [7].

Specially designed and fabricated test specimens can provide realistic flaws that are similar to actual flaws in terms of how they interact with a particular nondestructive evaluation (NDE) technique. Artificial test blocks allow the isolation of certain testing problems as well as the variation of certain parameters. Because conditions in the laboratory are controlled, the number of unknown variables can be decreased, making it possible to focus on specific aspects, investigate them in detail, and gain further information on the capabilities and limitations of each method. To minimize artifacts caused by boundary effects, the dimensions of the specimens should not be too compact. Representative large heavily reinforced PCC specimens would allow for comparative testing to evaluate the state-of-the-art in NDE in this area and to identify additional developments necessary to address the challenges potentially found in NPPs. These types of specimens would also be useful for calibration and validation of new technology and processing techniques. 



\section{NEED FOR SUITABLE CONCRETE SPECIMENS}

Comparative testing of the various NDE concrete measurement techniques will require concrete specimens with known material properties, voids, internal microstructure flaws, and reinforcement locations. Ideally commercial NPPs undergoing the decommissioning process would be used for NDE comparison, since there are certain characteristics of NPP structures that are difficult to replicate [7]. They are also exposed to known degradation mechanisms, including different levels of radiation, temperature, and chemical reactions that provide the most realistic concrete aging specimens. Concrete fabricated some 40 to 50 years ago is difficult to reproduce using fabricated test blocks, since old cements were generally coarser than present-day cement. Fine cements set and hydrate quickly, generating a high heat release at an early age that can cause thermal cracking and potentially delayed ettringite formation if not cured correctly, and the original admixture (plasticizer, etc.) may no longer be available [8]-[10]. Exclusive use of commercial NPPs to evaluate the effectiveness of NDE techniques is not feasible for a variety of reasons. Commercial NPPs do not always provide the accessibility to collect data using all potential NDE techniques. Destructive forensic activities necessary to validate discrepancies and limitations in the NDE results are also not typically feasible. Alternative methods such as transporting NPP samples to a laboratory environment could theoretically provide the necessary access and forensic capabilities. However, the lateral dimensions required to mitigate boundary effects for NDE specimens over $3 \mathrm{ft}$ thick often make transportation of specimens impractical.

Few applications other than NPPs require critical concrete structures as thick and heavily reinforced. This limits the existing entities with experience in conducting NDE on thick and reinforced concrete structures. Research reactors such as the High Flux Isotope Reactor (HFIR) located at Oak Ridge National Laboratory (ORNL) possess thick, heavily reinforced concrete structures. However, these structures are not as extensive as those of commercial NPPs. This creates a need to construct specimens for performing NDE technique assessments, research, and training.

The environment of specimen construction should represent the access conditions of an in-service containment structure for evaluation of techniques requiring only one-sided testing. Since it is not feasible to build specimens at the scale of in-service structures, compact sample specimens must be built while still replicating the NDE needs of real structures. This includes minimizing artifacts caused by boundary effects. Although significant NDE research has been conducted on thin PCC structures to assess pavement, bridge deck, and other infrastructure applications, construction of large reinforced concrete specimens specifically for NDE comparisons is less common [5], [6]. Comparative studies have shown various NDE techniques to be successful in identifying the types of internal characteristics of interest, requiring only one-sided access [2], [11]-[Fig. 19]. However, these applications are typically conducted to evaluate thin sections ( $\sim \mathrm{ft}$ thick), while NPP containment walls are often much thicker (over $3 \mathrm{ft}$ thick). Even though previous results for thinner structures show promise in the ability to nondestructively evaluate internal characteristics, the results need to be validated for thicker and more heavily reinforced structures. There are two major NDE challenges associated with the fabrication and evaluation of thicker structures:

- Low signal-to-noise ratio with greater depths due to heterogeneous material such as PCC with a dense and complex arrangement of reinforcements.

- Effects from vertical boundaries at similar distances to the region of interest (ROI). 
A background on elastic wave propagation, which is the basis of the MIRA ultrasonic technique used for initial data collection of the specimen built in this study, allows for a discussion of these challenges. When exposed to a short duration external impact, concrete reacts approximately like an elastic solid medium, where the distortion and subsequent movements in the concrete can be described using three general modes of wave propagation categorized by the coverage and direction of particle motion with respect to propagation direction: p-waves, s-waves, and r-waves.

The compression (also known as longitudinal or primary) waves (p-waves) have particle motion parallel with the direction of wave propagation. The four transverse (also known as shear) waves (s-waves) have particle motion perpendicular to the wave propagation direction. The Rayleigh waves (r-waves) have retrograde elliptical particle motion. The r-waves propagate along the surface, whereas the p- and s-waves propagate throughout the body of the solid in a hyperbolic nature [20]. The reflection of p- and s-waves depends on changes in acoustic impedance from internal characteristics of concrete structures. P- and swaves are useful in evaluating internal characteristics of concrete structures with only one-sided access because changes in subsurface properties such as flaws, inclusions, or layer boundaries cause reflections back to the surface.

If concrete is approximated as an isotropic and elastic medium, the relationship between elastic parameters (Modulus, Poisson's ratio), density, and wave velocity in concrete has the form shown in Eqs. (1) through (3) [20]:

$$
\begin{aligned}
& C_{P}=\sqrt{\frac{E(1-\mu)}{(1-\mu)(1-2 \mu) \rho}}, \\
& C_{S}=\sqrt{\frac{E}{2(1+\mu) \rho}}, \\
& C_{R}=C_{S} \frac{0.87+1.12 \mu}{1+\mu},
\end{aligned}
$$

where $E$ is Young's modulus of elasticity, $\mu$ is Poisson's ratio, $\rho$ is density, $C_{P}$ is the compression or pressure wave (p-wave) velocity, $C_{S}$ is the transverse or shear wave (s-wave) velocity, and $C_{R}$ is the Rayleigh wave (r-wave) velocity. Assuming the shear wave response is being evaluated and a typical value for Poisson's ratio in concrete, $\mu=0.2$, the velocity of the other wave types has the following relationship with respect to shear waves:

$$
C_{S}=0.61 C_{P}=1.09 C_{R}
$$

Table 1gives additional information, including the particle motion, relative wave speeds, and energy content of the various wave types [19]. 
Table 1. Wave type information

\begin{tabular}{|c|l|l|c|c|}
\hline Wave type & \multicolumn{1}{|c|}{ Particle motion } & $\begin{array}{c}\text { Propagation } \\
\text { medium }\end{array}$ & $\begin{array}{c}\text { Relative wave } \\
\text { speed, } \boldsymbol{\mu = 0 . 2}\end{array}$ & $\begin{array}{c}\text { Energy content } \\
\text { (\%) }\end{array}$ \\
\hline p-wave & $\begin{array}{l}\text { Parallel to propagation } \\
\text { direction }\end{array}$ & $\begin{array}{l}\text { Solid, liquid, or } \\
\text { gas body wave }\end{array}$ & 0.61 & 7 \\
\hline s-wave & $\begin{array}{l}\text { Perpendicular to } \\
\text { propagation direction }\end{array}$ & Solid body wave & 1 & 26 \\
\hline r-wave & Retrograde elliptical & Surface wave & 1.09 & 67 \\
\hline
\end{tabular}

A few observations can be made from these relationships with regard to use of elastic waves for evaluation of thick reinforced concrete structures. Since acoustic impedance is positively correlated to the stiffness of the material, elastic waves are extremely proficient at characterizing interfaces such as cracks, voids, or delamination where the change in acoustic impedance from concrete to air is extremely high. However, since PCC is composed of air voids and aggregates, elastic waves can also experience significant attenuation that limits the penetration depth. For example, since the p-wave has the lowest amount of energy from a point source impact, it may not achieve the necessary penetration depth required to characterize the thick concrete specimen due to the low energy content. However, the ability to propagate in all types of media may provide air-coupled possibilities [15]. S-waves have significantly higher energy content, allowing for greater penetration depth in heterogeneous media such as concrete. However, they require a solid material for propagation, creating a need for ground coupling. Moreover, because they are similar in velocity to r-waves, boundary effect interference may be a problem.

The Elastodynamic Finite Integration Technique (EFIT), developed at the University of Kassel, is an effective tool for investigating both the penetration depth and the boundary effect challenges in evaluating thick reinforced concrete structures [21]. The tool has been used to compare elastic wave propagation in a $2 \mathrm{D}$ concrete model assuming $0 \%$ and $1 \%$ air porosity from a $200 \mathrm{kHz}$ center frequency point source. It was reported that the reflections from simulated inclusions and back wall reflections were less clear with porosity and that the signal-to-noise ratio decreased with depth. While evaluation based on lower frequency content may resolve this difficulty, the general trend of increased attenuation and a decreased signal-to-noise ratio with depth holds true. Therefore, the same internal defects that can be identified in thin concrete structures may require improved filtering or processing techniques for identification at greater depth due to the decreased signal-to-noise ratio. This is especially true for heavily reinforced concrete structures where scattering and reflection of the wavefront occurs at the boundaries between concrete and steel [22]. This decreasing signal-to-noise ratio with depth is a significant challenge that needs to be addressed for effective nondestructive characterization of aging concrete material in NPP containment structures.

Unlike the loss of signal-to-noise ratio with greater penetration depth problem, which needs to be addressed solely by the NDE technique, the boundary effect problem is less of an issue for in-service inspection of commercial NPP containment structures, where lateral boundaries are less prevalent. However, boundary effects are more critical for thicker concrete structures with regards to specimen design. For many NDE techniques, the first reflected wave received is assumed to be from internal changes in acoustic impedance, or the rear surface, assuming the structure is infinitely expanded in the lateral direction. This assumption is generally valid for evaluation of continuous structures such as NPP containment walls and for internal interrogation of specimens of thin structures such as bridge decks or pavements. However, to use this assumption and properly represent NPP containment structures, thicker concrete structure specimens require higher restrictions for allowable vertical boundaries. 
If a vertical boundary is located sufficiently close to the sensor position in relation to the depth of interrogation interest, reflections of surface and body waves are present within the same time window [23]. In these cases, the boundary effects must either be eliminated through specimen and/or equipment design, or be taken into account in time-domain signal and spectrum analysis to mitigate systematic errors.

Since the ability of each NDE technique to account for boundary effects is not critical for in-service inspection, it is preferable to design the specimen to mitigate boundary effects, which are also representative of the lack of vertical boundaries for NPP containment structures. The exact lateral dimensions required of the specimen for this assumption to be valid are directly related to the ROI depth (often the thickness of the specimen) and the NDE technique used. For example, an NDE technique based on point-source elastic wave propagation used to evaluate a $3 \mathrm{ft}$ long, $3 \mathrm{ft}$ tall, $4 \mathrm{ft}$ thick specimen of a containment wall may have difficulty detecting a defect at $3 \mathrm{ft}$ depth due to boundary effects. However, the same technique may be able to detect the same defect when testing an in-service containment wall where the boundary effects do not affect the measurements.

Since specimens should be designed to test the ability of the technique to mitigate boundary effects, the most extreme case, evaluation of s-waves from a point source, should be taken into account for specimen design considerations. This is considered the most extreme case since a point source creates elastic waves with a full 180 degree divergence, and the s-wave velocity is very similar to the surface r-wave velocity, which also contains the highest energy content, as noted in Table 1. In this case, the distance from the source location to the rear surface must be the minimum dimension. This type of challenge was investigated using 3D EFIT simulation of a point-source elastic wave field in a $2 \times 1.5 \times 0.5 \mathrm{~m}^{3}$ steelreinforced concrete model with polystyrene inclusions [20]. Considering the energy content of r-waves shown in Table 1, it follows that the circular r-wave reflected by the lateral boundaries in the simulation lead to geometrical effects in time-domain signal and spectrum analysis. These boundary effects can cause systematic errors in internal flaw detection or thickness determination. This need to mitigate boundary effects creates transportation challenges for the use of concrete specimens removed from operating reactors to accommodate an increased lateral-to-depth dimension ratio that allows for representative testing.

Fortunately, concrete specimens fabricated under laboratory conditions to control various properties can be used as substitutes for specimens obtained from nuclear structures. Fabricated test blocks allow the isolation, as well as the variation, of certain test parameters. Under controlled laboratory conditions, the number of variables can be decreased, making it possible to investigate specific defects in detail as well as gain further information on the capabilities and limitations of the techniques. The laboratory environment also allows for forensic investigation of the specimen in locations where there are suspected discrepancies in as-built characteristics compared to as-designed features, although these activities should be limited so that the specimen is available for future calibration/verification of other methods after the initial comparative testing. The reinforced PCC specimen was constructed as part of this study to address the limitations discussed in this section. 


\section{TYPICAL PARAMETERS FOR CONCRETE USED IN NPPS}

\subsection{TYPICAL CONTAINMENT WALL DESIGN}

The concrete structures in NPP are thick in cross section and heavily reinforced with steel. Fig. 1 illustrates one type of NPP concrete and steel reinforced containment structure, which has the following specifications [7]:

- Wall thickness: 3 to $4 \mathrm{ft}$

- Dome thickness: $3 \mathrm{ft}$

- Floor thickness: $2 \mathrm{ft}$ if there are rock anchors; if not, $10 \mathrm{ft}$

- Inside diameter: $150 \mathrm{ft}$

- Liner thickness: $1 / 4$ to 3/8 in.

- Height: 150 to $200 \mathrm{ft}$

- Volume: $2.5 \times 10^{6}$ cubic $\mathrm{ft}$

- Liner material: carbon steel

- Containment shape: vertical right cylinder with hemispherical or shallow dome

- Concrete cover over bottom liner: 2 to $3 \mathrm{ft}$

- Reinforcing material: mild steel

- \#18 bars (2.257 in. diameter, 4.00 in. $^{2}$ cross-sectional area)

- $\quad$ \#8 bars (1.000 in. diameter, 0.79 in. $^{2}$ cross-sectional area)

In this typical containment structure, the upper reinforced concrete containment is typically $3.5 \mathrm{ft}$ thick with two layers of \#18 steel reinforcing bars on 12 in. centers vertically and horizontally on each face at a distance of $6 \mathrm{in}$. from each face of a containment structure ranging from 150 to $200 \mathrm{ft}$ tall with a diameter of approximately $150 \mathrm{ft}$. The lower cylindrical walls of the containment are even larger in order to carry the entire dead load, including the upper containment, to the base slab. The walls are at least $4 \mathrm{ft}$ thick with \#18 vertical bars at 12 in. spacing for each face and \#18 horizontal bars on both sides of the vertical reinforcing; the ties are made of \#8 bars. 

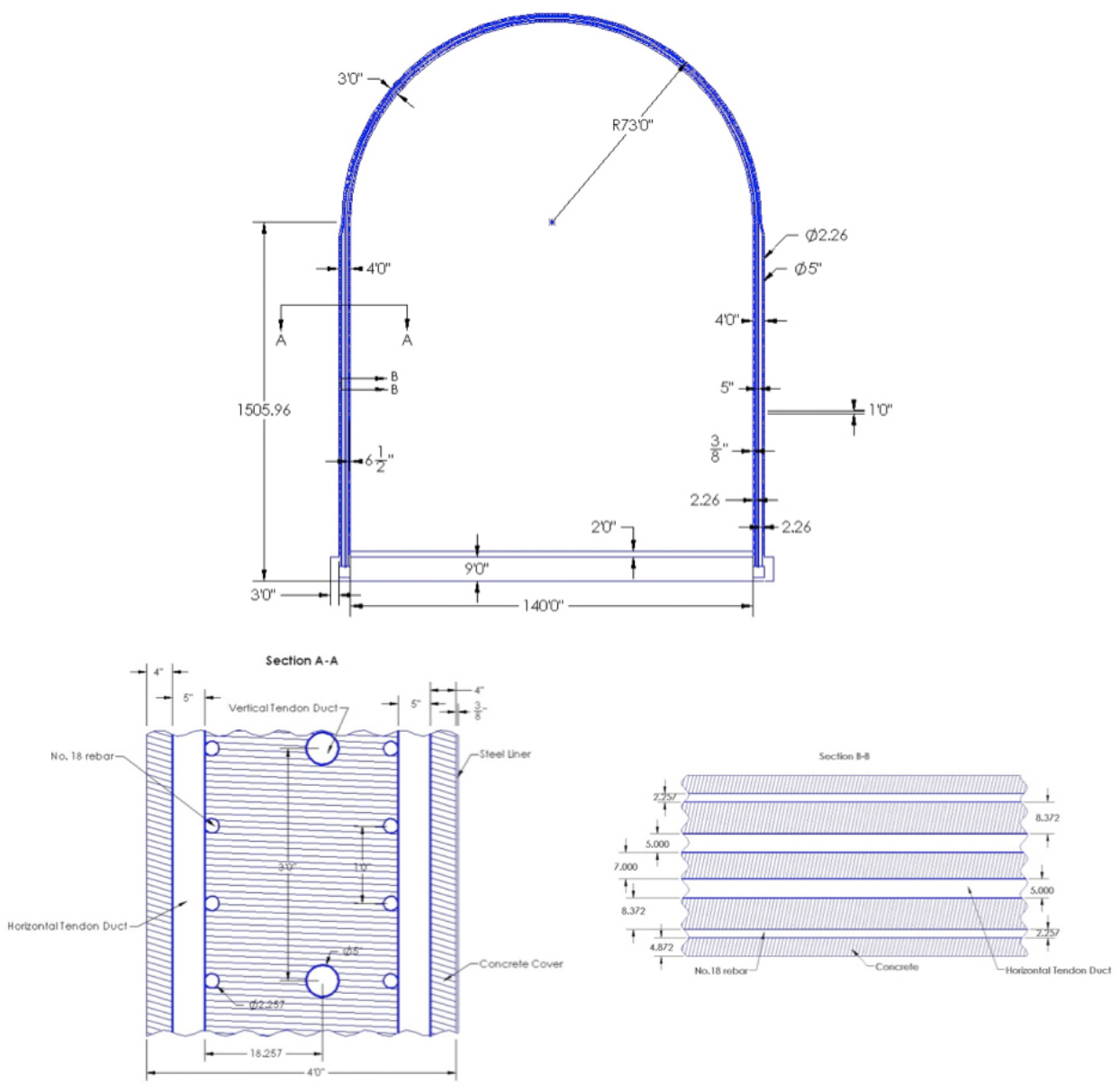

Fig. 1. Example NPP containment structure [4].

\subsection{PREVIOUS CONCRETE FAILURES IN NPP CONTAINMENT STRUCTURES}

Ideally, from an NDE point of view, the NPP specimen should have realistic and known distress. A few representative sections have experienced known distress. Concrete defects observed in power plant containment structures are tabulated by Braverman et al. [24]. It is observed that degradation in the form of cracking, spalling, and general deterioration was commonly observed, and the technique of identification was typically visual inspection. Delamination in the form of stress-corrosion cracks at the level of reinforcements is also generally a concern for the nuclear containment structures [25]-[28].

Fig. 2 shows in situ the cross section of the Crystal River Nuclear Plant (CR3) containment structure with tendon ducts and reinforcements where a delamination gap was observed in the vertical plane of the horizontal tendons, approximately $10 \mathrm{in}$. from the outer surface, of the CR3 containment wall [29]. One form of subsurface defect (delamination at the level of tendon ducts) that is possible for this type of containment structure can be observed in Fig. 2. 


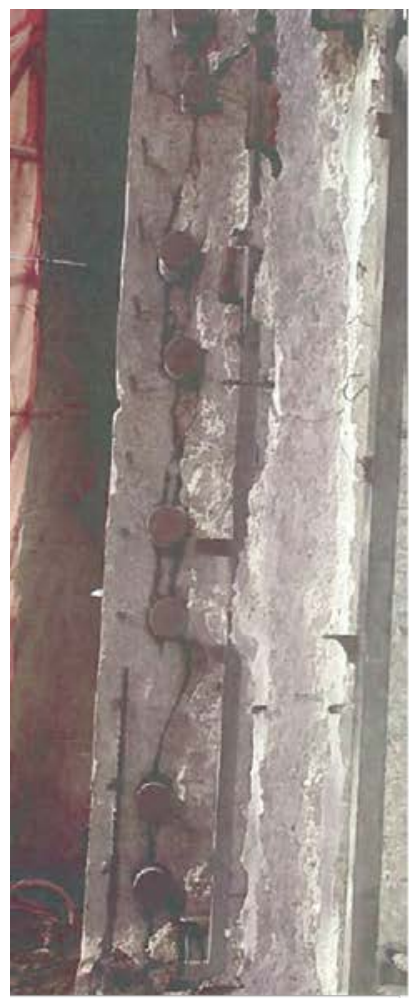

[A]

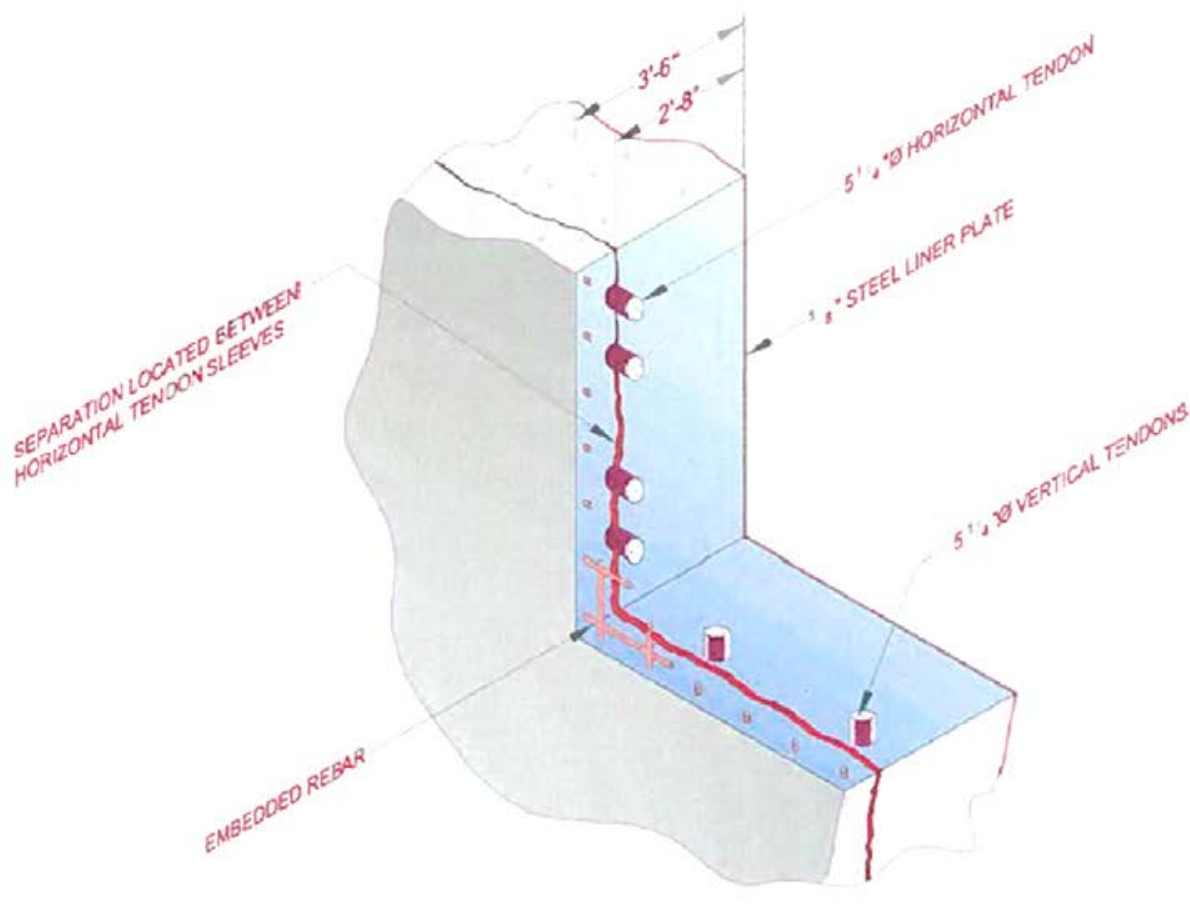

$[B]$

Fig. 2. Delamination crack running from horizontal tendons at approximately 10 in. deep; [A] photograph and [B] sketch[25].

Fig. 3 shows the Davis-Besse Nuclear Power Station [30]. Fig. 4 shows the general layout of the structure with reinforcements. Fig. 5 shows example laminar subsurface cracking at the depth of the reinforcement. This interface was visible due to hydro demolition to create an opening in the shield building, and the crack width is possibly larger than the delaminated condition prior to the cutting. Core bore samples, such as that shown in Fig. 6, show the crack condition unaffected by the hydro demolition process. 


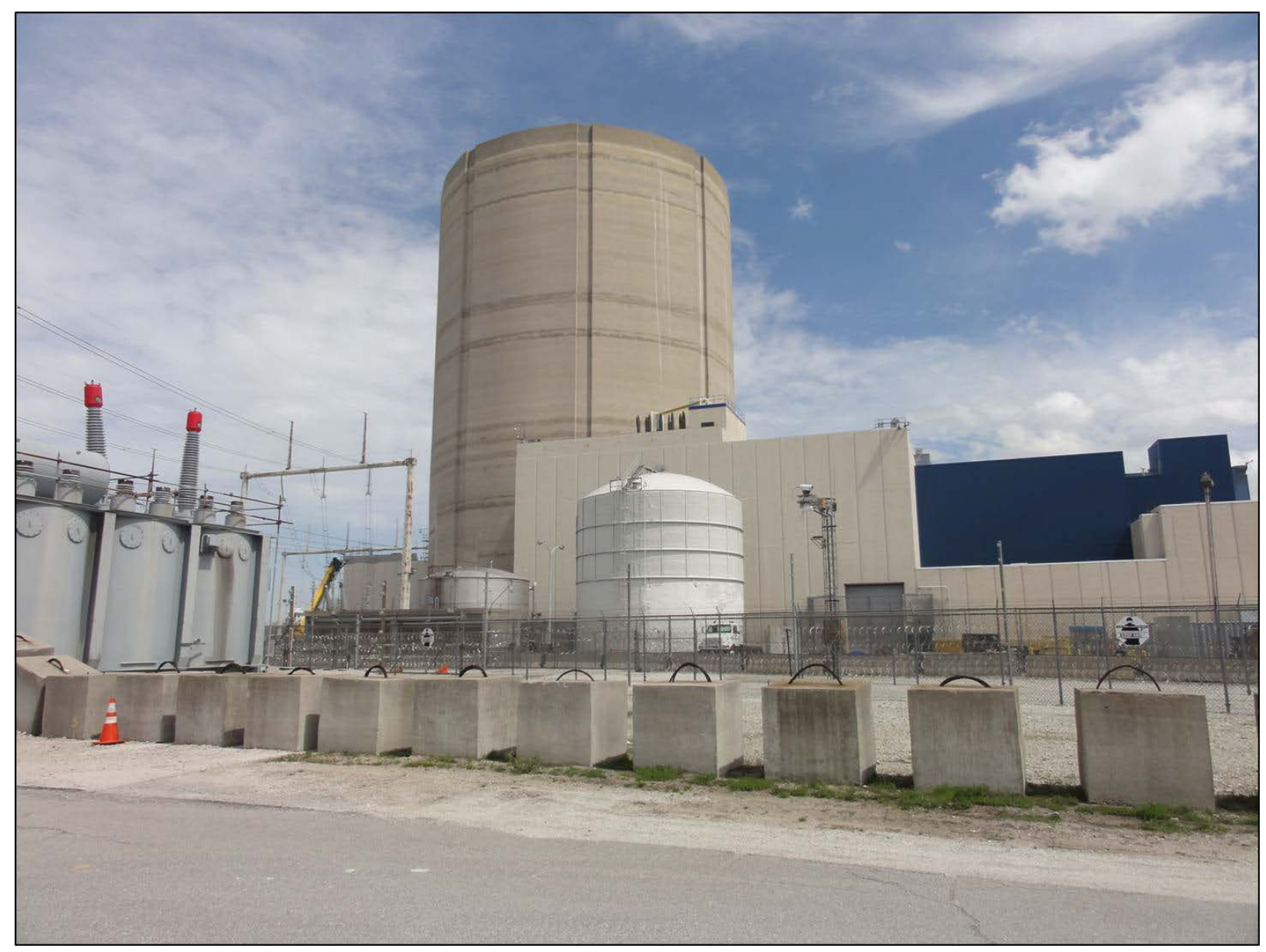

Fig. 3. Davis-Besse Nuclear Power Station [30].

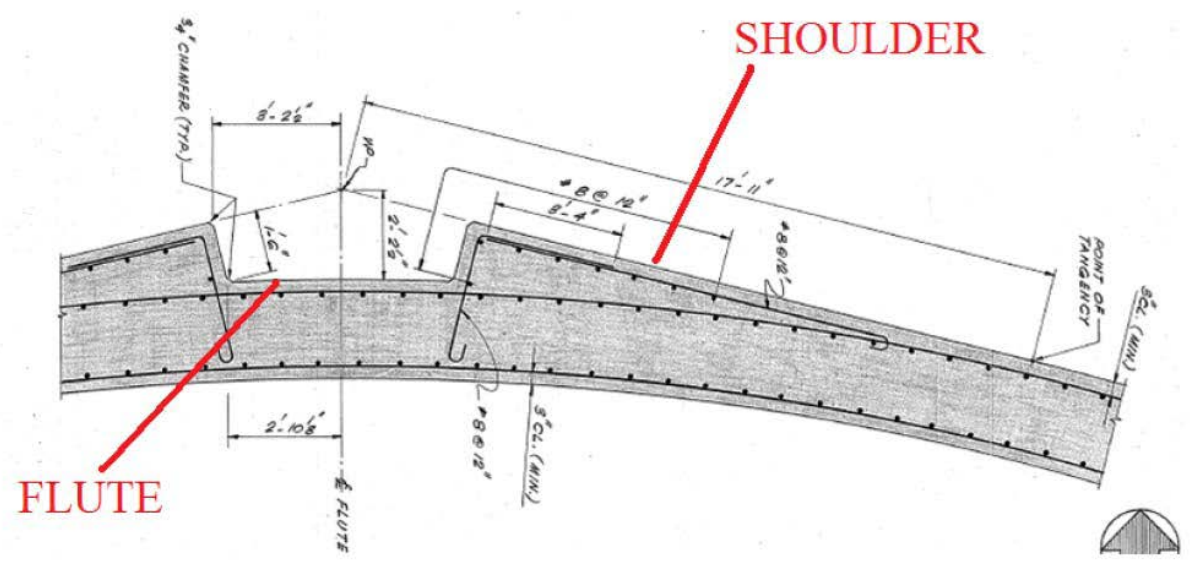

Fig. 4. View of the Davis-Besse Nuclear Power Station layout and reinforcement scheme [30]. 


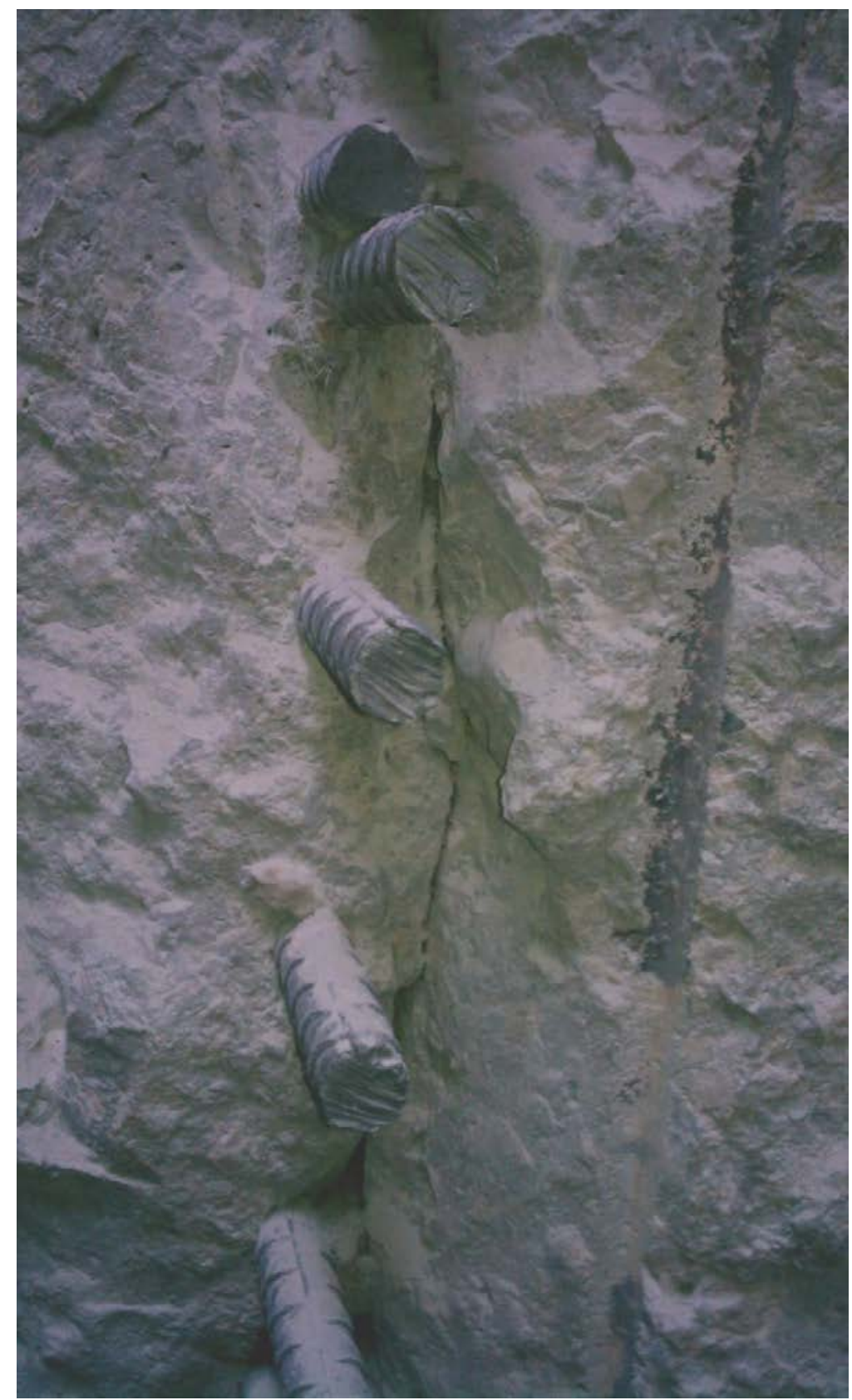

Fig. 5. Laminar cracking at reinforcements from an interface cut out by hydrodemolition [30]. 


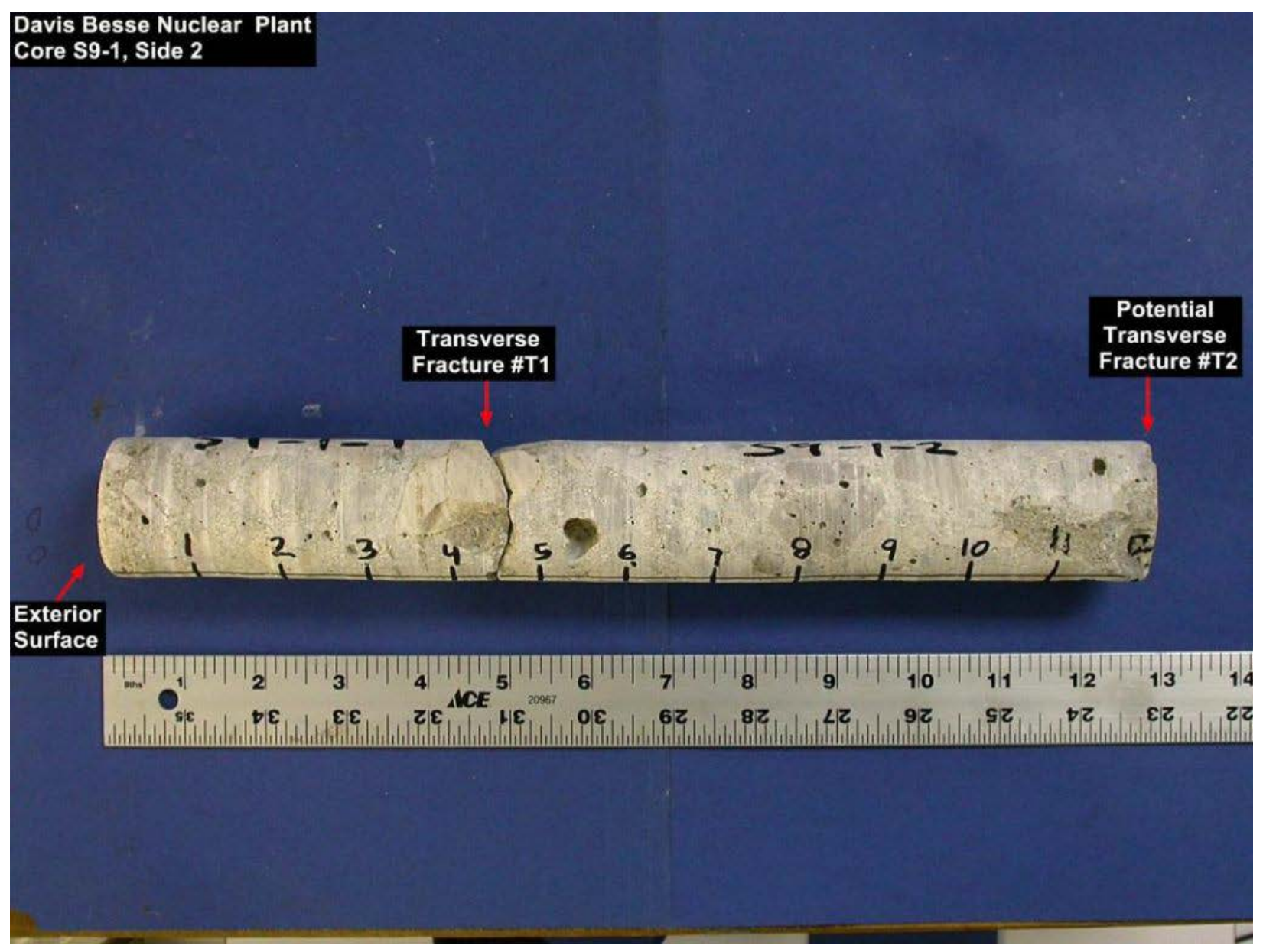

Fig. 6. Laminar cracking at a core location [30].

However, as detailed in ORNL/TM-2013/223 [6] and discussed in the "Need for Suitable Concrete Specimens" section, there are problems associated with use of in-service specimens for validation of NDE methods. The acquisition of such samples can be prohibitive due to the costs of transporting such a large concrete structure, lack of on-site access for research teams to collect data for comparative testing, lack of an ability to verify internal characteristics, and, in some cases, problems with transfer of ownership for a potentially radioactive specimen [5]-[6]. This leads to the lack of readily available samples of thick and heavily reinforced concrete for performing NDE, research, and training. 


\section{DESIGN REQUIREMENTS FOR THE REPRESENTATIVE NPP CONCRETE SAMPLE}

Suitable concrete specimens that are representative of NPP concrete cross sections are needed for NDE and testing of instrumentation and measurement techniques. Adequate test blocks/specimens play a key role, since they can provide defined conditions under which the different NDE concrete measurement methods can be evaluated. Material properties as well as the location of reinforcement, tendon ducts, and test flaws must be well documented. The artificial blocks can provide more defined conditions, since the critical parameters can be controlled during the block fabrication.

Due to the controlled conditions in the laboratory, the number of unknown variables can be decreased, making it possible to focus on specific aspects, investigate them in detail, and gain further information on the capabilities and limitations of the techniques. Comparative testing on the various NDE techniques will require concrete specimens with known material properties, voids, internal microstructure flaws, and reinforcement locations. These specimens can be artificially created under laboratory conditions where the various properties can be controlled. Since no available large concrete specimens are representative of NPP concrete structures are available for forensic verification activities, a detailed design is presented in this section after two major design concerns are reviewed.

\subsection{BOUNDARY EFFECT CONCERNS}

To minimize artifacts caused by boundary effects discussed in the previous sections, the dimensions of the specimen should not be too compact. The minimum dimensions of the test specimen are directly related to the thickness of the specimen. If the ultrasonic wave is modeled as a spherical propagation from the point source (as shown in previous sections), the distance from the source location to the rear surface must be the minimum dimension. However, the exact size of the specimen needed to address boundary effects depends on the NDE technique used. For example, multiple ultrasonic transducers can be utilized to beamform the generated ultrasonic wave to give it more directional propagation properties (Fig. 7) for a four element beamformed wave with no shading. If shading is applied, additional directionality is obtained.

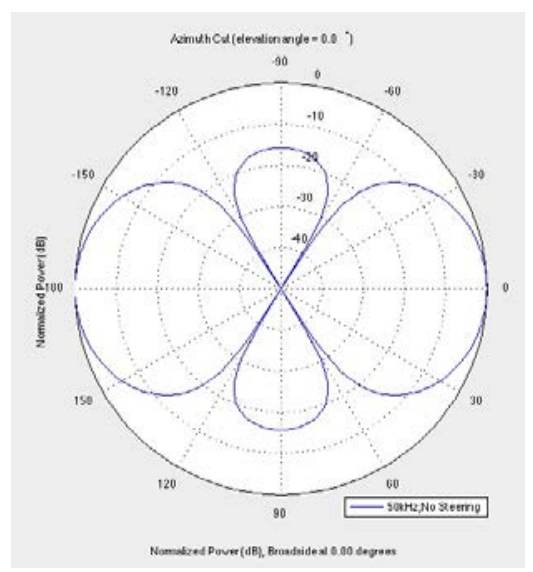

Fig. 7. Beam pattern from a four element ultrasonic array.

This type of focusing can assist in both boundary effects and depth of penetration concerns. To illustrate this, a simple example scan was taken by an ultrasonic linear array system, MIRA (shown in Fig. 8[A]), at the University of Minnesota Structures Laboratory. The scan was taken for an approximately 1-m-thick reinforced concrete specimen with inclusions at approximately $0.15 \mathrm{~m}$ and $0.4 \mathrm{~m}$ depths, respectively. Fig. 8[B]-[D] give cross-sectional imaging reconstructions of the same scan data showing the relative reflectivity throughout the depth using different inputs. 
It can be observed from Fig. 8[B] that the shallower inclusion (bordered by the solid black box) is properly represented by a round black increase in reflectivity, while the deeper inclusion and interface at the reinforced concrete structure thickness depth are underrepresented (bordered by the dashed box). It can be observed from Fig. 8[C] that the shallower inclusion (bordered by the dashed black box) is not properly represented due to a saturation in reflectivity, while the deeper inclusion and interface at the reinforced concrete structure thickness depth (bordered by the solid black box) are properly represented by a round black increase in reflectivity and oblong increase in reflectivity, respectively. By properly accounting for attenuating effects, the volumetric imaging and signal interpretation strategies can be adjusted accordingly. Fig. 8[D] shows how a volumetric reconstruction accounting for these types of effects might be applied to properly represent both shallow and deep characteristics, showing proper reflectivity at both inclusions and the back wall reflection at the thickness of the concrete structure.

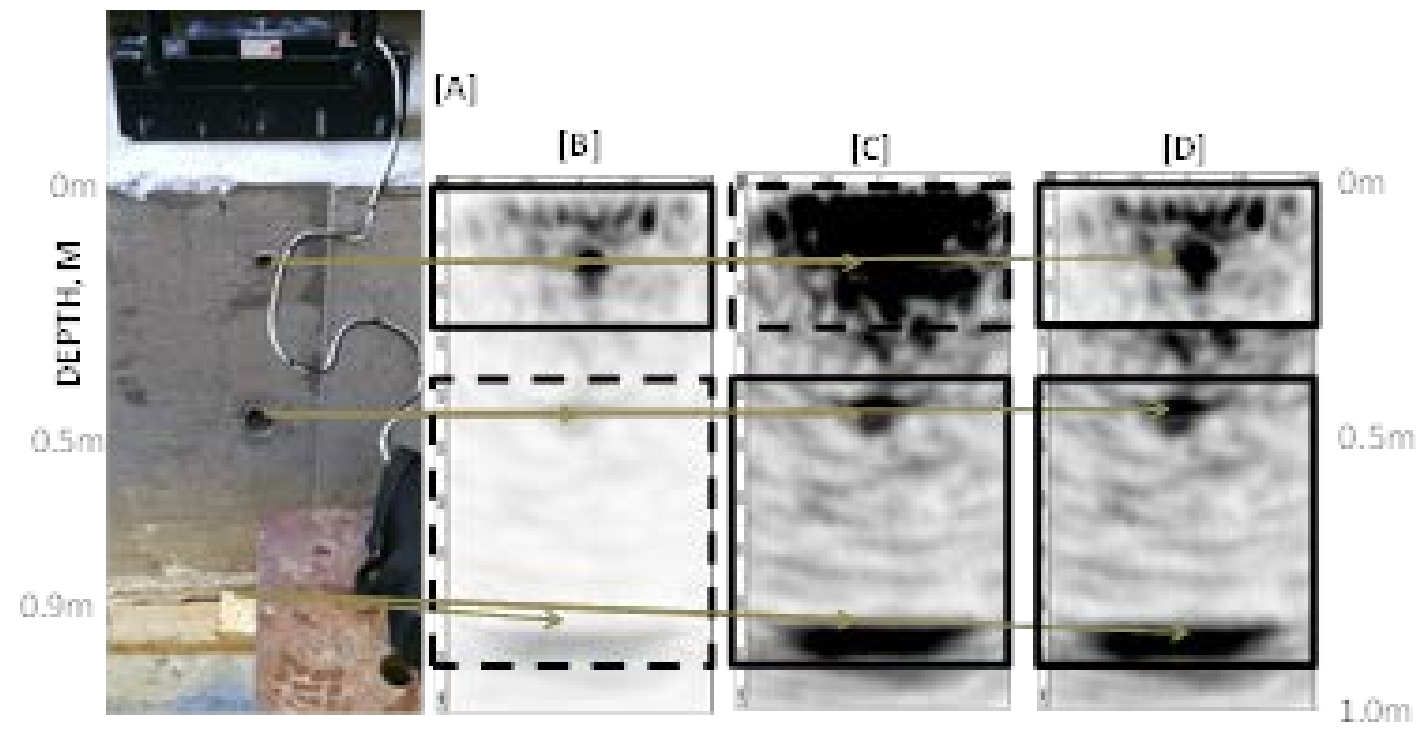

Fig. 8. [A] Picture of MIRA ultrasound scanner with an approximately 1-m-thick reinforced concrete sample with corresponding reconstructions that indicate the $[B]$ shallow features, $[C]$ deep features, and $[D]$ all features.

It is clear that using ultrasonic arrays for NDE with volumetric imaging techniques may reduce the necessary size of the concrete specimen; the lateral distance from the ultrasonic linear array is over five times smaller than the depth to the clear back wall reflection at the thickness of the specimen. However, since there are not a large number of vertical boundaries in aging concrete in NPP structures, an inability to handle boundary effects should not eliminate a technique from potential use for in-service inspection (also described in the Section 2). Therefore, the specimen should also be designed to evaluate techniques that do not have directional capabilities. A major design requirement is that the depth of the ROI in the specimen should be significantly larger than the remaining lateral dimensions. The ability of each technique to handle vertical boundary effects will not affect the validation activities if designed using this constraint, especially for defects near the center of the lateral boundaries of the specimen.

It should also be noted that, while boundary effects from finite lateral structure dimensions are generally not a concern in NPP containment structures, this does not suggest that directional capabilities do not affect the ability of various NDE methods to evaluate thick reinforced structures, even at locations where no lateral vertical boundaries are present. Signal directionality can assist in evaluating defects near or below reinforcement, especially if the signal is focused along a plane of interest parallel and centered between adjacent reinforcement. 
Beyond mitigation of interference of internal structure characteristics, such as reinforcement patterns for evaluation of defects, the focusing can also allow for greater penetration depth by increasing the energy along the focused plane. Therefore, since focusing capabilities are of interest, defects were also placed in locations that approach the lateral boundaries, in addition to the defects with no potential boundary effects near the center of the lateral dimensions of the various specimens. This will leverage the availability of an already cast specimen to increase the number and type of defects that can be tested by methods with directional capabilities and provide information about the extent of the focusing capabilities. Defects near a free vertical boundary provide the most controlled evaluation of the extent of directional focusing capabilities of the various techniques. In this case, care must be taken to separate the portion of the results that provide information on beam focusing capabilities of the various methods versus results that provide information about the ability of the methods to evaluate degradation assuming infinite lateral dimensions. It should be ensured that comparisons made near finite lateral dimensions do not lead to conclusions that negatively direct a realistic assessment of the current capabilities of each method to assess in-service NPP containment walls.

\subsection{LARGE SPECIMEN CONCERNS}

While the design of the large concrete specimen mitigates some of the boundary effect concerns, it creates some additional complexities involved with forming such a large reinforced concrete specimen. Beyond the efforts required to cast and properly consolidate a large concrete specimen, the ability to maneuver and transport the specimen can be restrictive. Often specimens need to be tilted to allow for actuator loading at the correct orientation or, in this case, NDE data collection access. Additionally, at times the specimen needs to be cast in a different location than the testing location to mitigate concrete truck or other access issues. The weight of the specimen is a major factor in this regard, where reinforced concrete is typically $150 \mathrm{lb} / \mathrm{ft}^{3}$ and $162 \mathrm{lb} / \mathrm{ft}^{3}$ assuming a reported NPP 5\% steel by volume ratio [6]. This can be restrictive for the use of a typical structural laboratory crane having 20 ton (40,000 lb) load capacity. There can also be restrictions due to the large specimen dimensions even if the specimen is cast in the location and orientation necessary to conduct the testing. While infrastructure specimens can easily be cut to desired dimensions along the thickness cross section for disposal, this technique is not straightforward for disposal of specimens meeting NPP containment structure thickness requirements. This can also create restrictions beyond the weight limits of crane operation. For example, the smallest dimension of the specimen and crane fixture mechanism is required to be less than the smallest dimension of clearance between the crane and floor along the path to the disposal site, assuming the specimen is instrumented to allow for rotation to the desired specimen orientation.

Fortunately, laboratories such as the University of Minnesota (UMN) Department of Civil Engineering have facilities that specialize in large concrete structure construction and testing. This includes the Theodore V. Galambos Structural Engineering Laboratory (UMN-TGL) and the Multi-Axial Subassemblage Testing (MAST) System laboratory. These facilities frequently test various heavily reinforced concrete structures up to $28.75 \mathrm{ft}(8.7 \mathrm{~m})$ in height and $20 \times 20 \mathrm{ft}(6.1 \times 6.1 \mathrm{~m})$ and provide large-scale concrete specimens for research that can be nondestructively evaluated and compared with forensics at various damage stages. 



\section{CONSTRUCTED SPECIMEN DESIGN}

The specimen constructed in this study is based on the prioritized conceptual designs from the preliminary report as well as a more detailed look at the mobility and safety implications of constructing the specimen at the University of Minnesota.

\subsection{SPECIMEN DIMENSIONS AND REINFORCEMENT}

Concrete structures in NPPs are typically 3 to $4 \mathrm{ft}$ thick along the wall and dome of the containment structure. Therefore, a specimen thickness of $3 \mathrm{ft} 4 \mathrm{in}$. $(1.016 \mathrm{~m})$ was chosen. This thickness is consistent with NPP containment wall specifications. The height and width of the specimen was constricted to $7 \mathrm{ft}$ to accommodate use of a maximum 20 ton crane while still mitigating boundary effects.

NPP concrete is normally embedded with heavily reinforced cross sections using mild steel \#18 bars (2.257 in. diameter, 4.00 in. $^{2}$ cross-sectional area) and \#8 bars (1.000 in. diameter, 0.79 in. $^{2}$ crosssectional area) at 6 to 12 in. spacing. The type and spacing of the reinforcement has a significant effect on the shielding evaluation of defects below the level of reinforcements, especially when using ground penetrating radar (GPR), since electromagnetic waves are extremely sensitive to metal. While elastic wave-based methods are less sensitive to reinforcement than GPR, characterization of defects within more heavily reinforced structures are more difficult than for less heavily reinforced structures. The constructed specimen contained \#18 rebar at 12 in. spacing in both horizontal and vertical orientation. This provides a realistic reinforcement size that also allows for space between reinforcement for semi-controlled evaluation of the effects of concrete depth on defect characterization. This will also allow for differentiation between complexities caused by dense levels of reinforcement versus complexities caused by depth of penetration within concrete, while still observing the effects of the uniquely large diameter reinforcement used in NPP structures. Fig. 9 is a photograph of the \#18 reinforcement size and arrangement at an intermediate stage of the formwork process.

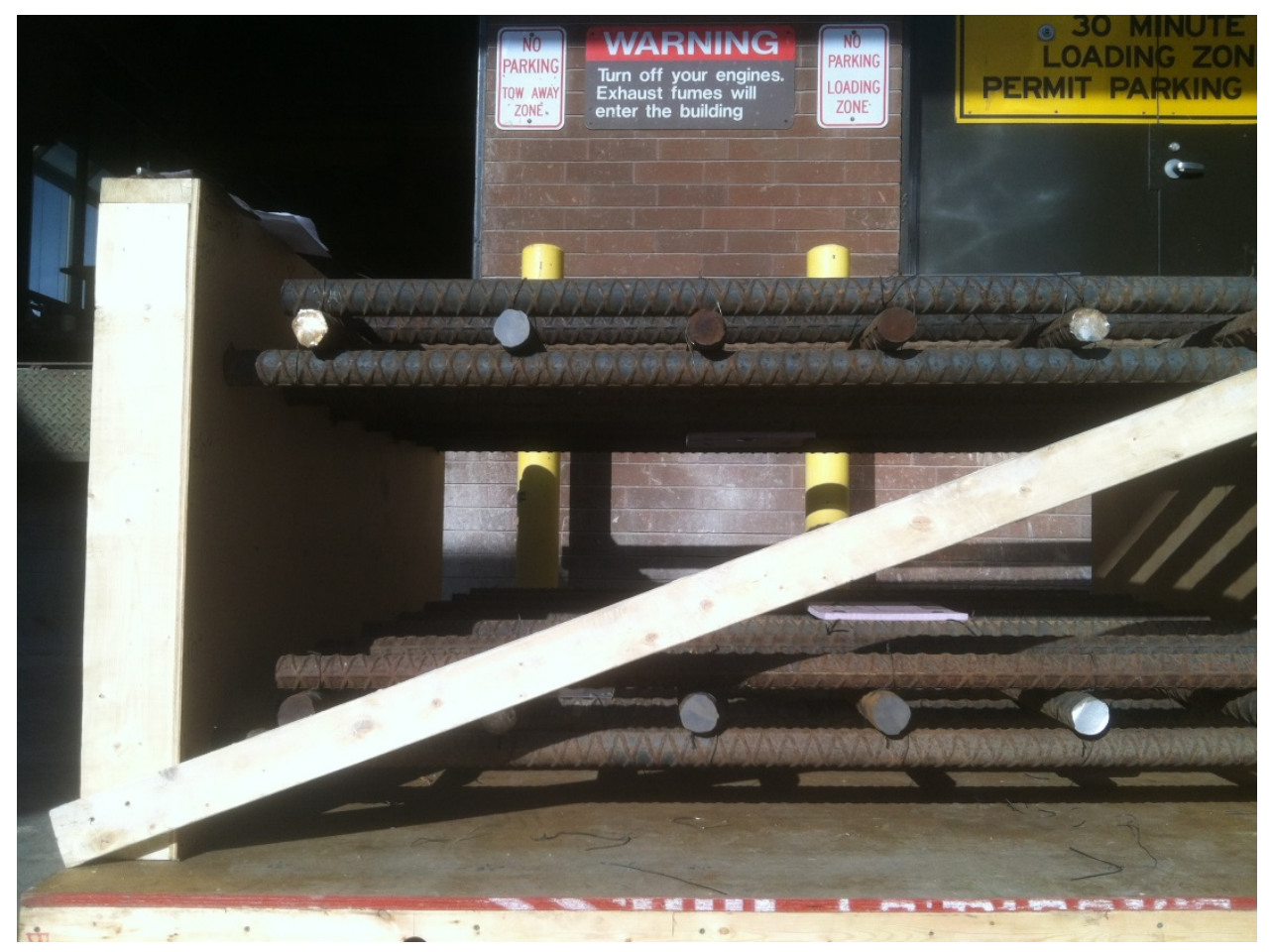

Fig. 9. \#18 reinforcement used to replicate the large diameter mesh used in NPP containment walls. 
The constructed concrete dimensions and reinforcement pattern chosen to represent a typical NPP containment structure in this report are shown in Fig. 10, Fig. 11, and Fig. 12. The cross-sectional view shown in Fig. 10 shows the two vertical rebar, 7 in. from edge to center, and horizontal rebar placed throughout the length of the wall, leaving 4 in. of concrete cover from the shallowest rebar edge to the testing surface. The horizontal rebar is designed to have $5 \mathrm{in}$. of concrete cover from both ends in each direction to allow for proper concrete cover as can be observed from Fig. 11. One rebar on each side of the vertical rebar creates a shadowing effect test for the NDE methods while mimicking the reinforcement pattern of a typical NPP containment wall. Some of the reinforcement extended to the outside of the specimen to allow for fixing the reinforcement and defects to the correct location within the formwork. However, all figures show the reinforcements ending within the evaluated area. The reinforcement pattern is similar for both sides of access, allowing evaluation of the effect of different depths using only one defect by comparing results of testing on both sides of the specimen. While testing and preliminary analysis were conducted on both sides of the specimen, it should be noted that each data set is treated independently and any potential method should not require two-sided access to ensure that the specimen is used to simulate realistic containment wall access conditions.

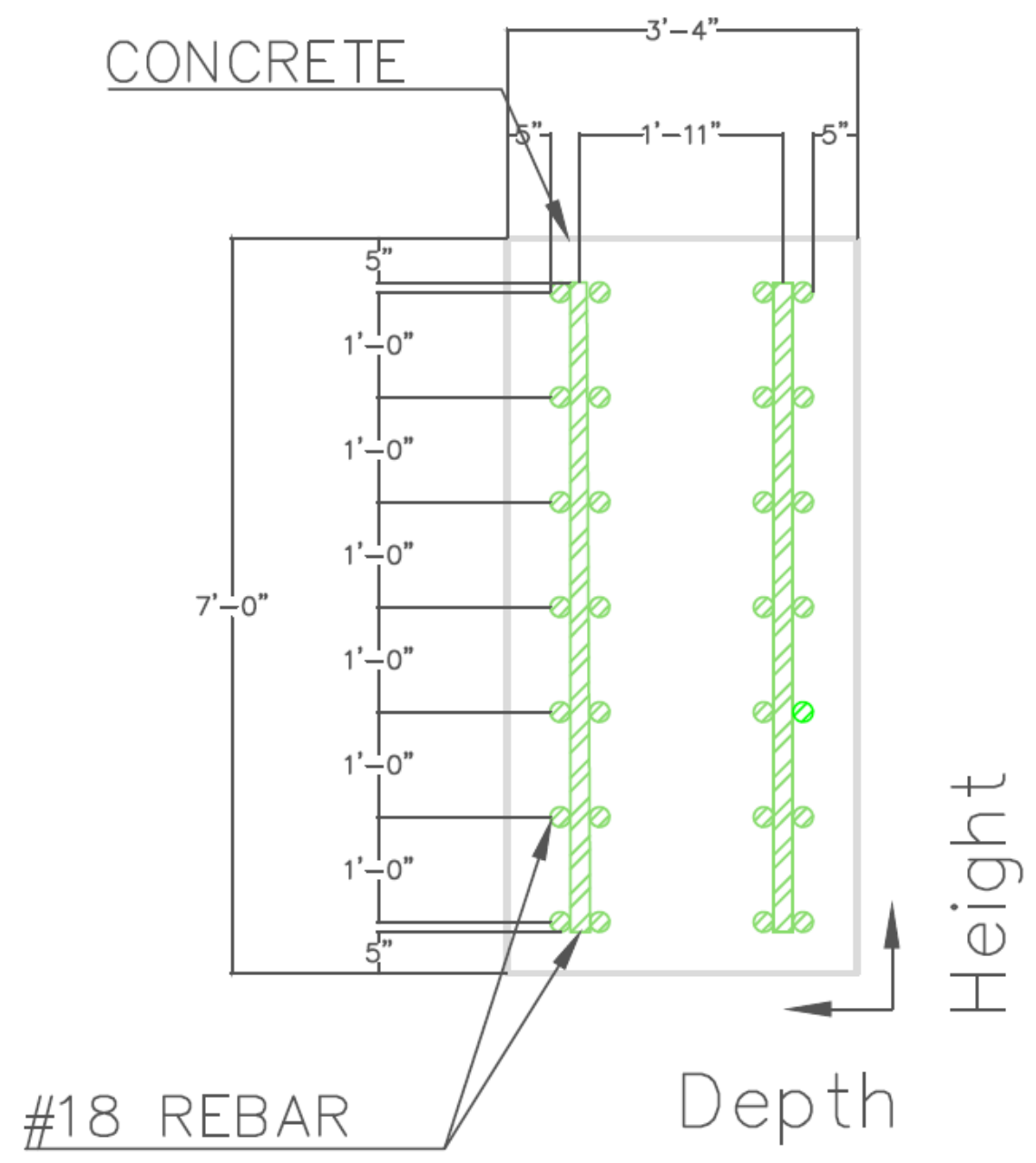

Fig. 10. Concrete dimensions and reinforcement cross section to represent an NPP containment wall. 


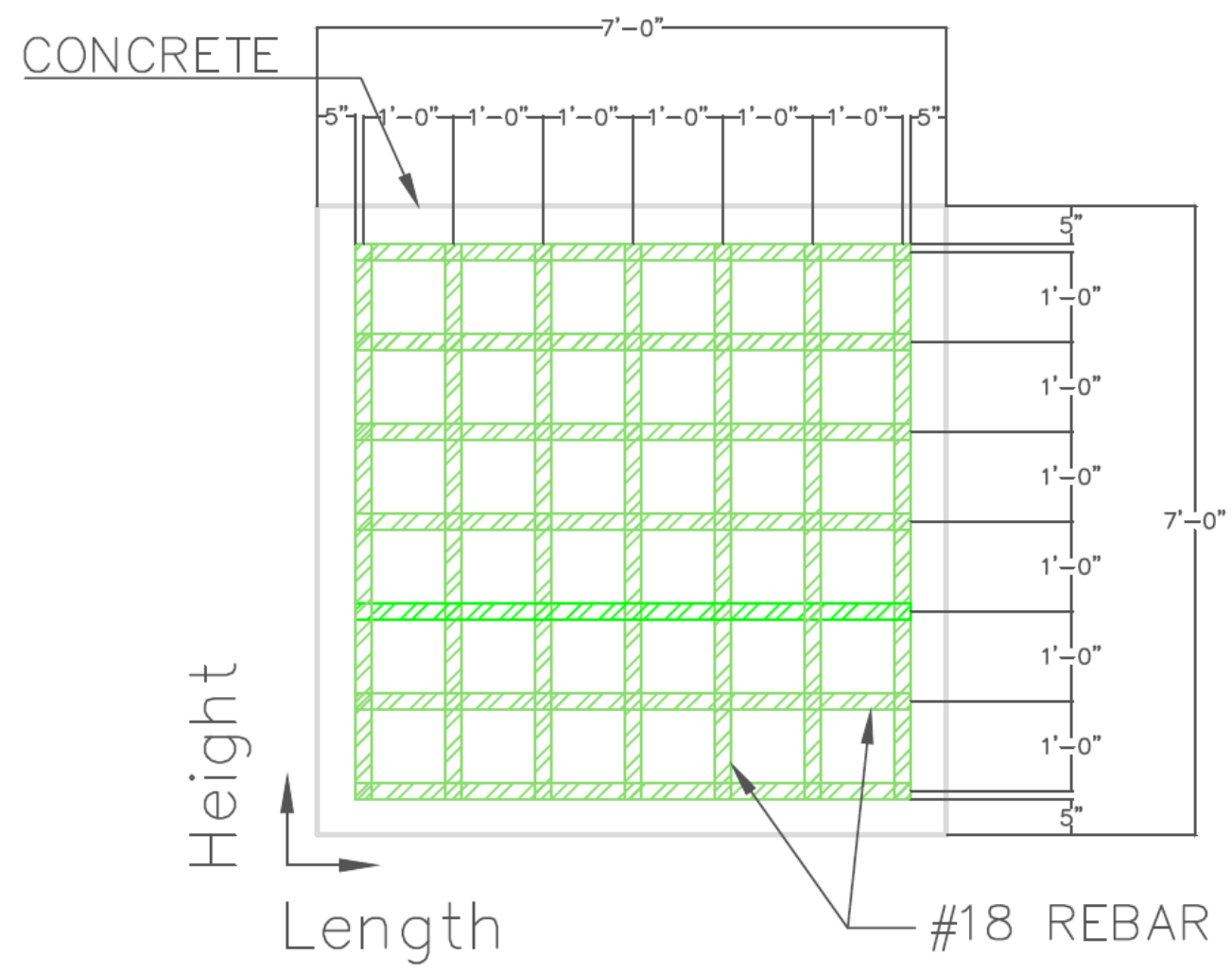

Fig. 11. Length/height view of the proposed concrete dimensions and reinforcement to represent an NPP containment wall.

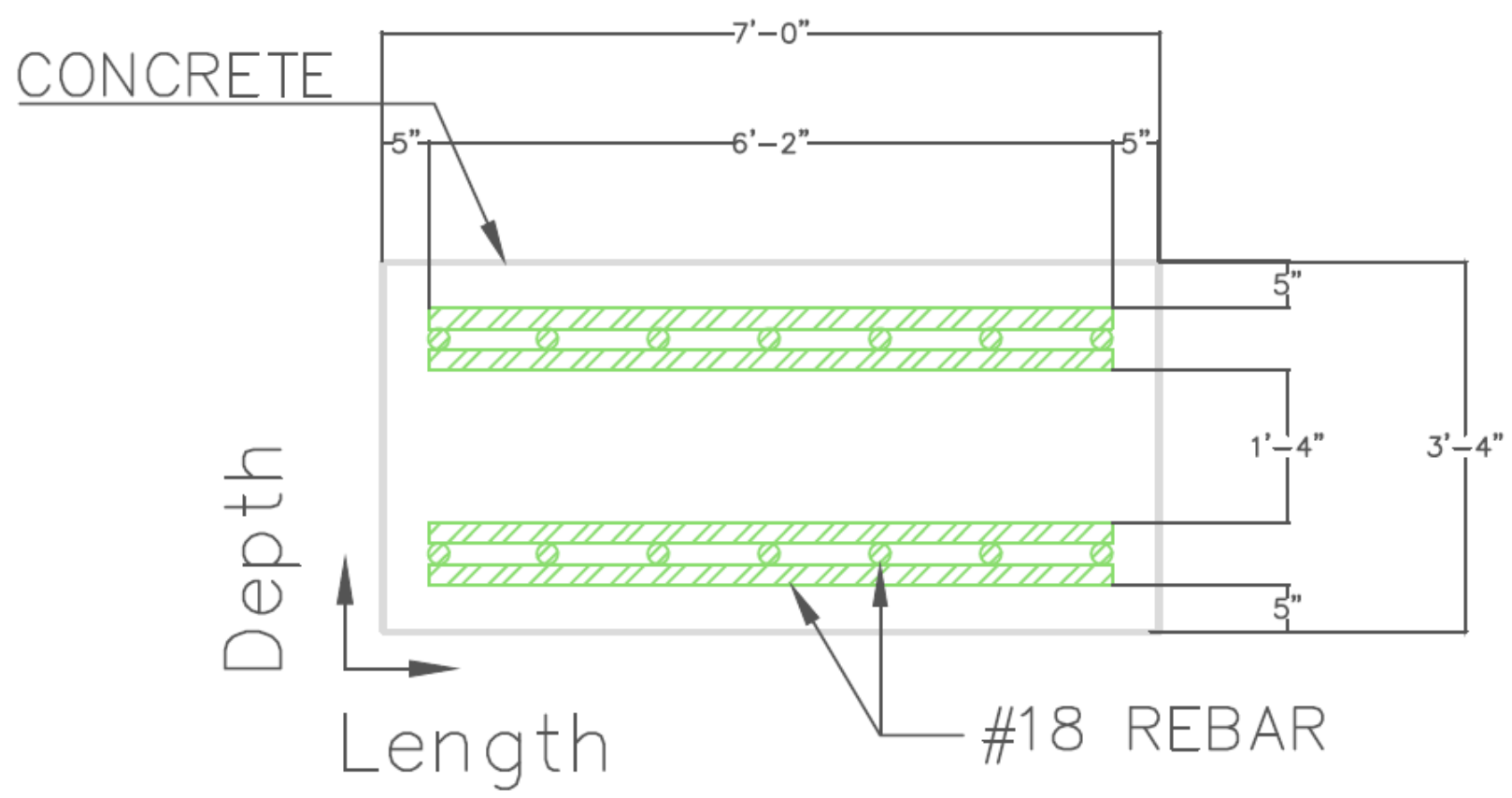

Fig. 12. Length/depth view of the proposed concrete dimensions and reinforcement to represent an NPP containment wall. 


\subsection{SPECIMEN MATERIAL AND SIMULATED DEFECTS}

Concrete material properties of the various NPP concrete structures depend on the materials used during construction of the specimen. The material used at each NPP site is variable depending on the distance from aggregate and cement sources in the area. Additionally, the early age material properties resulting from each mix design are different than the material properties 50 years after the beginning of the curing process. Theoretically, the mix design of example NPP locations could be replicated to determine the effect of material properties on NDE techniques. However, since the mix designs are variable from site to site, materials from 50 years ago are not easily obtained, and early age properties are not representative of older concrete, the proposed mix was designed to make the PCC matrix surrounding the simulated defects and reinforcement pattern as controlled and consistent as possible. By taking into account the large size of the specimen, complex nature of the reinforcement and simulated defects, and goals of limiting the variables other than the known defect and reinforcement locations, a self-consolidating (SCC) performance-based mix was chosen. The placement of the proprietary Cemstone SCC mix provided consistent consolidation and low stress on embedded defects without the need for vibration. This also mitigated concerns of the simulated defects damaging or moving from their desired location during the pour. A photograph of the SCC pouring process is shown in Fig. 13. The spread of the SCC mix allowed for minimal movement during placement.

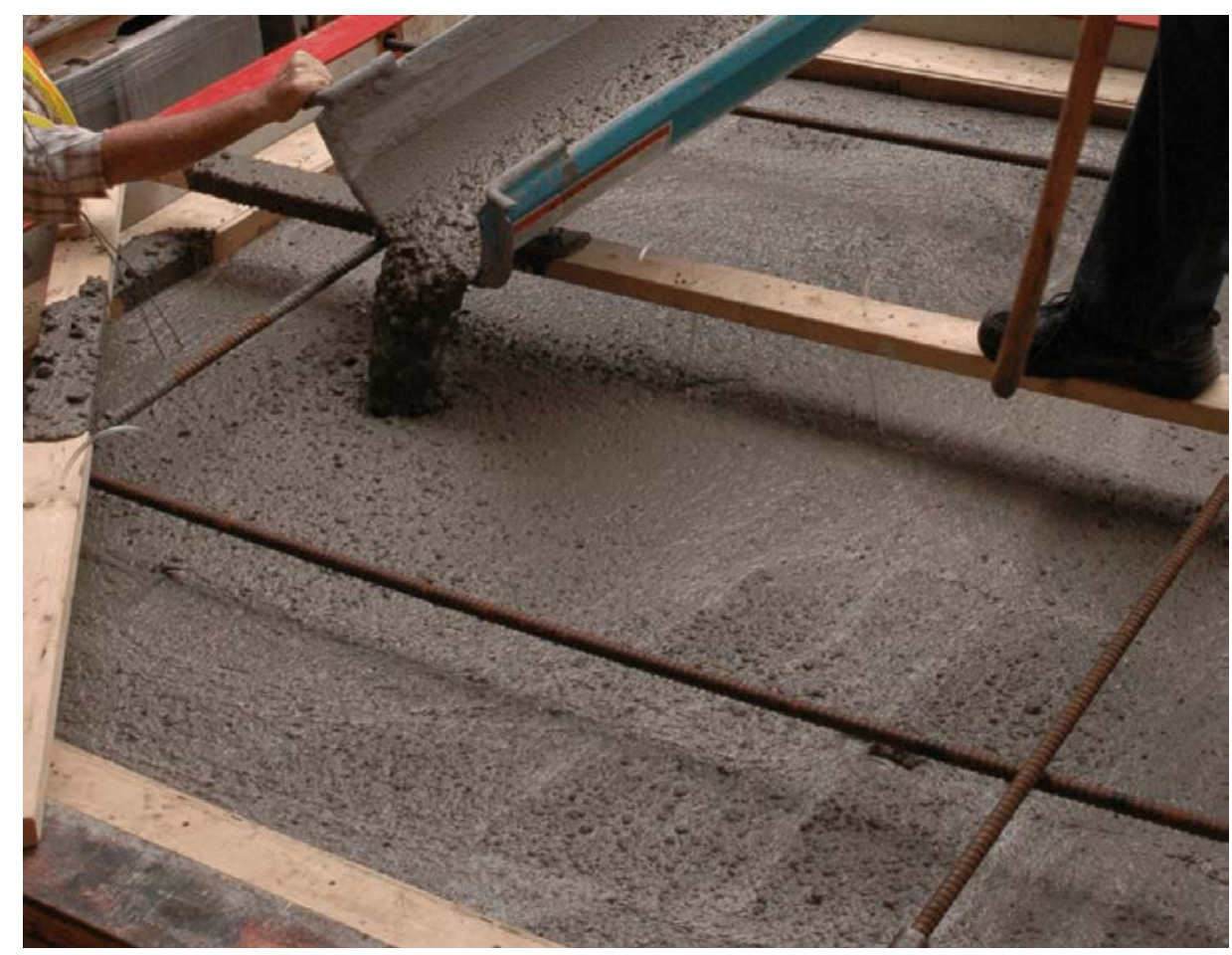

Fig. 13. Use of SCC to allow for consolidation without affecting simulated defects.

The simulated defects were embedded to determine how the current state-of-the-practice NDE techniques will be able to determine various forms of degradation in NPP concrete structures. This is a difficult task since, to date, limited comparisons of state-of-the-art methods have been conducted at the size and reinforcement density of LWR containment structures on controlled specimens, or verified through forensic activities. The constructed specimen is designed to allow for assessment of controlled benchmark defects from previous studies in a more heavily reinforced concrete structure as well as evaluation of realistic defects to ensure that the correct type of features for effective NPP NDE are included. 
The controlled defects embedded in the proposed wall sample include sufficiently challenging inclusions to ensure that limitations of even the most advanced methods can be quantified. At the same time, some of the proposed defects are designed to be identifiable by a majority of the methods. This will ensure that the methods not close to the desired achievement can be eliminated from consideration, while the baseline level of achievement of the methods performing well can be identified.

The realistic defects are designed to represent activities that could have occurred during the construction process and/or cumulative deterioration and degradation of the concrete with time. Some of the agingrelated degradation mechanisms cannot be reproduced due to time constraints, while more realistic construction defect simulation can cause less repeatable results and can be difficult to quantify. However, designing the defects solely to be repeatable and not realistic can lead to the wrong conclusions when evaluating the various NDE techniques. For example, the NDE attributes determined to be desirable based on good performance on the test block may not be useful for evaluation of commercial concrete NPP structures if the defects are not realistic enough.

With these factors in mind, the defects are designed to give a mix of realistic and controlled defects for assessment of both the necessary measures needed to overcome the challenges with more heavily reinforced concrete structures, while also ensuring that the correct type of features for effective NPP evaluation. Many of the defects match the defects referenced above in this report from previous studies of thinner structures. The following list gives the defect type associated with each ID number shown in Table 2.

1. Porous half cylinder (no cover)

2. Porous half cylinder (no cover)

3. Porous half cylinder (no cover)

4. Porous half cylinder (cover)

5. Porous half cylinder (cover and crack)

6. PVC

7. PVC

8. Dissolving Styrofoam (thick)

9. Foam (thick)

10. Styrofoam (thin)

11. Plexiglass

12. Dissolving Styrofoam (medium)

13. Styrofoam (medium)

14. Plexiglass

15. Dissolving Styrofoam (thin)

16. Lumber $(2 \times 4)$

17. Gloves

18. Debond duct tape (one layer)

19. Debond duct tape (multi-layer)

20. Moving rebar 
Table 2. Defect size and location

\begin{tabular}{|c|c|c|c|c|c|c|c|}
\hline \multirow[b]{2}{*}{ ID } & \multicolumn{4}{|c|}{ Center location, mm } & \multicolumn{3}{|c|}{ Specimen dimensions, mm } \\
\hline & Length, $x$ & Height, y & R_Depth, z & S_Depth, z & Length, $x$ & Height, y & Thickness, z \\
\hline D01 & 610 & 1676 & 930 & 86 & 152 & 102 & 51 \\
\hline D02 & 1372 & 610 & 140 & 876 & 102 & 229 & 51 \\
\hline D03 & 1067 & 1524 & 876 & 140 & 127 & 102 & 51 \\
\hline D04 & 1524 & 457 & 86 & 930 & 152 & 102 & 51 \\
\hline D05 & 610 & 1372 & 194 & 822 & 152 & 102 & 51 \\
\hline D06 & 711 & 1067 & 508 & 508 & 2134 & 51 & 51 \\
\hline D07 & 1422 & 1067 & 508 & 508 & 2134 & 51 & 51 \\
\hline D08 & 914 & 914 & 197 & 819 & 203 & 203 & 51 \\
\hline D09 & 1219 & 1219 & 248 & 768 & 203 & 203 & 51 \\
\hline D10 & 1219 & 305 & 908 & 108 & 305 & 305 & 6 \\
\hline D11 & 914 & 1829 & 111 & 905 & 305 & 305 & 2 \\
\hline D12 & 1219 & 914 & 283 & 733 & 305 & 305 & 13 \\
\hline D13 & 914 & 1219 & 733 & 283 & 305 & 305 & 13 \\
\hline D14 & 1600 & 1600 & 848 & 168 & 152 & 152 & 2 \\
\hline D15 & 533 & 533 & 168 & 848 & 152 & 152 & 6 \\
\hline D16 & 1753 & 1829 & 197 & 819 & 330 & 89 & 38 \\
\hline D17 & 1372 & 1524 & 168 & 848 & 102 & 102 & 57 \\
\hline D18 & 1905 & 1067 & 140 & 876 & 127 & 57 & 57 \\
\hline D19 & 1753 & 1067 & 140 & 876 & 127 & 57 & 57 \\
\hline D20 & 1067 & 762 & 140 & 876 & 1981 & 57 & 76 \\
\hline
\end{tabular}

The embedded defects arrangement for the specimen can be observed in Fig. 15, Fig. 16, and Fig. 17 (Fig. 14 is a legend identifying the various defects). Fig. 15 shows the length and height view of the specimen. Fig. 16 shows the height and depth view of the defects, while Fig. 17 shows the length and depth face view of the defect arrangement. It can be observed that the defects are spaced to the extent possible to decrease the chance of adjacent inclusions affecting the measurements while concentrating the defects away from boundaries. It should also be noted that none of the defects are overlapping, although some appear to be, if the spacing between them is in the dimension not shown in the figure. The vertical reinforcement in the lighter green color denotes Defect 20, which is designated for movement during the casting process to simulate increasingly disturbed concrete. 


\begin{tabular}{|c|c|c|}
\hline \multicolumn{3}{|c|}{ DEFECT TABLE } \\
\hline ID NUMBER & DESCRIPTION & LABE \\
\hline D1 & $\begin{array}{l}\text { POROUS HALF CYUNDER } \\
\text { (NO COVER) }\end{array}$ & \\
\hline D2 & $\begin{array}{c}\text { POROUS HALF CYUNDER } \\
\text { (COVER) }\end{array}$ & \\
\hline D3 & $\begin{array}{l}\text { POROUS HALF CYUNDER } \\
\text { (NO COVER) }\end{array}$ & \\
\hline D4 & $\begin{array}{c}\text { POROUS HALF CYUNDER } \\
\text { (COVER) }\end{array}$ & \\
\hline D5 & $\begin{array}{l}\text { POROUS HALF CYUNDER } \\
\text { (COVER \& CRACK) }\end{array}$ & \\
\hline D6 & PVC & \\
\hline D7 & PVC & \\
\hline D8 & $\begin{array}{l}\text { DISSOLVNG STYROFOAM } \\
\text { (THICK) }\end{array}$ & 曲些肃 \\
\hline D9 & STYROFOAM (THICK) & \\
\hline D10 & STYROFOAM (THIN) & \\
\hline D11 & PLEXIGLASS & 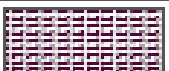 \\
\hline D12 & $\begin{array}{l}\text { DISSOLVNG STYROFOAM } \\
\text { (MEDIUM) }\end{array}$ & DDP \\
\hline D13 & STYROFOAM (MEDIUM) & \\
\hline D14 & PLEXIGLASS & 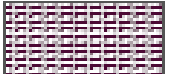 \\
\hline D15 & $\begin{array}{c}\text { DISSOLUNG STYROFOAM } \\
(T H I N)\end{array}$ & 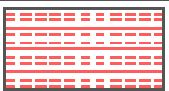 \\
\hline D16 & LUMBER (2X4) & \\
\hline D17 & GLOVES & \\
\hline D18 & $\begin{array}{l}\text { DEBOND DUCT TAPE } \\
\text { (ONE LAYER) }\end{array}$ & \\
\hline D19 & $\begin{array}{l}\text { DEBOND DUCT TAPE } \\
\text { (MULT-LAYER) }\end{array}$ & \\
\hline D20 & MOUNG REBAR & \\
\hline
\end{tabular}

Fig. 14. Legend identifying the various defects. 


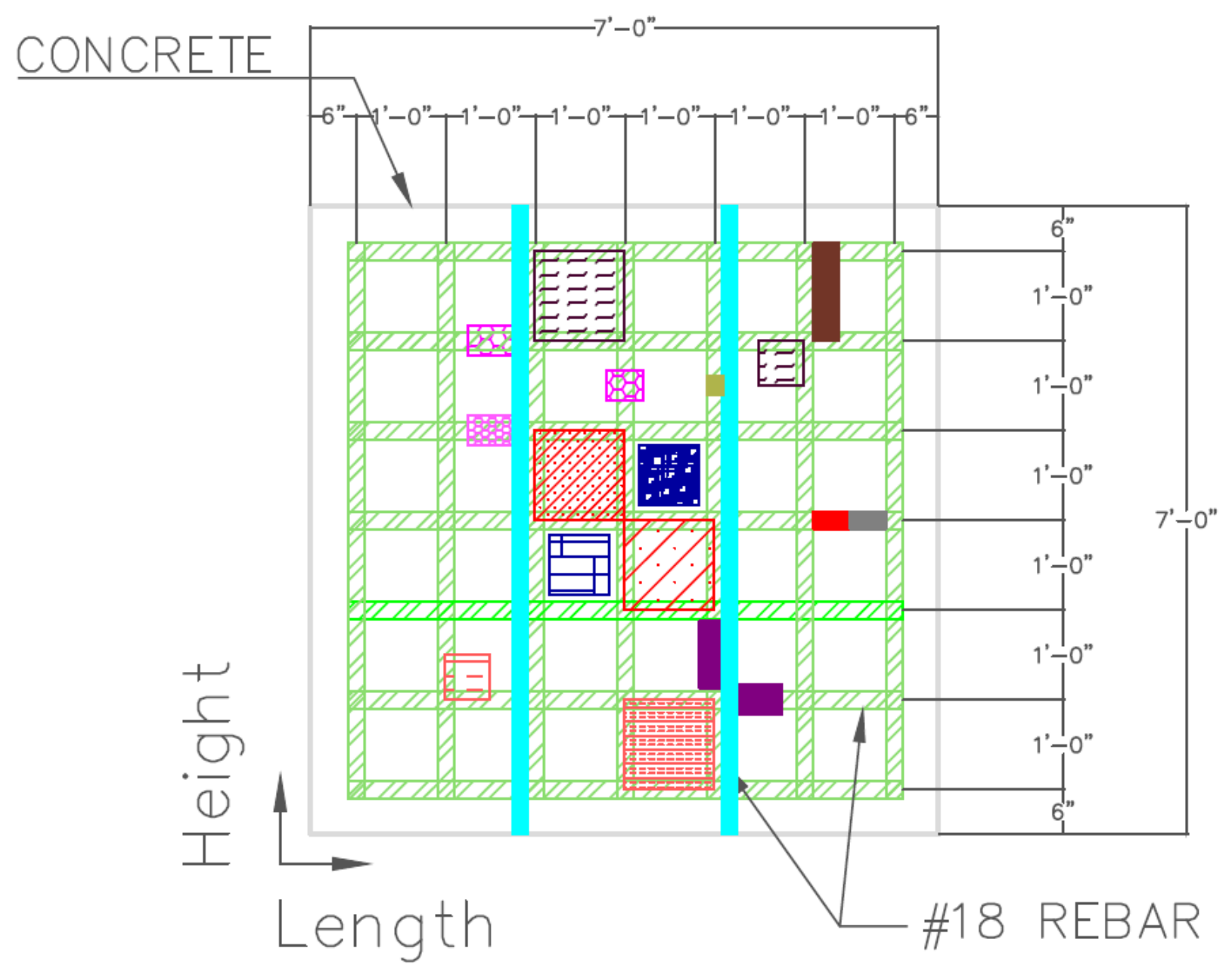

Fig. 15. Length/height view of the defects outlined in A- 6 . 


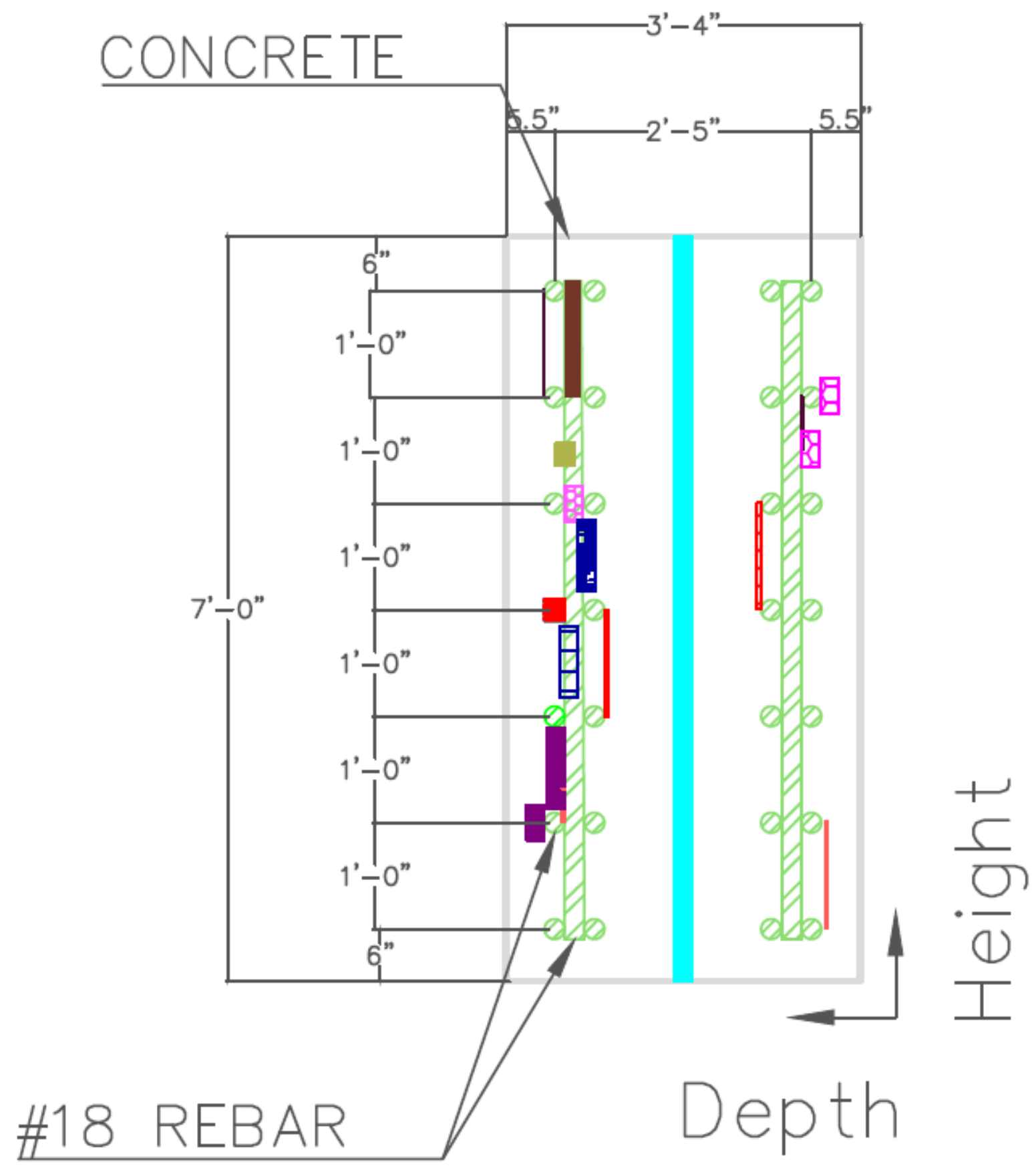

Fig. 16. Height/depth view of the defects outlined in A- 6 . 


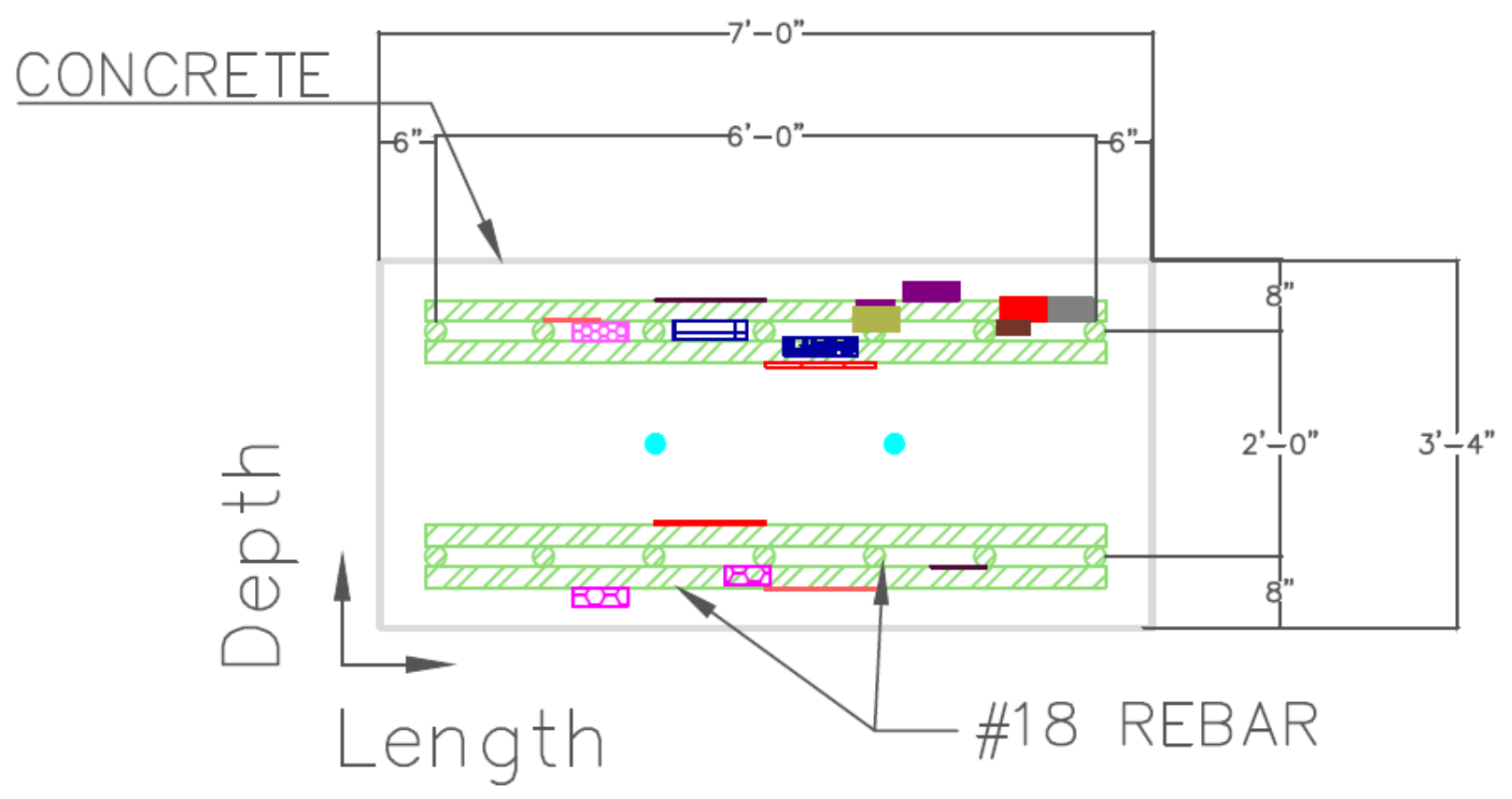

Fig. 17. Length/depth view of the defects outlined in A- 6.

Fig. 18 and Fig. 19 show example controlled (D12) and realistic (D03) defects, respectively. Similar to some of the defects from Yehia et al., D12 is made of Styrofoam with a tube inserted. The tube was placed to allow for pouring acetone into the Styrofoam after the concrete pour. This is designed to leave a controlled voided area in the specimen similar to the large delaminations shown in Fig. 2. Porous concrete such as D03 was embedded to create more realistic smaller voids that can be caused by many different concrete aging mechanisms such as alkali-silica reaction or deterioration. Some of these defects were precovered with concrete paste to keep the SCC from infiltrating the concrete voids during construction.

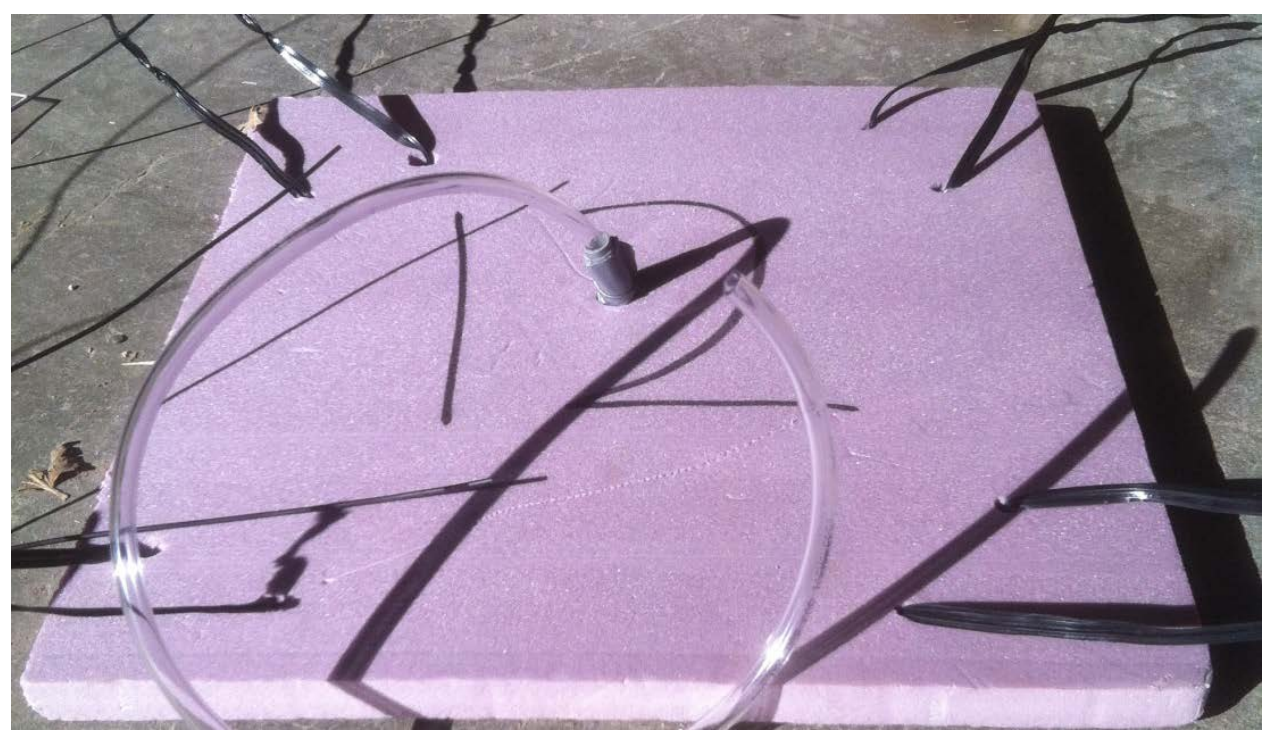

Fig. 18. Example controlled defect simulating a void with dissolving Styrofoam (D12). 


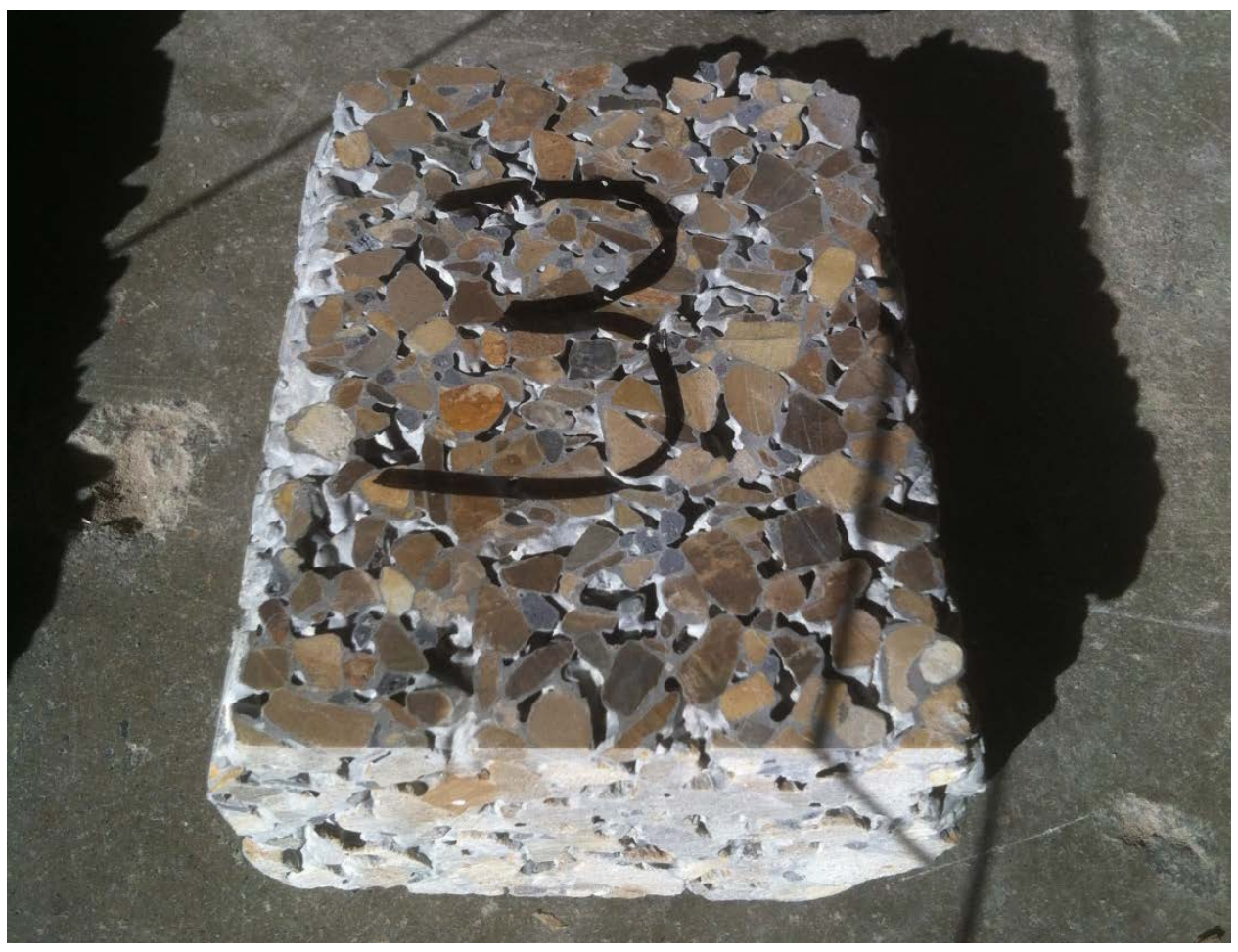

Fig. 19. Example realistic defect simulating concrete damage with embedded porous concrete (D03).

As is seen in some of the analysis figures that follow, the porous concrete with no cover is extremely difficult to identify. While it is impossible to nondestructively determine positively, it is believed that the SCC filled the voids so in essence the voids disappear. 



\section{DATA COLLECTION}

MIRA ultrasound measurements were taken independently on both sides of the specimen thickness. The ultrasonic tomography method presented in this report eliminates some of the issues associated with use of ultrasound to evaluate complex structures like reinforced concrete. One improvement is the use of dry point contact (DPC) transducers, which eliminates the issues associated with using liquid couples and only one signal. The dry point contact is possible due to the small size of the contact zone of each transducer (less than 1-2 mm [0.04-0.08 in.]). For this contact zone size, the transducer produces an oscillating force that can be treated as a point force. In this case, a contact liquid couple is not required for transmission of the shear wave to the tested medium [32].

The transducers have been developed with the capability of transmitting relatively low frequency (operating at $50 \mathrm{kHz}$ in this study) shear waves to penetrate to the necessary depths without relying on liquid coupling. Each transducer consists of two parallel piezoelectric elements surrounded by a liquid composite material. The transducer can be used as a transmitter or receiver. Each transmitting-receiving pair radiates and receives a transformable short duration shear wave impulse. The shear wave impulses are generated by oscillating the piezo-elements at a 180 degree time lag (antiphase). Self-reverberation noise is kept to a low level by damping with a liquid composite material that surrounds the piezoelements [32].

Use of these transducers allows for multiple measurement pairs in each scan, with the version used in this study (MIRA version 1) incorporating 10 channels, each composed of four transmitting and receiving transducers in a linear array. This linear array operates in a multi-static nature, allowing for 45 transmitting and receiving pair time-of-flight measurements in less than three seconds per scan. The spacing between adjacent transducer channels is $40 \mathrm{~mm}$ (1.6 in.). Thus, horizontally spaced measurement pairs in each MIRA scan include nine pairs at $40 \mathrm{~mm}$ (1.6 in.) spacing, eight pairs at $80 \mathrm{~mm}$ (3.1 in.) spacing, seven pairs at $120 \mathrm{~mm}$ (4.7 in.) spacing, six pairs at $160 \mathrm{~mm}$ (6.3 in.) spacing, five pairs at $200 \mathrm{~mm}$ (7.9 in.) spacing, four pairs at $240 \mathrm{~mm}$ (9.4 in.) spacing, three pairs at $280 \mathrm{~mm}$ (11.0 in.) spacing, two pairs at $320 \mathrm{~mm}$ (12.6 in.) spacing, and one pair at $360 \mathrm{~mm}$ (14.2 in.) spacing.

The data obtained from the transducers can be processed using SAFT to produce an image called a SAFT B-scan, which gives a visual representation of the cross section of the scanned area from multiple measurement pairs in each scan. The superposition of multiple transducer pair measurements and calculation of instantaneous amplitude along each reconstruction column allow for a dimensionless relative reflectivity map of changes in acoustic impedance below the scan locations [2]. The use of overlapping measurements along the cross section of each individual SAFT-B scan can allow for an extended reconstruction of the entire width and length of the specimen. To allow for a systematic testing procedure, the specimen was gridded in $102 \mathrm{~mm}$ (4 in.) squares covering the $84 \mathrm{in} . \times 84 \mathrm{in}$. total length and width of the specimen. Since the width of the device is approximately $407 \mathrm{~mm}$ (16 in.), the first and last measurement of each reconstruction was centered $203.2 \mathrm{~mm}$ (8 in.) from the edge, resulting in a total of 18 overlapping measurements within each extended reconstruction covering the entire dimension of the specimen in the direction of the current set of scans. Due to boundary effects near the edge of the specimen, the first and last extended scans were taken centered 6 in. away from the edge perpendicular to the scan orientation, resulting in a total of 19 extended reconstructions per orientation. The extended ultrasound reconstruction of an entire span is referred to herein as a synthetic aperture focusing technique panoramic (SAFT-PAN). Tables 7 and 8 give the data information as well as the orientation and location of each SAFT-PAN. The first and last SAFT-PAN in each orientation was centered $152.4 \mathrm{~mm}$ (6 in.) from the edge. Representations of data collection for both orientations of the device used to generate SAFT-PAN are given in Fig. 20 and Fig. 21. Fig. 20 represents the data collected to create a SAFT-PAN reconstruction spanning the length direction on the smooth surface. 
Fig. 21 represents the data collected to create a SAFT-PAN reconstruction spanning the height direction. Each individual scan covers approximately 16 in. Measurements were taken in 4 in. step sizes within each SAFT-PAN, allowing for use of overlapping measurements to create the full reconstruction. The scans were taken following two rules for consistency in creating and labeling SAFT-PAN. All scans within a panoramic reconstruction moved left to right in the direction of increasing length or height, depending on the current orientation, and subsequent SAFT-PAN move in the direction the device is facing. To fulfill these criteria, the testing procedure included increasing SAFT-PAN moving in opposite directions when testing on the rough and smooth side. Schematics for the testing procedures to create SAFT-PAN for rough and smooth side measurements are shown in Fig. 22 through Fig. 25. Fig. 22 and Fig. 23 show the length orientation SAFT-PAN for the rough and smooth sides, respectively. Fig. 24 and Fig. 25 show the height orientation SAFT-PAN for the rough and smooth sides, respectively. 
Table 3. Smooth side testing locations (December 16, 2014)

\begin{tabular}{|c|c|c|c|c|c|c|}
\hline Band & Orientation & $\begin{array}{l}\text { Starting } \\
\text { position }\end{array}$ & $\begin{array}{r}\text { Ending } \\
\text { position }\end{array}$ & Pan\# & $\begin{array}{c}\text { Height } \\
\text { location }(\mathrm{mm})\end{array}$ & $\begin{array}{c}\text { Length } \\
\text { location (mm) }\end{array}$ \\
\hline 1 & length & 1 & 18 & 1 & 1981 & all \\
\hline 2 & length & 19 & 36 & 2 & 1880 & all \\
\hline 3 & length & 37 & 54 & 3 & 1778 & all \\
\hline 4 & length & 55 & 72 & 4 & 1676 & all \\
\hline 5 & length & 73 & 90 & 5 & 1575 & all \\
\hline 6 & length & 91 & 108 & 6 & 1473 & all \\
\hline 7 & length & 109 & 126 & 7 & 1372 & all \\
\hline 8 & length & 127 & 144 & 8 & 1270 & all \\
\hline 9 & length & 145 & 162 & 9 & 1168 & all \\
\hline 10 & length & 163 & 180 & 10 & 1067 & all \\
\hline 11 & length & 181 & 198 & 11 & 965 & all \\
\hline 12 & length & 199 & 216 & 12 & 864 & all \\
\hline 13 & length & 217 & 234 & 13 & 762 & all \\
\hline 14 & length & 235 & 252 & 14 & 660 & all \\
\hline 15 & length & 253 & 270 & 15 & 559 & all \\
\hline 16 & length & 271 & 288 & 16 & 457 & all \\
\hline 17 & length & 289 & 306 & 17 & 356 & all \\
\hline 18 & length & 307 & 324 & 18 & 254 & all \\
\hline 1 & length & 325 & 342 & 19 & 152 & all \\
\hline 1 & height & 1 & 18 & 1 & all & 152 \\
\hline 2 & height & 19 & 36 & 2 & all & 254 \\
\hline 3 & height & 37 & 54 & 3 & all & 356 \\
\hline 4 & height & 55 & 72 & 4 & all & 457 \\
\hline 5 & height & 73 & 90 & 5 & all & 559 \\
\hline 6 & height & 91 & 108 & 6 & all & 660 \\
\hline 7 & height & 109 & 126 & 7 & all & 762 \\
\hline 8 & height & 127 & 144 & 8 & all & 864 \\
\hline 9 & height & 145 & 162 & 9 & all & 965 \\
\hline 10 & height & 163 & 180 & 10 & all & 1067 \\
\hline 11 & height & 181 & 198 & 11 & all & 1168 \\
\hline 12 & height & 199 & 216 & 12 & all & 1270 \\
\hline 13 & height & 217 & 234 & 13 & all & 1372 \\
\hline 14 & height & 235 & 252 & 14 & all & 1473 \\
\hline 15 & height & 253 & 270 & 15 & all & 1575 \\
\hline 16 & height & 271 & 288 & 16 & all & 1676 \\
\hline 17 & height & 289 & 306 & 17 & all & 1778 \\
\hline 18 & height & 307 & 324 & 18 & all & 1880 \\
\hline 19 & height & 325 & 342 & 19 & all & 1981 \\
\hline
\end{tabular}


Table 4. Rough side testing locations (November 25, 2014)

\begin{tabular}{|c|c|c|c|c|c|c|}
\hline Band & Orientation & $\begin{array}{l}\text { Starting } \\
\text { position }\end{array}$ & $\begin{array}{r}\text { Ending } \\
\text { position }\end{array}$ & Pan\# & $\begin{array}{c}\text { Height } \\
\text { location }(\mathrm{mm})\end{array}$ & $\begin{array}{c}\text { Length } \\
\text { location }(\mathrm{mm})\end{array}$ \\
\hline 1 & length & 1 & 18 & 1 & 152 & all \\
\hline 2 & length & 19 & 36 & 2 & 254 & all \\
\hline 3 & length & 37 & 54 & 3 & 356 & all \\
\hline 4 & length & 55 & 72 & 4 & 457 & all \\
\hline 5 & length & 73 & 90 & 5 & 559 & all \\
\hline 6 & length & 91 & 108 & 6 & 660 & all \\
\hline 7 & length & 109 & 126 & 7 & 762 & all \\
\hline 8 & length & 127 & 144 & 8 & 864 & all \\
\hline 9 & length & 145 & 162 & 9 & 965 & all \\
\hline 10 & length & 163 & 180 & 10 & 1067 & all \\
\hline 11 & length & 181 & 198 & 11 & 1168 & all \\
\hline 12 & length & 199 & 216 & 12 & 1270 & all \\
\hline 13 & length & 217 & 234 & 13 & 1372 & all \\
\hline 14 & length & 235 & 252 & 14 & 1473 & all \\
\hline 15 & length & 253 & 270 & 15 & 1575 & all \\
\hline 16 & length & 271 & 288 & 16 & 1676 & all \\
\hline 17 & length & 289 & 306 & 17 & 1778 & all \\
\hline 18 & length & 307 & 324 & 18 & 1880 & all \\
\hline 19 & length & 325 & 342 & 19 & 1981 & all \\
\hline 1 & height & 1 & 18 & 1 & all & 1981 \\
\hline 2 & height & 19 & 36 & 2 & all & 1880 \\
\hline 1 & height & 37 & 54 & 3 & all & 1778 \\
\hline 2 & height & 55 & 72 & 4 & all & 1676 \\
\hline 3 & height & 73 & 90 & 5 & all & 1575 \\
\hline 4 & height & 91 & 108 & 6 & all & 1473 \\
\hline 5 & height & 109 & 126 & 7 & all & 1372 \\
\hline 6 & height & 127 & 144 & 8 & all & 1270 \\
\hline 7 & height & 145 & 162 & 9 & all & 1168 \\
\hline 8 & height & 163 & 180 & 10 & all & 1067 \\
\hline 9 & height & 181 & 198 & 11 & all & 965 \\
\hline 10 & height & 199 & 216 & 12 & all & 864 \\
\hline 11 & height & 217 & 234 & 13 & all & 762 \\
\hline 12 & height & 235 & 252 & 14 & all & 660 \\
\hline 13 & height & 253 & 270 & 15 & all & 559 \\
\hline 14 & height & 271 & 288 & 16 & all & 457 \\
\hline 15 & height & 289 & 306 & 17 & all & 356 \\
\hline 16 & height & 307 & 324 & 18 & all & 254 \\
\hline 17 & height & 325 & 342 & 19 & all & 152 \\
\hline
\end{tabular}




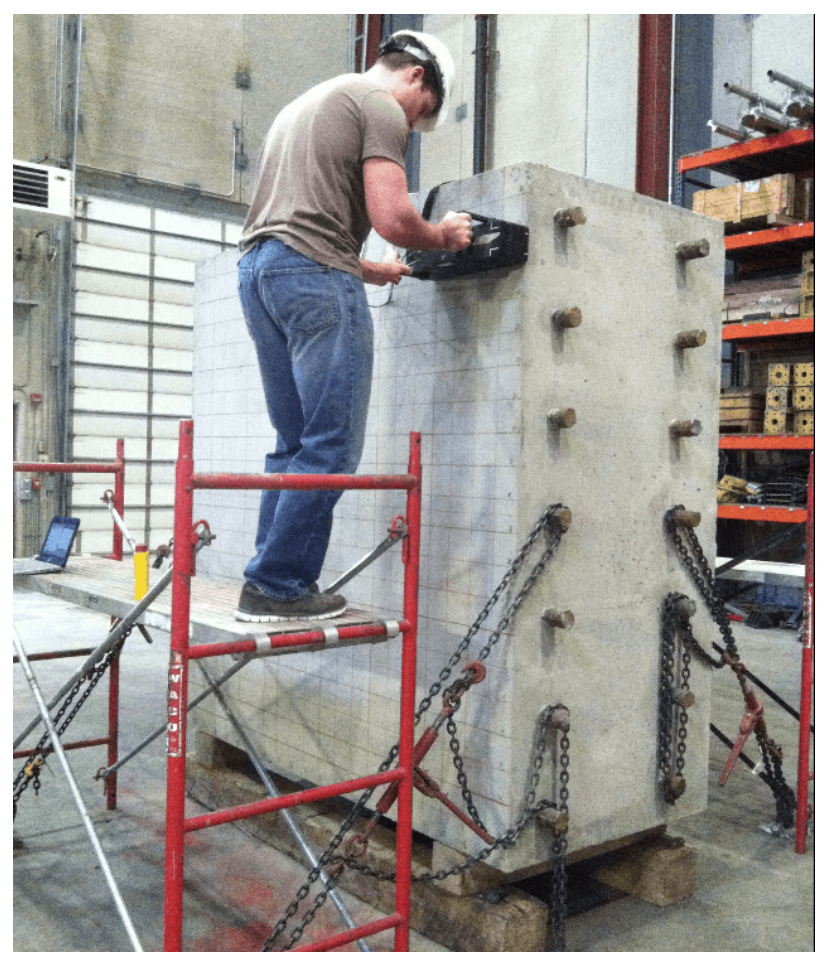

Fig. 20. Length data collection used to create a SAFT-PAN reconstruction.

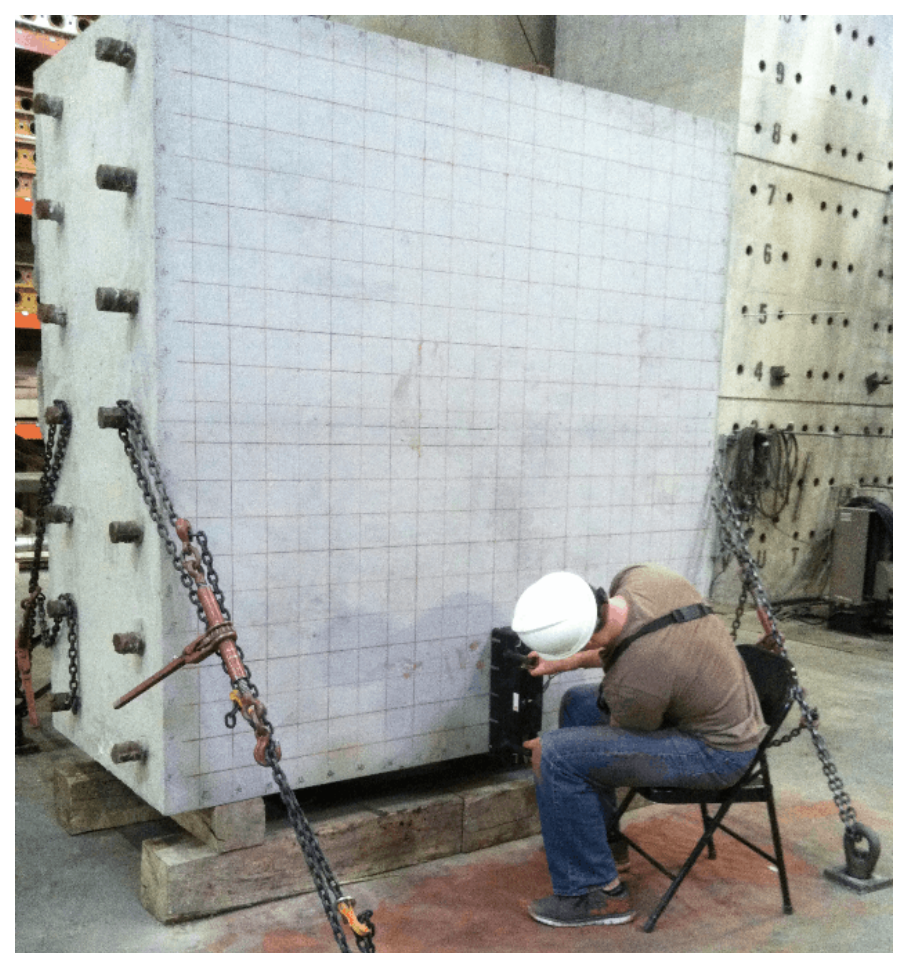

Fig. 21. Height data collection used to create a SAFT-PAN reconstruction. 


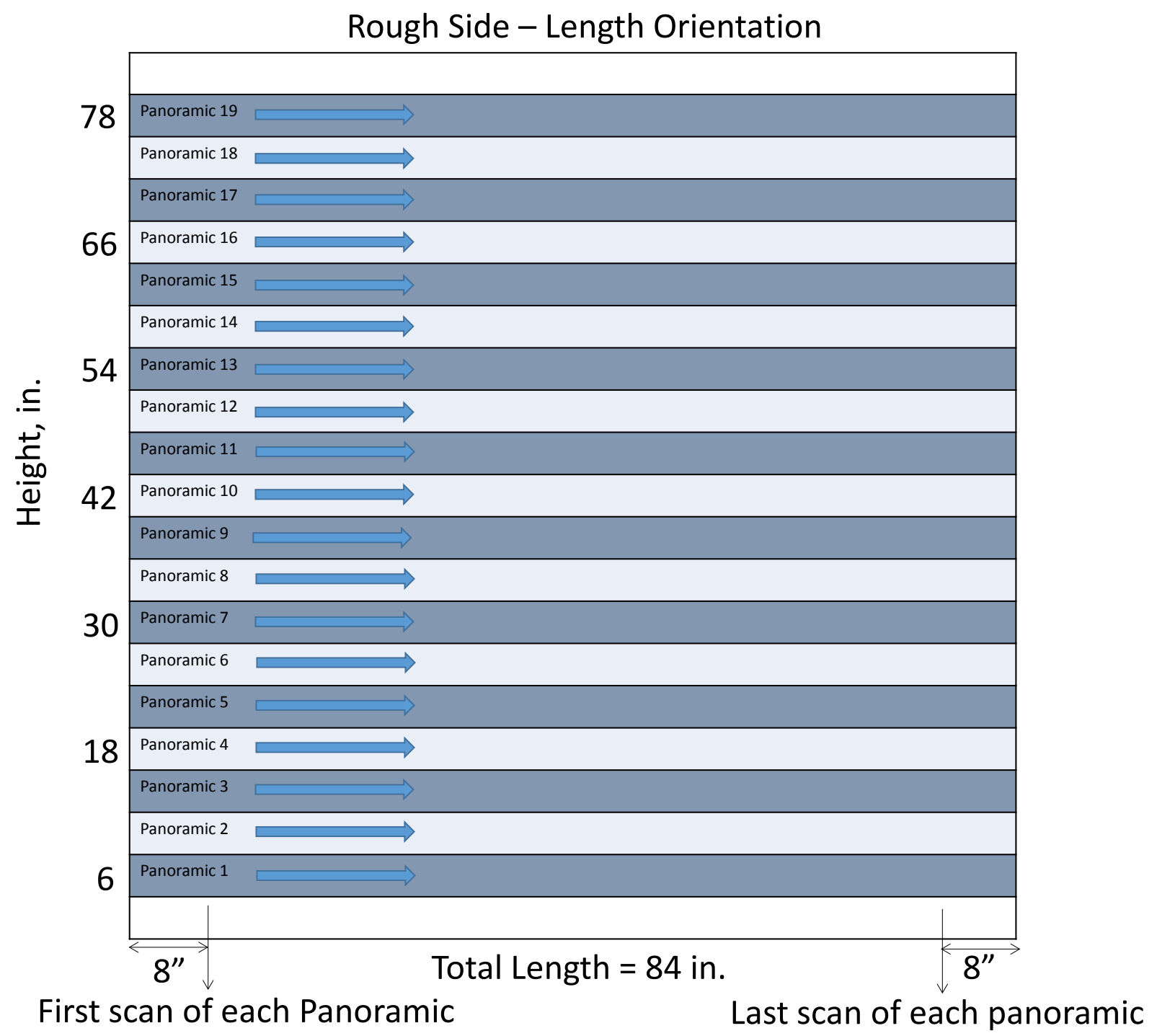

Fig. 22. Rough-side measurement procedure for length orientation measurements. 


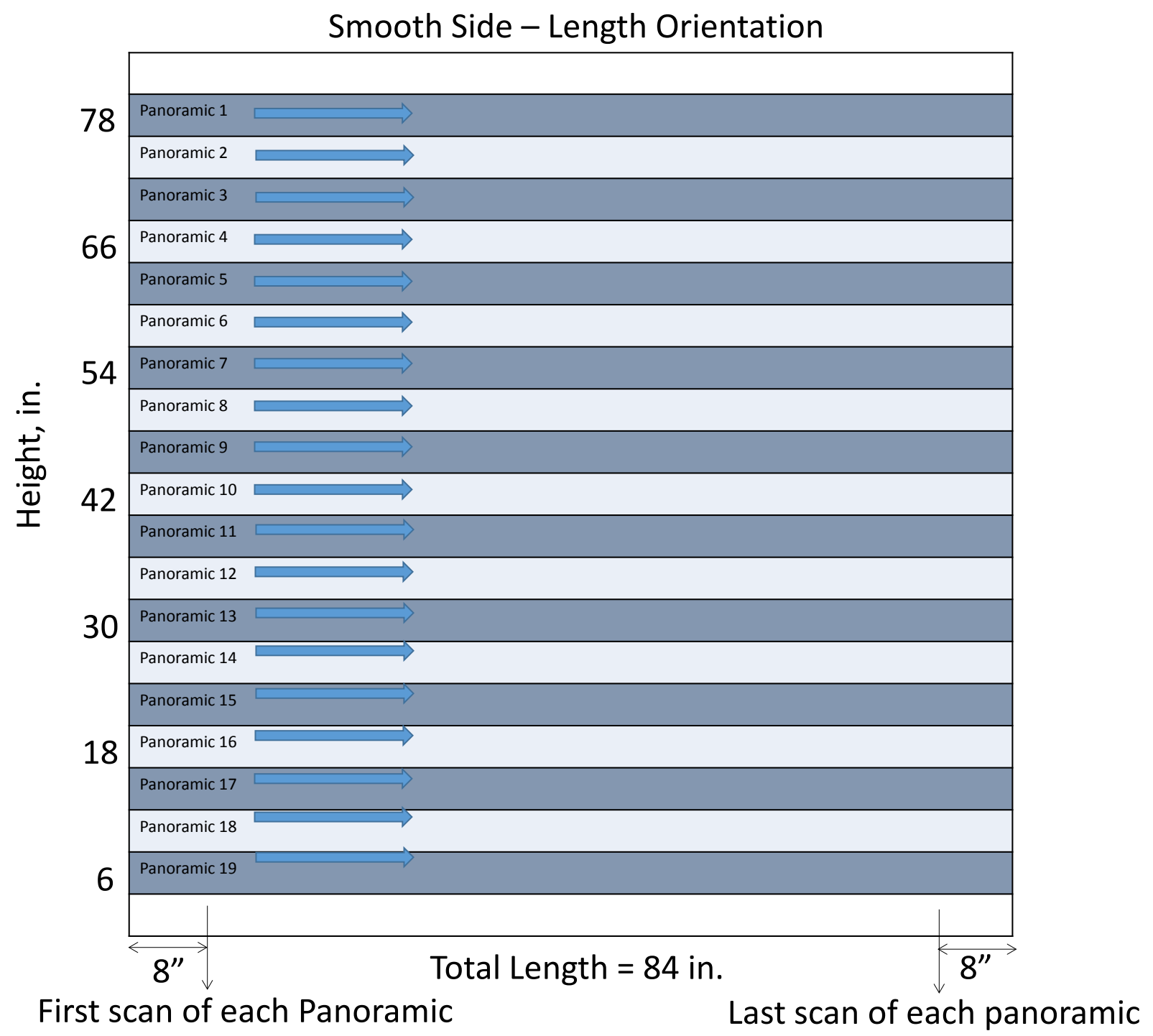

Fig. 23. Smooth-side measurement procedure for length orientation measurements. 


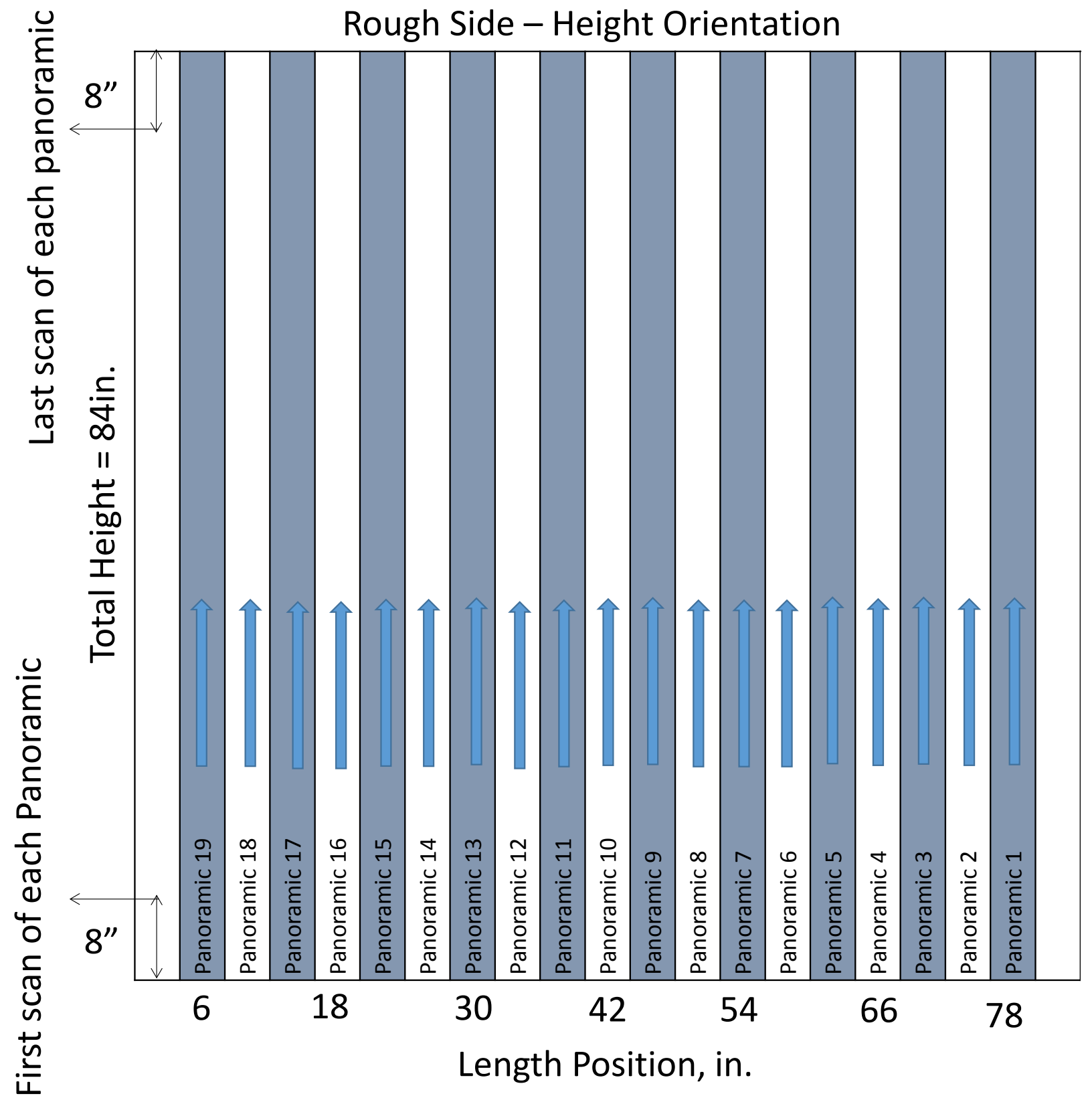

Fig. 24. Rough-side measurement procedure for height orientation measurements. 


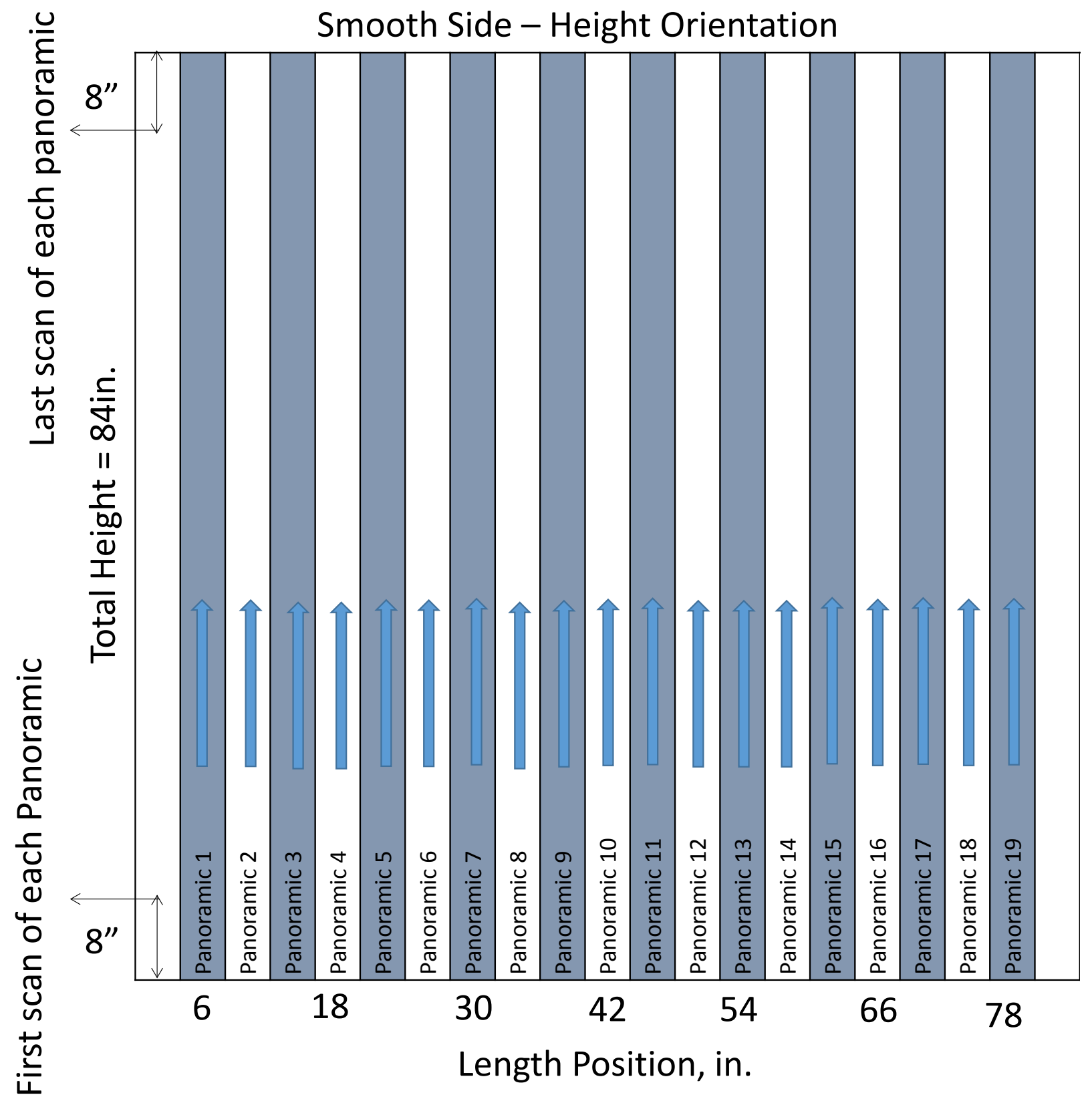

Fig. 25. Smooth-side measurement procedure for height orientation measurements. 



\section{WAVELET FREQUENCY-BANDING METHOD}

The sampling rate of the commercial ultrasonic inspection system used in the collection of the analyzed data is $1 \mathrm{MHz}$. Based on Nyquist criteria the collected data contains $500 \mathrm{kHz}$ of bandwidth. This allows for an alias free spectral content of $0 \mathrm{~Hz}$ (DC) through $500 \mathrm{kHz}$. The frequency banding method [31] used is based on wavelet packet decomposition. Ultimately, the end effect is that the bandwidth (spectral content) of a decomposed node is divided between the two resulting nodes. As an illustration, Node 0 is the starting point of the decomposition. It contains all $500 \mathrm{kHz}$ of bandwidth. Node 0 is then decomposed into two new nodes, Node 1 and Node 2. Node 1 contains the lower half of the bandwidth, $0 \mathrm{~Hz}$ through $250 \mathrm{kHz}$. Likewise, Node 2 contains the upper half, $250 \mathrm{kHz}$ through $500 \mathrm{kHz}$. There is a small amount of frequency leakage between the two new nodes at the dividing point, $250 \mathrm{kHz}$. At this point, the decomposition continues following the same principle. The bandwidth (spectral content) of Node 1 is split to form Nodes 3 and 4 . Node 2 is split to form Nodes 5 and 6. In all cases, each child node contains half of the bandwidth (spectral content) of its parent.

Fig. 26 illustrates this decomposition to a depth of six levels. Prior research in [31] indicates the nodes in blue showed the most promise, so analysis focused on those 16 nodes. Due to space constraints, only nodes $0,16,18,32$, and 34 were chosen and included in Appendix A and B. Detailed analysis is presented in the following sections, which shows how the best nodes were selected.

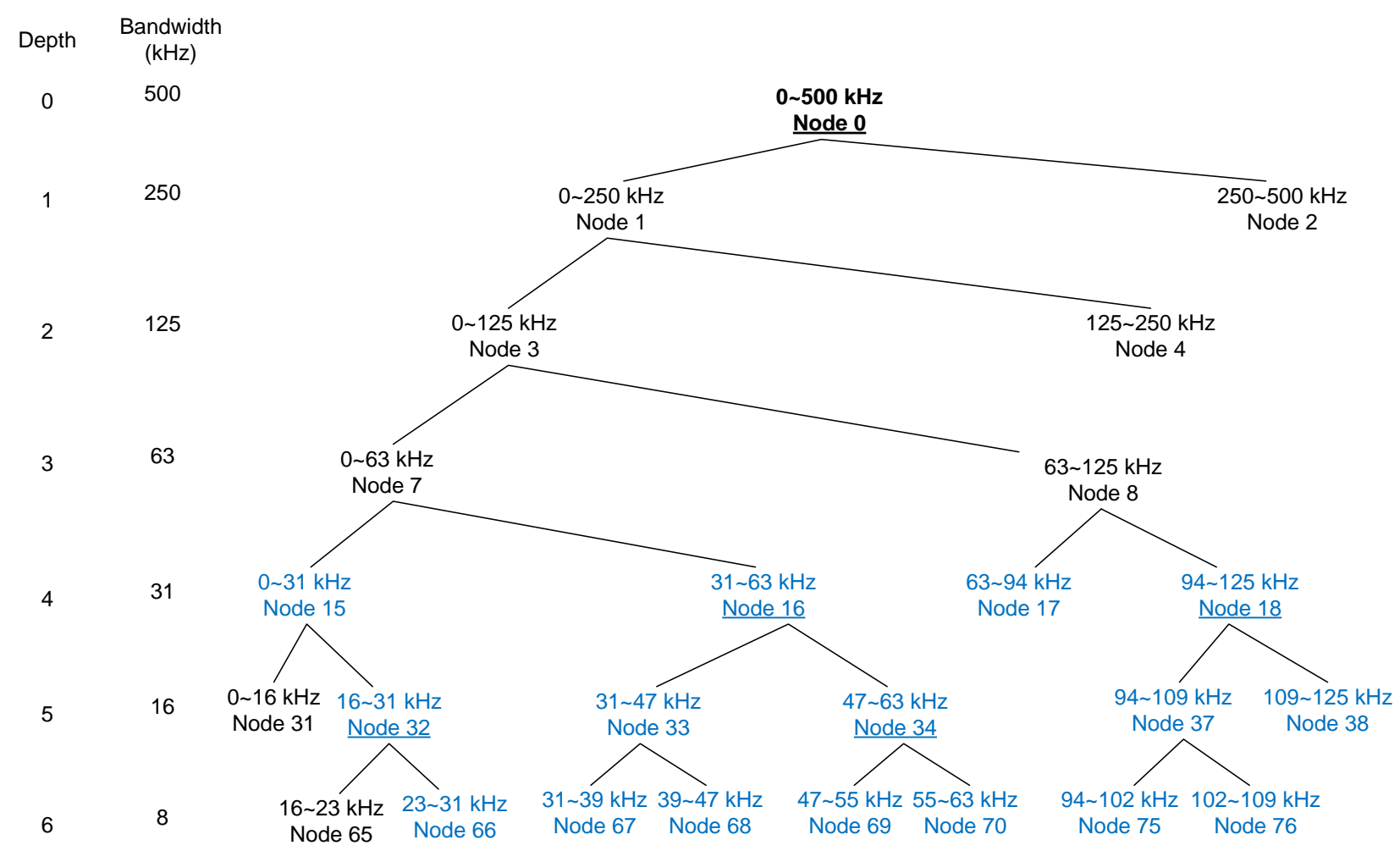

Fig. 26. Our wavelet decomposition. Nodes in blue were analyzed. Underlined nodes appear in Appendix A and $B$. 



\section{NODE SELECTION}

The selection of nodes used in this analysis were based on the original work done in [31]. Those nodes where selected using several pieces of information:

1. The knowledge that the ultrasonic array system used produces an ultrasonic pulse with a nominal center frequency of $50 \mathrm{kHz}$.

2. The percentage of total signal energy contained in each node.

3. Child nodes of parent nodes containing the nominal center frequency, so as to narrow frequency band around nominal center frequency or even divide the band containing the nominal center frequency.

4. Child nodes of a parent node containing a high energy percentage. Even if the node did not contain the nominal center frequency.

Several of these criteria where specifically chosen to open up the potential for the future exploration of the possibility of a frequency-to-defect-type relationship existing.

It should be mentioned that the concrete specimens that were used in the development of the frequencybanding algorithm, [31], were 10-inches thick, so it was anticipated that some of the choices made would likely not transition well to the 40 -inch thick specimen examined in this report. The most significant, and expected, difference is that far less energy is reflected back to the instrument in the 40 -inch case versus the 10-inch case. 



\section{ANALYSIS APPROACH}

Table 5 lists the scanlines, which are also the panoramic-SAFT index numbers, for each defect. Thus, each defect should be visible from the rough and smooth side, both in horizontal and vertical orientations. If a defect is in the middle of two scans, both scans are examined. For example, looking at Defect 1 from the Rough side, the horizontal orientation scanline indices are 14 and 15, since it lies in between those indices, which is indicated in Table 5 as 14.5. Using Table 2, an overlay of the estimated dimension for each defect on SAFT images is characterized as a white box. For each defect, first we analyze the shallow cover, then the deep cover. An analyst looked at each panoramic image resulting from different nodes, and assigned the following grading scale: 0 for defect not visible, 1 for defect partially visible, and 2 for defect clearly visible. The grading scale was "adaptive”, in the sense that spots on the images corresponding to defects that reside closer to the ultrasonic linear array will be "brighter" than those for defects further from the linear array. That is, using the color code in the images, a red spot might be expected for defects seen well above the first layer of rebar mesh, but a fainter green spot might be expected for defects seen below the second layer of rebar mesh.

Table 5. Adjacent SAFT-Panoramic scan information.

\begin{tabular}{|c|c|c|c|c|}
\hline \multirow[t]{2}{*}{$\begin{array}{l}\text { Defect } \\
\text { ID }\end{array}$} & \multicolumn{2}{|c|}{$\begin{array}{c}\text { Rough Side } \\
\text { Adjacent } \\
\text { Scanline } \backslash \text { Panoramic-SAFT } \\
\text { Index Numbers }\end{array}$} & \multicolumn{2}{|c|}{$\begin{array}{c}\text { Smooth Side } \\
\text { Adjacent } \\
\text { Scanline } \backslash \text { Panoramic-SAFT } \\
\text { Index Numbers }\end{array}$} \\
\hline & $\begin{array}{l}\text { Horizontal } \\
\text { orientation }\end{array}$ & $\begin{array}{c}\text { Vertical } \\
\text { orientation }\end{array}$ & $\begin{array}{l}\text { Horizontal } \\
\text { orientation }\end{array}$ & $\begin{array}{c}\text { Vertical } \\
\text { orientation }\end{array}$ \\
\hline 1 & 14.5 & 16.0 & 5.5 & 4.0 \\
\hline 2 & 7.0 & 5.5 & 13.0 & 14.5 \\
\hline 3 & 10.0 & 14.5 & 10.0 & 5.5 \\
\hline 4 & 5.5 & 4.0 & 14.5 & 16.0 \\
\hline 5 & 14.5 & 13.0 & 5.5 & 7.0 \\
\hline 6 & 13.5 & 10.0 & 6.5 & 10.0 \\
\hline 7 & 6.5 & 10.0 & 13.5 & 10.0 \\
\hline 8 & 11.5 & 8.5 & 8.5 & 11.5 \\
\hline 9 & 8.5 & 11.5 & 11.5 & 8.5 \\
\hline 10 & 8.5 & 2.5 & 11.5 & 17.5 \\
\hline 11 & 11.5 & 17.5 & 8.5 & 2.5 \\
\hline 12 & 8.5 & 8.5 & 11.5 & 11.5 \\
\hline 13 & 11.5 & 11.5 & 8.5 & 8.5 \\
\hline 14 & 4.8 & 15.3 & 15.3 & 4.7 \\
\hline 15 & 15.3 & 4.8 & 4.8 & 15.2 \\
\hline 16 & 3.3 & 17.5 & 16.8 & 2.5 \\
\hline 17 & 7.0 & 14.5 & 13.0 & 5.5 \\
\hline 18 & 1.8 & 10.0 & 18.3 & 10.0 \\
\hline 19 & 3.3 & 10.0 & 16.8 & 10.0 \\
\hline 20 & 10.0 & 7.0 & 10.0 & 13.0 \\
\hline
\end{tabular}





\section{GRADING}

The method of determining performance was by visually inspecting the 16 nodes illustrated in Fig. 26 for each defect from each side and orientation. If the center of a defect lied in the middle of two panoramics, both panoramics were analyzed. The following section displays results for each individual defect, whereas the section after that summarizes the results across all defects.

\subsection{INDIVIDUAL 20 DEFECTS}

A three level grading system was chosen where a 0 meant the defect could not be seen, a 1 meant the defect could partially be seen, and a 2 meant the defect could clearly be seen. Analysis was split between the shallow, deep, and combined shallow and deep sides. The Shallow side is presented on the left part of the analysis table, and the deep side is presented on the right. The "Shallow Total" is the normalized sum of all perspectives for that node. For example, for Defect 1, the Shallow Total for Node 0 is the score for Smooth horizontal Set 4, Smooth vertical 5, and Smooth vertical 6. It is normalized to prevent defects that straddle both scans, such as Defect 8, which has 4, from being artificially higher than a defect that does not straddle any scans, such as Defect 18, which has 2. The "Deep Total" is similarly the sum of all the perspectives for that node. The "Combined Total" is the sum of the Shallow and Deep Totals. The Shallow Total is represented in blue color in the bar chart, while the Deep Total is represented in red color in the bar chart. In this report, the analysis charts for all defects are presented, while only the analysis table for Defect 1 is presented.

For each defect, selected nodes deemed "better" than the regular SAFT method, the images of node 0 followed by some of the "better" nodes are presented. 


\subsubsection{Defect 1}

Table 6. Defect 1 analysis table

\begin{tabular}{|c|c|c|c|c|c|c|c|c|c|}
\hline \multicolumn{10}{|c|}{ D1-Porous Half cylinder (no cover) } \\
\hline & \multicolumn{4}{|c|}{ Shallow } & \multicolumn{4}{|c|}{ Deep } & \multirow{4}{*}{$\begin{array}{c}\text { Combined } \\
\text { Total }\end{array}$} \\
\hline & \multicolumn{4}{|c|}{ Smooth - 86 mm depth } & \multicolumn{4}{|c|}{ Rough - $930 \mathrm{~mm}$ depth } & \\
\hline & Hor & \multicolumn{2}{|c|}{ Ver } & \multirow{2}{*}{$\begin{array}{c}\text { Shallow } \\
\text { Total }\end{array}$} & \multirow{2}{*}{$\begin{array}{c}\text { Hor } \\
\text { Set } 16\end{array}$} & \multicolumn{2}{|c|}{ Ver } & \multirow{2}{*}{$\begin{array}{l}\text { Deep } \\
\text { Total }\end{array}$} & \\
\hline Node & Set 4 & Set 5 & Set 6 & & & Set 14 & Set 15 & & \\
\hline 0 & 0 & 0 & 0 & 0.00 & 0 & 0 & 0 & 0.00 & 0.00 \\
\hline 15 & 0 & 0 & 0 & 0.00 & 0 & 0 & 0 & 0.00 & 0.00 \\
\hline 16 & 2 & 0 & 0 & 0.67 & 0 & 0 & 0 & 0.00 & 0.67 \\
\hline 17 & 0 & 0 & 0 & 0.00 & 0 & 0 & 0 & 0.00 & 0.00 \\
\hline 18 & 0 & 0 & 0 & 0.00 & 0 & 0 & 0 & 0.00 & 0.00 \\
\hline 32 & 0 & 0 & 0 & 0.00 & 0 & 0 & 0 & 0.00 & 0.00 \\
\hline 33 & 0 & 0 & 0 & 0.00 & 0 & 0 & 0 & 0.00 & 0.00 \\
\hline 34 & 2 & 0 & 0 & 0.67 & 0 & 0 & 0 & 0.00 & 0.67 \\
\hline 37 & 0 & 0 & 0 & 0.00 & 0 & 0 & 0 & 0.00 & 0.00 \\
\hline 38 & 0 & 0 & 0 & 0.00 & 0 & 0 & 0 & 0.00 & 0.00 \\
\hline 66 & 0 & 0 & 0 & 0.00 & 0 & 0 & 0 & 0.00 & 0.00 \\
\hline 67 & 0 & 0 & 0 & 0.00 & 0 & 0 & 0 & 0.00 & 0.00 \\
\hline 68 & 0 & 0 & 0 & 0.00 & 0 & 0 & 0 & 0.00 & 0.00 \\
\hline 69 & 0 & 0 & 0 & 0.00 & 0 & 0 & 0 & 0.00 & 0.00 \\
\hline 70 & 0 & 0 & 0 & 0.00 & 0 & 0 & 0 & 0.00 & 0.00 \\
\hline 75 & 0 & 0 & 0 & 0.00 & 0 & 0 & 0 & 0.00 & 0.00 \\
\hline 76 & 0 & 0 & 0 & 0.00 & 0 & 0 & 0 & 0.00 & 0.00 \\
\hline
\end{tabular}

\section{D1-Porous Half Cylinder (No Cover)}

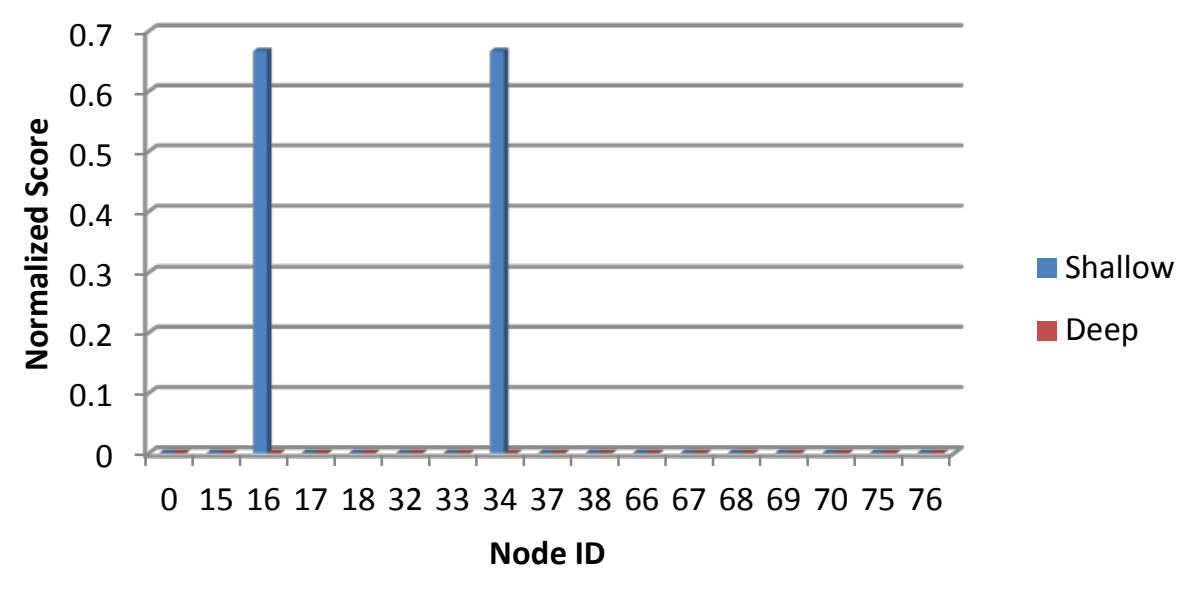

Fig. 27. Defect 1 analysis chart illustrating the normalized score for each node. 


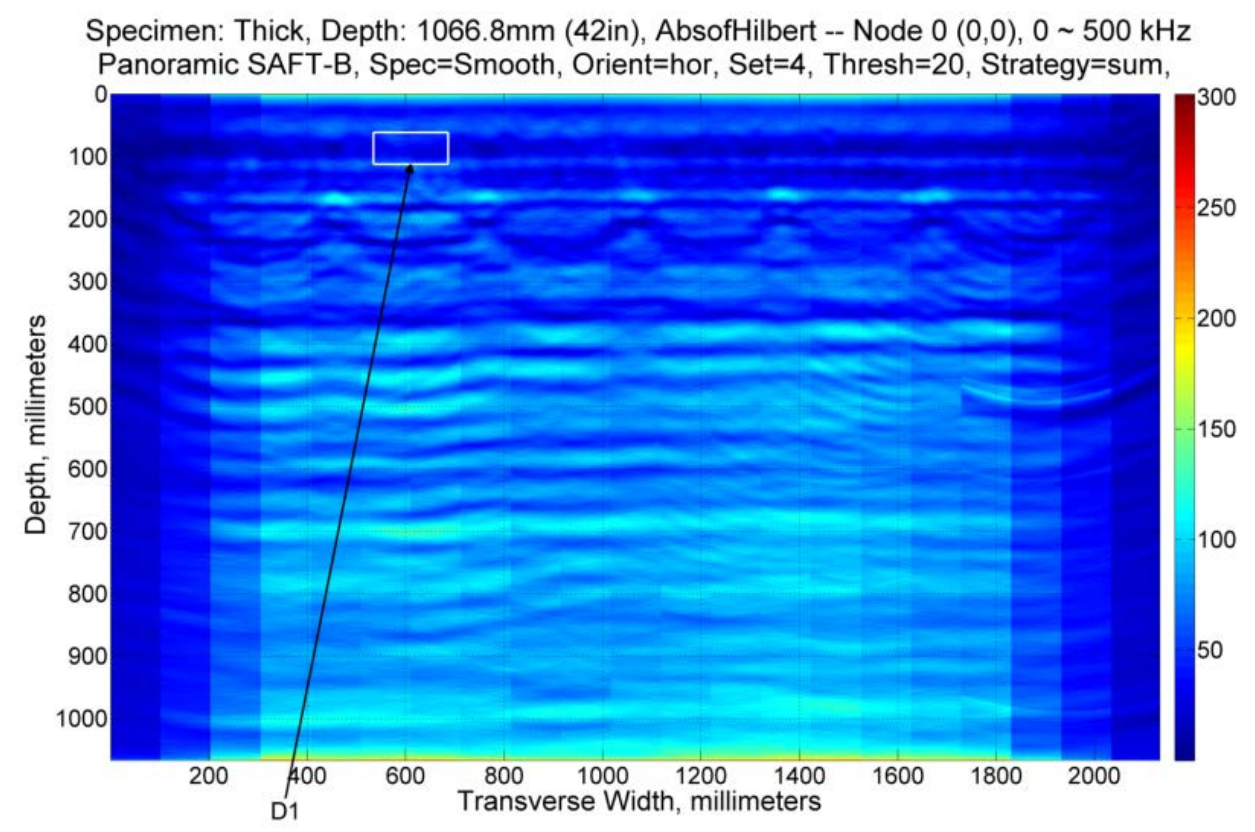

(a)

Specimen: Thick, Depth: 1066.8mm (42in), AbsofHilbert -- Node $16(4,1), 31.25 \sim 62.5 \mathrm{kHz}$ Panoramic SAFT-B, Spec=Smooth, Orient=hor, Set=4, Thresh=20, Strategy=sum,

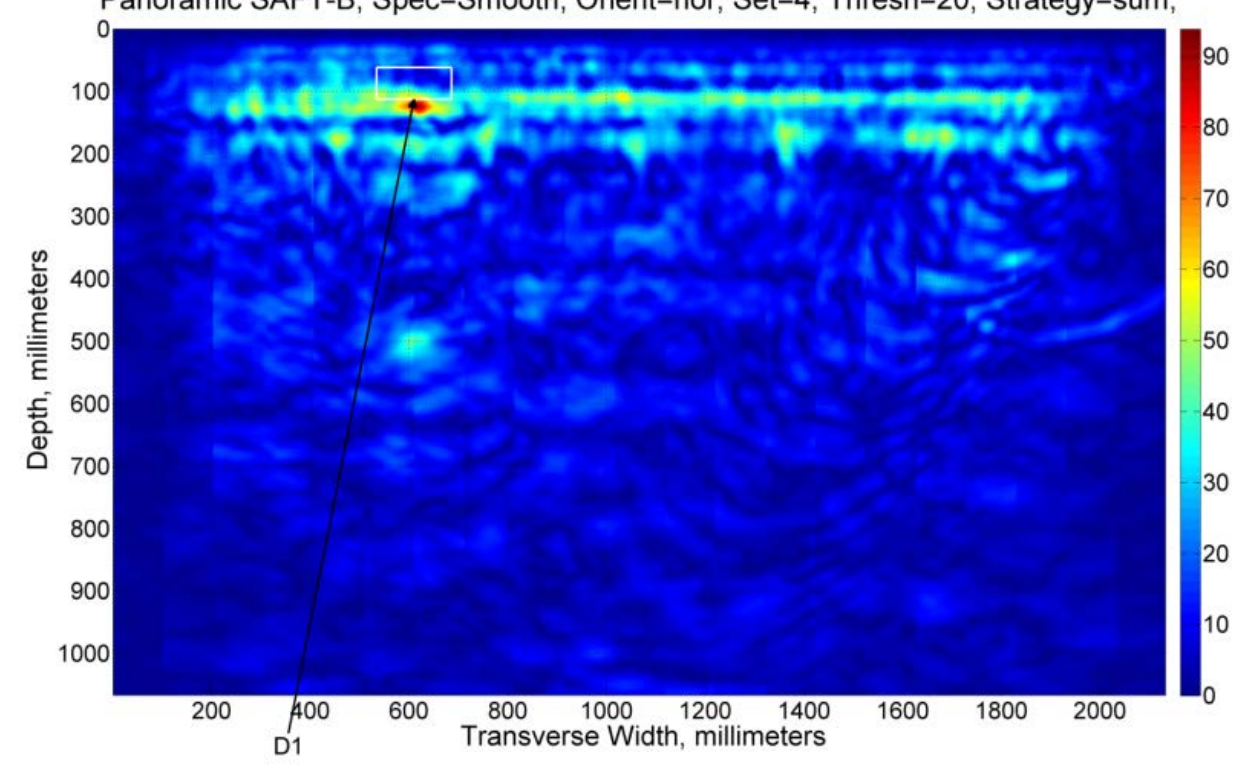

(b)

Fig. 28. Shallow cover improved detectability for Defect 1 for node 16 (b) compared to regular SAFT (a). 


\subsubsection{Defect 2}

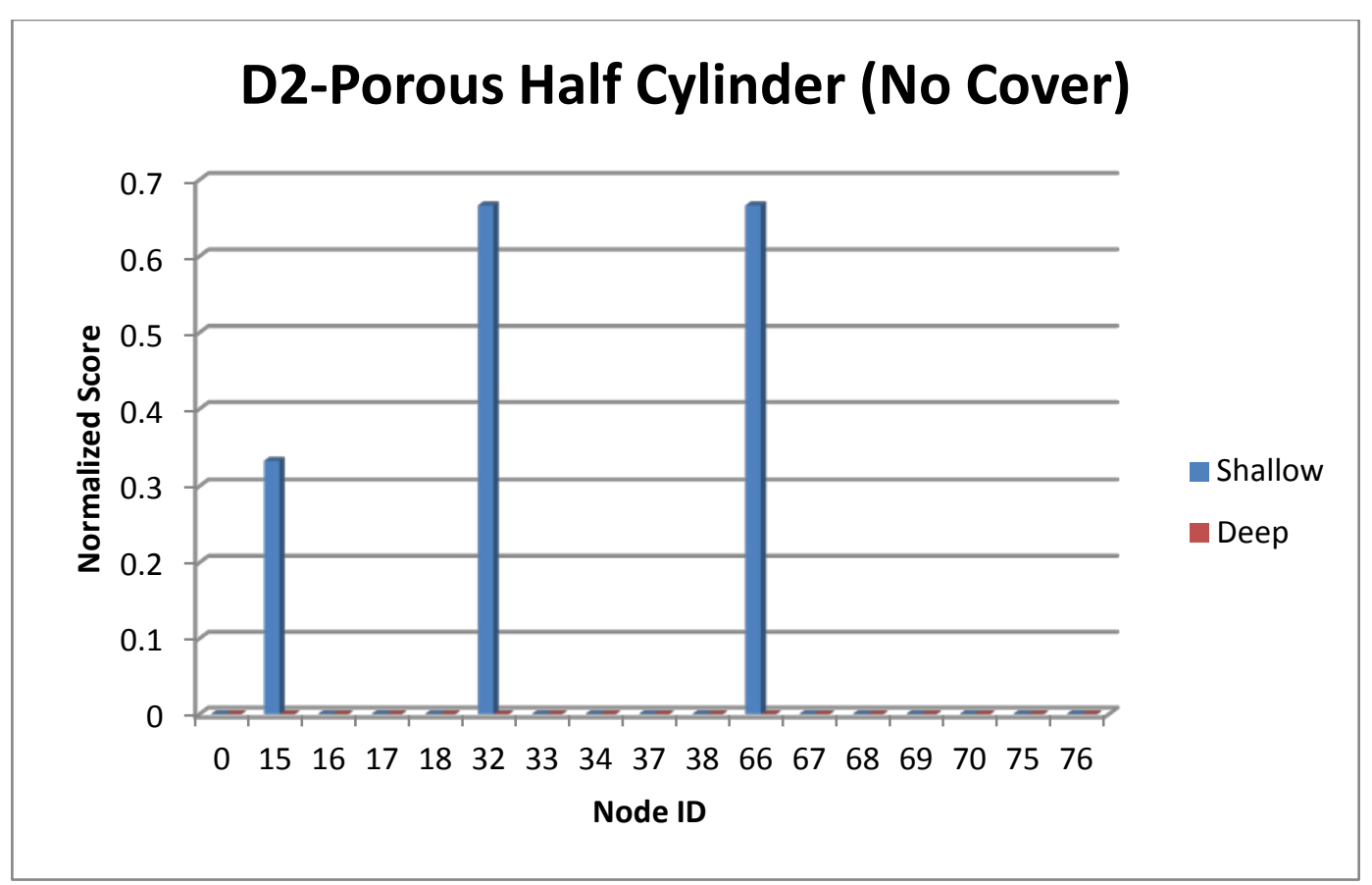

Fig. 29. Defect 2 analysis chart illustrating the normalized score for each node. 


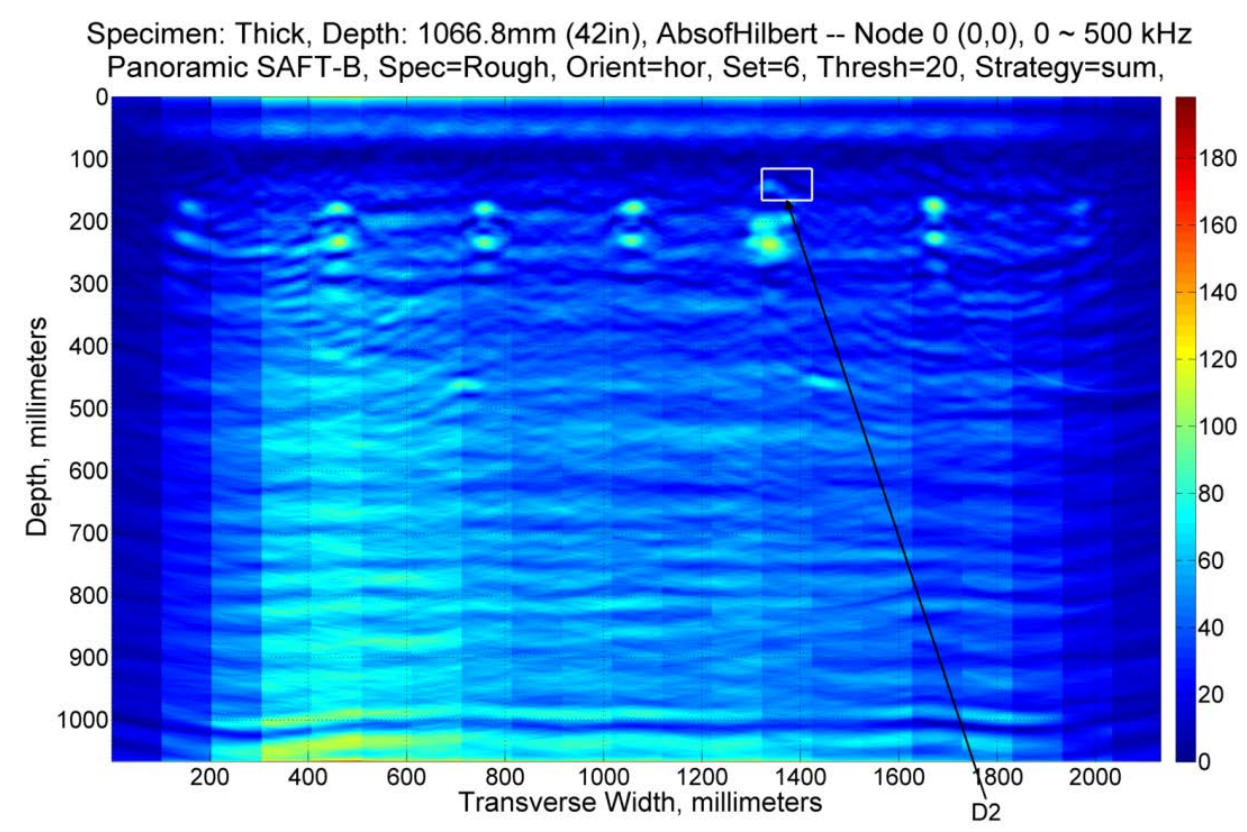

(a)

Specimen: Thick, Depth: 1066.8mm (42in), AbsofHilbert -- Node $32(5,1), 15.625 \sim 31.25 \mathrm{kHz}$ Panoramic SAFT-B, Spec=Rough, Orient $=$ hor, Set $=6$, Thresh $=20$, Strategy $=$ sum,

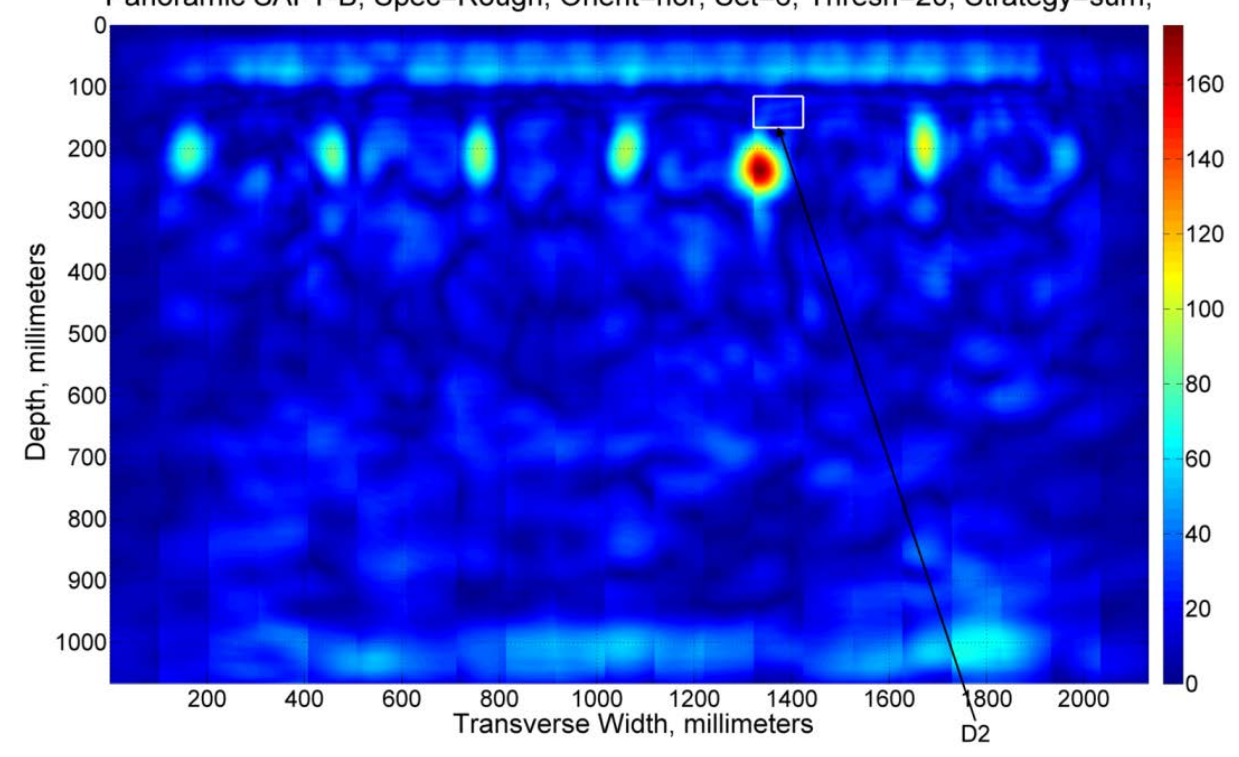

(b)

Fig. 30. Shallow cover improved detectability for Defect 2 for node 32 (b) compared to regular SAFT (a), horizontal orientation. 


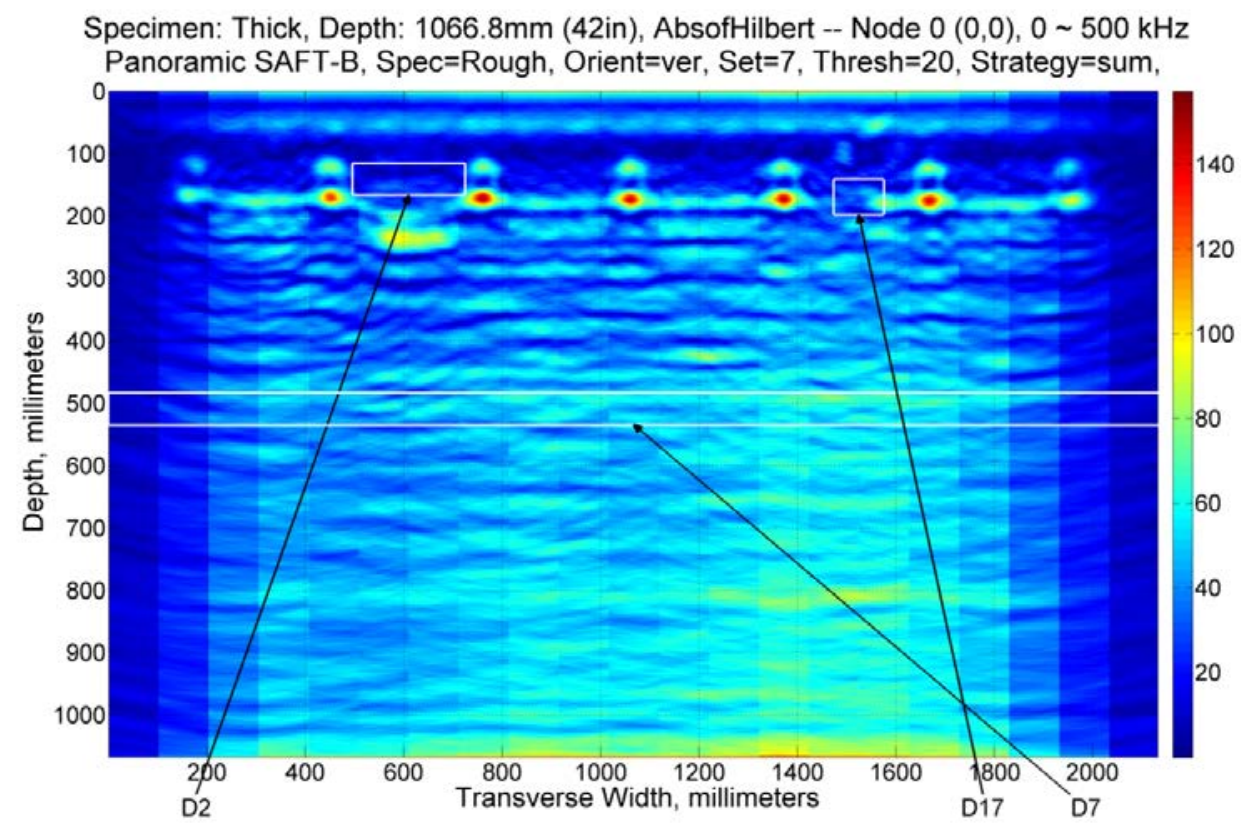

(a)

Specimen: Thick, Depth: 1066.8mm (42in), AbsofHilbert -- Node $32(5,1), 15.625 \sim 31.25 \mathrm{kHz}$

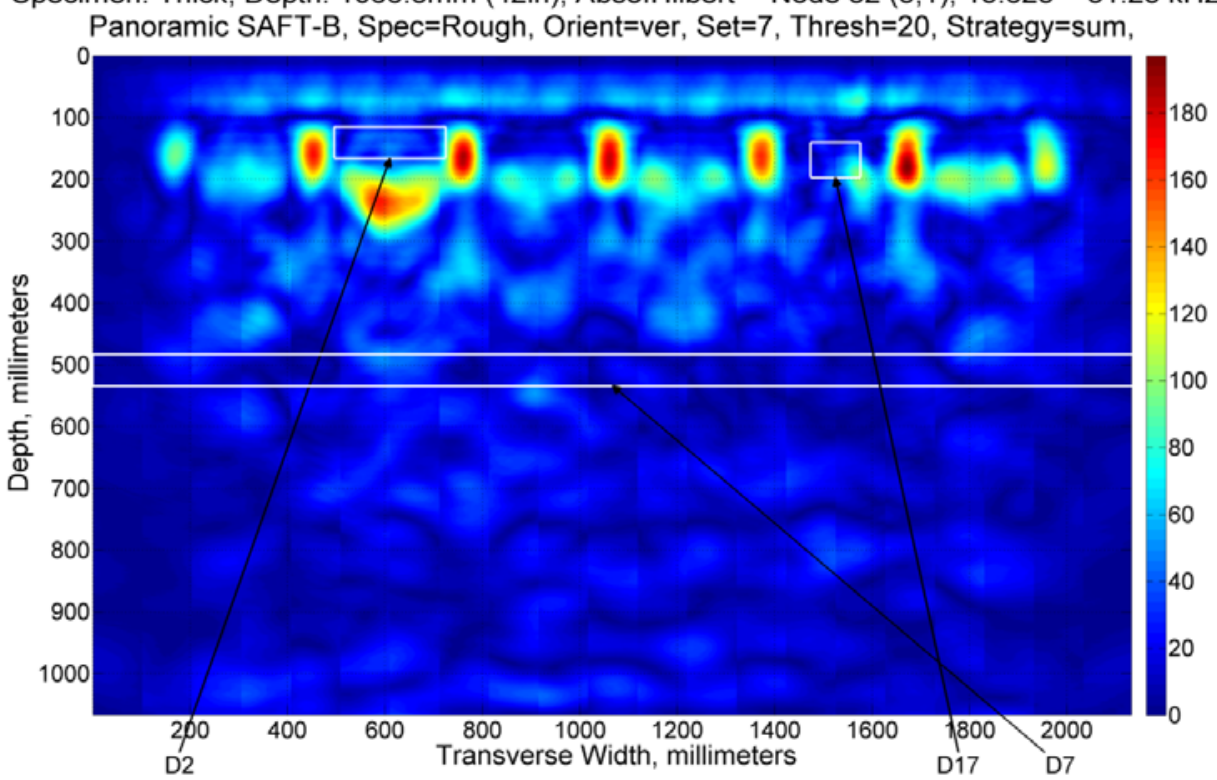

(b)

Fig. 31. Shallow cover improved detectability for Defect 2 for node 32 (b) compared to regular SAFT (a), vertical orientation. 


\subsubsection{Defect 3}

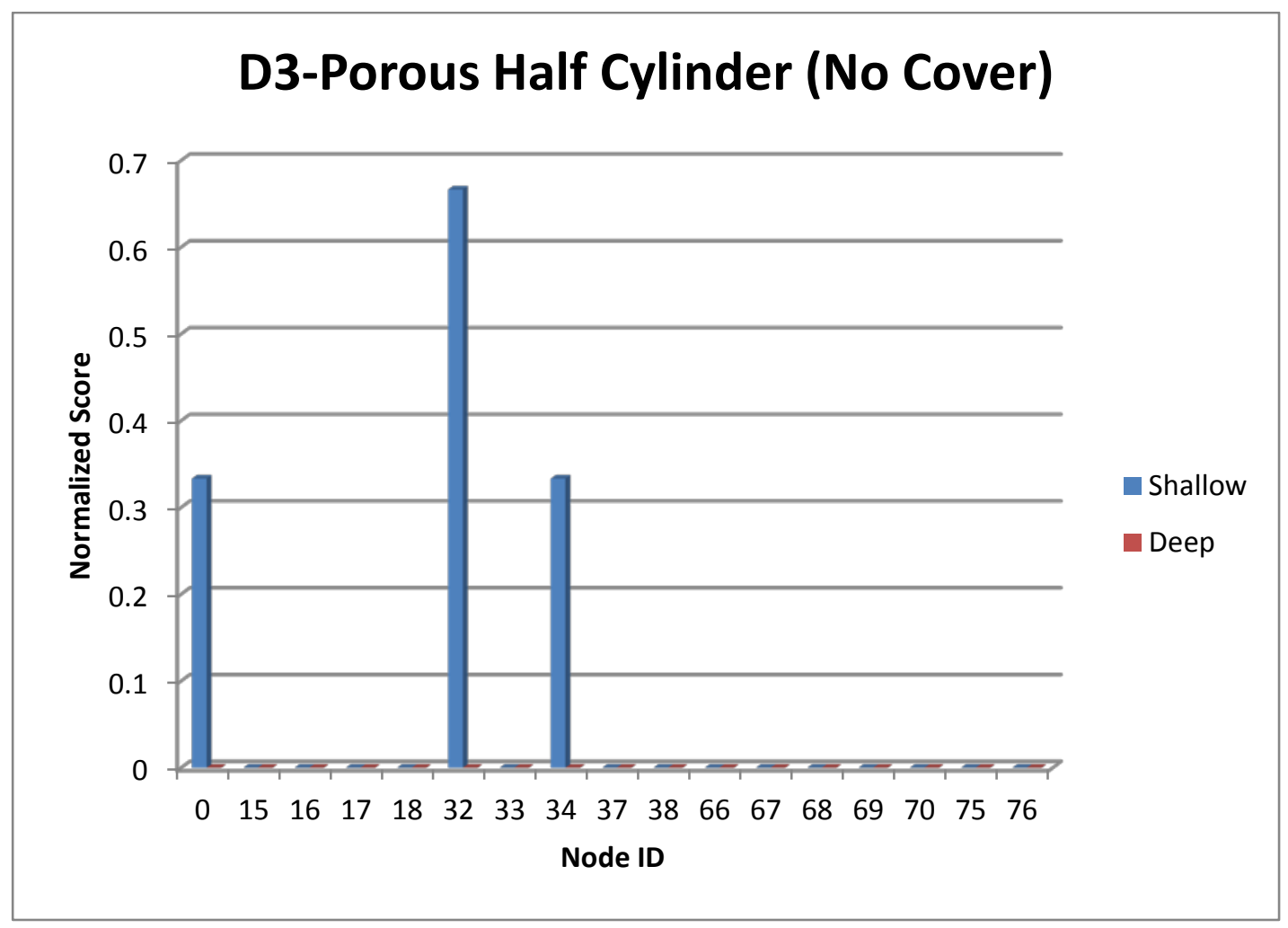

Fig. 32. Defect 3 analysis chart illustrating the normalized score for each node. 


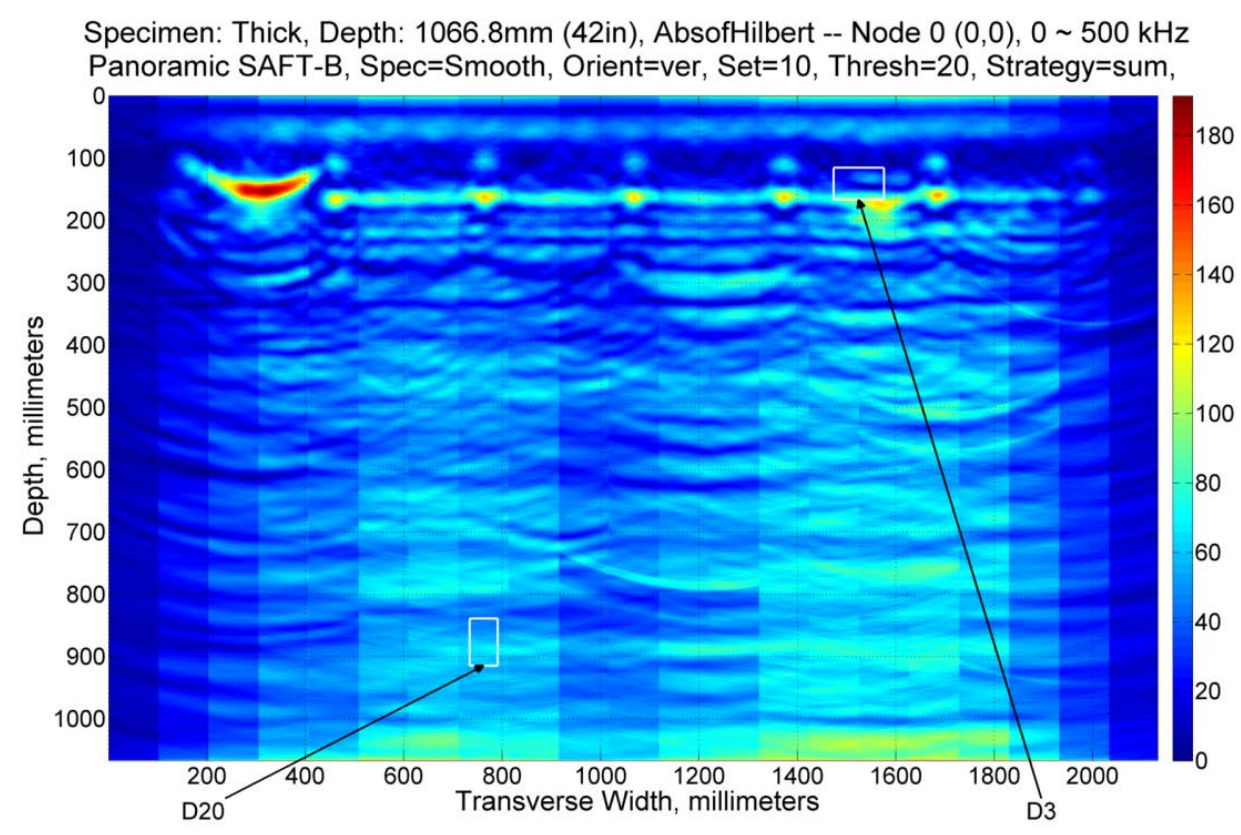

(a)

Specimen: Thick, Depth: 1066.8mm (42in), AbsofHilbert -- Node $32(5,1), 15.625 \sim 31.25 \mathrm{kHz}$

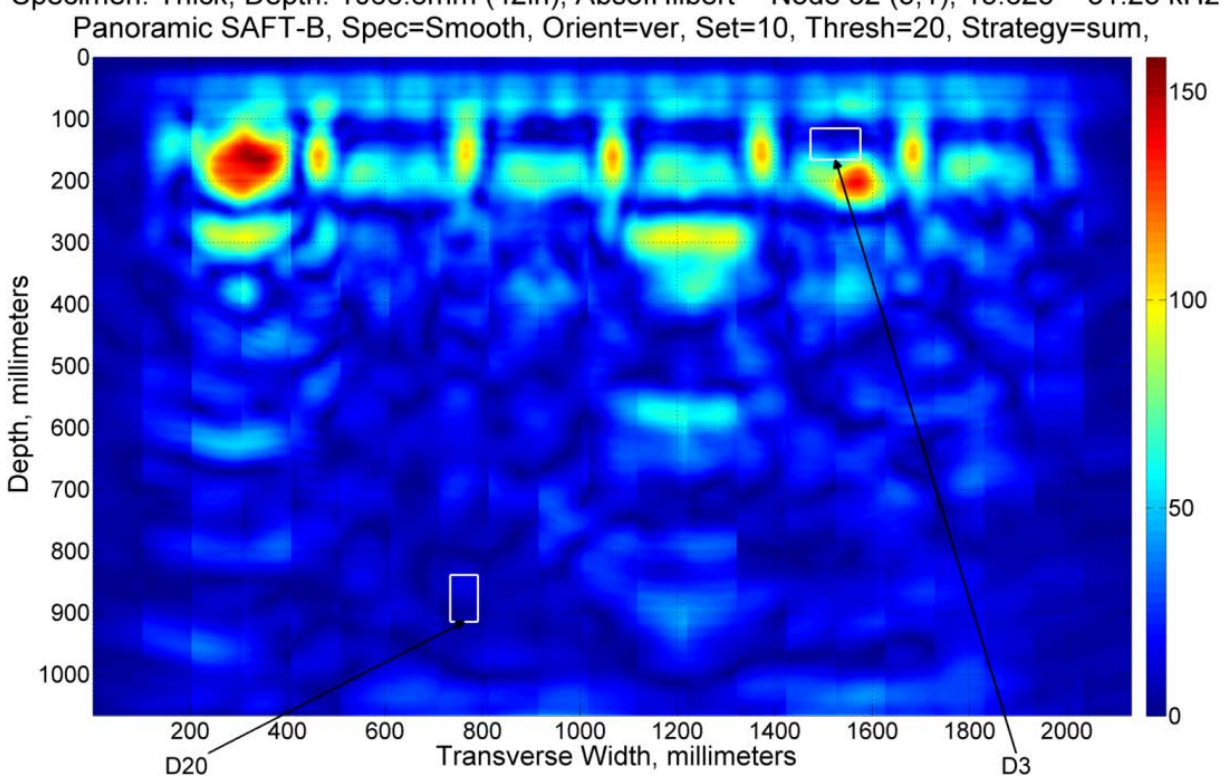

(b)

Fig. 33. Shallow cover improved detectability for Defect 3 for node 32 (b) compared to regular SAFT (a), vertical orientation. 
10.1.4 Defect 4

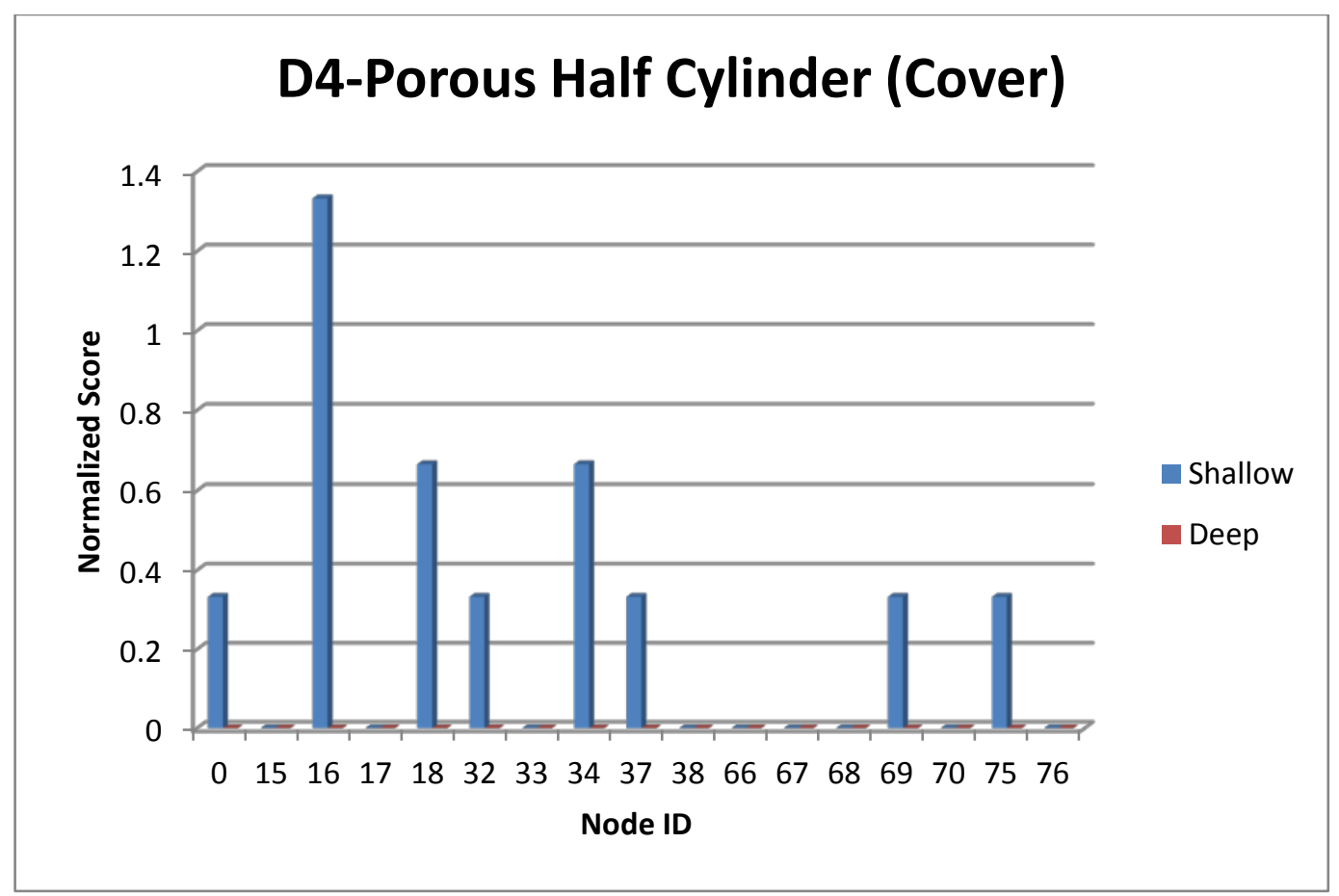

Fig. 34. Defect 4 analysis chart illustrating the normalized score for each node. 


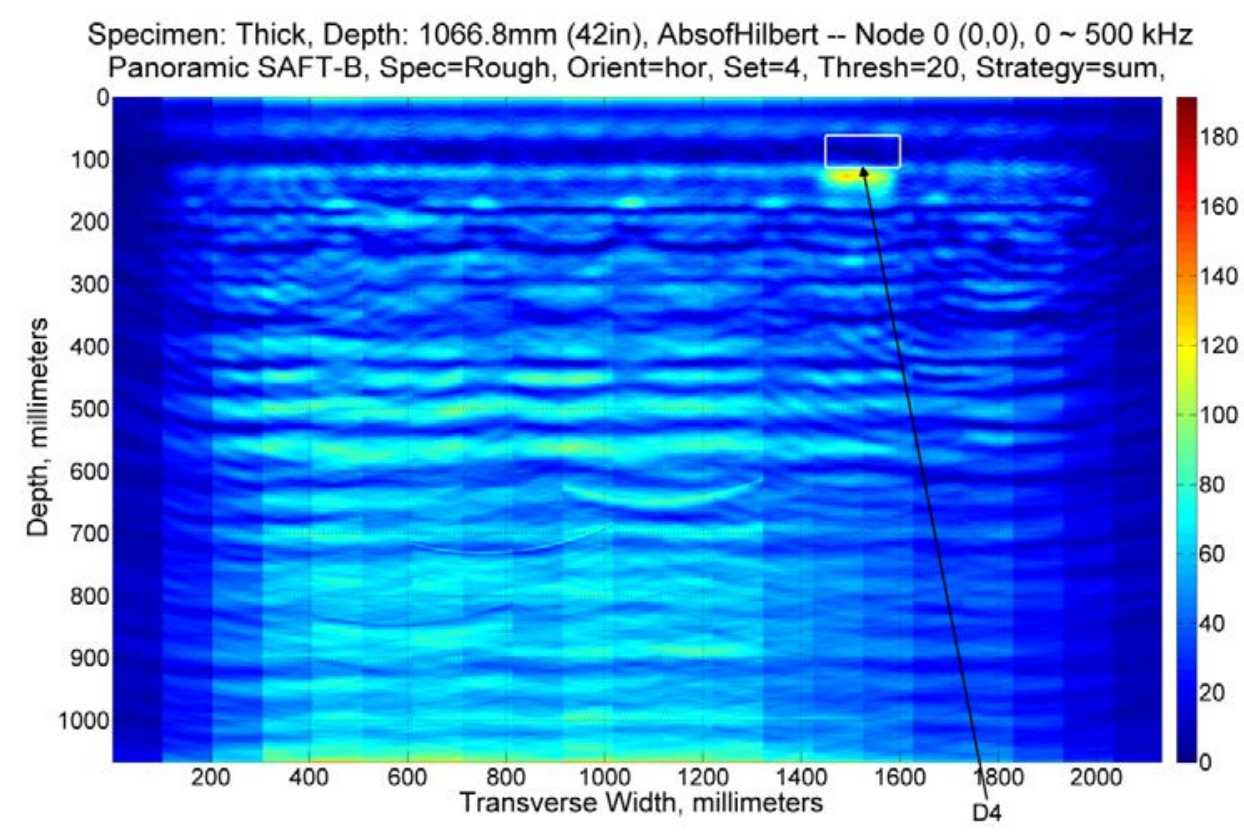

(a)

Specimen: Thick, Depth: 1066.8mm (42in), AbsofHilbert -- Node $16(4,1), 31.25 \sim 62.5 \mathrm{kHz}$ Panoramic SAFT-B, Spec=Rough, Orient=hor, Set $=4$, Thresh=20, Strategy $=$ sum,

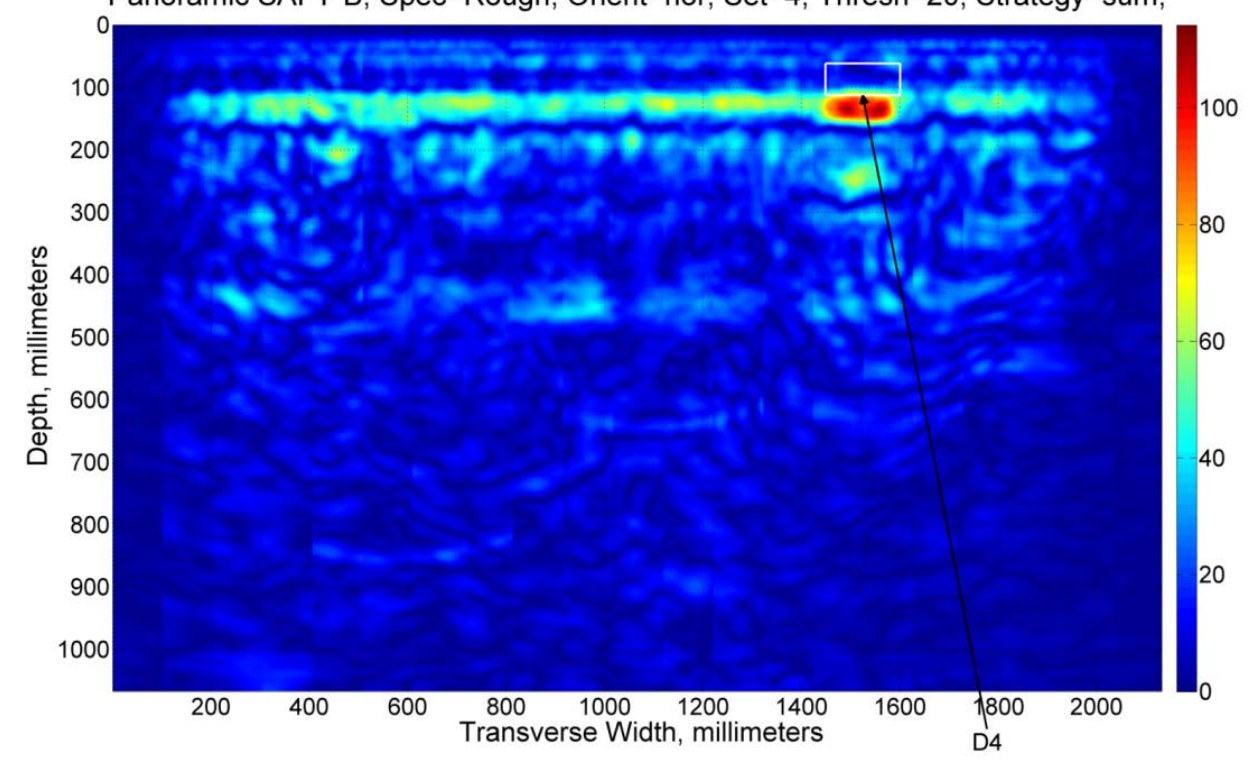

(b)

Fig. 35. Shallow cover improved detectability for Defect 4 for node 16 (b) compared to regular SAFT (a), horizontal orientation. 
Specimen: Thick, Depth: 1066.8mm (42in), AbsofHilbert -- Node $0(0,0), 0 \sim 500 \mathrm{kHz}$

Panoramic SAFT-B, Spec=Rough, Orient=ver, Set=5, Thresh=20, Strategy=sum,

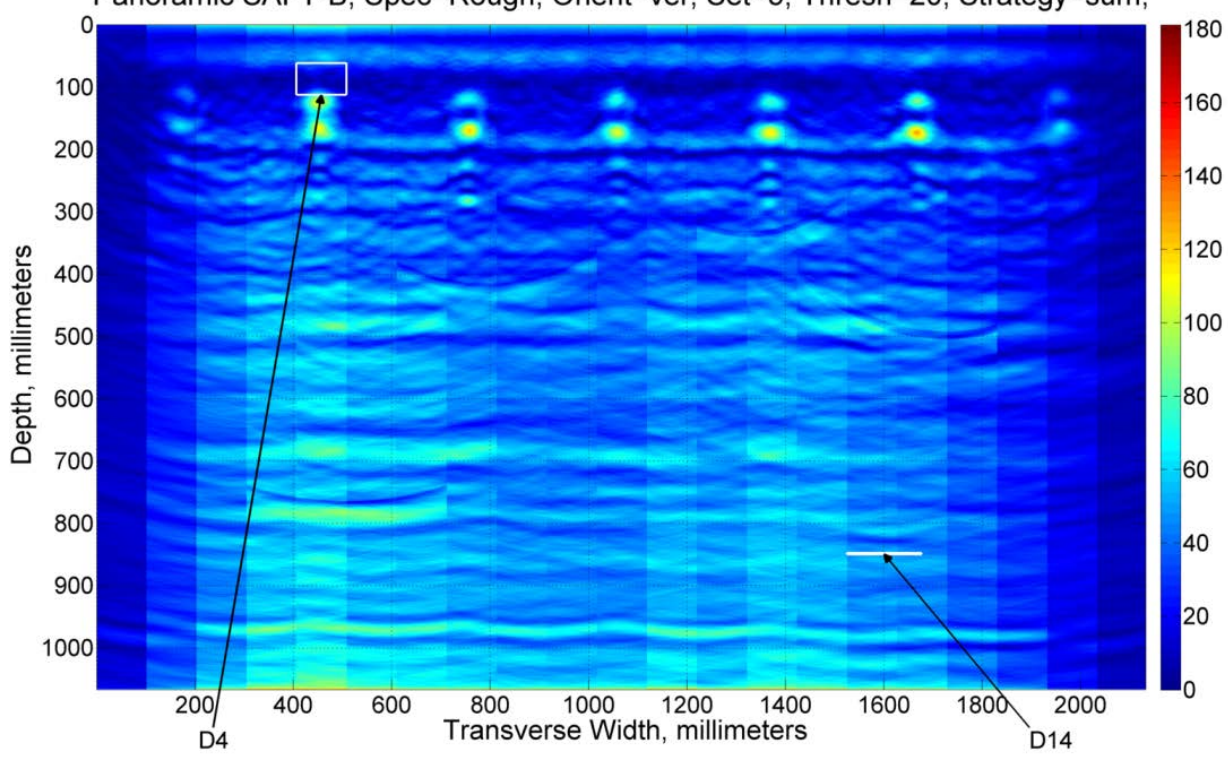

(a)

Specimen: Thick, Depth: 1066.8mm (42in), AbsofHilbert -- Node $16(4,1), 31.25 \sim 62.5 \mathrm{kHz}$ Panoramic SAFT-B, Spec=Rough, Orient=ver, Set=5, Thresh=20, Strategy=sum,

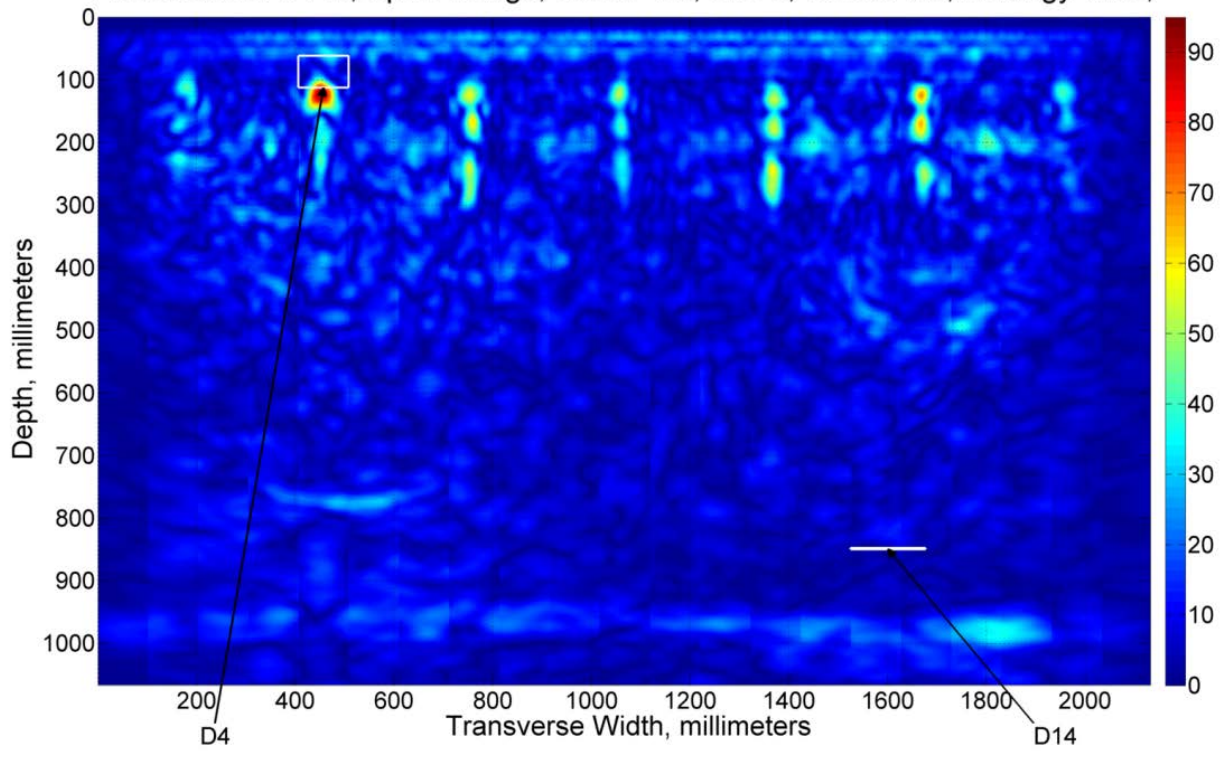

(b)

Fig. 36. Shallow cover improved detectability for Defect 4 for node 16 (b) compared to regular SAFT (a), vertical orientation \#1. 
Specimen: Thick, Depth: 1066.8mm (42in), AbsofHilbert -- Node $0(0,0), 0 \sim 500 \mathrm{kHz}$ Panoramic SAFT-B, Spec=Rough, Orient=ver, Set=6, Thresh=20, Strategy=sum,

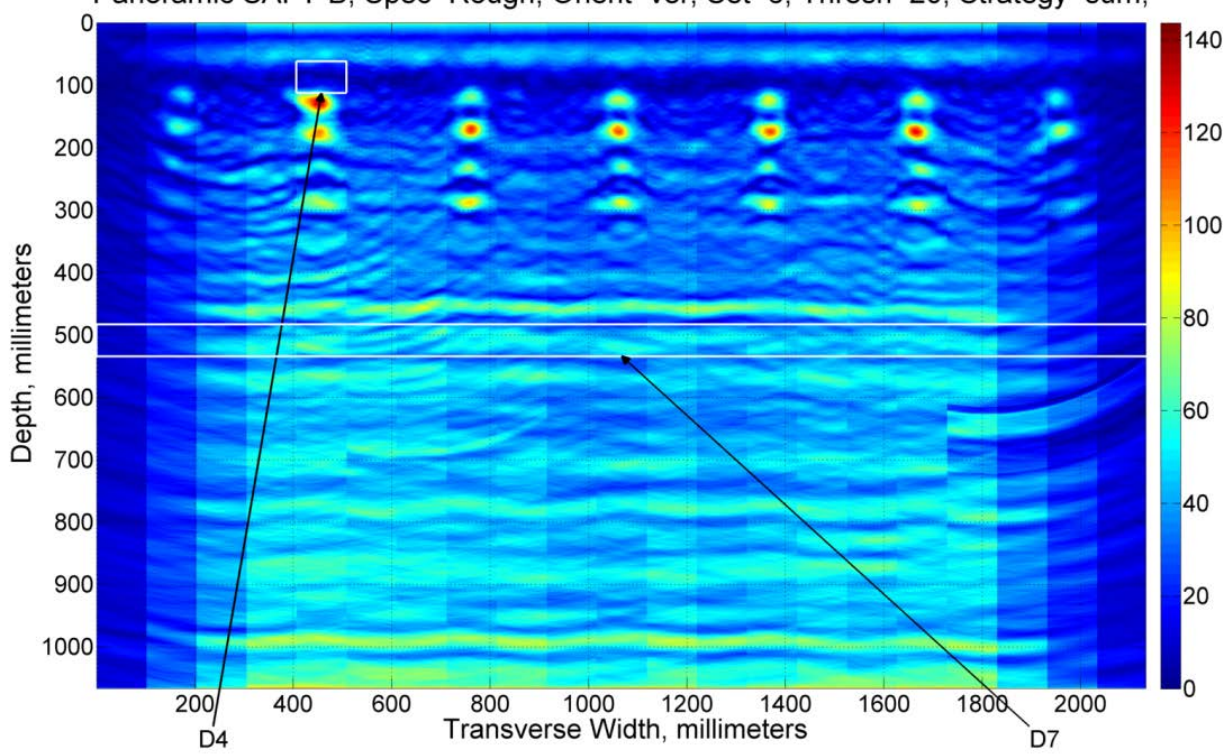

(a)

Specimen: Thick, Depth: 1066.8mm (42in), AbsofHilbert -- Node $16(4,1), 31.25 \sim 62.5 \mathrm{kHz}$ Panoramic SAFT-B, Spec=Rough, Orient=ver, Set=6, Thresh=20, Strategy=sum,

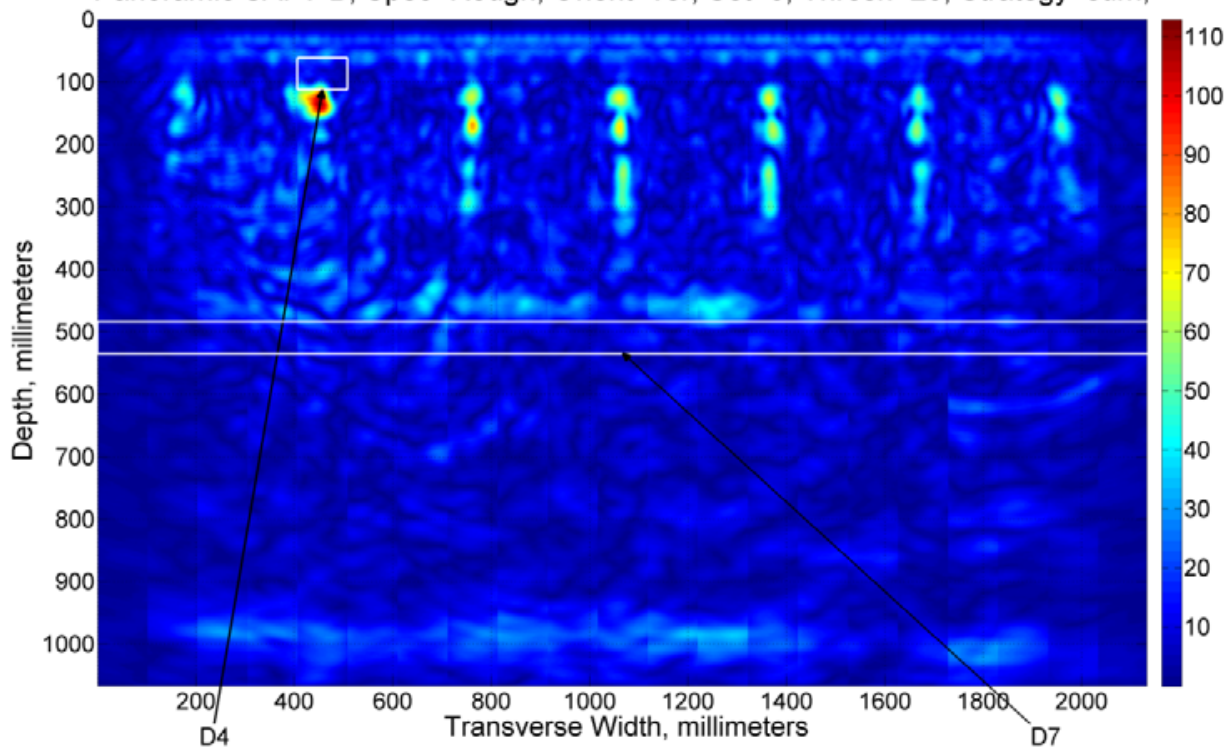

(b)

Fig. 37. Shallow cover improved detectability for Defect 4 for node 16 (b) compared to regular SAFT (a), vertical orientation \#2. 


\subsubsection{Defect 5}

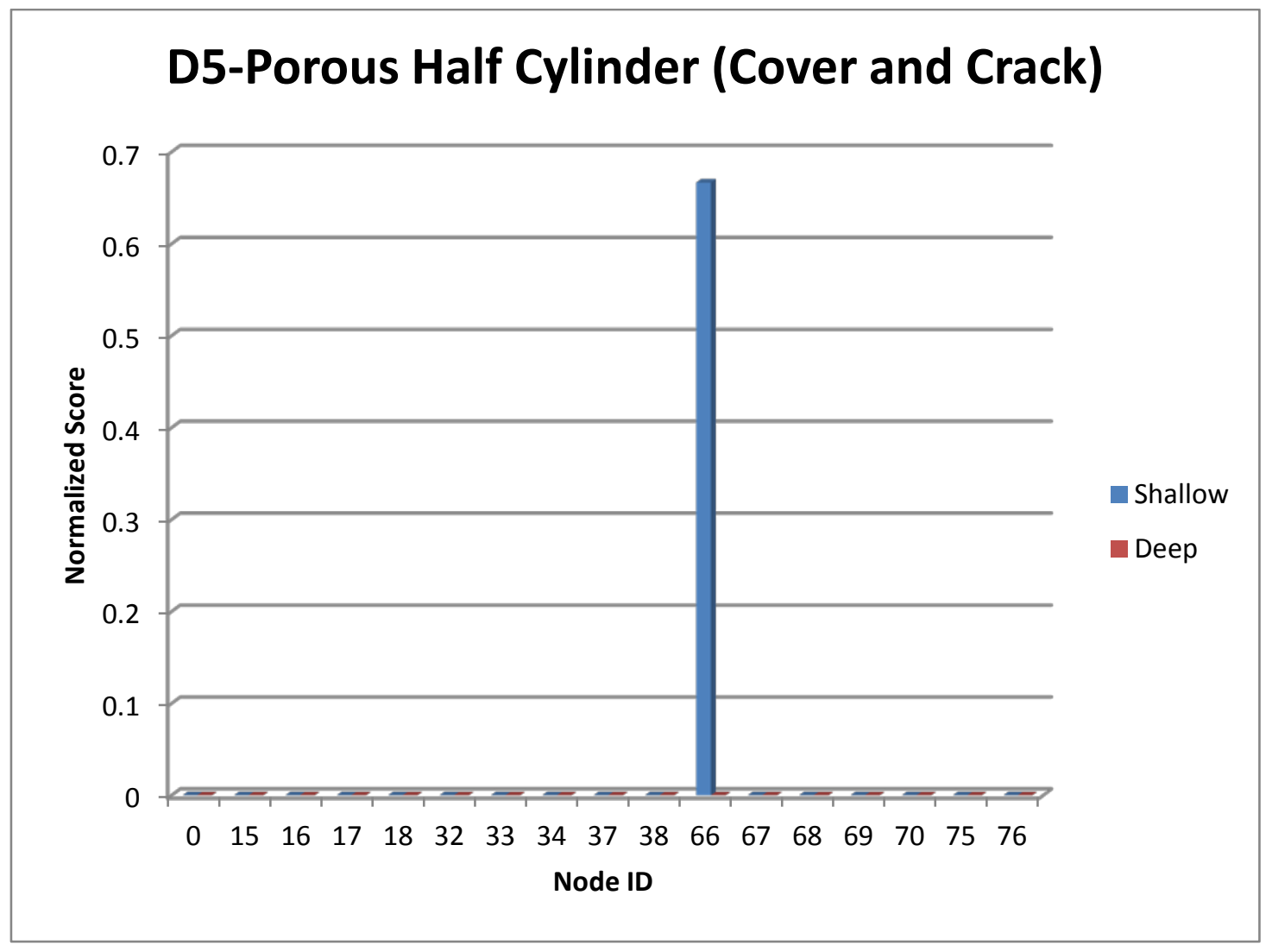

Fig. 38. Defect 5 analysis chart illustrating the normalized score for each node. 


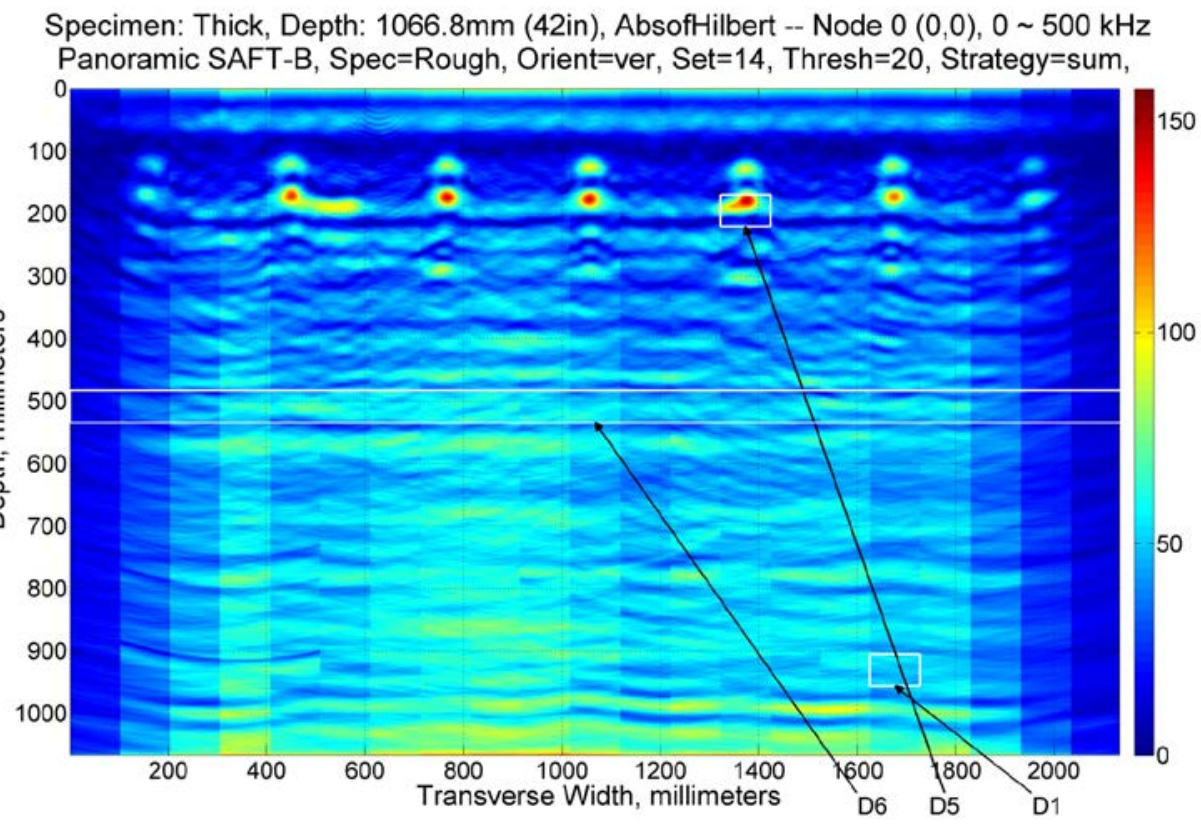

(a)

Specimen: Thick, Depth: 1066.8mm (42in), AbsofHilbert -- Node 66 (6,3), $23.4375 \sim 31.25 \mathrm{kHz}$

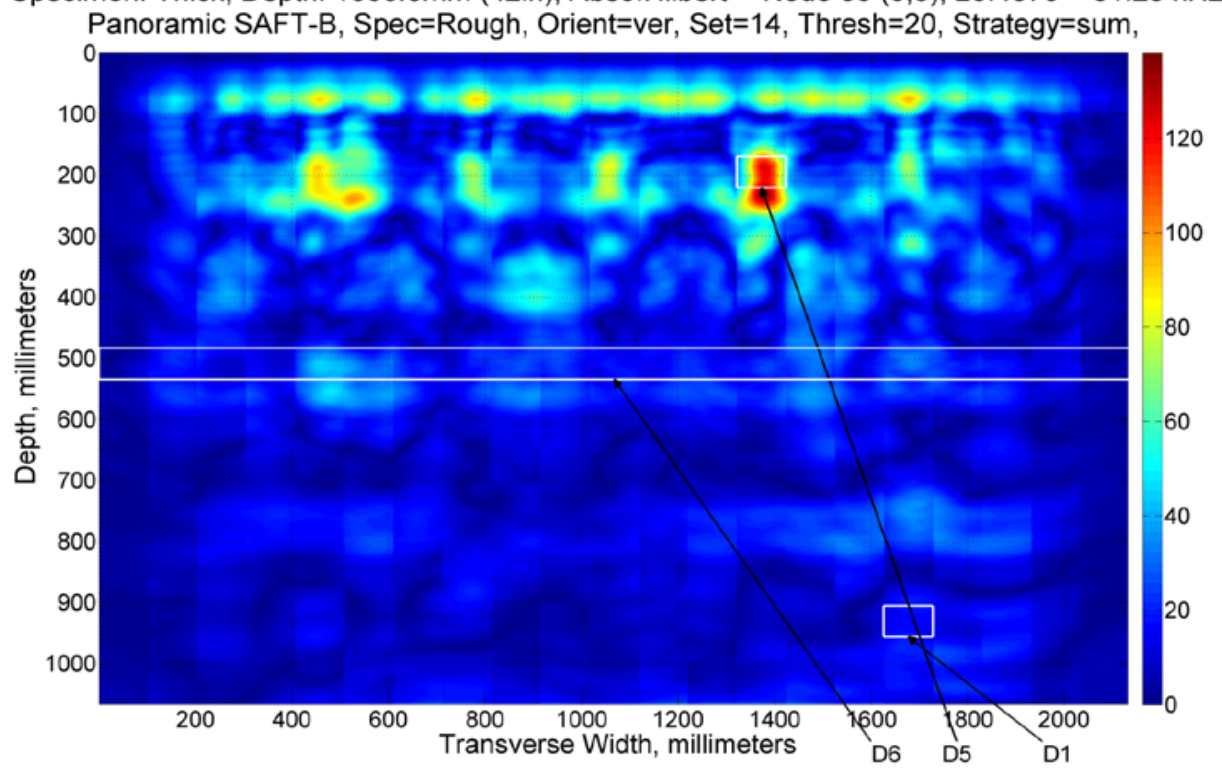

(b)

Fig. 39. Shallow cover improved detectability for Defect 5 for node 66 (b) compared to regular SAFT (a), vertical orientation \#1. 
Specimen: Thick, Depth: 1066.8mm (42in), AbsofHilbert -- Node $0(0,0), 0 \sim 500 \mathrm{kHz}$

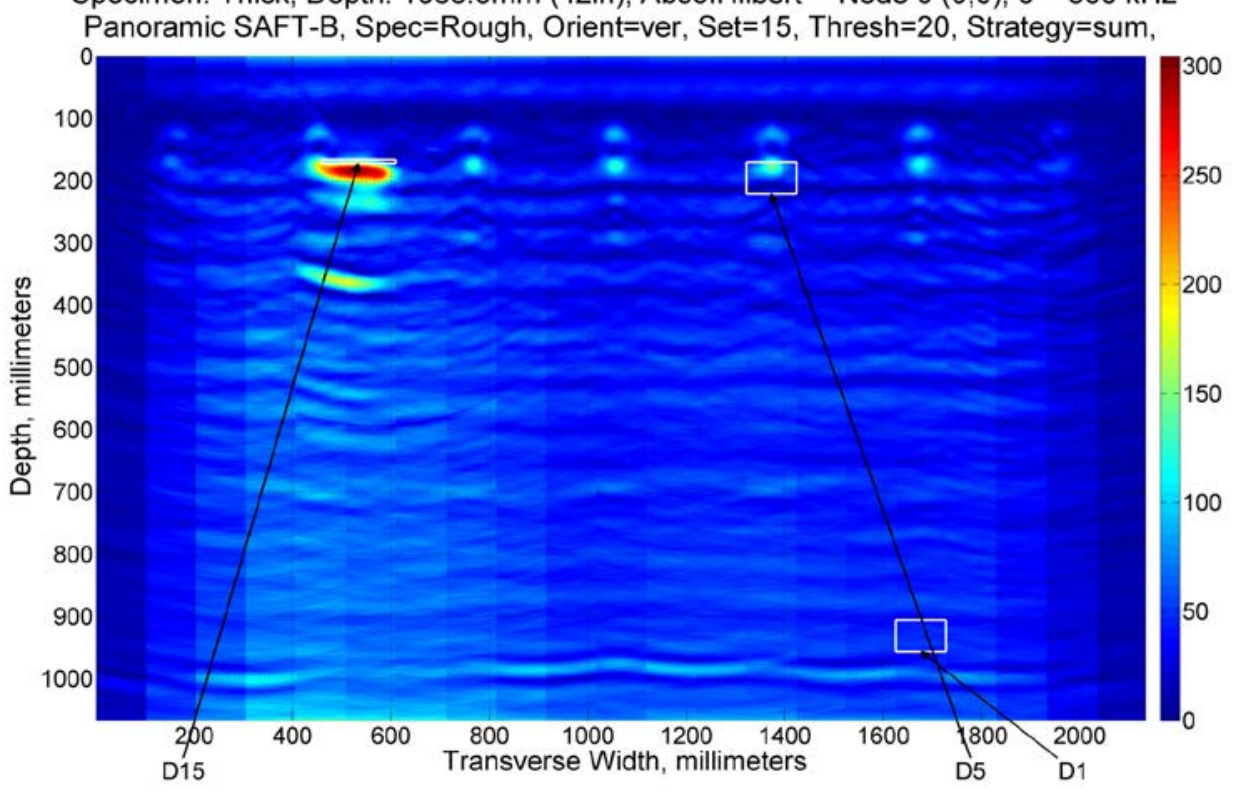

(a)

Specimen: Thick, Depth: 1066.8mm (42in), AbsofHilbert -- Node 66 (6,3), $23.4375 \sim 31.25 \mathrm{kHz}$ Panoramic SAFT-B, Spec=Rough, Orient=ver, Set=15, Thresh=20, Strategy=sum,

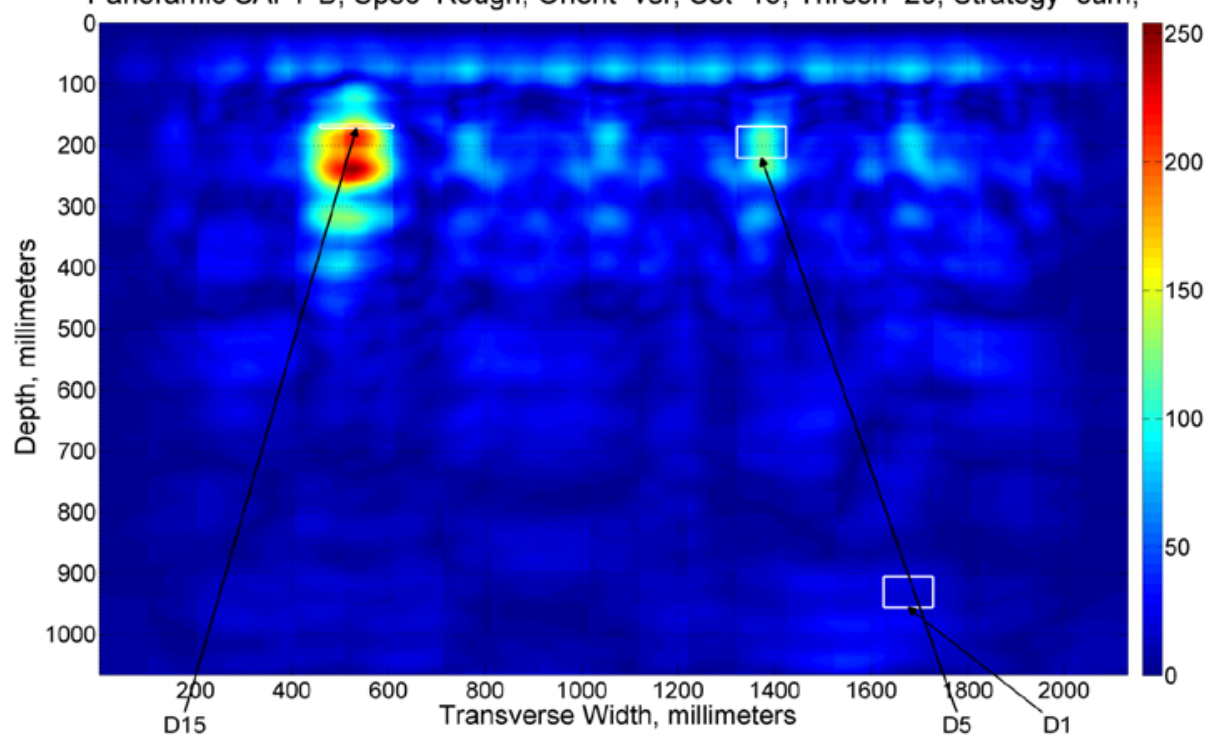

(b)

Fig. 40. Shallow cover improved detectability for Defect 5 for node 66 (b) compared to regular SAFT (a), vertical orientation \#2. 


\subsubsection{Defect 6}

Note that Defect 6 does not have a shallow or deep, because the center is in the midpoint of the concrete. So, Defect 6 analysis is categorized into the rough and smooth concrete sides.

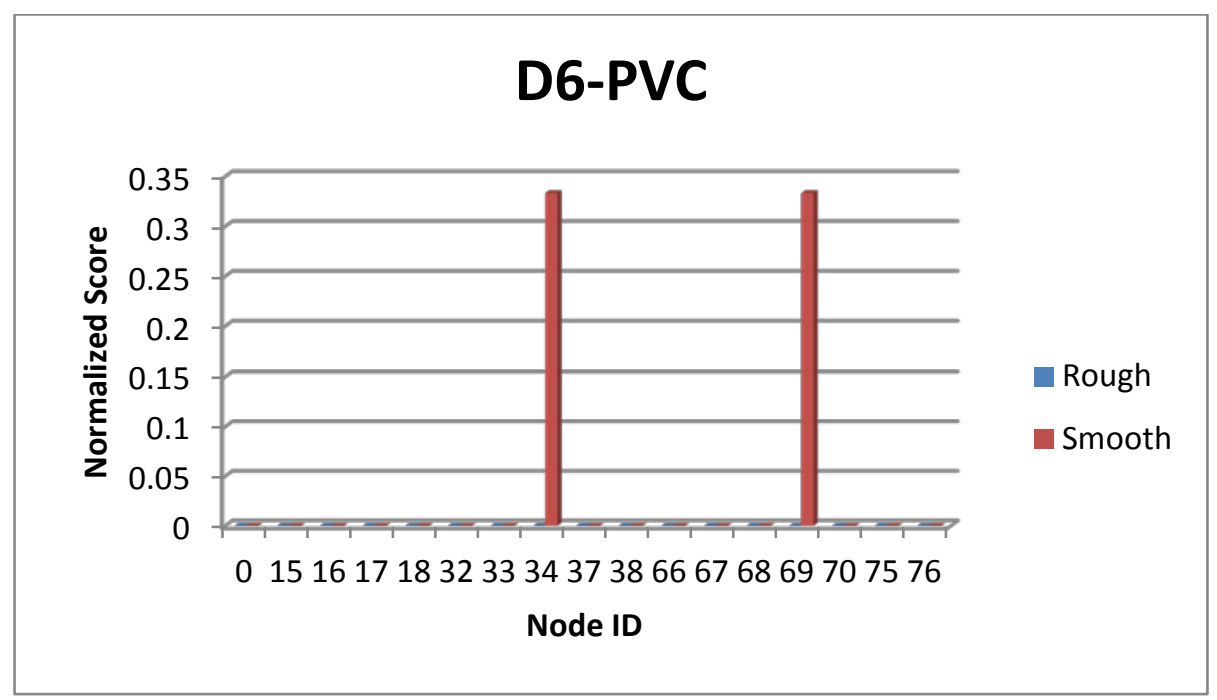

Fig. 41. Defect 6 analysis chart illustrating the normalized score for each node. 


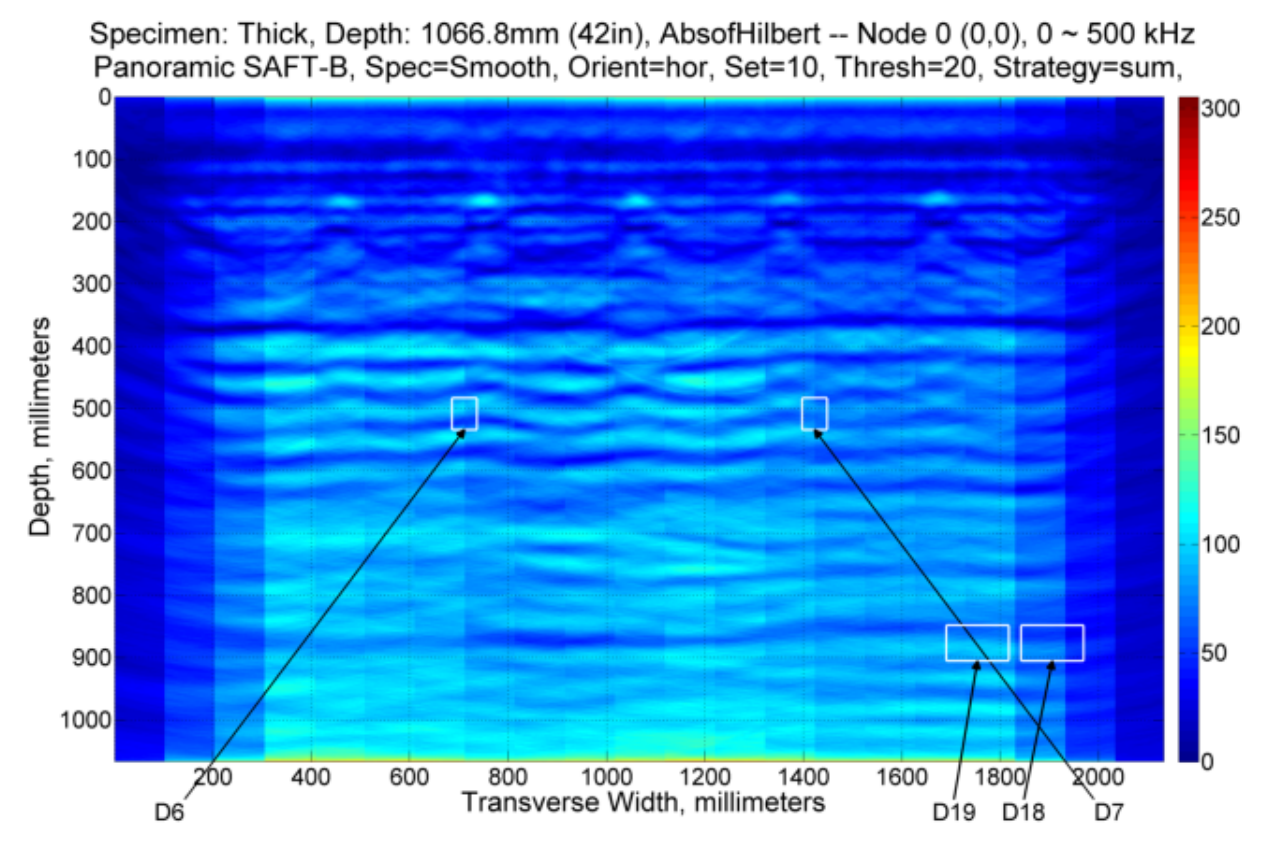

(a)

Specimen: Thick, Depth: 1066.8mm (42in), AbsofHilbert -- Node 69 (6,6), $39.0625 \sim 46.875 \mathrm{kHz}$

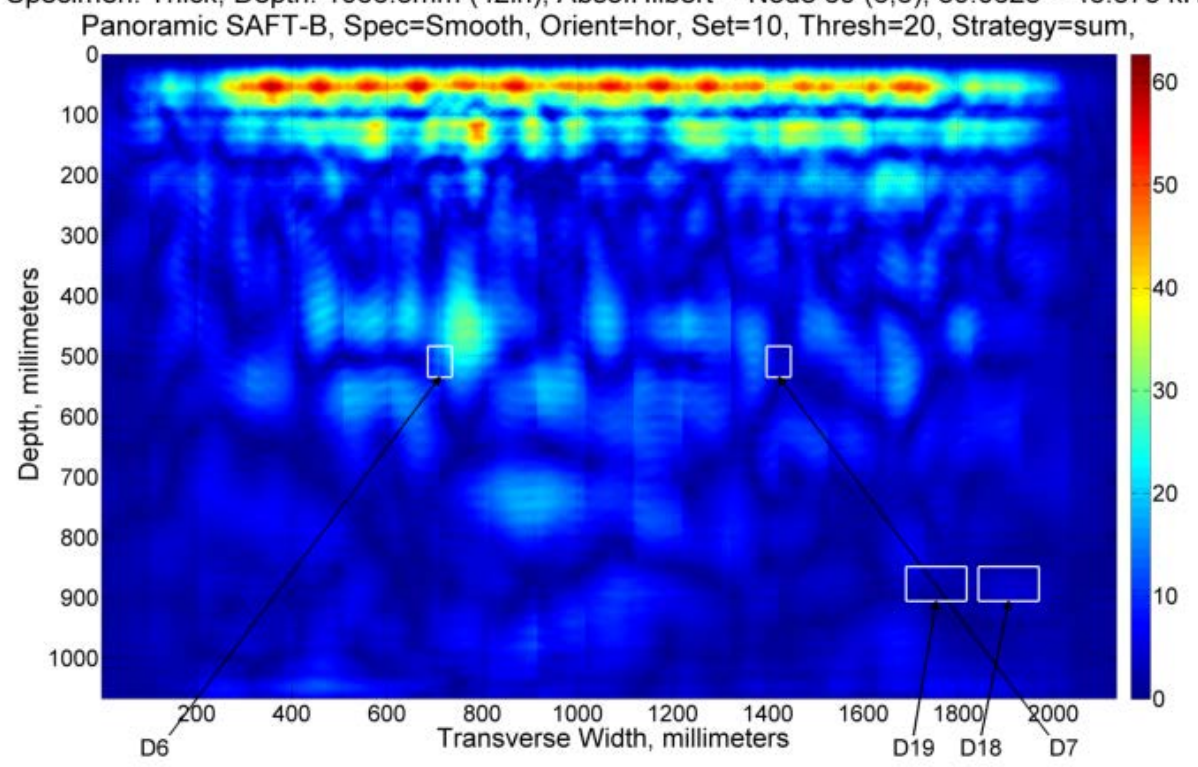

(b)

Fig. 42. Smooth side improved detectability for Defect 6 for node 69 (b) compared to regular SAFT (a). 


\subsubsection{Defect 7}

Note Defect 7 does not have a shallow or deep, because the center is in the midpoint of the concrete. So, Defect 7 analysis is categorized into the rough and smooth concrete sides.

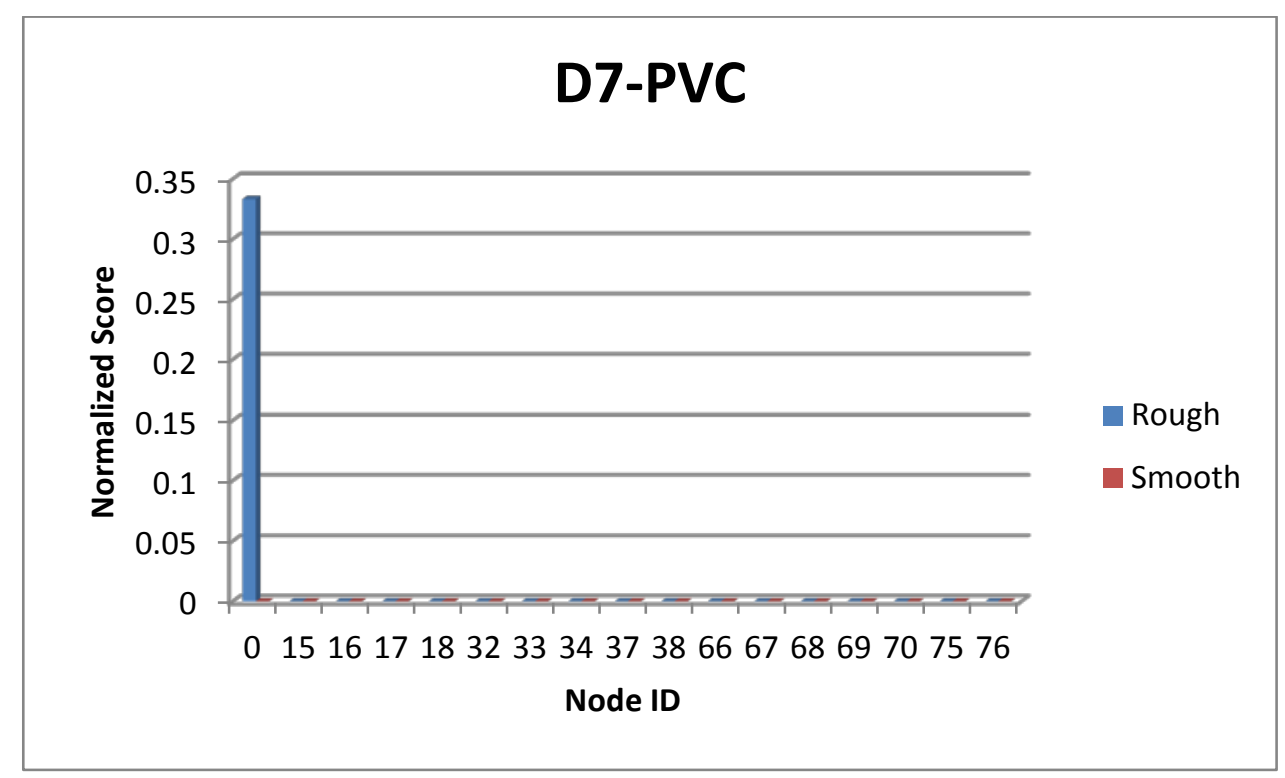

Fig. 43. Defect 7 analysis chart illustrating the normalized score for each node.

10.1.8 Defect 8

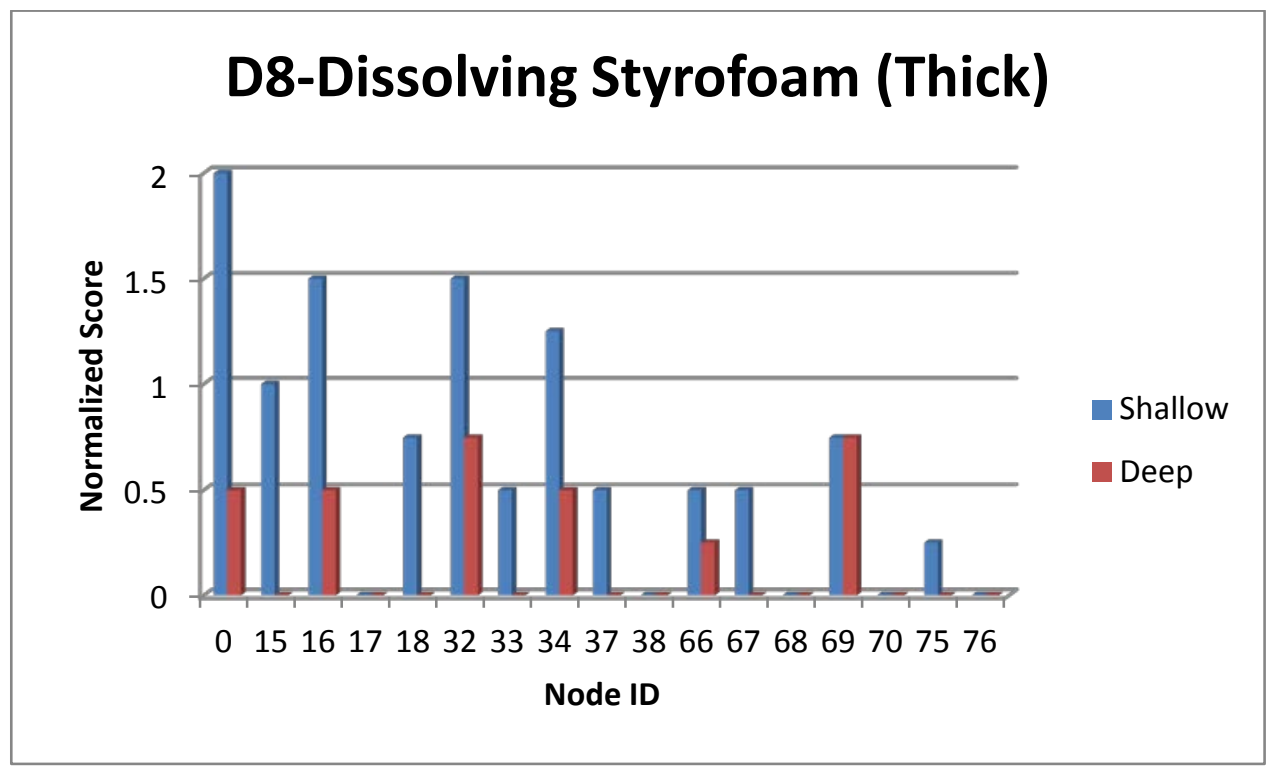

Fig. 44. Defect 8 analysis chart illustrating the normalized score for each node. 


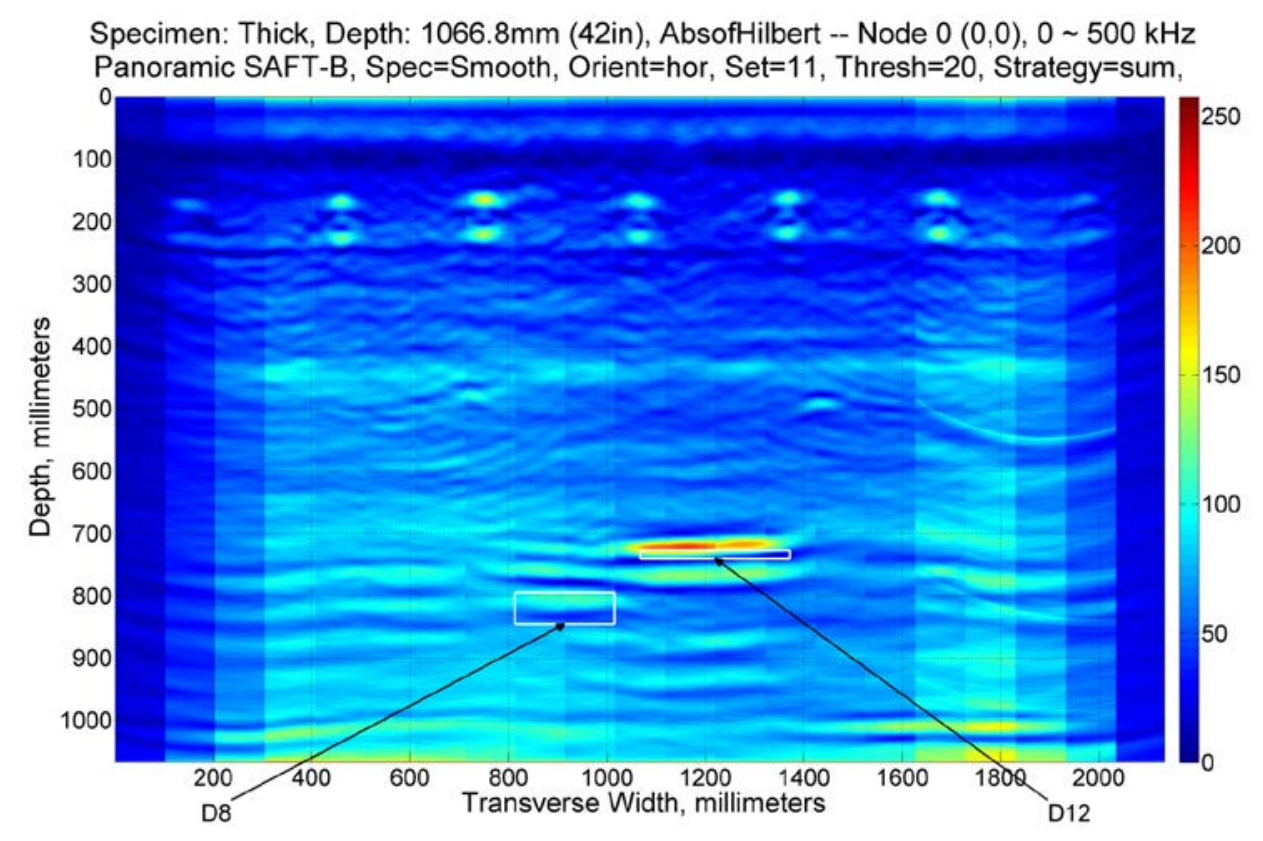

(a)

Specimen: Thick, Depth: 1066.8mm (42in), AbsofHilbert -- Node $32(5,1), 15.625 \sim 31.25 \mathrm{kHz}$

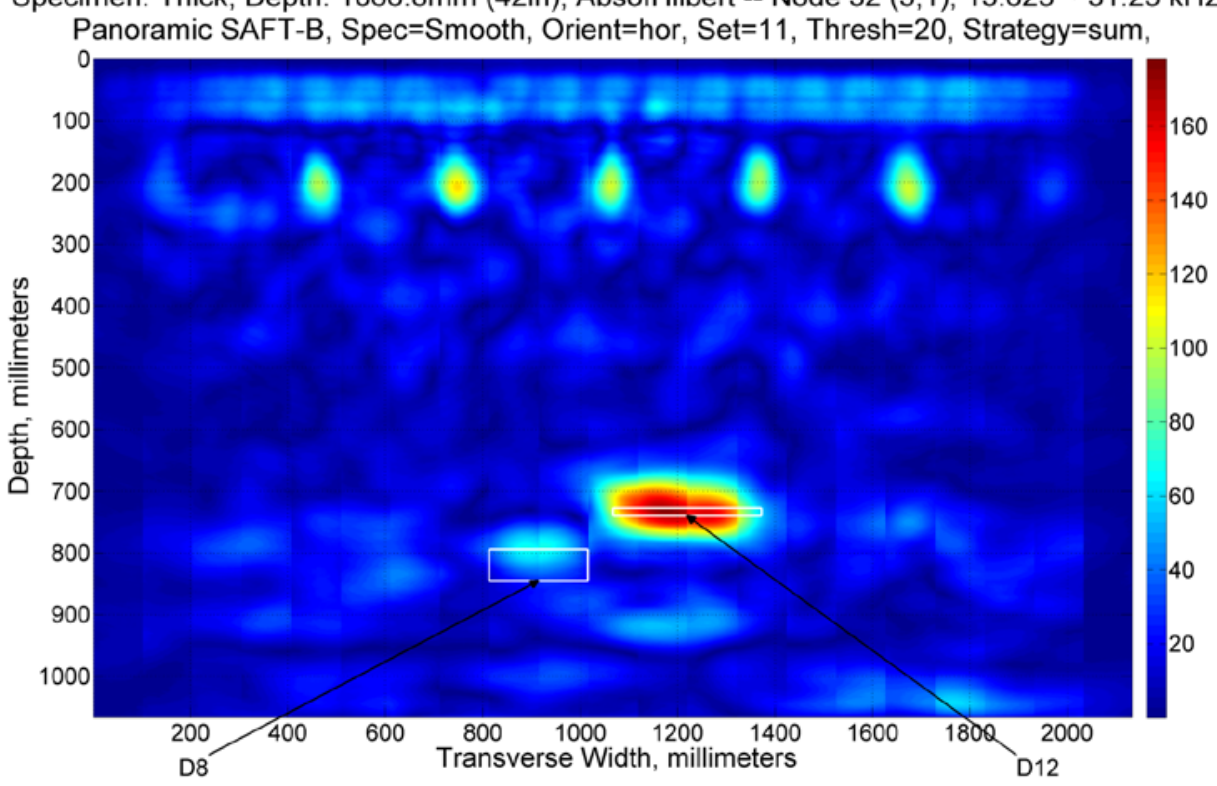

(b)

Fig. 45. Deep cover improved detectability for Defect 8 for node 32 (b) compared to regular SAFT (a), horizontal orientation. 


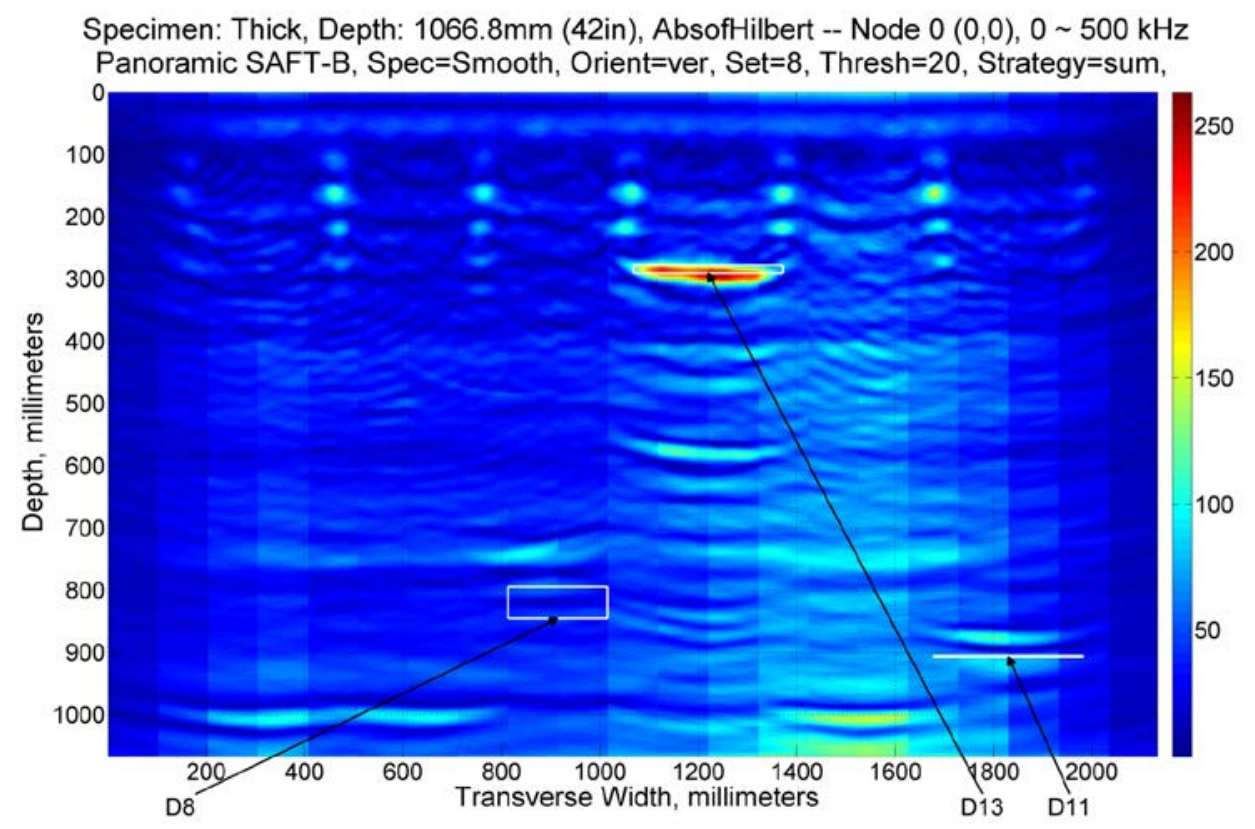

(a)

Specimen: Thick, Depth: 1066.8mm (42in), AbsofHilbert -- Node $66(6,3), 23.4375 \sim 31.25 \mathrm{kHz}$

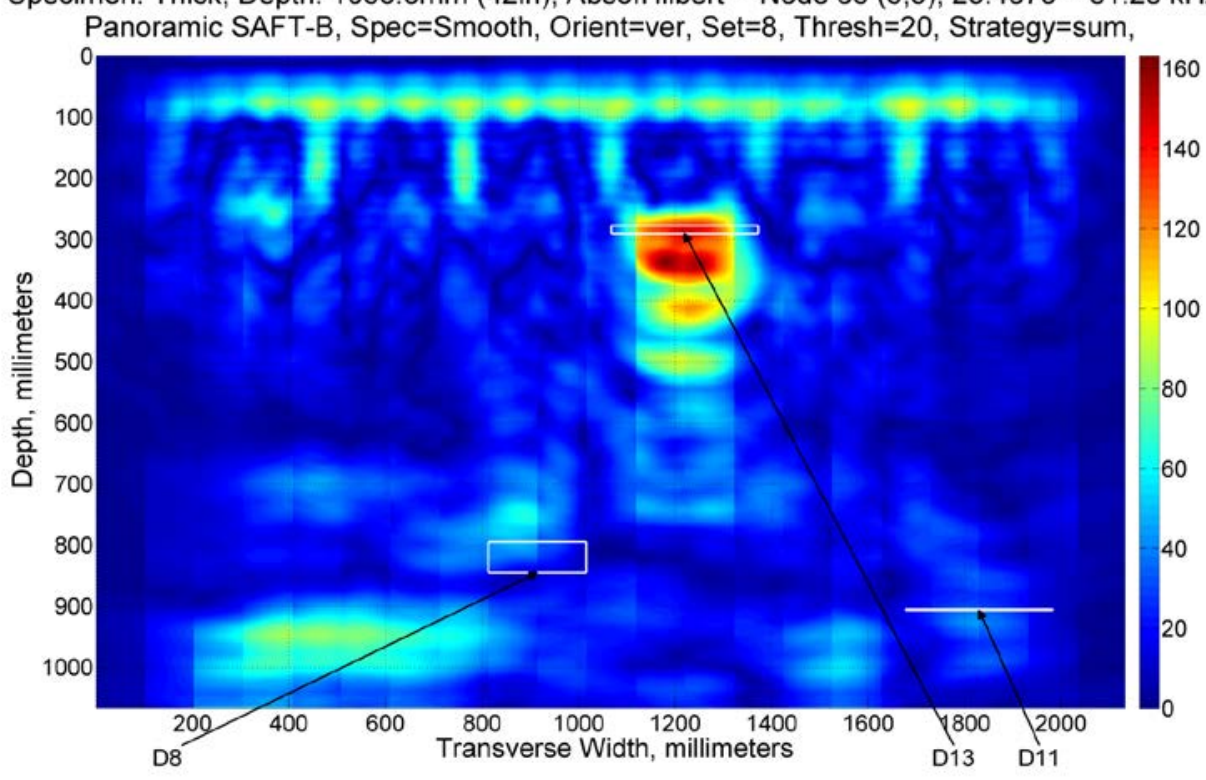

(b)

Fig. 46. Deep cover improved detectability for Defect 8 for node 66 (b) compared to regular SAFT (a), vertical orientation. 


\subsubsection{Defect 9}

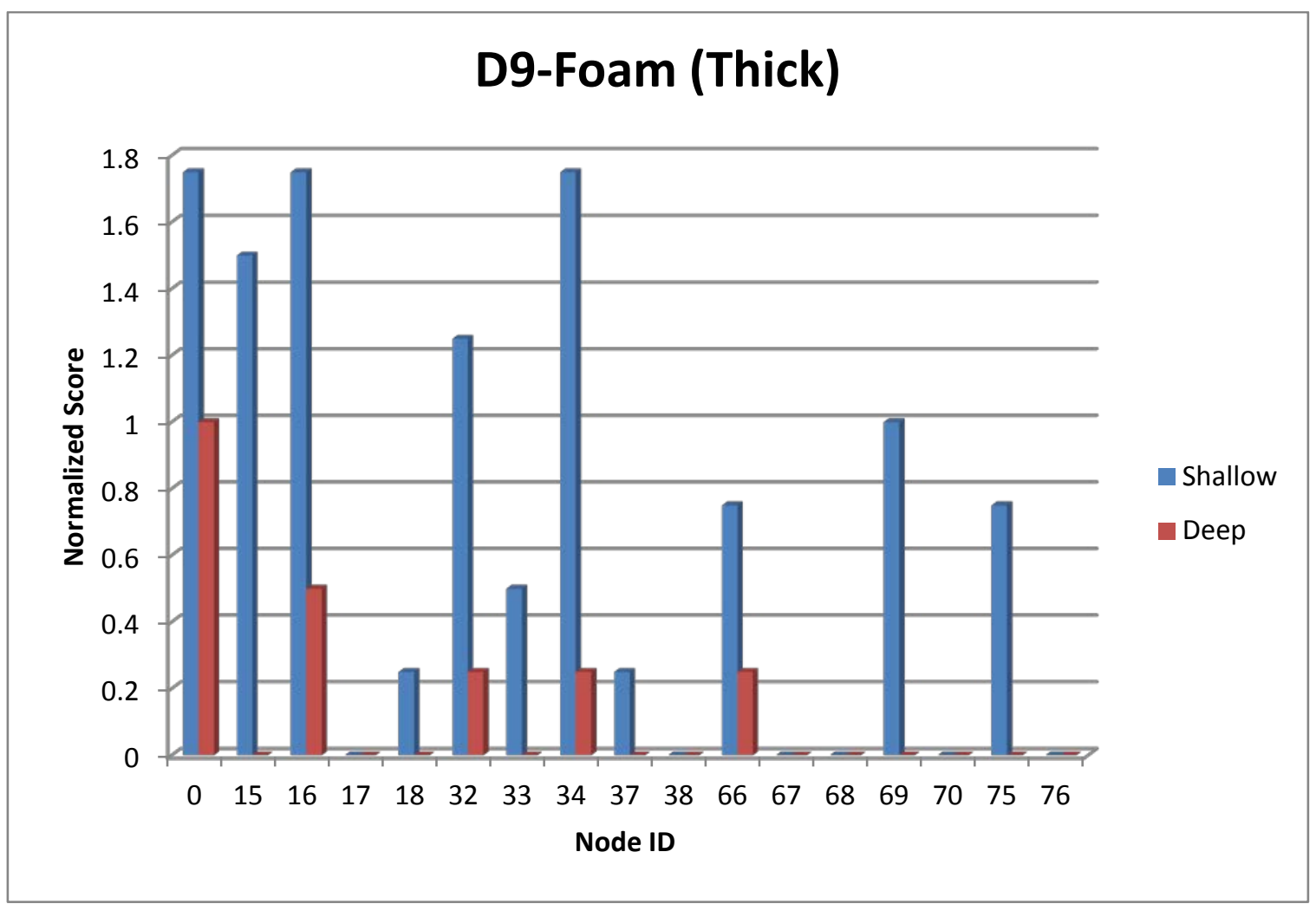

Fig. 47. Defect 9 analysis chart illustrating the normalized score for each node 
Specimen: Thick, Depth: 1066.8mm (42in), AbsofHilbert -- Node $0(0,0), 0 \sim 500 \mathrm{kHz}$ Panoramic SAFT-B, Spec=Smooth, Orient=ver, Set=11, Thresh=20, Strategy=sum,

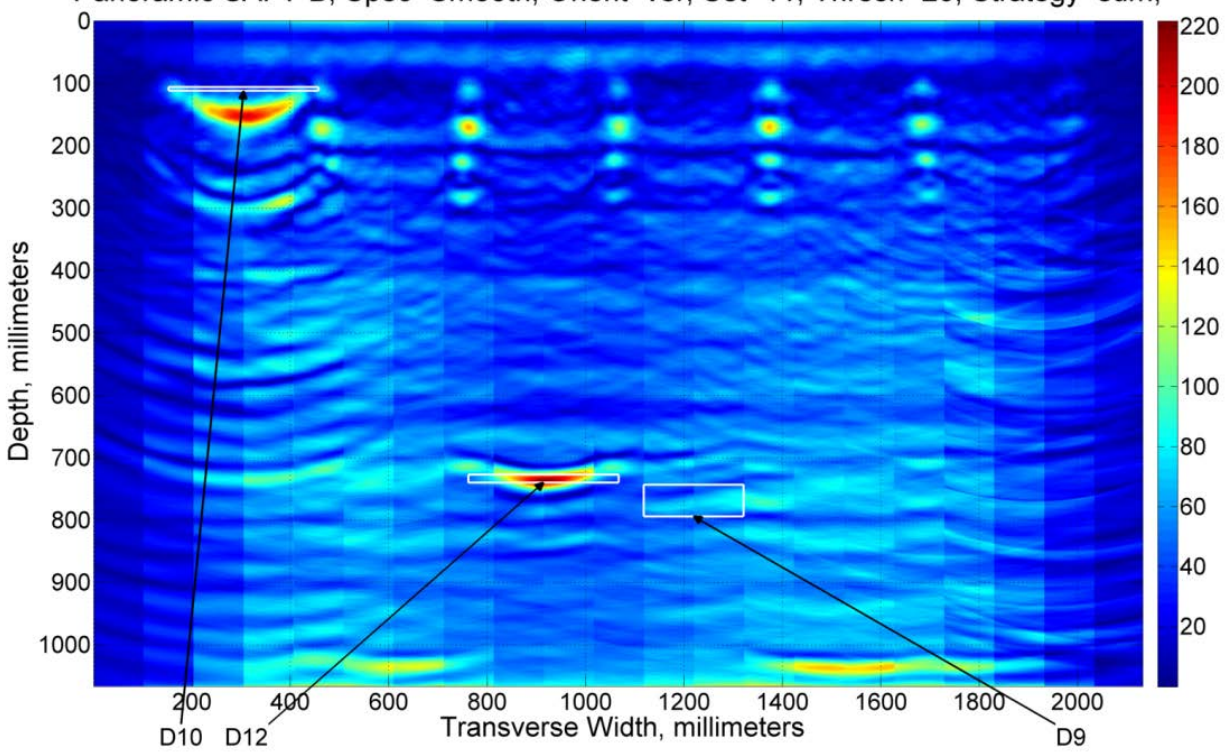

(a)

Specimen: Thick, Depth: 1066.8mm (42in), AbsofHilbert -- Node $66(6,3), 23.4375 \sim 31.25 \mathrm{kHz}$ Panoramic SAFT-B, Spec=Smooth, Orient=ver, Set=11, Thresh=20, Strategy=sum,

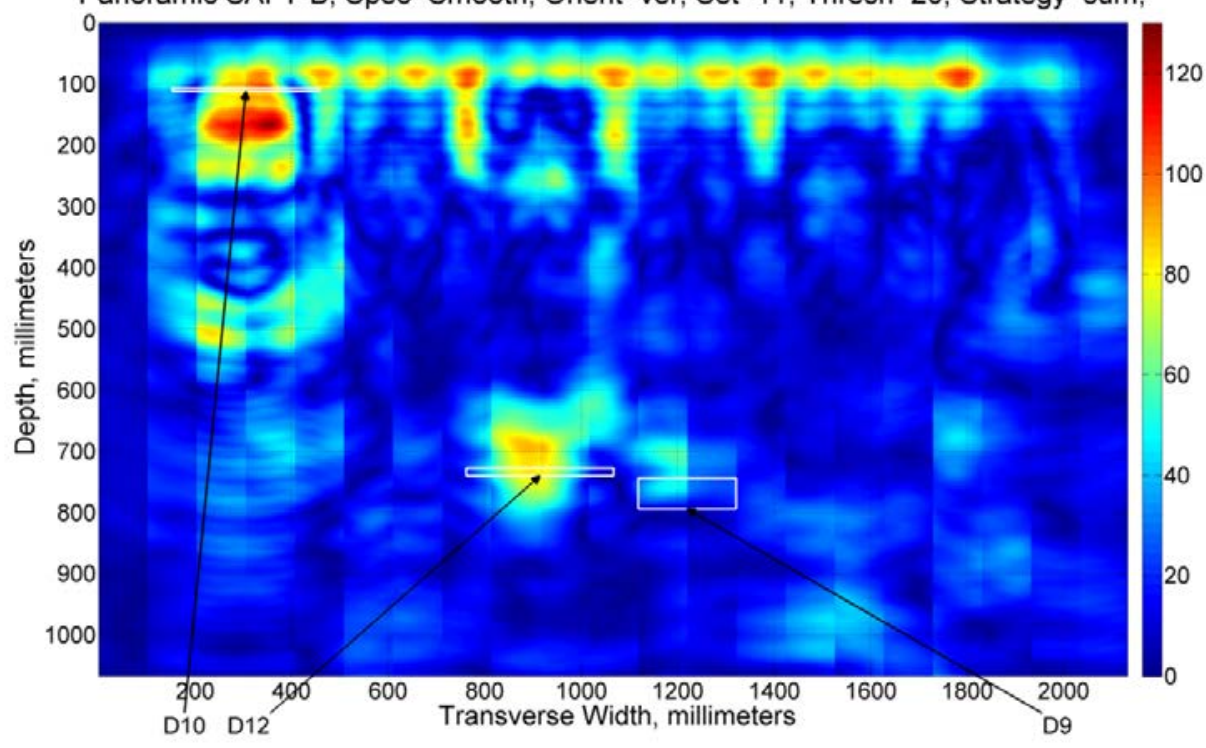

(b)

Fig. 48. Deep cover improved detectability for Defect 9 for node 66 (b) compared to regular SAFT (a). 
10.1.10 Defect 10

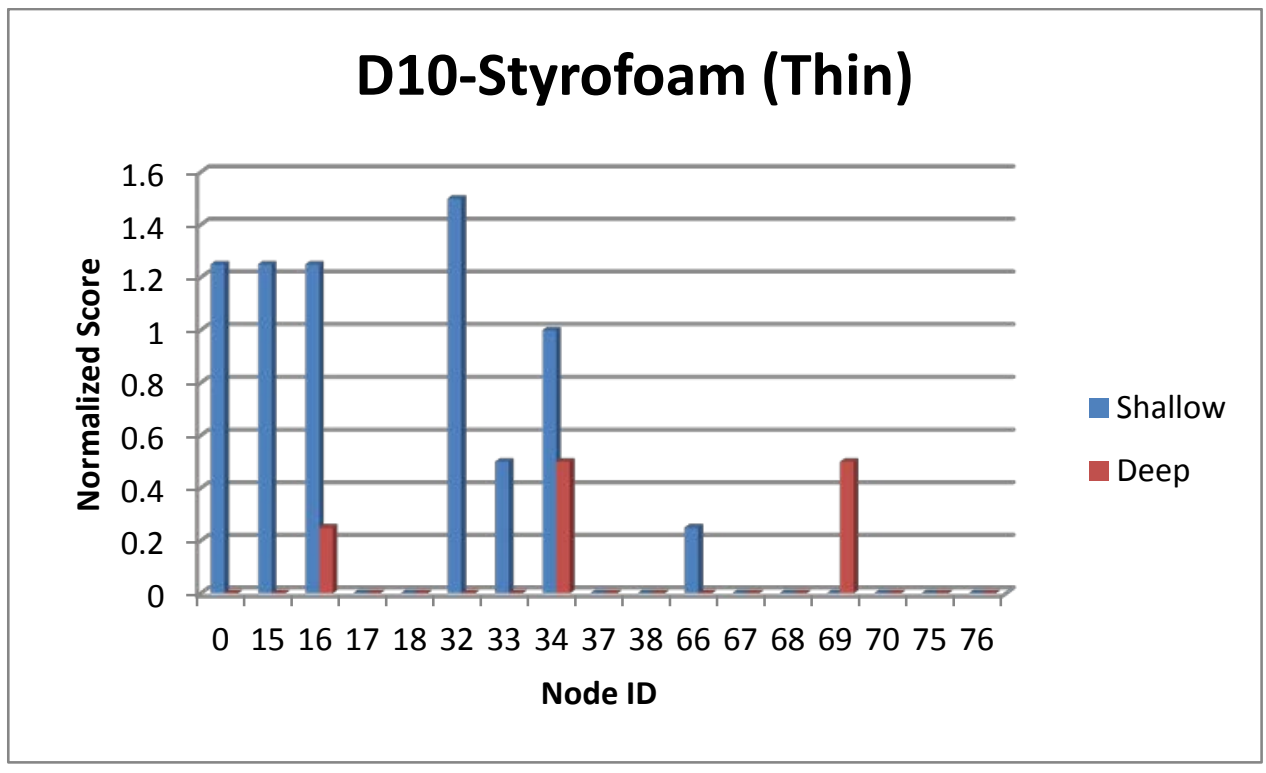

Fig. 49. Defect 10 analysis chart illustrating the normalized score for each node. 


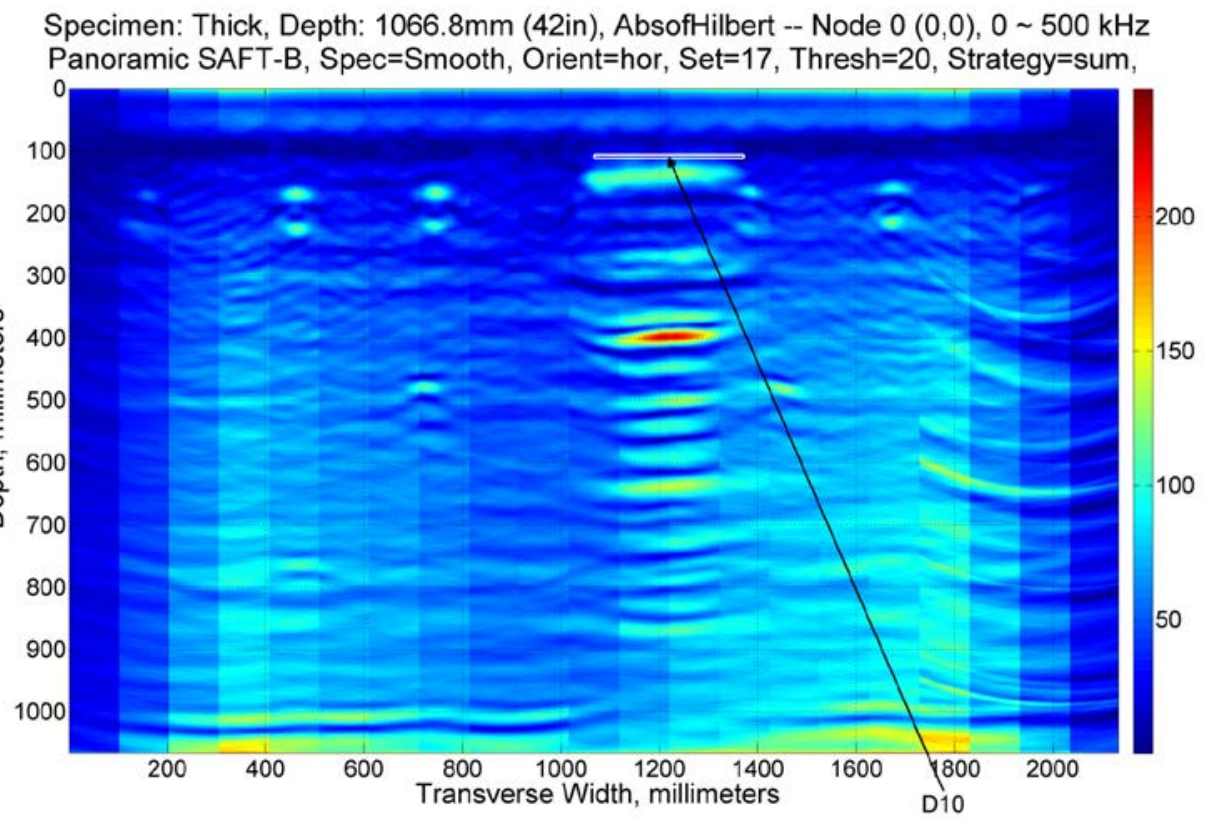

(a)

Specimen: Thick, Depth: 1066.8mm (42in), AbsofHilbert -- Node $32(5,1), 15.625 \sim 31.25 \mathrm{kHz}$

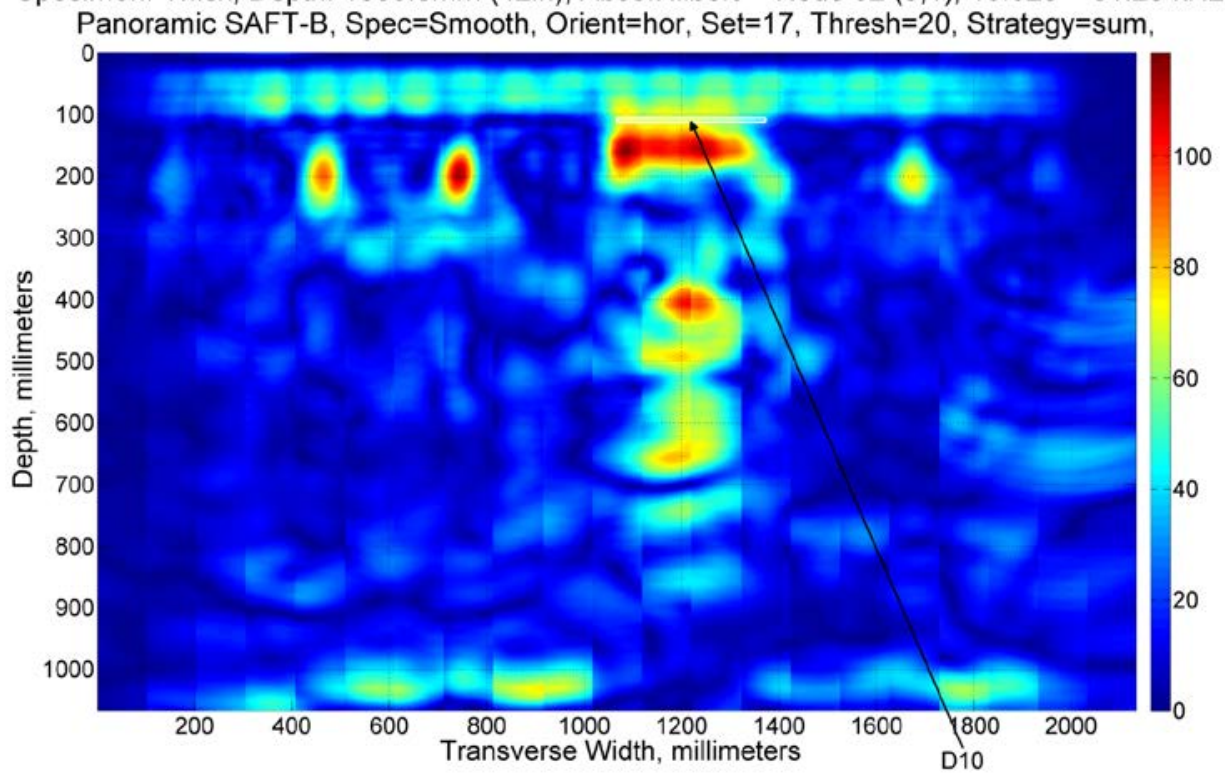

(b)

Fig. 50. Shallow cover improved detectability for Defect 10 for node 32 (b) compared to regular SAFT (a), horizontal orientation \#1. 


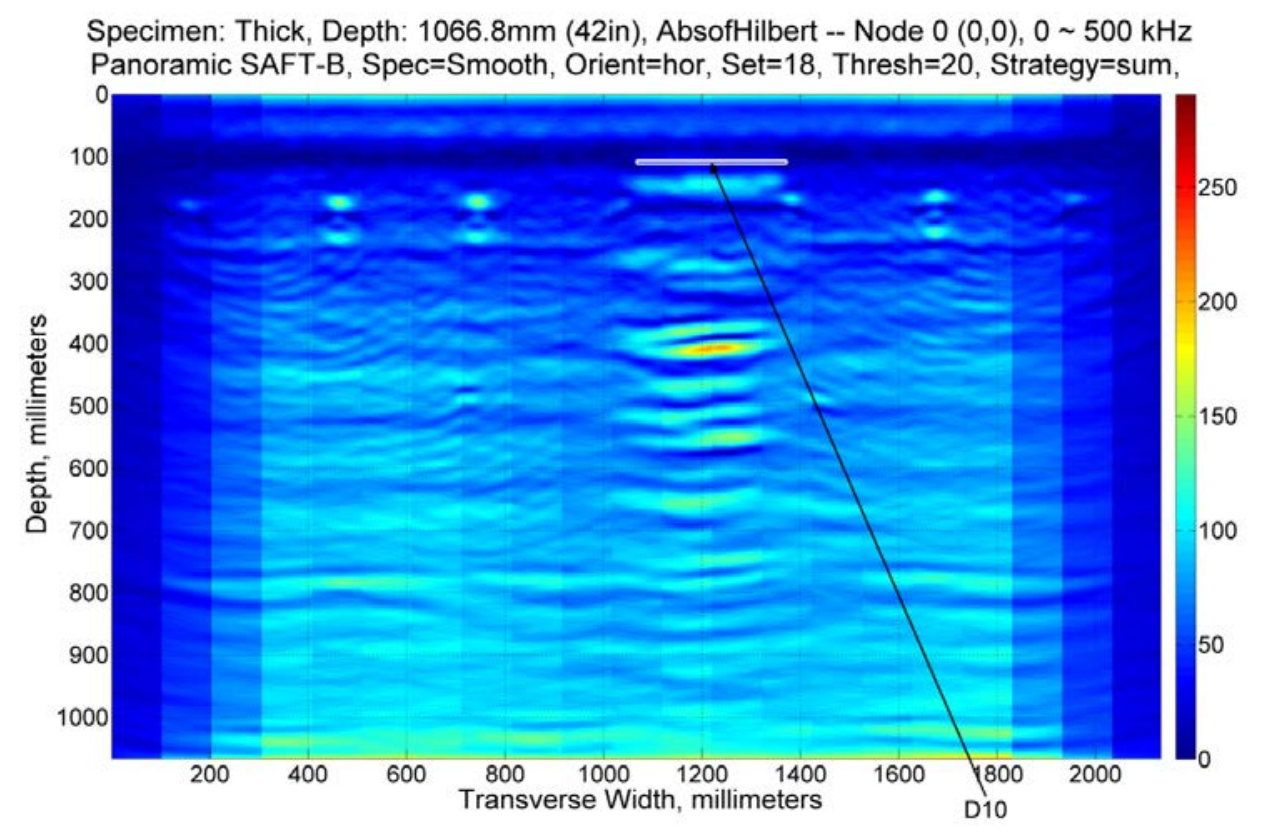

(a)

Specimen: Thick, Depth: 1066.8mm (42in), AbsofHilbert -- Node $32(5,1), 15.625 \sim 31.25 \mathrm{kHz}$

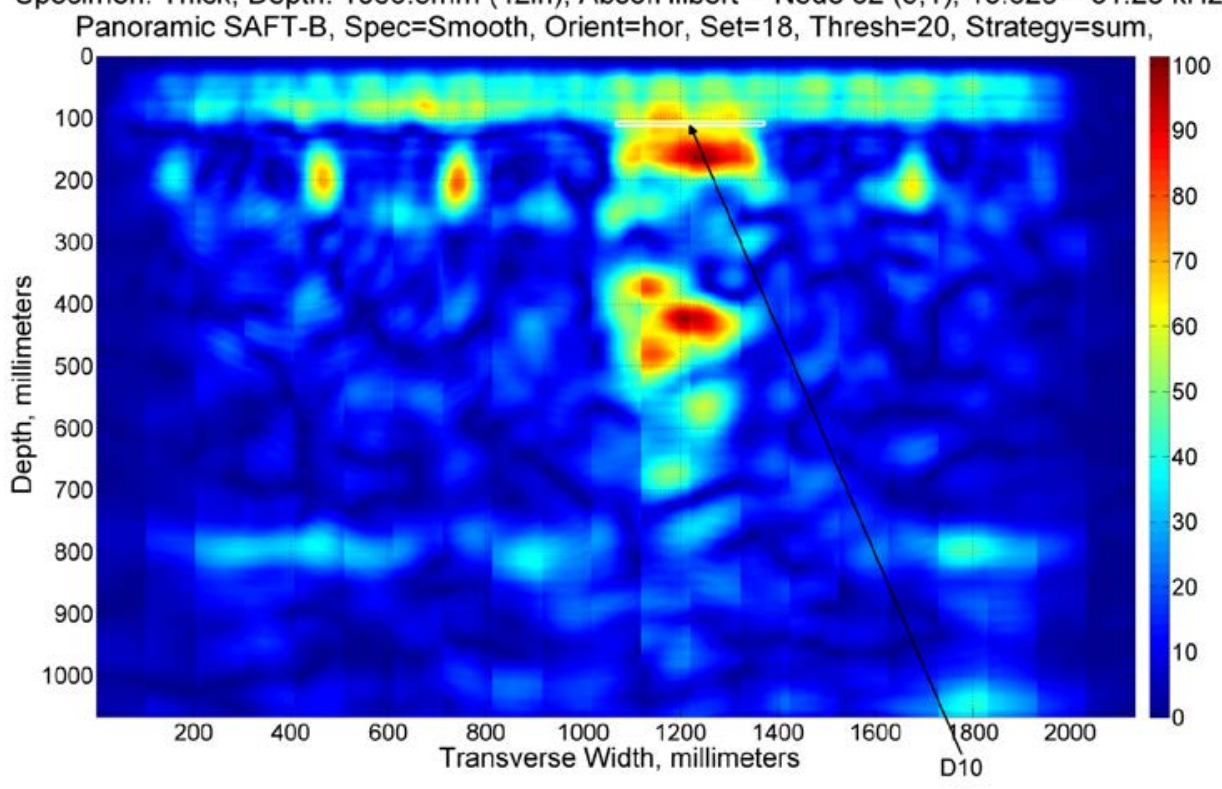

(b)

Fig. 51. Shallow cover improved detectability for Defect 10 for node 32 (b) compared to regular SAFT (a), horizontal orientation \#2. 
Specimen: Thick, Depth: 1066.8mm (42in), AbsofHilbert -- Node $0(0,0), 0 \sim 500 \mathrm{kHz}$ Panoramic SAFT-B, Spec=Rough, Orient=hor, Set=2, Thresh=20, Strategy=sum,

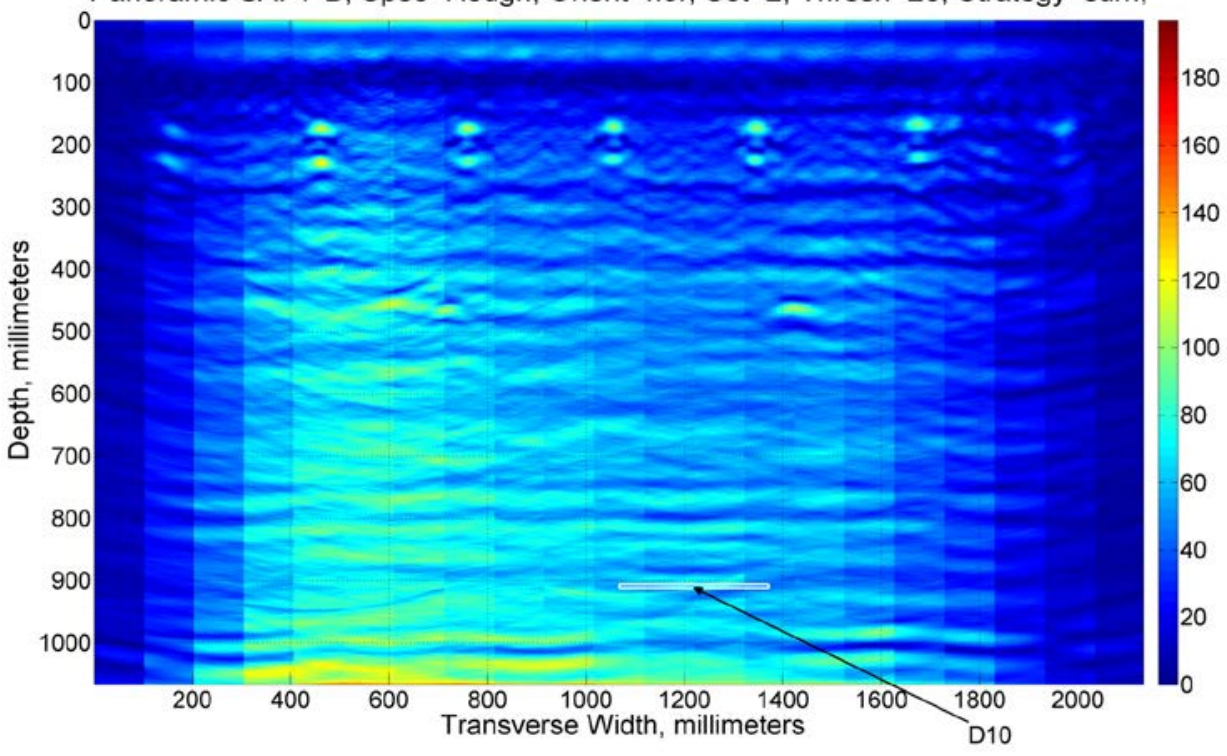

(a)

Specimen: Thick, Depth: 1066.8mm (42in), AbsofHilbert -- Node 34 (5,3), $46.875 \sim 62.5 \mathrm{kHz}$ Panoramic SAFT-B, Spec=Rough, Orient=hor, Set=2, Thresh $=20$, Strategy $=$ sum,

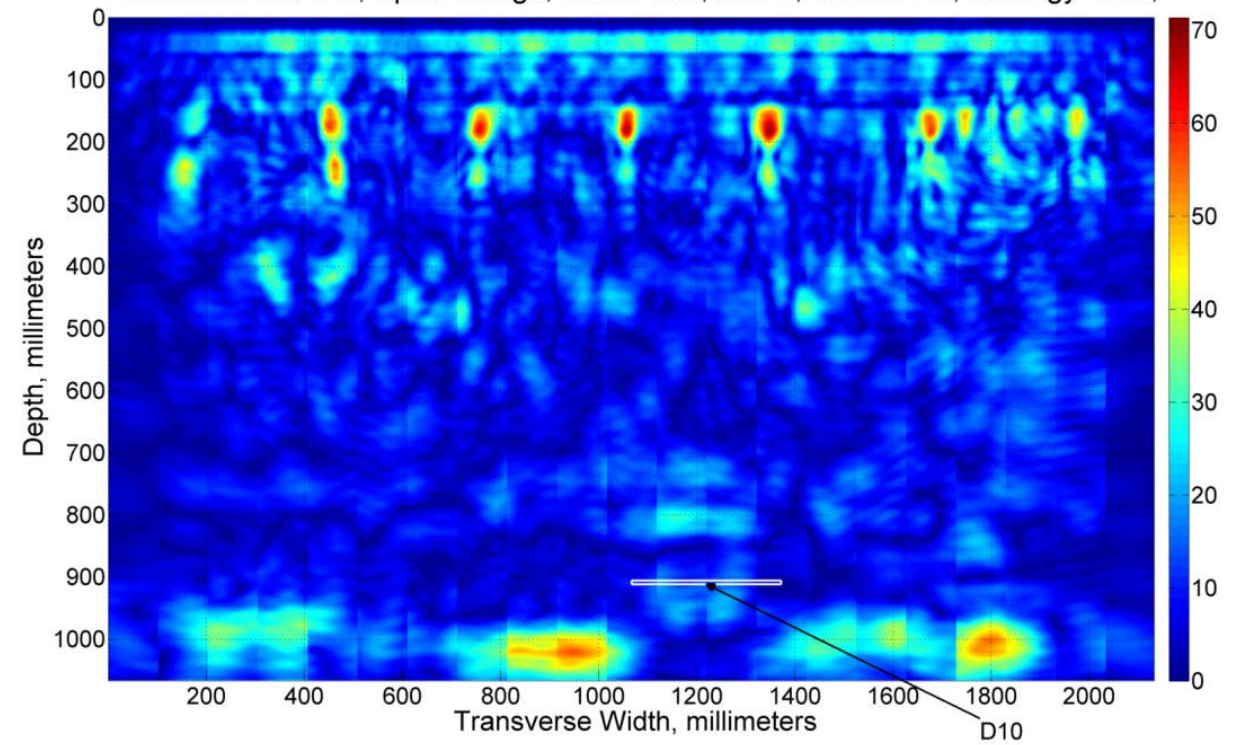

(b)

Fig. 52. Deep cover improved detectability for Defect 10 for node 34 (b) compared to regular SAFT (a), horizontal orientation \#1. 
Specimen: Thick, Depth: 1066.8mm (42in), AbsofHilbert -- Node $0(0,0), 0 \sim 500 \mathrm{kHz}$ Panoramic SAFT-B, Spec=Rough, Orient=hor, Set=3, Thresh=20, Strategy=sum,

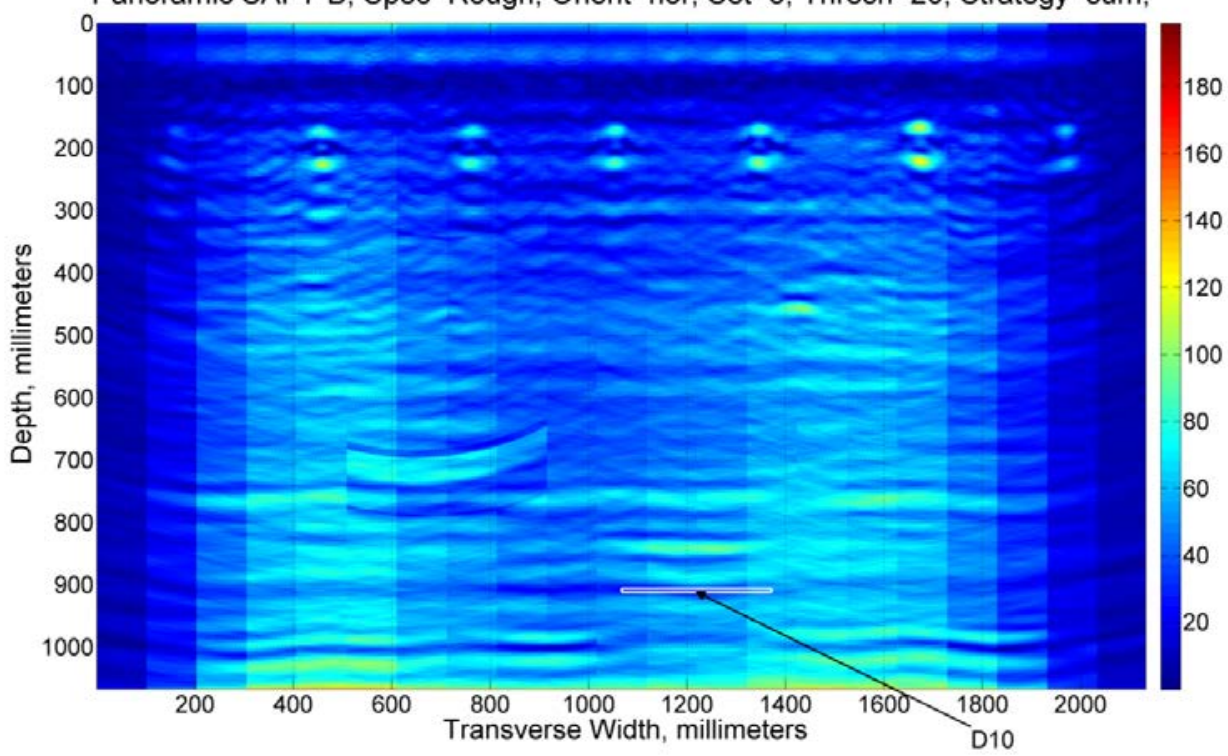

(a)

Specimen: Thick, Depth: 1066.8mm (42in), AbsofHilbert -- Node 34 (5,3), $46.875 \sim 62.5 \mathrm{kHz}$ Panoramic SAFT-B, Spec=Rough, Orient=hor, Set=3, Thresh=20, Strategy=sum,

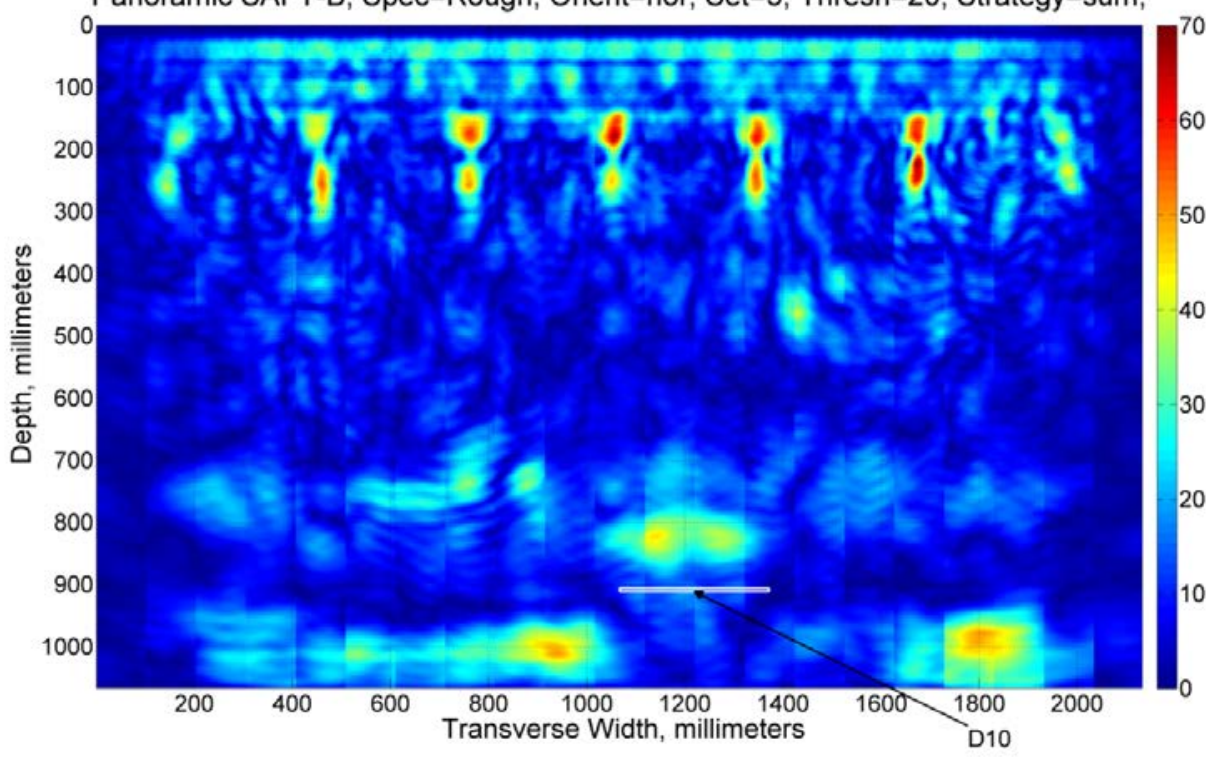

(b)

Fig. 53. Deep cover improved detectability for Defect 10 for node 34 (b) compared to regular SAFT (a), horizontal orientation \#2. 
10.1.11 Defect 11

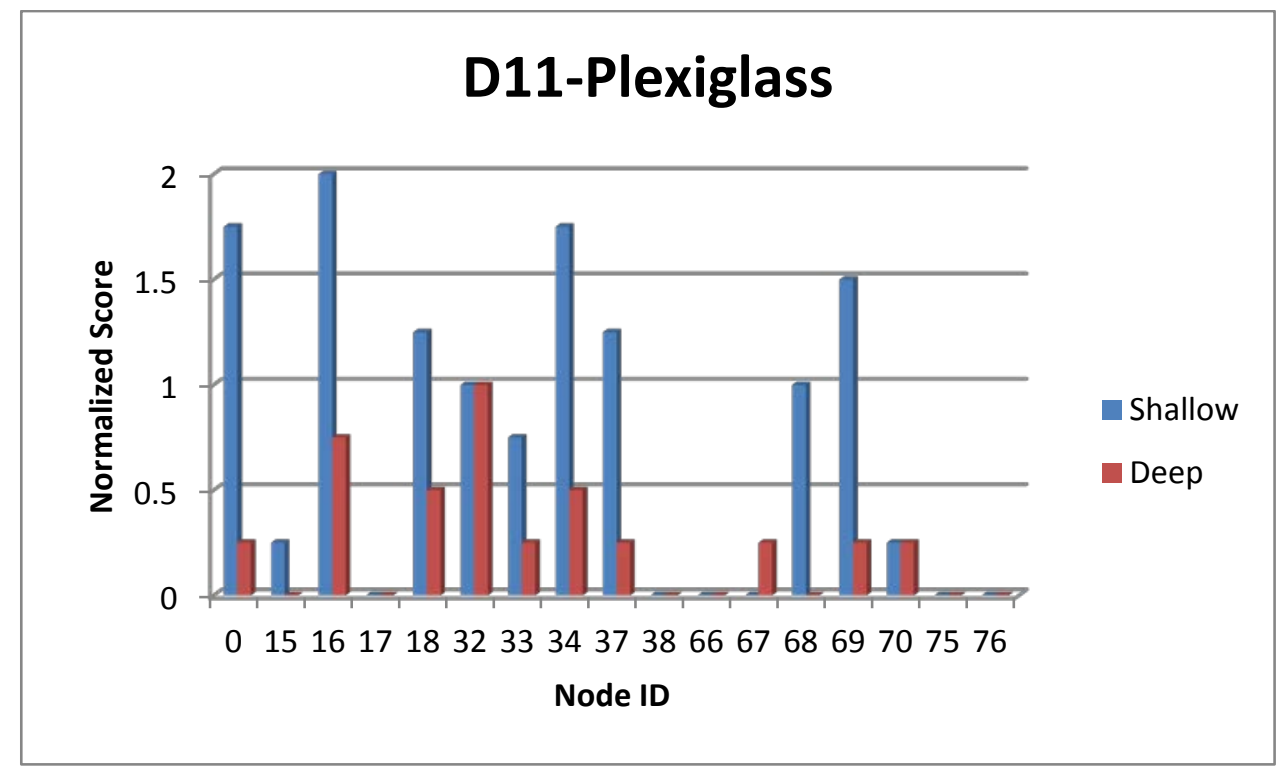

Fig. 54. Defect 11 analysis chart illustrating the normalized score for each node. 


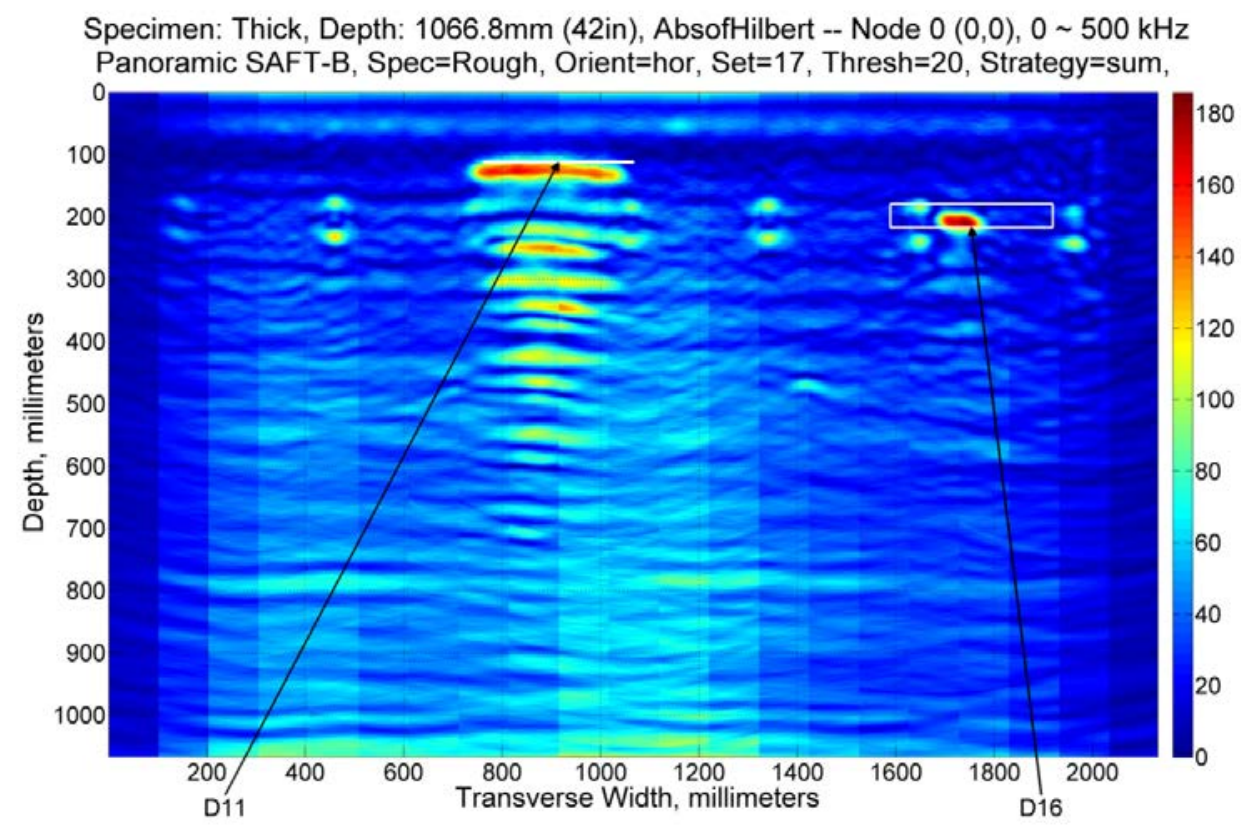

(a)

Specimen: Thick, Depth: 1066.8mm (42in), AbsofHilbert -- Node $18(4,3), 93.75 \sim 125 \mathrm{kHz}$ Panoramic SAFT-B, Spec=Rough, Orient=hor, Set=17, Thresh=20, Strategy=sum,

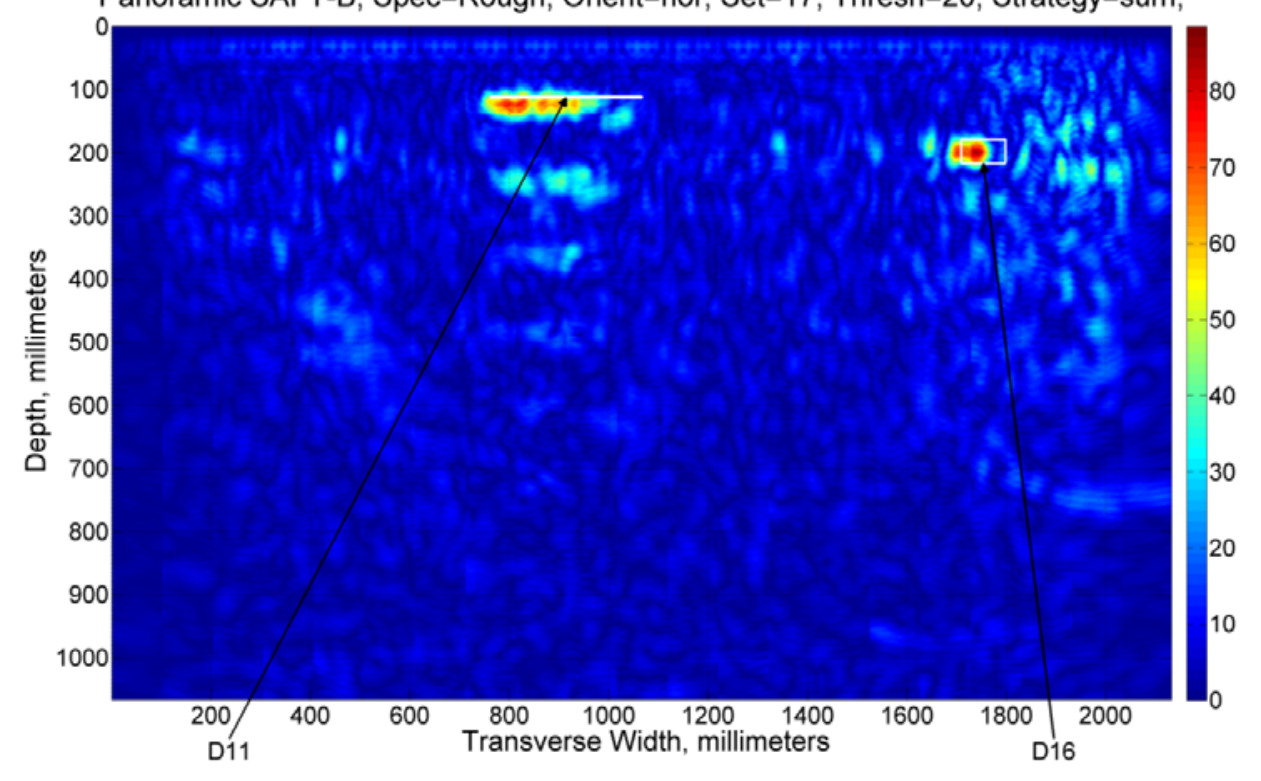

(b)

Fig. 55. Shallow cover improved detectability (reduced reverberation artifact) for Defect 11 for node 18 (b) compared to regular SAFT (a), horizontal orientation. 


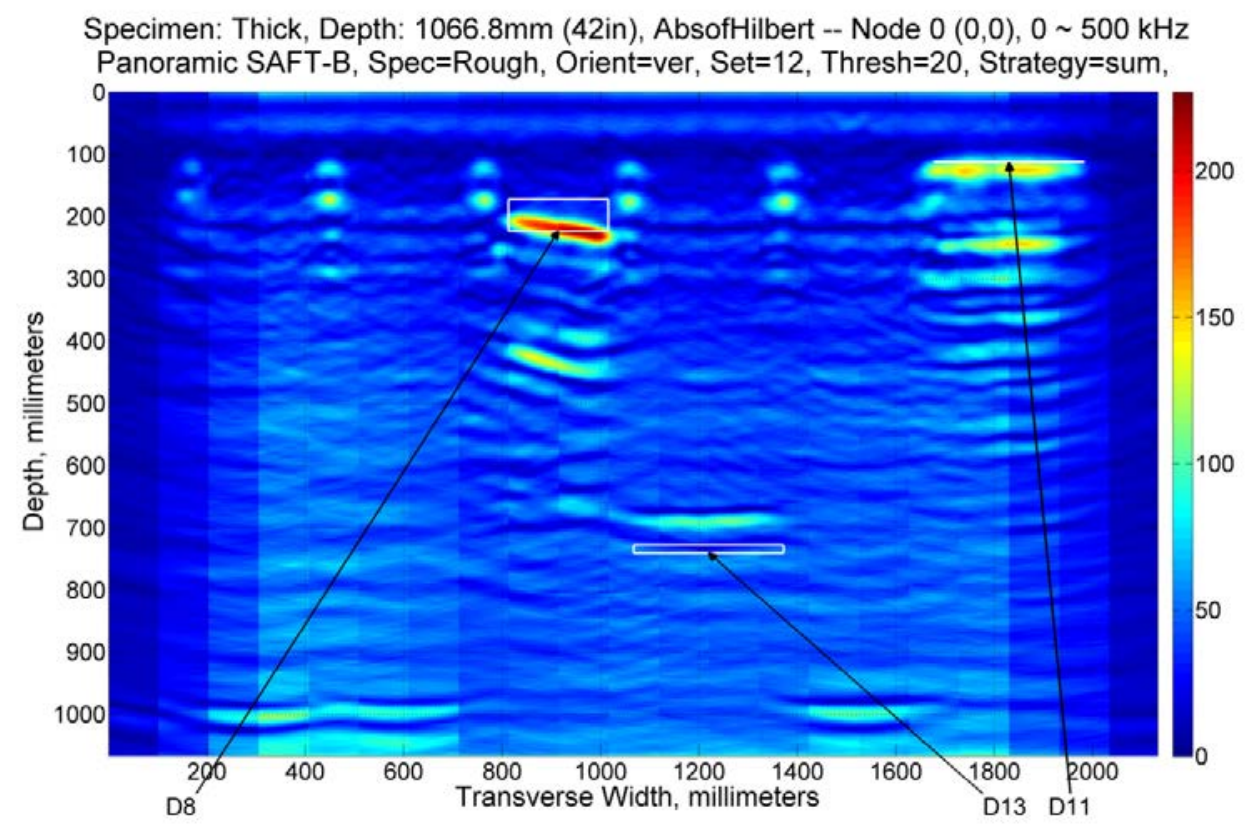

(a)

Specimen: Thick, Depth: 1066.8mm (42in), AbsofHilbert -- Node $16(4,1), 31.25 \sim 62.5 \mathrm{kHz}$

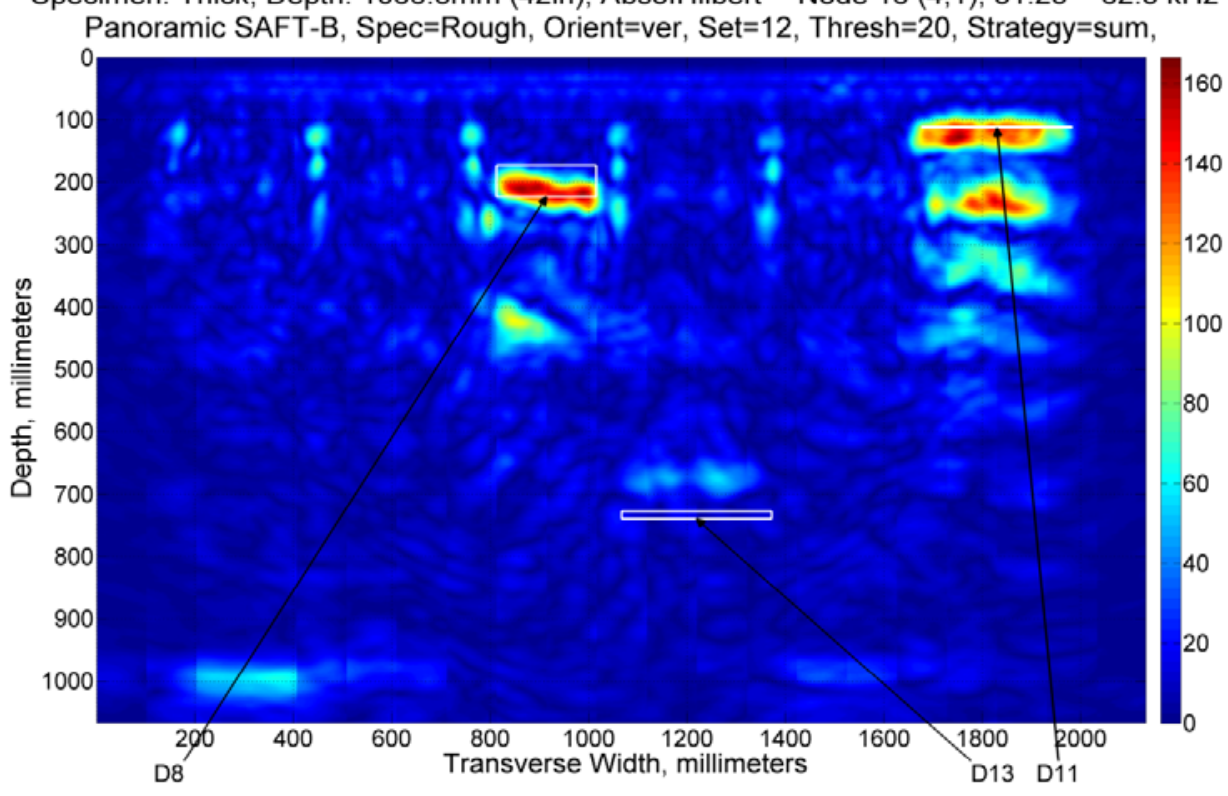

(b)

Fig. 56. Shallow cover improved detectability for Defect 11 for node 16 (b) compared to regular SAFT (a), vertical orientation 


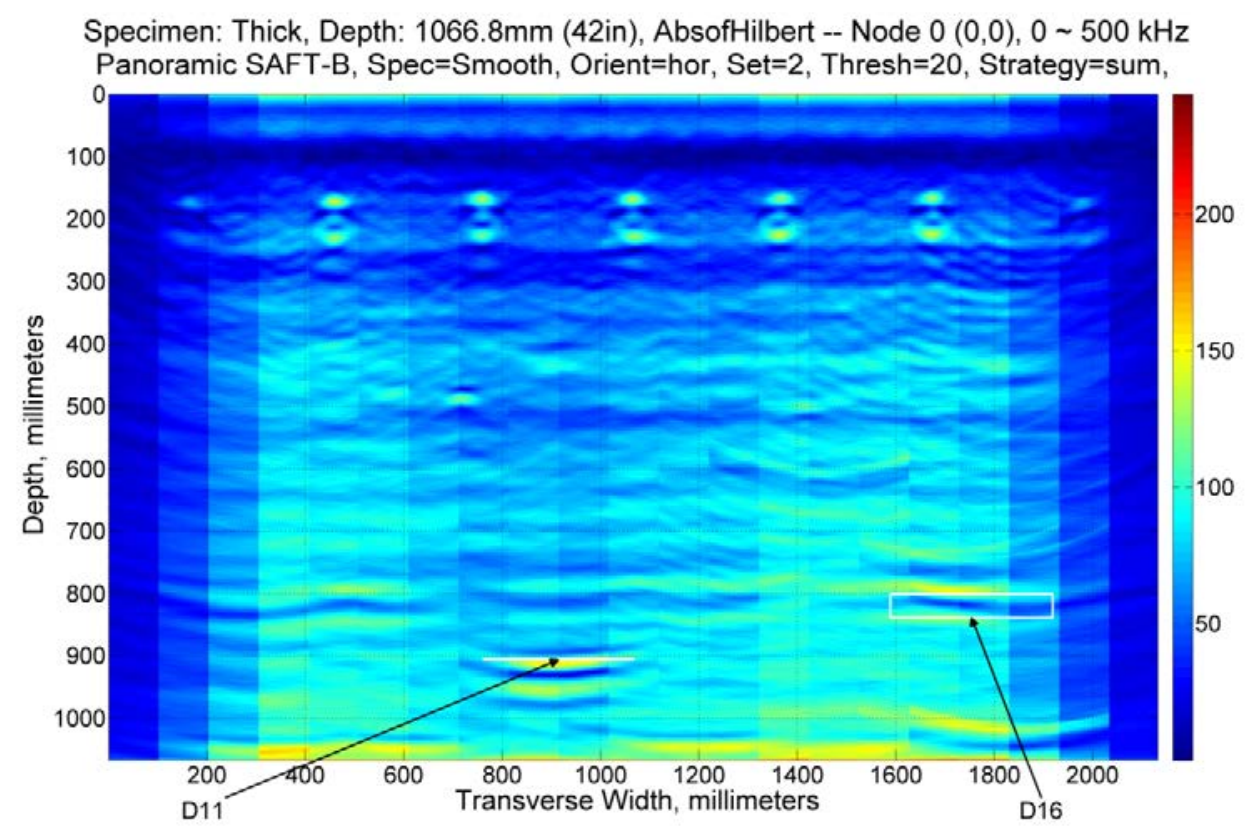

(a)

Specimen: Thick, Depth: 1066.8mm (42in), AbsofHilbert -- Node $16(4,1), 31.25 \sim 62.5 \mathrm{kHz}$

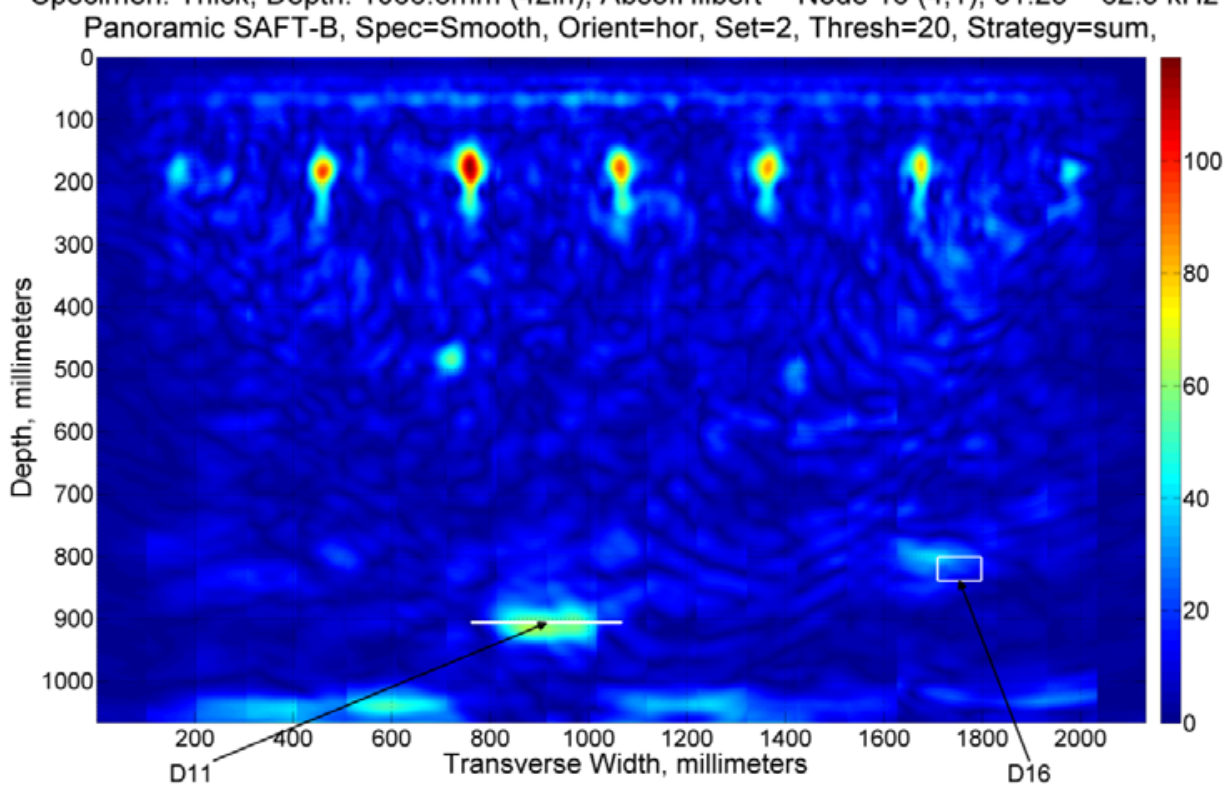

(b)

Fig. 57. Deep cover improved detectability for Defect 11 for node 18 (b) compared to regular SAFT (a), horizontal orientation. 
Specimen: Thick, Depth: 1066.8mm (42in), AbsofHilbert -- Node $0(0,0), 0 \sim 500 \mathrm{kHz}$

Panoramic SAFT-B, Spec=Smooth, Orient=ver, Set=8, Thresh=20, Strategy=sum,

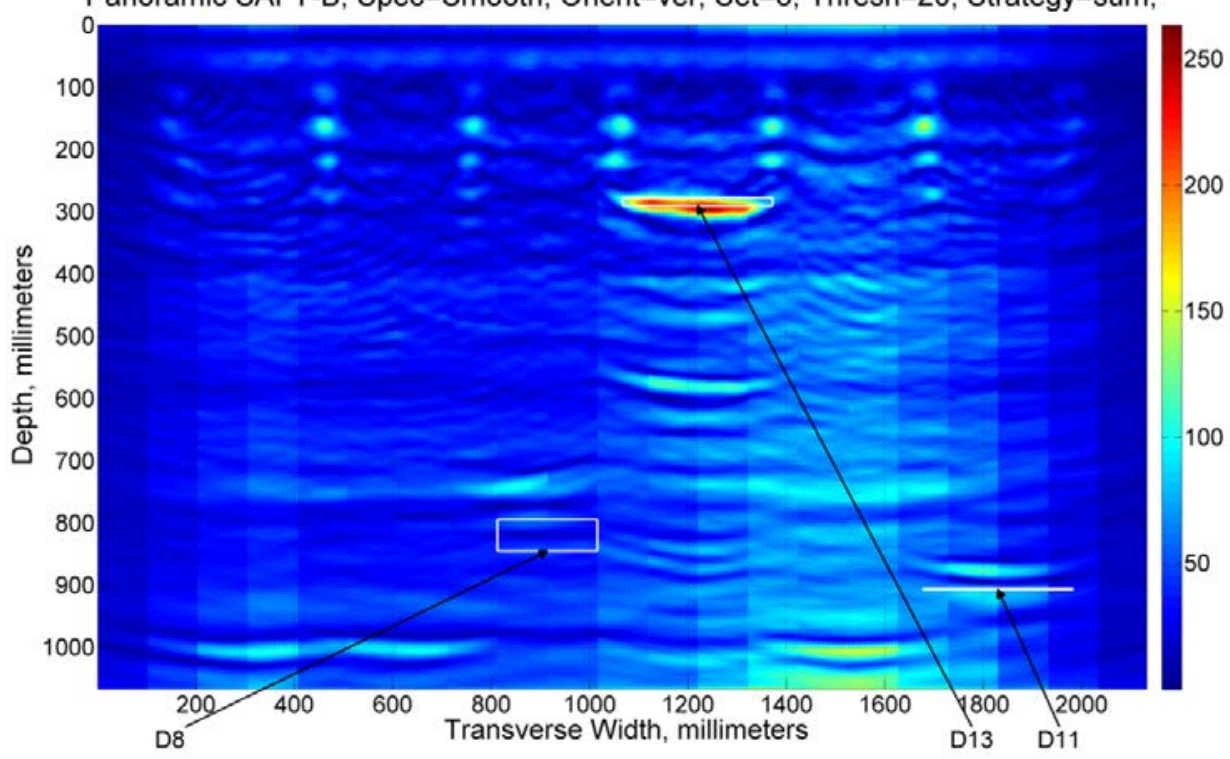

(a)

Specimen: Thick, Depth: 1066.8mm (42in), AbsofHilbert -- Node $16(4,1), 31.25 \sim 62.5 \mathrm{kHz}$ Panoramic SAFT-B, Spec $=$ Smooth, Orient $=$ ver, Set $=8$, Thresh $=20$, Strategy $=$ sum,

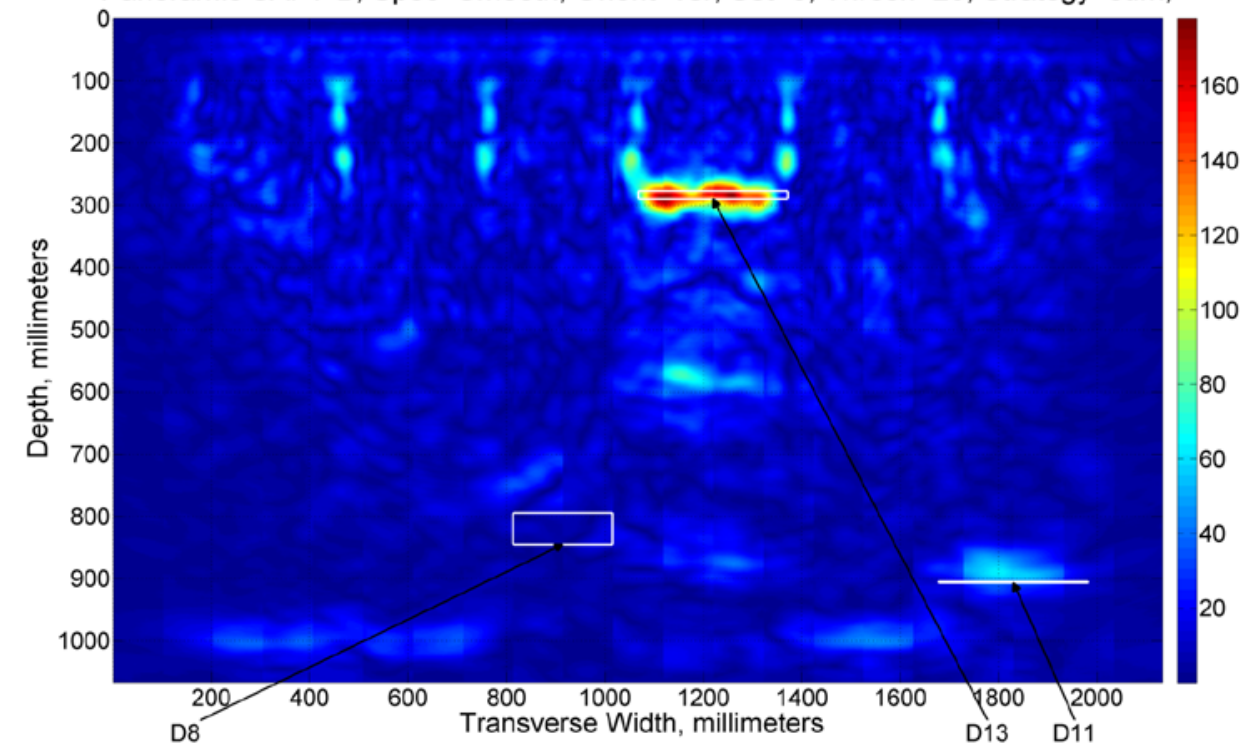

(b)

Fig. 58. Deep cover improved detectability for Defect 11 for node 18 (b) compared to regular SAFT (a), vertical orientation. 


\subsubsection{Defect 12}

Defect 12 was easily seen by regular SAFT, so there was not much room for improvement from other frequency bands.

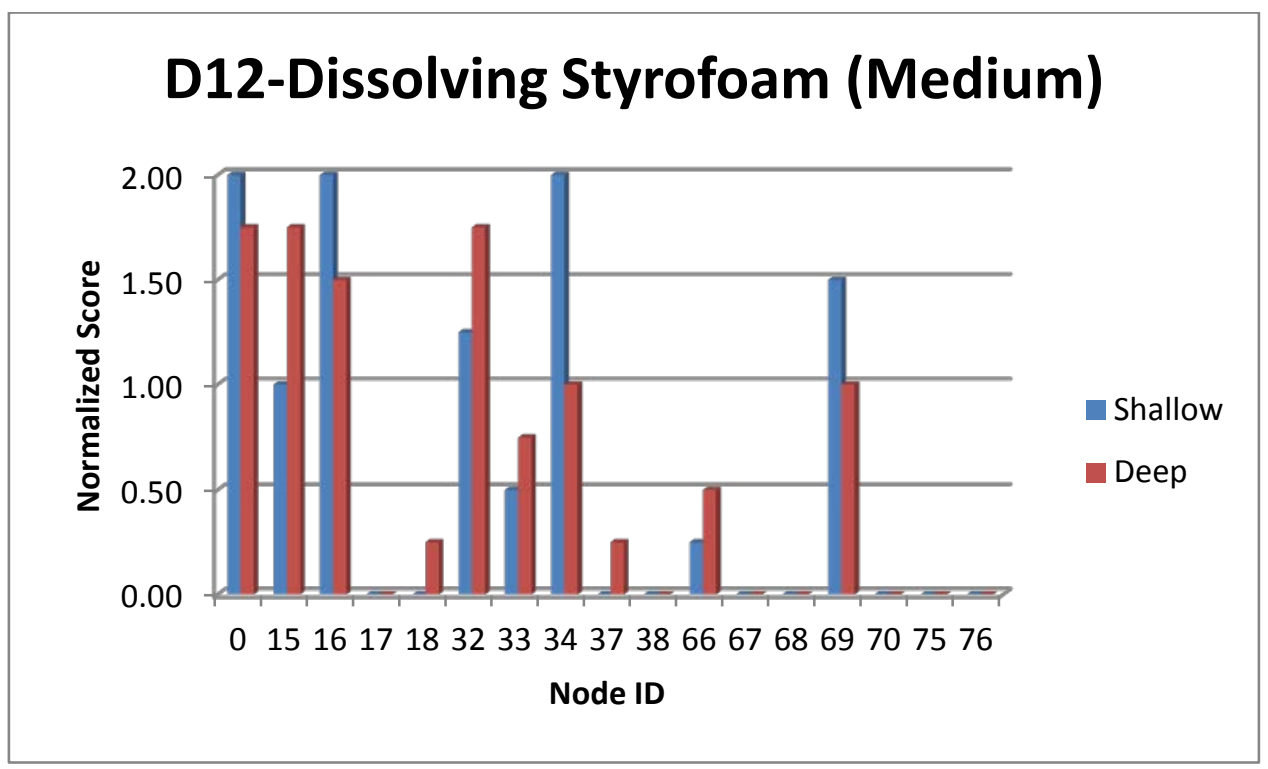

Fig. 59. Defect 12 analysis chart illustrating the normalized score for each node. 


\subsubsection{Defect 13}

Defect 13 was easily seen by regular SAFT, so there was not much room for improvement from other frequency bands.

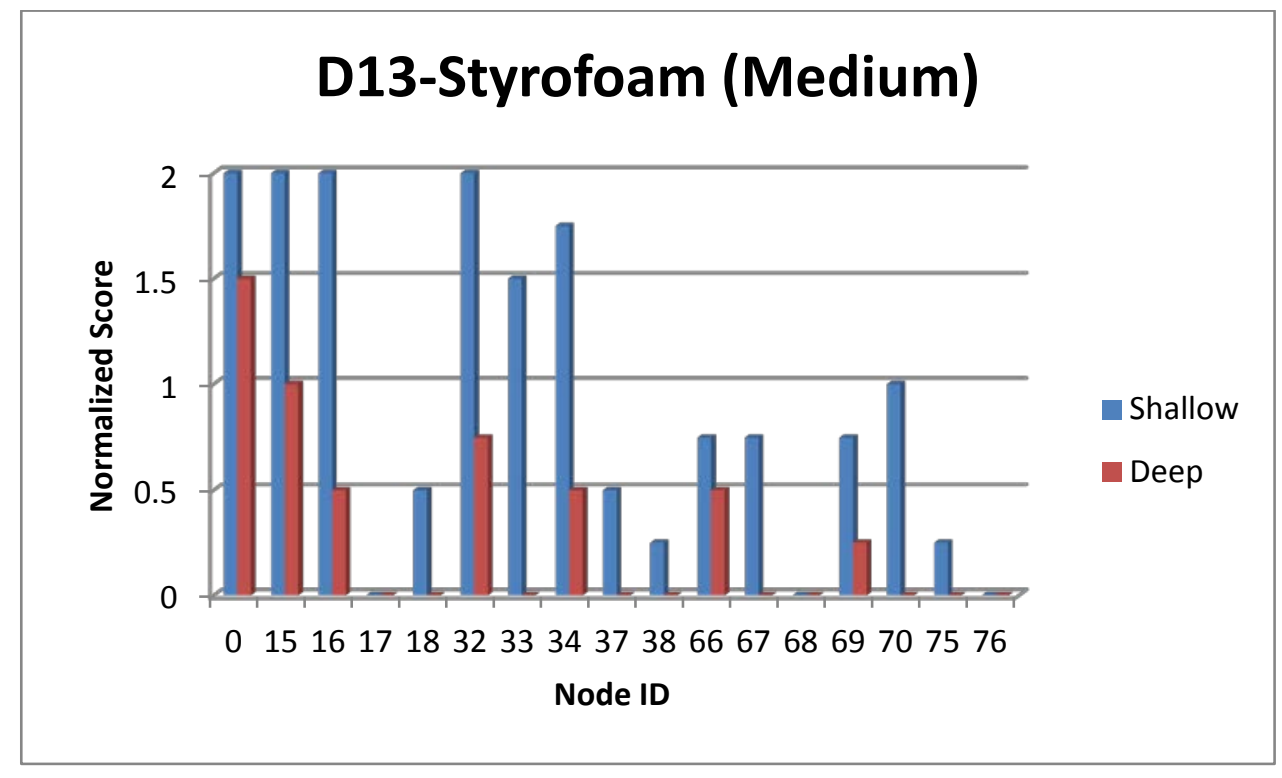

Fig. 60. Defect 13 analysis chart illustrating the normalized score for each node. 
10.1.14 Defect 14

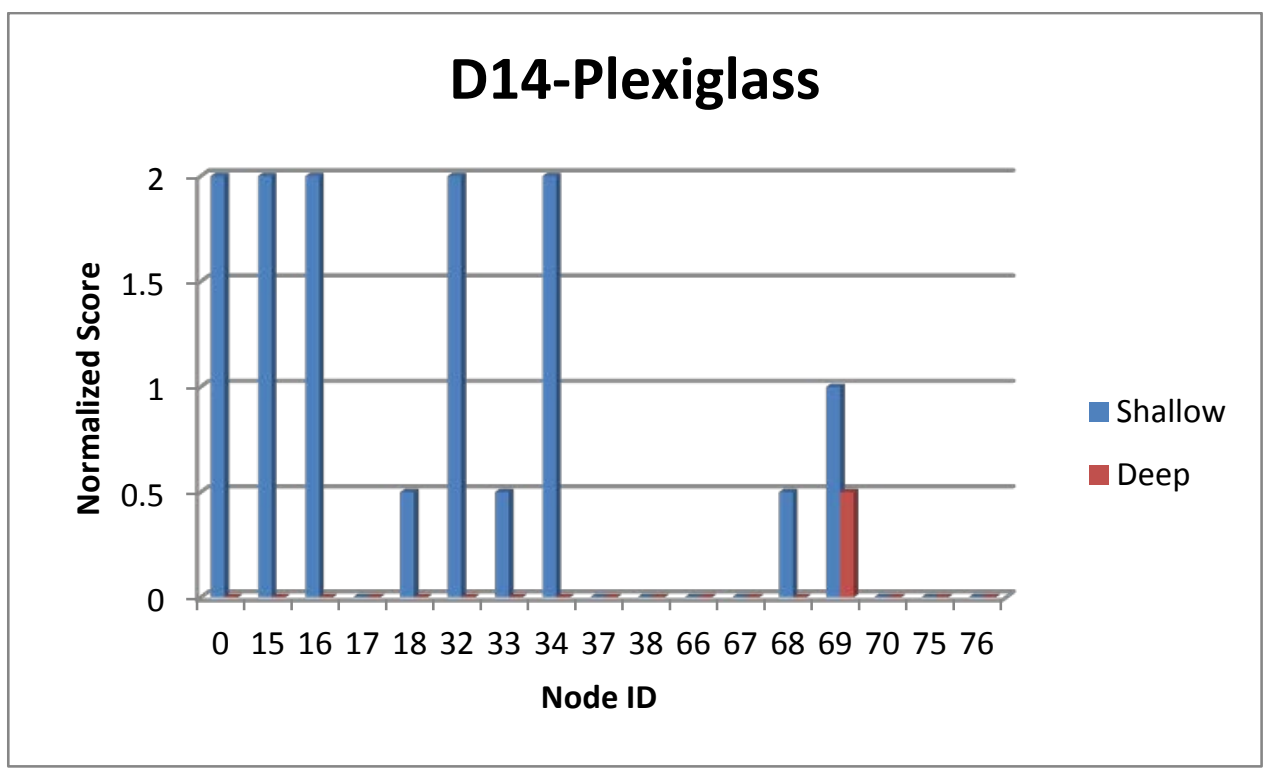

Fig. 61. Defect 14 analysis chart illustrating the normalized score for each node. 


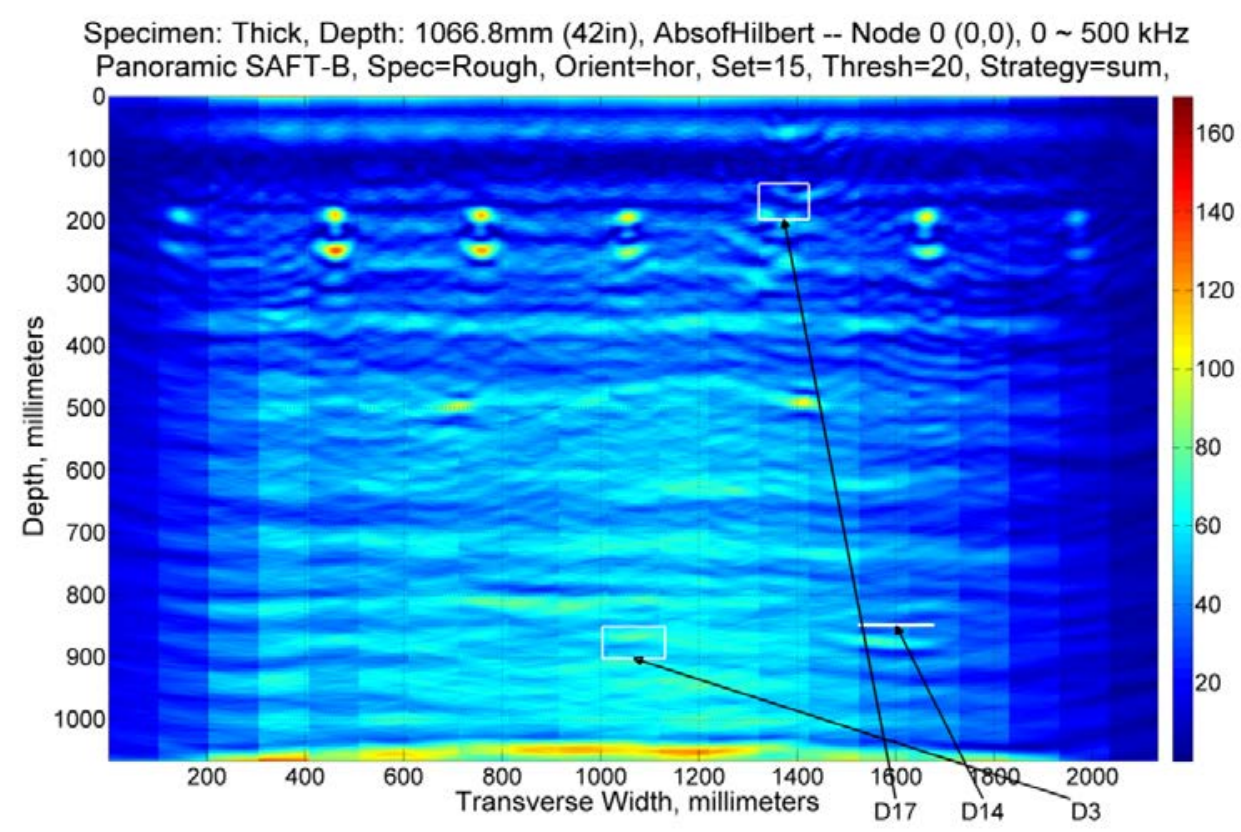

(a)

Specimen: Thick, Depth: 1066.8mm (42in), AbsofHilbert -- Node $69(6,6), 39.0625 \sim 46.875 \mathrm{kHz}$ Panoramic SAFT-B, Spec=Rough, Orient=hor, Set $=15$, Thresh $=20$, Strategy $=$ sum,

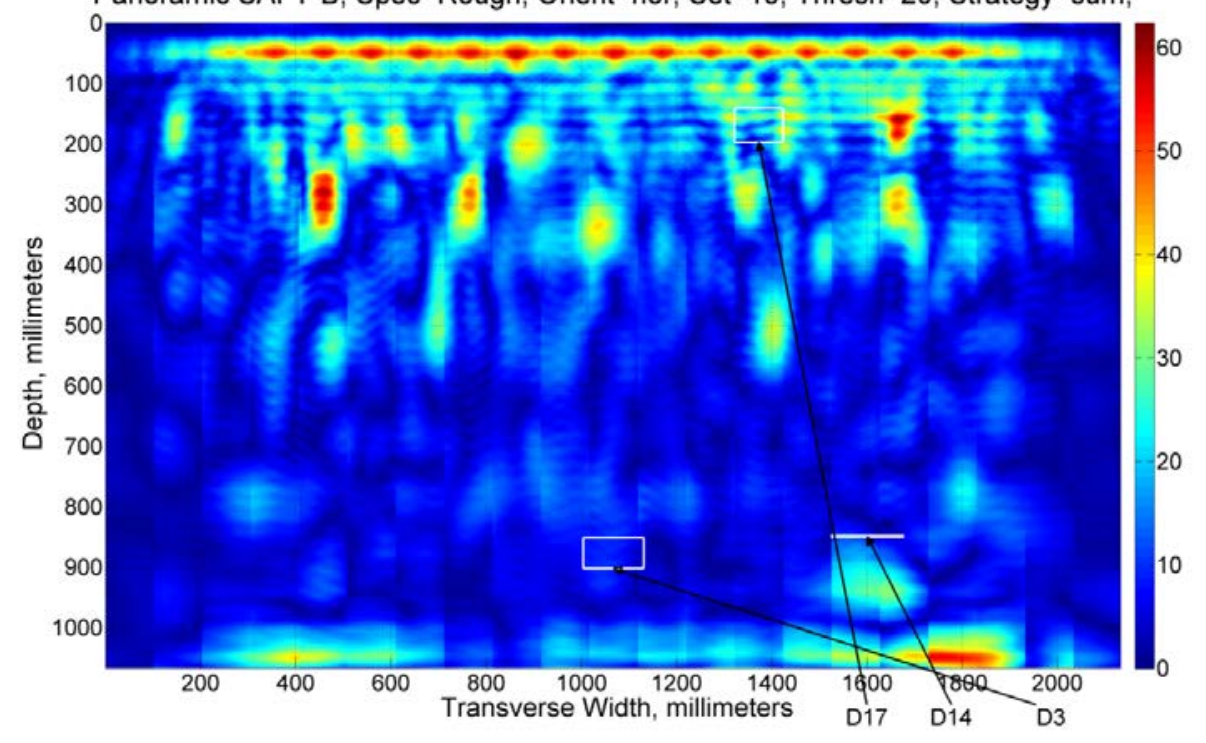

(b)

Fig. 62. Deep cover improved detectability for Defect 14 for node 69 (b) compared to regular SAFT (a). 
10.1.15 Defect 15

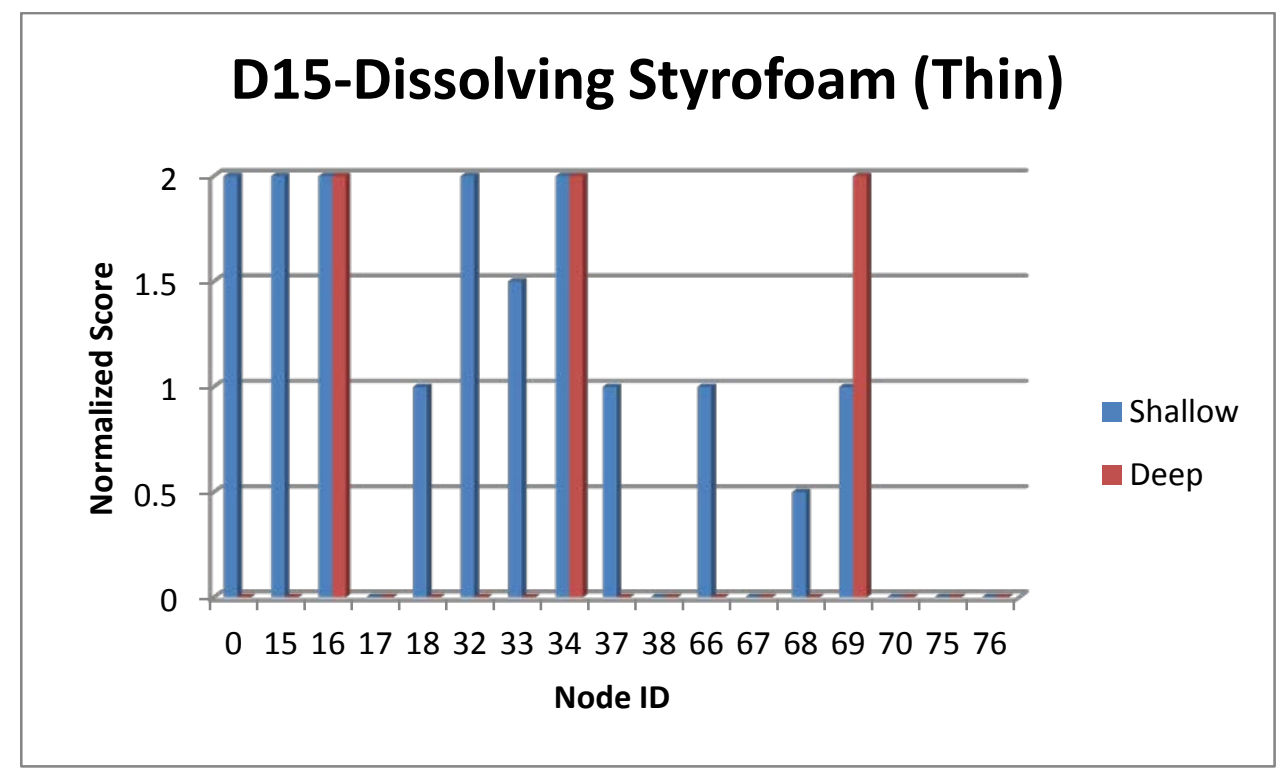

Fig. 63. Defect 15 analysis chart illustrating the normalized score for each node. 


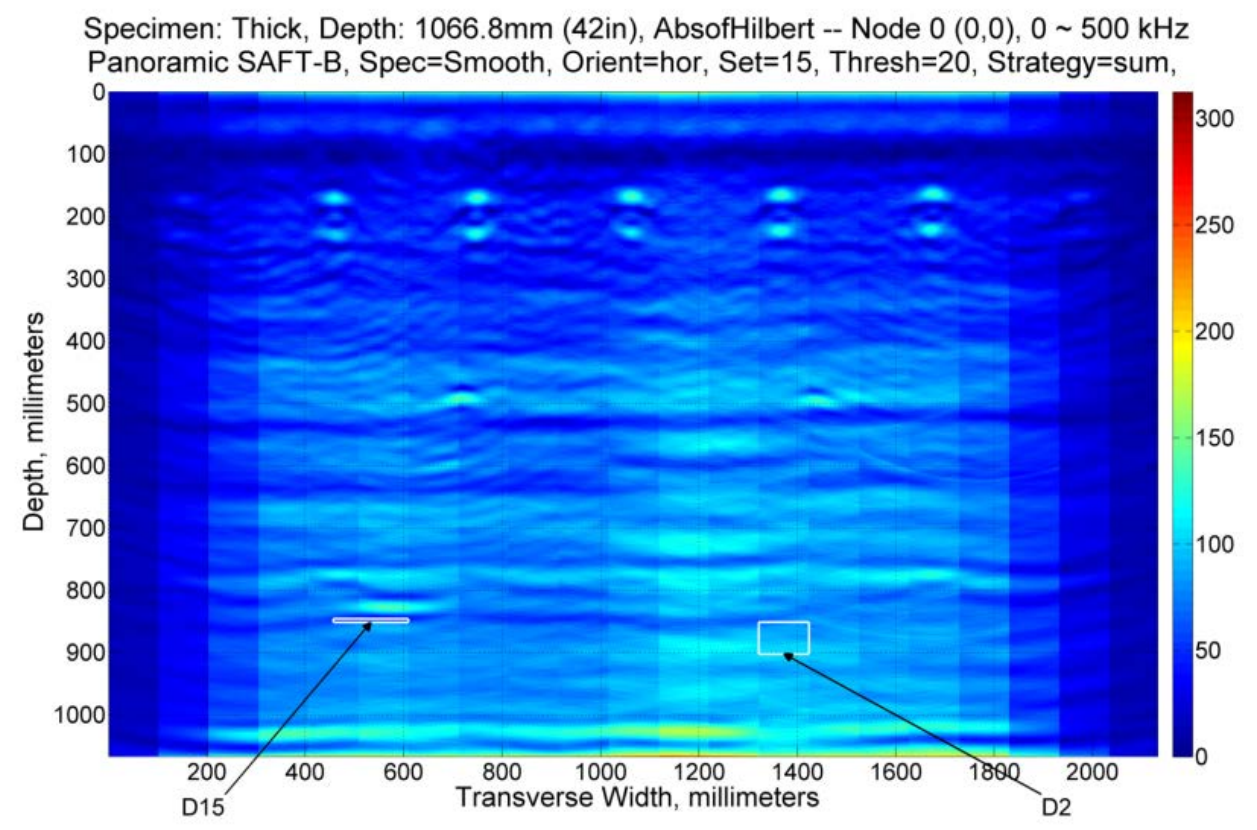

(a)

Specimen: Thick, Depth: 1066.8mm (42in), AbsofHilbert -- Node $16(4,1), 31.25 \sim 62.5 \mathrm{kHz}$

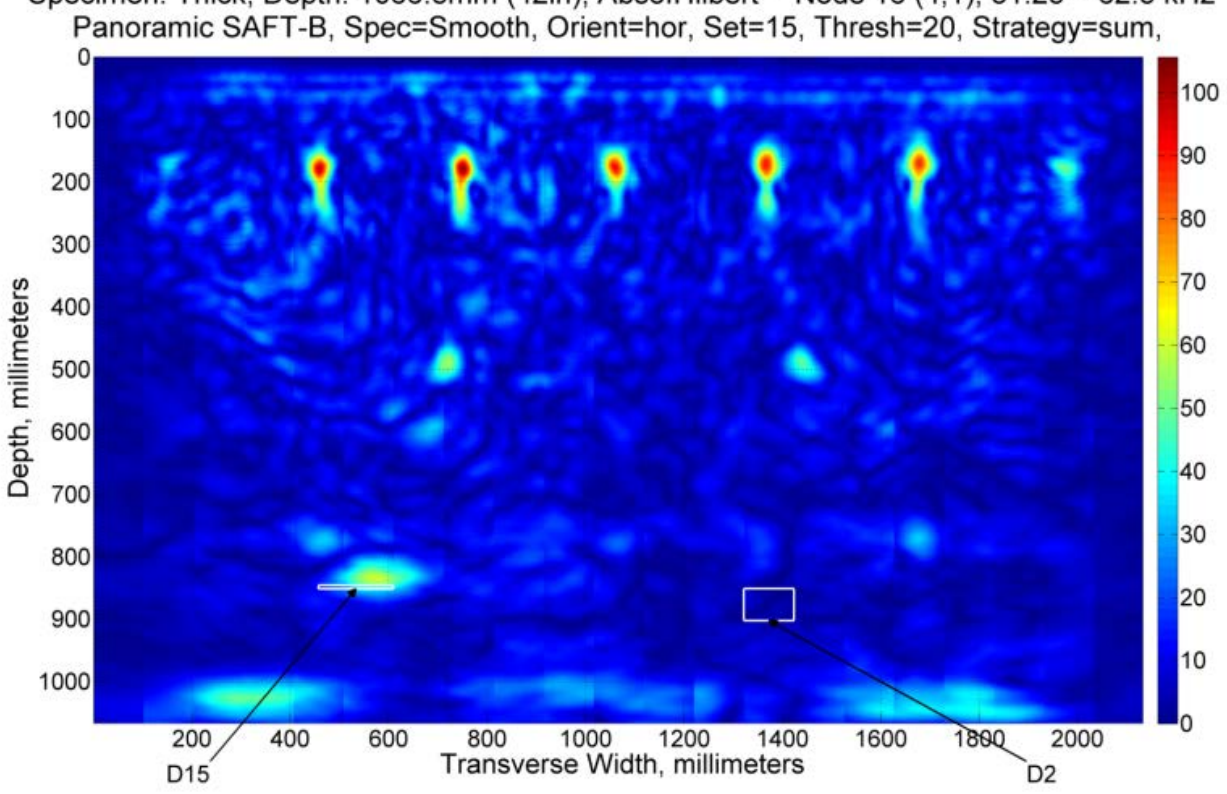

(b)

Fig. 64. Deep cover improved detectability for Defect 15 for node 16 (b) compared to regular SAFT (a), horizontal orientation. 


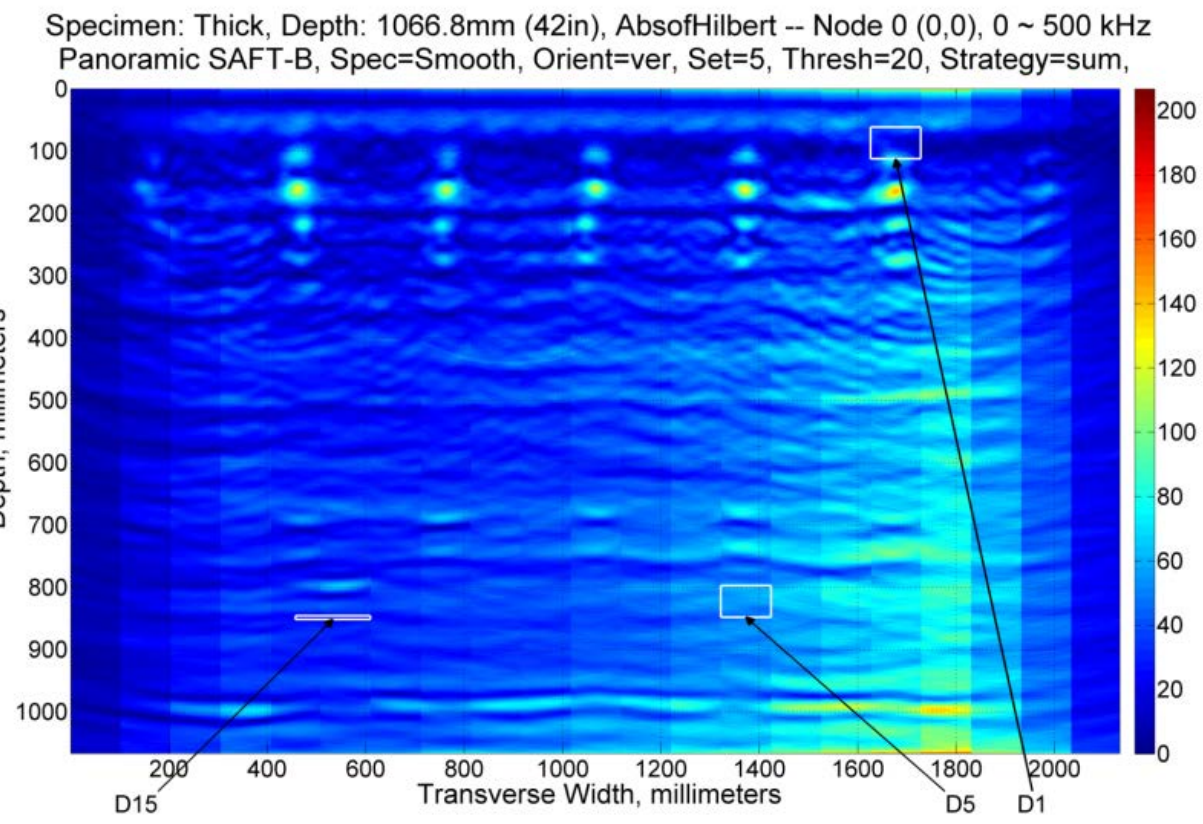

(a)

Specimen: Thick, Depth: 1066.8mm (42in), AbsofHilbert -- Node $16(4,1), 31.25 \sim 62.5 \mathrm{kHz}$

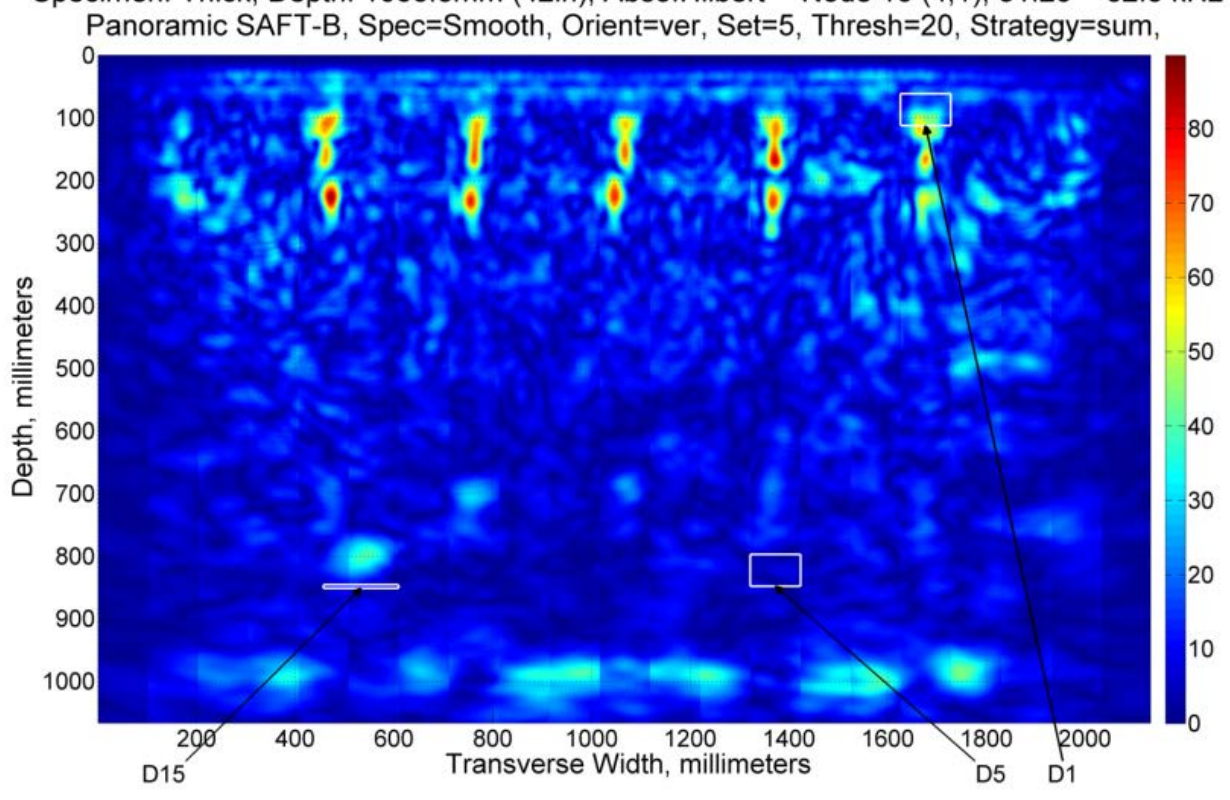

(b)

Fig. 65. Deep cover improved detectability for Defect 15 for node 16 (b) compared to regular SAFT (a), vertical orientation. 
10.1.16 Defect 16

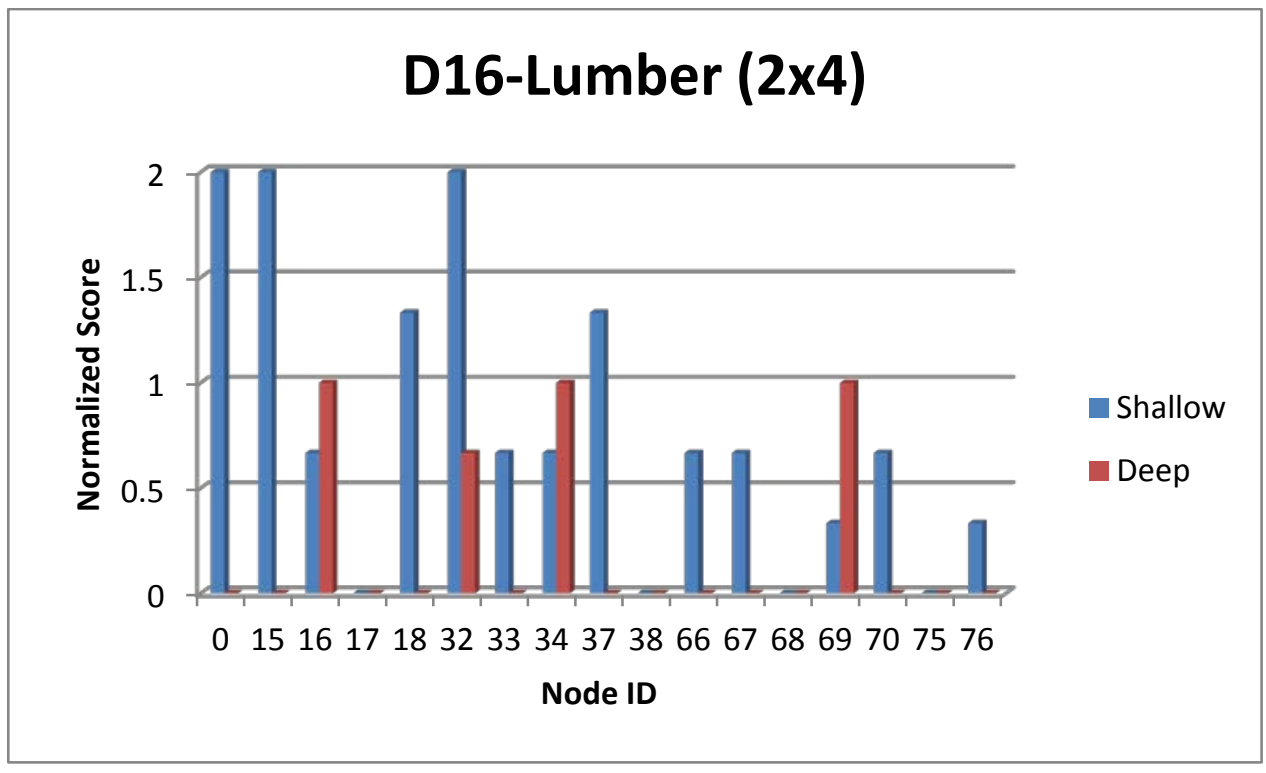

Fig. 66. Defect 16 analysis chart illustrating the normalized score for each node. 


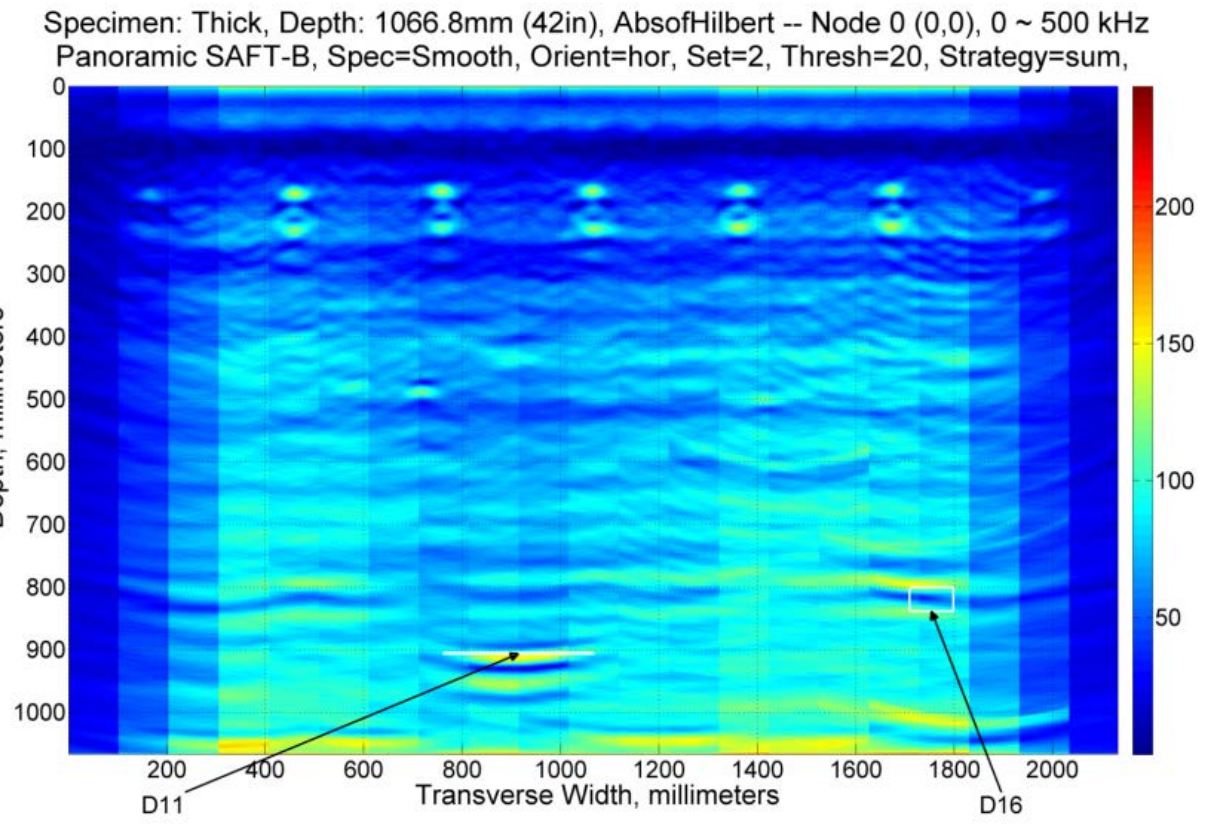

(a)

Specimen: Thick, Depth: 1066.8mm (42in), AbsofHilbert -- Node $32(5,1), 15.625 \sim 31.25 \mathrm{kHz}$ Panoramic SAFT-B, Spec=Smooth, Orient=hor, Set=2, Thresh=20, Strategy=sum,

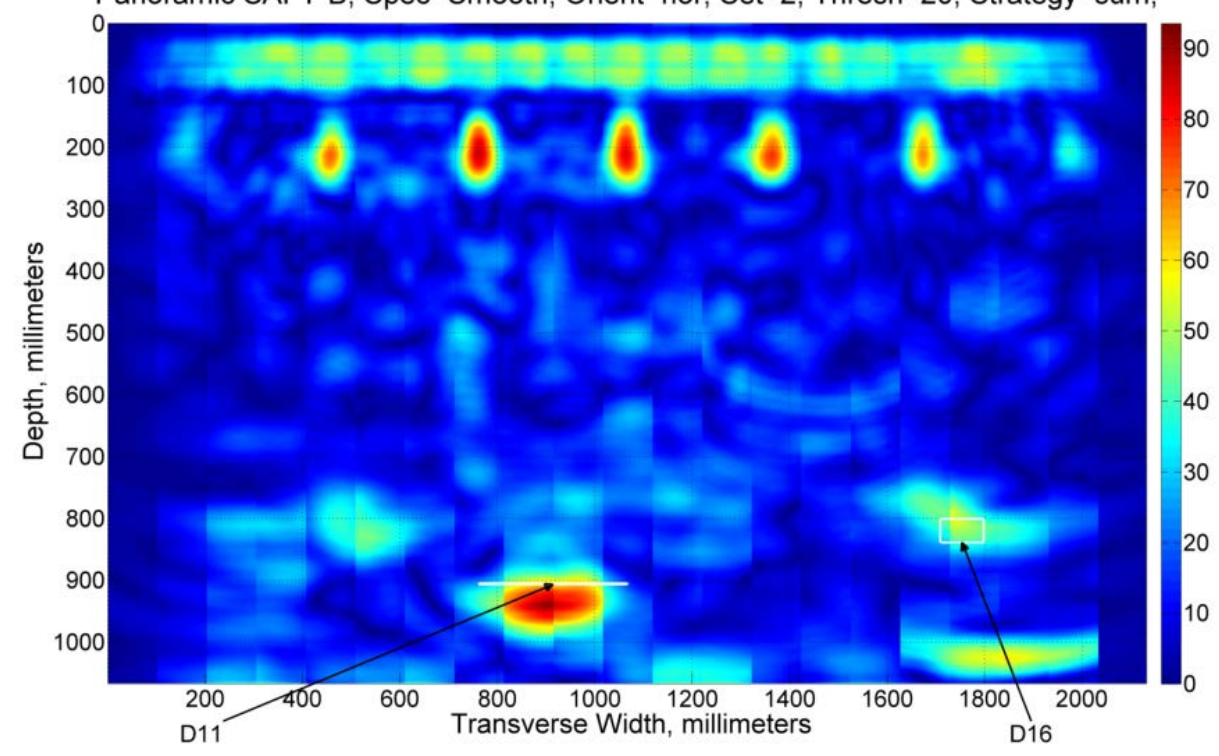

(b)

Fig. 67. Deep cover improved detectability for Defect 6 for node 32 (b) compared to regular SAFT (a), horizontal orientation \#1 


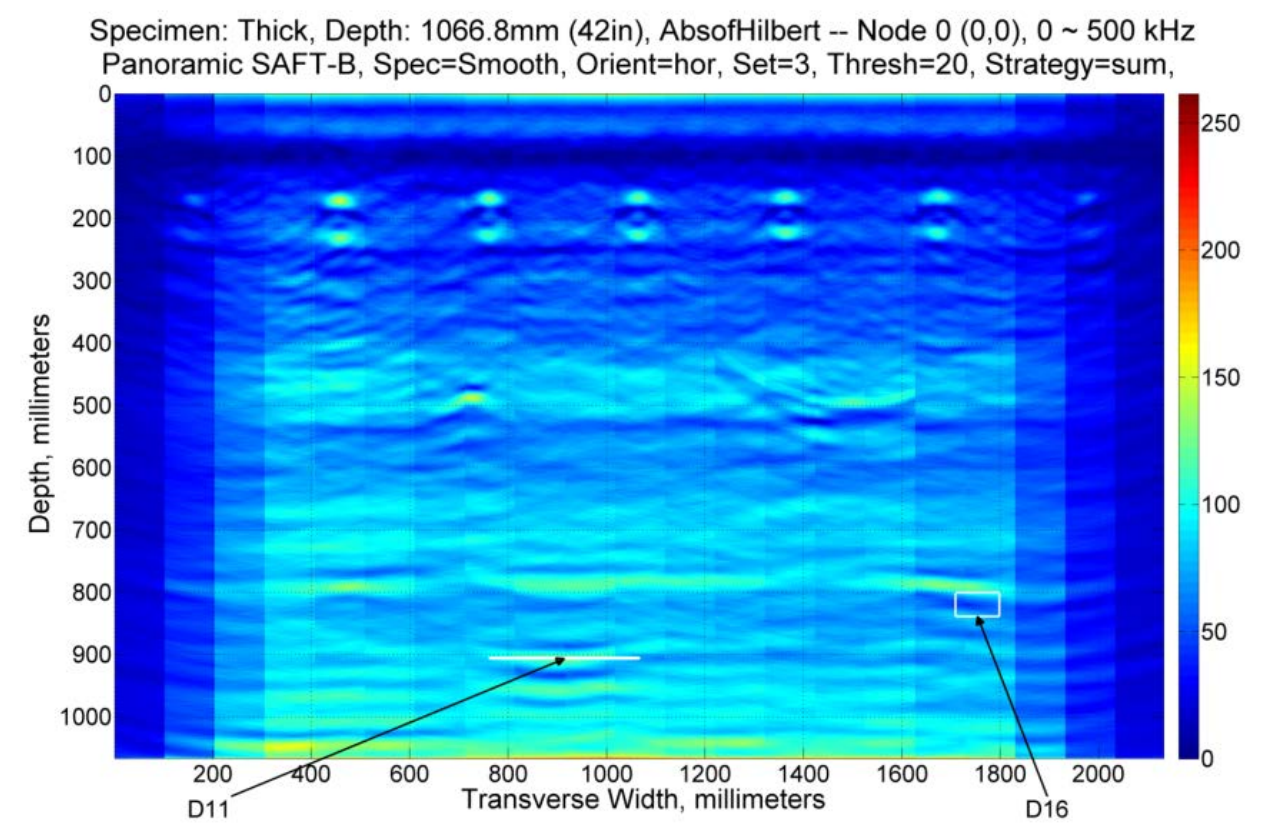

(a)

Specimen: Thick, Depth: 1066.8mm (42in), AbsofHilbert -- Node $32(5,1), 15.625 \sim 31.25 \mathrm{kHz}$ Panoramic SAFT-B, Spec=Smooth, Orient=hor, Set $=3$, Thresh=20, Strategy=sum,

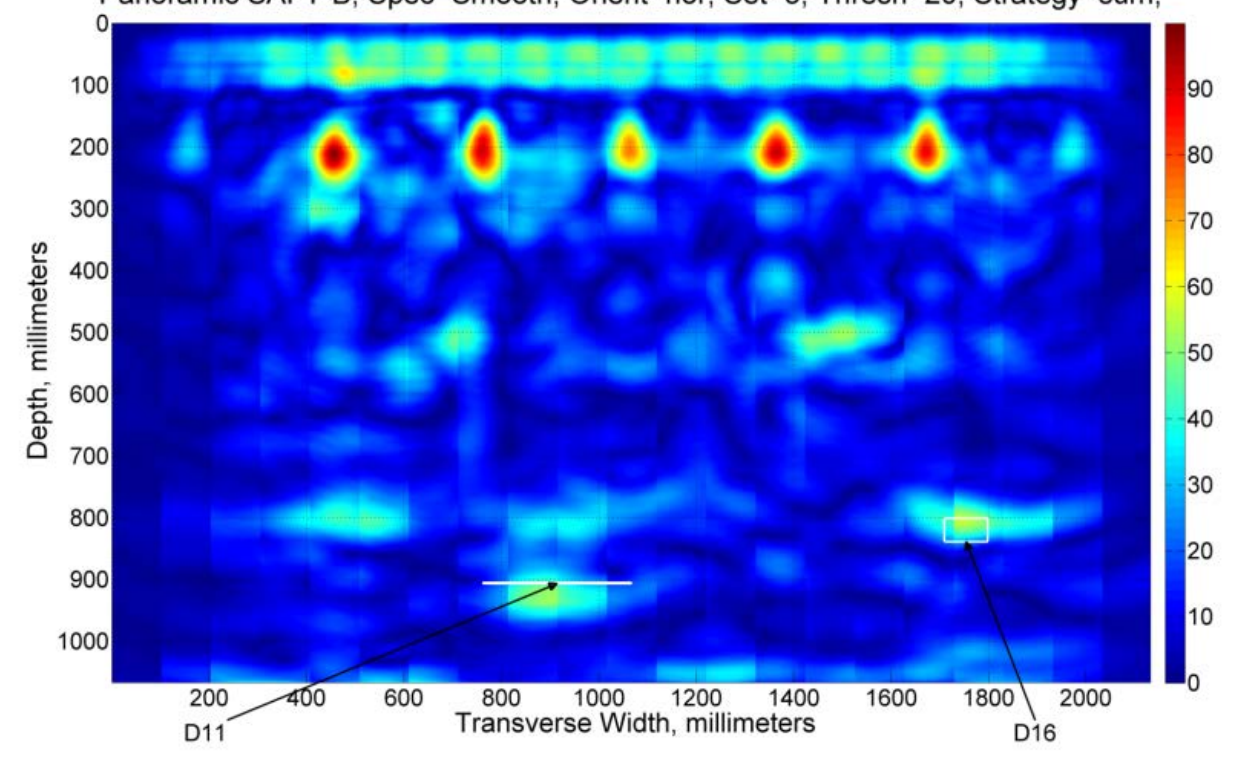

(b)

Fig. 68. Deep cover improved detectability for Defect 6 for node 32 (b) compared to regular SAFT (a), horizontal orientation \#2. 


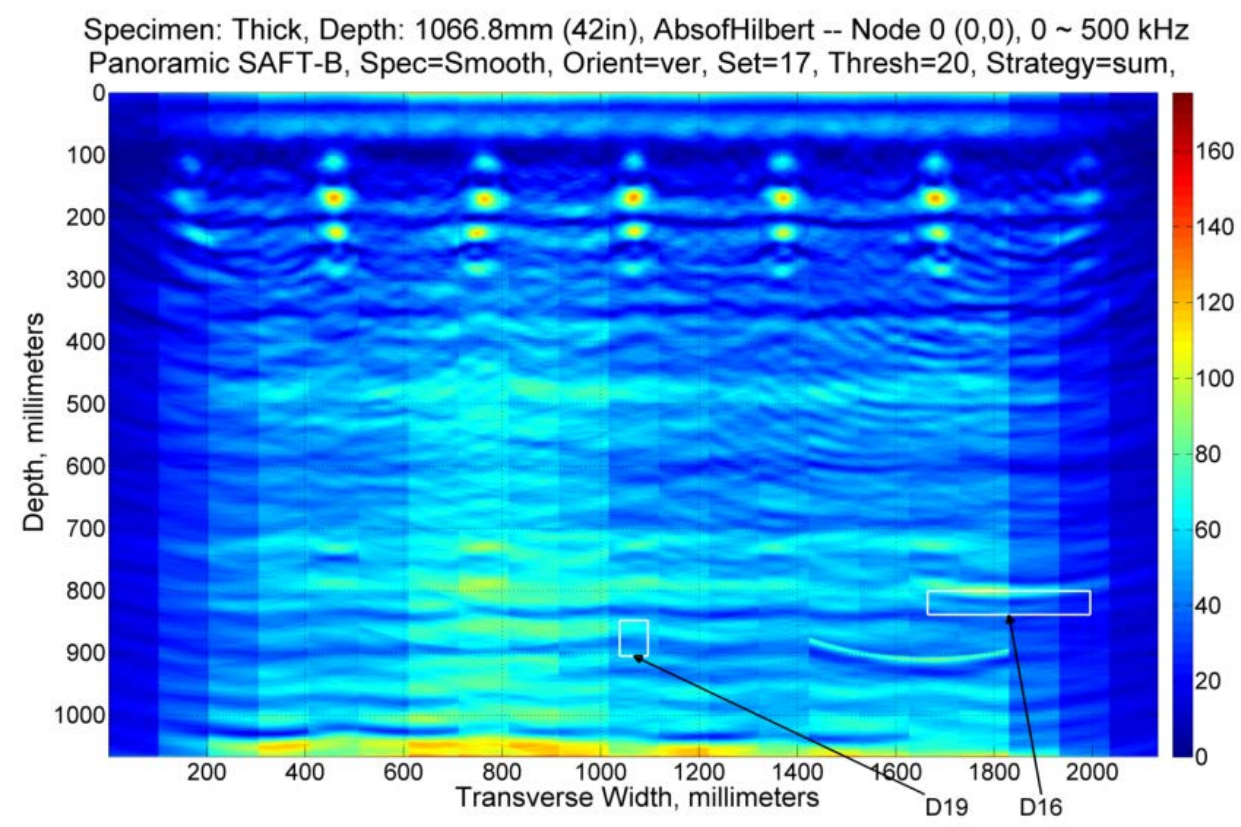

(a)

Specimen: Thick, Depth: 1066.8mm (42in), AbsofHilbert -- Node $16(4,1), 31.25 \sim 62.5 \mathrm{kHz}$

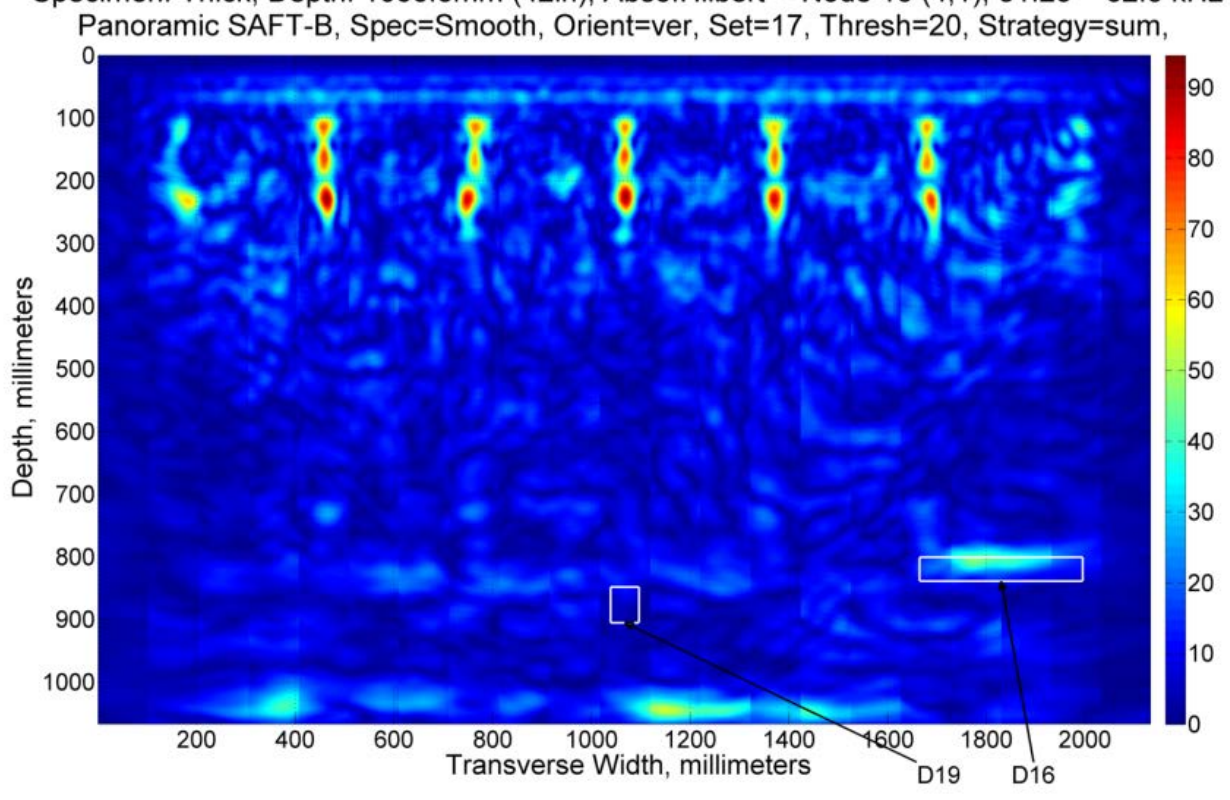

(b)

Fig. 69. Deep cover improved detectability for Defect 6 for node 16 (b) compared to regular SAFT (a), vertical orientation. 


\subsubsection{Defect 17}

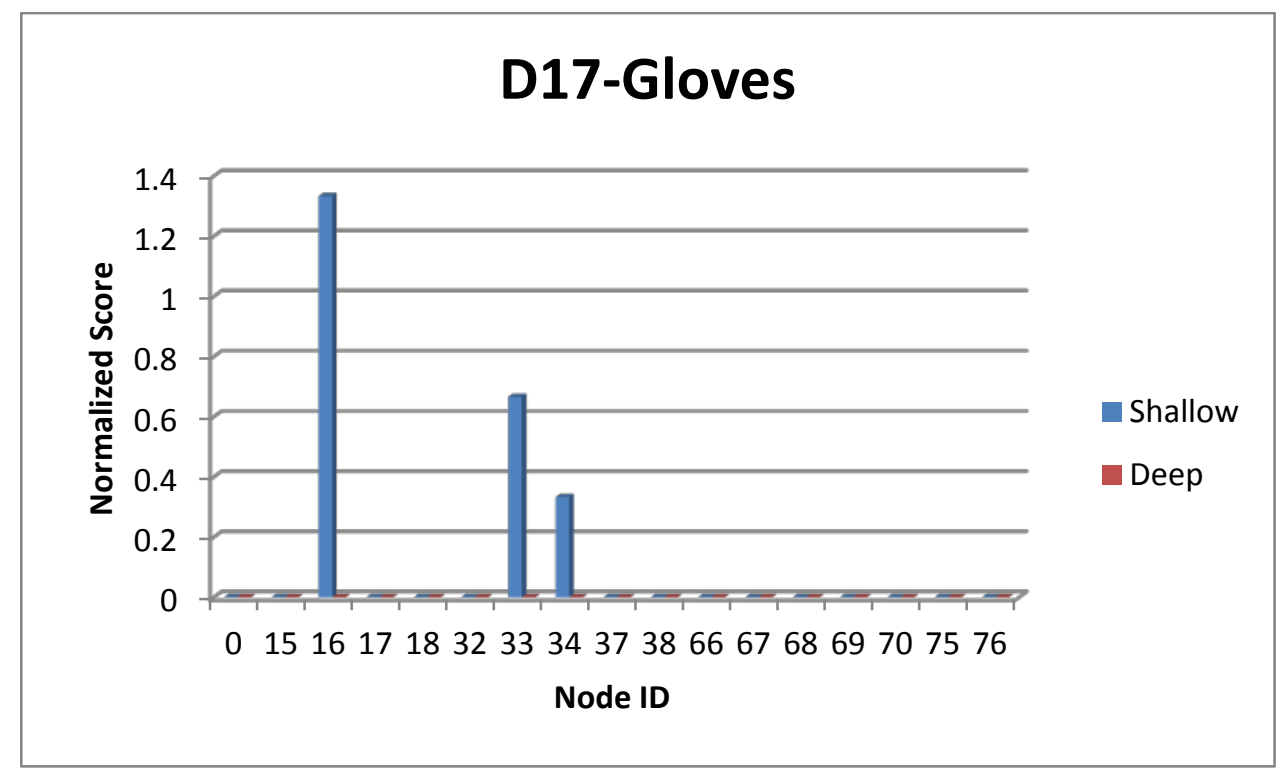

Fig. 70. Defect 17 analysis chart illustrating the normalized score for each node. 


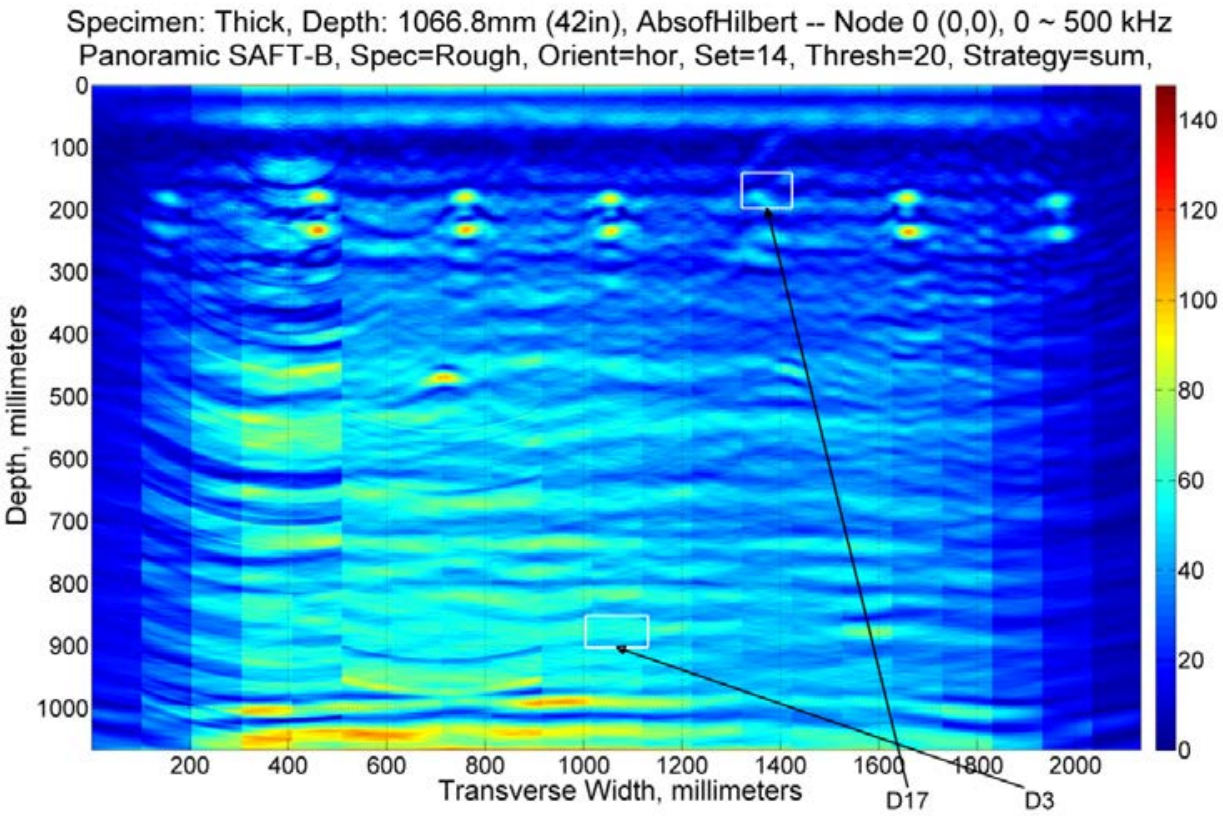

(a)

Specimen: Thick, Depth: 1066.8mm (42in), AbsofHilbert -- Node $16(4,1), 31.25 \sim 62.5 \mathrm{kHz}$ Panoramic SAFT-B, Spec=Rough, Orient $=$ hor, Set $=14$, Thresh $=20$, Strategy=sum,

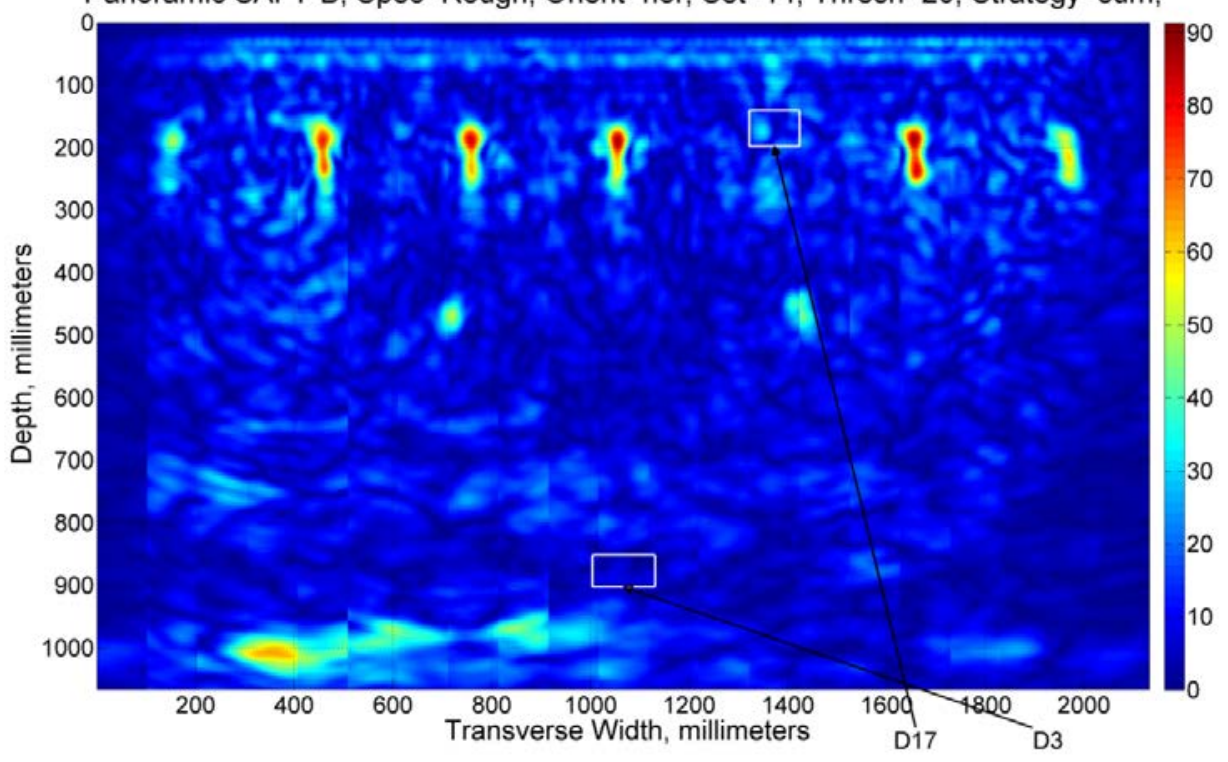

(b)

Fig. 71. Shallow cover improved detectability for Defect 17 for node 16 (b) compared to regular SAFT (a), horizontal orientation \#1. The glove absorbs the signal so the rebar behind it is not visible. 


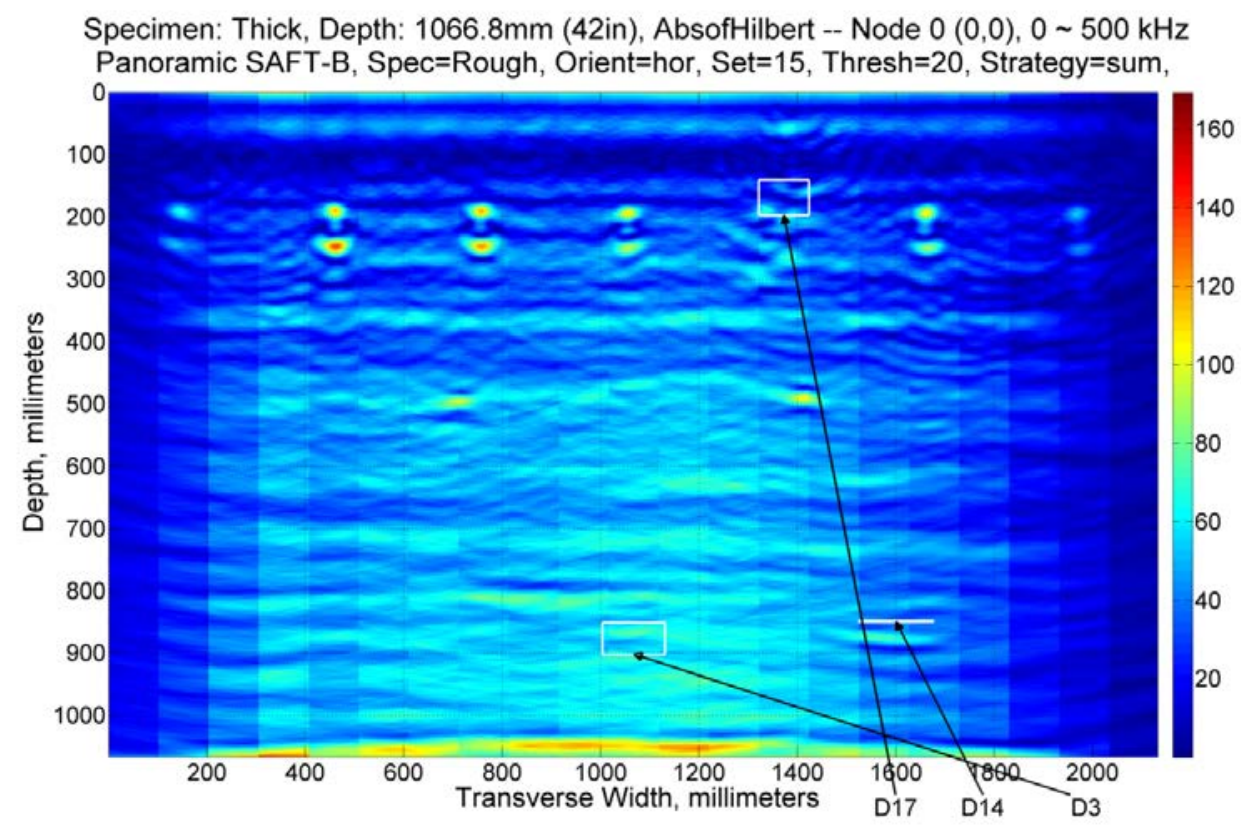

(a)

Specimen: Thick, Depth: 1066.8mm (42in), AbsofHilbert -- Node $16(4,1), 31.25 \sim 62.5 \mathrm{kHz}$ Panoramic SAFT-B, Spec=Rough, Orient=hor, Set=15, Thresh=20, Strategy=sum,

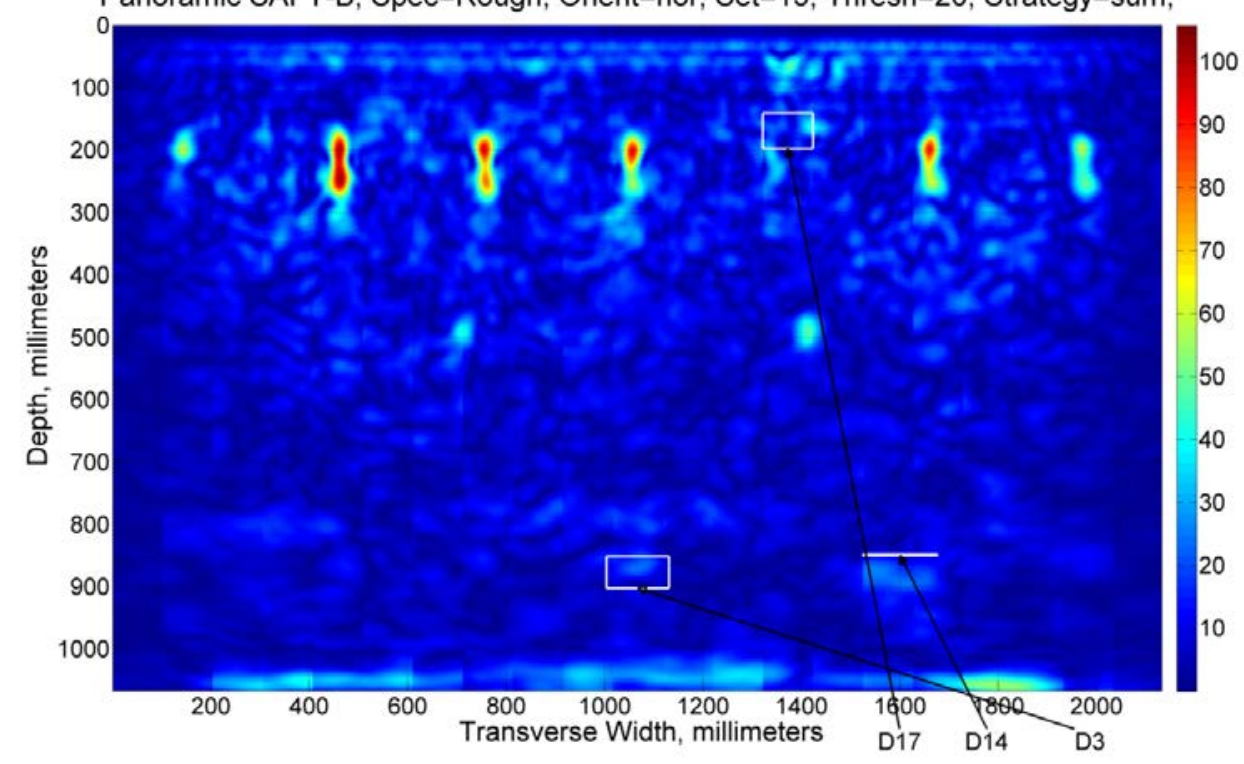

(b)

Fig. 72. Shallow cover improved detectability for Defect 17 for node 16 (b) compared to regular SAFT (a), horizontal orientation \#2. The glove absorbs the signal so the rebar behind it is not visible. 
10.1.18 Defect 18

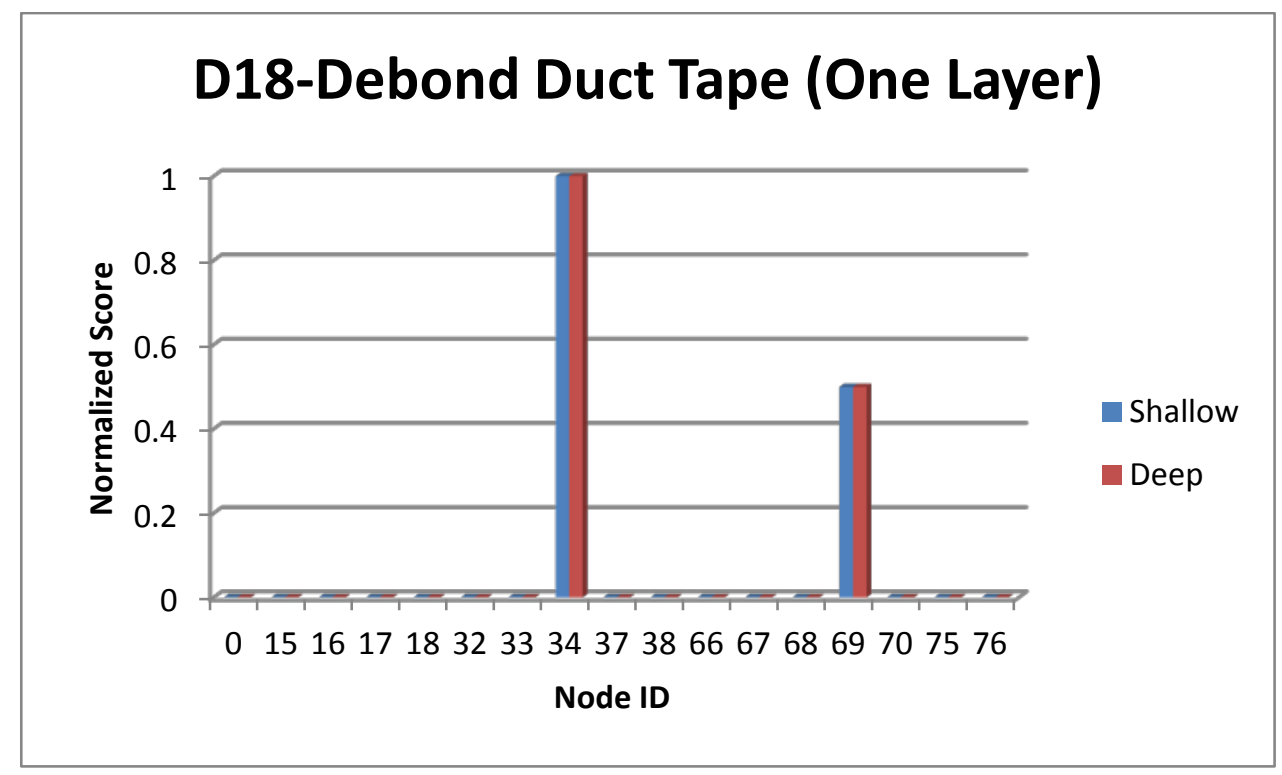

Fig. 73. Defect 18 analysis chart illustrating the normalized score for each node. 


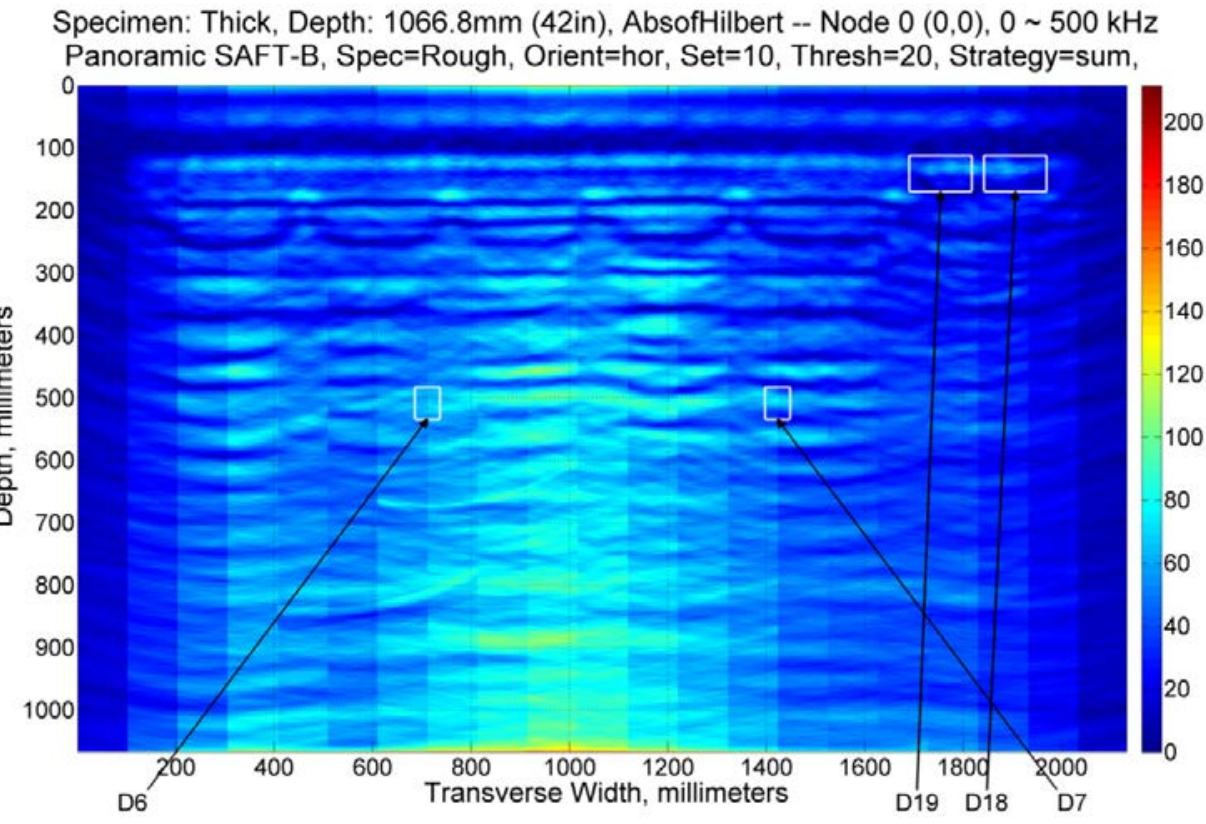

(a)

Specimen: Thick, Depth: 1066.8mm (42in), AbsofHilbert -- Node $34(5,3), 46.875 \sim 62.5 \mathrm{kHz}$ Panoramic SAFT-B, Spec=Rough, Orient $=$ hor, Set $=10$, Thresh $=20$, Strategy $=s u m$,

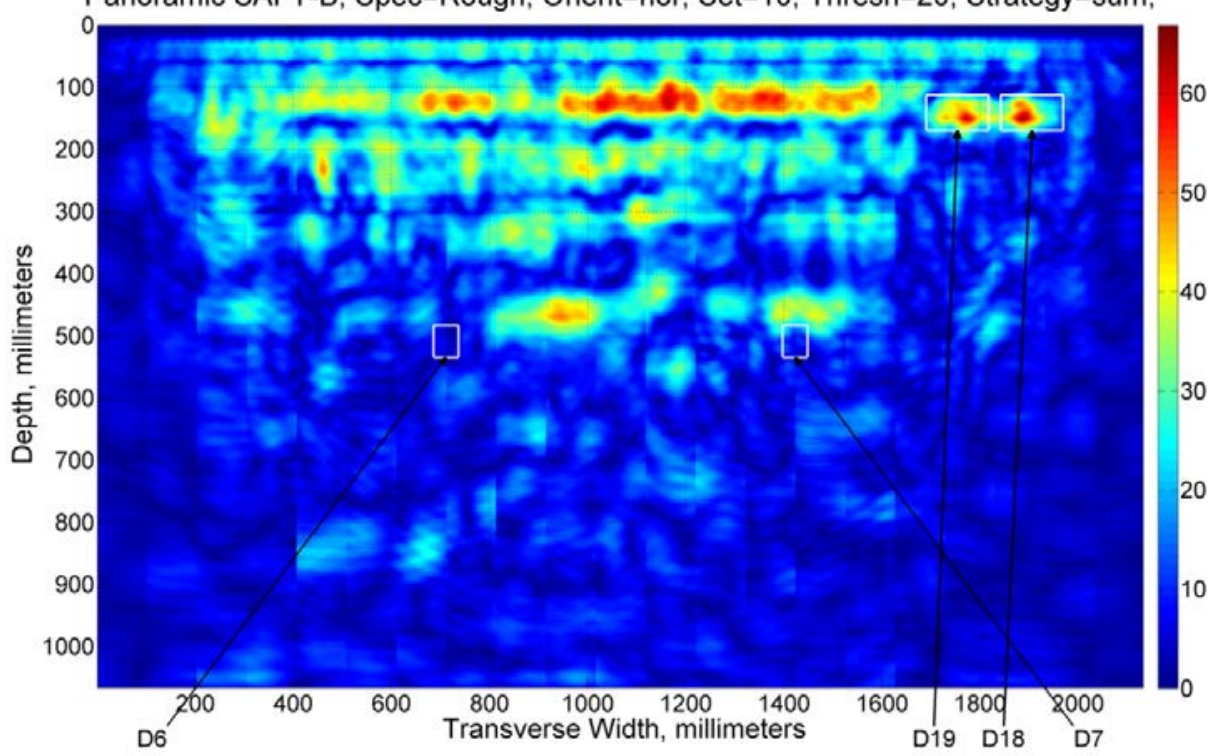

(b)

Fig. 74. Shallow cover improved detectability for Defect 18 for node 34 (b) compared to regular SAFT (a), horizontal orientation \#2. 


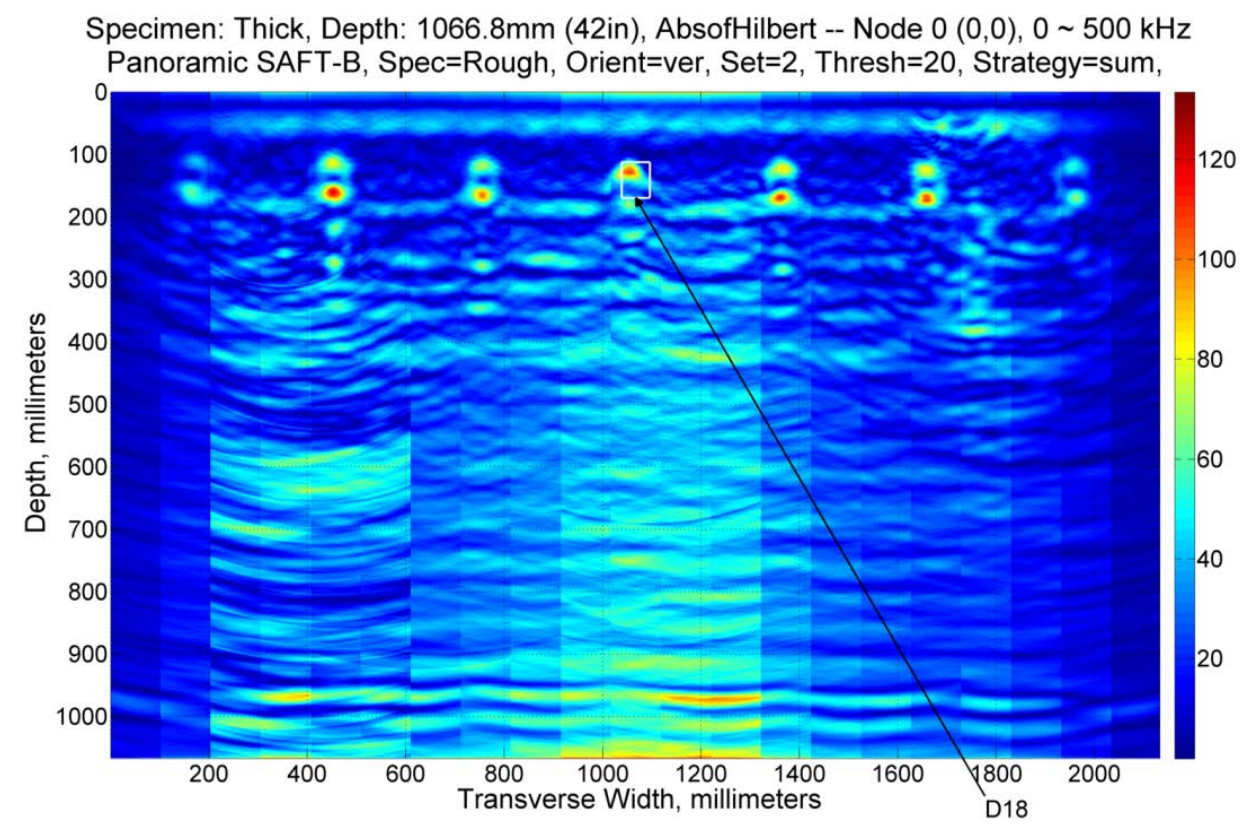

(a)

Specimen: Thick, Depth: 1066.8mm (42in), AbsofHilbert -- Node $34(5,3), 46.875 \sim 62.5 \mathrm{kHz}$

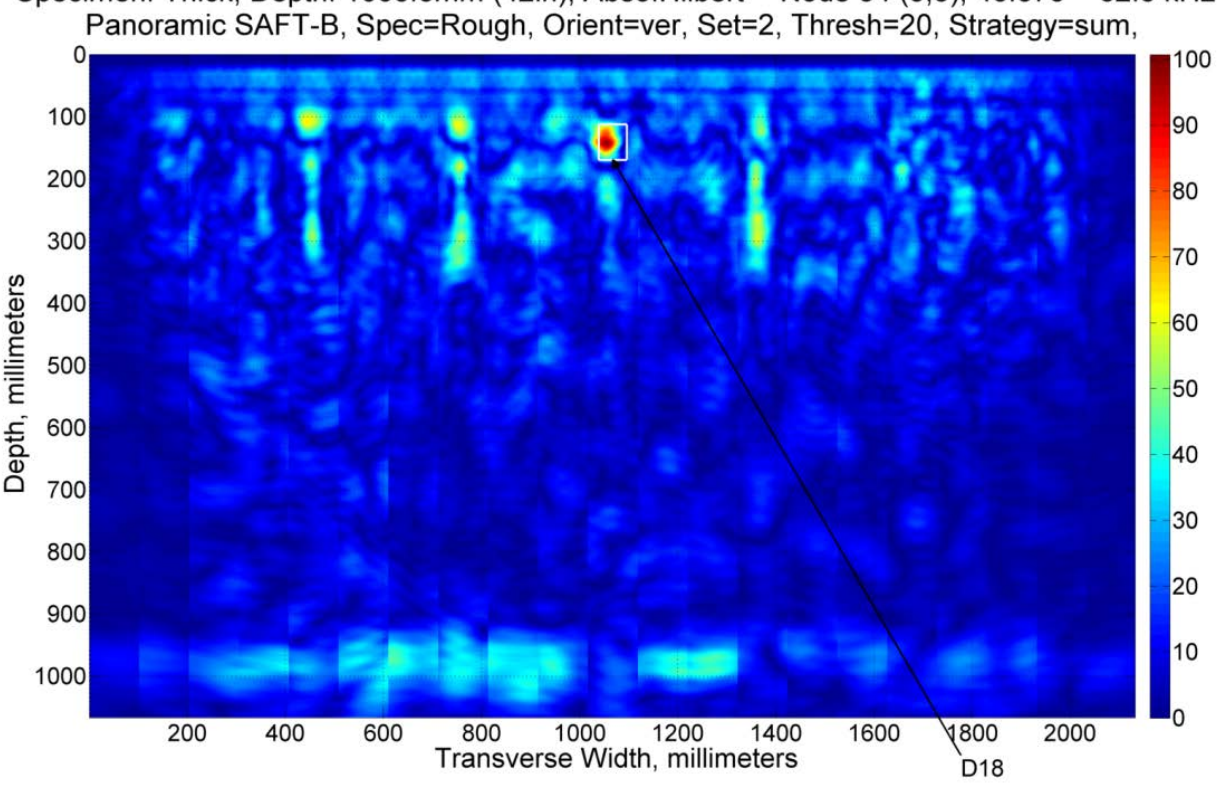

(b)

Fig. 75. Shallow cover improved detectability for Defect 18 for node 34 (b) compared to regular SAFT (a), horizontal orientation \#2. 
10.1.19 Defect 19

\section{D19-Debond Duct Tape (Multi-Layer)}

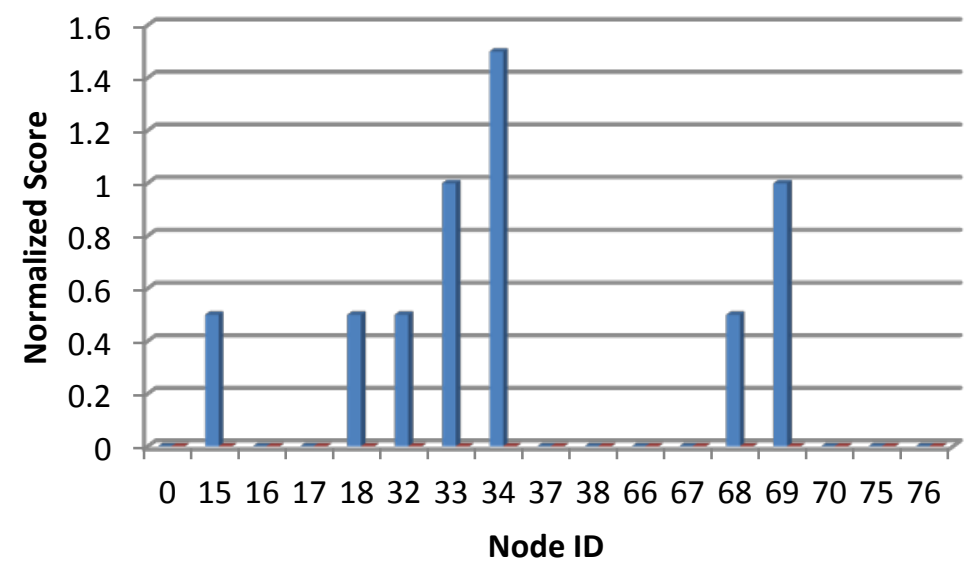

Shallow

Deep

Fig. 76. Defect 19 analysis chart illustrating the normalized score for each node. 


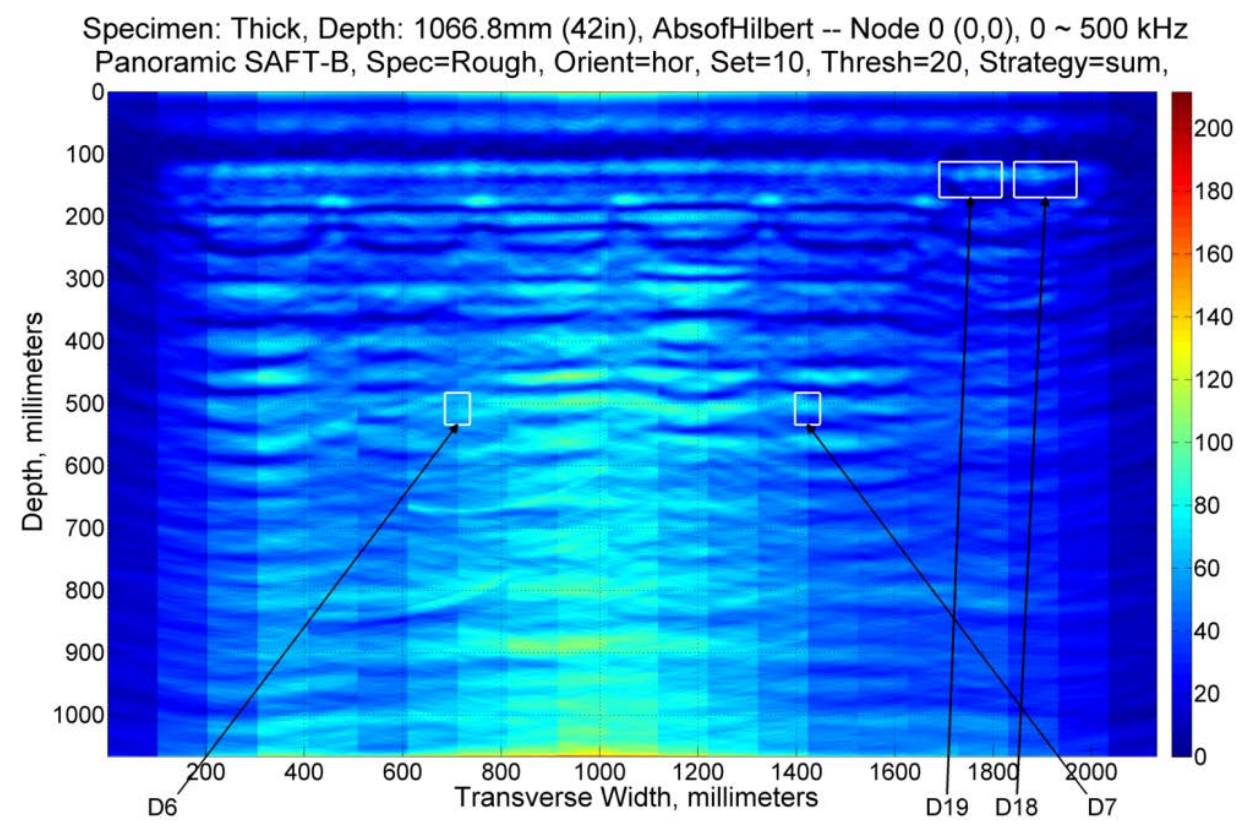

(a)

Specimen: Thick, Depth: 1066.8mm (42in), AbsofHilbert -- Node $34(5,3), 46.875 \sim 62.5 \mathrm{kHz}$ Panoramic SAFT-B, Spec=Rough, Orient=hor, Set=10, Thresh=20, Strategy=sum,

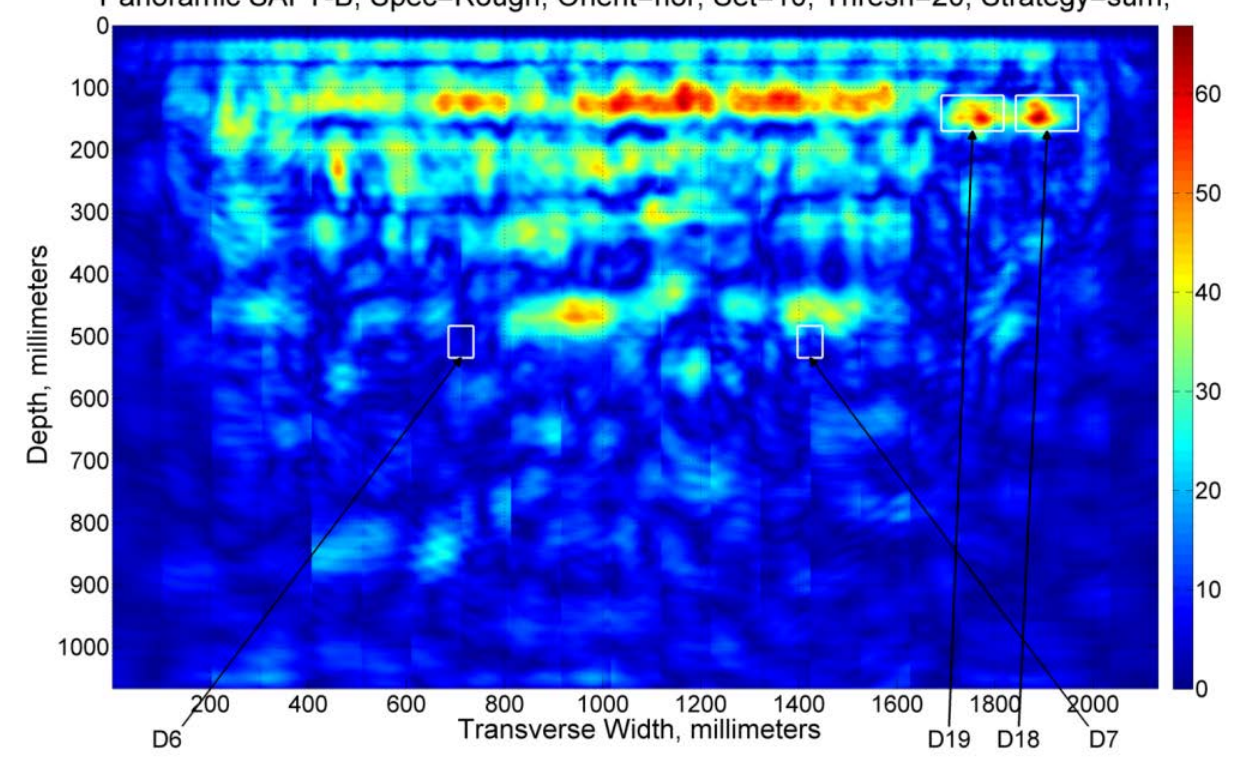

(b)

Fig. 77. Shallow cover improved detectability for Defect 19 for node 34 (b) compared to regular SAFT (a), horizontal orientation. 


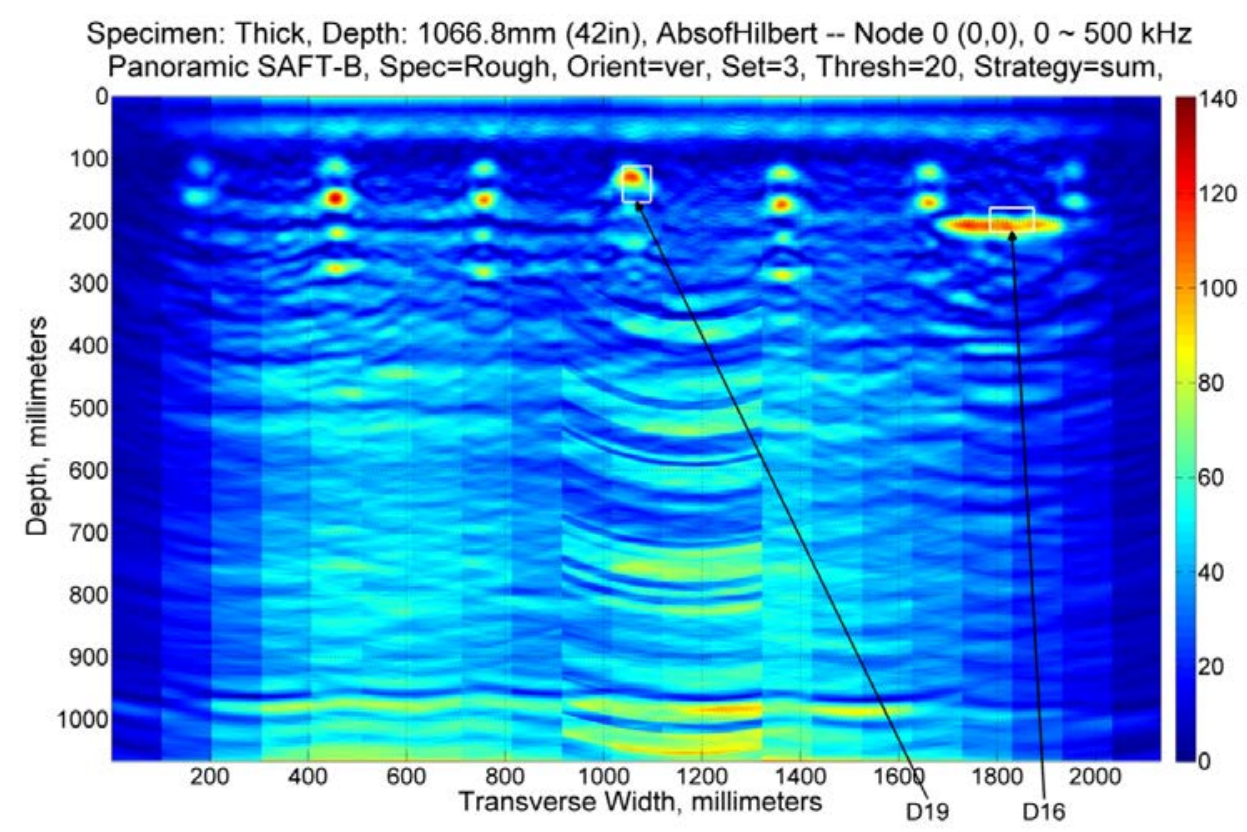

(a)

Specimen: Thick, Depth: 1066.8mm (42in), AbsofHilbert -- Node 34 (5,3), $46.875 \sim 62.5 \mathrm{kHz}$ Panoramic SAFT-B, Spec=Rough, Orient=ver, Set $=3$, Thresh $=20$, Strategy $=s u m$,

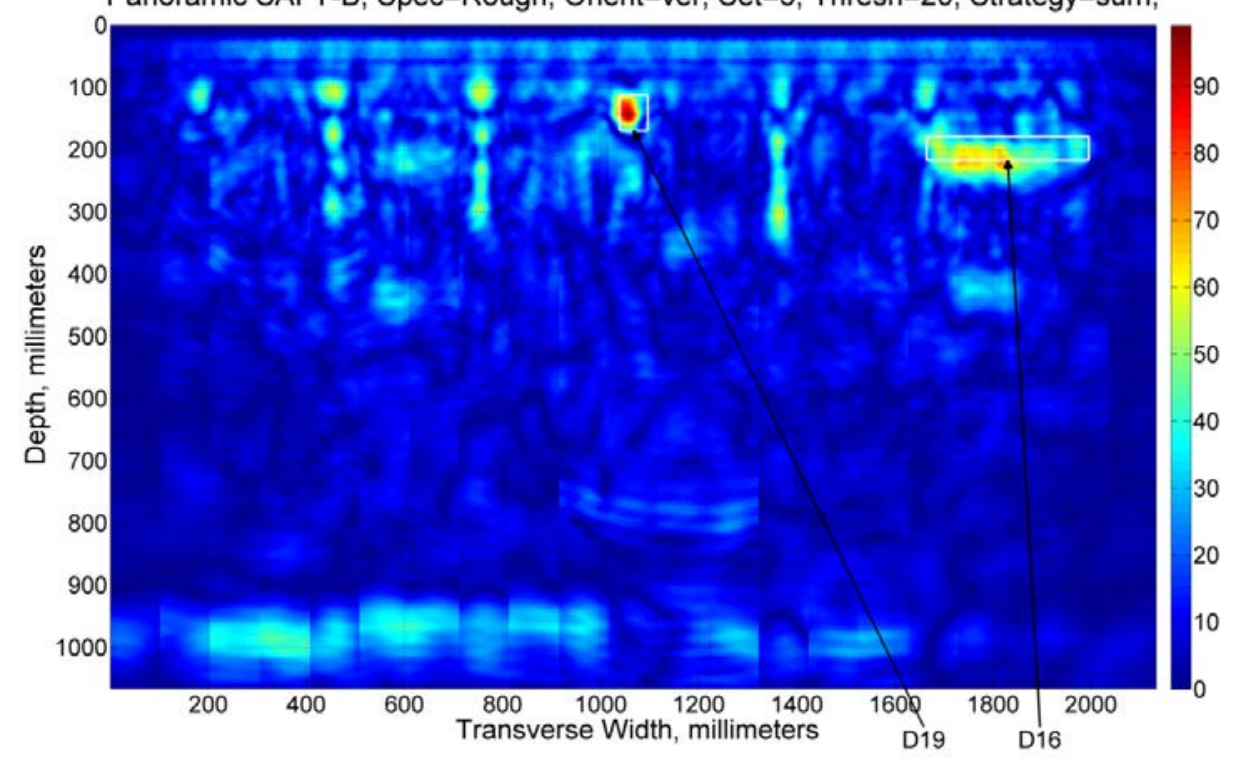

(b)

Fig. 78. Shallow cover improved detectability for Defect 19 for node 34 (b) compared to regular SAFT (a), vertical orientation. 
10.1.20 Defect 20

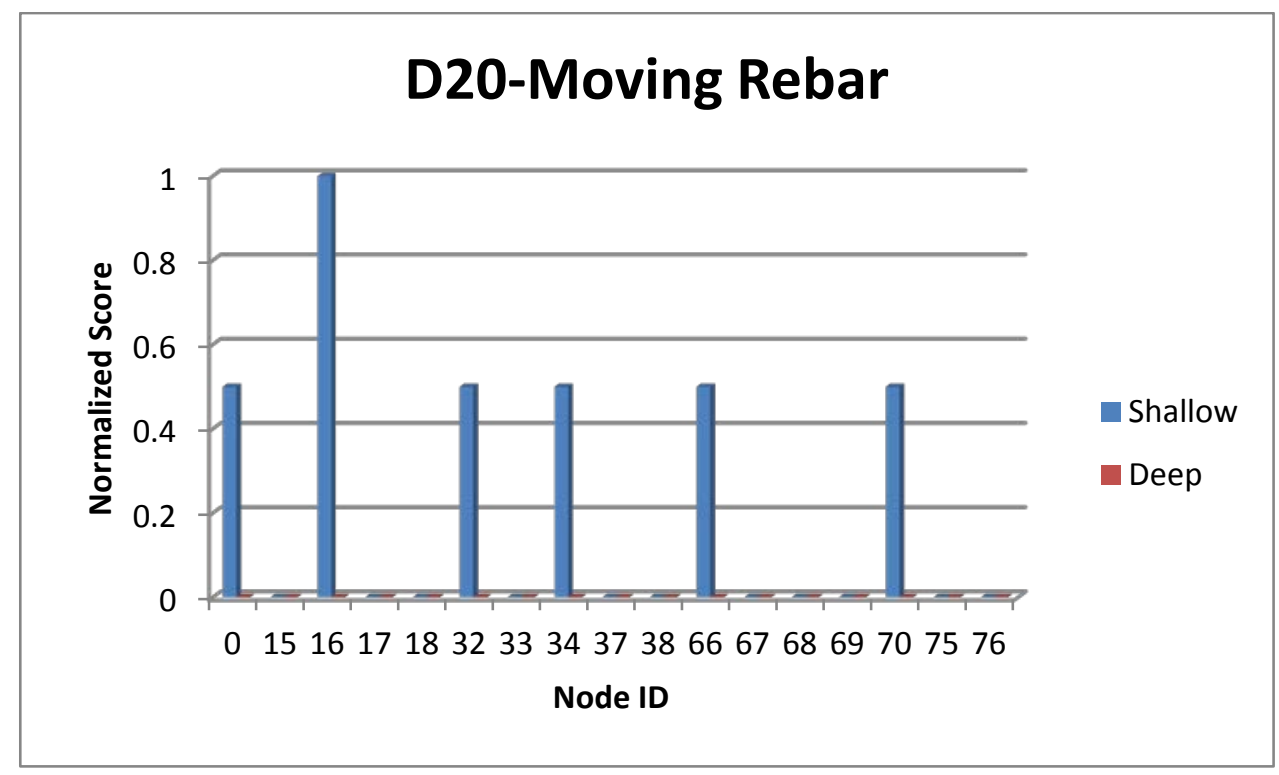

Fig. 79. Defect 20 analysis chart illustrating the normalized score for each node. 
Specimen: Thick, Depth: 1066.8mm (42in), AbsofHilbert -- Node $0(0,0), 0 \sim 500 \mathrm{kHz}$ Panoramic SAFT-B, Spec=Rough, Orient=hor, Set=7, Thresh=20, Strategy=sum,

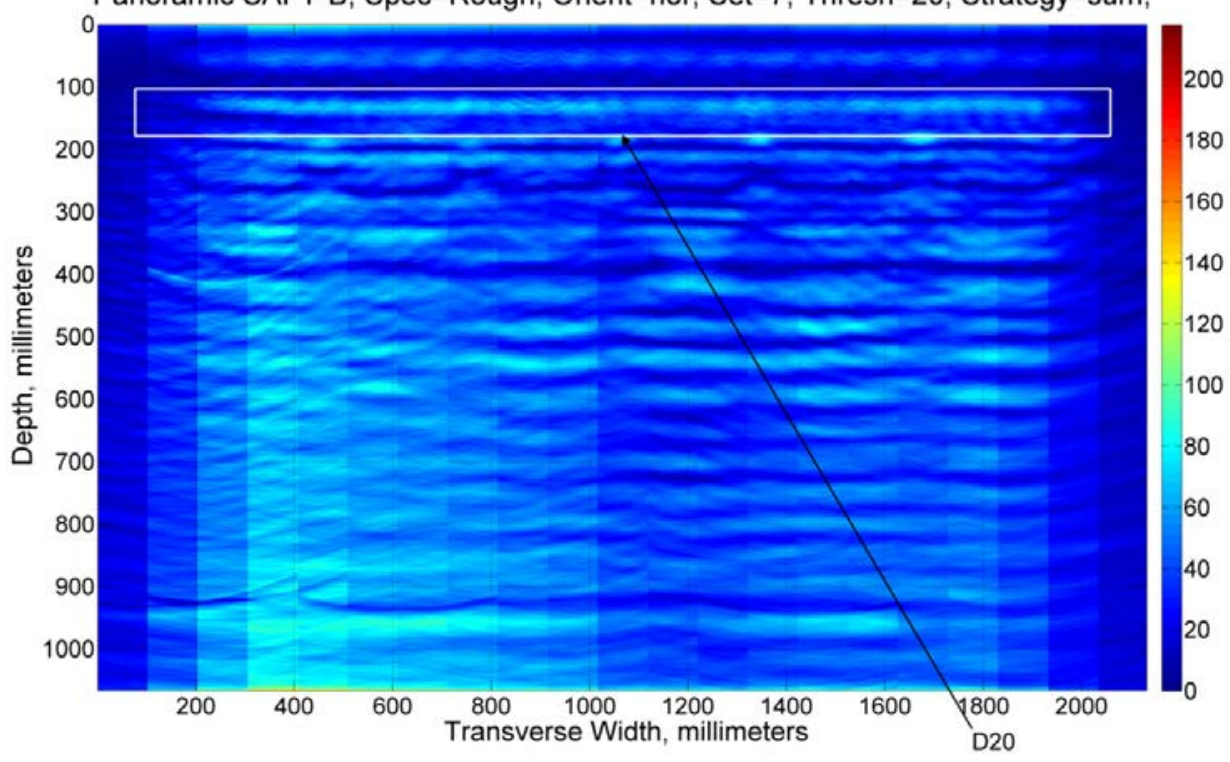

(a)

Specimen: Thick, Depth: 1066.8mm (42in), AbsofHilbert -- Node $16(4,1), 31.25 \sim 62.5 \mathrm{kHz}$ Panoramic SAFT-B, Spec=Rough, Orient=hor, Set=7, Thresh=20, Strategy=sum,

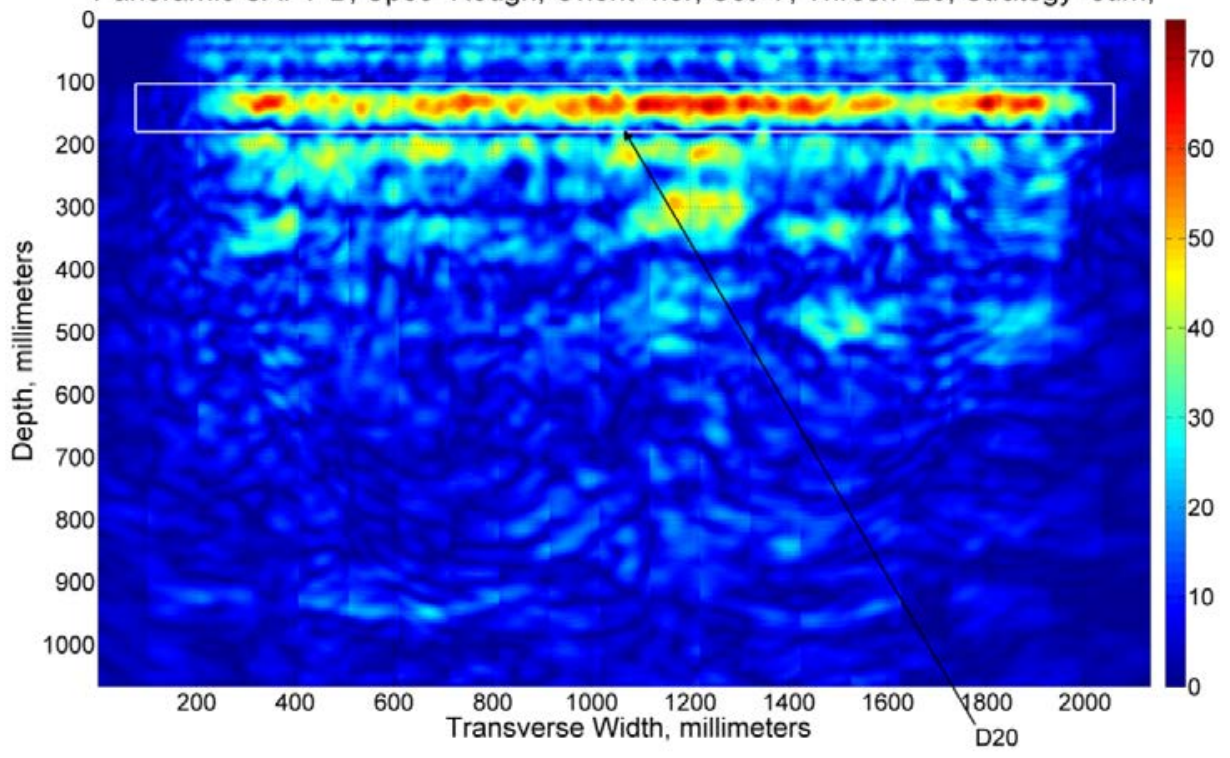

(b)

Fig. 80. Shallow cover improved detectability for Defect 20 for node 16 (b) compared to regular SAFT (a). 


\subsection{DEFECT SUMMARIES}

Information from all 20 defects from the shallow, deep, and combined shallow and deep are presented in this section.

\subsubsection{Shallow}

Table 7 aggregates the "Shallow Total" column from all 20 defects. Summing across rows gives a sense of detectability by node, and summing down columns gives a sense of detectability of each defect.

Table 7. Shallow side performance summary for all defects.

\begin{tabular}{|c|c|c|c|c|c|c|c|c|c|c|c|c|c|c|c|c|c|c|c|c|c|}
\hline \multicolumn{22}{|c|}{ Shallow } \\
\hline & \multicolumn{20}{|c|}{ Defect } & \multirow[b]{2}{*}{ Total } \\
\hline & 1 & 2 & 3 & 4 & 5 & 6 & 7 & 8 & 9 & 10 & 11 & 12 & 13 & 14 & 15 & 16 & 17 & 18 & 19 & 20 & \\
\hline \multicolumn{22}{|c|}{ Node } \\
\hline 0 & 0.00 & 0.00 & 0.33 & 0.33 & 0.00 & 0.00 & 0.33 & 2.00 & 1.75 & 1.25 & 1.75 & 2.00 & 2.00 & 2.00 & 2.00 & 2.00 & 0.00 & 0.00 & 0.00 & 0.50 & 18.25 \\
\hline 15 & 0.00 & 0.33 & 0.00 & 0.00 & 0.00 & 0.00 & 0.00 & 1.00 & 1.50 & 1.25 & 0.25 & 1.00 & 2.00 & 2.00 & 2.00 & 2.00 & 0.00 & 0.00 & 0.50 & 0.00 & 13.83 \\
\hline 16 & 0.67 & 0.00 & 0.00 & 1.33 & 0.00 & 0.00 & 0.00 & 1.50 & 1.75 & 1.25 & 2.00 & 2.00 & 2.00 & 2.00 & 2.00 & 0.67 & 1.33 & 0.00 & 0.00 & 1.00 & 19.50 \\
\hline 17 & 0.00 & 0.00 & 0.00 & 0.00 & 0.00 & 0.00 & 0.00 & 0.00 & 0.00 & 0.00 & 0.00 & 0.00 & 0.00 & 0.00 & 0.00 & 0.00 & 0.00 & 0.00 & 0.00 & 0.00 & 0.00 \\
\hline 18 & 0.00 & 0.00 & 0.00 & 0.67 & 0.00 & 0.00 & 0.00 & 0.75 & 0.25 & 0.00 & 1.25 & 0.00 & 0.50 & 0.50 & 1.00 & 1.33 & 0.00 & 0.00 & 0.50 & 0.00 & 6.75 \\
\hline 32 & 0.00 & 0.67 & 0.67 & 0.33 & 0.00 & 0.00 & 0.00 & 1.50 & 1.25 & 1.50 & 1.00 & 1.25 & 2.00 & 2.00 & 2.00 & 2.00 & 0.00 & 0.00 & 0.50 & 0.50 & 17.17 \\
\hline 33 & 0.00 & 0.00 & 0.00 & 0.00 & 0.00 & 0.00 & 0.00 & 0.50 & 0.50 & 0.50 & 0.75 & 0.50 & 1.50 & 0.50 & 1.50 & 0.67 & 0.67 & 0.00 & 1.00 & 0.00 & 8.58 \\
\hline 34 & 0.67 & 0.00 & 0.33 & 0.67 & 0.00 & 0.00 & 0.00 & 1.25 & 1.75 & 1.00 & 1.75 & 2.00 & 1.75 & 2.00 & 2.00 & 0.67 & 0.33 & 1.00 & 1.50 & 0.50 & 19.17 \\
\hline 37 & 0.00 & 0.00 & 0.00 & 0.33 & 0.00 & 0.00 & 0.00 & 0.50 & 0.25 & 0.00 & 1.25 & 0.00 & 0.50 & 0.00 & 1.00 & 1.33 & 0.00 & 0.00 & 0.00 & 0.00 & 5.17 \\
\hline 38 & 0.00 & 0.00 & 0.00 & 0.00 & 0.00 & 0.00 & 0.00 & 0.00 & 0.00 & 0.00 & 0.00 & 0.00 & 0.25 & 0.00 & 0.00 & 0.00 & 0.00 & 0.00 & 0.00 & 0.00 & 0.25 \\
\hline 66 & 0.00 & 0.67 & 0.00 & 0.00 & 0.67 & 0.00 & 0.00 & 0.50 & 0.75 & 0.25 & 0.00 & 0.25 & 0.75 & 0.00 & 1.00 & 0.67 & 0.00 & 0.00 & 0.00 & 0.50 & 6.00 \\
\hline 67 & 0.00 & 0.00 & 0.00 & 0.00 & 0.00 & 0.00 & 0.00 & 0.50 & 0.00 & 0.00 & 0.00 & 0.00 & 0.75 & 0.00 & 0.00 & 0.67 & 0.00 & 0.00 & 0.00 & 0.00 & 1.92 \\
\hline 68 & 0.00 & 0.00 & 0.00 & 0.00 & 0.00 & 0.00 & 0.00 & 0.00 & 0.00 & 0.00 & 1.00 & 0.00 & 0.00 & 0.50 & 0.50 & 0.00 & 0.00 & 0.00 & 0.50 & 0.00 & 2.50 \\
\hline 69 & 0.00 & 0.00 & 0.00 & 0.33 & 0.00 & 0.00 & 0.00 & 0.75 & 1.00 & 0.00 & 1.50 & 1.50 & 0.75 & 1.00 & 1.00 & 0.33 & 0.00 & 0.50 & 1.00 & 0.00 & 9.67 \\
\hline 70 & 0.00 & 0.00 & 0.00 & 0.00 & 0.00 & 0.00 & 0.00 & 0.00 & 0.00 & 0.00 & 0.25 & 0.00 & 1.00 & 0.00 & 0.00 & 0.67 & 0.00 & 0.00 & 0.00 & 0.50 & 2.42 \\
\hline 75 & 0.00 & 0.00 & 0.00 & 0.33 & 0.00 & 0.00 & 0.00 & 0.25 & 0.75 & 0.00 & 0.00 & 0.00 & 0.25 & 0.00 & 0.00 & 0.00 & 0.00 & 0.00 & 0.00 & 0.00 & 1.58 \\
\hline 76 & 0.00 & 0.00 & 0.00 & 0.00 & 0.00 & 0.00 & 0.00 & 0.00 & 0.00 & 0.00 & 0.00 & 0.00 & 0.00 & 0.00 & 0.00 & 0.33 & 0.00 & 0.00 & 0.00 & 0.00 & 0.33 \\
\hline Total & 1.33 & 1.67 & 1.33 & 4.33 & 0.67 & 0.00 & 0.33 & 11.00 & 11.50 & 7.00 & 12.75 & 10.50 & 16.00 & 12.50 & 16.00 & 13.33 & 2.33 & 1.50 & 5.50 & 3.50 & \\
\hline
\end{tabular}


Fig. 81 shows the normalized values in the far right column of Table 7 normalized and sorted in descending order.

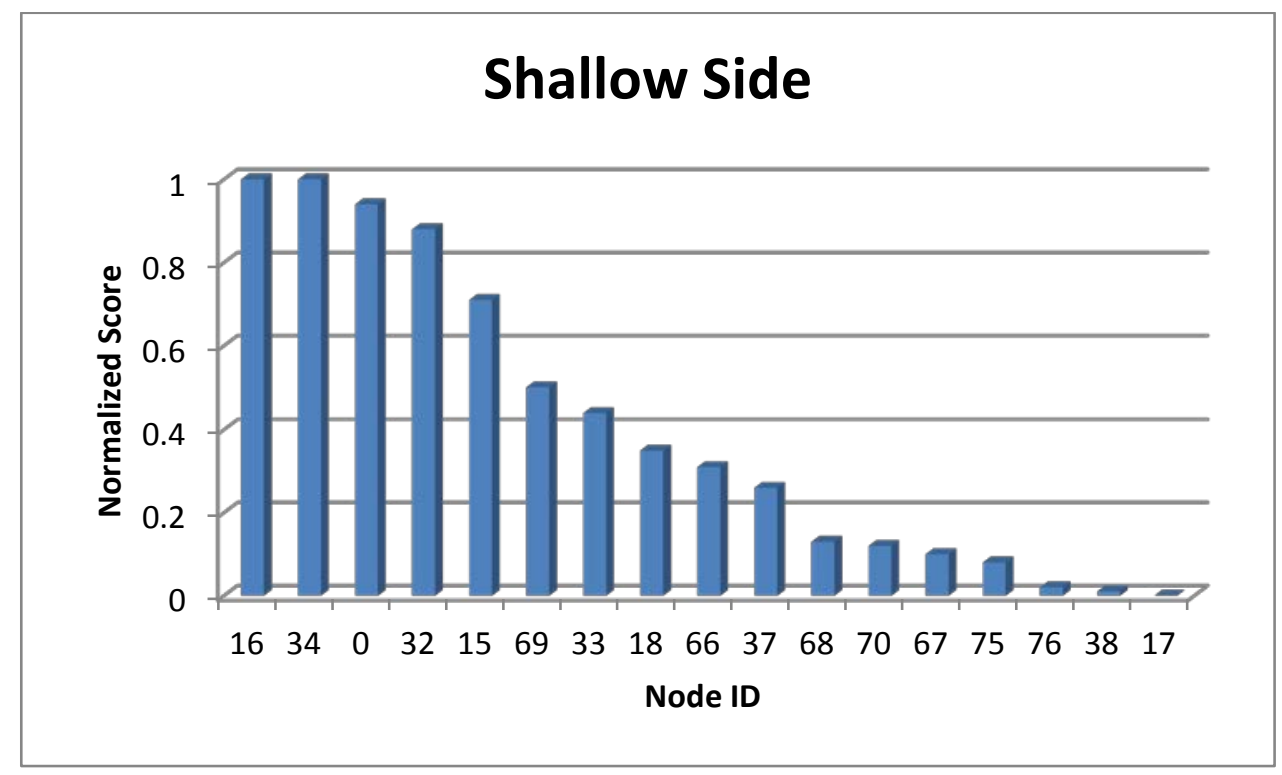

Fig. 81. Best node for shallow side sorted by normalized total across all defects.

Fig. 82 shows the normalized values in the bottom row of Table 7, normalized and sorted in descending order.

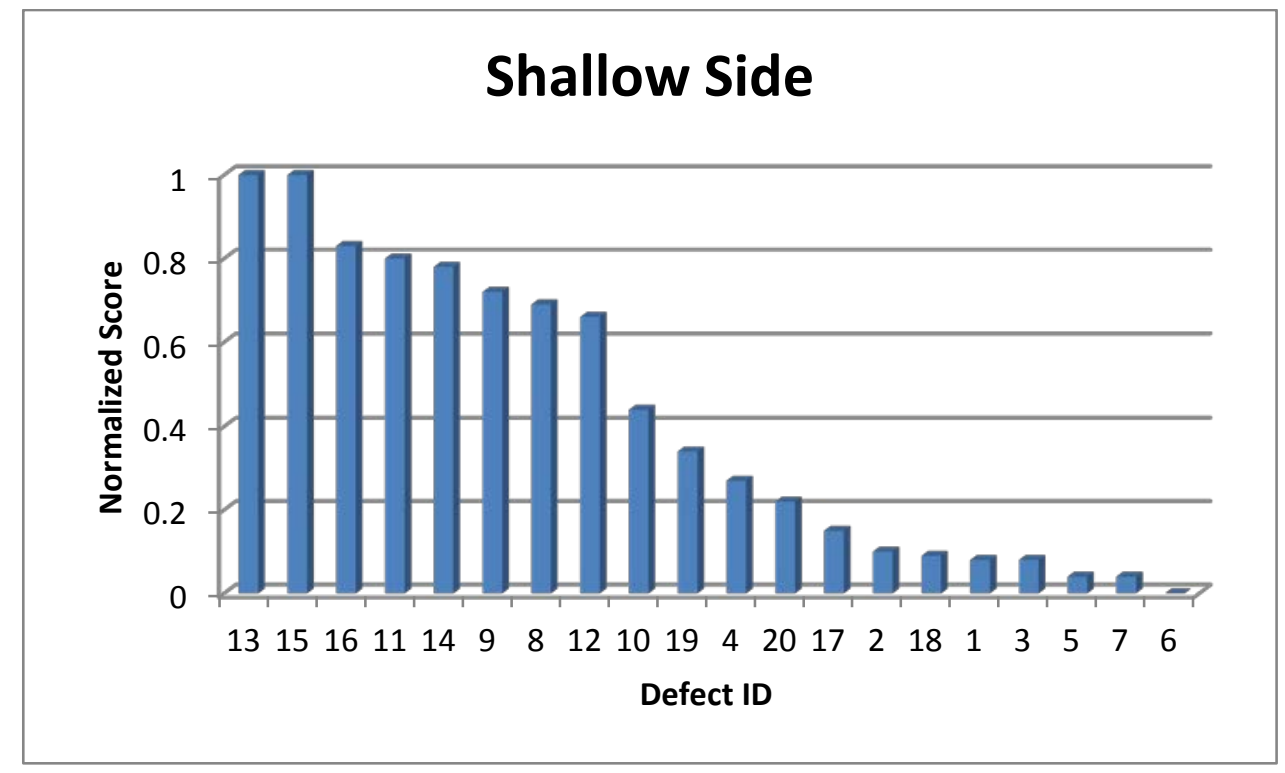

Fig. 82. Most detectible defect for shallow side sorted by normalized total across all nodes. 


\subsubsection{Deep}

Table 8 aggregates the "Deep Total” column from all 20 defects. Summing across rows gives a sense of detectability by node, and summing down columns gives a sense of detectability of each defect.

Table 8. Deep side performance summary for all defects.

\begin{tabular}{|c|c|c|c|c|c|c|c|c|c|c|c|c|c|c|c|c|c|c|c|c|c|}
\hline \multicolumn{22}{|c|}{ Deep } \\
\hline & \multicolumn{20}{|c|}{ Defect } & \multirow[b]{2}{*}{ Total } \\
\hline & 1 & 2 & 3 & 4 & 5 & 6 & 7 & 8 & 9 & 10 & 11 & 12 & 13 & 14 & 15 & 16 & 17 & 18 & 19 & 20 & \\
\hline \multicolumn{22}{|c|}{ Node } \\
\hline $\mathbf{0}$ & 0.00 & 0.00 & 0.00 & 0.00 & 0.00 & 0.00 & 0.00 & 0.50 & 1.00 & 0.00 & 0.25 & 1.75 & 1.50 & 0.00 & 0.00 & 0.00 & 0.00 & 0.00 & 0.00 & 0.00 & 5.00 \\
\hline 15 & 0.00 & 0.00 & 0.00 & 0.00 & 0.00 & 0.00 & 0.00 & 0.00 & 0.00 & 0.00 & 0.00 & 1.75 & 1.00 & 0.00 & 0.00 & 0.00 & 0.00 & 0.00 & 0.00 & 0.00 & 2.75 \\
\hline 16 & 0.00 & 0.00 & 0.00 & 0.00 & 0.00 & 0.00 & 0.00 & 0.50 & 0.50 & 0.25 & 0.75 & 1.50 & 0.50 & 0.00 & 2.00 & 1.00 & 0.00 & 0.00 & 0.00 & 0.00 & 7.00 \\
\hline 17 & 0.00 & 0.00 & 0.00 & 0.00 & 0.00 & 0.00 & 0.00 & 0.00 & 0.00 & 0.00 & 0.00 & 0.00 & 0.00 & 0.00 & 0.00 & 0.00 & 0.00 & 0.00 & 0.00 & 0.00 & 0.00 \\
\hline 18 & 0.00 & 0.00 & 0.00 & 0.00 & 0.00 & 0.00 & 0.00 & 0.00 & 0.00 & 0.00 & 0.50 & 0.25 & 0.00 & 0.00 & 0.00 & 0.00 & 0.00 & 0.00 & 0.00 & 0.00 & 0.75 \\
\hline 32 & 0.00 & 0.00 & 0.00 & 0.00 & 0.00 & 0.00 & 0.00 & 0.75 & 0.25 & 0.00 & 1.00 & 1.75 & 0.75 & 0.00 & 0.00 & 0.67 & 0.00 & 0.00 & 0.00 & 0.00 & 5.17 \\
\hline 33 & 0.00 & 0.00 & 0.00 & 0.00 & 0.00 & 0.00 & 0.00 & 0.00 & 0.00 & 0.00 & 0.25 & 0.75 & 0.00 & 0.00 & 0.00 & 0.00 & 0.00 & 0.00 & 0.00 & 0.00 & 1.00 \\
\hline 34 & 0.00 & 0.00 & 0.00 & 0.00 & 0.00 & 0.33 & 0.00 & 0.50 & 0.25 & 0.50 & 0.50 & 1.00 & 0.50 & 0.00 & 2.00 & 1.00 & 0.00 & 0.00 & 0.00 & 0.00 & 6.58 \\
\hline 37 & 0.00 & 0.00 & 0.00 & 0.00 & 0.00 & 0.00 & 0.00 & 0.00 & 0.00 & 0.00 & 0.25 & 0.25 & 0.00 & 0.00 & 0.00 & 0.00 & 0.00 & 0.00 & 0.00 & 0.00 & 0.50 \\
\hline 38 & 0.00 & 0.00 & 0.00 & 0.00 & 0.00 & 0.00 & 0.00 & 0.00 & 0.00 & 0.00 & 0.00 & 0.00 & 0.00 & 0.00 & 0.00 & 0.00 & 0.00 & 0.00 & 0.00 & 0.00 & 0.00 \\
\hline 66 & 0.00 & 0.00 & 0.00 & 0.00 & 0.00 & 0.00 & 0.00 & 0.25 & 0.25 & 0.00 & 0.00 & 0.50 & 0.50 & 0.00 & 0.00 & 0.00 & 0.00 & 0.00 & 0.00 & 0.00 & 1.50 \\
\hline 67 & 0.00 & 0.00 & 0.00 & 0.00 & 0.00 & 0.00 & 0.00 & 0.00 & 0.00 & 0.00 & 0.25 & 0.00 & 0.00 & 0.00 & 0.00 & 0.00 & 0.00 & 0.00 & 0.00 & 0.00 & 0.25 \\
\hline 68 & 0.00 & 0.00 & 0.00 & 0.00 & 0.00 & 0.00 & 0.00 & 0.00 & 0.00 & 0.00 & 0.00 & 0.00 & 0.00 & 0.00 & 0.00 & 0.00 & 0.00 & 0.00 & 0.00 & 0.00 & 0.00 \\
\hline 69 & 0.00 & 0.00 & 0.00 & 0.00 & 0.00 & 0.33 & 0.00 & 0.75 & 0.00 & 0.50 & 0.25 & 1.00 & 0.25 & 0.50 & 2.00 & 1.00 & 0.00 & 0.00 & 0.00 & 0.00 & 6.58 \\
\hline 70 & 0.00 & 0.00 & 0.00 & 0.00 & 0.00 & 0.00 & 0.00 & 0.00 & 0.00 & 0.00 & 0.25 & 0.00 & 0.00 & 0.00 & 0.00 & 0.00 & 0.00 & 0.00 & 0.00 & 0.00 & 0.25 \\
\hline 75 & 0.00 & 0.00 & 0.00 & 0.00 & 0.00 & 0.00 & 0.00 & 0.00 & 0.00 & 0.00 & 0.00 & 0.00 & 0.00 & 0.00 & 0.00 & 0.00 & 0.00 & 0.00 & 0.00 & 0.00 & 0.00 \\
\hline 76 & 0.00 & 0.00 & 0.00 & 0.00 & 0.00 & 0.00 & 0.00 & 0.00 & 0.00 & 0.00 & 0.00 & 0.00 & 0.00 & 0.00 & 0.00 & 0.00 & 0.00 & 0.00 & 0.00 & 0.00 & 0.00 \\
\hline Total & 0.00 & 0.00 & 0.00 & 0.00 & 0.00 & 0.67 & 0.00 & 3.25 & 2.25 & 1.25 & 4.25 & 10.50 & 5.00 & 0.50 & 6.00 & 3.67 & 0.00 & 0.00 & 0.00 & 0.00 & \\
\hline
\end{tabular}


Fig. 83 shows the normalized values in the far right column of Table 8, normalized and sorted in descending order.

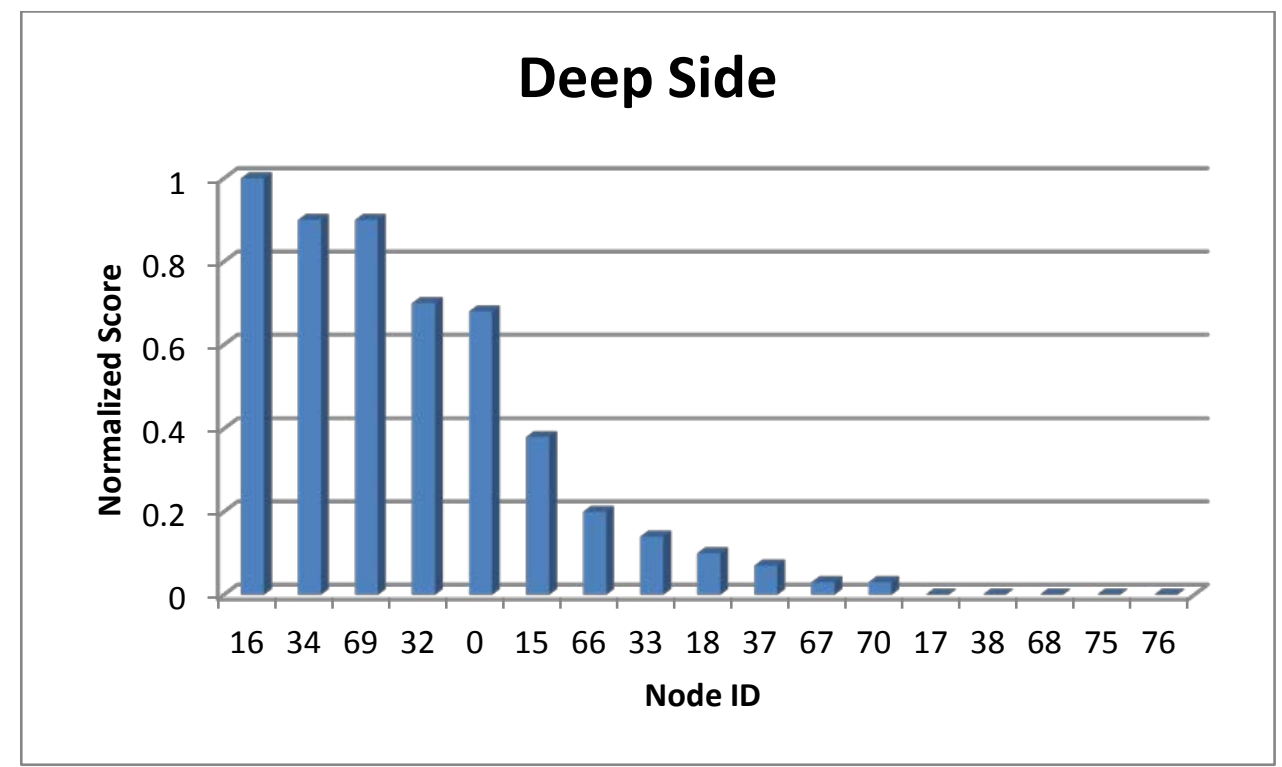

Fig. 83. Best node for deep side sorted by normalized total across all defects.

Fig. 84 shows the normalized values in the bottom row of Table 8, normalized and sorted in descending order.

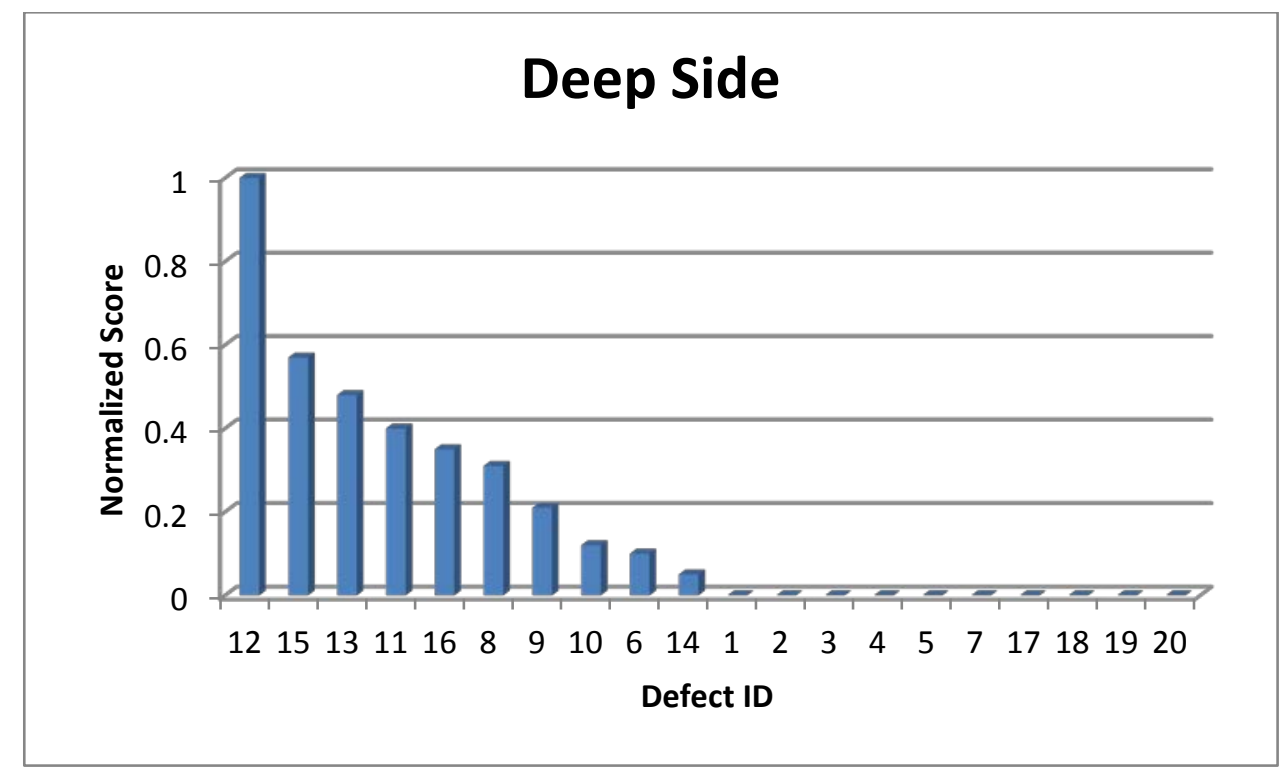

Fig. 84. Most detectible defect for deep side sorted by normalized total across all nodes. 
10.2.3 Combined Shallow and Deep

Table 9 aggregates the "Combined Shallow and Deep Total” column from all 20 defects. Summing across rows gives a sense of detectability by node, and summing down columns gives a sense of detectability of each defect.

Table 9. Combined Shallow and deep side performance summary for all defects.

\begin{tabular}{|c|c|c|c|c|c|c|c|c|c|c|c|c|c|c|c|c|c|c|c|c|c|}
\hline \multicolumn{22}{|c|}{ Combined Shallow and Deep } \\
\hline & \multicolumn{20}{|c|}{ Defect } & \multirow[b]{2}{*}{ Total } \\
\hline & 1 & 2 & 3 & 4 & 5 & 6 & 7 & 8 & 9 & 10 & 11 & 12 & 13 & 14 & 15 & 16 & 17 & 18 & 19 & 20 & \\
\hline \multicolumn{22}{|l|}{ Node } \\
\hline $\mathbf{0}$ & 0.00 & 0.00 & 0.33 & 0.33 & 0.00 & 0.00 & 0.33 & 2.50 & 2.75 & 1.25 & 2.00 & 3.75 & 3.50 & 2.00 & 2.00 & 2.00 & 0.00 & 0.00 & 0.00 & 0.50 & 23.25 \\
\hline 15 & 0.00 & 0.33 & 0.00 & 0.00 & 0.00 & 0.00 & 0.00 & 1.00 & 1.50 & 1.25 & 0.25 & 2.75 & 3.00 & 2.00 & 2.00 & 2.00 & 0.00 & 0.00 & 0.50 & 0.00 & 16.58 \\
\hline 16 & 0.67 & 0.00 & 0.00 & 1.33 & 0.00 & 0.00 & 0.00 & 2.00 & 2.25 & 1.50 & 2.75 & 3.50 & 2.50 & 2.00 & 4.00 & 1.67 & 1.33 & 0.00 & 0.00 & 1.00 & 26.50 \\
\hline 17 & 0.00 & 0.00 & 0.00 & 0.00 & 0.00 & 0.00 & 0.00 & 0.00 & 0.00 & 0.00 & 0.00 & 0.00 & 0.00 & 0.00 & 0.00 & 0.00 & 0.00 & 0.00 & 0.00 & 0.00 & 0.00 \\
\hline 18 & 0.00 & 0.00 & 0.00 & 0.67 & 0.00 & 0.00 & 0.00 & 0.75 & 0.25 & 0.00 & 1.75 & 0.25 & 0.50 & 0.50 & 1.00 & 1.33 & 0.00 & 0.00 & 0.50 & 0.00 & 7.50 \\
\hline 32 & 0.00 & 0.67 & 0.67 & 0.33 & 0.00 & 0.00 & 0.00 & 2.25 & 1.50 & 1.50 & 2.00 & 3.00 & 2.75 & 2.00 & 2.00 & 2.67 & 0.00 & 0.00 & 0.50 & 0.50 & 22.33 \\
\hline 33 & 0.00 & 0.00 & 0.00 & 0.00 & 0.00 & 0.00 & 0.00 & 0.50 & 0.50 & 0.50 & 1.00 & 1.25 & 1.50 & 0.50 & 1.50 & 0.67 & 0.67 & 0.00 & 1.00 & 0.00 & 9.58 \\
\hline 34 & 0.67 & 0.00 & 0.33 & 0.67 & 0.00 & 0.33 & 0.00 & 1.75 & 2.00 & 1.50 & 2.25 & 3.00 & 2.25 & 2.00 & 4.00 & 1.67 & 0.33 & 1.00 & 1.50 & 0.50 & 25.75 \\
\hline 37 & 0.00 & 0.00 & 0.00 & 0.33 & 0.00 & 0.00 & 0.00 & 0.50 & 0.25 & 0.00 & 1.50 & 0.25 & 0.50 & 0.00 & 1.00 & 1.33 & 0.00 & 0.00 & 0.00 & 0.00 & 5.67 \\
\hline 38 & 0.00 & 0.00 & 0.00 & 0.00 & 0.00 & 0.00 & 0.00 & 0.00 & 0.00 & 0.00 & 0.00 & 0.00 & 0.25 & 0.00 & 0.00 & 0.00 & 0.00 & 0.00 & 0.00 & 0.00 & 0.25 \\
\hline 66 & 0.00 & 0.67 & 0.00 & 0.00 & 0.67 & 0.00 & 0.00 & 0.75 & 1.00 & 0.25 & 0.00 & 0.75 & 1.25 & 0.00 & 1.00 & 0.67 & 0.00 & 0.00 & 0.00 & 0.50 & 7.50 \\
\hline 67 & 0.00 & 0.00 & 0.00 & 0.00 & 0.00 & 0.00 & 0.00 & 0.50 & 0.00 & 0.00 & 0.25 & 0.00 & 0.75 & 0.00 & 0.00 & 0.67 & 0.00 & 0.00 & 0.00 & 0.00 & 2.17 \\
\hline 68 & 0.00 & 0.00 & 0.00 & 0.00 & 0.00 & 0.00 & 0.00 & 0.00 & 0.00 & 0.00 & 1.00 & 0.00 & 0.00 & 0.50 & 0.50 & 0.00 & 0.00 & 0.00 & 0.50 & 0.00 & 2.50 \\
\hline 69 & 0.00 & 0.00 & 0.00 & 0.33 & 0.00 & 0.33 & 0.00 & 1.50 & 1.00 & 0.50 & 1.75 & 2.50 & 1.00 & 1.50 & 3.00 & 1.33 & 0.00 & 0.50 & 1.00 & 0.00 & 16.25 \\
\hline 70 & 0.00 & 0.00 & 0.00 & 0.00 & 0.00 & 0.00 & 0.00 & 0.00 & 0.00 & 0.00 & 0.50 & 0.00 & 1.00 & 0.00 & 0.00 & 0.67 & 0.00 & 0.00 & 0.00 & 0.50 & 2.67 \\
\hline 75 & 0.00 & 0.00 & 0.00 & 0.33 & 0.00 & 0.00 & 0.00 & 0.25 & 0.75 & 0.00 & 0.00 & 0.00 & 0.25 & 0.00 & 0.00 & 0.00 & 0.00 & 0.00 & 0.00 & 0.00 & 1.58 \\
\hline 76 & 0.00 & 0.00 & 0.00 & 0.00 & 0.00 & 0.00 & 0.00 & 0.00 & 0.00 & 0.00 & 0.00 & 0.00 & 0.00 & 0.00 & 0.00 & 0.33 & 0.00 & 0.00 & 0.00 & 0.00 & 0.33 \\
\hline Total & 1.33 & 1.67 & 1.33 & 4.33 & 0.67 & 0.67 & 0.33 & 14.25 & 13.75 & 8.25 & 17.00 & 21.00 & 21.00 & 13.00 & 22.00 & 17.00 & 2.33 & 1.50 & 5.50 & 3.50 & \\
\hline
\end{tabular}


Fig. 85 shows the normalized values in the far right column of Table 9, normalized and sorted in descending order.

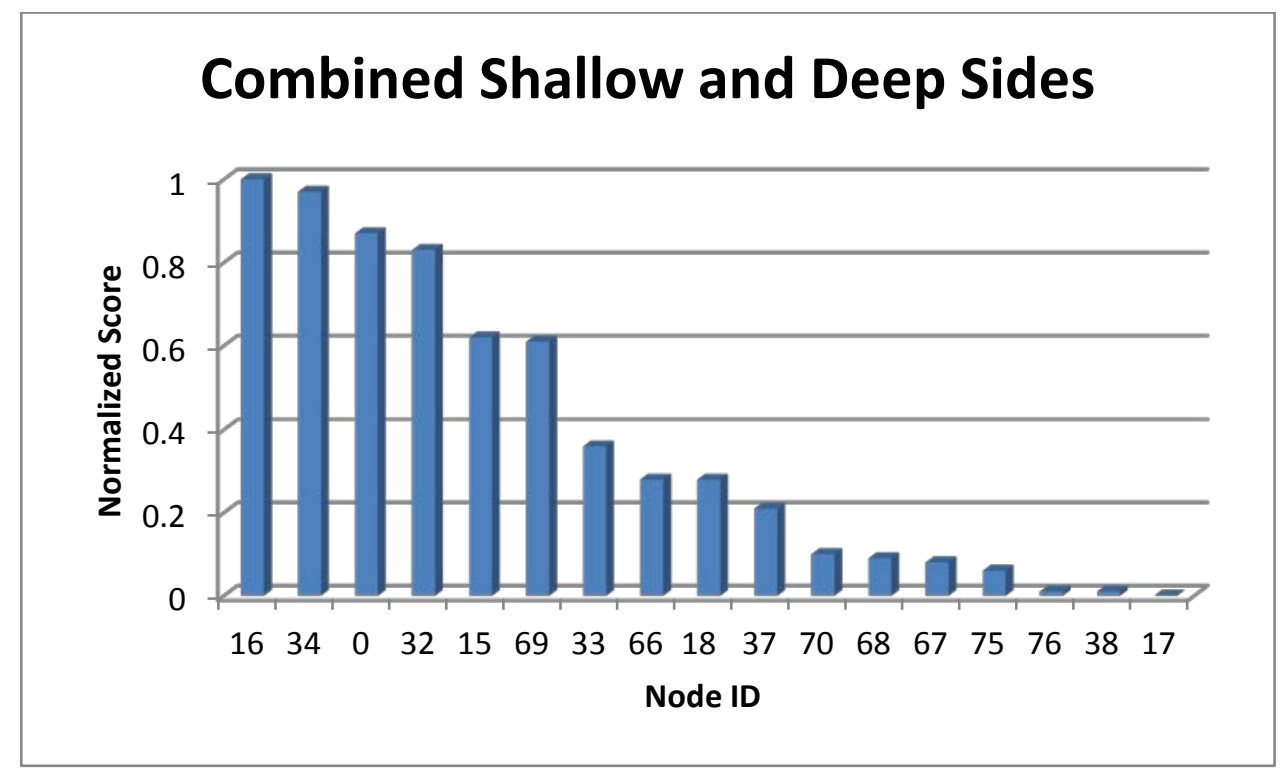

Fig. 85. Best node for combined shallow and deep sides sorted by normalized total across all defects.

Fig. 86 shows the normalized values in the bottom row of Table 9, normalized and sorted in descending order.

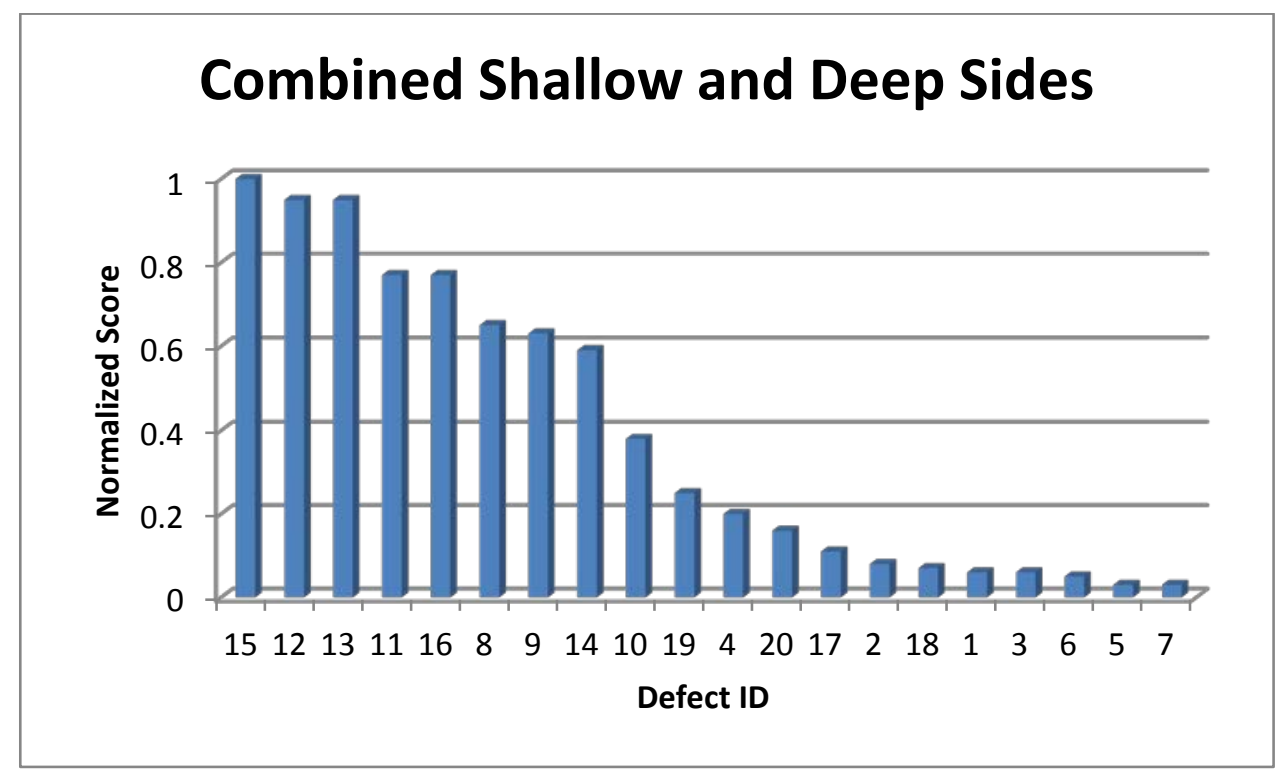

Fig. 86. Most detectible defect for combined shallow and deep sides sorted by normalized total across all nodes. 


\section{WAVELET FREQUENCY-BANDING METHOD CONCLUSIONS}

\subsection{BEST NODES}

Looking at Fig. 81, if you pick a normalized threshold, such as 0.5 , you can see that these 6 nodes are the most promising out of the 16 for the shallow side: 16, 34, 0, 32, 15, 69.

Similarly, Fig. 83 suggests these 5 nodes are the most promising out of the 16 for the deep side: 16, 34, 69, 32, 0.

Likewise, Fig. 85 suggests these 6 nodes are the most promising out of the 16 for the combined shallow and deep side: $16,34,0,32,15,69$.

One observation is that the same nodes are generally the best both from the shallow and deep sides. It could help detection if nodes 16, 34, 32, 69, and 15 were viewed in addition to regular SAFT.

\subsection{MOST DETECTIBLE DEFECTS}

Fig. 82 and Fig. 84 show that defects were obviously more detectible from the shallow side than the deep side. If you pick a normalized threshold, say 0.3 , you can say you detect these defects pretty well from the shallow side: $13,15,16,11,14,9,8,12,10,9$

And these from the deep side: 12, 15, 13, 11, 16, 8.

The additional wavelet analysis proved useful for several defects, but some were still undetectable no matter which node was used. 



\section{MODEL-BASED ITERATIVE RECONSTRUCTION}

Model-Based Iterative Reconstruction (MBIR) is an image reconstruction framework that embraces the integrated imaging philosophy, where the hardware and software are tailored to provide the most informative measurement. The method has been applied to the reconstruction of X-ray Computed Tomography (CT) with a superior image quality than state-of-the-art filter back projection techniques. MBIR shows equivalent image quality even after X-ray dose reductions of up to $80 \%$. This reduction in $\mathrm{X}$-ray dose is a testament of the robustness of the system in the presence of noise and sparse information collection, which are usually the interrogation conditions for thick concrete. Consequently, we are pioneering the first implementation of the MBIR algorithm for ultrasound signals. In contrast to delayand-sum approaches like SAFT, where reconstructed pixel intensities are an integration of signal amplitudes under poor wave propagation assumptions (i.e., constant acoustic speed), our end goal is to include MBIR comprehensive models for the acoustic system and media (e.g., approximations of wave propagation models for longitudinal and shear waves that include changes in acoustic speed throughout the media). In addition, MBIR will iterate until it finds the intensity reflectivity coefficients (IRC) over the field of view that best fits the data. Our end-goal is to contribute to a reconstruction method that increases the depth range of current ultrasonic probes and image quality, and provides quantitative measurements of the physical properties of the medium under interrogation. For this initial implementation, we define a forward model for acoustic p-wave propagation from an ultrasonic phased array with the following assumptions: the media is homogenous, and the Born approximation and coherent integration hold. This chapter provides a detailed description of the forward, inverted, and discretized models. Finally, we offer a toy reconstruction sample with synthetic data.

\subsection{THE MBIR FRAMEWORK}

MBIR is a powerful probabilistic tool that has been proven to be very effective for reconstruction in many applications. The method has been extensively applied to the reconstruction of X-ray CT with an image quality superior to state-of-the-art filter back projection techniques. MBIR models the system and reconstructs the solution using the inverse problem method. MBIR works by designing a probabilistic model for the measurements, known as the forward model, and a probabilistic model for the object, known as the prior model. Both models are used to formulate an objective function, i.e., the cost function, such as the maximum a posteriori (MAP) cost function. A typical MBIR problem revolves around finding

$$
\widetilde{x}=\frac{\operatorname{argmin}}{x}\{f(x)\}=\frac{\operatorname{argmin}}{x}\{-\log p(y \mid x)-\log p(x)\}
$$

where $x$ is the unknown (ie., IRC values) that we would like to estimate, $f(x)$ is the cost function, $y$ is the measured data, $\tilde{x}$ is the estimate of $x, p(y \mid x)$ is the forward model, and $p(x)$ is the prior model [33].

The final step in MBIR is to optimize the cost function. While more accurate models produce high quality solutions, they make the cost function more complicated. This in return makes solving the inverse problem very computationally expensive. Therefore, many iterative reconstruction algorithms have been adopted to speed up the computation, such as Gradient Descent (GD), Conjugate Gradient (CG), Iterative Coordinate Descent (ICD), etc.

There are many iterative reconstruction methods, such as iterative maximum likelihood estimation (MLE) [34], and simultaneous iterative reconstruction technique (SIRT) [35]. The main difference between MBIR and MLE is that MLE does not require a prior model of the object, which can be sensitive to random variation in data. The prior model is necessary to regulate the estimation and reduce variance. However, this will require having an accurate prior model. Similarly, SIRT does not require a prior model, either, and does not require a probabilistic model for the measurements [36]. 
MBIR has been used in many tomographic applications. It has been used in X-Ray (CT) [37], positron emission tomography (PET) [38], and electron tomography [39]. However, MBIR has not been applied to ultrasound tomography yet. There are a few iterative non-MBIR reconstruction methods for ultrasound, such as the Born Iterative Method (BIM), which is combined with Total Variation (TV) minimization to reconstruct ultrasound computed tomography (UCT) using compressed sensing (CS) [40], and the Iterative Inverse Non-uniform Fast Fourier Transform (NUFFT), which is used in diffraction UCT [41]. While these methods can produce good results, they are intended for transmission measurements. In this paper, we show an MBIR implementation for one-sided ultrasound applications.

\subsection{MBIR FORWARD AND PRIOR MODELS}

To apply MBIR to the ultrasonic signals, we need to formulate the forward model $p_{y \mid x}(y \mid x)$ and the prior model $p(x)$, where $y$ is the observed data and $x$ is the unknown IRC. The forward model $-\log \left(p_{y \mid x}(y \mid x)\right)$ is approximated by

$$
\frac{1}{2}(y-A x)^{T} \Lambda(y-A x)
$$

where $\Lambda$ is an diagonal matrix with statistical weights and $A$ is the system matrix defined below.

In order to compute the system matrix $A$, we will consider an ultrasound system that probes a position $p \in \mathbb{R}^{3}$ in a homogeneous medium [42] by transmitting a signal from the $i^{\text {th }}$ transducer located at position $r_{i} \in \mathbb{R}^{3}$ and receives the reflected signal at the $j^{\text {th }}$ transducer located at position $r_{j} \in \mathbb{R}^{3}$.

If the medium is linear, homogeneous, and isotropic, then the transfer function from the transmitter to the point $p$ is given by

$$
G\left(r_{i}, p, f\right)=\tau_{1} \tau_{2} \exp \left\{-\propto(f)+j \beta(f) \| p-r_{i}\right.
$$

where

$$
\propto(f) \approx \propto_{0}|f|
$$

is the rate of attenuation in Nepers per $\mathrm{m} \mathrm{Hz}$,

$$
\beta(f)) \approx \frac{2 \pi f}{c}
$$

is the phase delay due to propagation through the medium [43]. Similarly, the transfer function from the point $p$ to the receiver is given by

$$
G\left(p, r_{i}, f\right)=\tau_{1} \tau_{2} \exp \left\{-(\propto(f)+j \beta(f))|| p-r_{j} \|\right\}
$$

Let $s(t)$ be the transmitted signal, and let $x(p)$ be the IRC of the voxel at location $p$ that we would like to measure. Then, the received signal due to reflections from the voxel $x(p)$ at location $p$ is given by

$$
\begin{aligned}
Y_{i, j}(p, f) & =S(f) G\left(r_{i}, p, f\right) x(p)(G)\left(p, r_{j}, f\right) \\
& \left.=\tau_{1}^{2} \tau_{2}^{2} x(p) S(f) \exp \left\{-\propto_{0} c|f|+j 2 \pi f\right) T_{i, j}(p)\right\}
\end{aligned}
$$

where $S(f)$ is the Fourier transform of $s(t)$, and 


$$
T_{i, j}(p)=|| p-r_{i}\|+\| p-r_{j}
$$

From Eq. 1, the system point spread function (PSF) is defined as

$$
h\left(T_{i, j}(p), t\right)=F^{-1}\left\{\tau_{1}^{2} \tau_{2}^{2} S(f) \exp \left\{-\propto_{0} c|f| T_{i, j}(p)\right\}\right\}
$$

Then, the received signal amplitude at time $t$ is given by

$$
y_{i, j}(p, t)=h\left(T_{i, j}(p), t-T_{i, j}(p)\right) x(p)
$$

Finally, the full signal transmitted and received by transducer $i$ and $j$, respectively is computed by integrating over $p$.

$$
y_{i, j}(t)=\int_{\mathbb{R}^{3}} y_{i, j}(p, t) d p=\int_{\mathbb{R}^{3}} A_{i, j}\left(T_{i, j}(p), t\right) x(p) d p
$$

where the system matrix is defined as

$$
\boldsymbol{A}_{i, j}\left(\boldsymbol{T}_{i, j}(\boldsymbol{p}), \boldsymbol{t}\right)=\boldsymbol{h}\left(\boldsymbol{T}_{i, j}(\boldsymbol{p}), \boldsymbol{t}-\boldsymbol{T}_{i, j}(\boldsymbol{p})\right)
$$

Fig. 87 illustrates the system matrix $A$ coefficients for the row corresponding a time of 133us. The forward model was generated for a single transducer pair located at the bottom-left and top-left of the propagation plane. The input signal $s(t)$ is a 5-cycle sine wave tapered with a Hanning Window. As expected, the coefficients' amplitudes are constant at equal propagation distances. Similarly, the coefficients at system matrix rows corresponding to a longer propagation time are smaller due to attenuation.

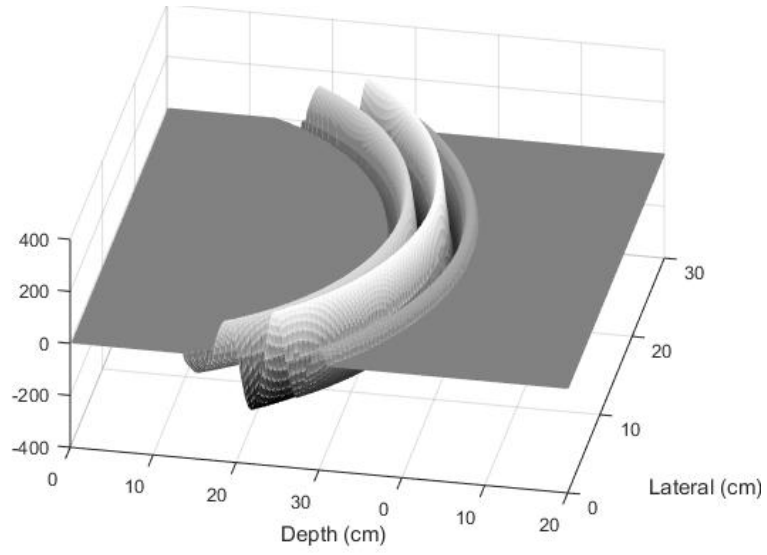

Fig. 87. Illustration of system matrix coefficient amplitudes for two transducers at time 133us.

The prior model is assumed a q-Generalized Gaussian Markov Random Fields (q-GGMRF) [44], which is defined as

$$
U(x)=-\log (p(x)) \approx \frac{1}{v \sigma^{v}} \sum_{j, k \in C} b_{i, k} \rho\left(x_{i}-x_{k}\right) \text { and } \rho(\Delta)=\frac{|\Delta|^{u}}{1+|\Delta / 50|^{u-v}}
$$

where $\sigma$ is a scalar determined empirically and controls the prior influence on the solution, $C$ is the set of pixels in the neighborhood of $x$, and $1 \leq u \leq v \leq 2$. 


\subsection{DISCRETIZATION}

The Quadrature Method [45] was employed for discretization of the forward model. If we observe until $t=T_{R}$, where $T_{R}$ is the maximum expected roundtrip time for the ultrasonic signal, then, there is no need to integrate over $\mathbb{R}^{3}$. It suffices to integrate over a region where sound propagates for $T_{R} / 2$ seconds. This makes

$$
p \in \Omega=\left\{\left[\begin{array}{l}
p_{1} \\
p_{2} \\
p_{3}
\end{array}\right] ; 0 \leq p_{1} \leq M_{1},-M_{2} \leq p_{2} \leq M_{2},-M_{3} \leq p_{3} \leq M_{3}\right\}
$$

where $M_{1}, M_{2}$, and $M_{3}$ are chosen in a way to ensure all points outside $\Omega$ are neglected in the integral of Eq. 3. Let $\Delta_{1}, \Delta_{2}$, and $\Delta_{3}$ be the distance between each pixel along the three dimensional axes. Let

$$
m_{1}=\frac{M_{1}}{\Delta_{1}}, m_{2}=\frac{M_{2}}{\Delta_{2}}, m_{3}=\frac{M_{3}}{\Delta_{3}}
$$

Consequently, we can discretize the system matrix and object to reconstruct by

$$
\begin{aligned}
& A_{i, j}\left[k_{1}, k_{2}, k_{3, n}\right]=\Delta_{1} \Delta_{2} \Delta_{3} A_{i, j}\left(\Delta_{1} k_{1}, \Delta_{2} k_{2} \Delta_{3} k_{3}, T_{0} n\right) \\
& x\left[k_{1}, k_{2}, k_{3}\right]=x\left(\Delta_{1} k_{1}, \Delta_{2} k_{2}, \Delta_{3} k_{3}\right.
\end{aligned}
$$

then

$$
\begin{gathered}
y_{i, j}[n]=y_{i, j}\left(T_{0} n\right) \\
\approx \Delta_{x} \Delta_{y} \Delta_{z} \sum_{k_{3}=-m_{3}}^{m_{3}} \sum_{k_{2}=-m_{2}}^{m_{2}} \sum_{k_{1}=1}^{m_{1}} A_{i, j}\left(\Delta_{1} k_{1}, \Delta_{2} k_{2}, \Delta_{3} k_{3} T_{0} n\right) x\left(\Delta_{1} k_{1} \Delta_{2} k_{2}, \Delta_{3} k_{3}\right) \\
=\sum_{k_{3}=-m_{3}}^{m_{3}} \sum_{k_{2}=-m_{2}}^{m_{2}} \sum_{k_{1}=1}^{m_{1}} A_{i, j}\left[k_{1}, k_{2}, k_{3}, n\right] x\left[k_{1}, k_{2}, k_{3}\right]
\end{gathered}
$$

where $T_{0}$ is the time sampling rate. The same approach was employed to discretize the prior model $U(p)$ to $U\left[k_{1}, k_{2}, k_{3}\right]$. This permits the construction of the forward model for each transducer pair $<i, j>$ as

$$
Y_{i, j}=A_{i, j} X
$$

where

$$
Y_{i, j}=\left[\begin{array}{c}
r_{i, j}[1] \\
r_{i, j}[2] \\
\vdots \\
r_{i, j}[N-1] \\
r_{i, j}[N]
\end{array}\right]_{N \times 1}
$$




$$
X=\left[\begin{array}{c}
X\left[1,-m_{2},-m_{3}\right. \\
X\left[2,-m_{2},-m_{3}\right. \\
\vdots \\
X\left[m_{1},-m_{2},-m_{3}\right. \\
X\left[1,1-m_{2},-m_{3}\right. \\
\vdots \\
X\left[m_{1}, m_{2},-m_{3}\right] \\
X\left[1,-m_{2}, 1-m_{3}\right. \\
\vdots \\
X\left[m_{1}, m_{2}, m_{3}\right]
\end{array}\right]_{\left(m_{1}\left(2 m_{2}+1\right)\left(2 m_{3}+1\right)\right) \times 1}
$$

And

$$
A_{i, j}=\left[\begin{array}{ccccc}
{\left[A_{i, j}\left[1,-m_{2},-m_{3}, 1\right]\right.} & \cdots & A_{i, j}\left[m_{1}-m_{2},-m_{3}, 1\right] & \cdots & A_{i, j}\left[m_{1}, m_{2}, m_{3}, 1\right] \\
A_{i, j}\left[1,-m_{2},-m_{3}, 2\right] & \cdots & A_{i, j}\left[m_{1},-m_{2},-m_{3}, 2\right] & \cdots & A_{i, j}\left[m_{1}, m_{2}, m_{3}, 2\right] \\
& & \vdots & & \\
A_{i, j}\left[1,-m_{2},-m_{3}, N\right] & \cdots & A_{i, j}\left[1,-m_{2},-m_{3}, N\right] & \cdots & A_{i, j}\left[m_{1}, m_{2}, m_{3}, N\right]
\end{array}\right]_{N \times\left(m_{1}\left(2 m_{2}+1\right)\left(2 m_{3}+1\right)\right)}
$$

Note that multiple transducer pairs can be added to the MBIR forward model by concatenating their corresponding forward models and measurements to $A$ and $Y$, respectively. The number of pairs is limited by the amount of computational memory available. However, our initial assessment of the system matrix indicates that the matrix is sparse. There are methods for sparse large matrices that process such matrices under constrained computational resources [47]. Consequently, we do not foresee computational memory to be a limiting factor when designing our ultrasonic phased array. Finally, we can define our optimization function as

$$
\tilde{x}=\frac{\operatorname{argmin}}{x}\left\{\frac{1}{2}(Y-A X)^{T} \Lambda(Y-A X)+U\right\}
$$

\subsection{SYNTHETIC RESULTS}

The initial forward model was tested with synthetic data. This test was performed to confirm the correctness of the propagation model and to have better insight on the influence of the q-GGMRF prior model on the solution. For the initial test, we assumed a homogenous cement slab with an acoustic speed of 3,680 m/s and attenuation coefficient of 4.8e-5 Nepers / $\mathrm{m} \mathrm{Hz}$. The ultrasound system is defined as non-focused isotropic sources and sensors, where the input signal is a single-cycle sine wave with a central frequency of $100 \mathrm{KHz}$ and a bandwidth of $150 \mathrm{KHz}$. Consequently, the expected axial resolution is approximately $2 \mathrm{~cm}$. Two phantom test samples were generated for the synthetic test (See Error! Reference source not found.). The positive pixel intensities in the phantom images correspond to the IRC values for changes in impedance (i.e., interfaces where the current material ends and a new material starts, for example, the cement to steel interface with an IRC of 0.525). Fig. 88 (a) shows a onedimensional object with two interfaces at $35 \mathrm{~cm}$ and $65 \mathrm{~cm}$ deep with IRC amplitudes of 0.525 and 0.95 , respectively. For this phantom, transmission and receiving were performed by a transducer at the origin (i.e., 0cm). Fig. 88 (b) illustrates a two-dimensional phantom sample with two impedance changes at $18 \mathrm{~cm}$ and $35 \mathrm{~cm}$ deep with IRC amplitudes of 0.525 and 0.95 , respectively. Ten transducers were placed at the origin - one at each row. Note that the first interface extends the full width of the medium and the second interface is a small, highly reflective impurity. 


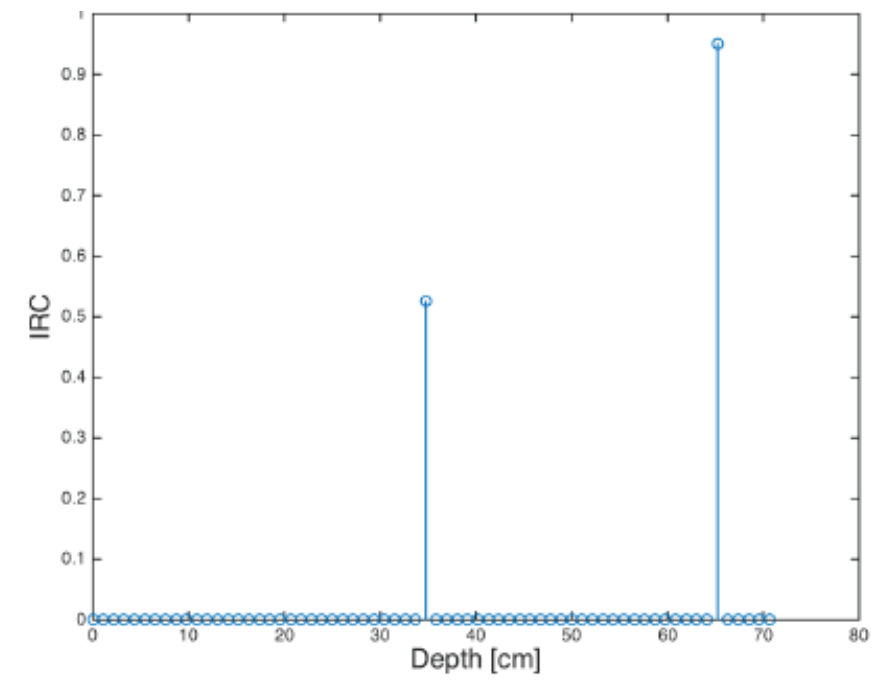

(a)

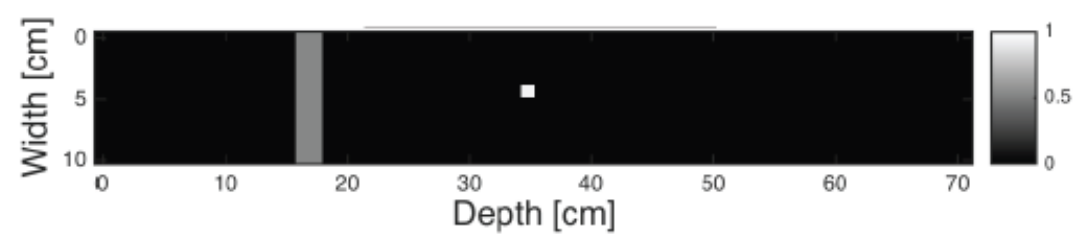

(b)

Fig. 88. Phantom samples used for the synthetic test. (a) One-dimensional object with two impedance changes interfaces. (b) Two-dimensional object with two embedded interfaces of different widths and lengths.

The synthetic signal was generated using conventional sound propagation models. Diffraction and modeconversion (i.e., p-wave to s-wave conversion) were not included in the current simulation. We added Gaussian noise to the synthetic signal to emulate electrical noise in the components of the ultrasound system. Fig. 89 (a) shows a plot of the synthetic signal generated from the phantom in Fig. 88 (a). We selected Iterative Coordinate Descent (ICD) as the optimization algorithm [44]. Typically, this algorithm shows fast and stable convergence. Usually, the algorithm is initialized with a low frequency (i.e., blurred) version of the object. The low frequency initial object estimate could be obtained from back projection $\tilde{x}=A^{T} Y$ or from other reconstruction techniques such as SAFT. Initializing the object with a quick low frequency estimate is the ideal reconstruction workflow given that ICD emphasizes high frequency components during convergence. For the one-dimensional case, in Fig. 89 (b), the reconstruction algorithm obtained IRC values of 0.536 and 0.953 for the first and second interfaces, respectively. The ICD algorithm ran for 500 iterations. For the two-dimensional case, in Fig. 89 (c), the estimated interface IRC coefficients converged to 0.524 and 0.878 after 5000 iterations. Although these initial results are very encouraging, we can improve the accuracy of our reconstructions by adjusting our prior model regularization parameters. 


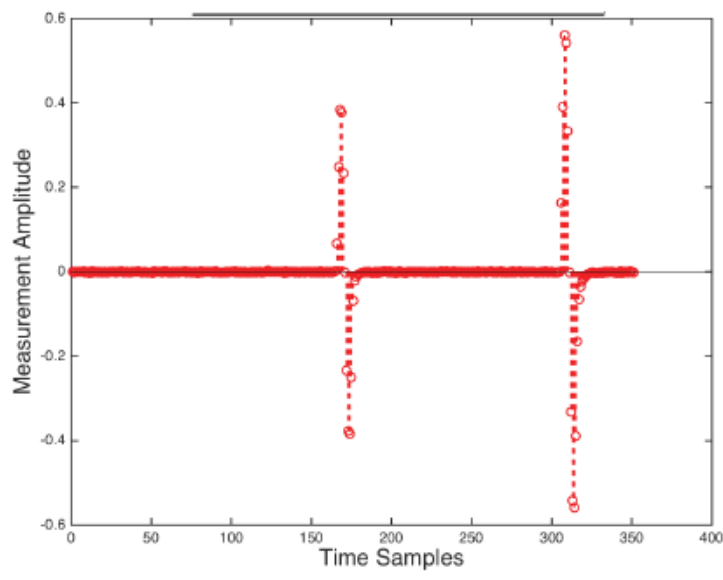

(a)

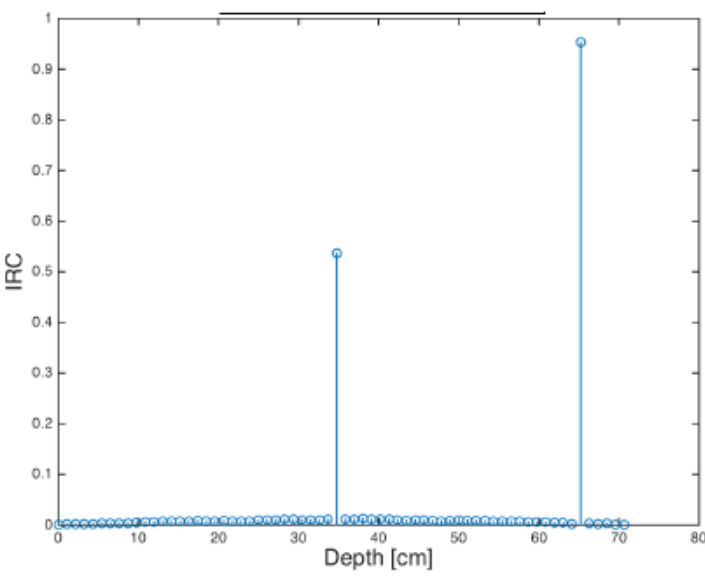

(b)

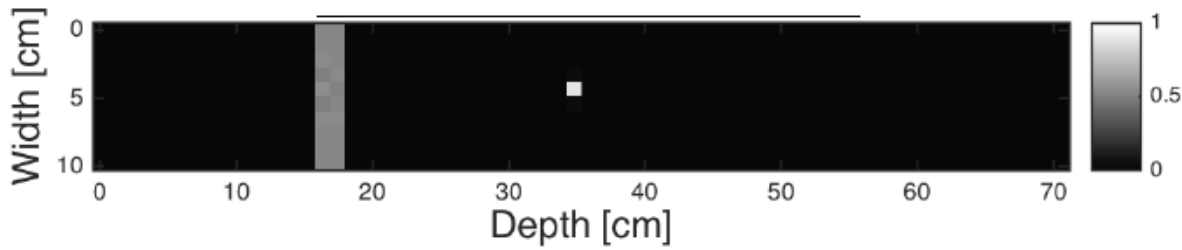

(c)

Fig. 89. (a) Synthetic measured reflections for the phantom in Figure 2(a), and reconstruction results for the (b) one-dimensional and (c) two-dimensional phantoms.

\subsection{MBIR CLOSING REMARKS}

We have developed an initial implementation of the MBIR algorithm for ultrasonic signals. The current algorithm assumes isotropic sources and sensors with longitudinal waves (i.e., p-waves) propagating in a homogenous medium. There are a few features that need incorporation to the current forward model in order to successfully reconstruct real data. For example, actual transmitters are not isotropic; the input signal strength typically decreases for the sound vectors away from the transducer optical axis. Our endgoal is to reconstruct complex and heterogeneous solid objects. Consequently, future implementations of the forward model most include diffraction and conversion-mode models. Our biggest challenge is to incorporate these complex features while keeping the forward model tractable and computational efficient. 



\section{CONCLUSION}

The uniqueness of concrete structures associated with NPPs creates distinctive challenges in NDE. Comparative NDE of various defects in reinforced concrete specimens is a key component in identifying the most promising techniques and directing the research and development efforts needed to characterize concrete degradation in commercial NPPs. This requires access to the specimens for data collection using state-of-the-art technology. Validation data is needed to properly evaluate the effectiveness of the techniques. In this case, the various defects should be created, well defined, and/or feasible to evaluate forensically. It is also critical that the evaluation specimen and embedded defects are representative of inservice NPP structure concrete. Past studies have shown that it is not feasible to meet all of these needs through field removal or evaluation of currently available fabricated specimens.

In the past, multiple comparative NDE studies have been conducted on reinforced concrete structures that are not as thick or heavily reinforced as typical commercial NPP reinforced concrete structures. Results of the comparative studies on these specimens showed the promise of various techniques in evaluating concrete degradation, providing the basis of the conceptual designs from this study. The results from the comparative testing on the thinner structures must be validated under NPP reinforced concrete conditions, where difficulties such as a lower signal-to-noise ratio with greater depth of penetration need to be resolved.

Using the specimen that was specifically fabricated to be representative of a NPP containment structure, this report shows the improvements that can be obtained using frequency banded SAFT. While standard SAFT techniques are adequate for many defects with shallow concrete cover, some defects that are located under deep concrete cover are not easily identified using the standard SAFT techniques. For many defects, particularly defects under deep cover, the use of frequency banded SAFT improves the detectability over standard SAFT. In addition to the improved detectability, the frequency banded SAFT also provides improved scan depth resolution that can be important in determining the suitability of a particular structure to perform its designed safety function.

Using a linear array ultrasound instrument with a $50 \mathrm{kHz}$ center frequency data was collected from the thick specimen and processed. For the defects in the specimen, wavelet nodes containing the frequencies $13.25-62.5 \mathrm{kHz}$ and second harmonic, $93.75-125 \mathrm{kHz}$ offer promise for multiple types of defects at both deep and shallow depths. Improvements include seeing an intensity corresponding to a defect with our technique that is either not visible at all in regular, full frequency content SAFT, or an improvement in clarity or brightness over the SAFT images rendered using the full frequency content. However, further research is needed to better understand in what circumstances (depth, defect type, etc.) which wavelet decomposition node is best.

Additional investigation is also needed to determine why some "bright spots" are defects, and some are not, i.e. false positives. Data fusion, using data from multiple sources using different technologies, may be needed to address these issues. 



\section{REFERENCES}

1. L. J. Bond, "Old Reactors, New Tricks," IEEE Spectrum 49(8), 30-35 (2012).

2. Dwight A. Clayton, Cyrus M. Smith, Christopher Ferraro, Jordan Nelson, Lev Khazanovich, Kyle Hoegh, Satish Chintakunta, John Popovics, Hajin Choi, and Suyun Ham, "Evaluation of Ultrasonic Techniques on Concrete Structures,” ORNL/TM-2013/430, Oak Ridge National Laboratory, 2013.

3. D. A. Clayton K. Hoegh, L. Khazanovich, and M. S. Hileman, "Preliminary Conceptual Design of Thick Concrete Nondestructive Evaluation Specimen” ORNL/TM-2014/136, Oak Ridge National Laboratory, 2014.

4. D. A. Clayton, K. Hoegh, and L. Khazanovich, “Thick Concrete Specimen Construction, Testing, and Preliminary Analysis,” ORNL/TM-2015/72, Oak Ridge National Laboratory, 2015.

5. D. A. Clayton and M. S. Hileman, "Light Water Reactor Sustainability Non-Destructive Evaluation for Concrete Research and Development Roadmap,” ORNL/TM-2012/360, Oak Ridge National Laboratory, 2012.

6. D. A. Clayton and C. M. Smith, "Summary of Large Concrete Samples," ORNL/TM-2013/223, Oak Ridge National Laboratory, 2013.

7. D. J. Naus et al., "Summary and conclusions of a program addressing aging of nuclear power plant concrete structures," Nuclear Engineering and Design 194(1), 73-96 (1999).

8. D. J. Naus, C. B. Oland, and E. G. Arndt, "Ageing management of safety-related concrete structures to provide improved bases for continuing the service of nuclear power plants." Materials and Structures 24(4), 308-316 (1991).

9. J. I. Braverman, C. A. Miller, B. R. Ellingwood, D. J. Naus, C. H. Hofmayer, P. Bezler, and T. Y. Chang, "Structural performance of degraded reinforced concrete members," in Transaction 17th International Conference on Structural Mecha, March 2001.

10. D. J. Naus, C. B. Oland, and E. G. Arndt, "Ageing management of safety-related concrete structures to provide improved bases for continuing the service of nuclear power plants," Materials and Structures 24(4), 308-316 (1991).

11. D. Algernon, D. Hiltunen, and C. Ferraro, "Validation of Nondestructive Testing Equipment for Concrete," University of Florida Department of Civil and Coastal Engineering, 2010.

12. Sherif Yehia et al., "Detection of common defects in concrete bridge decks using nondestructive evaluation techniques," Journal of Bridge Engineering 12(2), 215-225 (2007).

13. Mary Sansalone and Nicholas J. Carino, "Detecting delaminations in concrete slabs with and without overlays using the impact-echo method," ACI Materials Journal 86(2) (1989).

14. Masanori Asano et al., "Impact acoustics methods for defect evaluation in concrete," NDT-CE 2003, Proc., BB 85-CD, DGZIP, 2003.

15. Jinying Zhu and John S. Popovics, "Imaging concrete structures using air-coupled impact-echo," Journal of Engineering Mechanics 133(6), 628-640 (2007).

16. F. Weritz et al., "Investigation of concrete structures with pulse phase thermography," Materials and Structures 38(9), 843-849 (2005).

17. Ralf Beutel et al., "Comparative performance tests and validation of NDT methods for concrete testing," Journal of Nondestructive Evaluation 27(1-3), 59-65 (2008). 
18. Christiane Maierhofer et al., "Complementary application of radar, impact-echo, and ultrasonics for testing concrete structures and metallic tendon ducts," Transportation Research Record: Journal of the Transportation Research Board 1892(1), 170-1 (2004).

19. Alexander Taffe, Kerstin Borchardt, and Herbert Wiggenhauser, "Specimen for the improvement of NDT methods - Design and construction of a Large Concrete Slab for NDT methods at BAM," International Symposium Non-Destructive Testing in Civil Engineering, (2003).

20. Frank Schubert and Bernd Köhler, "Ten lectures on impact-echo," Journal of Nondestructive Evaluation 27(1-3), 5-21 (2008).

21. P. Fellinger et al., "Numerical modeling of elastic wave propagation and scattering with EFITelastodynamic finite integration technique," Wave Motion 21(1), 47-66 (1995).

22. Frank Schubert and René Marklein, "Numerical computation of ultrasonic wave propagation in concrete using the elastodynamic finite integration technique (EFIT)," Ultrasonics Symposium, 2002. IEEE Proceedings Vol. 1, 2002.

23. Frank Schubert, Herbert Wiggenhauser, and Regine Lausch, "On the accuracy of thickness measurements in impact-echo testing of finite concrete specimens-numerical and experimental results," Ultrasonics 42(1), 897-901 (2004).

24. J. L. Braverman, C. A. Miller, C. H. Hofmayer, B. R. Ellingwood, D. J. Naus, and T. Y. Chang, "Degradation assessment of structures and passive components at nuclear power plants," Nuclear Engineering and Design 228(1), 283-304 (2004).

25. C. Chiu et al., Performance Improvement International, "Root Cause Assessment Crystal River Unit 3 Containment Concrete Delamination,” Crystal River Nuclear Plant - Special Inspection Report 05000302/2009007, US Nuclear Regulatory Commission (NRC) Special Inspection at Crystal River Unit 3 Nuclear Plant, 2010.

26. H. Ashar and G. Bagchi, "Assessment of inservice conditions of safety-related nuclear plant structures,” Washington, DC: US Nuclear Regulatory Commission, 1995.

27. Frederick L. Moreadith and Richard E. Pages, "Delaminated prestressed concrete dome: investigation and repair," Journal of Structural Engineering 109(5), 1235-1249 (1983).

28. Shen Wang and Javeed A. Munshi, "Design of radial reinforcement for prestressed concrete containments," Nuclear Engineering and Design 255, 153-161 (2013).

29. C. Chiu et al., Performance Improvement International, "Root Cause Assessment Crystal River Unit 3 Containment Concrete Delamination,” Crystal River Nuclear Plant - Special Inspection Report 05000302/2009007, US Nuclear Regulatory Commission (NRC) Special Inspection at Crystal River Unit 3 Nuclear Plant, 2010.

30. S.A. Reynolds, "Davis-Besse Nuclear Power Station - Inspection to Evaluate the Root Cause Evaluation and Corrective Actions for Cracking in the Reinforced Concrete Shield Building of the Containment System,” Nuclear Regulatory Commission Inspection Report 05000346/2012009(DRS), 2012.

31. Albright, Austin P. and Dwight A. Clayton. "The Benefits of Using Time-Frequency Analysis with Synthetic Aperture Focusing Technique." In Review of Progress in Quantitative Nondestructive Evaluation, 2015.

32. V. G. Shevaldykin, A. A. Samokrutov, and V. N. Kozlov, "Ultrasonic Low-Frequency Transducers with Dry Dot Contact and Their Applications for Evaluation of Concrete Structures,” IEEE Ultrasonics Symposium Proceedings 1 \& 2, pp. 793-798, 2002. 
33. C. Bouman, Model Based Image Processing, 1st ed. (Purdue University, 2013) https://engineering.purdue.edu/ bouman/publications/pdf/MBIP-book.pdf.

34. 33.Q. Wang and G. Ren, IEEE Transactions on Wireless Communications 14, 2778-2787 May (2015).

35. 34.Z. Haiyan, S. Xiuli, Q. Xuerui, F. Shixuan, L. Xiao, and L. Donghui, "Lamb wave tomography combining simulated annealing and simultaneous iterative reconstruction technique,” in Microwave Conference (2008), pp. 741-744.

36. 35.S. Venkatakrishnan, L. Drummy, M. Jackson, M. D. Graef, J. Simmons, and C. Bouman, IEEE Transactions on Image Processing 22, 4532-4544 November (2013).

37. Z. Yu, J. Thibault, C. Bouman, K. Sauer, and J. Hsieh, IEEE Transactions on Image Processing 20, 161- 175 January (2011).

38. M. E. Kamasak, C. A. Bouman, E. D. Morris, and K. Sauer, IEEE Transactions on Medical Imaging 24, 636-650May (2005).

39. Z. H. Levine, A. J. Kearsley, and J. G. Hagedorn, J. Res. Nat. Inst. Stand. Technol 11, 411417Nov (2006).

40. R. V. Sloun, A. Pandharipande, M. Mischi, and L. Demi, IEEE Transactions on Biomedical Engineering 62, pp.1660-1664 June (2015).

41. J. A. Fessler, "Iterative tomographic image reconstruction using nonuniform fast fourier transforms," Technical Report (Communications and Signal Processing Laboratory Department of Electrical Engineering Computer Science, University of Michigan, 2001).

42. S. J. Norton and M. Linzer, IEEE Transactions on Biomedical Engineering BME-28, 202-220 February (1981).

43. A. Kak and K. A. Dines, IEEE Transactions on Biomedical Engineering BME-25, 321-344 July (1978).

44. J.-B. Thibault, K. Sauer, C. Bouman, and J. Hsieh, Medical Physics 34, 4526-4544 November (2007).

45. L. Soldo and N. Wider, Discretization of Linear Inverse Problems,

46. https://www.math.ethz.ch/education/bachelor/seminars/hs2010/ipip/slides.6.pdf ( 2011).

47. N. Bell and M. Garland, "Implementing sparse matrix-vector multiplication on throughputoriented processors," in Proceedings of the Conference on High Performance Computing Networking, Storage and Analysis, SC ’09 (ACM, New York, NY, USA, 2009), pp. 18:1-18:11. 

APPENDIX A. ROUGH SIDE DEFECT IMAGES 



\section{HORIZONTAL}

\section{SET 1 - NO DEFECT}

Specimen: Thick, Depth: 1066.8mm (42in), AbsofHilbert -- Node $0(0,0), 0 \sim 500 \mathrm{kHz}$ Panoramic SAFT-B, Spec=Rough, Orient=hor, Set=1, Thresh=20, Strategy=sum,

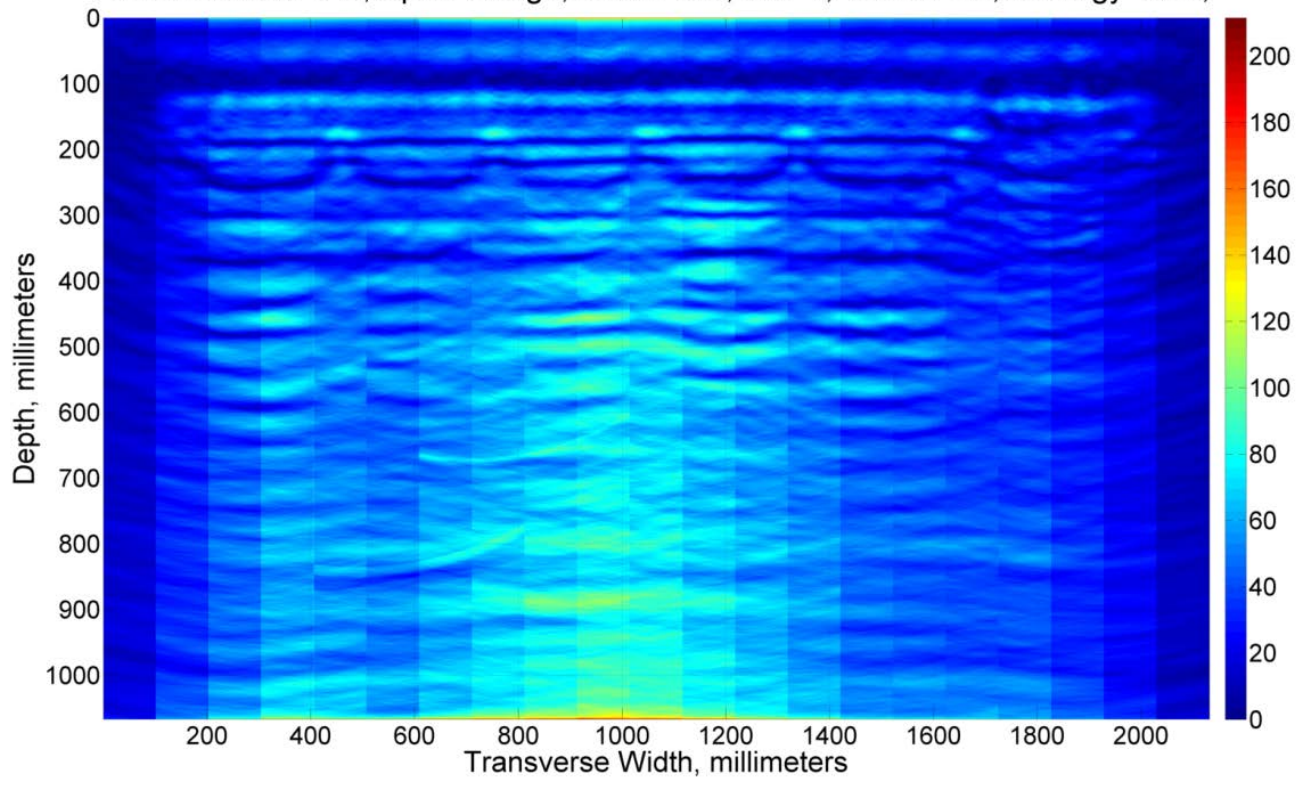

Fig. A. 1. Horizontal Set 1, Node 0.

Specimen: Thick, Depth: 1066.8mm (42in), AbsofHilbert -- Node $16(4,1), 31.25 \sim 62.5 \mathrm{kHz}$ Panoramic SAFT-B, Spec=Rough, Orient=hor, Set=1, Thresh=20, Strategy=sum,

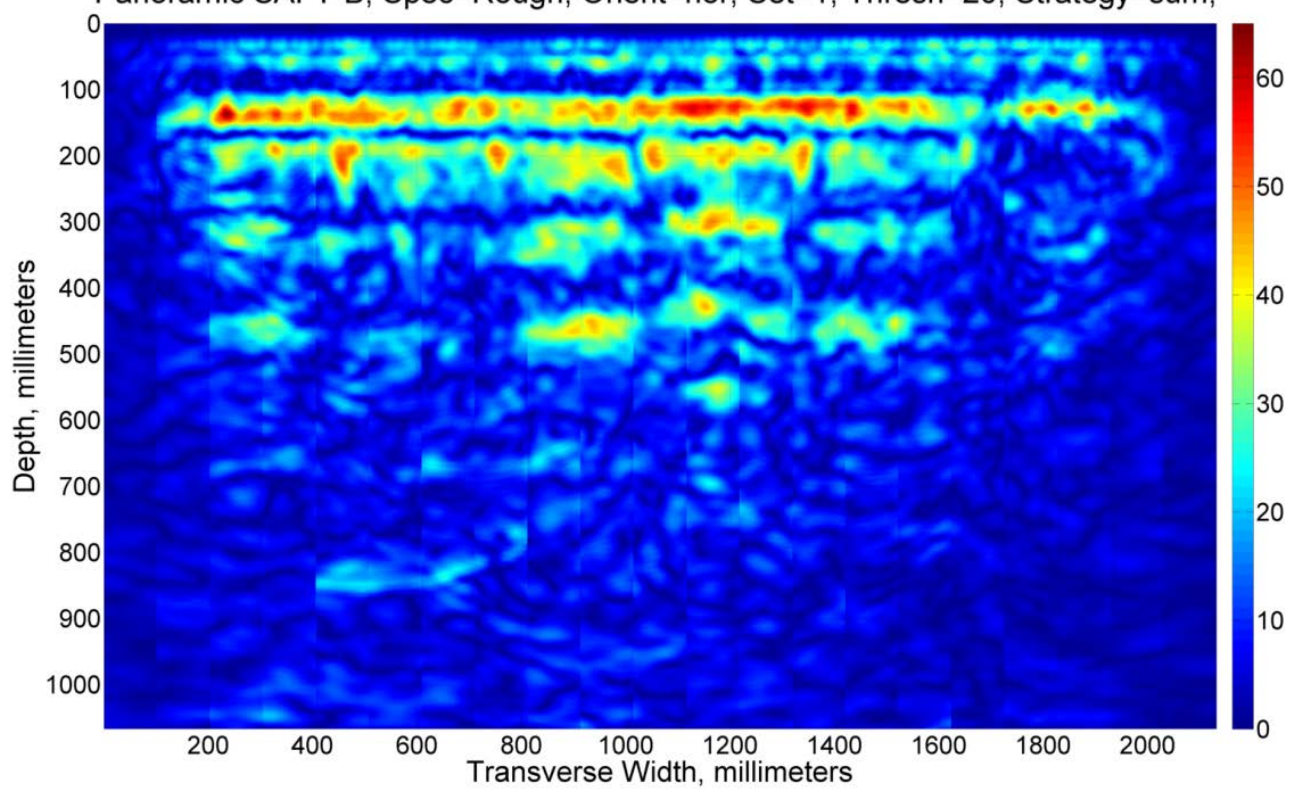

Fig. A. 2. Horizontal Set 1, Node 16. 


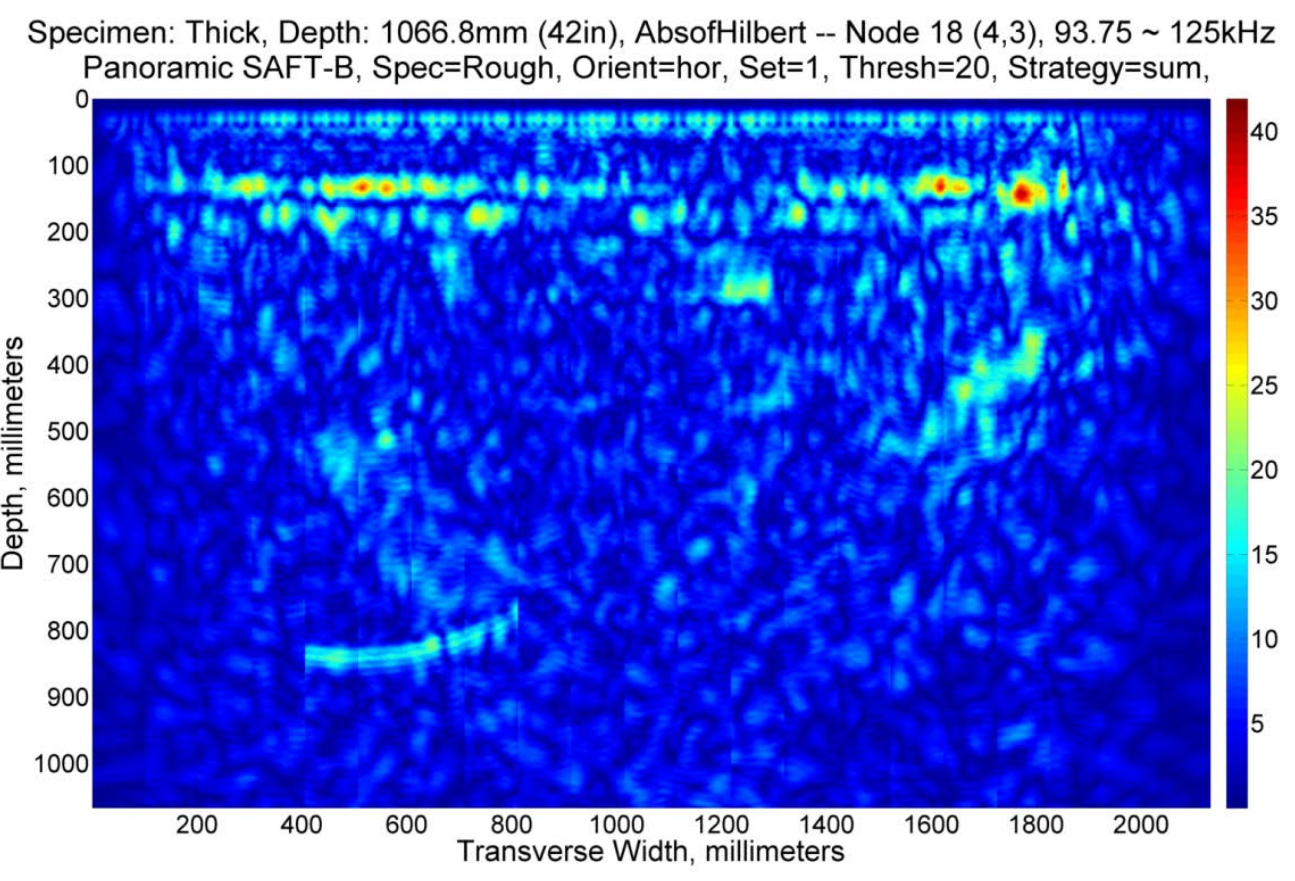

Fig. A. 3. Horizontal Set 1, Node 18.

Specimen: Thick, Depth: 1066.8mm (42in), AbsofHilbert -- Node 32 (5,1), $15.625 \sim 31.25 \mathrm{kHz}$

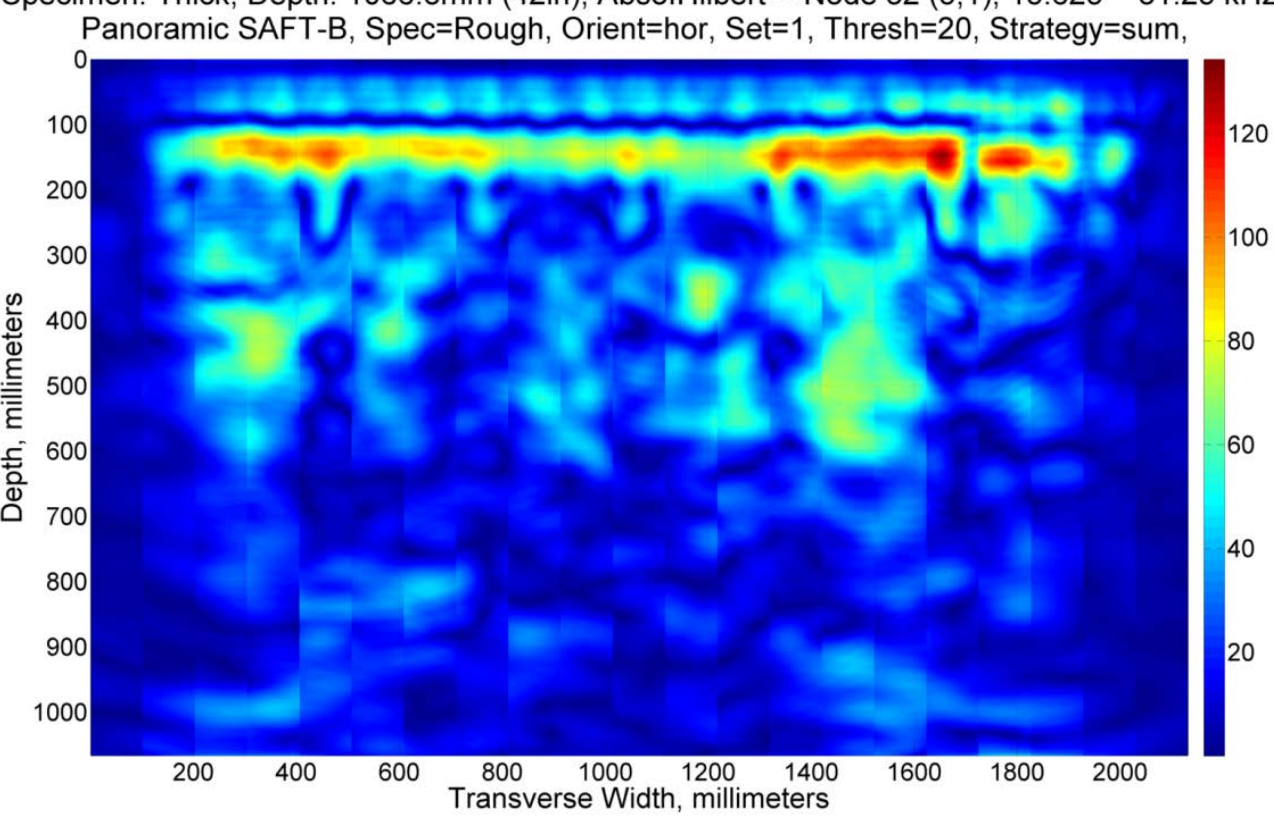

Fig. A. 4. Horizontal Set 1, Node 32. 


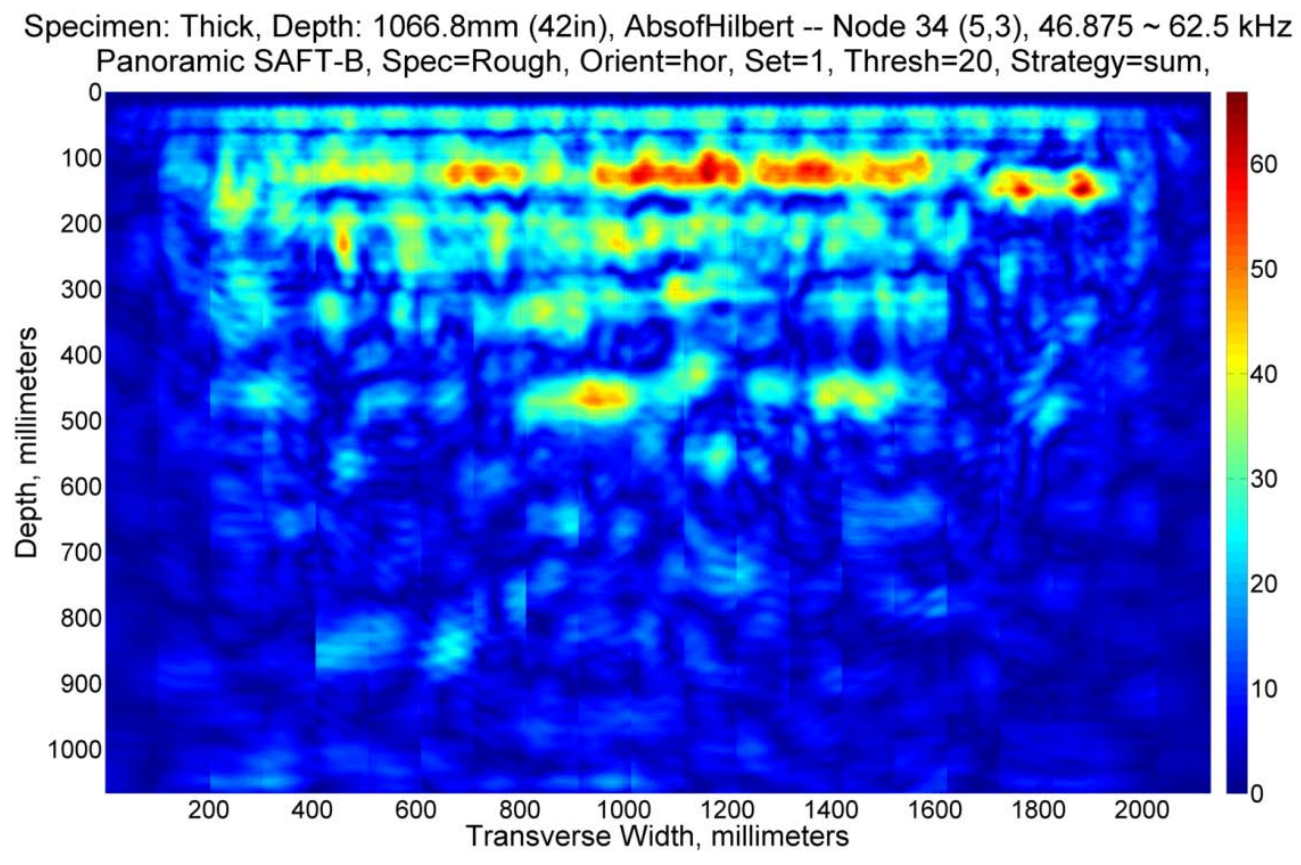

Fig. A. 5. Horizontal Set 1, Node 34 .

\section{SET 2 - DEFECT 10}

Specimen: Thick, Depth: 1066.8mm (42in), AbsofHilbert -- Node $0(0,0), 0 \sim 500 \mathrm{kHz}$ Panoramic SAFT-B, Spec=Rough, Orient=hor, Set=2, Thresh=20, Strategy=sum,

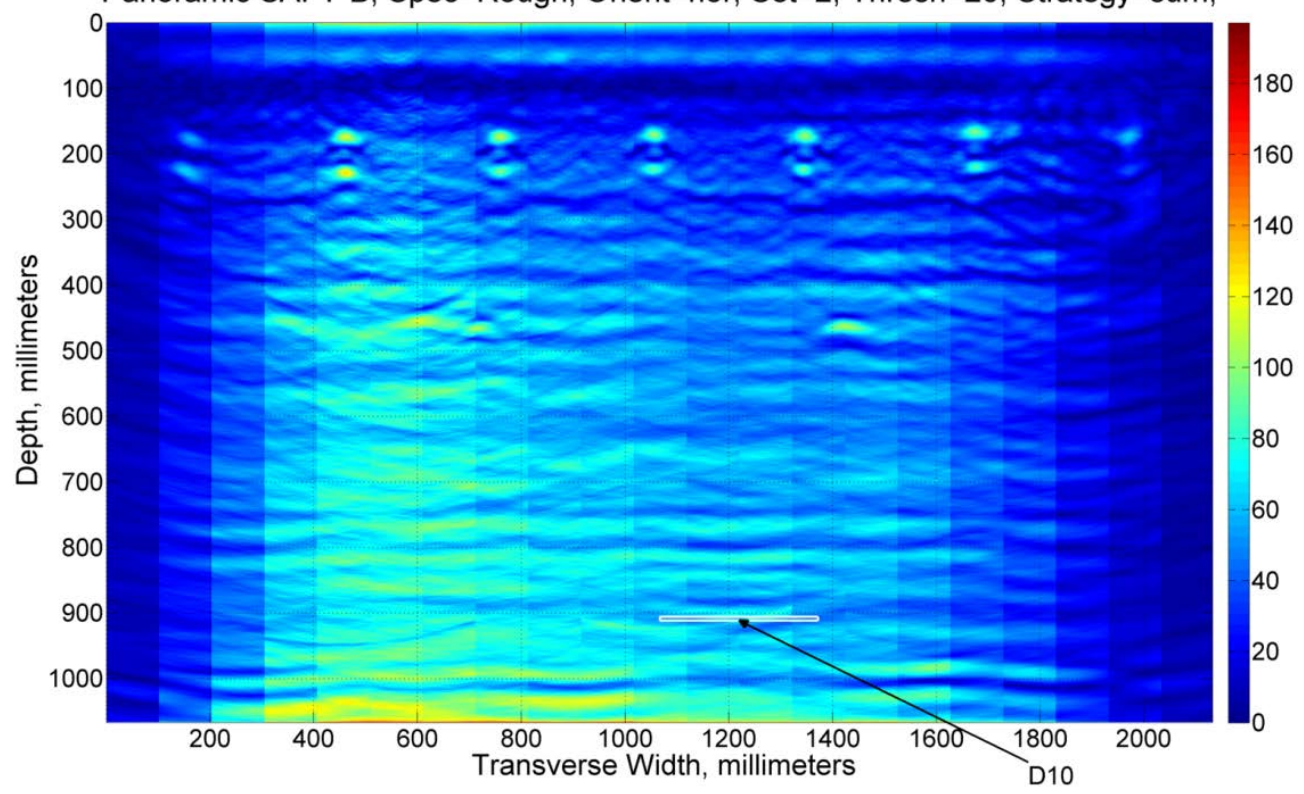

Fig. A. 6. Horizontal Set 2, Node 0. 


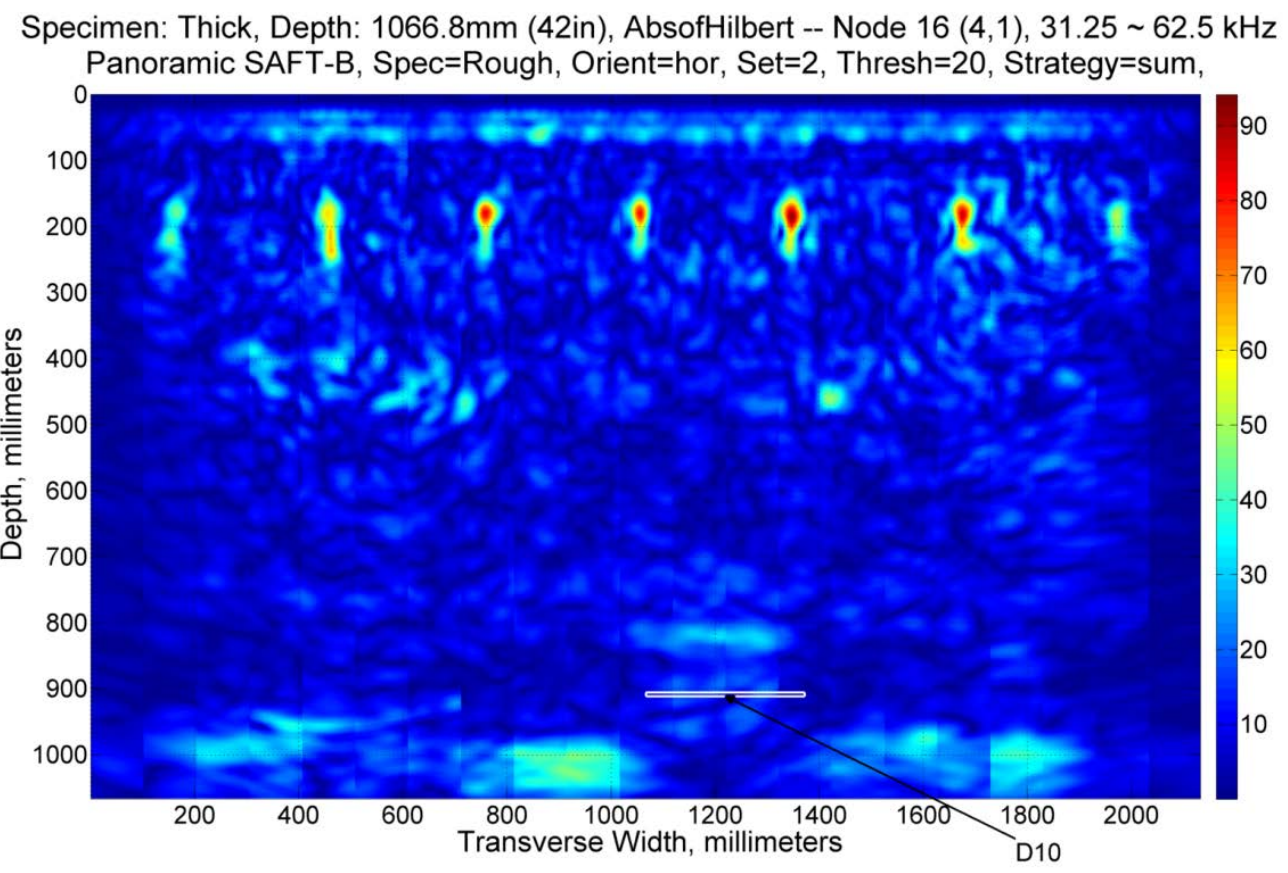

Fig. A. 7. Horizontal Set 2, Node 16.

Specimen: Thick, Depth: 1066.8mm (42in), AbsofHilbert -- Node $18(4,3), 93.75 \sim 125 \mathrm{kHz}$ Panoramic SAFT-B, Spec=Rough, Orient=hor, Set=2, Thresh=20, Strategy=sum,

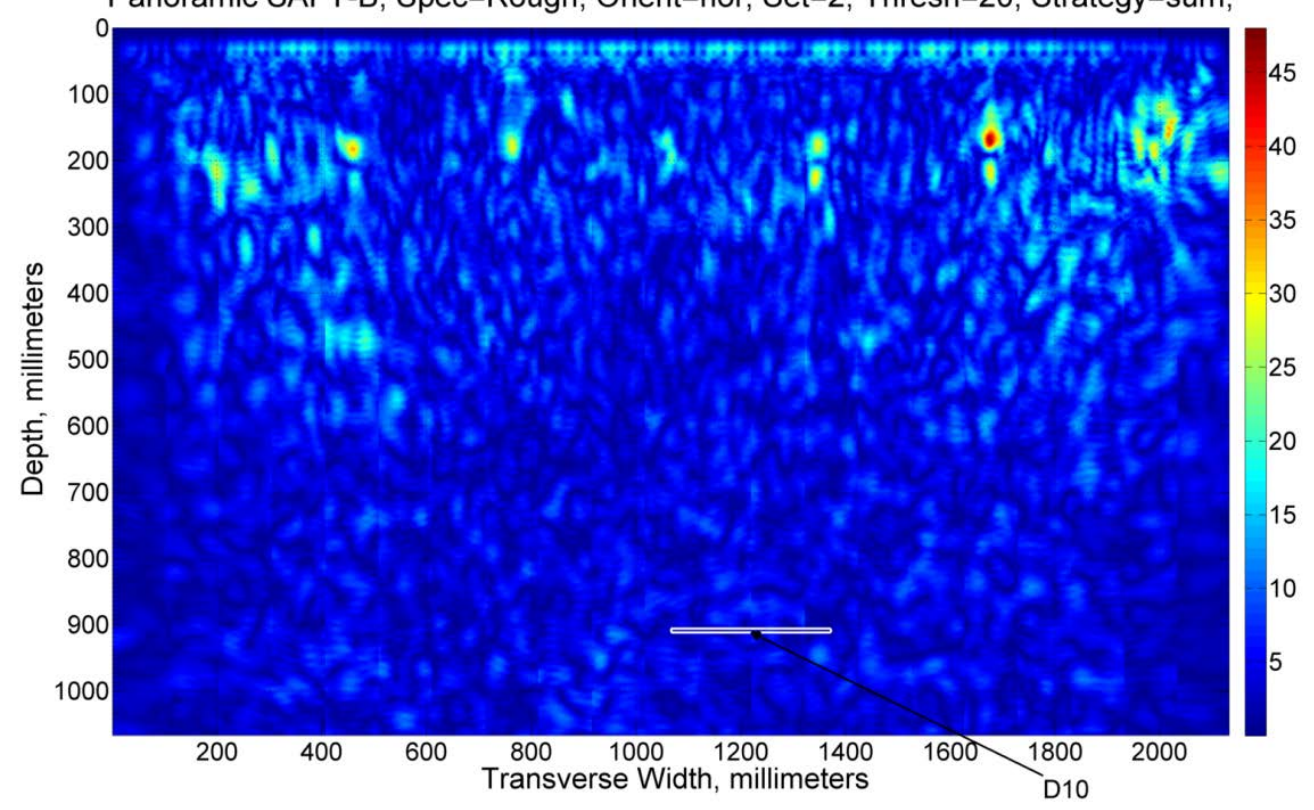

Fig. A. 8. Horizontal Set 2, Node 18. 
Specimen: Thick, Depth: 1066.8mm (42in), AbsofHilbert -- Node 32 (5,1), $15.625 \sim 31.25 \mathrm{kHz}$ Panoramic SAFT-B, Spec=Rough, Orient=hor, Set=2, Thresh=20, Strategy=sum,

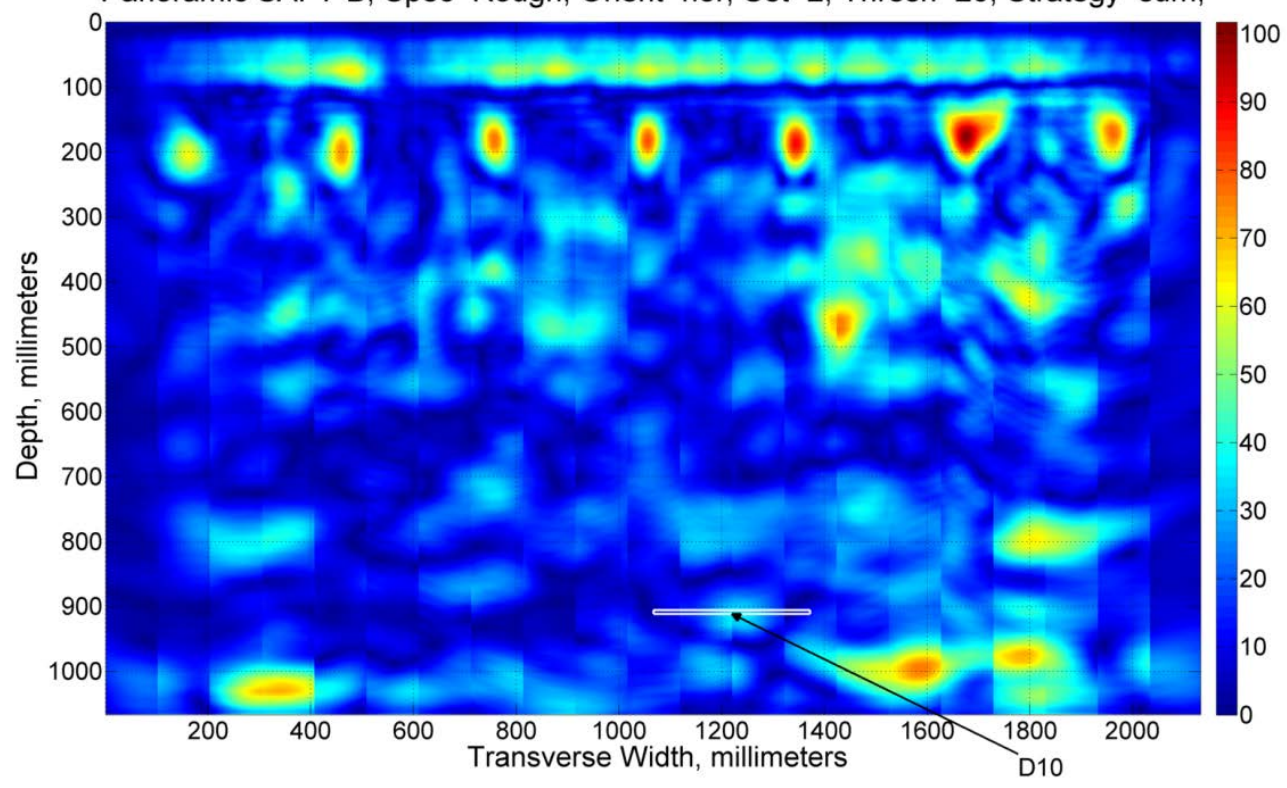

Fig. A. 9. Horizontal Set 2, Node 32 .

Specimen: Thick, Depth: 1066.8mm (42in), AbsofHilbert -- Node $34(5,3), 46.875 \sim 62.5 \mathrm{kHz}$ Panoramic SAFT-B, Spec=Rough, Orient=hor, Set=2, Thresh=20, Strategy=sum,

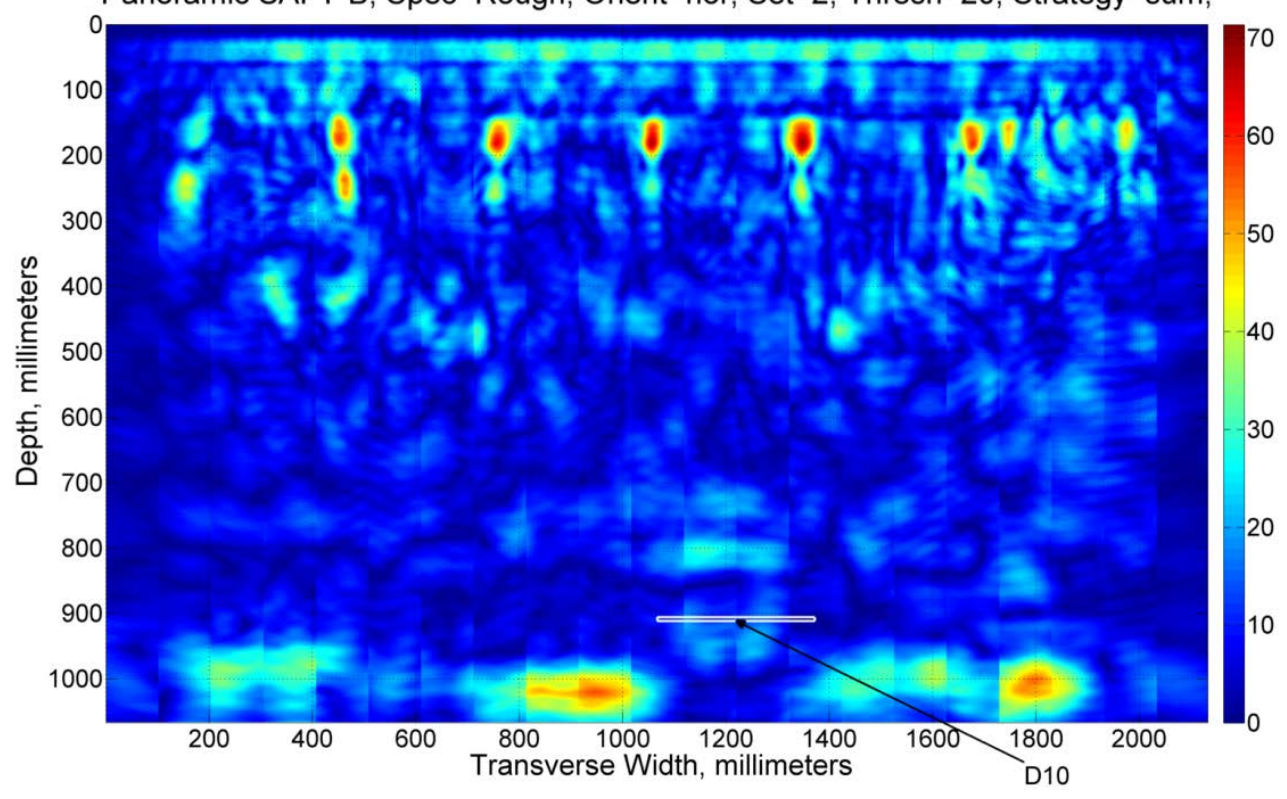

Fig. A. 10. Horizontal Set 2, Node 34. 


\section{SET 3 - DEFECT 10}

Specimen: Thick, Depth: 1066.8mm (42in), AbsofHilbert -- Node $0(0,0), 0 \sim 500 \mathrm{kHz}$ Panoramic SAFT-B, Spec=Rough, Orient=hor, Set=3, Thresh=20, Strategy=sum,

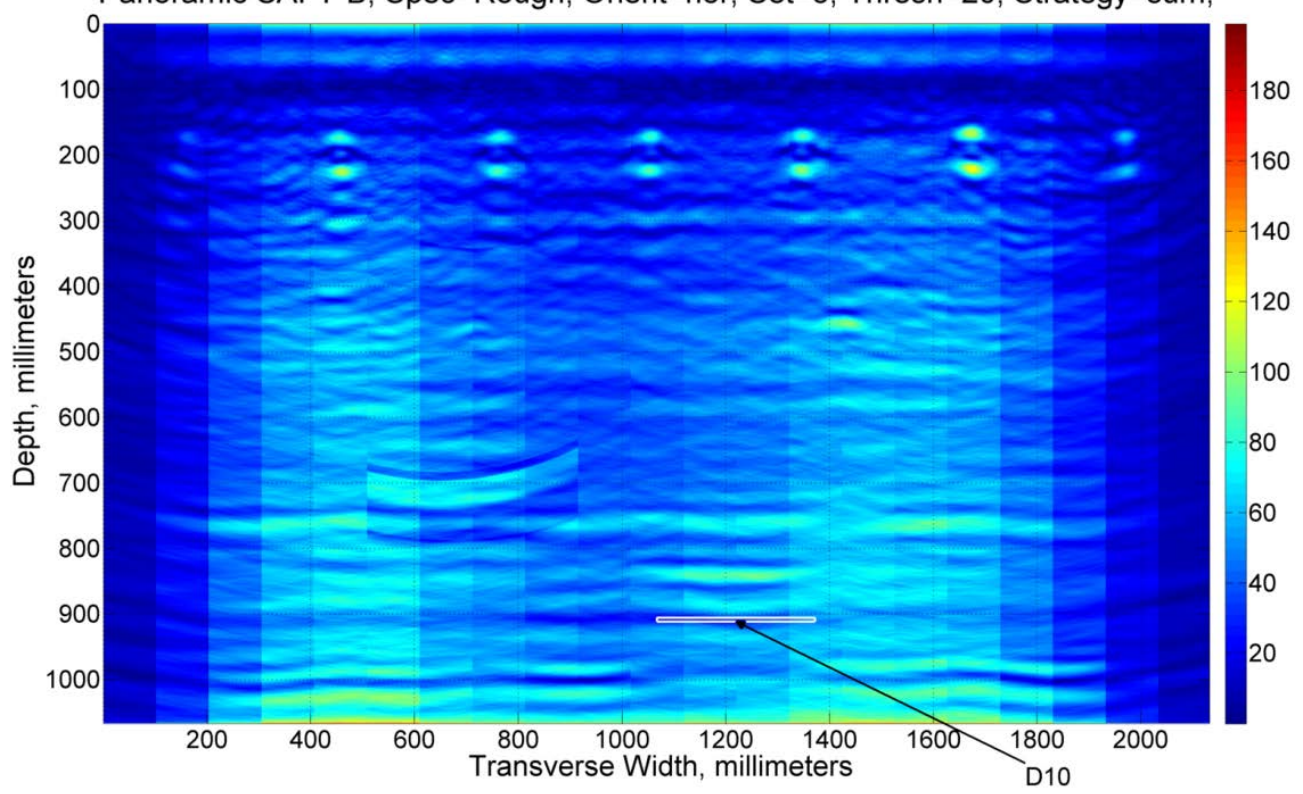

Fig. A. 11. Horizontal Set 3, Node 0.

Specimen: Thick, Depth: 1066.8mm (42in), AbsofHilbert -- Node $16(4,1), 31.25 \sim 62.5 \mathrm{kHz}$ Panoramic SAFT-B, Spec=Rough, Orient=hor, Set=3, Thresh=20, Strategy=sum,

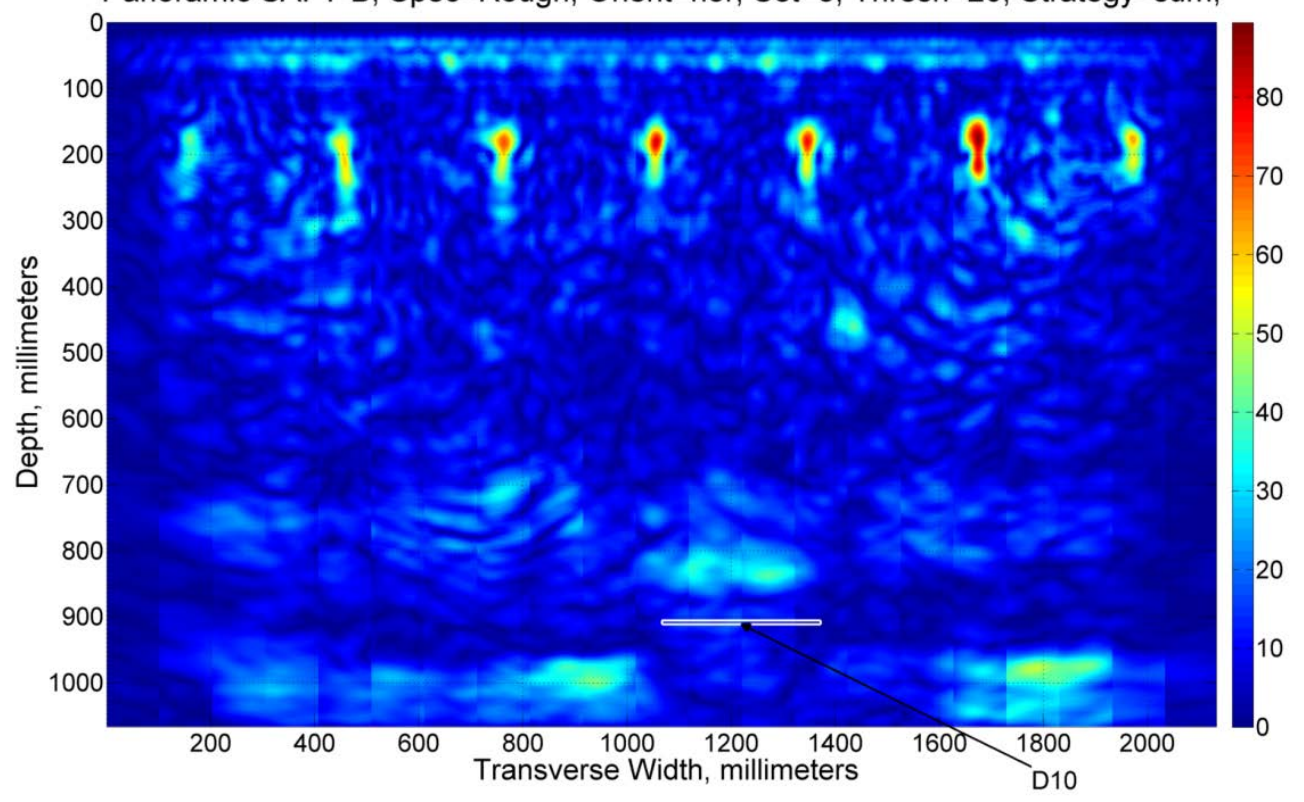

Fig. A. 12. Horizontal Set 3, Node 16. 
Specimen: Thick, Depth: 1066.8mm (42in), AbsofHilbert -- Node $18(4,3), 93.75 \sim 125 \mathrm{kHz}$ Panoramic SAFT-B, Spec=Rough, Orient=hor, Set=3, Thresh=20, Strategy=sum,

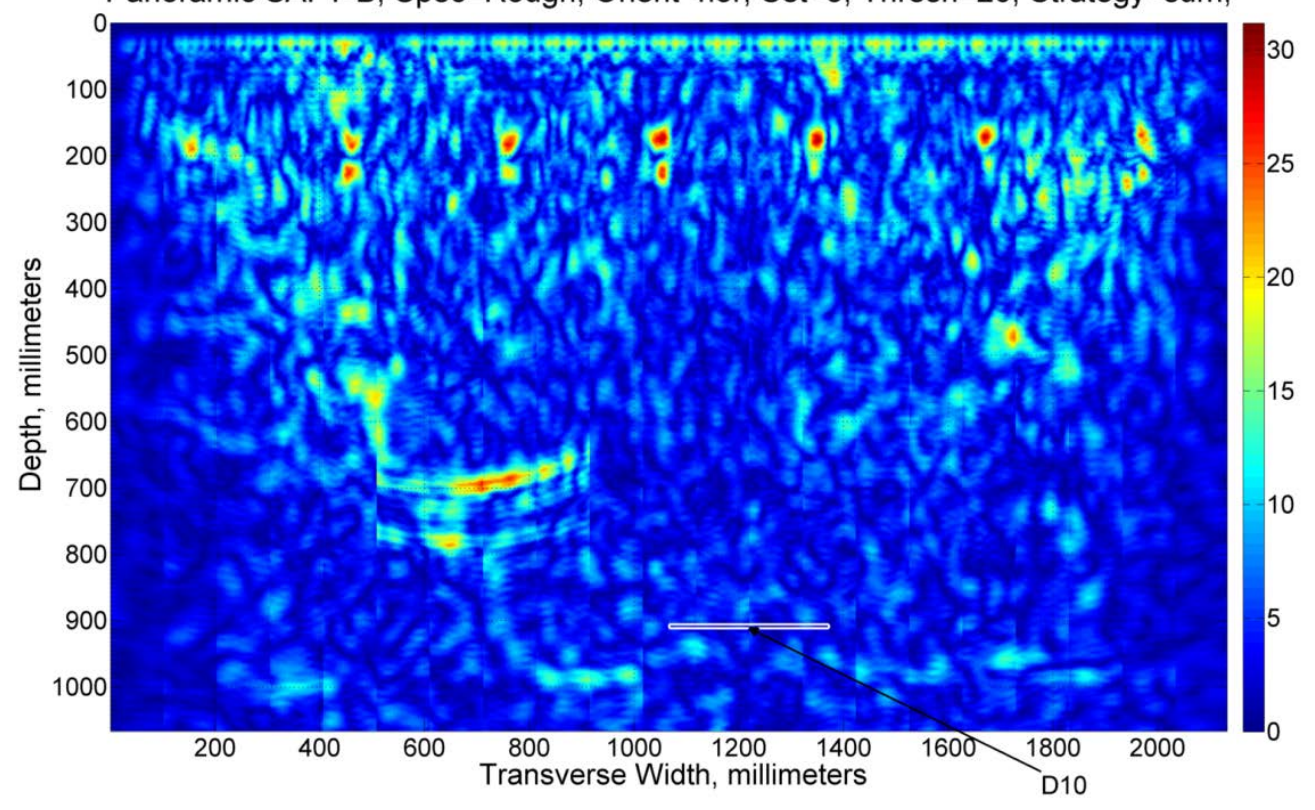

Fig. A. 13. Horizontal Set 3, Node 18.

Specimen: Thick, Depth: 1066.8mm (42in), AbsofHilbert -- Node $32(5,1), 15.625 \sim 31.25 \mathrm{kHz}$

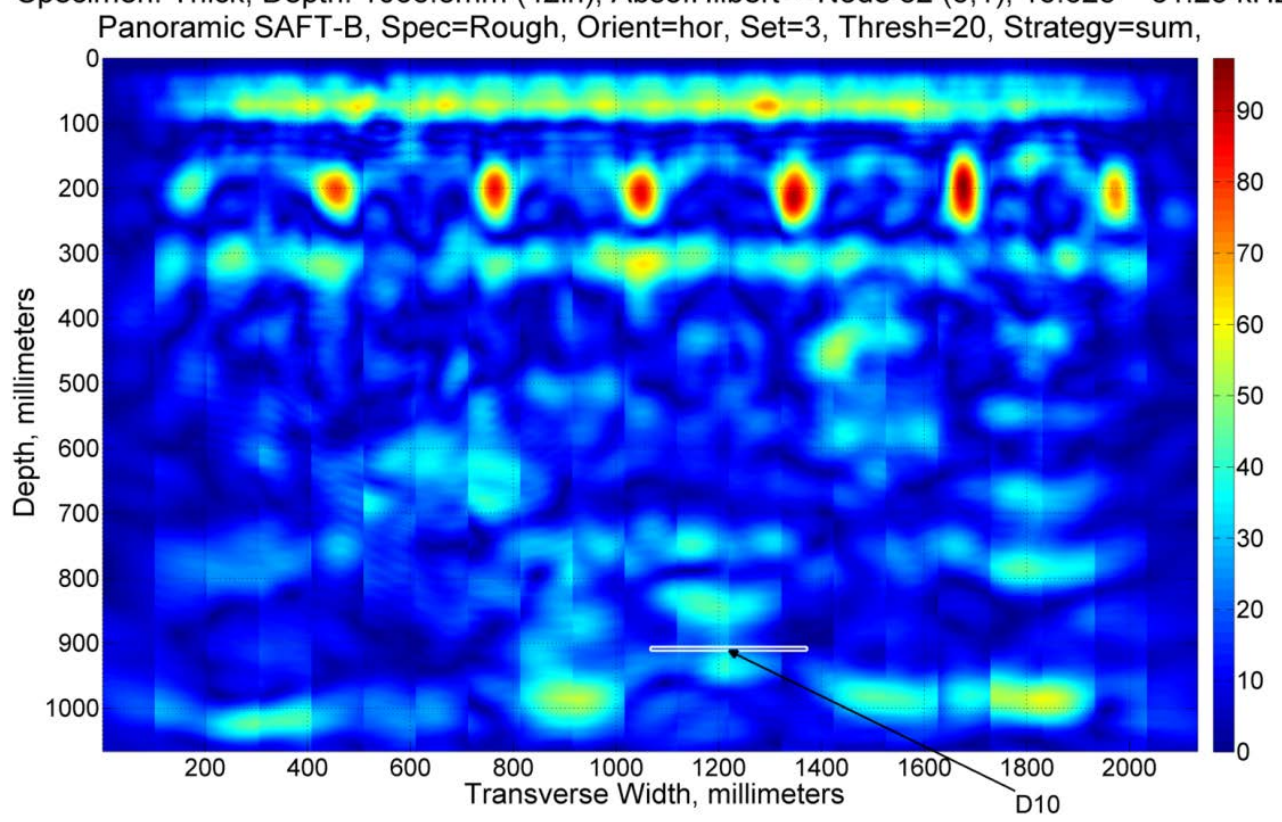

Fig. A. 14. Horizontal Set 3, Node 32. 


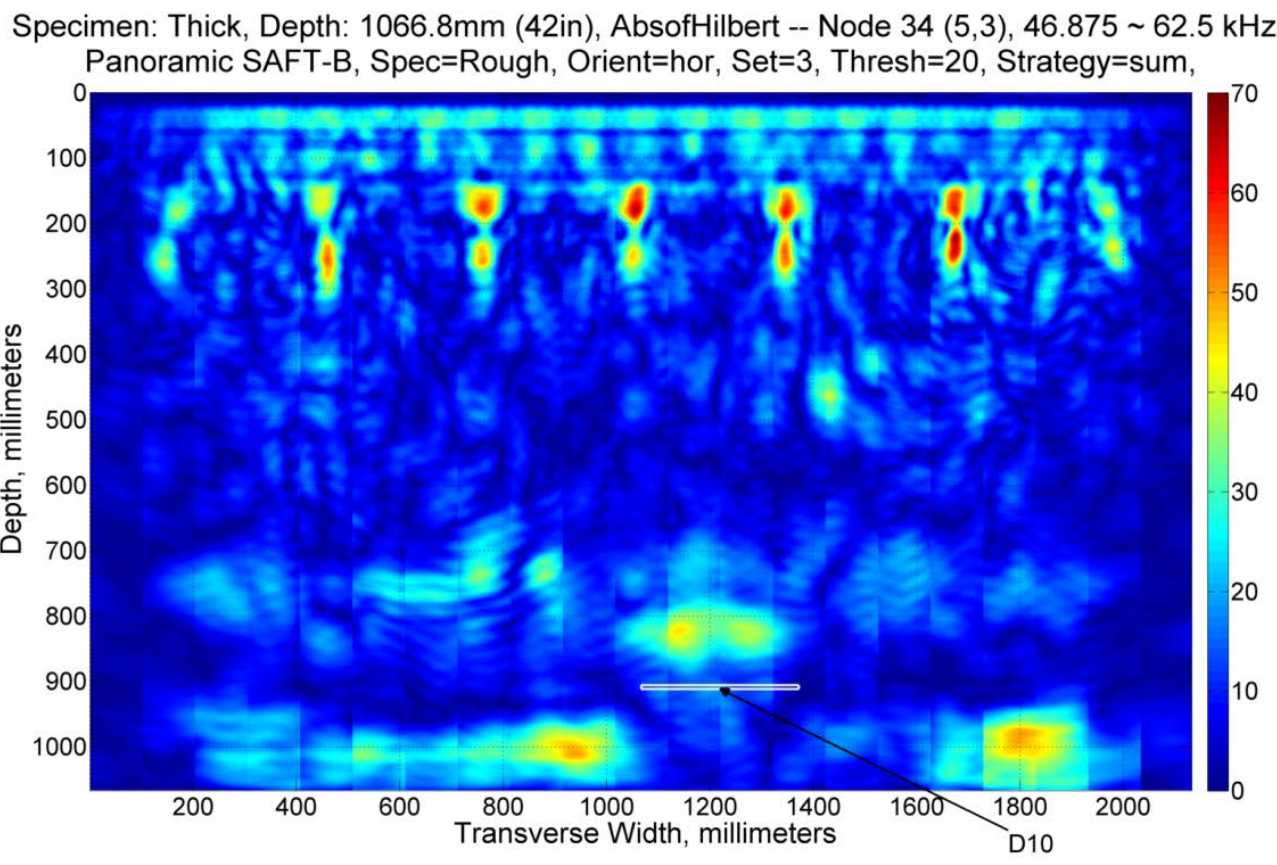

Fig. A. 15. Horizontal Set 3, Node 34.

\section{SET 4 - DEFECT 4}

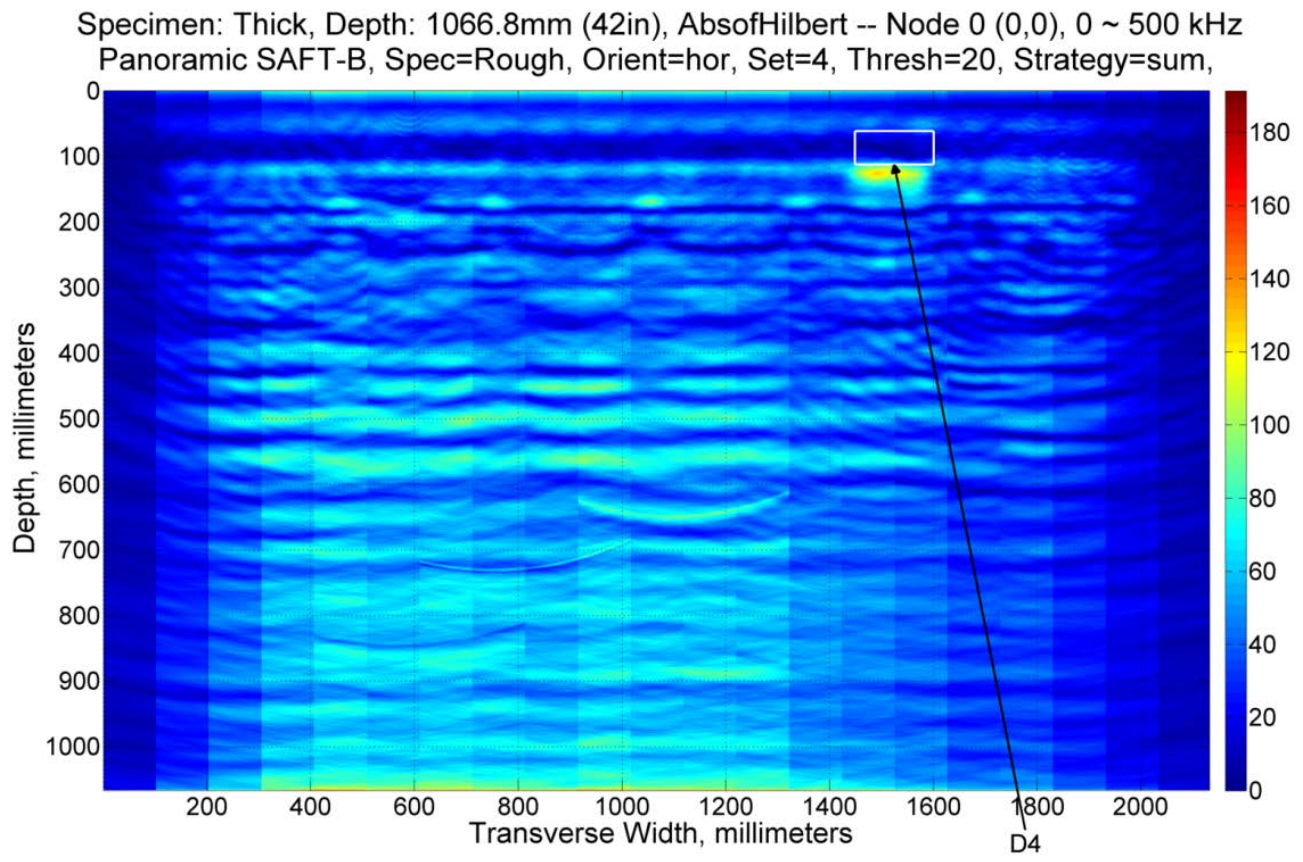

Fig. A. 16. Horizontal Set 4, Node 0. 
Specimen: Thick, Depth: 1066.8mm (42in), AbsofHilbert -- Node $18(4,3), 93.75 \sim 125 \mathrm{kHz}$

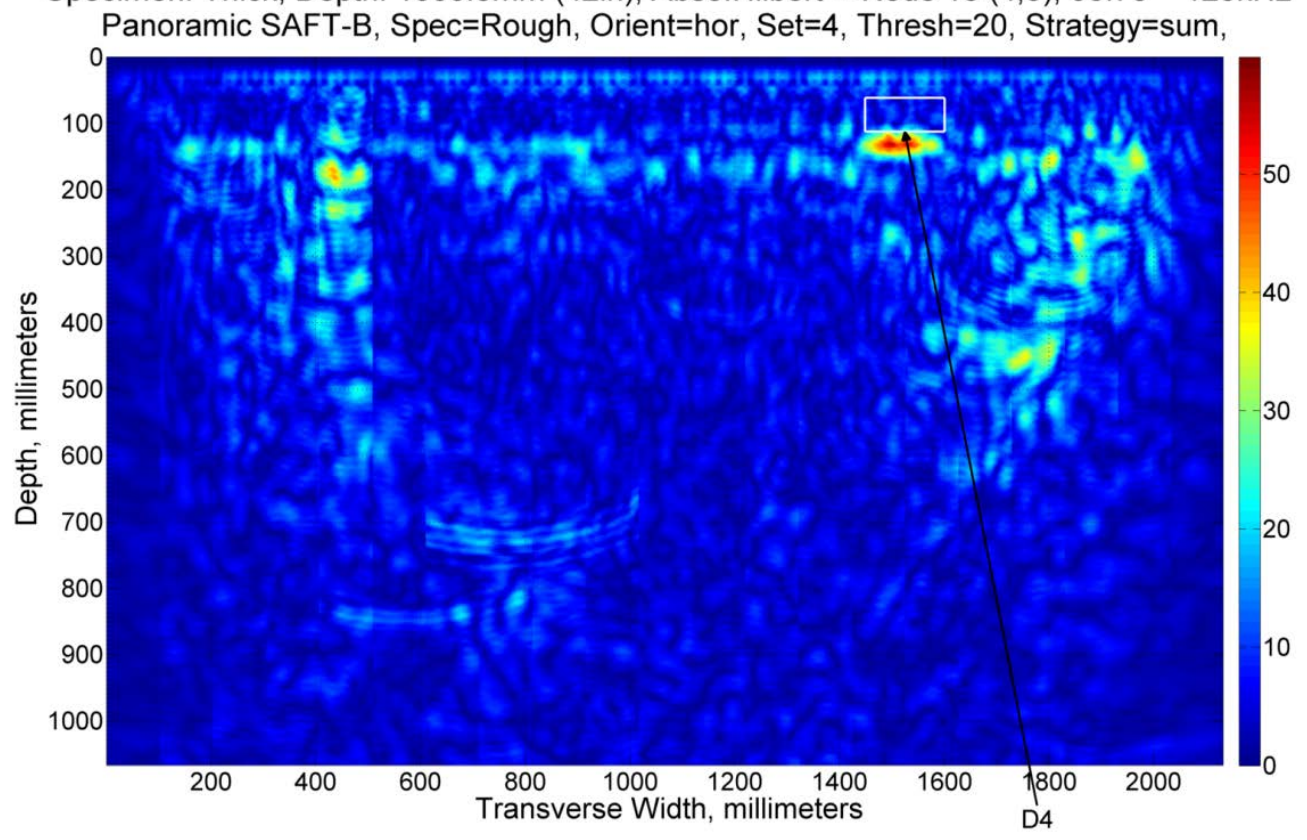

Fig. A. 17. Horizontal Set 4, Node 18.

Specimen: Thick, Depth: 1066.8mm (42in), AbsofHilbert -- Node $16(4,1), 31.25 \sim 62.5 \mathrm{kHz}$ Panoramic SAFT-B, Spec=Rough, Orient=hor, Set=4, Thresh=20, Strategy=sum,

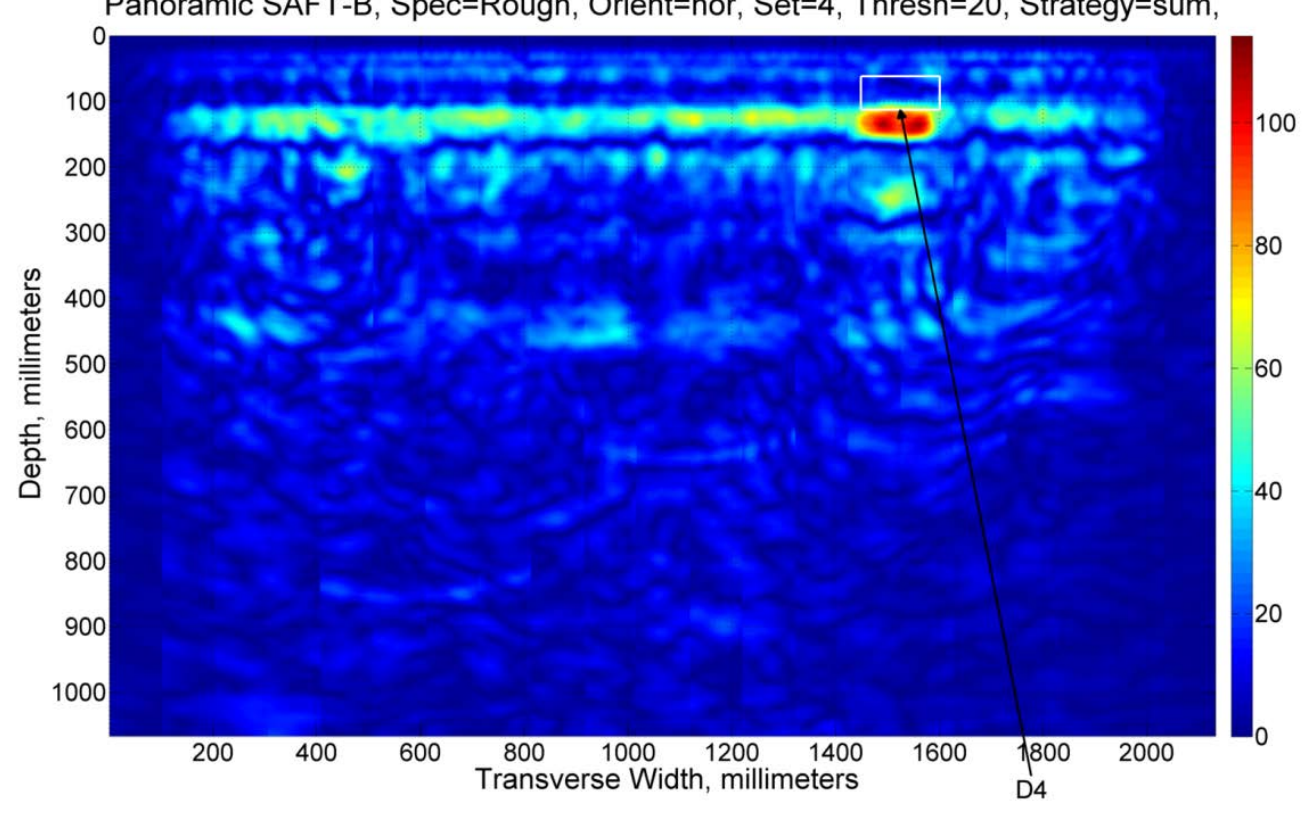

Fig. A. 18. Horizontal Set 4, Node 16. 


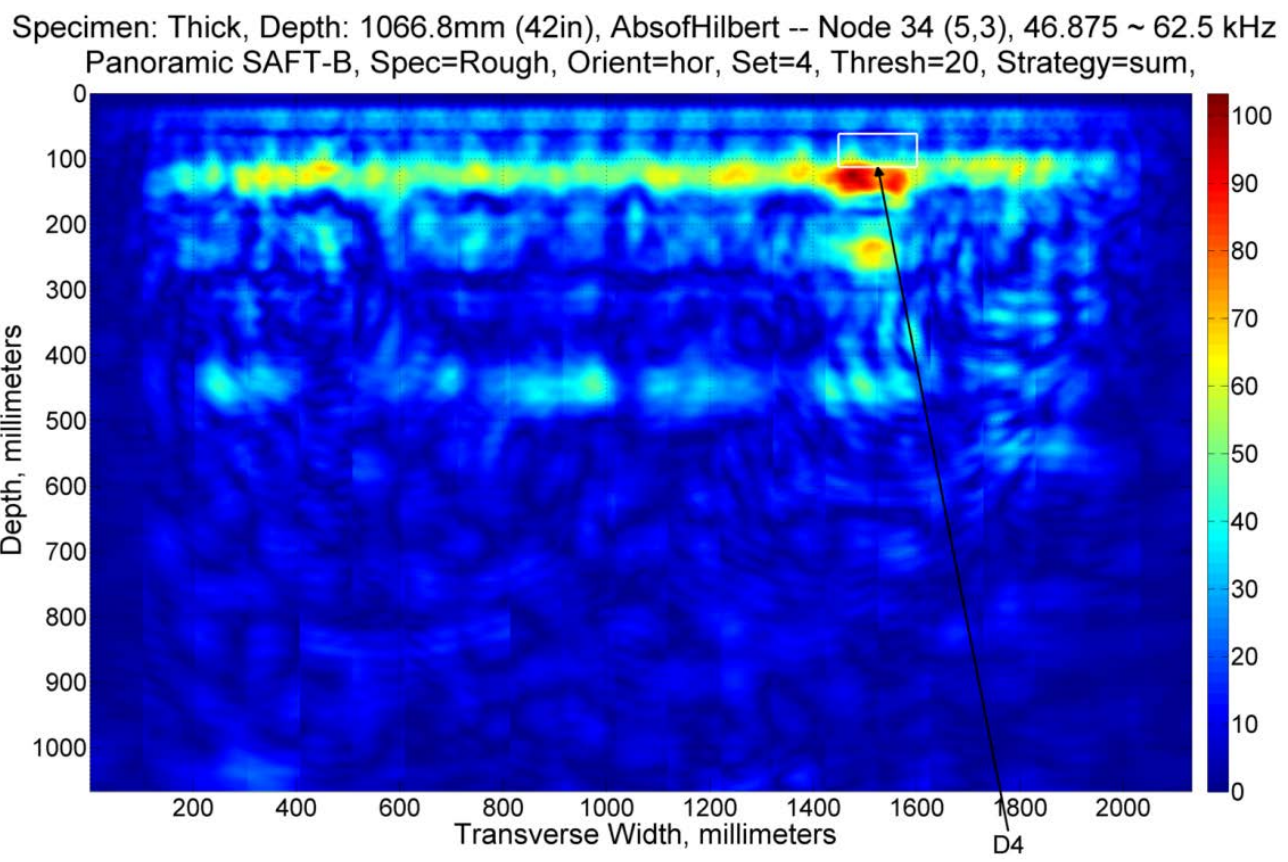

Fig. A. 19. Horizontal Set 4, Node 34.

Specimen: Thick, Depth: 1066.8mm (42in), AbsofHilbert -- Node $32(5,1), 15.625 \sim 31.25 \mathrm{kHz}$ Panoramic SAFT-B, Spec=Rough, Orient=hor, Set=4, Thresh=20, Strategy=sum,

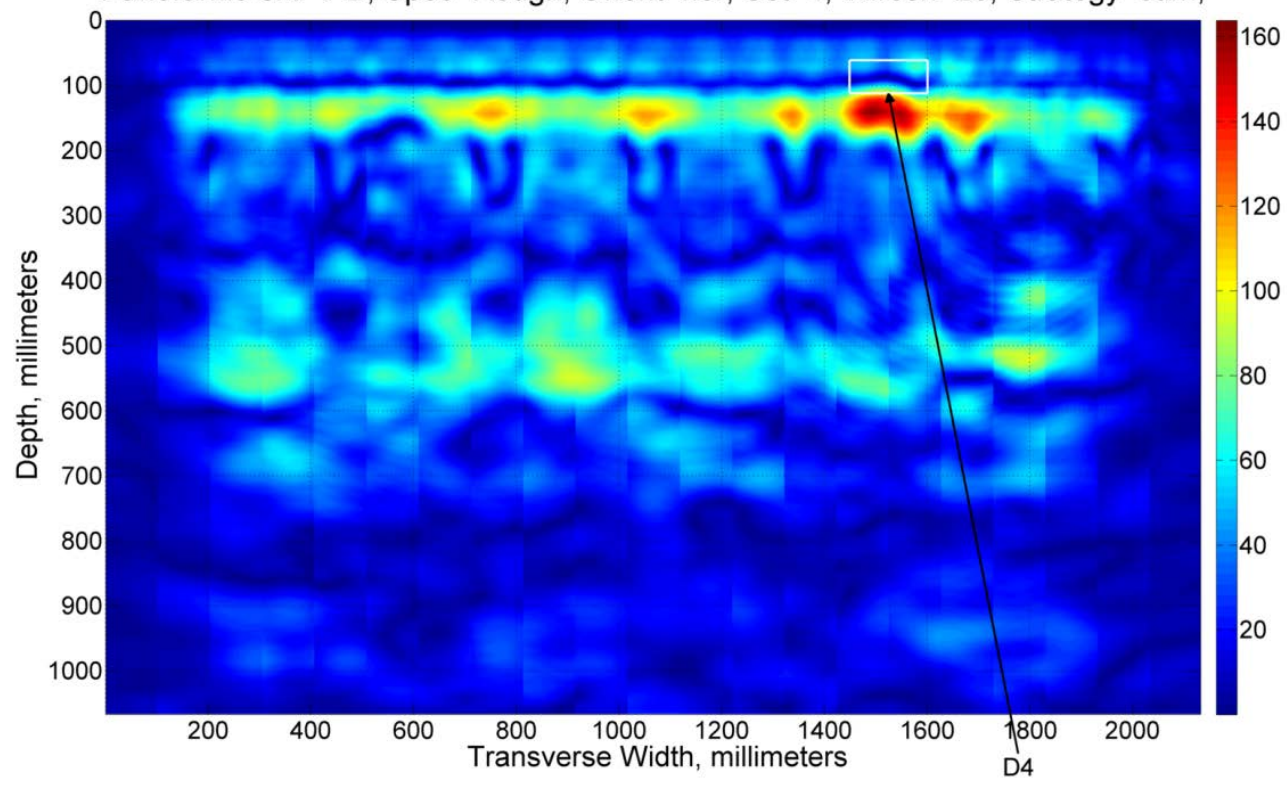

Fig. A. 20. Horizontal Set 4, Node 32 . 
Specimen: Thick, Depth: 1066.8mm (42in), AbsofHilbert -- Node $0(0,0), 0 \sim 500 \mathrm{kHz}$ Panoramic SAFT-B, Spec=Rough, Orient=hor, Set=5, Thresh=20, Strategy=sum,

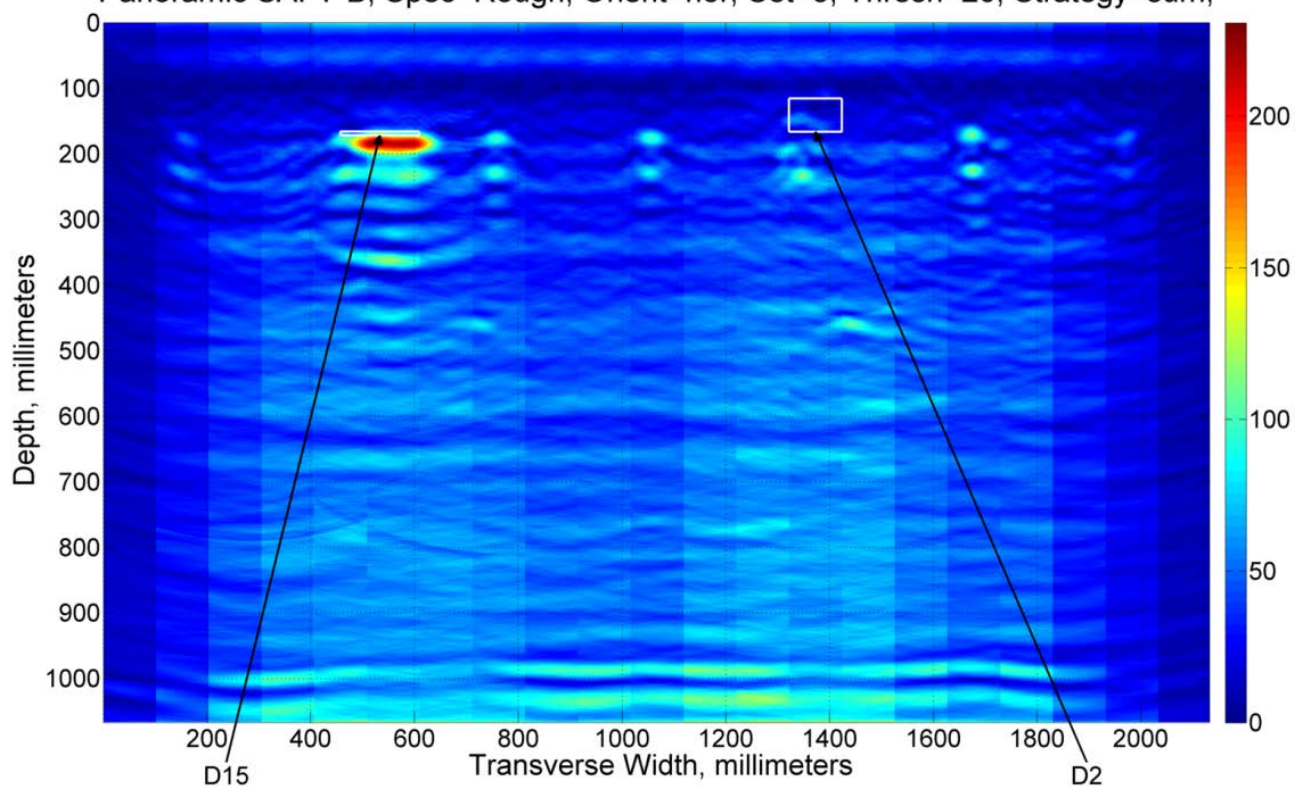

Fig. A. 21. Horizontal Set 5, Node 0.

Specimen: Thick, Depth: 1066.8mm (42in), AbsofHilbert -- Node $16(4,1), 31.25 \sim 62.5 \mathrm{kHz}$ Panoramic SAFT-B, Spec=Rough, Orient=hor, Set=5, Thresh=20, Strategy=sum,

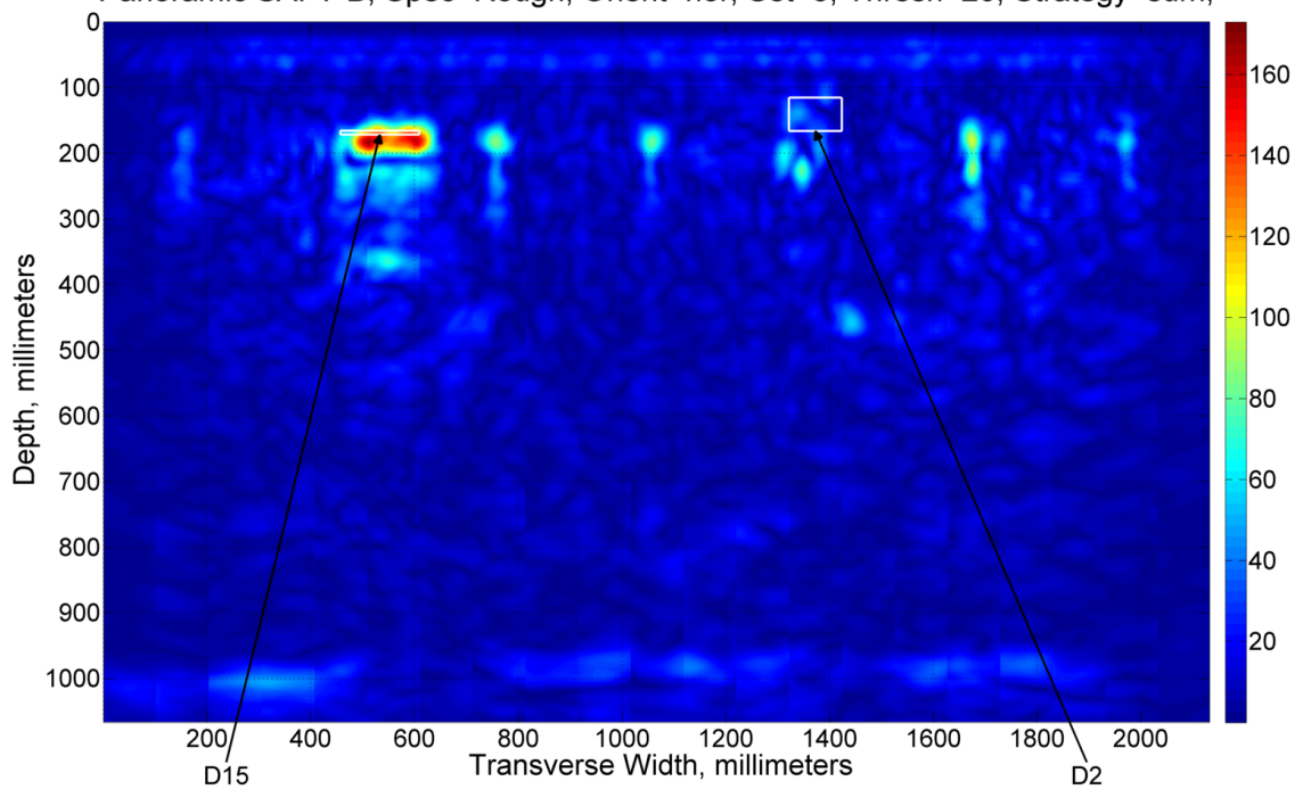

Fig. A. 22. Horizontal Set 5, Node 16. 
Specimen: Thick, Depth: 1066.8mm (42in), AbsofHilbert -- Node $18(4,3), 93.75 \sim 125 \mathrm{kHz}$ Panoramic SAFT-B, Spec=Rough, Orient=hor, Set=5, Thresh=20, Strategy=sum,

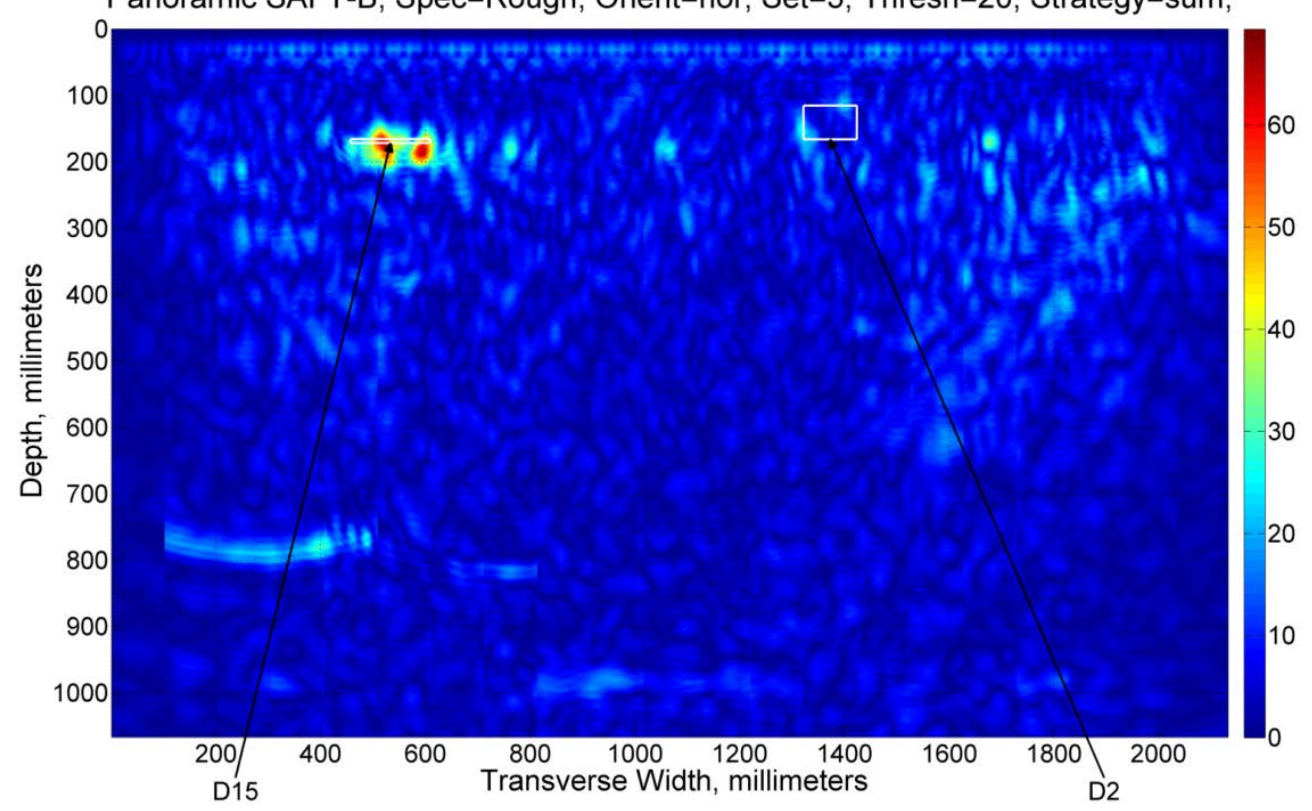

Fig. A. 23. Horizontal Set 5, Node 18.

Specimen: Thick, Depth: 1066.8mm (42in), AbsofHilbert -- Node $32(5,1), 15.625 \sim 31.25 \mathrm{kHz}$ Panoramic SAFT-B, Spec=Rough, Orient=hor, Set=5, Thresh=20, Strategy=sum,

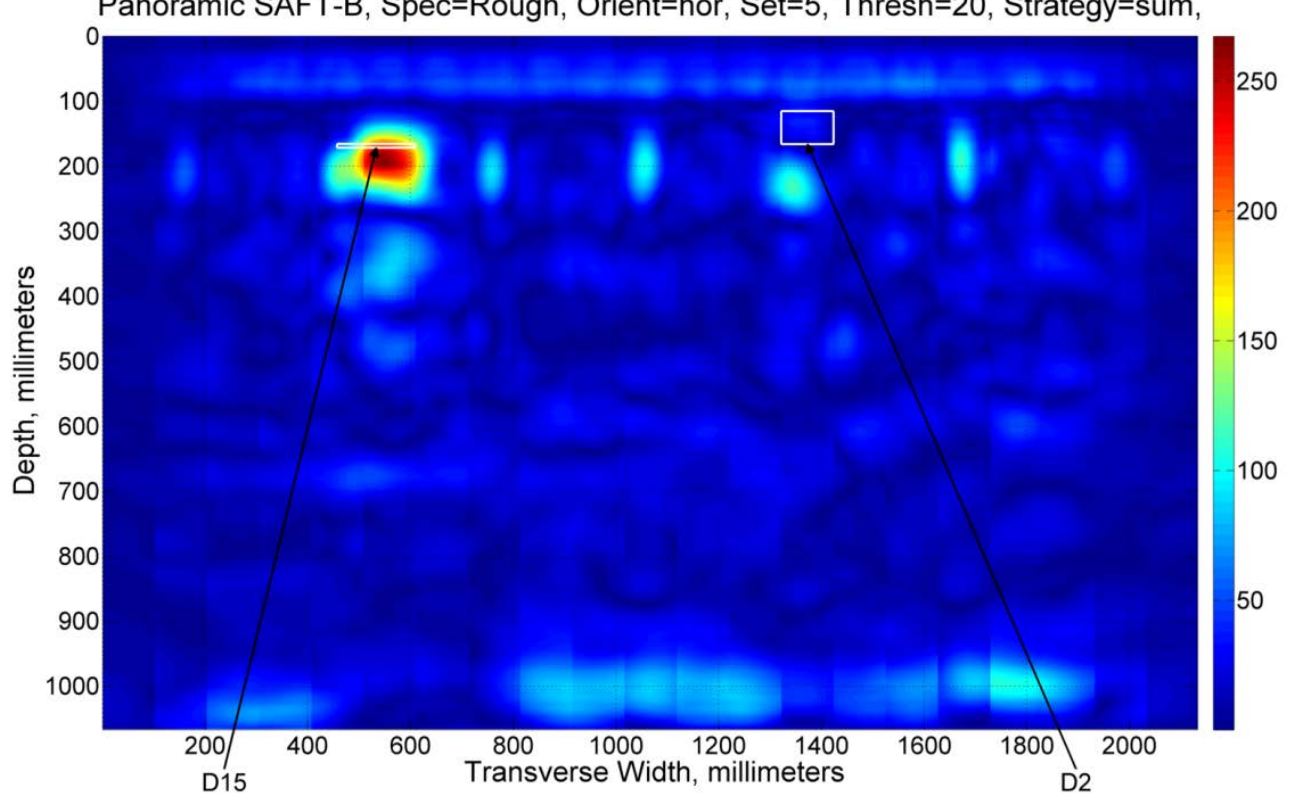

Fig. A. 24. Horizontal Set 5, Node 32. 


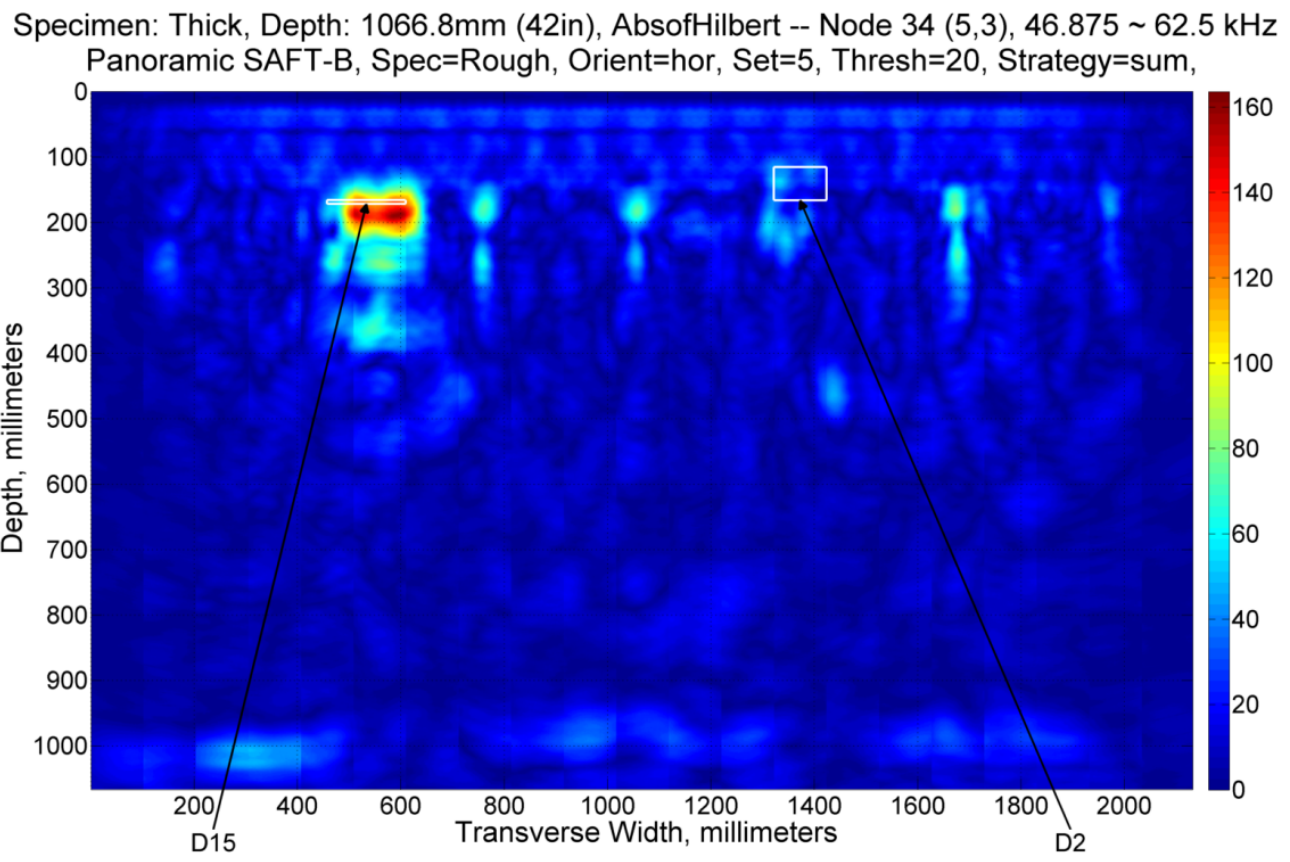

Fig. A. 25. Horizontal Set 5, Node 34.

\section{SET 6 - DEFECT 2}

Specimen: Thick, Depth: 1066.8mm (42in), AbsofHilbert -- Node $0(0,0), 0 \sim 500 \mathrm{kHz}$ Panoramic SAFT-B, Spec=Rough, Orient=hor, Set=6, Thresh=20, Strategy=sum,

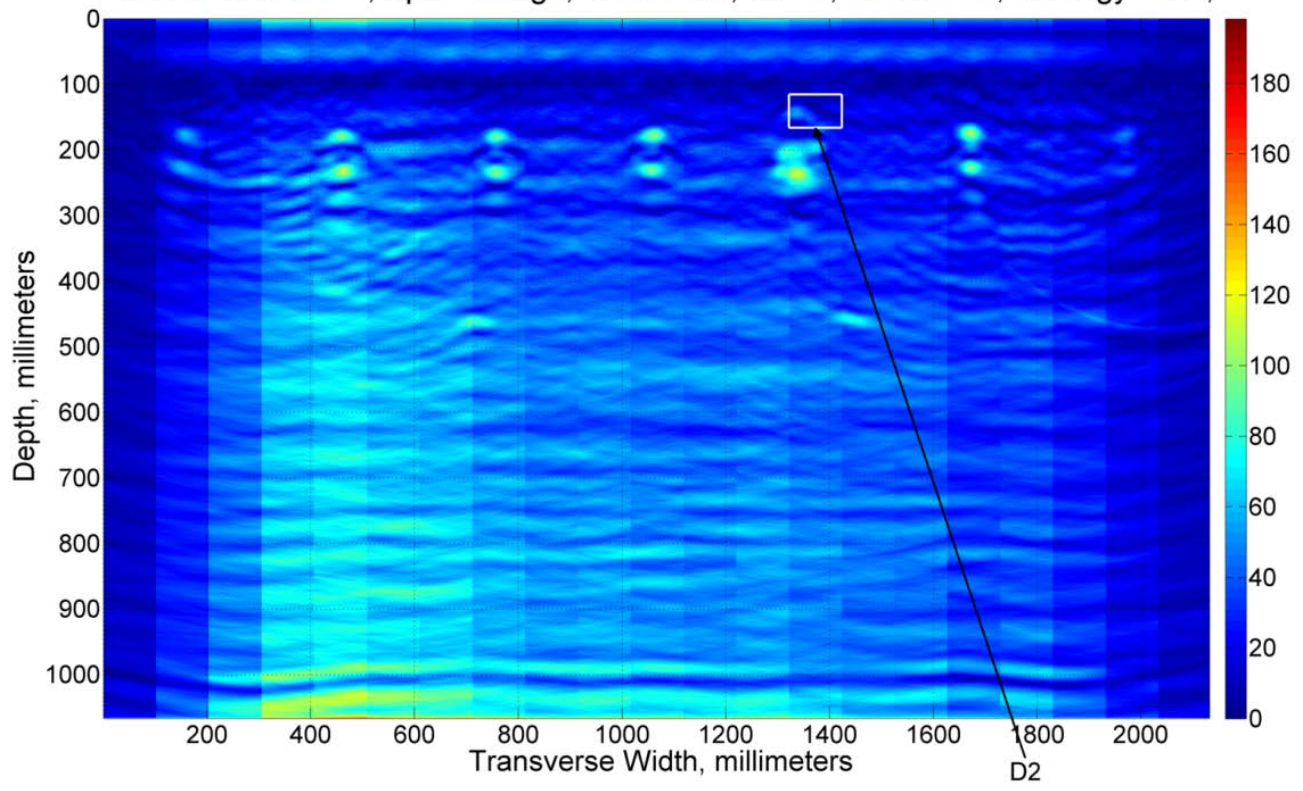

Fig. A. 26. Horizontal Set 6, Node 0. 
Specimen: Thick, Depth: 1066.8mm (42in), AbsofHilbert -- Node $16(4,1), 31.25 \sim 62.5 \mathrm{kHz}$ Panoramic SAFT-B, Spec=Rough, Orient=hor, Set=6, Thresh=20, Strategy=sum,

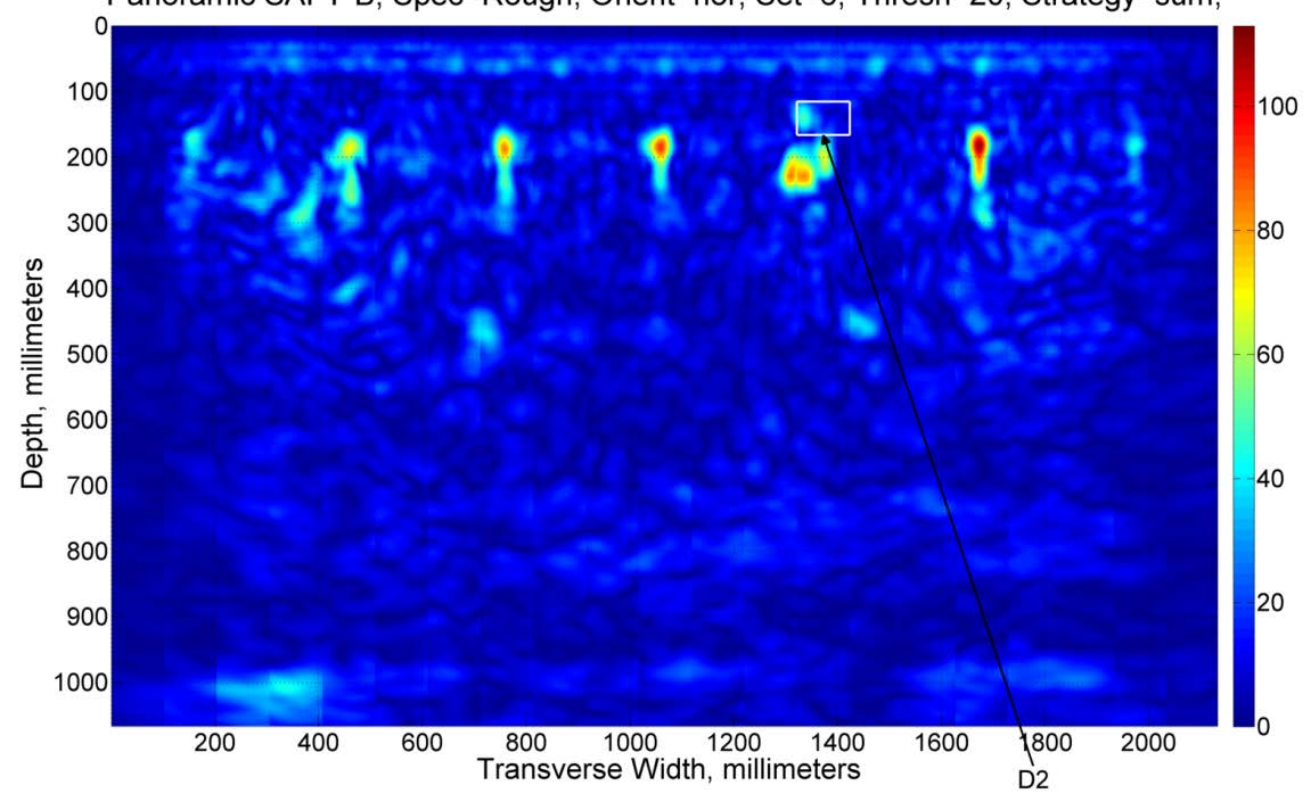

Fig. A. 27. Horizontal Set 6, Node 16.

Specimen: Thick, Depth: 1066.8mm (42in), AbsofHilbert -- Node $18(4,3), 93.75 \sim 125 \mathrm{kHz}$ Panoramic SAFT-B, Spec=Rough, Orient=hor, Set=6, Thresh=20, Strategy=sum,

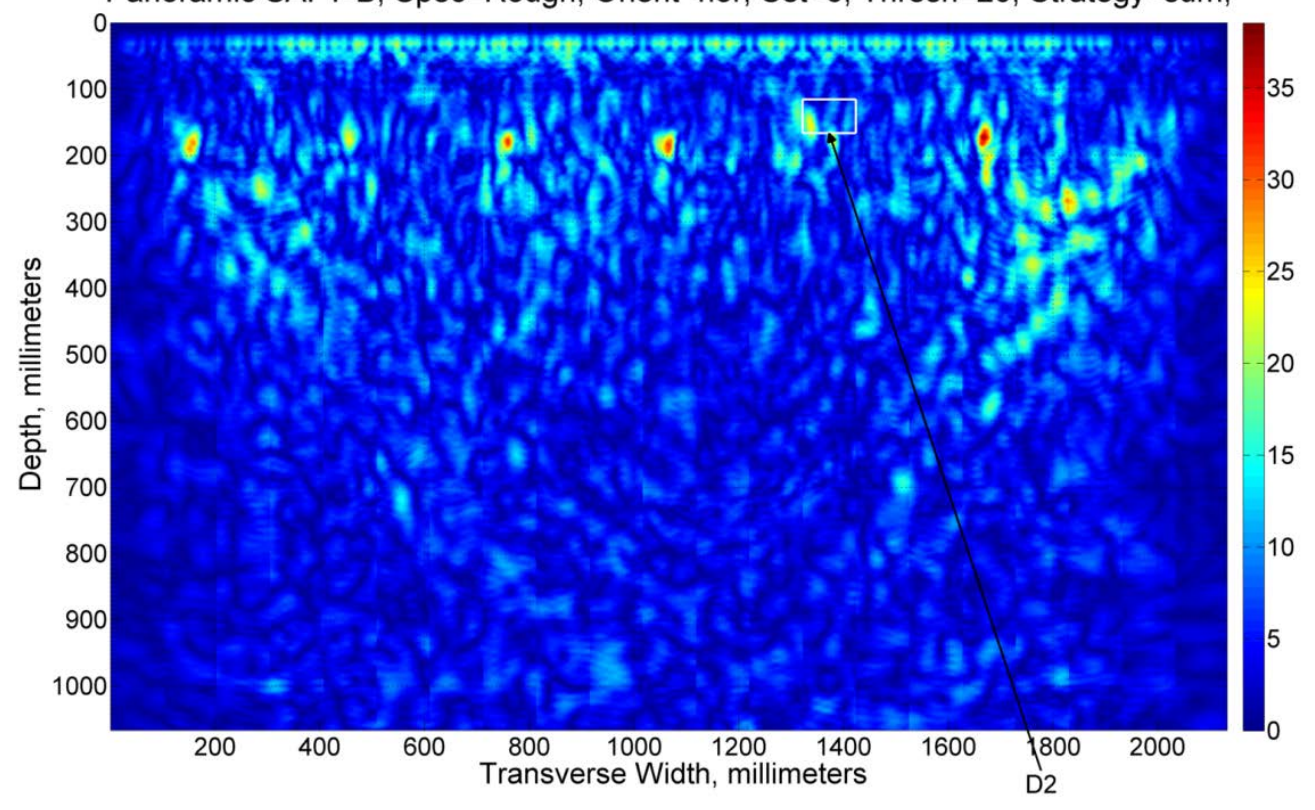

Fig. A. 28. Horizontal Set 6, Node 18. 


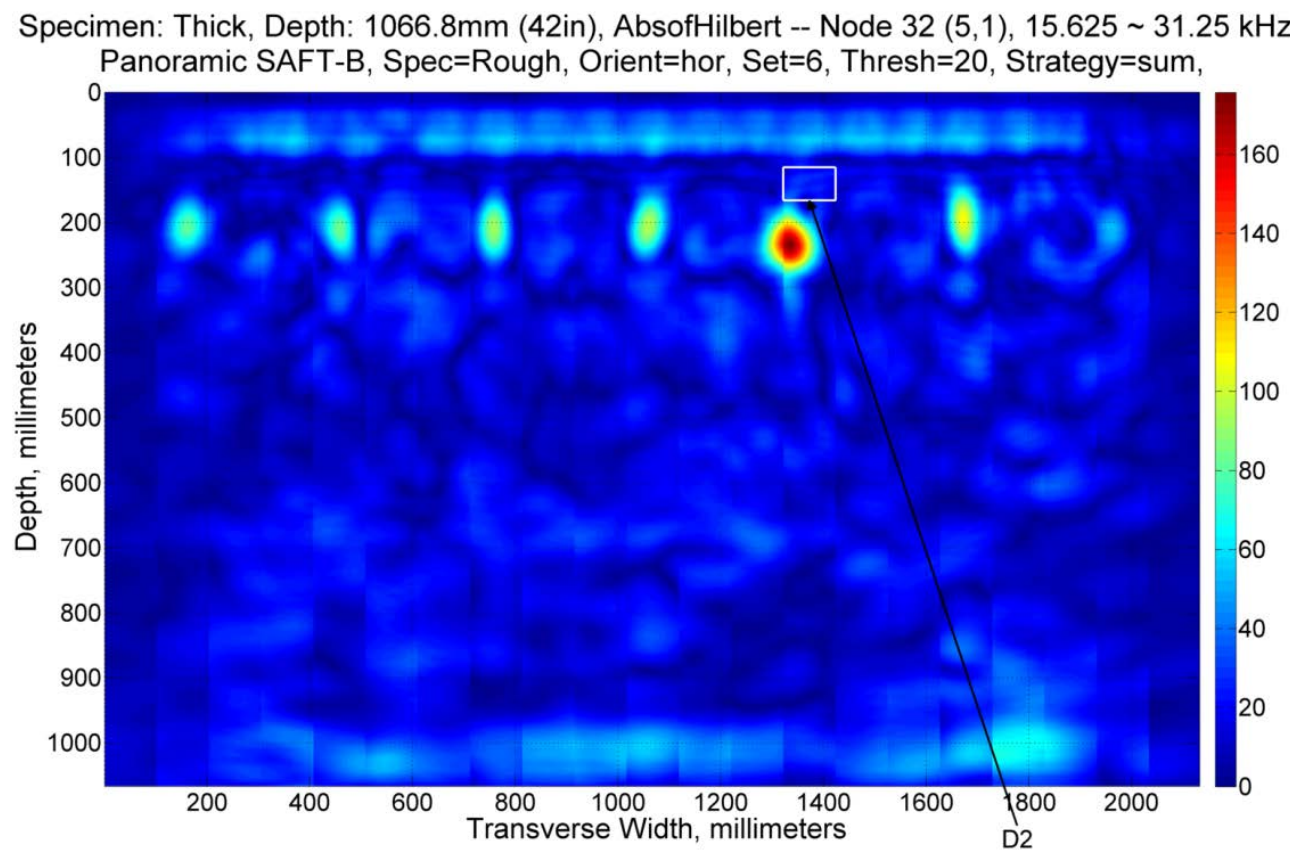

Fig. A. 29. Horizontal Set 6, Node 32.

Specimen: Thick, Depth: 1066.8mm (42in), AbsofHilbert -- Node $34(5,3), 46.875 \sim 62.5 \mathrm{kHz}$ Panoramic SAFT-B, Spec=Rough, Orient=hor, Set=6, Thresh=20, Strategy=sum,

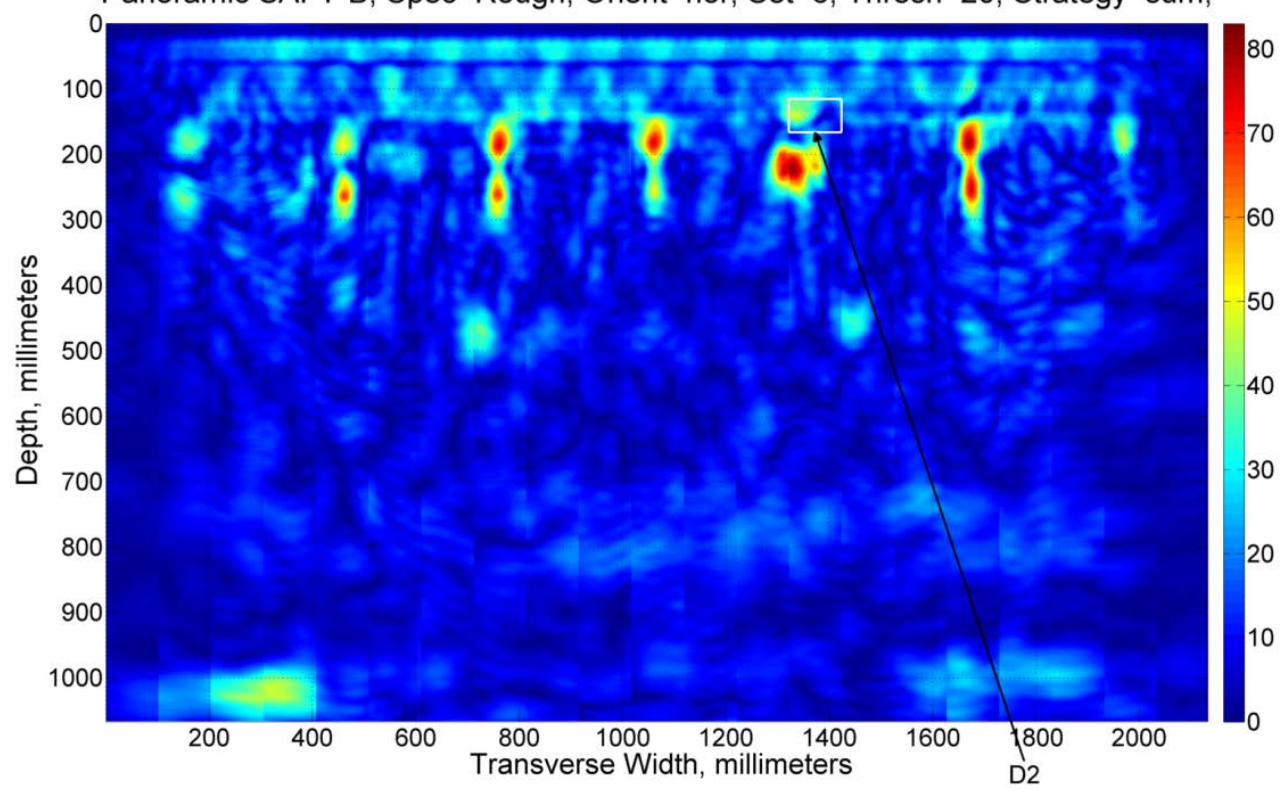

Fig. A. 30. Horizontal Set 6, Node 34. 


\section{SET 7 - DEFECT 20}

Specimen: Thick, Depth: 1066.8mm (42in), AbsofHilbert -- Node $0(0,0), 0 \sim 500 \mathrm{kHz}$ Panoramic SAFT-B, Spec=Rough, Orient=hor, Set=7, Thresh=20, Strategy=sum,

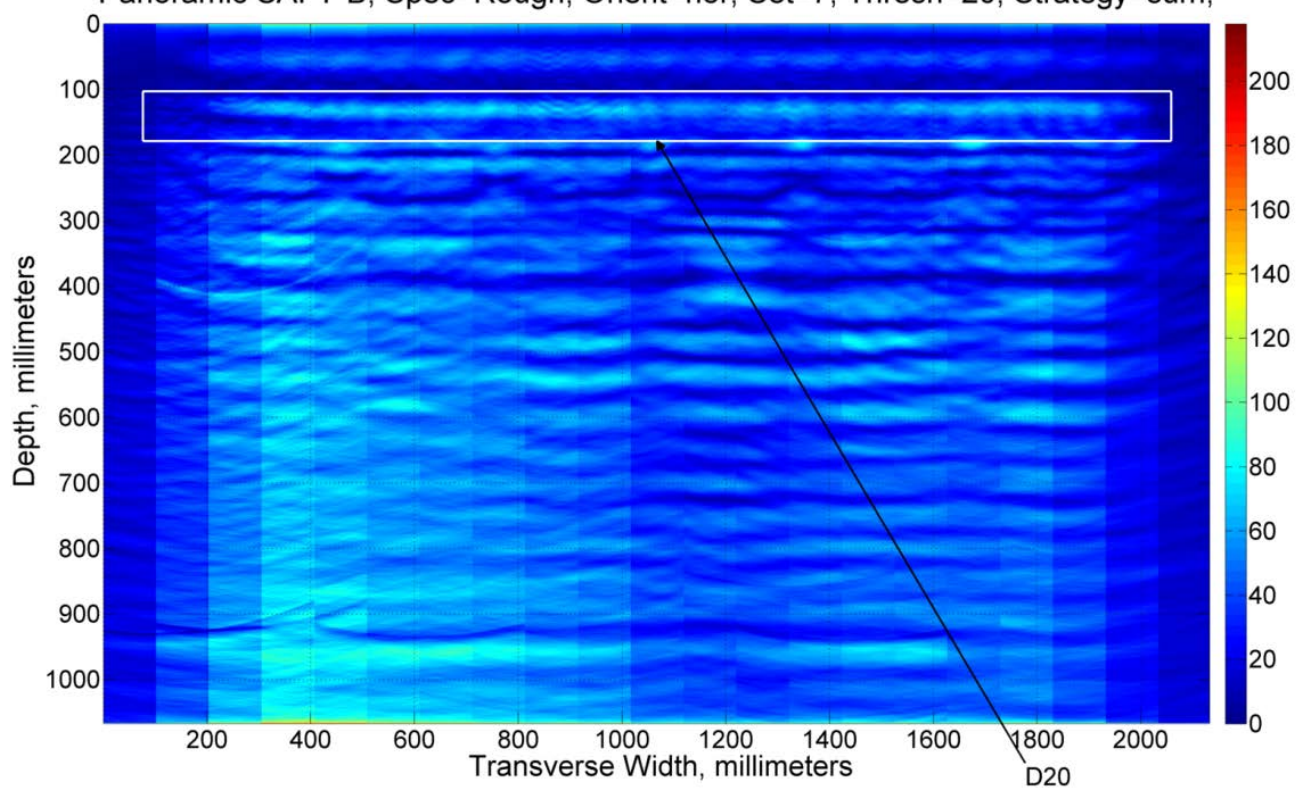

Fig. A. 31. Horizontal Set 7, Node 0.

Specimen: Thick, Depth: 1066.8mm (42in), AbsofHilbert -- Node $16(4,1), 31.25 \sim 62.5 \mathrm{kHz}$ Panoramic SAFT-B, Spec=Rough, Orient=hor, Set=7, Thresh=20, Strategy=sum,

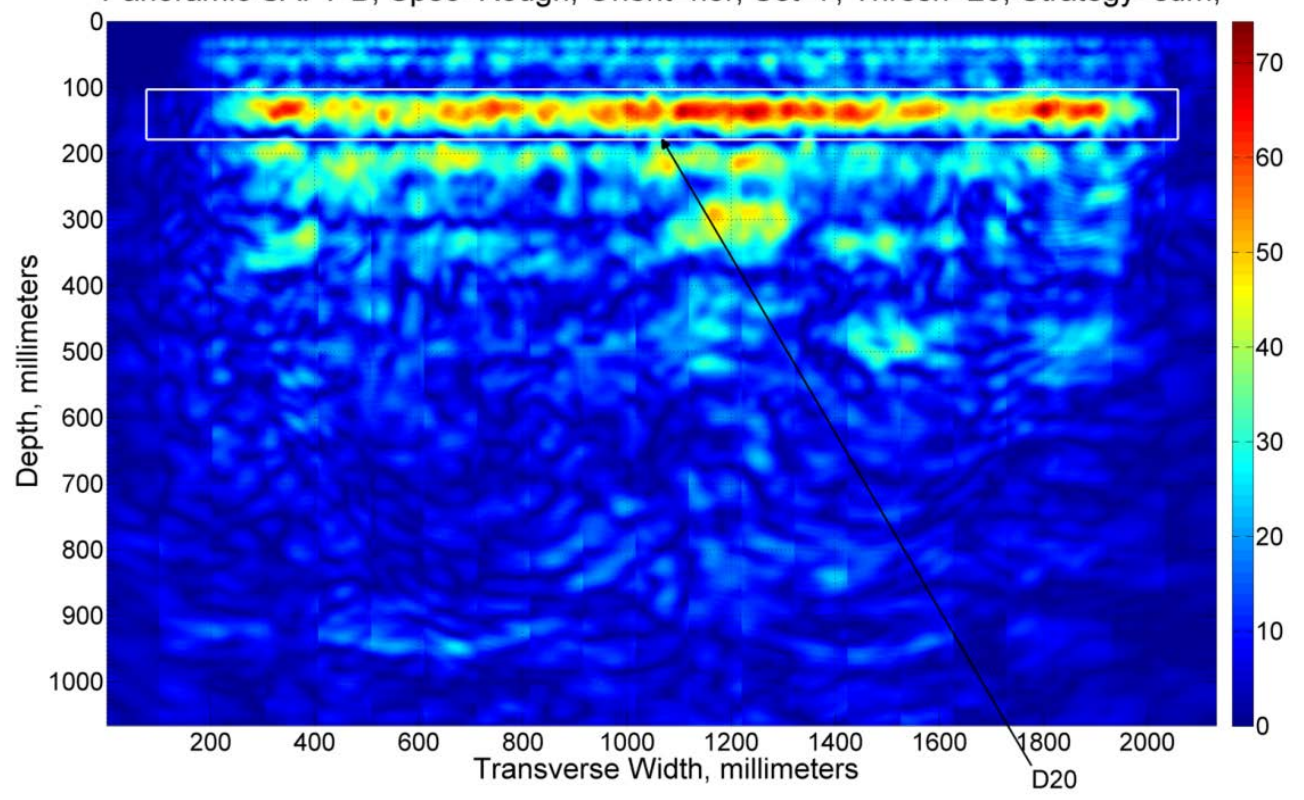

Fig. A. 32. Horizontal Set 7, Node 16. 


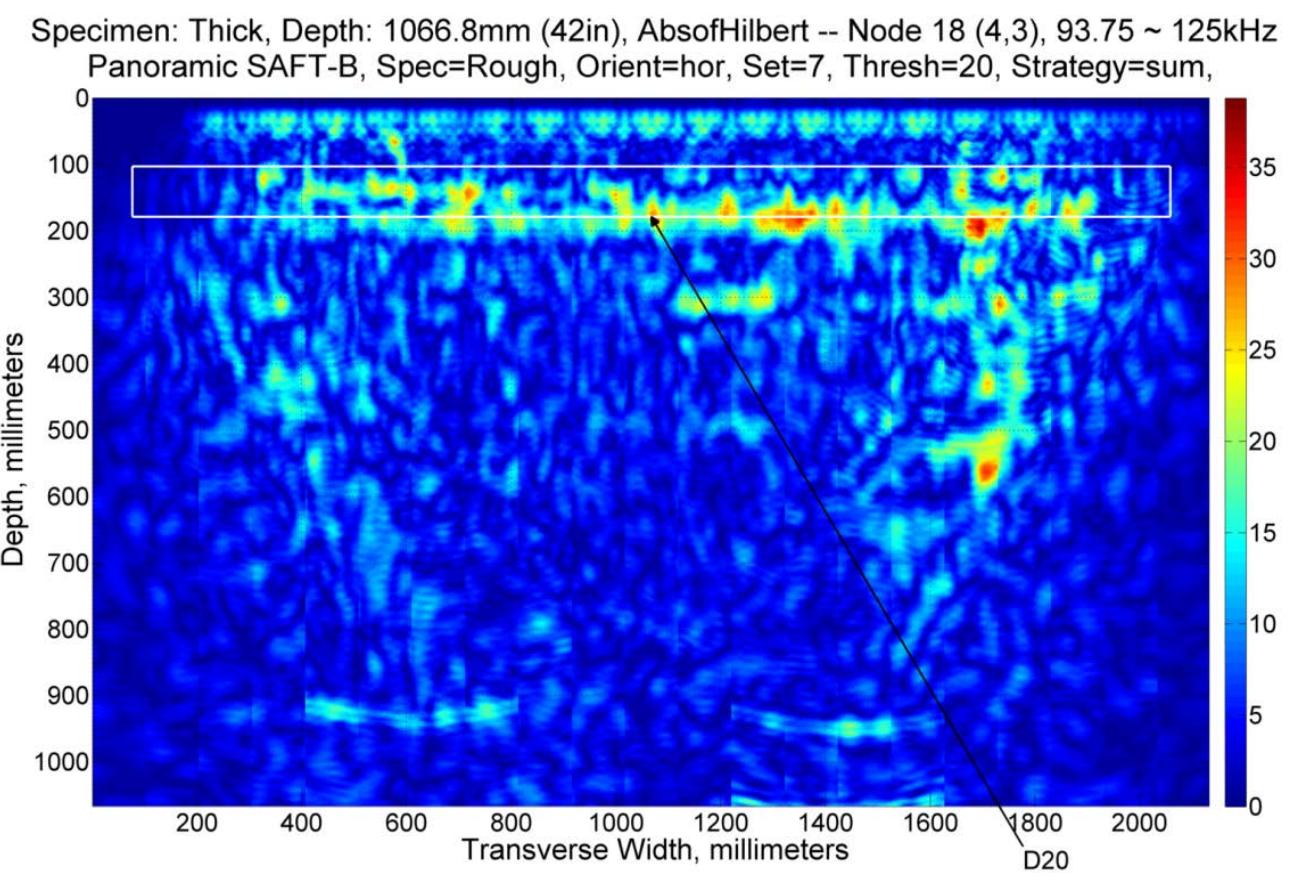

Fig. A. 33. Horizontal Set 7, Node 18.

Specimen: Thick, Depth: 1066.8mm (42in), AbsofHilbert -- Node 32 (5,1), $15.625 \sim 31.25 \mathrm{kHz}$

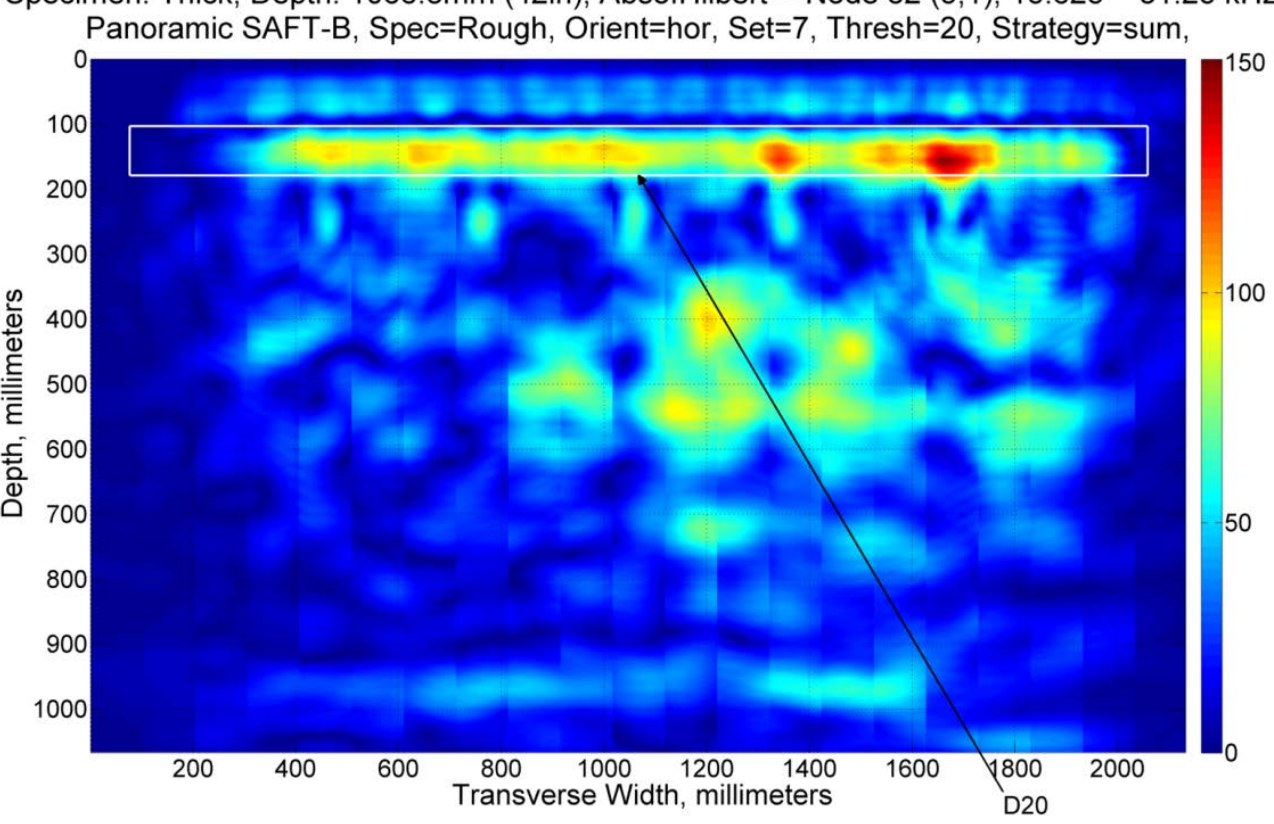

Fig. A. 34. Horizontal Set 7, Node 32. 


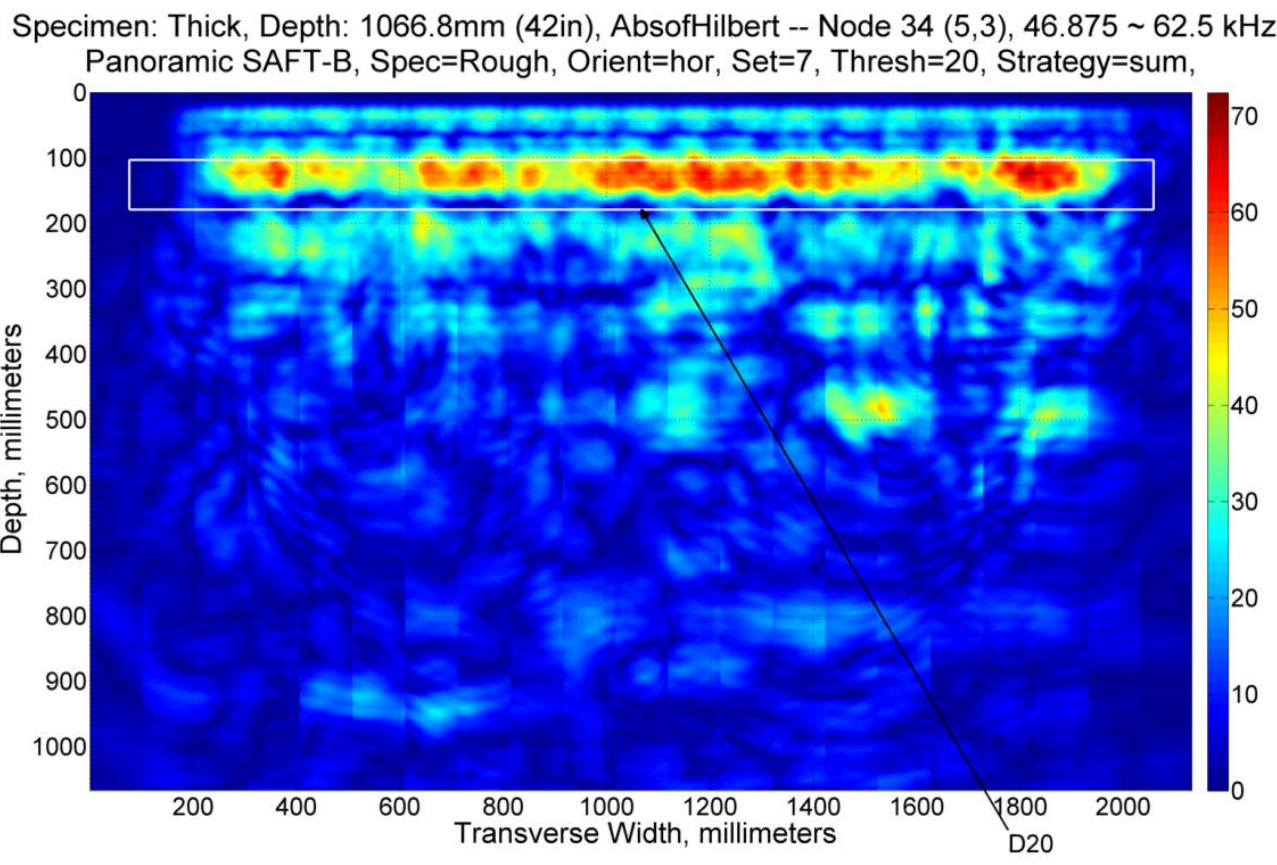

Fig. A. 35. Horizontal Set 7, Node 34.

\section{SET 8 - DEFECT 8, DEFECT 12}

Specimen: Thick, Depth: 1066.8mm (42in), AbsofHilbert -- Node $0(0,0), 0 \sim 500 \mathrm{kHz}$ Panoramic SAFT-B, Spec=Rough, Orient=hor, Set=8, Thresh=20, Strategy=sum,

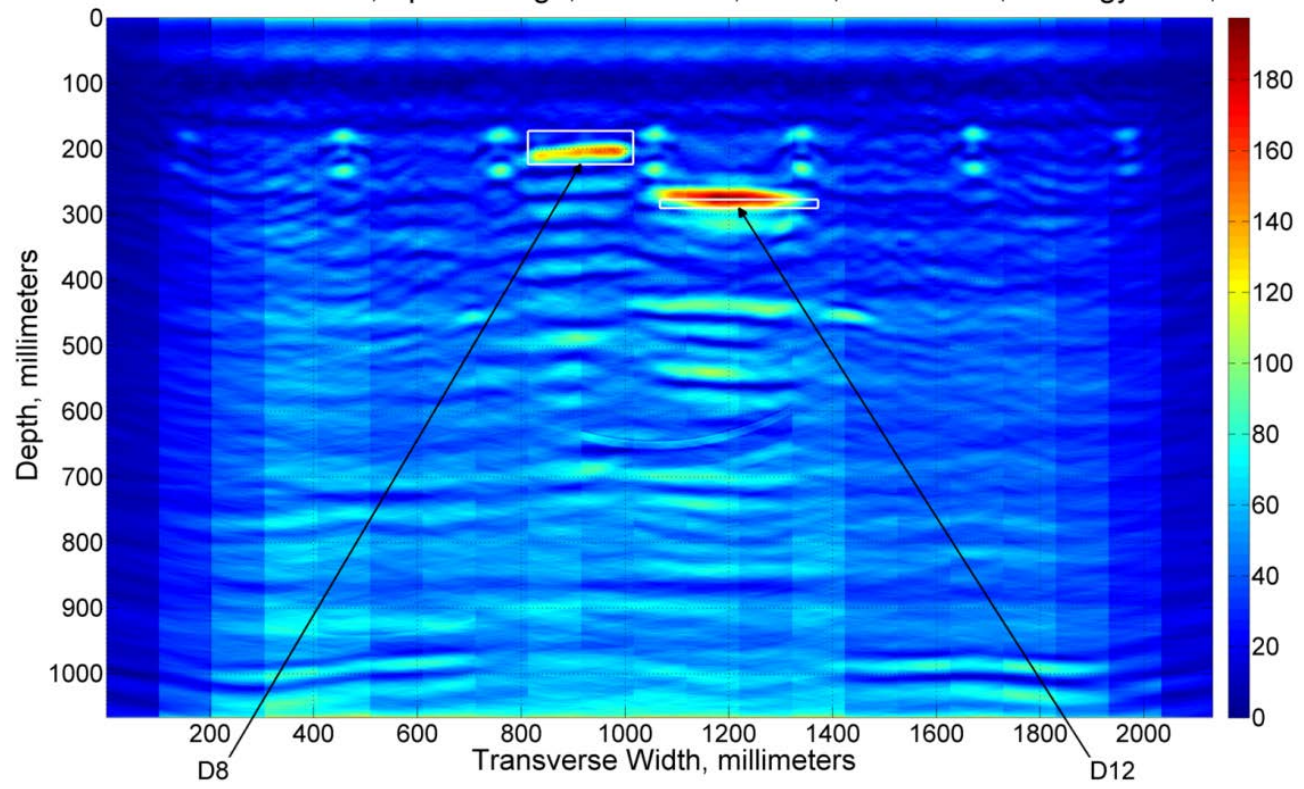

Fig. A. 36. Horizontal Set 8, Node 0 . 
Specimen: Thick, Depth: 1066.8mm (42in), AbsofHilbert -- Node $16(4,1), 31.25 \sim 62.5 \mathrm{kHz}$ Panoramic SAFT-B, Spec=Rough, Orient=hor, Set=8, Thresh=20, Strategy=sum,

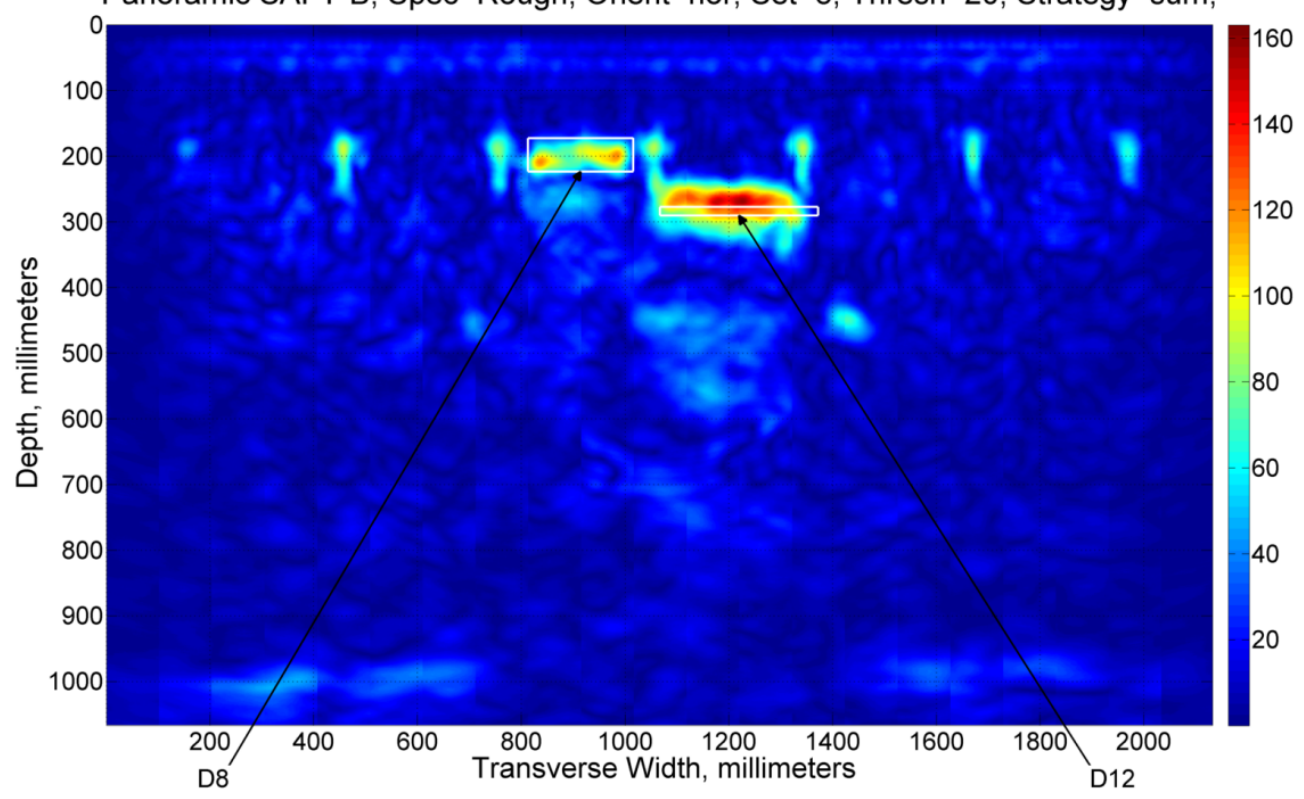

Fig. A. 37. Horizontal Set 8, Node 16.

Specimen: Thick, Depth: 1066.8mm (42in), AbsofHilbert -- Node $18(4,3), 93.75 \sim 125 \mathrm{kHz}$ Panoramic SAFT-B, Spec=Rough, Orient=hor, Set=8, Thresh=20, Strategy=sum,

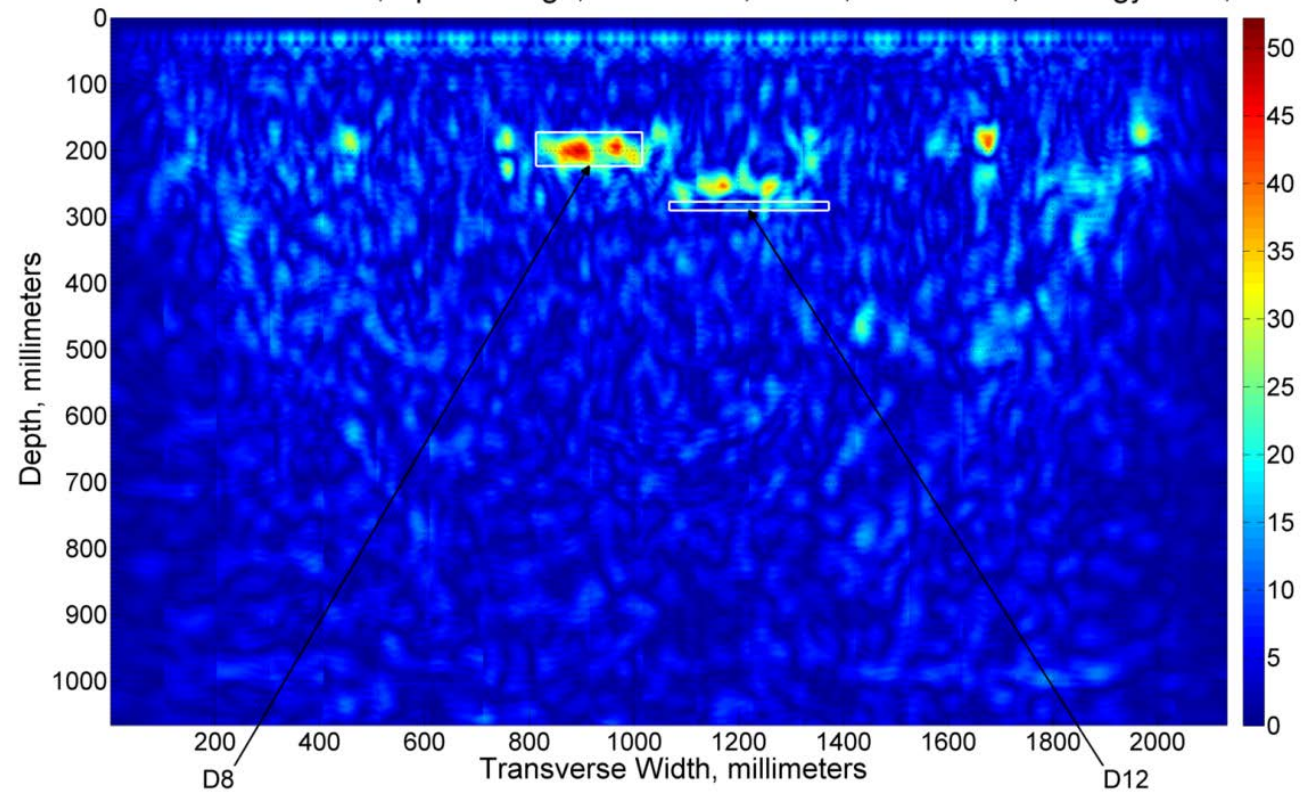

Fig. A. 38. Horizontal Set 8, Node 18. 
Specimen: Thick, Depth: 1066.8mm (42in), AbsofHilbert -- Node $32(5,1), 15.625 \sim 31.25 \mathrm{kHz}$ Panoramic SAFT-B, Spec=Rough, Orient=hor, Set=8, Thresh=20, Strategy=sum,

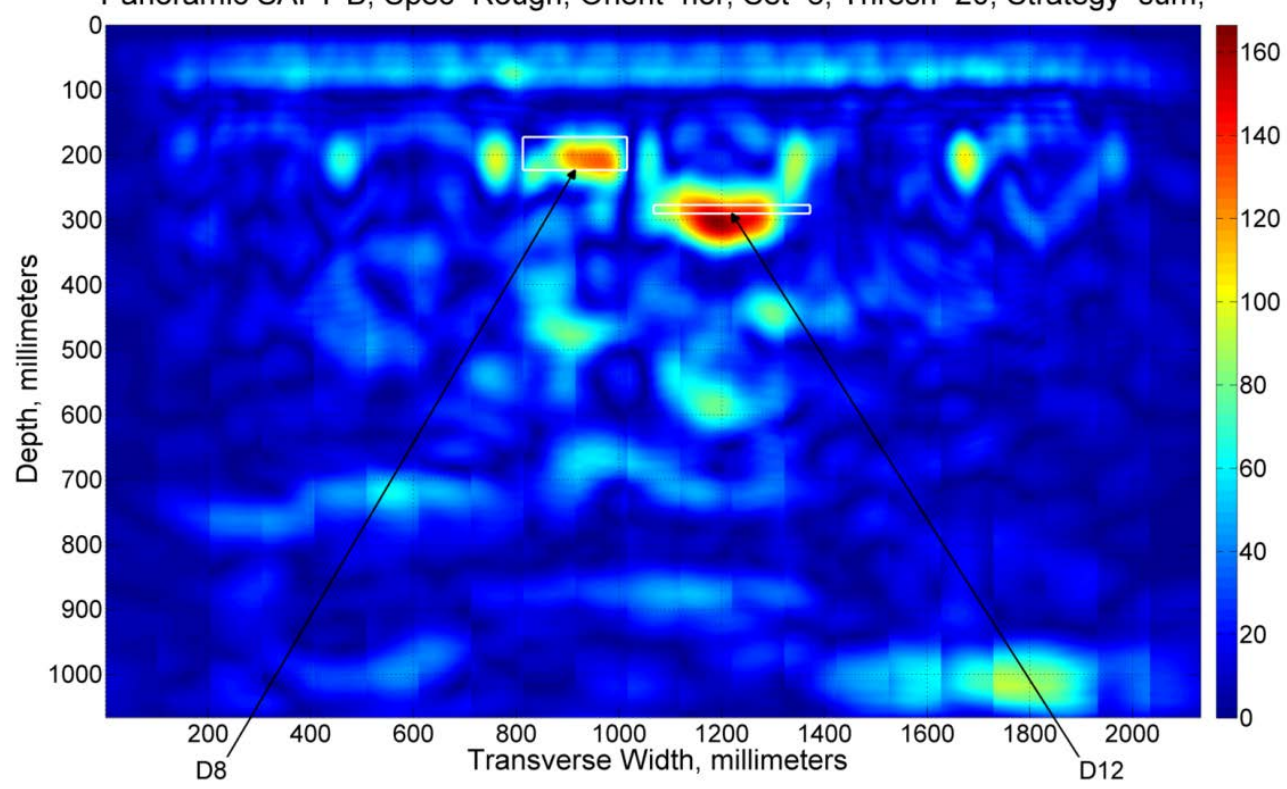

Fig. A. 39. Horizontal Set 8, Node 32.

Specimen: Thick, Depth: 1066.8mm (42in), AbsofHilbert -- Node $34(5,3), 46.875 \sim 62.5 \mathrm{kHz}$ Panoramic SAFT-B, Spec=Rough, Orient=hor, Set=8, Thresh=20, Strategy=sum,

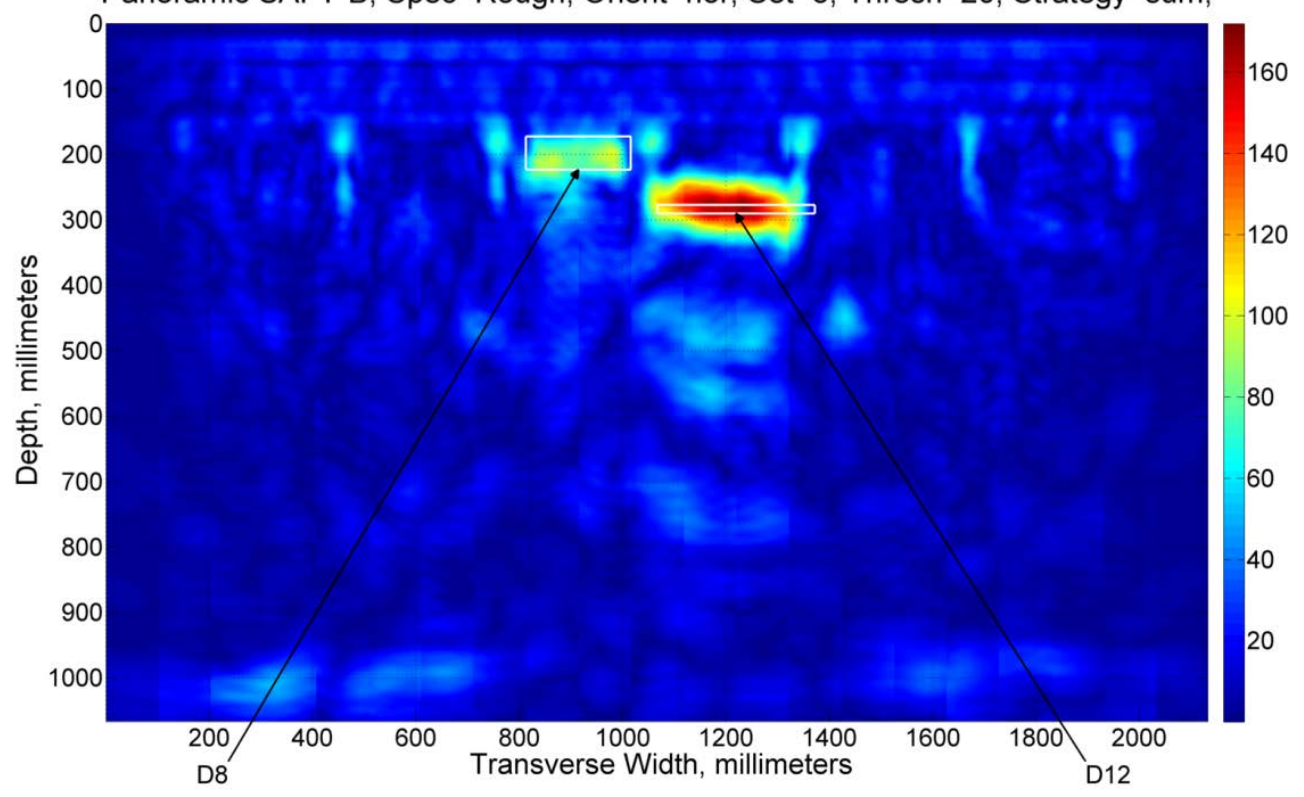

Fig. A. 40. Horizontal Set 8, Node 34. 
Specimen: Thick, Depth: 1066.8mm (42in), AbsofHilbert -- Node $0(0,0), 0 \sim 500 \mathrm{kHz}$ Panoramic SAFT-B, Spec=Rough, Orient=hor, Set=9, Thresh=20, Strategy=sum,

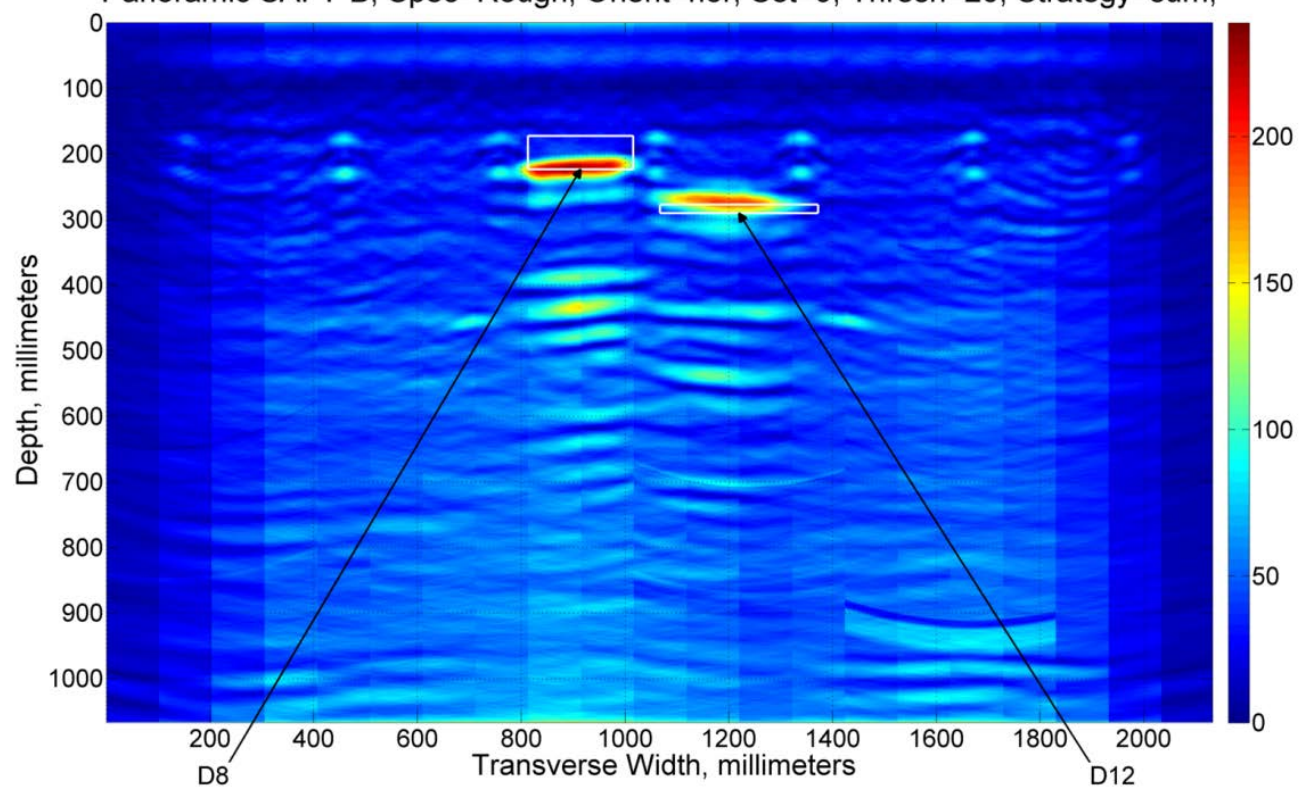

Fig. A. 41. Horizontal Set 9, Node 0.

Specimen: Thick, Depth: 1066.8mm (42in), AbsofHilbert -- Node $16(4,1), 31.25 \sim 62.5 \mathrm{kHz}$ Panoramic SAFT-B, Spec=Rough, Orient=hor, Set=9, Thresh=20, Strategy=sum,

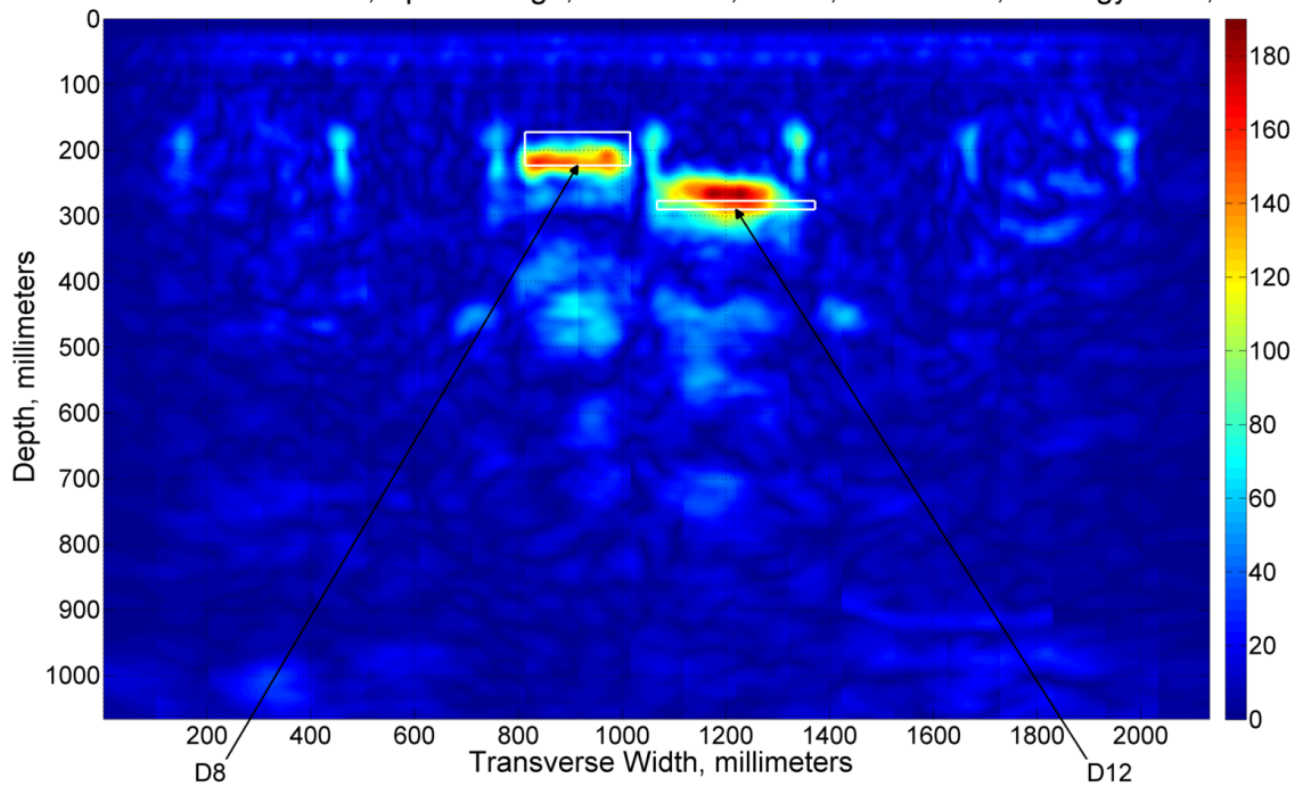

Fig. A. 42. Horizontal Set 9, Node 16. 


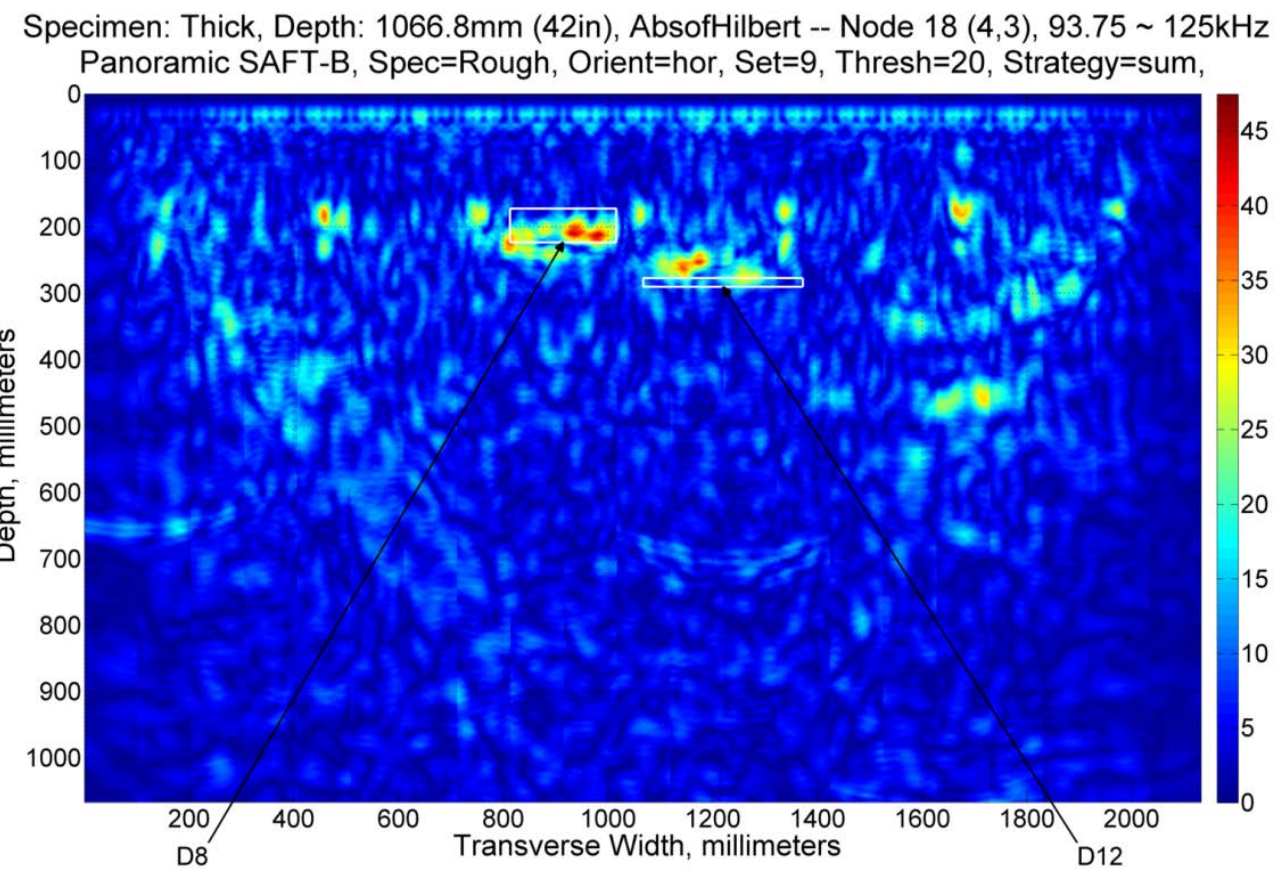

Fig. A. 43. Horizontal Set 9, Node 18.

Specimen: Thick, Depth: 1066.8mm (42in), AbsofHilbert -- Node $32(5,1), 15.625 \sim 31.25 \mathrm{kHz}$

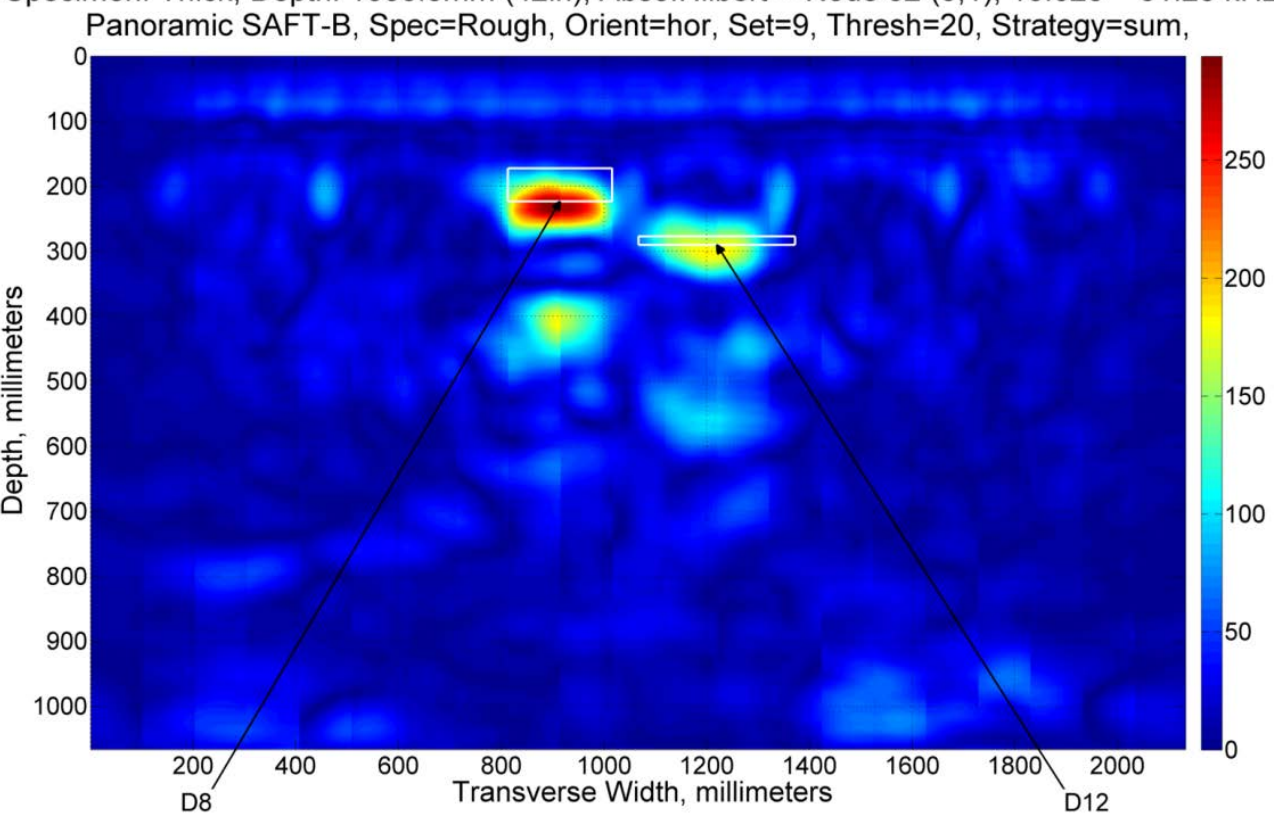

Fig. A. 44. Horizontal Set 9, Node 32. 


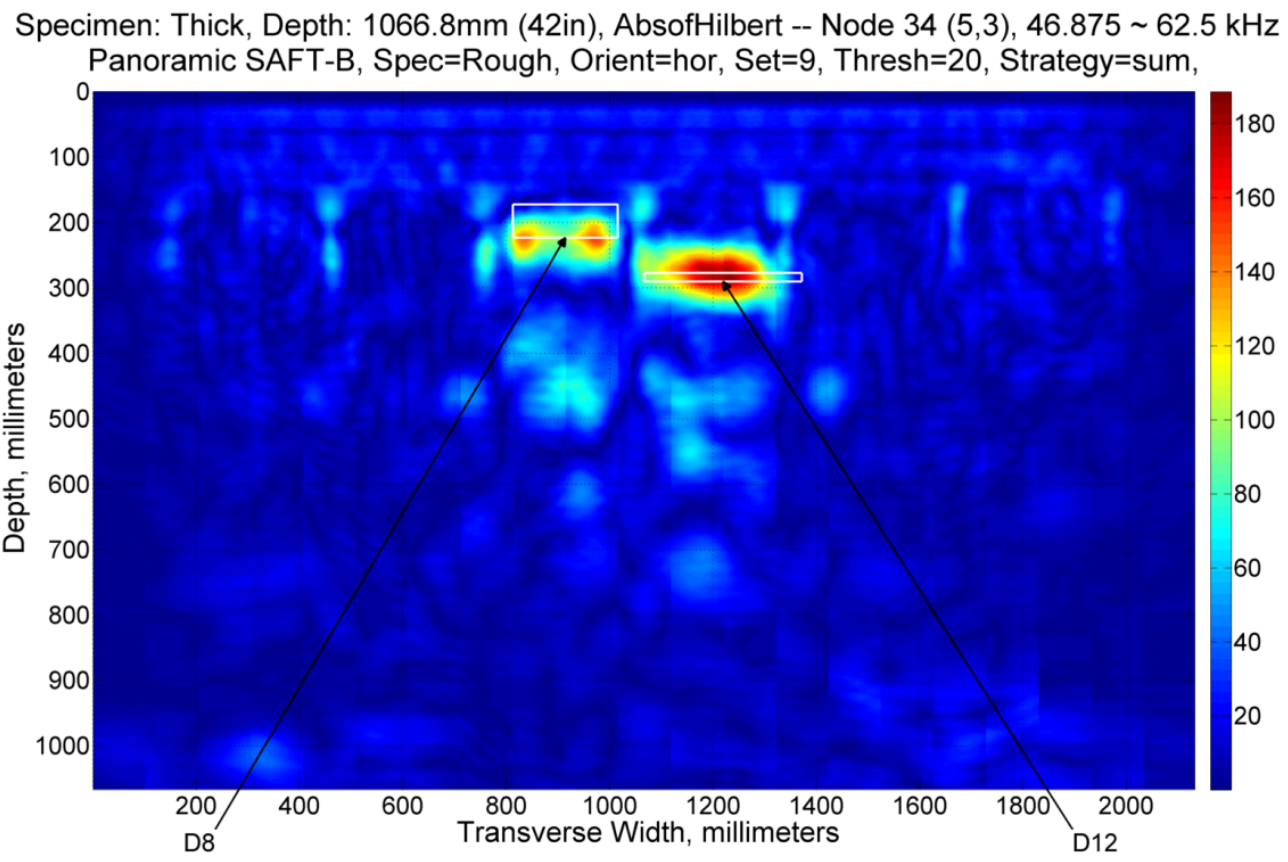

Fig. A. 45. Horizontal Set 9, Node 34.

\section{SET 10 - DEFECT 6, DEFECT 7}

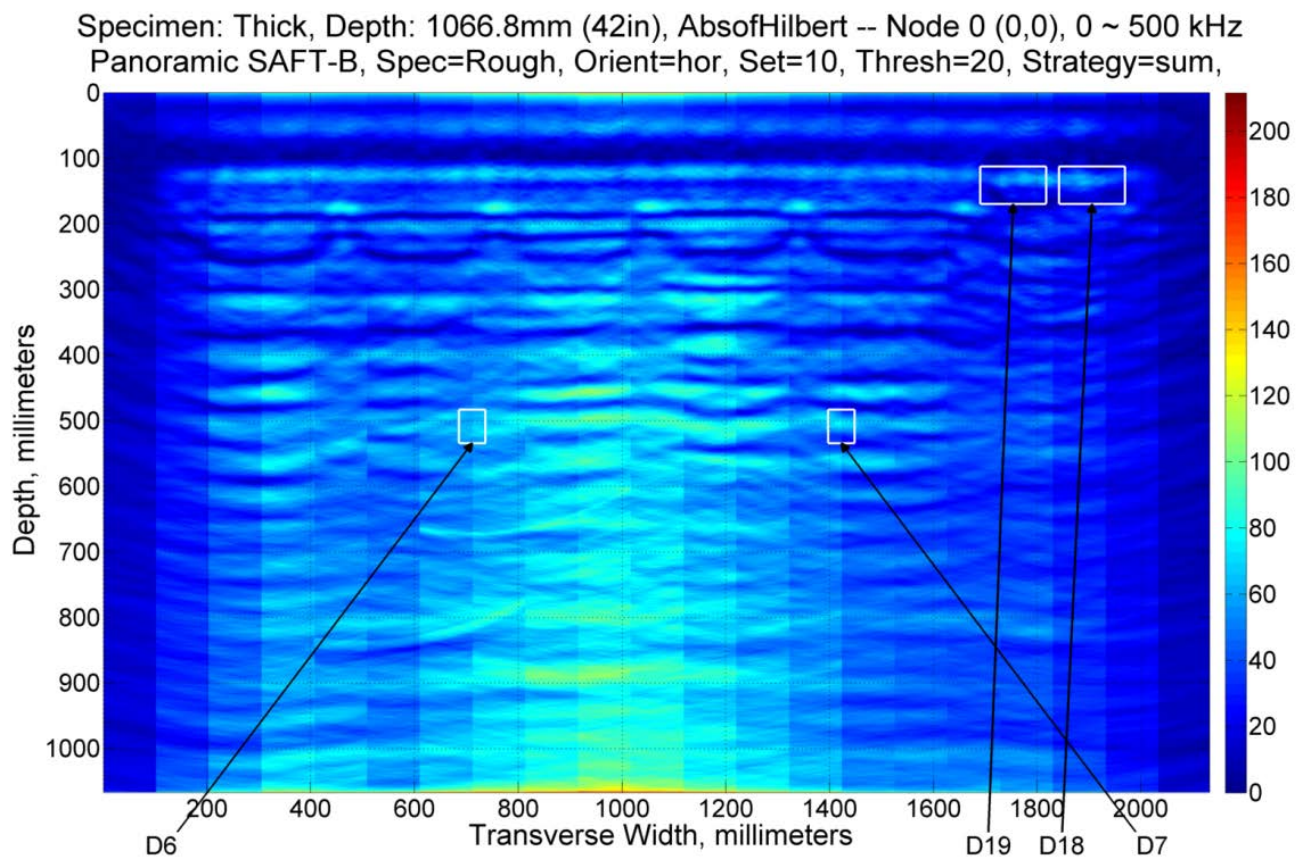

Fig. A. 46. Horizontal Set 10, Node 0. 


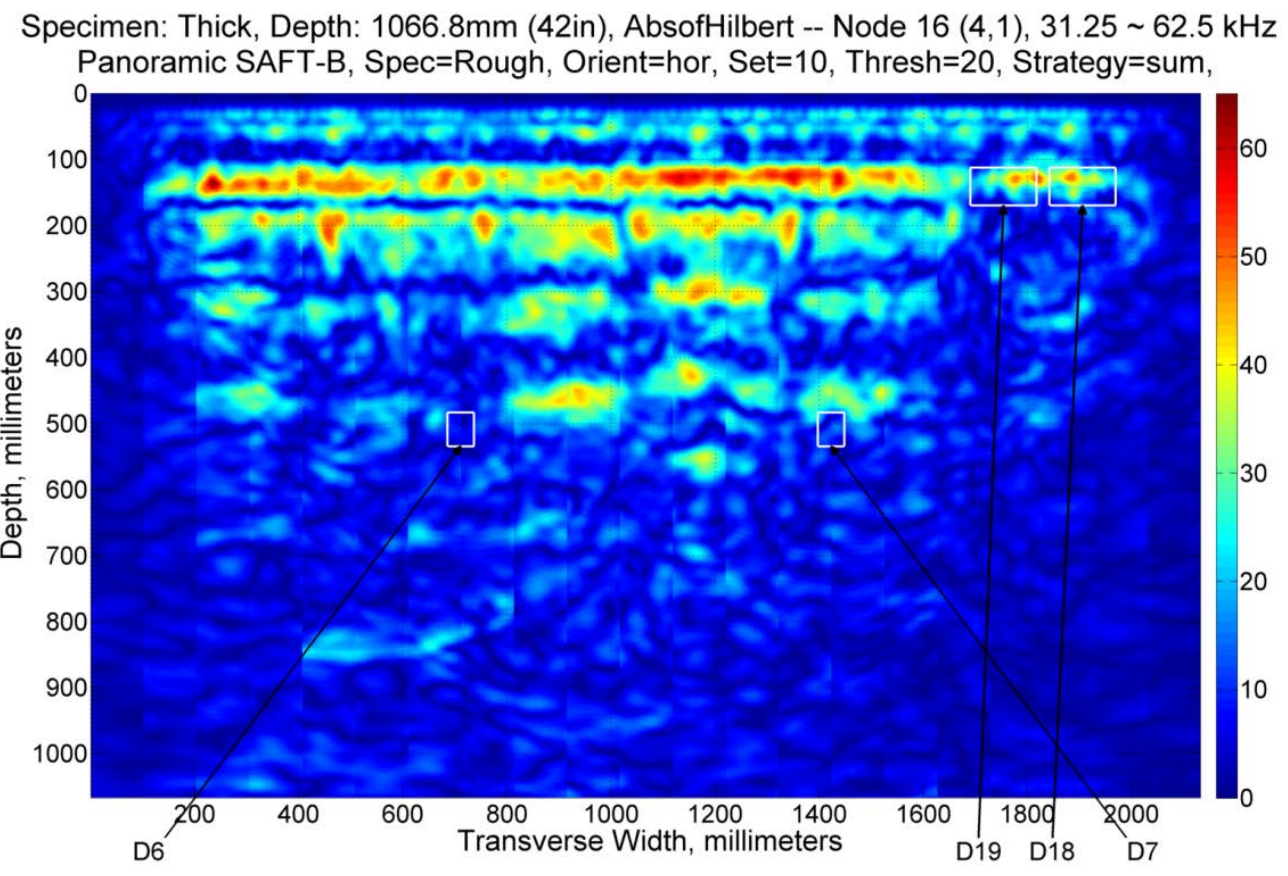

Fig. A. 47. Horizontal Set 10, Node 16.

Specimen: Thick, Depth: 1066.8mm (42in), AbsofHilbert -- Node $18(4,3), 93.75 \sim 125 \mathrm{kHz}$

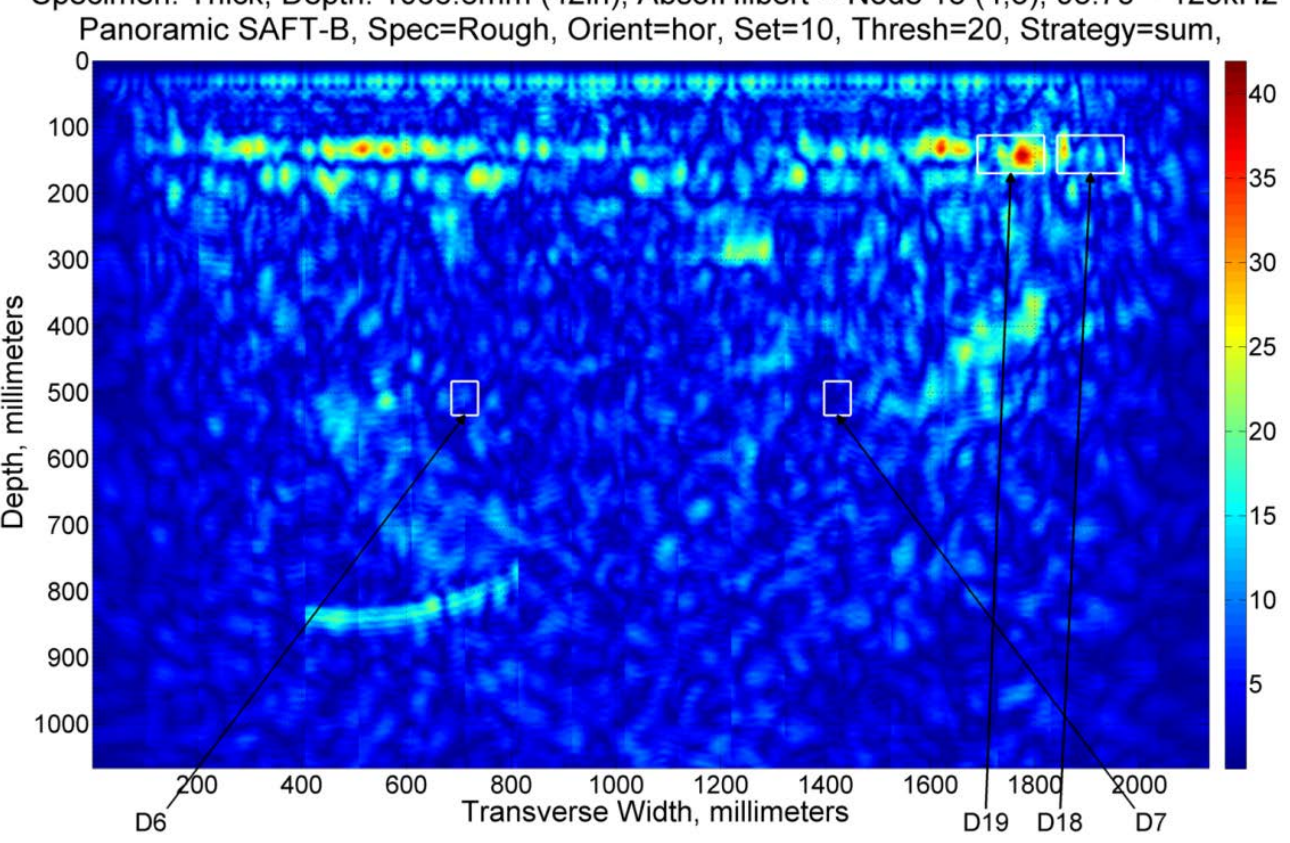

Fig. A. 48. Horizontal Set 10, Node 18. 


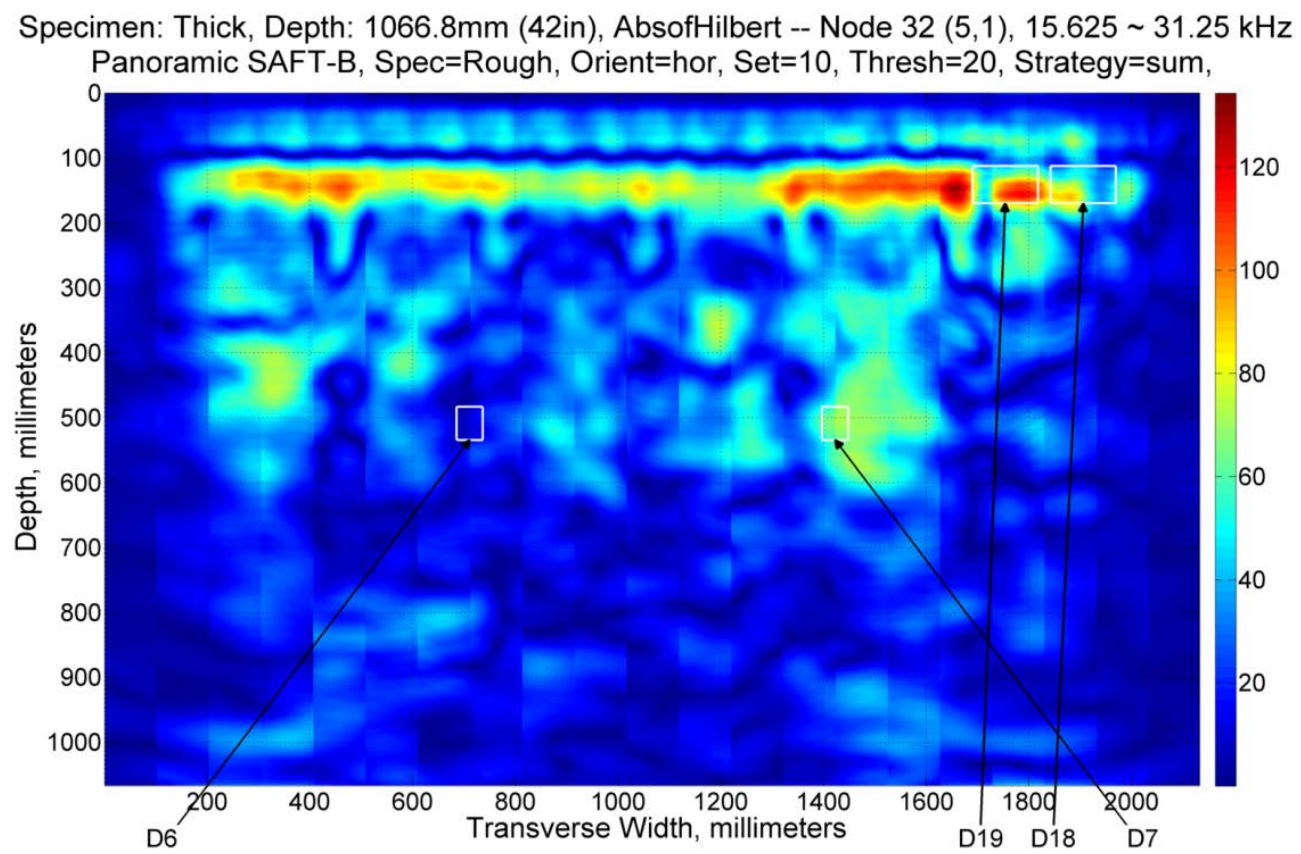

Fig. A. 49. Horizontal Set 10, Node 32.

Specimen: Thick, Depth: 1066.8mm (42in), AbsofHilbert -- Node $34(5,3), 46.875 \sim 62.5 \mathrm{kHz}$ Panoramic SAFT-B, Spec=Rough, Orient=hor, Set=10, Thresh=20, Strategy=sum,

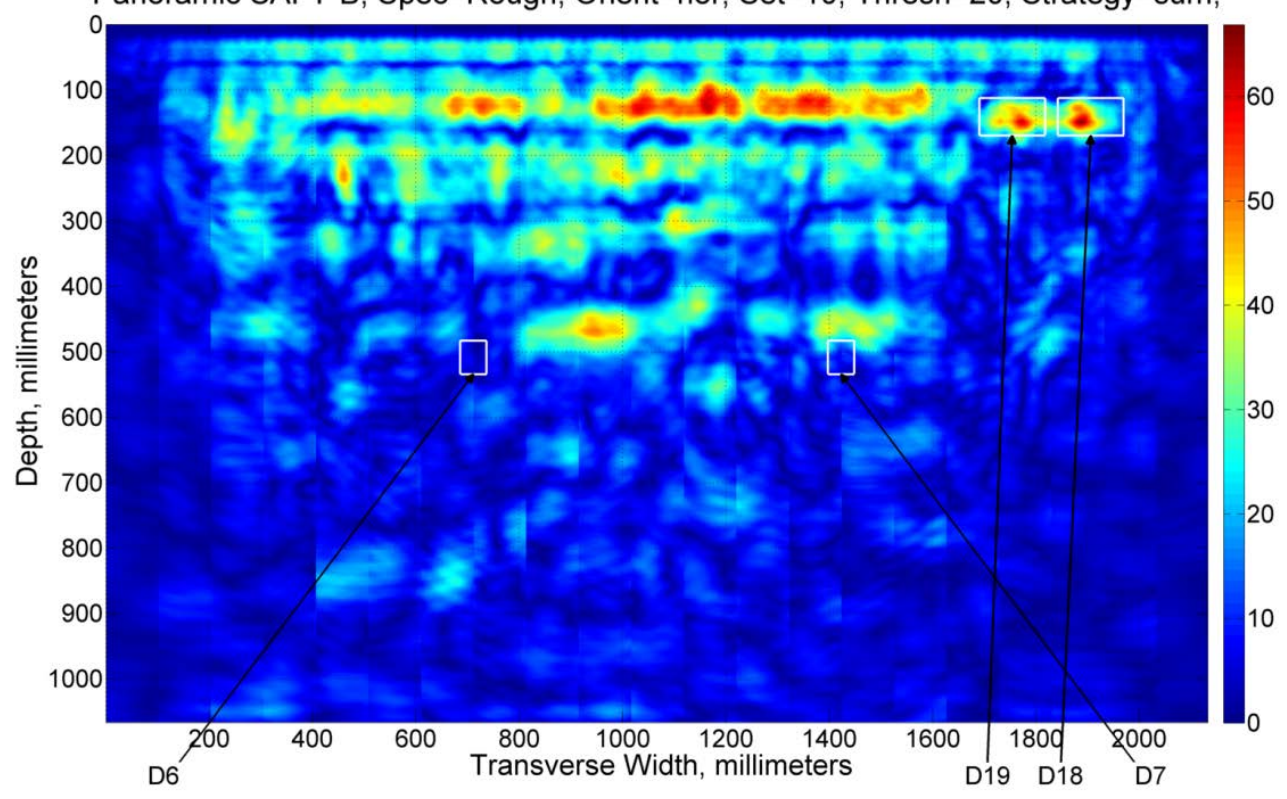

Fig. A. 50. Horizontal Set 10, Node 34. 


\section{SET 11 - DEFECT 9, DEFECT 13}

Specimen: Thick, Depth: 1066.8mm (42in), AbsofHilbert -- Node $0(0,0), 0 \sim 500 \mathrm{kHz}$ Panoramic SAFT-B, Spec=Rough, Orient=hor, Set=11, Thresh=20, Strategy=sum

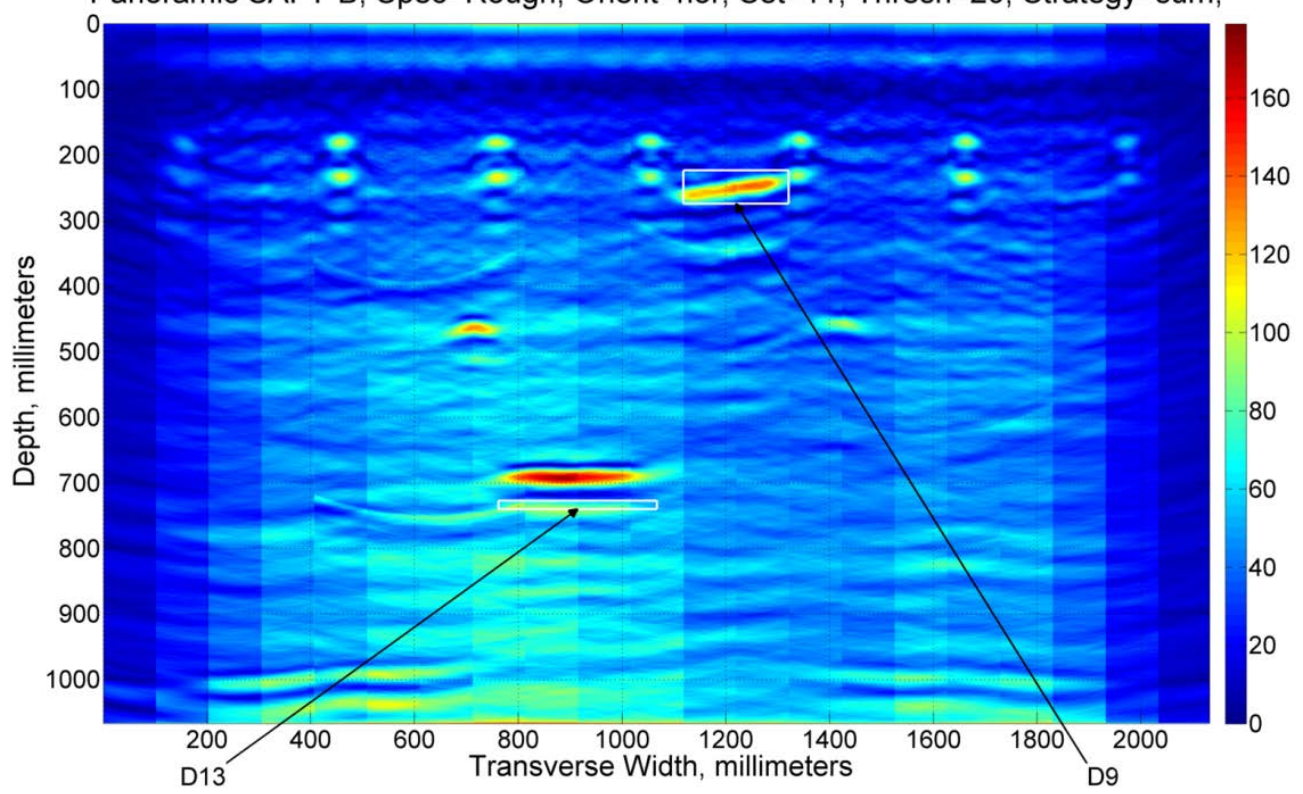

Fig. A. 51. Horizontal Set 11, Node 0.

Specimen: Thick, Depth: 1066.8mm (42in), AbsofHilbert -- Node $16(4,1), 31.25 \sim 62.5 \mathrm{kHz}$ Panoramic SAFT-B, Spec=Rough, Orient=hor, Set=11, Thresh=20, Strategy=sum,

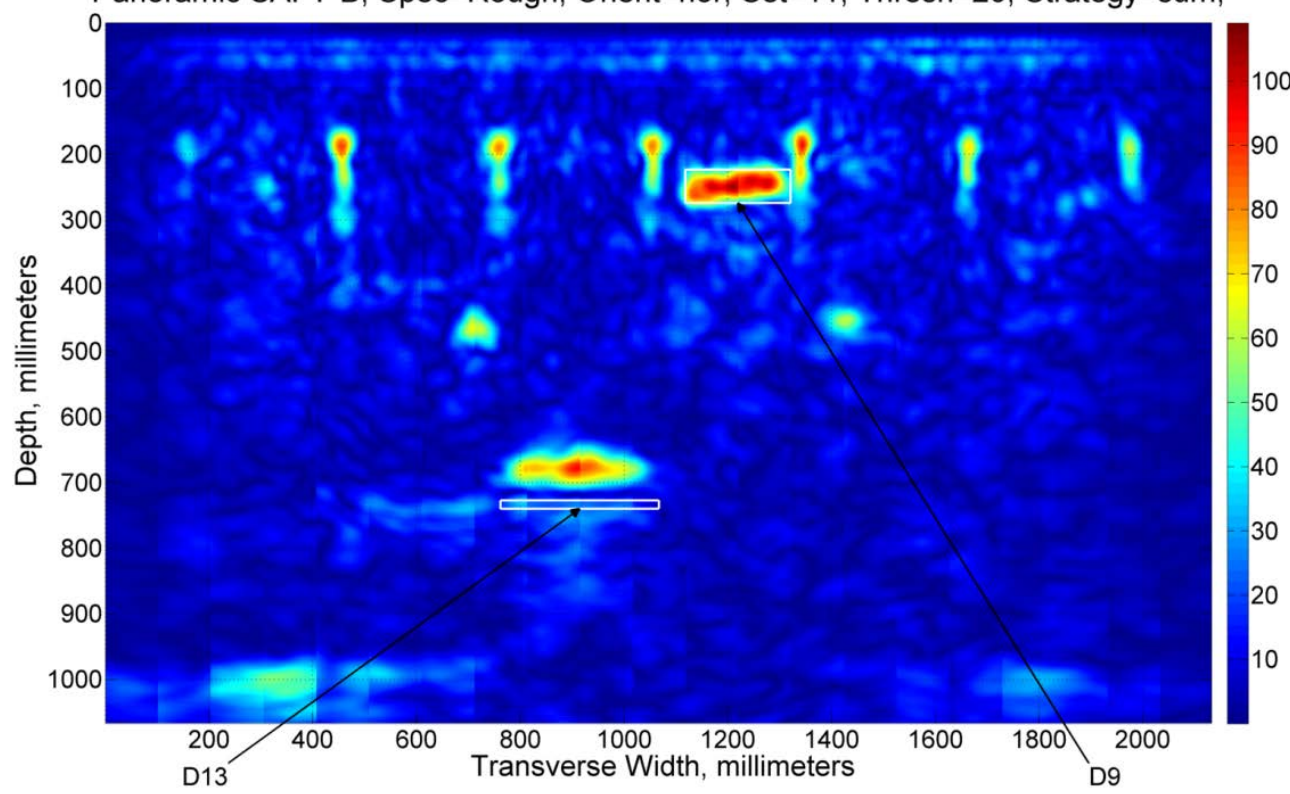

Fig. A. 52. Horizontal Set 11, Node 16. 
Specimen: Thick, Depth: 1066.8mm (42in), AbsofHilbert -- Node $18(4,3), 93.75 \sim 125 \mathrm{kHz}$ Panoramic SAFT-B, Spec=Rough, Orient=hor, Set=11, Thresh=20, Strategy=sum,

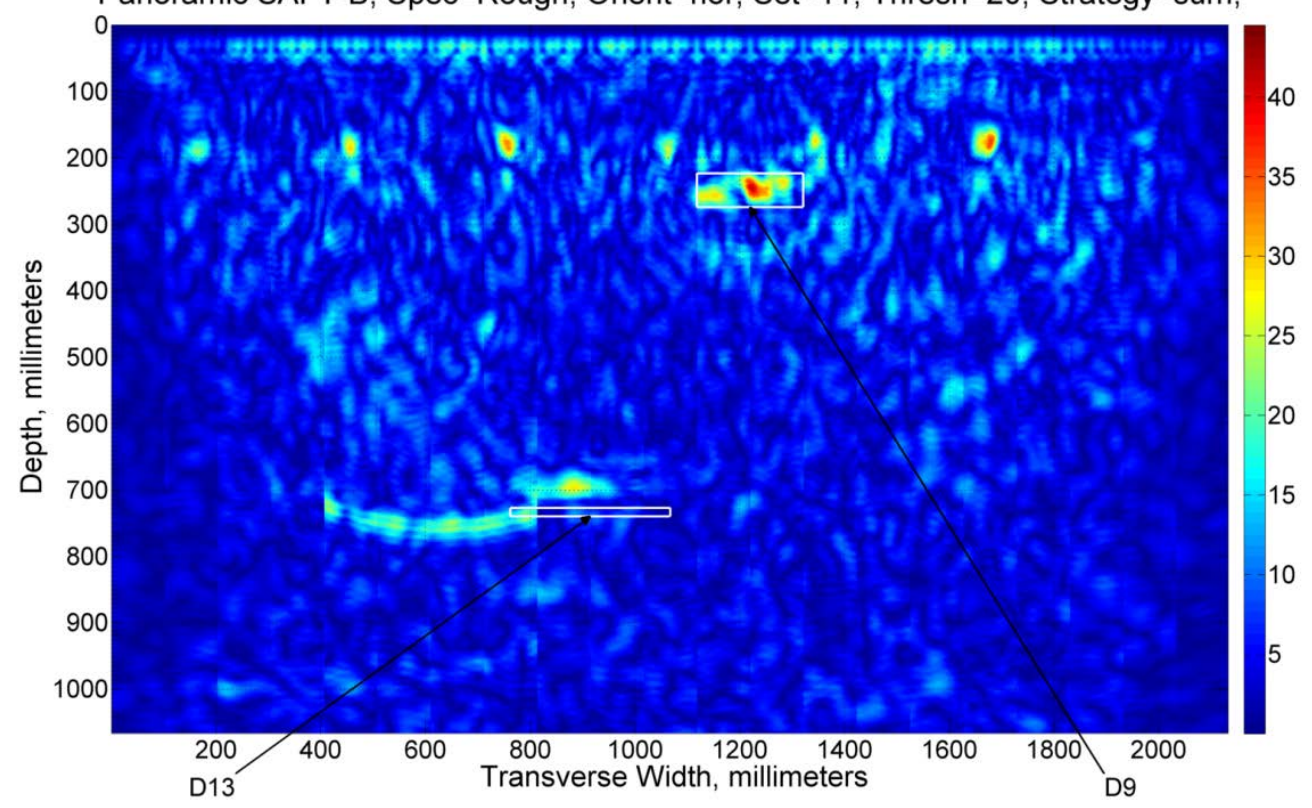

Fig. A. 53. Horizontal Set 11, Node 18.

Specimen: Thick, Depth: 1066.8mm (42in), AbsofHilbert -- Node $32(5,1), 15.625 \sim 31.25 \mathrm{kHz}$ Panoramic SAFT-B, Spec=Rough, Orient=hor, Set=11, Thresh=20, Strategy=sum,

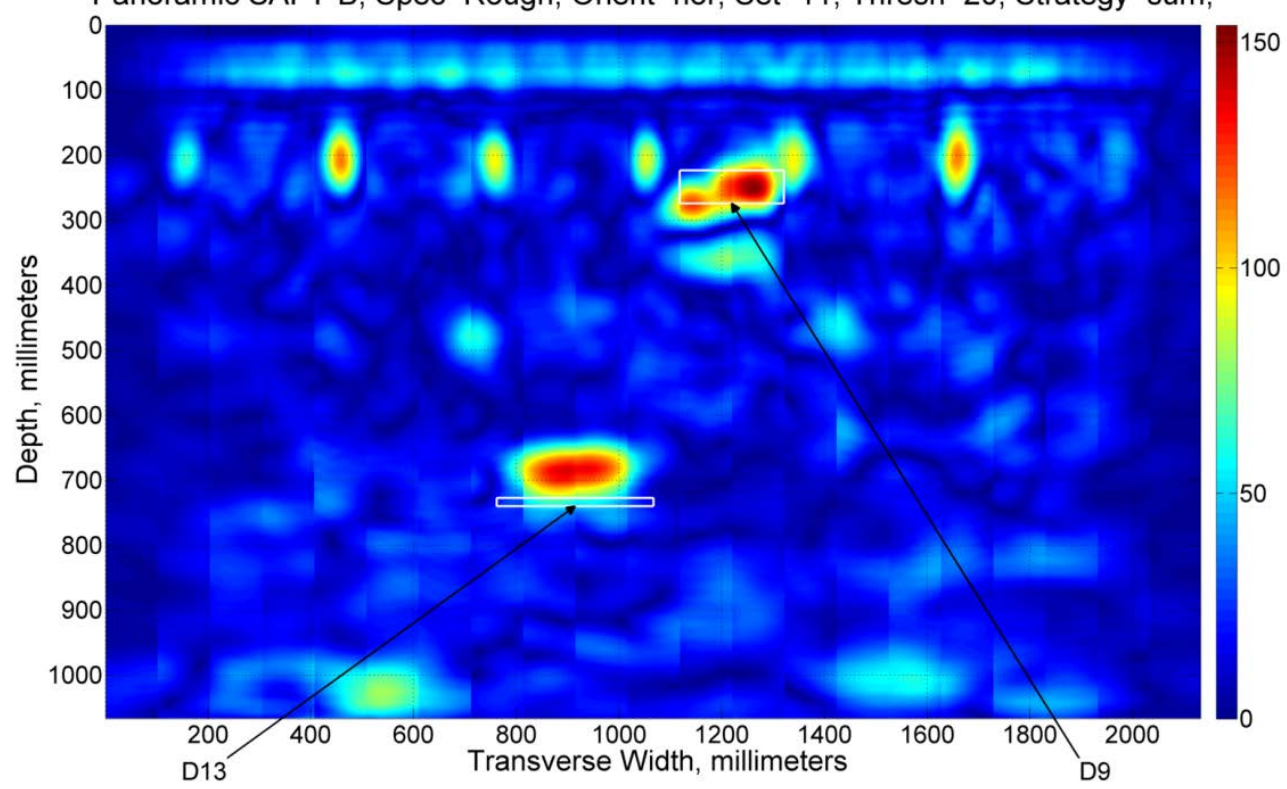

Fig. A. 54. Horizontal Set 11, Node 32. 


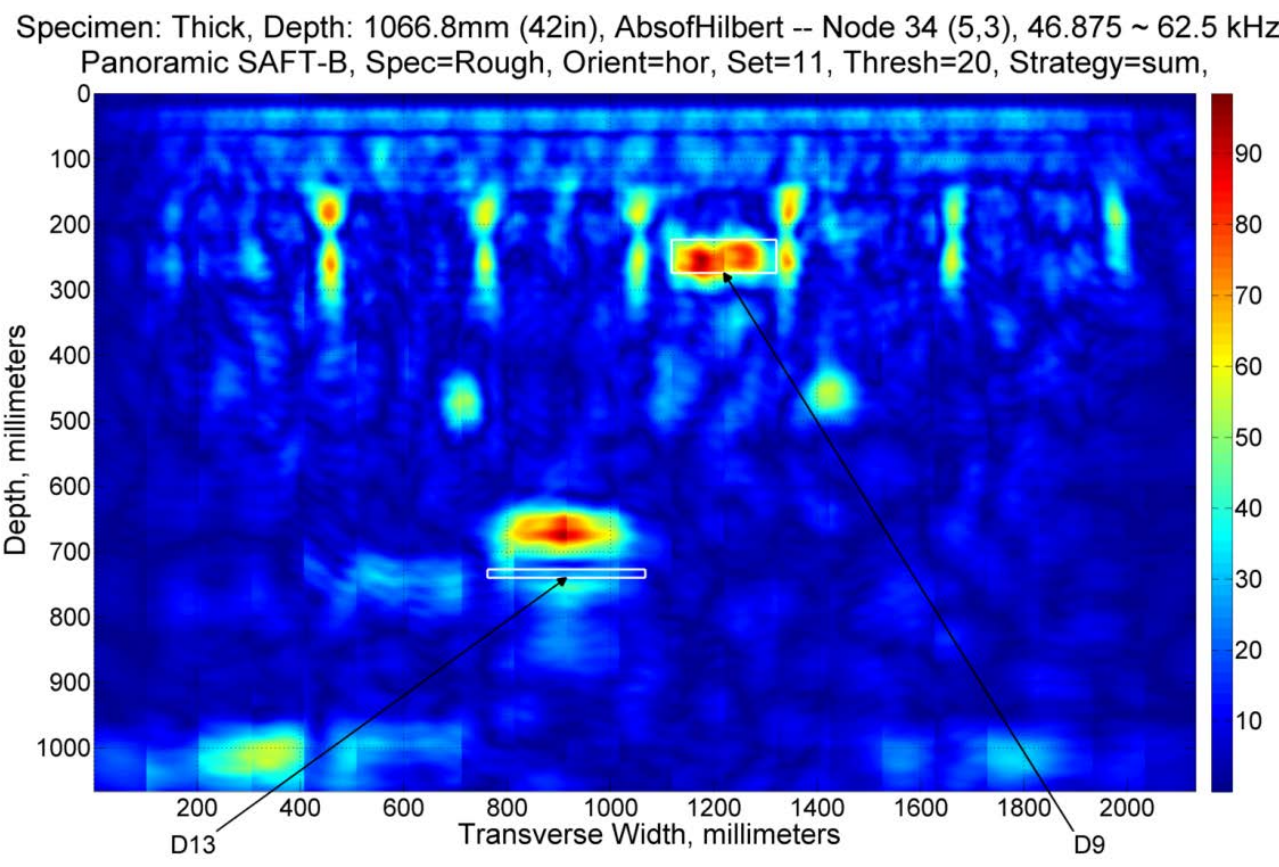

Fig. A. 55. Horizontal Set 11, Node 34.

\section{SET 12 - DEFECT 9, DEFECT 13}

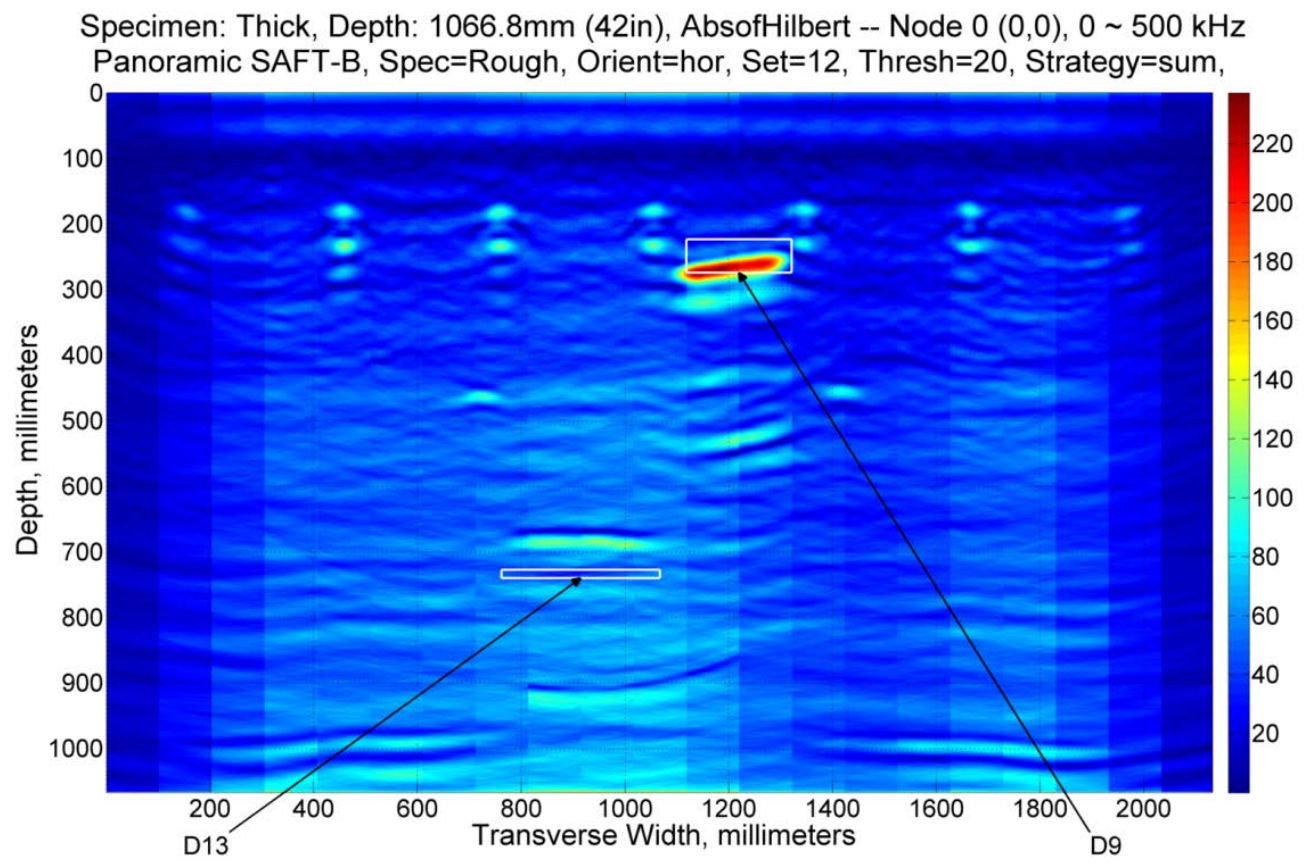

Fig. A. 56. Horizontal Set 12, Node 0. 


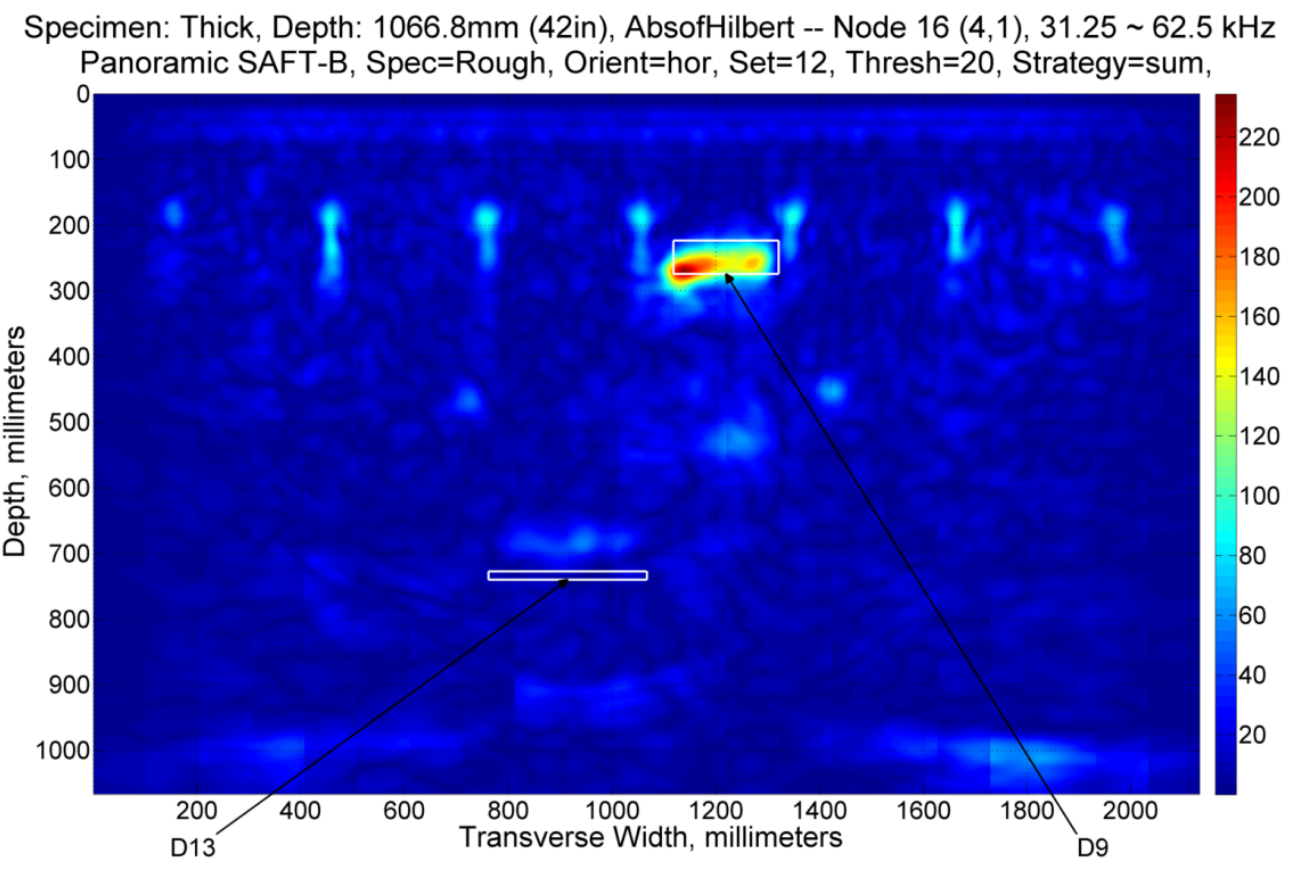

Fig. A. 57. Horizontal Set 12, Node 16.

Specimen: Thick, Depth: 1066.8mm (42in), AbsofHilbert -- Node $18(4,3), 93.75 \sim 125 \mathrm{kHz}$ Panoramic SAFT-B, Spec=Rough, Orient=hor, Set=12, Thresh=20, Strategy=sum,

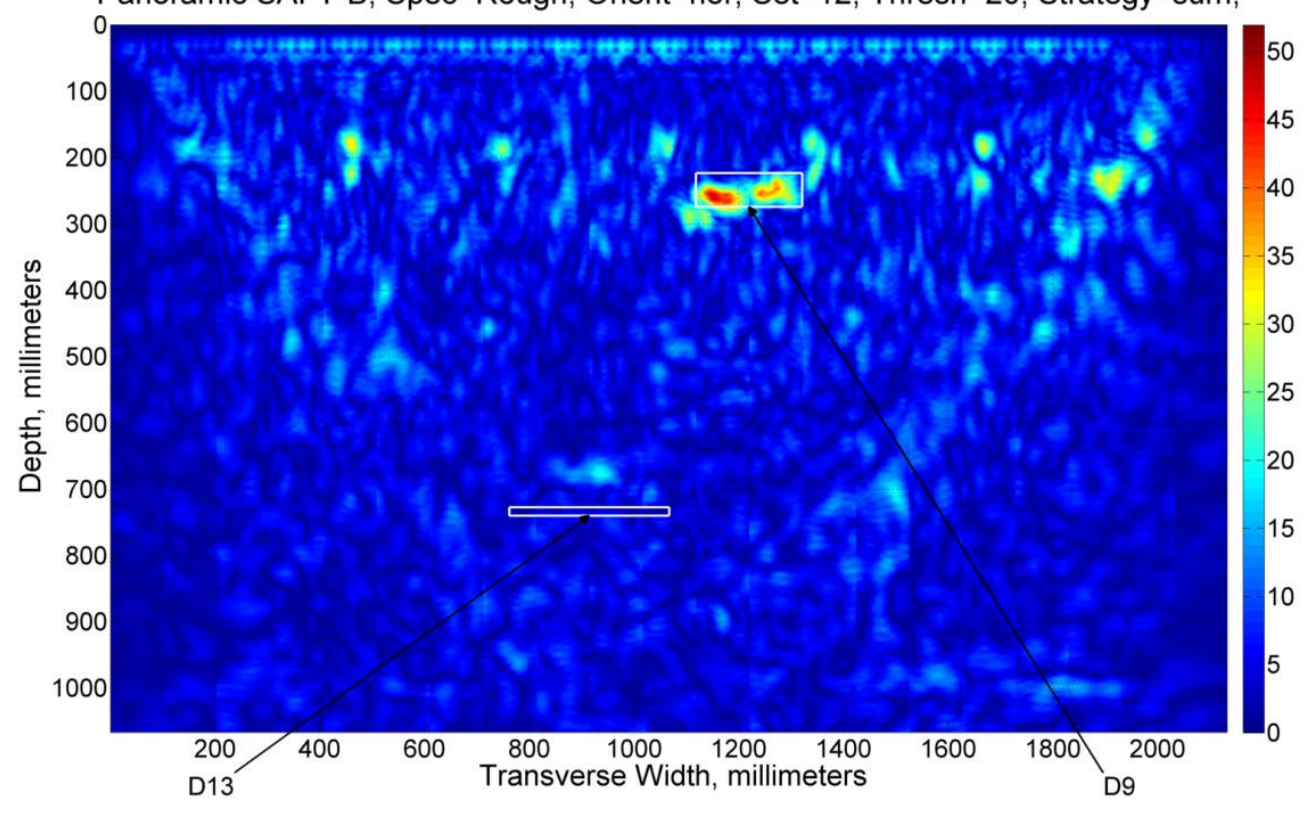

Fig. A. 58. Horizontal Set 12, Node 18. 


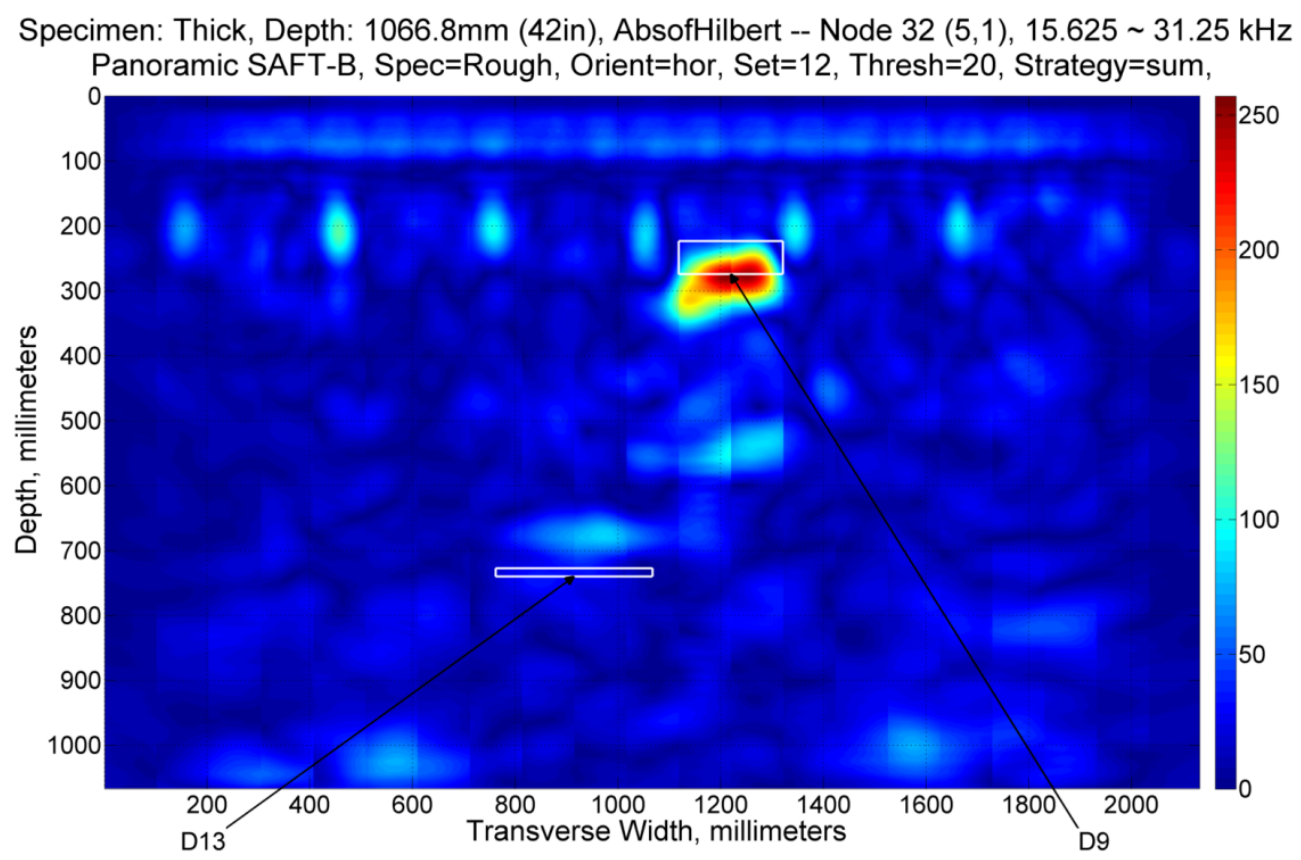

Fig. A. 59. Horizontal Set 12, Node 32.

Specimen: Thick, Depth: 1066.8mm (42in), AbsofHilbert -- Node 34 (5,3), $46.875 \sim 62.5 \mathrm{kHz}$ Panoramic SAFT-B, Spec=Rough, Orient=hor, Set=12, Thresh=20, Strategy=sum,

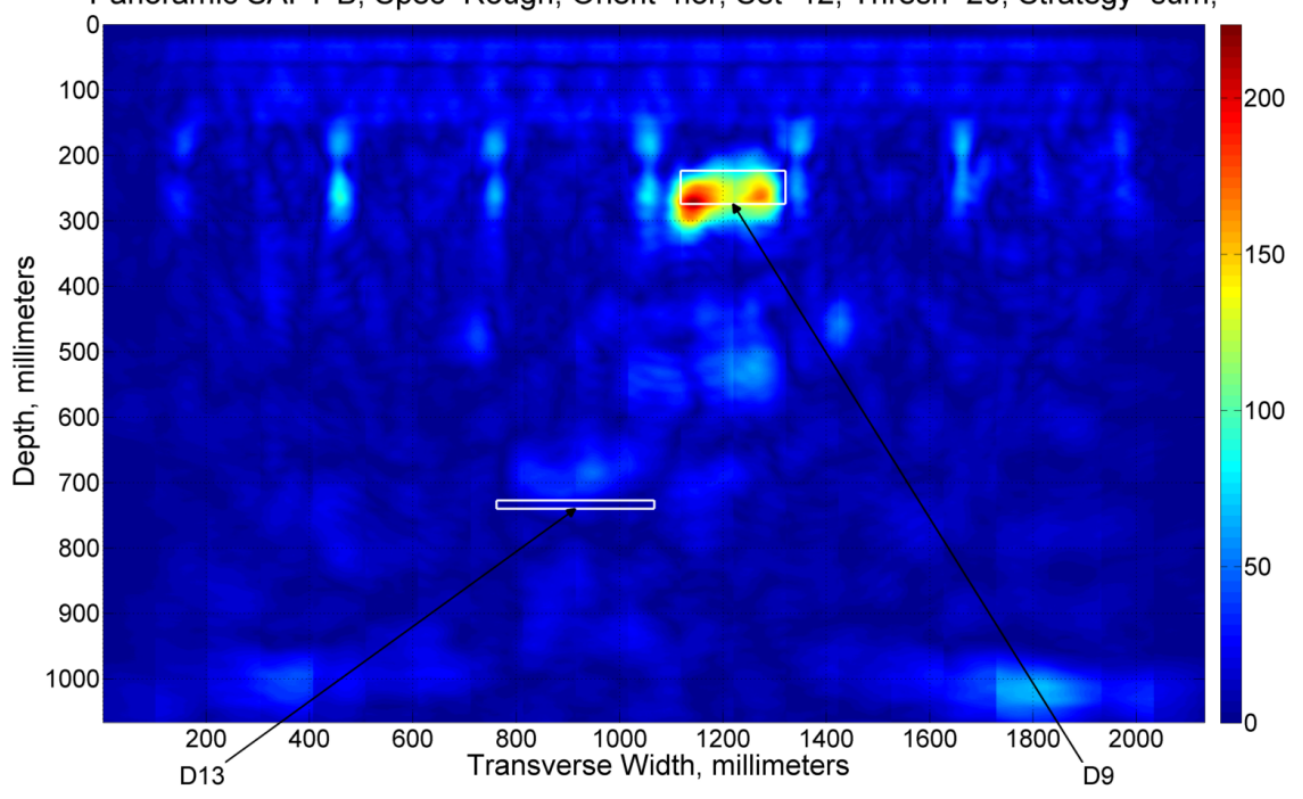

Fig. A. 60. Horizontal Set 12, Node 34 . 


\section{SET 13 - DEFECT 5}

Specimen: Thick, Depth: 1066.8mm (42in), AbsofHilbert -- Node $0(0,0), 0 \sim 500 \mathrm{kHz}$ Panoramic SAFT-B, Spec=Rough, Orient=hor, Set=13, Thresh=20, Strategy=sum

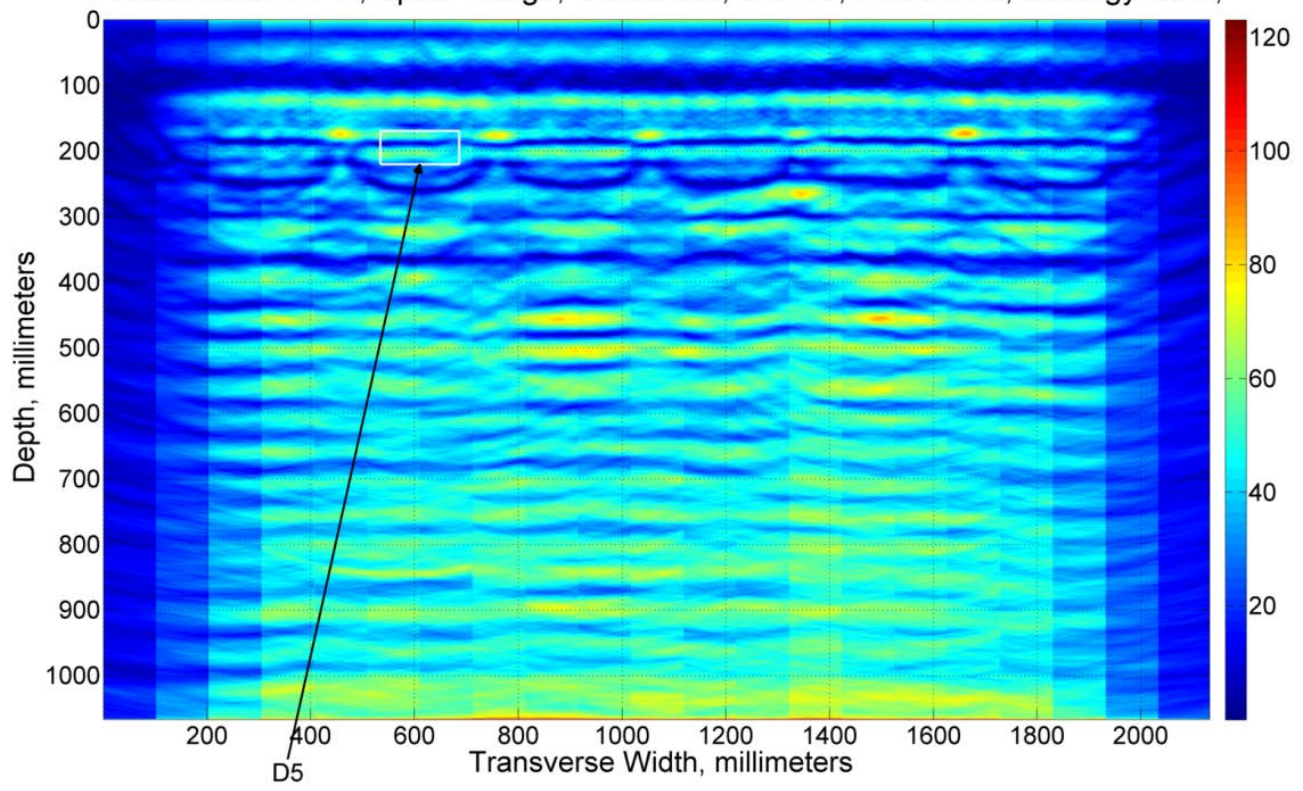

Fig. A. 61. Horizontal Set 13, Node 0.

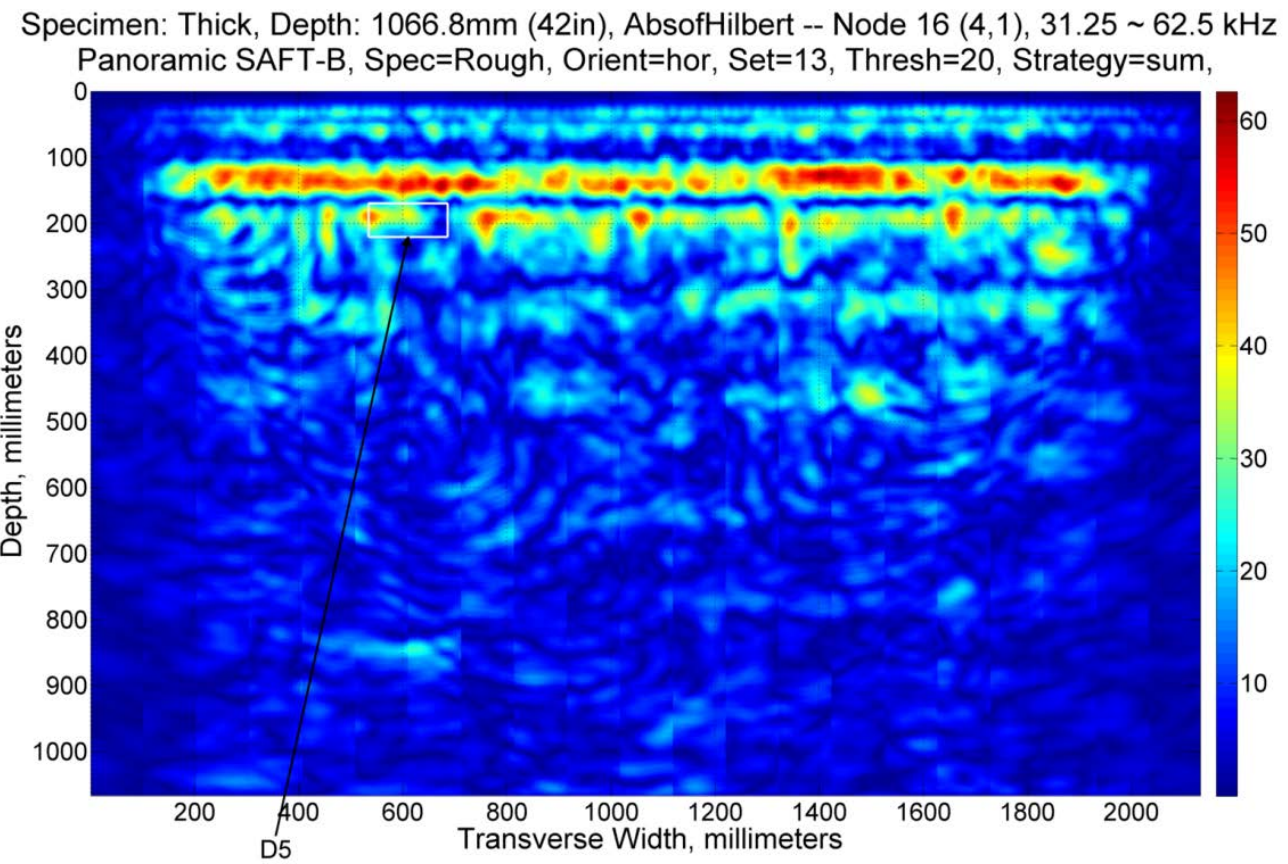

Fig. A. 62. Horizontal Set 13, Node 16. 
Specimen: Thick, Depth: 1066.8mm (42in), AbsofHilbert -- Node $18(4,3), 93.75 \sim 125 \mathrm{kHz}$ Panoramic SAFT-B, Spec=Rough, Orient=hor, Set=13, Thresh=20, Strategy=sum,

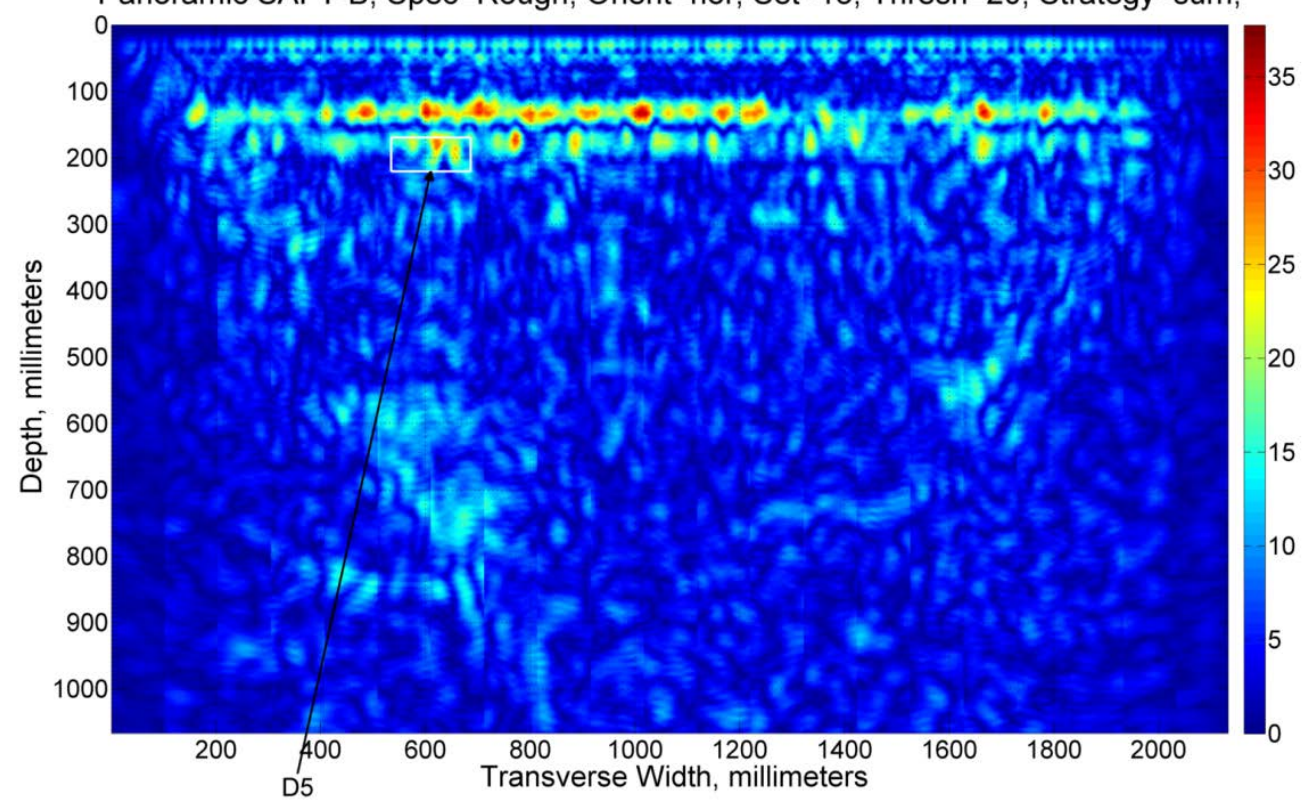

Fig. A. 63. Horizontal Set 13, Node 18.

Specimen: Thick, Depth: 1066.8mm (42in), AbsofHilbert -- Node $32(5,1), 15.625 \sim 31.25 \mathrm{kHz}$ Panoramic SAFT-B, Spec=Rough, Orient=hor, Set=13, Thresh=20, Strategy=sum,

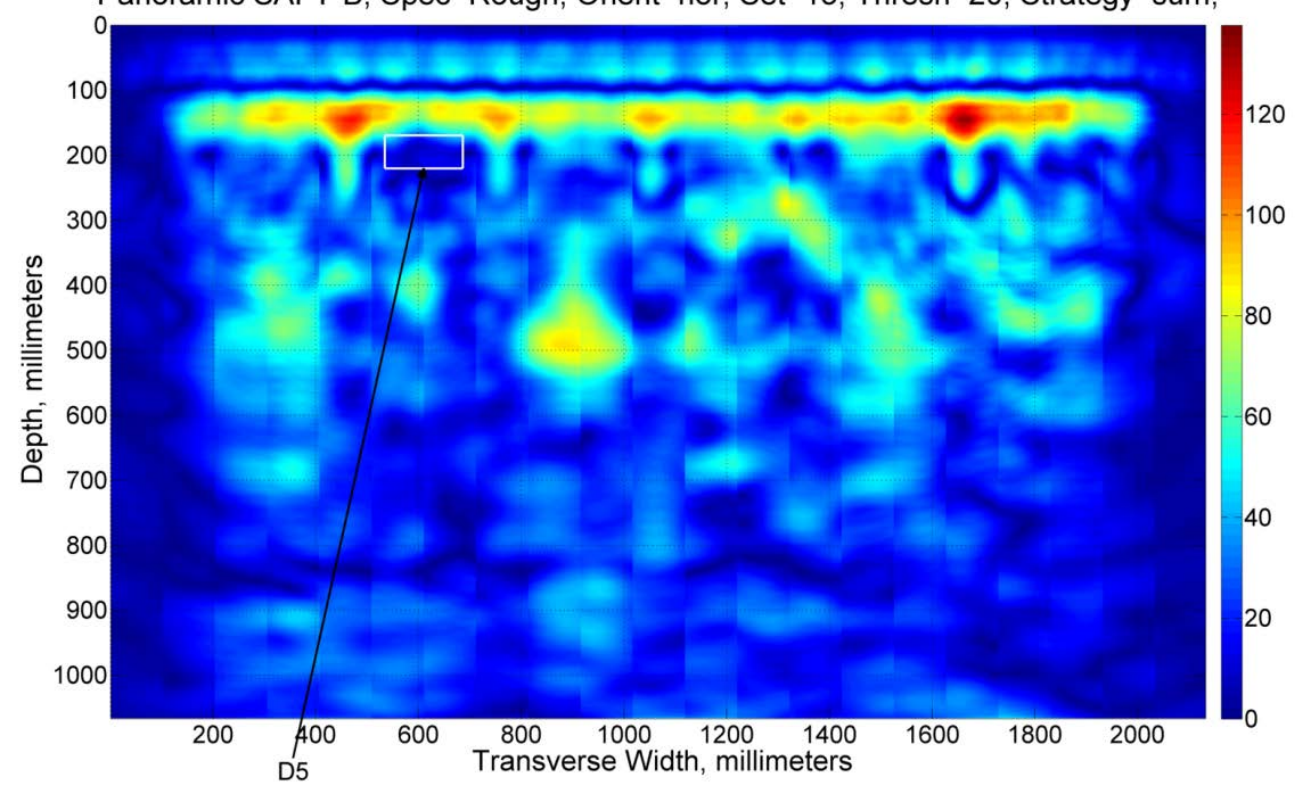

Fig. A. 64. Horizontal Set 13, Node 32. 


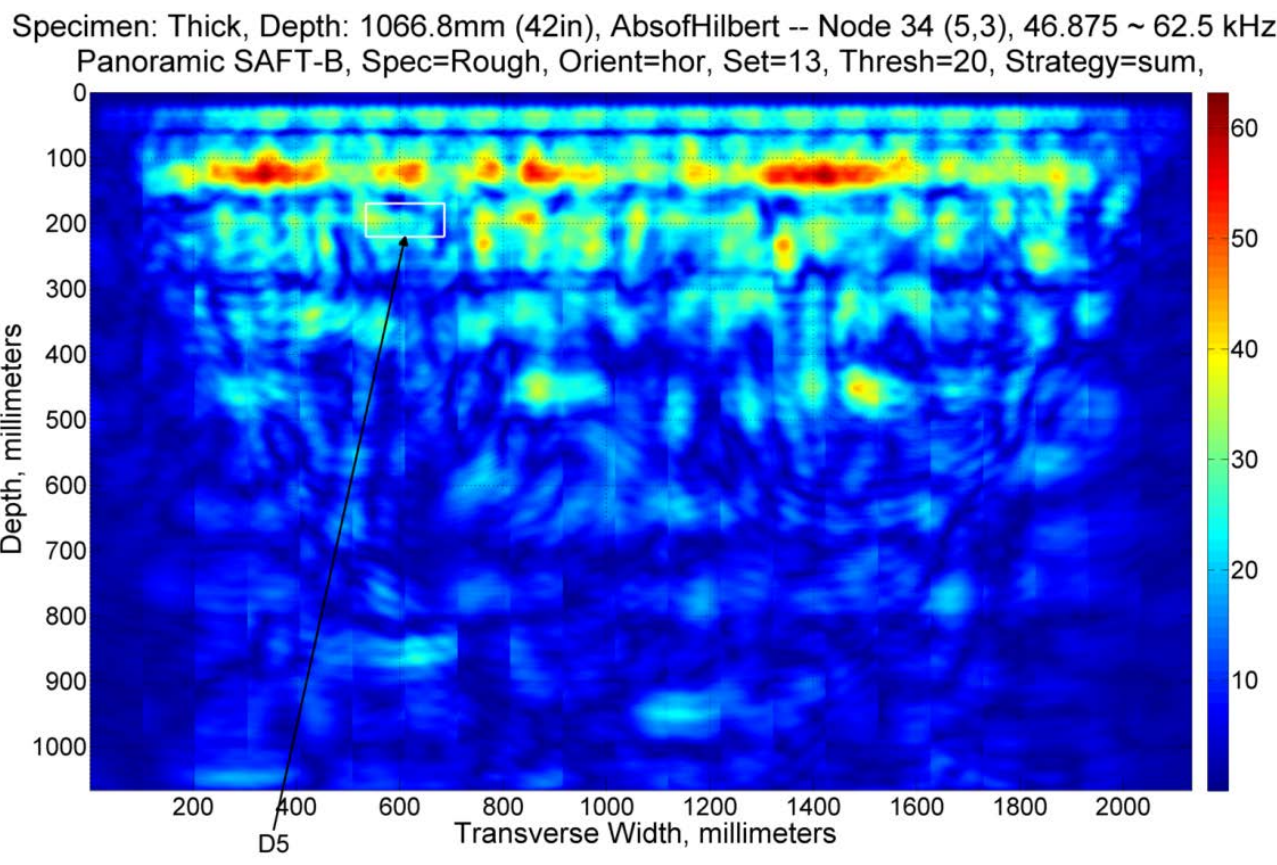

Fig. A. 65. Horizontal Set 13, Node 34.

\section{SET 14 - DEFECT 3, DEFECT 17}

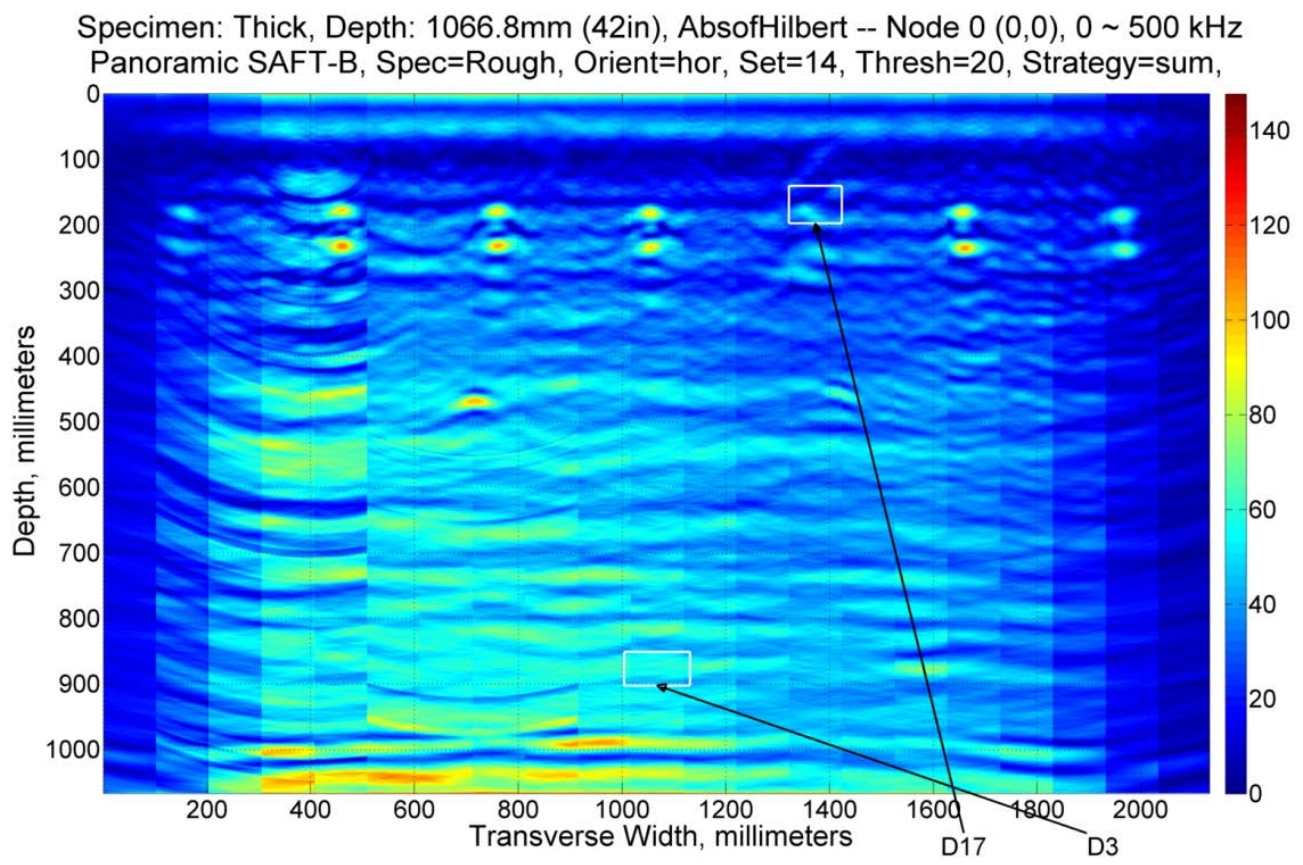

Fig. A. 66. Horizontal Set 14, Node 0. 


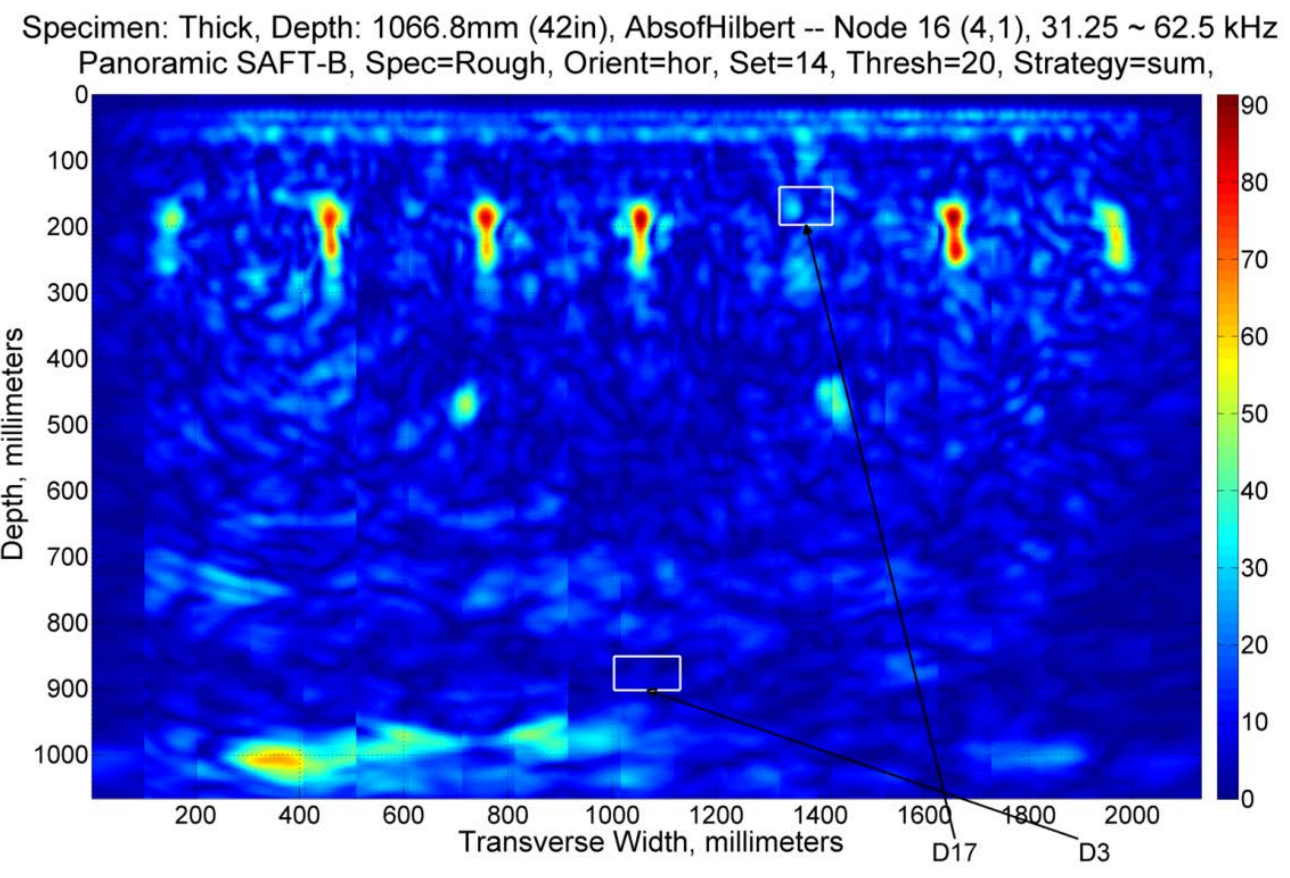

Fig. A. 67. Horizontal Set 14, Node 16.

Specimen: Thick, Depth: 1066.8mm (42in), AbsofHilbert -- Node $18(4,3), 93.75 \sim 125 \mathrm{kHz}$ Panoramic SAFT-B, Spec=Rough, Orient=hor, Set=14, Thresh=20, Strategy=sum,

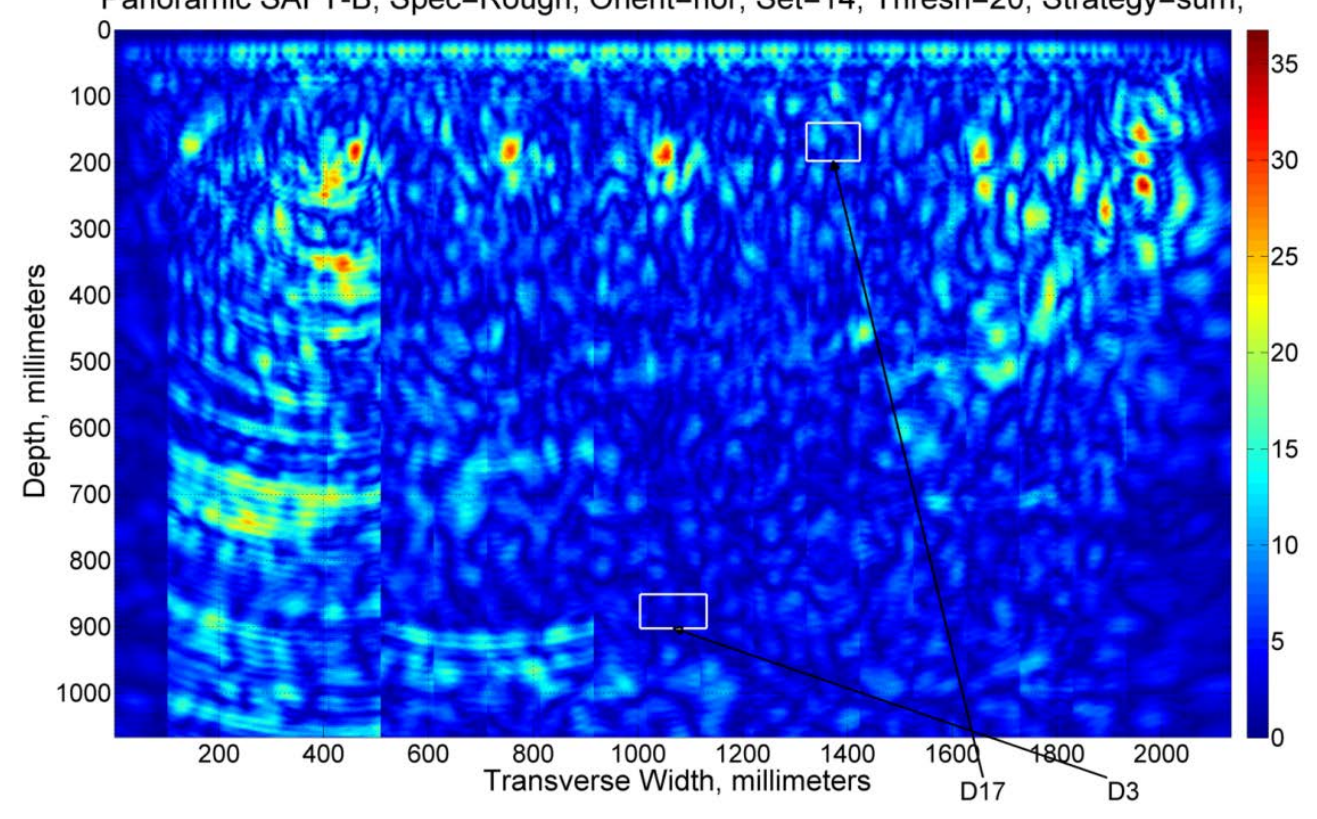

Fig. A. 68. Horizontal Set 14, Node 18. 


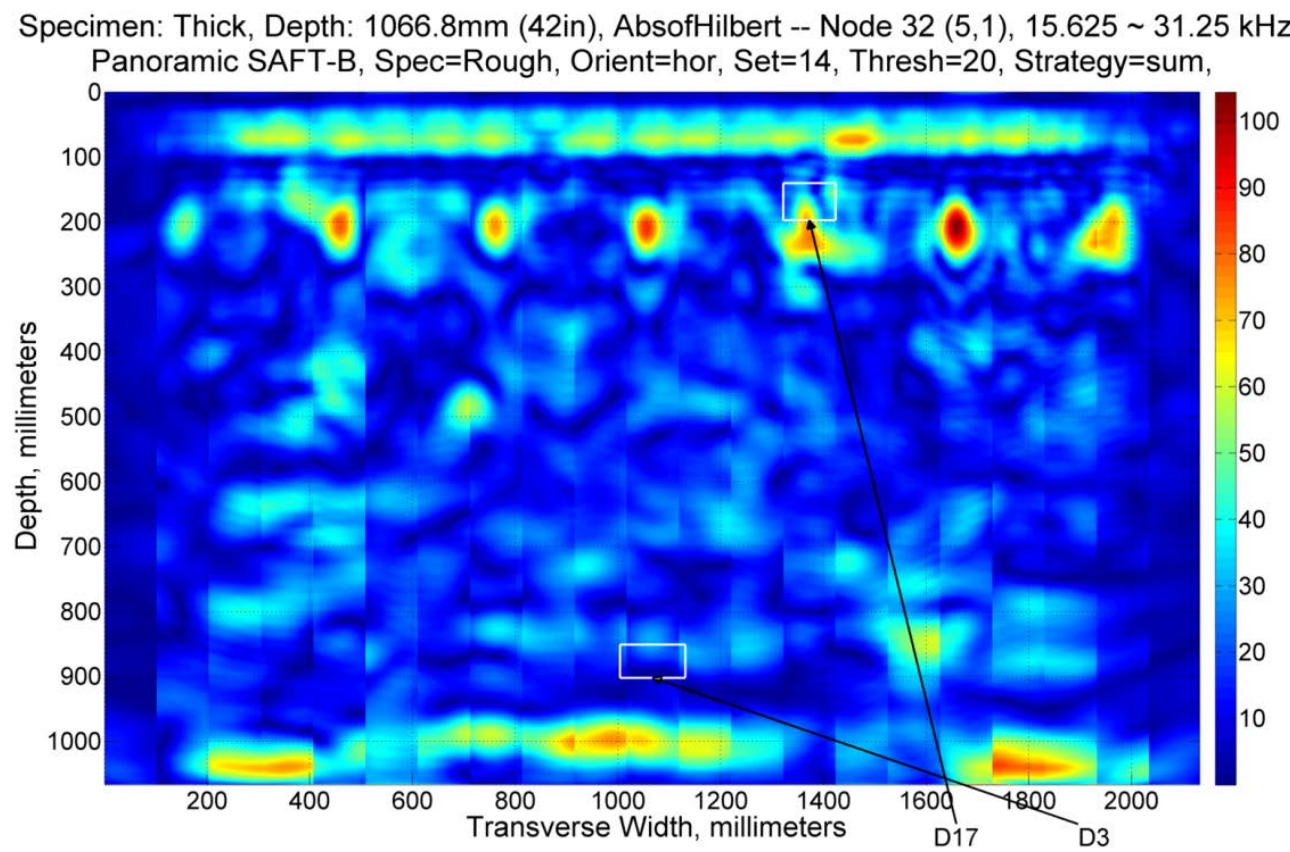

Fig. A. 69. Horizontal Set 14, Node 32.

Specimen: Thick, Depth: 1066.8mm (42in), AbsofHilbert -- Node $34(5,3), 46.875 \sim 62.5 \mathrm{kHz}$ Panoramic SAFT-B, Spec=Rough, Orient=hor, Set=14, Thresh=20, Strategy=sum,

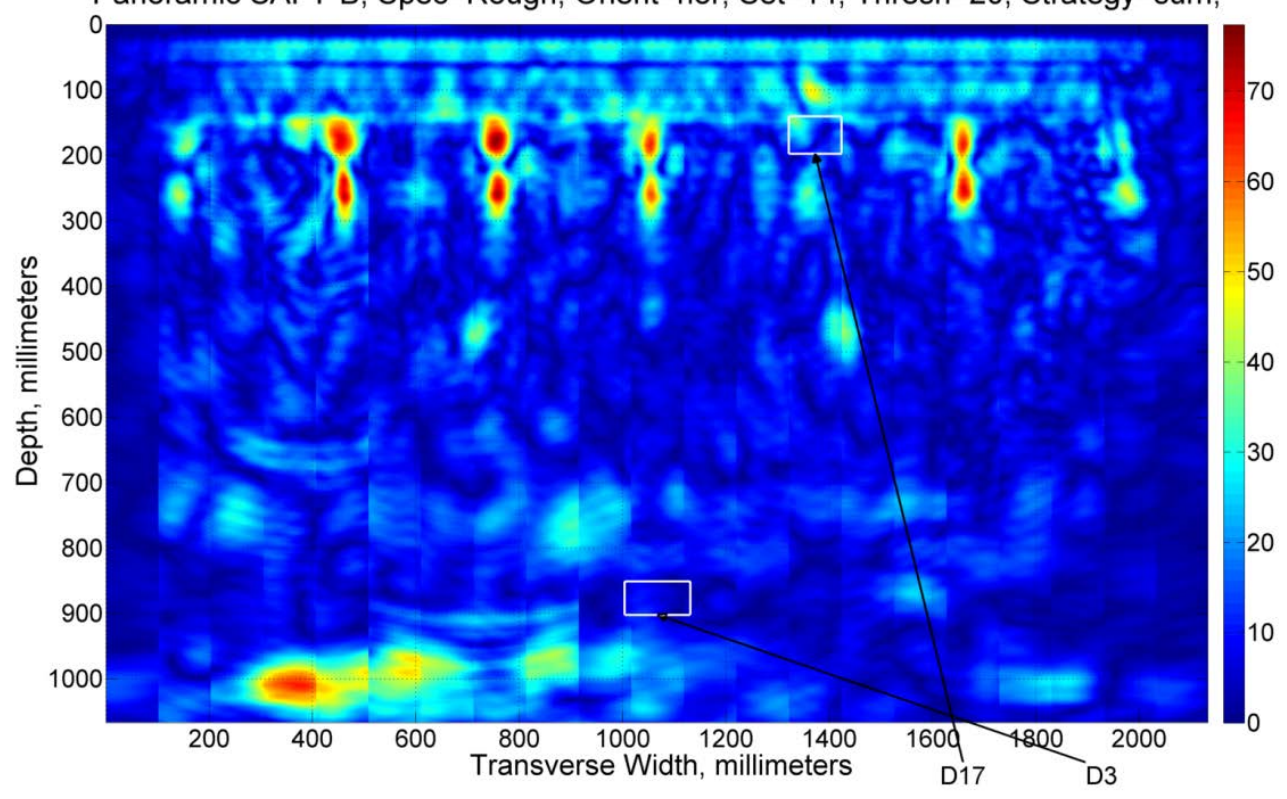

Fig. A. 70. Horizontal Set 14, Node 34. 


\section{SET 15 - DEFECT 3, DEFECT 14, DEFECT 17}

Specimen: Thick, Depth: 1066.8mm (42in), AbsofHilbert -- Node $0(0,0), 0 \sim 500 \mathrm{kHz}$ Panoramic SAFT-B, Spec=Rough, Orient=hor, Set=15, Thresh=20, Strategy=sum

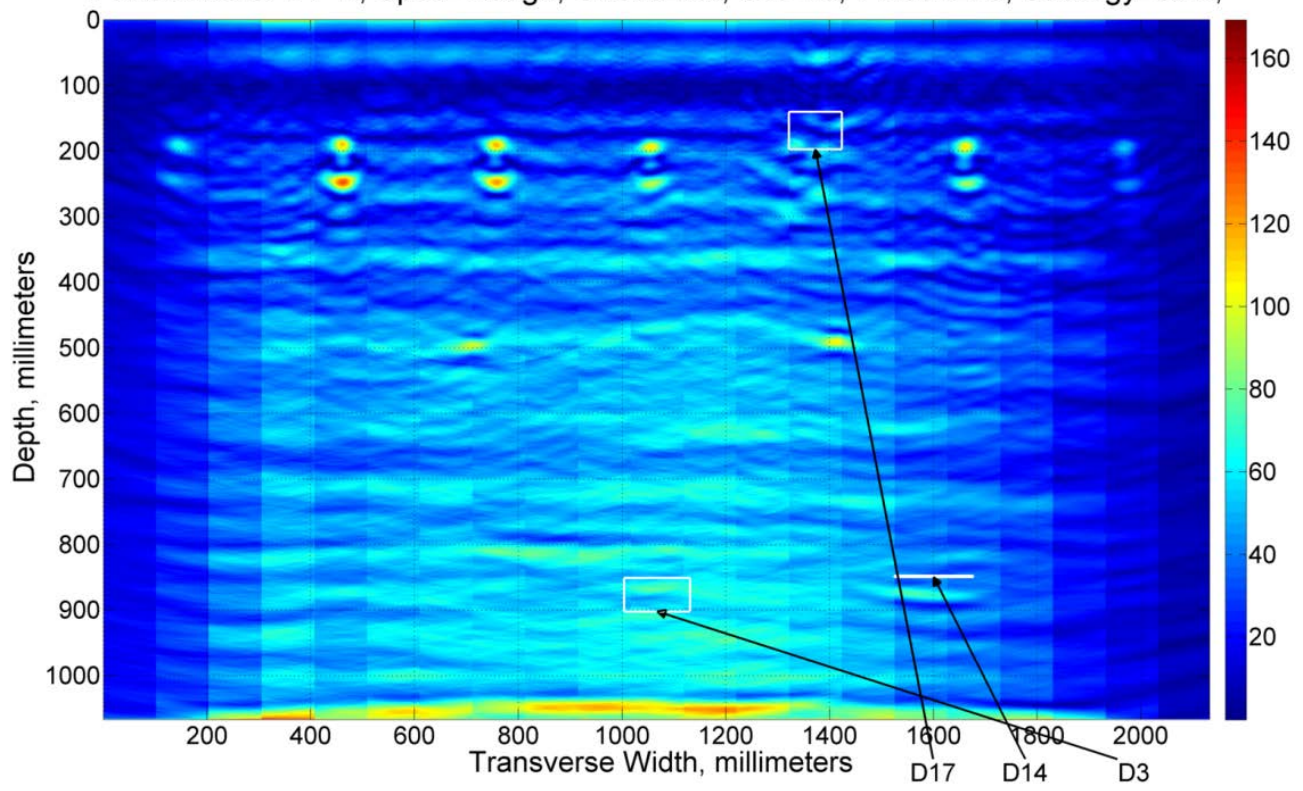

Fig. A. 71. Horizontal Set 15, Node 0.

Specimen: Thick, Depth: 1066.8mm (42in), AbsofHilbert -- Node $16(4,1), 31.25 \sim 62.5 \mathrm{kHz}$ Panoramic SAFT-B, Spec=Rough, Orient=hor, Set=15, Thresh=20, Strategy=sum

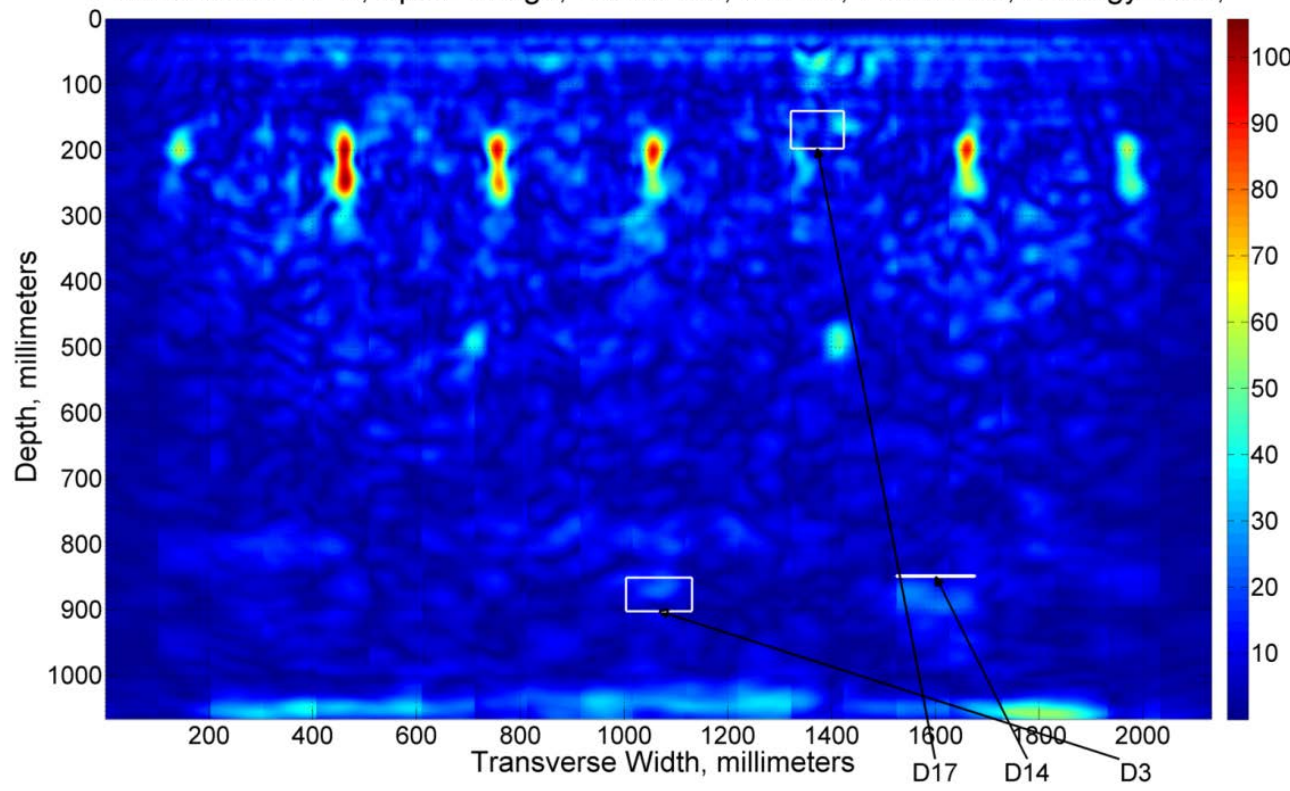

Fig. A. 72. Horizontal Set 15, Node 16. 
Specimen: Thick, Depth: 1066.8mm (42in), AbsofHilbert -- Node $18(4,3), 93.75 \sim 125 \mathrm{kHz}$ Panoramic SAFT-B, Spec=Rough, Orient=hor, Set=15, Thresh=20, Strategy=sum,

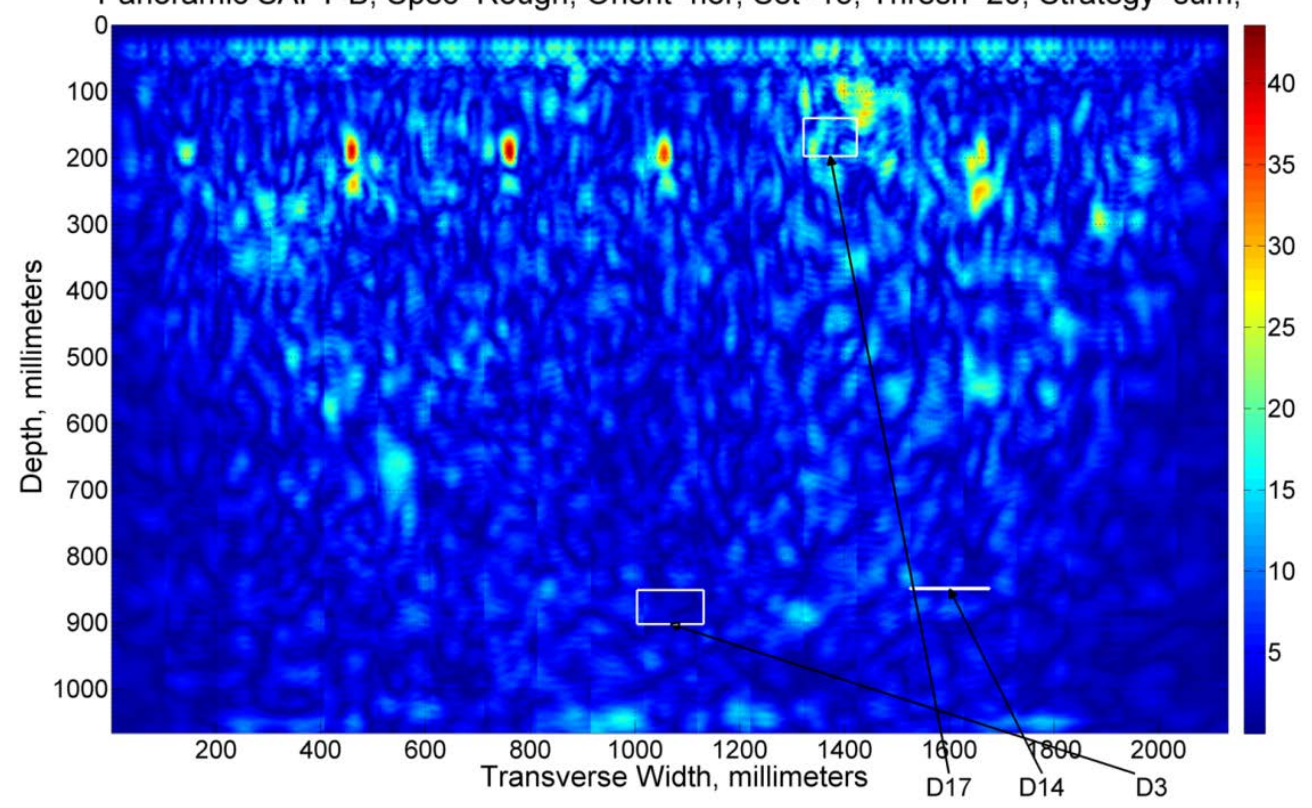

Fig. A. 73. Horizontal Set 15, Node 18.

Specimen: Thick, Depth: 1066.8mm (42in), AbsofHilbert -- Node $32(5,1), 15.625 \sim 31.25 \mathrm{kHz}$ Panoramic SAFT-B, Spec=Rough, Orient=hor, Set=15, Thresh=20, Strategy=sum,

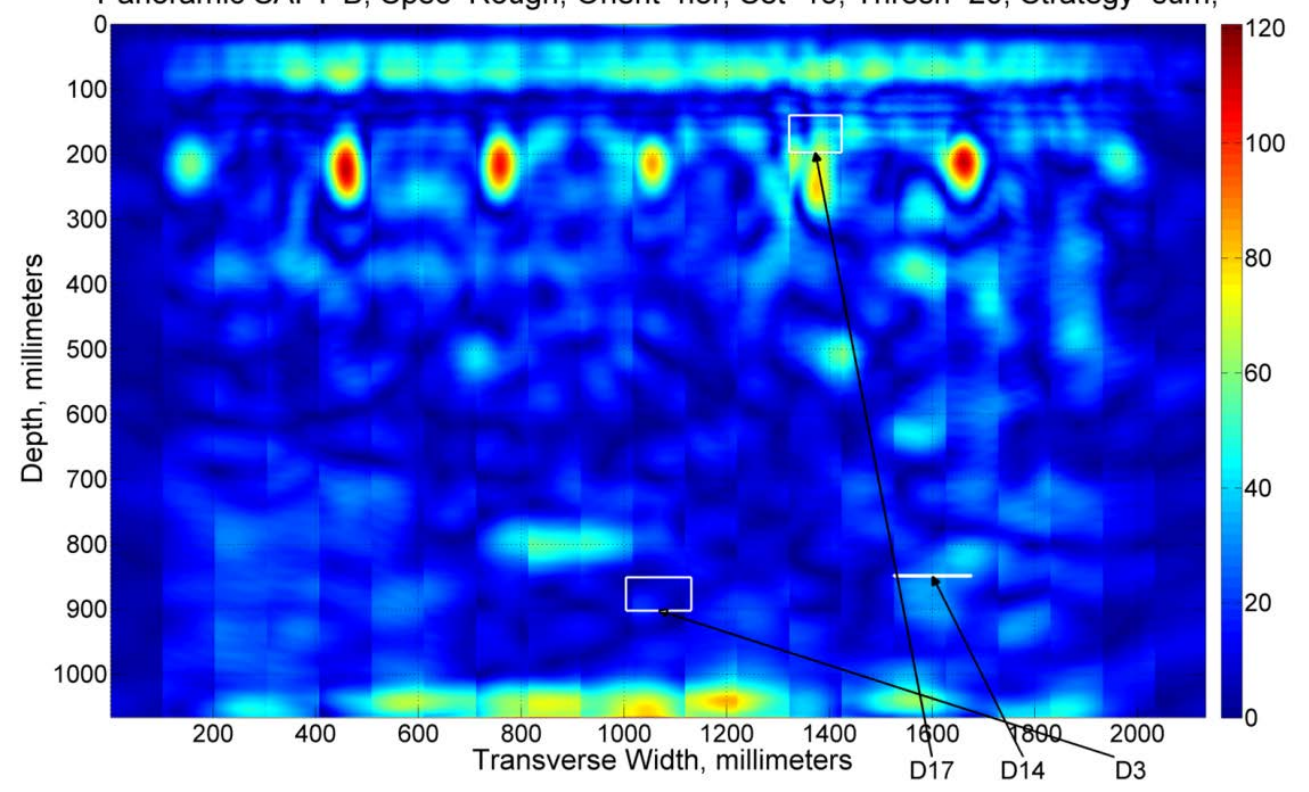

Fig. A. 74. Horizontal Set 15, Node 32. 


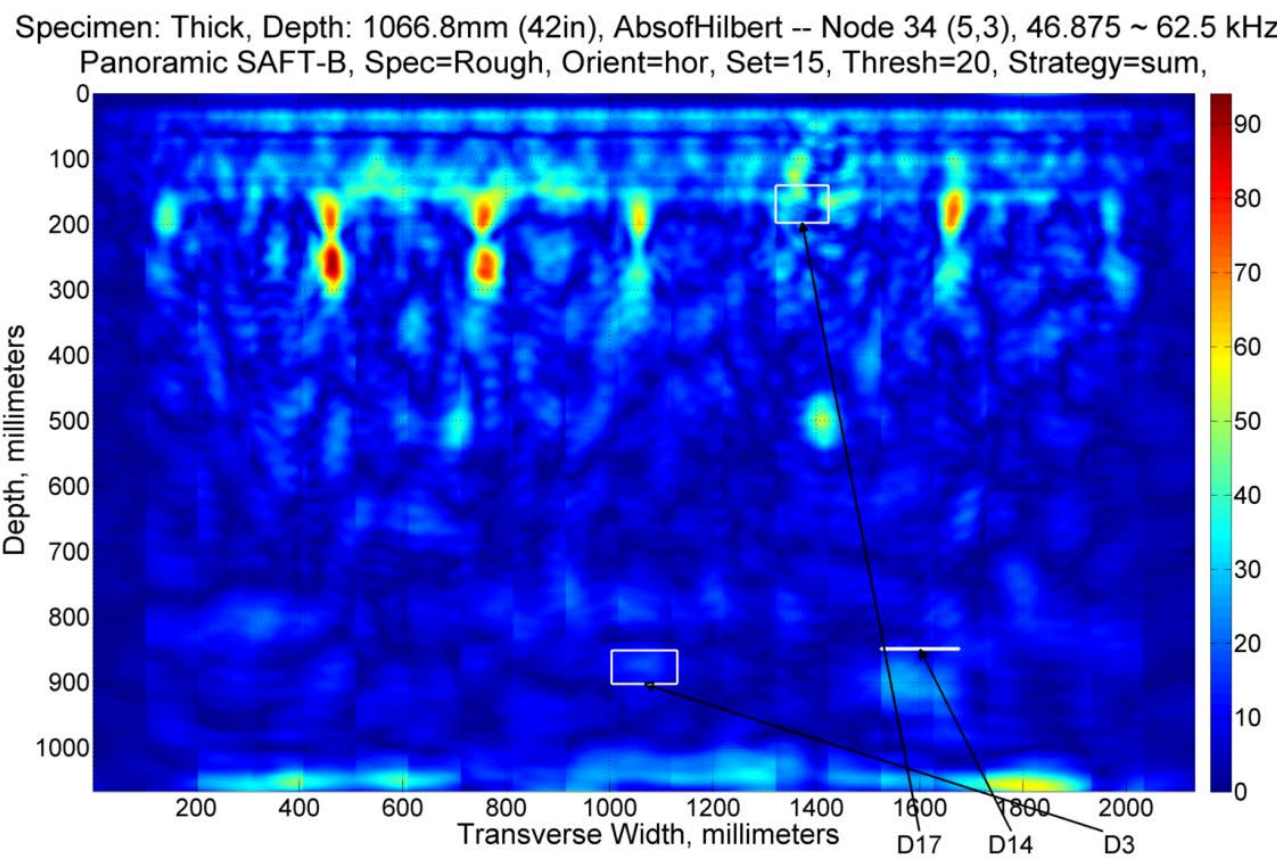

Fig. A. 75. Horizontal Set 15, Node 34.

\section{SET 16 - DEFECT 1}

Specimen: Thick, Depth: 1066.8mm (42in), AbsofHilbert -- Node $0(0,0), 0 \sim 500 \mathrm{kHz}$ Panoramic SAFT-B, Spec=Rough, Orient=hor, Set=16, Thresh=20, Strategy=sum,

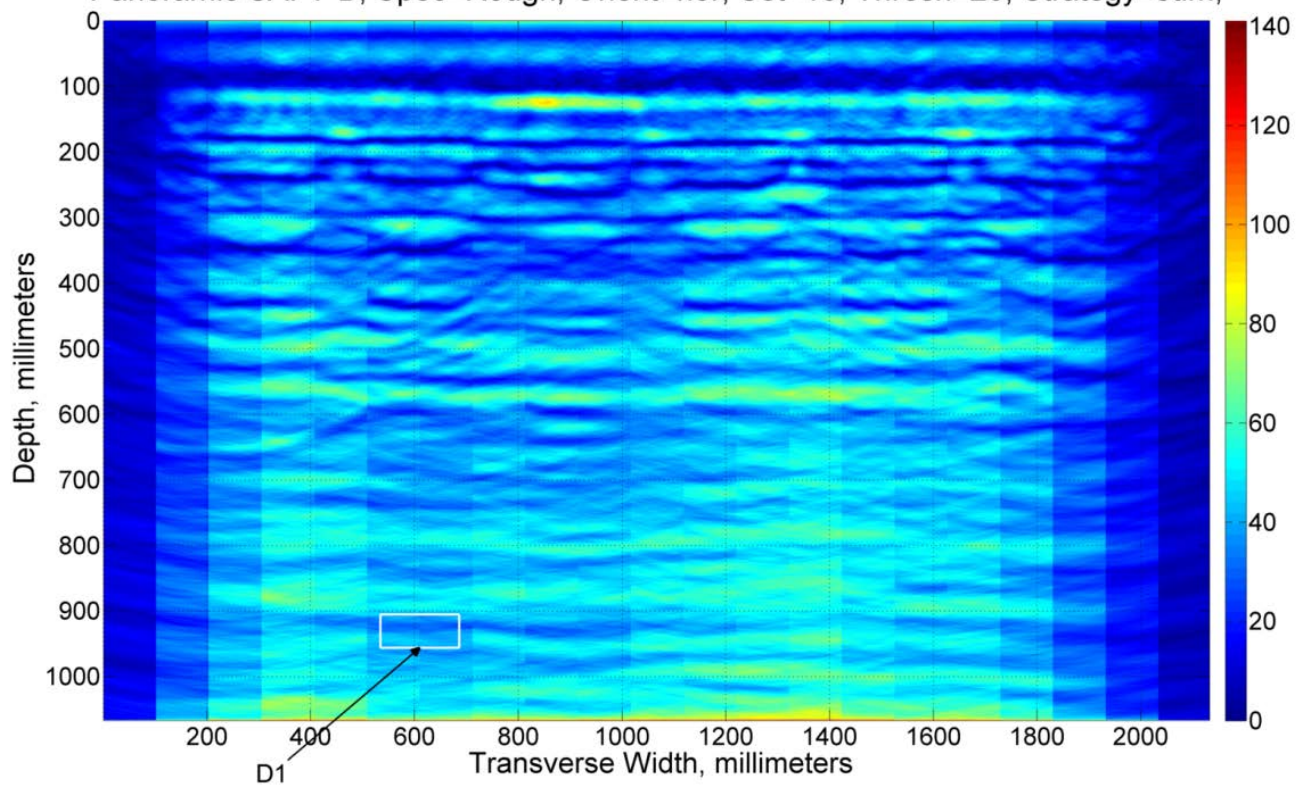

Fig. A. 76. Horizontal Set 16, Node 0. 


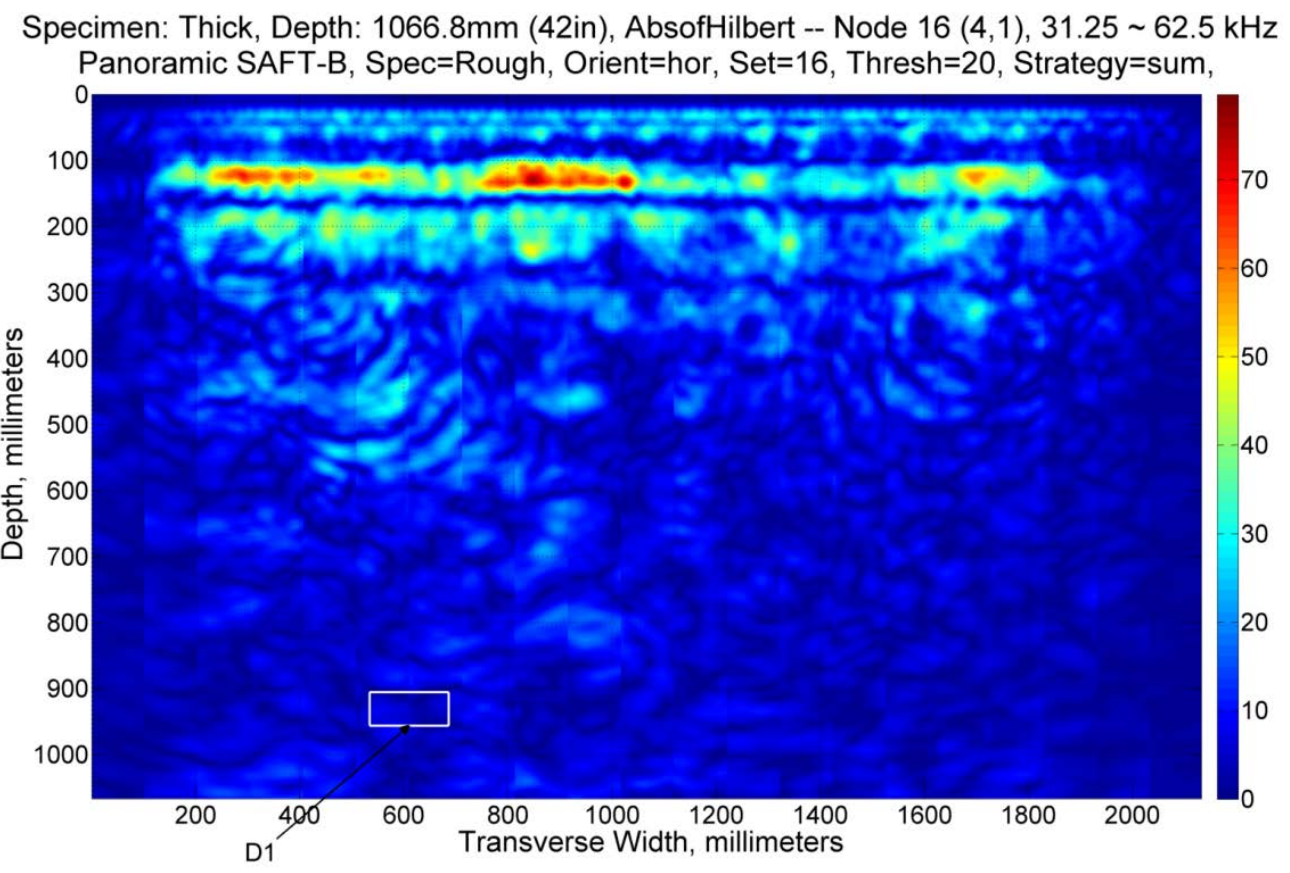

Fig. A. 77. Horizontal Set 16, Node 16.

Specimen: Thick, Depth: 1066.8mm (42in), AbsofHilbert -- Node 18 (4,3), $93.75 \sim 125 \mathrm{kHz}$ Panoramic SAFT-B, Spec=Rough, Orient=hor, Set=16, Thresh=20, Strategy=sum,

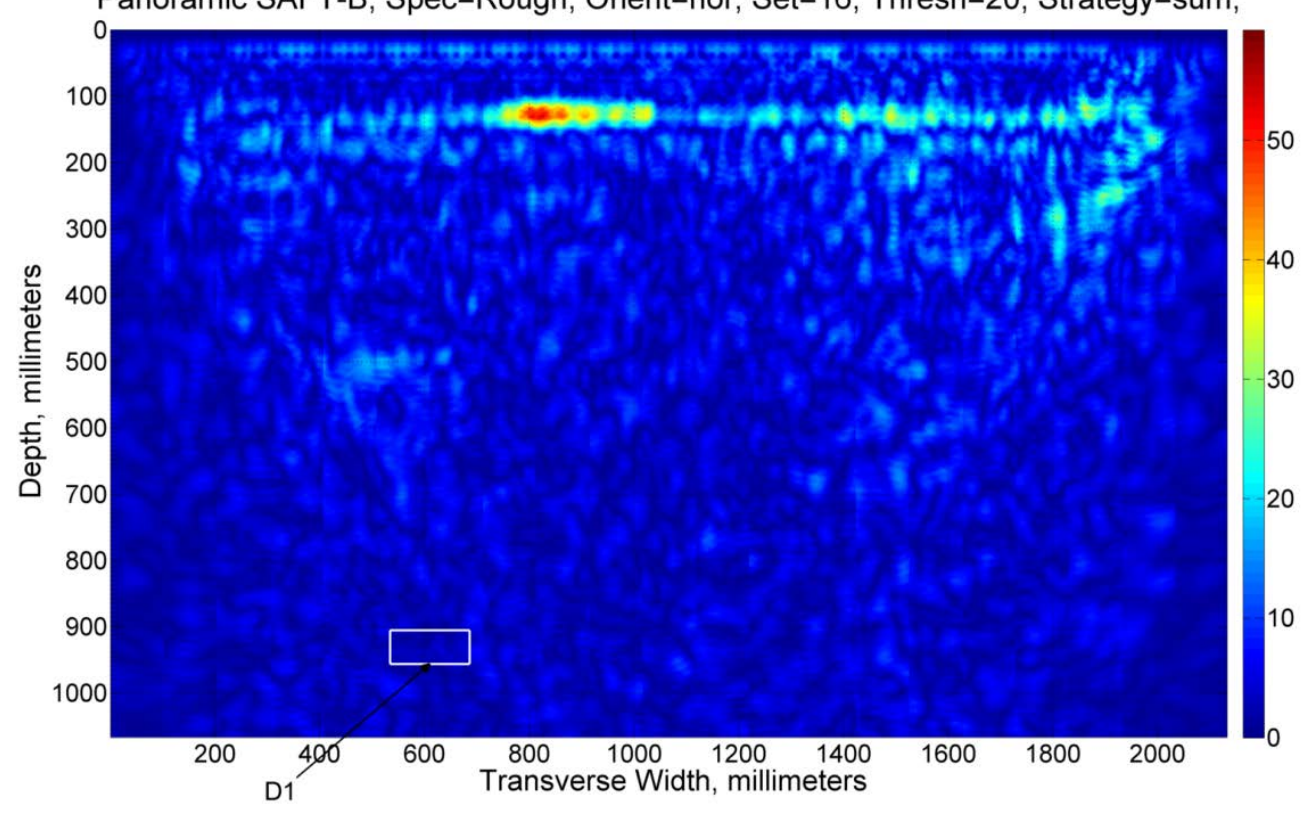

Fig. A. 78. Horizontal Set 16, Node 16. 


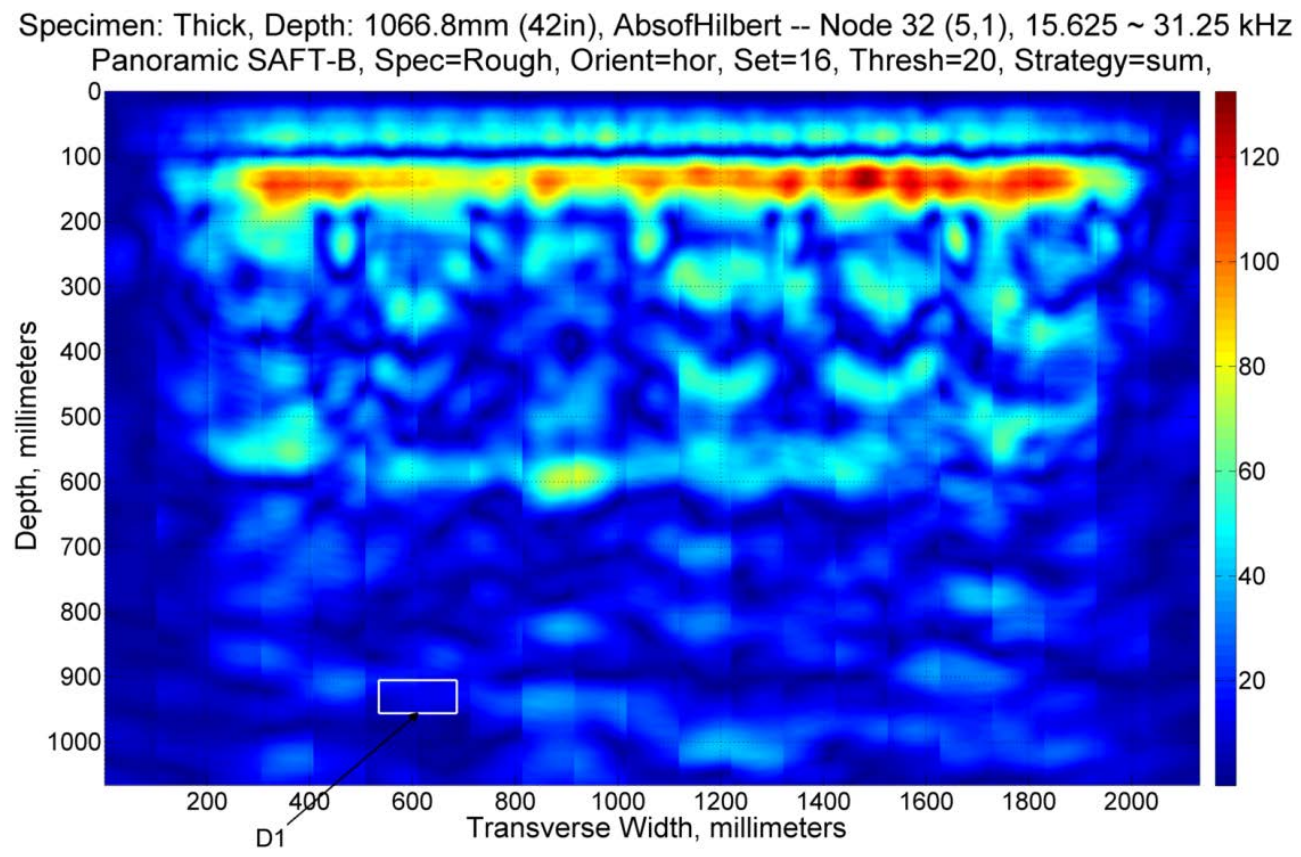

Fig. A. 79. Horizontal Set 16, Node 32.

Specimen: Thick, Depth: 1066.8mm (42in), AbsofHilbert -- Node 34 (5,3), $46.875 \sim 62.5 \mathrm{kHz}$ Panoramic SAFT-B, Spec=Rough, Orient=hor, Set=16, Thresh=20, Strategy=sum,

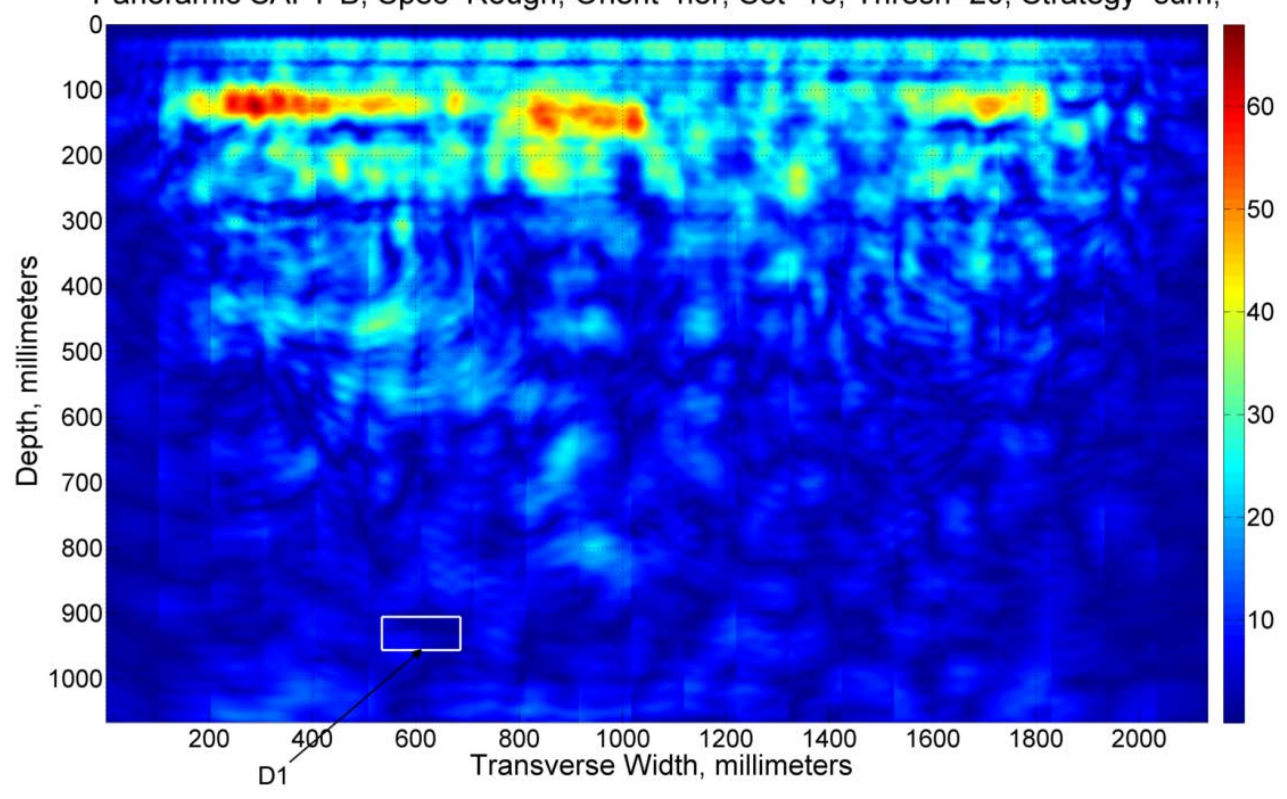

Fig. A. 80. Horizontal Set 16, Node 34. 


\section{SET 17 - DEFECT 11, DEFECT 16}

Specimen: Thick, Depth: 1066.8mm (42in), AbsofHilbert -- Node $0(0,0), 0 \sim 500 \mathrm{kHz}$ Panoramic SAFT-B, Spec=Rough, Orient=hor, Set=17, Thresh=20, Strategy=sum,

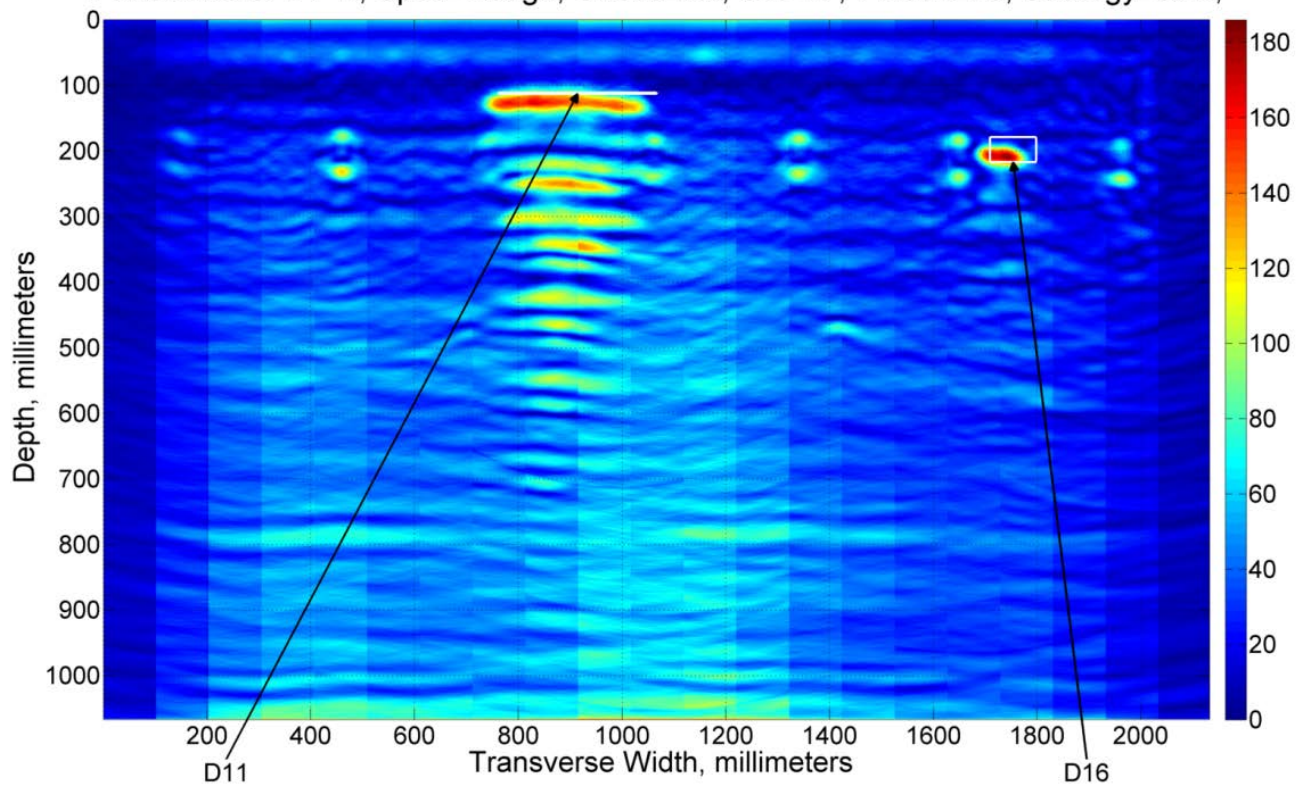

Fig. A. 81. Horizontal Set 17, Node 0.

Specimen: Thick, Depth: 1066.8mm (42in), AbsofHilbert -- Node $16(4,1), 31.25 \sim 62.5 \mathrm{kHz}$ Panoramic SAFT-B, Spec=Rough, Orient=hor, Set=17, Thresh=20, Strategy=sum,

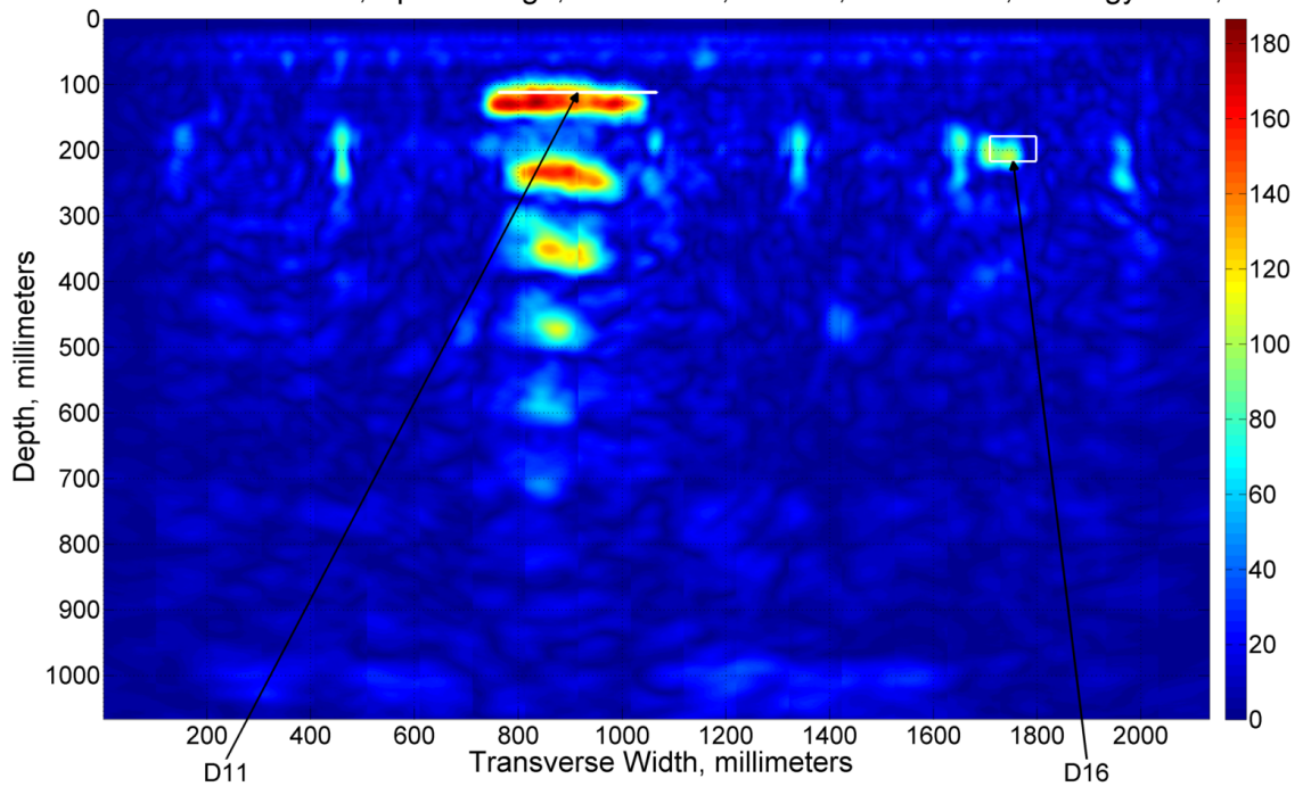

Fig. A. 82. Horizontal Set 17, Node 16. 
Specimen: Thick, Depth: 1066.8mm (42in), AbsofHilbert -- Node $18(4,3), 93.75 \sim 125 \mathrm{kHz}$ Panoramic SAFT-B, Spec=Rough, Orient=hor, Set=17, Thresh=20, Strategy=sum,

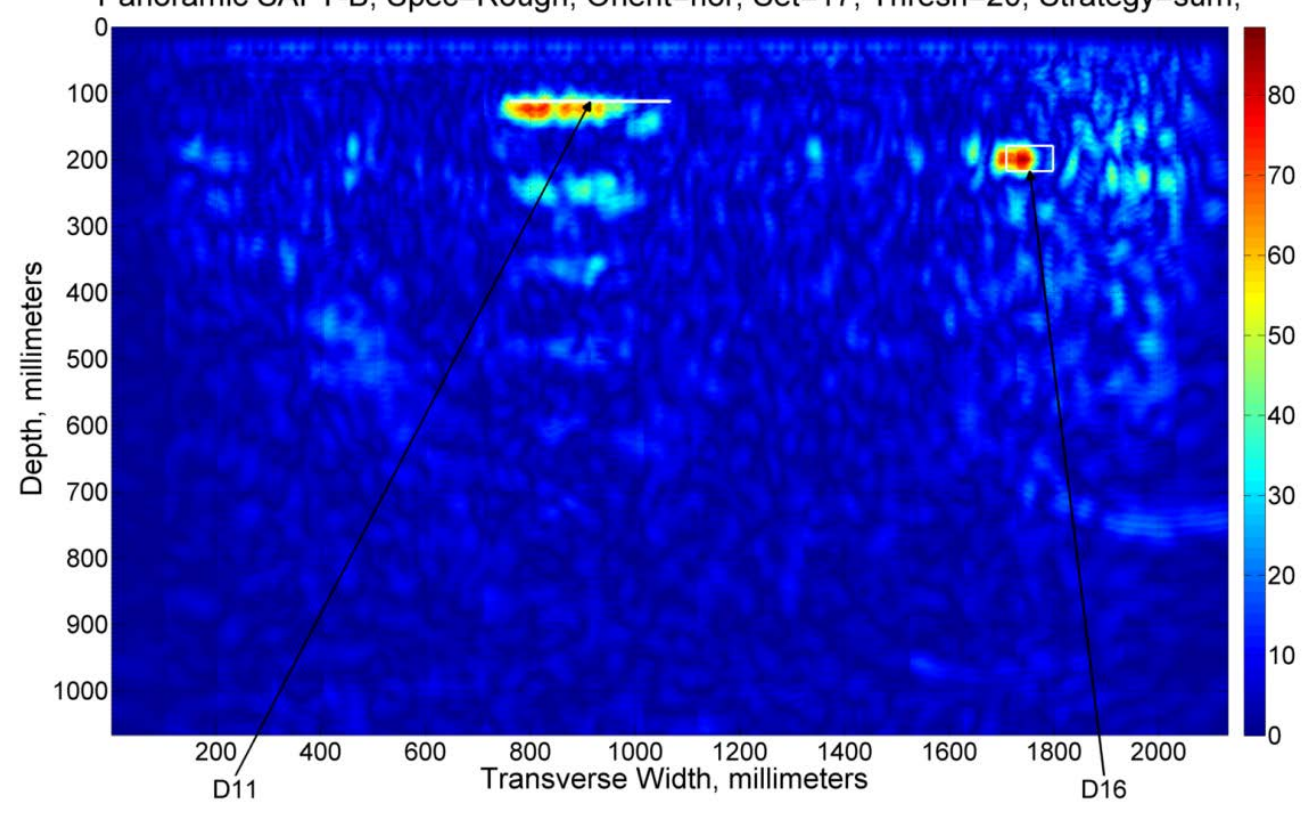

Fig. A. 83. Horizontal Set 17, Node 18.

Specimen: Thick, Depth: 1066.8mm (42in), AbsofHilbert -- Node $32(5,1), 15.625 \sim 31.25 \mathrm{kHz}$ Panoramic SAFT-B, Spec=Rough, Orient=hor, Set=17, Thresh=20, Strategy=sum,

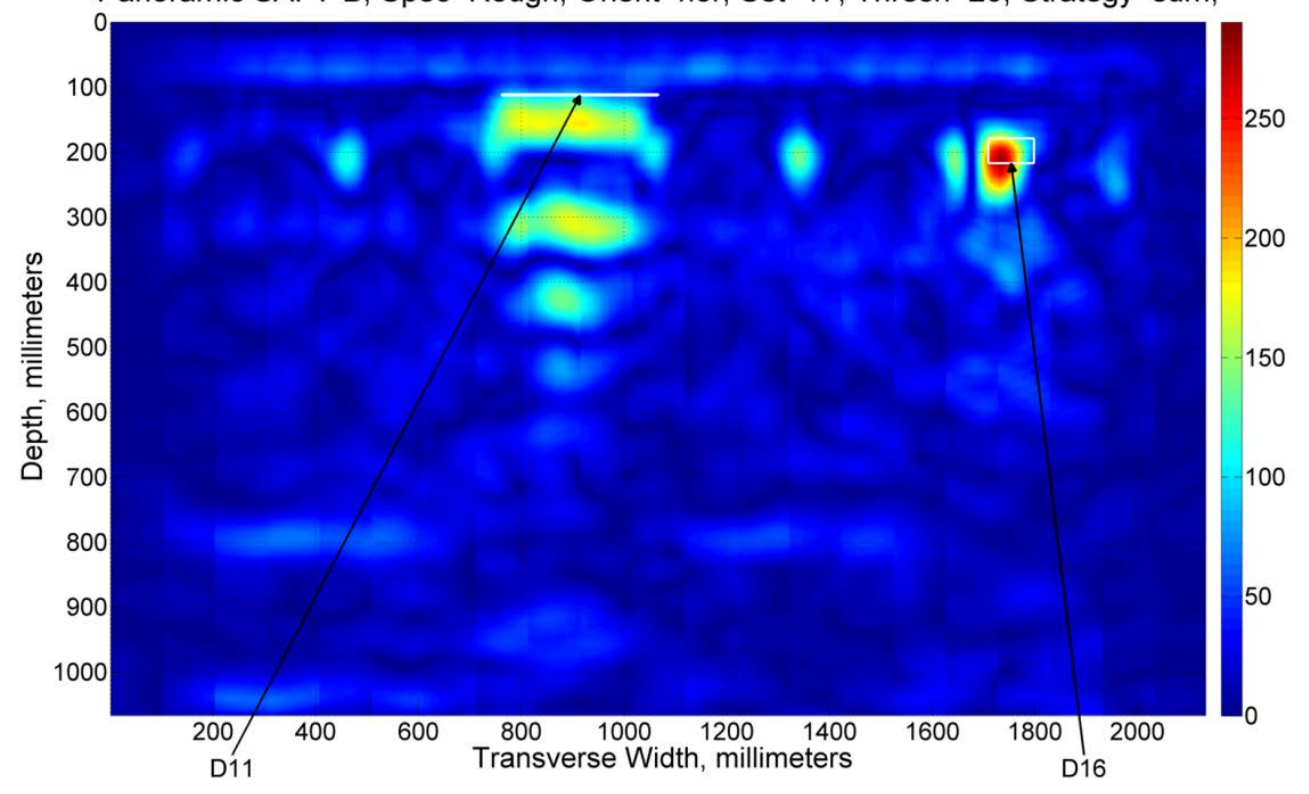

Fig. A. 84. Horizontal Set 17, Node 32. 


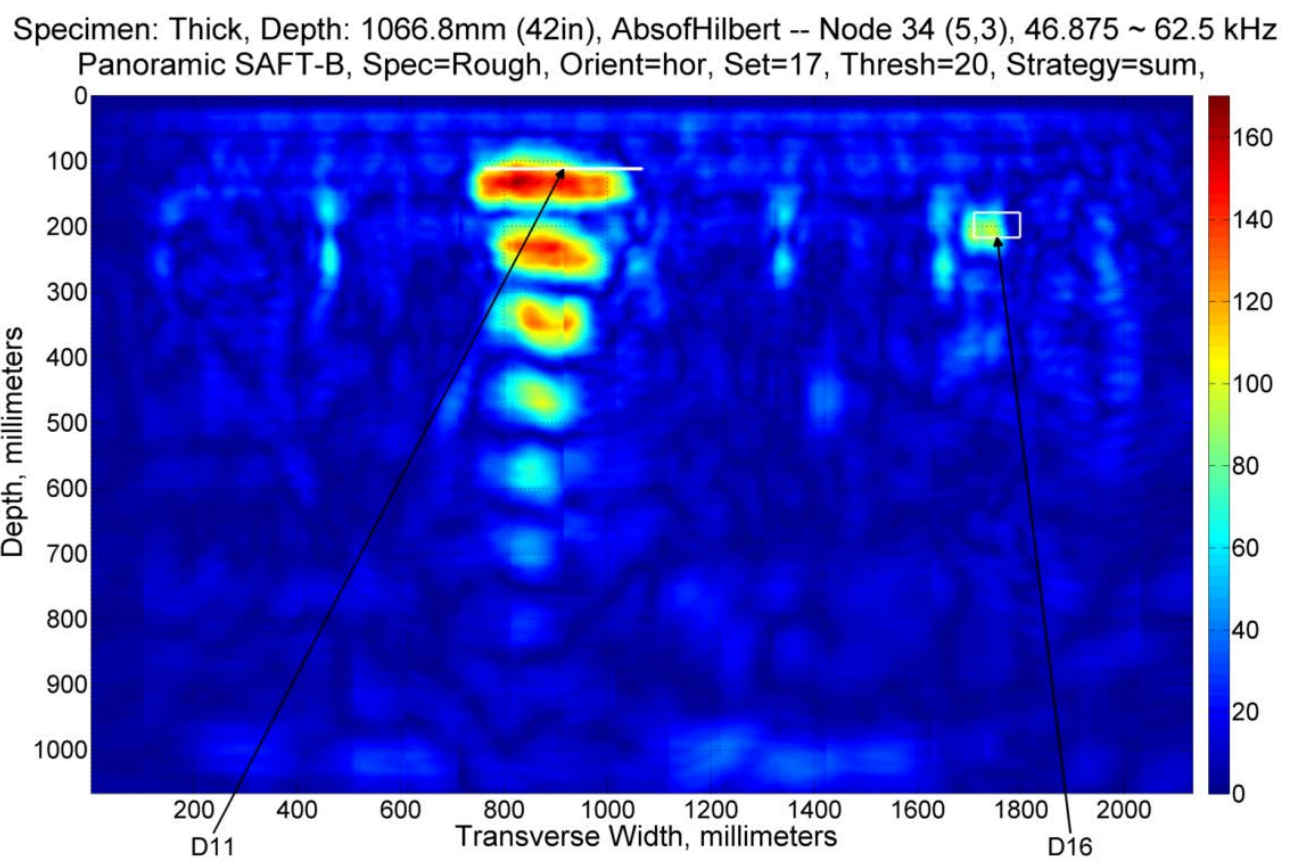

Fig. A. 85. Horizontal Set 17, Node 34.

\section{SET 18 - DEFECT 11, DEFECT 16}

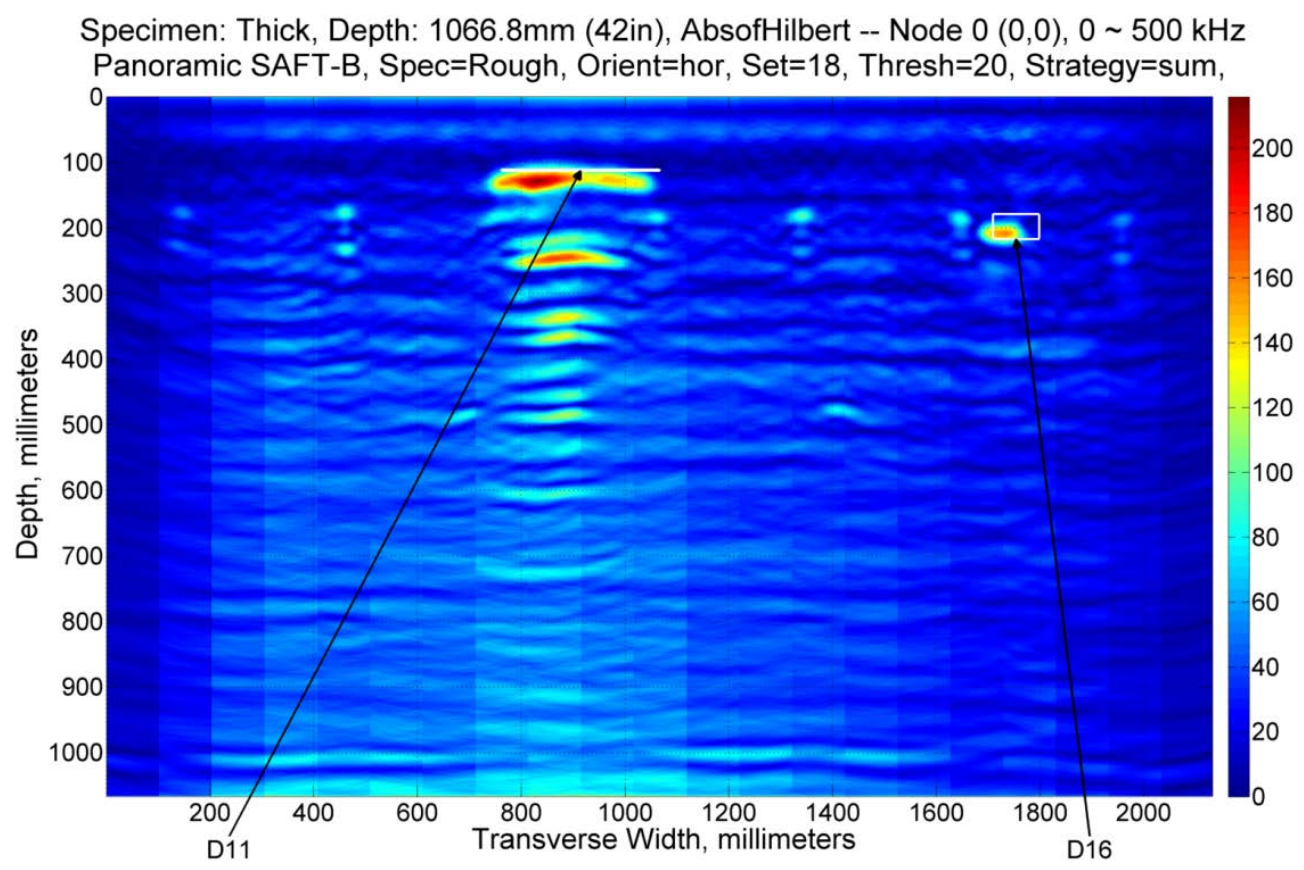

Fig. A. 86. Horizontal Set 18, Node 0. 
Specimen: Thick, Depth: 1066.8mm (42in), AbsofHilbert -- Node $16(4,1), 31.25 \sim 62.5 \mathrm{kHz}$ Panoramic SAFT-B, Spec=Rough, Orient=hor, Set=18, Thresh=20, Strategy=sum,

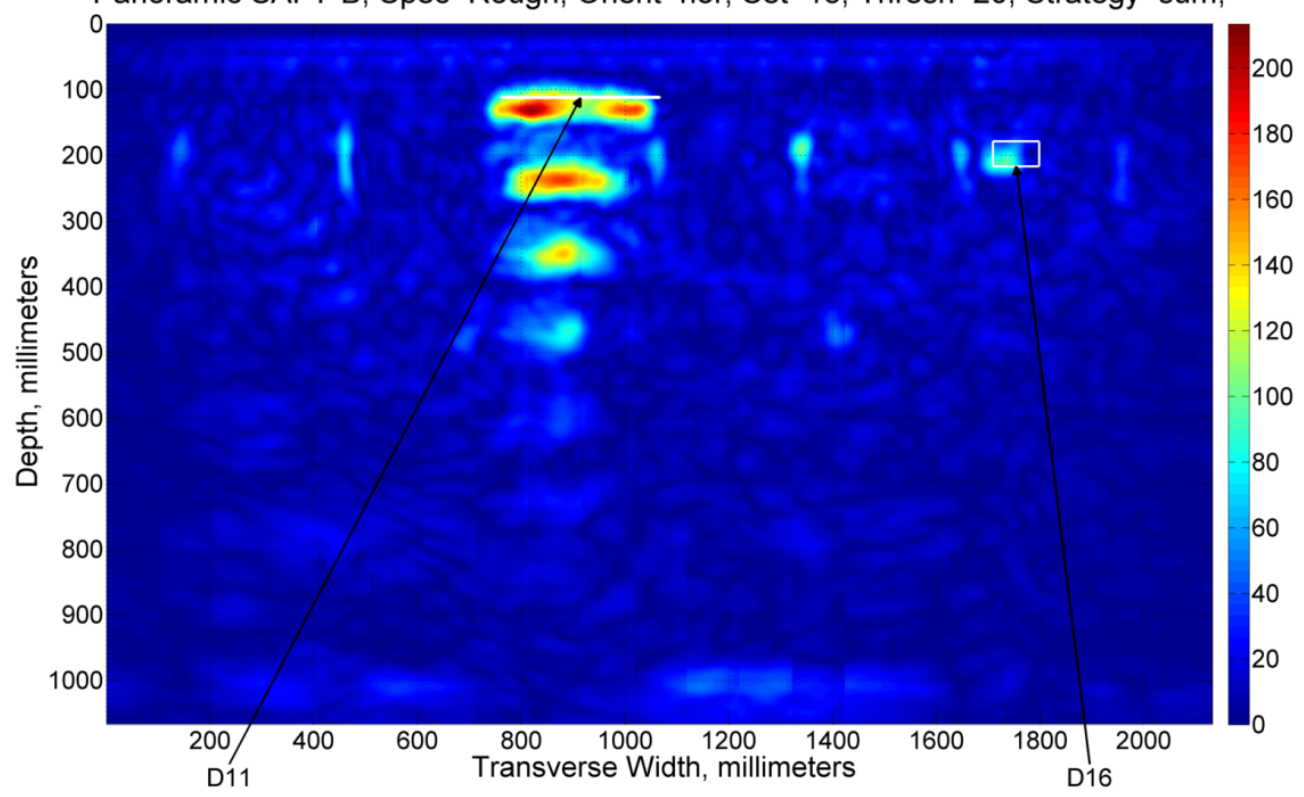

Fig. A. 87. Horizontal Set 18, Node 16.

Specimen: Thick, Depth: 1066.8mm (42in), AbsofHilbert -- Node $18(4,3), 93.75 \sim 125 \mathrm{kHz}$ Panoramic SAFT-B, Spec=Rough, Orient=hor, Set=18, Thresh=20, Strategy=sum,

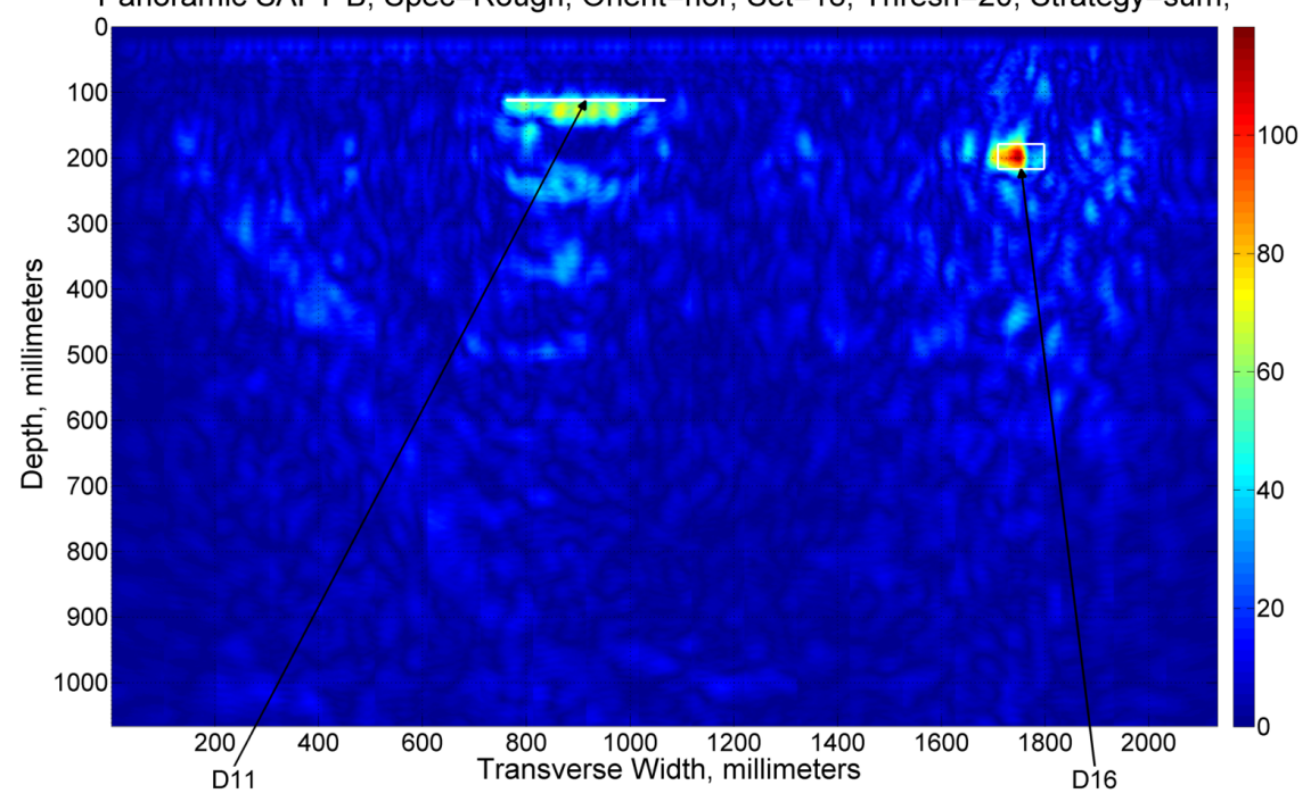

Fig. A. 88. Horizontal Set 18, Node 18. 


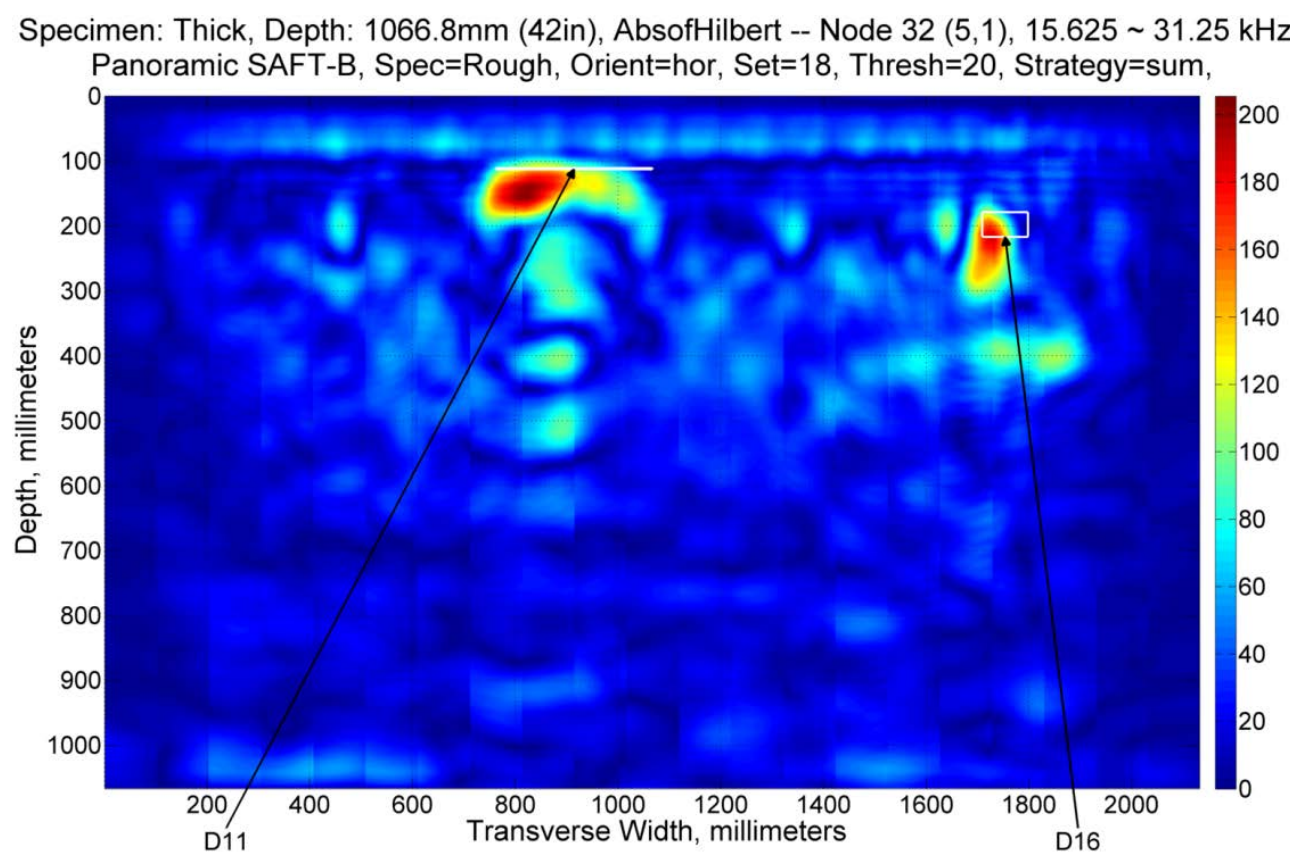

Fig. A. 89. Horizontal Set 18, Node 32.

Specimen: Thick, Depth: 1066.8mm (42in), AbsofHilbert -- Node 34 (5,3), $46.875 \sim 62.5 \mathrm{kHz}$ Panoramic SAFT-B, Spec=Rough, Orient=hor, Set=18, Thresh=20, Strategy=sum,

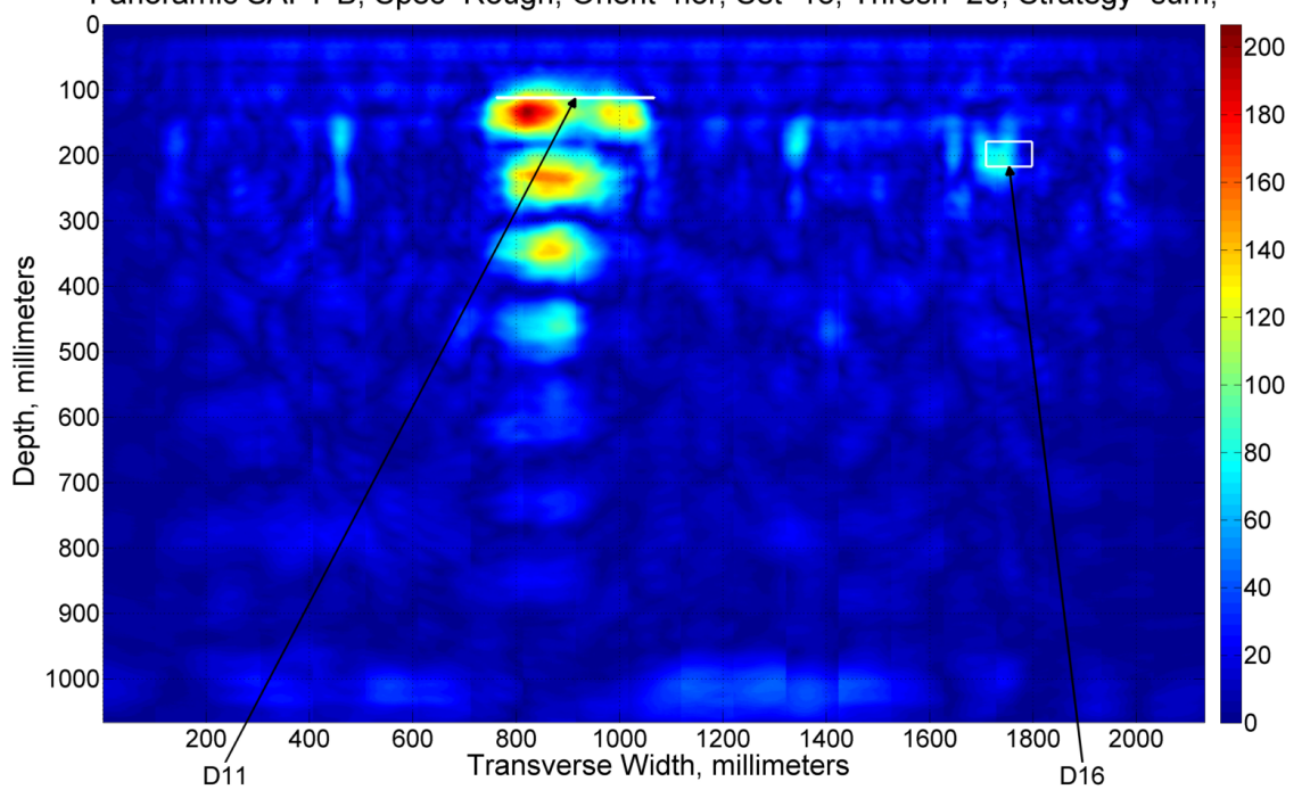

Fig. A. 90. Horizontal Set 18, Node 34 . 


\section{SET 19 - NO DEFECT}

Specimen: Thick, Depth: 1066.8mm (42in), AbsofHilbert -- Node $0(0,0), 0 \sim 500 \mathrm{kHz}$ Panoramic SAFT-B, Spec=Rough, Orient=hor, Set=19, Thresh=20, Strategy=sum,

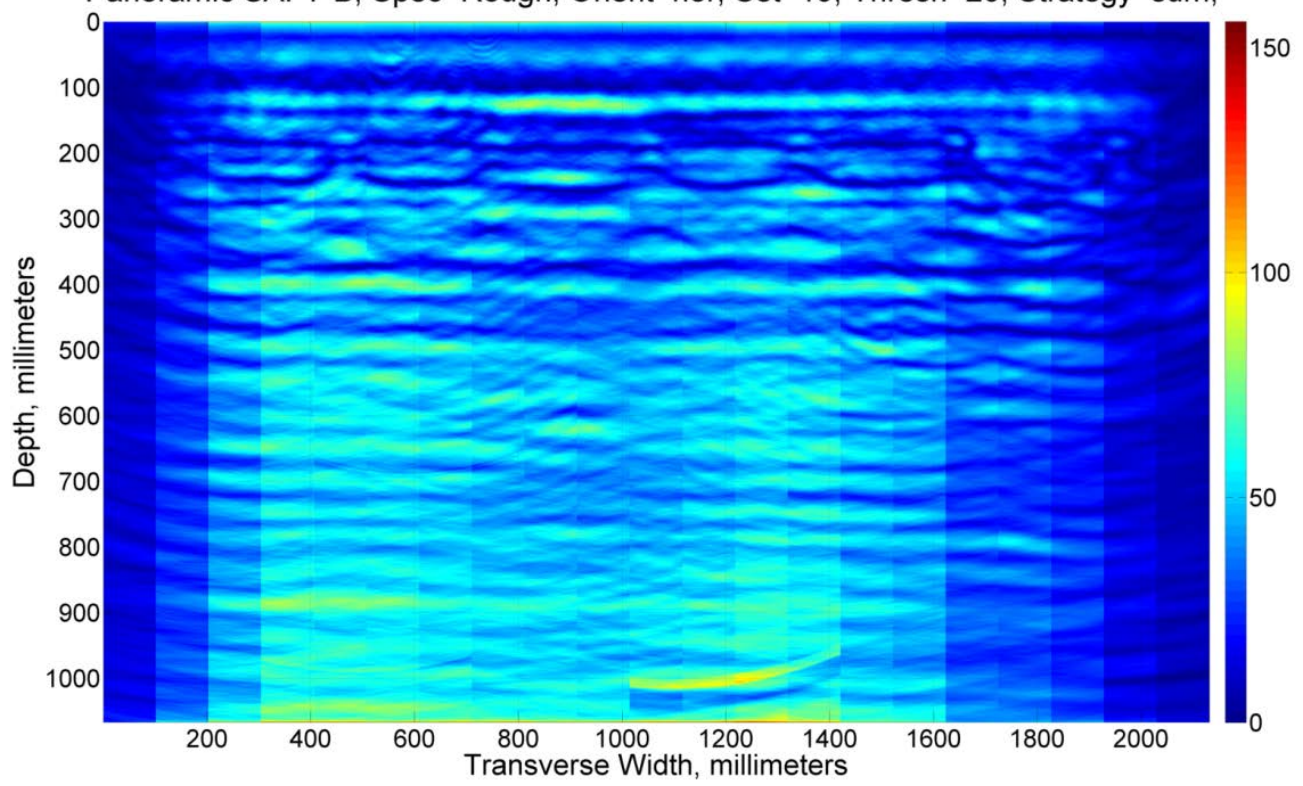

Fig. A. 91. Horizontal Set 19, Node 0.

Specimen: Thick, Depth: 1066.8mm (42in), AbsofHilbert -- Node $16(4,1), 31.25 \sim 62.5 \mathrm{kHz}$ Panoramic SAFT-B, Spec=Rough, Orient=hor, Set=19, Thresh=20, Strategy=sum,

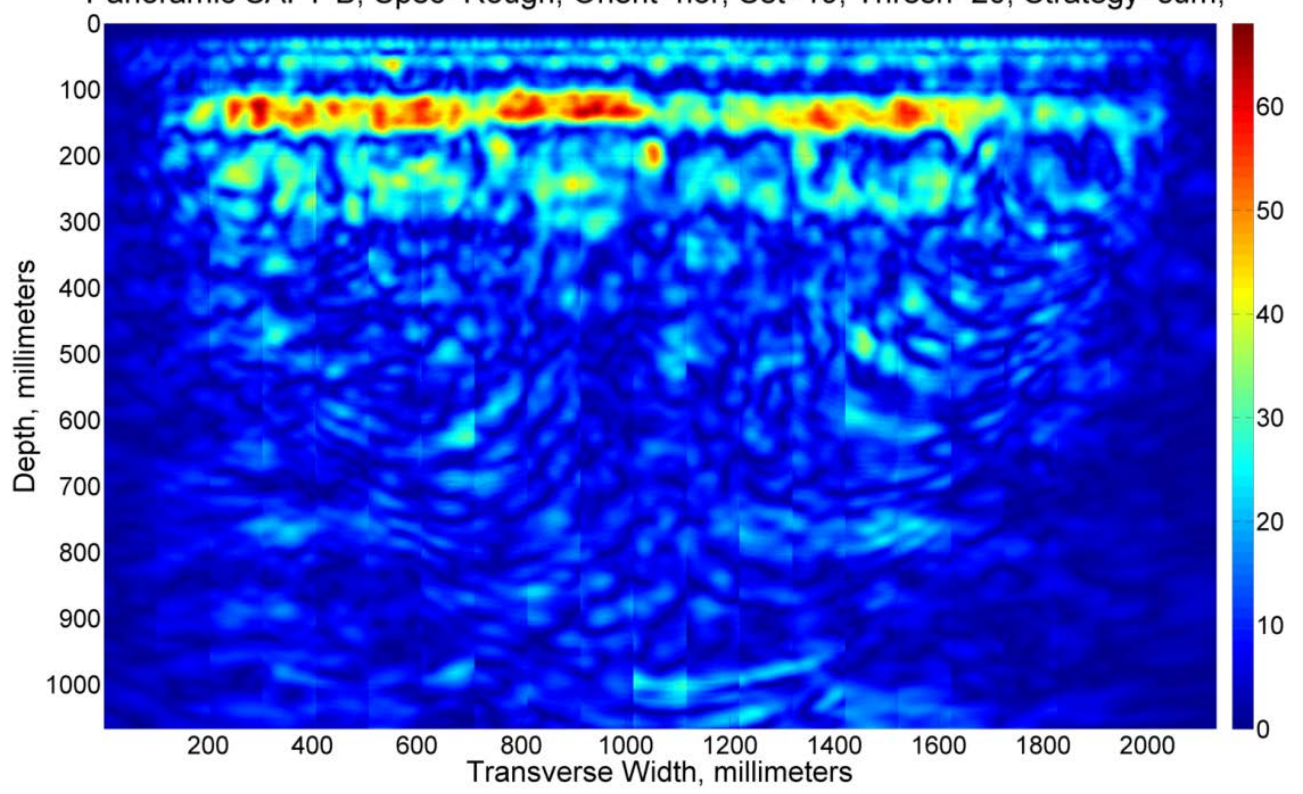

Fig. A. 92. Horizontal Set 19, Node 16. 
Specimen: Thick, Depth: 1066.8mm (42in), AbsofHilbert -- Node $18(4,3), 93.75 \sim 125 \mathrm{kHz}$ Panoramic SAFT-B, Spec=Rough, Orient=hor, Set=19, Thresh=20, Strategy=sum,

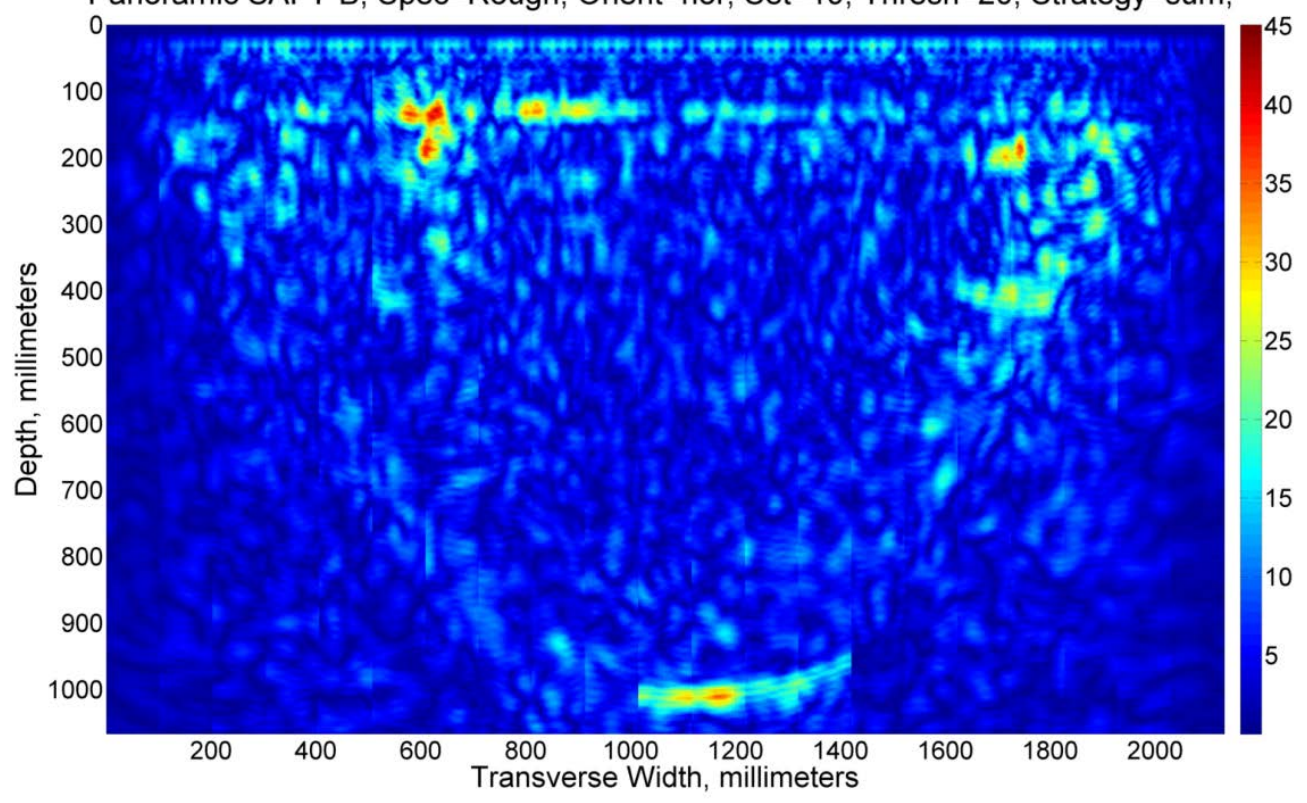

Fig. A. 93. Horizontal Set 19, Node 18.

Specimen: Thick, Depth: 1066.8mm (42in), AbsofHilbert -- Node $32(5,1), 15.625 \sim 31.25 \mathrm{kHz}$ Panoramic SAFT-B, Spec=Rough, Orient=hor, Set=19, Thresh=20, Strategy=sum,

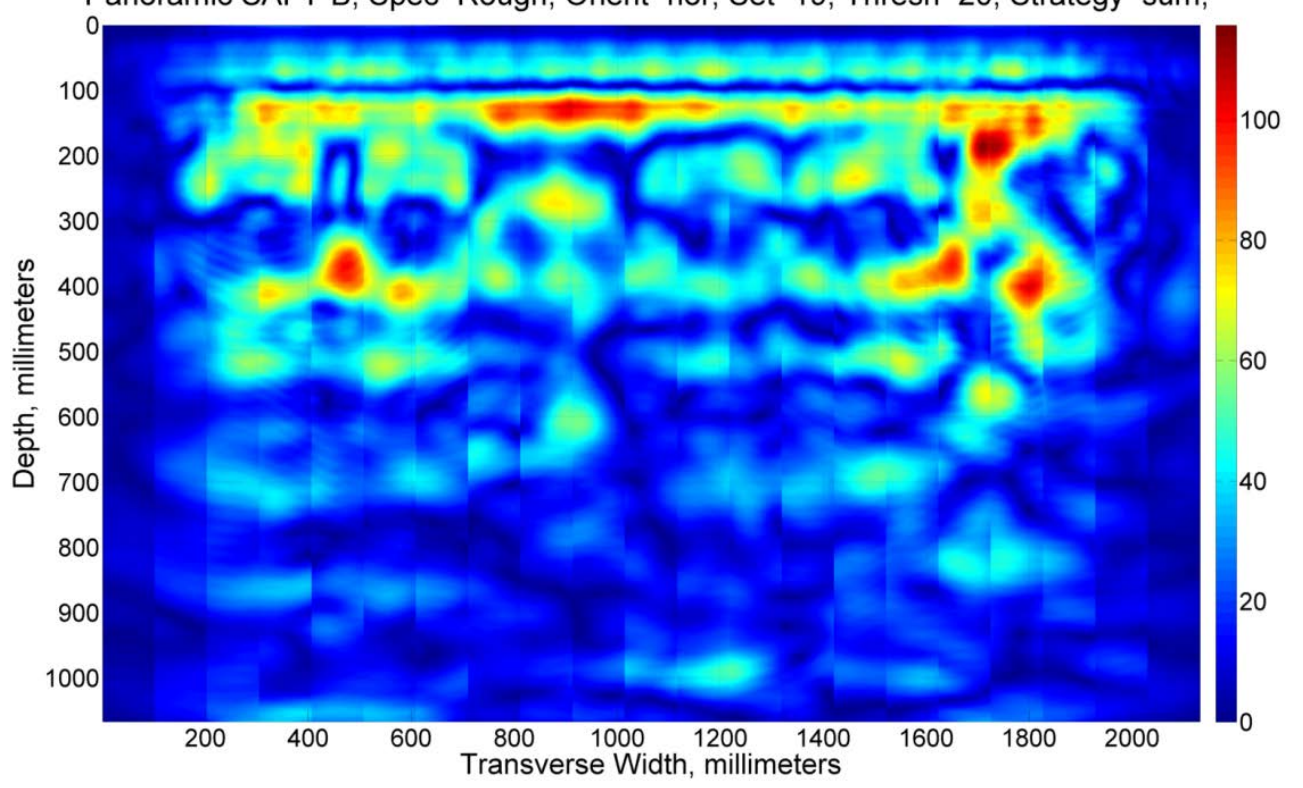

Fig. A. 94. Horizontal Set 19, Node 32. 


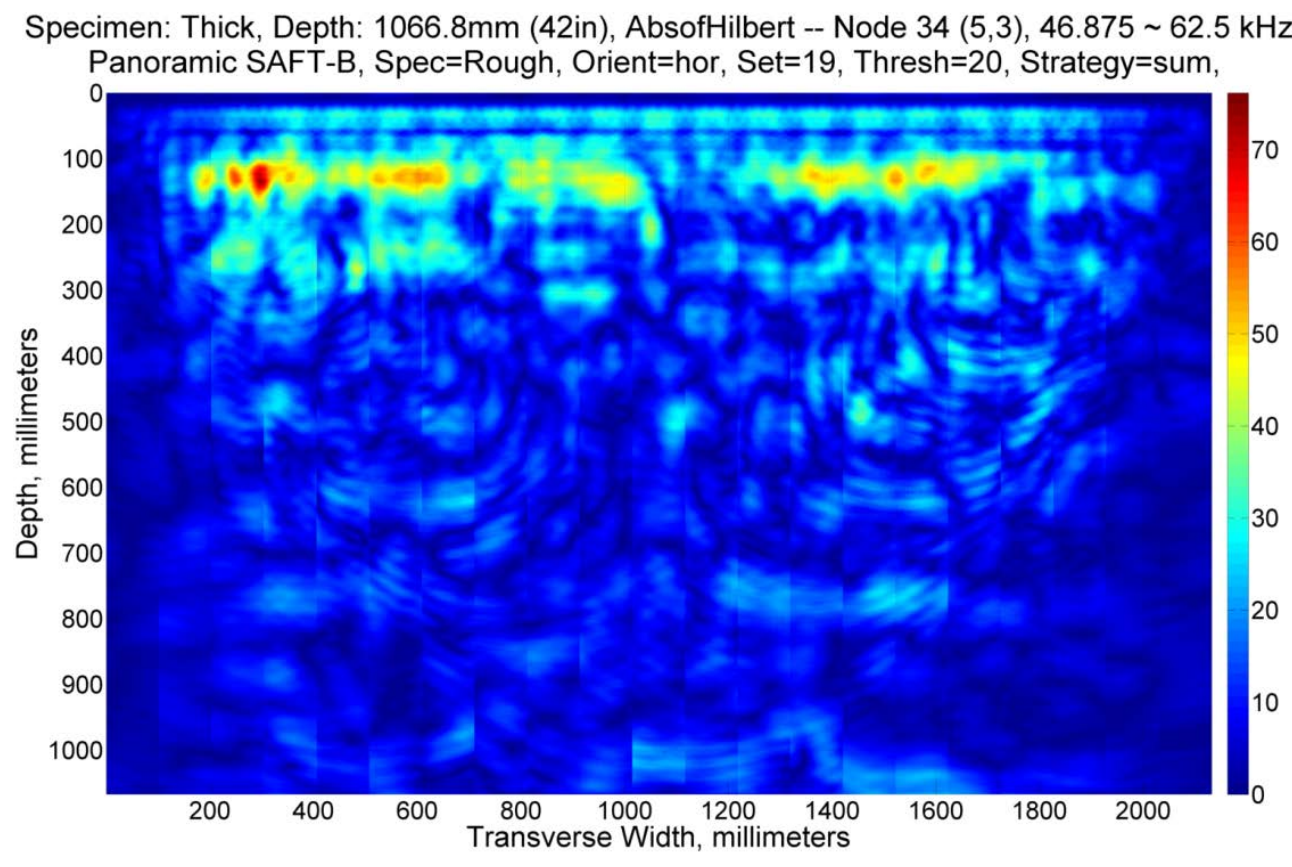

Fig. A. 95. Horizontal Set 19, Node 34. 


\section{VERTICAL}

\section{SET 1 - NO DEFECT}

Specimen: Thick, Depth: 1066.8mm (42in), AbsofHilbert -- Node $0(0,0), 0 \sim 500 \mathrm{kHz}$

Panoramic SAFT-B, Spec=Rough, Orient=ver, Set=1, Thresh=20, Strategy=sum,

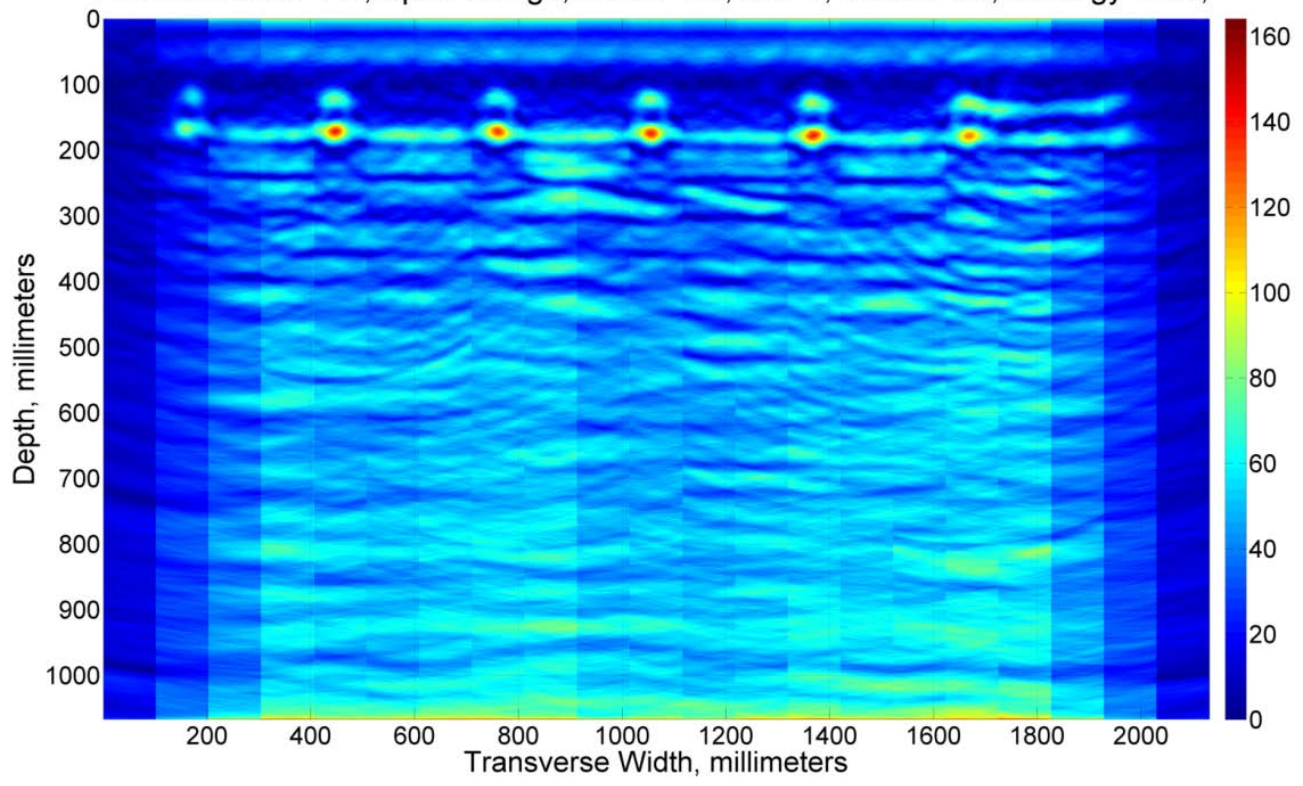

Fig. A. 96. Vertical Set 1, Node 0.

Specimen: Thick, Depth: 1066.8mm (42in), AbsofHilbert -- Node $16(4,1), 31.25 \sim 62.5 \mathrm{kHz}$ Panoramic SAFT-B, Spec=Rough, Orient=ver, Set=1, Thresh=20, Strategy=sum,

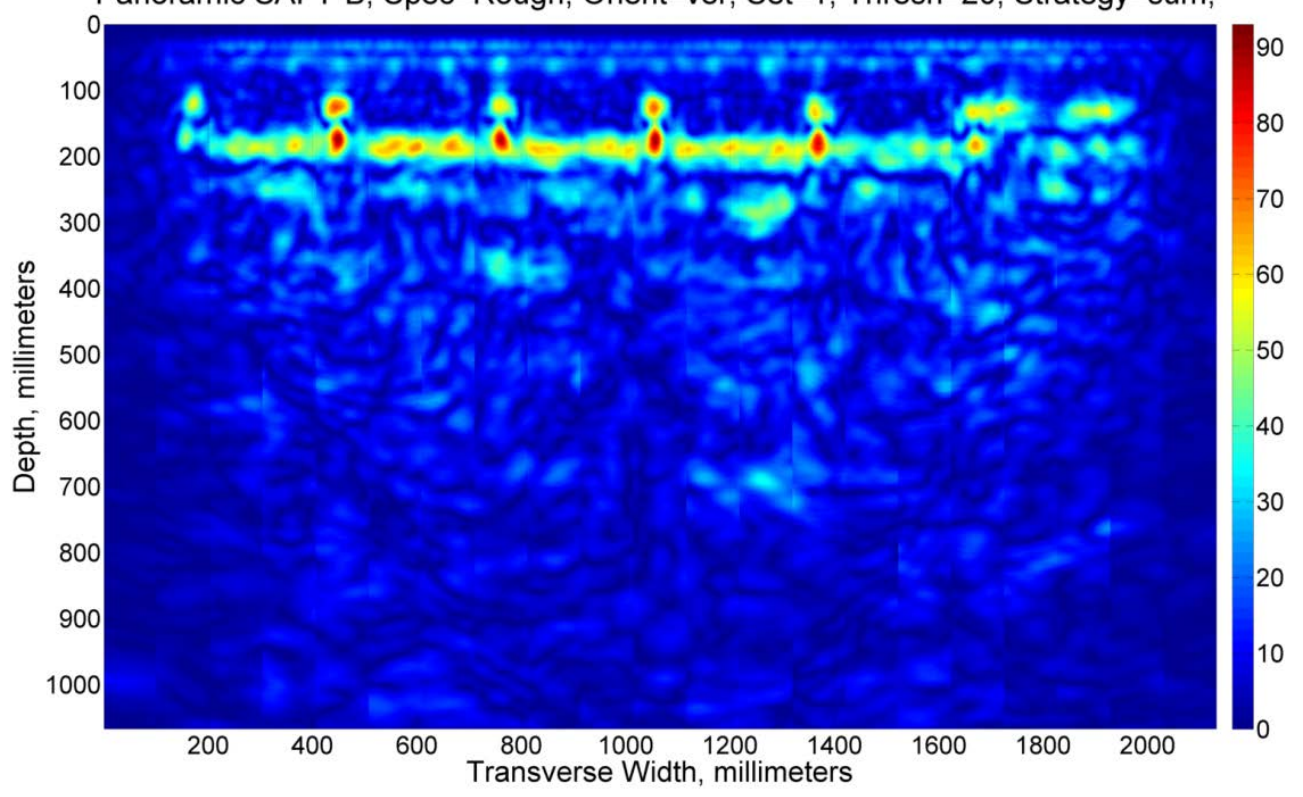

Fig. A. 97. Vertical Set 1, Node 16. 


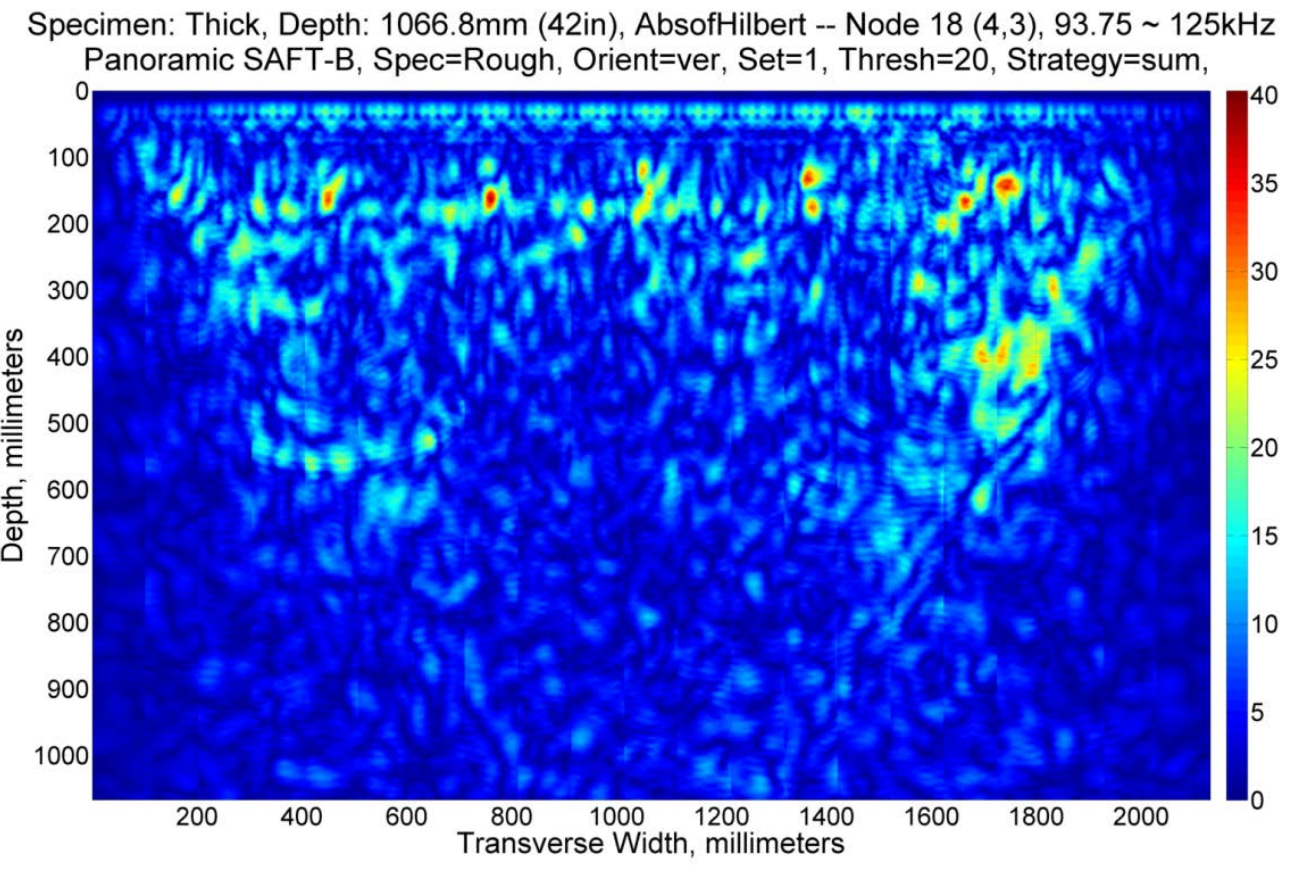

Fig. A. 98. Vertical Set 1, Node 18.

Specimen: Thick, Depth: 1066.8mm (42in), AbsofHilbert -- Node $32(5,1), 15.625 \sim 31.25 \mathrm{kHz}$

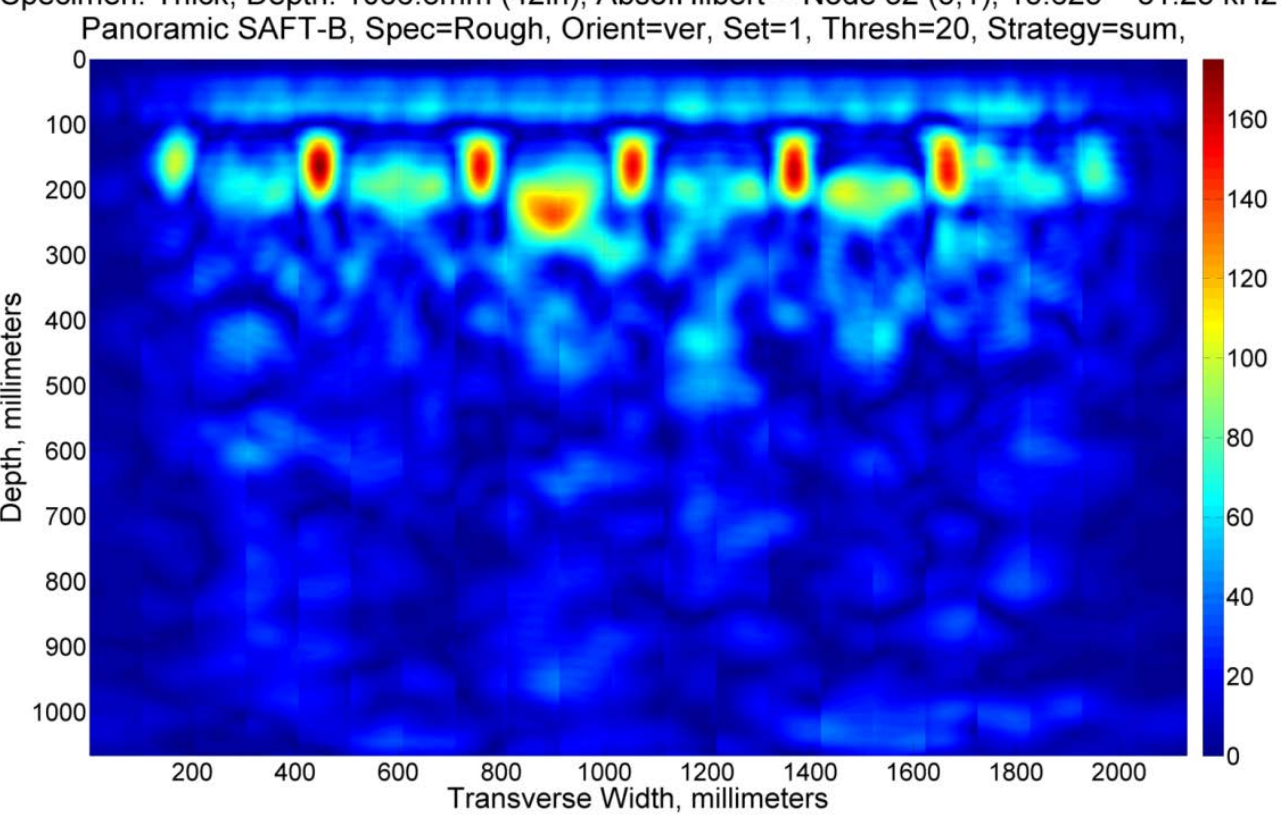

Fig. A. 99. Vertical Set 1, Node 32. 


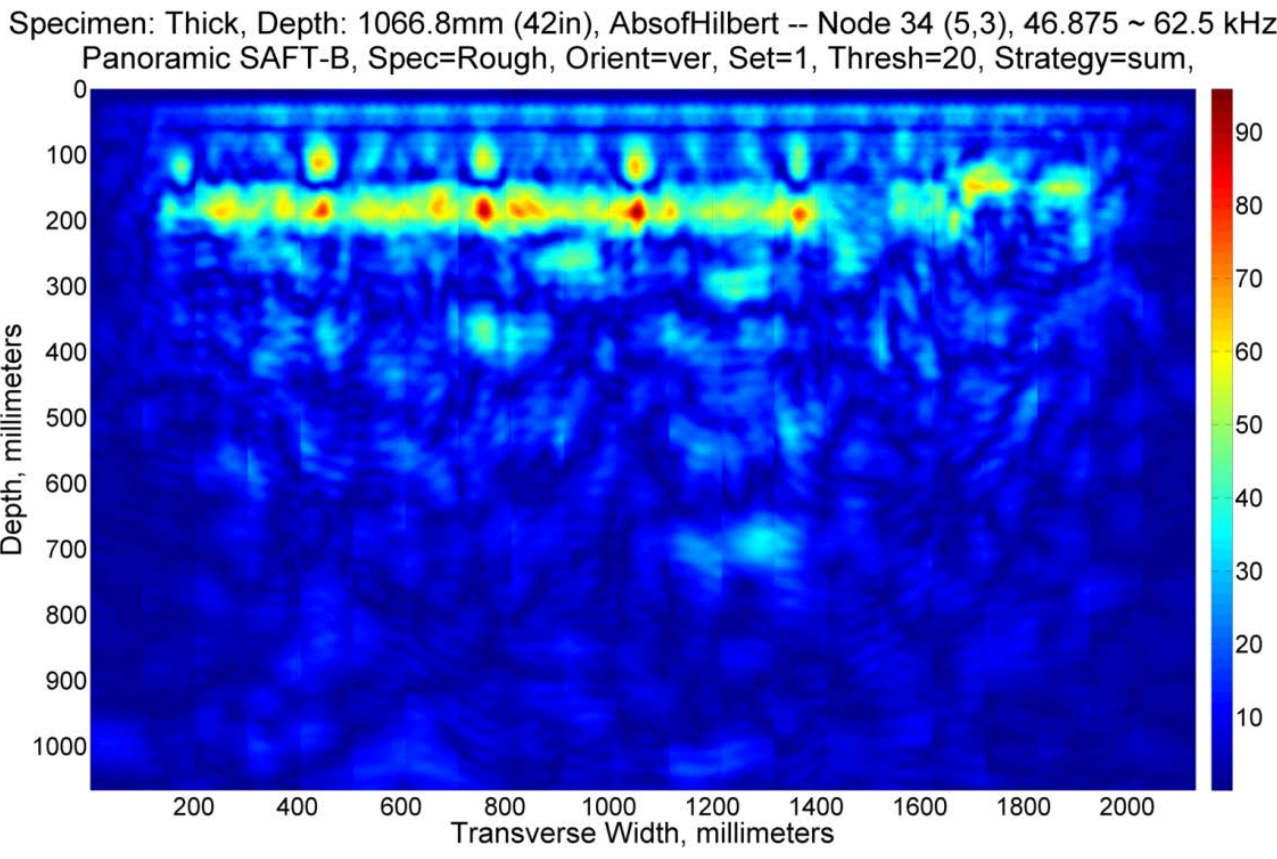

Fig. A. 100. Vertical Set 1, Node 34.

\section{SET 2 - DEFECT 18}

Specimen: Thick, Depth: 1066.8mm (42in), AbsofHilbert -- Node $0(0,0), 0 \sim 500 \mathrm{kHz}$ Panoramic SAFT-B, Spec=Rough, Orient=ver, Set=2, Thresh=20, Strategy=sum,

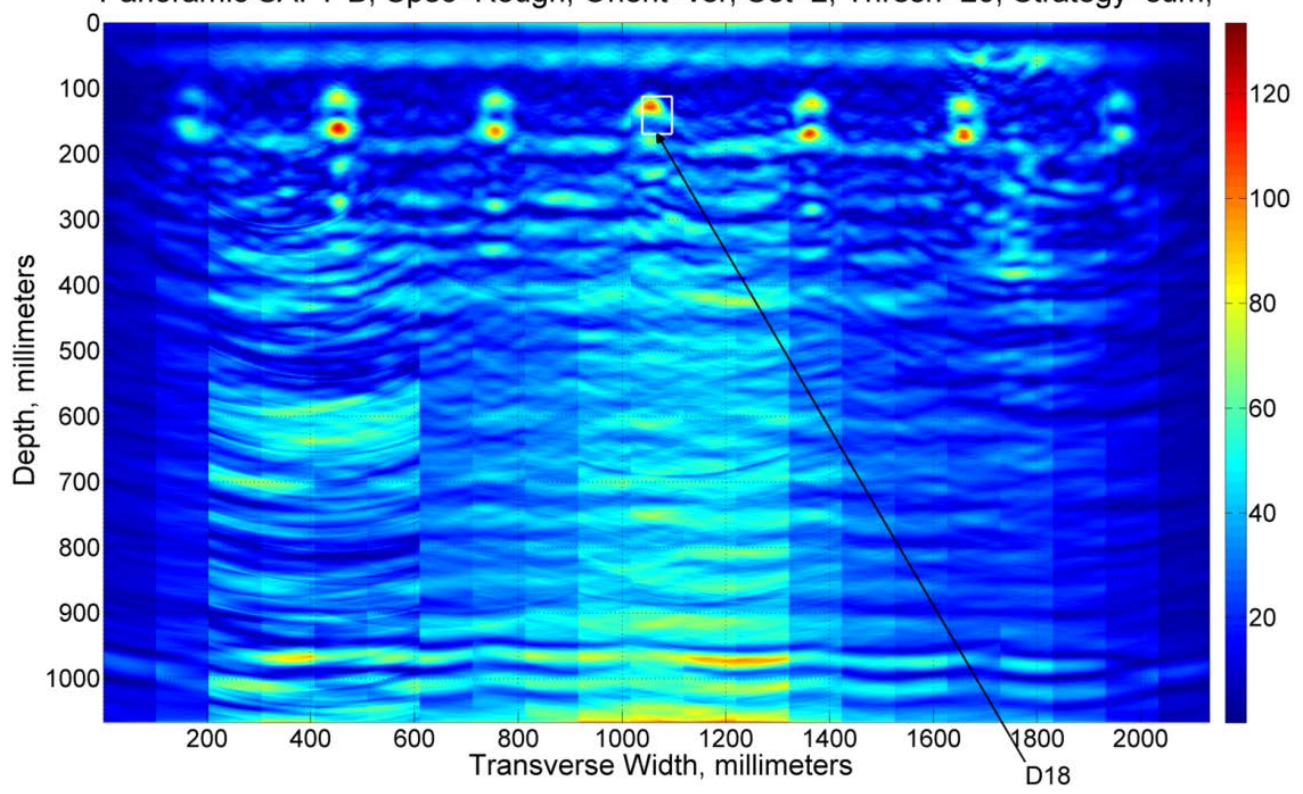

Fig. A. 101. Vertical Set 2, Node 0. 
Specimen: Thick, Depth: 1066.8mm (42in), AbsofHilbert -- Node $16(4,1), 31.25 \sim 62.5 \mathrm{kHz}$ Panoramic SAFT-B, Spec=Rough, Orient=ver, Set=2, Thresh=20, Strategy=sum,

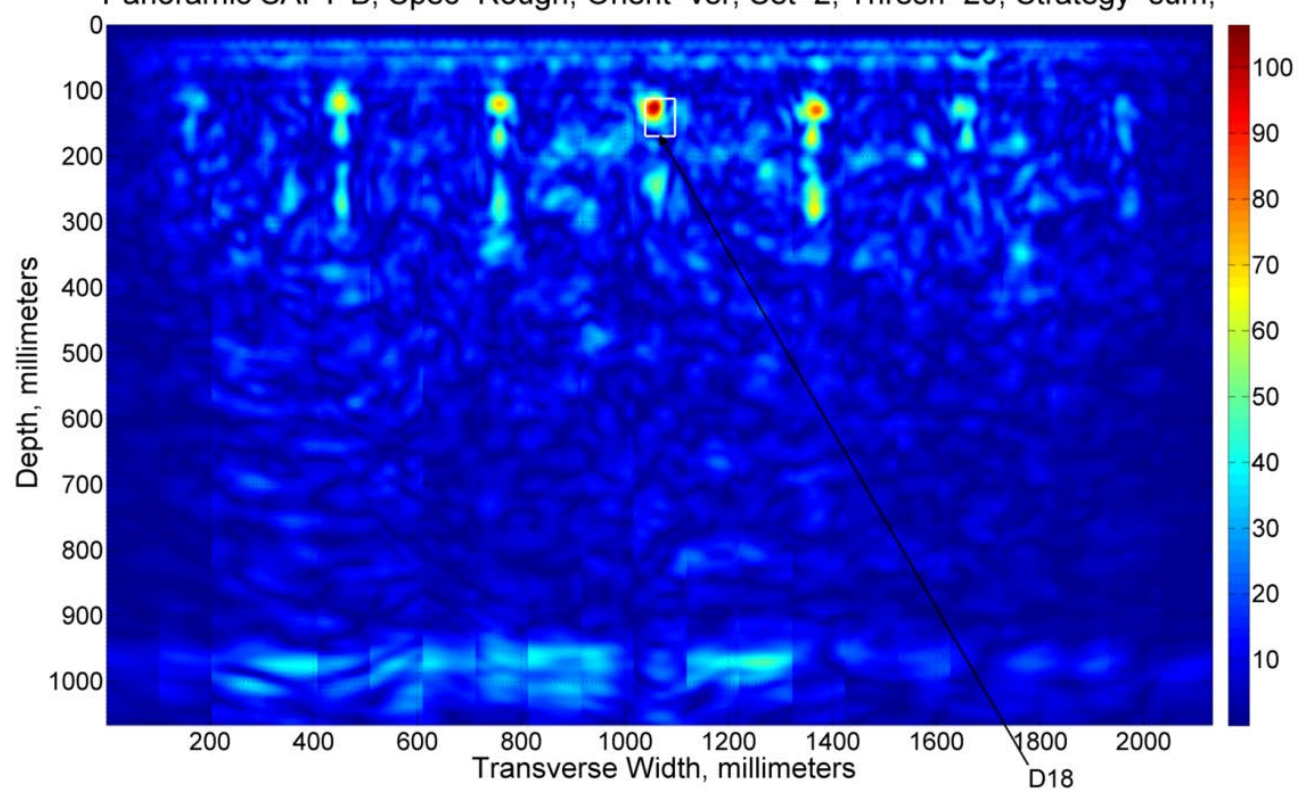

Fig. A. 102. Vertical Set 2, Node 16.

Specimen: Thick, Depth: 1066.8mm (42in), AbsofHilbert -- Node $18(4,3), 93.75 \sim 125 \mathrm{kHz}$ Panoramic SAFT-B, Spec=Rough, Orient=ver, Set=2, Thresh=20, Strategy=sum,

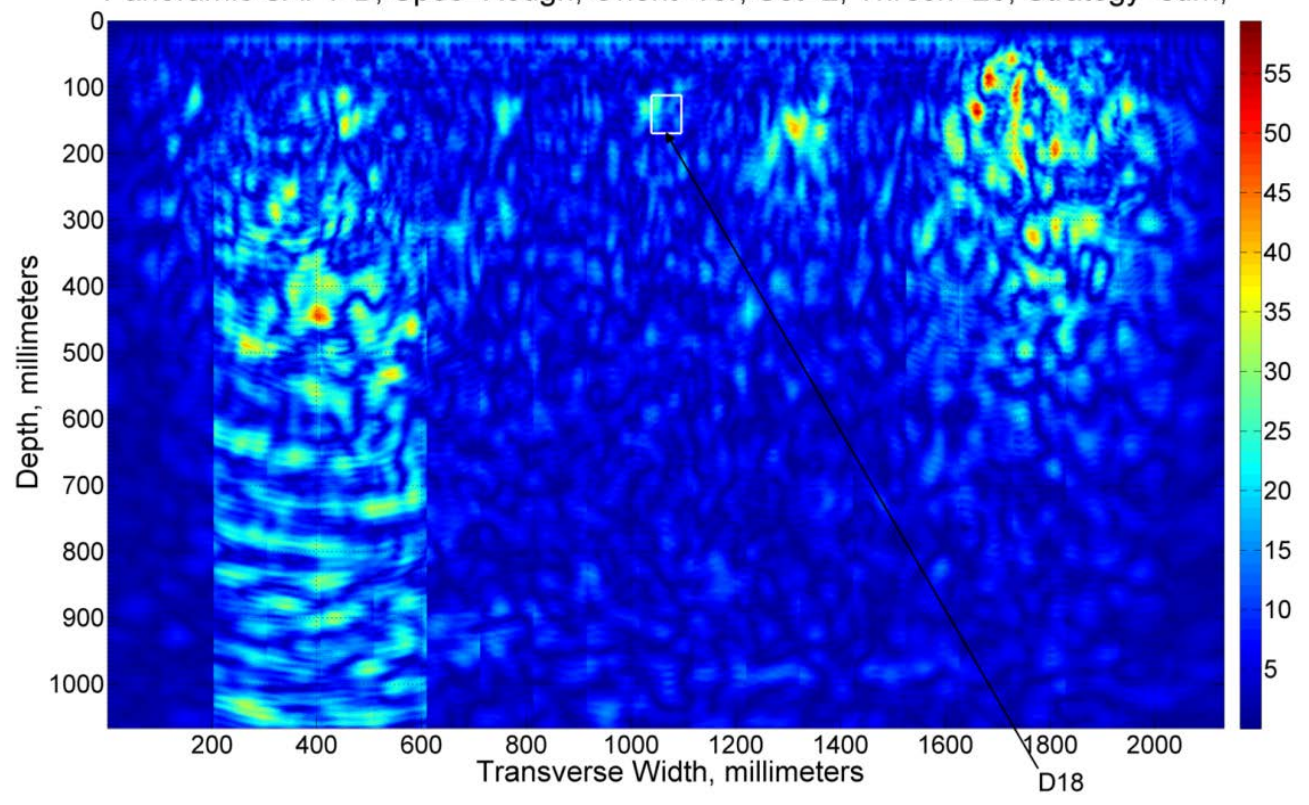

Fig. A. 103. Vertical Set 2, Node 18. 
Specimen: Thick, Depth: 1066.8mm (42in), AbsofHilbert -- Node 32 (5,1), $15.625 \sim 31.25 \mathrm{kHz}$ Panoramic SAFT-B, Spec=Rough, Orient=ver, Set=2, Thresh=20, Strategy=sum,

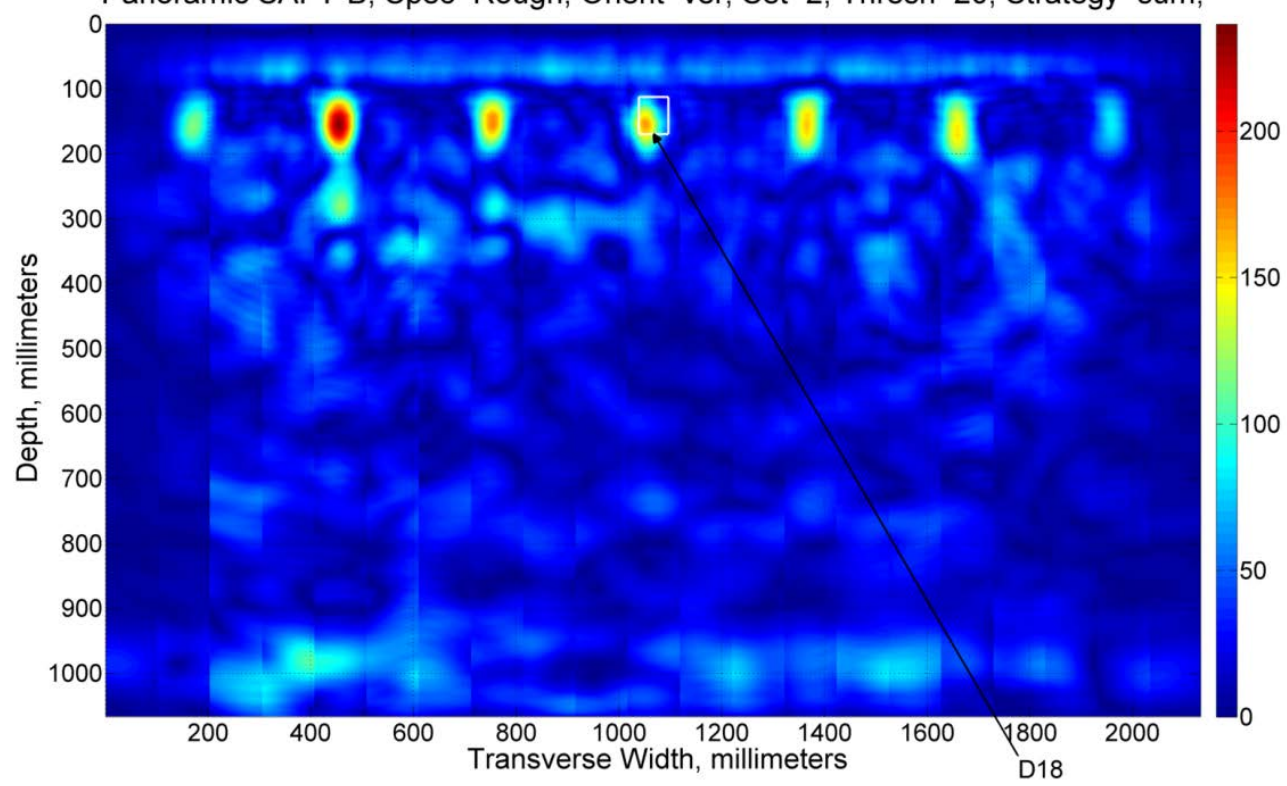

Fig. A. 104. Vertical Set 2, Node 32.

Specimen: Thick, Depth: 1066.8mm (42in), AbsofHilbert -- Node $34(5,3), 46.875 \sim 62.5 \mathrm{kHz}$ Panoramic SAFT-B, Spec=Rough, Orient=ver, Set=2, Thresh=20, Strategy=sum,

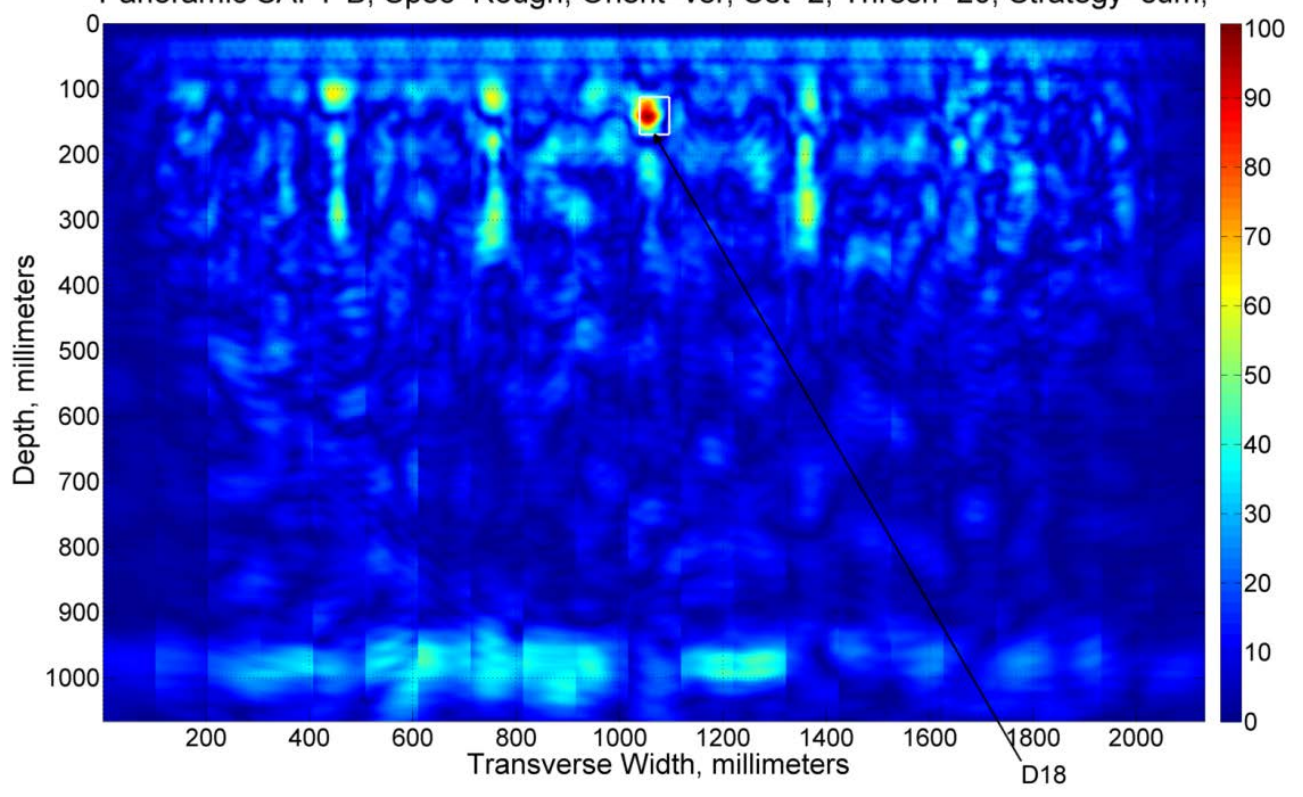

Fig. A. 105. Vertical Set 2, Node 34. 


\section{SET 3 - DEFECT 16, DEFECT 19}

Specimen: Thick, Depth: 1066.8mm (42in), AbsofHilbert -- Node $0(0,0), 0 \sim 500 \mathrm{kHz}$ Panoramic SAFT-B, Spec=Rough, Orient=ver, Set=3, Thresh=20, Strategy=sum,

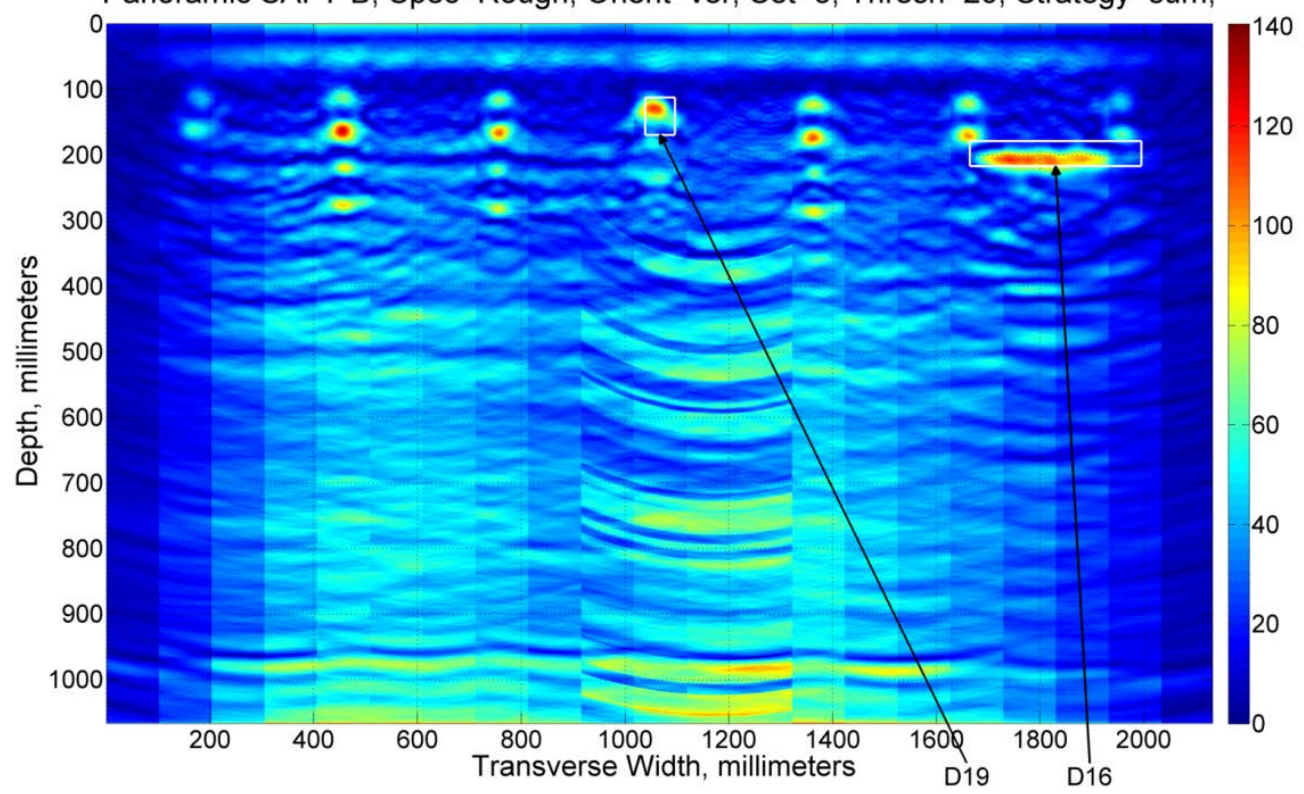

Fig. A. 106. Vertical Set 3, Node 0.

Specimen: Thick, Depth: 1066.8mm (42in), AbsofHilbert -- Node $16(4,1), 31.25 \sim 62.5 \mathrm{kHz}$ Panoramic SAFT-B, Spec=Rough, Orient=ver, Set=3, Thresh=20, Strategy=sum,

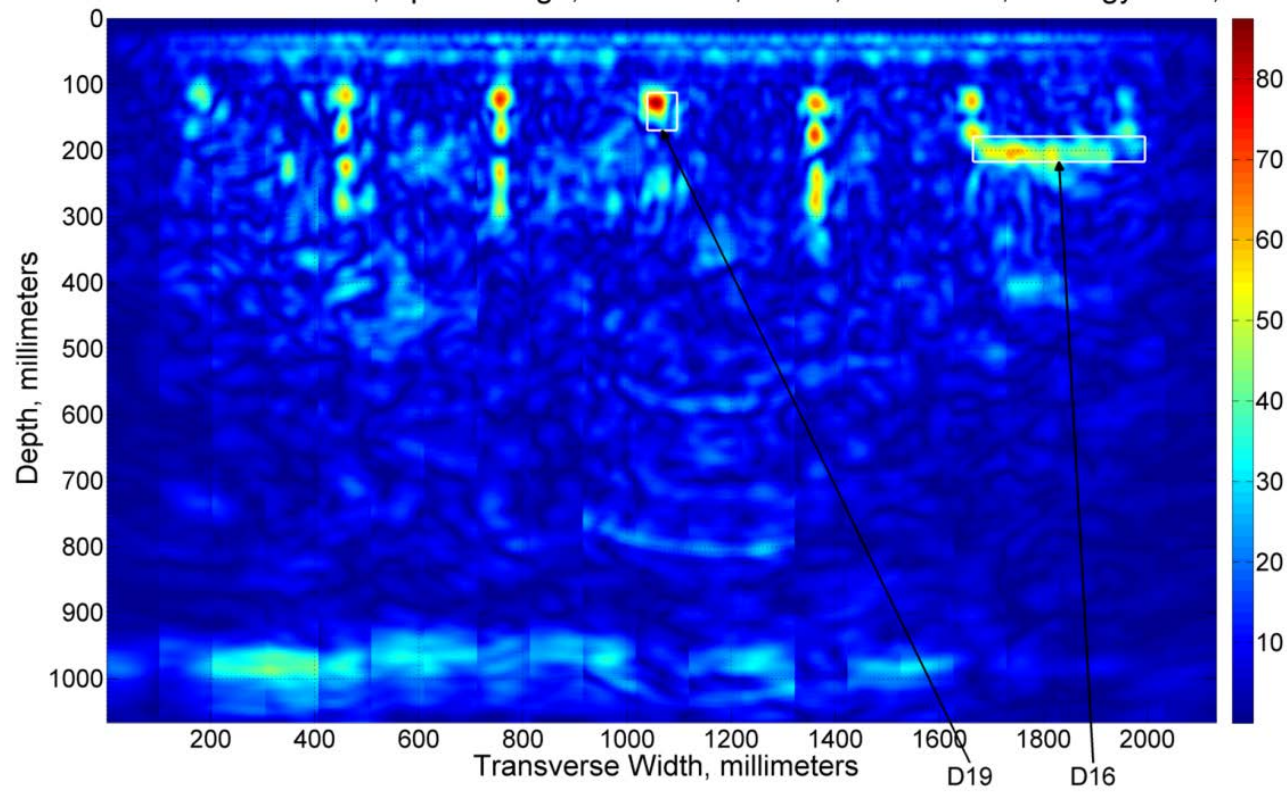

Fig. A. 107. Vertical Set 3, Node 16. 
Specimen: Thick, Depth: 1066.8mm (42in), AbsofHilbert -- Node $18(4,3), 93.75 \sim 125 \mathrm{kHz}$

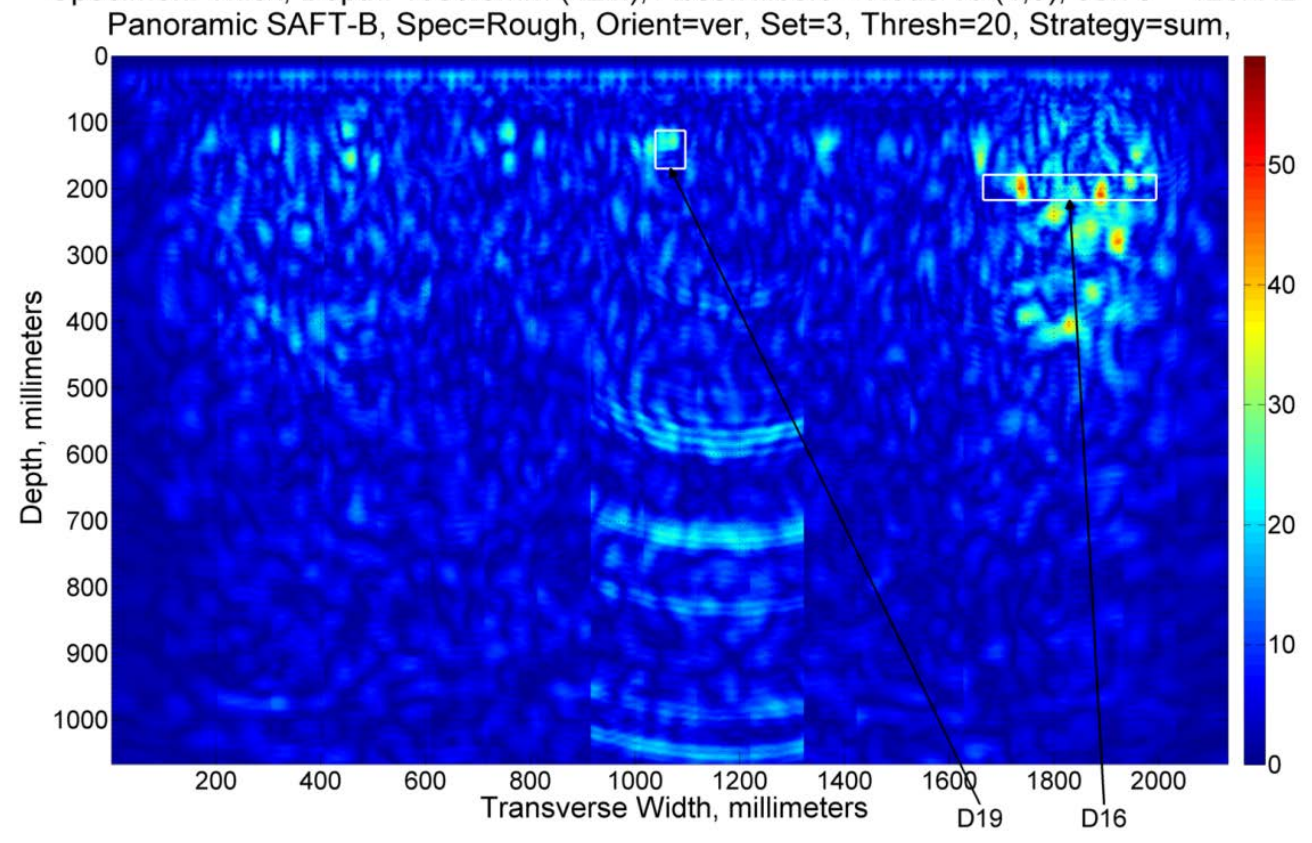

Fig. A. 108. Vertical Set 3, Node 18.

Specimen: Thick, Depth: 1066.8mm (42in), AbsofHilbert -- Node 32 (5,1), $15.625 \sim 31.25 \mathrm{kHz}$

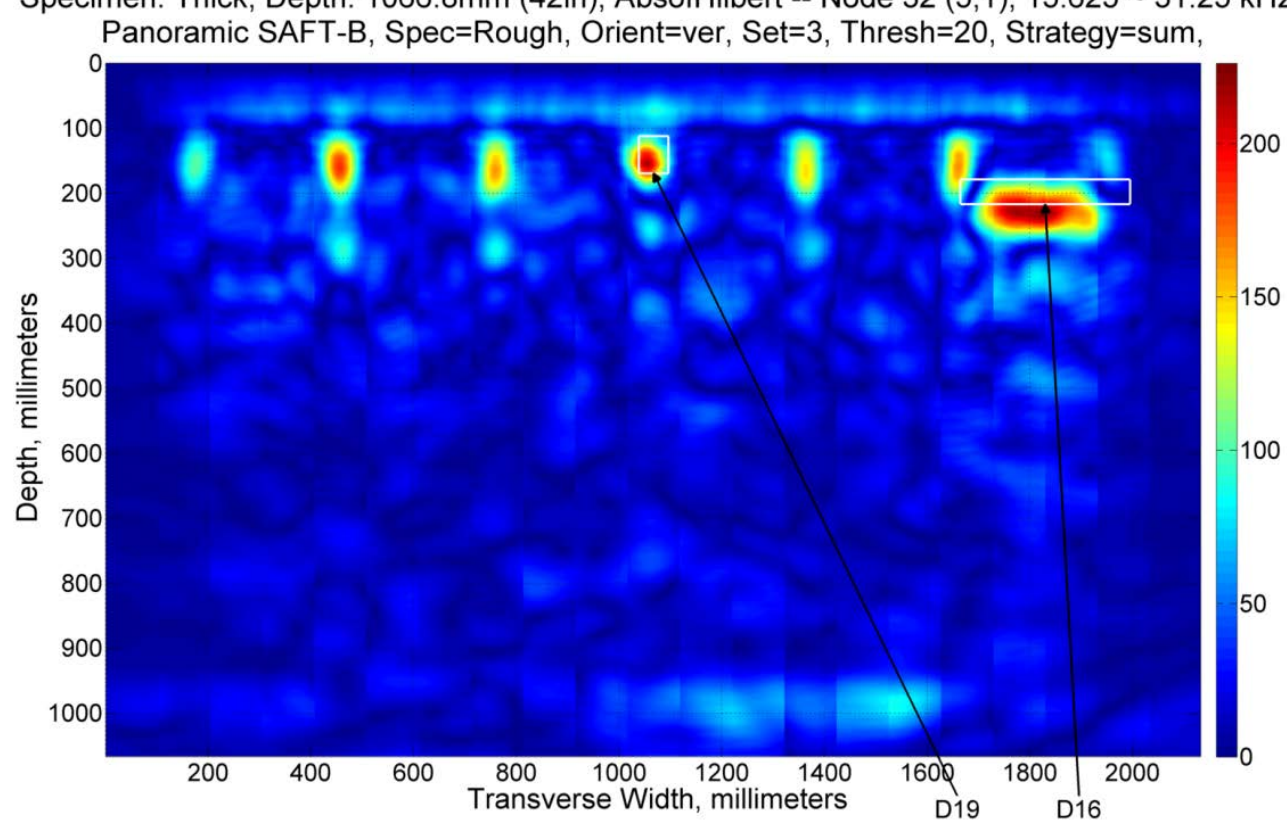

Fig. A. 109. Vertical Set 3, Node 32. 


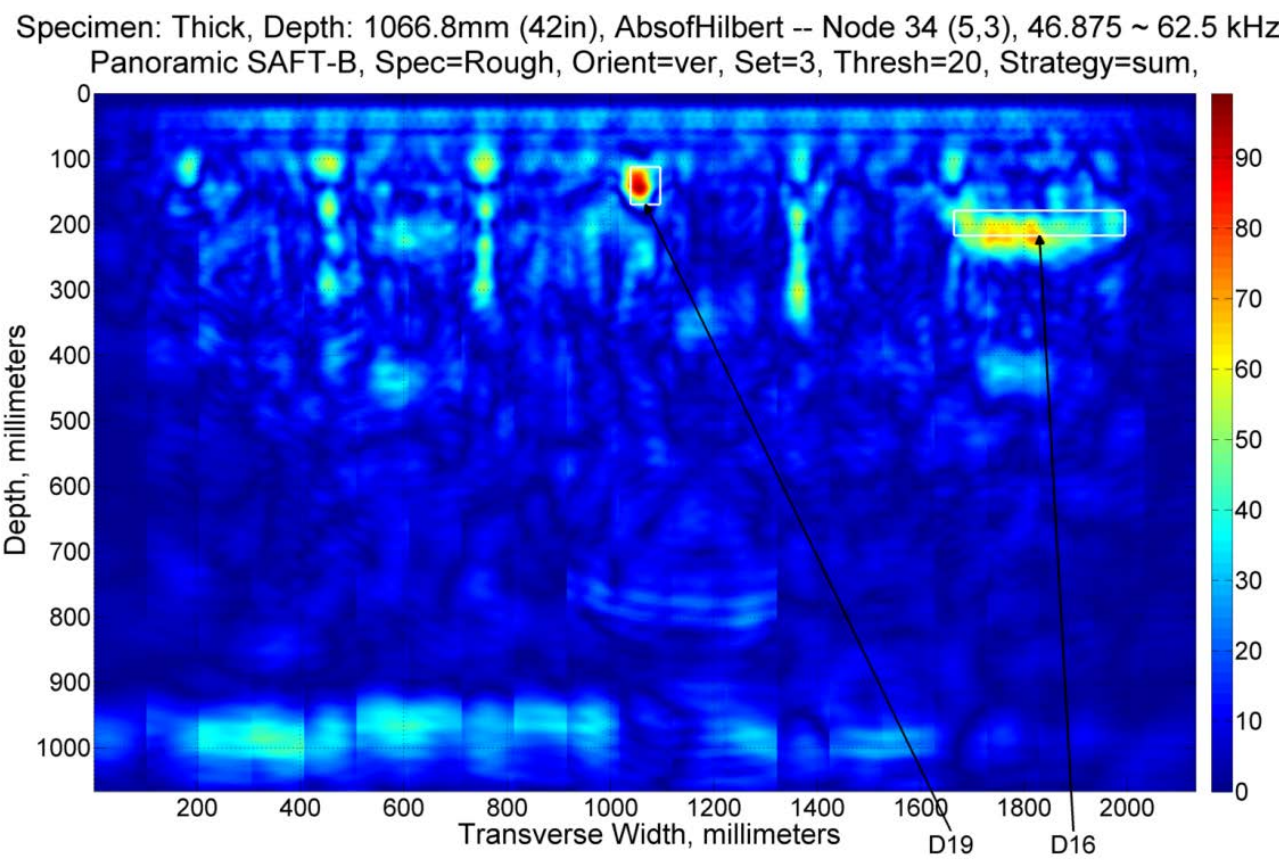

Fig. A. 110. Vertical Set 3, Node 34.

\section{SET 4 - NO DEFECT}

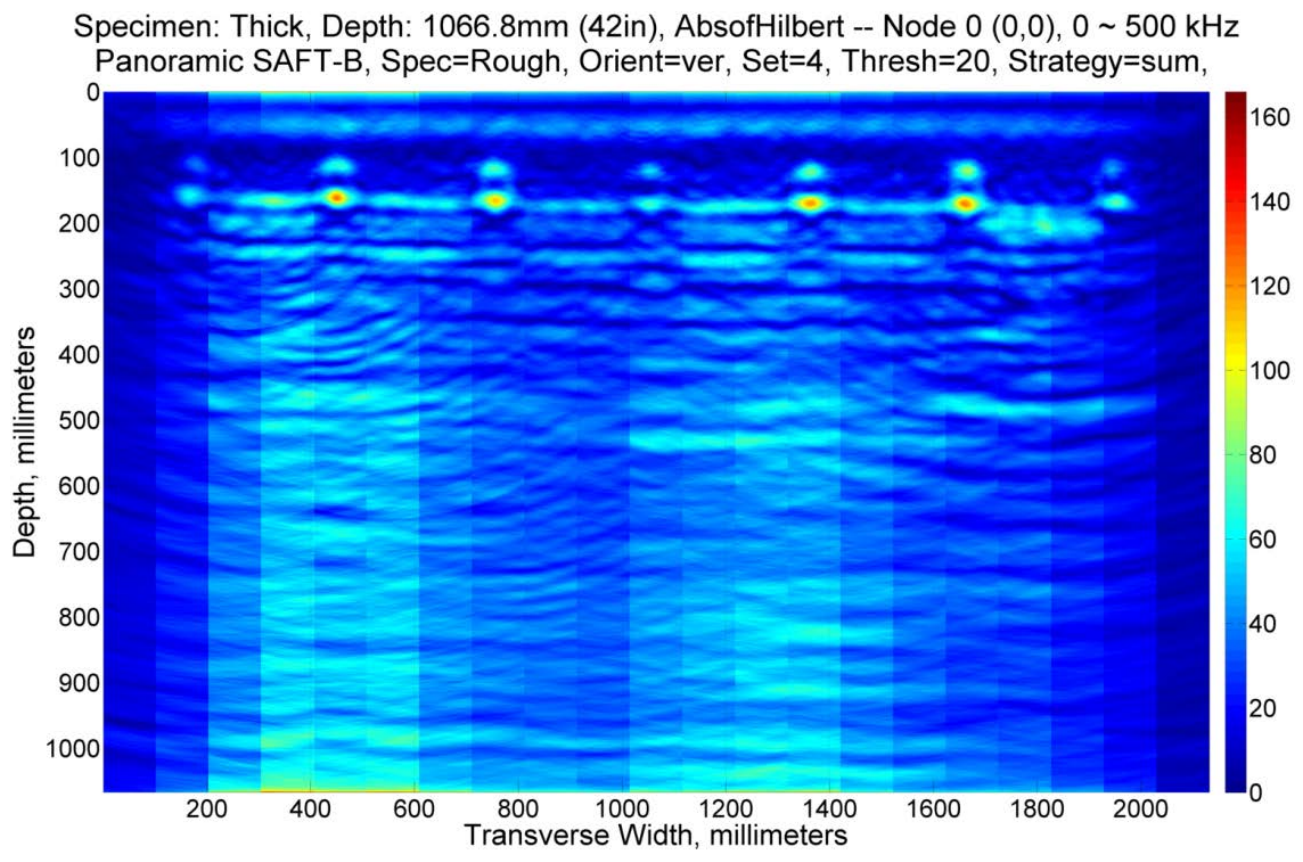

Fig. A. 111. Vertical Set 4, Node 0. 


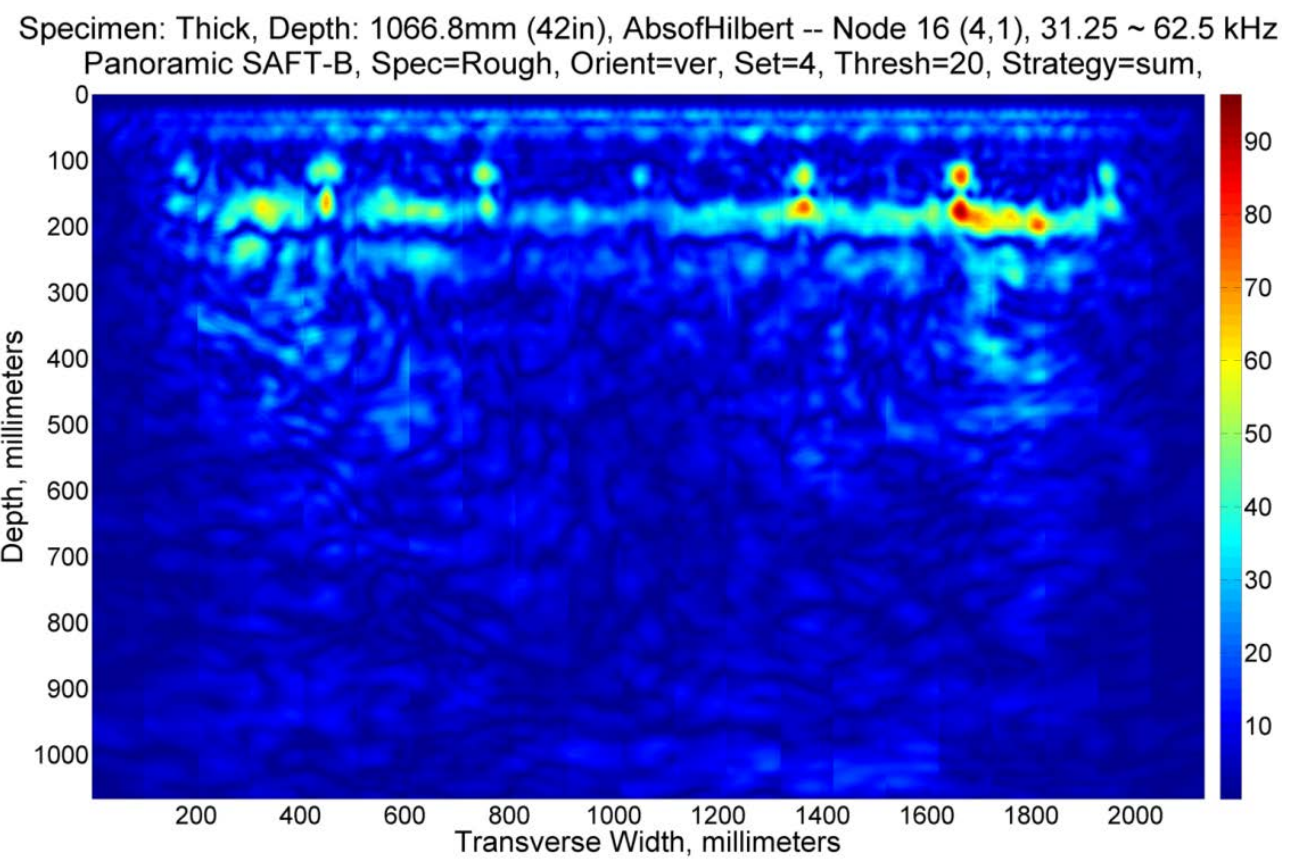

Fig. A. 112. Vertical Set 4, Node 16.

Specimen: Thick, Depth: 1066.8mm (42in), AbsofHilbert -- Node $18(4,3), 93.75 \sim 125 \mathrm{kHz}$

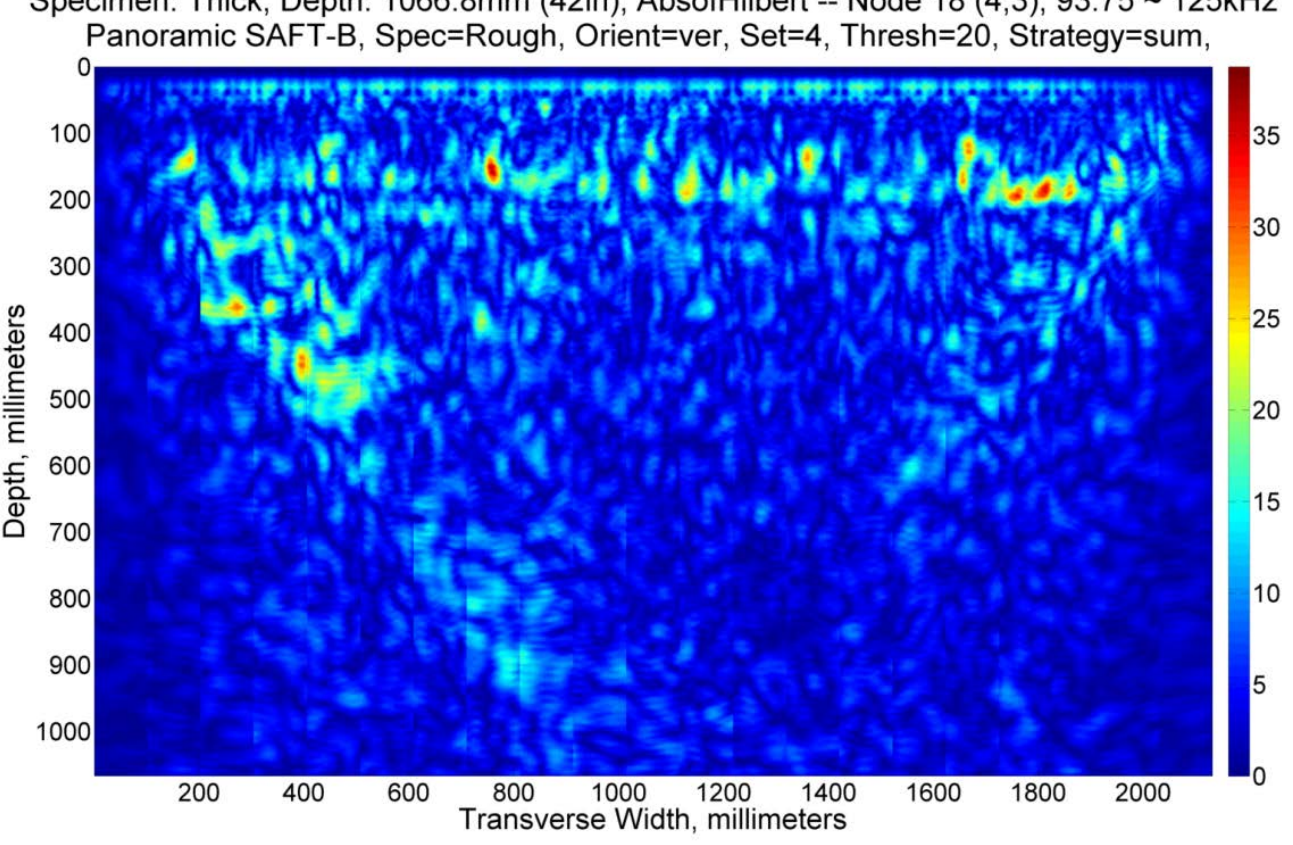

Fig. A. 113. Vertical Set 4, Node 18. 
Specimen: Thick, Depth: 1066.8mm (42in), AbsofHilbert -- Node 32 (5,1), $15.625 \sim 31.25 \mathrm{kHz}$ Panoramic SAFT-B, Spec=Rough, Orient=ver, Set=4, Thresh=20, Strategy=sum

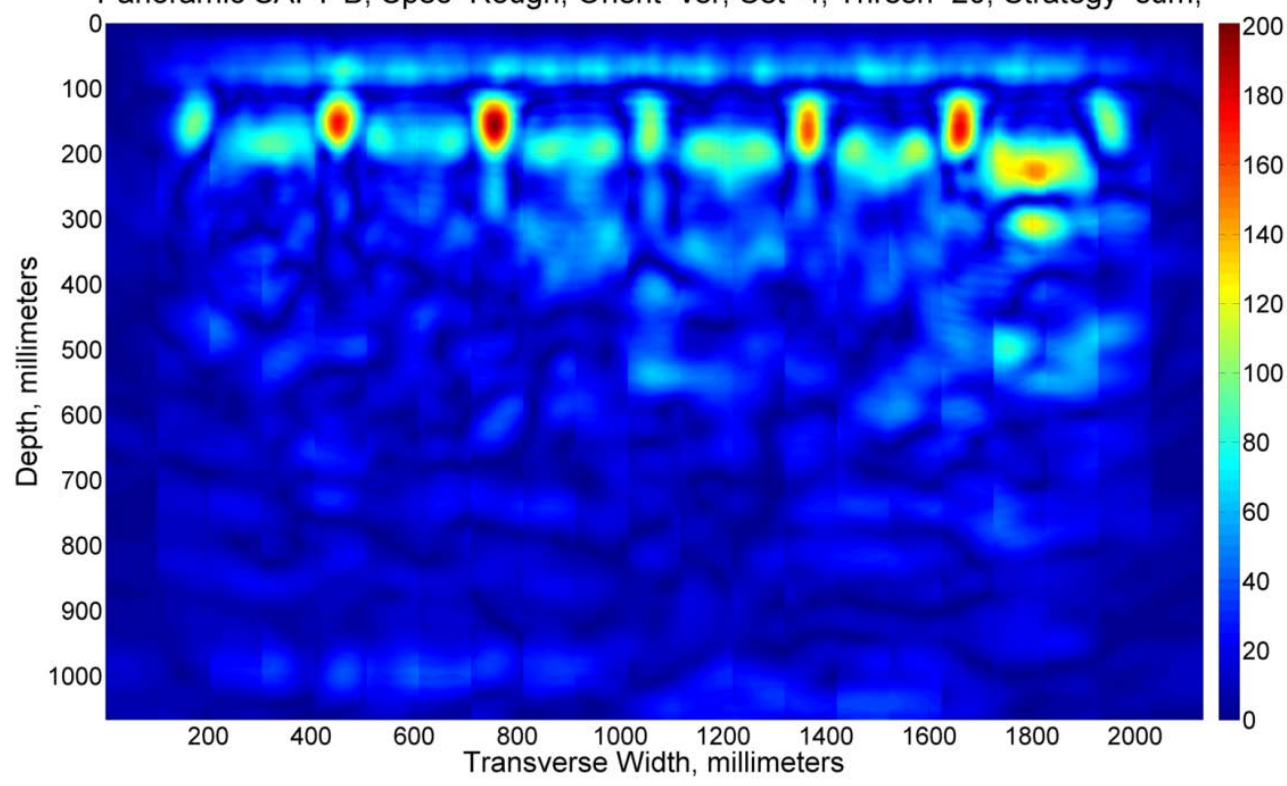

Fig. A. 114. Vertical Set 4, Node 32.

Specimen: Thick, Depth: 1066.8mm (42in), AbsofHilbert -- Node $34(5,3), 46.875 \sim 62.5 \mathrm{kHz}$ Panoramic SAFT-B, Spec=Rough, Orient=ver, Set=4, Thresh=20, Strategy=sum,

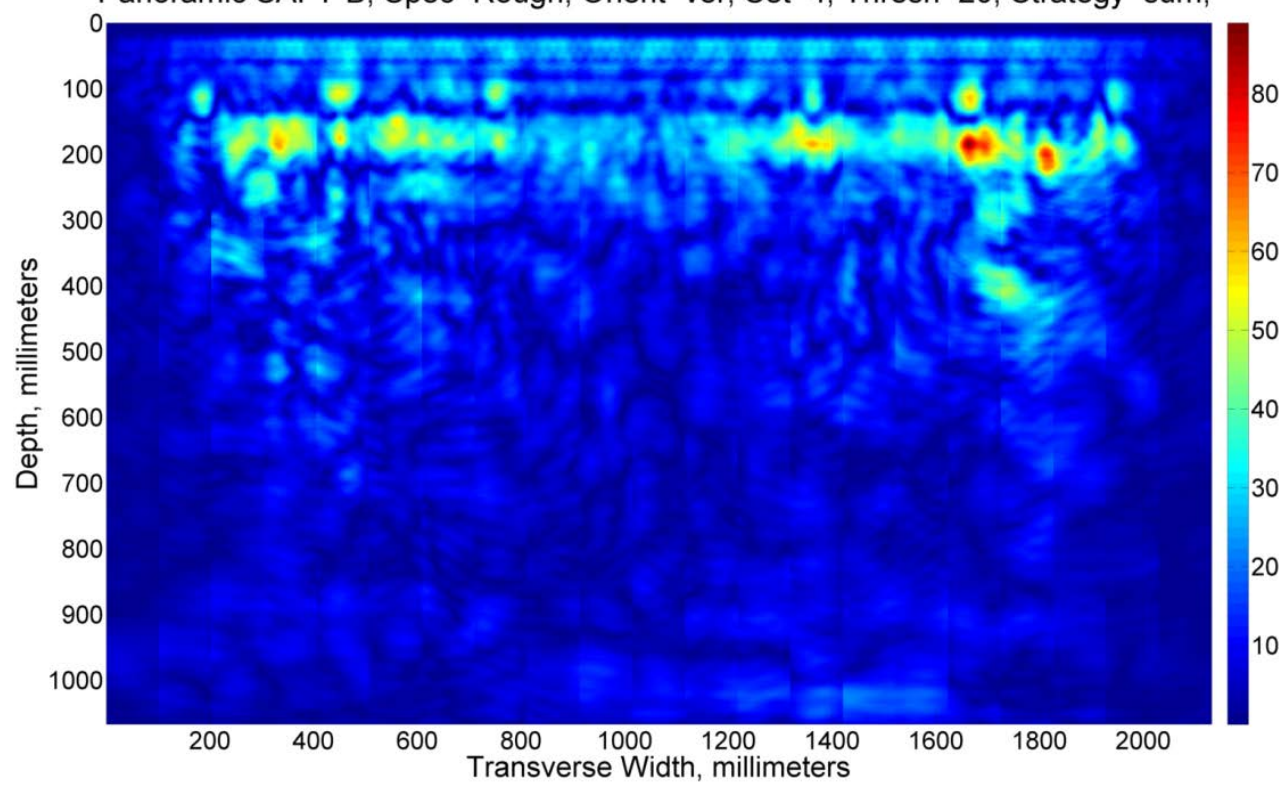

Fig. A. 115. Vertical Set 4, Node 34. 


\section{SET 5 - DEFECT 4, DEFECT 14}

Specimen: Thick, Depth: 1066.8mm (42in), AbsofHilbert -- Node $0(0,0), 0 \sim 500 \mathrm{kHz}$ Panoramic SAFT-B, Spec=Rough, Orient=ver, Set=5, Thresh=20, Strategy=sum,

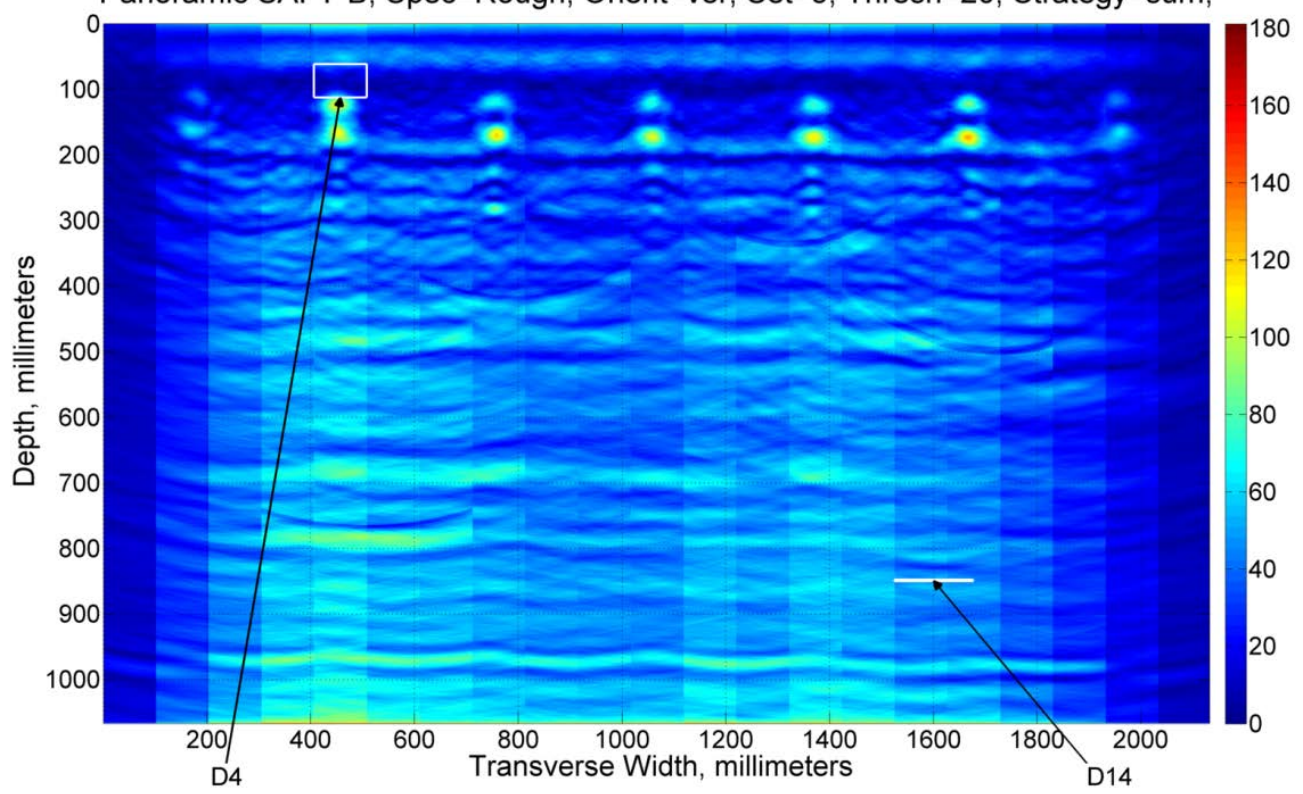

Fig. A. 116. Vertical Set 5, Node 0 .

Specimen: Thick, Depth: 1066.8mm (42in), AbsofHilbert -- Node $16(4,1), 31.25 \sim 62.5 \mathrm{kHz}$ Panoramic SAFT-B, Spec=Rough, Orient=ver, Set=5, Thresh=20, Strategy=sum,

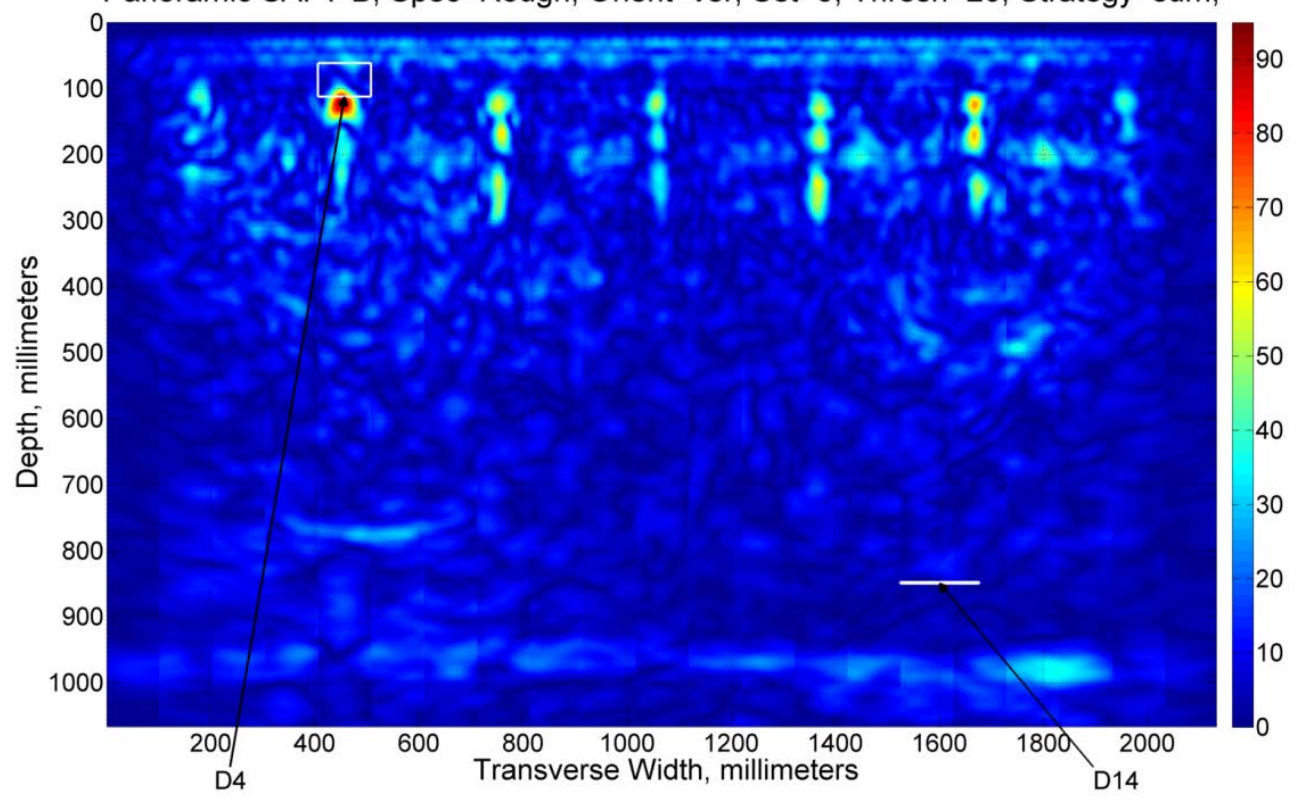

Fig. A. 117. Vertical Set 5, Node 16. 


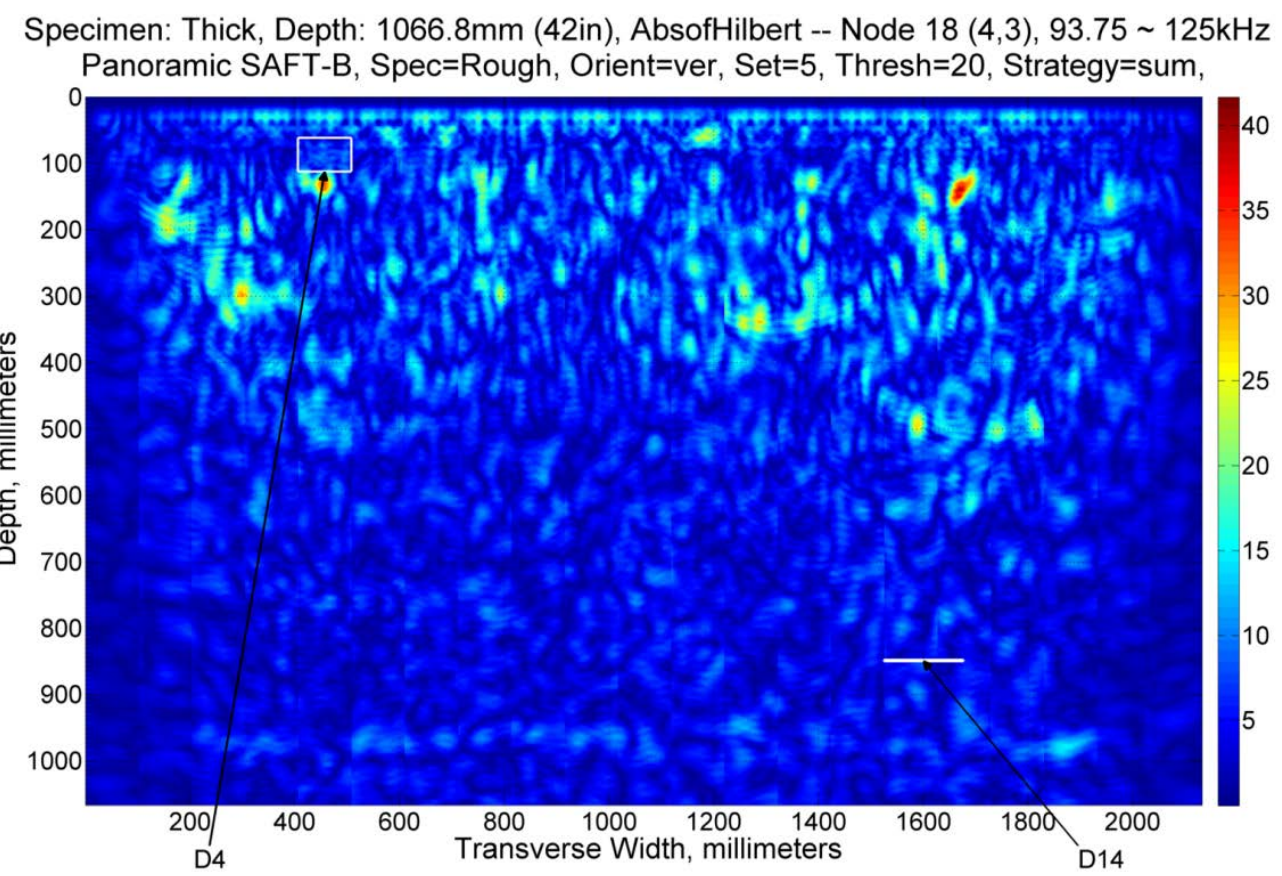

Fig. A. 118. Vertical Set 5, Node 18.

Specimen: Thick, Depth: 1066.8mm (42in), AbsofHilbert -- Node 32 (5,1), $15.625 \sim 31.25 \mathrm{kHz}$

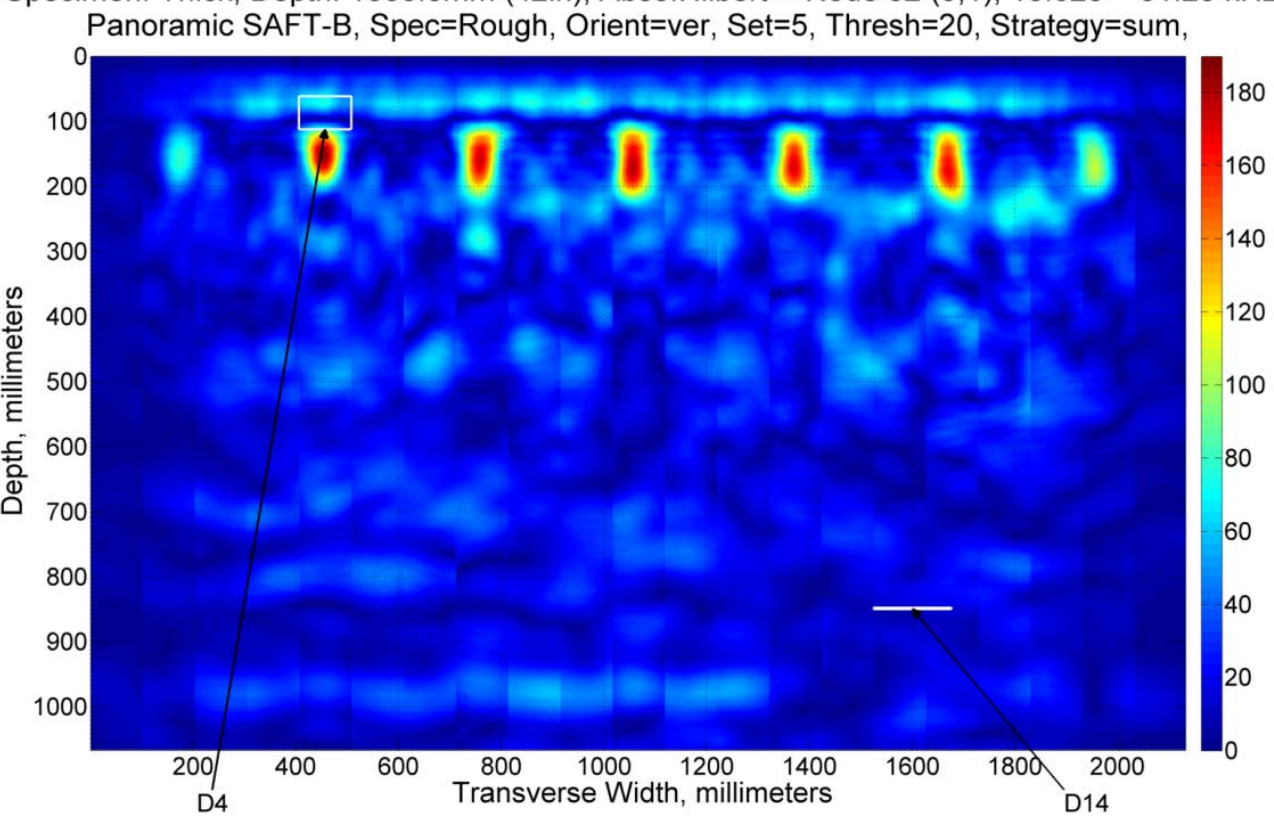

Fig. A. 119. Vertical Set 5, Node 32. 
Specimen: Thick, Depth: 1066.8mm (42in), AbsofHilbert -- Node $34(5,3), 46.875 \sim 62.5 \mathrm{kHz}$ Panoramic SAFT-B, Spec=Rough, Orient=ver, Set=5, Thresh=20, Strategy=sum,

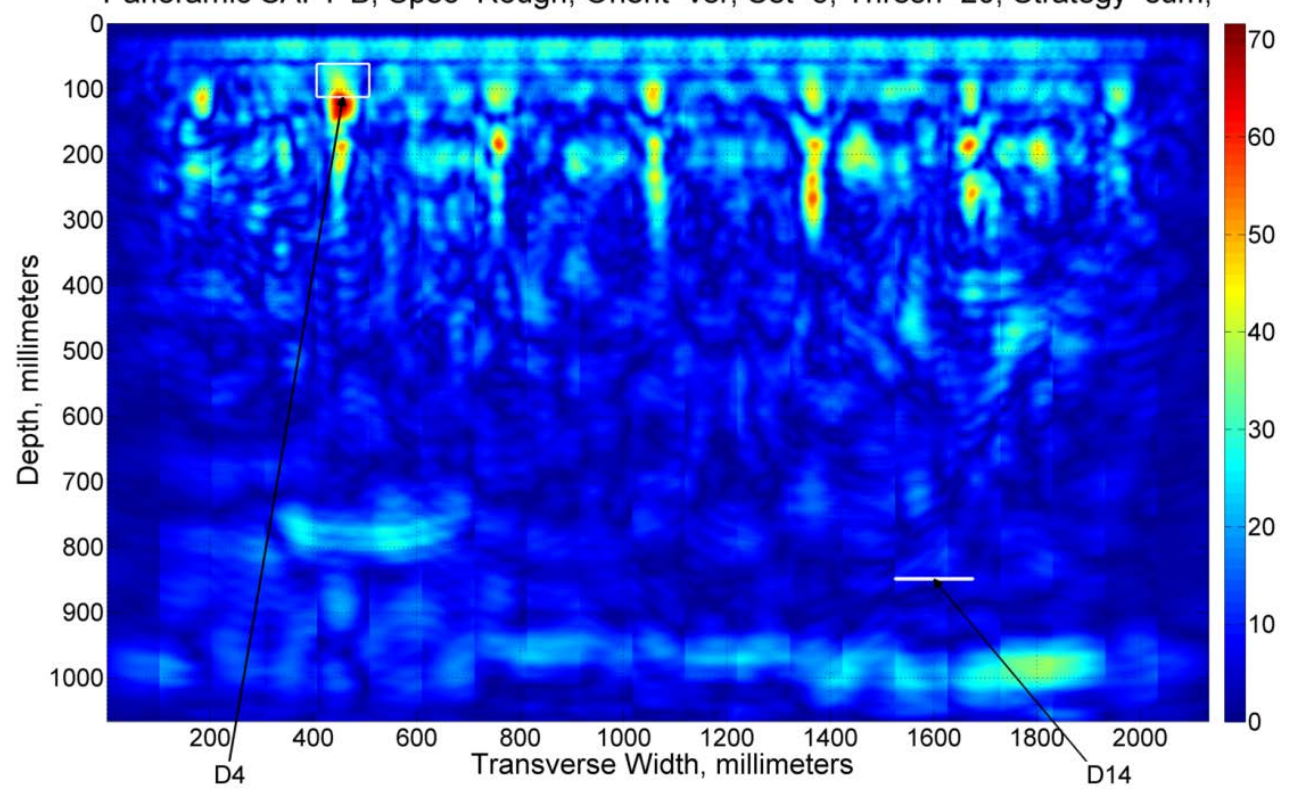

Fig. A. 120. Vertical Set 5, Node 34.

\section{SET 6 - DEFECT 4, DEFECT 7}

Specimen: Thick, Depth: 1066.8mm (42in), AbsofHilbert -- Node $0(0,0), 0 \sim 500 \mathrm{kHz}$ Panoramic SAFT-B, Spec=Rough, Orient=ver, Set=6, Thresh=20, Strategy=sum,

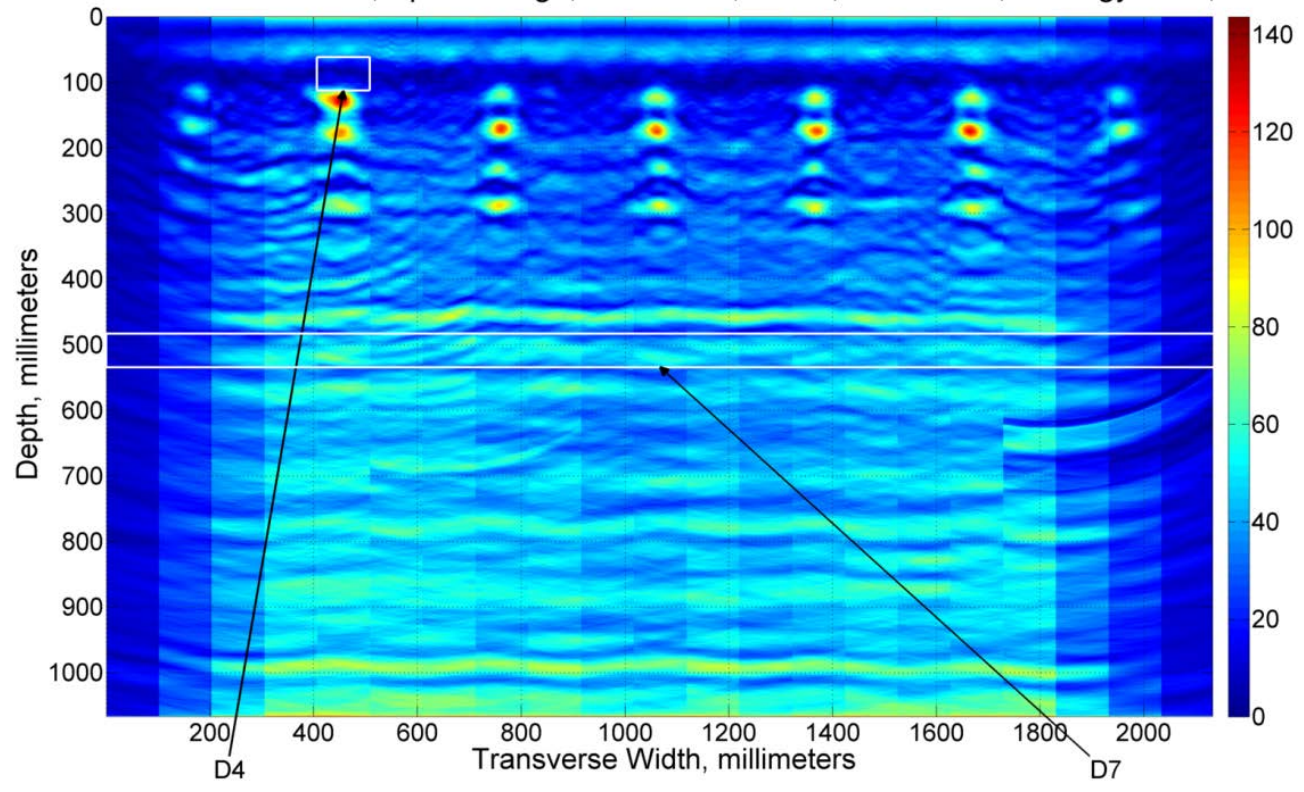

Fig. A. 121. Vertical Set 6, Node 0. 
Specimen: Thick, Depth: 1066.8mm (42in), AbsofHilbert -- Node $16(4,1), 31.25 \sim 62.5 \mathrm{kHz}$ Panoramic SAFT-B, Spec=Rough, Orient=ver, Set=6, Thresh=20, Strategy=sum,

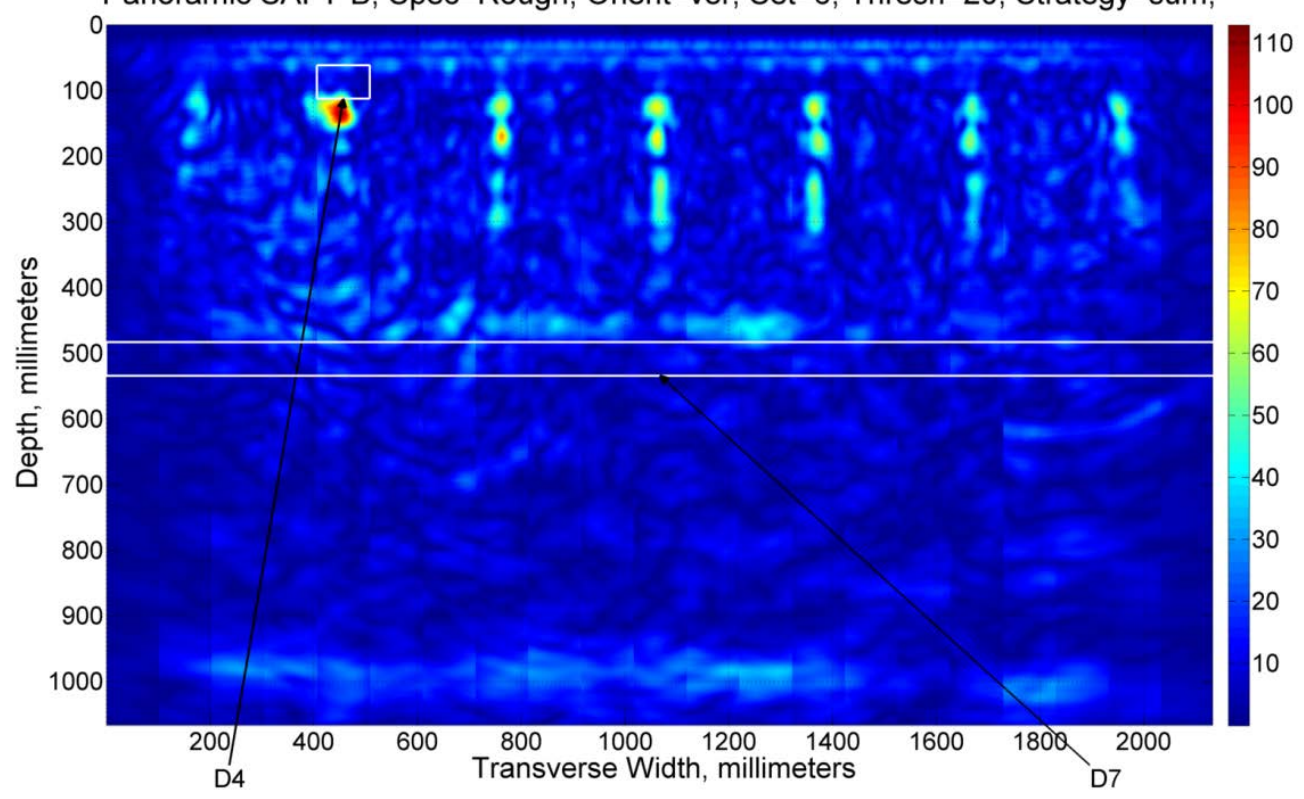

Fig. A. 122. Vertical Set 6, Node 16.

Specimen: Thick, Depth: 1066.8mm (42in), AbsofHilbert -- Node $18(4,3), 93.75 \sim 125 \mathrm{kHz}$ Panoramic SAFT-B, Spec=Rough, Orient=ver, Set=6, Thresh=20, Strategy=sum,

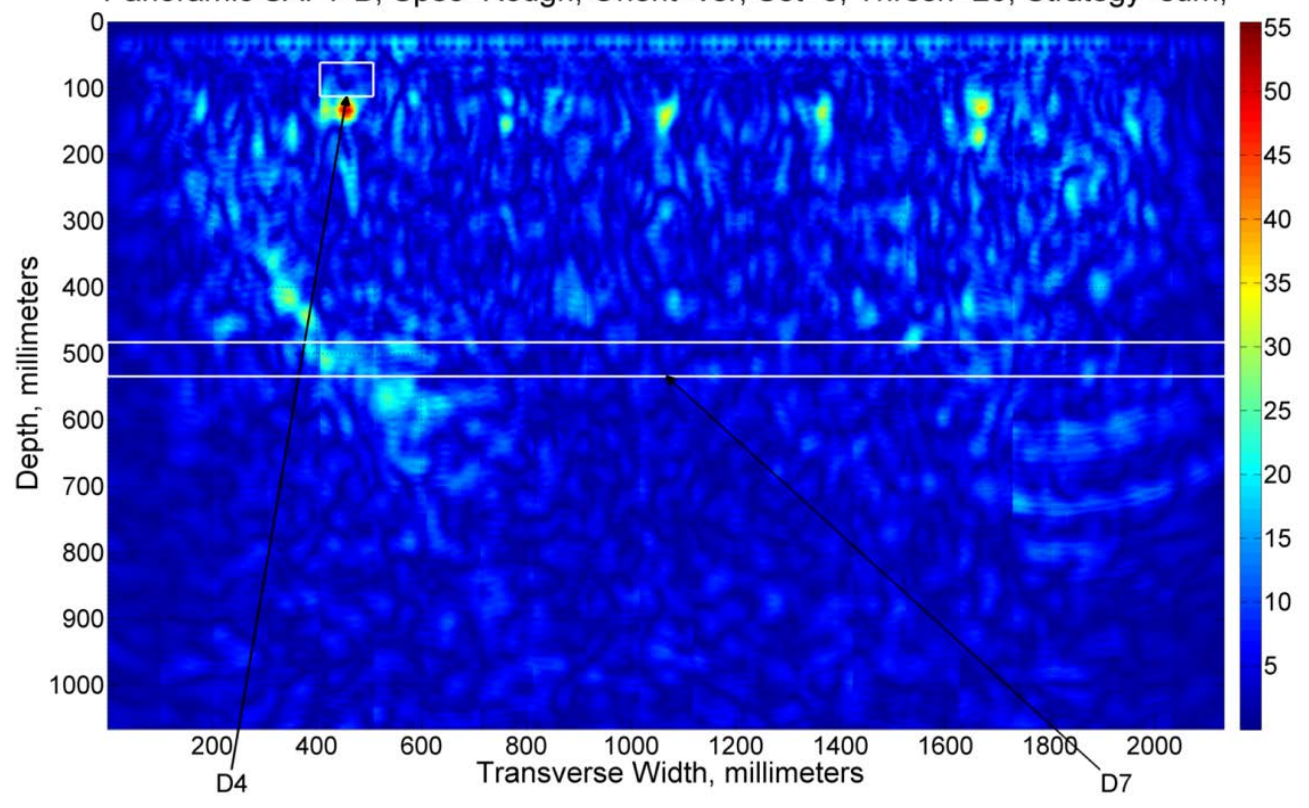

Fig. A. 123. Vertical Set 6, Node 18. 


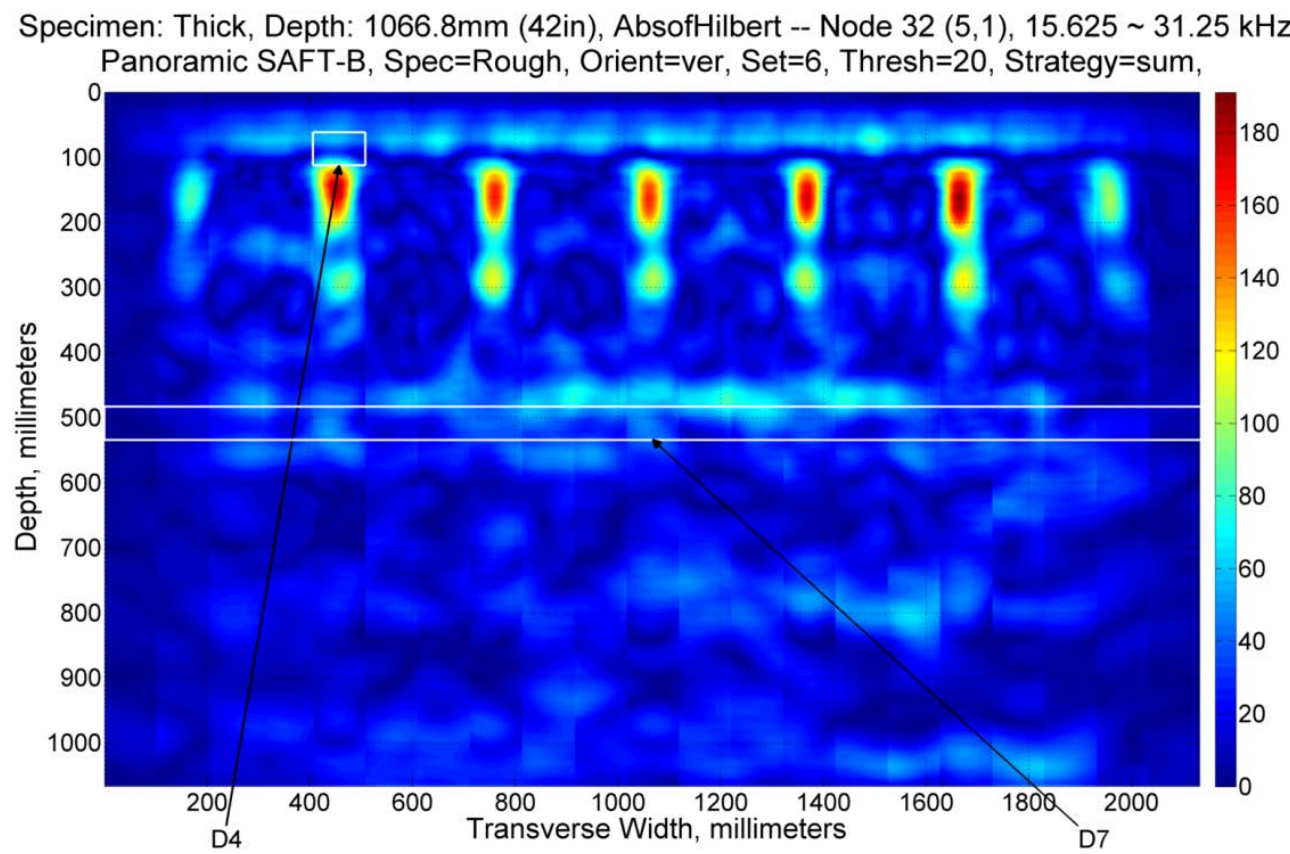

Fig. A. 124. Vertical Set 6, Node 32.

Specimen: Thick, Depth: 1066.8mm (42in), AbsofHilbert -- Node $34(5,3), 46.875 \sim 62.5 \mathrm{kHz}$ Panoramic SAFT-B, Spec=Rough, Orient=ver, Set=6, Thresh=20, Strategy=sum,

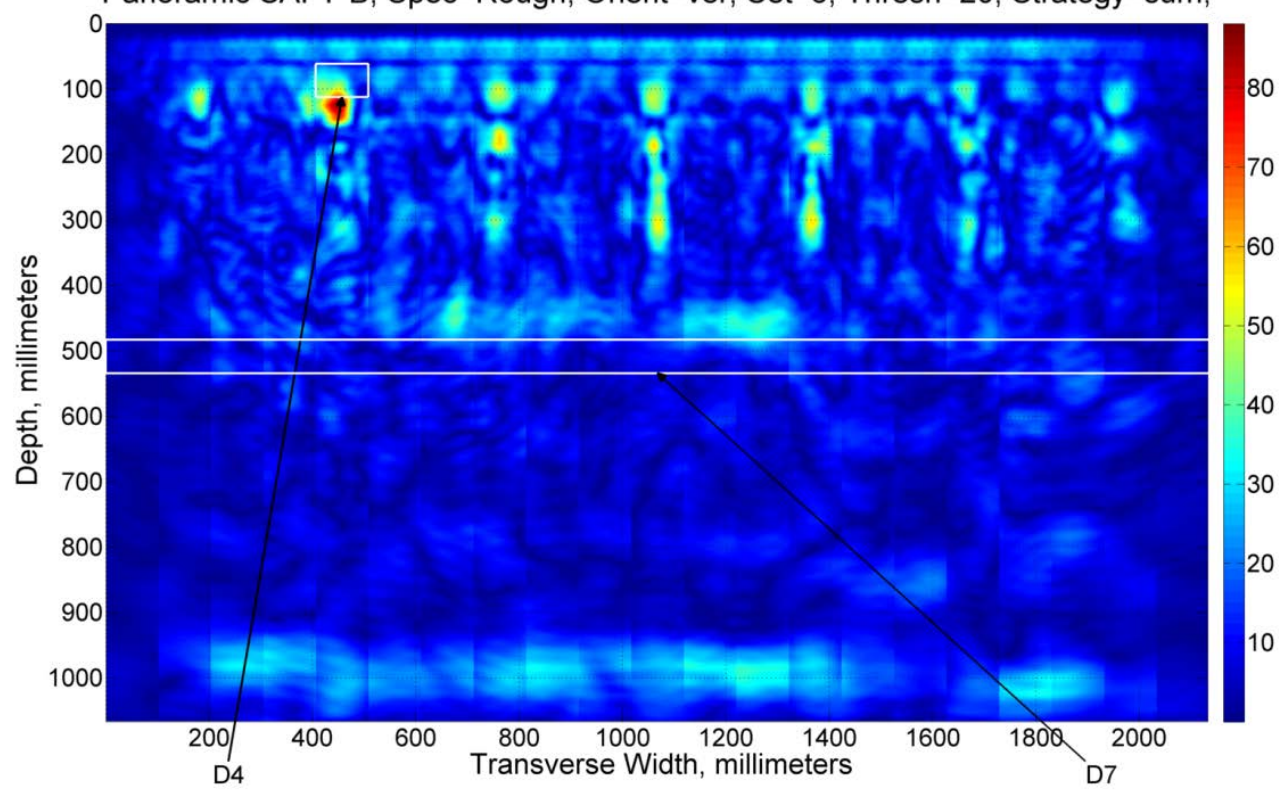

Fig. A. 125. Vertical Set 6, Node 34. 


\section{SET 7 - DEFECT 2, DEFECT 7, DEFECT 17}

Specimen: Thick, Depth: 1066.8mm (42in), AbsofHilbert -- Node $0(0,0), 0 \sim 500 \mathrm{kHz}$ Panoramic SAFT-B, Spec=Rough, Orient=ver, Set=7, Thresh=20, Strategy=sum,

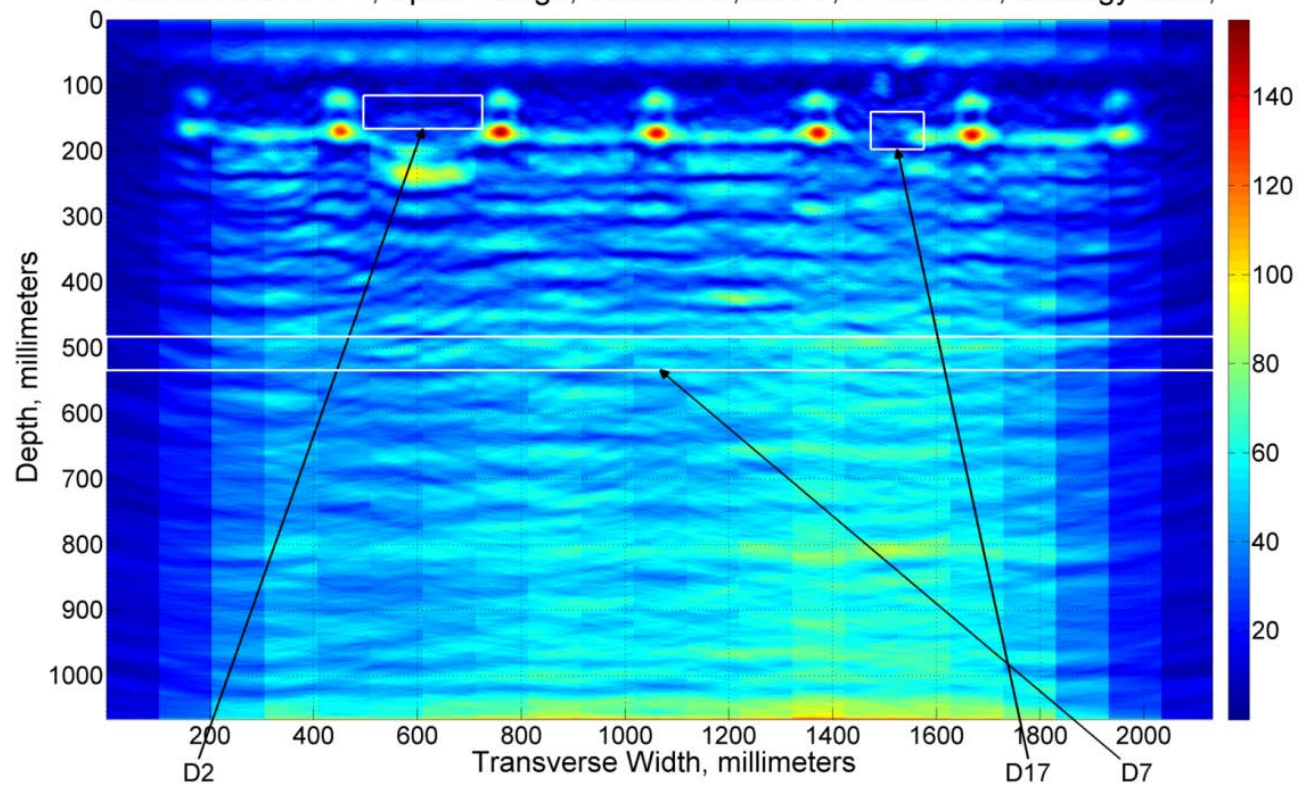

Fig. A. 126. Vertical Set 7, Node 0.

Specimen: Thick, Depth: 1066.8mm (42in), AbsofHilbert -- Node $16(4,1), 31.25 \sim 62.5 \mathrm{kHz}$ Panoramic SAFT-B, Spec=Rough, Orient=ver, Set=7, Thresh=20, Strategy=sum,

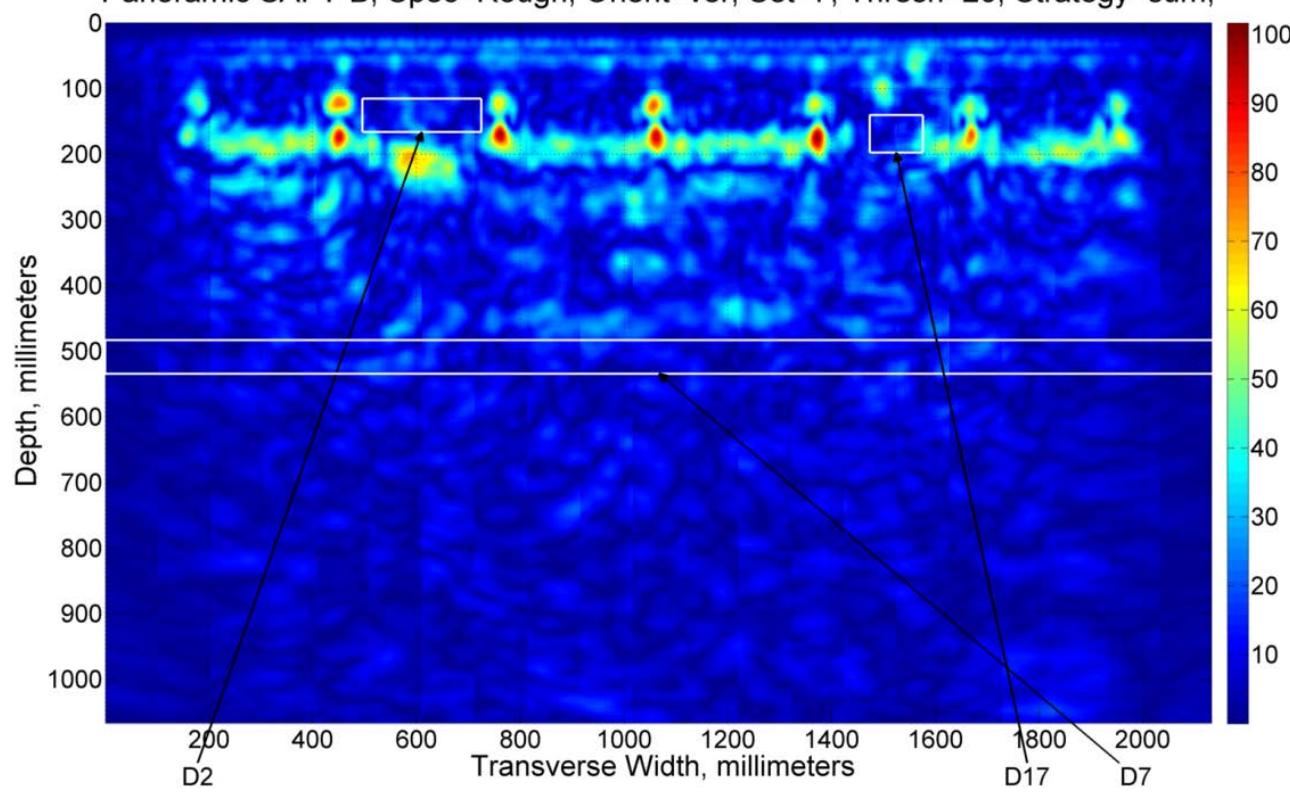

Fig. A. 127. Vertical Set 7, Node 16. 


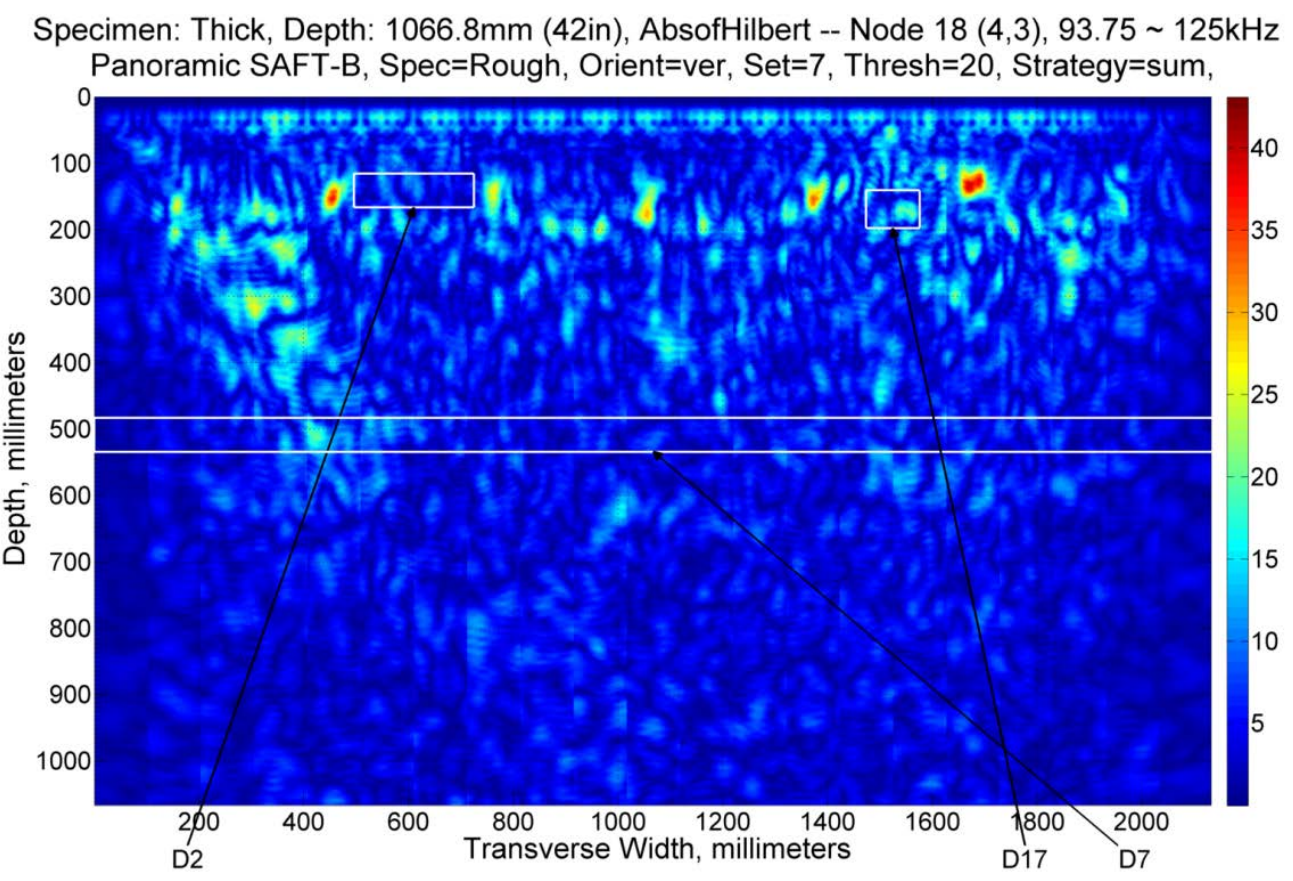

Fig. A. 128. Vertical Set 7, Node 18.

Specimen: Thick, Depth: 1066.8mm (42in), AbsofHilbert -- Node $32(5,1), 15.625 \sim 31.25 \mathrm{kHz}$

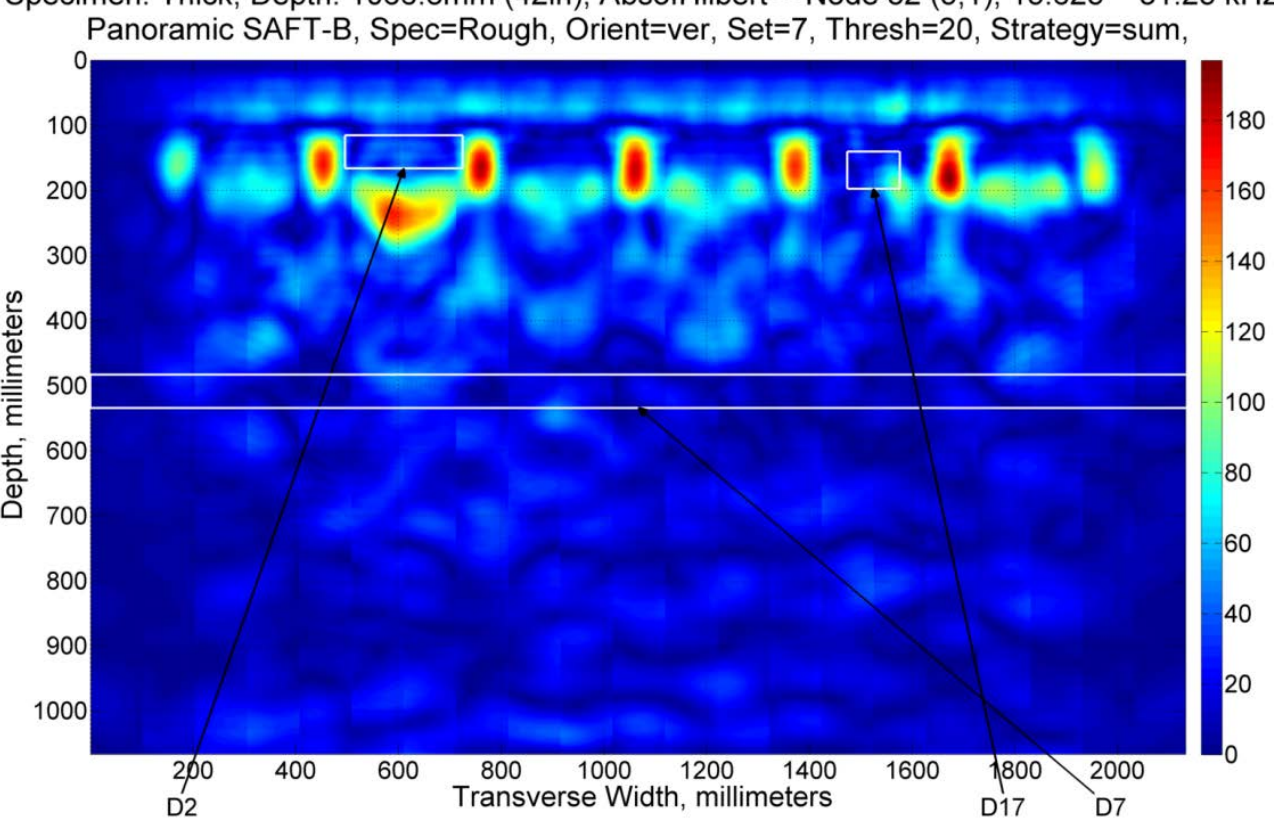

Fig. A. 129. Vertical Set 7, Node 32. 


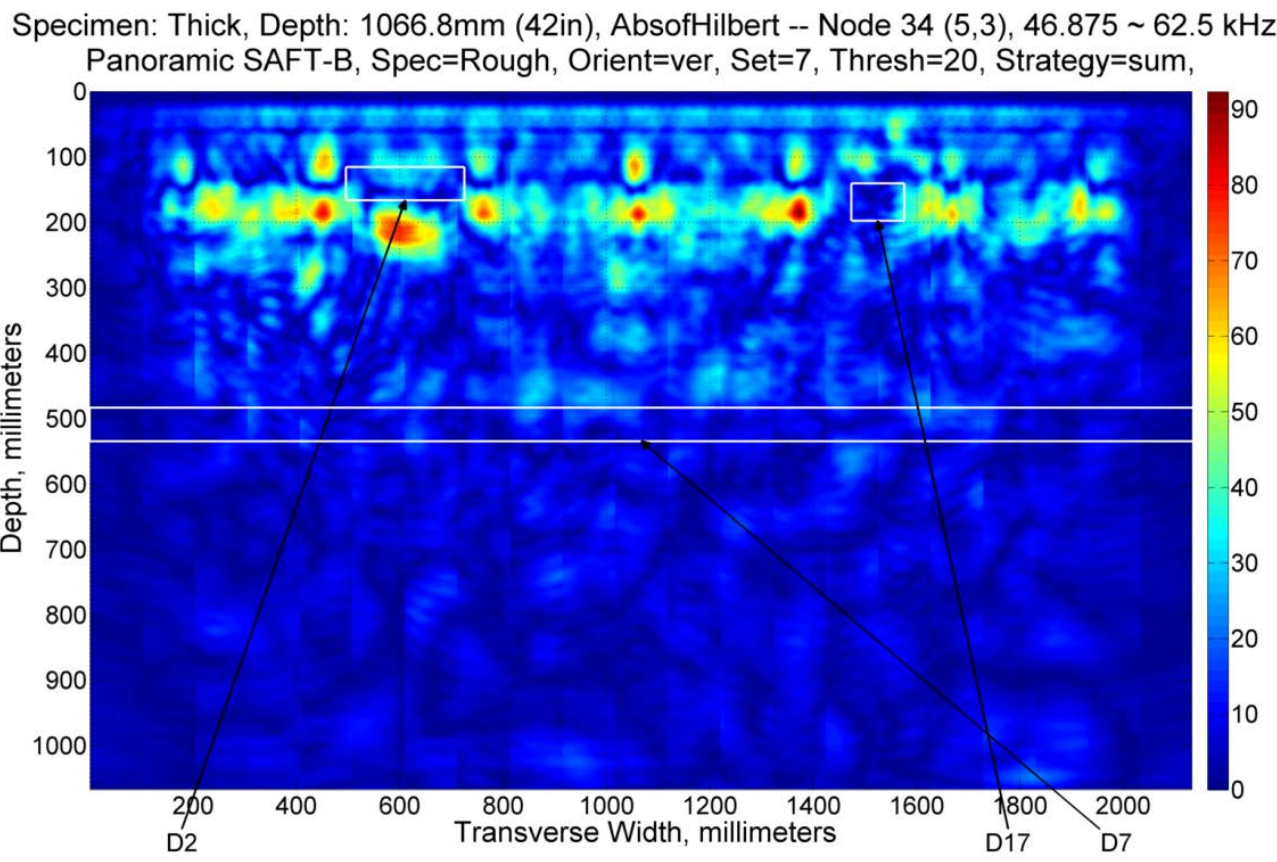

Fig. A. 130. Vertical Set 7, Node 34 .

\section{SET 8 - DEFECT 9, DEFECT 10, DEFECT 12}

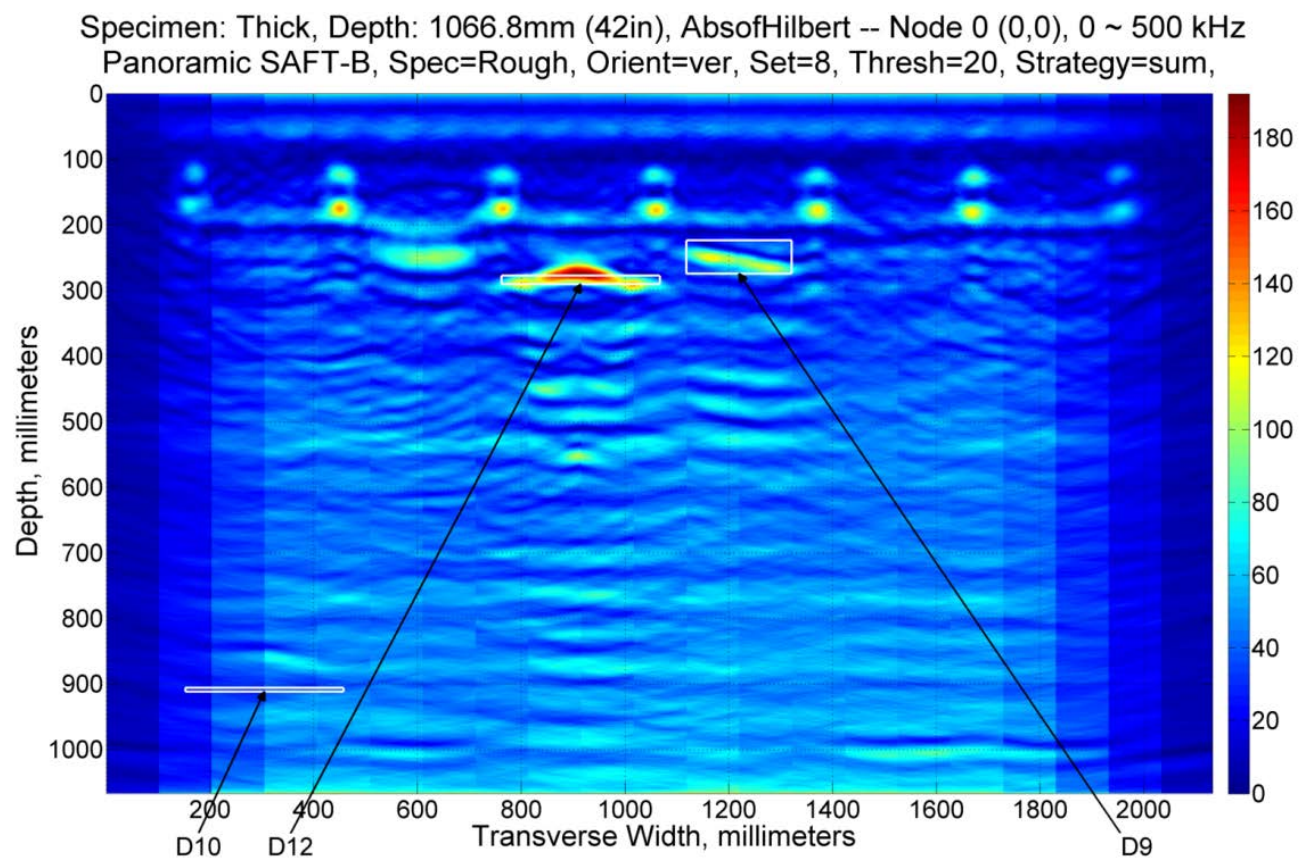

Fig. A. 131. Vertical Set 8, Node 0. 
Specimen: Thick, Depth: 1066.8mm (42in), AbsofHilbert -- Node $16(4,1), 31.25 \sim 62.5 \mathrm{kHz}$ Panoramic SAFT-B, Spec=Rough, Orient=ver, Set=8, Thresh=20, Strategy=sum,

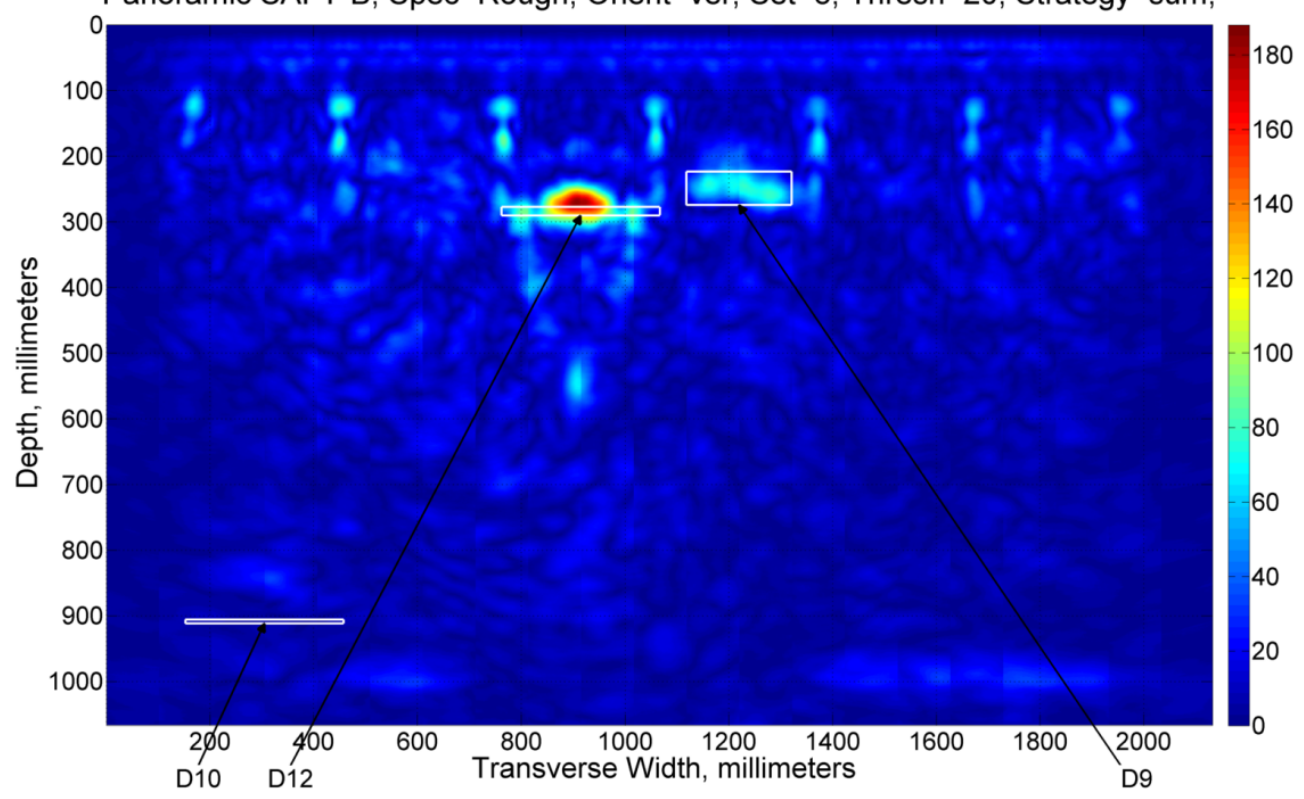

Fig. A. 132. Vertical Set 8, Node 16.

Specimen: Thick, Depth: 1066.8mm (42in), AbsofHilbert -- Node $18(4,3), 93.75 \sim 125 \mathrm{kHz}$ Panoramic SAFT-B, Spec=Rough, Orient $=v e r$, Set $=8$, Thresh $=20$, Strategy=sum,

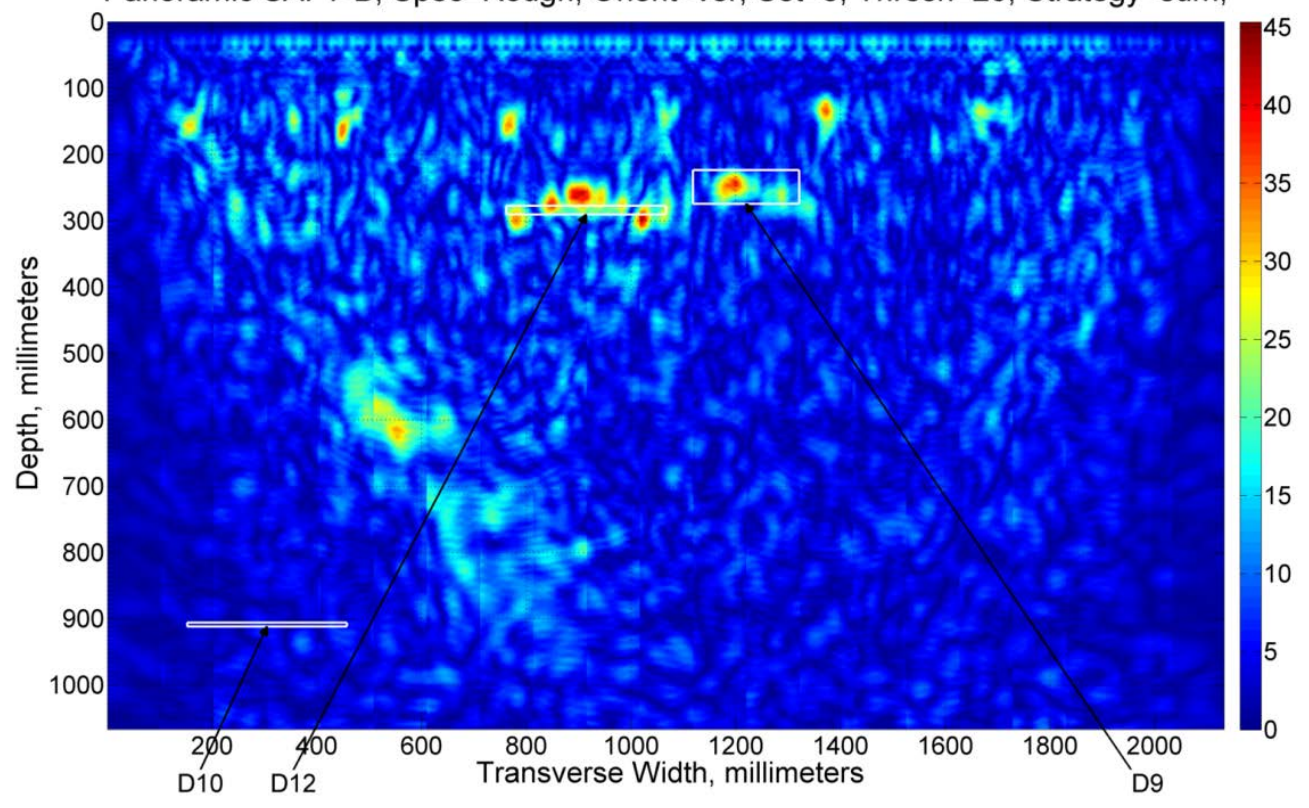

Fig. A. 133. Vertical Set 8, Node 18. 


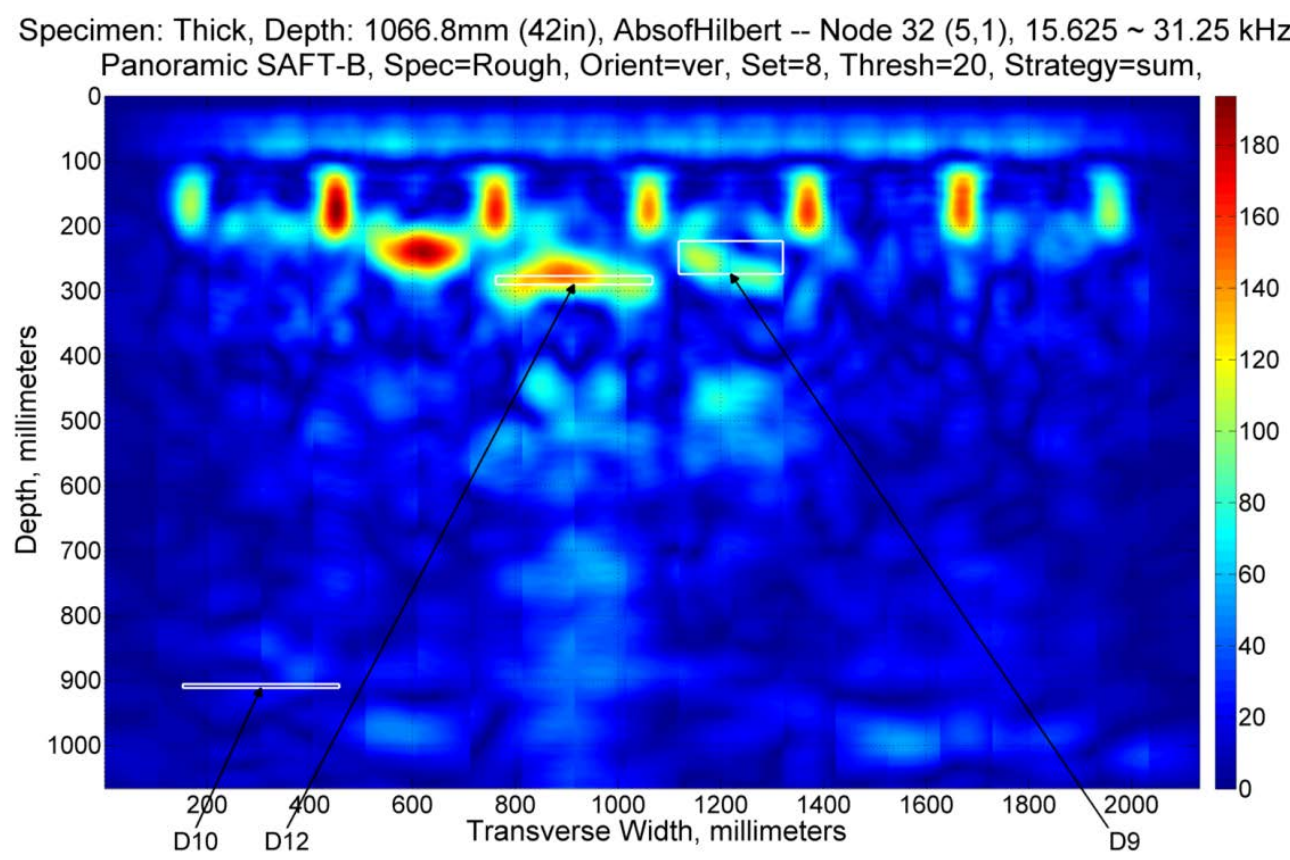

Fig. A. 134. Vertical Set 8, Node 32.

Specimen: Thick, Depth: 1066.8mm (42in), AbsofHilbert -- Node $34(5,3), 46.875 \sim 62.5 \mathrm{kHz}$ Panoramic SAFT-B, Spec=Rough, Orient=ver, Set=8, Thresh=20, Strategy=sum,

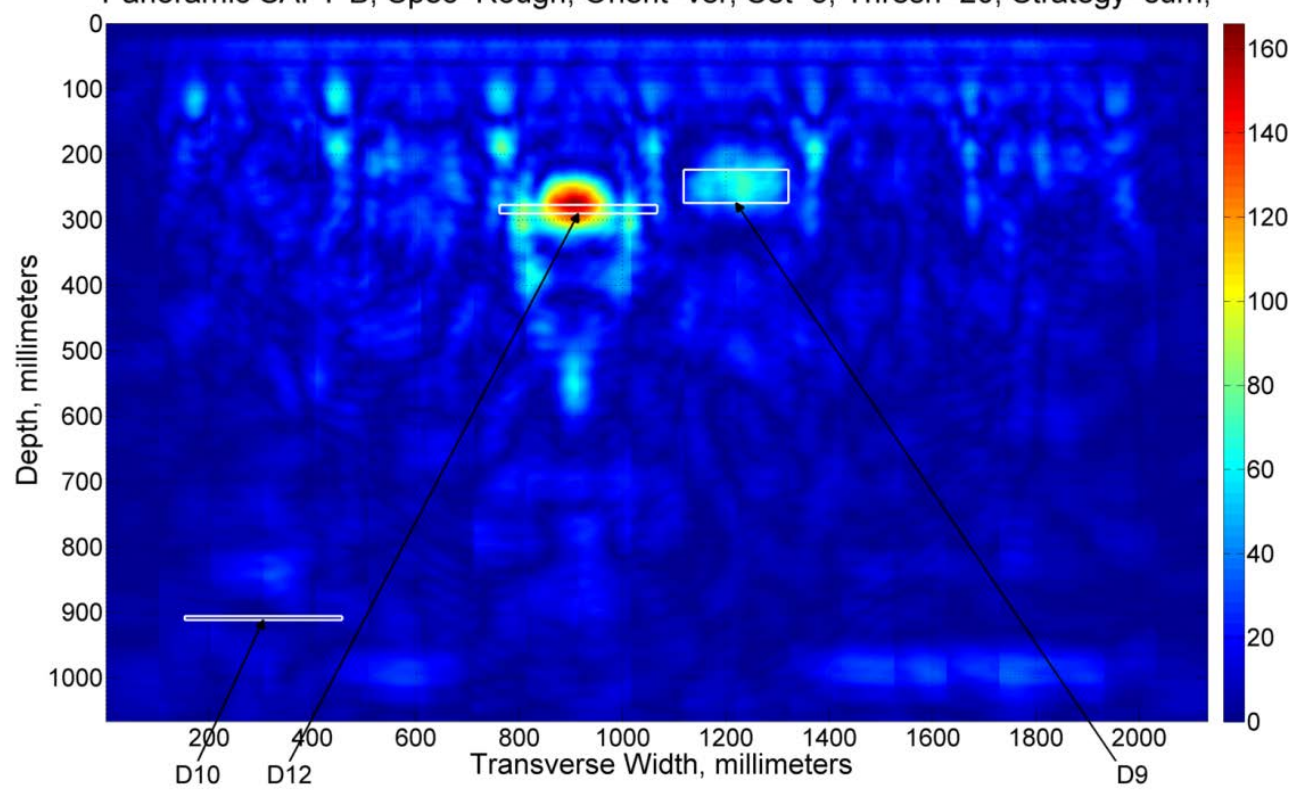

Fig. A. 135. Vertical Set 8, Node 34. 
Specimen: Thick, Depth: 1066.8mm (42in), AbsofHilbert -- Node $0(0,0), 0 \sim 500 \mathrm{kHz}$ Panoramic SAFT-B, Spec=Rough, Orient=ver, Set=9, Thresh=20, Strategy=sum,

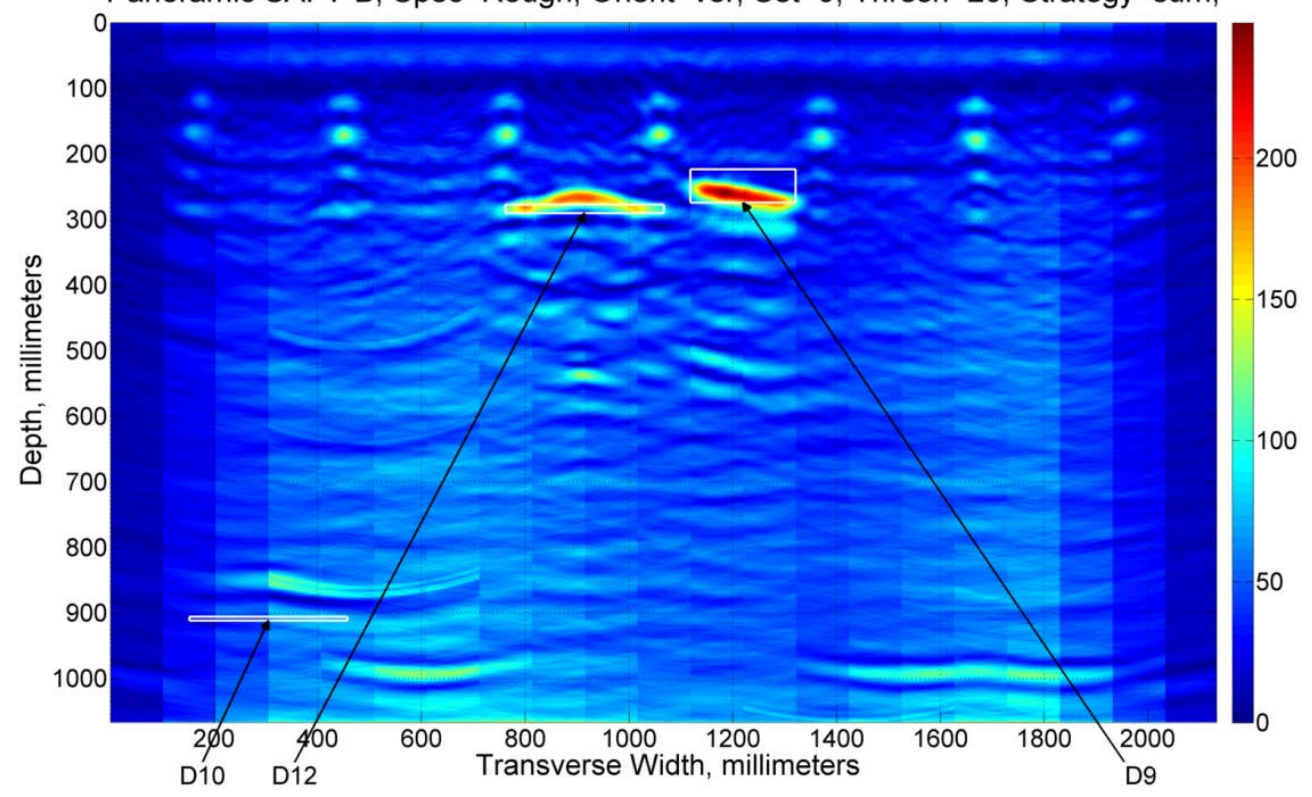

Fig. A. 136. Vertical Set 9, Node 0 .

Specimen: Thick, Depth: 1066.8mm (42in), AbsofHilbert -- Node $16(4,1), 31.25 \sim 62.5 \mathrm{kHz}$ Panoramic SAFT-B, Spec=Rough, Orient=ver, Set=9, Thresh=20, Strategy=sum,

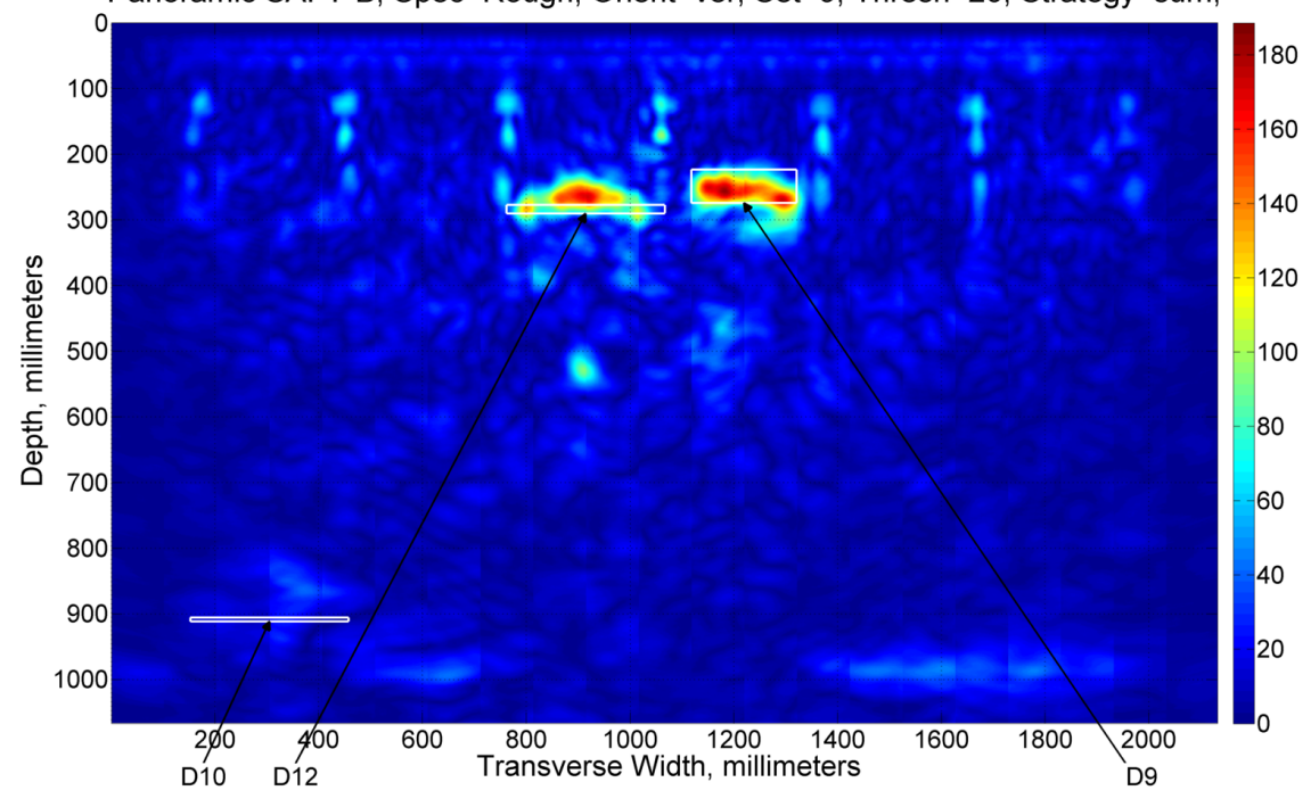

Fig. A. 137. Vertical Set 9, Node 16. 
Specimen: Thick, Depth: 1066.8mm (42in), AbsofHilbert -- Node $18(4,3), 93.75 \sim 125 \mathrm{kHz}$

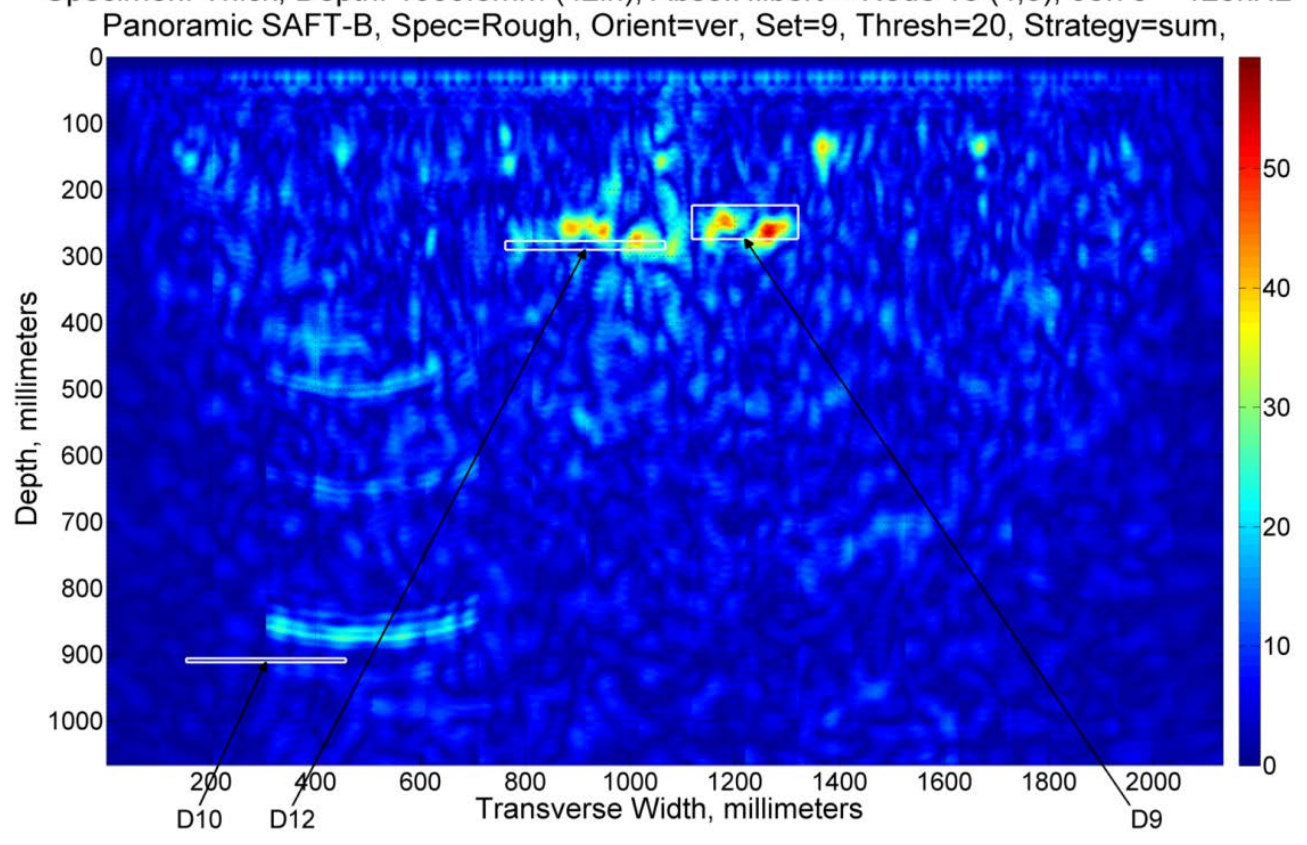

Fig. A. 138. Vertical Set 9, Node 18.

Specimen: Thick, Depth: 1066.8mm (42in), AbsofHilbert -- Node $32(5,1), 15.625 \sim 31.25 \mathrm{kHz}$

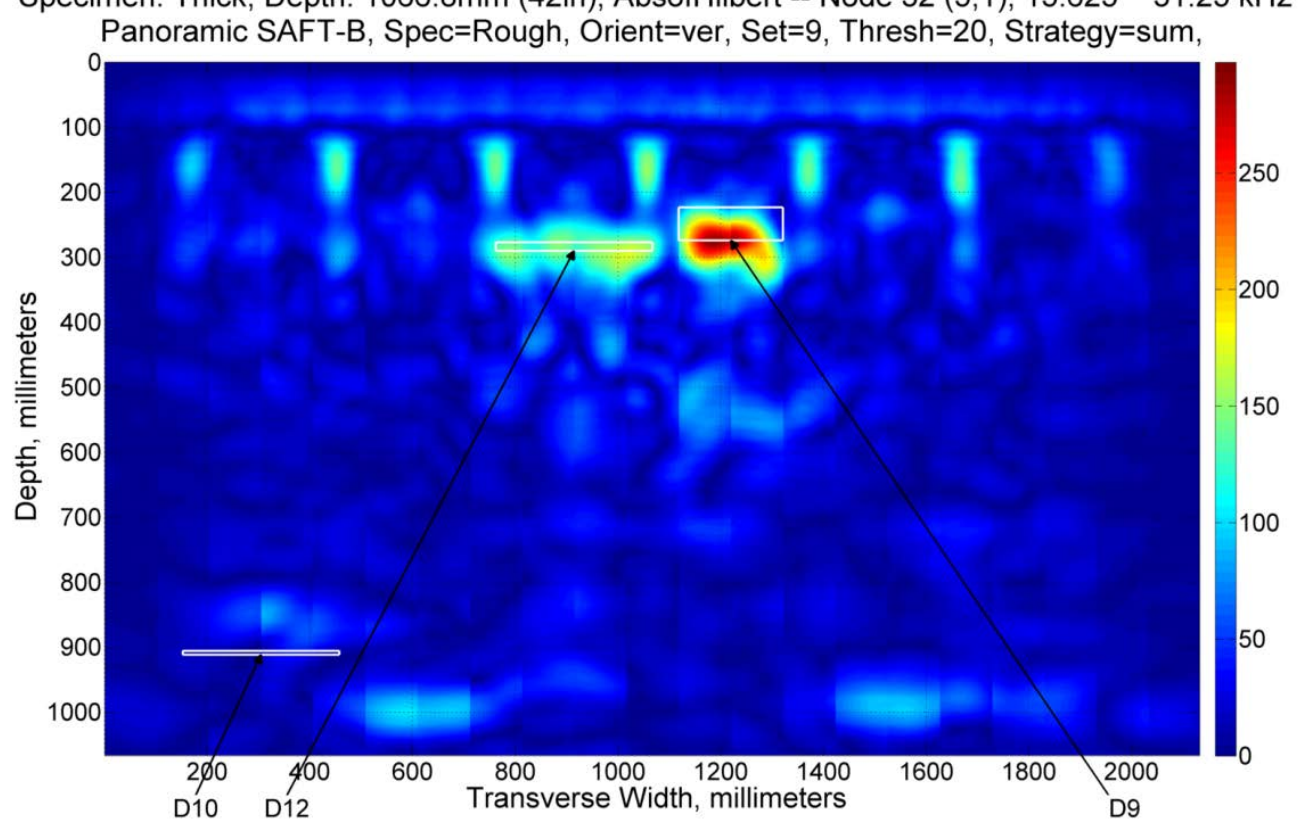

Fig. A. 139. Vertical Set 9, Node 32. 


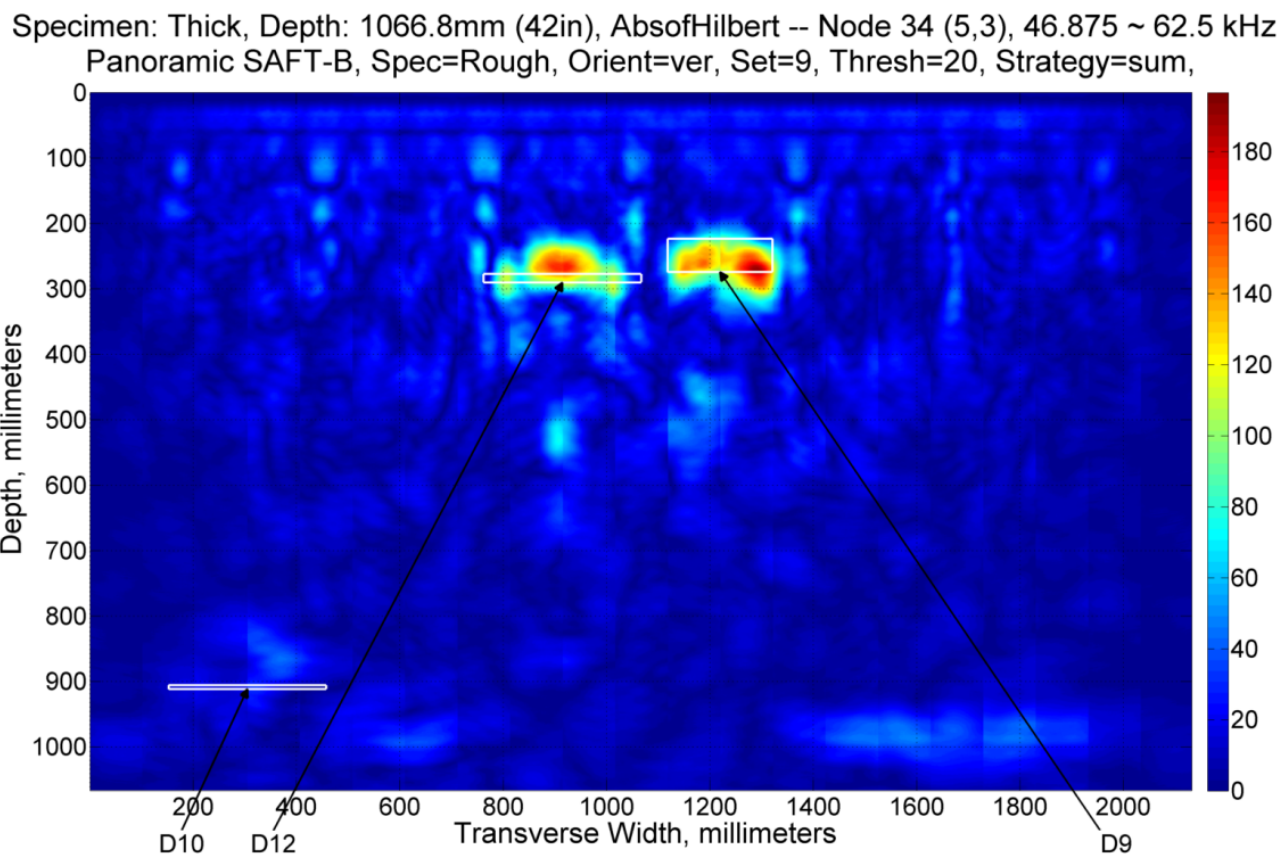

Fig. A. 140. Vertical Set 9, Node 34.

\section{SET 10 - DEFECT 3, DEFECT 20}

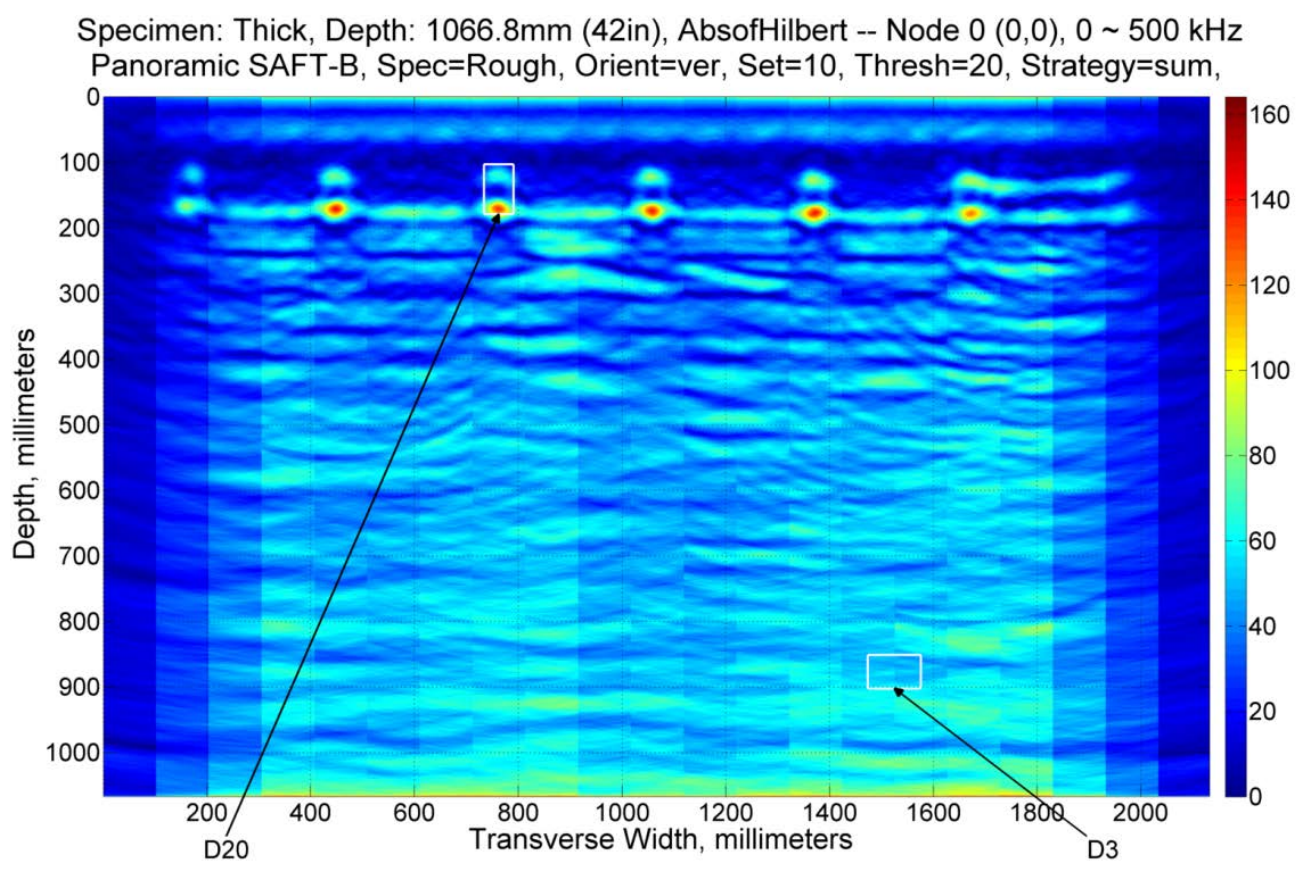

Fig. A. 141. Vertical Set 10, Node 0. 


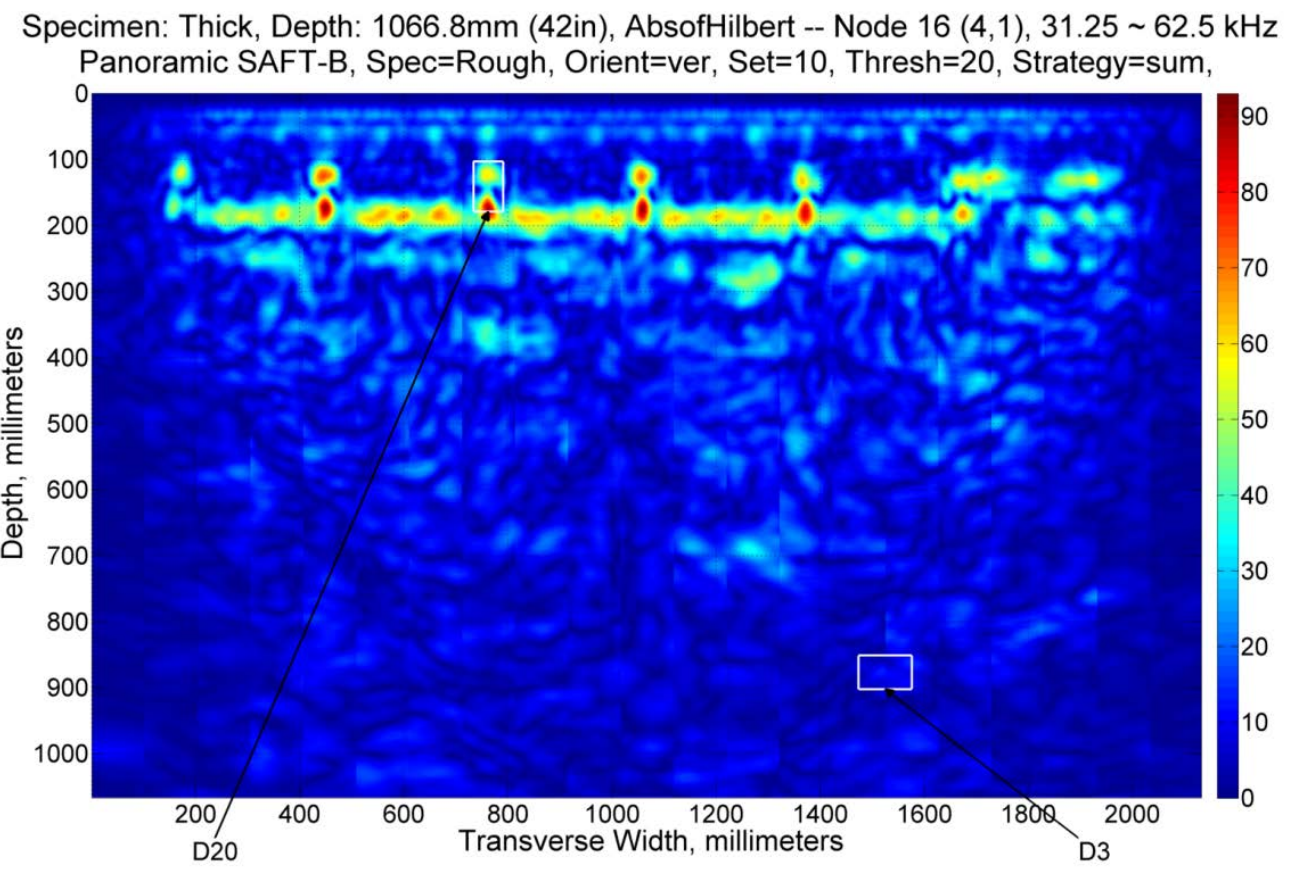

Fig. A. 142. Vertical Set 10, Node 16.

Specimen: Thick, Depth: 1066.8mm (42in), AbsofHilbert -- Node $18(4,3), 93.75 \sim 125 \mathrm{kHz}$

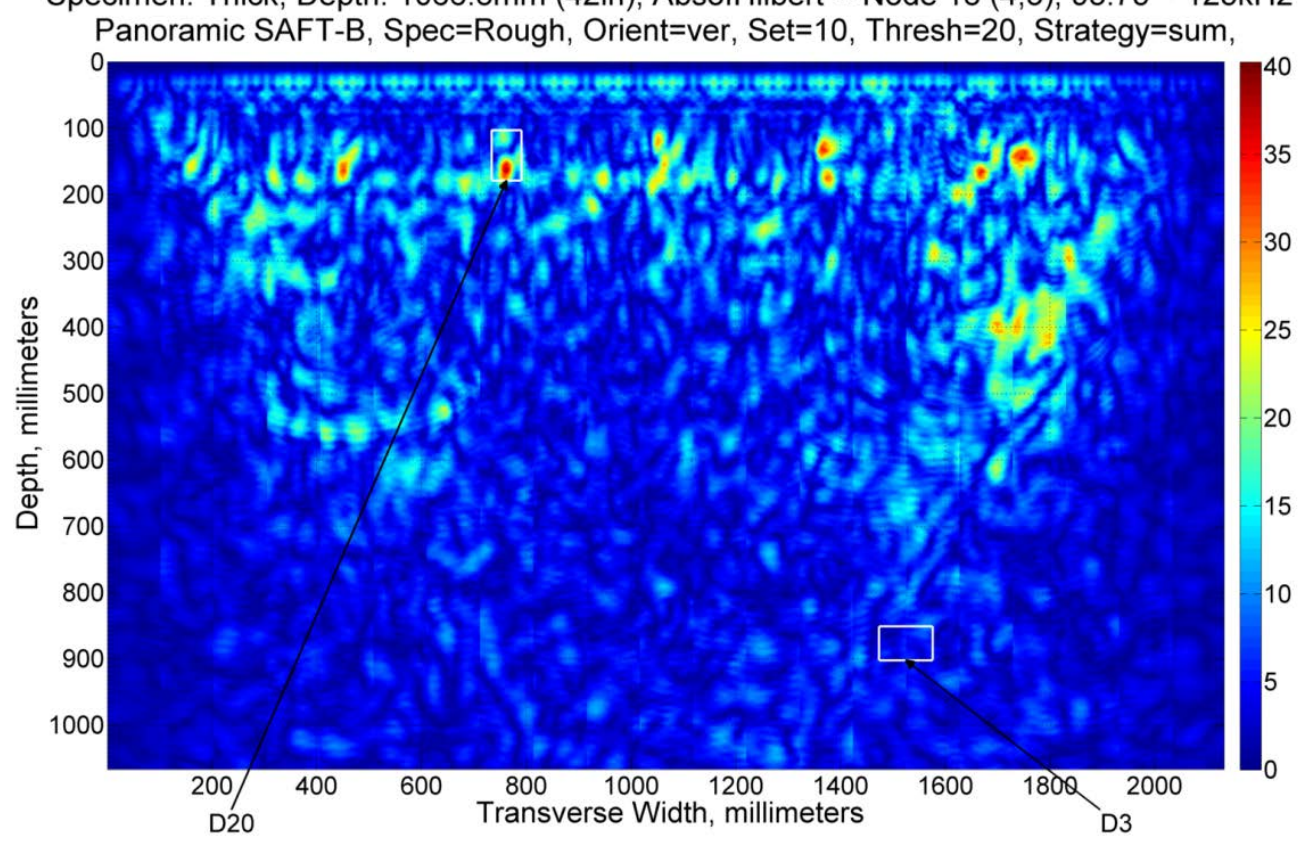

Fig. A. 143. Vertical Set 10, Node 18. 


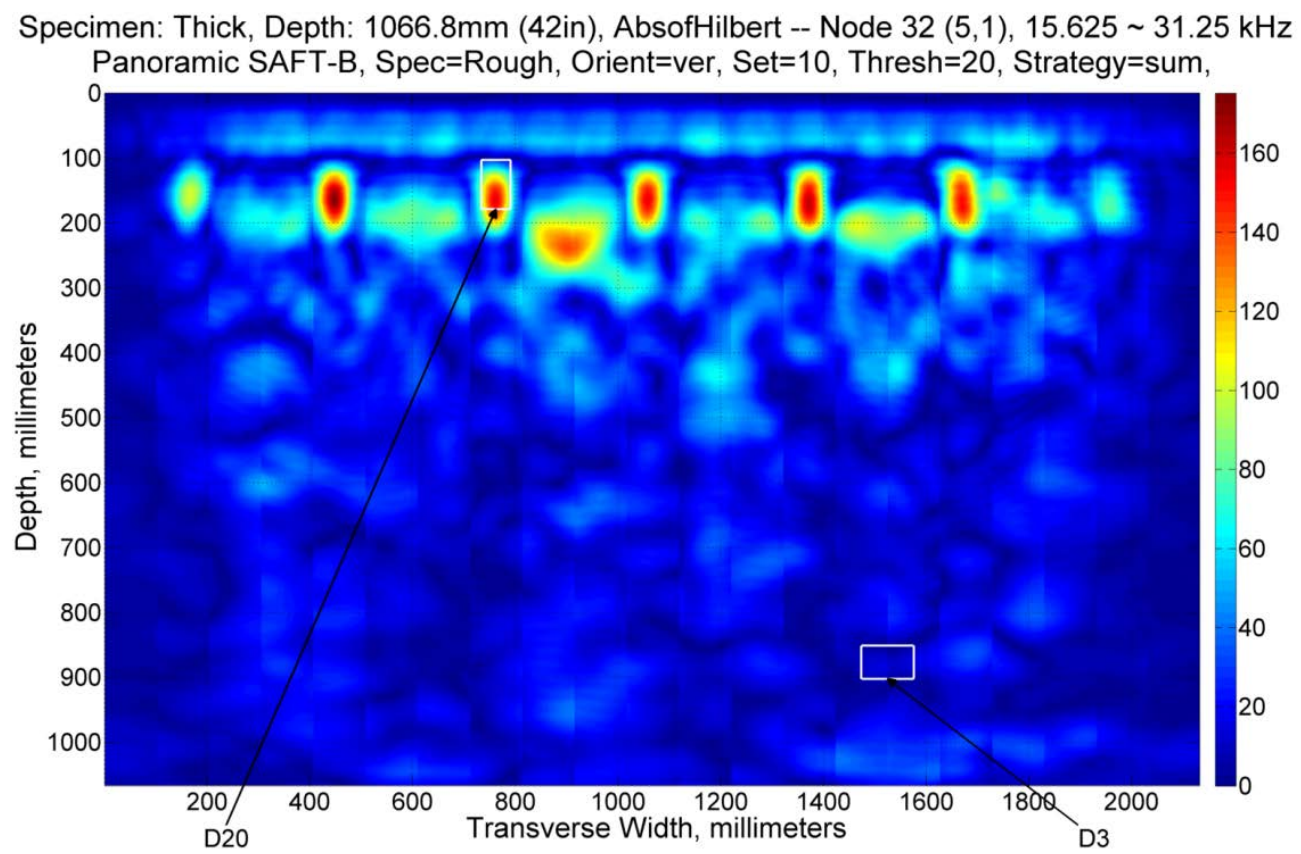

Fig. A. 144. Vertical Set 10, Node 32.

Specimen: Thick, Depth: 1066.8mm (42in), AbsofHilbert -- Node $34(5,3), 46.875 \sim 62.5 \mathrm{kHz}$ Panoramic SAFT-B, Spec=Rough, Orient=ver, Set=10, Thresh=20, Strategy=sum,

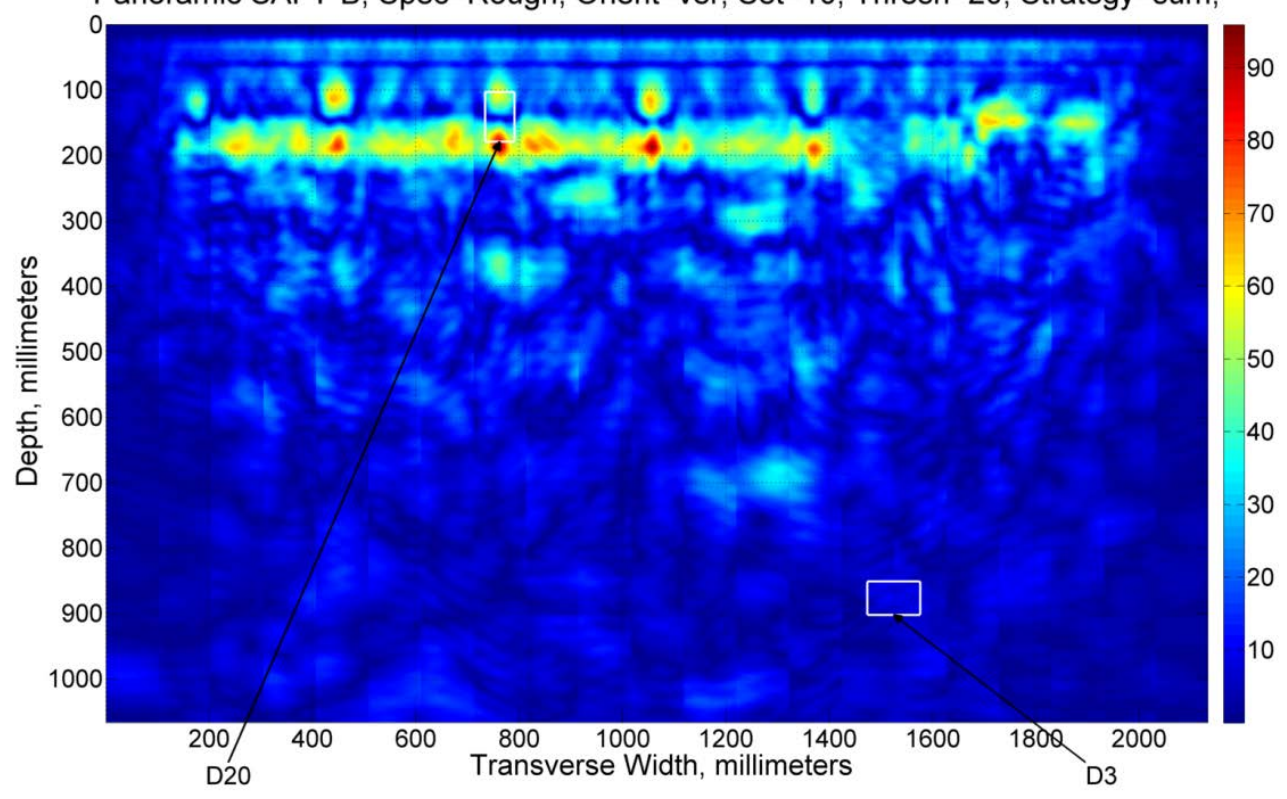

Fig. A. 145. Vertical Set 10, Node 34. 


\section{SET 11 - DEFECT 8, DEFECT 11, DEFECT 13}

Specimen: Thick, Depth: 1066.8mm (42in), AbsofHilbert -- Node $0(0,0), 0 \sim 500 \mathrm{kHz}$ Panoramic SAFT-B, Spec=Rough, Orient=ver, Set=11, Thresh=20, Strategy=sum,

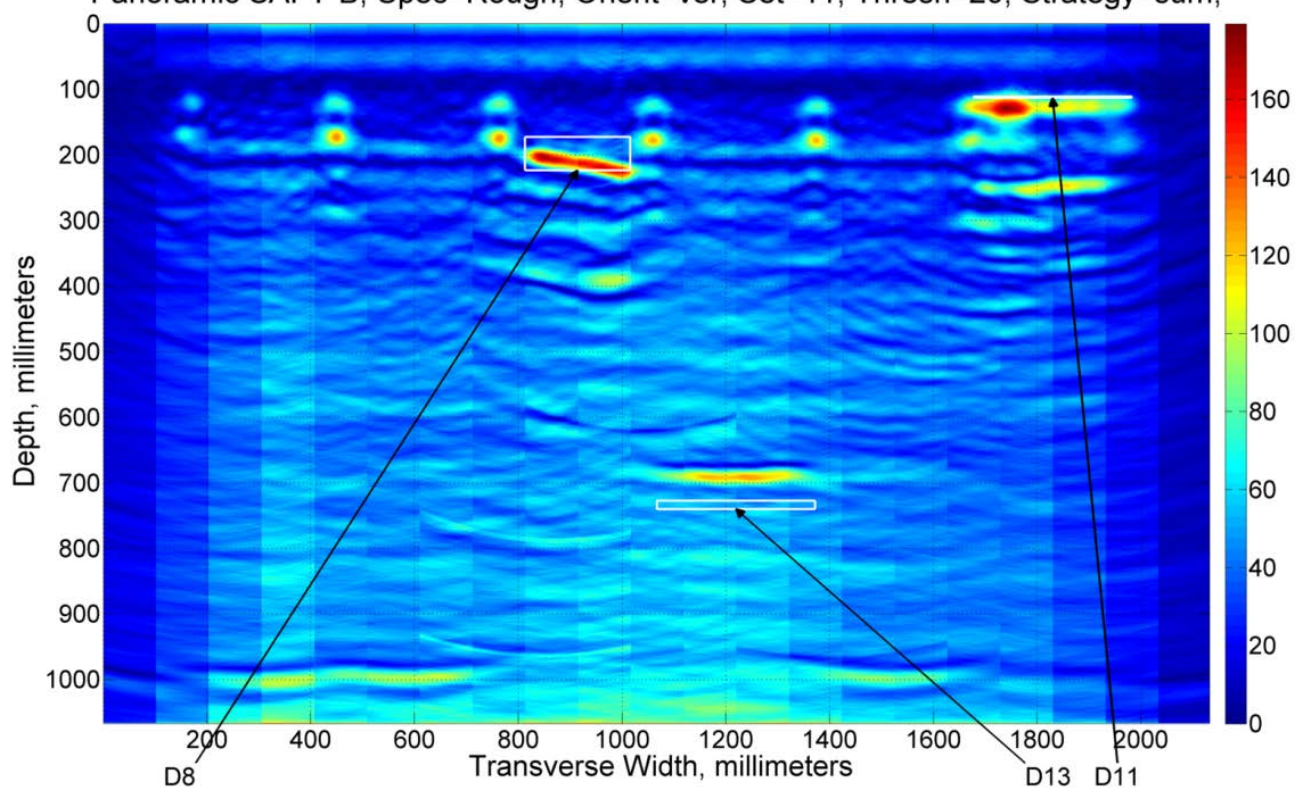

Fig. A. 146. Vertical Set 11, Node 0.

Specimen: Thick, Depth: 1066.8mm (42in), AbsofHilbert -- Node $16(4,1), 31.25 \sim 62.5 \mathrm{kHz}$ Panoramic SAFT-B, Spec=Rough, Orient=ver, Set=11, Thresh=20, Strategy=sum,

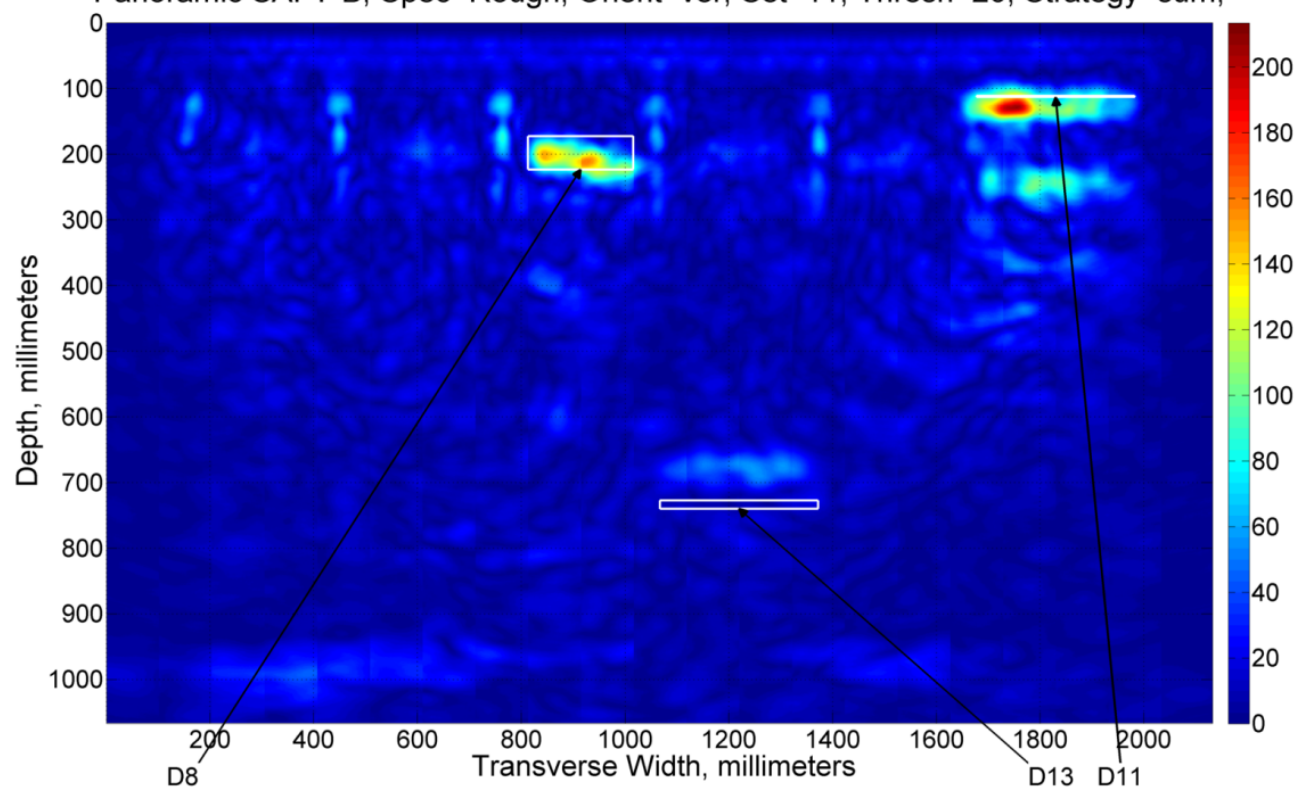

Fig. A. 147. Vertical Set 11, Node 16. 
Specimen: Thick, Depth: 1066.8mm (42in), AbsofHilbert -- Node $18(4,3), 93.75 \sim 125 \mathrm{kHz}$ Panoramic SAFT-B, Spec=Rough, Orient=ver, Set=11, Thresh=20, Strategy=sum,

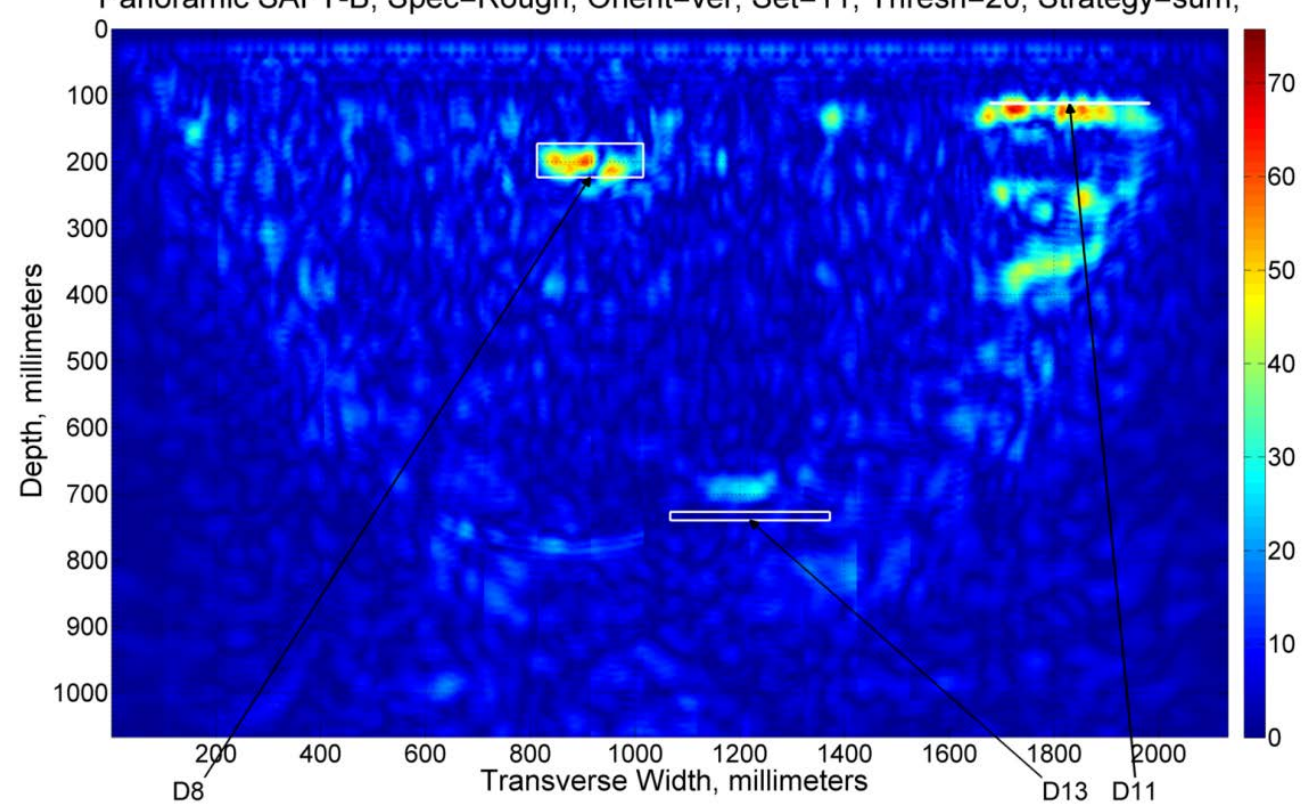

Fig. A. 148. Vertical Set 11, Node 18.

Specimen: Thick, Depth: 1066.8mm (42in), AbsofHilbert -- Node $32(5,1), 15.625 \sim 31.25 \mathrm{kHz}$ Panoramic SAFT-B, Spec=Rough, Orient=ver, Set=11, Thresh=20, Strategy=sum,

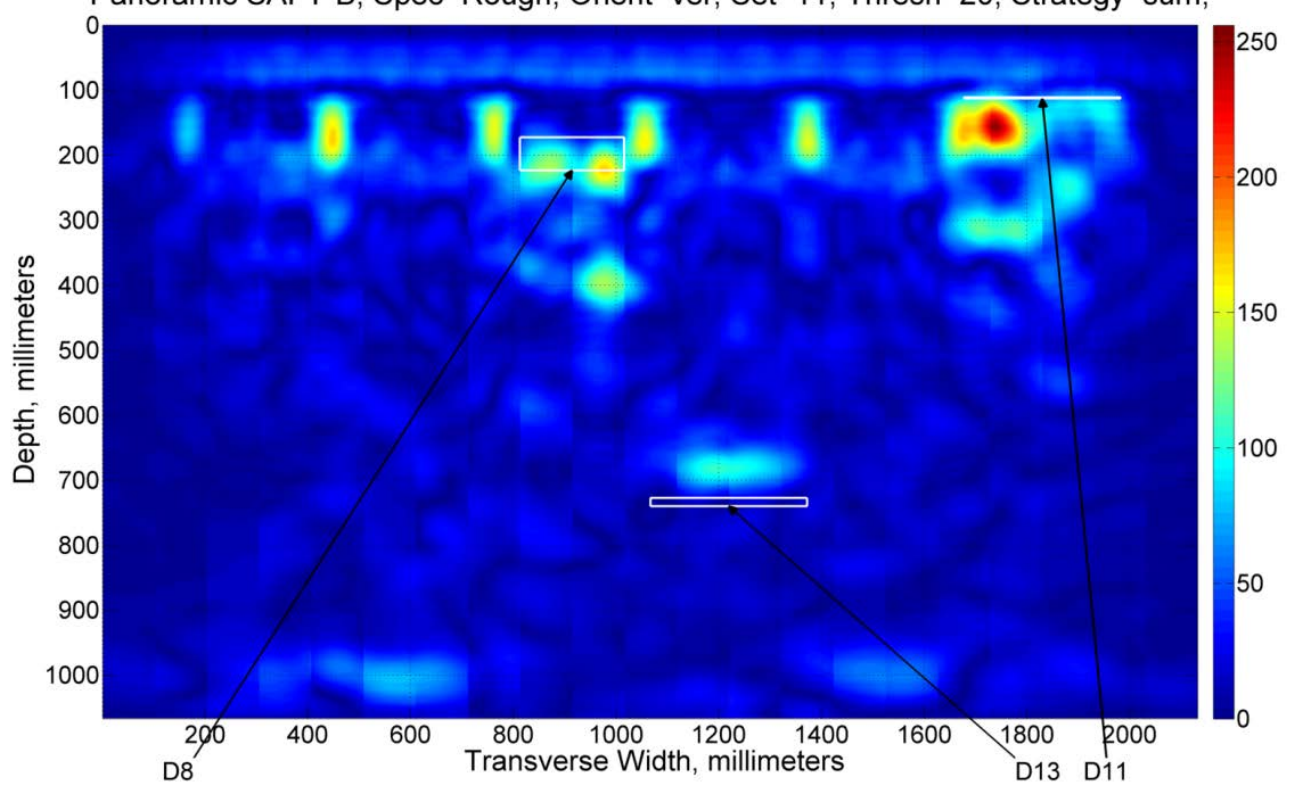

Fig. A. 149. Vertical Set 11, Node 32. 


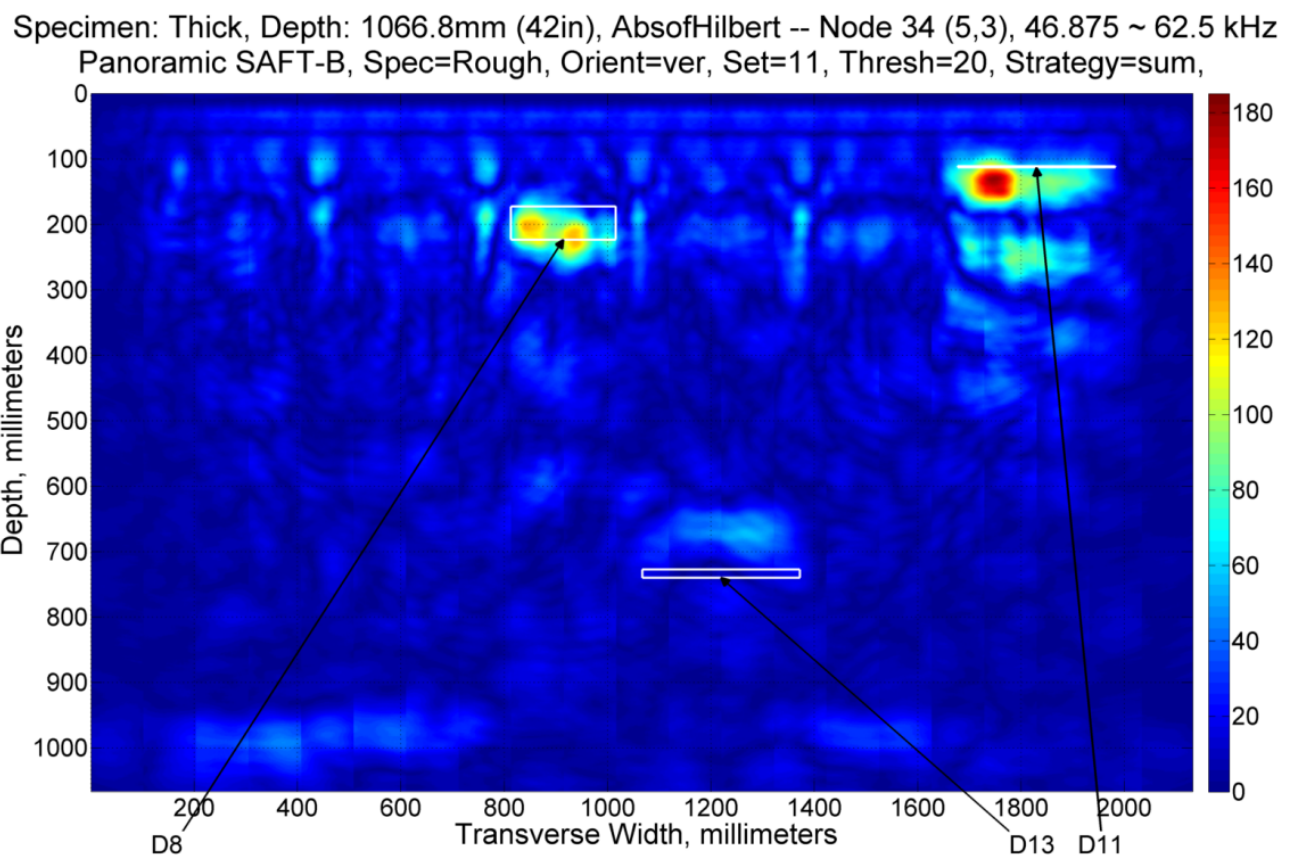

Fig. A. 150. Vertical Set 11, Node 34.

\section{SET 12 - DEFECT 8, DEFECT 11, DEFECT 13}

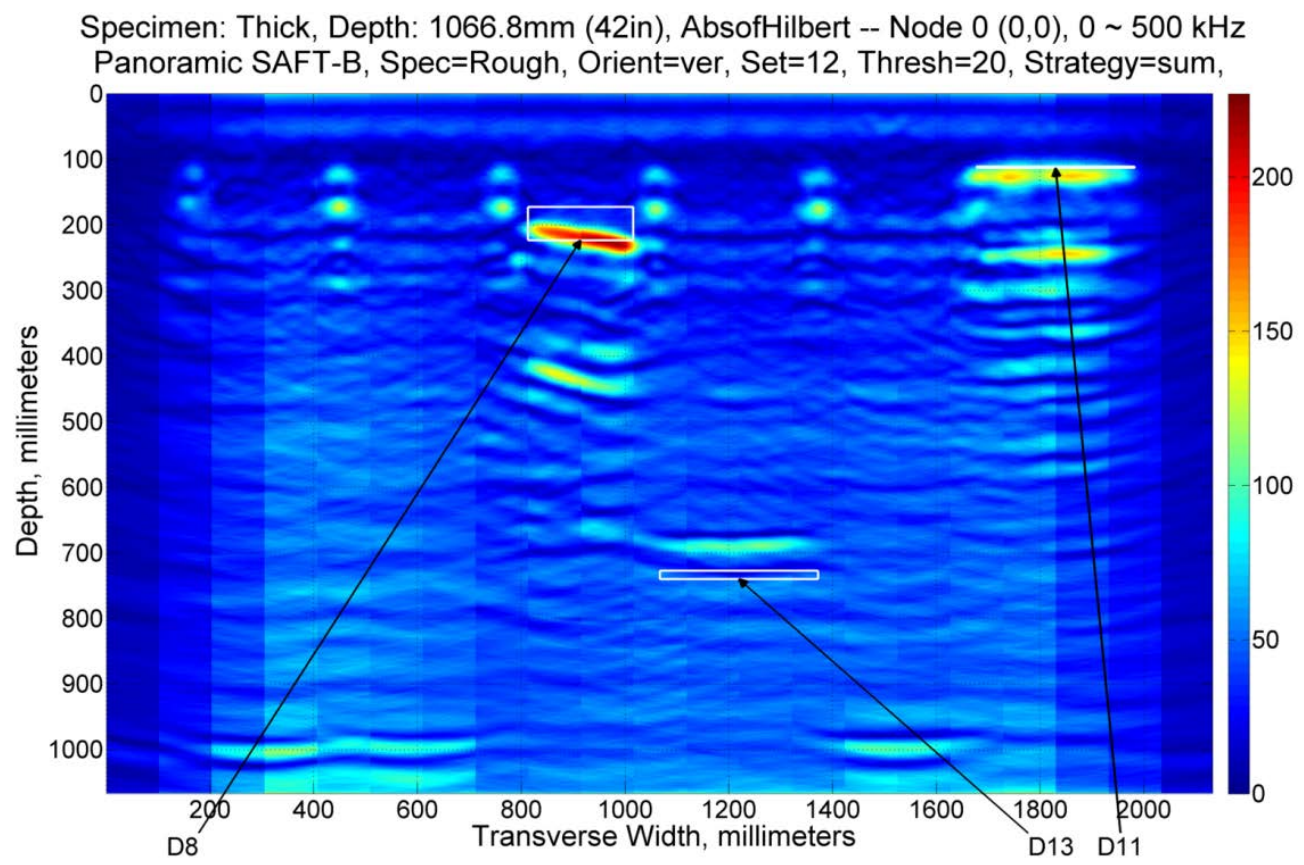

Fig. A. 151. Vertical Set 12, Node 0. 
Specimen: Thick, Depth: 1066.8mm (42in), AbsofHilbert -- Node $16(4,1), 31.25 \sim 62.5 \mathrm{kHz}$ Panoramic SAFT-B, Spec=Rough, Orient=ver, Set=12, Thresh=20, Strategy=sum,

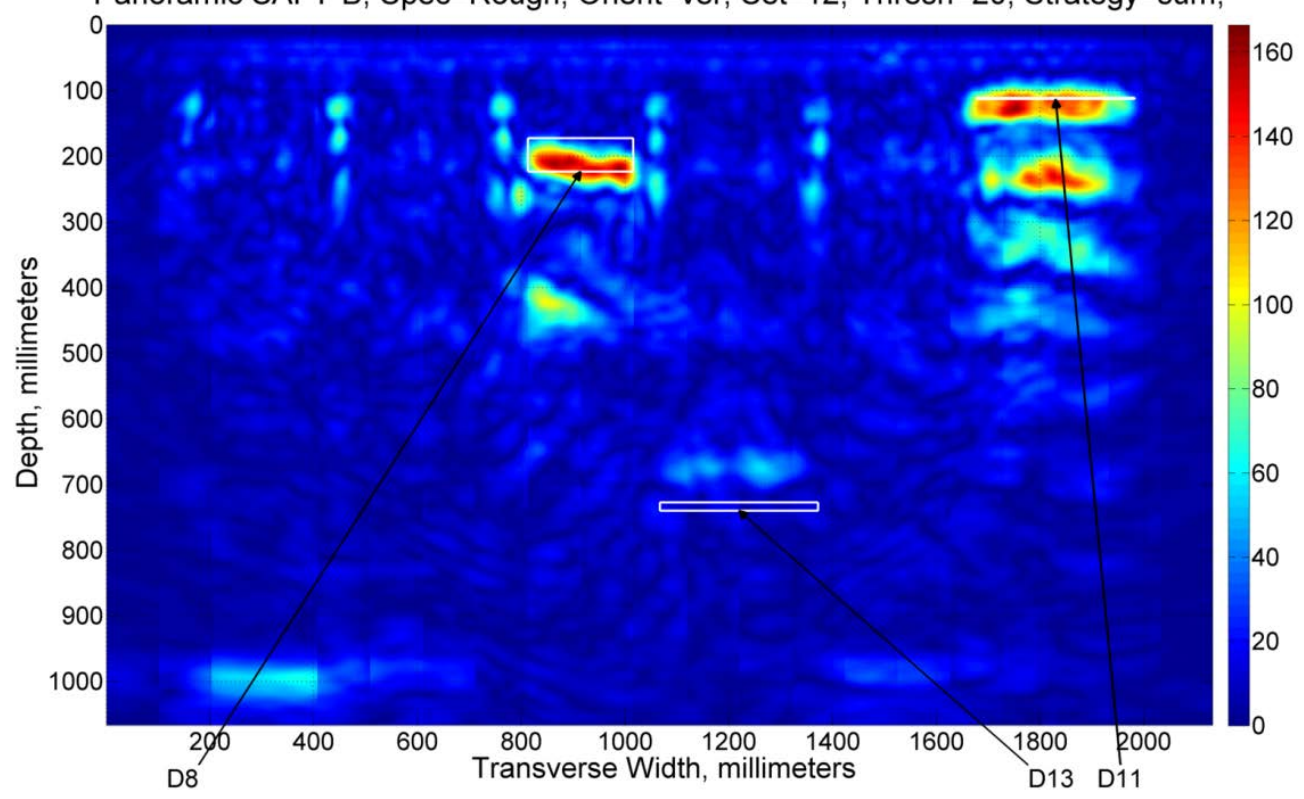

Fig. A. 152. Vertical Set 12, Node 16.

Specimen: Thick, Depth: 1066.8mm (42in), AbsofHilbert -- Node $18(4,3), 93.75 \sim 125 \mathrm{kHz}$ Panoramic SAFT-B, Spec=Rough, Orient=ver, Set=12, Thresh=20, Strategy=sum,

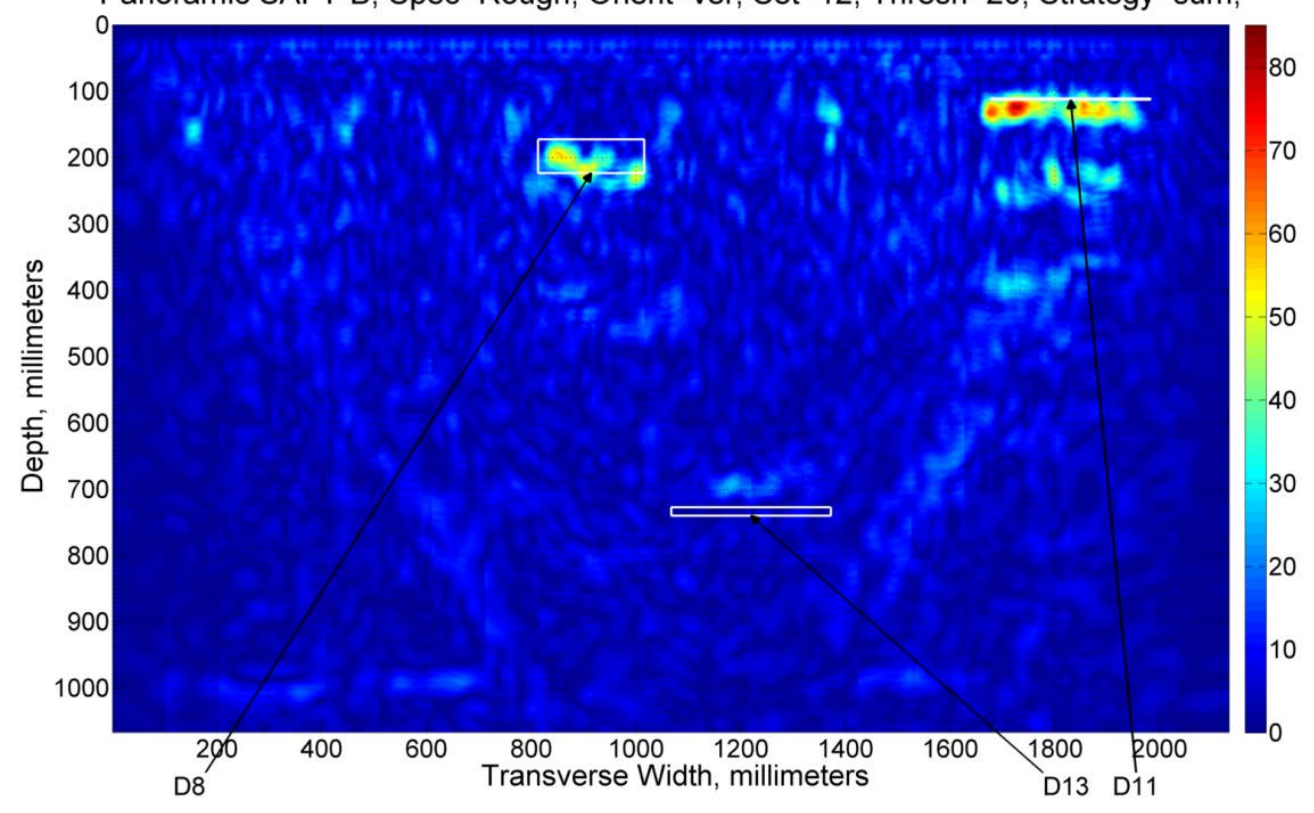

Fig. A. 153. Vertical Set 12, Node 18. 


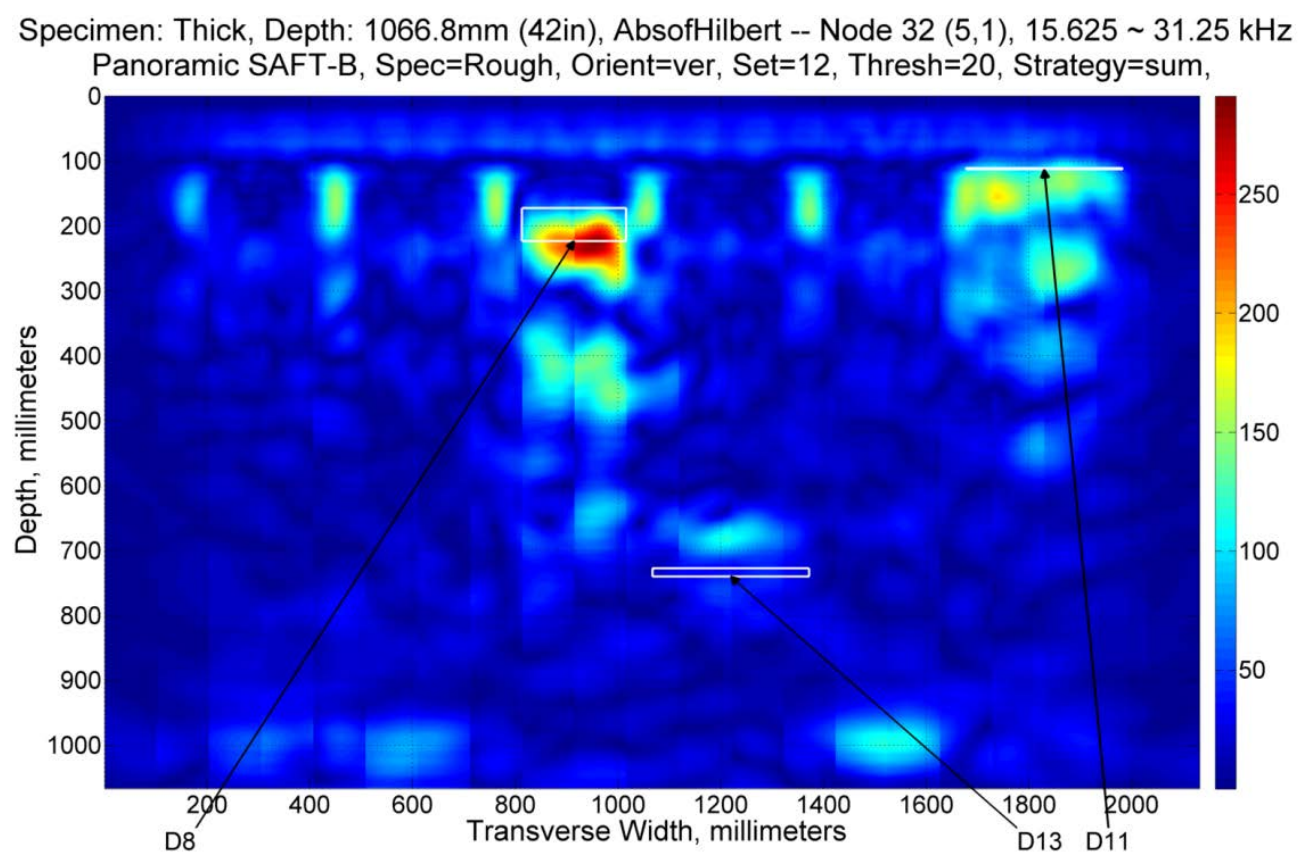

Fig. A. 154. Vertical Set 12, Node 32.

Specimen: Thick, Depth: 1066.8mm (42in), AbsofHilbert -- Node $34(5,3), 46.875 \sim 62.5 \mathrm{kHz}$ Panoramic SAFT-B, Spec=Rough, Orient=ver, Set=12, Thresh=20, Strategy=sum,

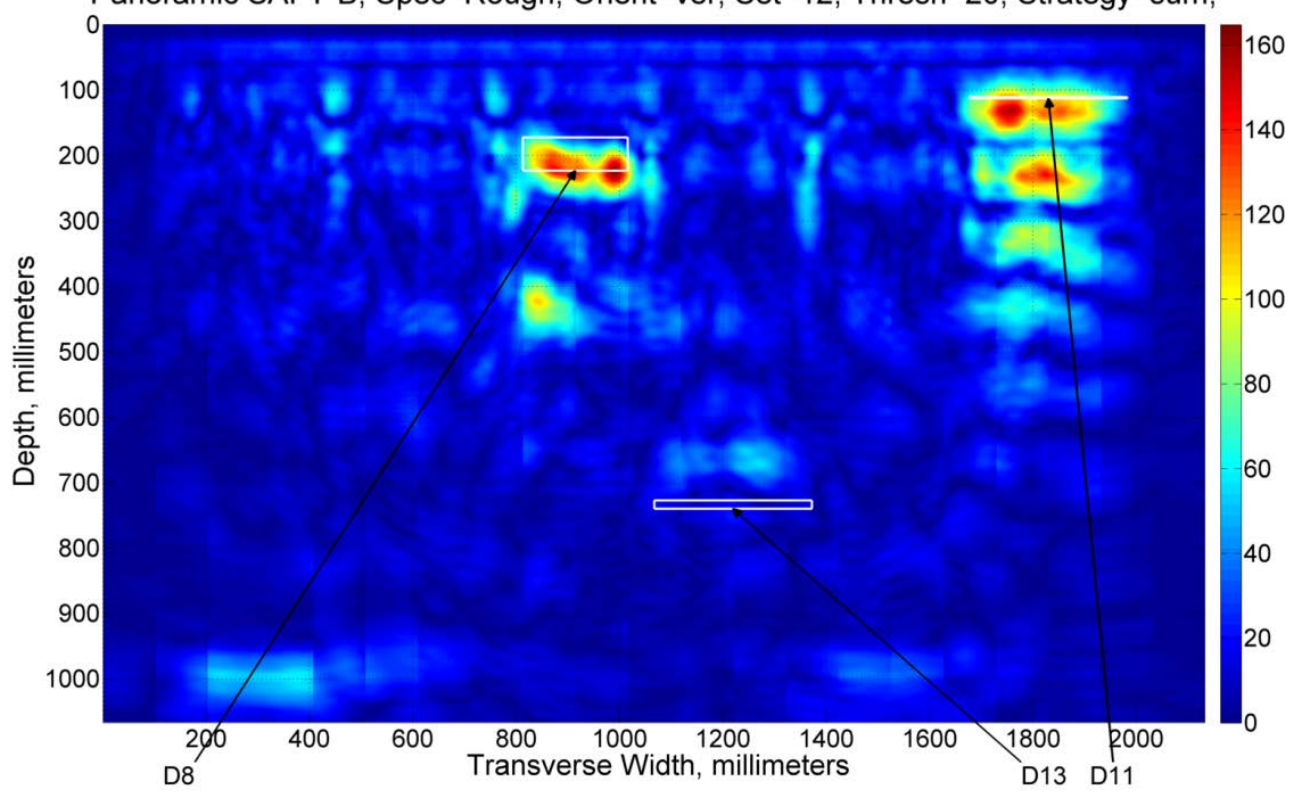

Fig. A. 155. Vertical Set 12, Node 34. 


\section{SET 13 - DEFECT 6}

Specimen: Thick, Depth: 1066.8mm (42in), AbsofHilbert -- Node $0(0,0), 0 \sim 500 \mathrm{kHz}$ Panoramic SAFT-B, Spec=Rough, Orient=ver, Set=13, Thresh=20, Strategy=sum,

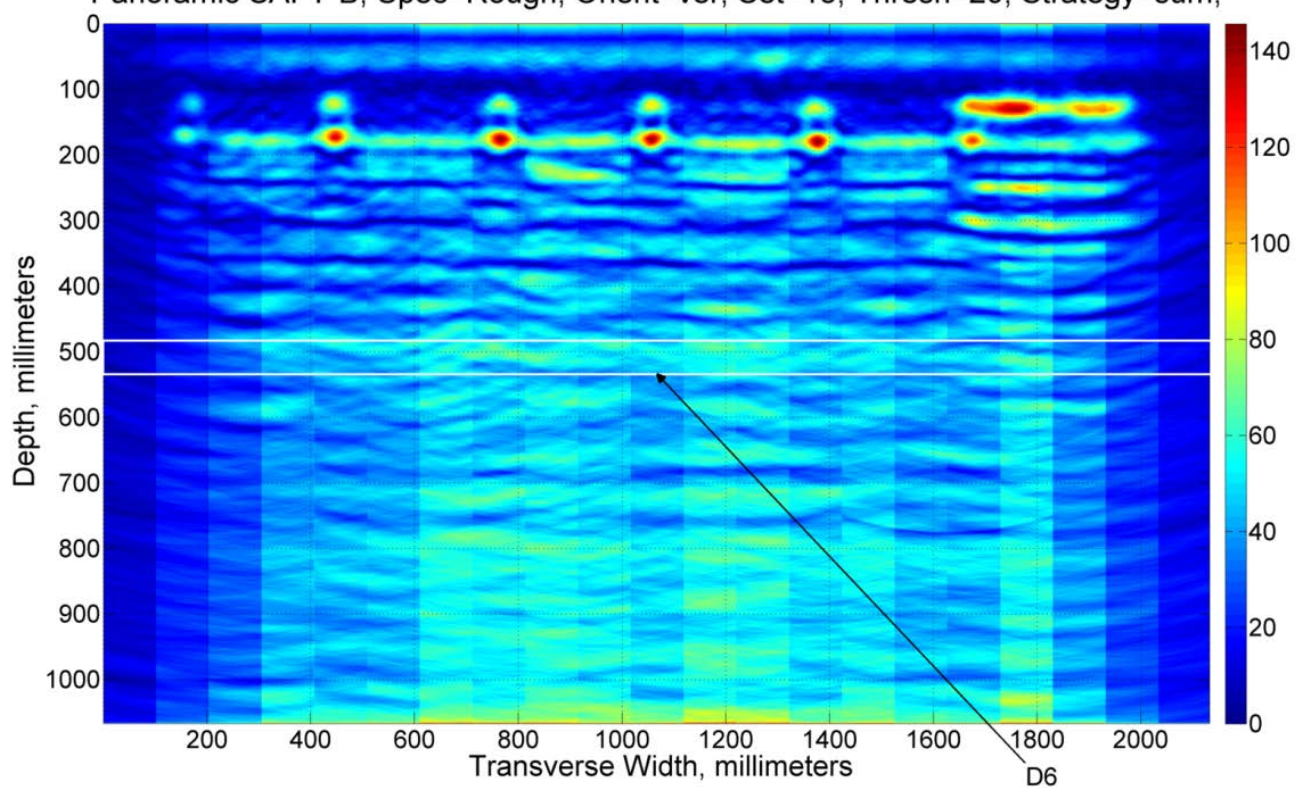

Fig. A. 156. Vertical Set 13, Node 0.

Specimen: Thick, Depth: 1066.8mm (42in), AbsofHilbert -- Node $16(4,1), 31.25 \sim 62.5 \mathrm{kHz}$ Panoramic SAFT-B, Spec=Rough, Orient=ver, Set $=13$, Thresh $=20$, Strategy=sum,

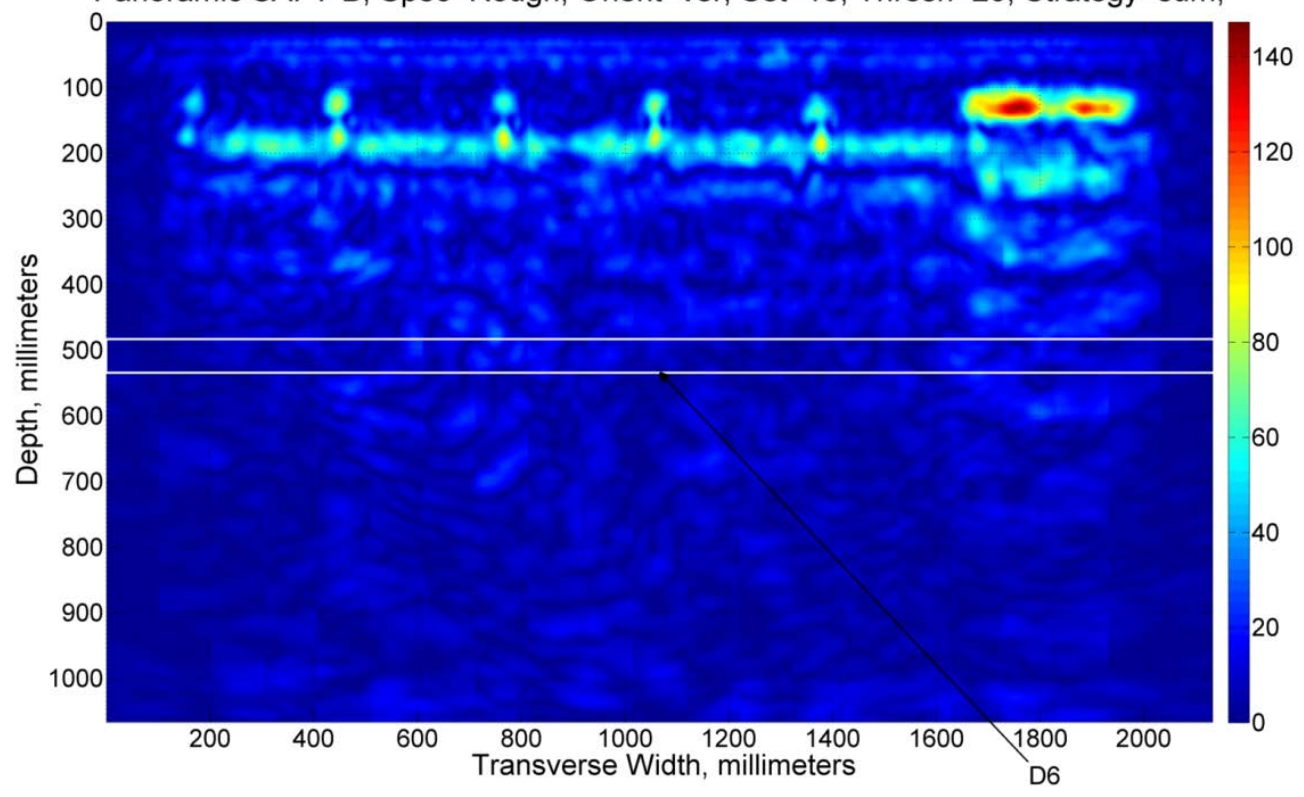

Fig. A. 157. Vertical Set 13, Node 16. 
Specimen: Thick, Depth: 1066.8mm (42in), AbsofHilbert -- Node $18(4,3), 93.75 \sim 125 \mathrm{kHz}$ Panoramic SAFT-B, Spec=Rough, Orient=ver, Set=13, Thresh=20, Strategy=sum,

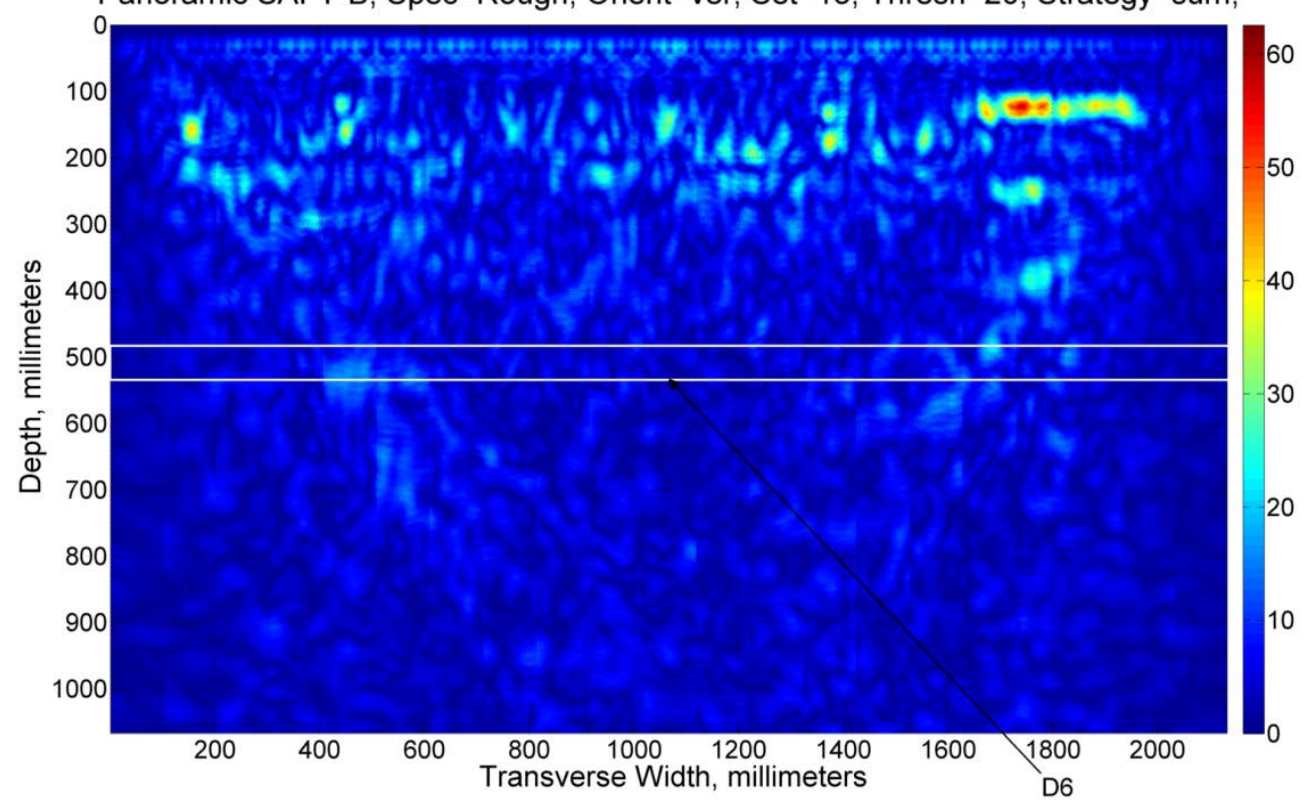

Fig. A. 158. Vertical Set 13, Node 18.

Specimen: Thick, Depth: 1066.8mm (42in), AbsofHilbert -- Node $32(5,1), 15.625 \sim 31.25 \mathrm{kHz}$ Panoramic SAFT-B, Spec=Rough, Orient=ver, Set=13, Thresh=20, Strategy=sum,

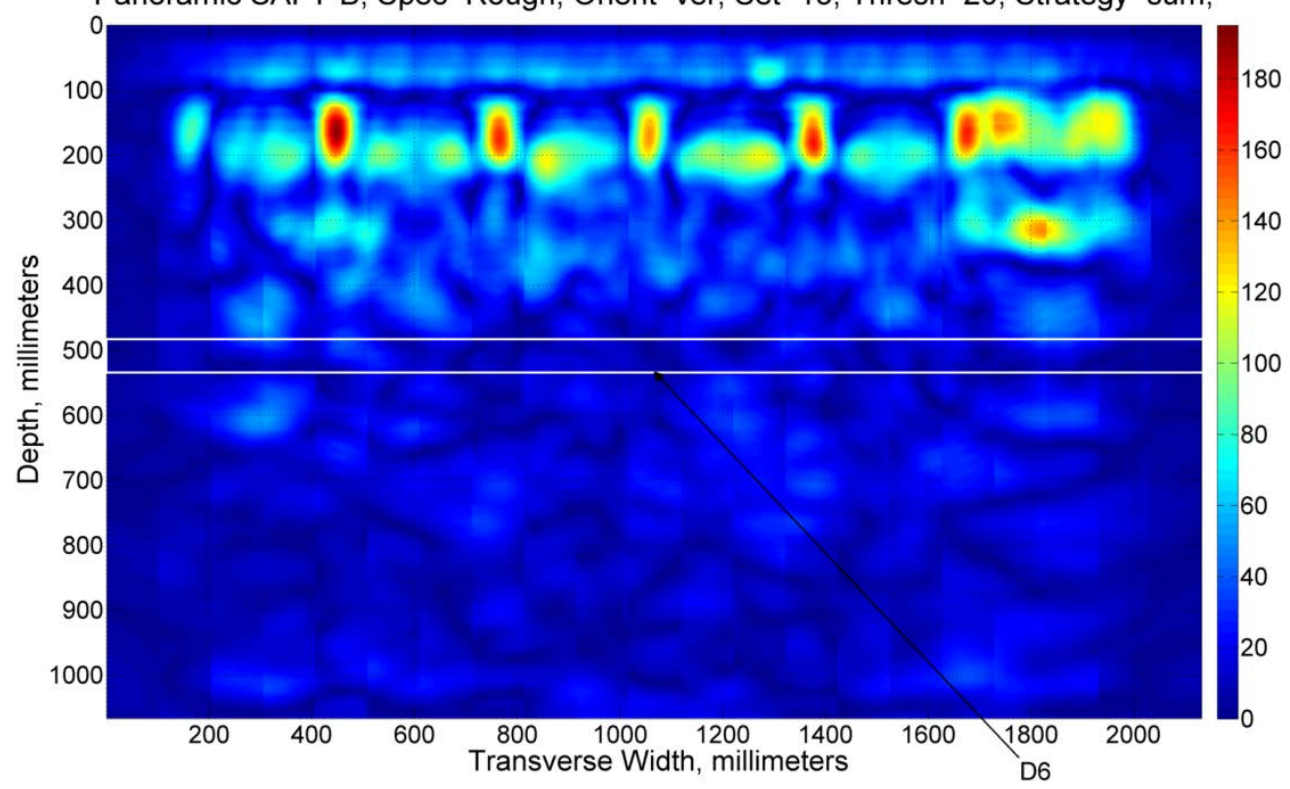

Fig. A. 159. Vertical Set 13 


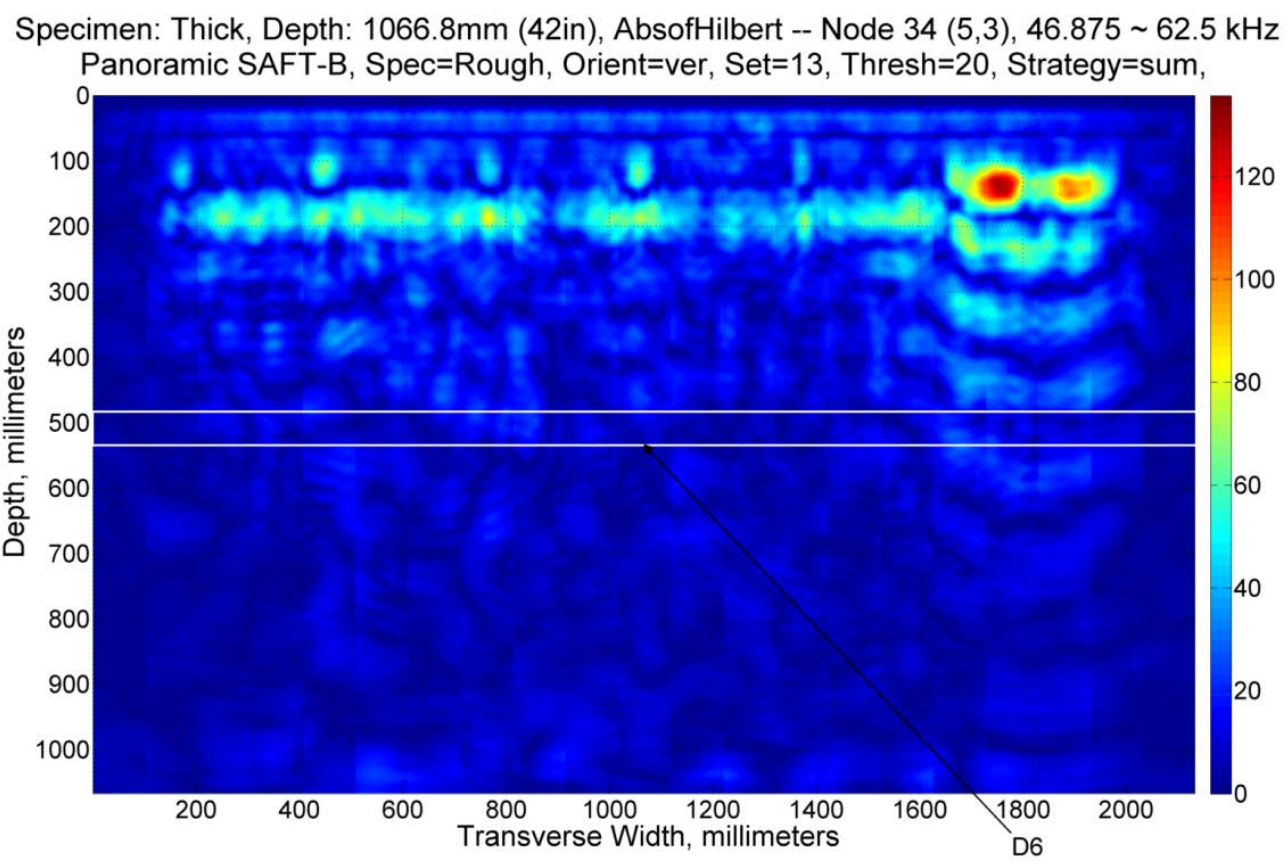

Fig. A. 160. Vertical Set 13, Node 34.

\section{SET 14 - DEFECT 1, DEFECT 5, DEFECT 6}

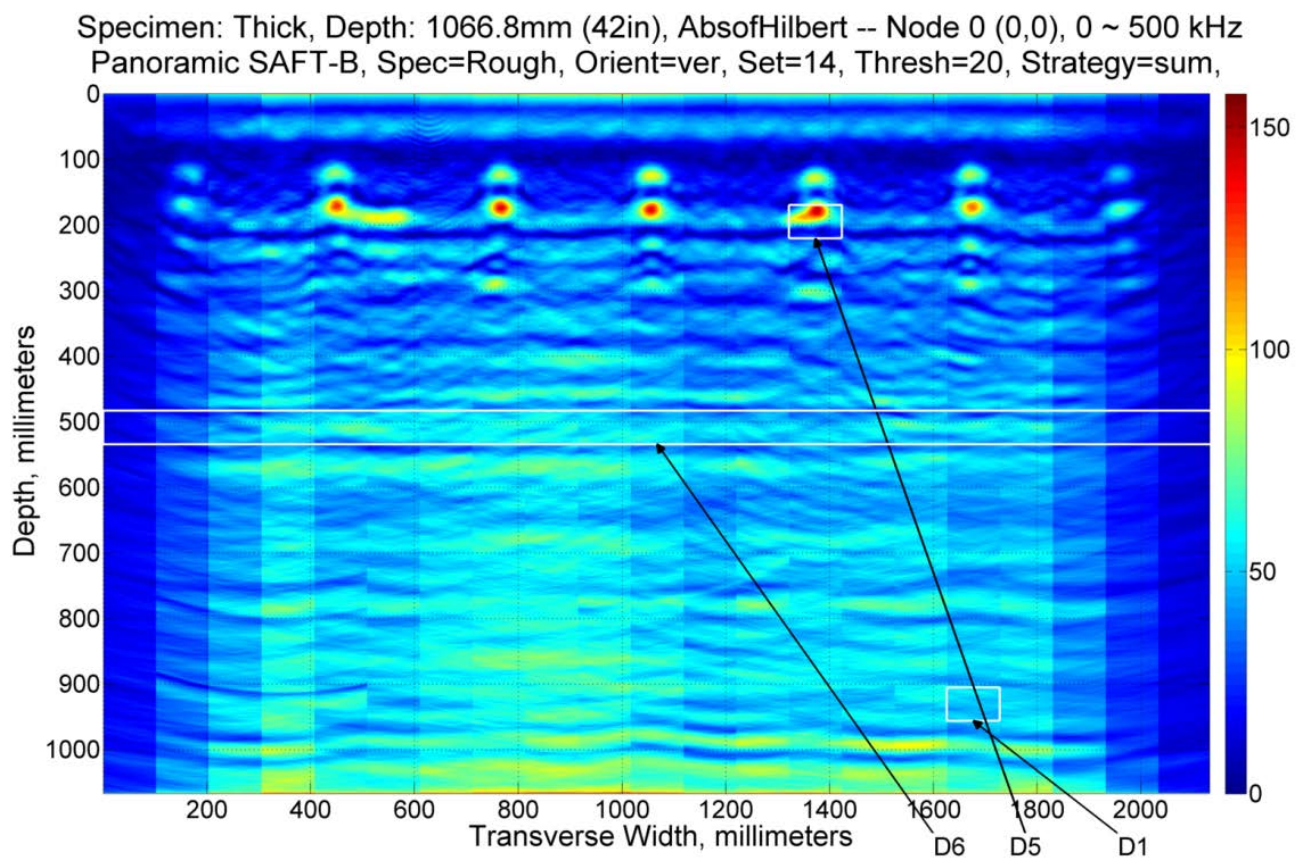

Fig. A. 161. Vertical Set 14, Node 0. 


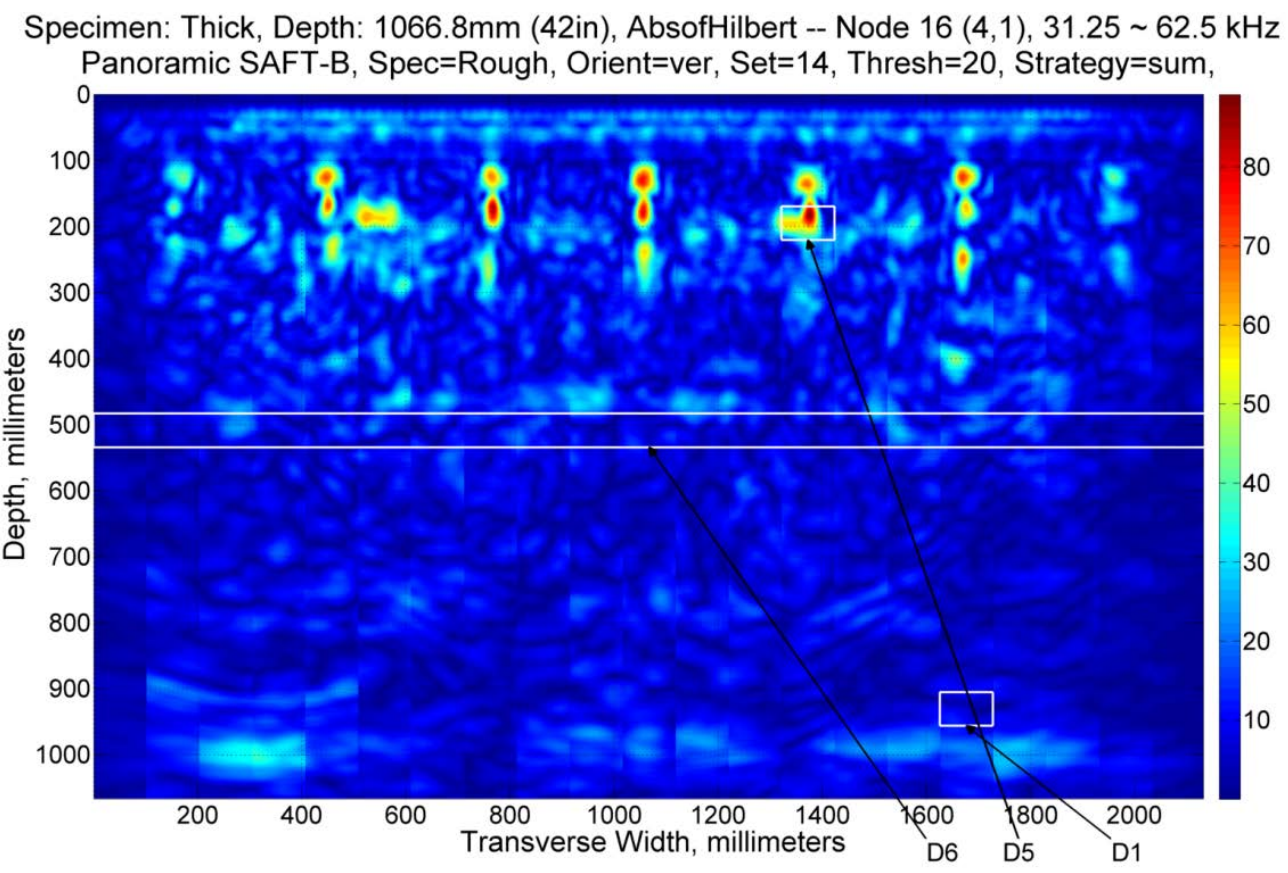

Fig. A. 162. Vertical Set 14, Node 16.

Specimen: Thick, Depth: 1066.8mm (42in), AbsofHilbert -- Node $18(4,3), 93.75 \sim 125 \mathrm{kHz}$

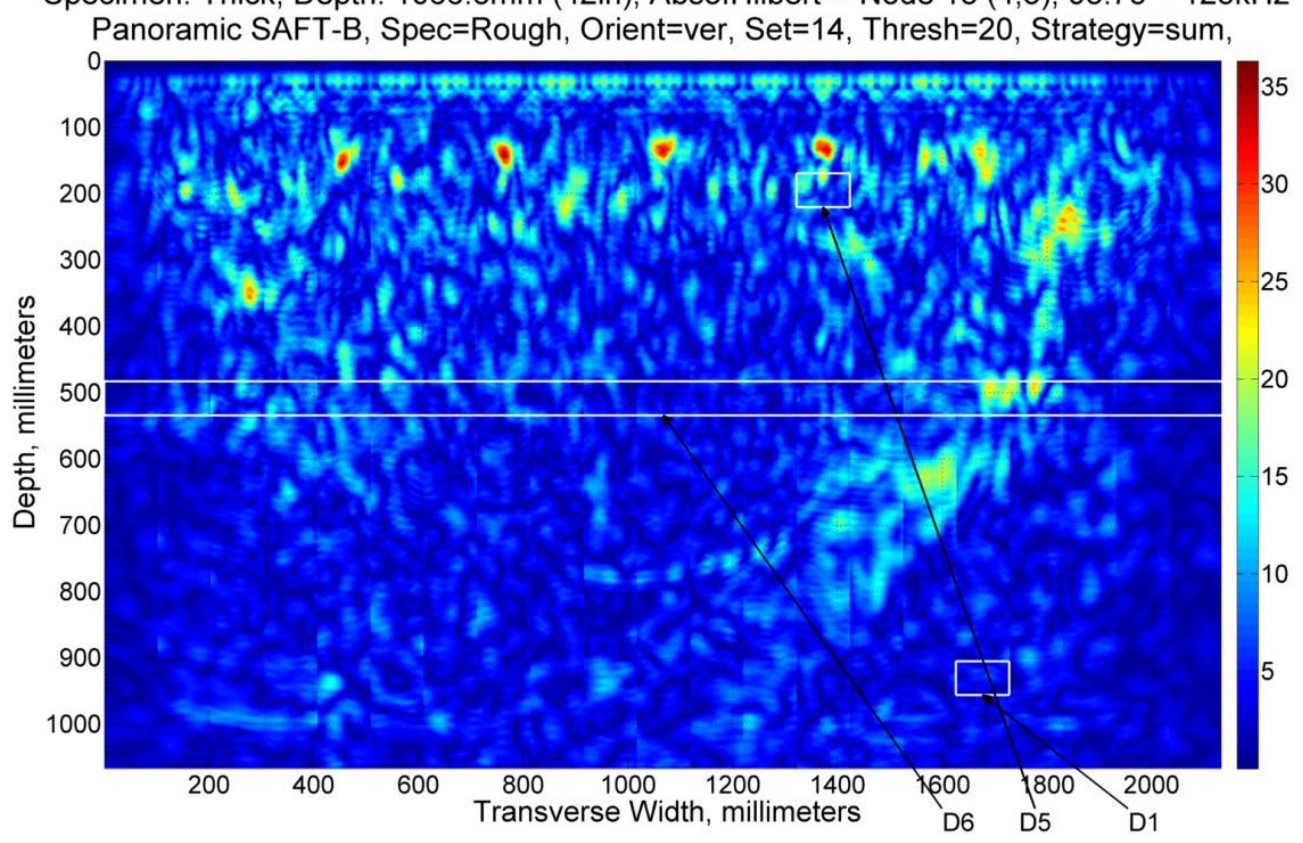

Fig. A. 163. Vertical Set 14, Node 18. 


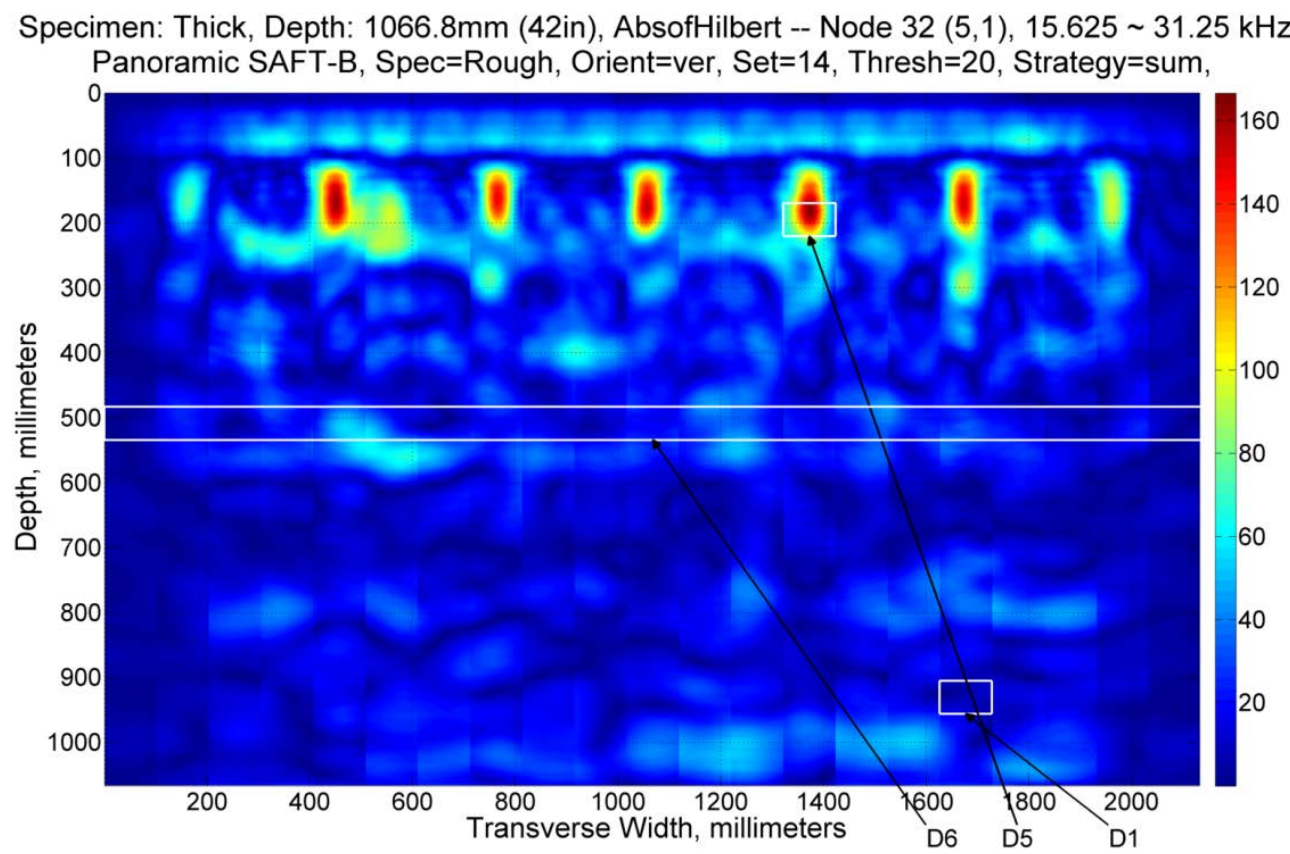

Fig. A. 164. Vertical Set 14, Node 32.

Specimen: Thick, Depth: 1066.8mm (42in), AbsofHilbert -- Node 34 (5,3), $46.875 \sim 62.5 \mathrm{kHz}$ Panoramic SAFT-B, Spec=Rough, Orient=ver, Set=14, Thresh=20, Strategy=sum,

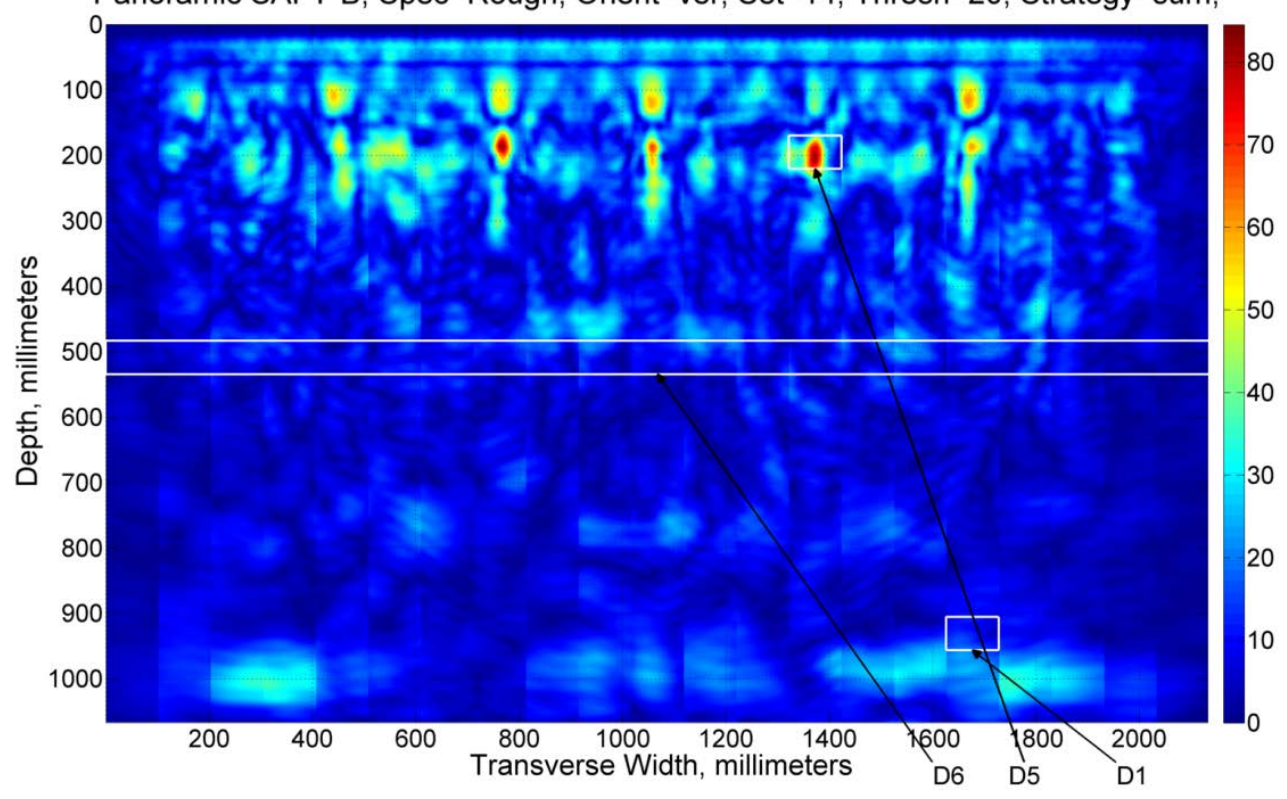

Fig. A. 165. Vertical Set 14, Node 34. 
Specimen: Thick, Depth: 1066.8mm (42in), AbsofHilbert -- Node $0(0,0), 0 \sim 500 \mathrm{kHz}$ Panoramic SAFT-B, Spec=Rough, Orient=ver, Set=15, Thresh=20, Strategy=sum,

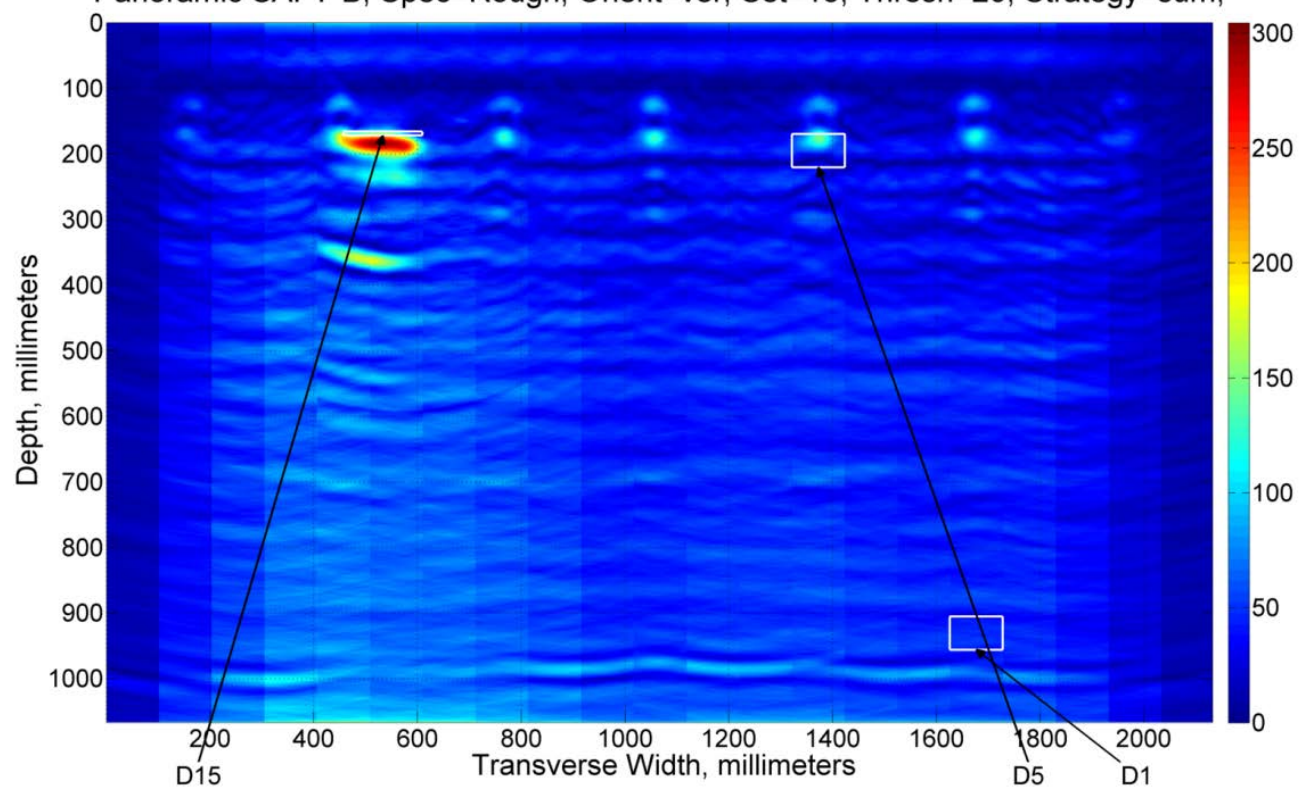

Fig. A. 166. Vertical Set 15, Node 0.

Specimen: Thick, Depth: 1066.8mm (42in), AbsofHilbert -- Node $16(4,1), 31.25 \sim 62.5 \mathrm{kHz}$ Panoramic SAFT-B, Spec=Rough, Orient=ver, Set $=15$, Thresh $=20$, Strategy=sum,

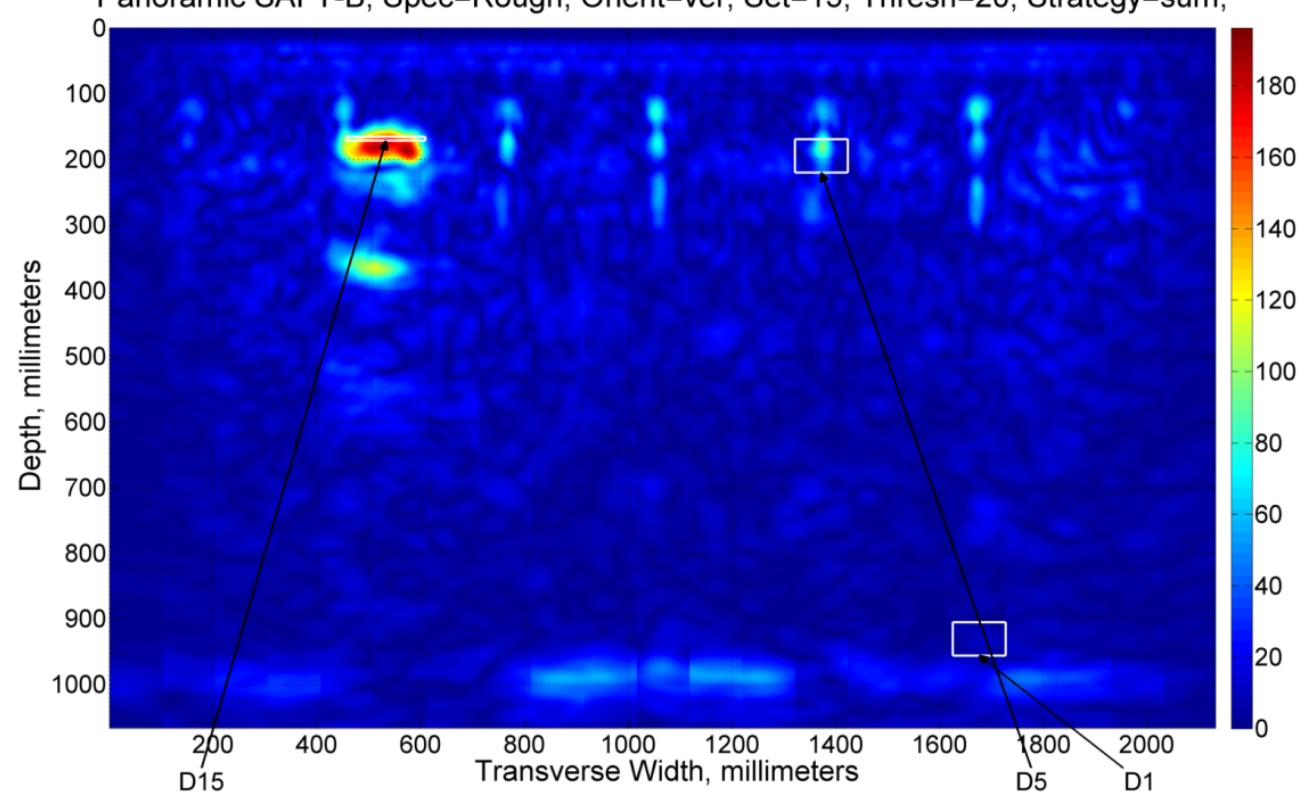

Fig. A. 167. Vertical Set 15, Node 16. 
Specimen: Thick, Depth: 1066.8mm (42in), AbsofHilbert -- Node $18(4,3), 93.75 \sim 125 \mathrm{kHz}$ Panoramic SAFT-B, Spec=Rough, Orient=ver, Set=15, Thresh=20, Strategy=sum,

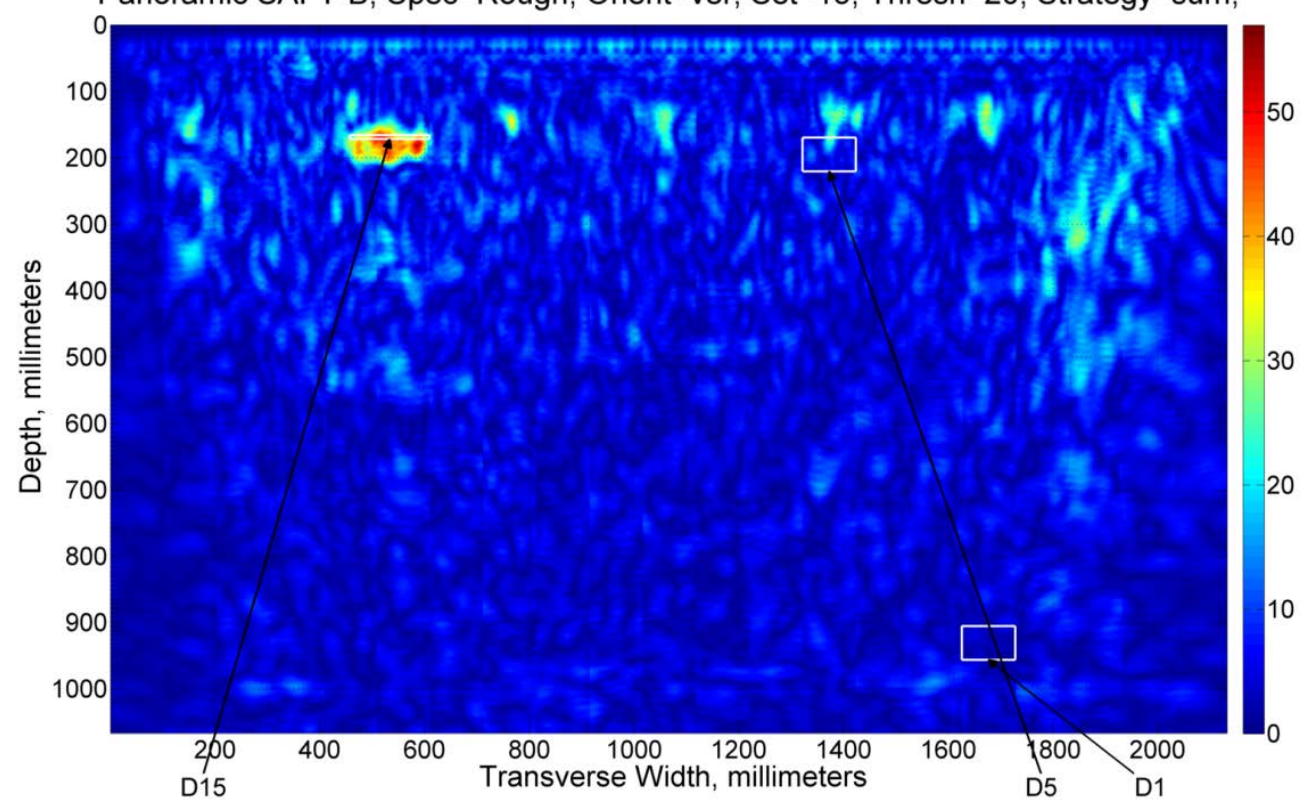

Fig. A. 168. Vertical Set 15, Node 18.

Specimen: Thick, Depth: 1066.8mm (42in), AbsofHilbert -- Node $32(5,1), 15.625 \sim 31.25 \mathrm{kHz}$ Panoramic SAFT-B, Spec=Rough, Orient=ver, Set=15, Thresh=20, Strategy=sum,

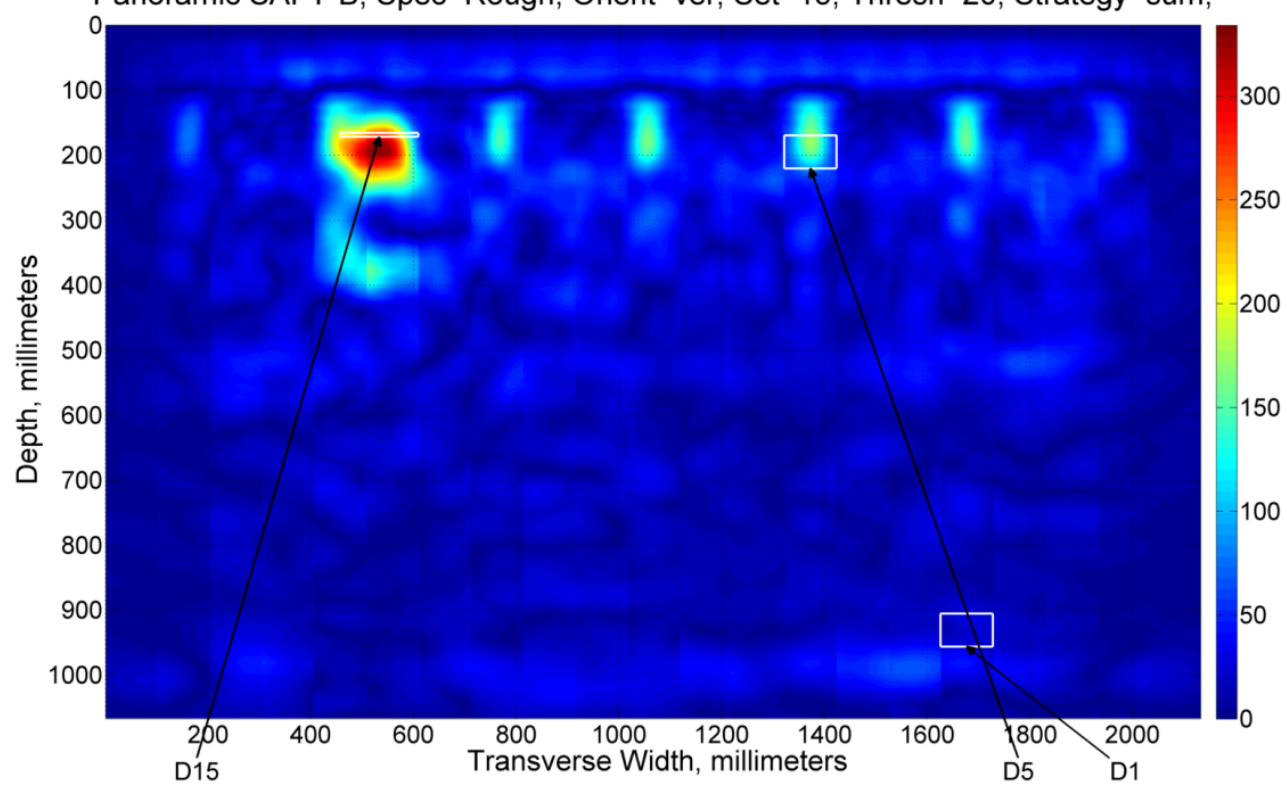

Fig. A. 169. Vertical Set 15, Node 32. 


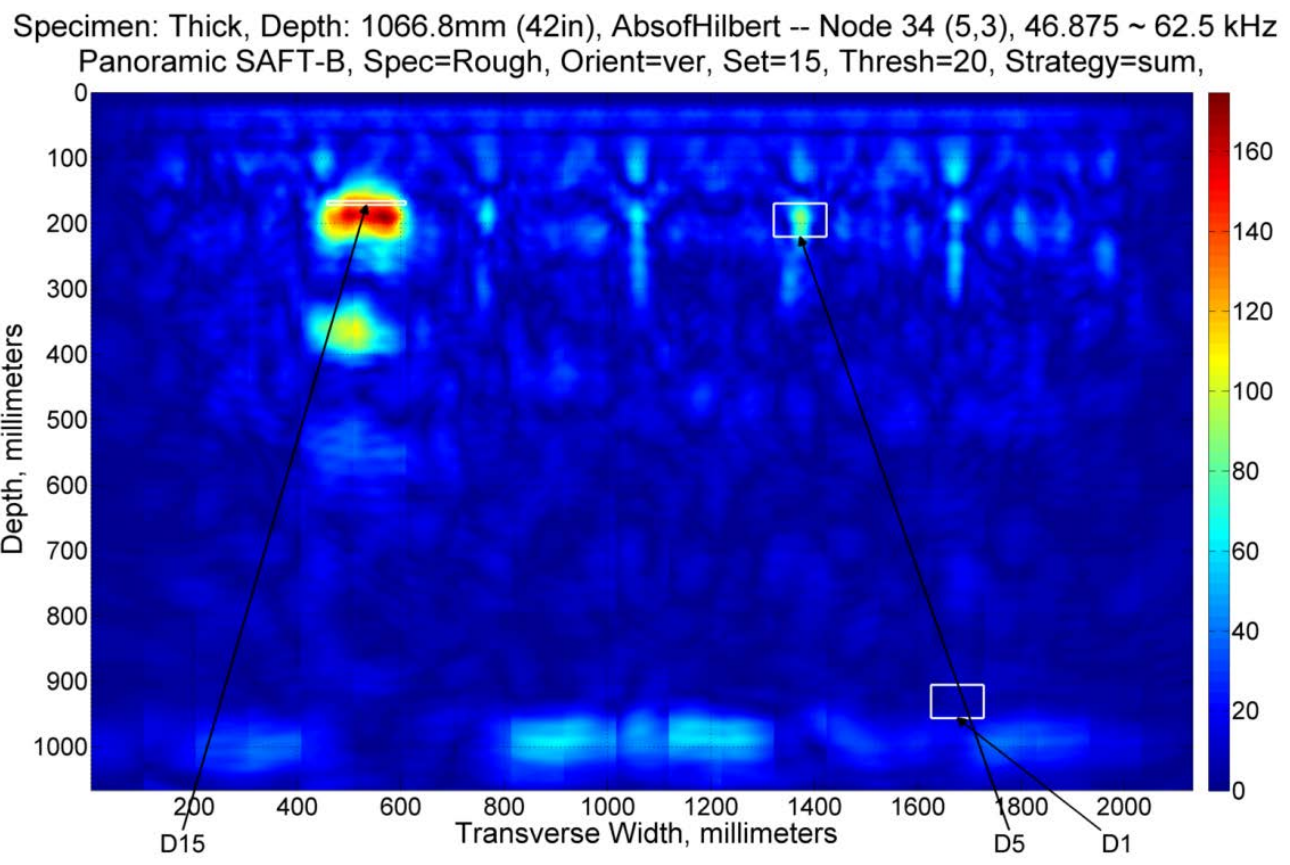

Fig. A. 170. Vertical Set 15, Node 34.

\section{SET 16 - NO DEFECT}

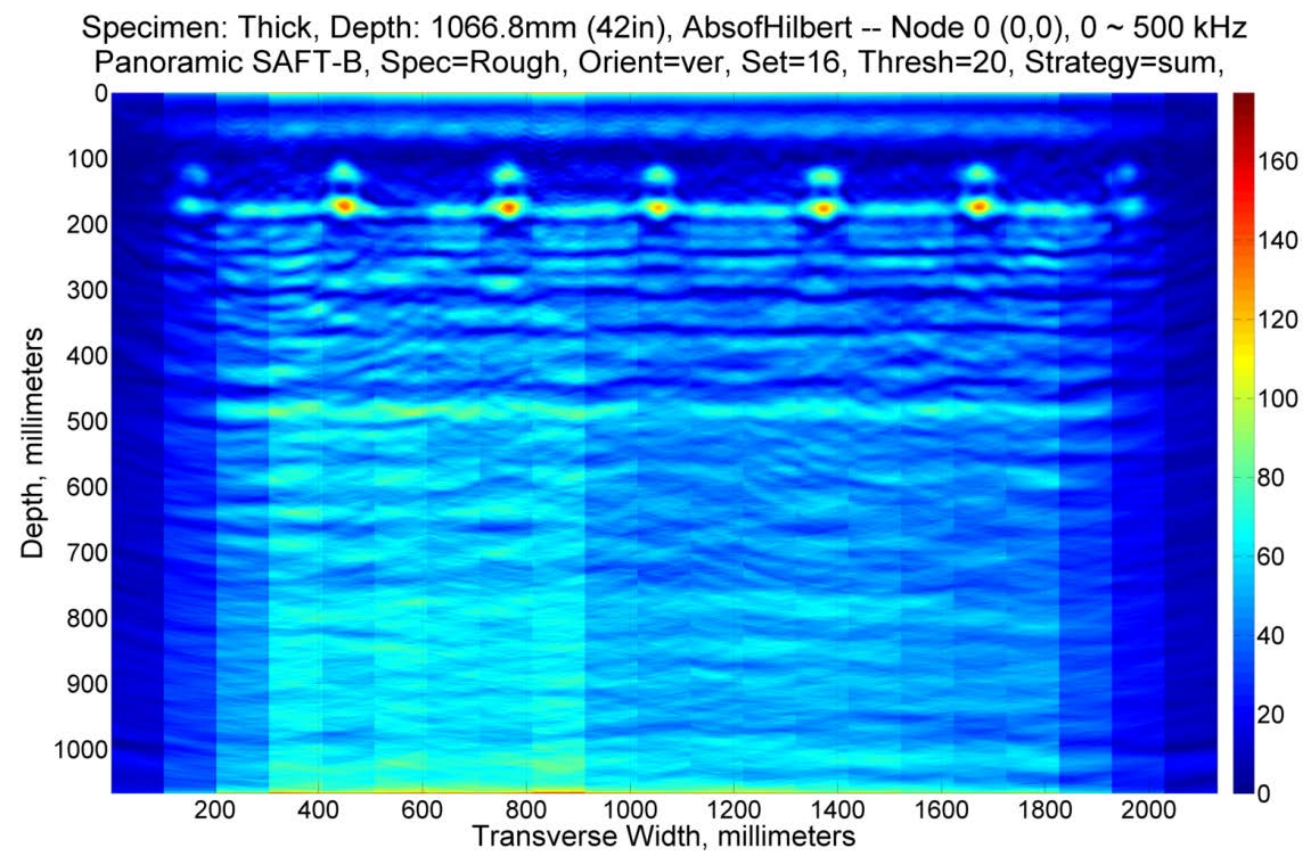

Fig. A. 171. Vertical Set 16, Node 0. 


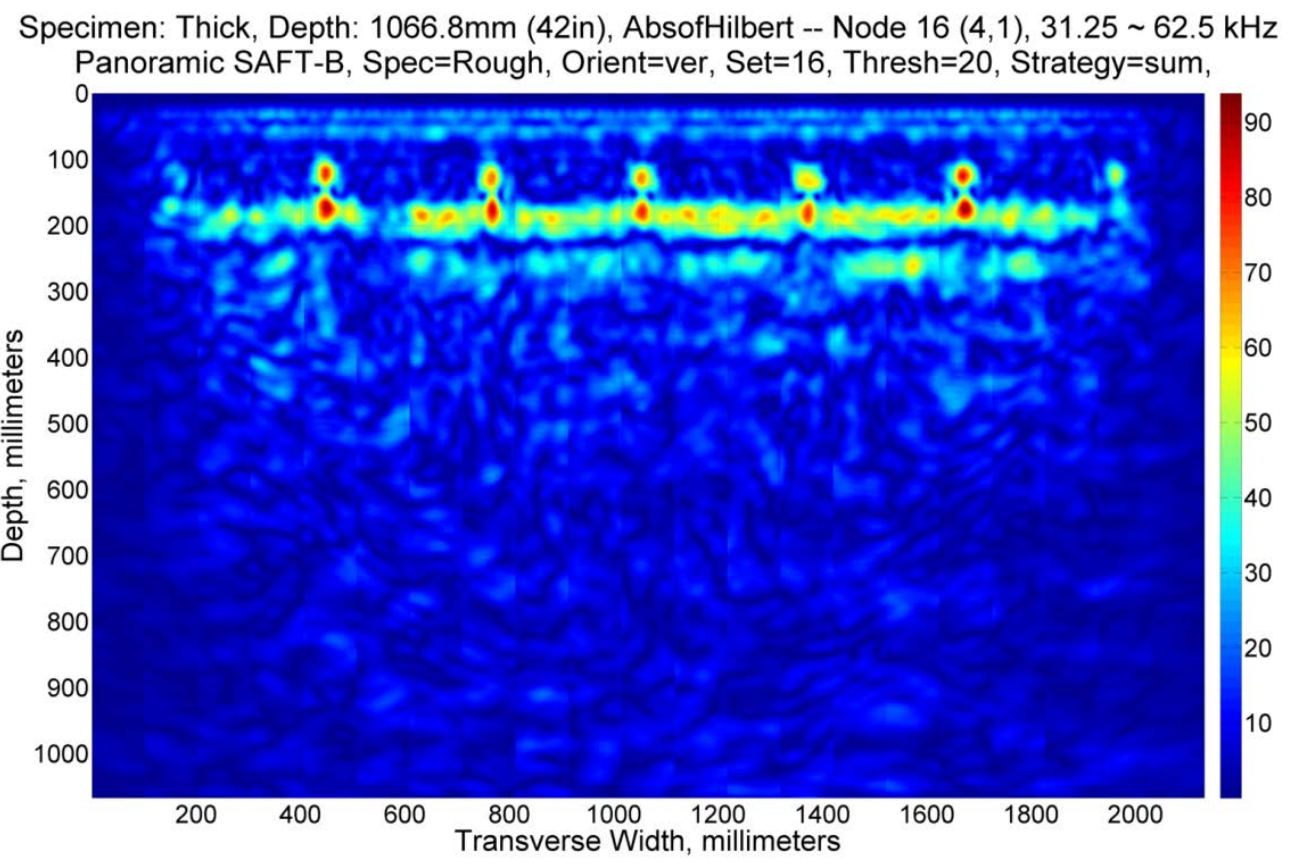

Fig. A. 172. Vertical Set 16, Node 16.

Specimen: Thick, Depth: 1066.8mm (42in), AbsofHilbert -- Node $18(4,3), 93.75 \sim 125 \mathrm{kHz}$ Panoramic SAFT-B, Spec=Rough, Orient=ver, Set=16, Thresh=20, Strategy=sum,

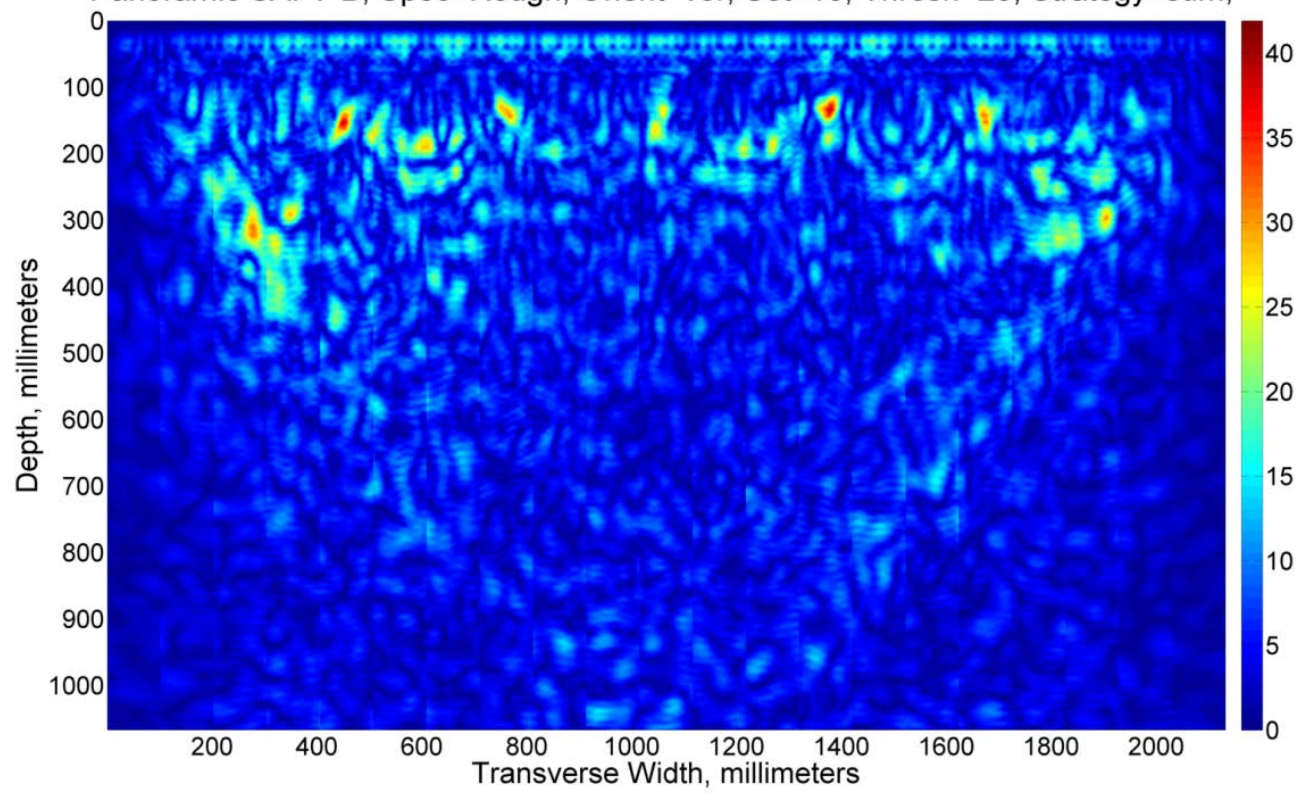

Fig. A. 173. Vertical Set 16, Node 18. 


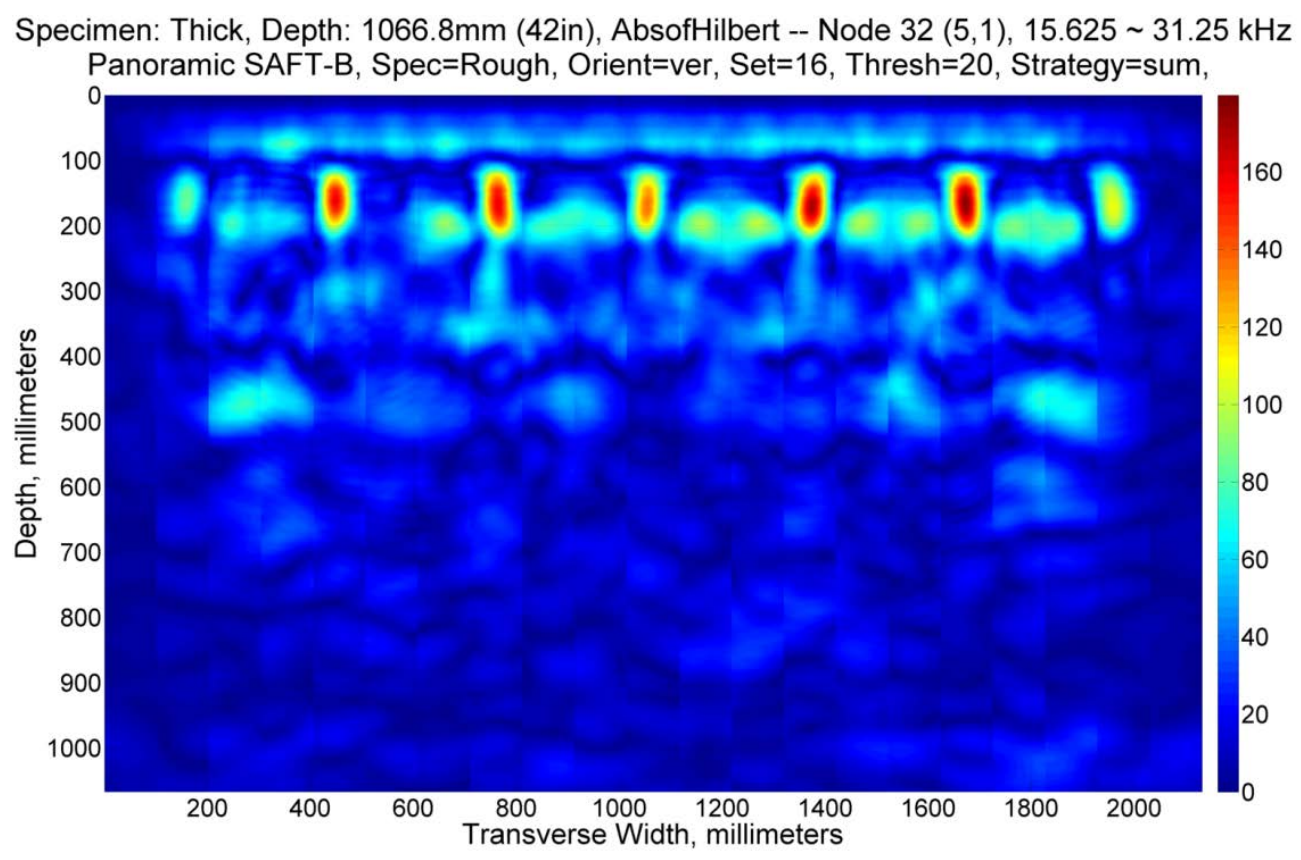

Fig. A. 174. Vertical Set 16, Node 32.

Specimen: Thick, Depth: 1066.8mm (42in), AbsofHilbert -- Node $34(5,3), 46.875 \sim 62.5 \mathrm{kHz}$ Panoramic SAFT-B, Spec=Rough, Orient=ver, Set=16, Thresh=20, Strategy=sum,

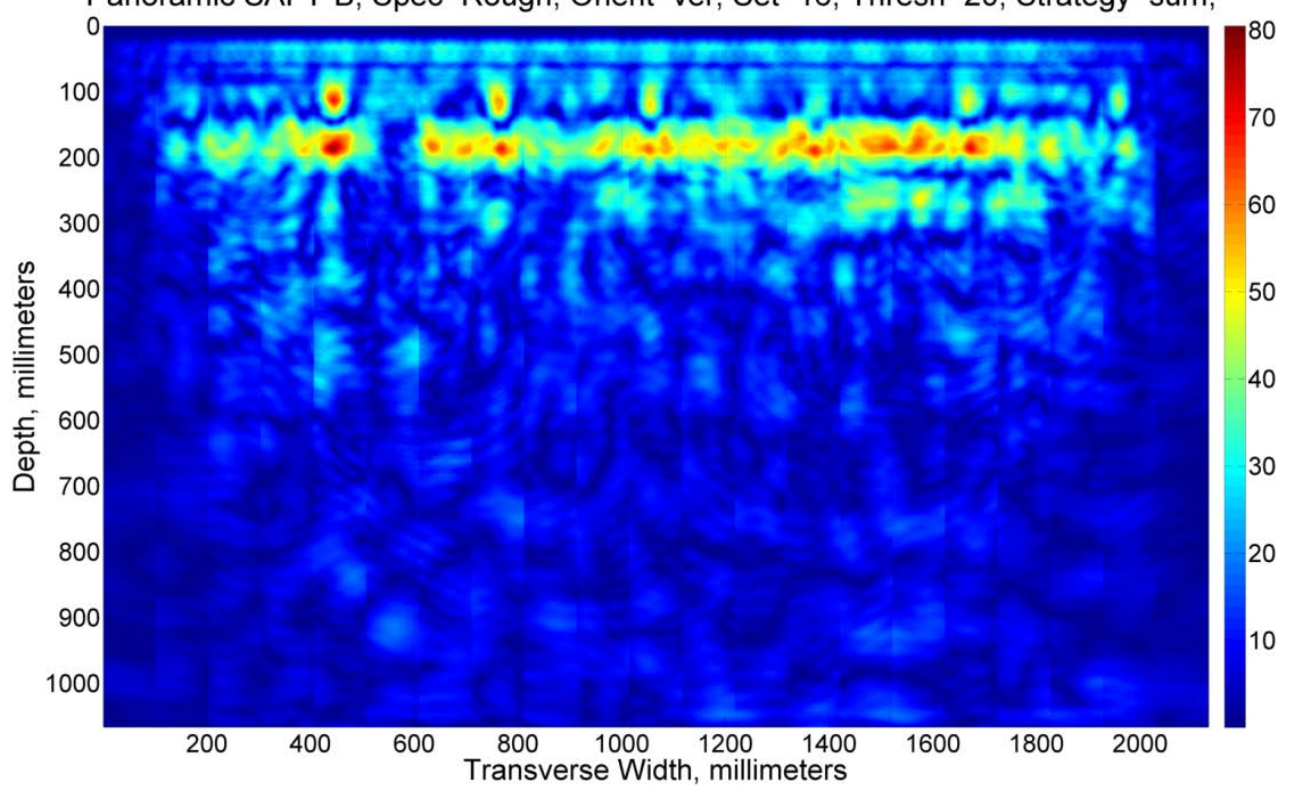

Fig. A. 175. Vertical Set 16, Node 34. 


\section{SET 17 - NO DEFECT}

Specimen: Thick, Depth: 1066.8mm (42in), AbsofHilbert -- Node $0(0,0), 0 \sim 500 \mathrm{kHz}$ Panoramic SAFT-B, Spec=Rough, Orient=ver, Set=17, Thresh=20, Strategy=sum,

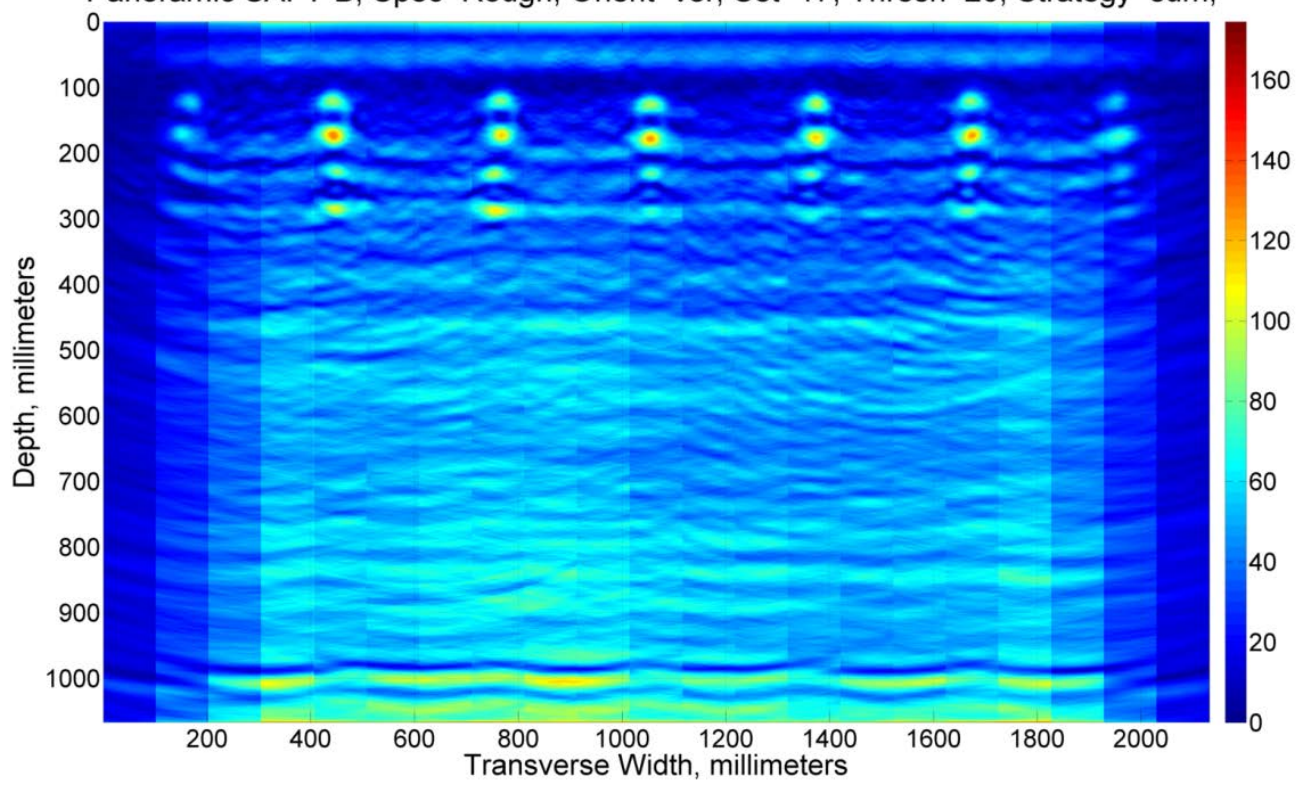

Fig. A. 176. Vertical Set 17, Node 0.

Specimen: Thick, Depth: 1066.8mm (42in), AbsofHilbert -- Node $16(4,1), 31.25 \sim 62.5 \mathrm{kHz}$ Panoramic SAFT-B, Spec=Rough, Orient=ver, Set=17, Thresh=20, Strategy=sum,

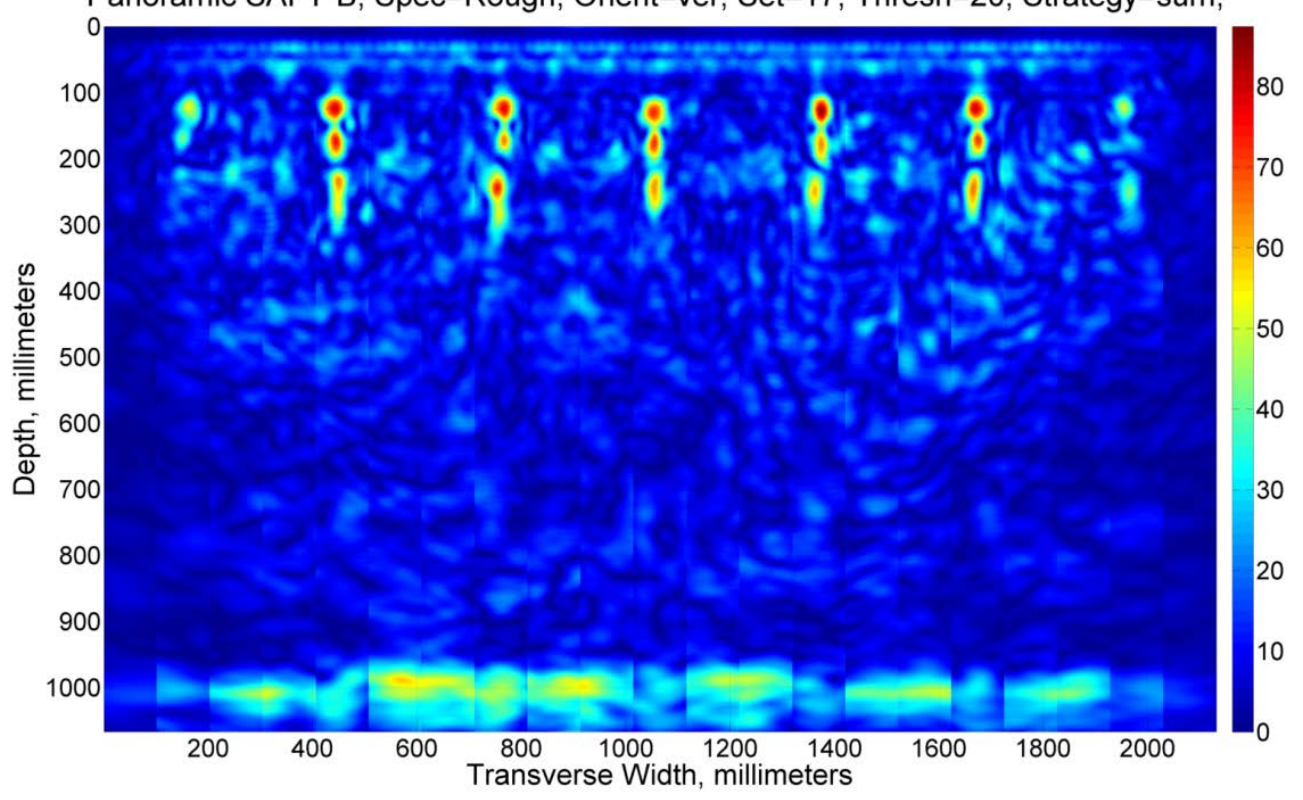

Fig. A. 177. Vertical Set 17, Node 16. 


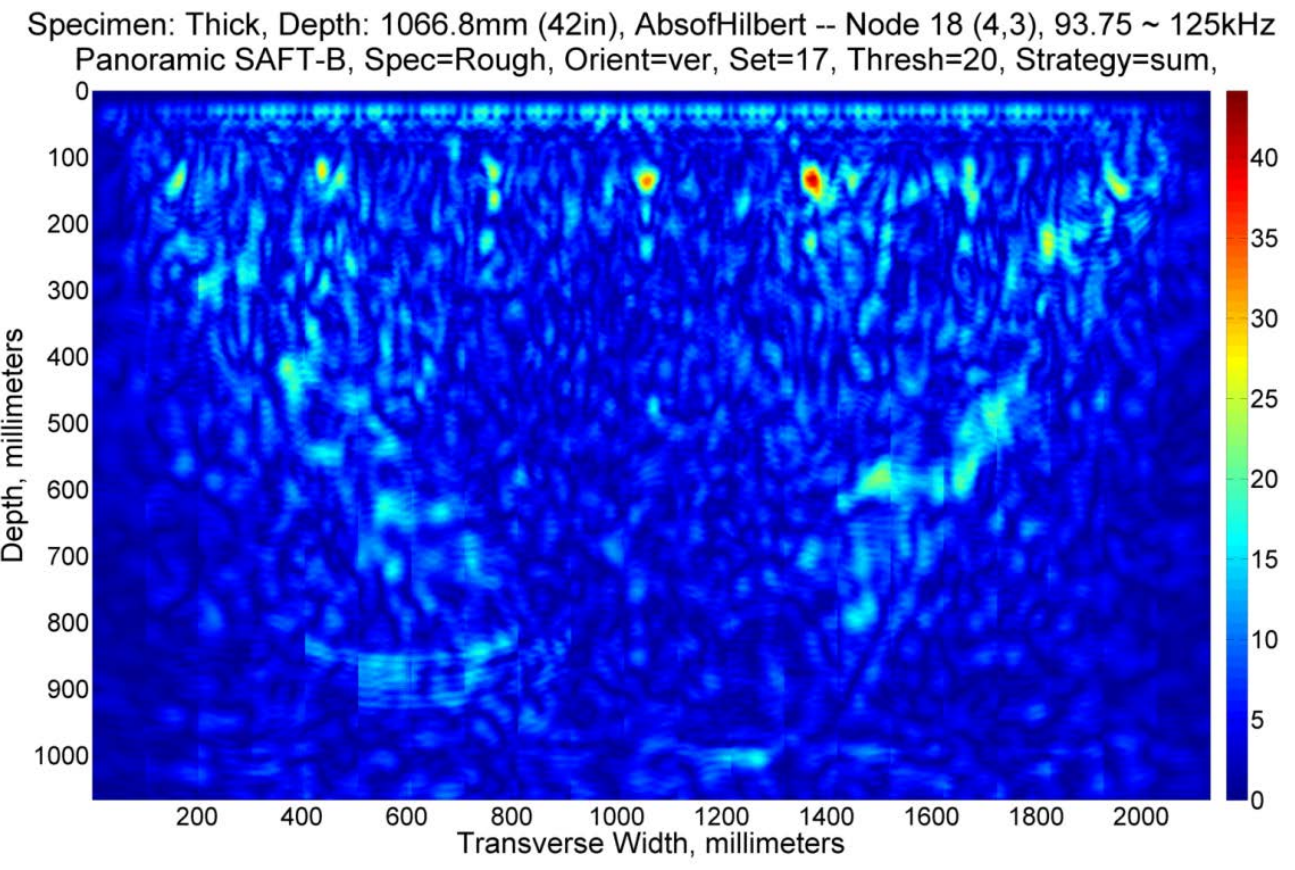

Fig. A. 178. Vertical Set 17, Node 18.

Specimen: Thick, Depth: 1066.8mm (42in), AbsofHilbert -- Node 34 (5,3), $46.875 \sim 62.5 \mathrm{kHz}$

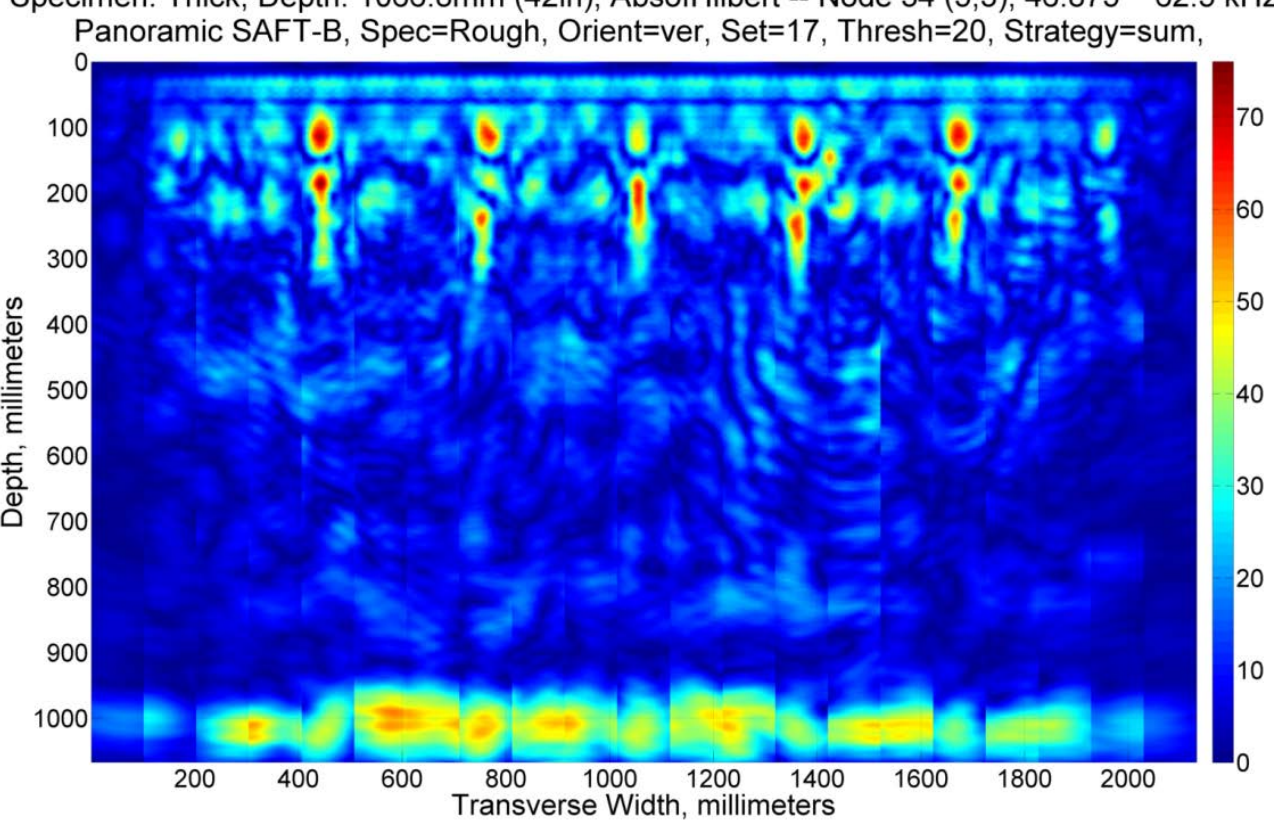

Fig. A. 179. Vertical Set 17, Node 34. 


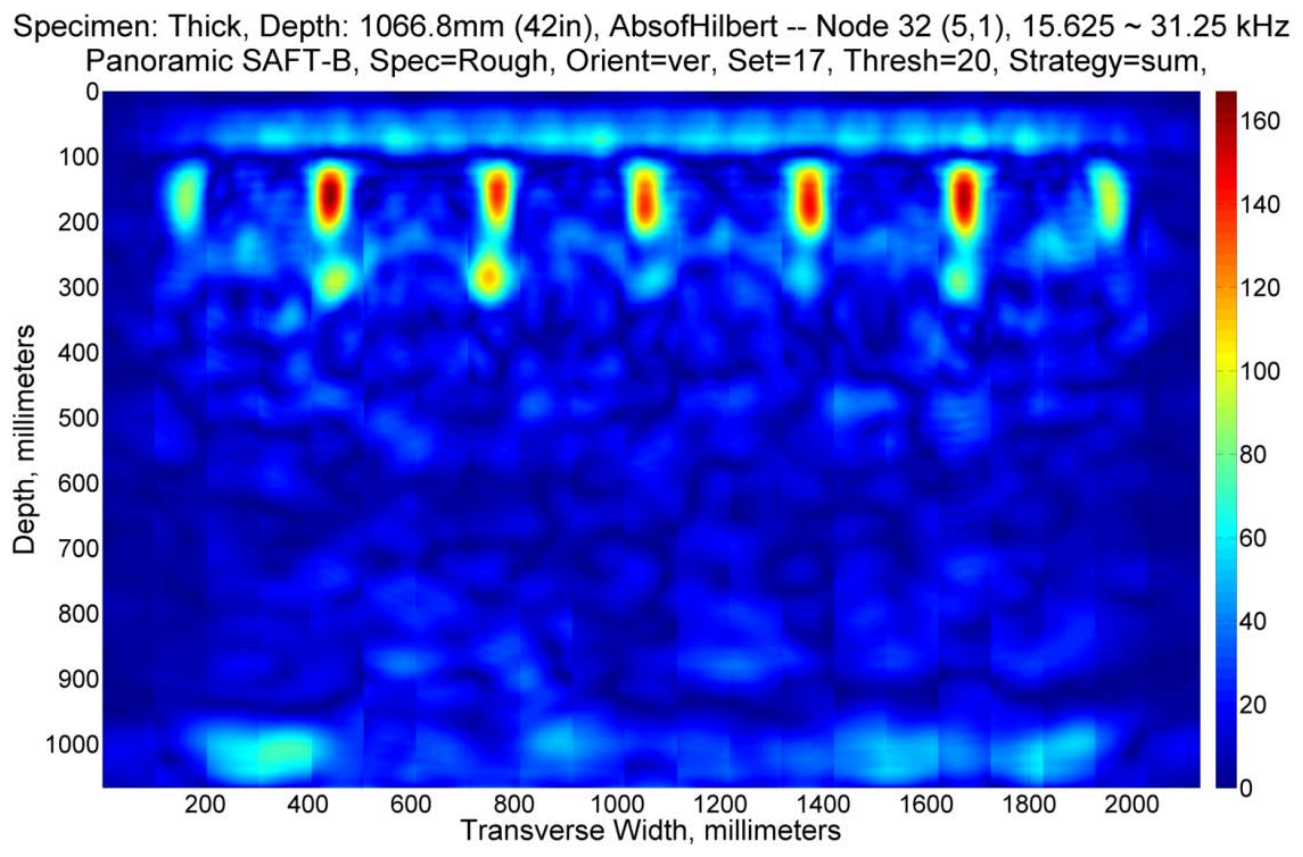

Fig. A. 180. Vertical Set 17, Node 32.

\section{SET 18 - NO DEFECT}

Specimen: Thick, Depth: 1066.8mm (42in), AbsofHilbert -- Node $0(0,0), 0 \sim 500 \mathrm{kHz}$ Panoramic SAFT-B, Spec=Rough, Orient=ver, Set=18, Thresh=20, Strategy=sum,

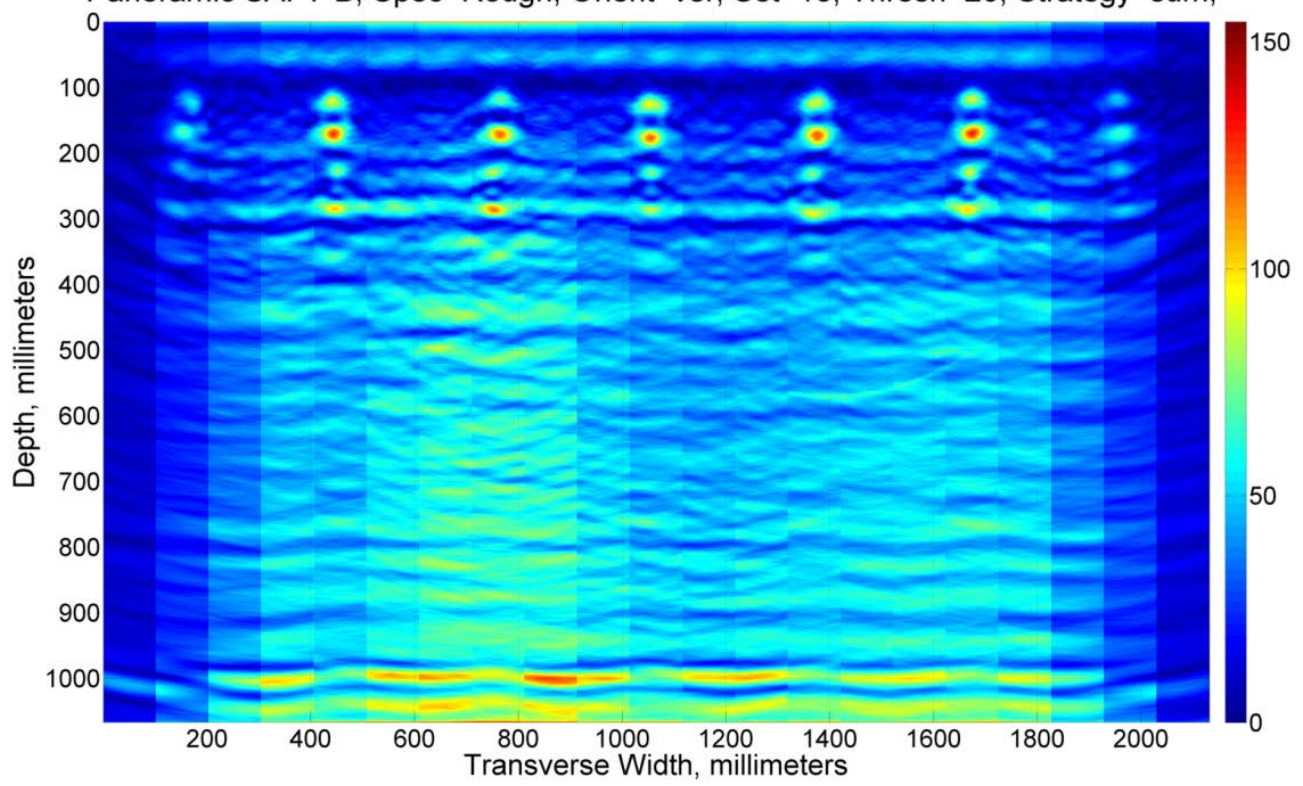

Fig. A. 181. Vertical Set 18, Node 0. 


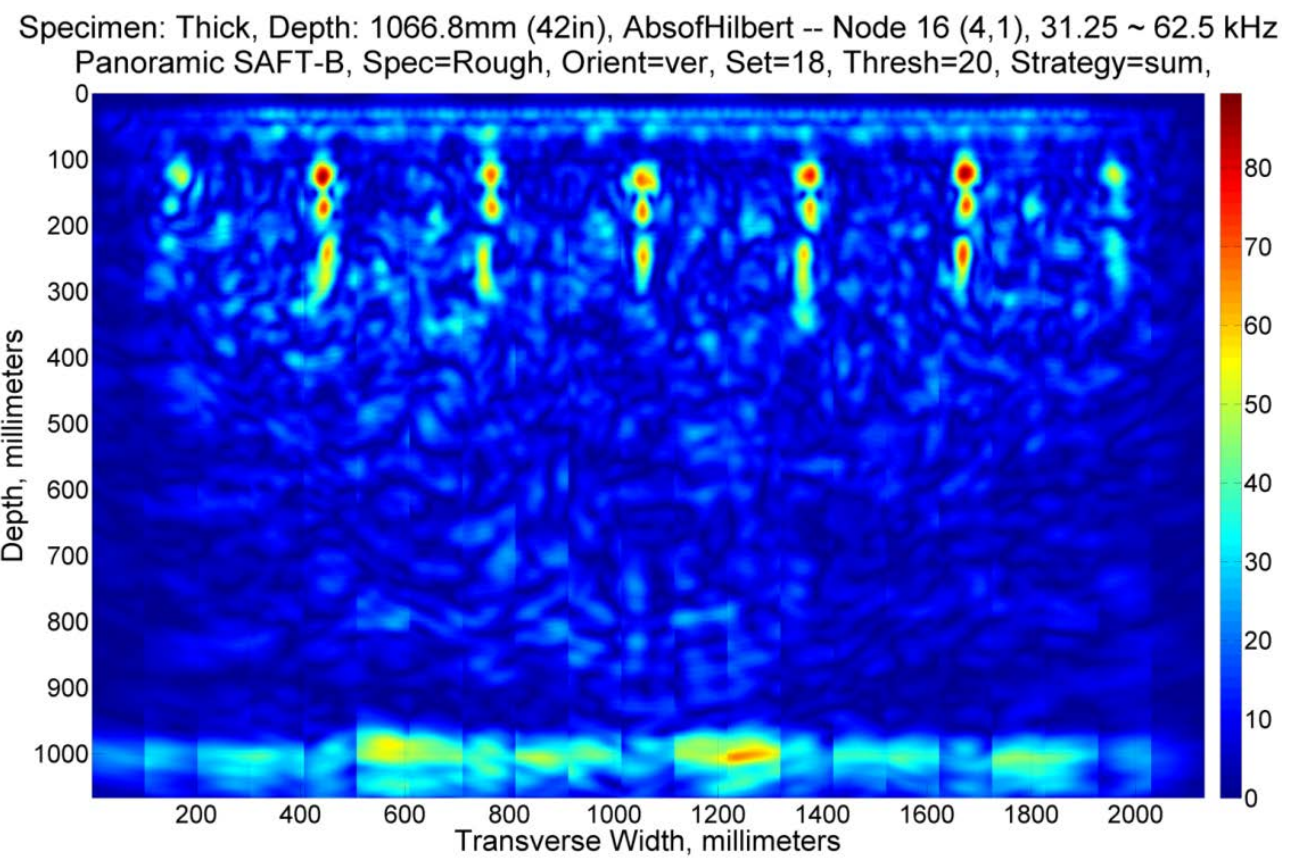

Fig. A. 182. Vertical Set 18, Node 16

Specimen: Thick, Depth: 1066.8mm (42in), AbsofHilbert -- Node $18(4,3), 93.75 \sim 125 \mathrm{kHz}$ Panoramic SAFT-B, Spec=Rough, Orient=ver, Set=18, Thresh=20, Strategy=sum,

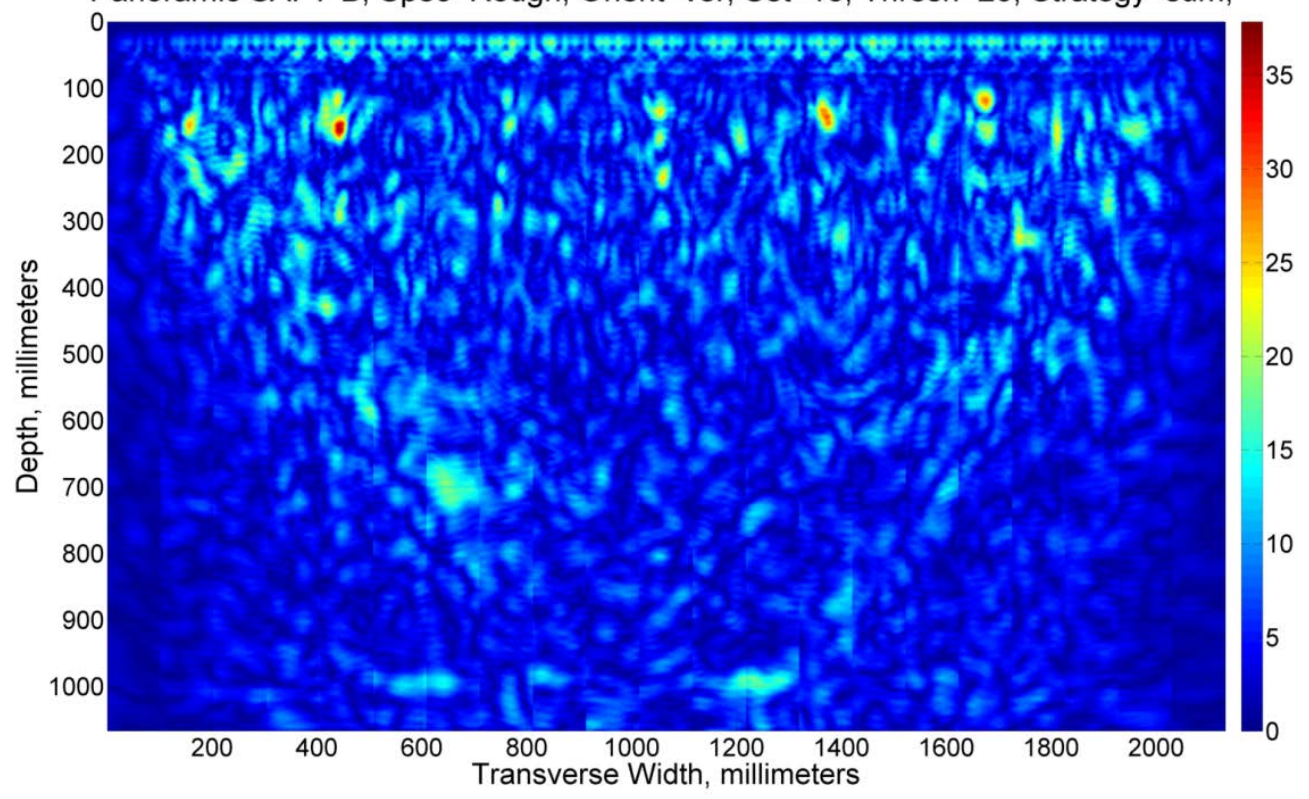

Fig. A. 183. Vertical Set 18, Node 18. 


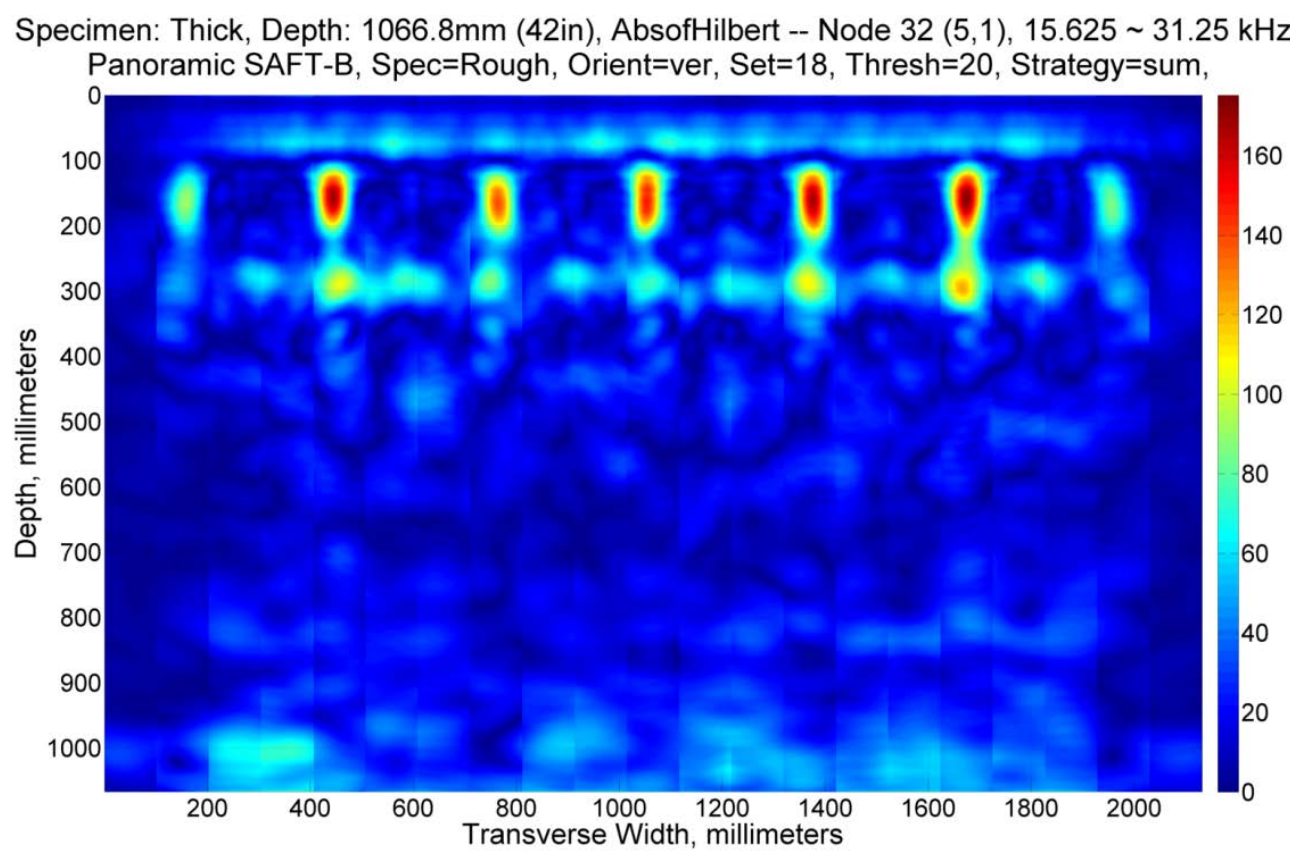

Fig. A. 184. Vertical Set 18, Node 32.

Specimen: Thick, Depth: 1066.8mm (42in), AbsofHilbert -- Node $34(5,3), 46.875 \sim 62.5 \mathrm{kHz}$ Panoramic SAFT-B, Spec=Rough, Orient=ver, Set=18, Thresh=20, Strategy=sum,

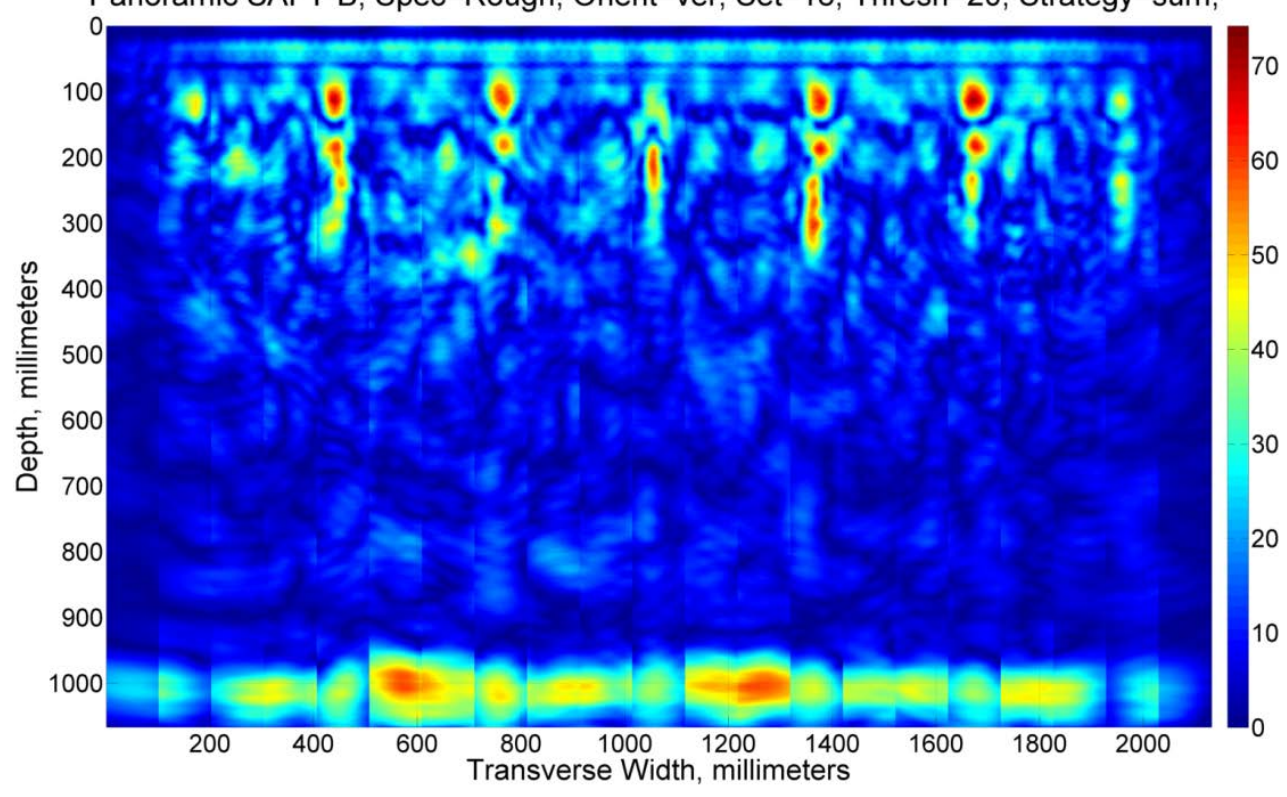

Fig. A. 185. Vertical Set 18, Node 34. 


\section{SET 19 - NO DEFECT}

Specimen: Thick, Depth: 1066.8mm (42in), AbsofHilbert -- Node $0(0,0), 0 \sim 500 \mathrm{kHz}$ Panoramic SAFT-B, Spec=Rough, Orient=ver, Set=19, Thresh=20, Strategy=sum,

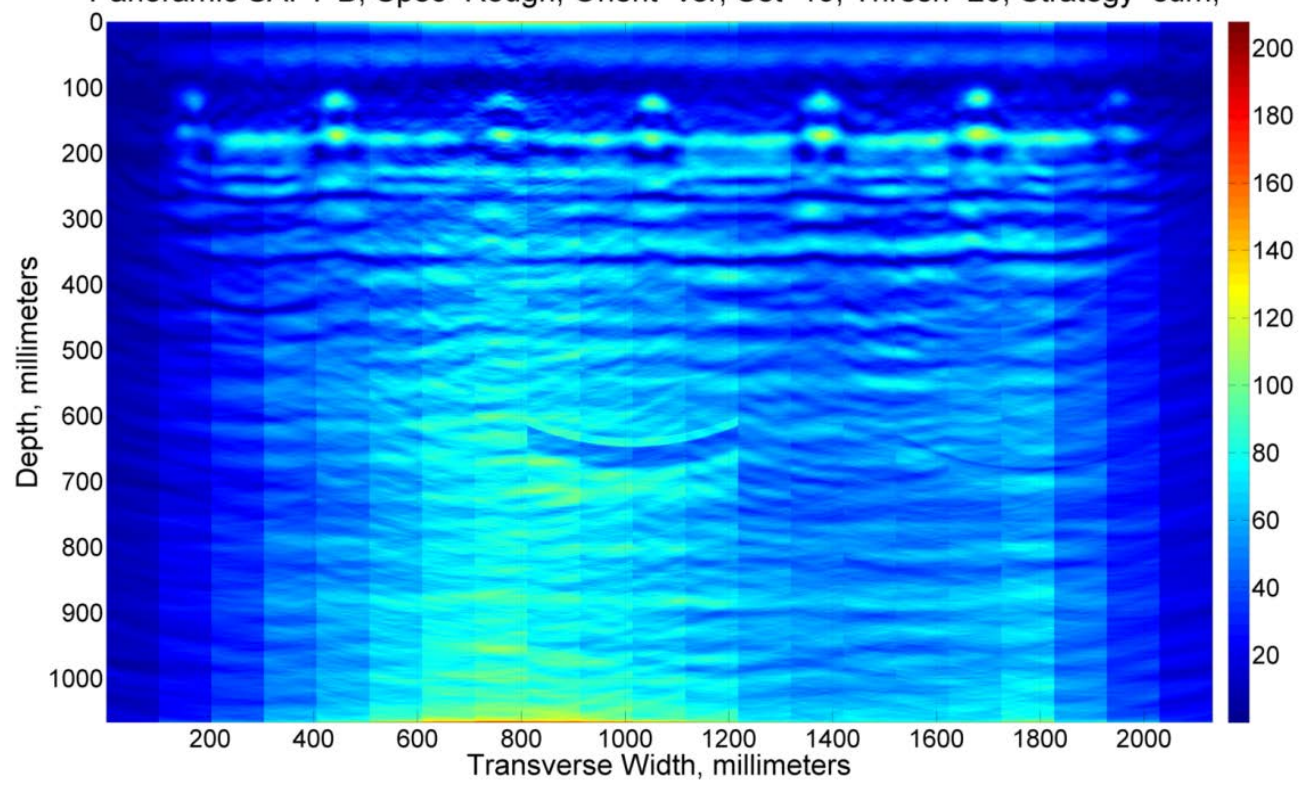

Fig. A. 186. Vertical Set 19, Node 0.

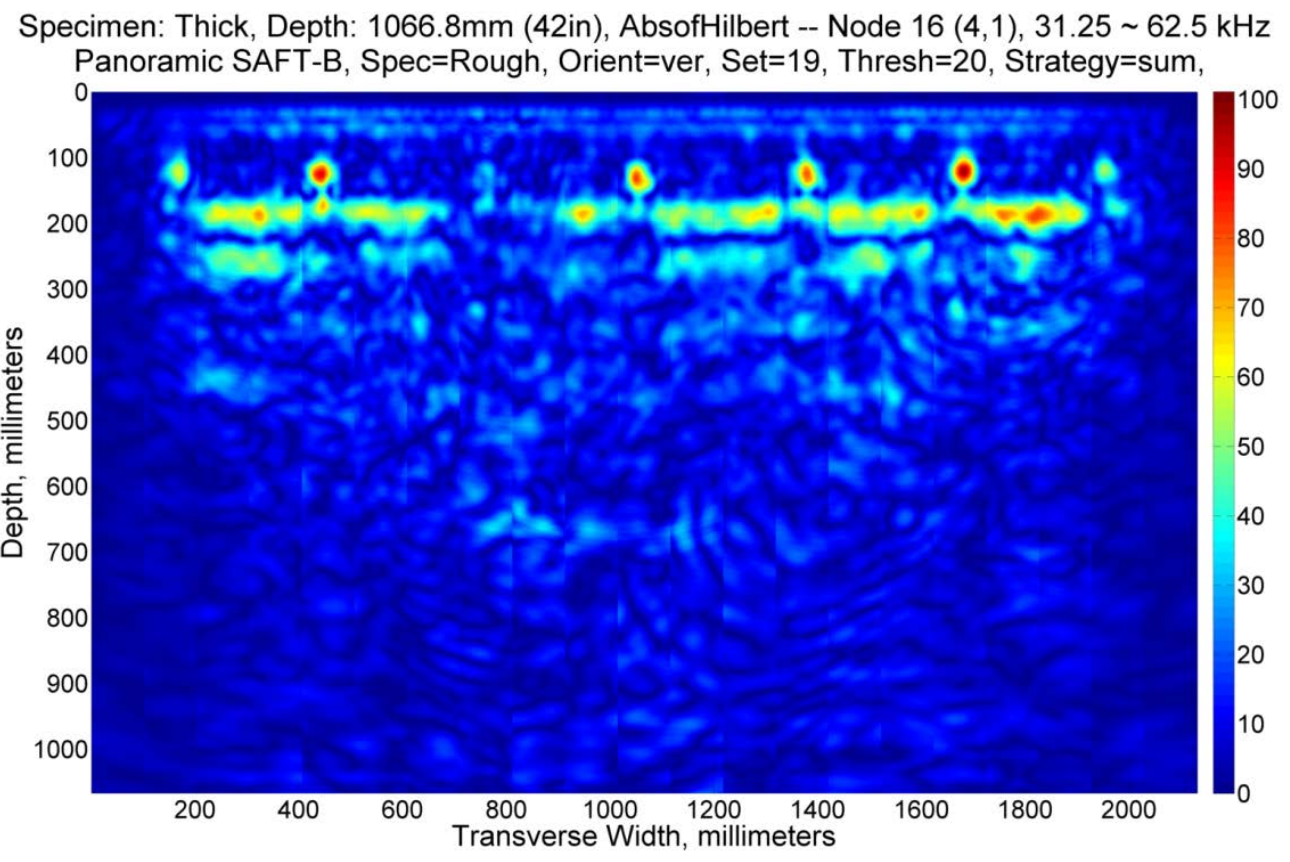

Fig. A. 187. Vertical Set 19, Node 16. 


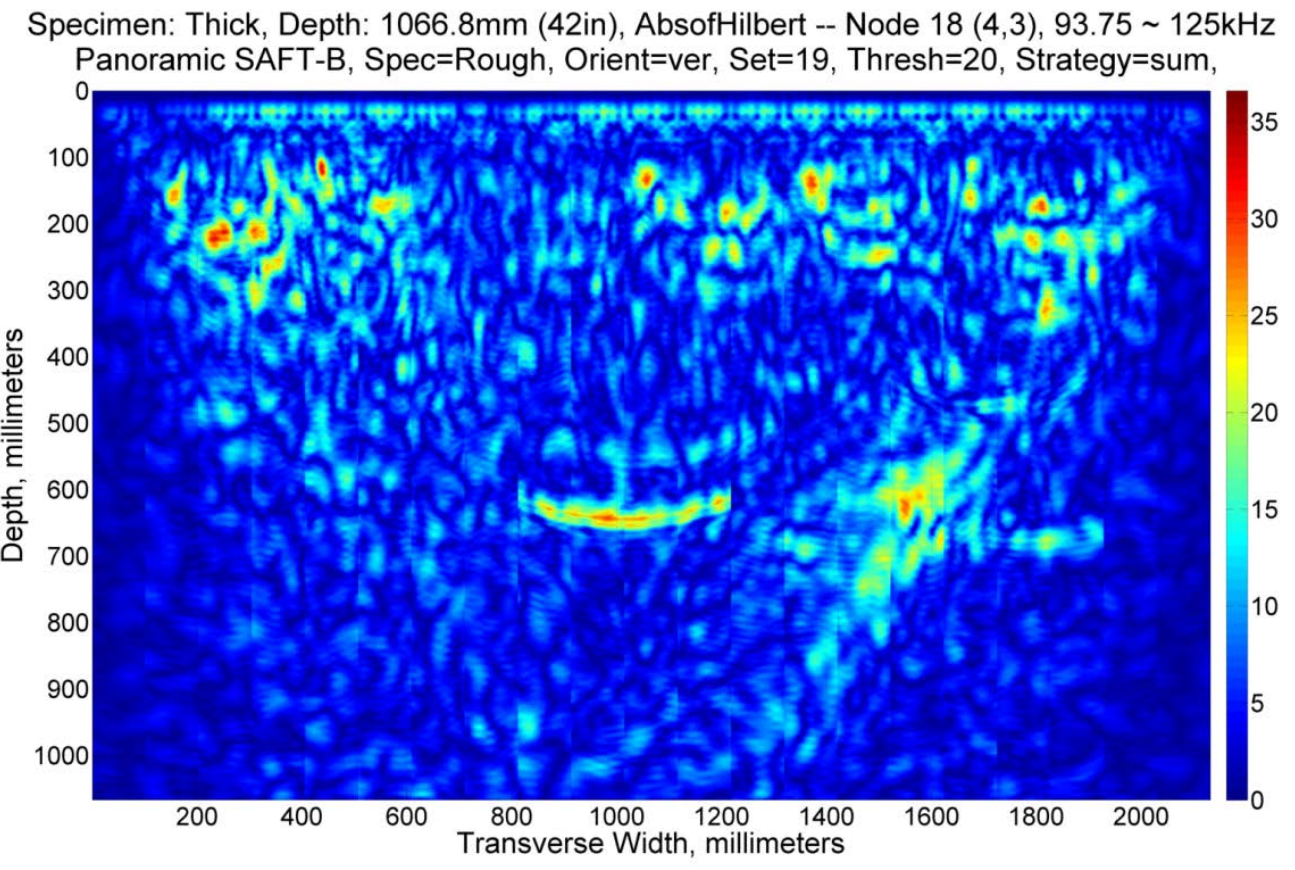

Fig. A. 188. Vertical Set 19, Node 18.

Specimen: Thick, Depth: 1066.8mm (42in), AbsofHilbert -- Node 34 (5,3), $46.875 \sim 62.5 \mathrm{kHz}$ Panoramic SAFT-B, Spec=Rough, Orient=ver, Set=19, Thresh=20, Strategy=sum,

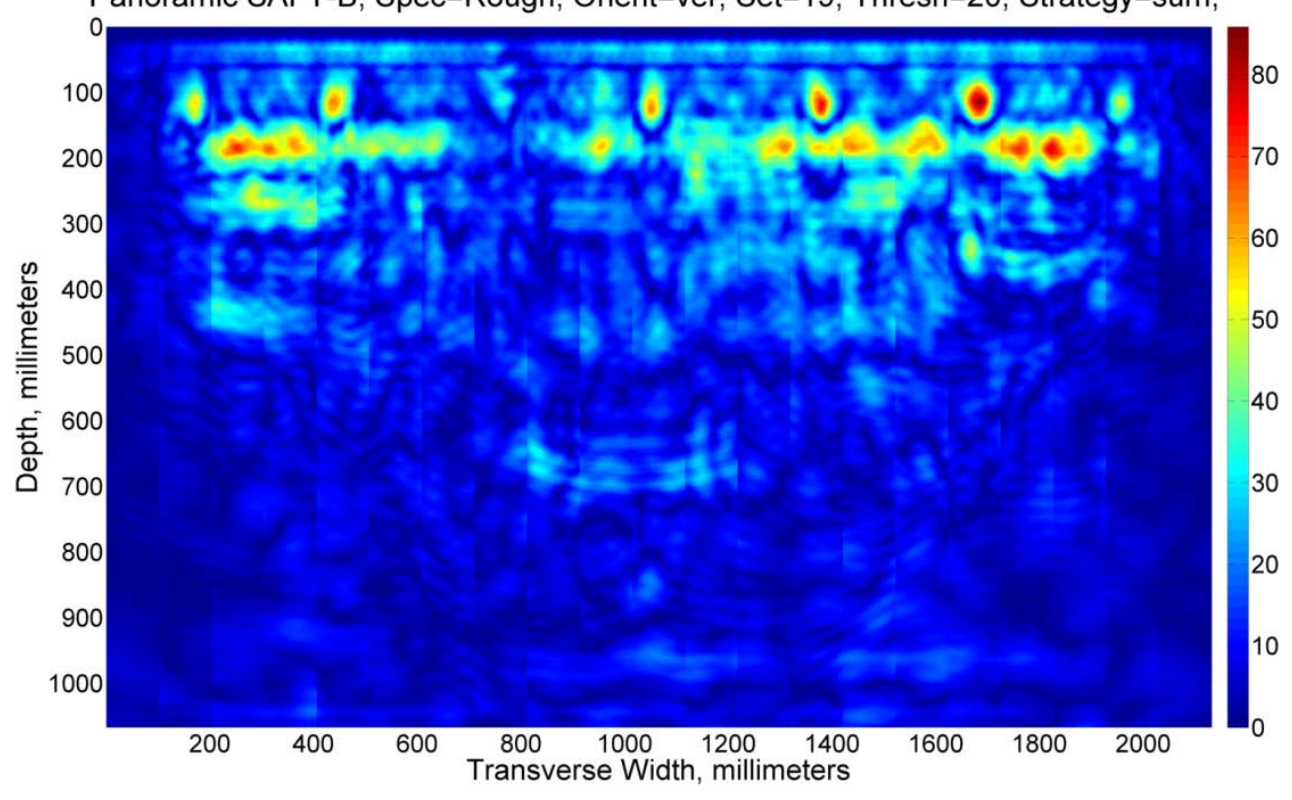

Fig. A. 189. Vertical Set 19, Node 34. 


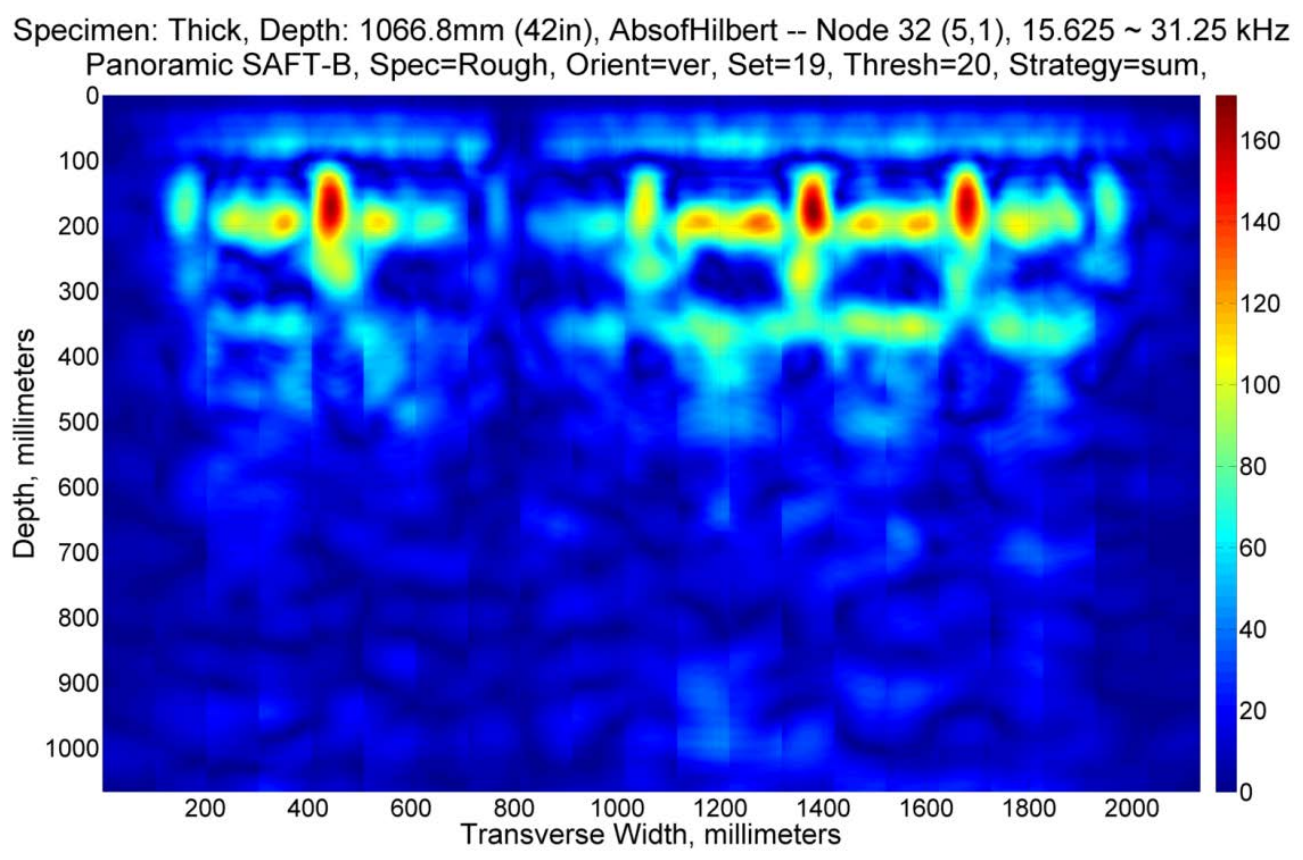

Fig. A. 190. Vertical Set 19, Node 32. 
APPENDIX B. SMOOTH SIDE DEFECT IMAGES 



\section{SMOOTH HORIZONTAL}

\section{SET ONE - NO DEFECT}

Specimen: Thick, Depth: 1066.8mm (42in), AbsofHilbert -- Node $0(0,0), 0 \sim 500 \mathrm{kHz}$ Panoramic SAFT-B, Spec=Smooth, Orient=hor, Set=1, Thresh=20, Strategy=sum,

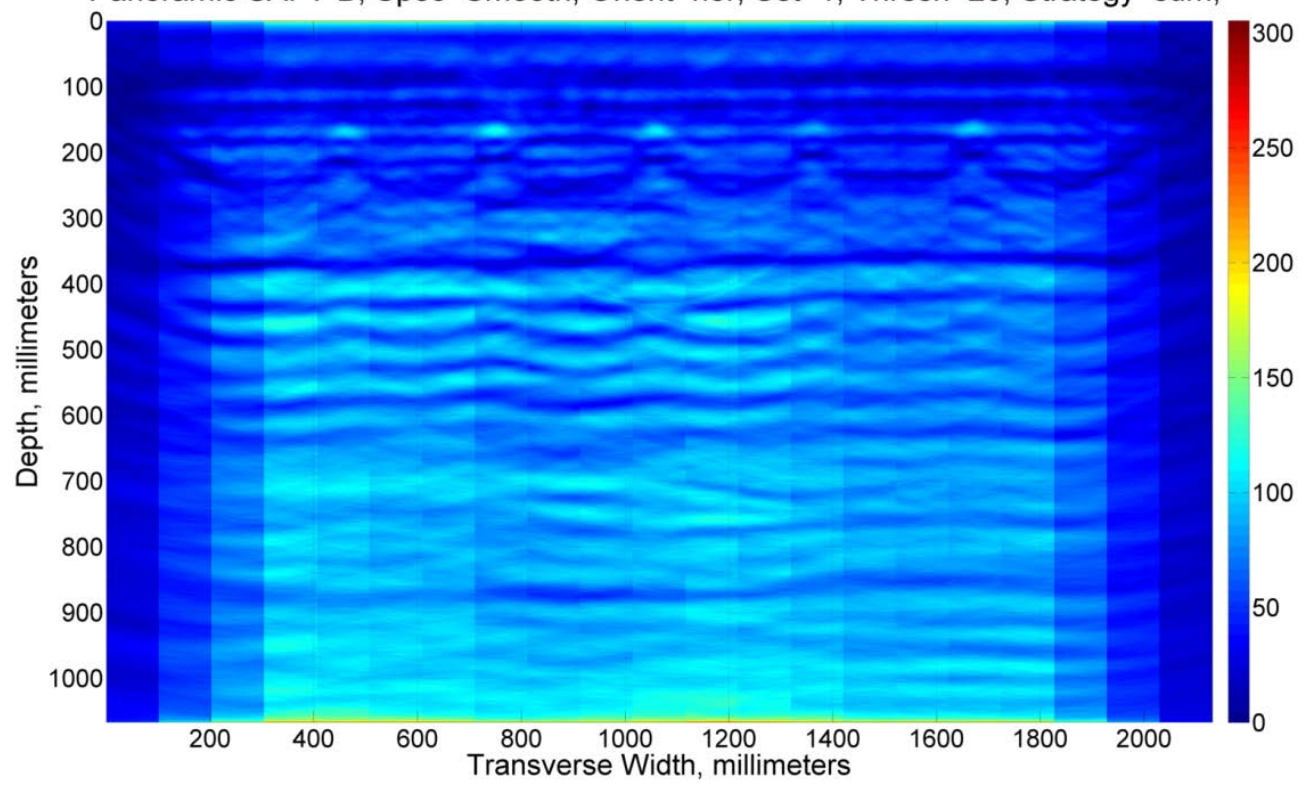

Fig. B. 1. Horizontal Set 1, Node 0.

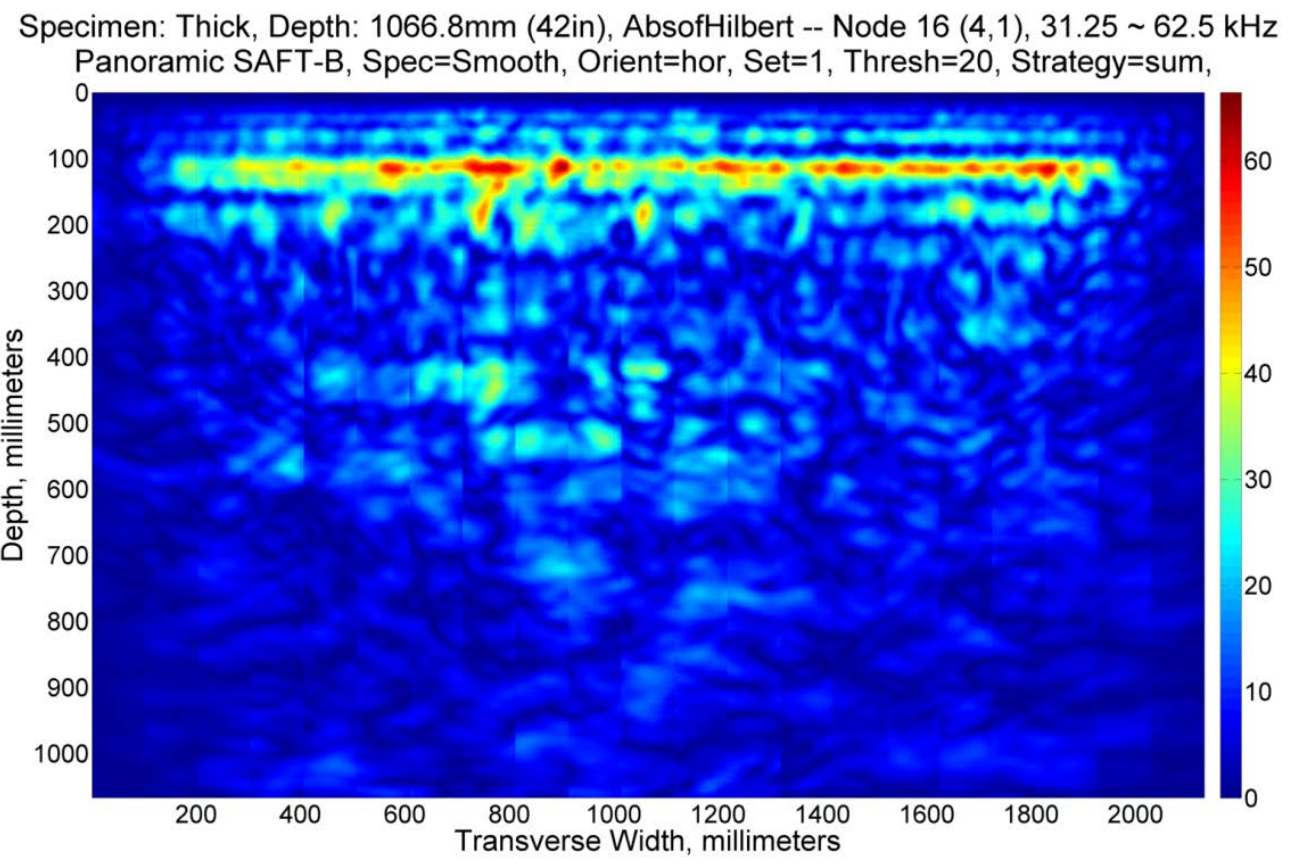

Fig. B. 2. Horizontal Set 1, Node 16. 
Specimen: Thick, Depth: 1066.8mm (42in), AbsofHilbert -- Node $18(4,3), 93.75 \sim 125 \mathrm{kHz}$ Panoramic SAFT-B, Spec=Smooth, Orient=hor, Set=1, Thresh=20, Strategy=sum,

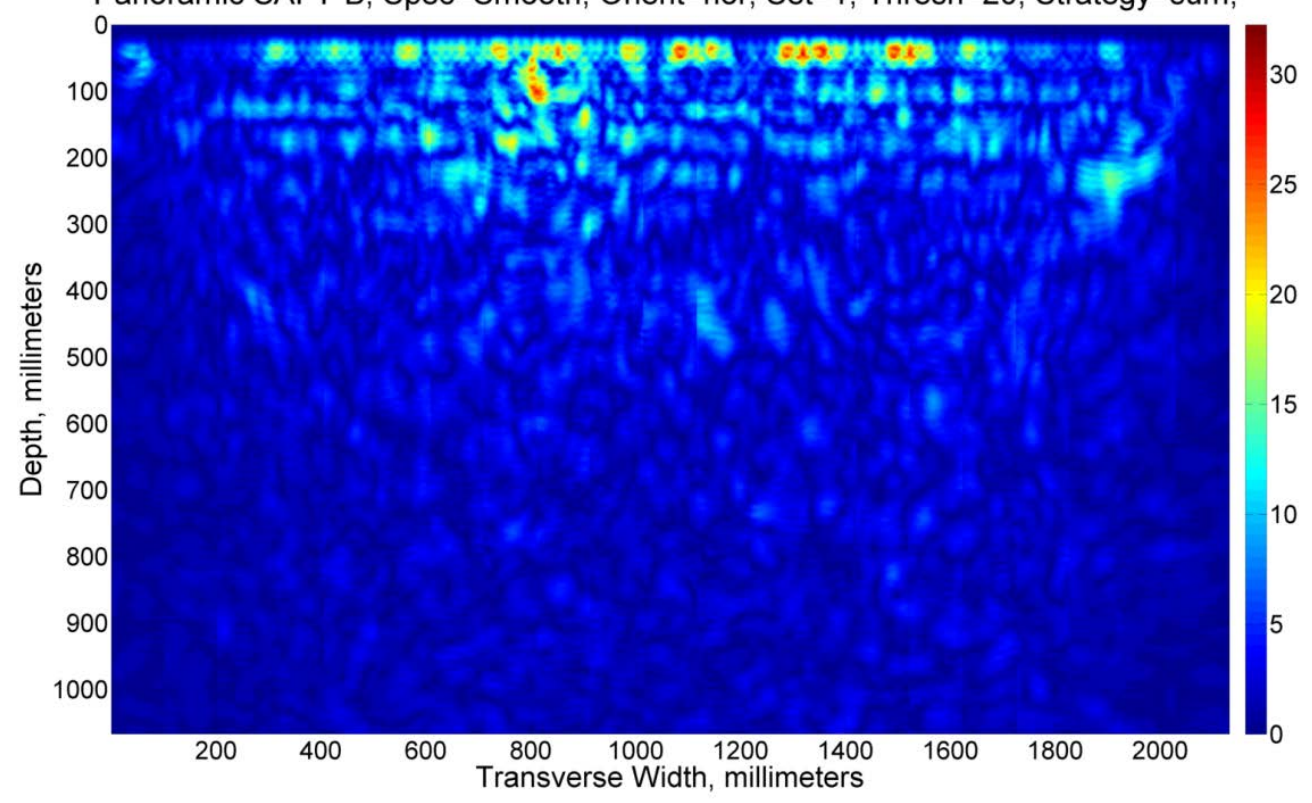

Fig. B. 3. Horizontal Set 1, Node 18.

Specimen: Thick, Depth: 1066.8mm (42in), AbsofHilbert -- Node $32(5,1), 15.625 \sim 31.25 \mathrm{kHz}$ Panoramic SAFT-B, Spec=Smooth, Orient=hor, Set=1, Thresh=20, Strategy=sum,

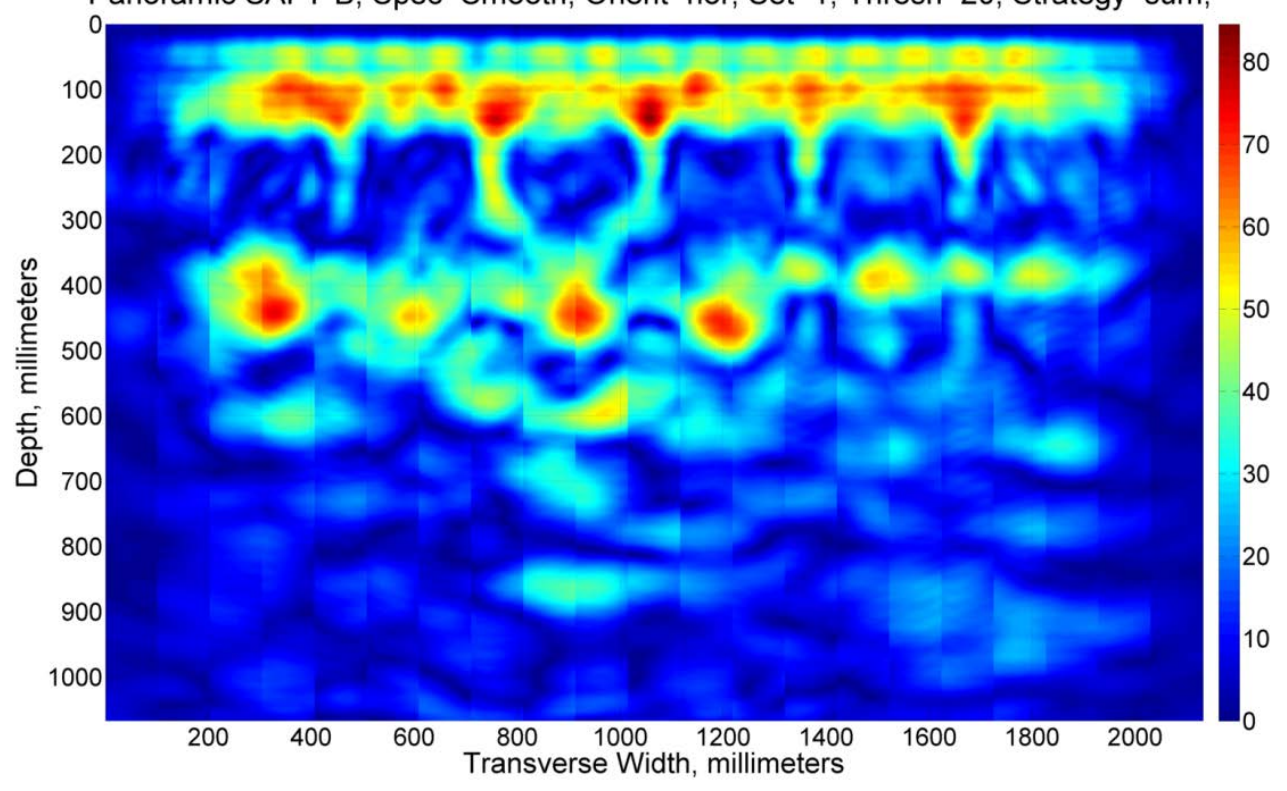

Fig. B. 4. Horizontal Set 1, Node 32. 


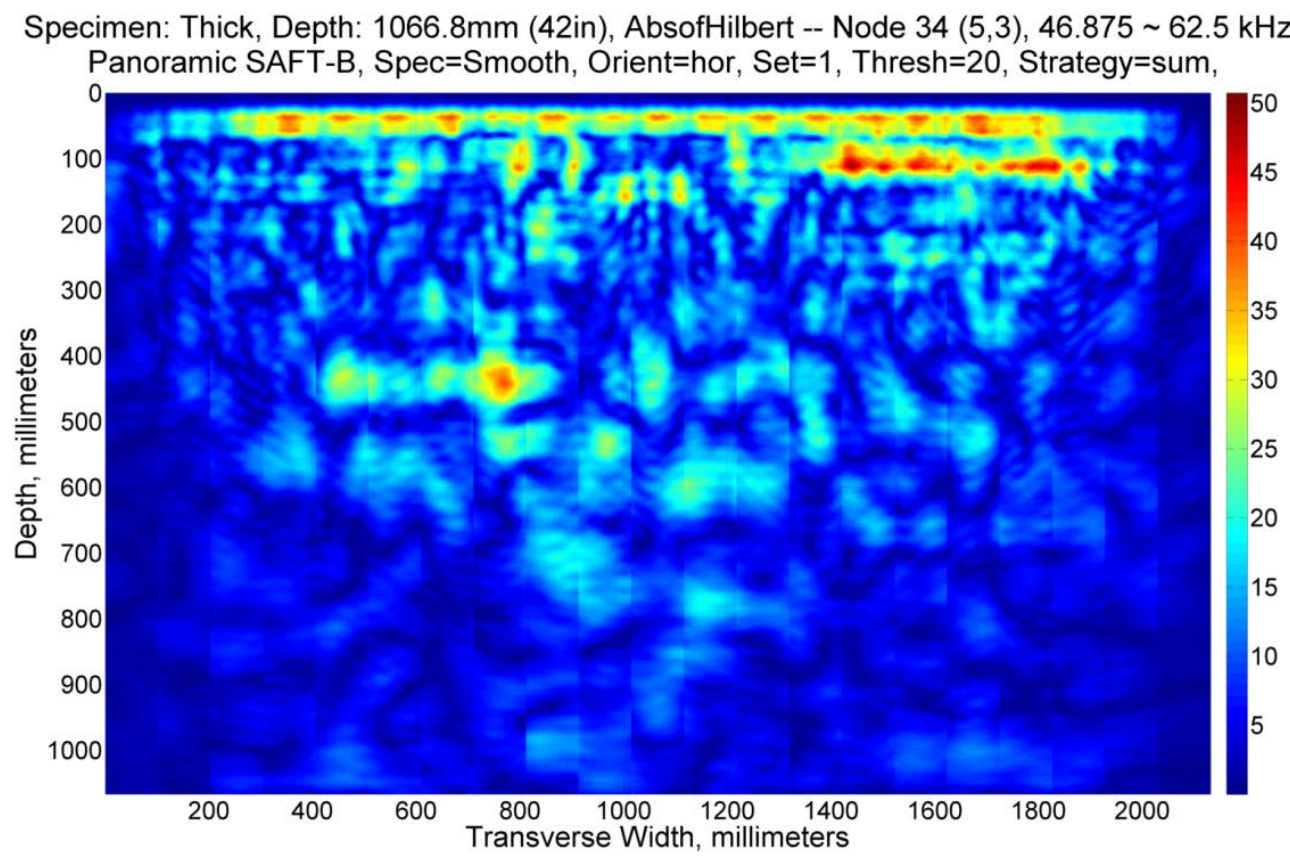

Fig. B. 5. Horizontal Set 1, Node 34 .

\section{SET TWO - DEFECT 11, DEFECT 16}

Specimen: Thick, Depth: 1066.8mm (42in), AbsofHilbert -- Node $0(0,0), 0 \sim 500 \mathrm{kHz}$ Panoramic SAFT-B, Spec=Smooth, Orient=hor, Set=2, Thresh=20, Strategy=sum,

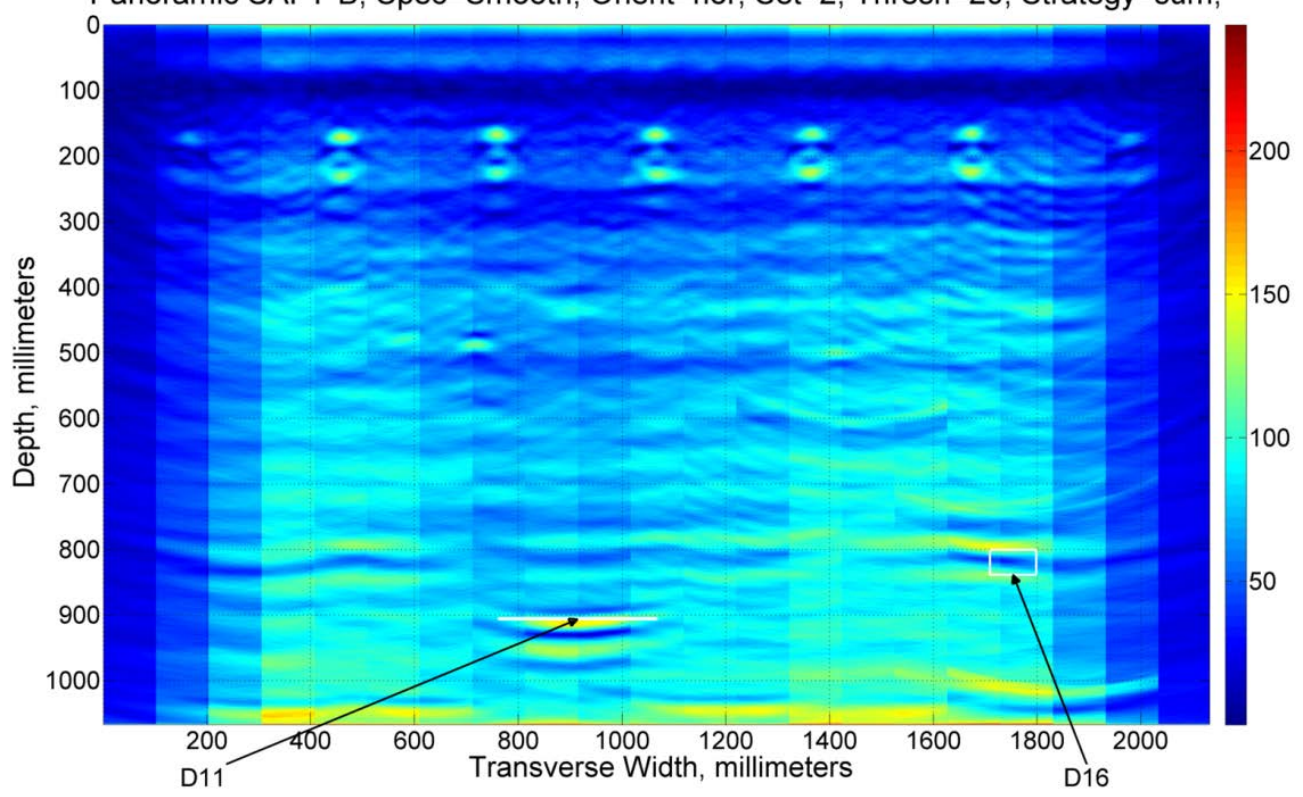

Fig. B. 6. Horizontal Set 2, Node 0. 


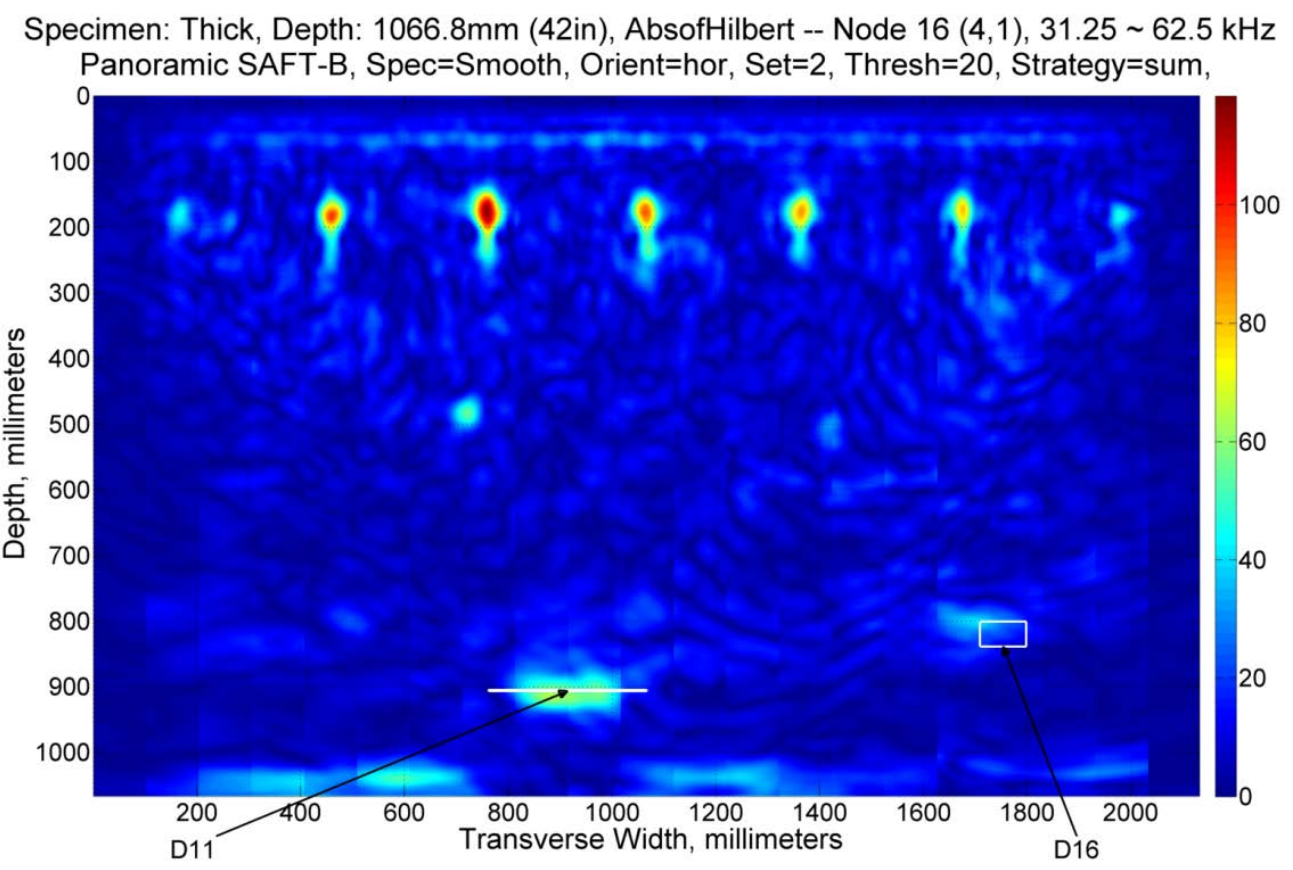

Fig. B. 7. Horizontal Set 2, Node 16.

Specimen: Thick, Depth: 1066.8mm (42in), AbsofHilbert -- Node $18(4,3), 93.75 \sim 125 \mathrm{kHz}$ Panoramic SAFT-B, Spec=Smooth, Orient=hor, Set=2, Thresh=20, Strategy=sum,

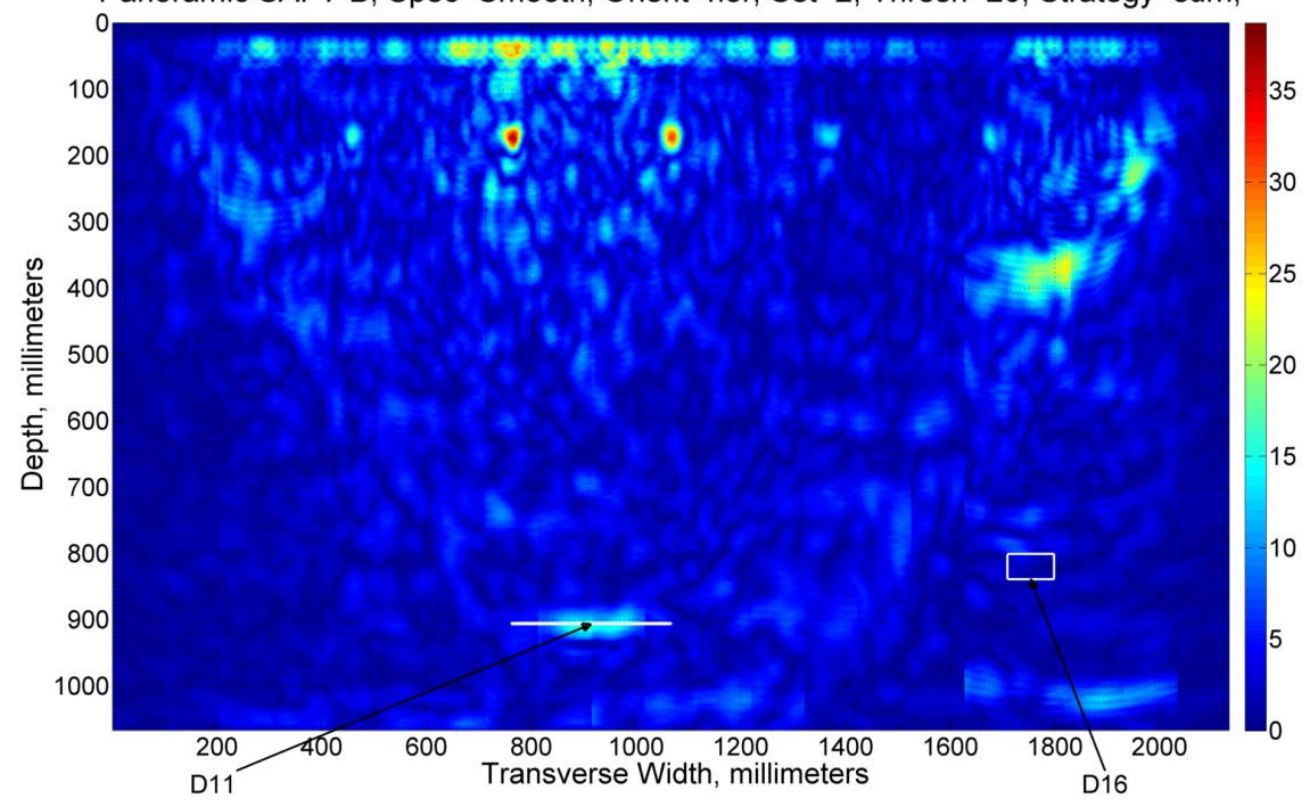

Fig. B. 8. Horizontal Set 2, Node 18. 


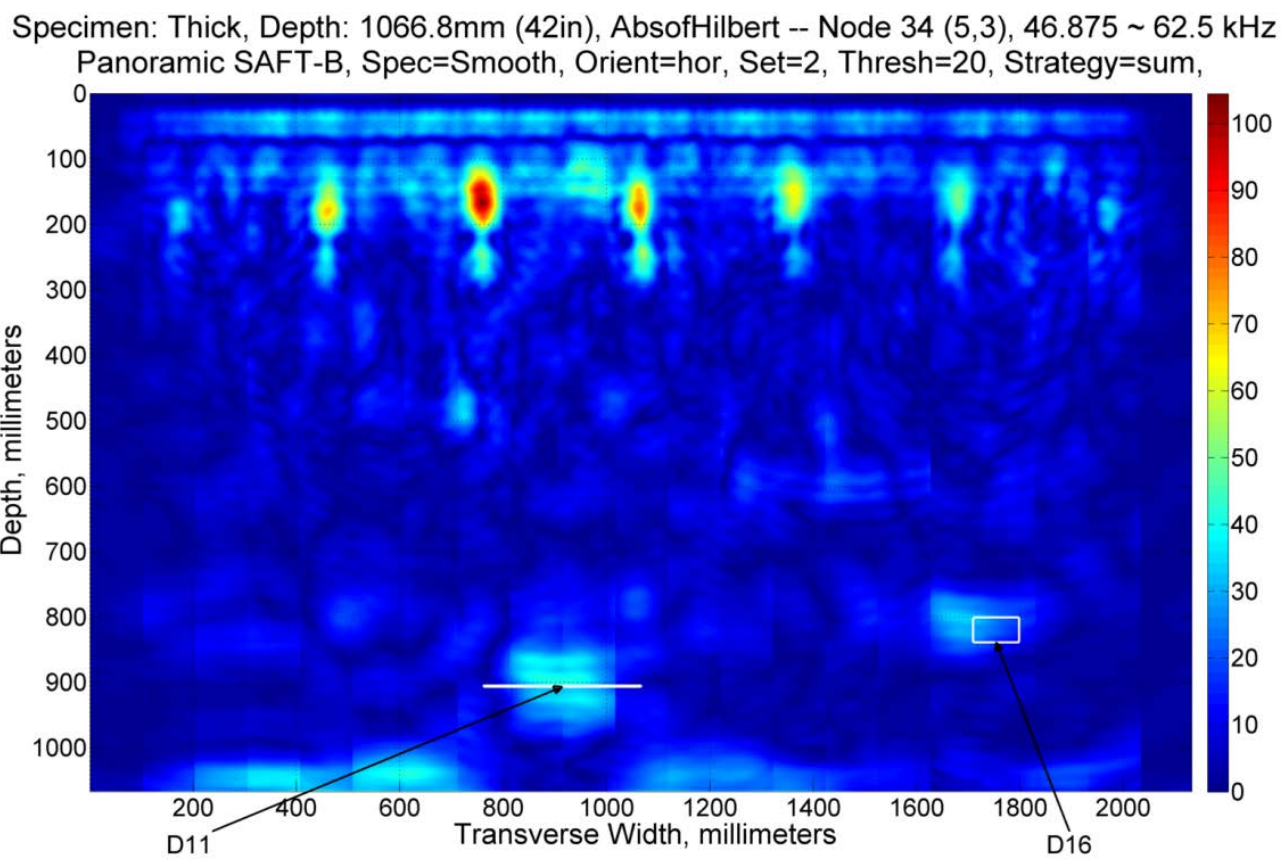

Fig. B. 9. Horizontal Set 2, Node 34 .

Specimen: Thick, Depth: 1066.8mm (42in), AbsofHilbert -- Node 32 (5,1), $15.625 \sim 31.25 \mathrm{kHz}$ Panoramic SAFT-B, Spec=Smooth, Orient=hor, Set=2, Thresh=20, Strategy=sum,

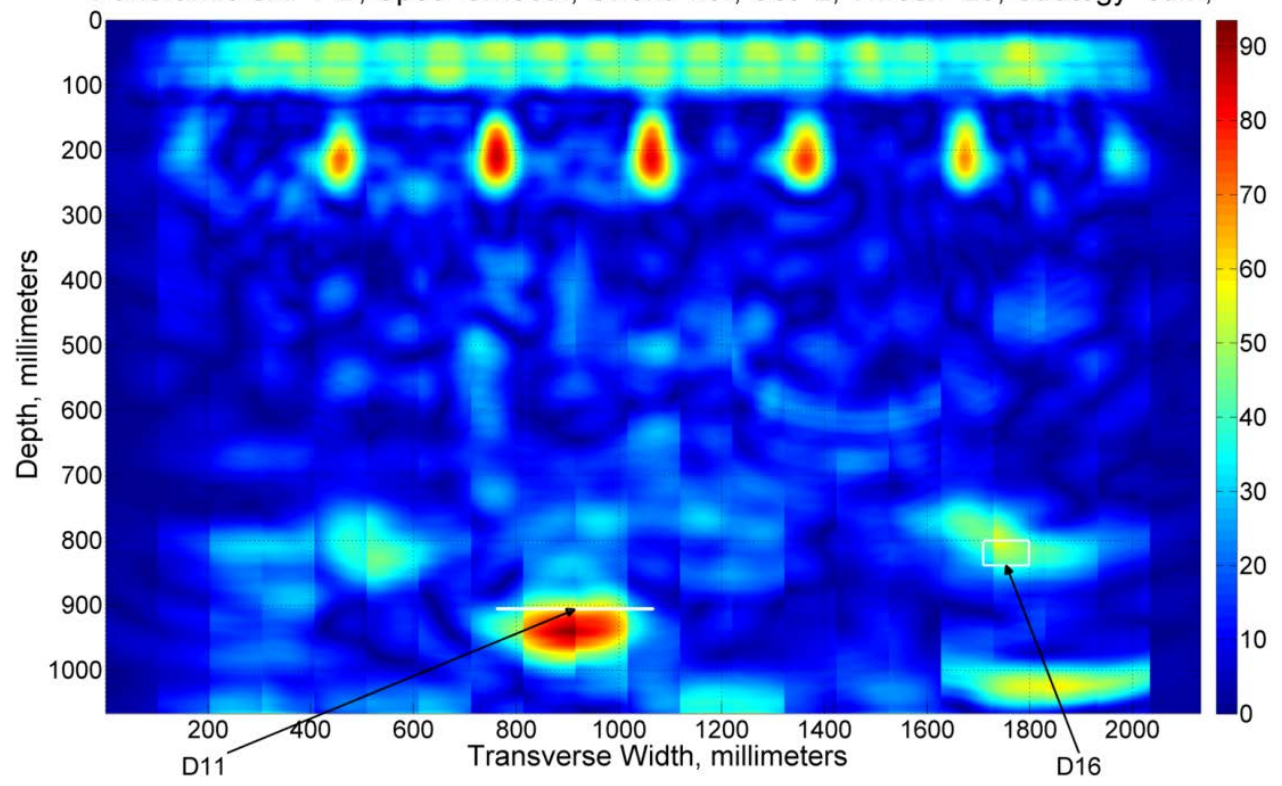

Fig. B. 10, Horizontal Set 2, Node 32. 


\section{SET THREE - DEFECT 11, DEFECT 16}

Specimen: Thick, Depth: 1066.8mm (42in), AbsofHilbert -- Node $0(0,0), 0 \sim 500 \mathrm{kHz}$ Panoramic SAFT-B, Spec=Smooth, Orient=hor, Set=3, Thresh=20, Strategy=sum,

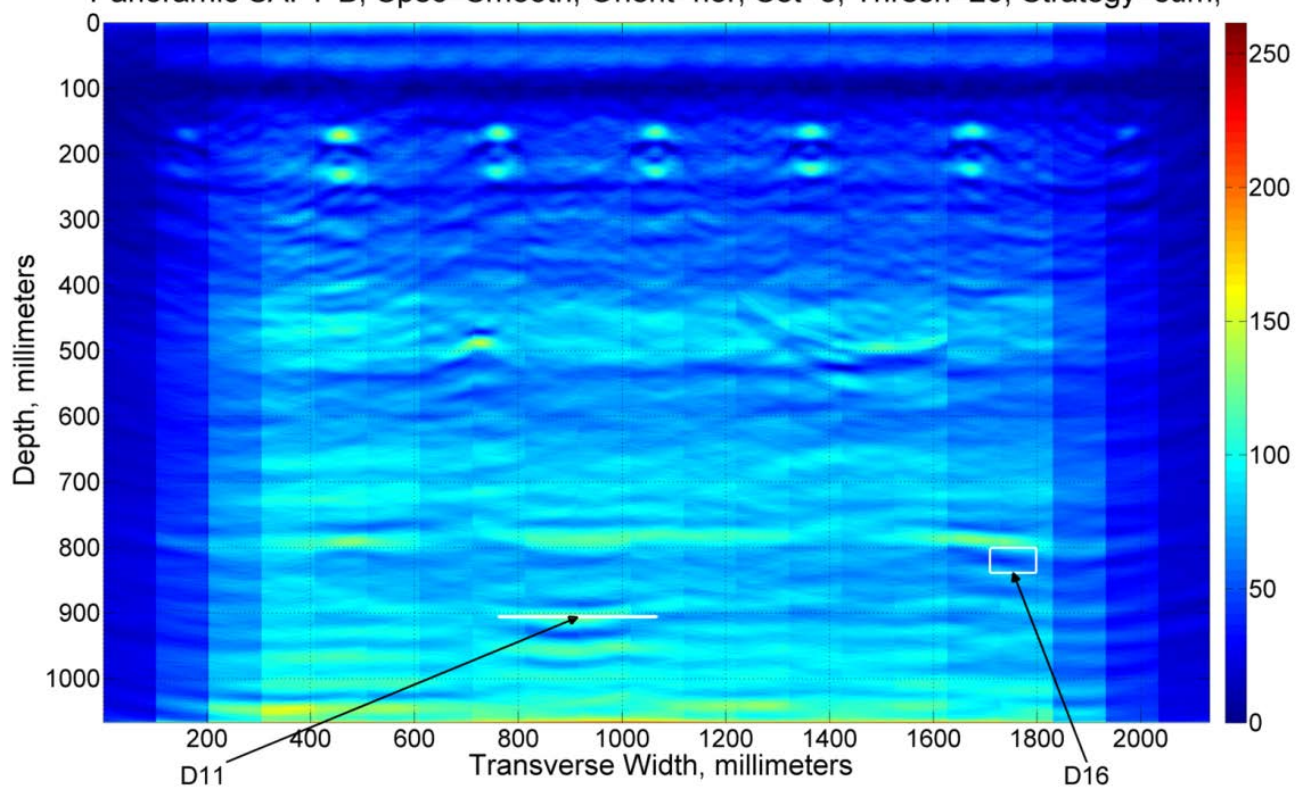

Fig. B. 11. Horizontal Set 3, Node 0.

Specimen: Thick, Depth: 1066.8mm (42in), AbsofHilbert -- Node $16(4,1), 31.25 \sim 62.5 \mathrm{kHz}$ Panoramic SAFT-B, Spec=Smooth, Orient=hor, Set=3, Thresh=20, Strategy=sum,

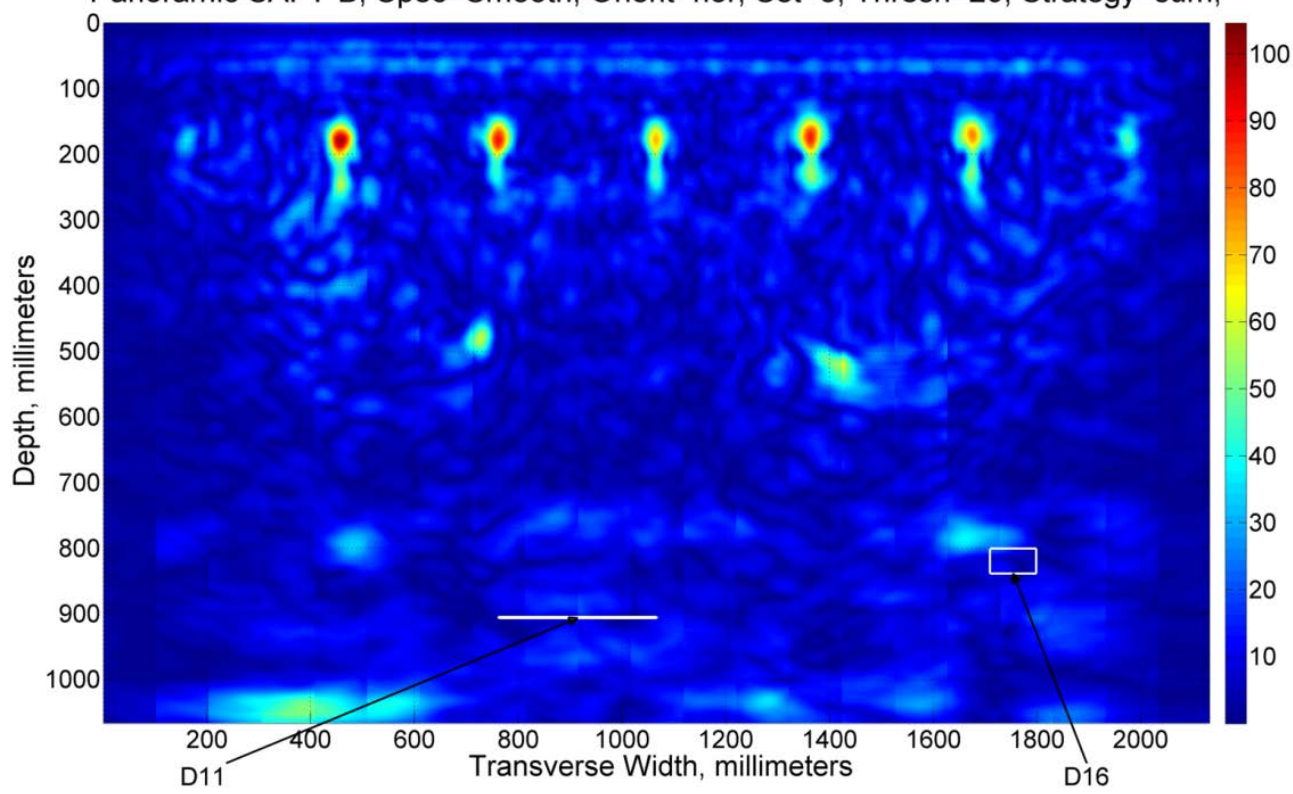

Fig. B. 12. Horizontal Set 3, Node 16. 
Specimen: Thick, Depth: 1066.8mm (42in), AbsofHilbert -- Node $18(4,3), 93.75 \sim 125 \mathrm{kHz}$ Panoramic SAFT-B, Spec=Smooth, Orient=hor, Set=3, Thresh=20, Strategy=sum,

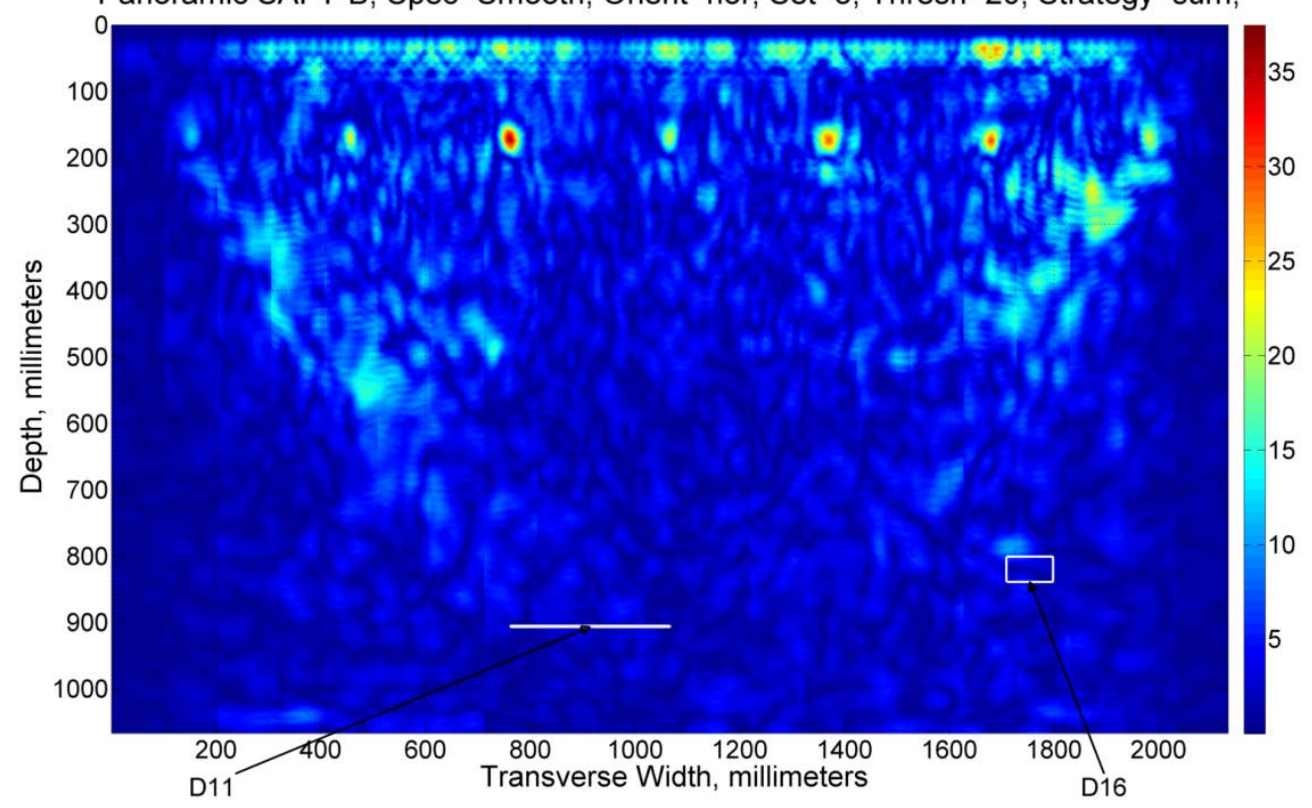

Fig. B. 13. Horizontal Set 3, Node 18.

Specimen: Thick, Depth: 1066.8mm (42in), AbsofHilbert -- Node 32 (5,1), $15.625 \sim 31.25 \mathrm{kHz}$ Panoramic SAFT-B, Spec=Smooth, Orient=hor, Set=3, Thresh=20, Strategy=sum,

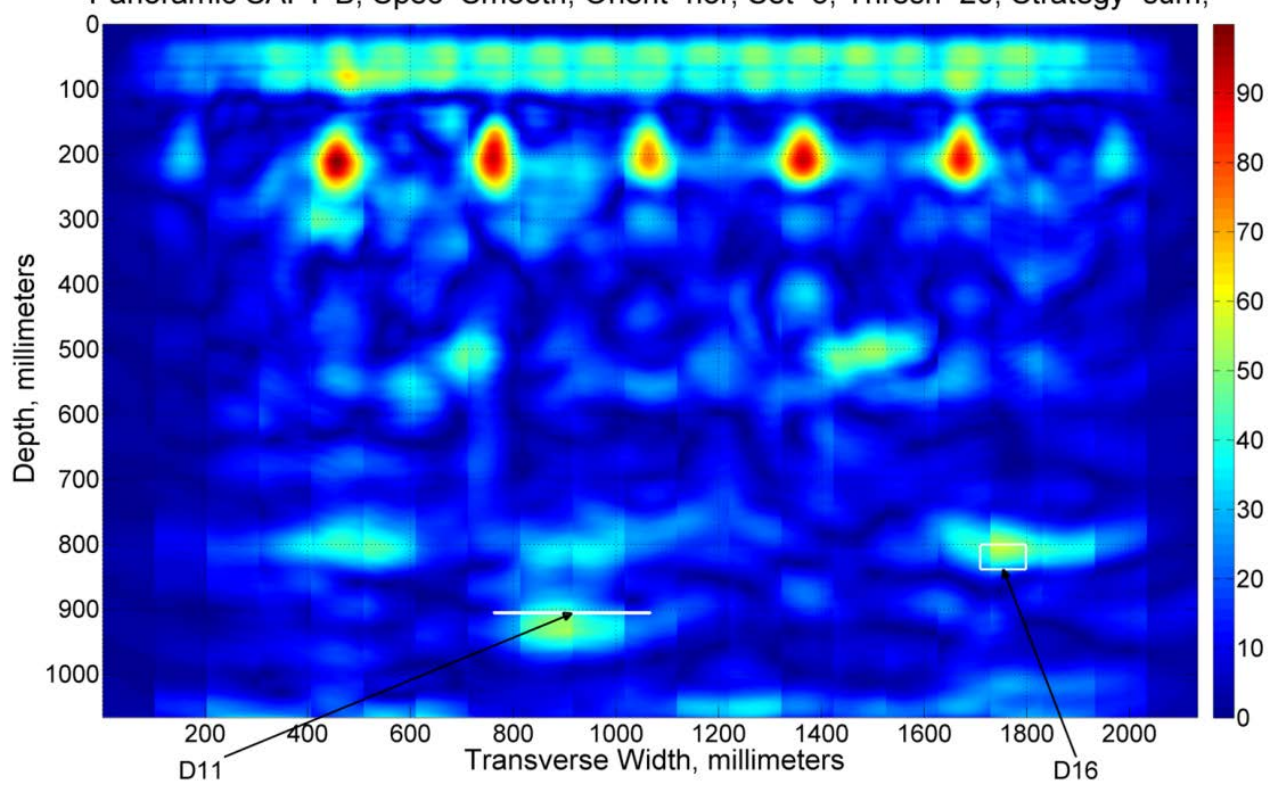

Fig. B. 14. Horizontal Set 3, Node 32. 


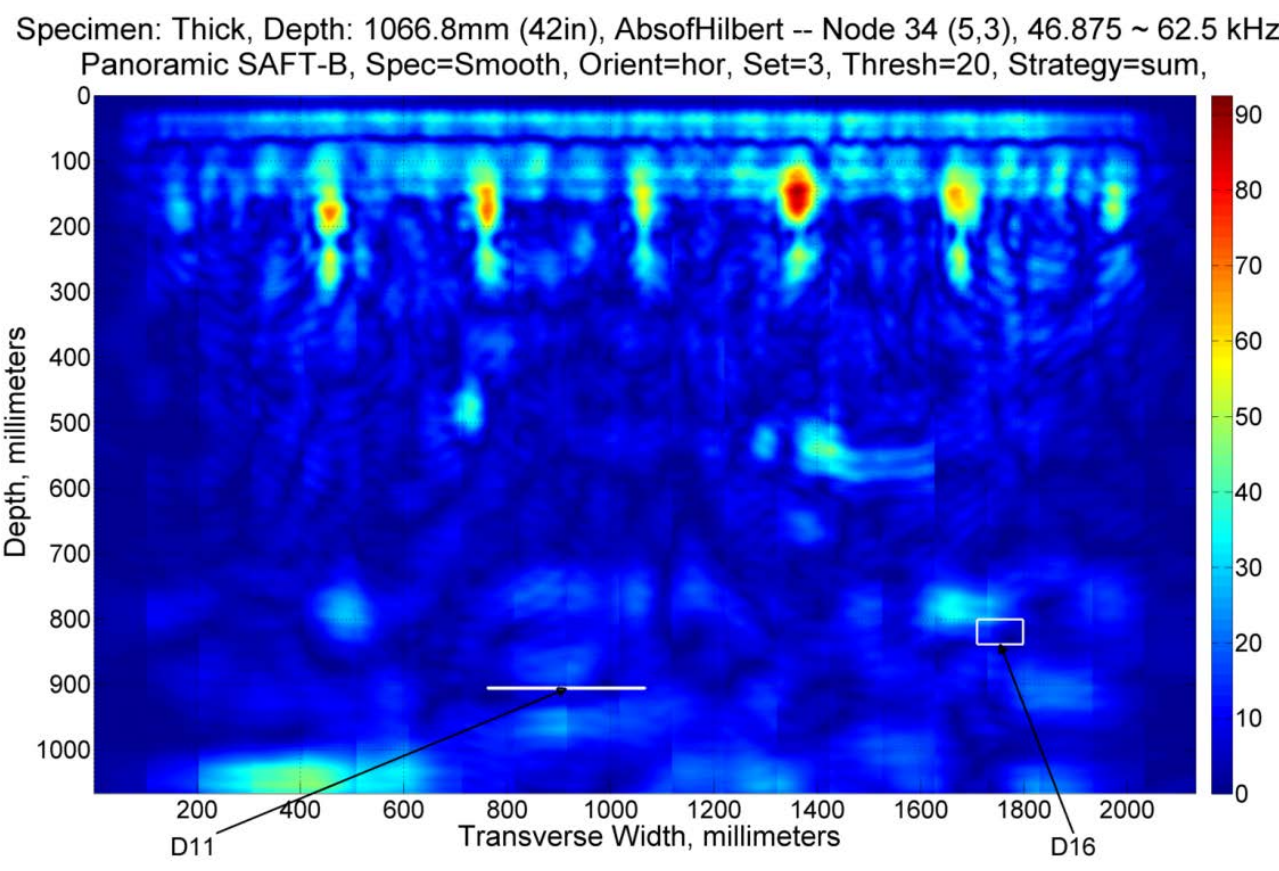

Fig. B. 15. Horizontal Set 3, Node 34.

\section{SET FOUR - DEFECT 1}

Specimen: Thick, Depth: 1066.8mm (42in), AbsofHilbert -- Node $0(0,0), 0 \sim 500 \mathrm{kHz}$ Panoramic SAFT-B, Spec=Smooth, Orient=hor, Set=4, Thresh=20, Strategy=sum,

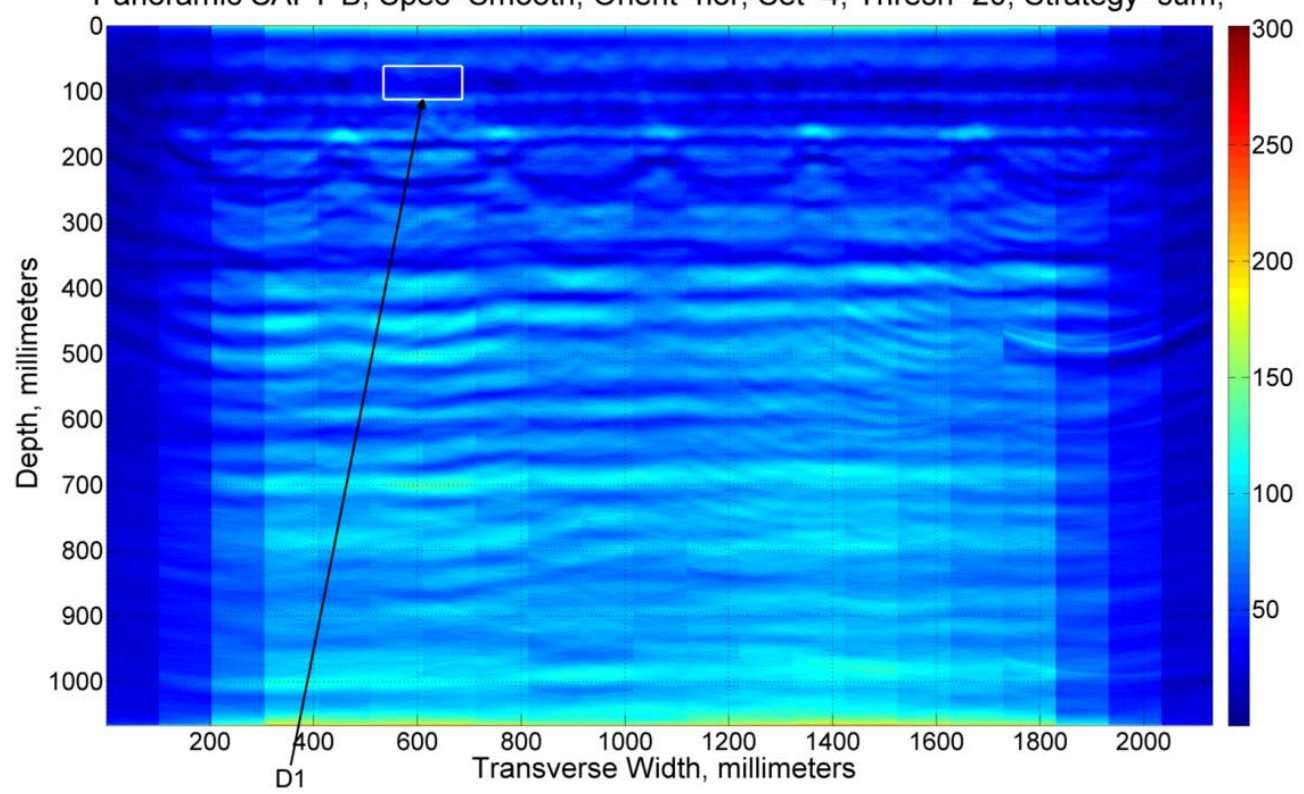

Fig. B. 16. Horizontal Set 4, Node 0. 


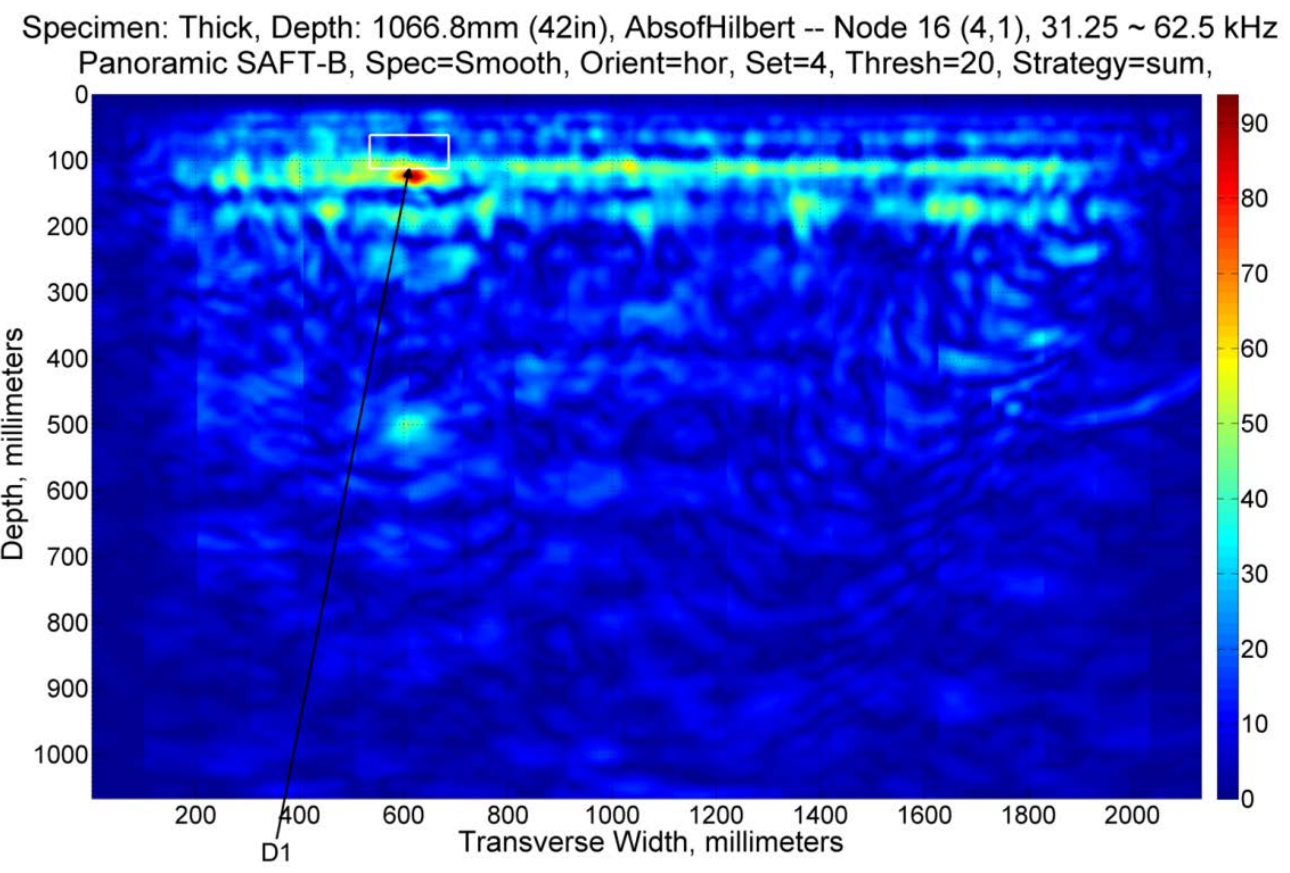

Fig. B. 17. Horizontal Set 4, Node 16.

Specimen: Thick, Depth: 1066.8mm (42in), AbsofHilbert -- Node 18 (4,3), $93.75 \sim 125 \mathrm{kHz}$ Panoramic SAFT-B, Spec=Smooth, Orient=hor, Set=4, Thresh=20, Strategy=sum,

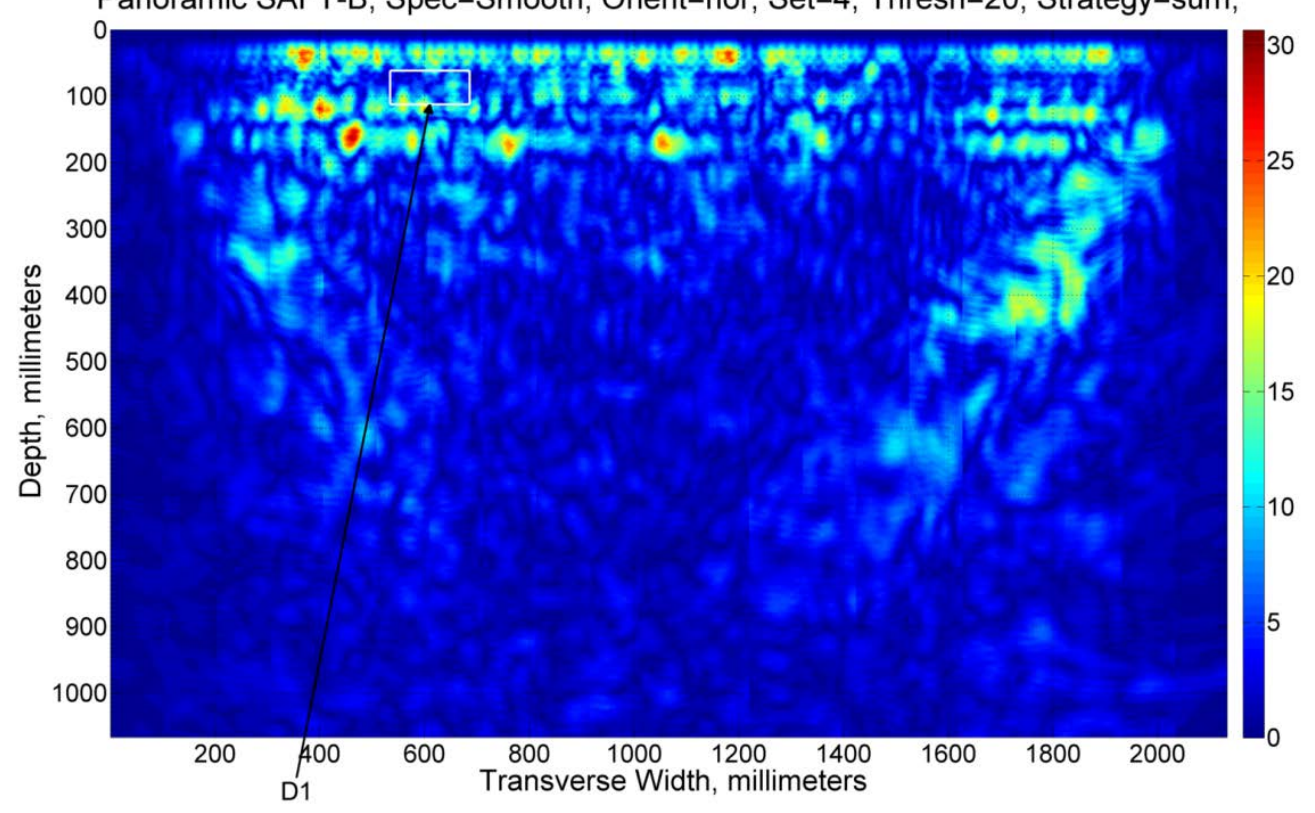

Fig. B. 18. Horizontal Set 4, Node 18. 


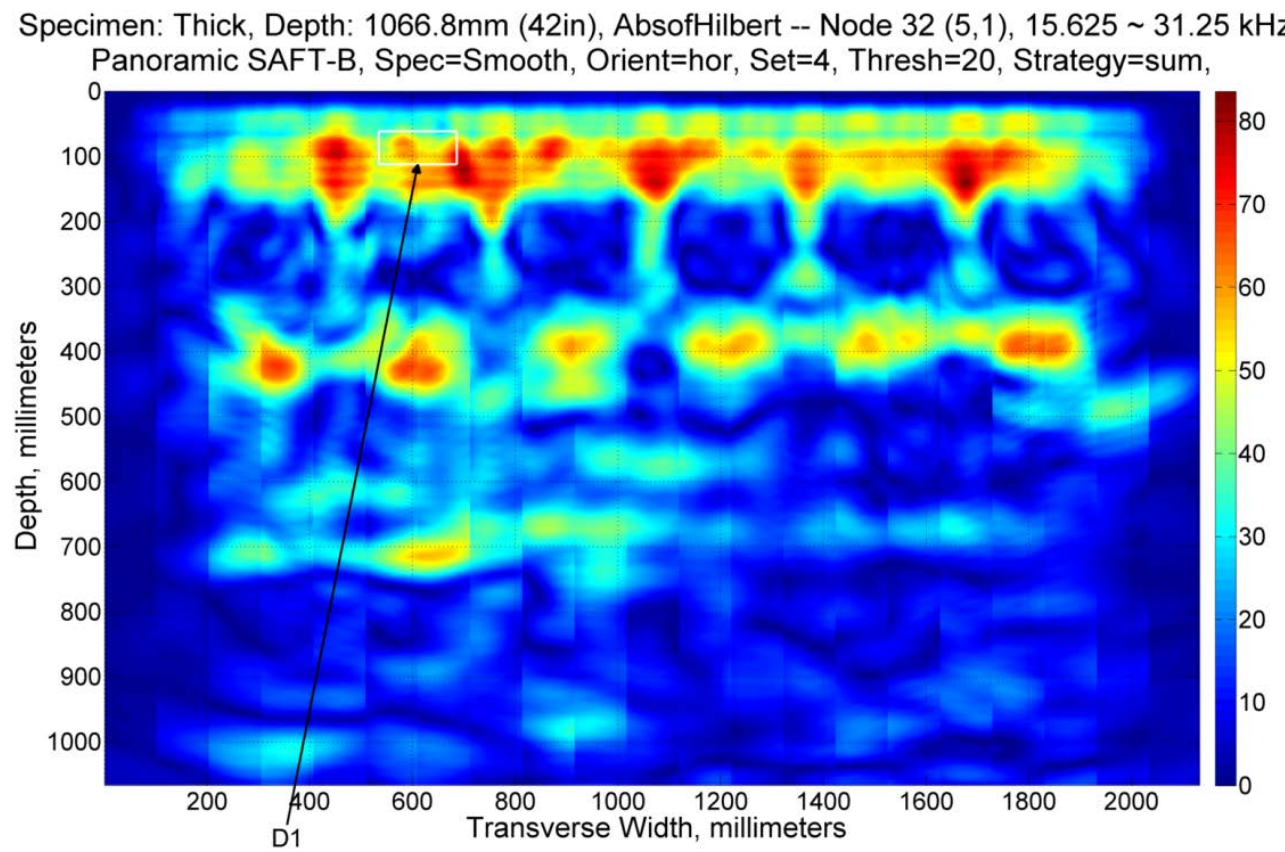

Fig. B. 19. Horizontal Set 4, Node 32.

Specimen: Thick, Depth: 1066.8mm (42in), AbsofHilbert -- Node 34 (5,3), $46.875 \sim 62.5 \mathrm{kHz}$ Panoramic SAFT-B, Spec=Smooth, Orient=hor, Set=4, Thresh=20, Strategy=sum,

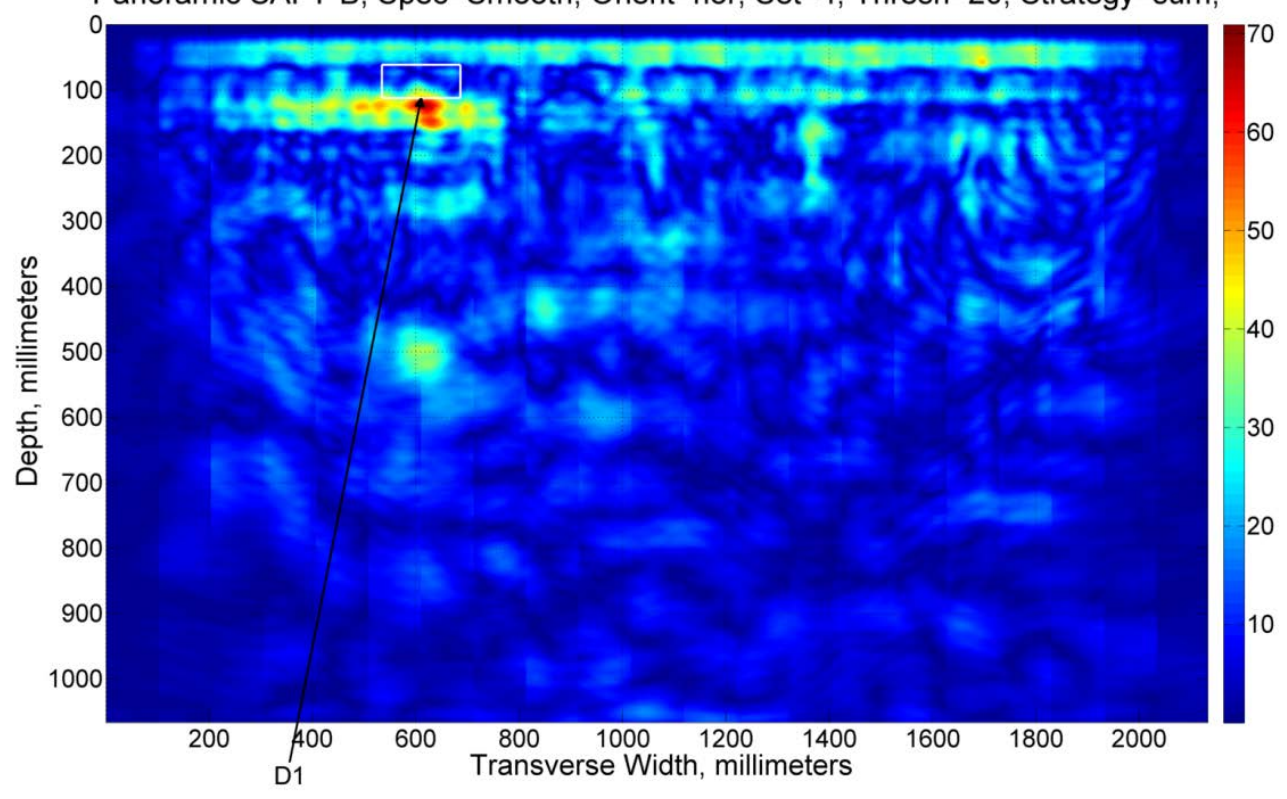

Fig. B. 20. Horizontal Set 4, Node 34. 


\section{SET FIVE - DEFECT 3, DEFECT 14, DEFECT 17}

Specimen: Thick, Depth: 1066.8mm (42in), AbsofHilbert -- Node $0(0,0), 0 \sim 500 \mathrm{kHz}$ Panoramic SAFT-B, Spec=Smooth, Orient=hor, Set=5, Thresh=20, Strategy=sum,

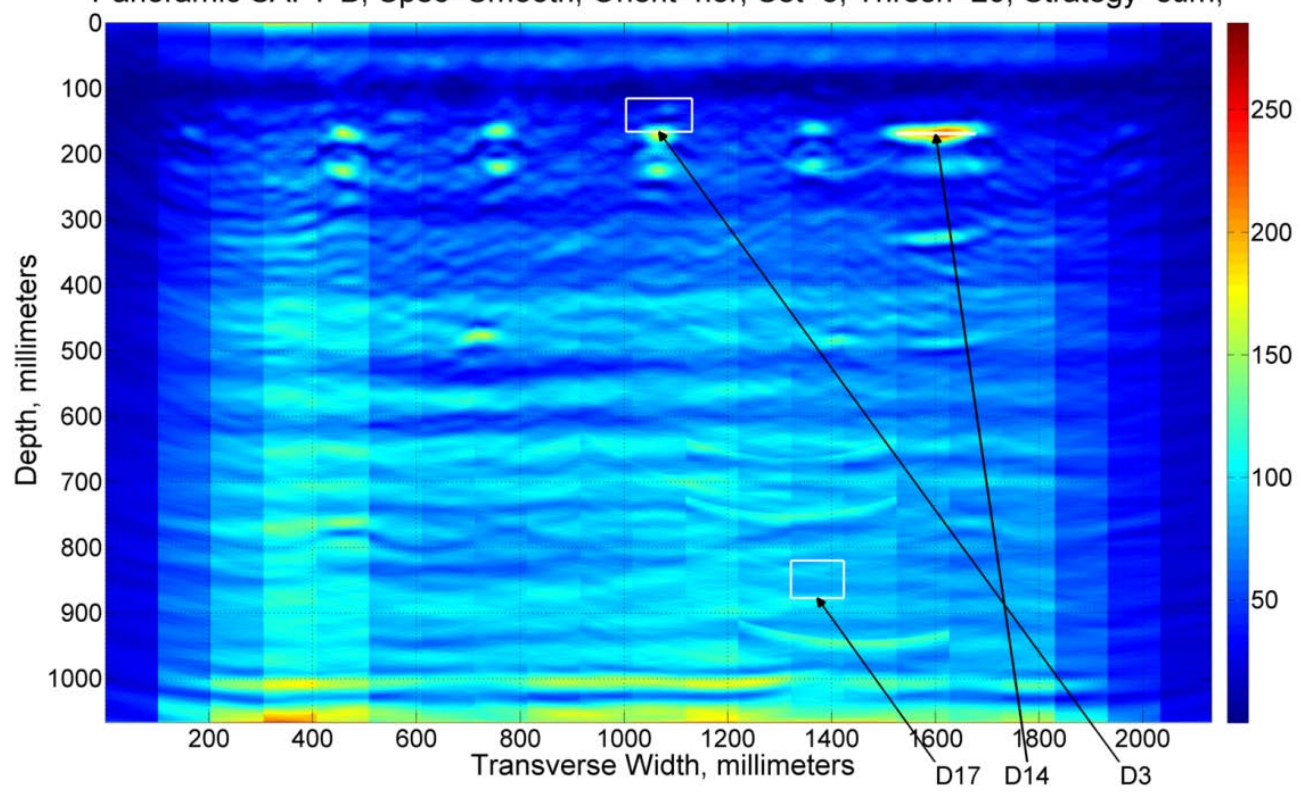

Fig. B. 21. Horizontal Set 5, Node 0.

Specimen: Thick, Depth: 1066.8mm (42in), AbsofHilbert -- Node $16(4,1), 31.25 \sim 62.5 \mathrm{kHz}$ Panoramic SAFT-B, Spec=Smooth, Orient=hor, Set=5, Thresh=20, Strategy=sum,

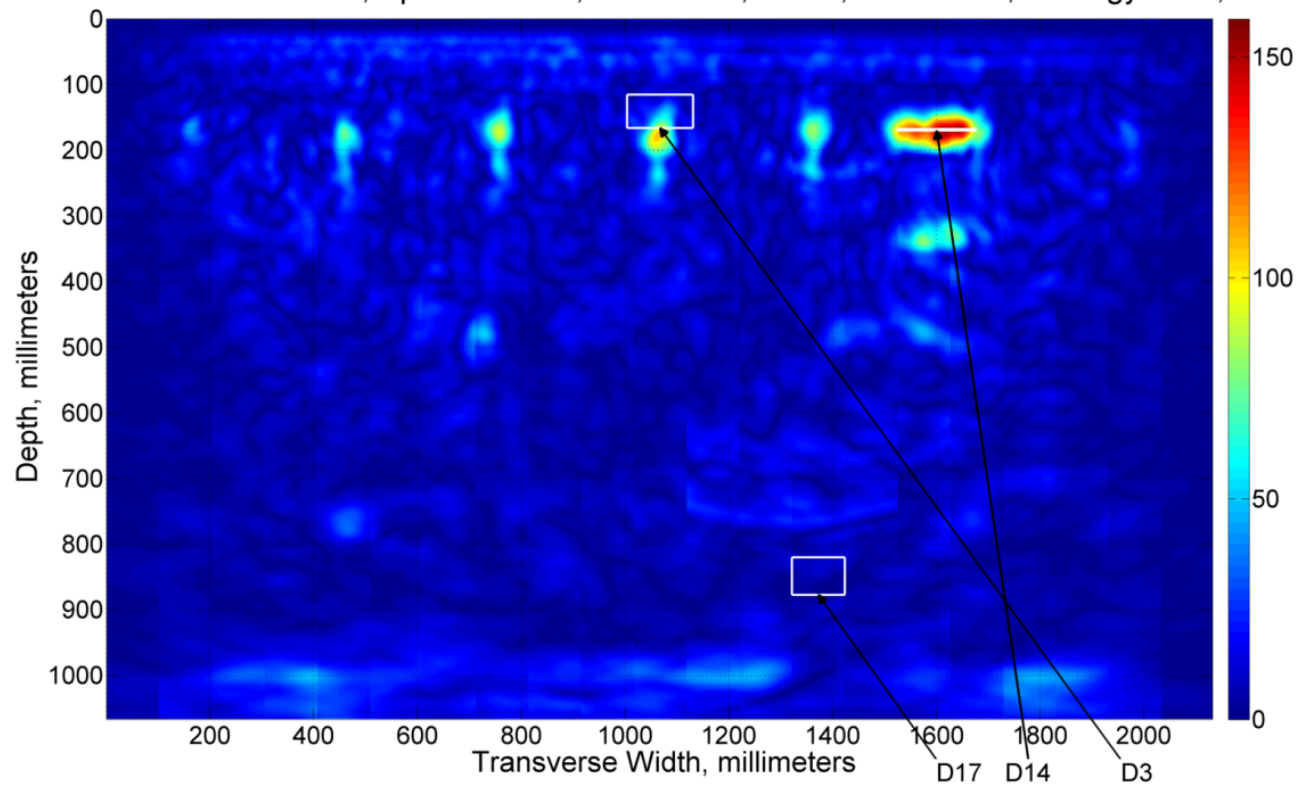

Fig. B. 22. Horizontal Set 5, Node 16. 
Specimen: Thick, Depth: 1066.8mm (42in), AbsofHilbert -- Node $18(4,3), 93.75 \sim 125 \mathrm{kHz}$ Panoramic SAFT-B, Spec=Smooth, Orient=hor, Set=5, Thresh=20, Strategy=sum,

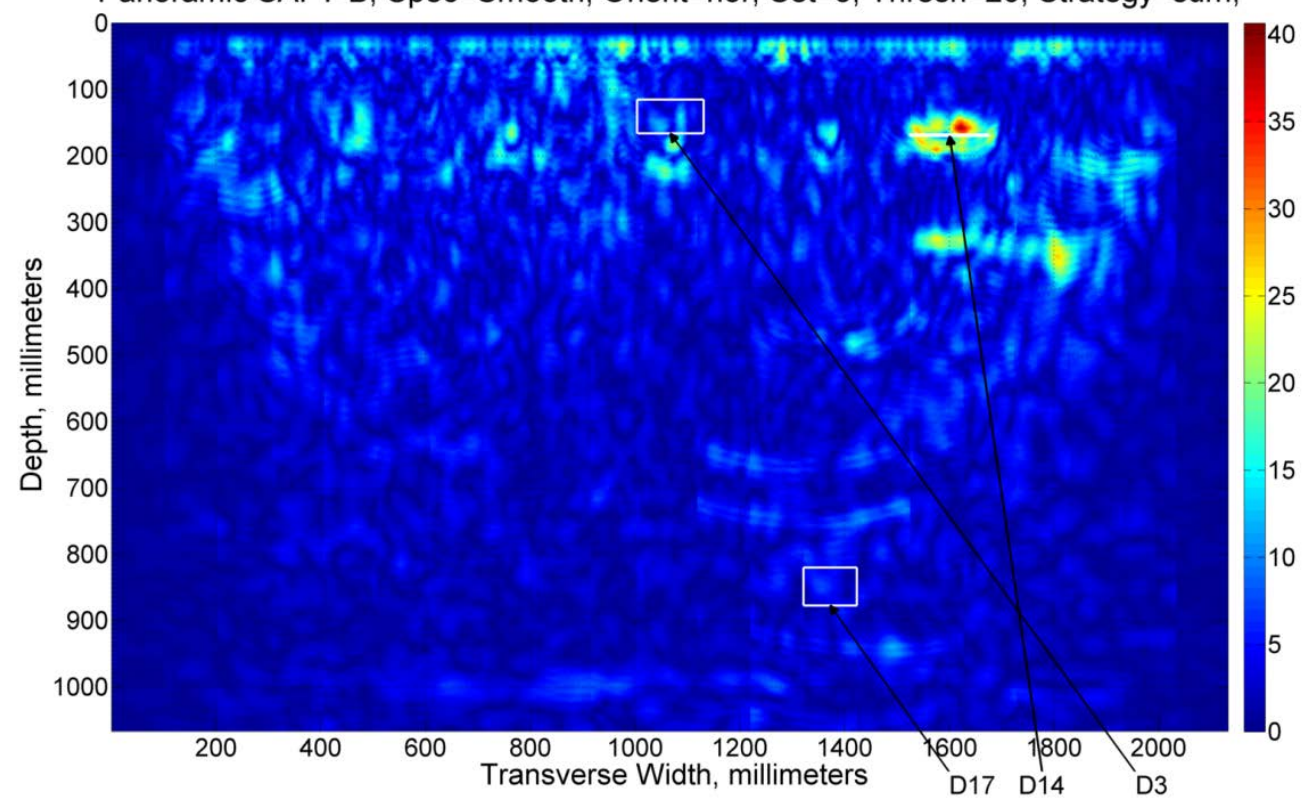

Fig. B. 23. Horizontal Set 5, Node 18.

Specimen: Thick, Depth: 1066.8mm (42in), AbsofHilbert -- Node $32(5,1), 15.625 \sim 31.25 \mathrm{kHz}$ Panoramic SAFT-B, Spec=Smooth, Orient=hor, Set=5, Thresh=20, Strategy=sum,

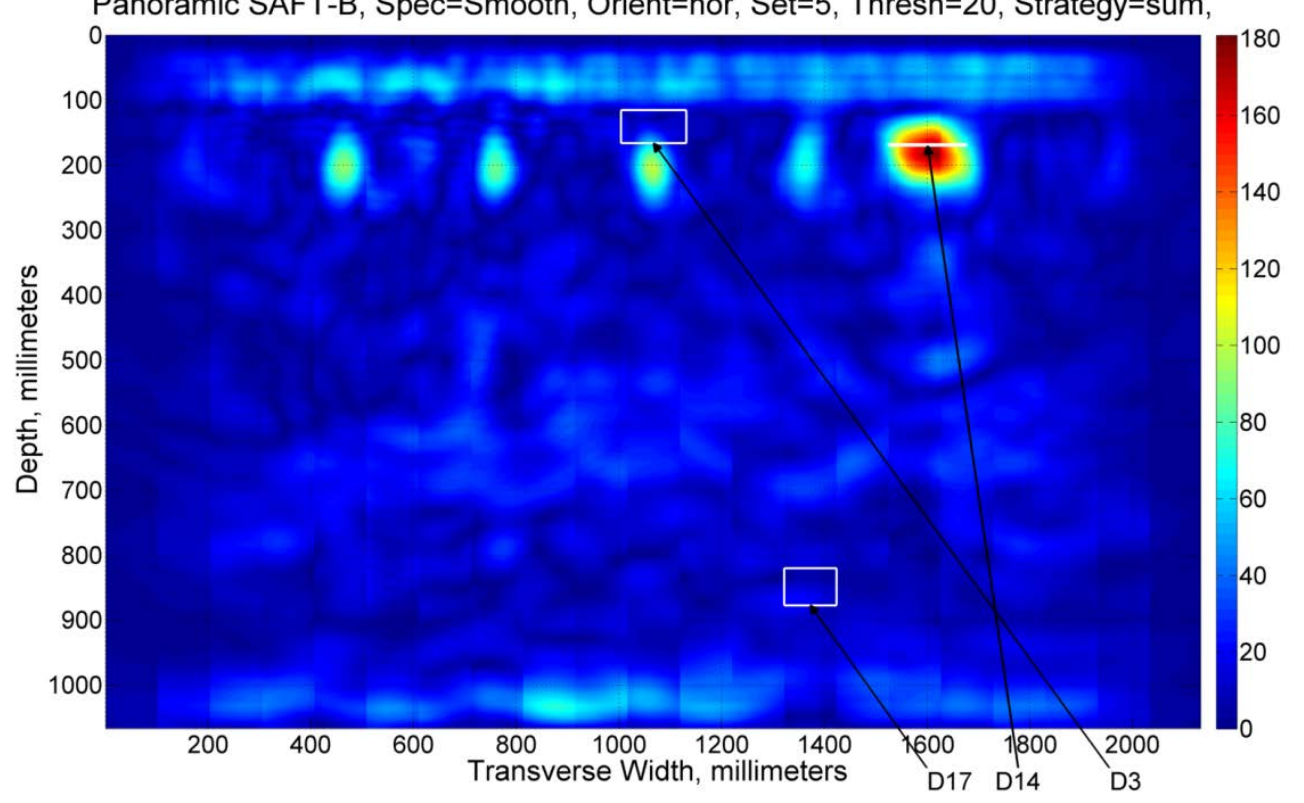

Fig. B. 24. Horizontal Set 5, Node 32. 


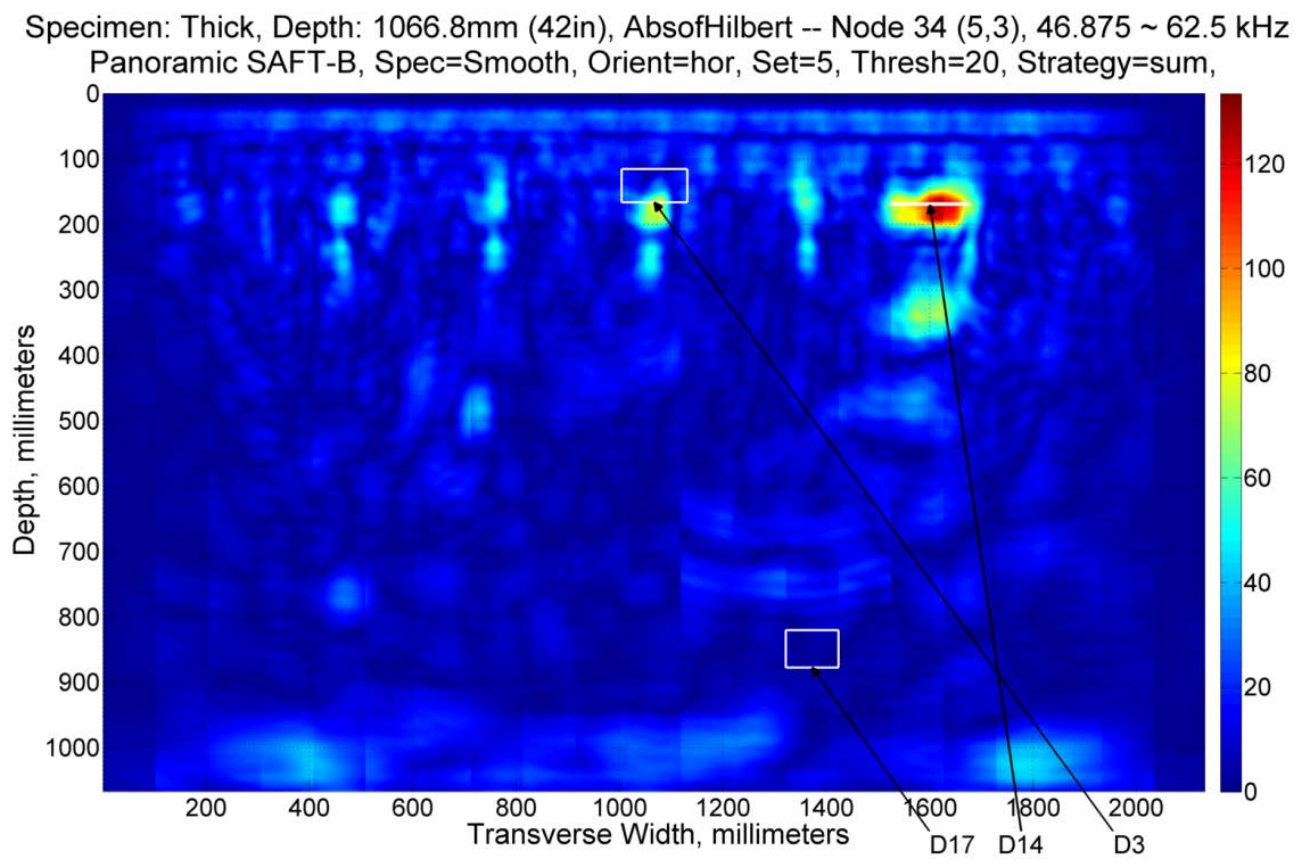

Fig. B. 25. Horizontal Set 5, Node 34.

\section{SET SIX - DEFECT 3, DEFECT 17}

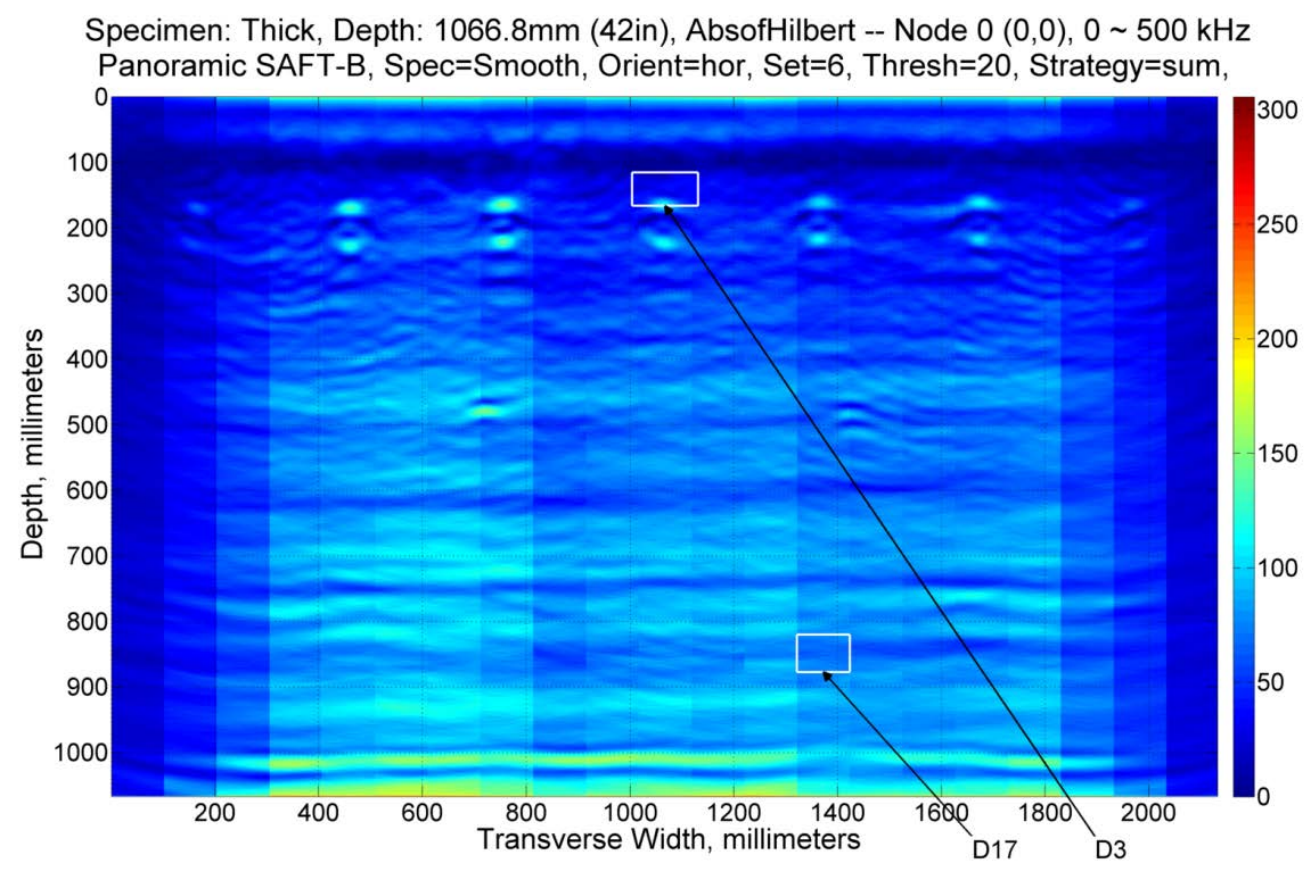

Fig. B. 26. Horizontal Set 6, Node 0. 
Specimen: Thick, Depth: 1066.8mm (42in), AbsofHilbert -- Node $16(4,1), 31.25 \sim 62.5 \mathrm{kHz}$ Panoramic SAFT-B, Spec=Smooth, Orient=hor, Set=6, Thresh=20, Strategy=sum

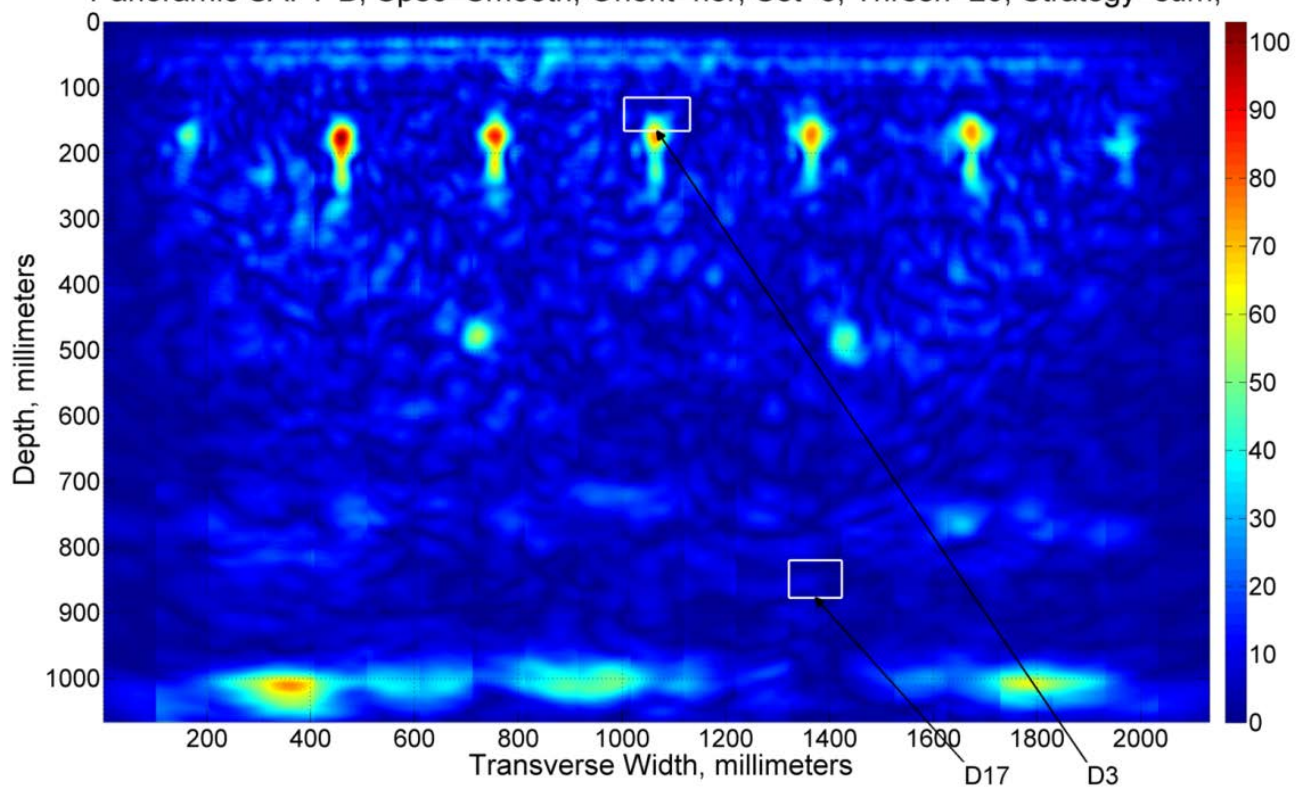

Fig. B. 27. Horizontal Set 6, Node 16.

Specimen: Thick, Depth: 1066.8mm (42in), AbsofHilbert -- Node $18(4,3), 93.75 \sim 125 \mathrm{kHz}$ Panoramic SAFT-B, Spec $=$ Smooth, Orient $=$ hor, Set $=6$, Thresh $=20$, Strategy=sum,

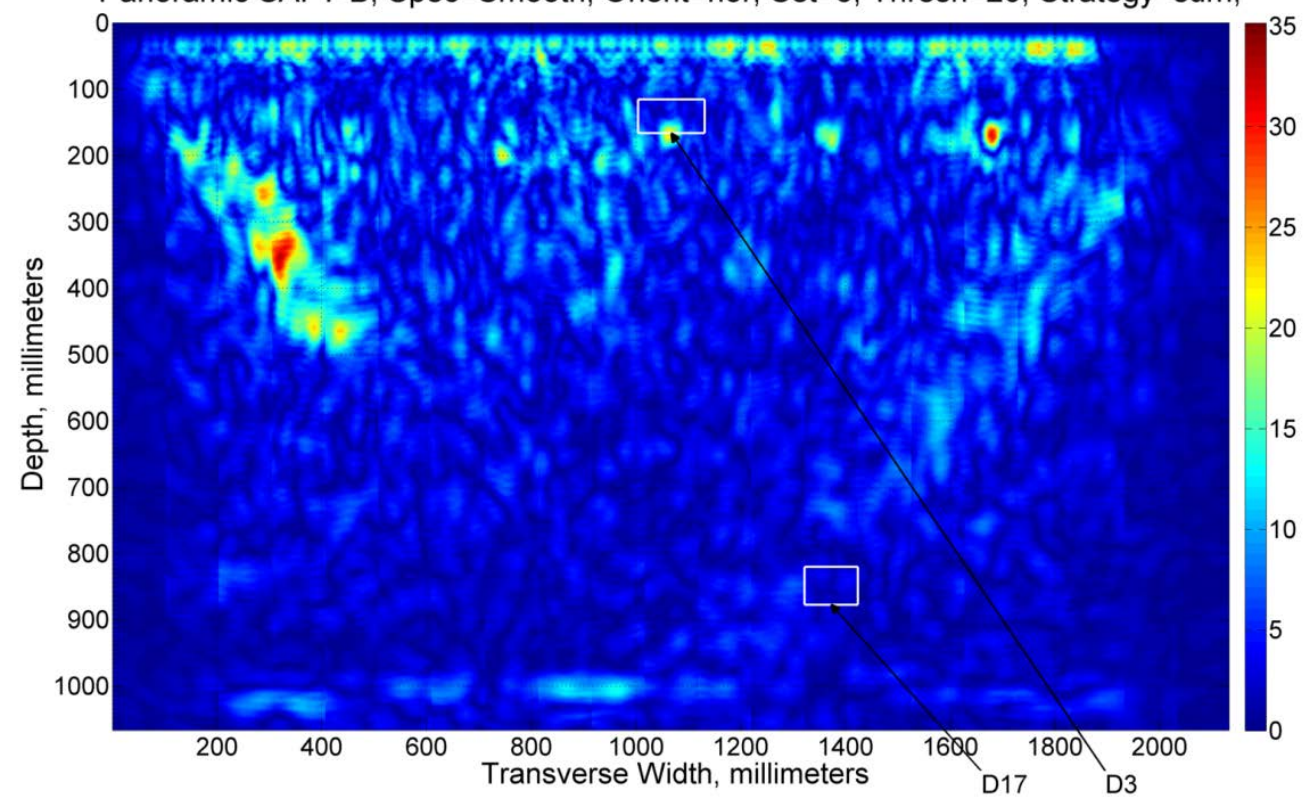

Fig. B. 28. Horizontal Set 6, Node 18. 
Specimen: Thick, Depth: 1066.8mm (42in), AbsofHilbert -- Node $32(5,1), 15.625 \sim 31.25 \mathrm{kHz}$ Panoramic SAFT-B, Spec=Smooth, Orient=hor, Set=6, Thresh=20, Strategy=sum,

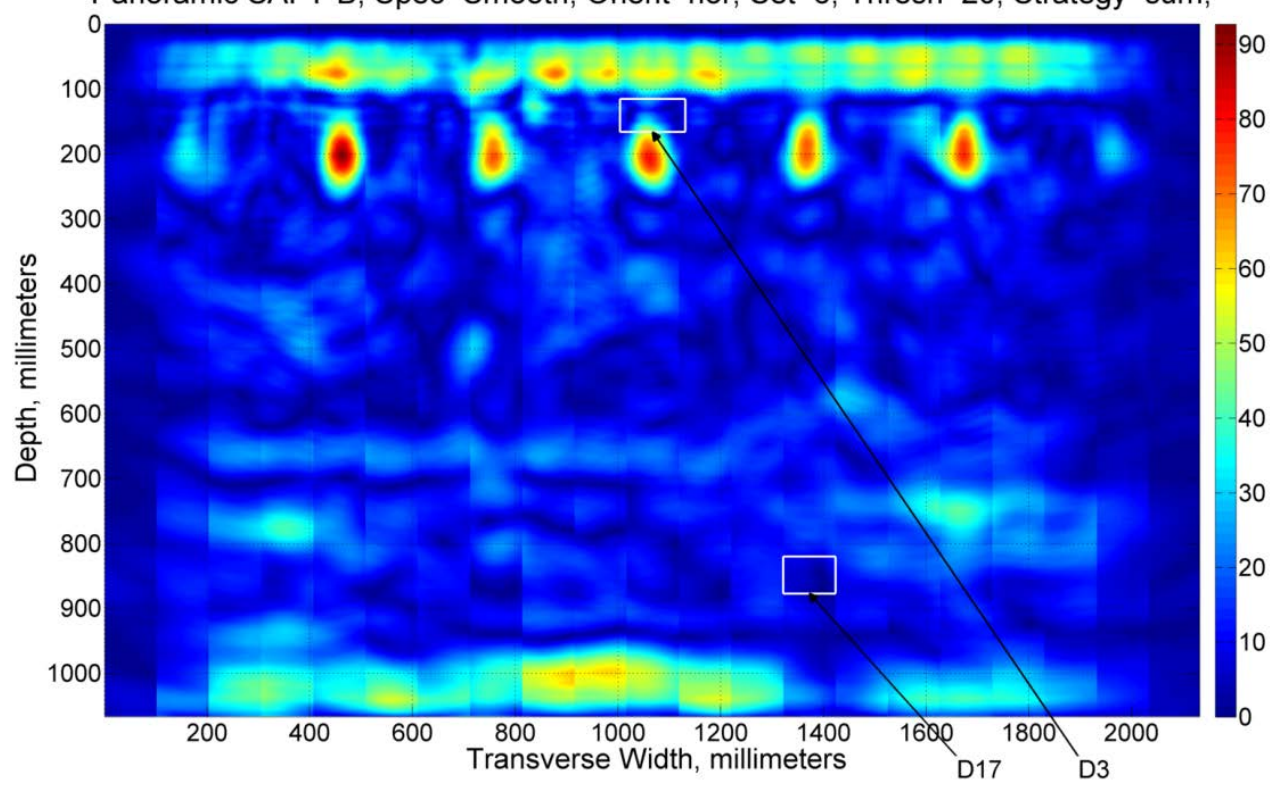

Fig. B. 29. Horizontal Set 6, Node 32.

Specimen: Thick, Depth: 1066.8mm (42in), AbsofHilbert -- Node 34 (5,3), $46.875 \sim 62.5 \mathrm{kHz}$ Panoramic SAFT-B, Spec=Smooth, Orient=hor, Set=6, Thresh=20, Strategy=sum,

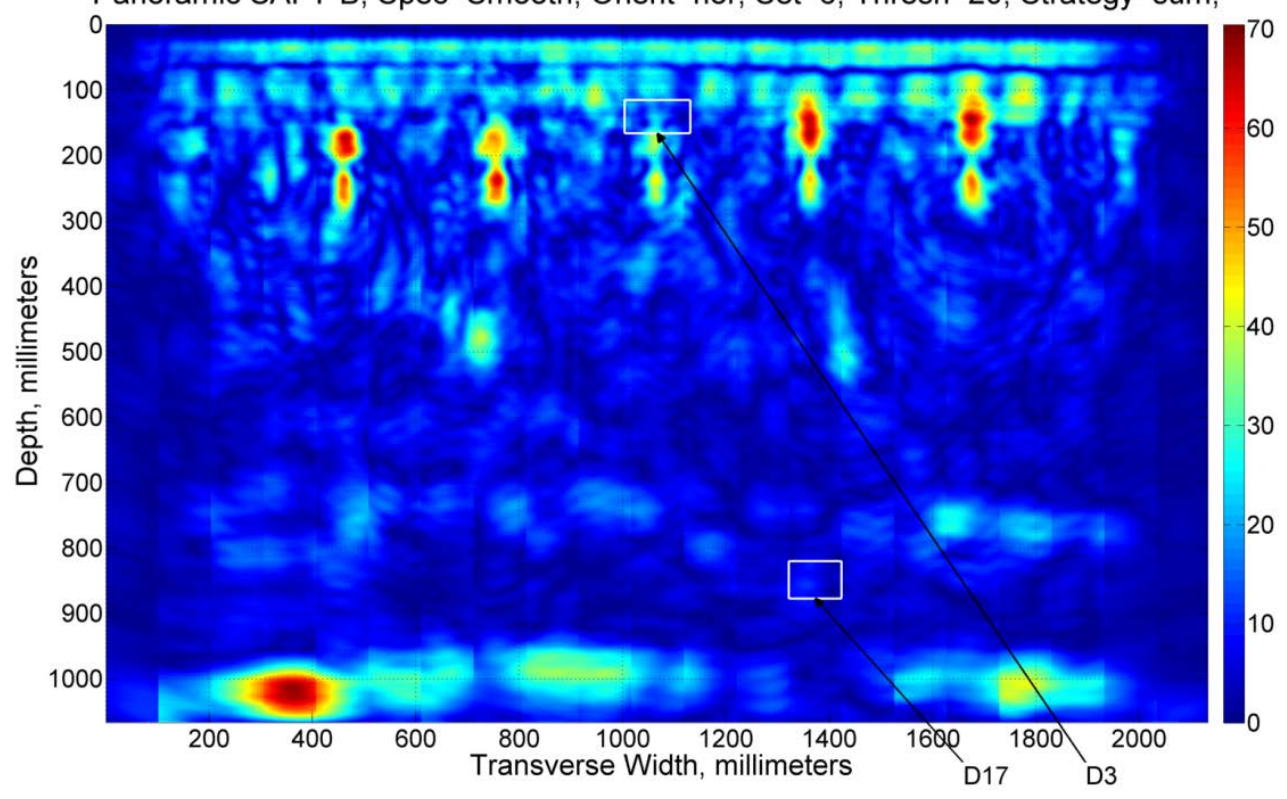

Fig. B. 30. Horizontal Set 6, Node 34. 


\section{SET SEVEN - DEFECT 5}

Specimen: Thick, Depth: 1066.8mm (42in), AbsofHilbert -- Node $0(0,0), 0 \sim 500 \mathrm{kHz}$ Panoramic SAFT-B, Spec=Smooth, Orient=hor, Set=7, Thresh=20, Strategy=sum,

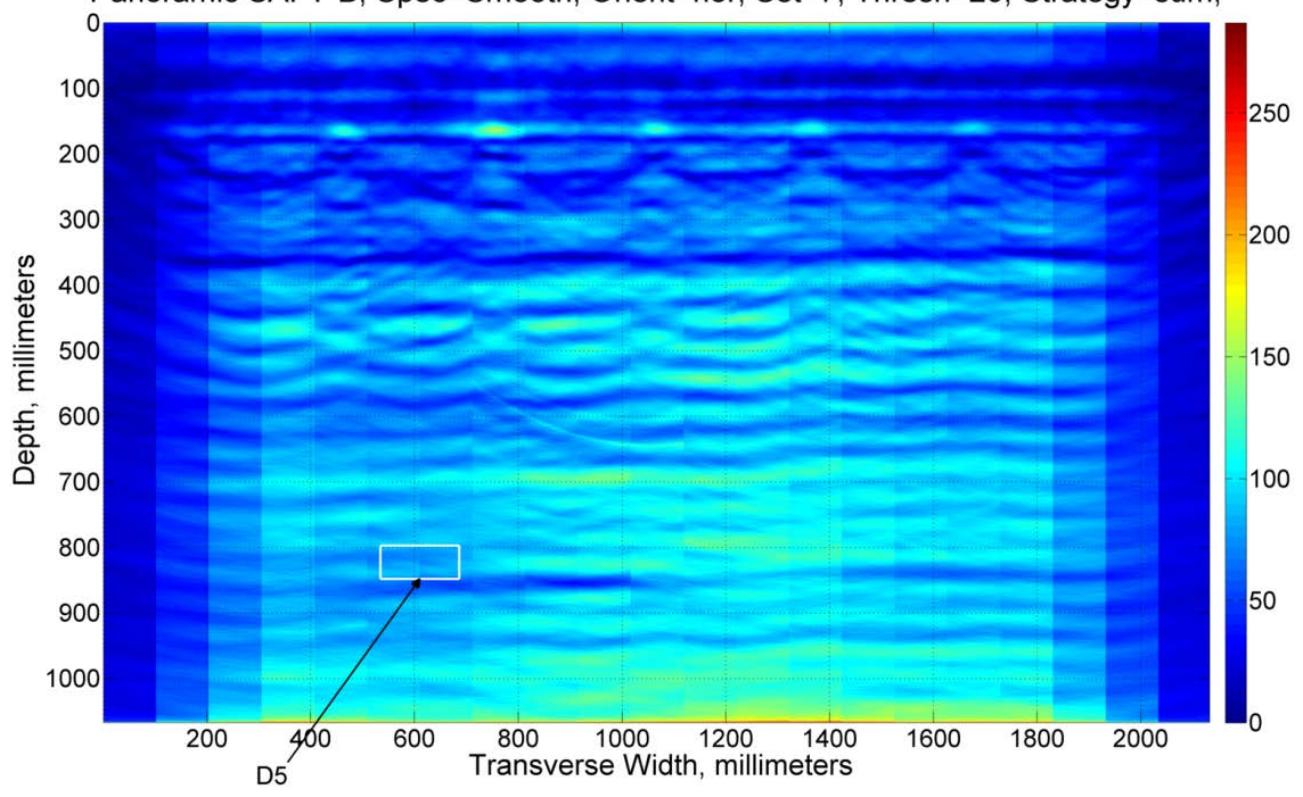

Fig. B. 31. Horizontal Set 7, Node 0.

Specimen: Thick, Depth: 1066.8mm (42in), AbsofHilbert -- Node $16(4,1), 31.25 \sim 62.5 \mathrm{kHz}$ Panoramic SAFT-B, Spec=Smooth, Orient=hor, Set=7, Thresh=20, Strategy=sum,

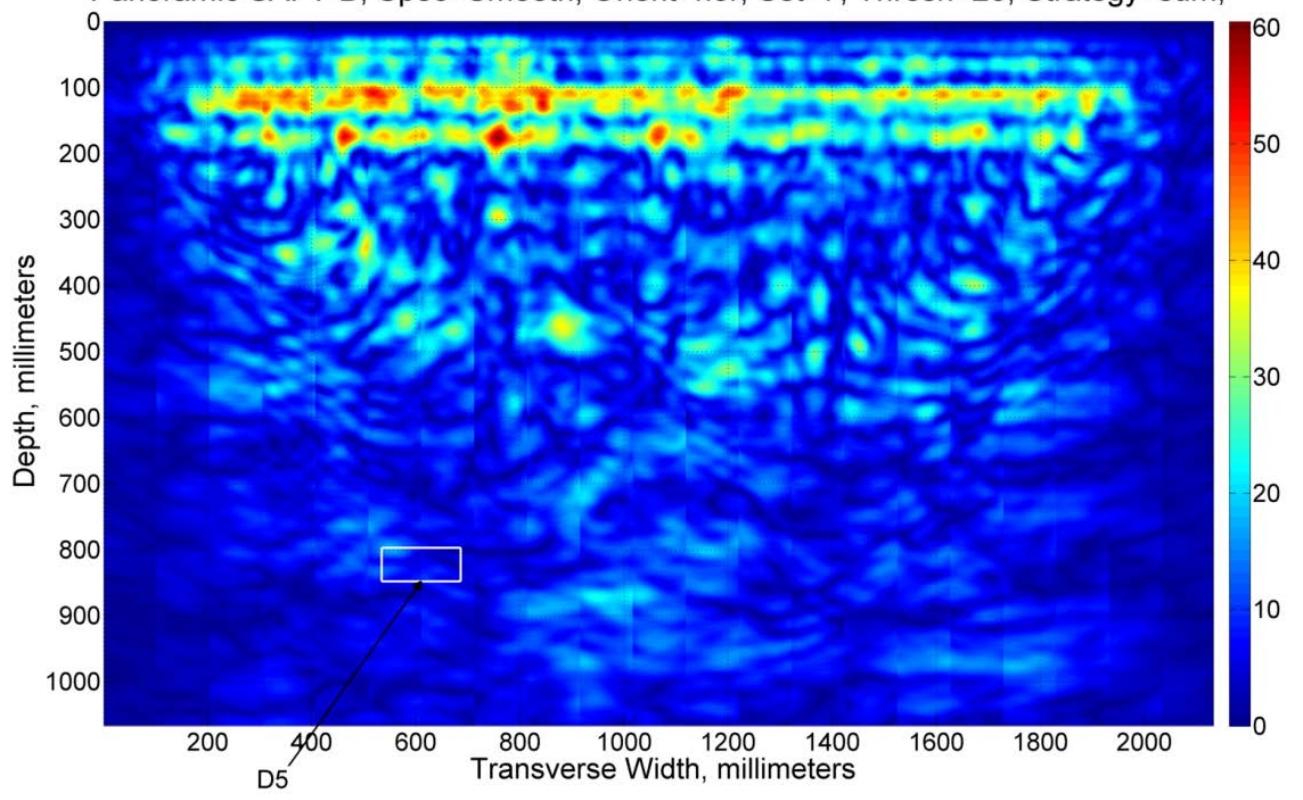

Fig. B. 32. Horizontal Set 7, Node 16. 
Specimen: Thick, Depth: 1066.8mm (42in), AbsofHilbert -- Node $18(4,3), 93.75 \sim 125 \mathrm{kHz}$ Panoramic SAFT-B, Spec=Smooth, Orient=hor, Set=7, Thresh=20, Strategy=sum,

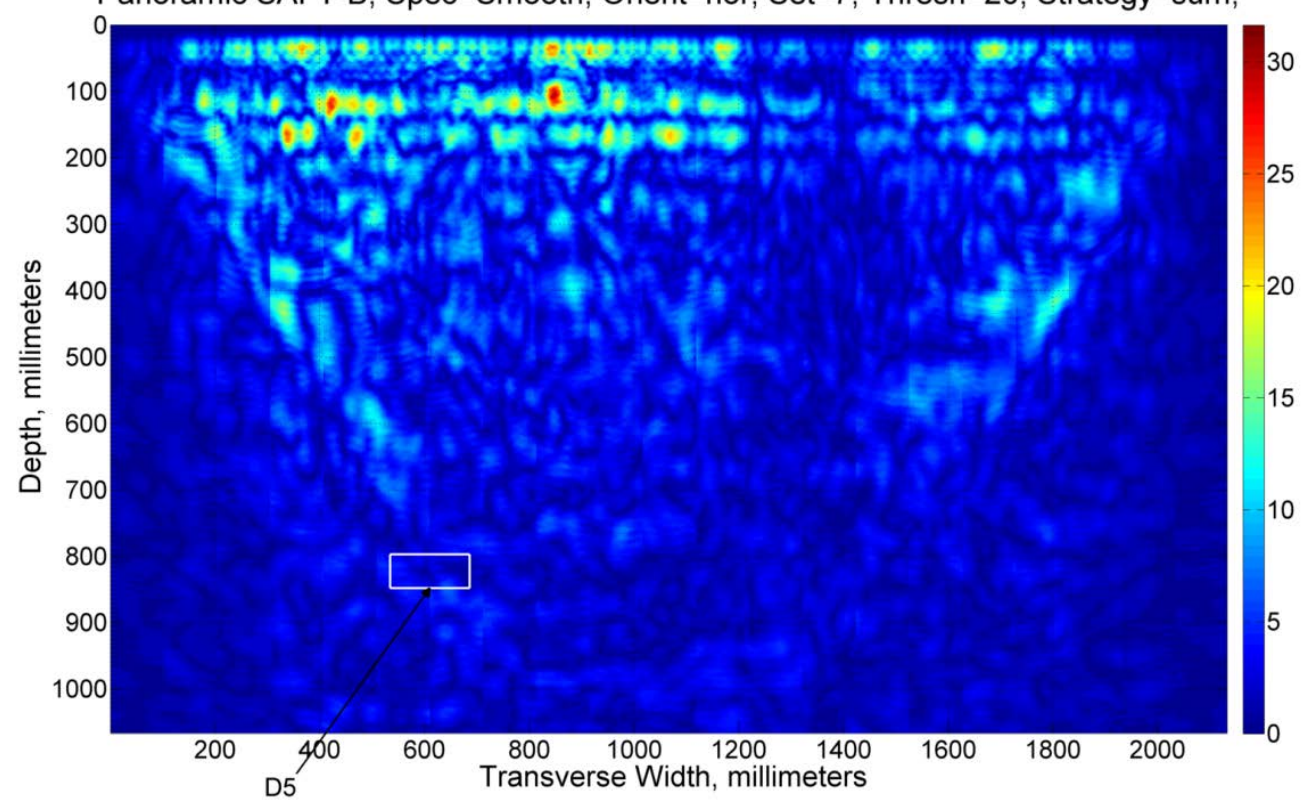

Fig. B. 33. Horizontal Set 7, Node 18.

Specimen: Thick, Depth: 1066.8mm (42in), AbsofHilbert -- Node $32(5,1), 15.625 \sim 31.25 \mathrm{kHz}$ Panoramic SAFT-B, Spec=Smooth, Orient=hor, Set=7, Thresh=20, Strategy=sum,

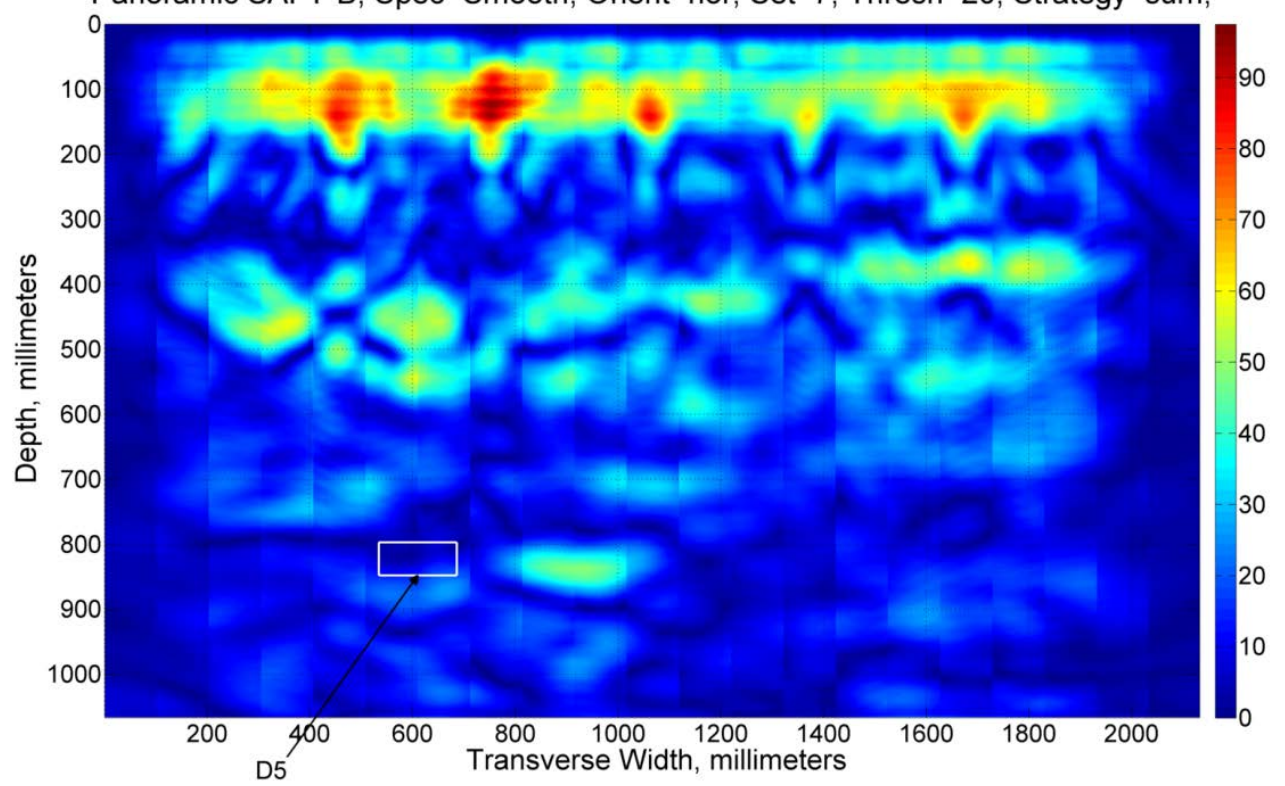

Fig. B. 34. Horizontal Set 7, Node 32. 


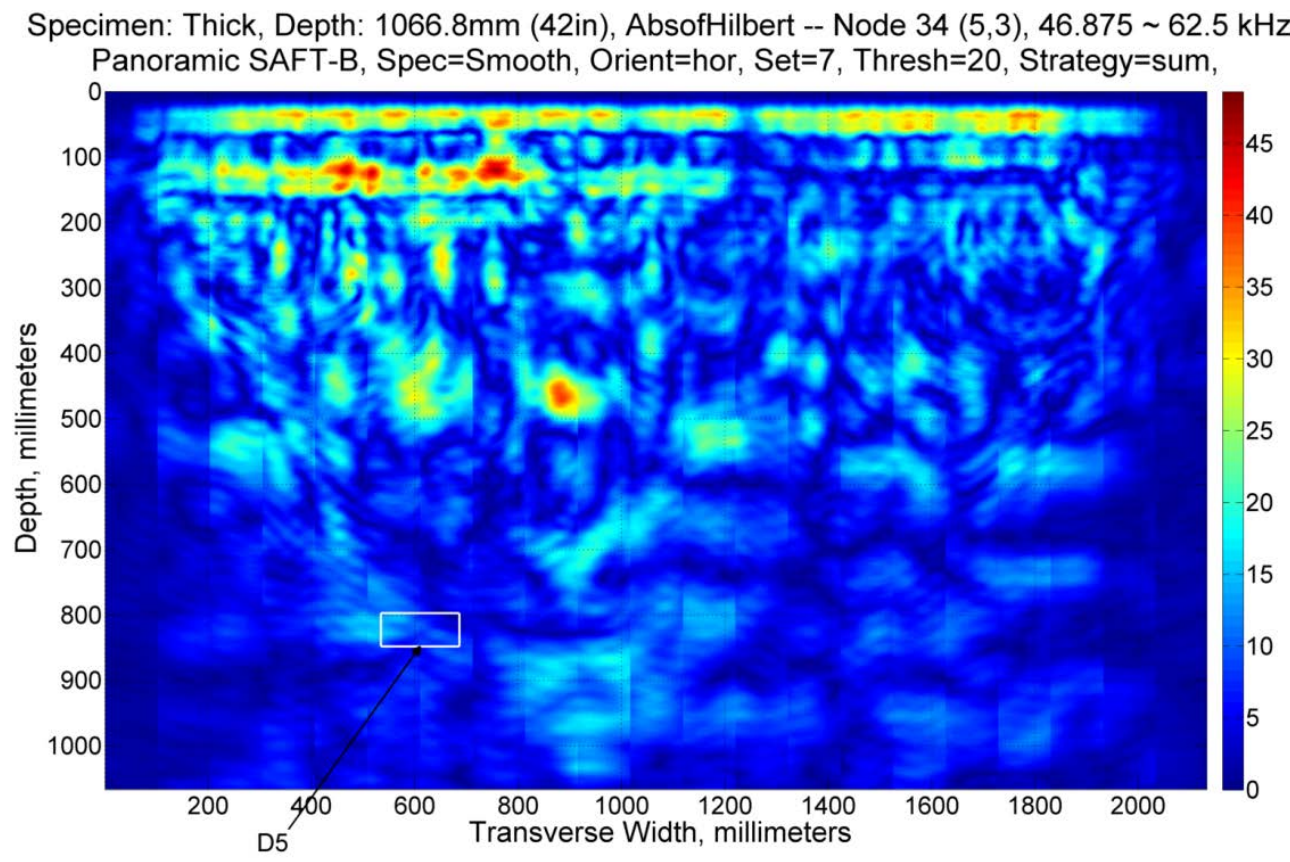

Fig. B. 35. Horizontal Set 7, Node 34.

\section{SET EIGHT - DEFECT 9, DEFECT 13}

Specimen: Thick, Depth: 1066.8mm (42in), AbsofHilbert -- Node $0(0,0), 0 \sim 500 \mathrm{kHz}$ Panoramic SAFT-B, Spec=Smooth, Orient=hor, Set=8, Thresh=20, Strategy=sum,

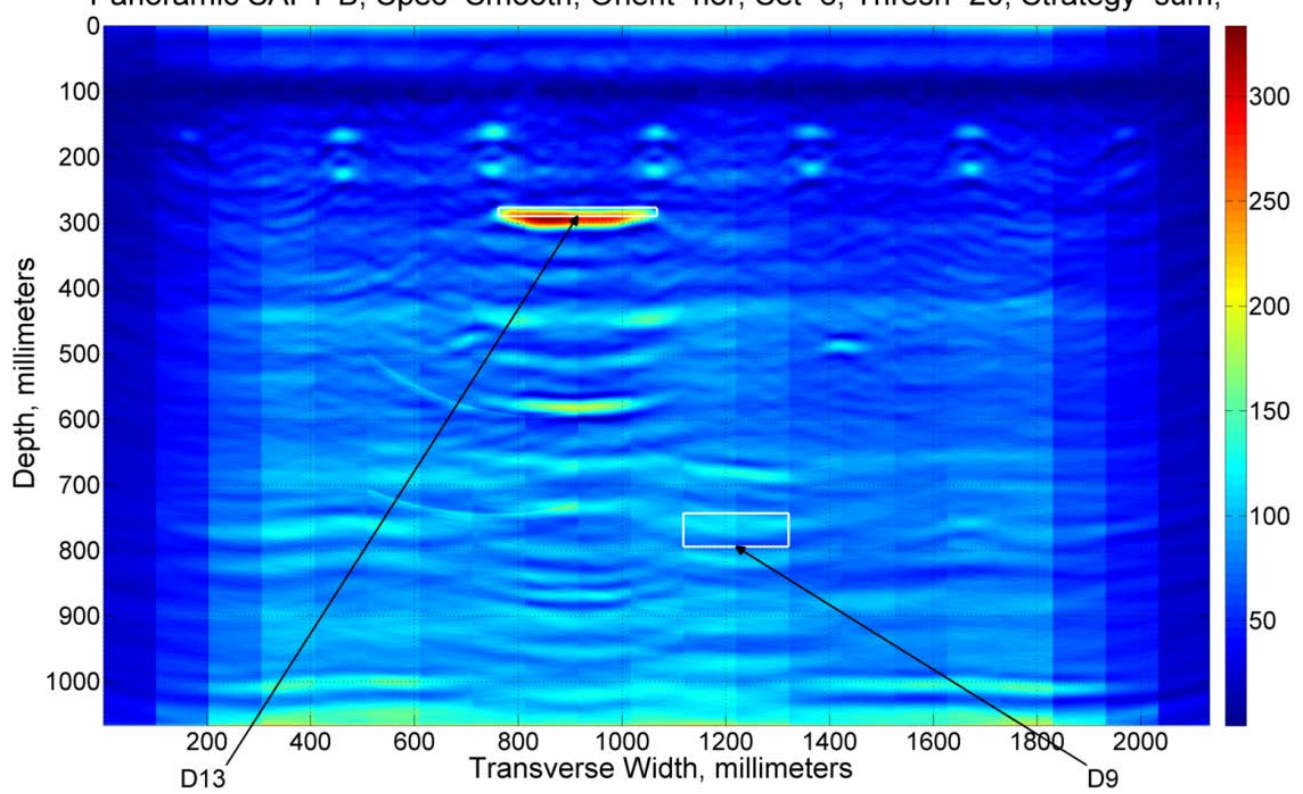

Fig. B. 36. Horizontal Set 8, Node 0. 


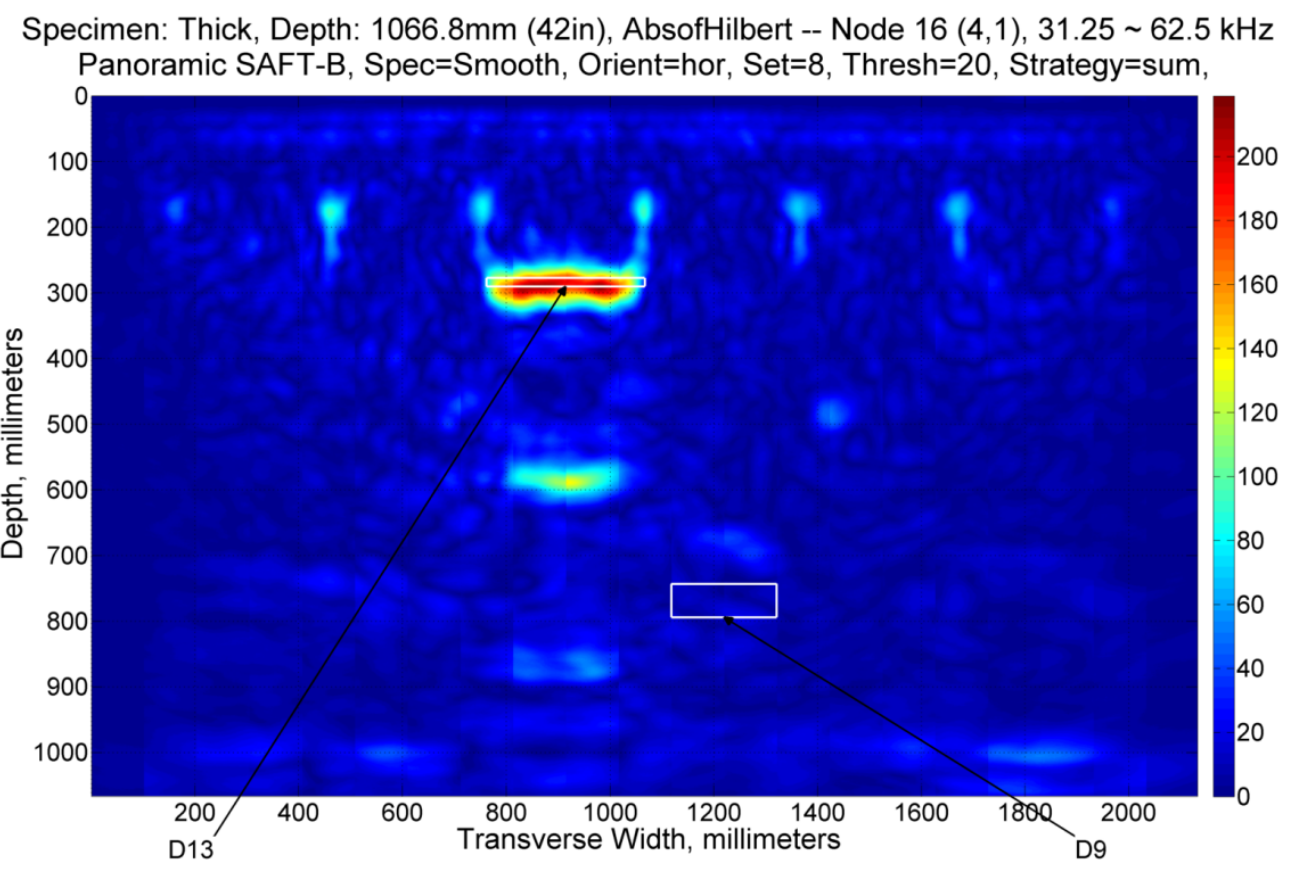

Fig. B. 37. Horizontal Set 8, Node 16.

Specimen: Thick, Depth: 1066.8mm (42in), AbsofHilbert -- Node $18(4,3), 93.75 \sim 125 \mathrm{kHz}$ Panoramic SAFT-B, Spec=Smooth, Orient=hor, Set=8, Thresh=20, Strategy=sum,

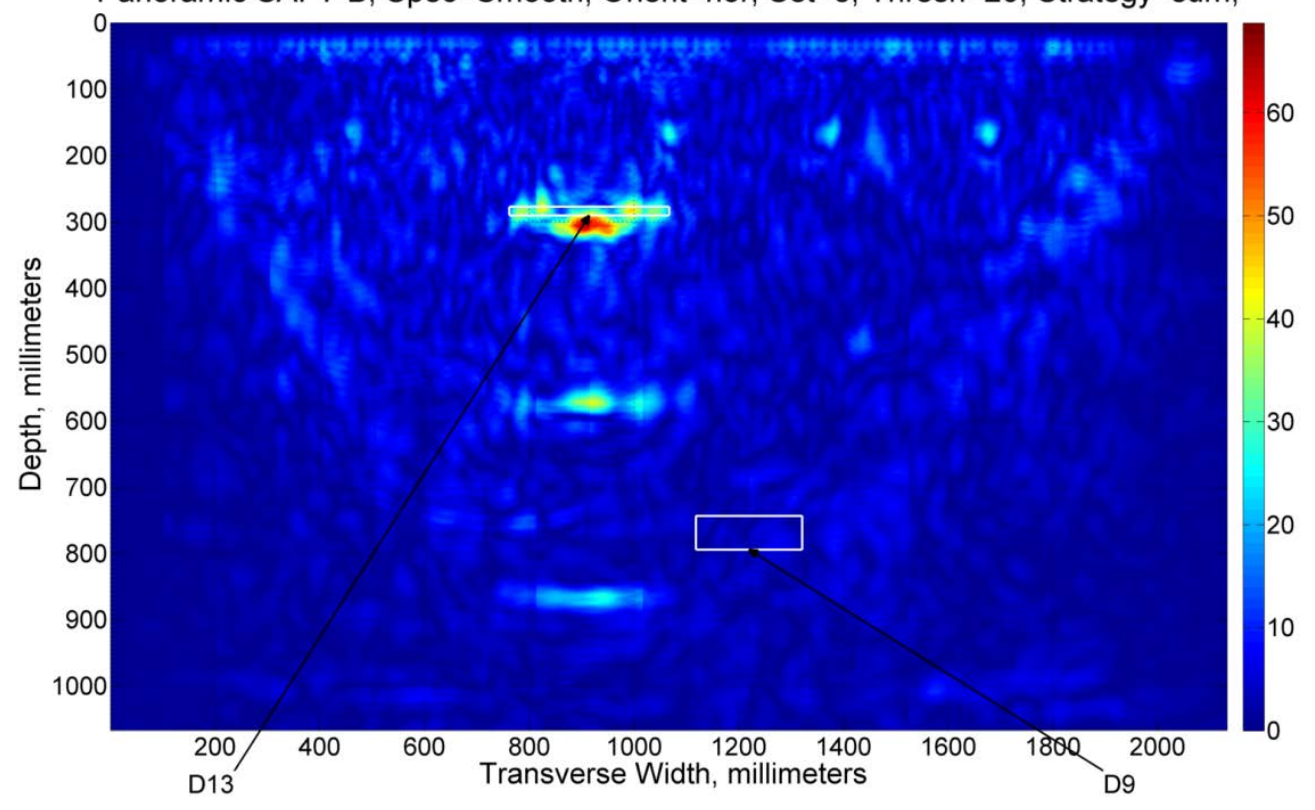

Fig. B. 38. Horizontal Set 8, Node 18. 


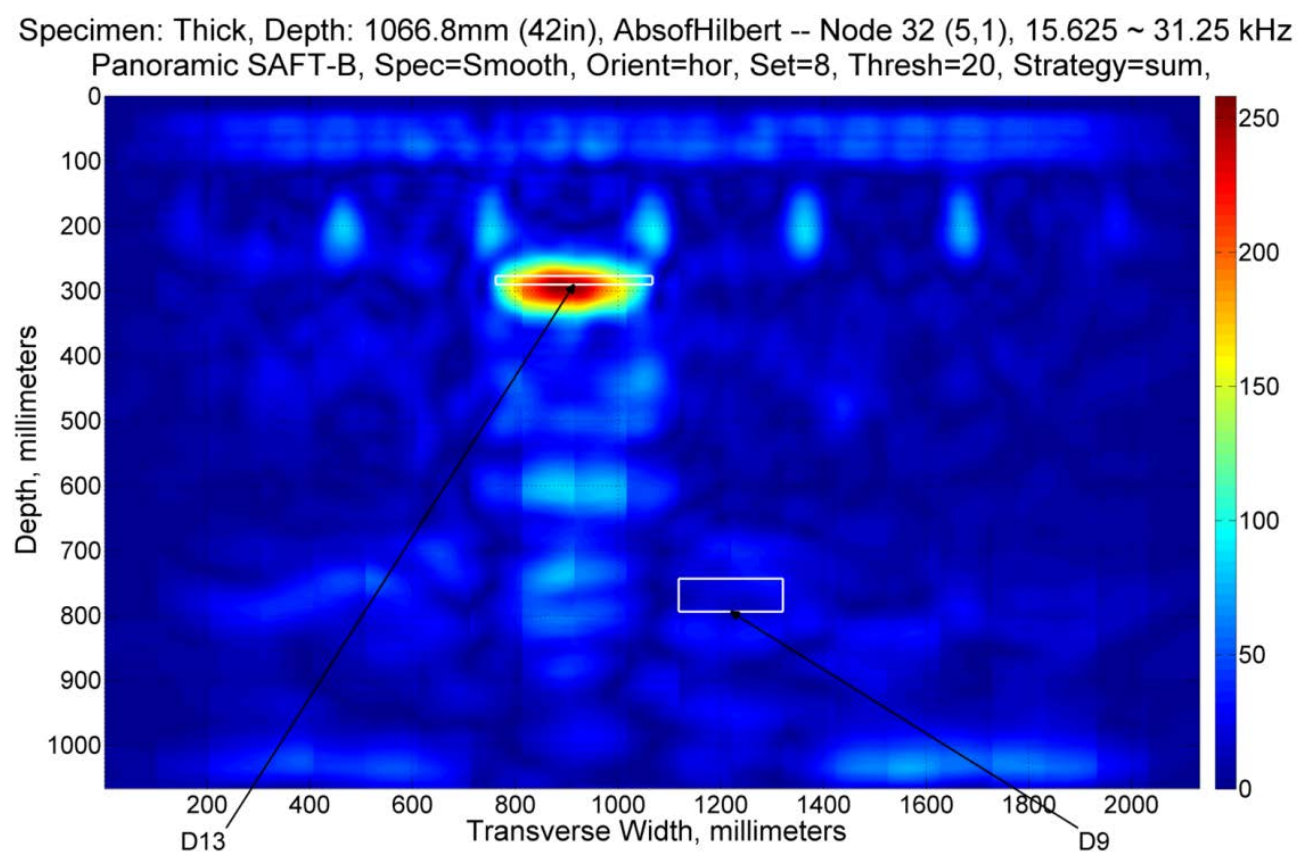

Fig. B. 39. Horizontal Set 8, Node 32.

Specimen: Thick, Depth: 1066.8mm (42in), AbsofHilbert -- Node $34(5,3), 46.875 \sim 62.5 \mathrm{kHz}$ Panoramic SAFT-B, Spec=Smooth, Orient=hor, Set=8, Thresh=20, Strategy=sum,

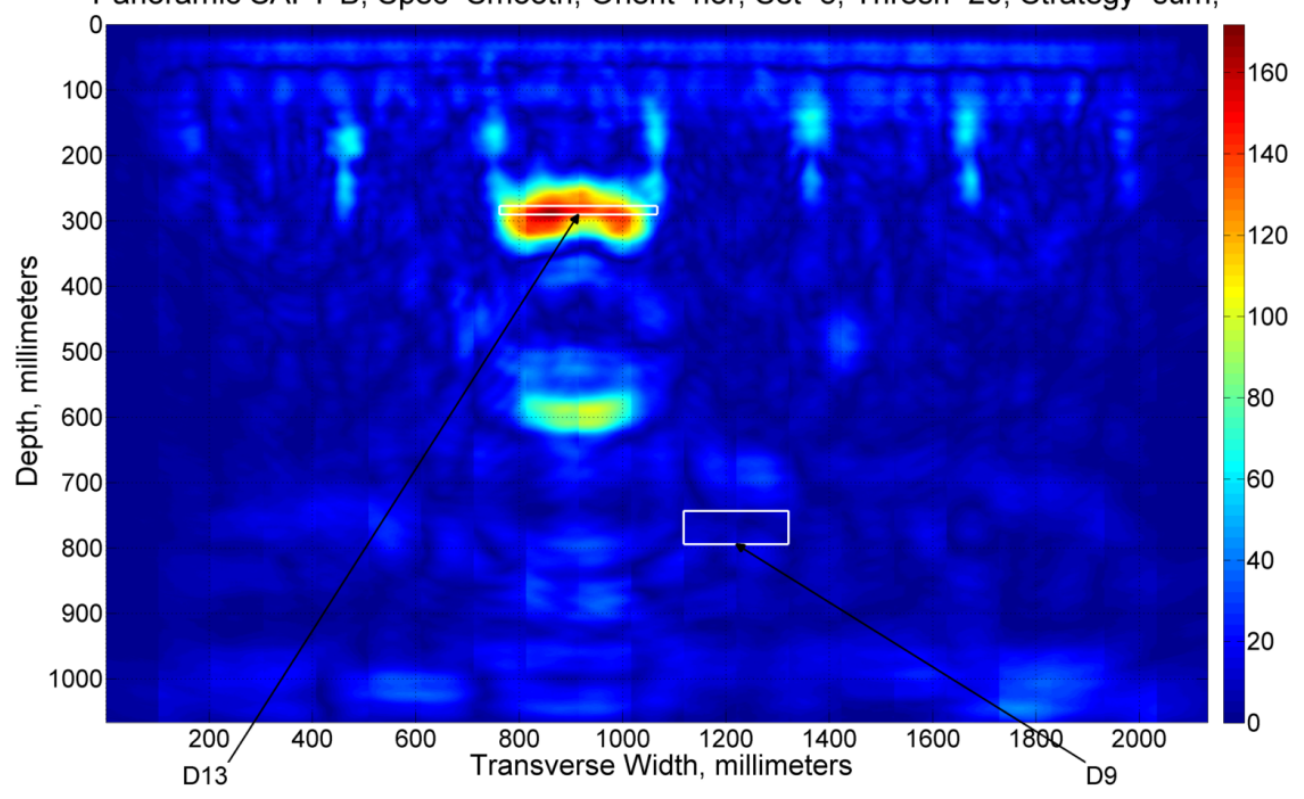

Fig. B. 40. Horizontal Set 8, Node 34. 


\section{SET NINE - DEFECT 9, DEFECT 13}

Specimen: Thick, Depth: 1066.8mm (42in), AbsofHilbert -- Node $0(0,0), 0 \sim 500 \mathrm{kHz}$ Panoramic SAFT-B, Spec=Smooth, Orient=hor, Set=9, Thresh=20, Strategy=sum,

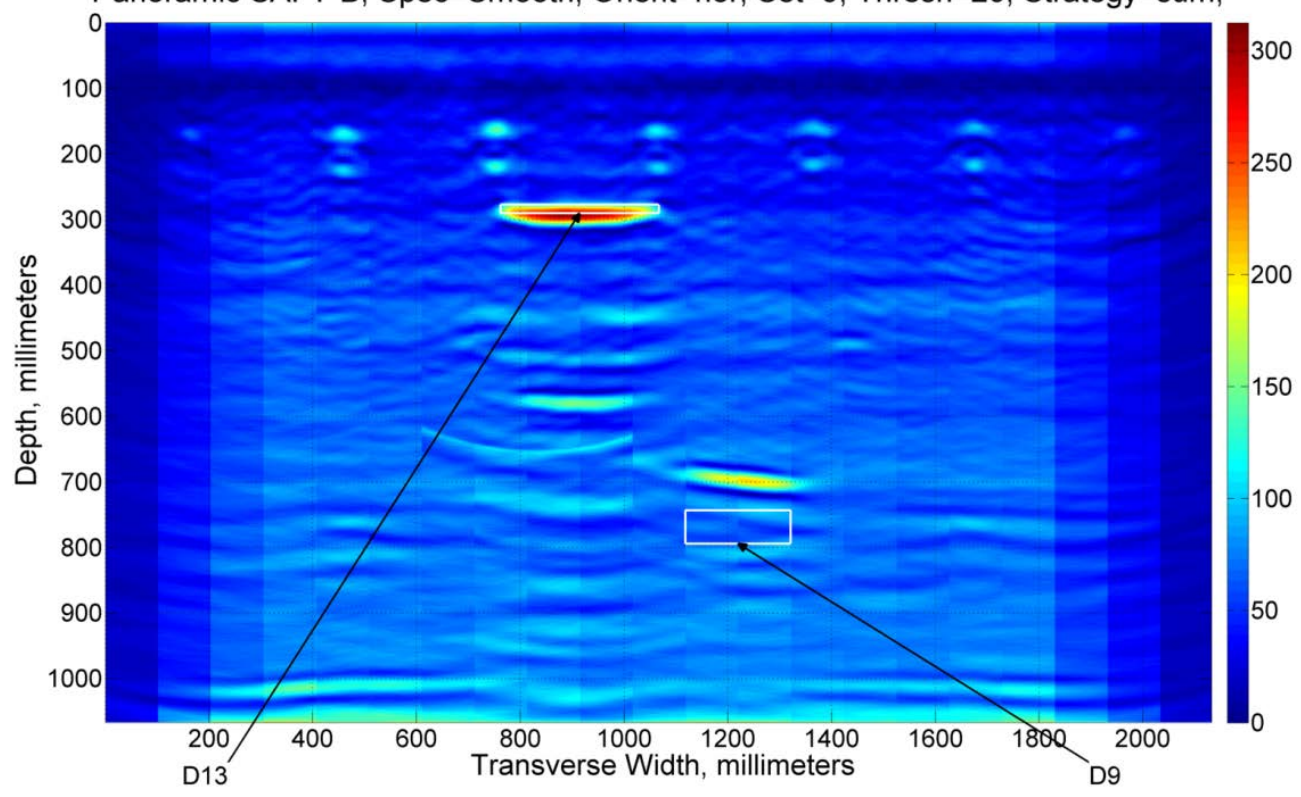

Fig. B. 41. Horizontal Set 9, Node 0.

Specimen: Thick, Depth: 1066.8mm (42in), AbsofHilbert -- Node $16(4,1), 31.25 \sim 62.5 \mathrm{kHz}$ Panoramic SAFT-B, Spec=Smooth, Orient=hor, Set $=9$, Thresh $=20$, Strategy=sum,

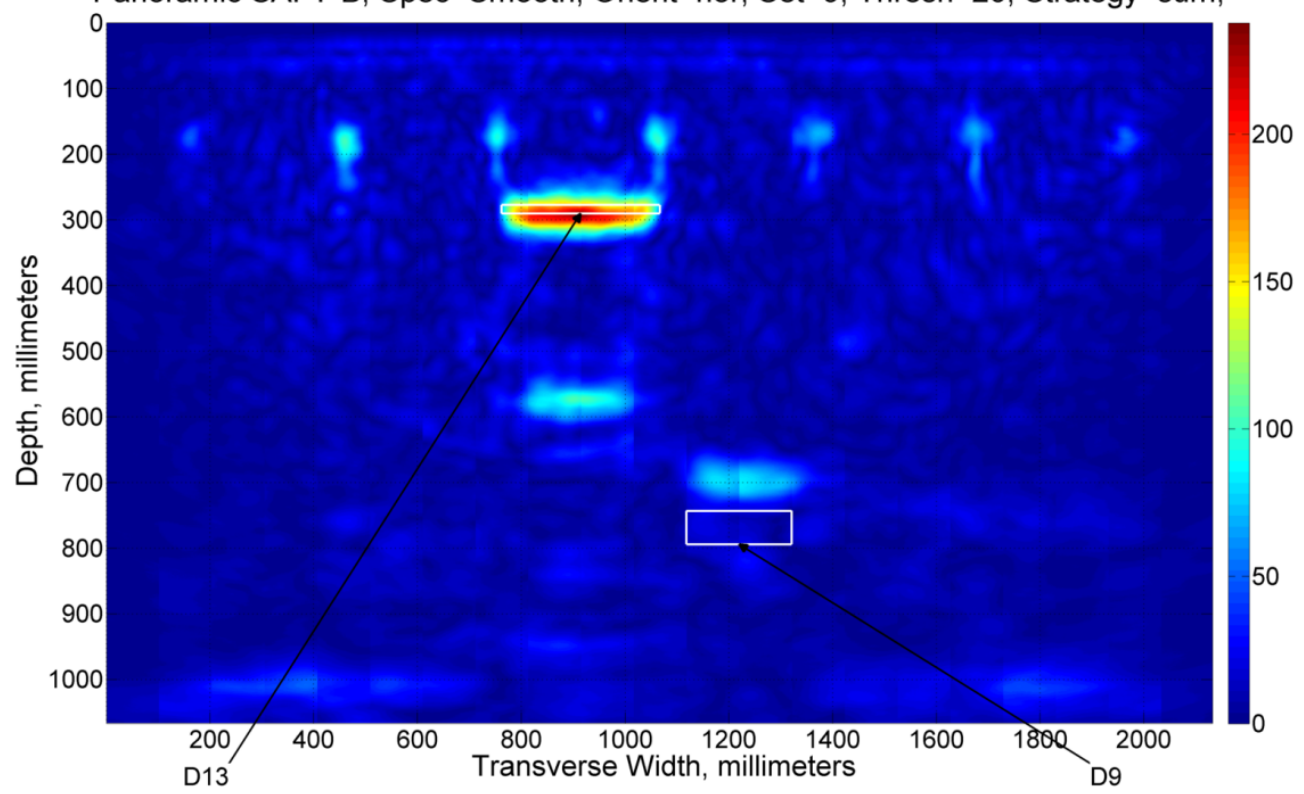

Fig. B. 42. Horizontal Set 9, Node 16. 
Specimen: Thick, Depth: 1066.8mm (42in), AbsofHilbert -- Node $18(4,3), 93.75 \sim 125 \mathrm{kHz}$ Panoramic SAFT-B, Spec=Smooth, Orient=hor, Set=9, Thresh=20, Strategy=sum,

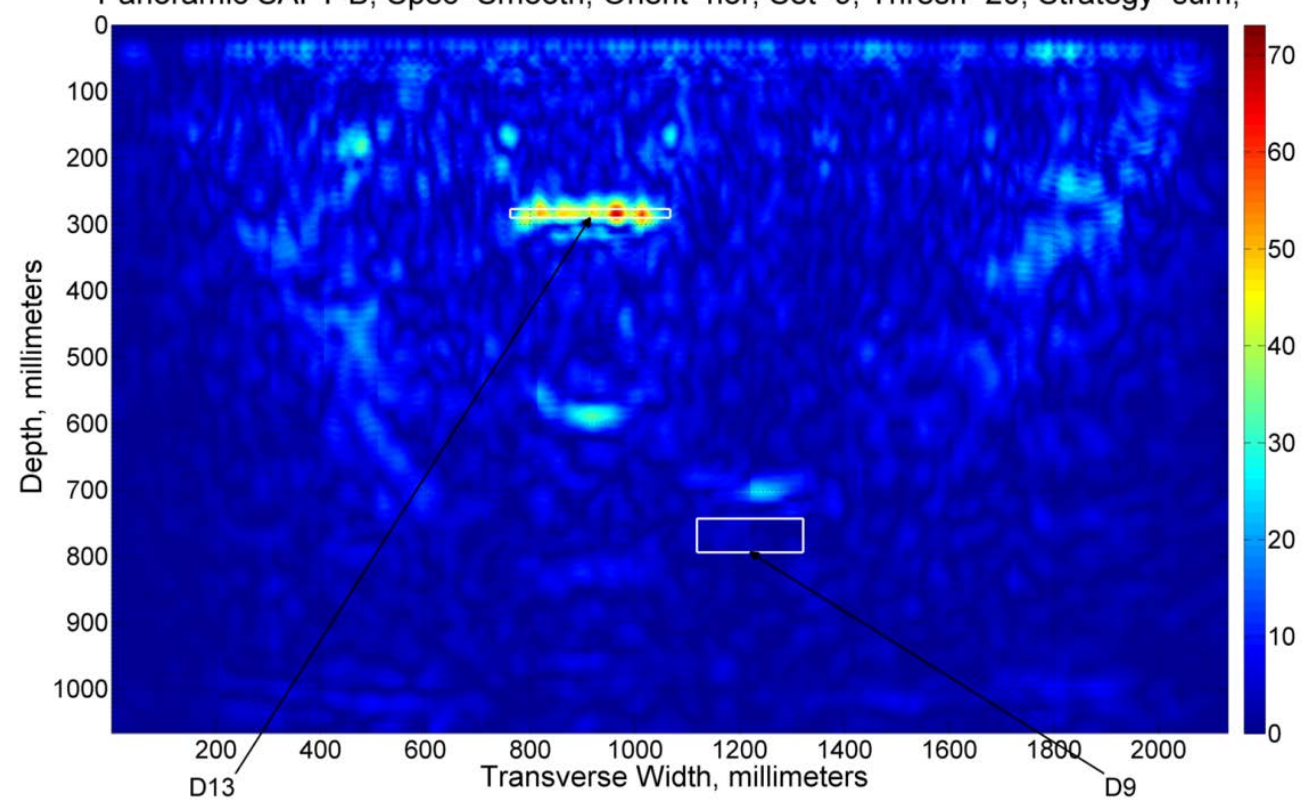

Fig. B. 43. Horizontal Set 9, Node 18.

Specimen: Thick, Depth: 1066.8mm (42in), AbsofHilbert -- Node 32 (5,1), $15.625 \sim 31.25 \mathrm{kHz}$ Panoramic SAFT-B, Spec=Smooth, Orient=hor, Set=9, Thresh=20, Strategy=sum,

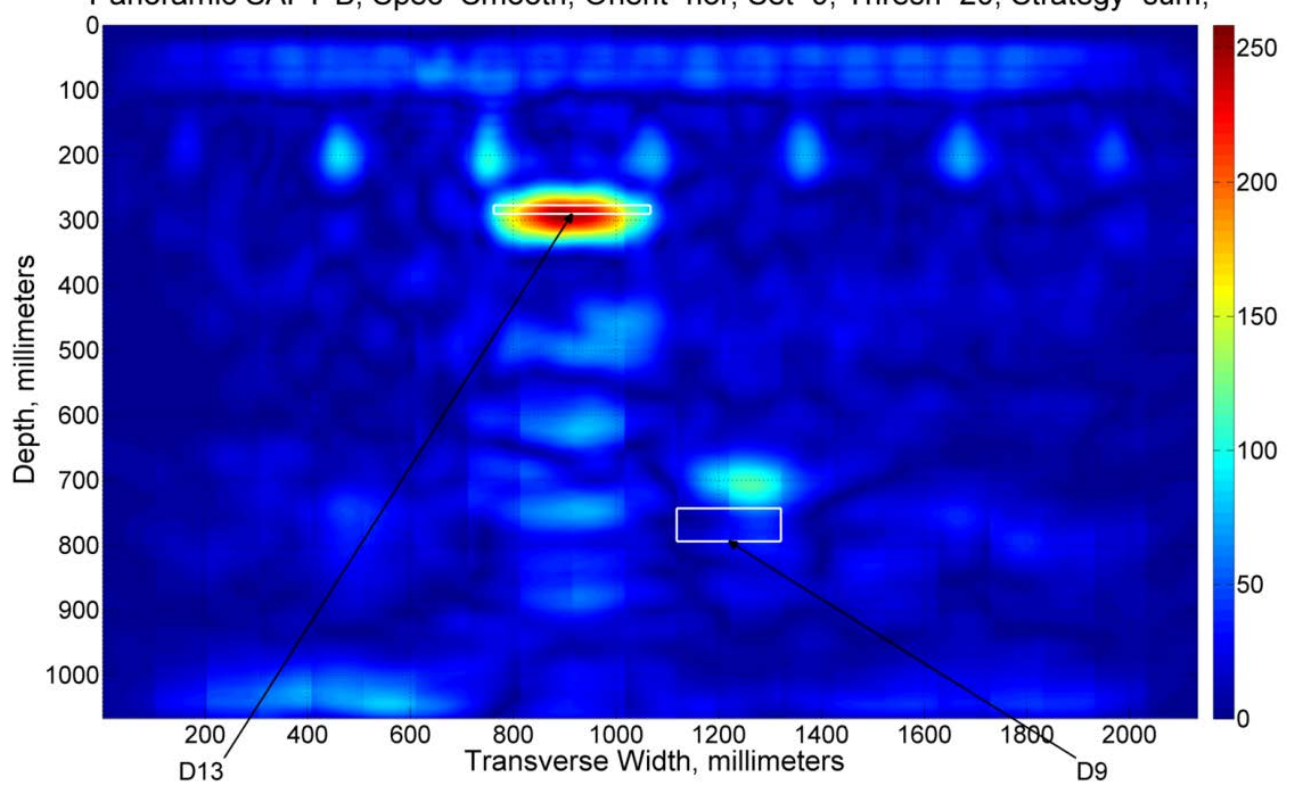

Fig. B. 44. Horizontal Set 9, Node 32. 


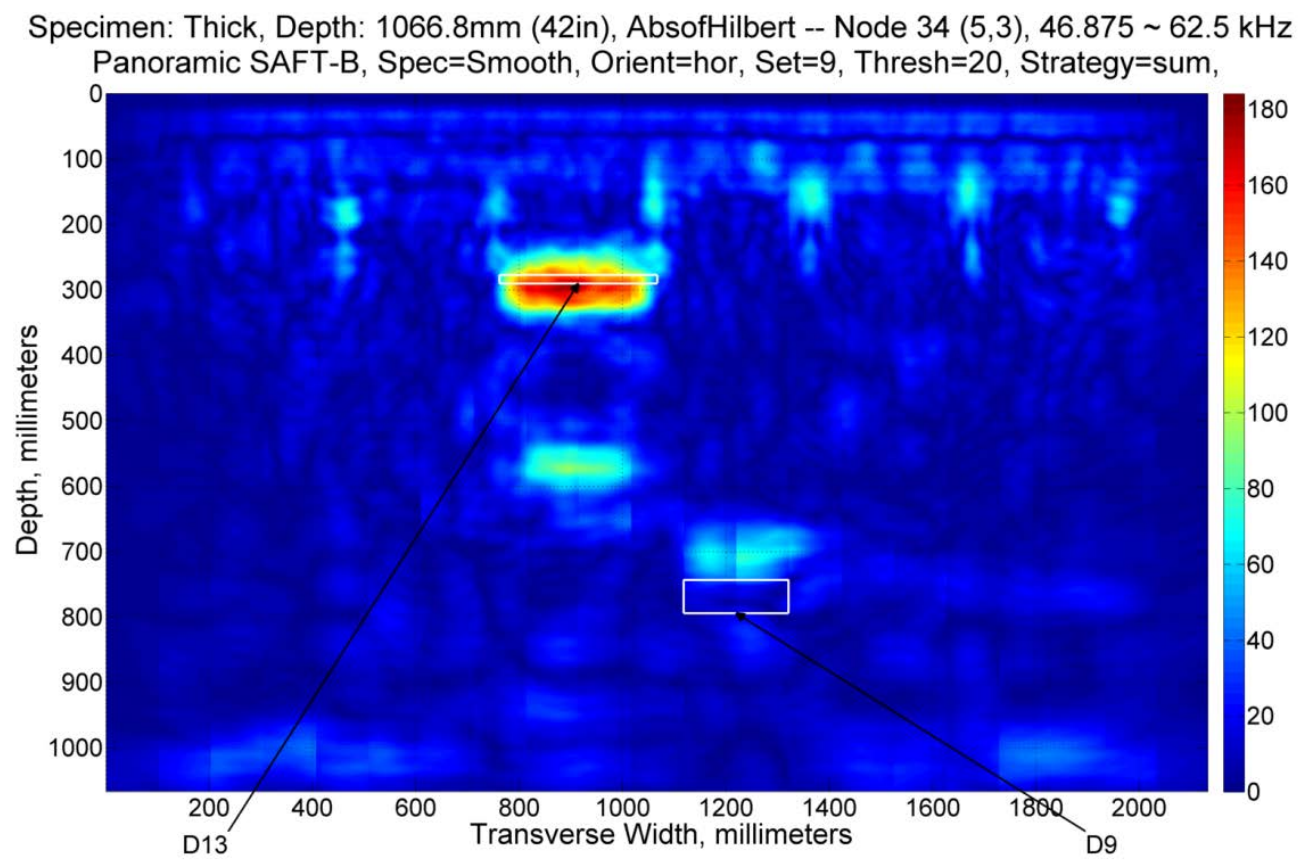

Fig. B. 45. Horizontal Set 9, Node 34.

\section{SET TEN - DEFECT 6, DEFECT 7, DEFECT 18, DEFECT 19}

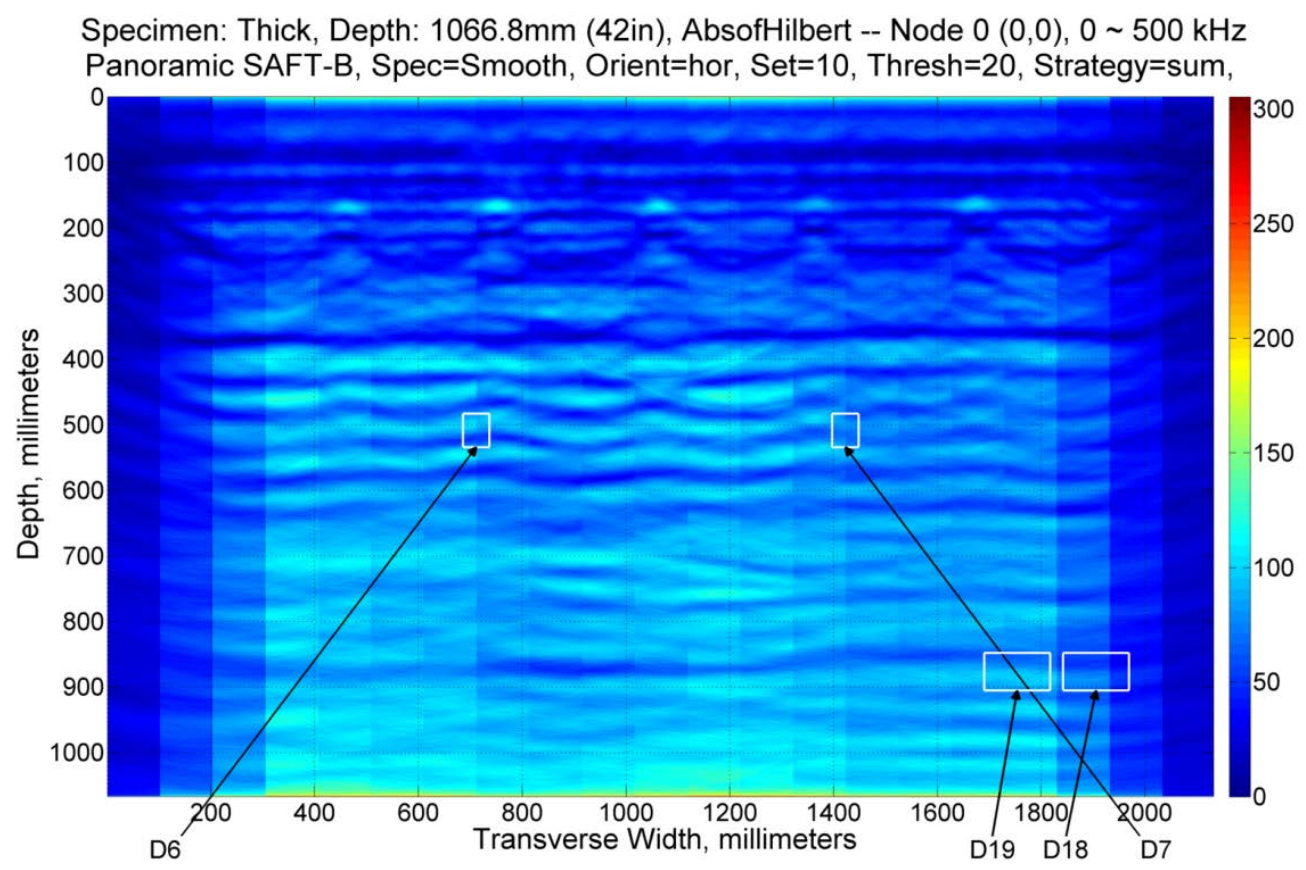

Fig. B. 46. Horizontal Set 10, Node 0. 


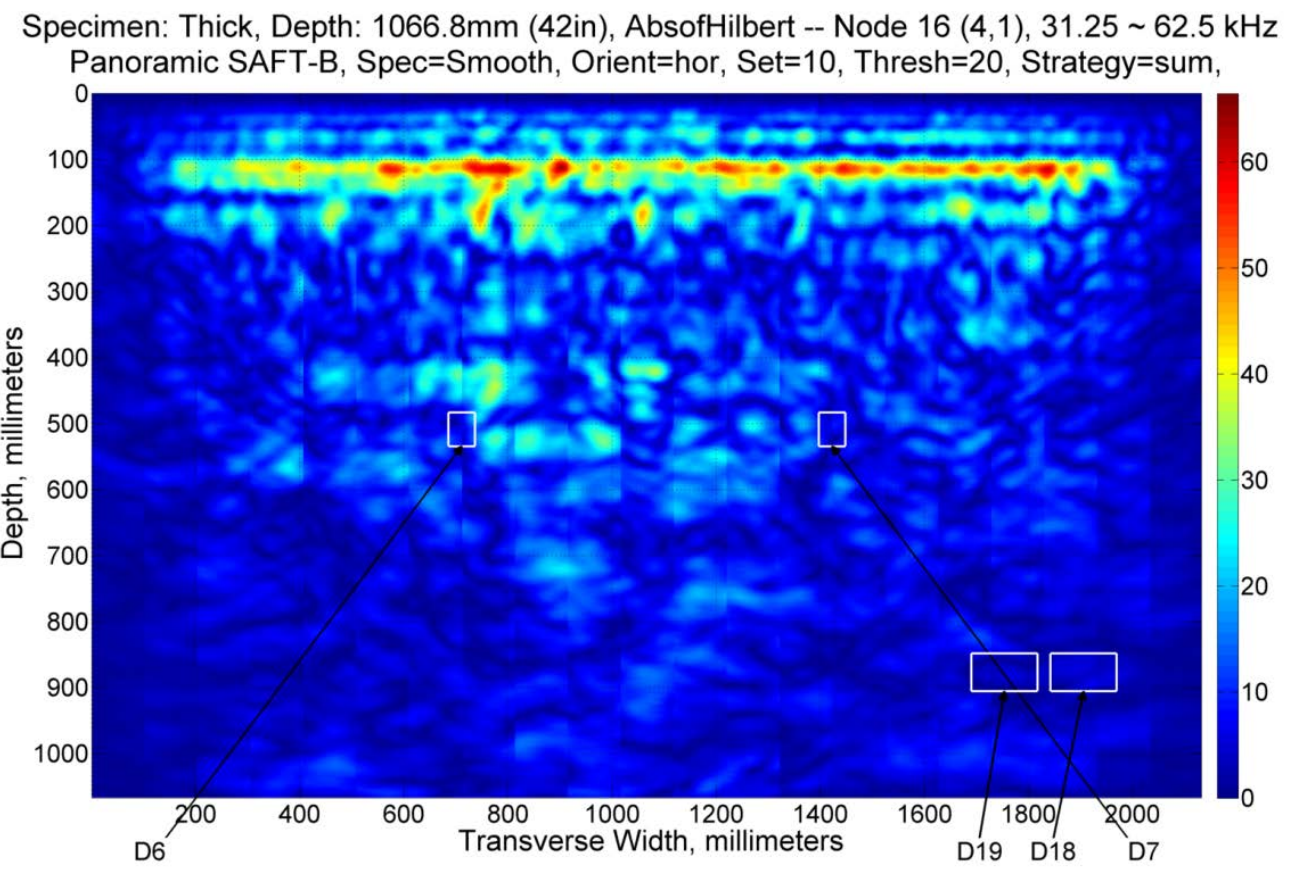

Fig. B. 47. Horizontal Set 10, Node 16.

Specimen: Thick, Depth: 1066.8mm (42in), AbsofHilbert -- Node $18(4,3), 93.75 \sim 125 \mathrm{kHz}$ Panoramic SAFT-B, Spec=Smooth, Orient=hor, Set=10, Thresh=20, Strategy=sum,

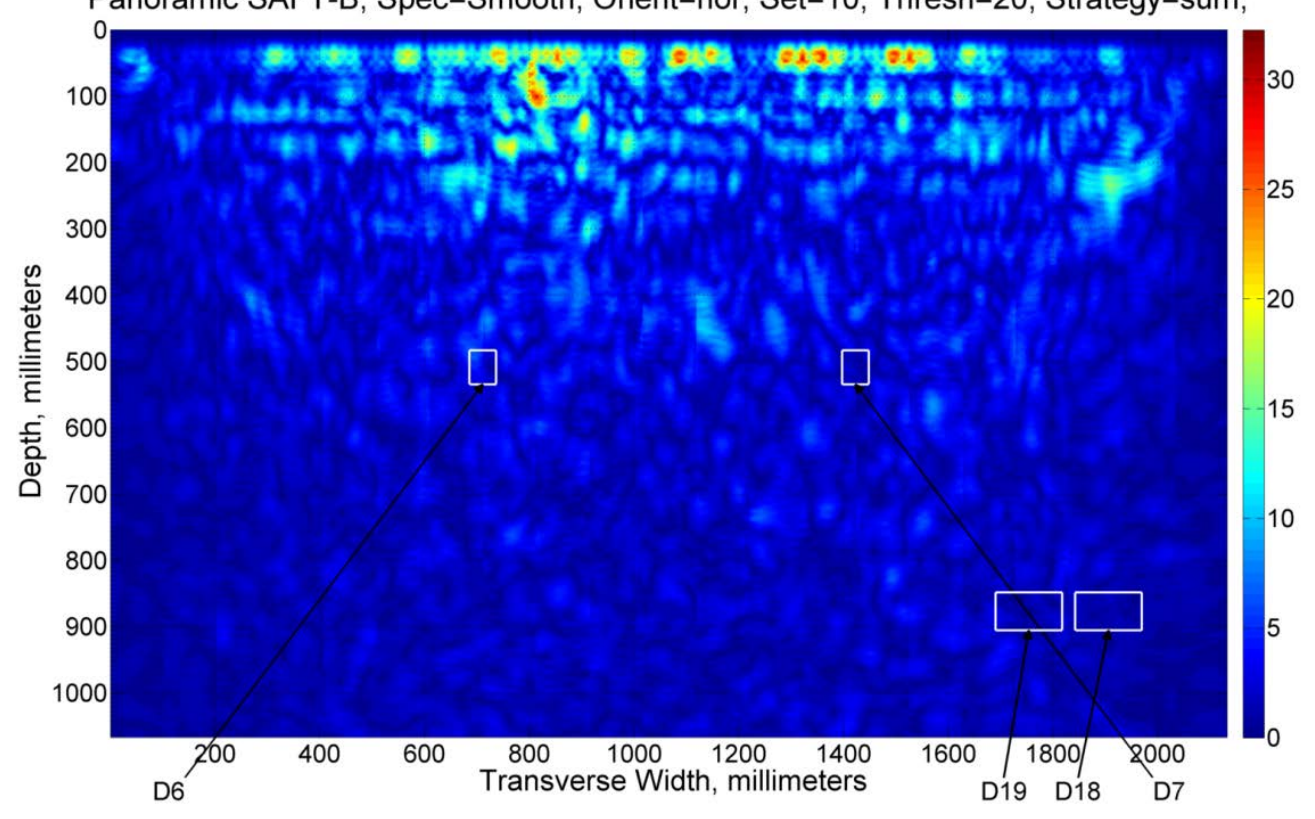

Fig. B. 48. Horizontal Set 10, Node 18. 


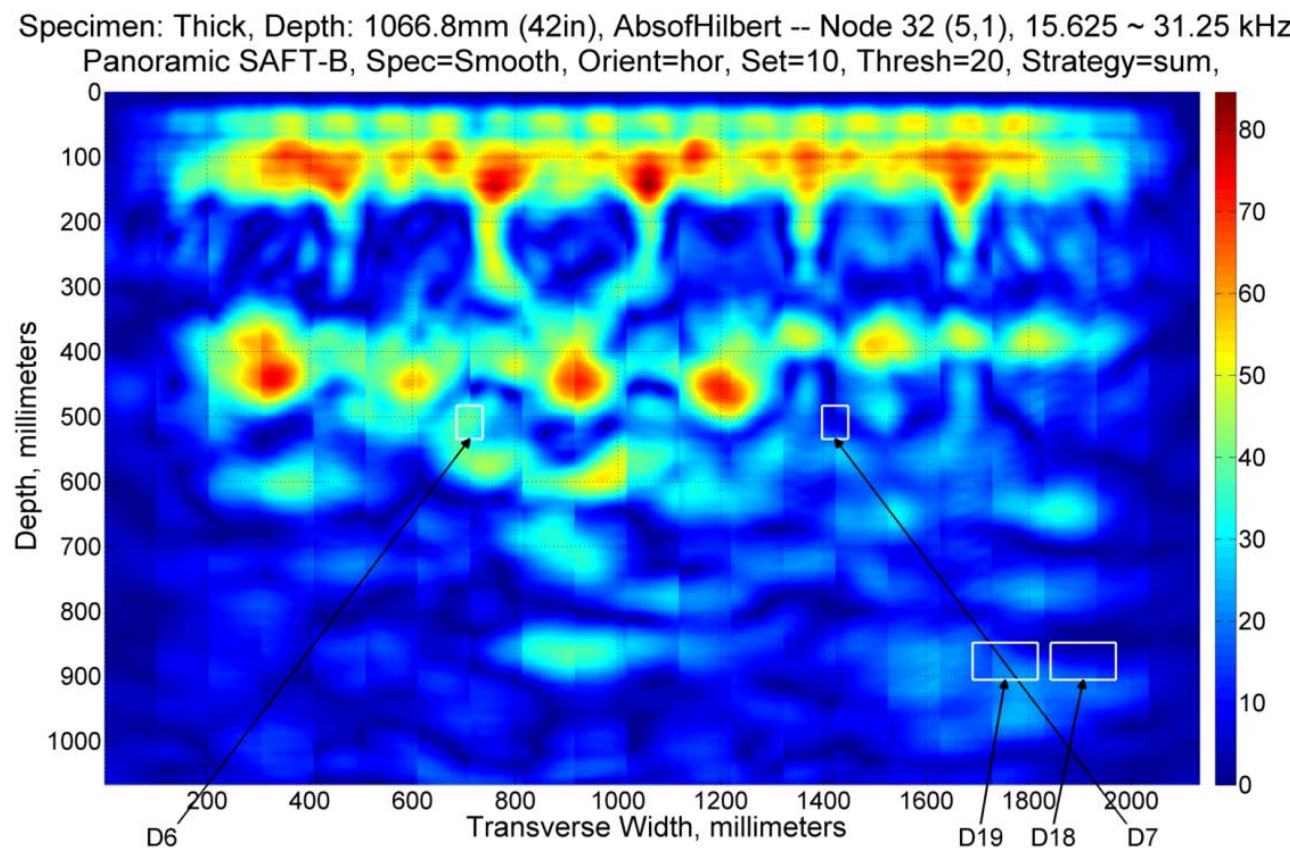

Fig. B. 49. Horizontal Set 10, Node 32.

Specimen: Thick, Depth: 1066.8mm (42in), AbsofHilbert -- Node $34(5,3), 46.875 \sim 62.5 \mathrm{kHz}$ Panoramic SAFT-B, Spec=Smooth, Orient=hor, Set=10, Thresh=20, Strategy=sum,

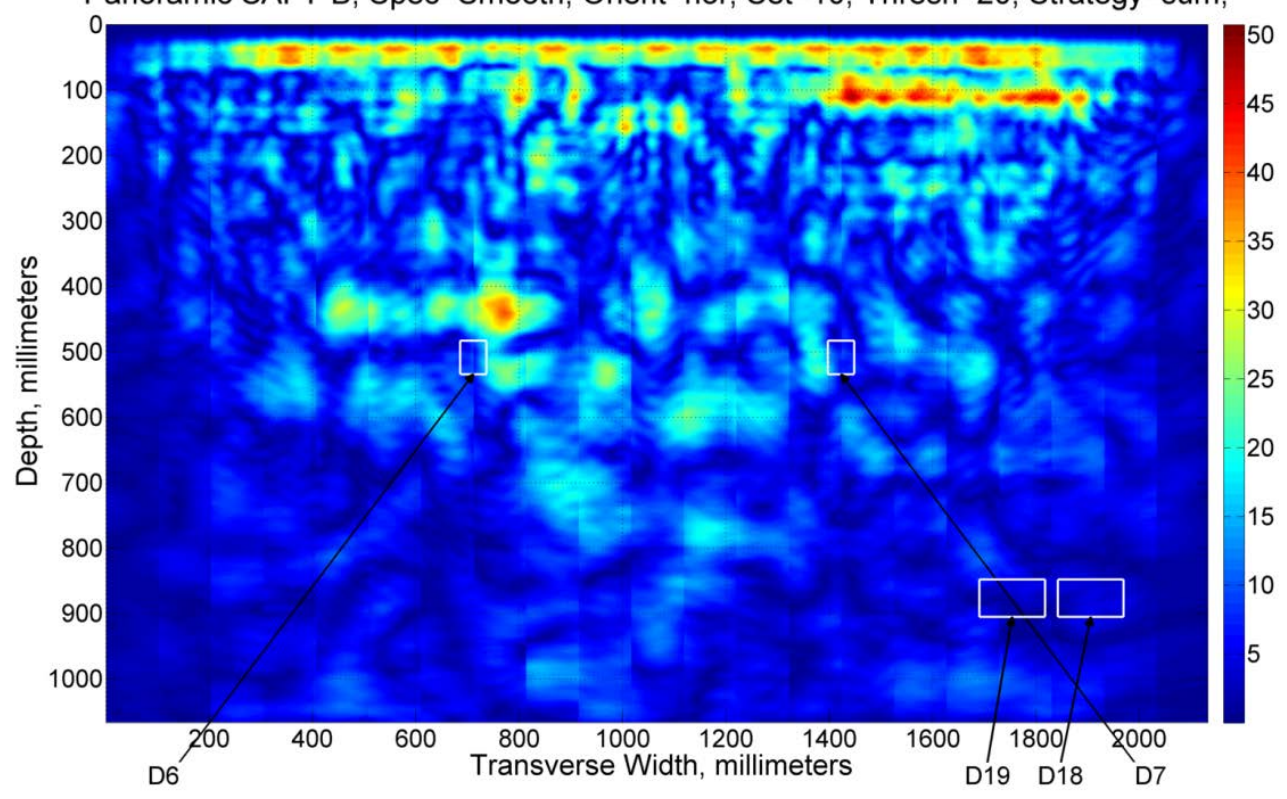

Fig. B. 50. Horizontal Set 10, Node 34. 


\section{SET ELEVEN - DEFECT 8, DEFECT 12}

Specimen: Thick, Depth: 1066.8mm (42in), AbsofHilbert -- Node $0(0,0), 0 \sim 500 \mathrm{kHz}$ Panoramic SAFT-B, Spec=Smooth, Orient=hor, Set=11, Thresh=20, Strategy=sum,

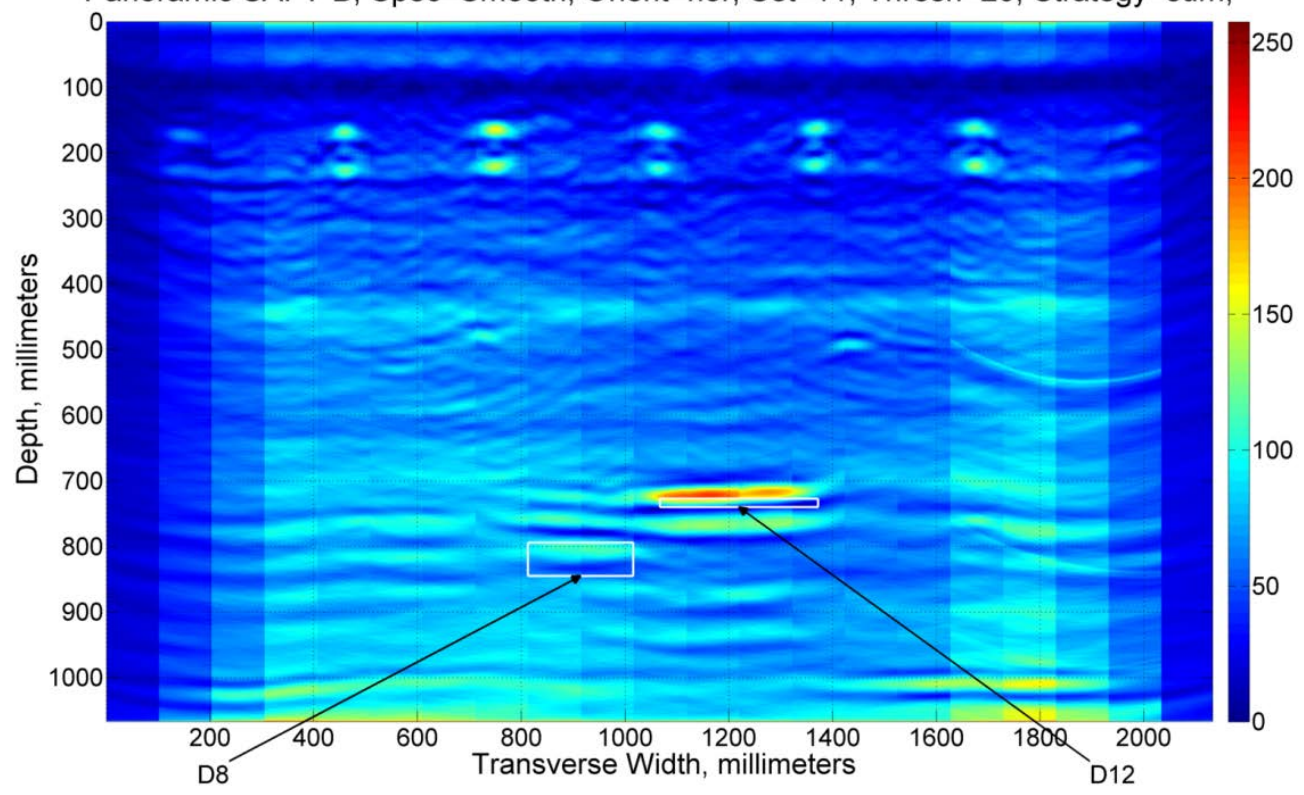

Fig. B. 51. Horizontal Set 11, Node 0.

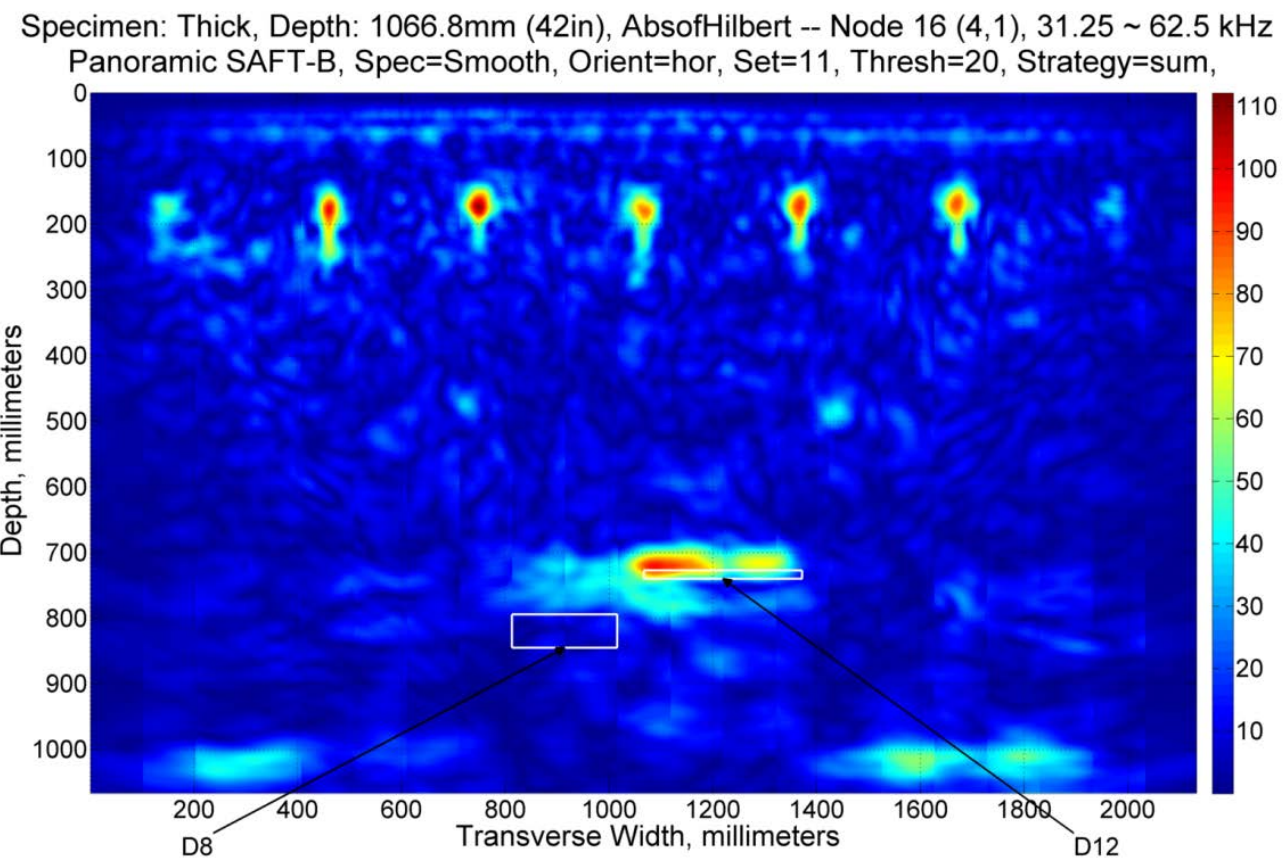

Fig. B. 52. Horizontal Set 11, Node 16. 


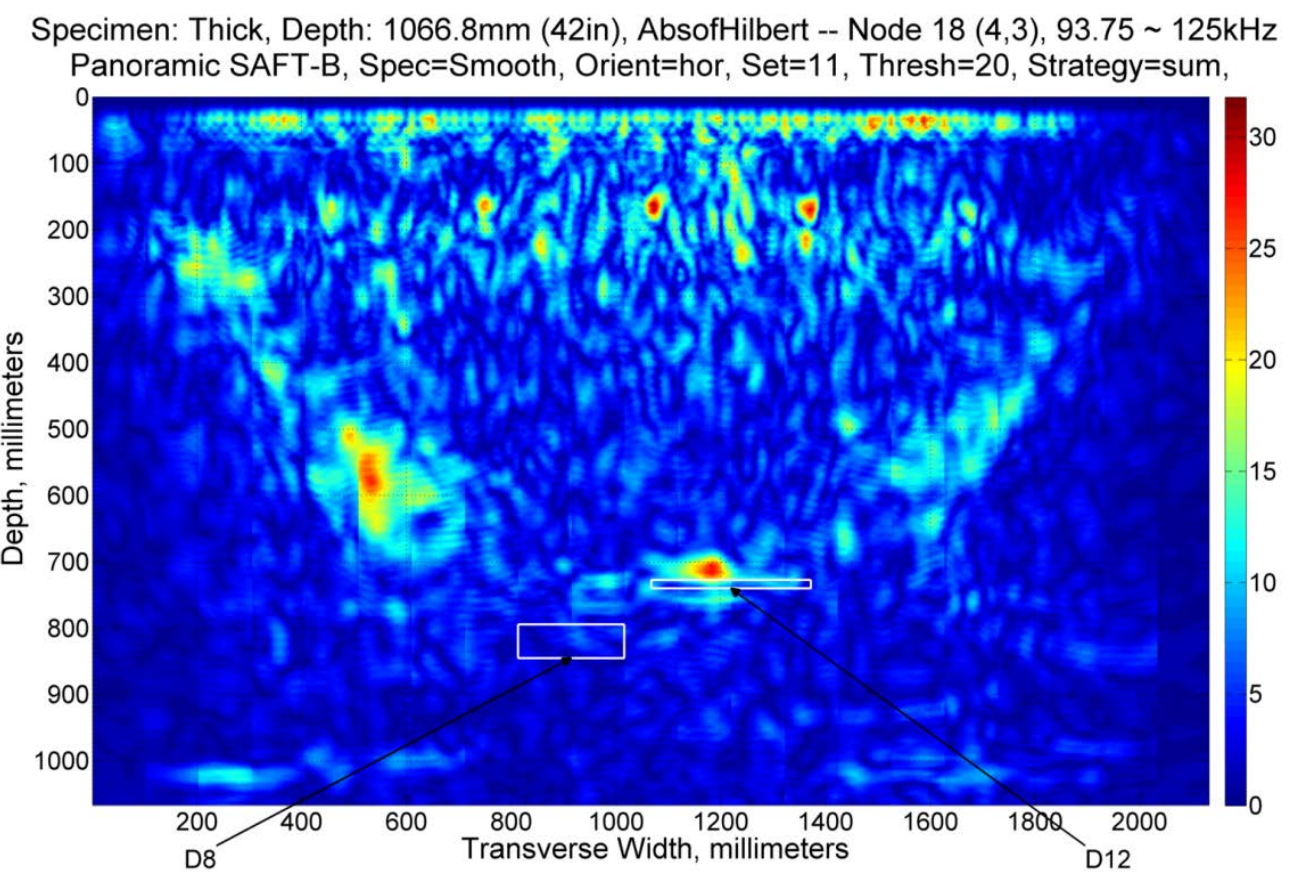

Fig. B. 53. Horizontal Set 11, Node 18.

Specimen: Thick, Depth: 1066.8mm (42in), AbsofHilbert -- Node $32(5,1), 15.625 \sim 31.25 \mathrm{kHz}$ Panoramic SAFT-B, Spec=Smooth, Orient=hor, Set=11, Thresh=20, Strategy=sum,

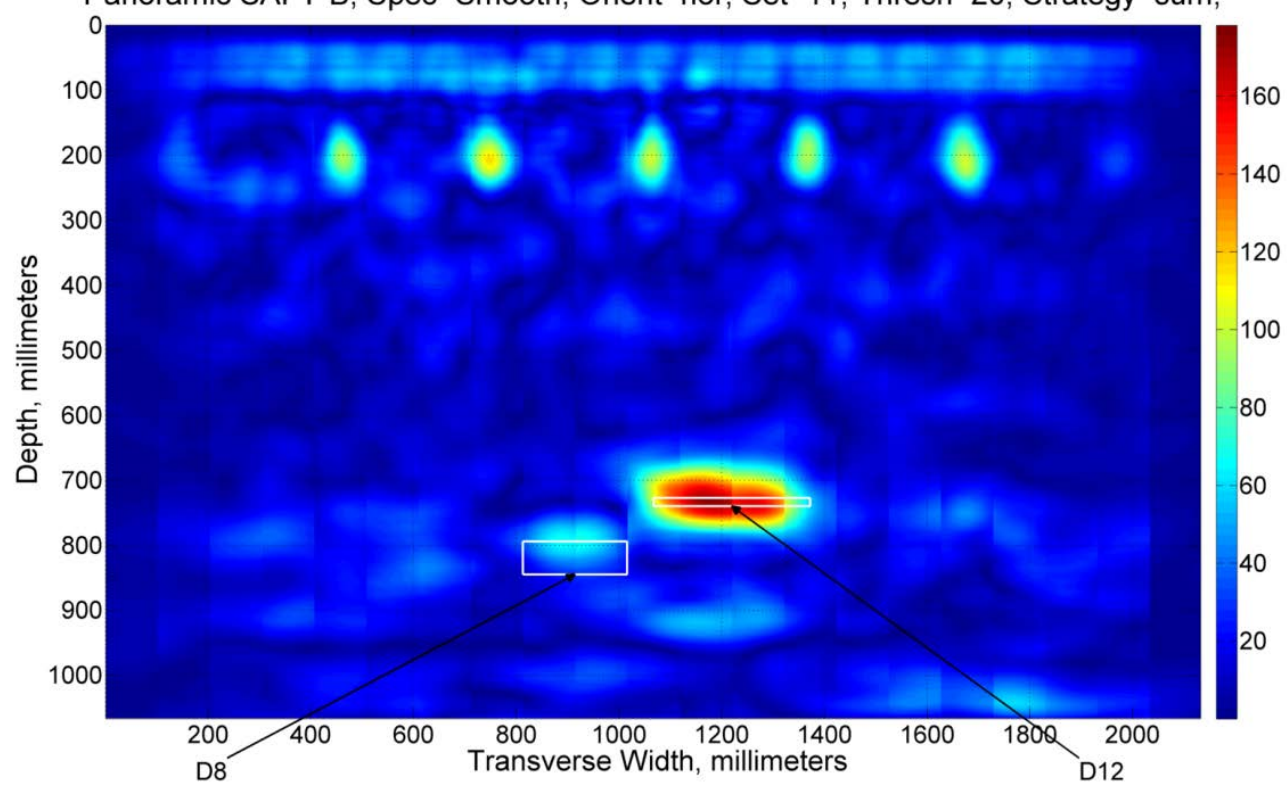

Fig. B. 54. Horizontal Set 11, Node 32. 


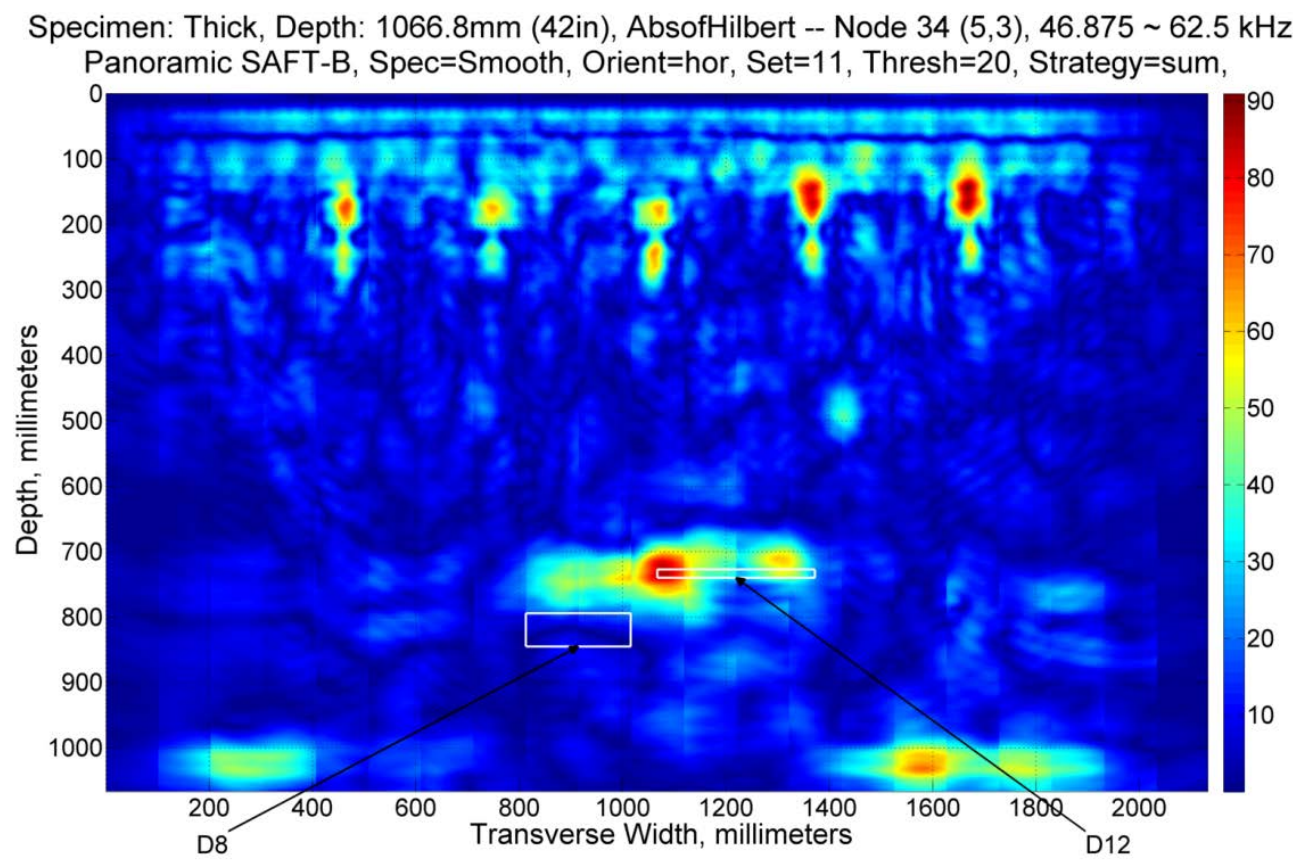

Fig. B. 55. Horizontal Set 11, Node 34.

\section{SET TWELVE - DEFECT 8, DEFECT 12}

Specimen: Thick, Depth: 1066.8mm (42in), AbsofHilbert -- Node $0(0,0), 0 \sim 500 \mathrm{kHz}$ Panoramic SAFT-B, Spec=Smooth, Orient=hor, Set=12, Thresh=20, Strategy=sum,

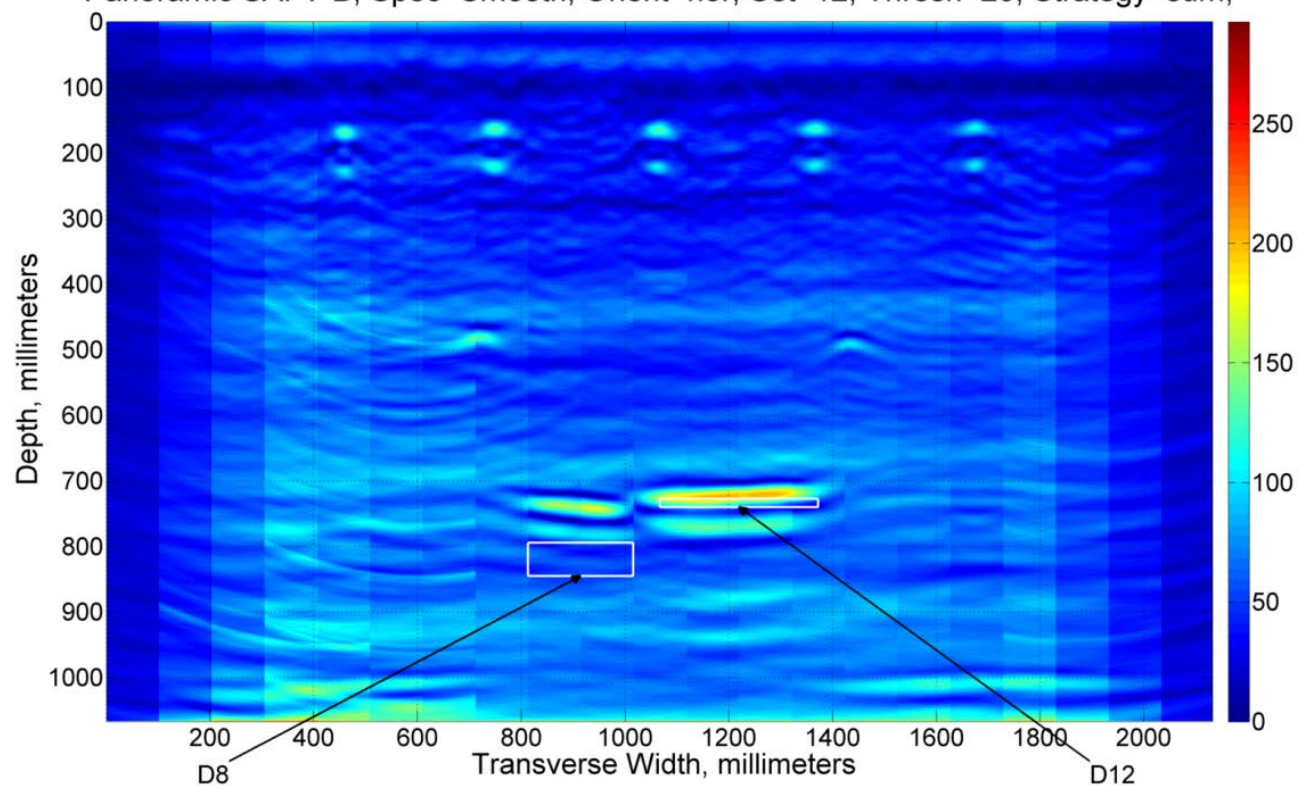

Fig. B. 56. Horizontal Set 12, Node 0. 


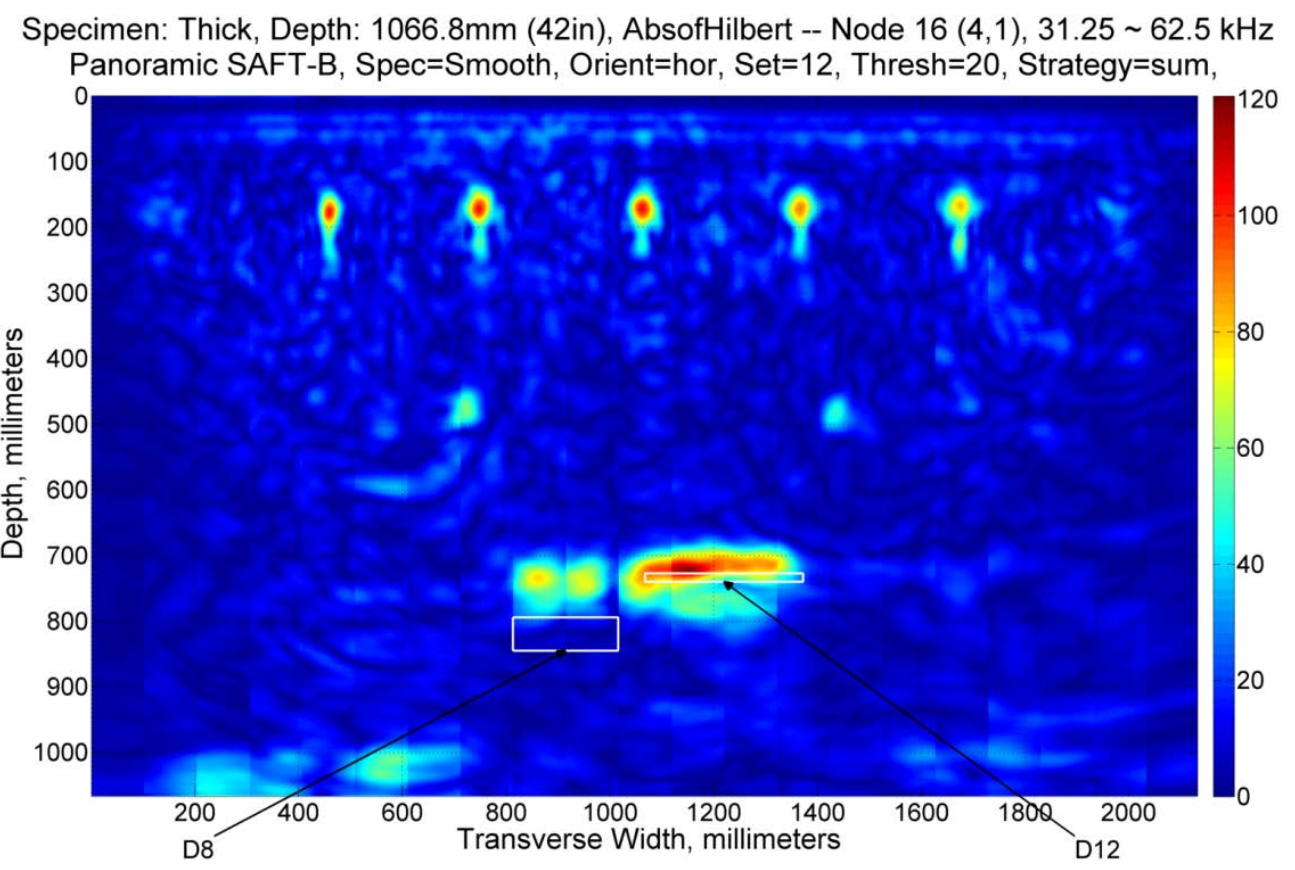

Fig. B. 57. Horizontal Set 12, Node 16.

Specimen: Thick, Depth: 1066.8mm (42in), AbsofHilbert -- Node $18(4,3), 93.75 \sim 125 \mathrm{kHz}$ Panoramic SAFT-B, Spec=Smooth, Orient=hor, Set=12, Thresh=20, Strategy=sum,

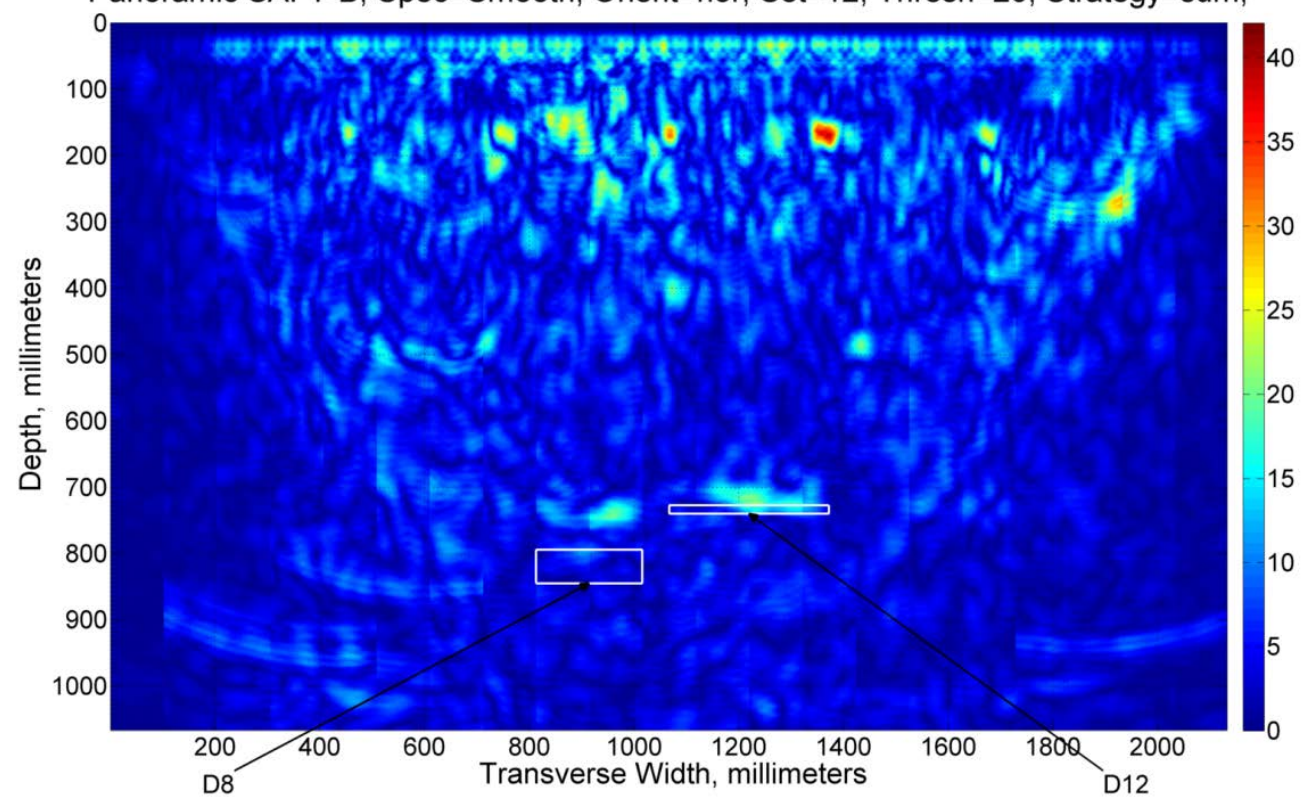

Fig. B. 58. Horizontal Set 12, Node 18. 


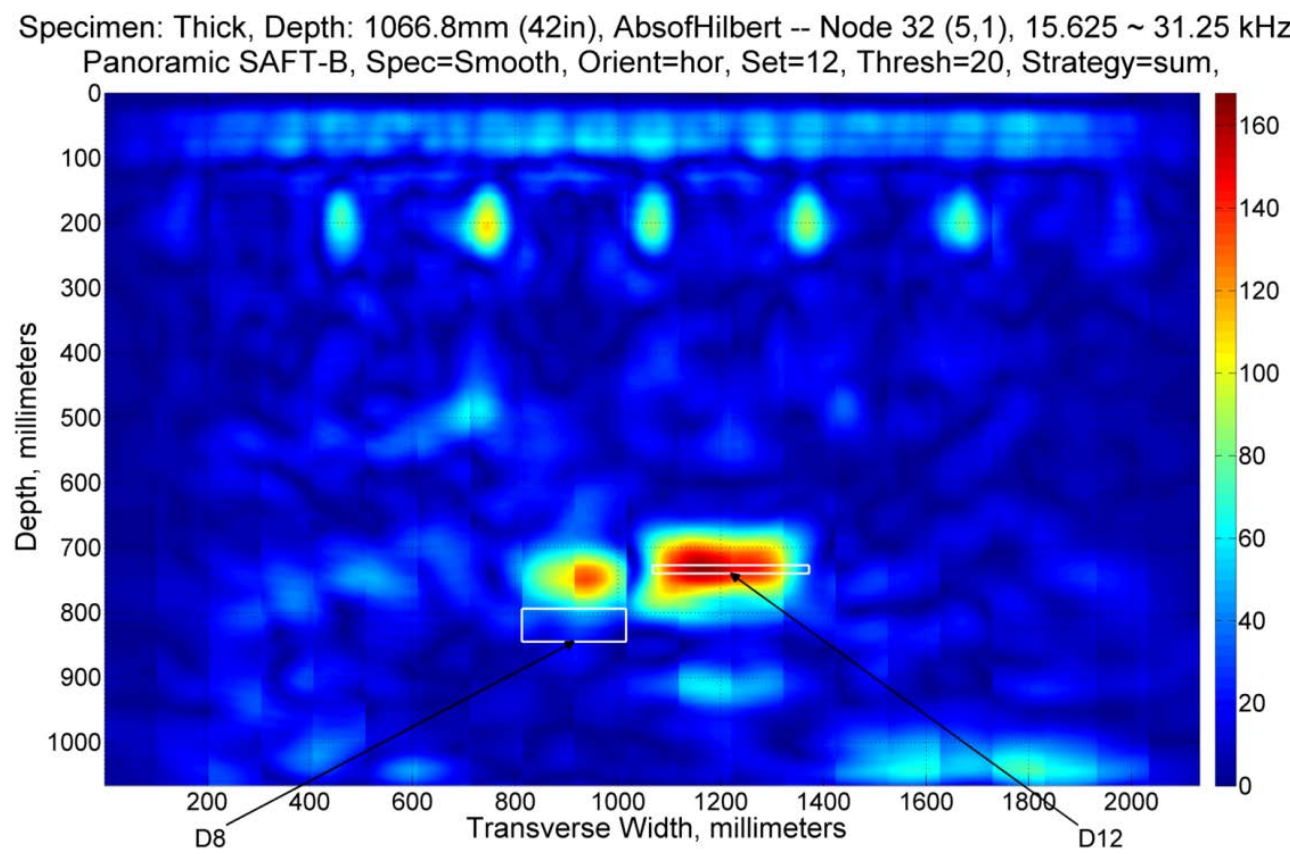

Fig. B. 59. Horizontal Set 12, Node 32.

Specimen: Thick, Depth: 1066.8mm (42in), AbsofHilbert -- Node 34 (5,3), $46.875 \sim 62.5 \mathrm{kHz}$ Panoramic SAFT-B, Spec=Smooth, Orient=hor, Set=12, Thresh=20, Strategy=sum,

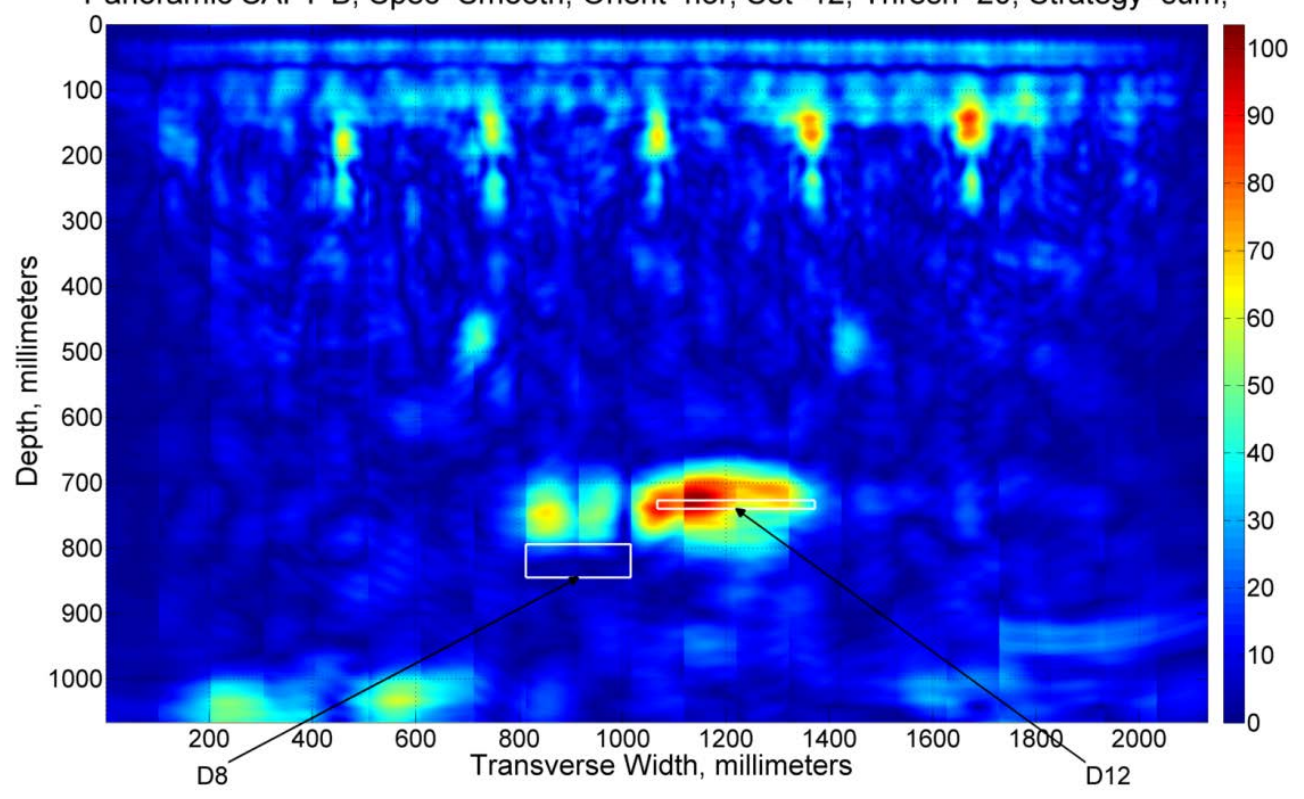

Fig. B. 60. Horizontal Set 12, Node 34. 


\section{SET THIRTEEN - DEFECT 20}

Specimen: Thick, Depth: 1066.8mm (42in), AbsofHilbert -- Node $0(0,0), 0 \sim 500 \mathrm{kHz}$ Panoramic SAFT-B, Spec=Smooth, Orient=hor, Set=13, Thresh=20, Strategy=sum,

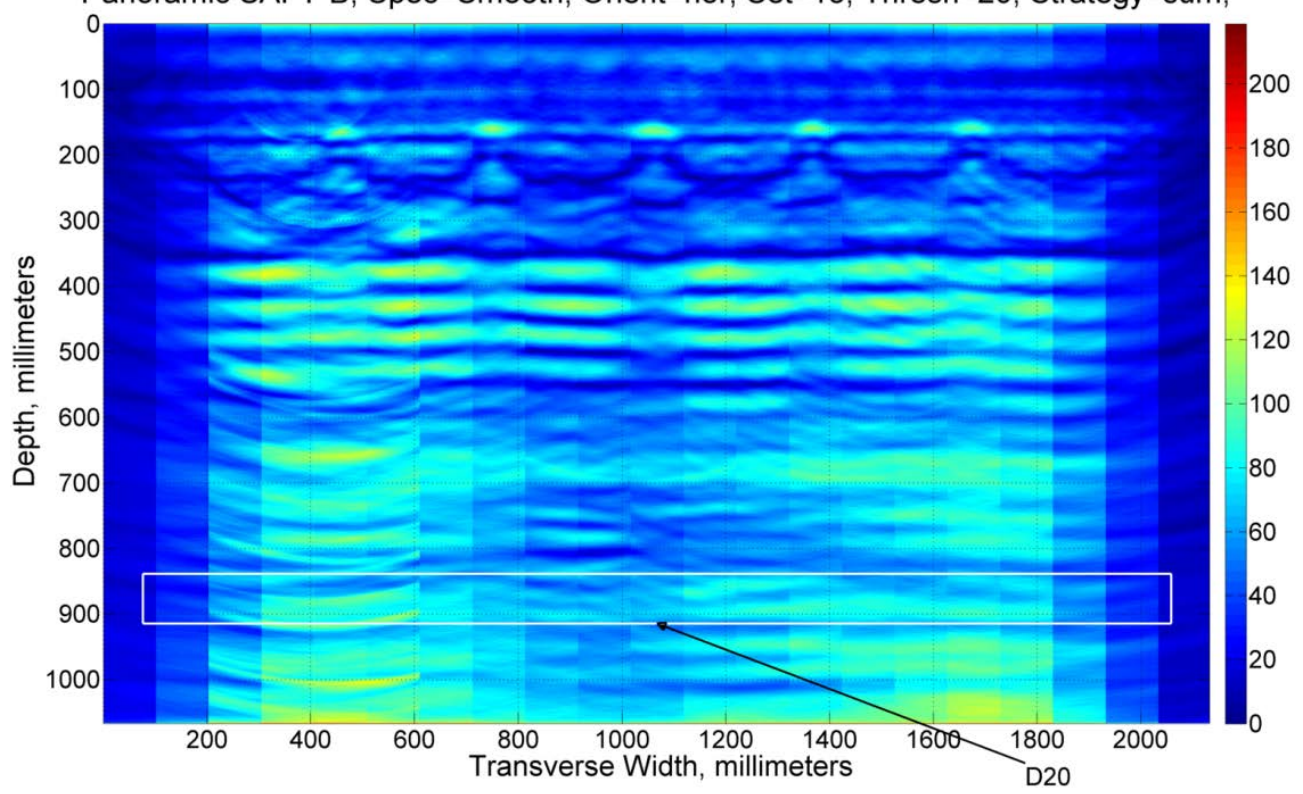

Fig. B. 61. Horizontal Set 13, Node 0.

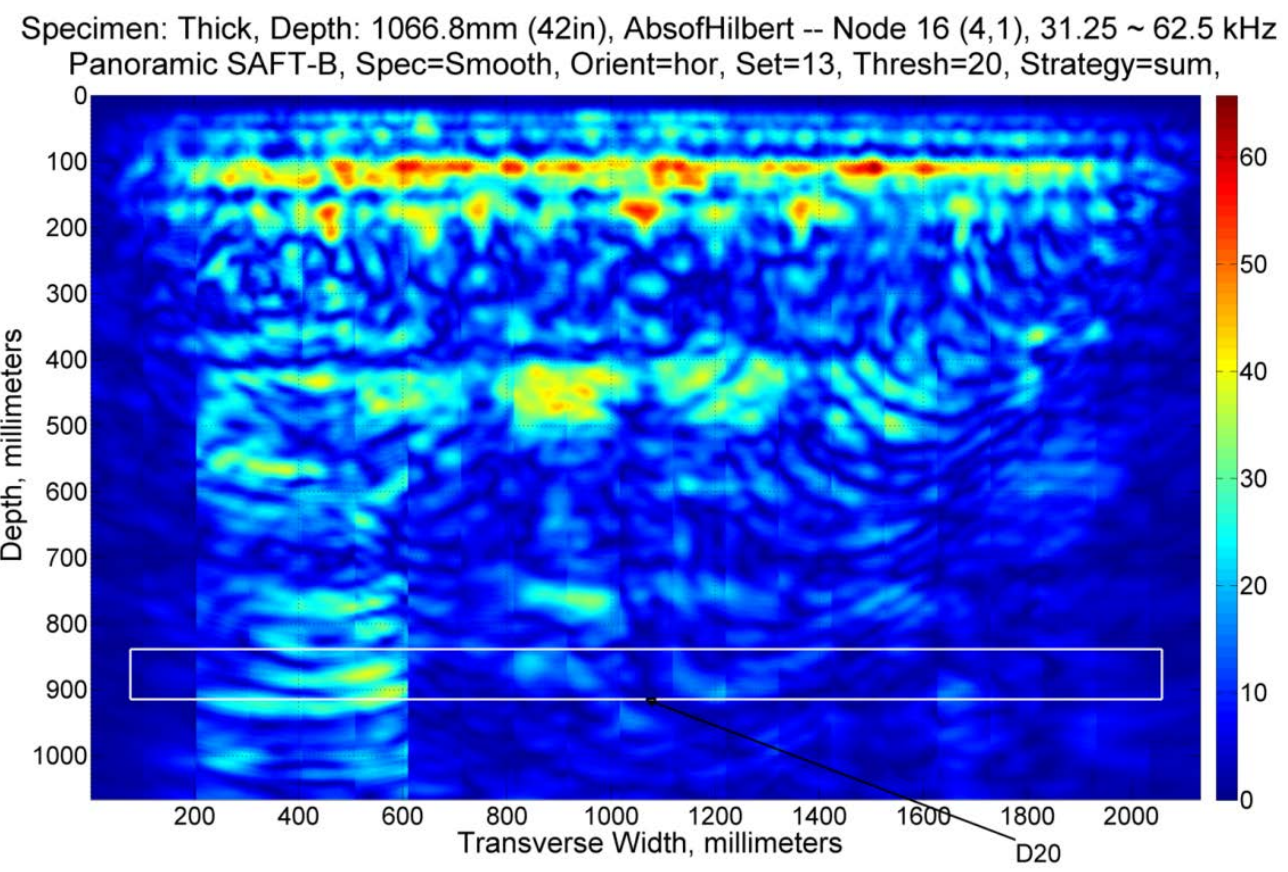

Fig. B. 62. Horizontal Set 13, Node 16. 


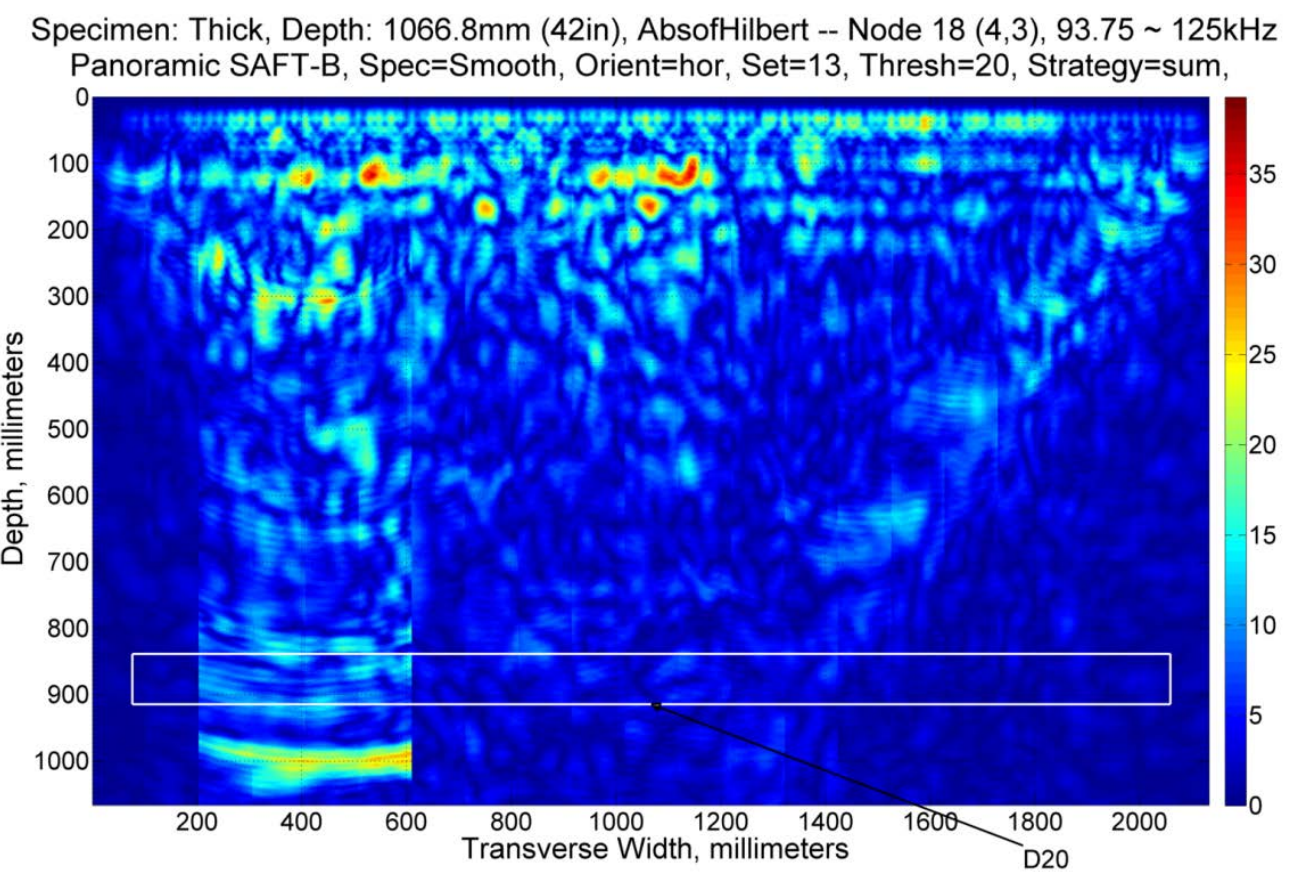

Fig. B. 63. Horizontal Set 13, Node 18.

Specimen: Thick, Depth: 1066.8mm (42in), AbsofHilbert -- Node $32(5,1), 15.625 \sim 31.25 \mathrm{kHz}$ Panoramic SAFT-B, Spec=Smooth, Orient=hor, Set=13, Thresh=20, Strategy=sum,

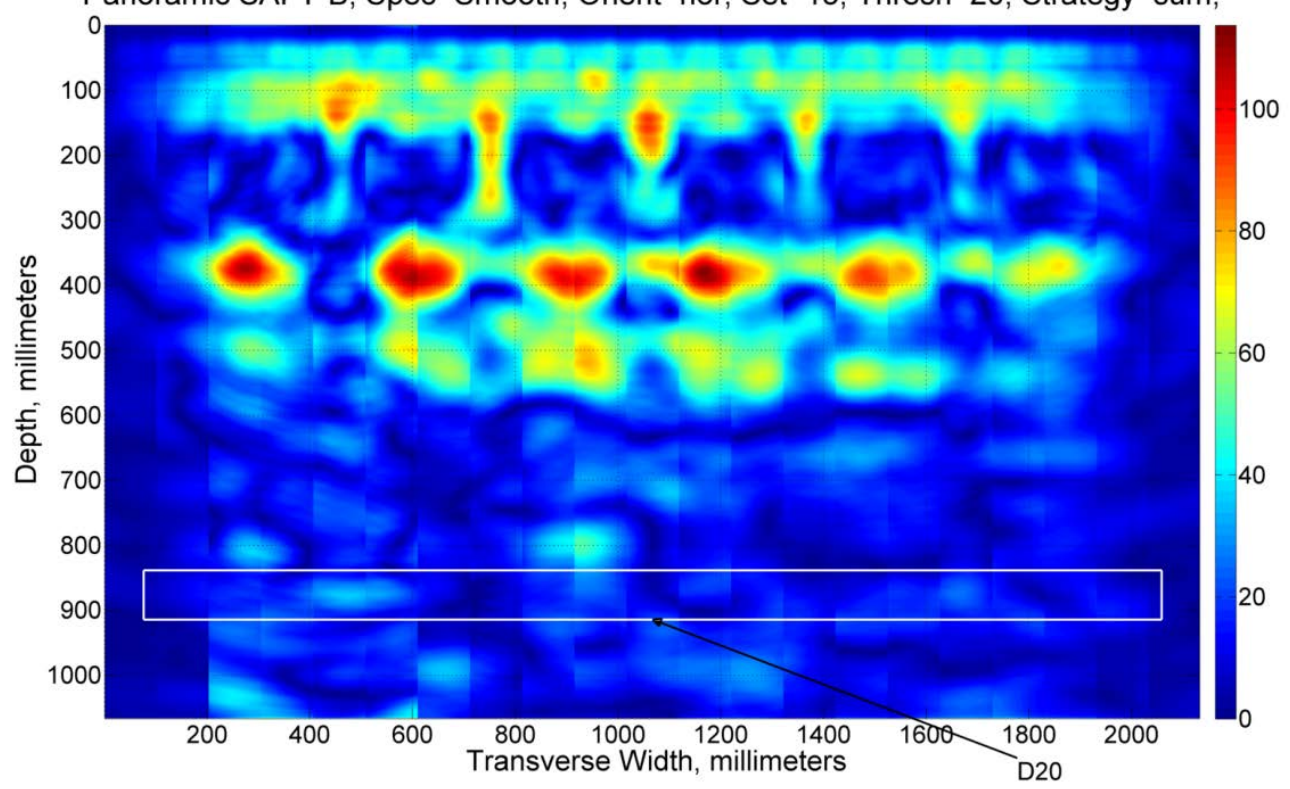

Fig. B. 64 Horizontal Set 13, Node 32. 


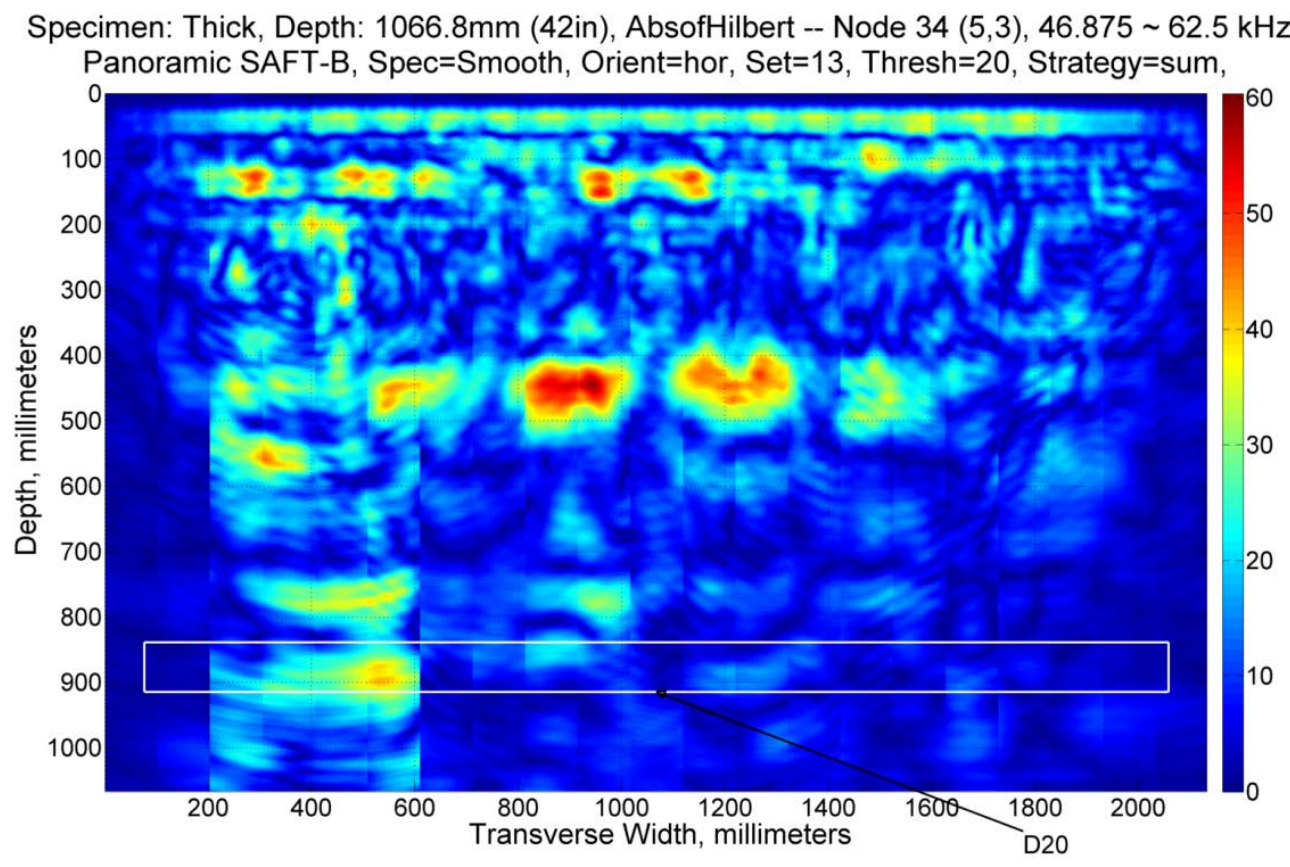

Fig. B. 65. Horizontal Set 13, Node 34.

\section{SET FOURTEEN - DEFECT 2}

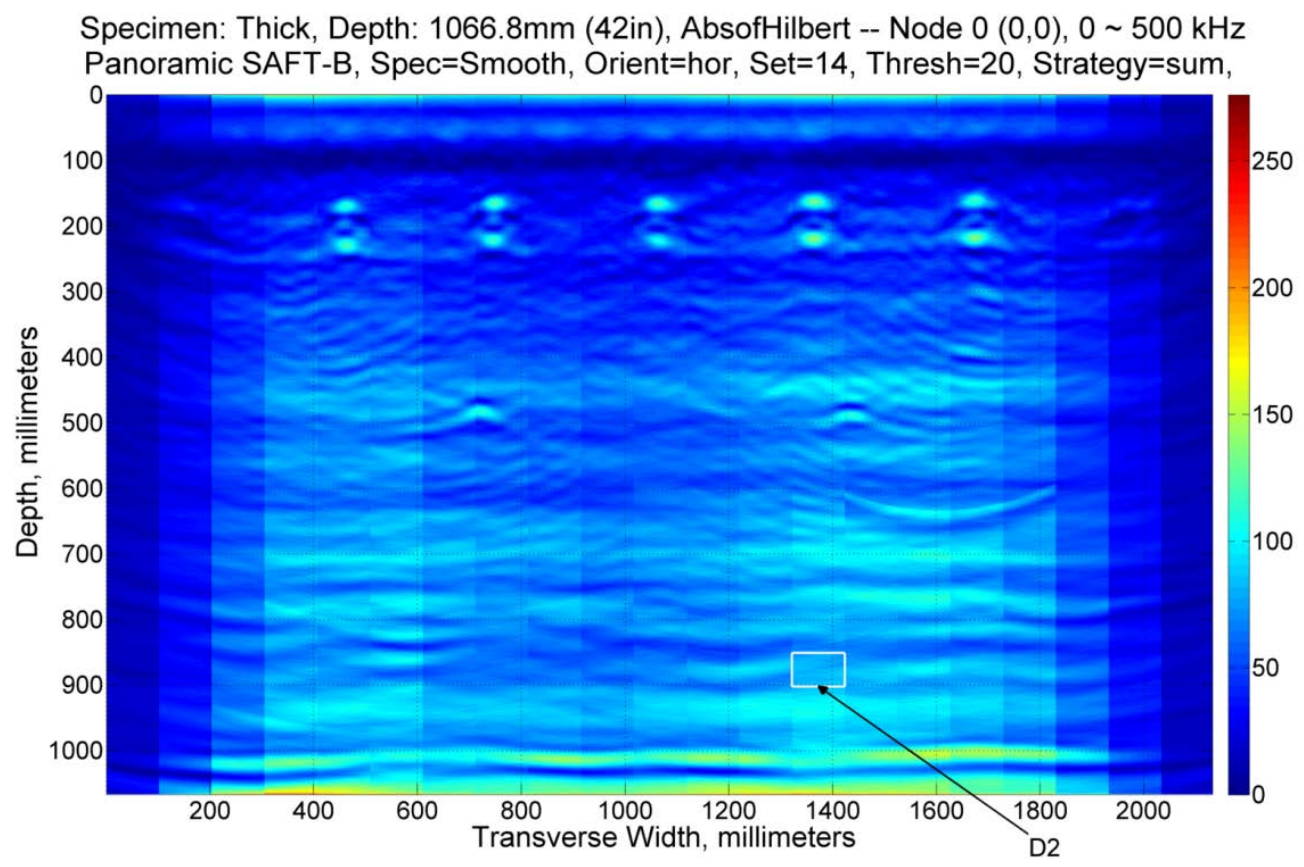

Fig. B. 66. Horizontal Set 14, Node 0. 


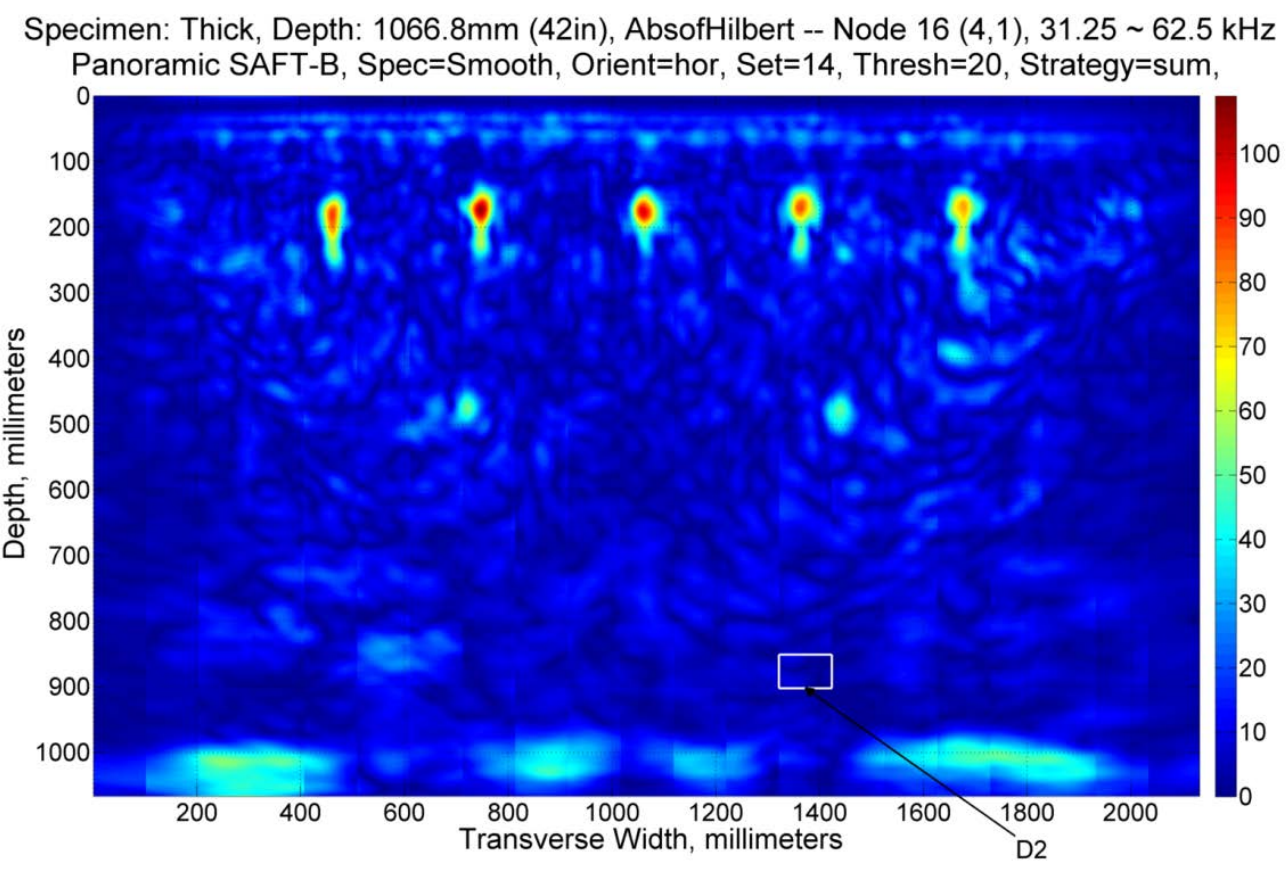

Fig. B. 67. Horizontal Set 14, Node 16.

Specimen: Thick, Depth: 1066.8mm (42in), AbsofHilbert -- Node $18(4,3), 93.75 \sim 125 \mathrm{kHz}$ Panoramic SAFT-B, Spec=Smooth, Orient=hor, Set=14, Thresh=20, Strategy=sum,

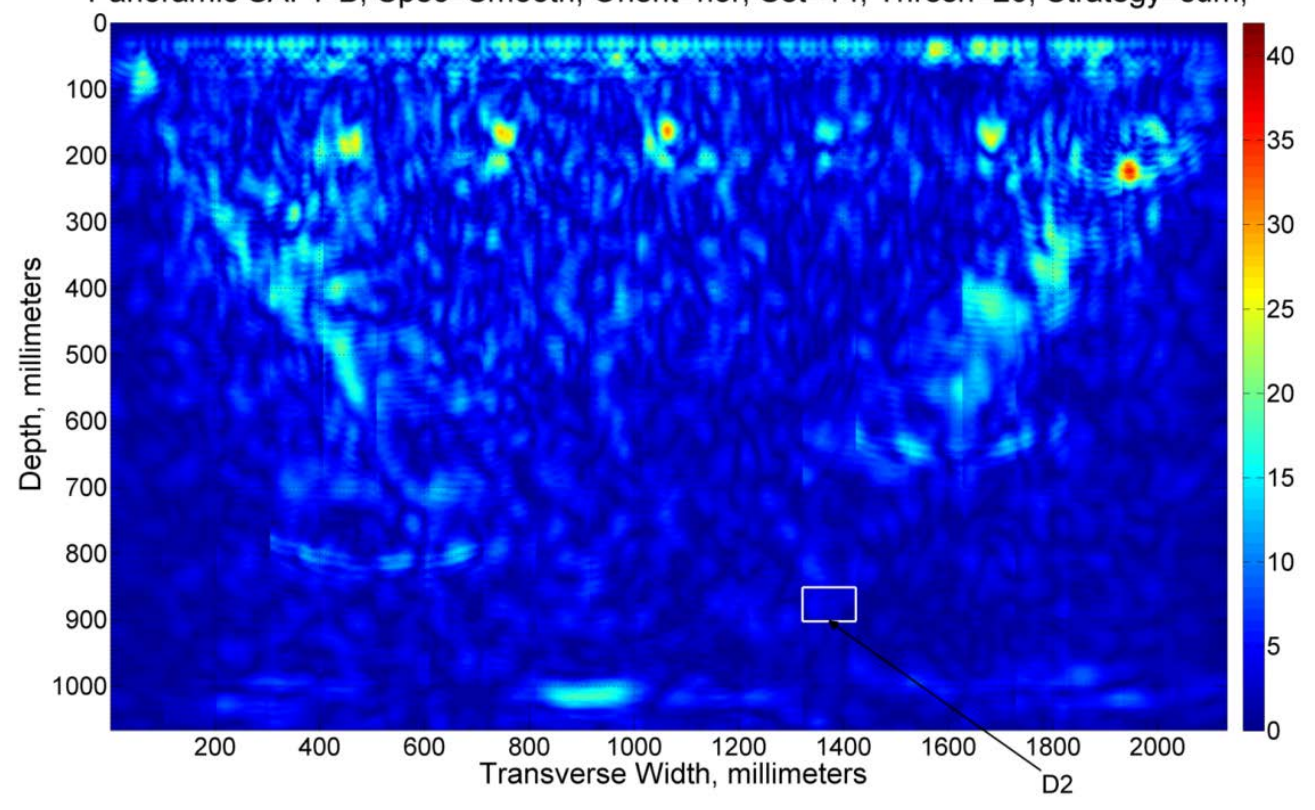

Fig. B. 68. Horizontal Set 14, Node 18. 


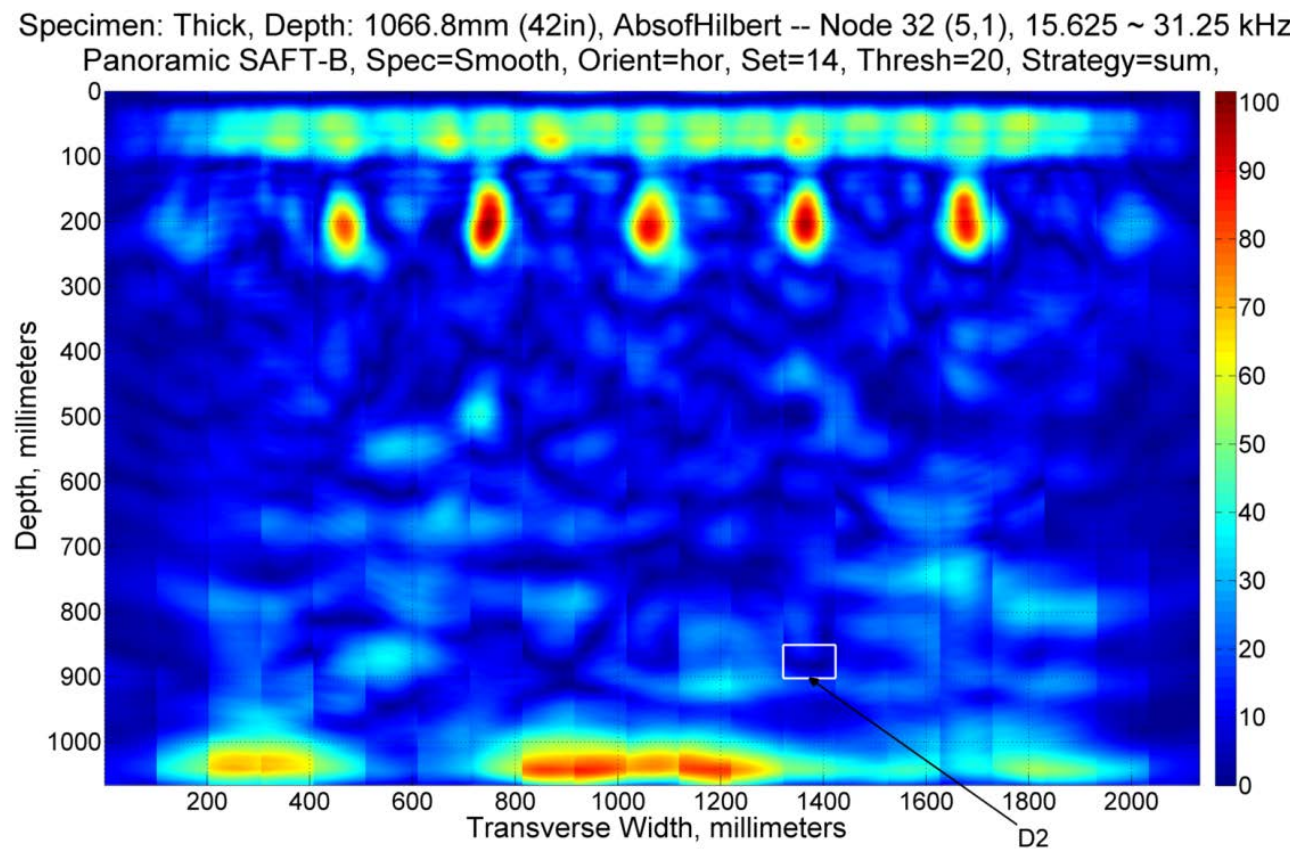

Fig. B. 69. Horizontal Set 14, Node 32.

Specimen: Thick, Depth: 1066.8mm (42in), AbsofHilbert -- Node 34 (5,3), $46.875 \sim 62.5 \mathrm{kHz}$ Panoramic SAFT-B, Spec=Smooth, Orient=hor, Set=14, Thresh=20, Strategy=sum,

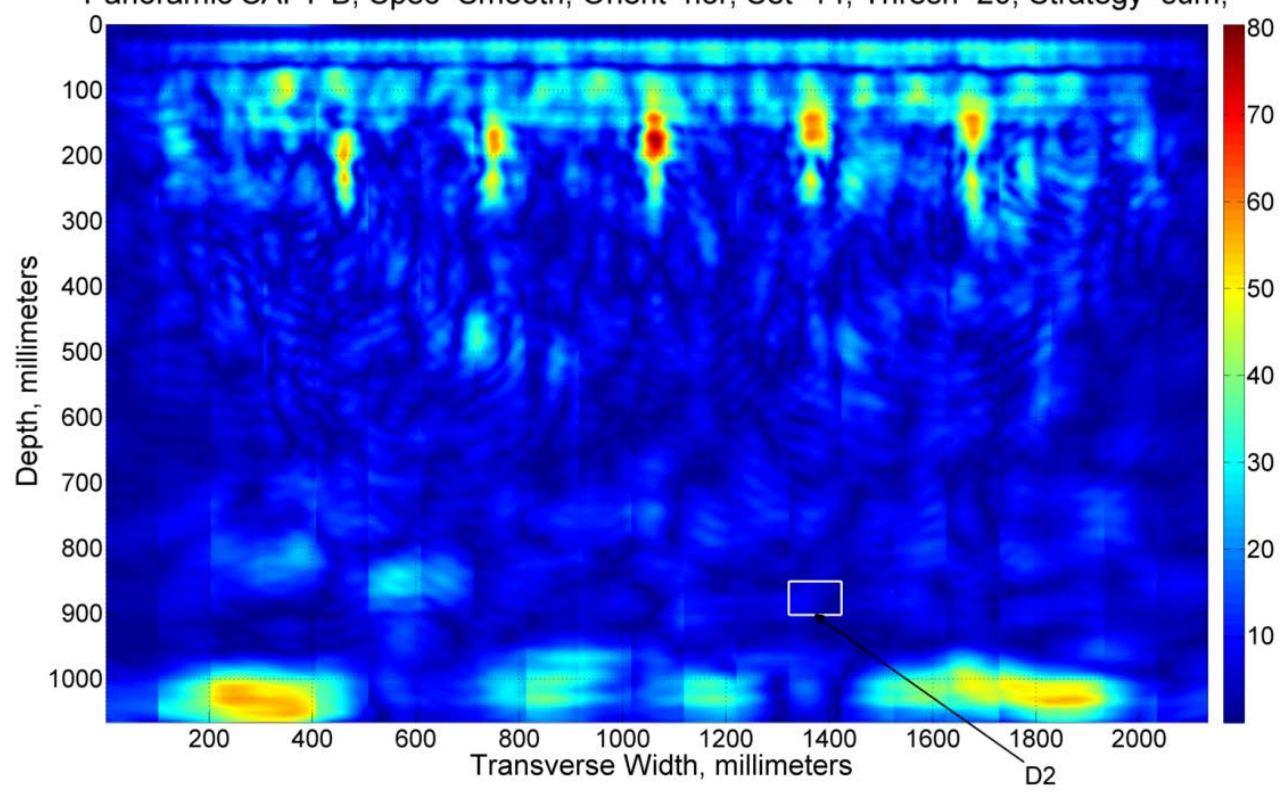

Fig. B. 70. Horizontal Set 14, Node 34. 


\section{SET FIFTEEN - DEFECT 2, DEFECT 15}

Specimen: Thick, Depth: 1066.8mm (42in), AbsofHilbert -- Node $0(0,0), 0 \sim 500 \mathrm{kHz}$ Panoramic SAFT-B, Spec=Smooth, Orient=hor, Set=15, Thresh=20, Strategy=sum,

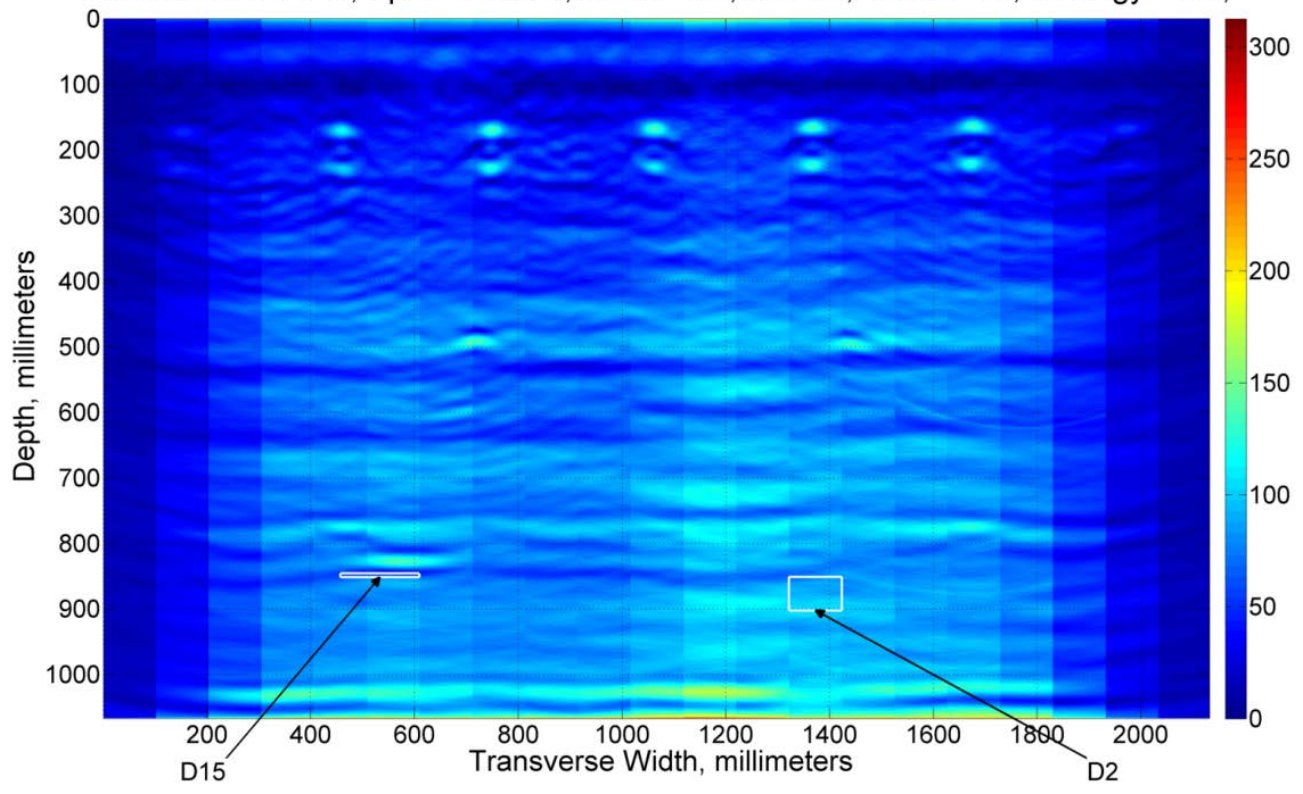

Fig. B. 71. Horizontal Set 15, Node 0.

Specimen: Thick, Depth: 1066.8mm (42in), AbsofHilbert -- Node $16(4,1), 31.25 \sim 62.5 \mathrm{kHz}$ Panoramic SAFT-B, Spec=Smooth, Orient=hor, Set=15, Thresh=20, Strategy=sum,

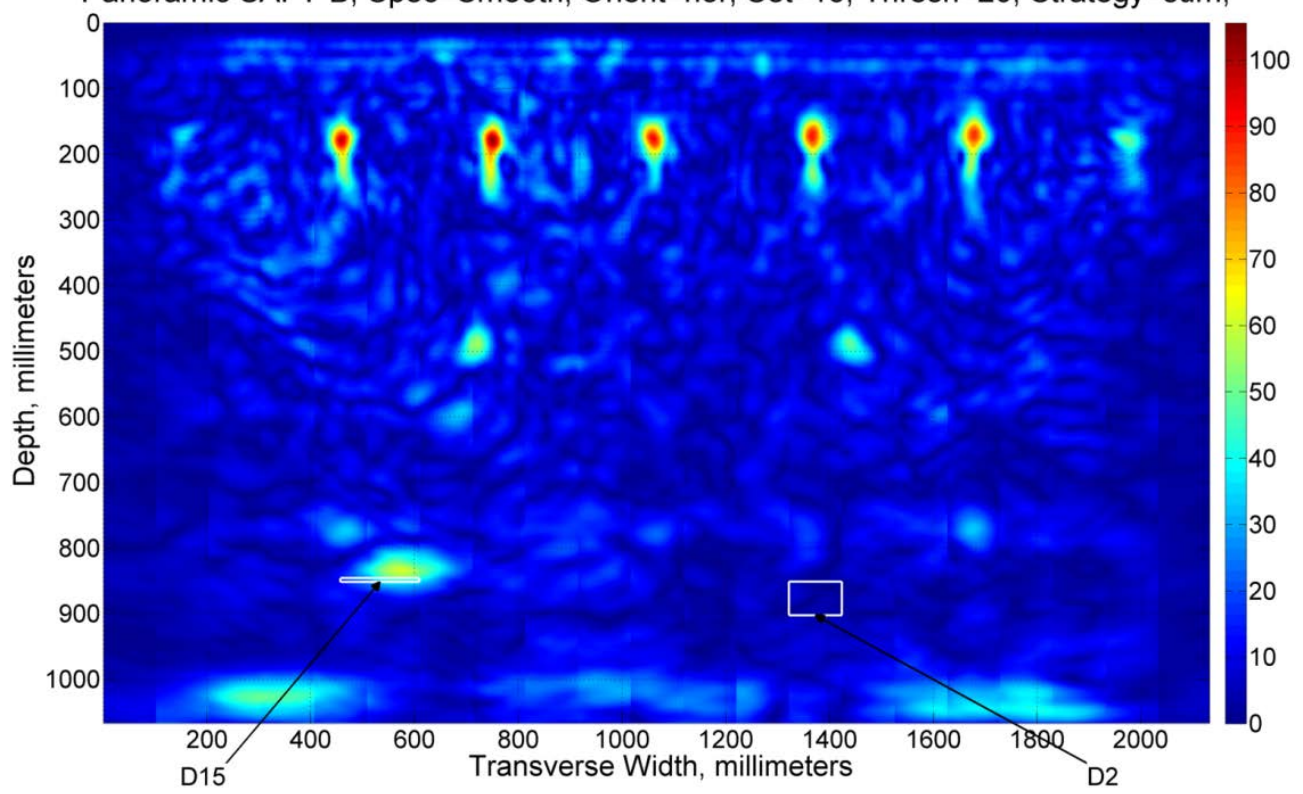

Fig. B. 72. Horizontal Set 15, Node 16. 


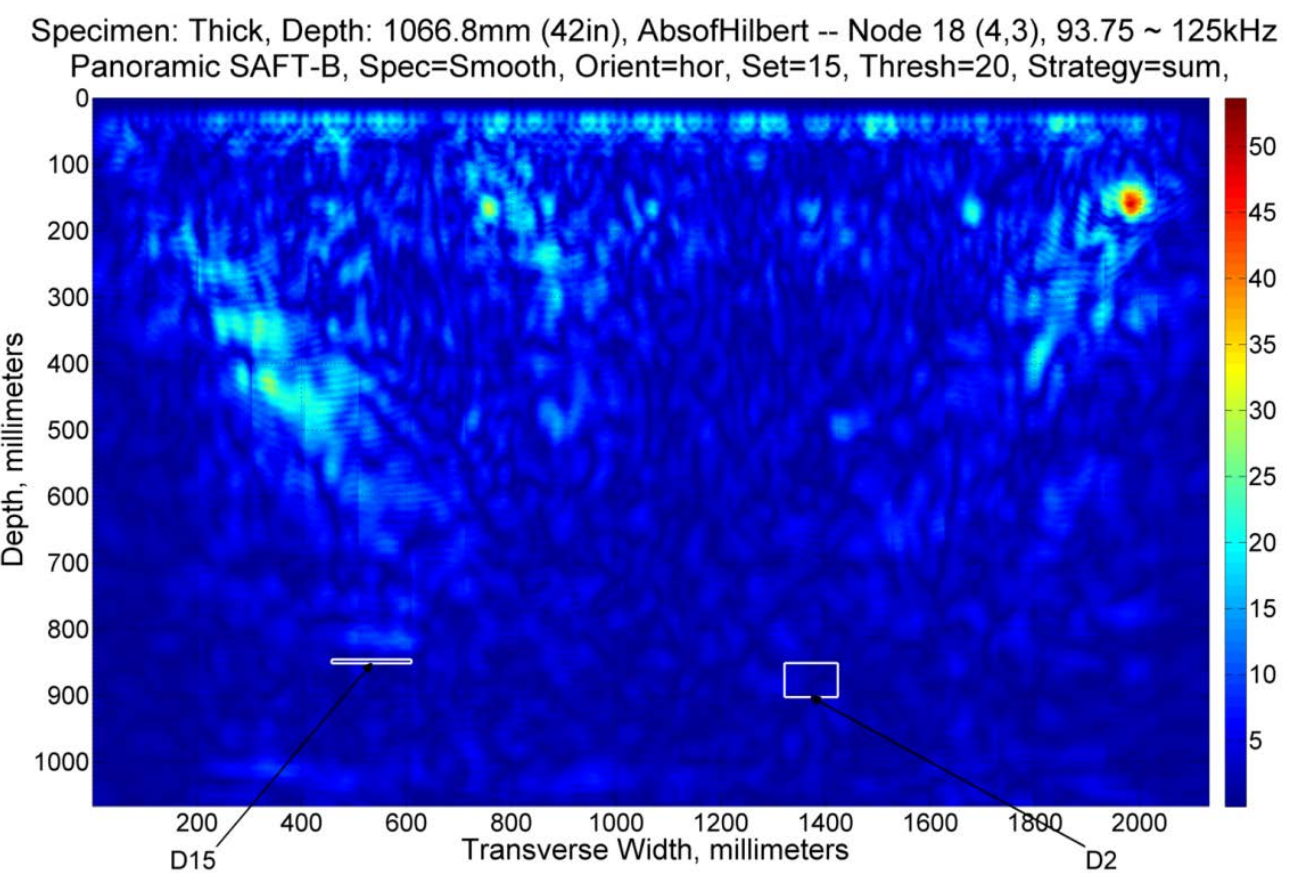

Fig. B. 73. Horizontal Set 15, Node 18.

Specimen: Thick, Depth: 1066.8mm (42in), AbsofHilbert -- Node $32(5,1), 15.625 \sim 31.25 \mathrm{kHz}$ Panoramic SAFT-B, Spec=Smooth, Orient=hor, Set=15, Thresh=20, Strategy=sum,

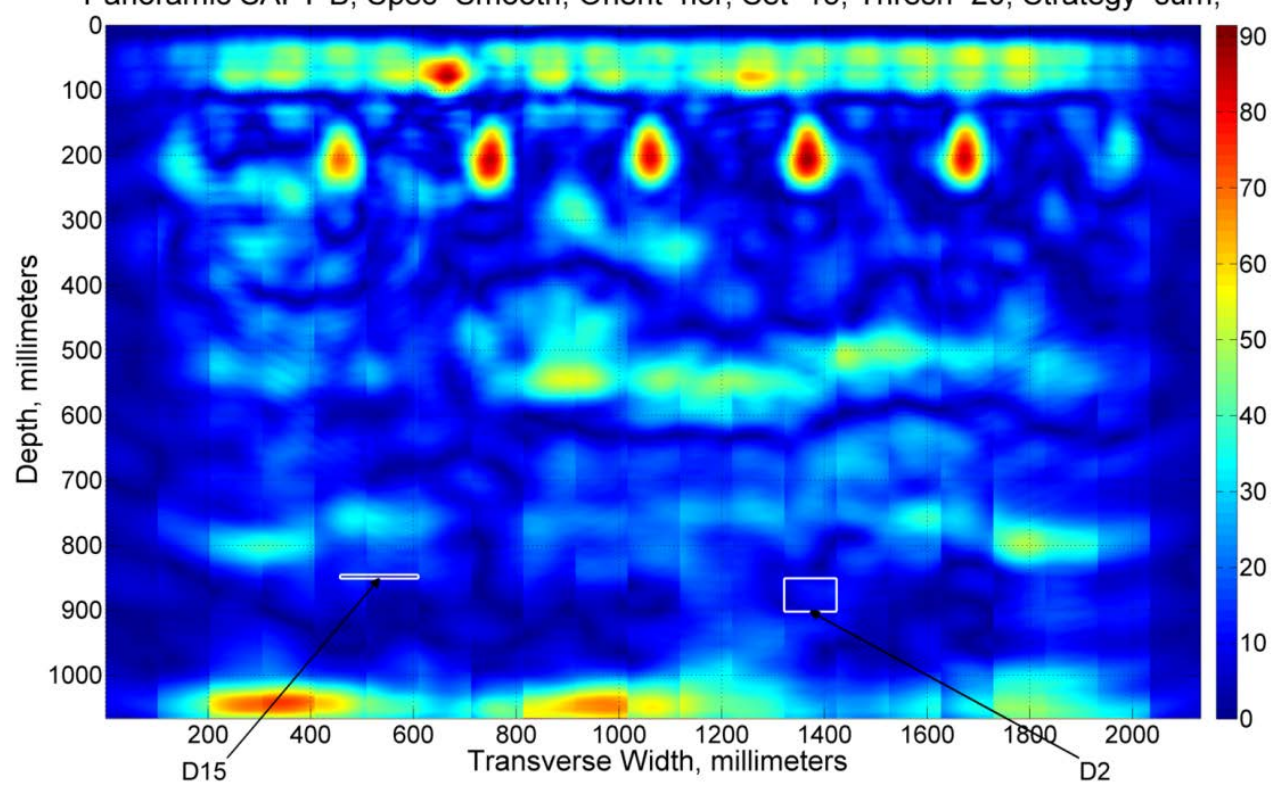

Fig. B. 74. Horizontal Set 15, Node 32. 


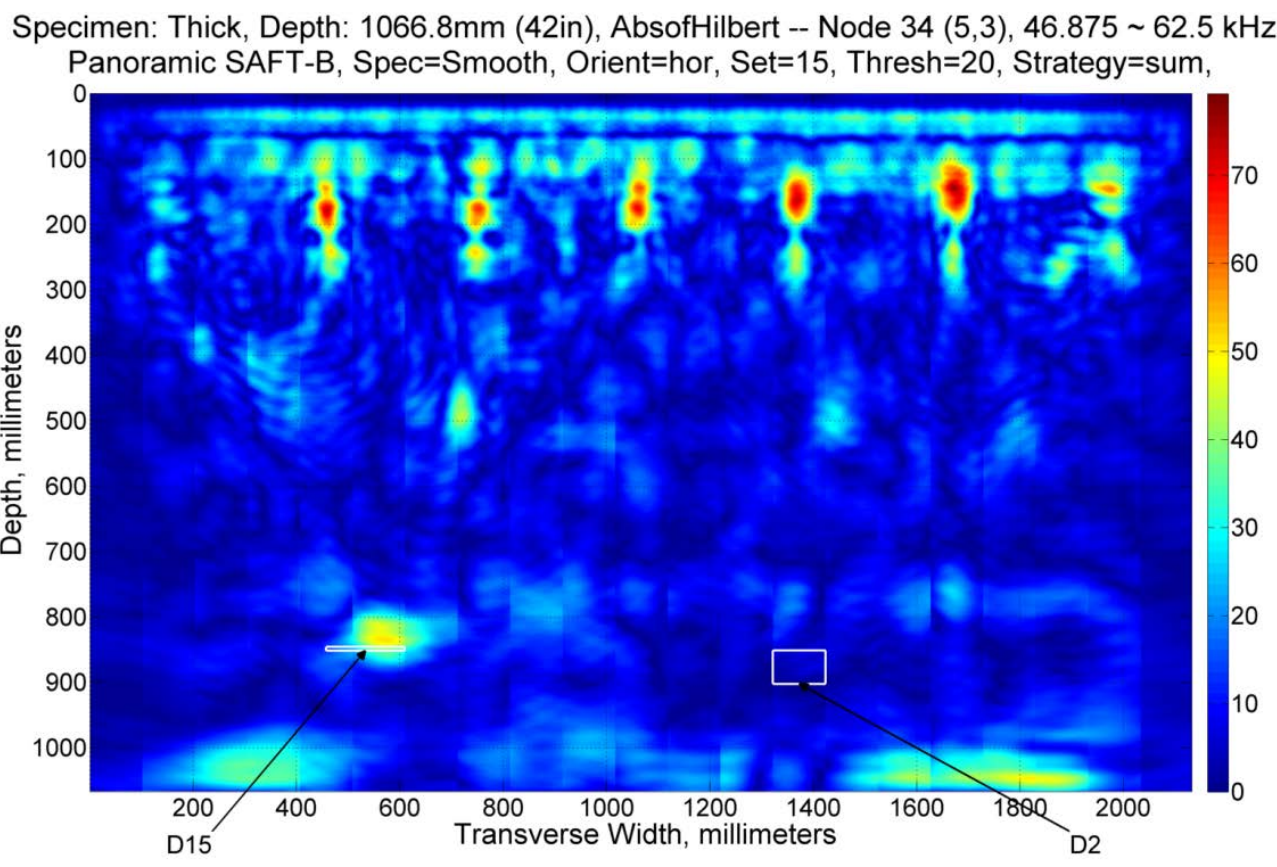

Fig. B. 75. Horizontal Set 15, Node 34.

\section{SET SIXTEEN - DEFECT 4}

Specimen: Thick, Depth: 1066.8mm (42in), AbsofHilbert -- Node $0(0,0), 0 \sim 500 \mathrm{kHz}$ Panoramic SAFT-B, Spec=Smooth, Orient=hor, Set=16, Thresh=20, Strategy=sum,

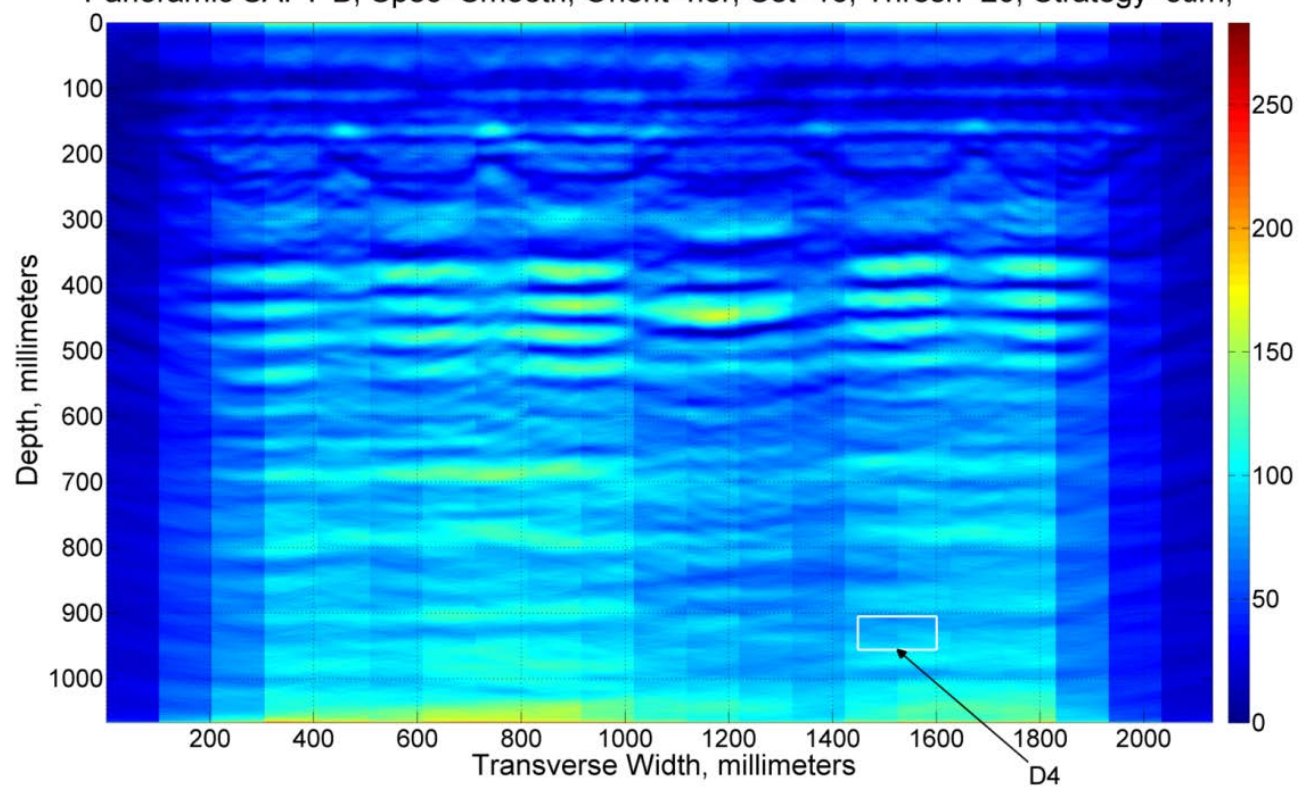

Fig. B. 76. Horizontal Set 16, Node 0. 


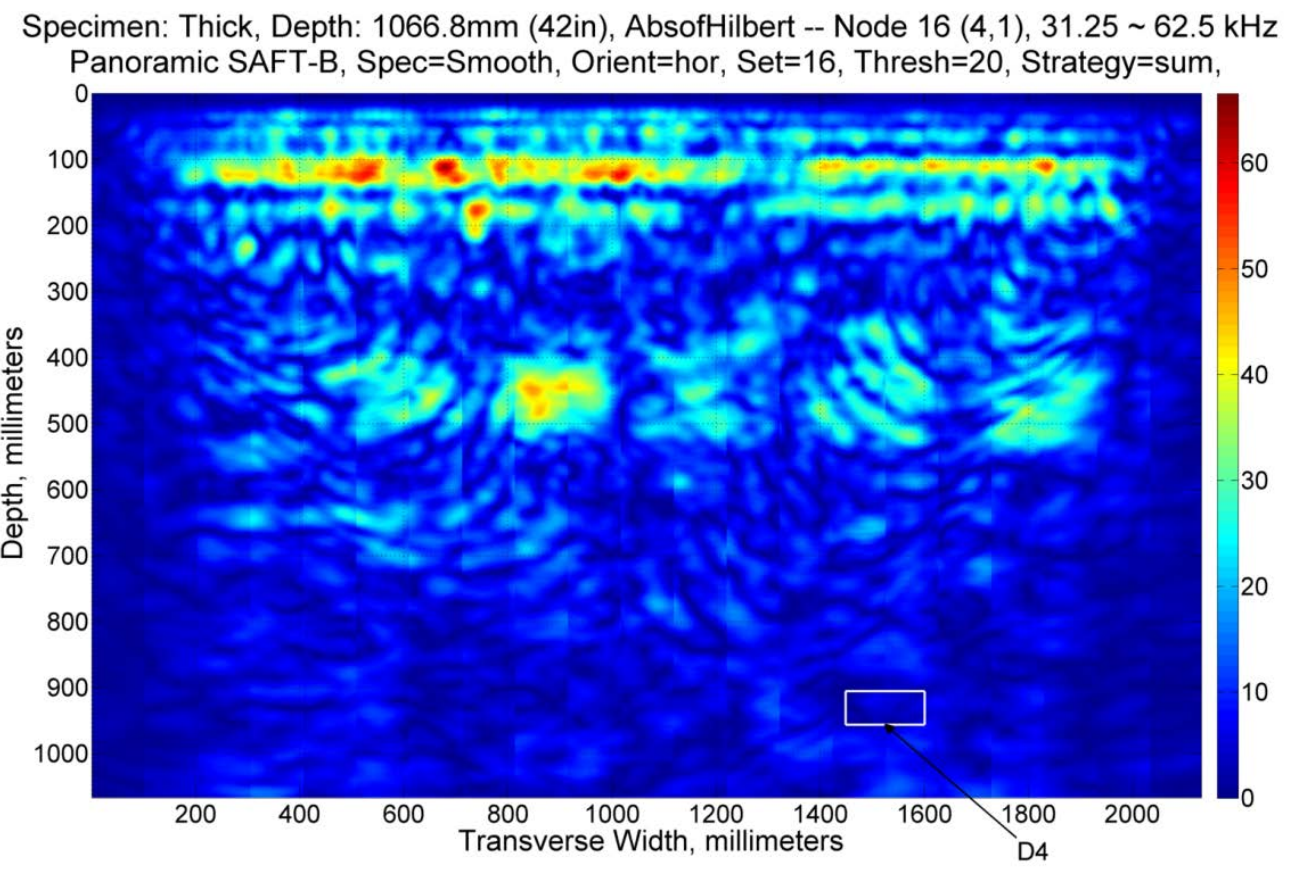

Fig. B. 77. Horizontal Set 16, Node 16.

Specimen: Thick, Depth: 1066.8mm (42in), AbsofHilbert -- Node $18(4,3), 93.75 \sim 125 \mathrm{kHz}$ Panoramic SAFT-B, Spec=Smooth, Orient=hor, Set=16, Thresh=20, Strategy=sum,

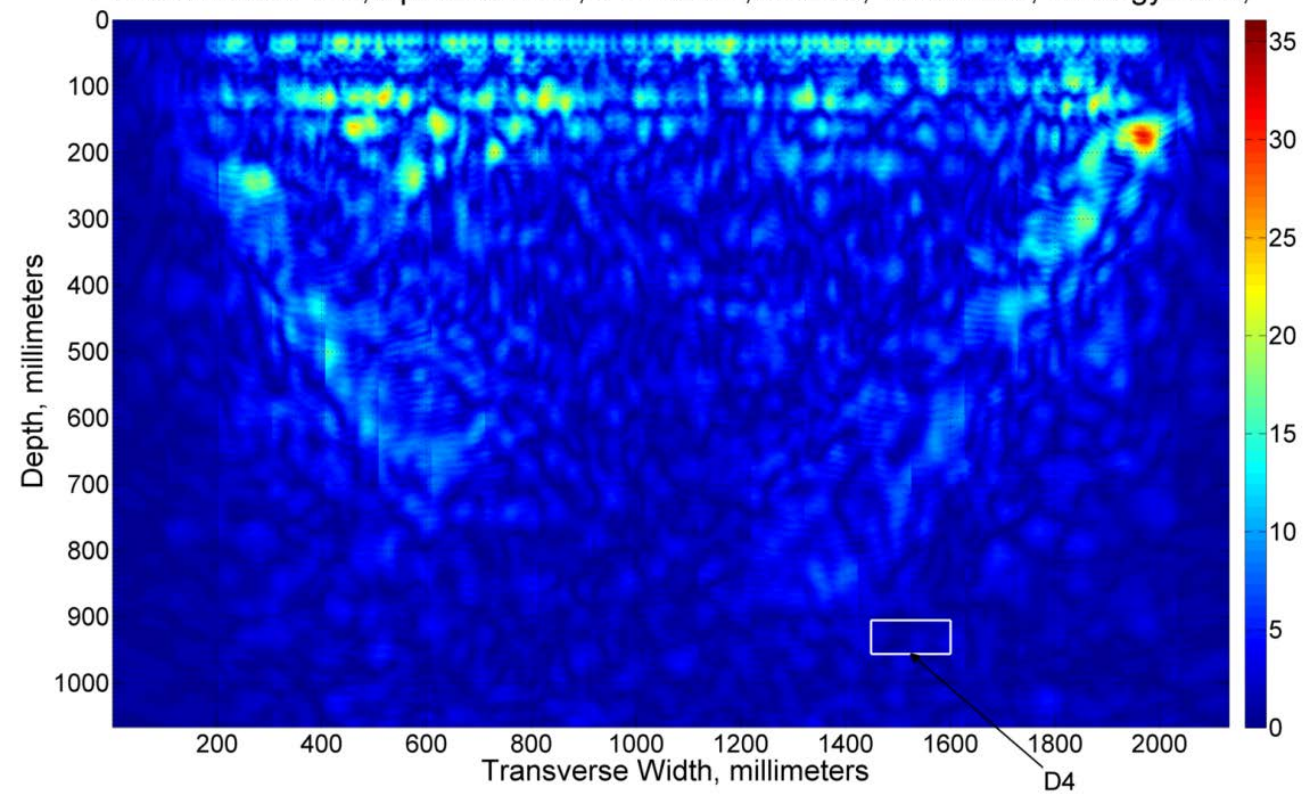

Fig. B. 78. Horizontal Set 16, Node 18. 


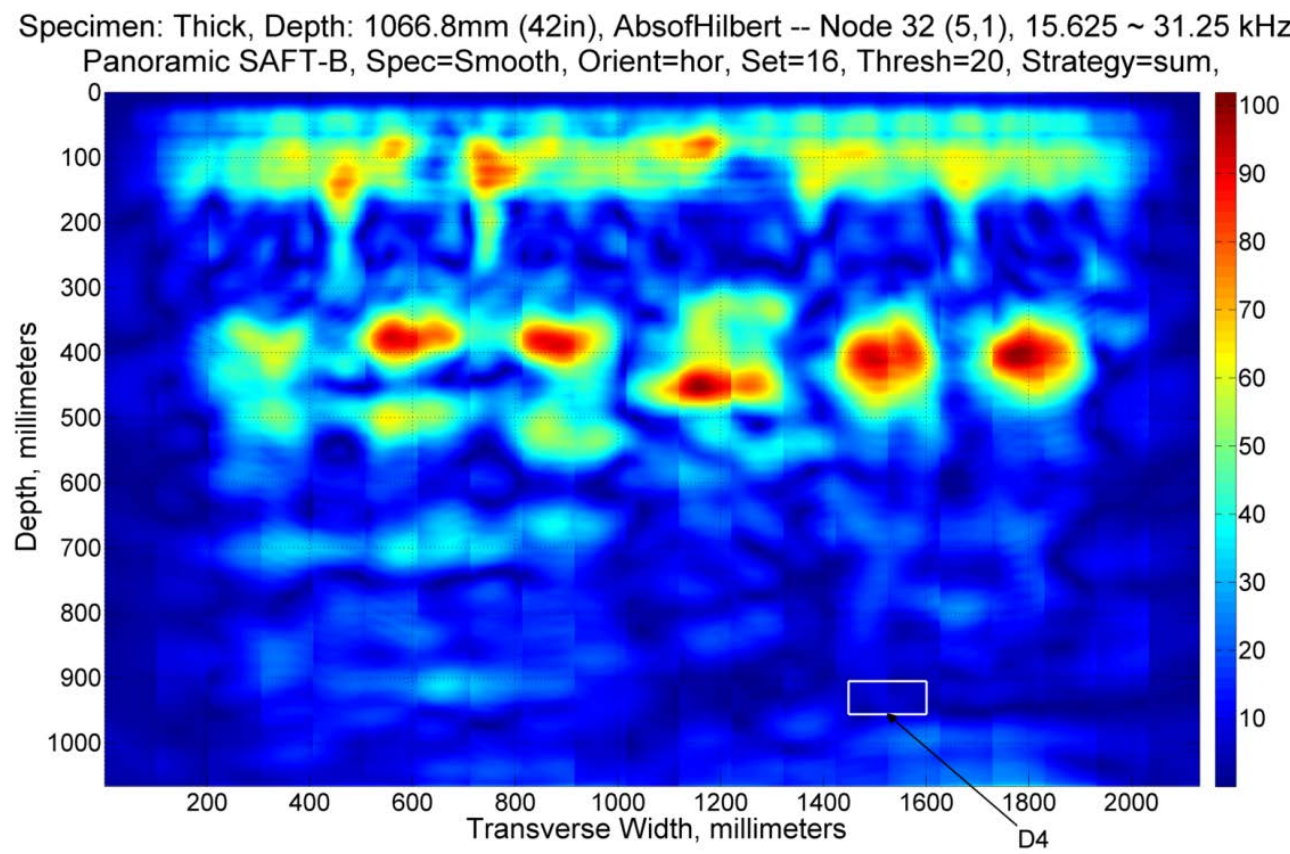

Fig. B. 79. Horizontal Set 16, Node 32.

Specimen: Thick, Depth: 1066.8mm (42in), AbsofHilbert -- Node $34(5,3), 46.875 \sim 62.5 \mathrm{kHz}$ Panoramic SAFT-B, Spec=Smooth, Orient=hor, Set=16, Thresh=20, Strategy=sum,

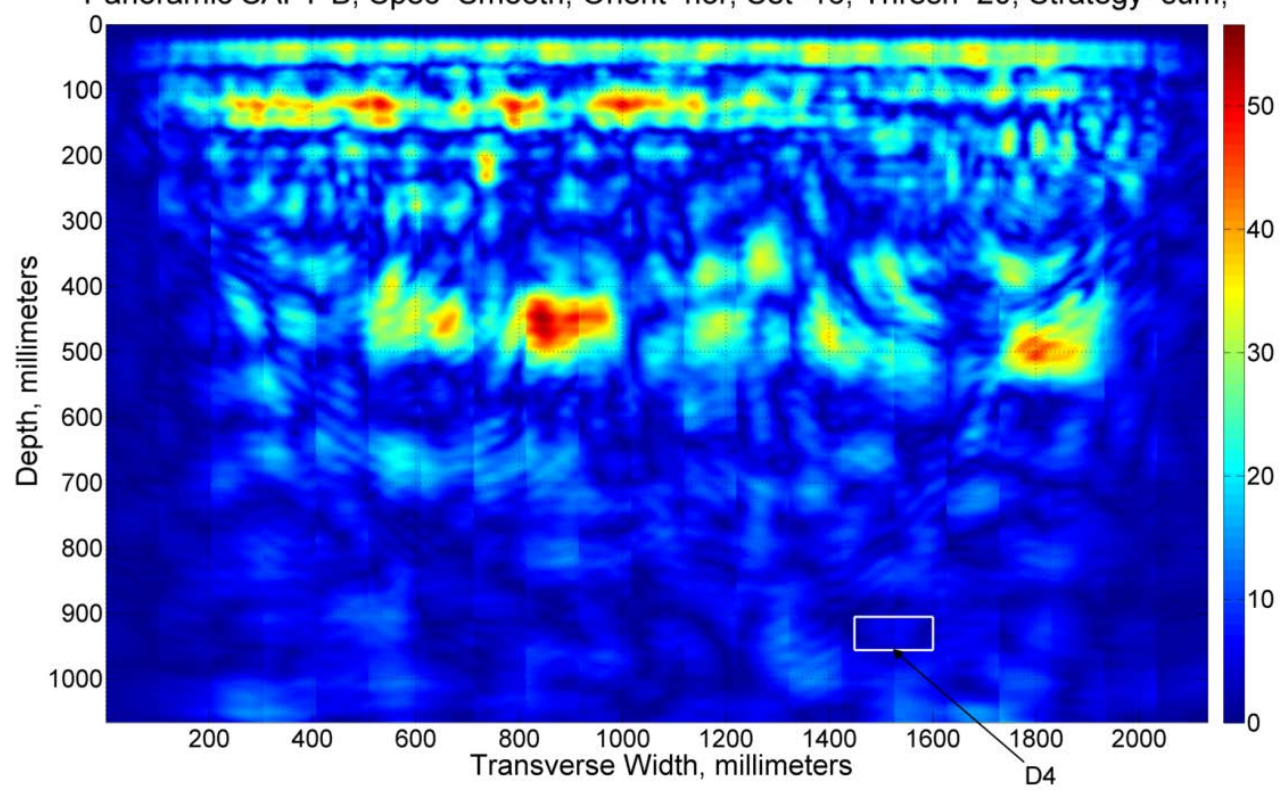

Fig. B. 80. Horizontal Set 16, Node 34. 


\section{SET SEVENTEEN - DEFECT 10}

Specimen: Thick, Depth: 1066.8mm (42in), AbsofHilbert -- Node $0(0,0), 0 \sim 500 \mathrm{kHz}$ Panoramic SAFT-B, Spec=Smooth, Orient=hor, Set=17, Thresh=20, Strategy=sum,

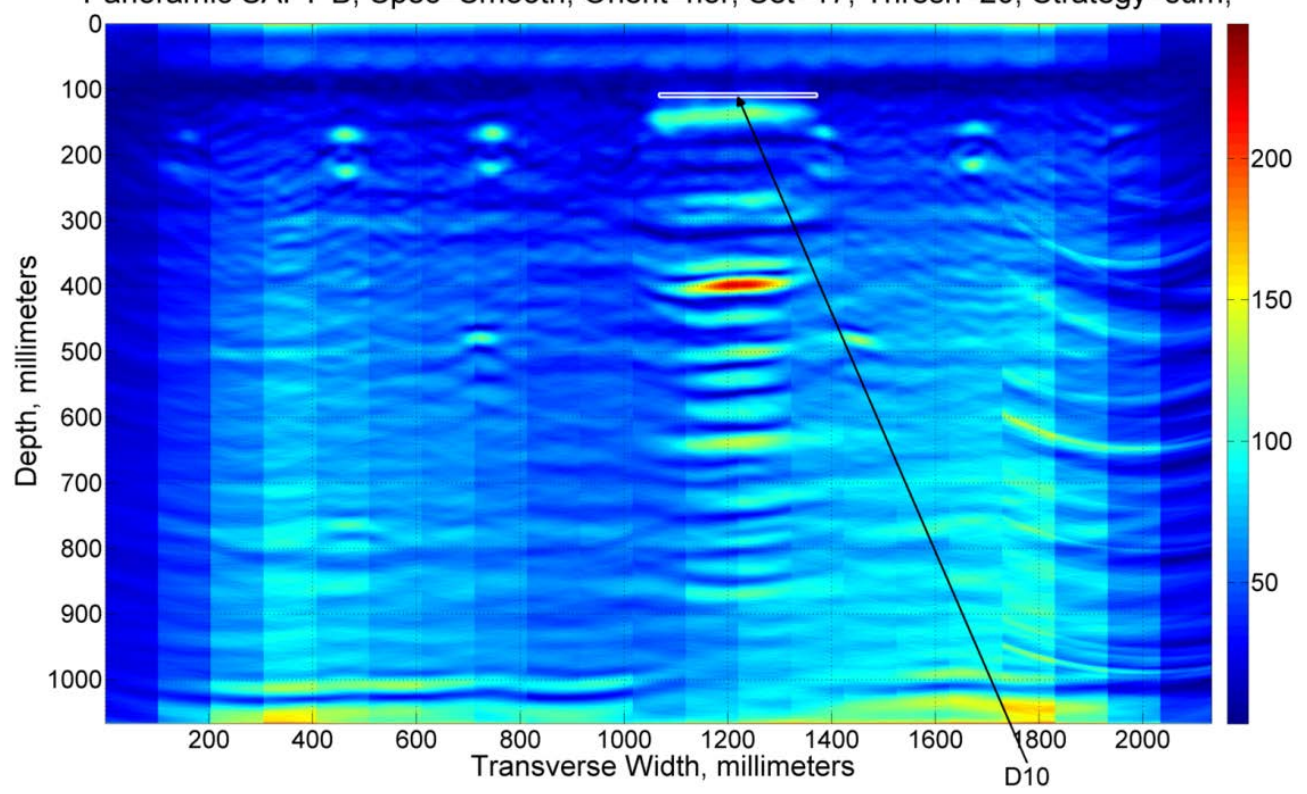

Fig. B. 81. Horizontal Set 17, Node 0.

Specimen: Thick, Depth: 1066.8mm (42in), AbsofHilbert -- Node $16(4,1), 31.25 \sim 62.5 \mathrm{kHz}$ Panoramic SAFT-B, Spec=Smooth, Orient=hor, Set=17, Thresh=20, Strategy=sum,

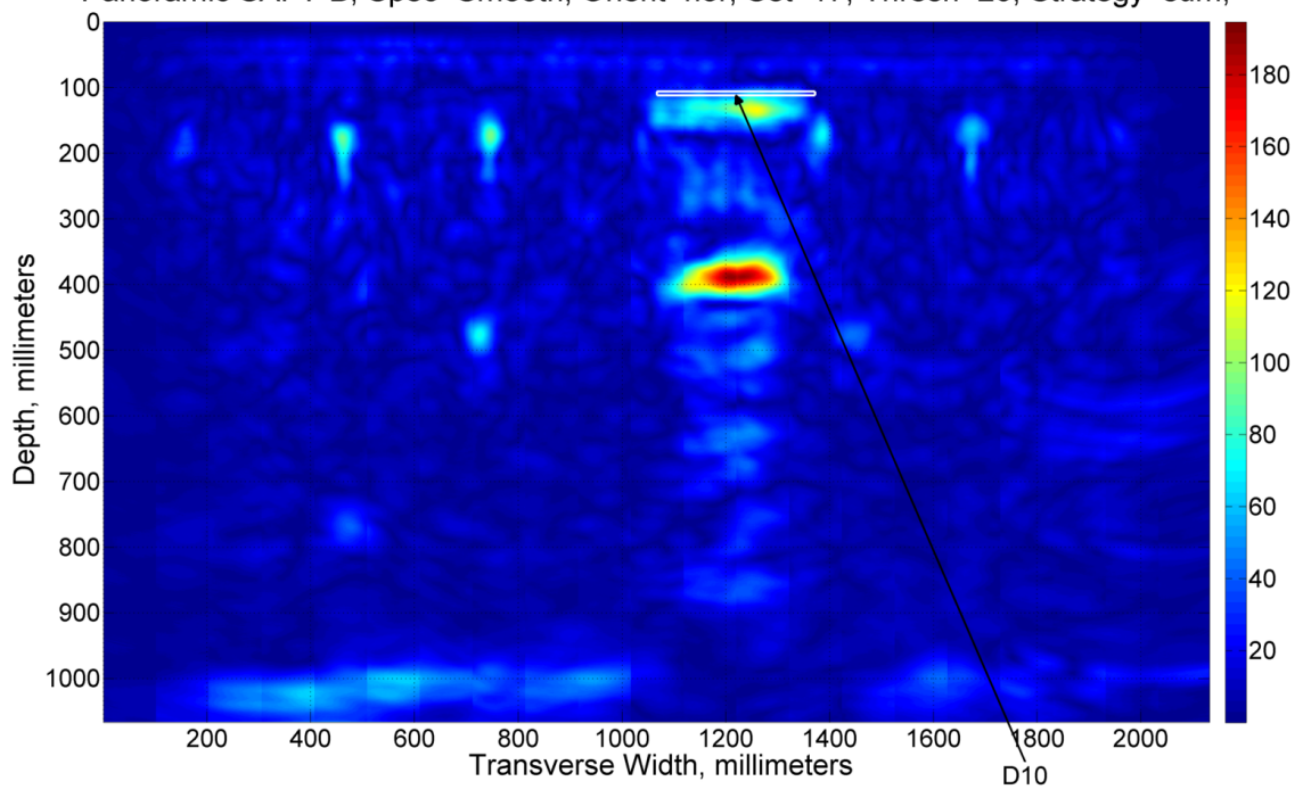

Fig. B. 82. Horizontal Set 17, Node 16. 


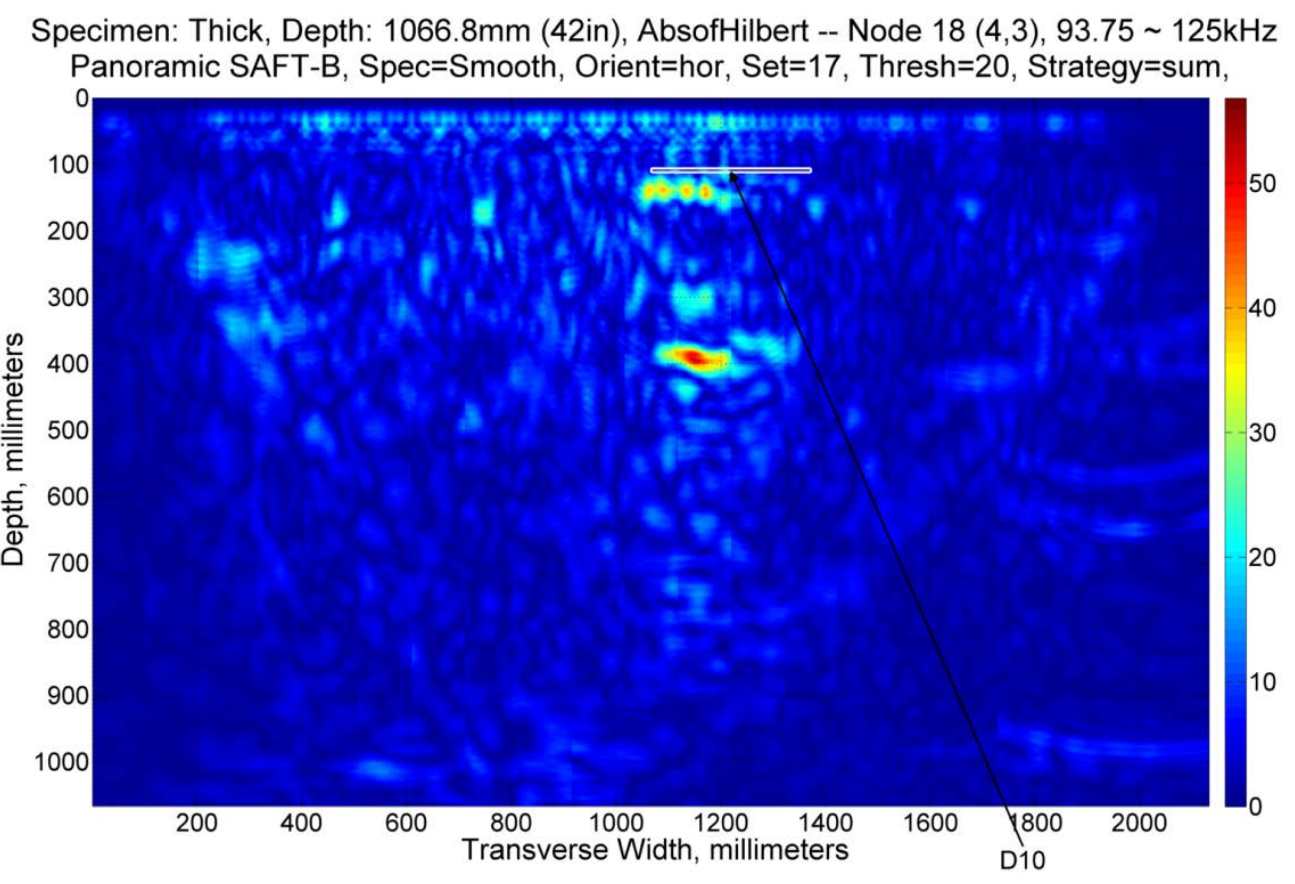

Fig. B. 83. Horizontal Set 17, Node 18.

Specimen: Thick, Depth: 1066.8mm (42in), AbsofHilbert -- Node $32(5,1), 15.625 \sim 31.25 \mathrm{kHz}$ Panoramic SAFT-B, Spec=Smooth, Orient=hor, Set=17, Thresh=20, Strategy=sum,

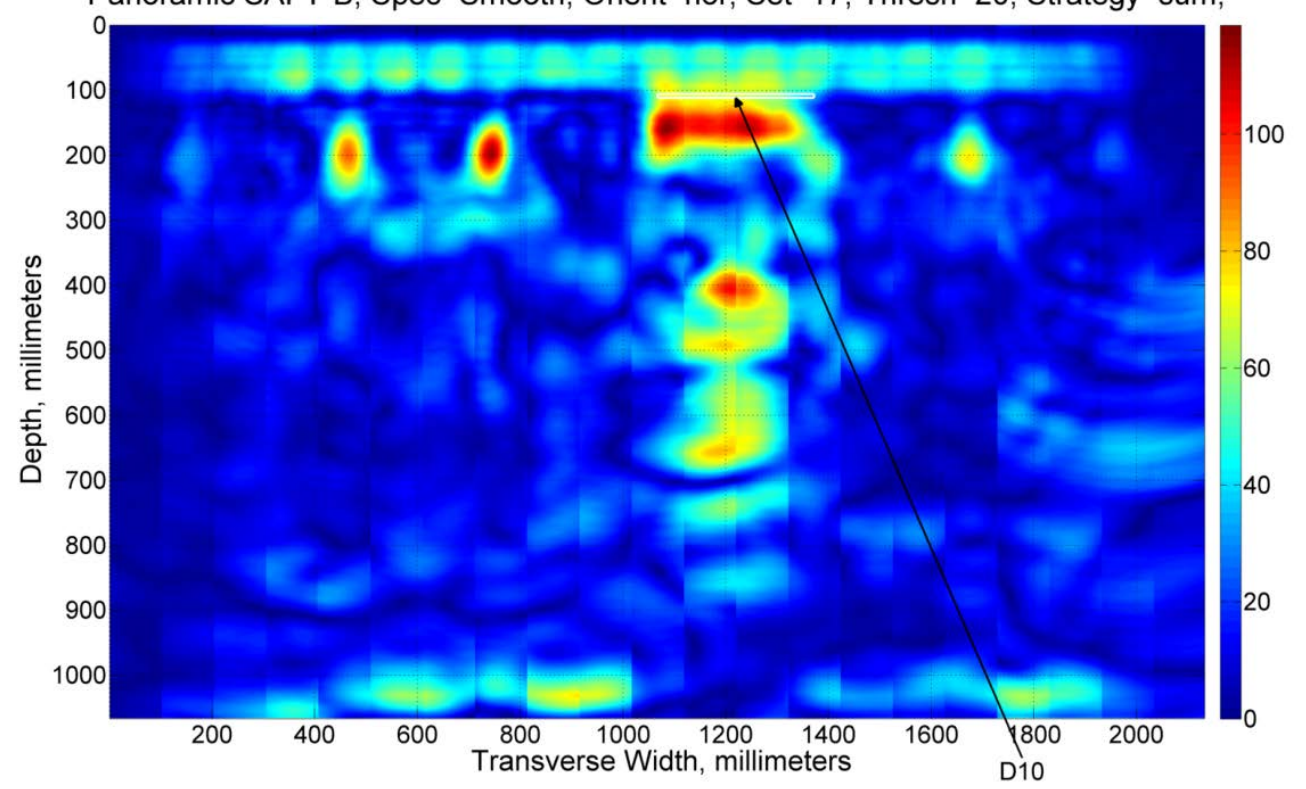

Fig. B. 84. Horizontal Set 17, Node 32. 


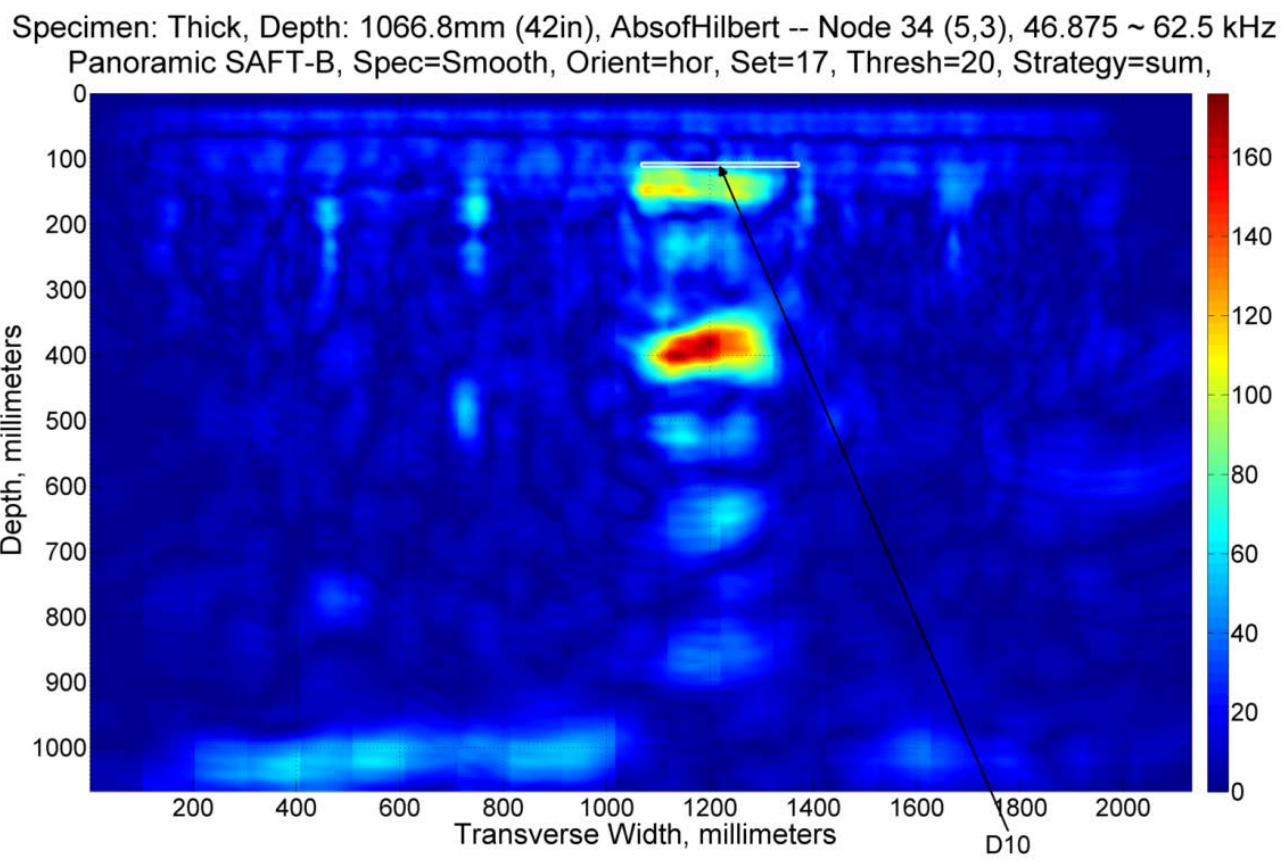

Fig. B. 85. Horizontal Set 17, Node 34.

\section{SET EIGHTEEN - DEFECT 10}

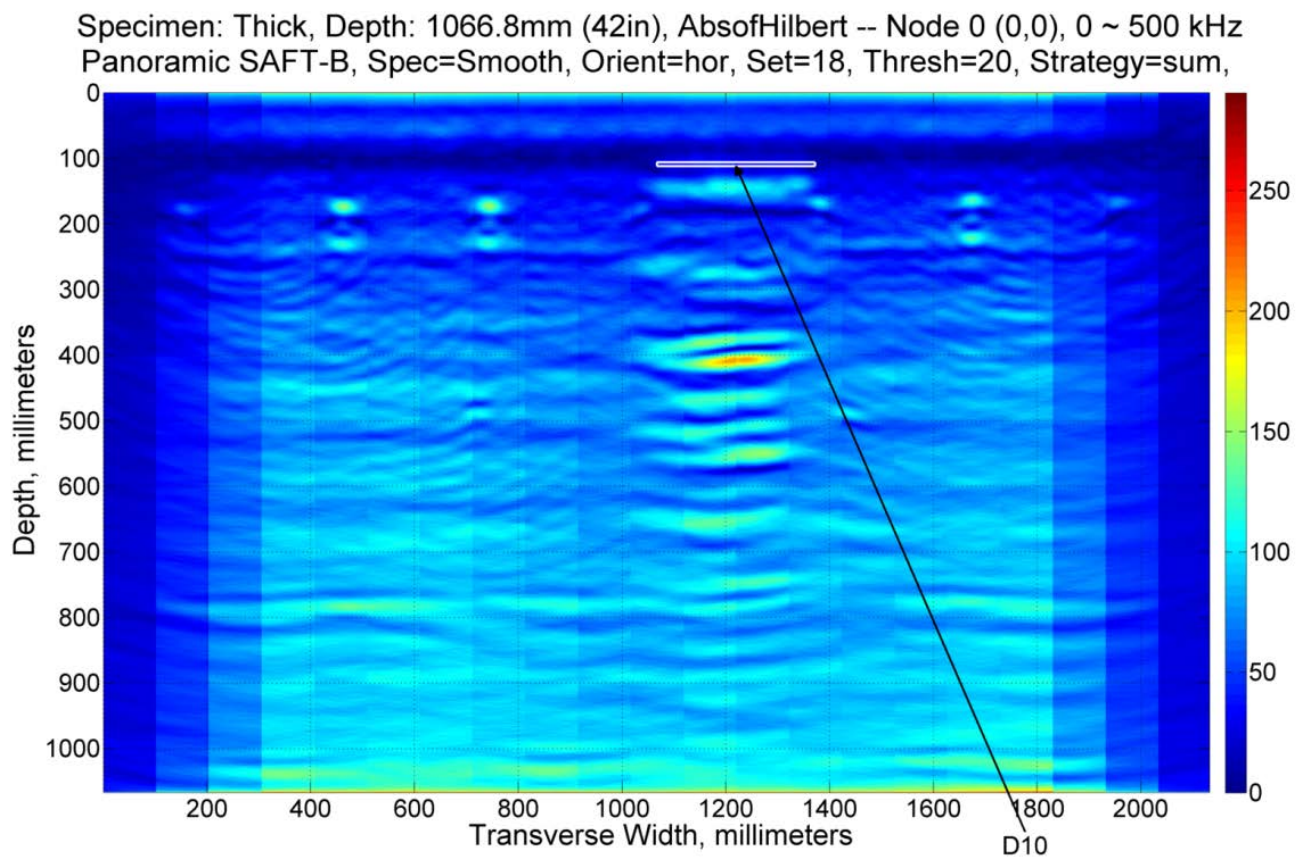

Fig. B. 86. Horizontal Set 18, Node 0. 


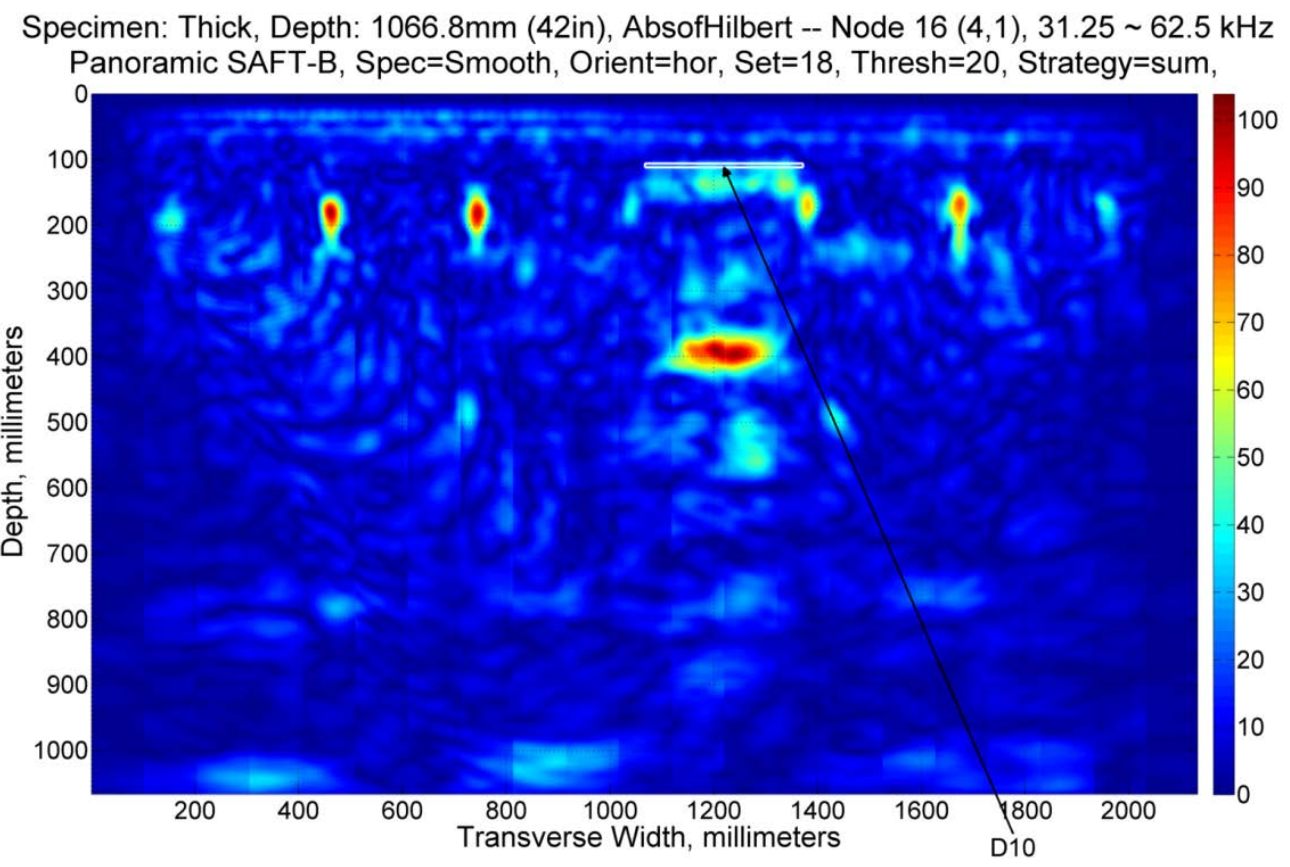

Fig. B. 87. Horizontal Set 18, Node 16.

Specimen: Thick, Depth: 1066.8mm (42in), AbsofHilbert -- Node $18(4,3), 93.75 \sim 125 \mathrm{kHz}$ Panoramic SAFT-B, Spec=Smooth, Orient $=$ hor, Set $=18$, Thresh $=20$, Strategy $=$ sum,

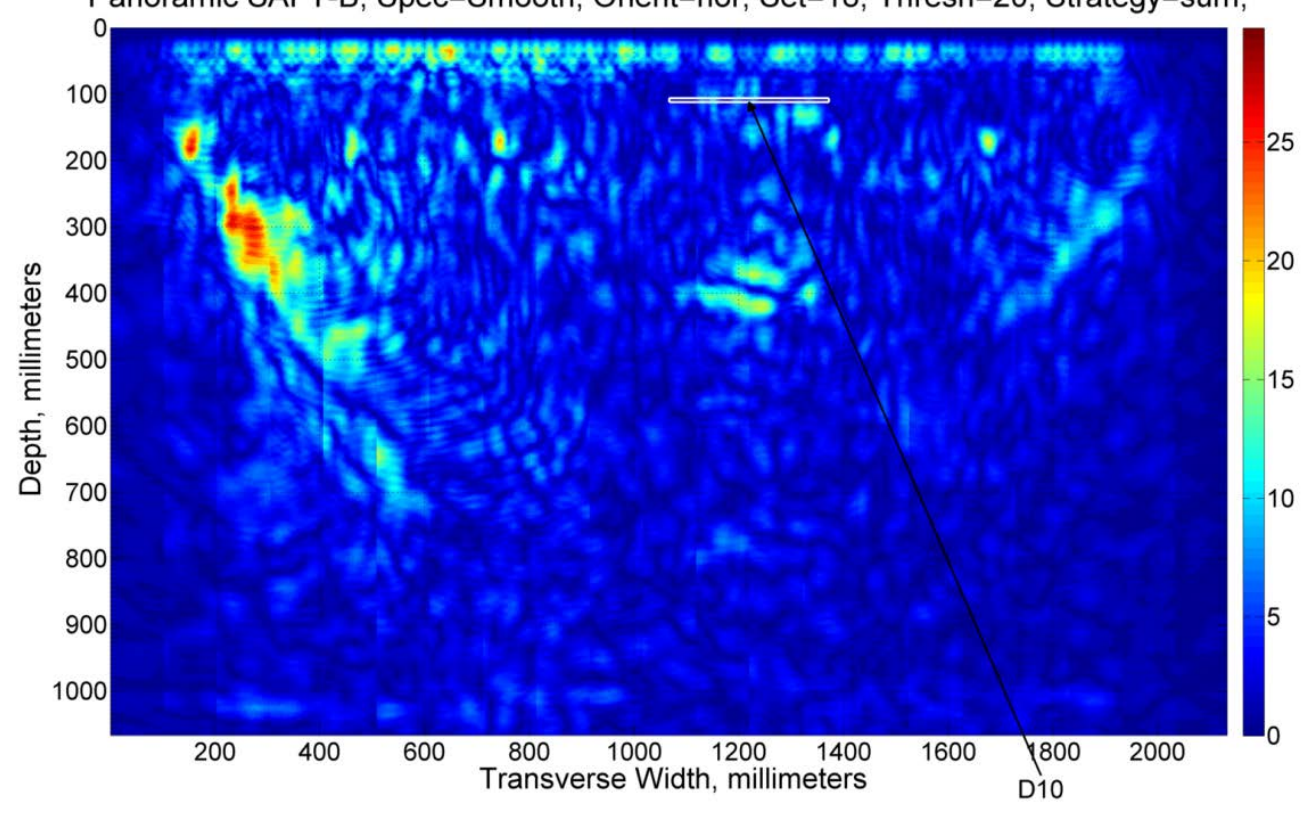

Fig. B. 88. Horizontal Set 18, Node 18. 


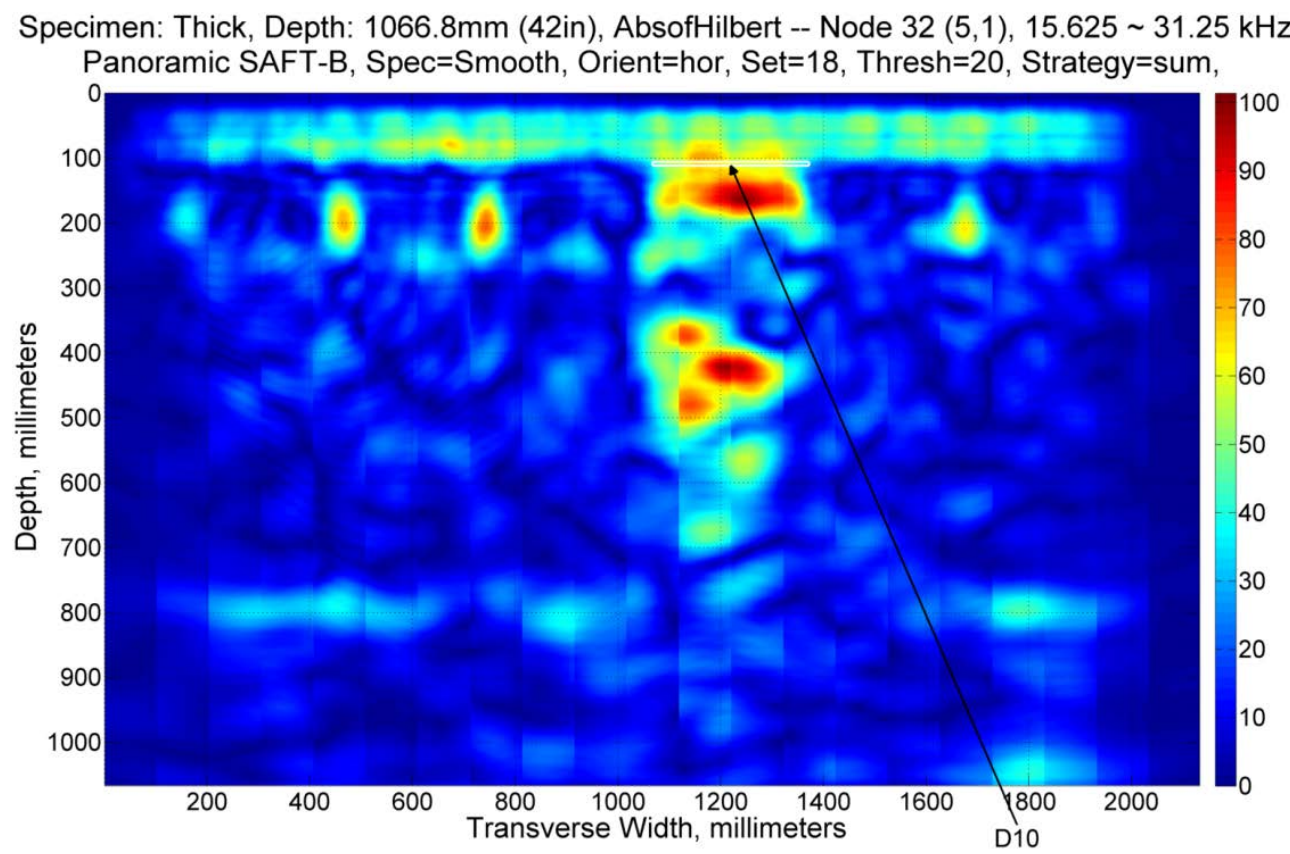

Fig. B. 89. Horizontal Set 18, Node 32.

Specimen: Thick, Depth: 1066.8mm (42in), AbsofHilbert -- Node 34 (5,3), $46.875 \sim 62.5 \mathrm{kHz}$ Panoramic SAFT-B, Spec=Smooth, Orient=hor, Set=18, Thresh=20, Strategy=sum,

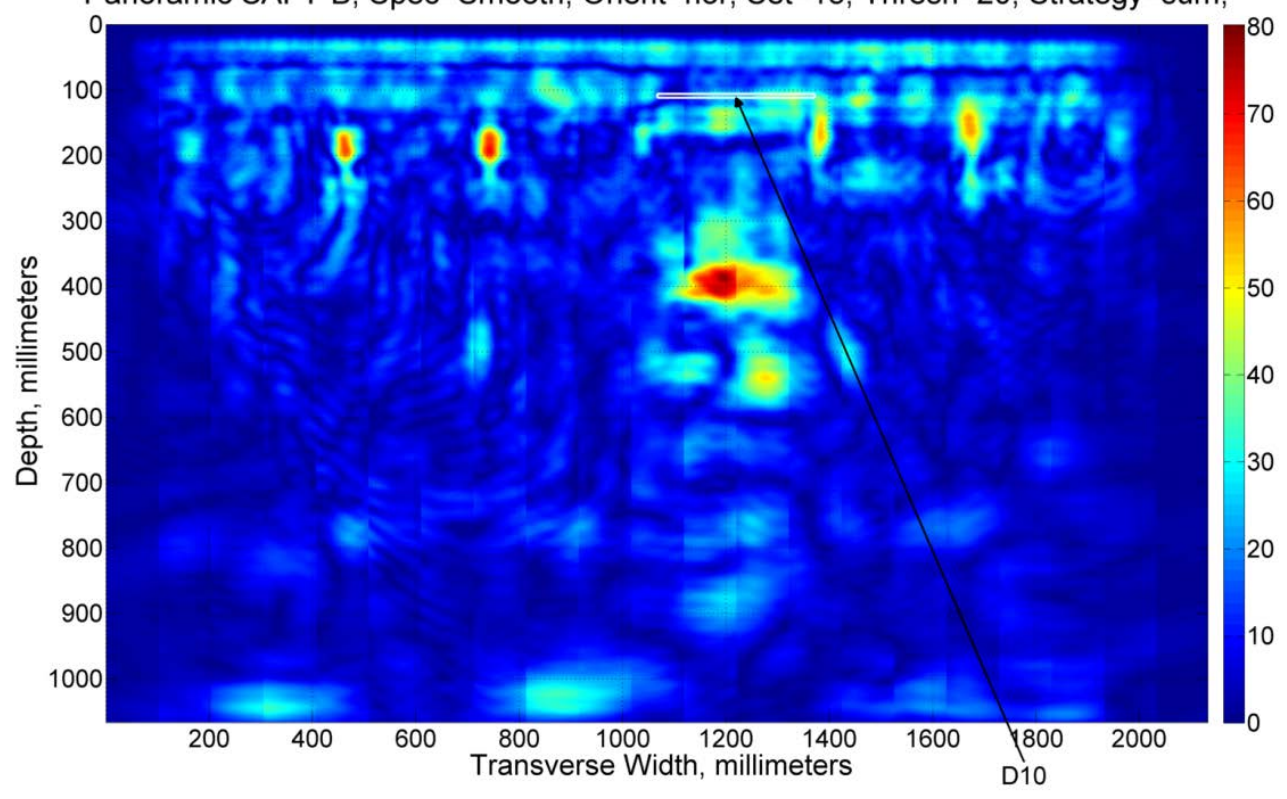

Fig. B. 90. Horizontal Set 18, Node 34. 


\section{SET NINETEEN - NO DEFECT}

Specimen: Thick, Depth: 1066.8mm (42in), AbsofHilbert -- Node $0(0,0), 0 \sim 500 \mathrm{kHz}$ Panoramic SAFT-B, Spec=Smooth, Orient=hor, Set=19, Thresh=20, Strategy=sum,

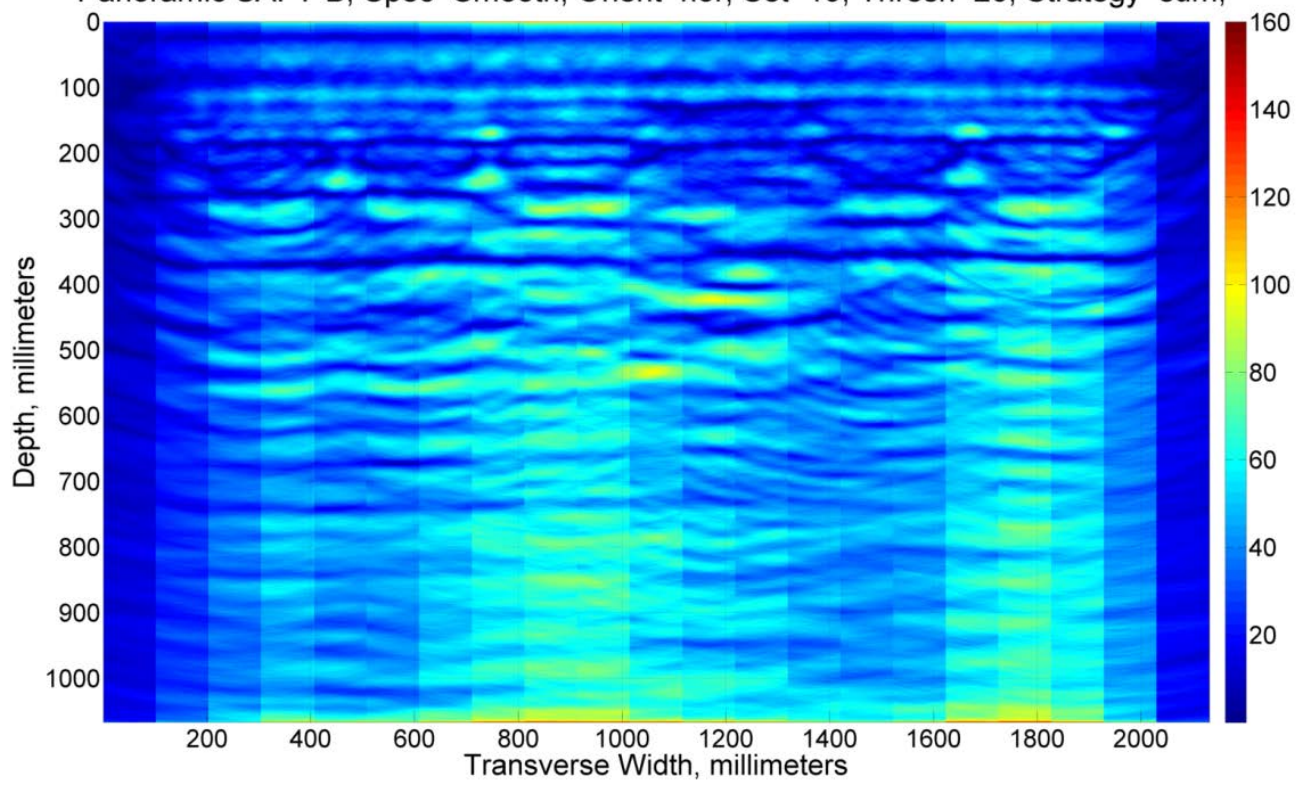

Fig. B. 91. Horizontal Set 19, Node 0.

Specimen: Thick, Depth: 1066.8mm (42in), AbsofHilbert -- Node $16(4,1), 31.25 \sim 62.5 \mathrm{kHz}$ Panoramic SAFT-B, Spec=Smooth, Orient=hor, Set=19, Thresh=20, Strategy=sum,

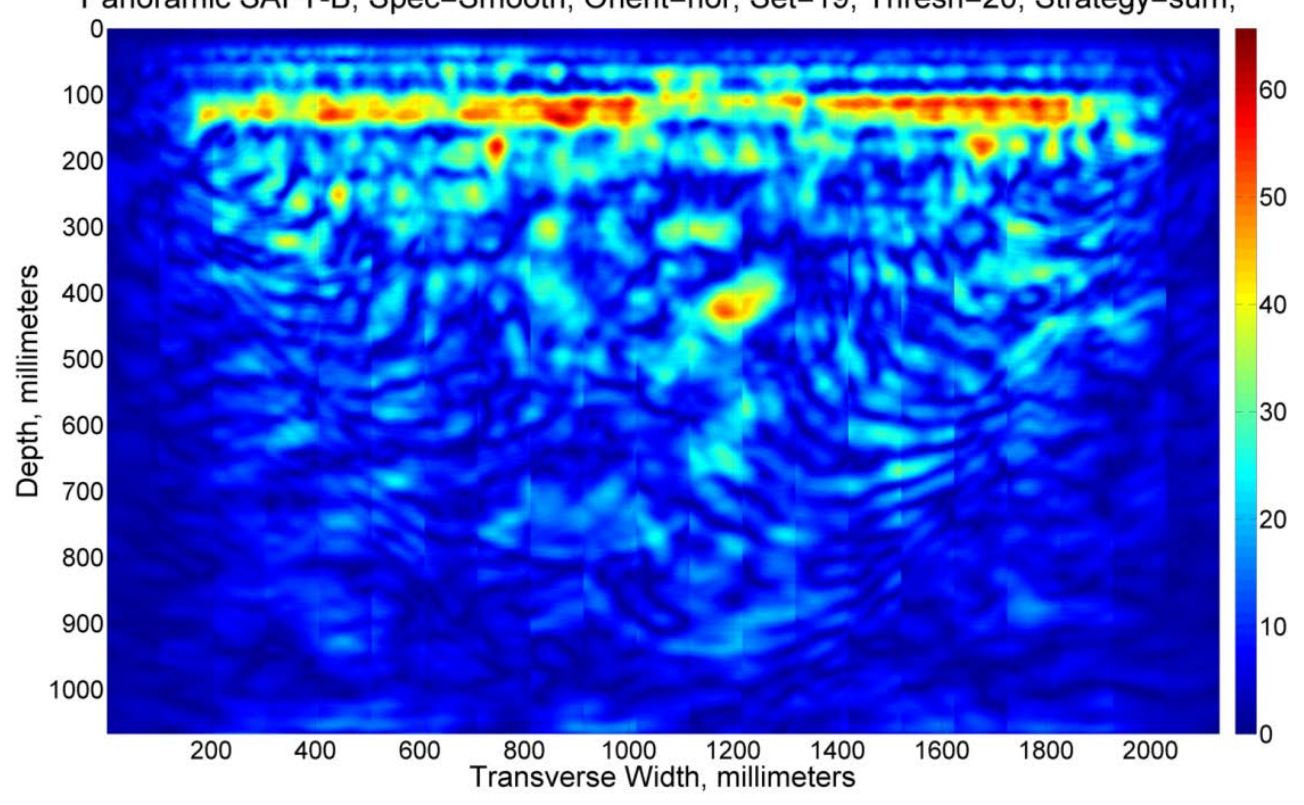

Fig. B. 92. Horizontal Set 19, Node 16. 


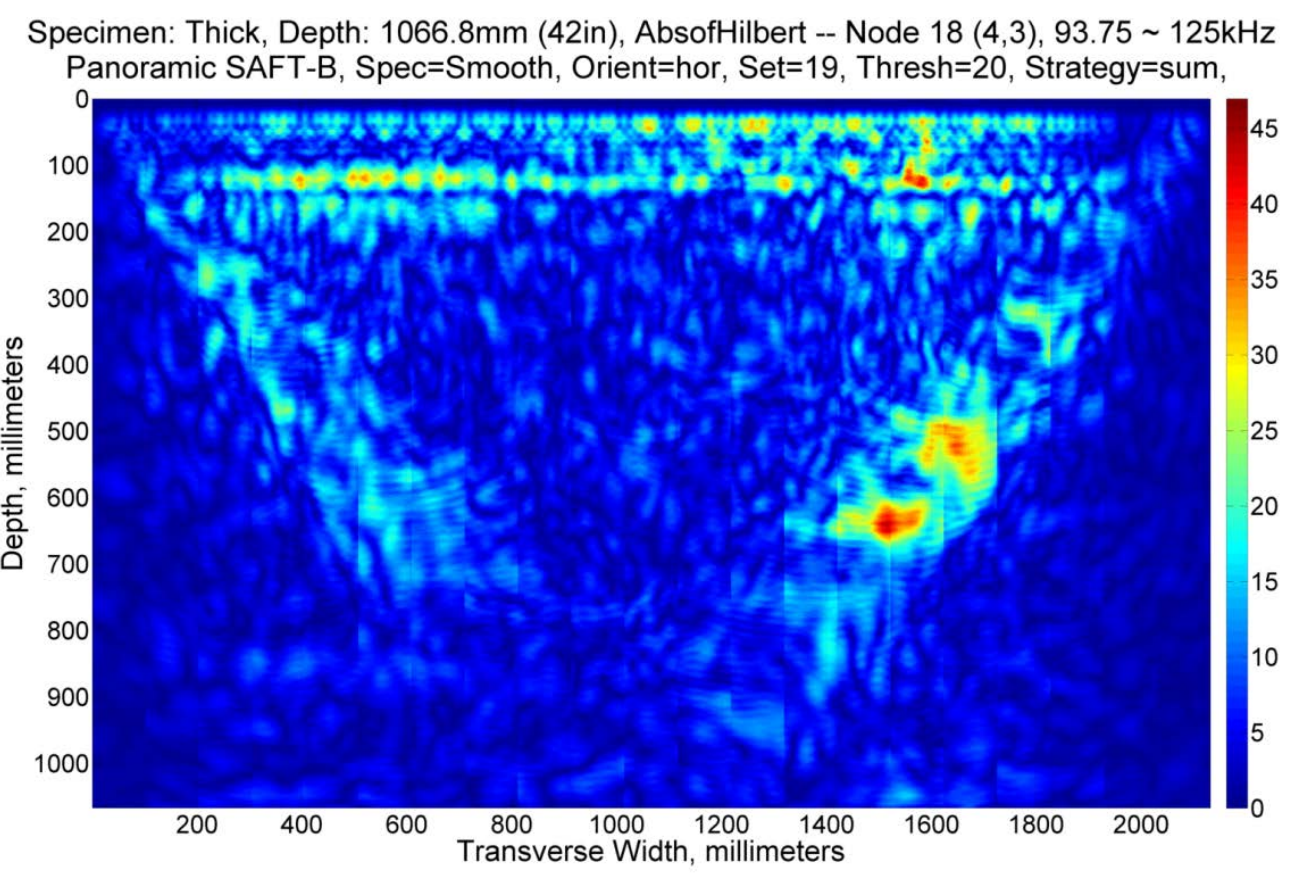

Fig. B. 93. Horizontal Set 19, Node 18.

Specimen: Thick, Depth: 1066.8mm (42in), AbsofHilbert -- Node 32 (5,1), $15.625 \sim 31.25 \mathrm{kHz}$ Panoramic SAFT-B, Spec=Smooth, Orient=hor, Set=19, Thresh=20, Strategy=sum,

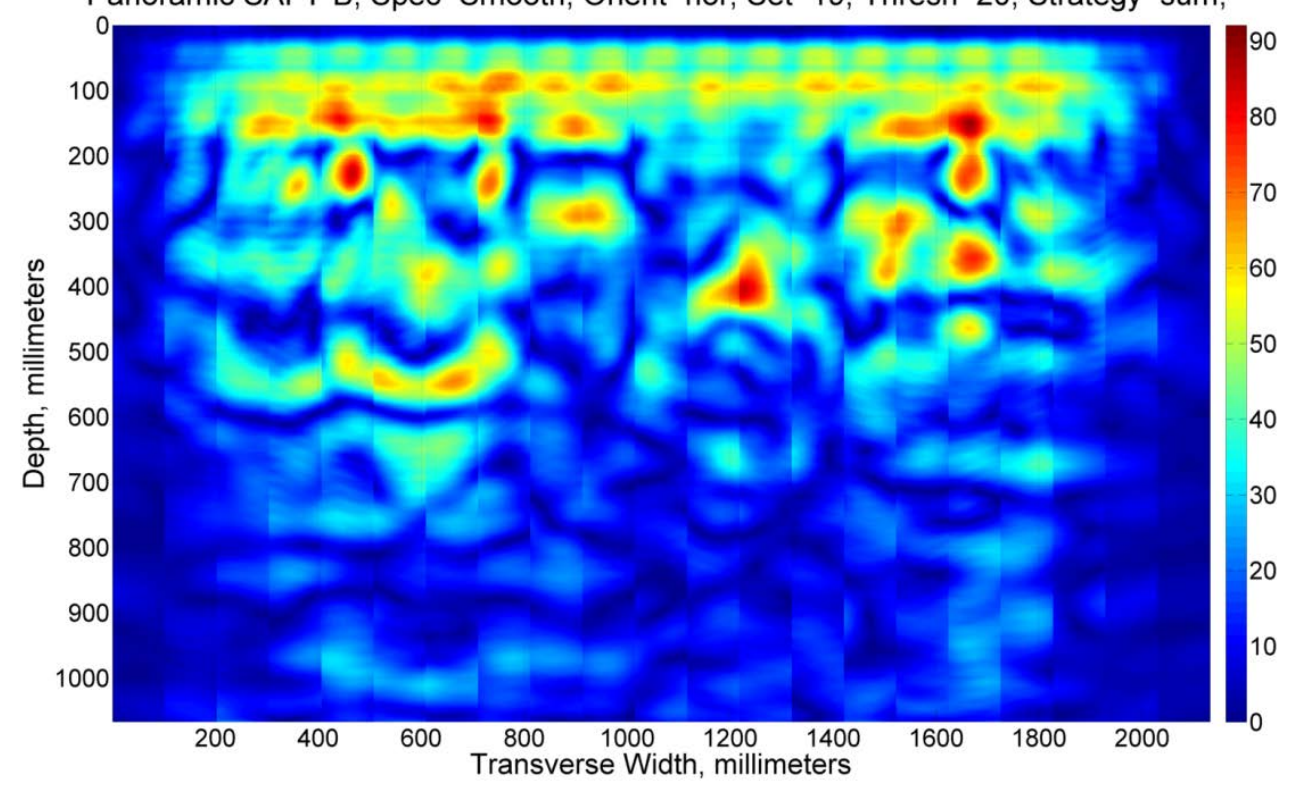

Fig. B. 94. Horizontal Set 19, Node 32. 


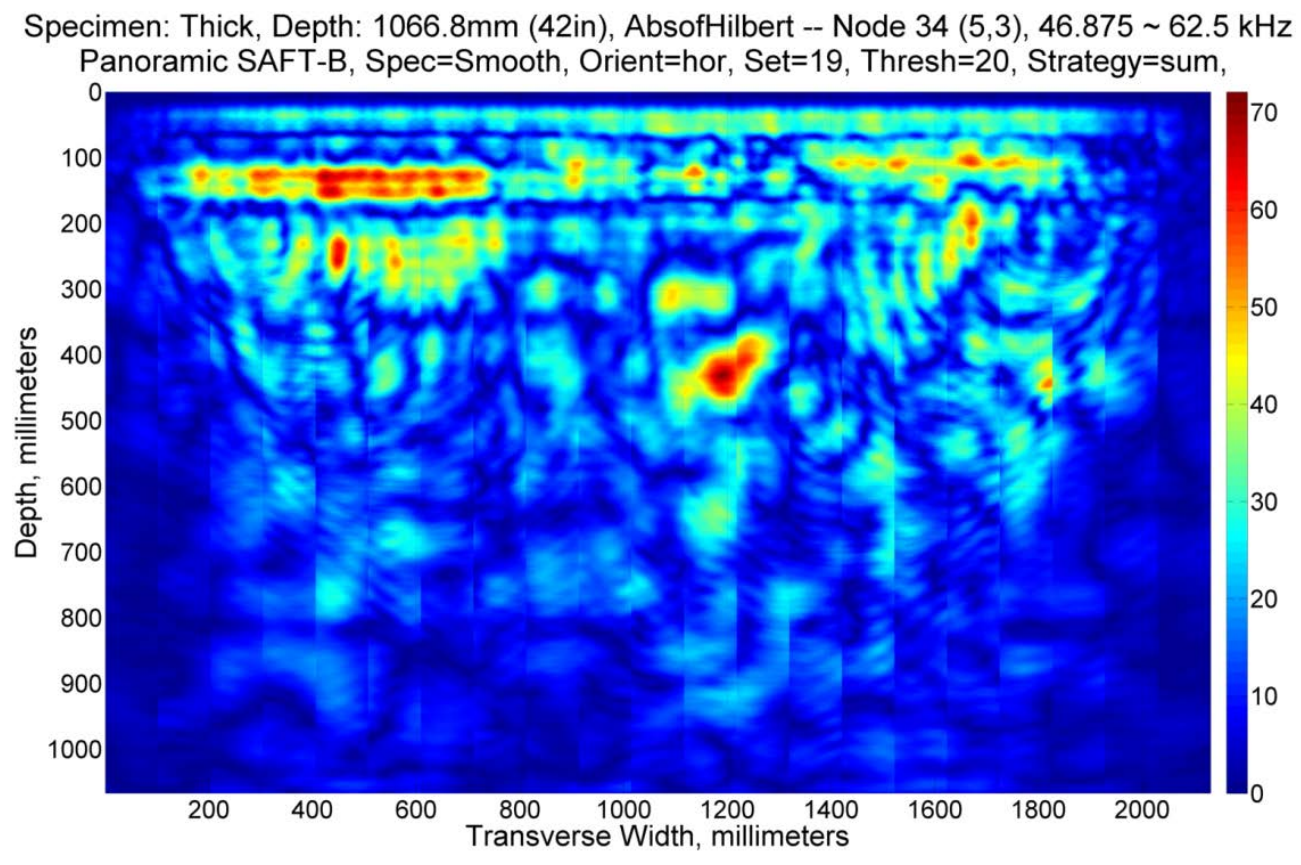

Fig. B. 95. Horizontal Set 19, Node 34. 


\section{SMOOTH VERTICAL}

\section{SET ONE - NO DEFECT}

Specimen: Thick, Depth: 1066.8mm (42in), AbsofHilbert -- Node $0(0,0), 0 \sim 500 \mathrm{kHz}$ Panoramic SAFT-B, Spec=Smooth, Orient=ver, Set=1, Thresh=20, Strategy=sum,

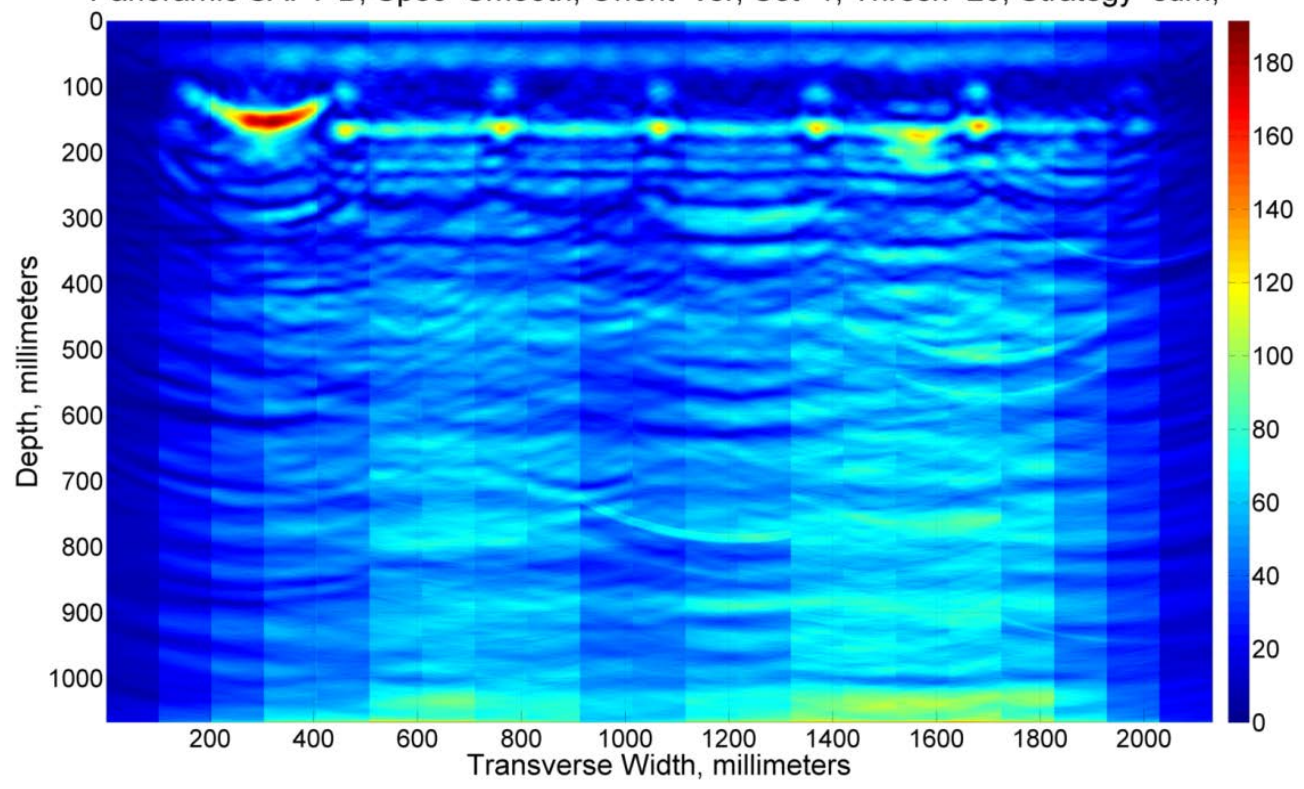

Fig. B. 96. Vertical Set 1, Node 0.

Specimen: Thick, Depth: 1066.8mm (42in), AbsofHilbert -- Node $16(4,1), 31.25 \sim 62.5 \mathrm{kHz}$ Panoramic SAFT-B, Spec=Smooth, Orient=ver, Set=1, Thresh=20, Strategy=sum,

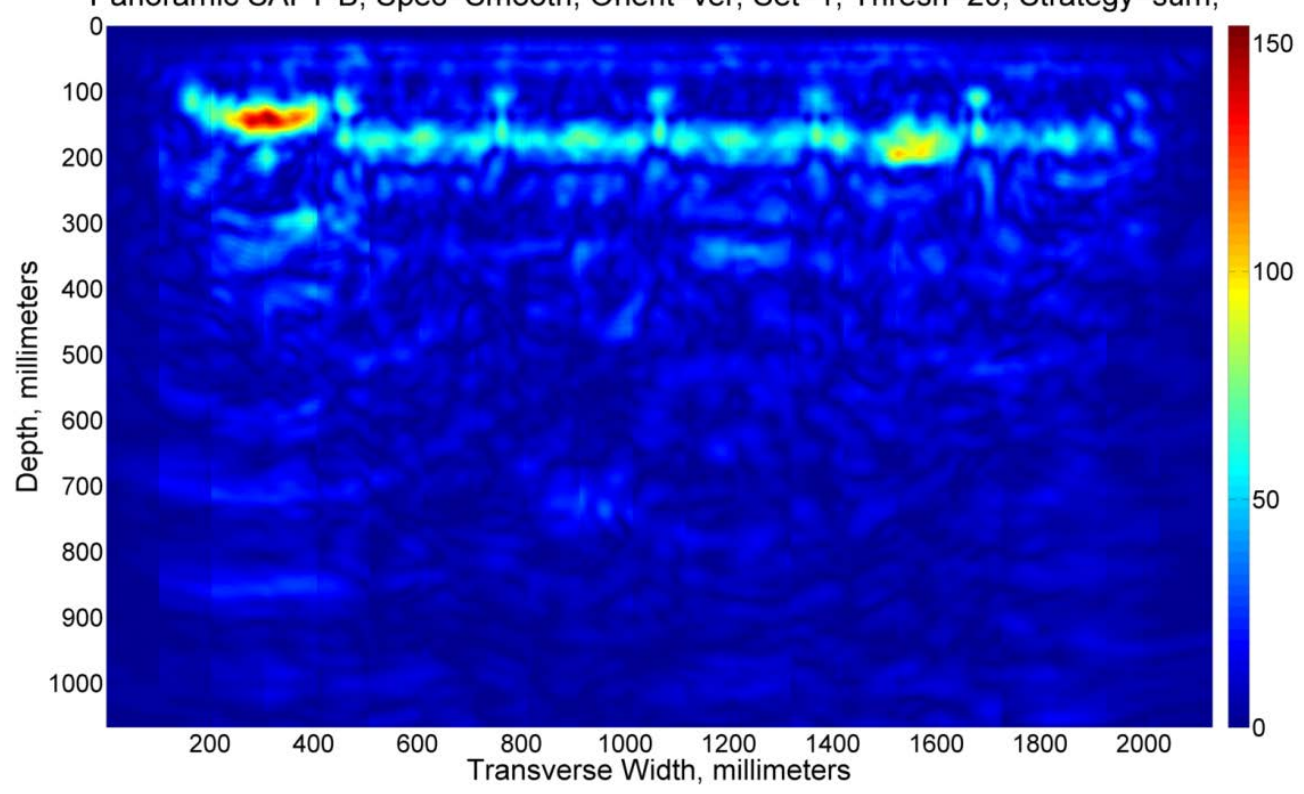

Fig. B. 97. Vertical Set 1, Node 16. 


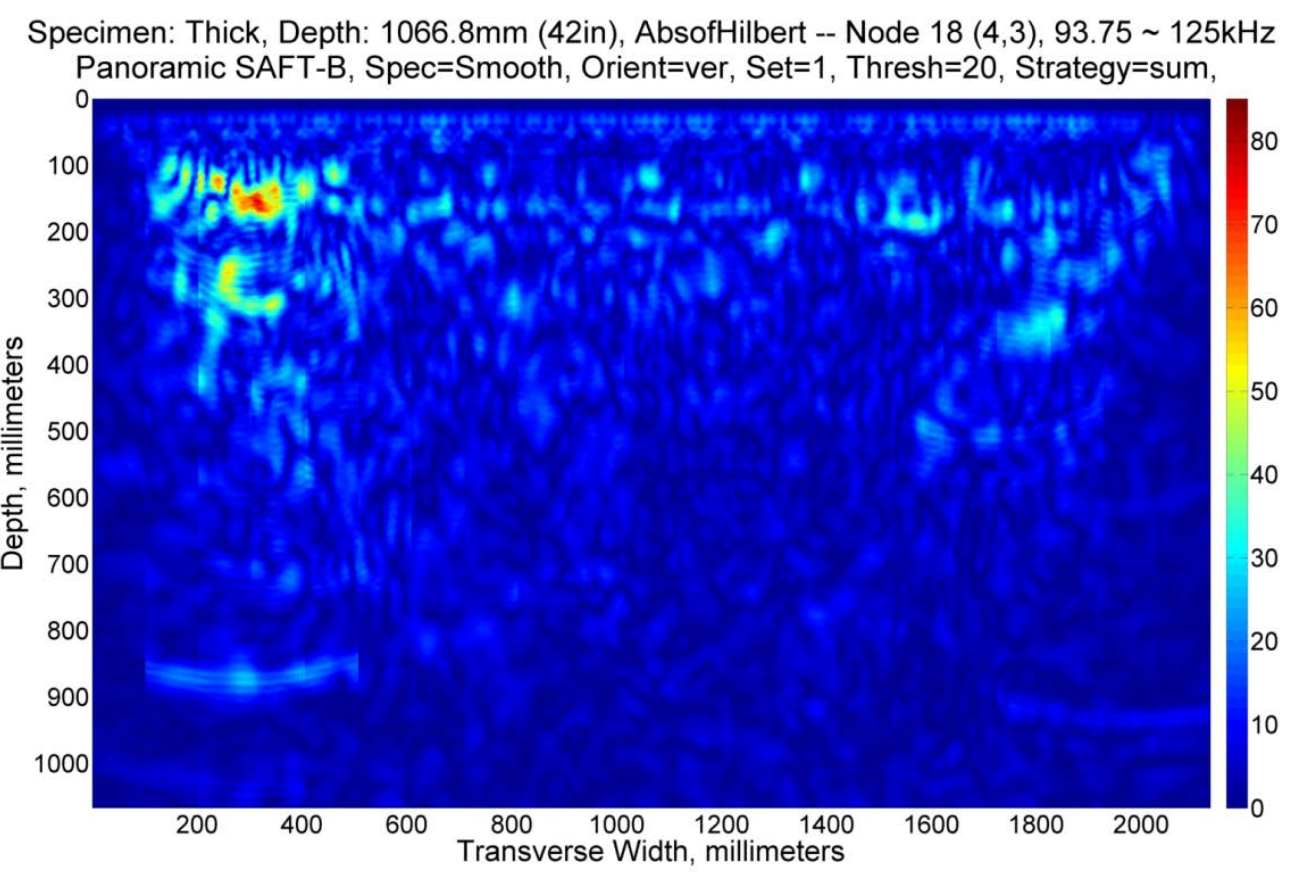

Fig. B. 98. Vertical Set 1, Node 18.

Specimen: Thick, Depth: 1066.8mm (42in), AbsofHilbert -- Node $32(5,1), 15.625 \sim 31.25 \mathrm{kHz}$ Panoramic SAFT-B, Spec=Smooth, Orient=ver, Set=1, Thresh=20, Strategy=sum,

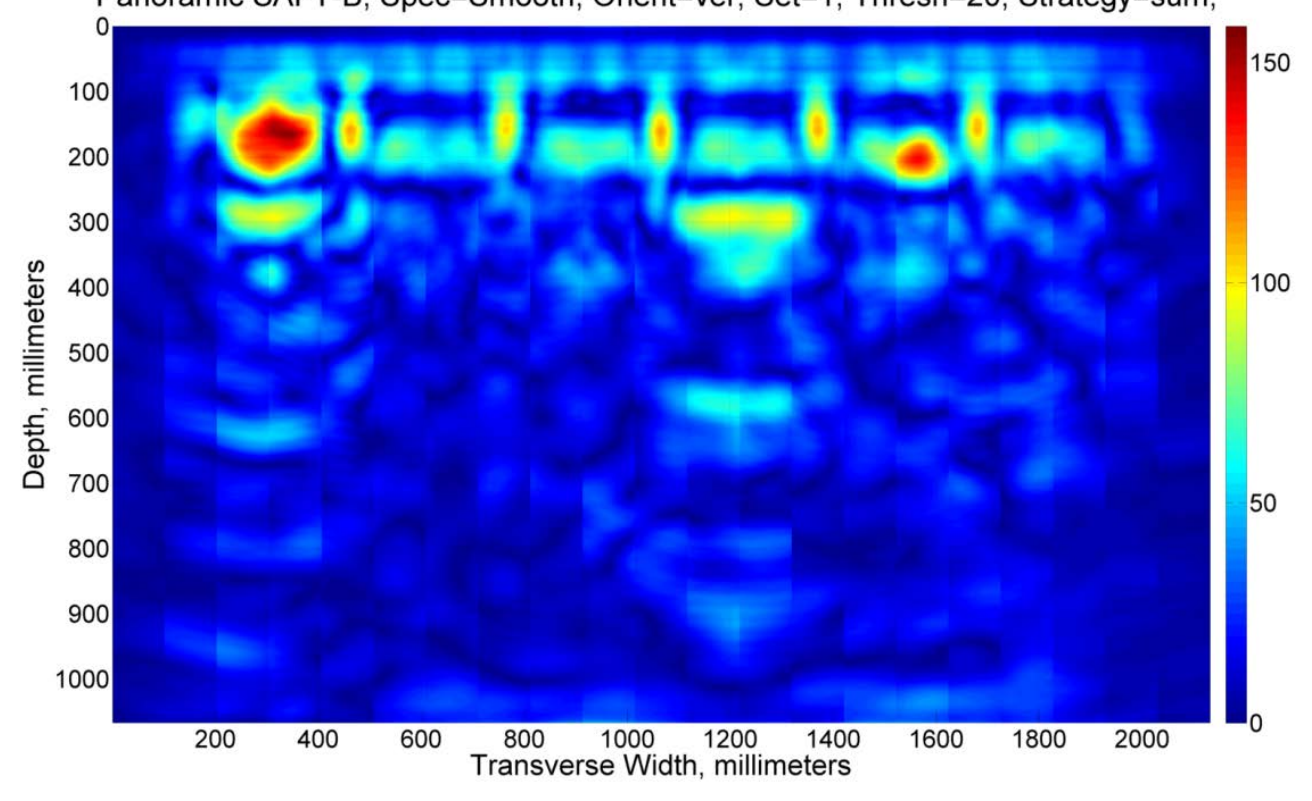

Fig. B. 99, Vertical Set 1, Node 32. 


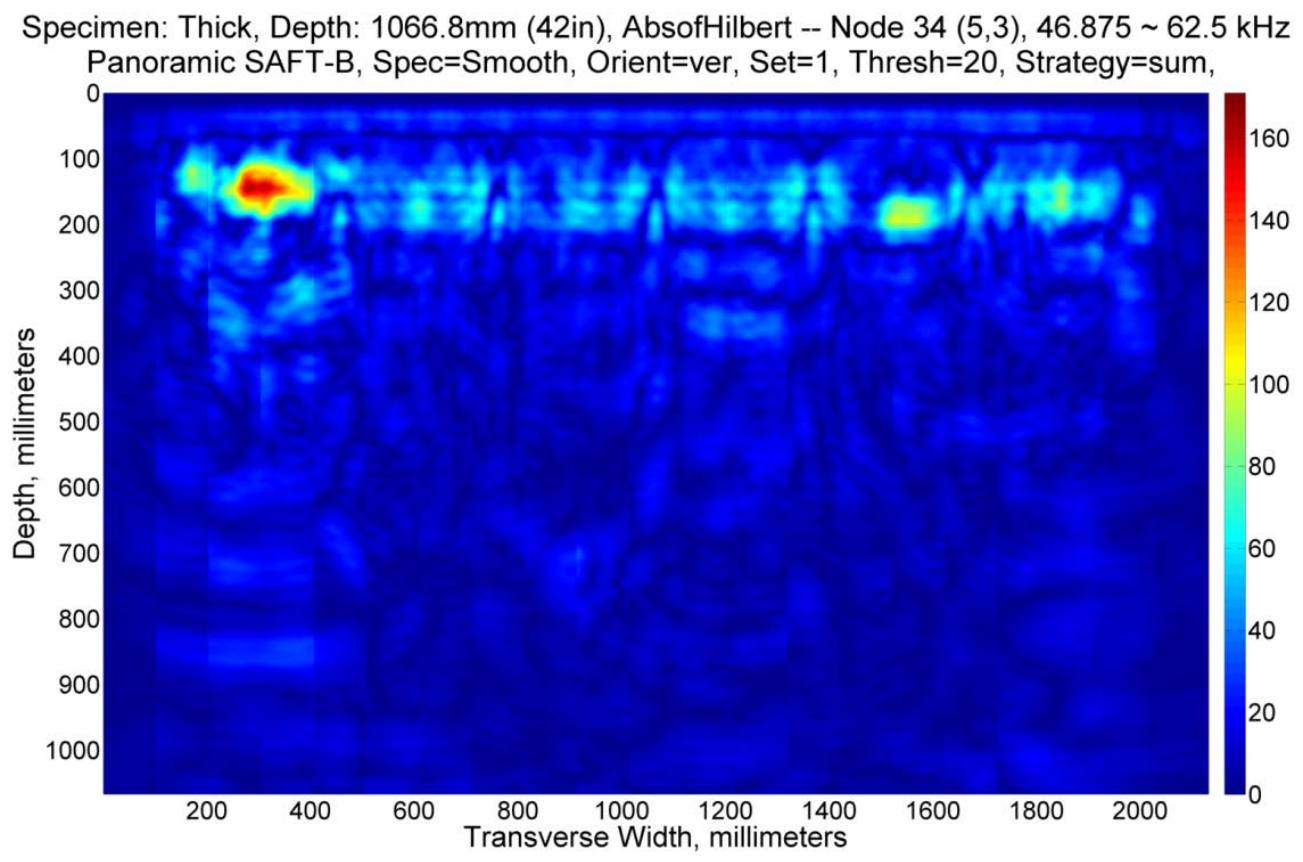

Fig. B. 100. Vertical Set 1, Node 34.

\section{SET TWO - NO DEFECT}

Specimen: Thick, Depth: 1066.8mm (42in), AbsofHilbert -- Node $0(0,0), 0 \sim 500 \mathrm{kHz}$ Panoramic SAFT-B, Spec=Smooth, Orient=ver, Set=2, Thresh=20, Strategy=sum,

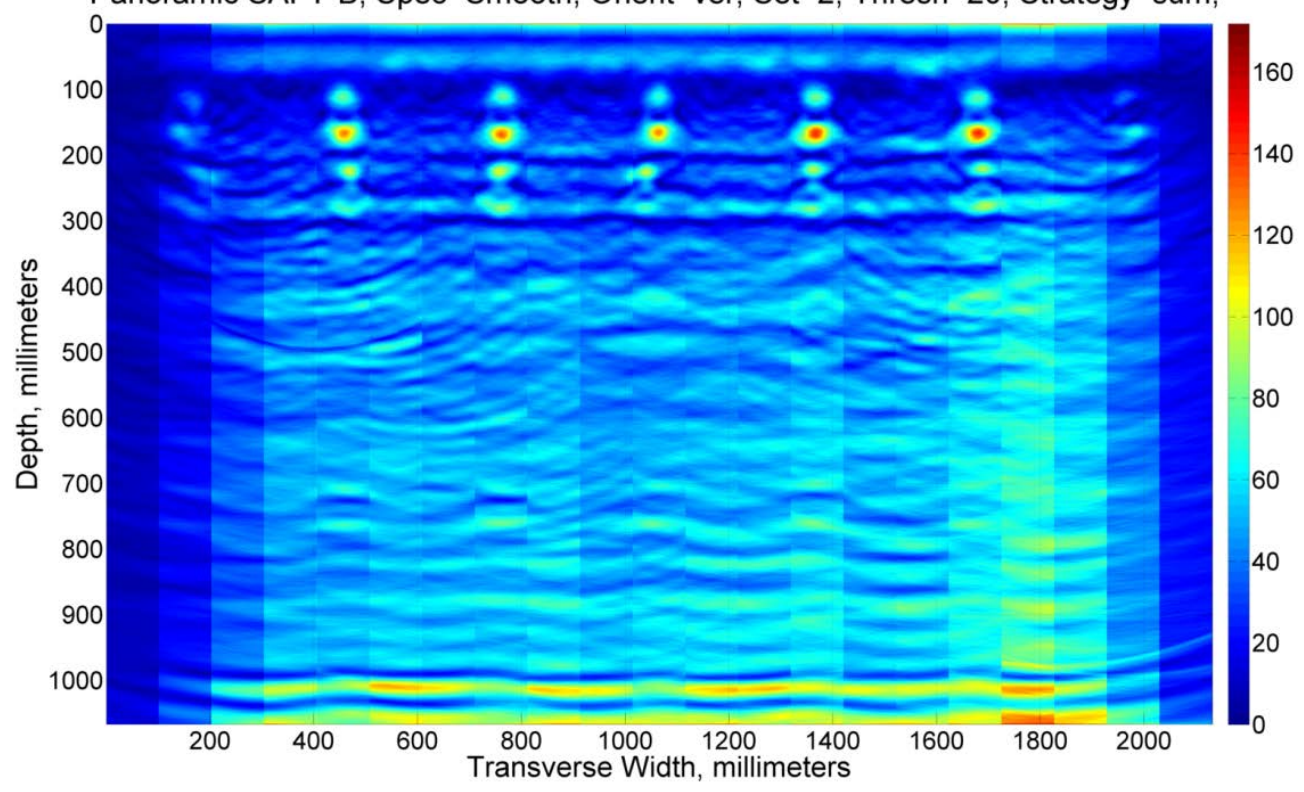

Fig. B. 101. Vertical Set 2, Node 0. 


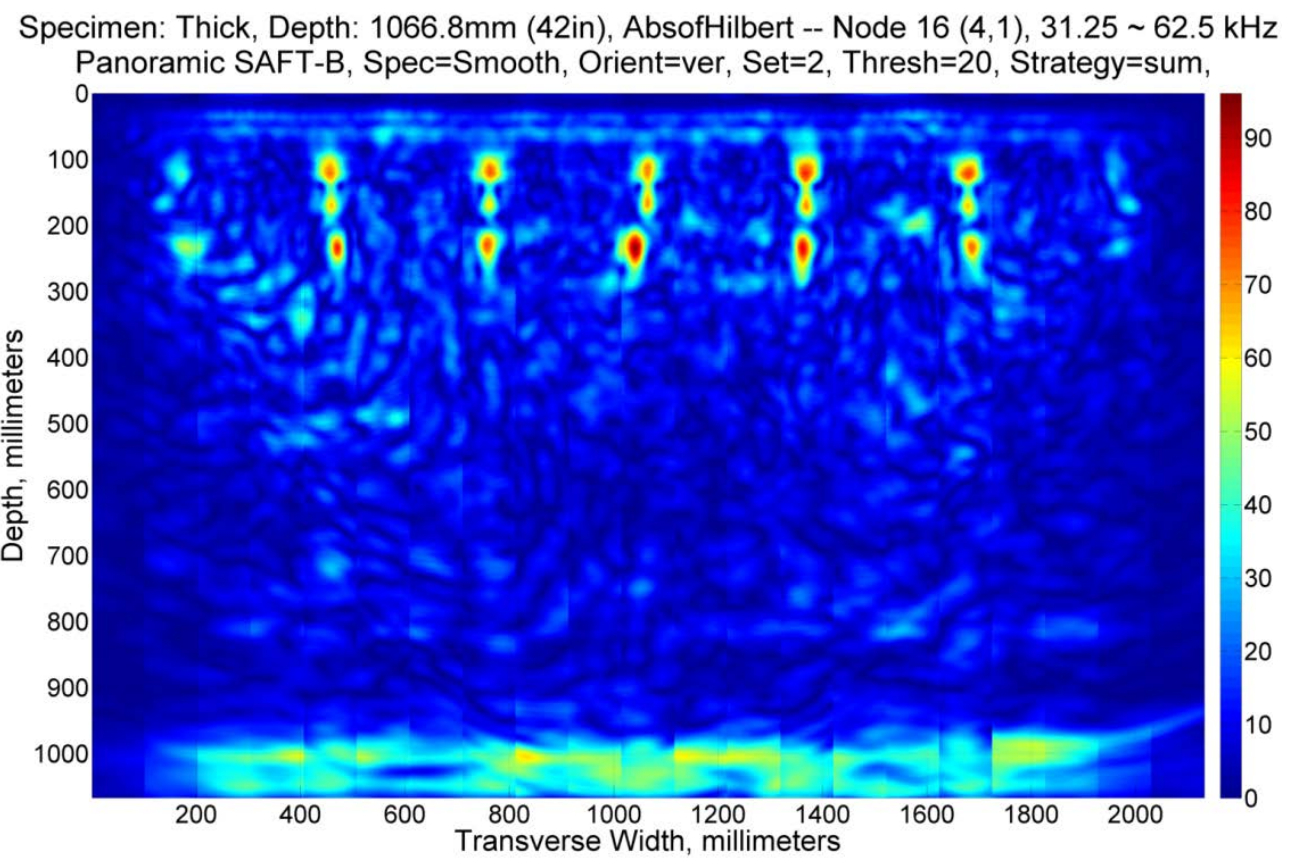

Fig. B. 102. Vertical Set 2, Node 16.

Specimen: Thick, Depth: 1066.8mm (42in), AbsofHilbert -- Node $18(4,3), 93.75 \sim 125 \mathrm{kHz}$ Panoramic SAFT-B, Spec=Smooth, Orient=ver, Set $=2$, Thresh=20, Strategy=sum,

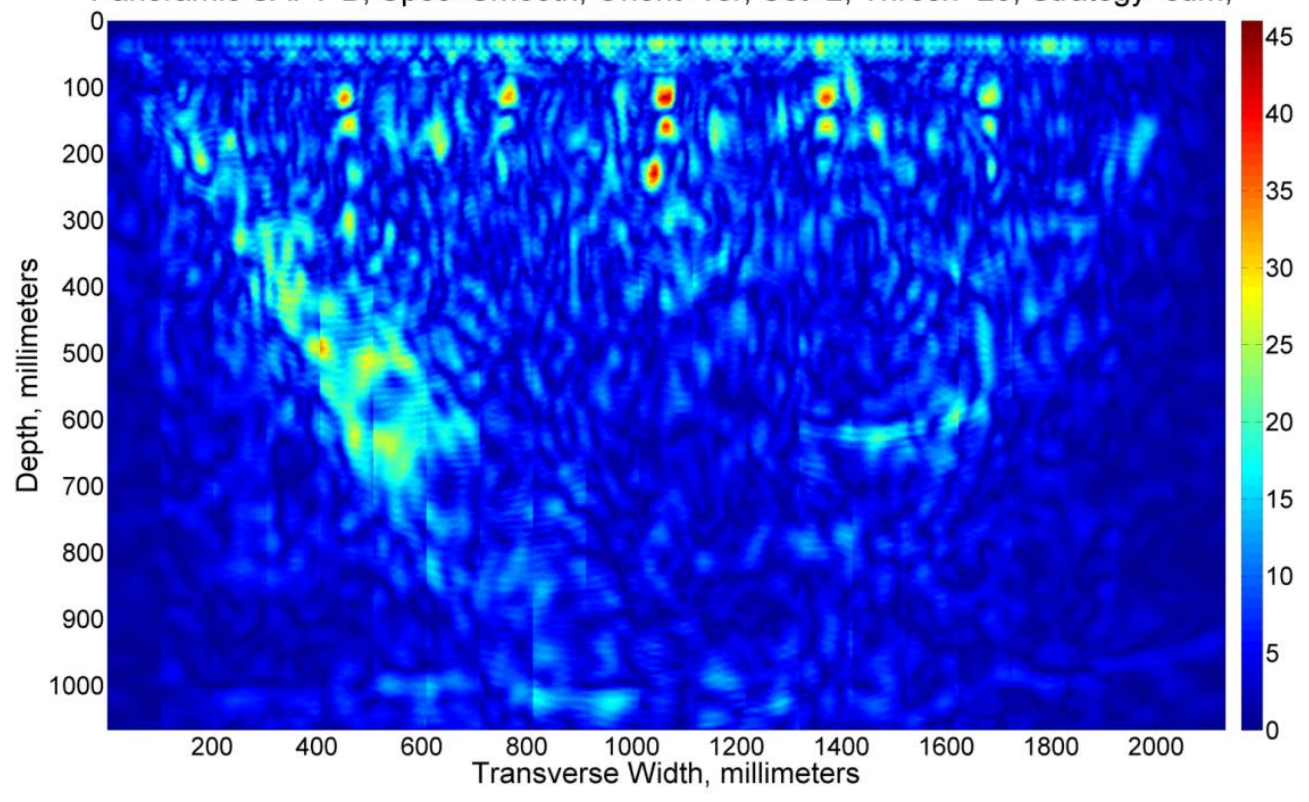

Fig. B. 103. Vertical Set 2, Node 18. 


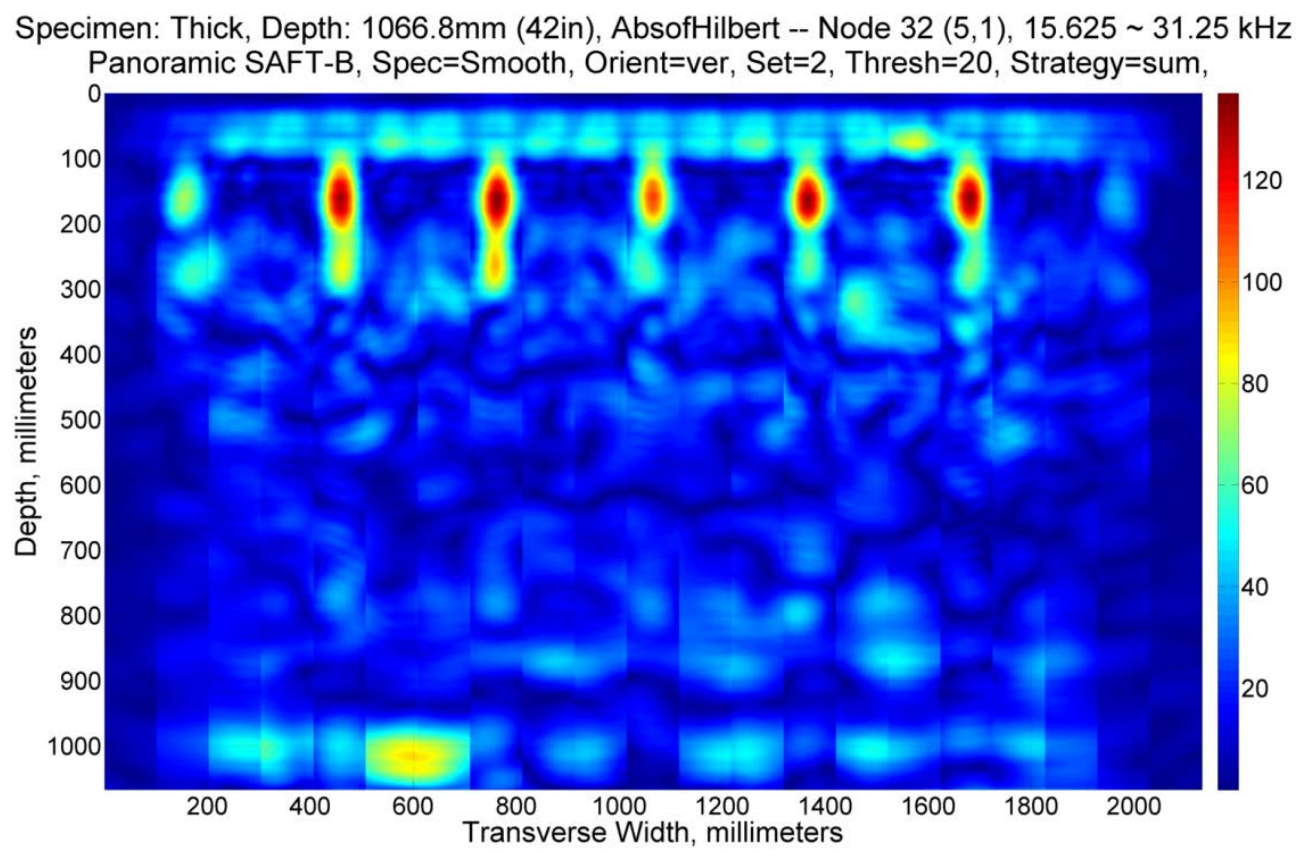

Fig. B. 104. Vertical Set 2, Node 32.

Specimen: Thick, Depth: 1066.8mm (42in), AbsofHilbert -- Node $34(5,3), 46.875 \sim 62.5 \mathrm{kHz}$ Panoramic SAFT-B, Spec=Smooth, Orient=ver, Set=2, Thresh=20, Strategy=sum,

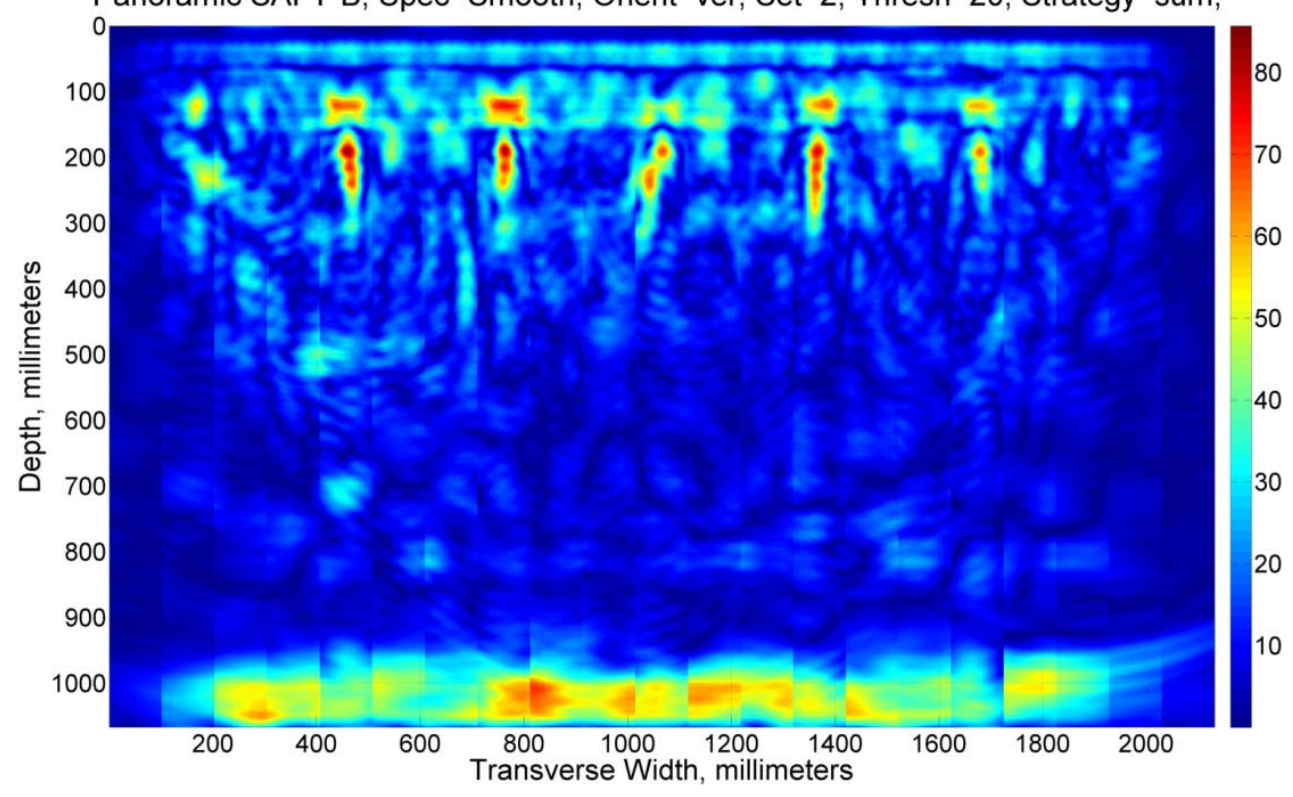

Fig. B. 105. Vertical Set 2, Node 34. 


\section{SET THREE - NO DEFECT}

Specimen: Thick, Depth: 1066.8mm (42in), AbsofHilbert -- Node $0(0,0), 0 \sim 500 \mathrm{kHz}$ Panoramic SAFT-B, Spec=Smooth, Orient=ver, Set=3, Thresh=20, Strategy=sum,

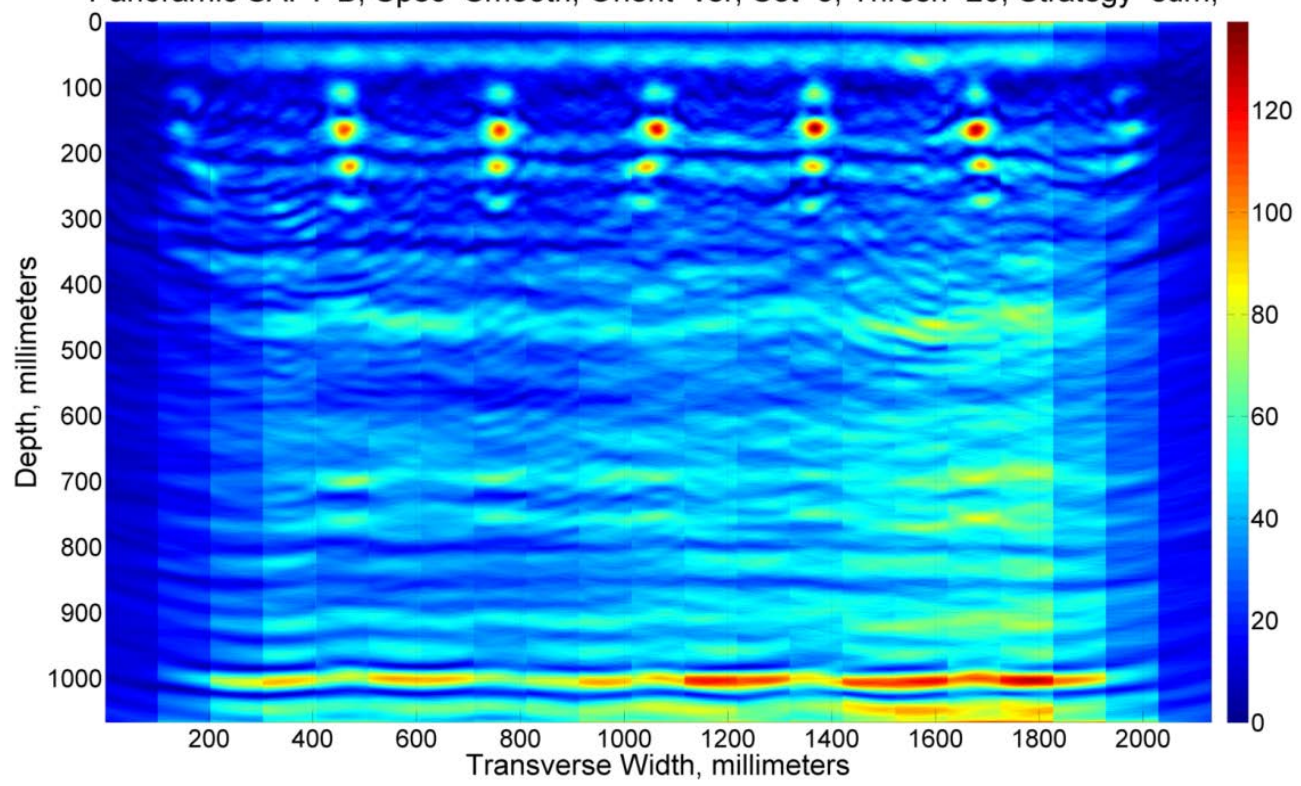

Fig. B. 106. Vertical Set 3, Node 0.

Specimen: Thick, Depth: 1066.8mm (42in), AbsofHilbert -- Node $16(4,1), 31.25 \sim 62.5 \mathrm{kHz}$ Panoramic SAFT-B, Spec=Smooth, Orient=ver, Set=3, Thresh=20, Strategy=sum,

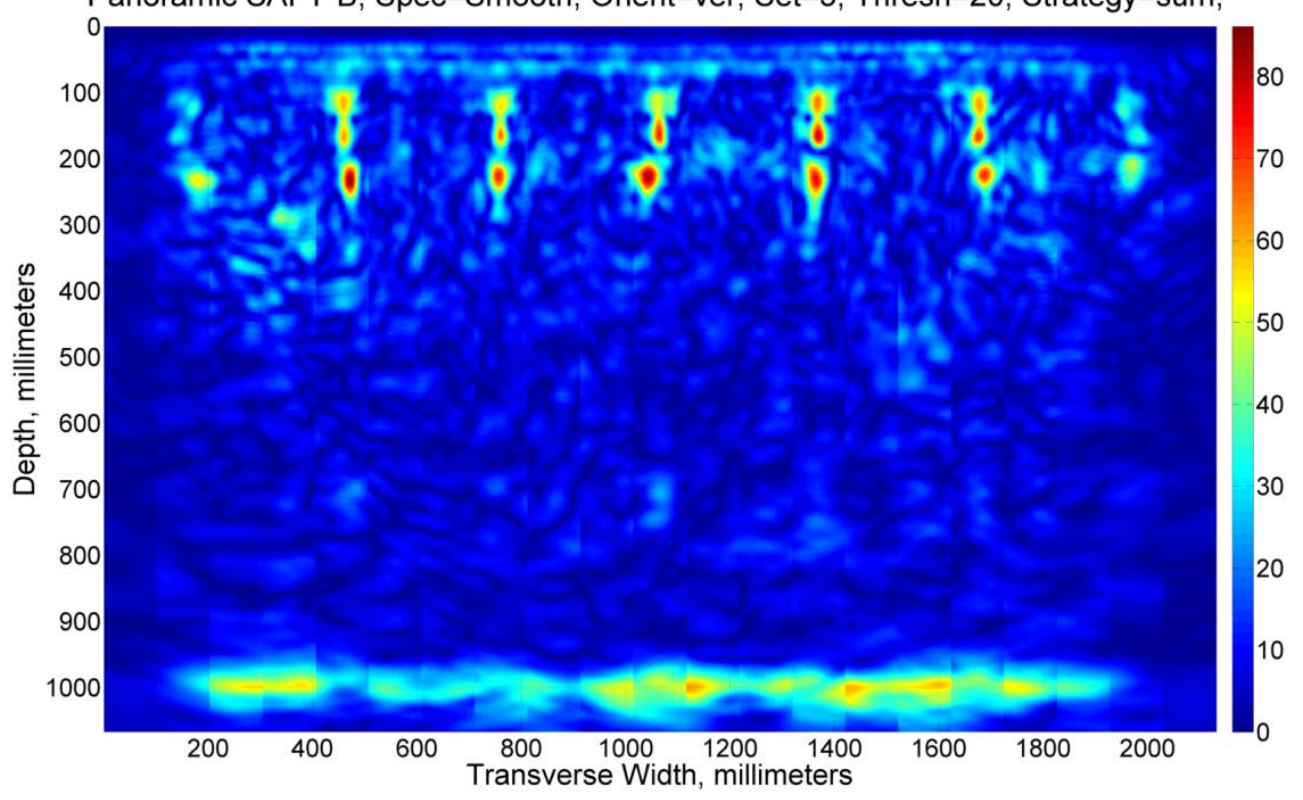

Fig. B. 107. Vertical Set 3, Node 16. 


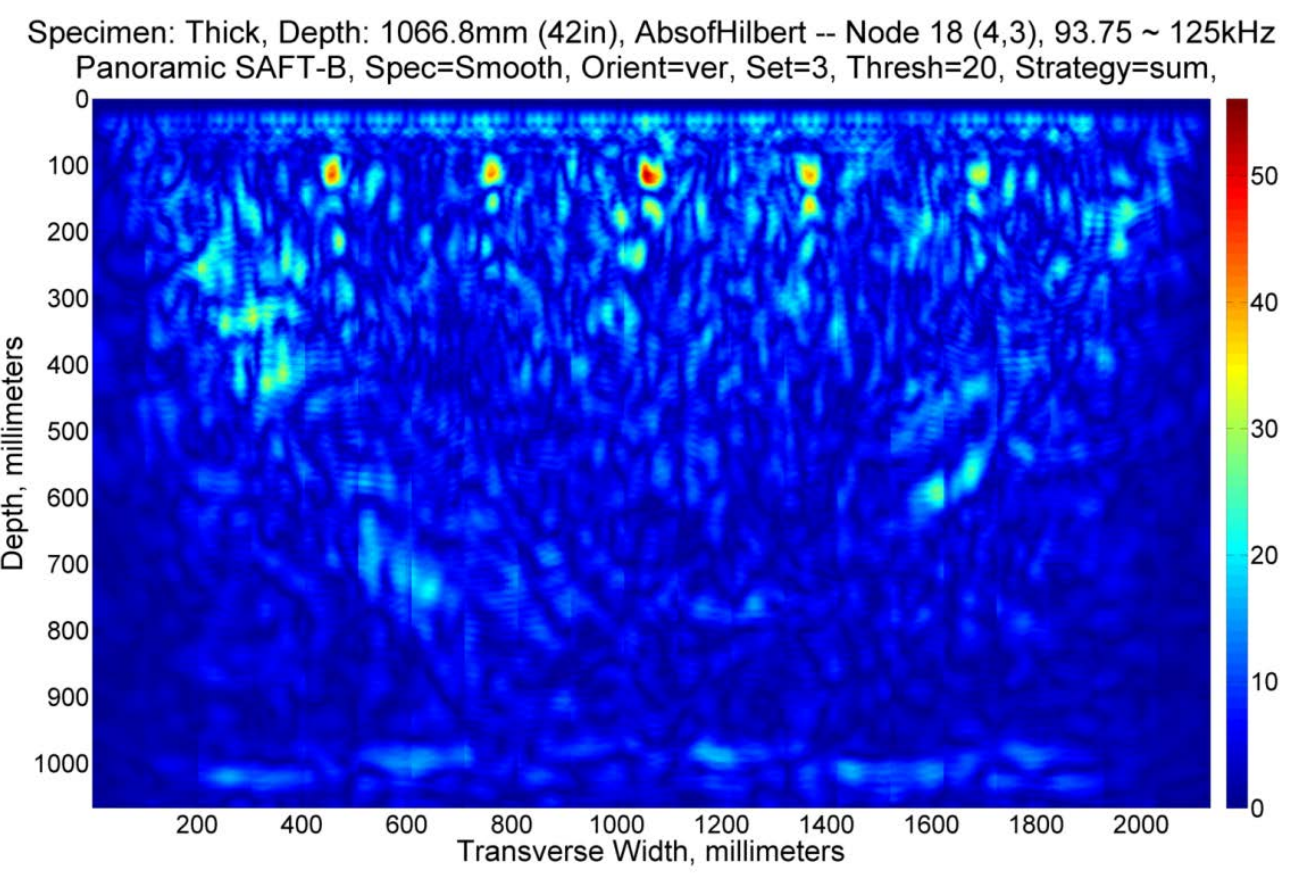

Fig. B. 108. Vertical Set 3, Node 18.

Specimen: Thick, Depth: 1066.8mm (42in), AbsofHilbert -- Node $32(5,1), 15.625 \sim 31.25 \mathrm{kHz}$ Panoramic SAFT-B, Spec=Smooth, Orient=ver, Set=3, Thresh=20, Strategy=sum,

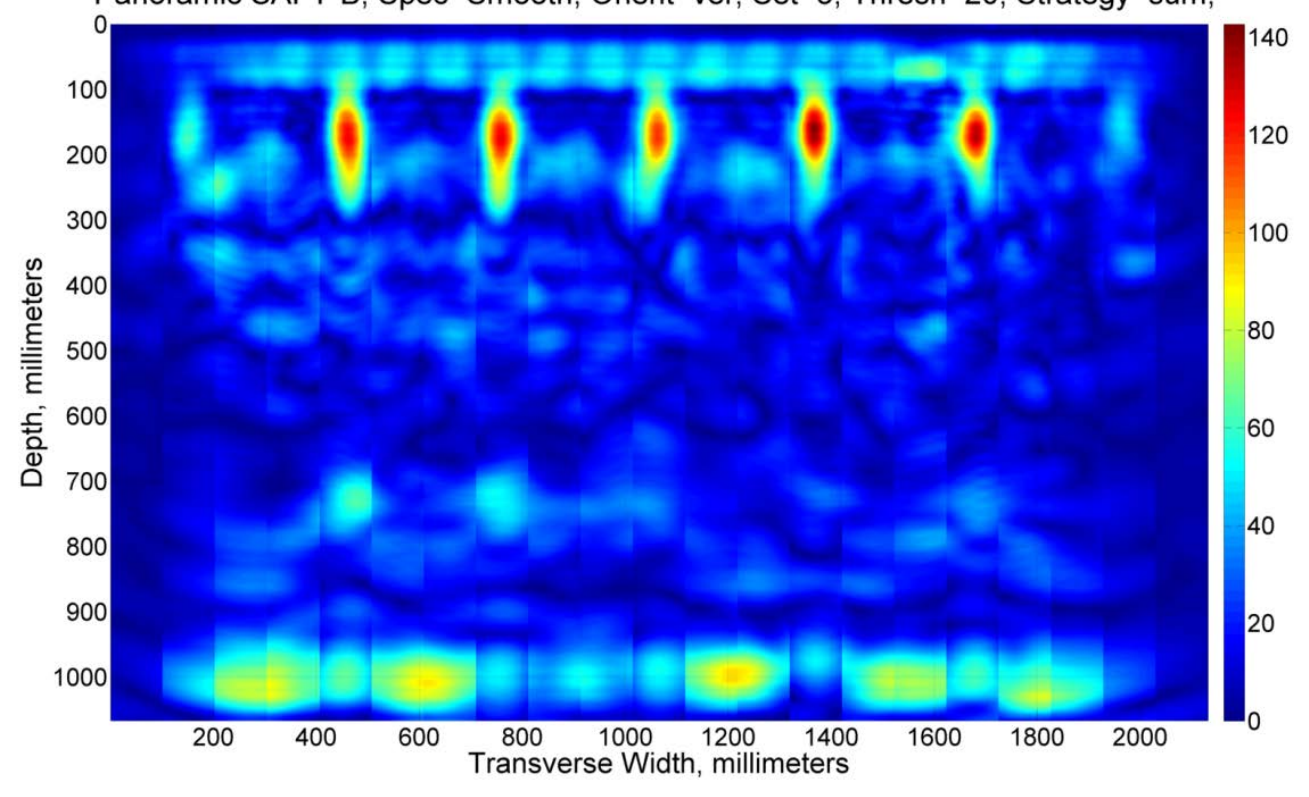

Fig. B. 109. Vertical Set 3, Node 32. 


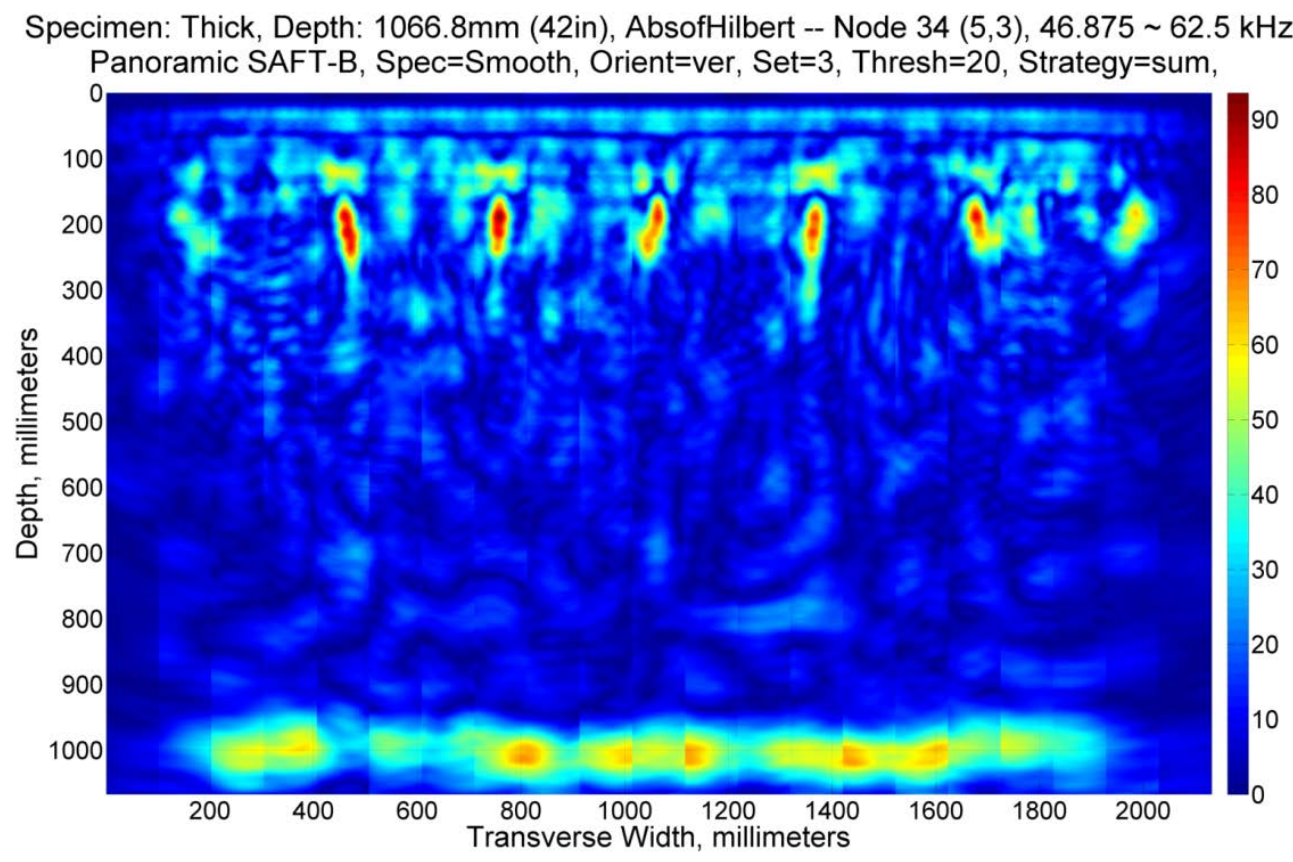

Fig. B. 110. Vertical Set 3, Node 34.

\section{SET FOUR - NO DEFECT}

Specimen: Thick, Depth: 1066.8mm (42in), AbsofHilbert -- Node $0(0,0), 0 \sim 500 \mathrm{kHz}$ Panoramic SAFT-B, Spec=Smooth, Orient=ver, Set=4, Thresh=20, Strategy=sum,

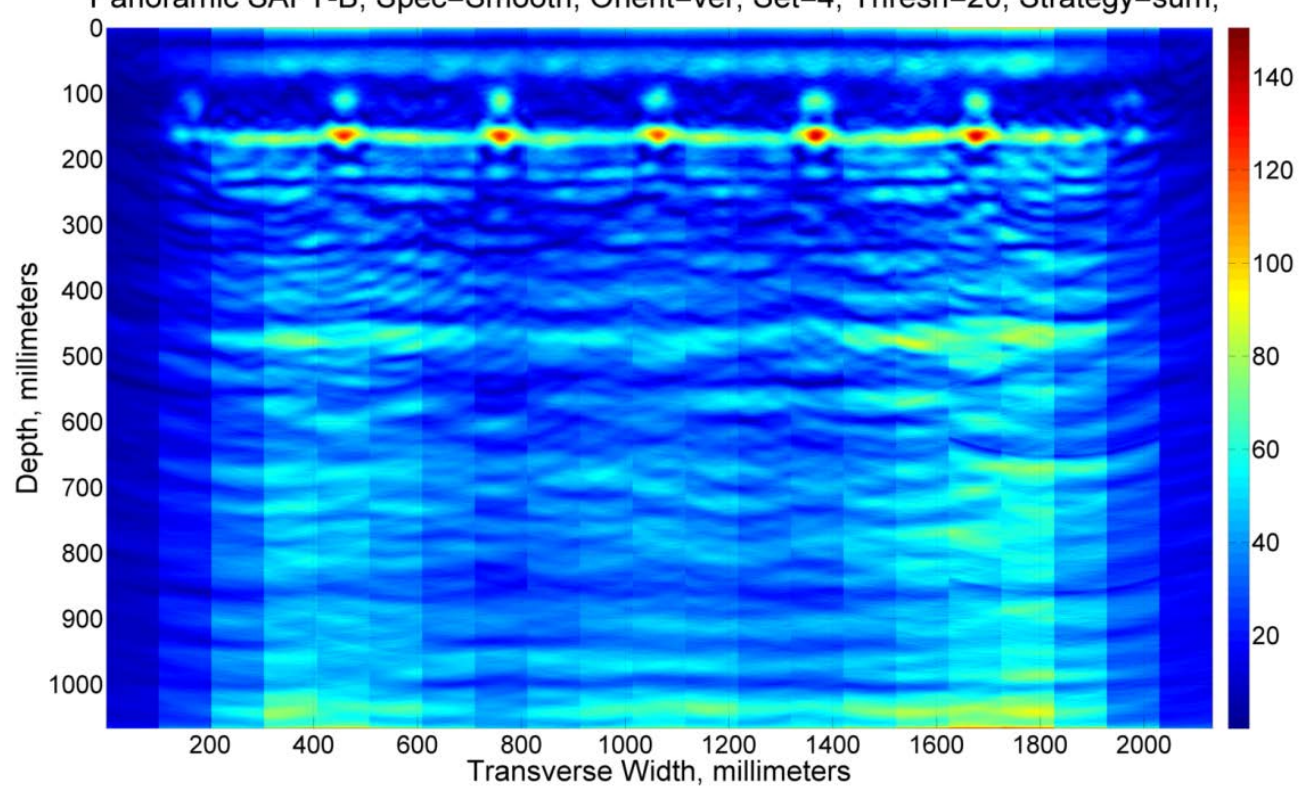

Fig. B. 111. Vertical Set 4, Node 0 . 


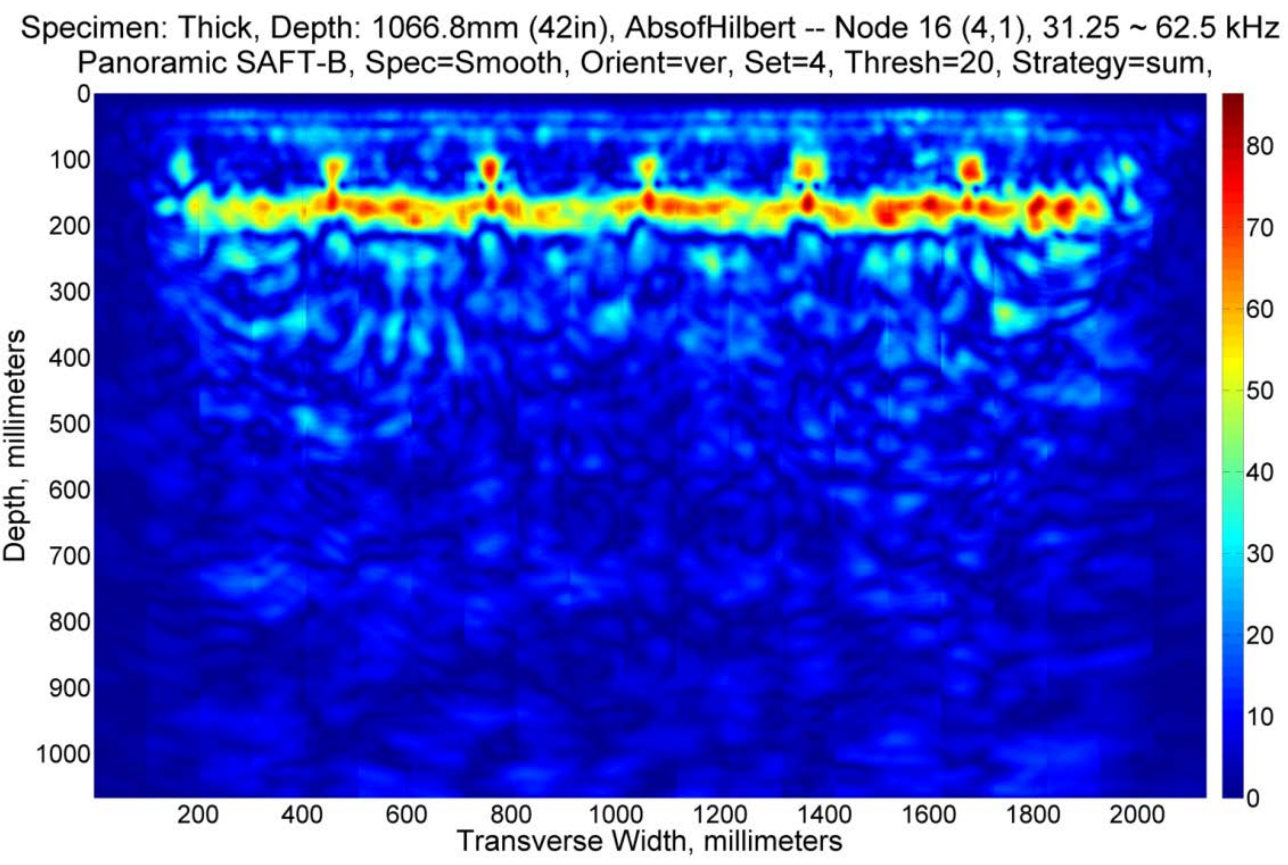

Fig. B. 112. Vertical Set 4, Node 16.

Specimen: Thick, Depth: 1066.8mm (42in), AbsofHilbert -- Node $18(4,3), 93.75 \sim 125 \mathrm{kHz}$ Panoramic SAFT-B, Spec=Smooth, Orient=ver, Set $=4$, Thresh $=20$, Strategy $=$ sum,

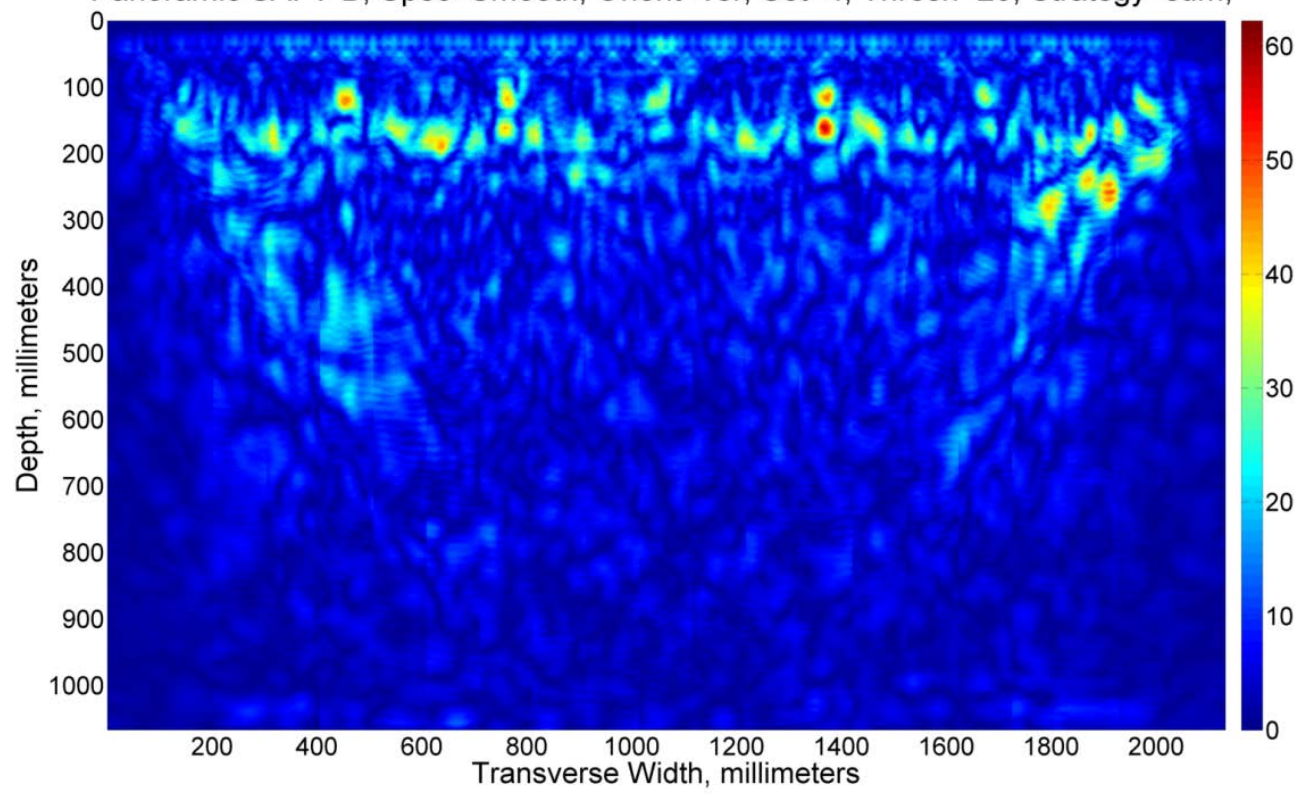

Fig. B. 113. Vertical Set 4, Node 18. 
Specimen: Thick, Depth: 1066.8mm (42in), AbsofHilbert -- Node 32 (5,1), $15.625 \sim 31.25 \mathrm{kHz}$ Panoramic SAFT-B, Spec=Smooth, Orient=ver, Set=4, Thresh=20, Strategy=sum,

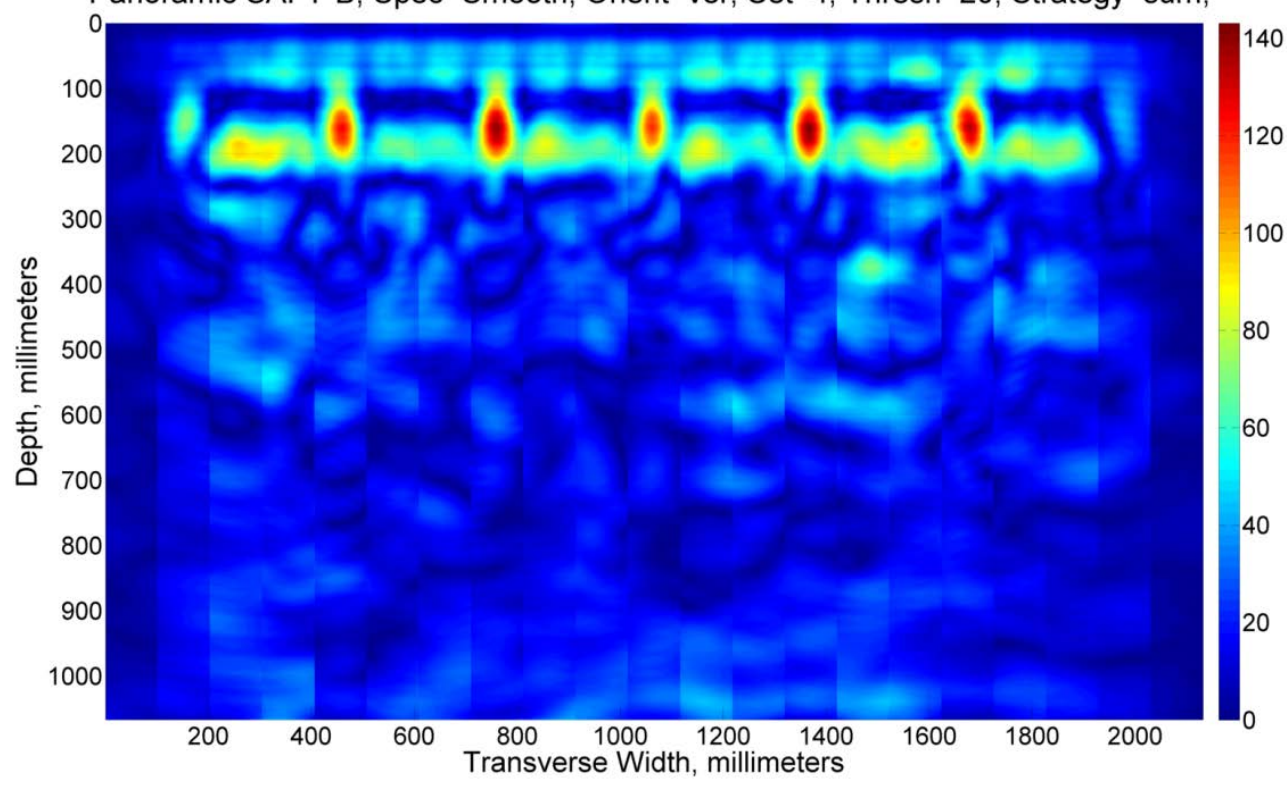

Fig. B. 114. Vertical Set 4, Node 32.

Specimen: Thick, Depth: 1066.8mm (42in), AbsofHilbert -- Node $34(5,3), 46.875 \sim 62.5 \mathrm{kHz}$ Panoramic SAFT-B, Spec=Smooth, Orient=ver, Set=4, Thresh=20, Strategy=sum,

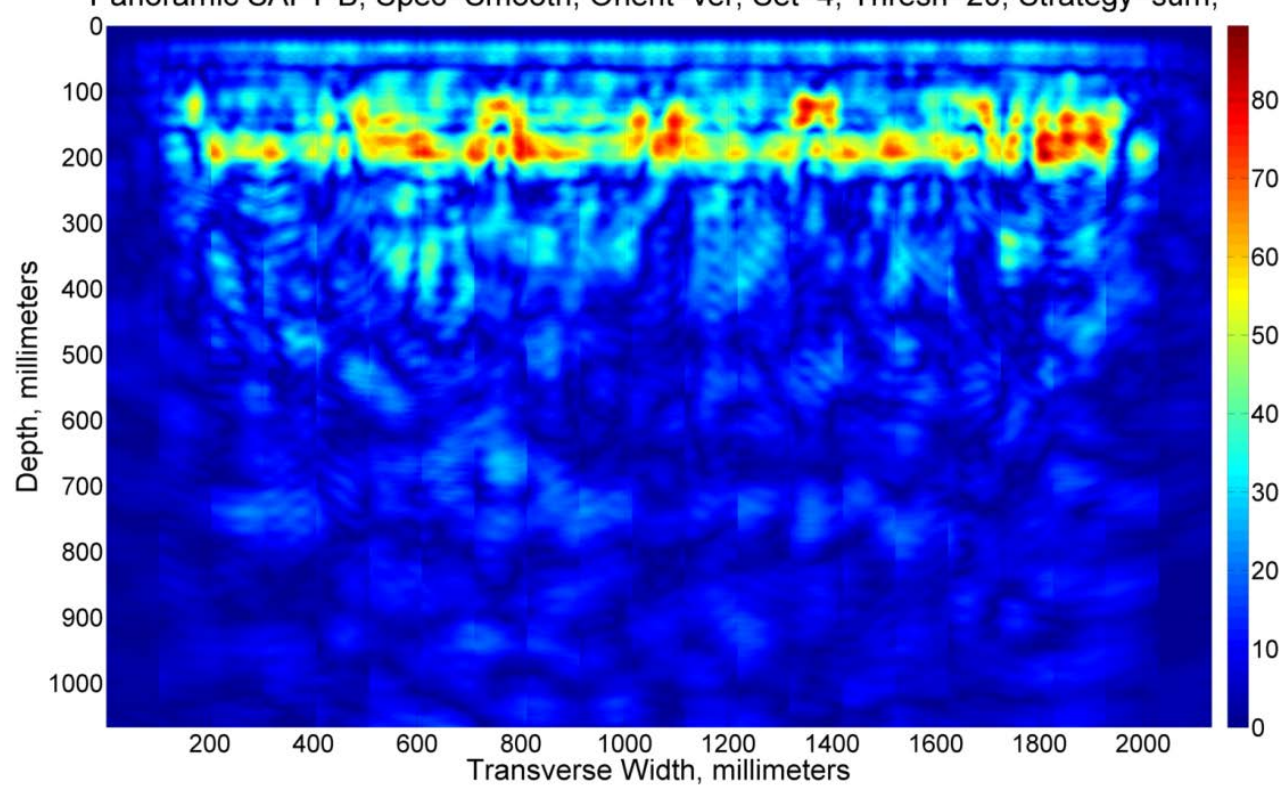

Fig. B. 115. Vertical Set 4, Node 34. 
Specimen: Thick, Depth: 1066.8mm (42in), AbsofHilbert -- Node $0(0,0), 0 \sim 500 \mathrm{kHz}$ Panoramic SAFT-B, Spec=Smooth, Orient=ver, Set=5, Thresh=20, Strategy=sum,

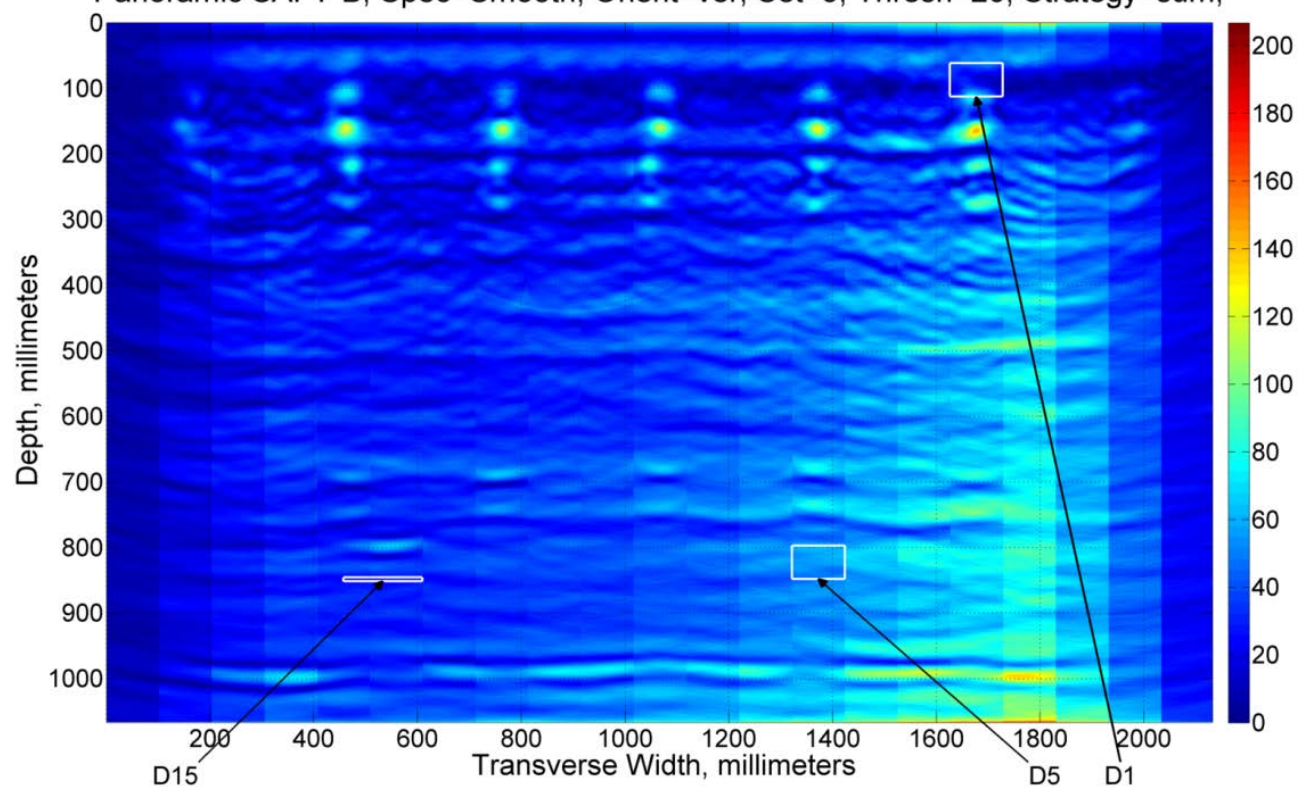

Fig. B. 116. Vertical Set 5, Node 0.

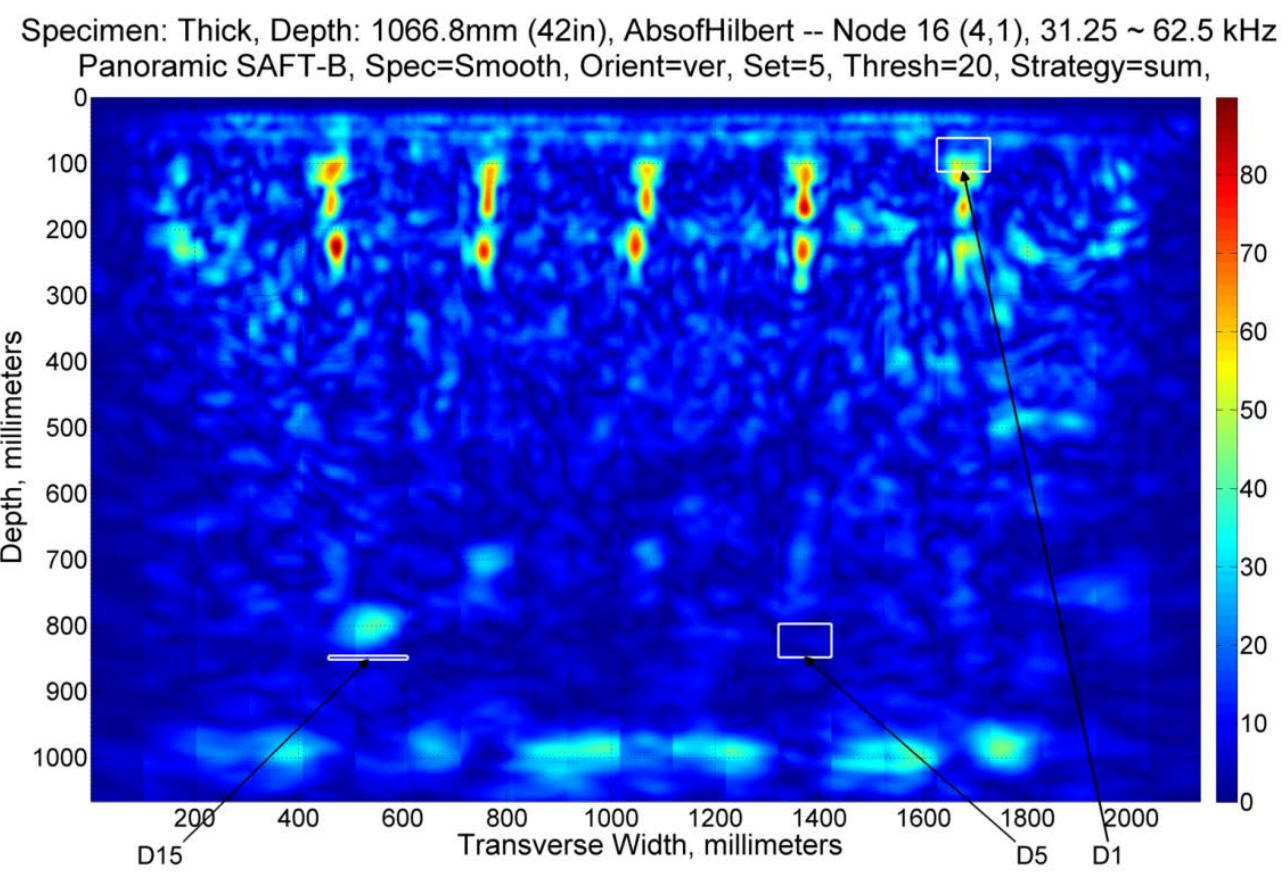

Fig. B. 117. Vertical Set 5, Node 16. 
Specimen: Thick, Depth: 1066.8mm (42in), AbsofHilbert -- Node $18(4,3), 93.75 \sim 125 \mathrm{kHz}$ Panoramic SAFT-B, Spec=Smooth, Orient=ver, Set=5, Thresh=20, Strategy=sum,

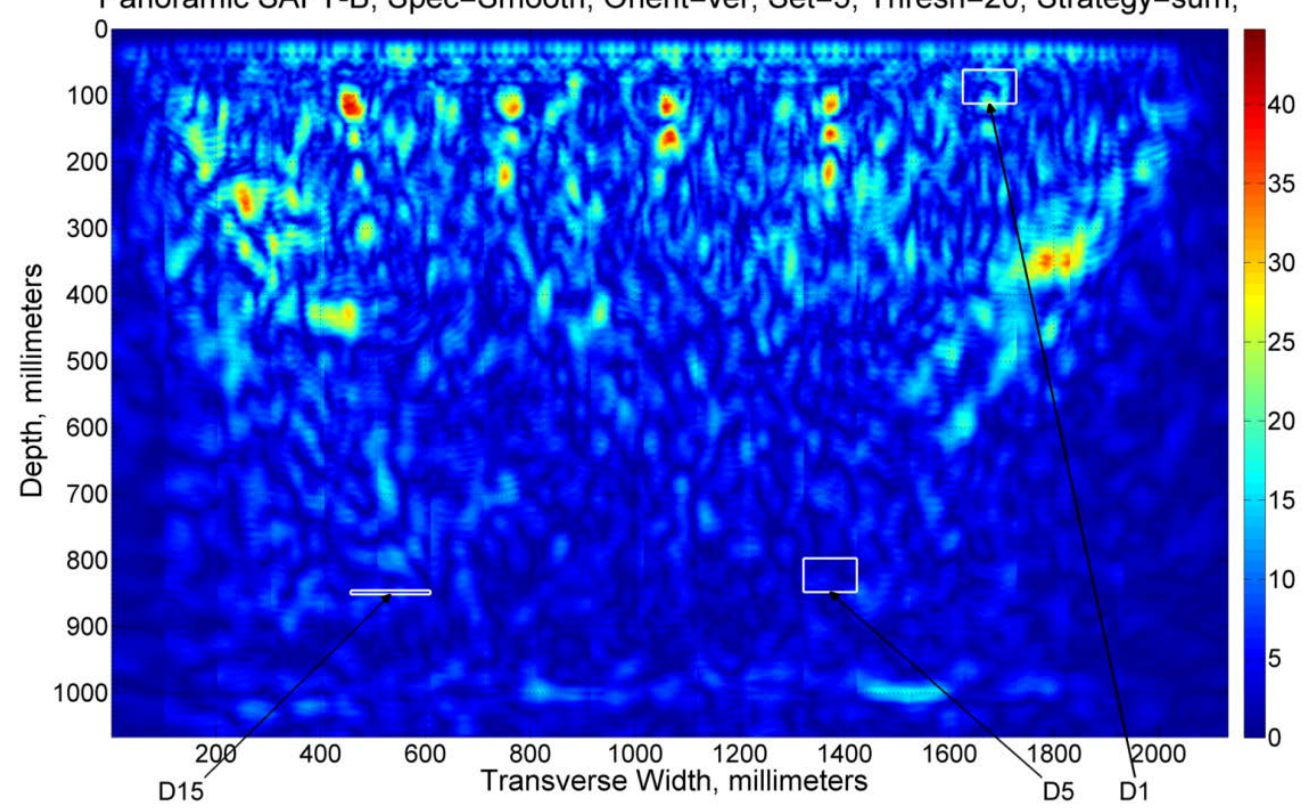

Fig. B. 118. Vertical Set 5, Node 18.

Specimen: Thick, Depth: 1066.8mm (42in), AbsofHilbert -- Node 32 (5,1), $15.625 \sim 31.25 \mathrm{kHz}$ Panoramic SAFT-B, Spec=Smooth, Orient=ver, Set=5, Thresh=20, Strategy=sum,

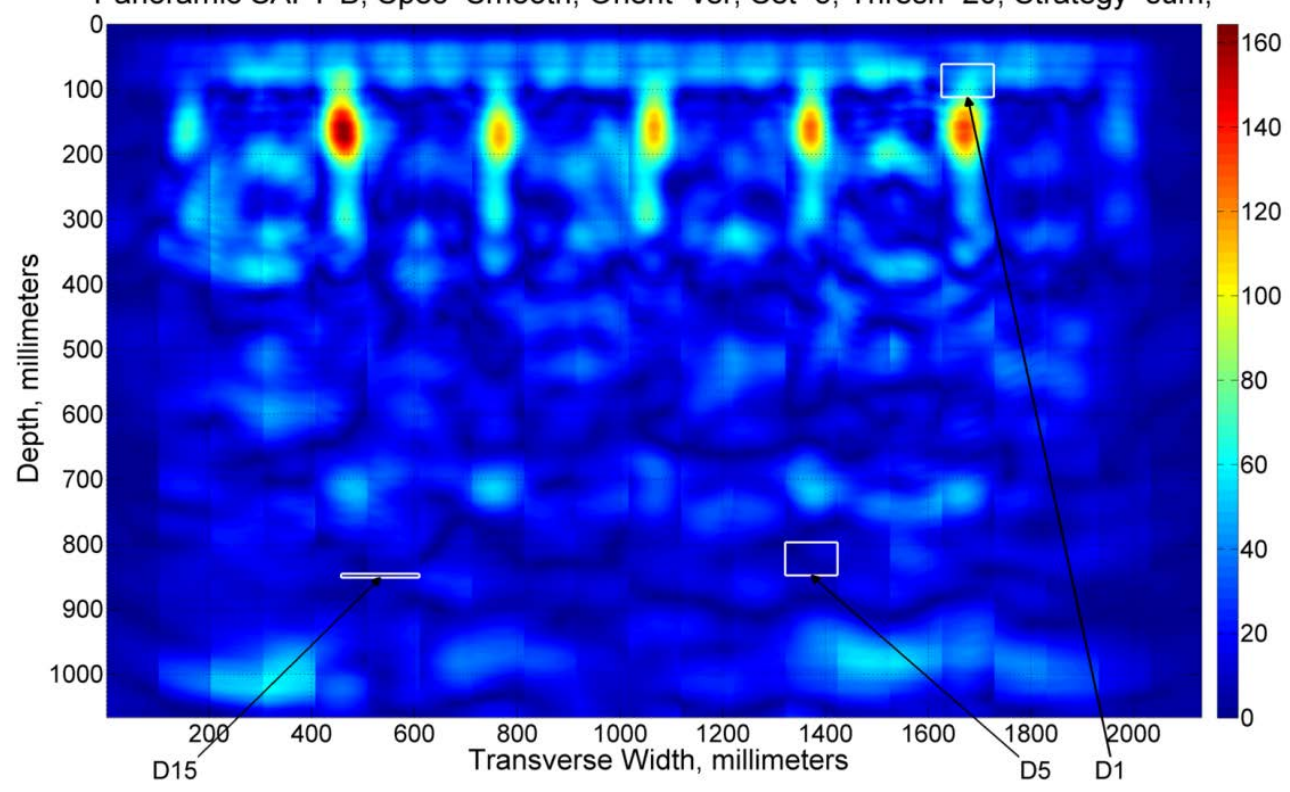

Fig. B. 119. Vertical Set 5, Node 32. 


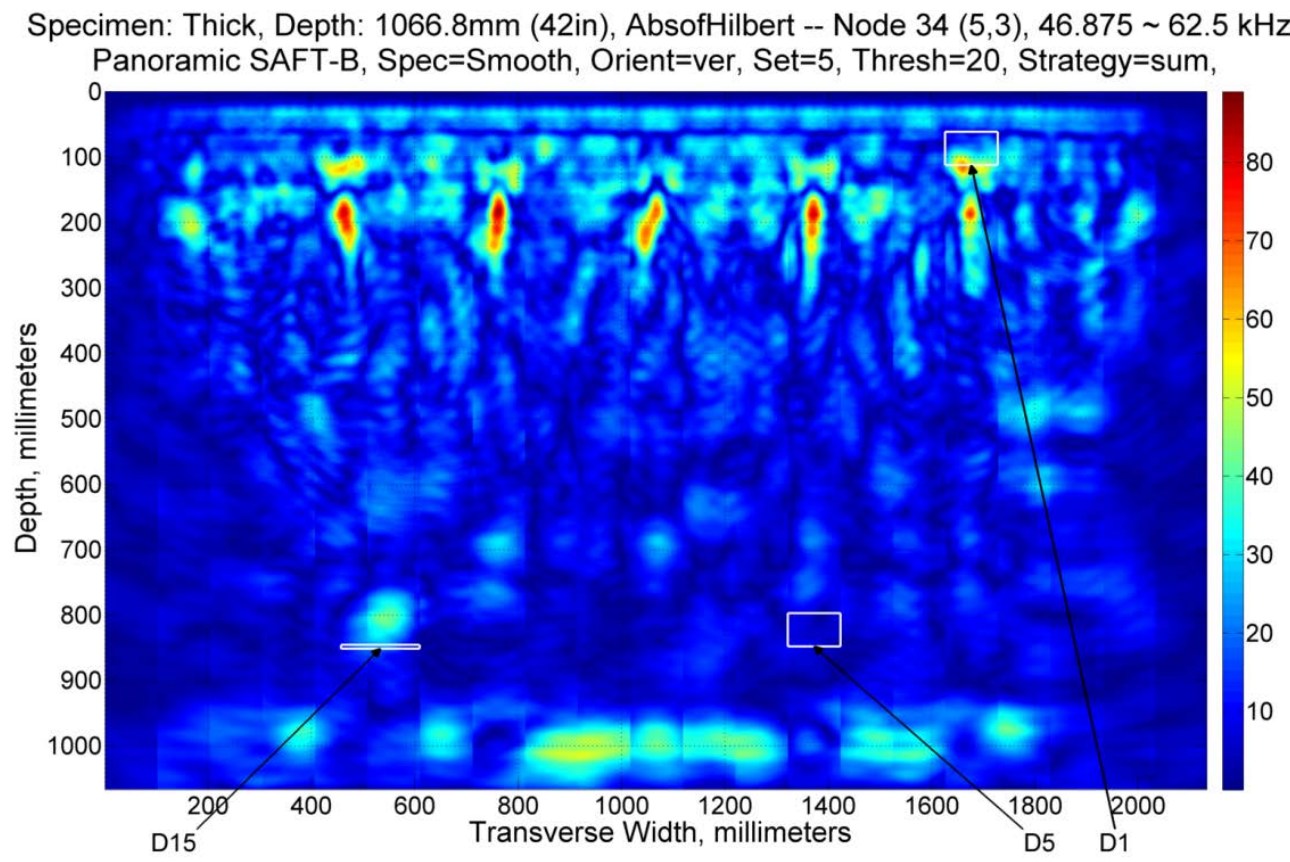

Fig. B. 120. Vertical Set 5, Node 34.

\section{SET SIX - DEFECT 1, DEFECT 5, DEFECT 6}

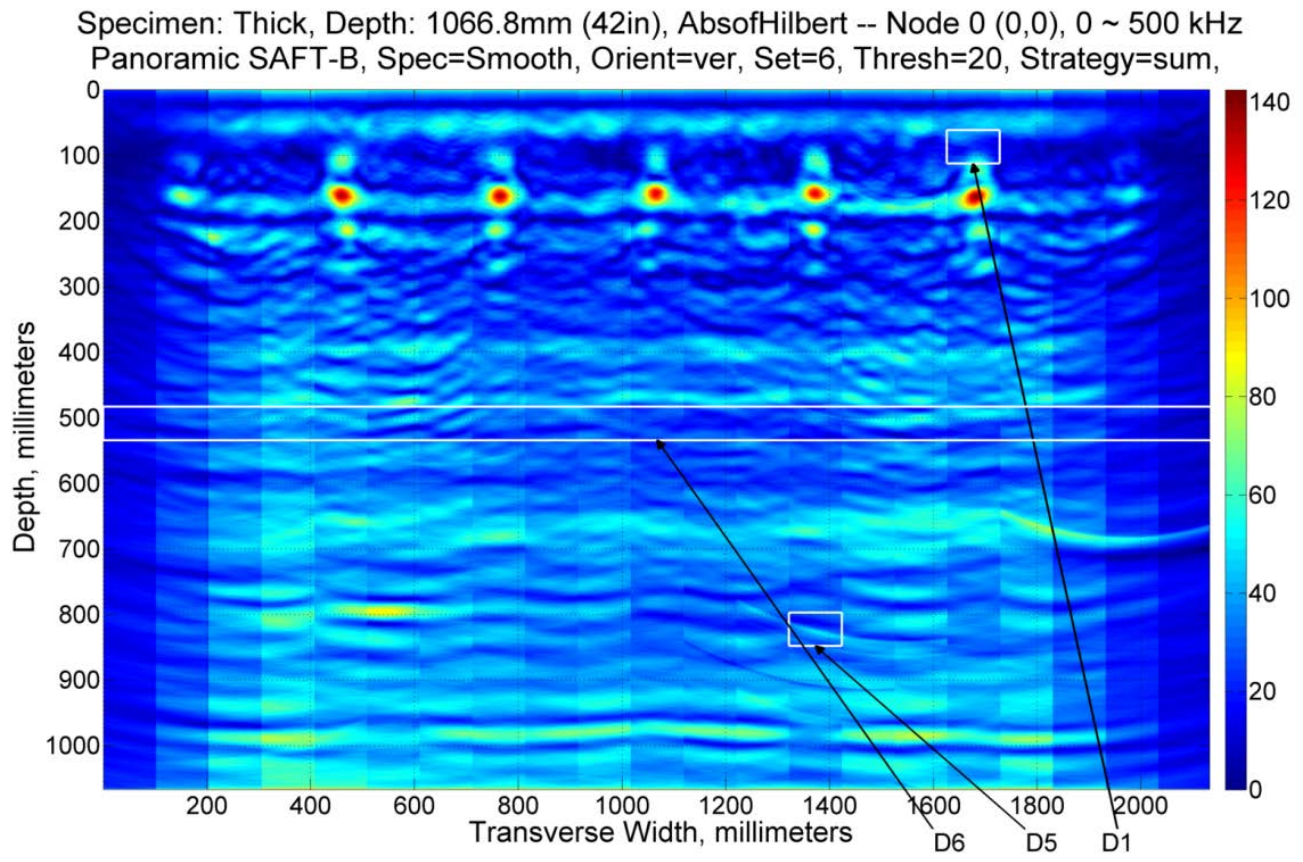

Fig. B. 121. Vertical Set 6, Node 0. 


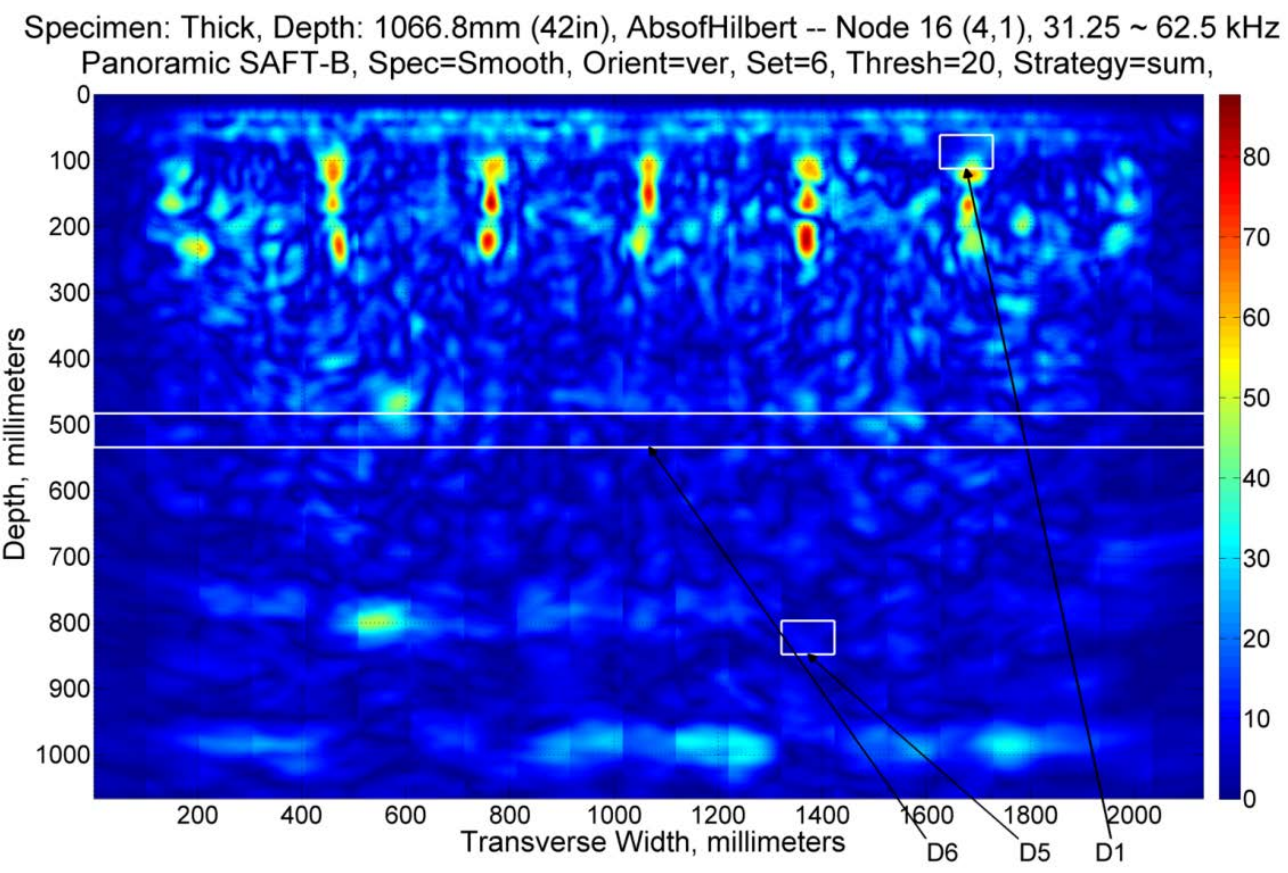

Fig. B. 122. Vertical Set 6, Node 16.

Specimen: Thick, Depth: 1066.8mm (42in), AbsofHilbert -- Node $18(4,3), 93.75 \sim 125 \mathrm{kHz}$

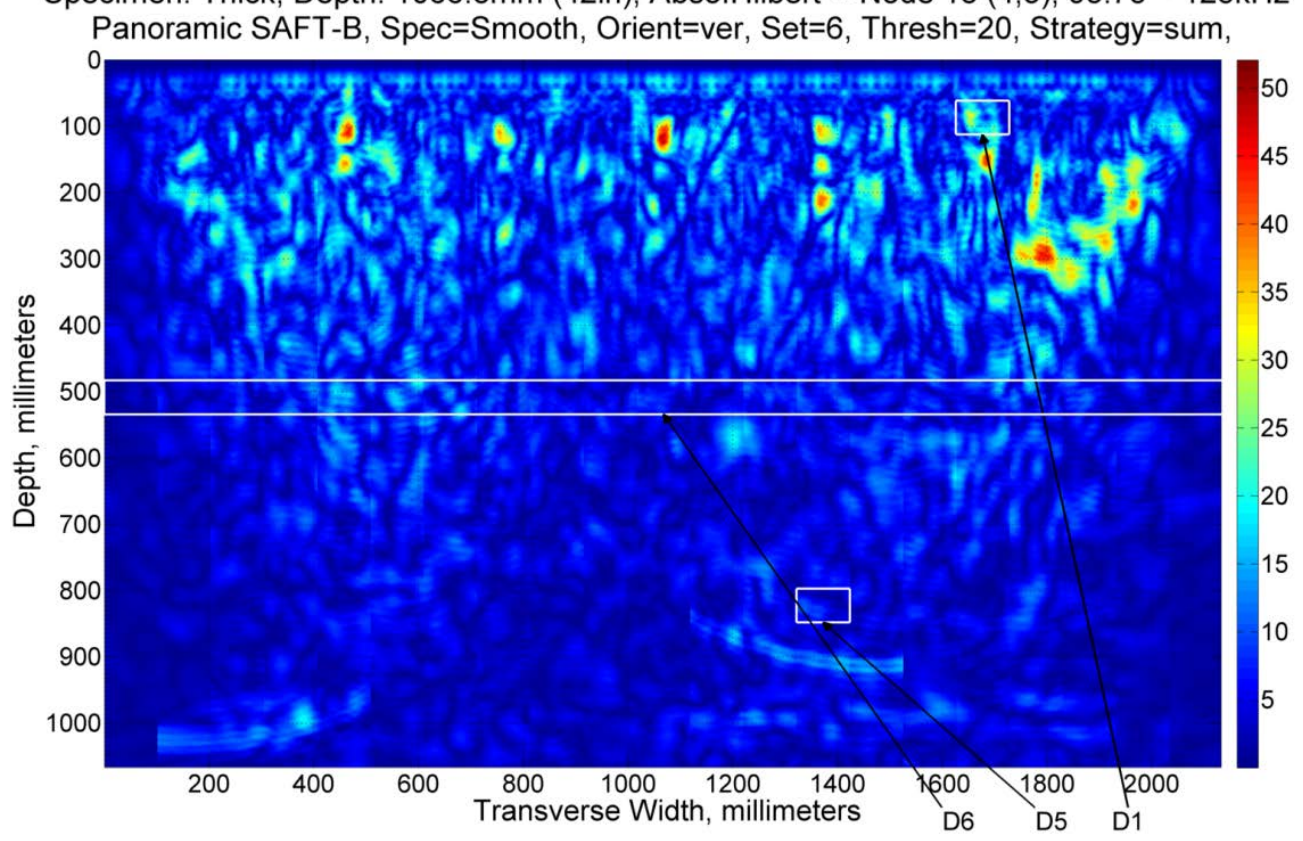

Fig. B. 123. Vertical Set 6, Node 18. 


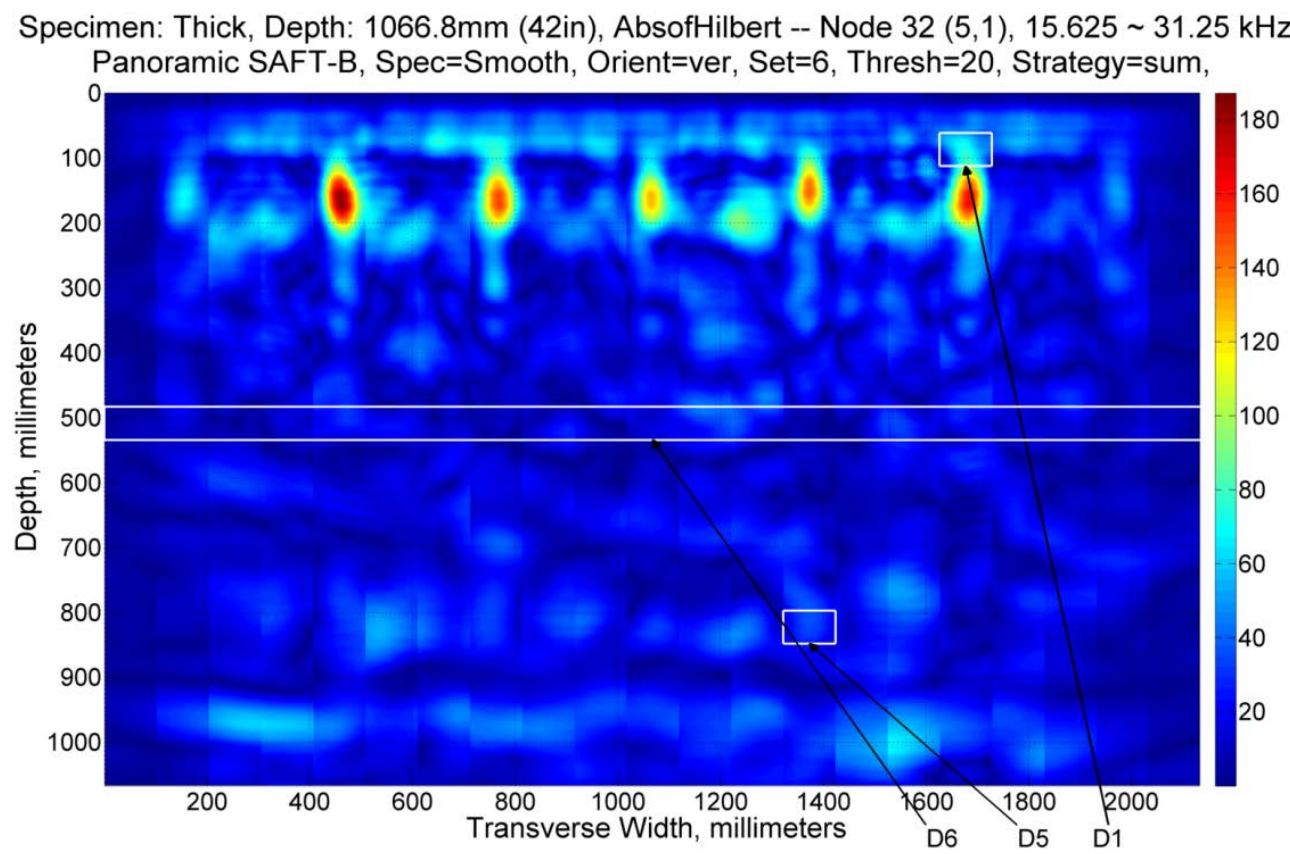

Fig. B. 124. Vertical Set 6, Node 32.

Specimen: Thick, Depth: 1066.8mm (42in), AbsofHilbert -- Node 34 (5,3), $46.875 \sim 62.5 \mathrm{kHz}$ Panoramic SAFT-B, Spec=Smooth, Orient=ver, Set=6, Thresh=20, Strategy=sum,

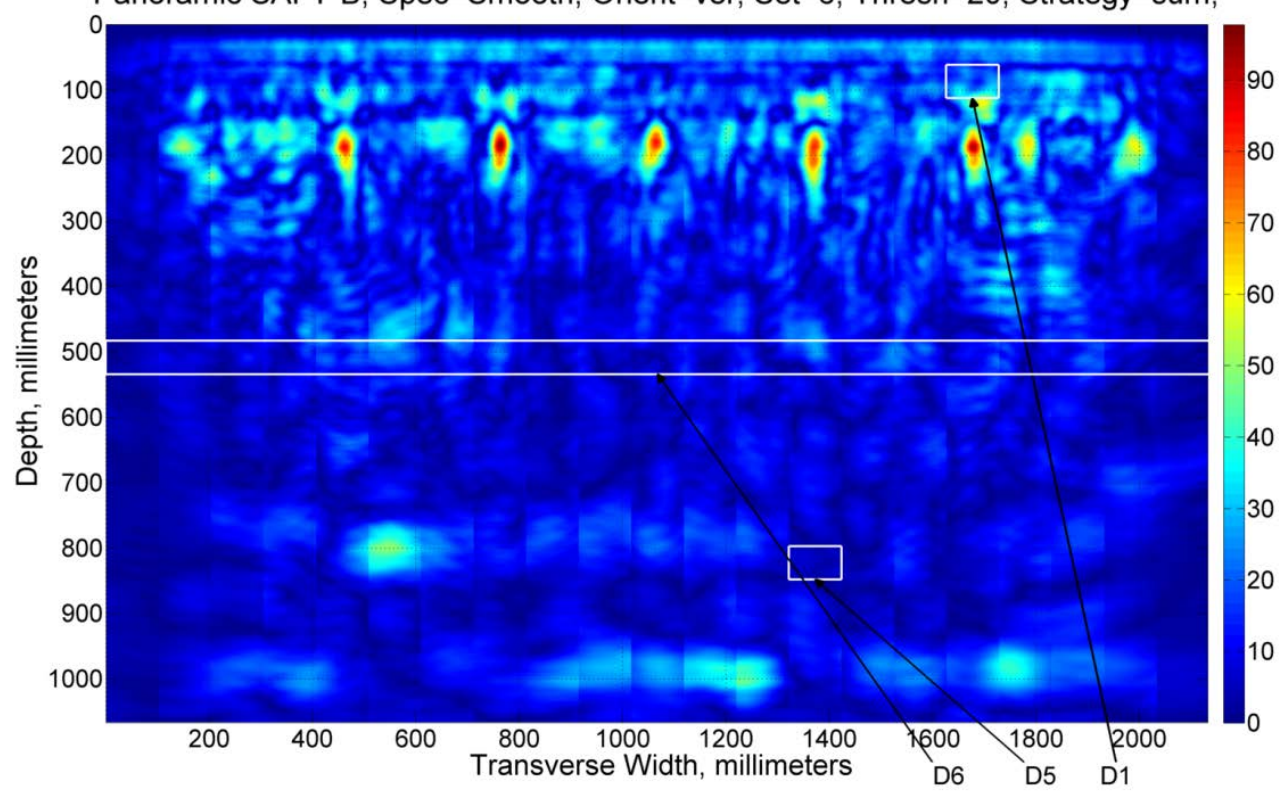

Fig. B. 125. Vertical Set 6, Node 34. 


\section{SET SEVEN - DEFECT 6}

Specimen: Thick, Depth: 1066.8mm (42in), AbsofHilbert -- Node $0(0,0), 0 \sim 500 \mathrm{kHz}$ Panoramic SAFT-B, Spec=Smooth, Orient=ver, Set=7, Thresh=20, Strategy=sum,

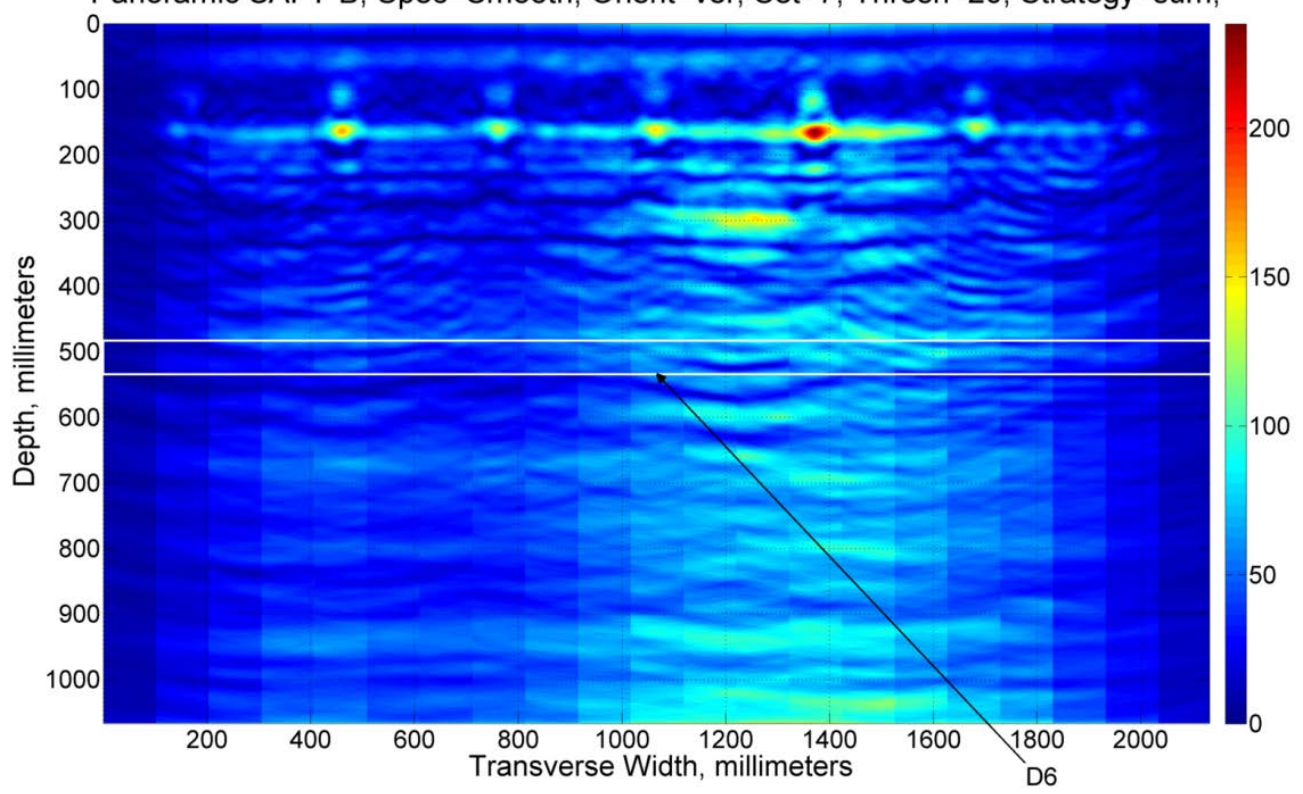

Fig. B. 126. Vertical Set 7, Node 0.

Specimen: Thick, Depth: 1066.8mm (42in), AbsofHilbert -- Node $16(4,1), 31.25 \sim 62.5 \mathrm{kHz}$ Panoramic SAFT-B, Spec=Smooth, Orient=ver, Set=7, Thresh=20, Strategy=sum,

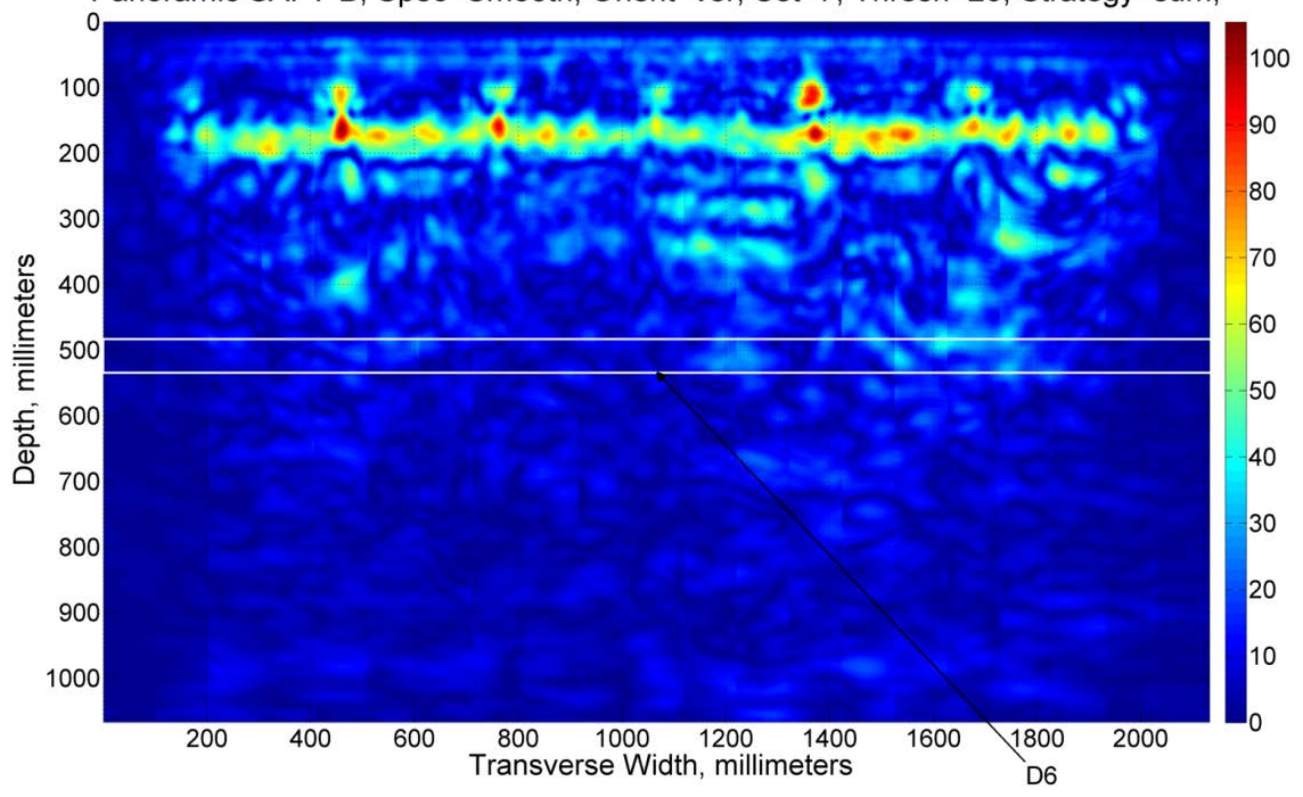

Fig. B. 127. Vertical Set 7, Node 16. 
Specimen: Thick, Depth: 1066.8mm (42in), AbsofHilbert -- Node $18(4,3), 93.75 \sim 125 \mathrm{kHz}$ Panoramic SAFT-B, Spec=Smooth, Orient=ver, Set=7, Thresh=20, Strategy=sum,

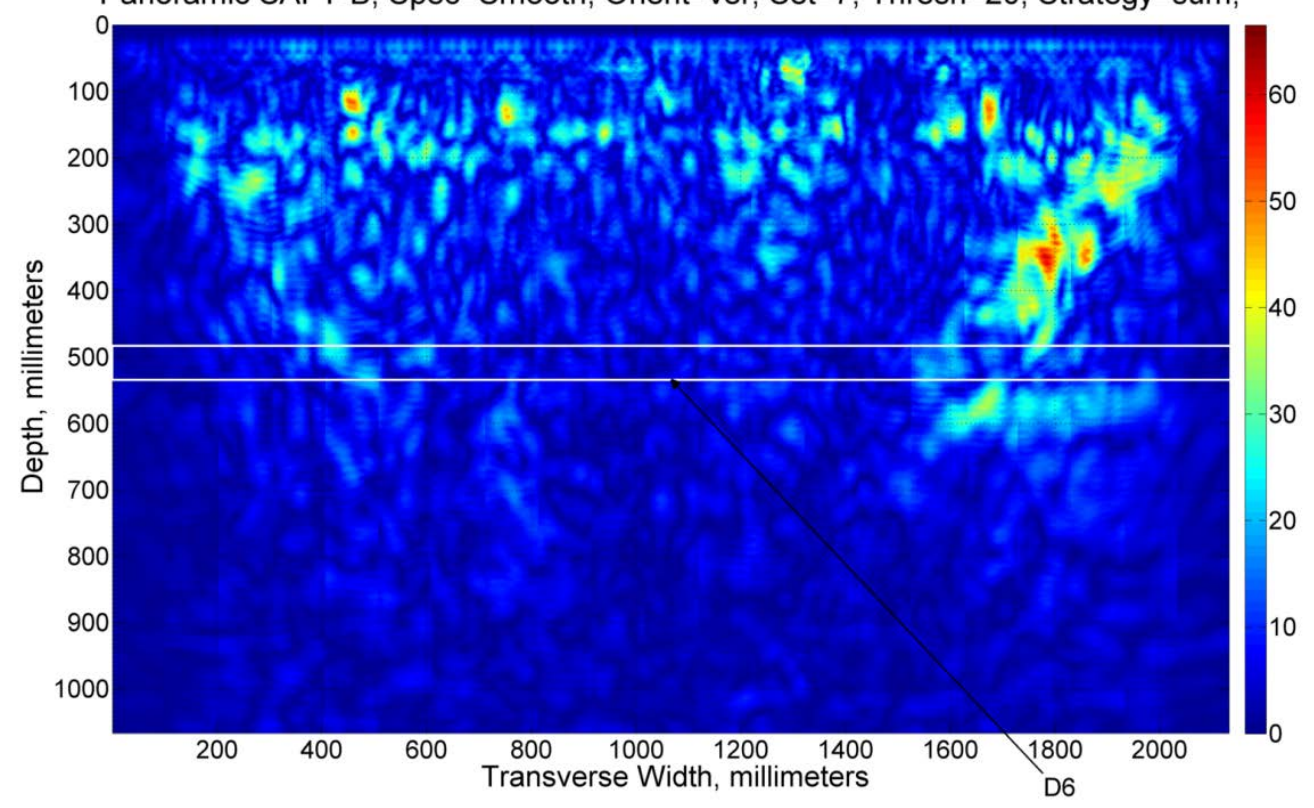

Fig. B. 128. Vertical Set 7, Node 18.

Specimen: Thick, Depth: 1066.8mm (42in), AbsofHilbert -- Node 32 (5,1), $15.625 \sim 31.25 \mathrm{kHz}$ Panoramic SAFT-B, Spec=Smooth, Orient=ver, Set=7, Thresh=20, Strategy=sum,

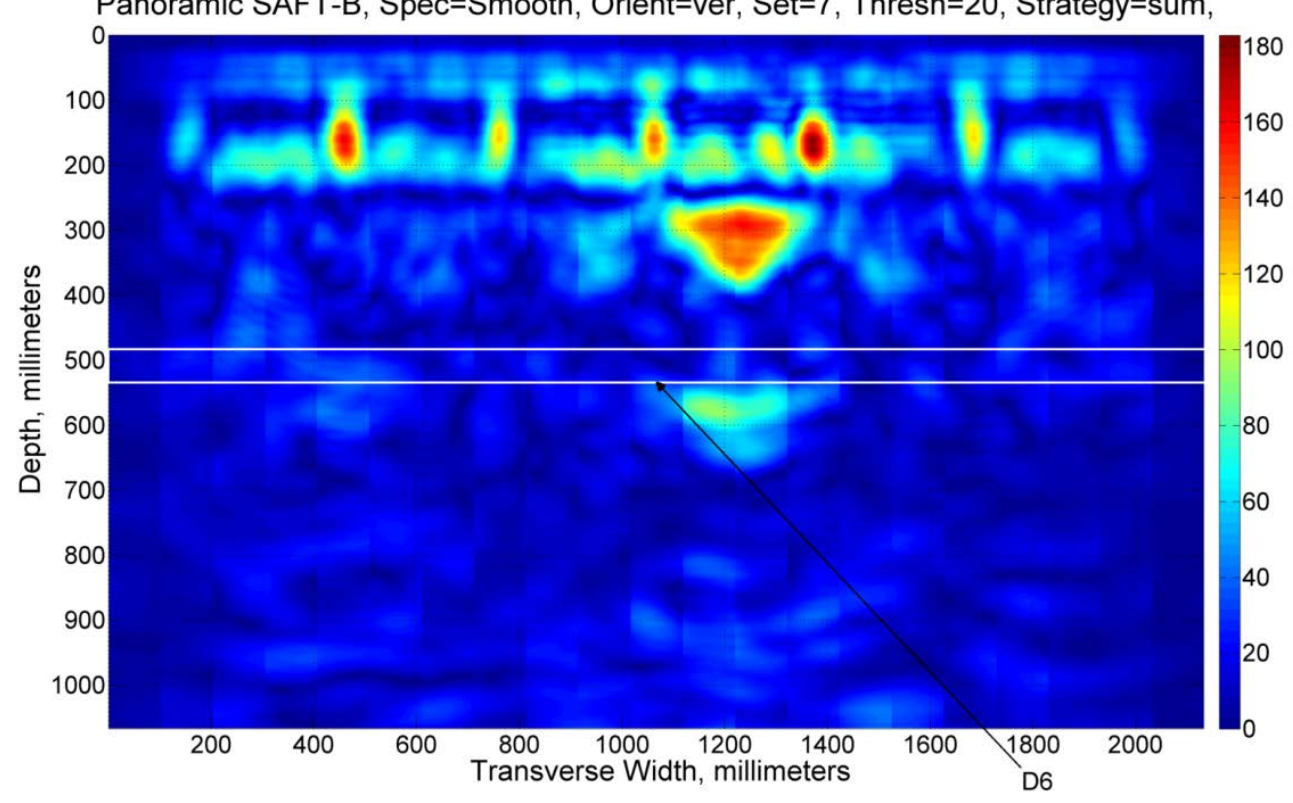

Fig. B. 129. Vertical Set 7, Node 32. 


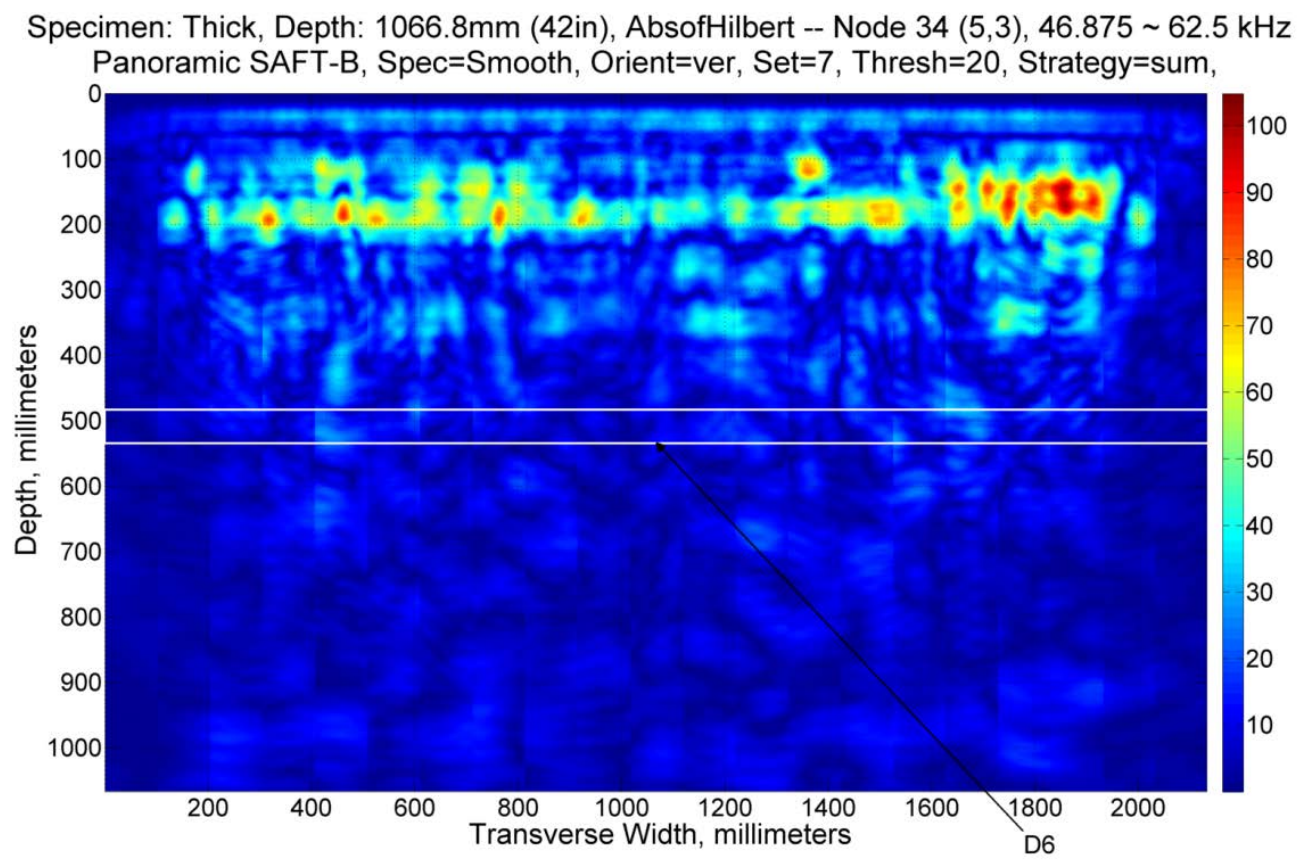

Fig. B. 130. Vertical Set 7, Node 34.

\section{SET EIGHT - DEFECT 8, DEFECT 11, DEFECT 13}

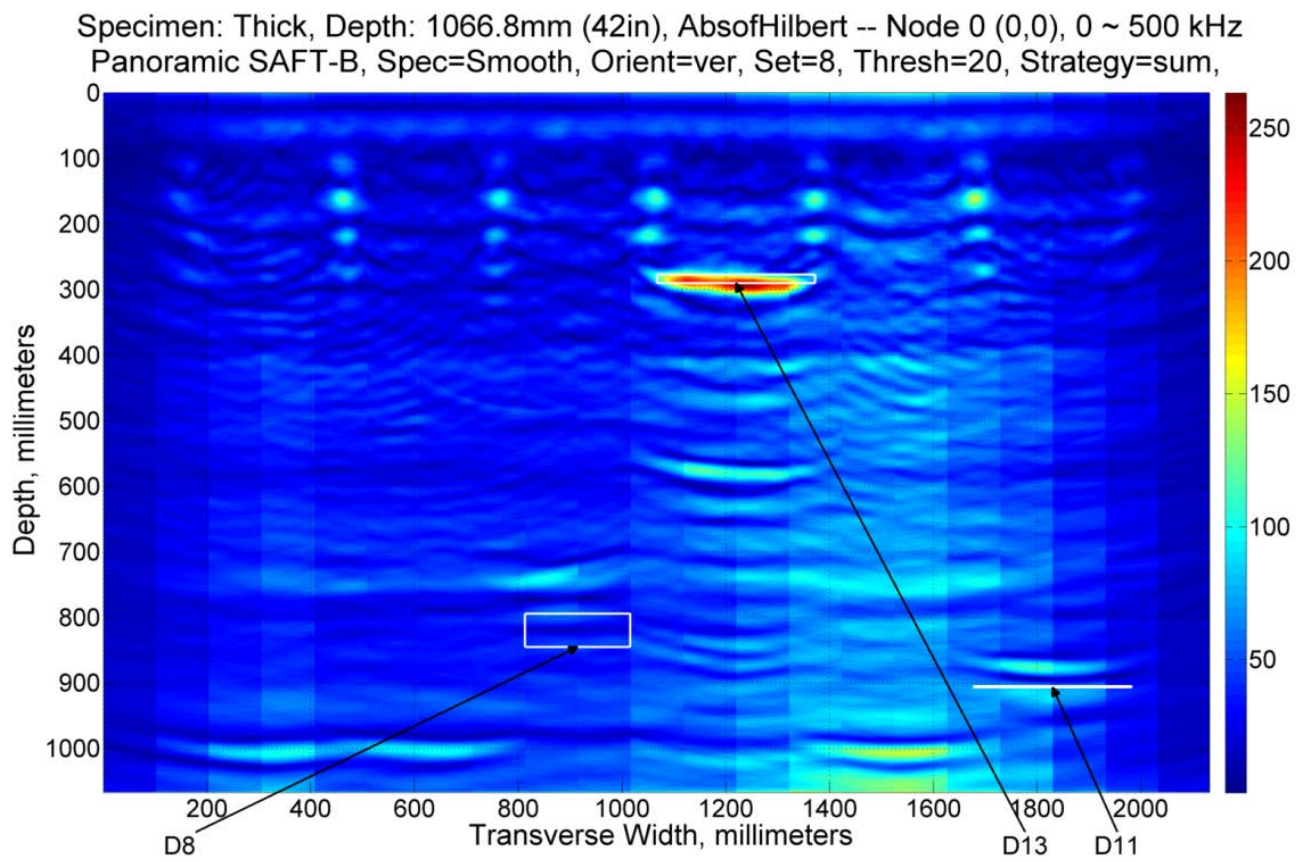

Fig. B. 131. Vertical Set 8, Node 0. 


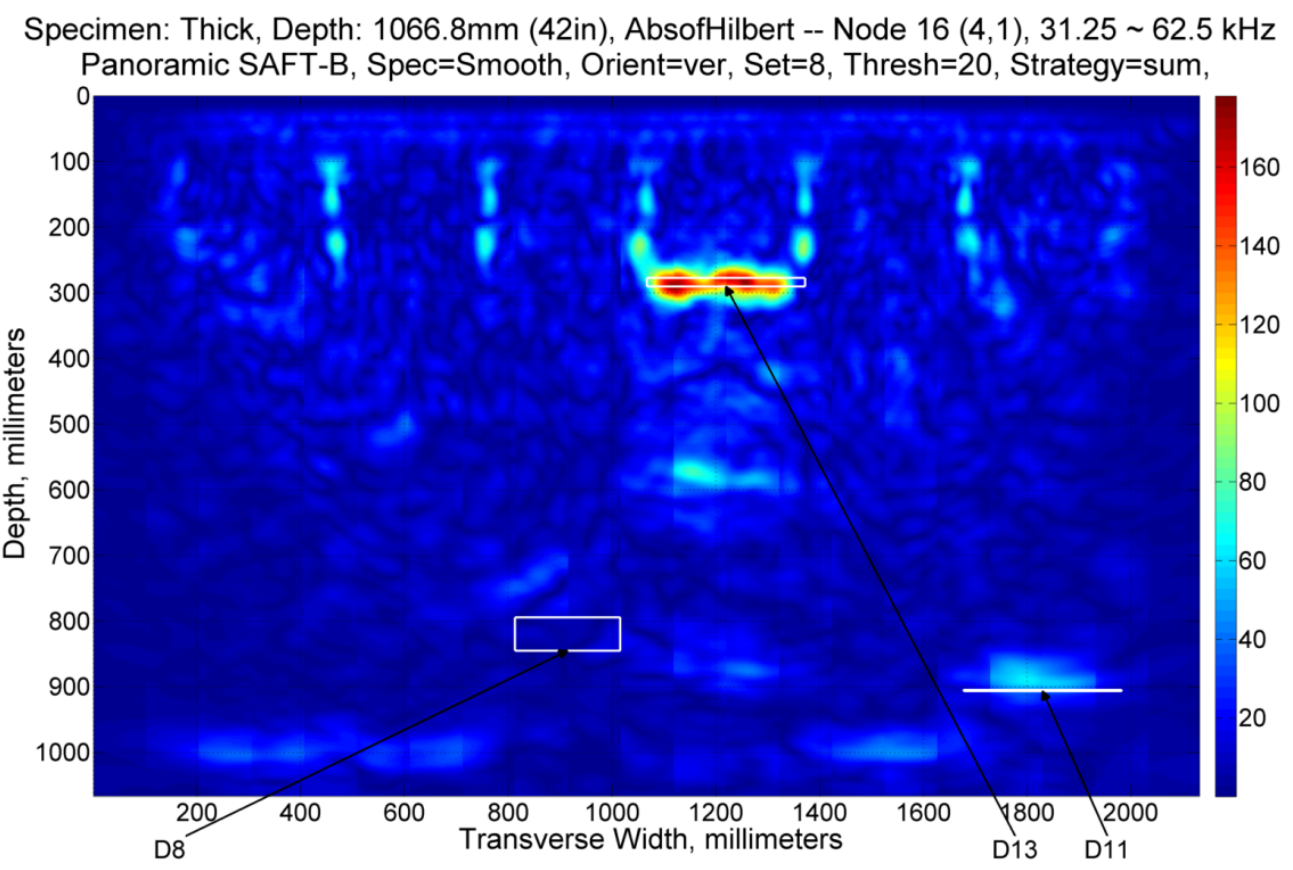

Fig. B. 132. Vertical Set 8, Node 16.

Specimen: Thick, Depth: 1066.8mm (42in), AbsofHilbert -- Node $18(4,3), 93.75 \sim 125 \mathrm{kHz}$

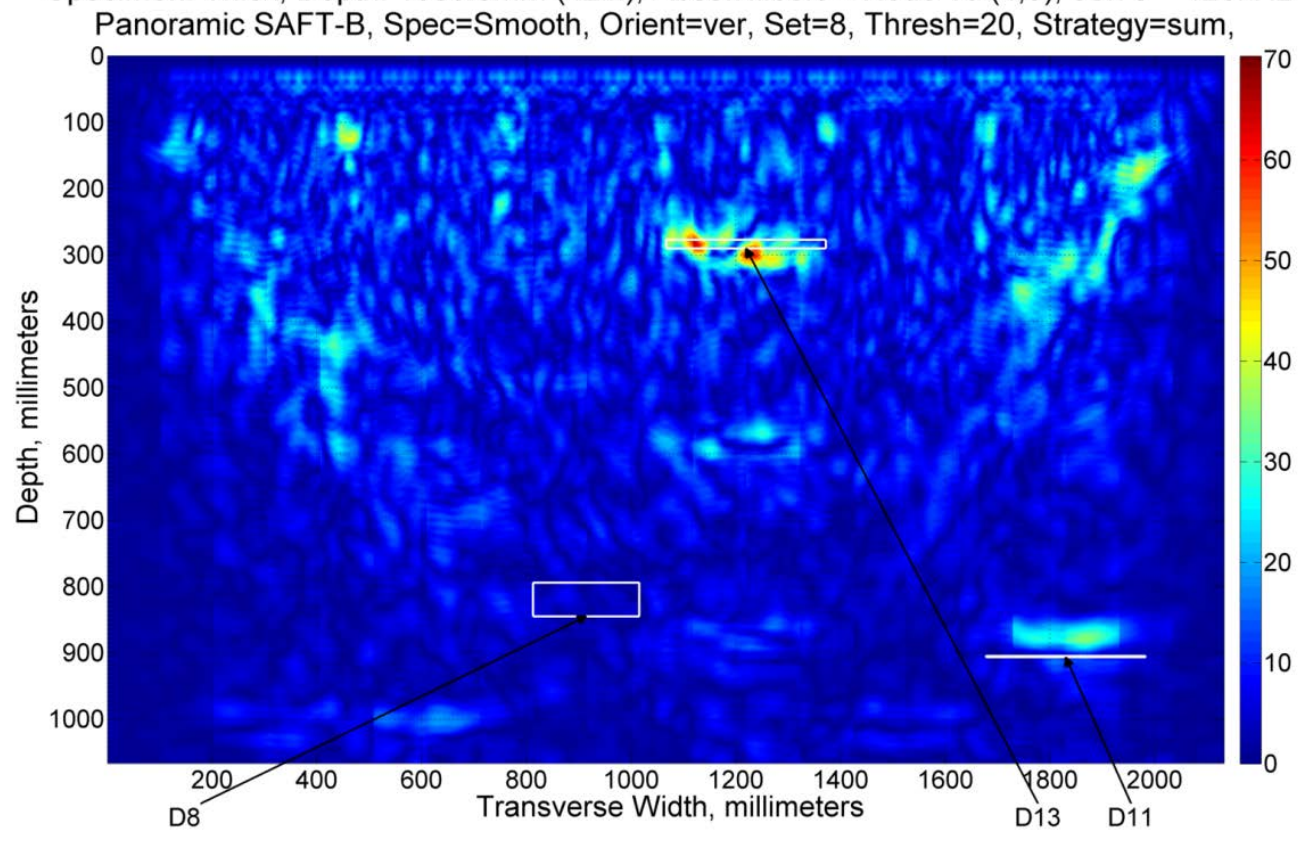

Fig. B. 133. Vertical Set 8, Node 18. 


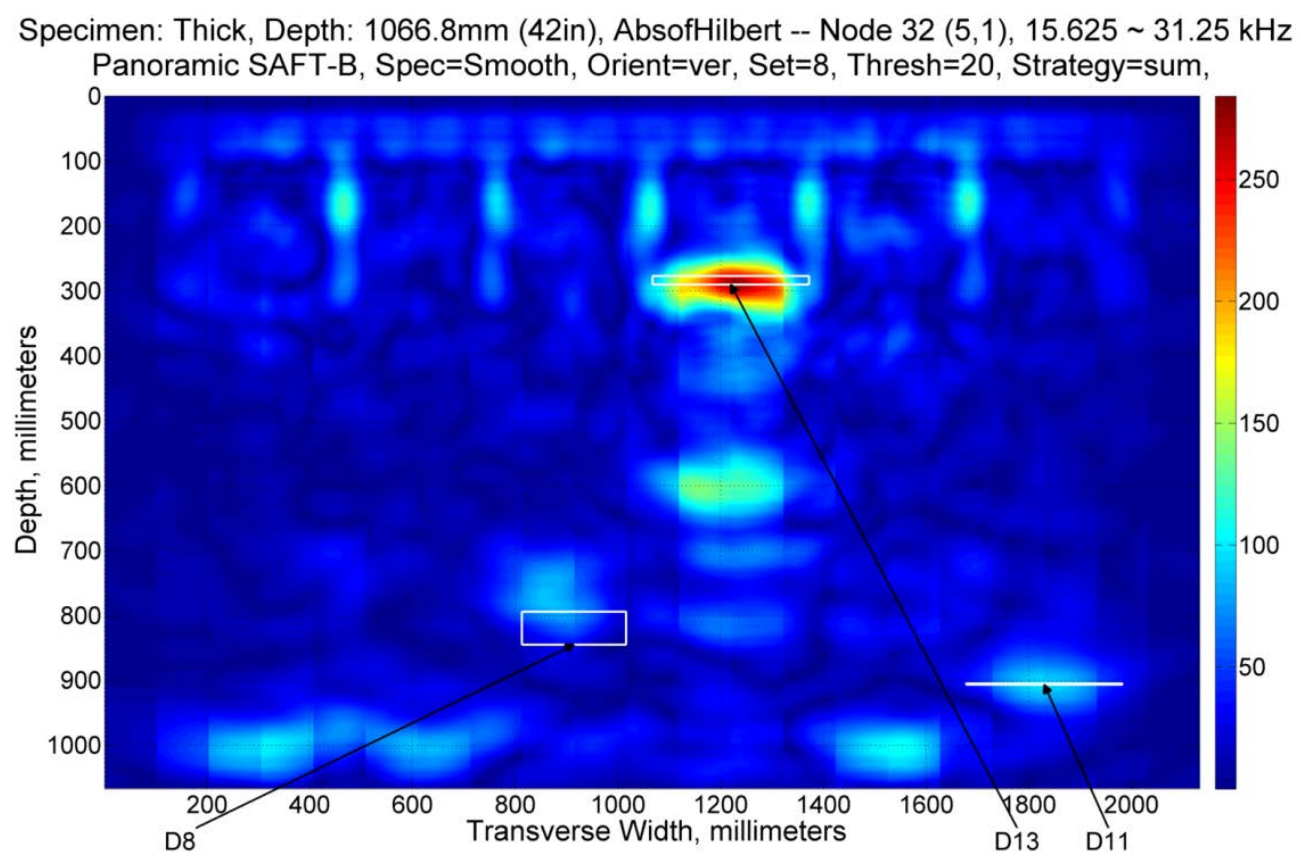

Fig. B. 134. Vertical Set 8, Node 32.

Specimen: Thick, Depth: 1066.8mm (42in), AbsofHilbert -- Node 34 (5,3), $46.875 \sim 62.5 \mathrm{kHz}$ Panoramic SAFT-B, Spec=Smooth, Orient=ver, Set=8, Thresh=20, Strategy=sum,

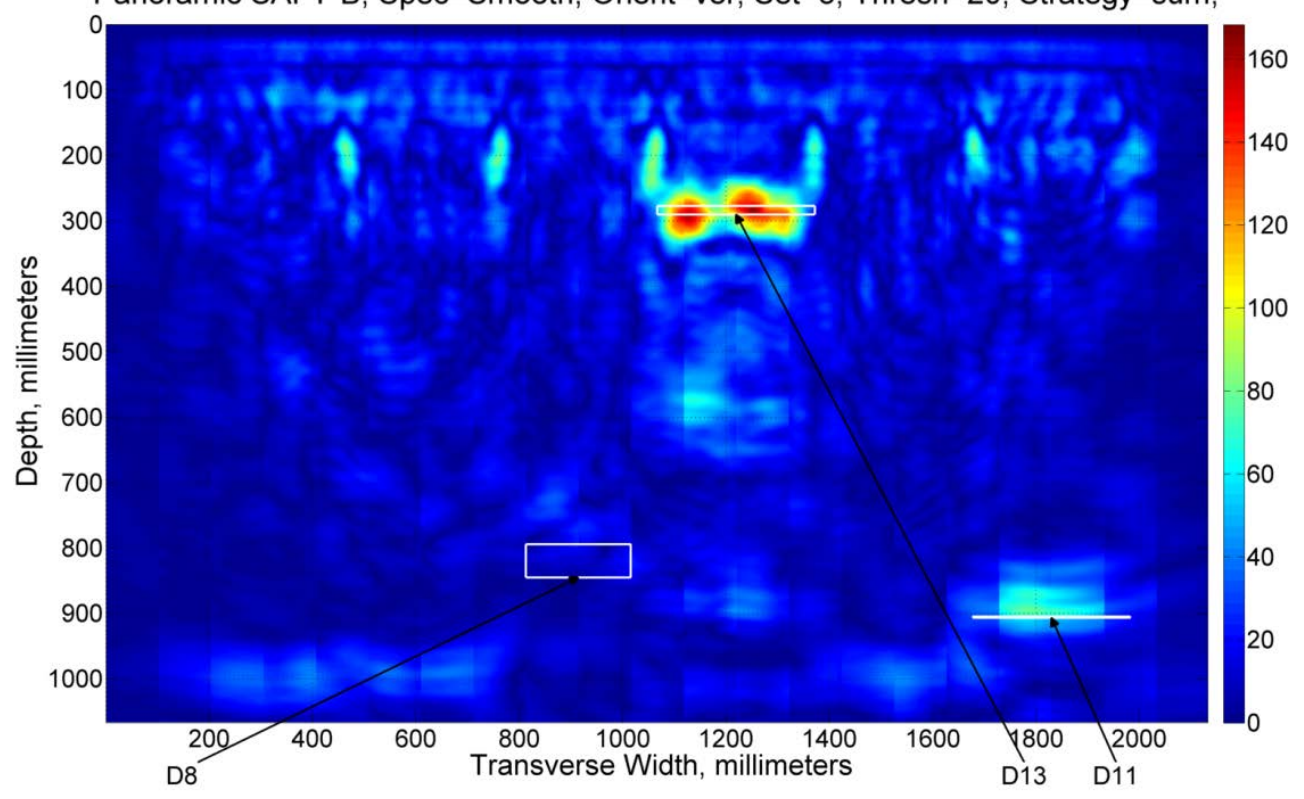

Fig. B. 135. Vertical Set 8, Node 34. 
Specimen: Thick, Depth: 1066.8mm (42in), AbsofHilbert -- Node $0(0,0), 0 \sim 500 \mathrm{kHz}$ Panoramic SAFT-B, Spec=Smooth, Orient=ver, Set=9, Thresh=20, Strategy=sum,

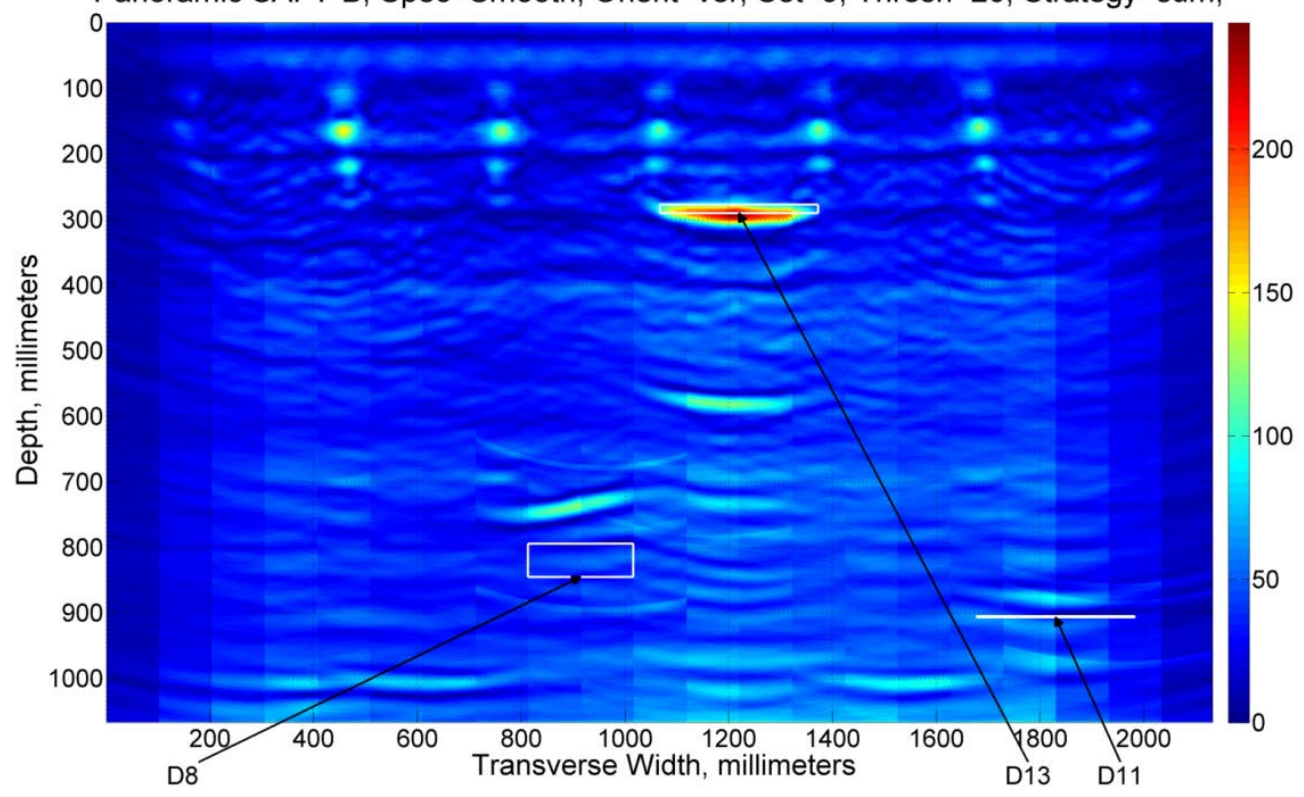

Fig. B. 136. Vertical Set 9, Node 0.

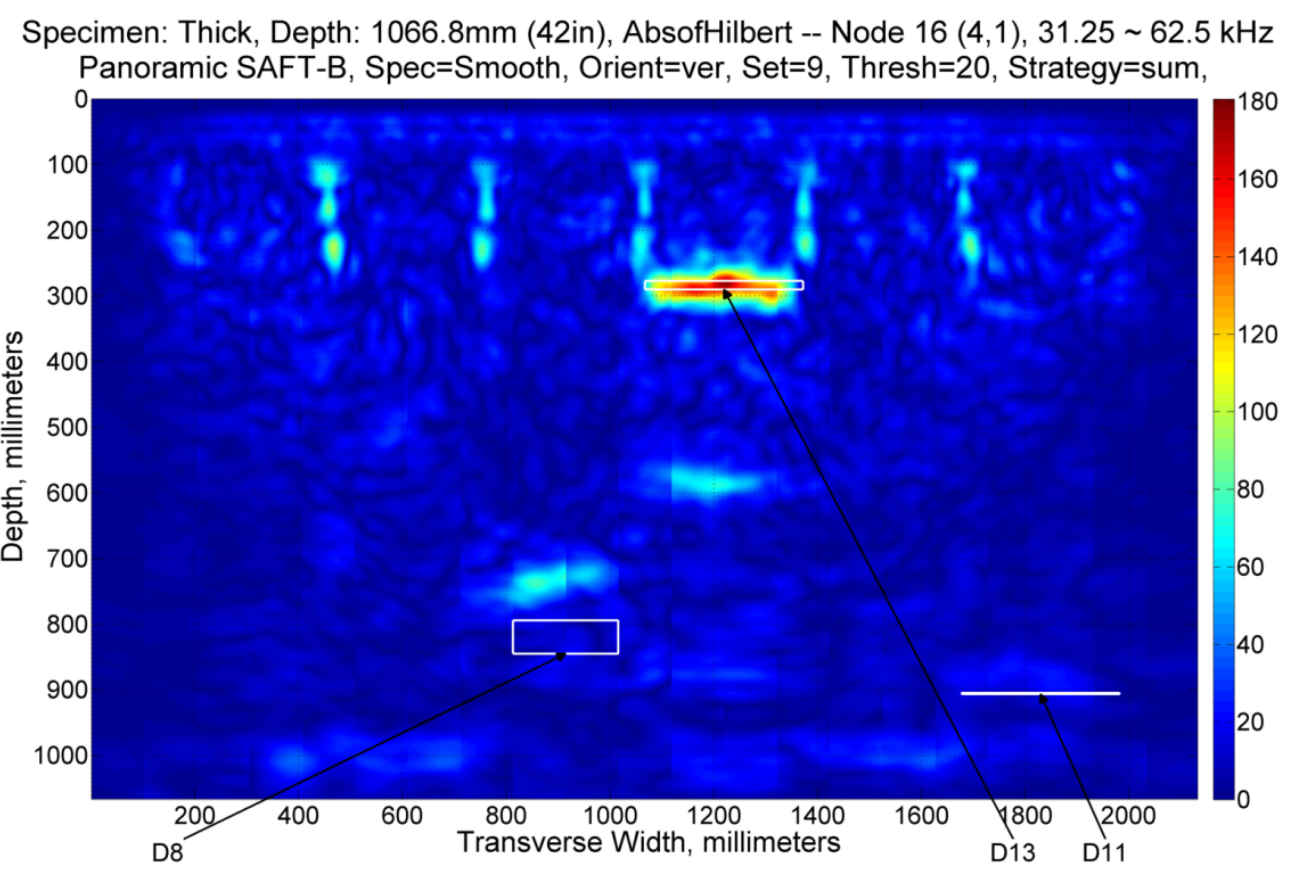

Fig. B. 137. Vertical Set 9, Node 16. 
Specimen: Thick, Depth: 1066.8mm (42in), AbsofHilbert -- Node $18(4,3), 93.75 \sim 125 \mathrm{kHz}$ Panoramic SAFT-B, Spec=Smooth, Orient=ver, Set=9, Thresh=20, Strategy=sum,

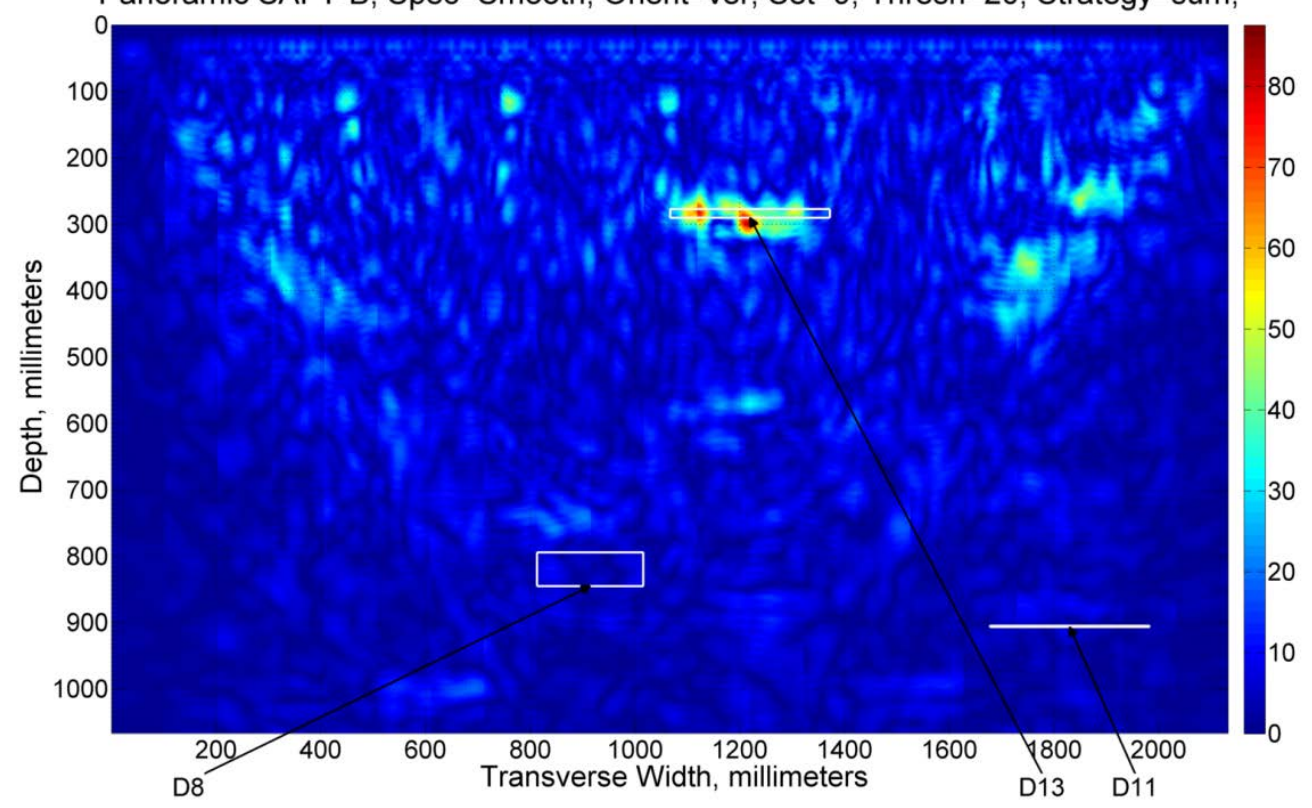

Fig. B. 138. Vertical Set 9, Node 18

Specimen: Thick, Depth: 1066.8mm (42in), AbsofHilbert -- Node $34(5,3), 46.875 \sim 62.5 \mathrm{kHz}$ Panoramic SAFT-B, Spec=Smooth, Orient=ver, Set=9, Thresh=20, Strategy=sum,

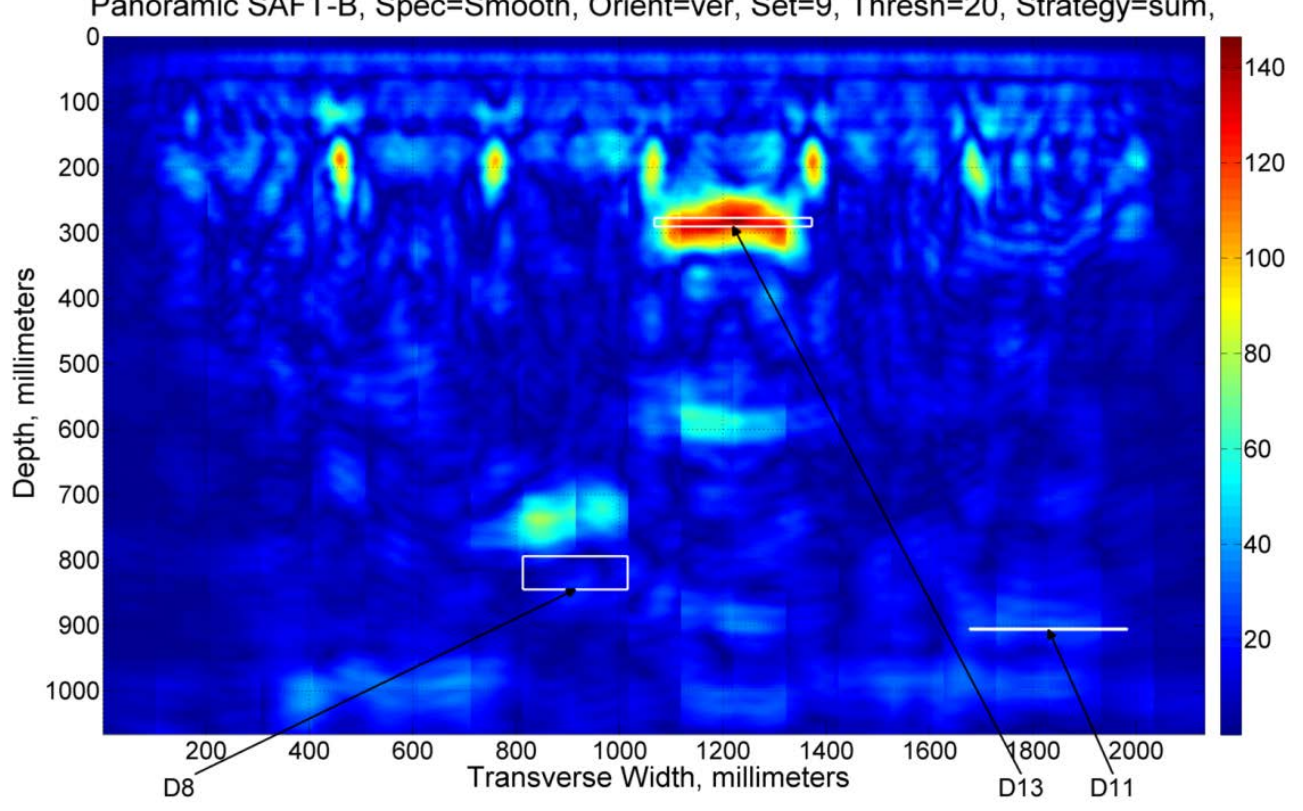

Fig. B. 139. Vertical Set 9, Set 34 . 
Specimen: Thick, Depth: 1066.8mm (42in), AbsofHilbert -- Node 32 (5,1), $15.625 \sim 31.25 \mathrm{kHz}$ Panoramic SAFT-B, Spec=Smooth, Orient=ver, Set=9, Thresh=20, Strategy=sum,

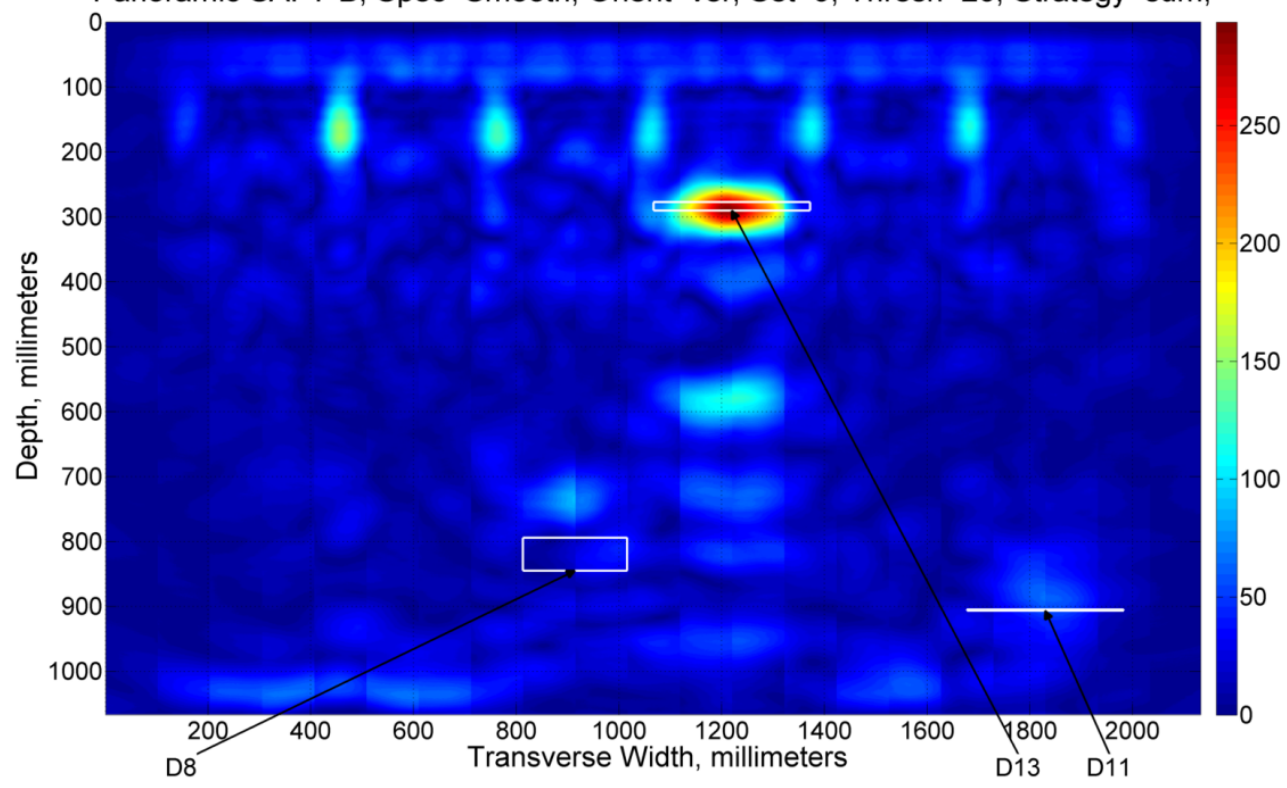

Fig. B. 140. Vertical Set 9, Node 32.

\section{SET TEN - DEFECT 3, DEFECT 20}

Specimen: Thick, Depth: 1066.8mm (42in), AbsofHilbert -- Node $0(0,0), 0 \sim 500 \mathrm{kHz}$ Panoramic SAFT-B, Spec=Smooth, Orient=ver, Set=10, Thresh=20, Strategy=sum,

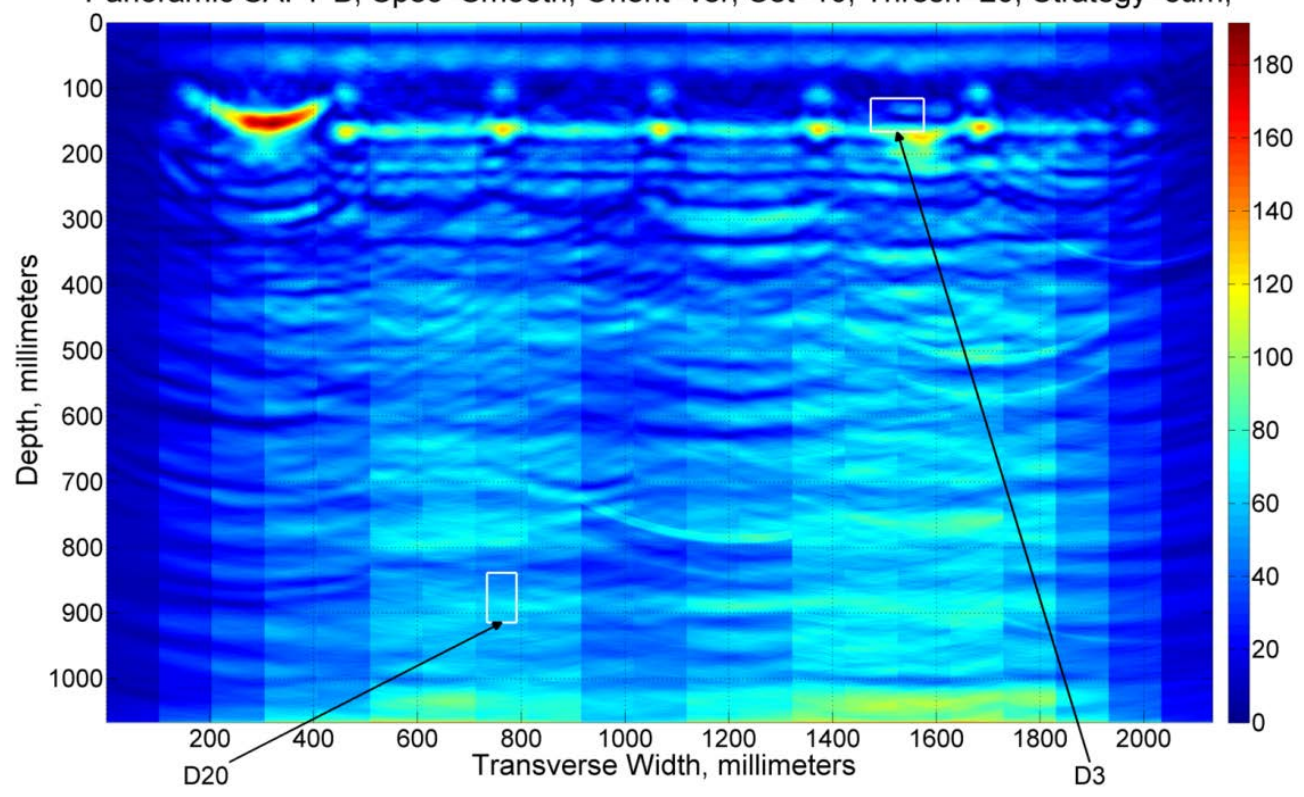

Fig. B. 141. Vertical Set 10, Node 0. 


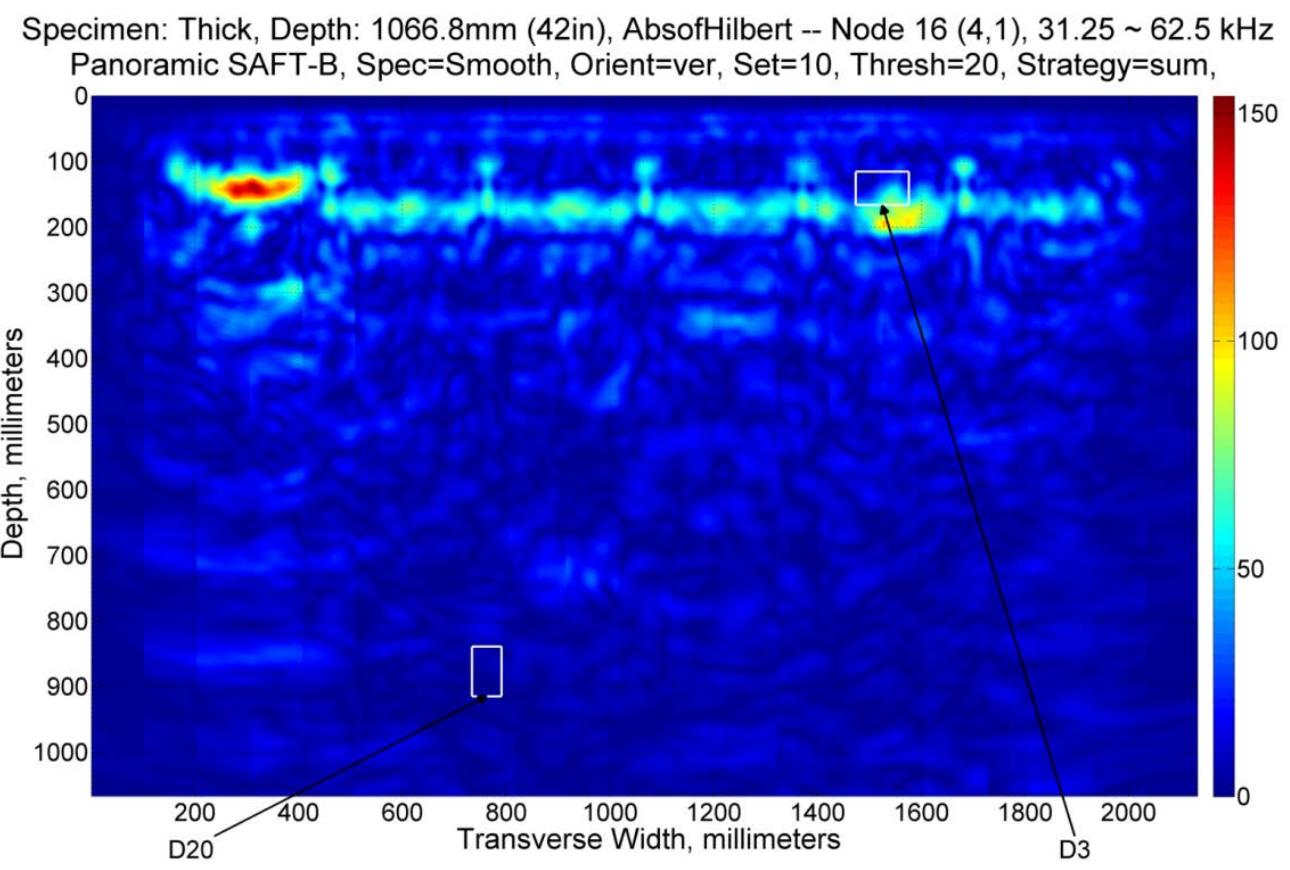

Fig. B. 142. Vertical Set 10, Node 16.

Specimen: Thick, Depth: 1066.8mm (42in), AbsofHilbert -- Node $18(4,3), 93.75 \sim 125 \mathrm{kHz}$ Panoramic SAFT-B, Spec=Smooth, Orient=ver, Set $=10$, Thresh $=20$, Strategy=sum,

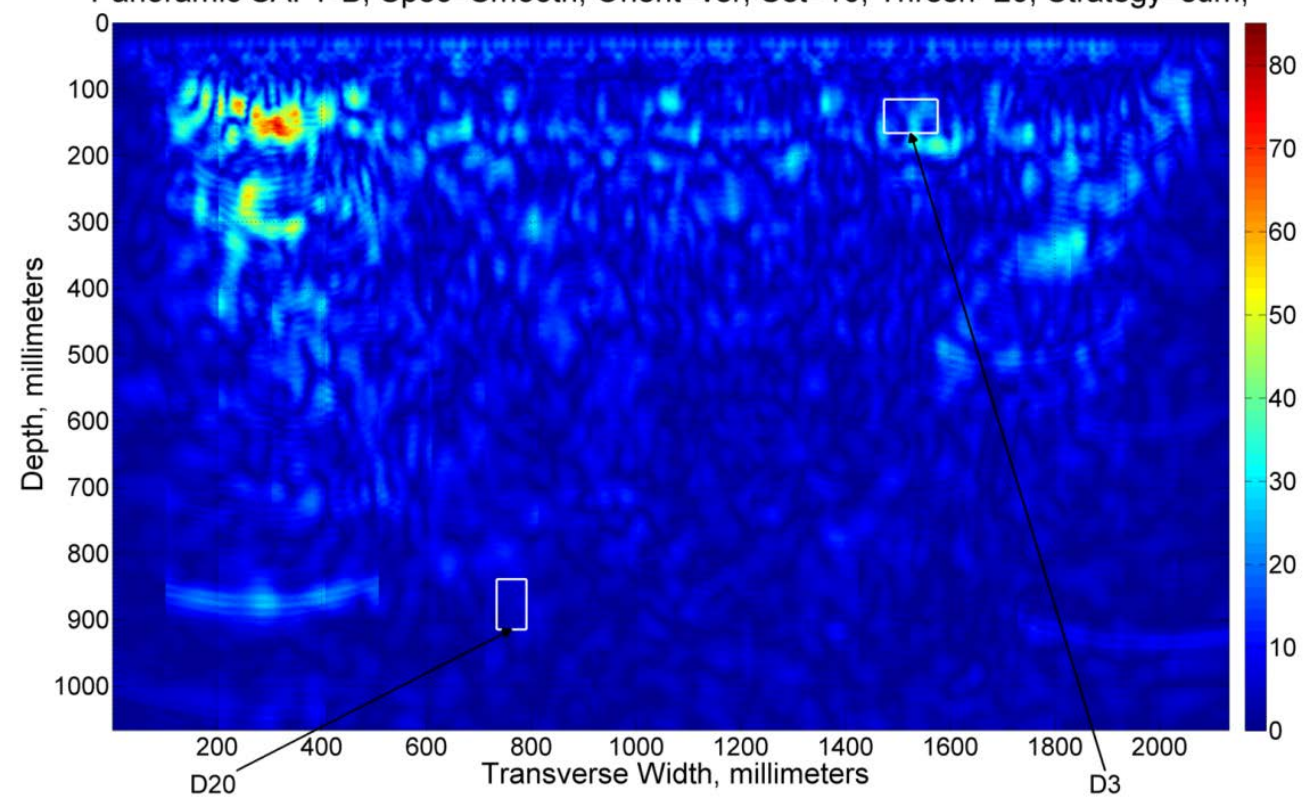

Fig. B. 143. Vertical Set 10, Node 18. 


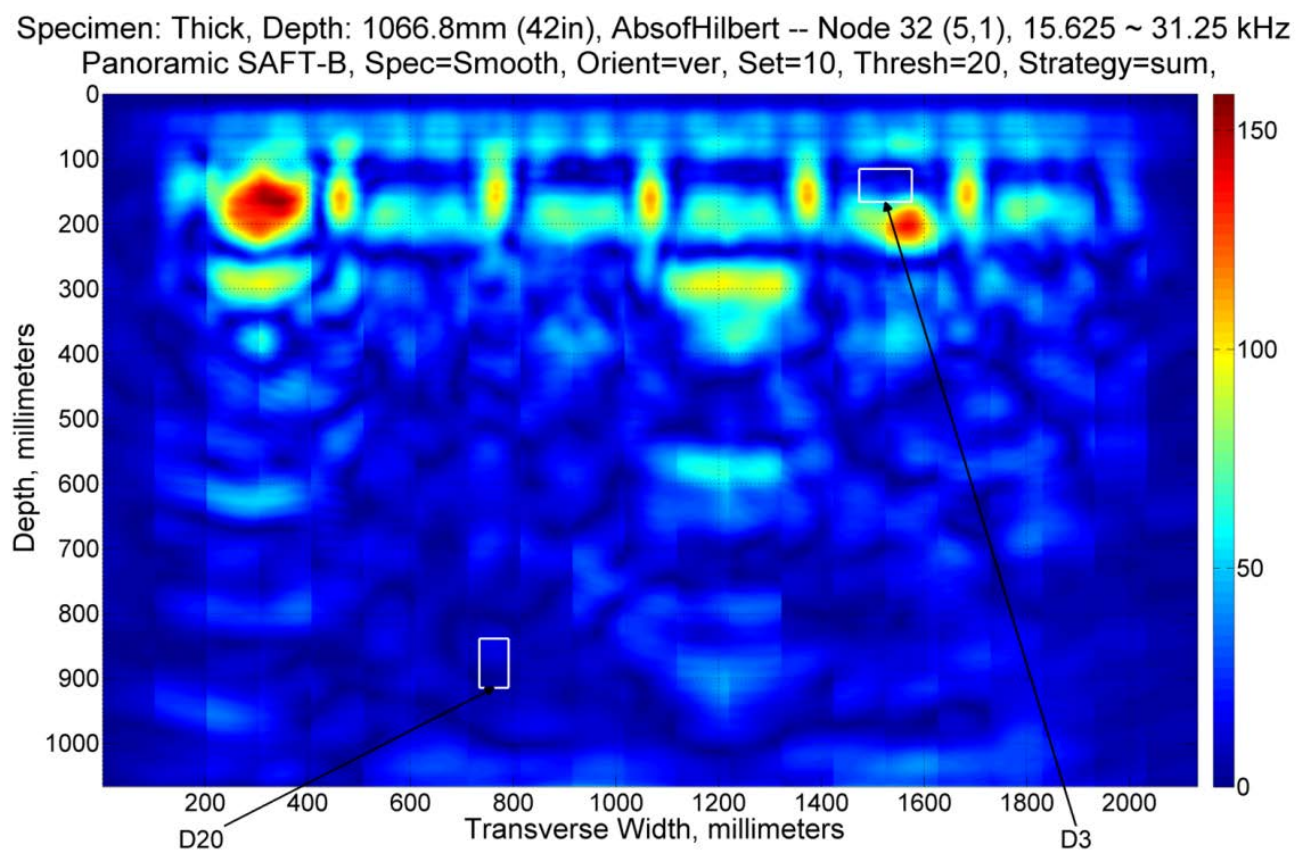

Fig. B. 144. Vertical Set 10, Node 32.

Specimen: Thick, Depth: 1066.8mm (42in), AbsofHilbert -- Node $34(5,3), 46.875 \sim 62.5 \mathrm{kHz}$ Panoramic SAFT-B, Spec=Smooth, Orient=ver, Set=10, Thresh=20, Strategy=sum,

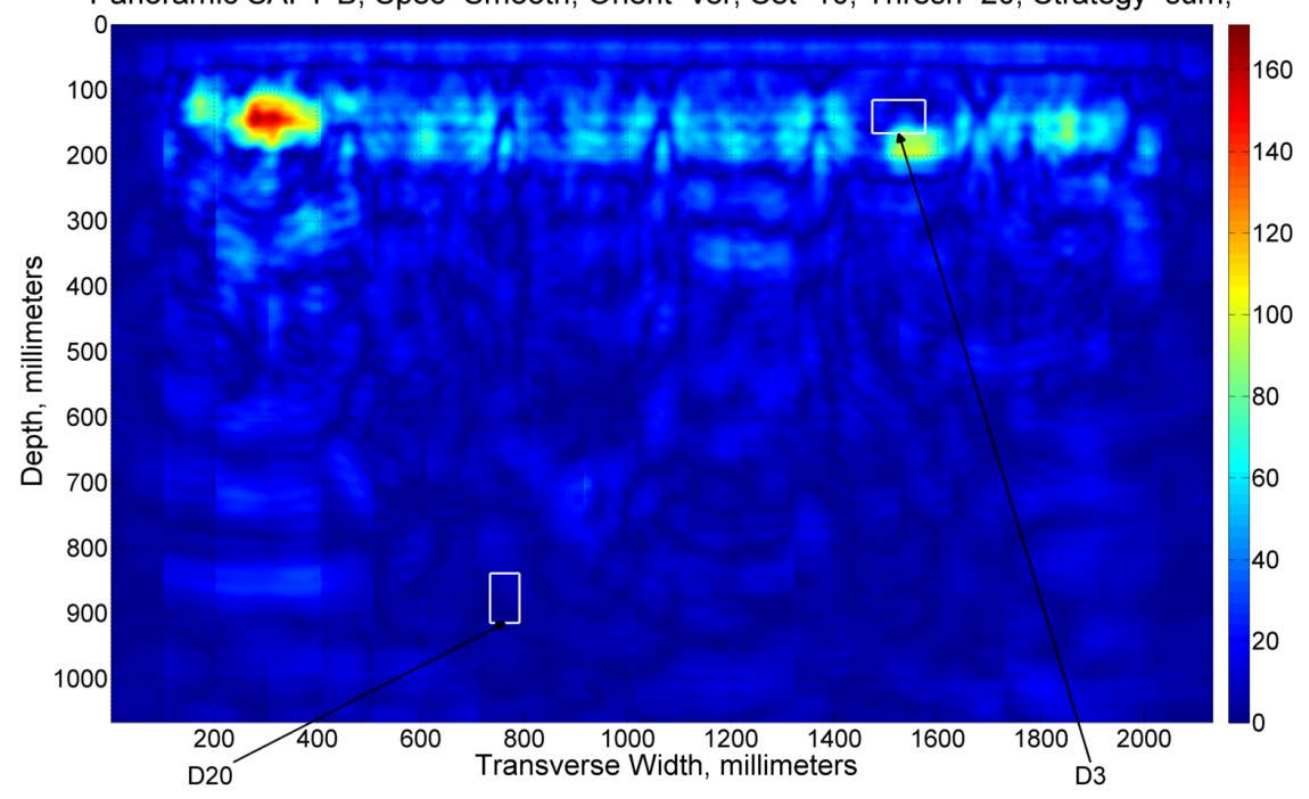

Fig. B. 145. Vertical Set 10, Node 34. 
Specimen: Thick, Depth: 1066.8mm (42in), AbsofHilbert -- Node $0(0,0), 0 \sim 500 \mathrm{kHz}$ Panoramic SAFT-B, Spec=Smooth, Orient=ver, Set=11, Thresh=20, Strategy=sum,

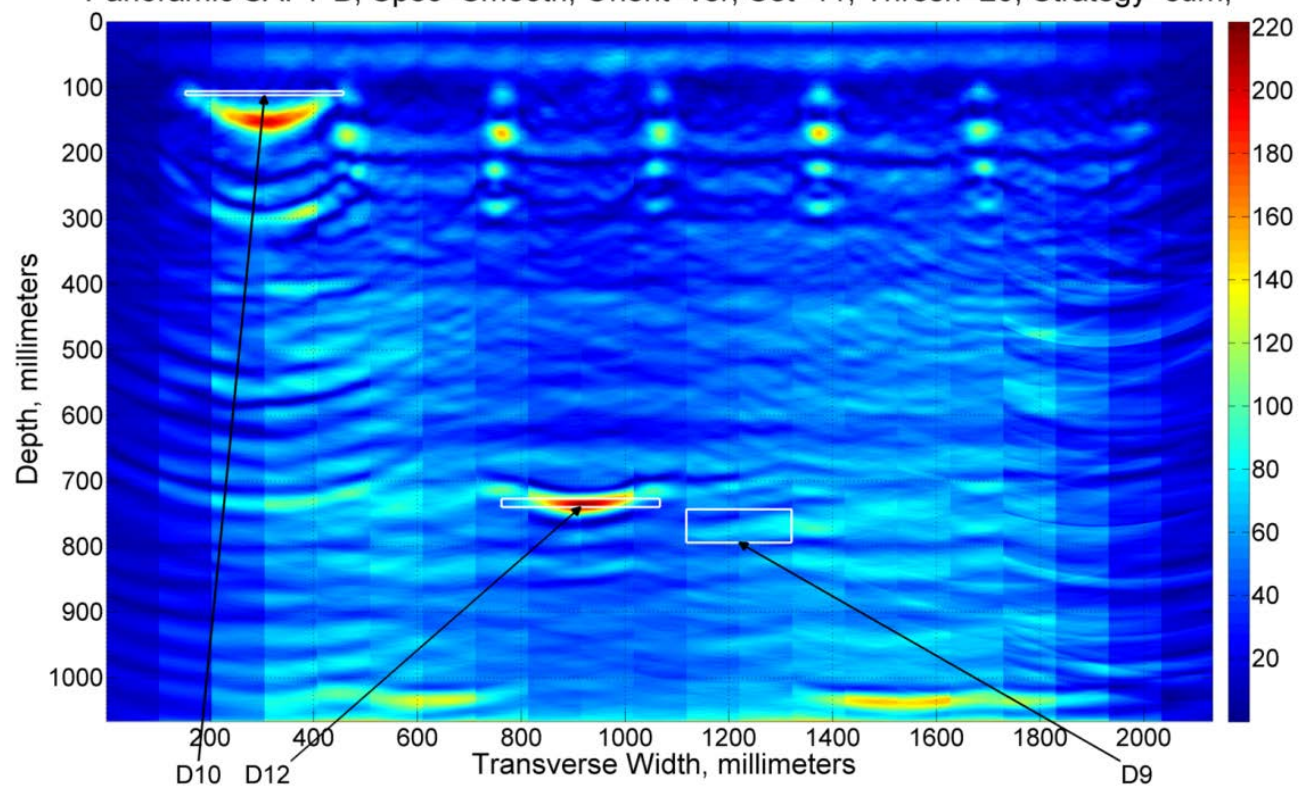

Fig. B. 146. Vertical Set 11, Node 0.

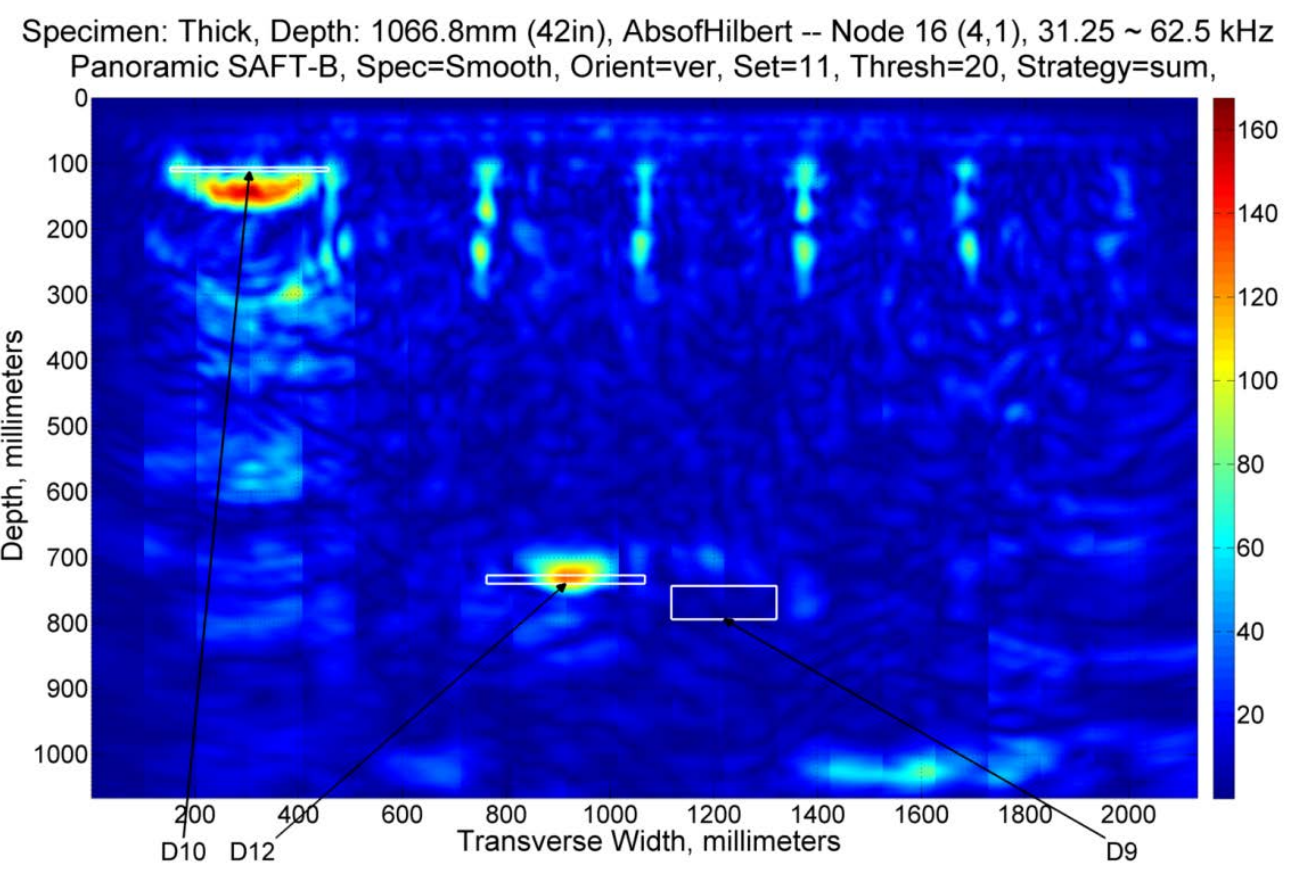

Fig. B. 147. Vertical Set 11, Node 16. 


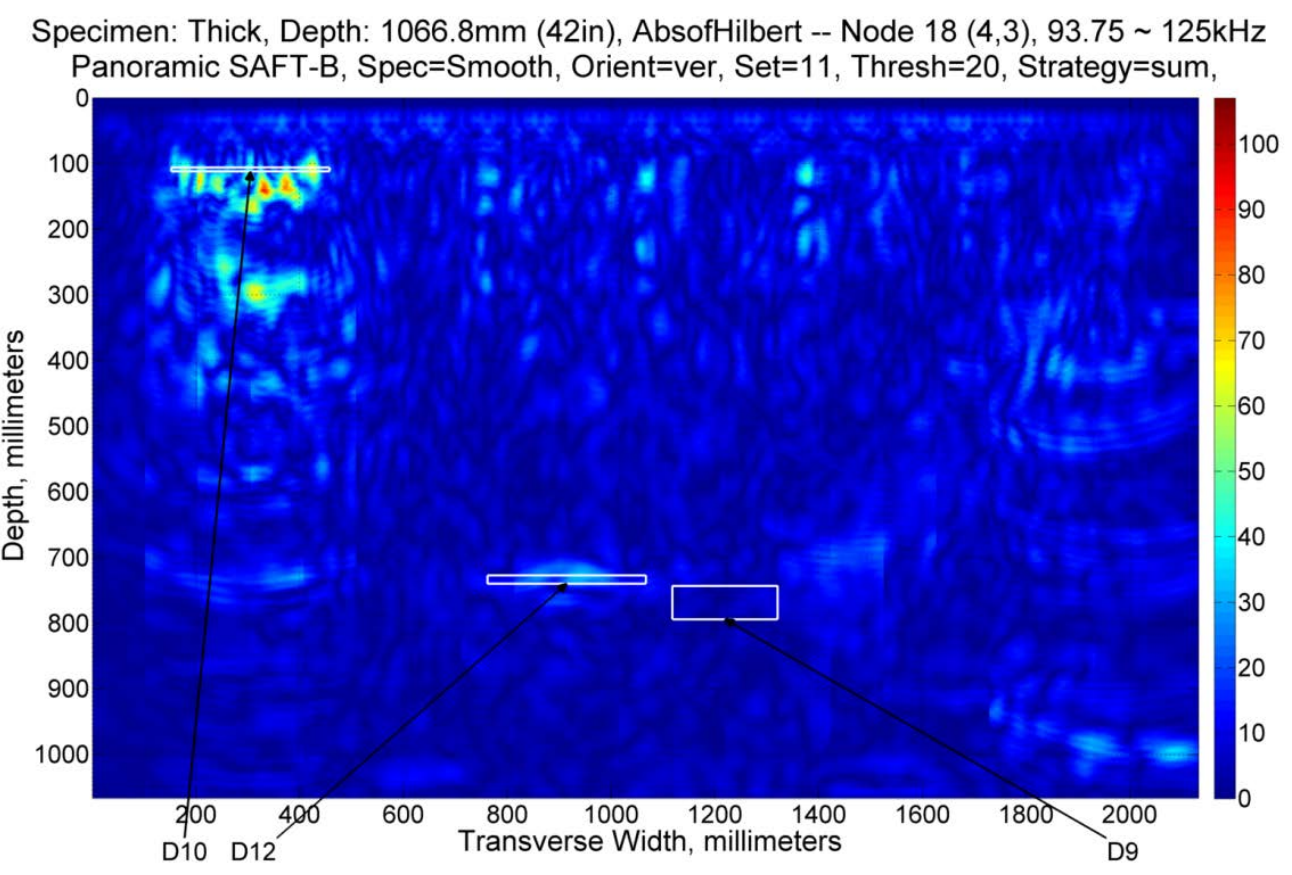

Fig. B. 148. Vertical Set 11, Node 18.

Specimen: Thick, Depth: 1066.8mm (42in), AbsofHilbert -- Node $32(5,1), 15.625 \sim 31.25 \mathrm{kHz}$ Panoramic SAFT-B, Spec=Smooth, Orient=ver, Set $=11$, Thresh $=20$, Strategy=sum,

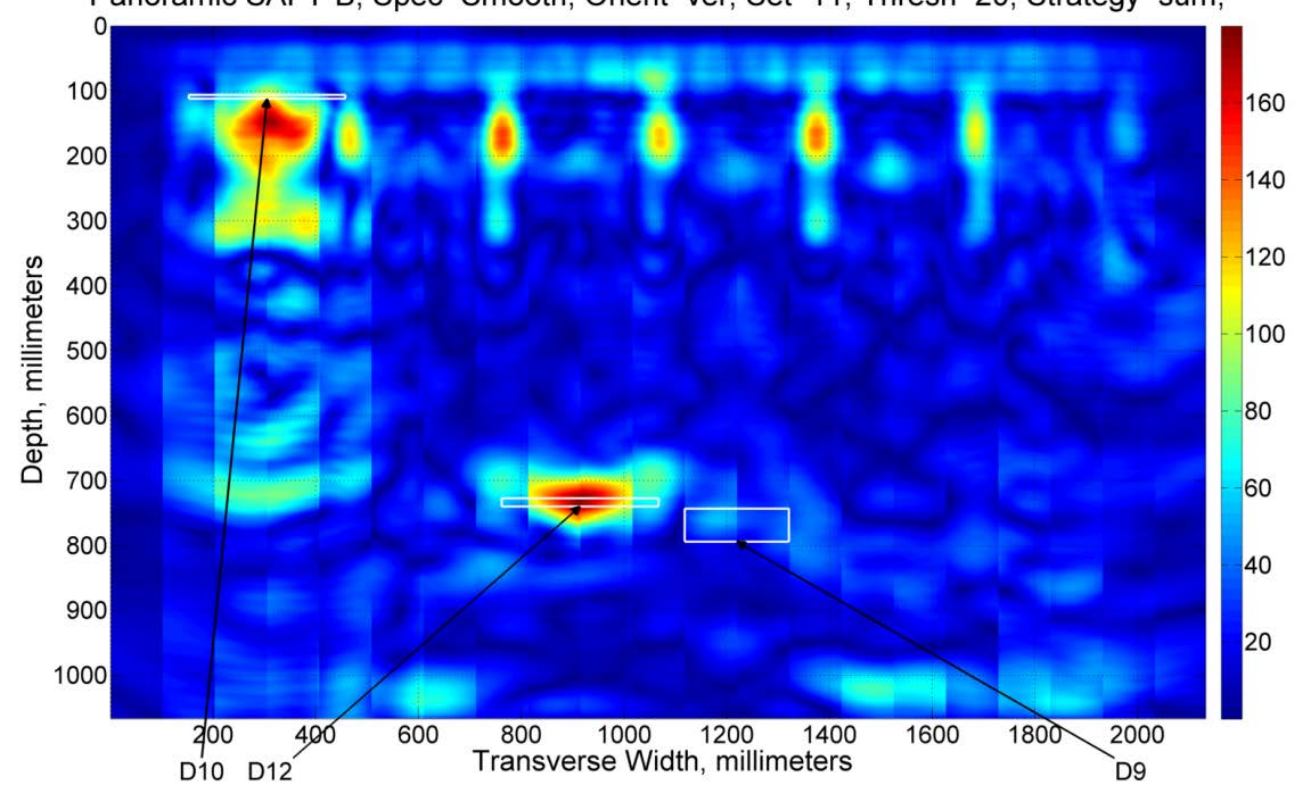

Fig. B. 149. Vertical Set 11, Node 32. 


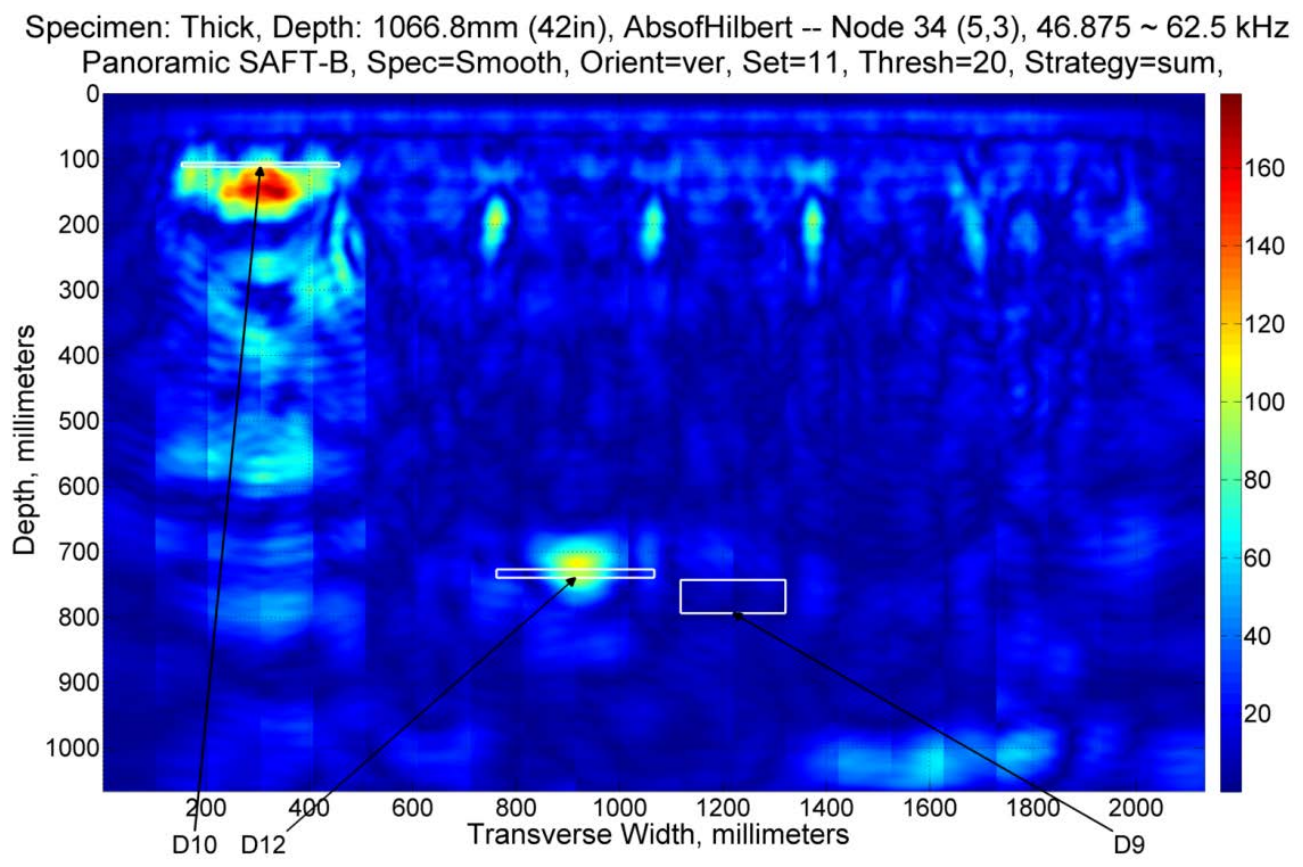

Fig. B. 150. Vertical Set 11, Node 34.

\section{SET TWELVE - DEFECT 9, DEFECT 10, DEFECT 12}

Specimen: Thick, Depth: 1066.8mm (42in), AbsofHilbert -- Node $0(0,0), 0 \sim 500 \mathrm{kHz}$ Panoramic SAFT-B, Spec=Smooth, Orient=ver, Set=12, Thresh=20, Strategy=sum,

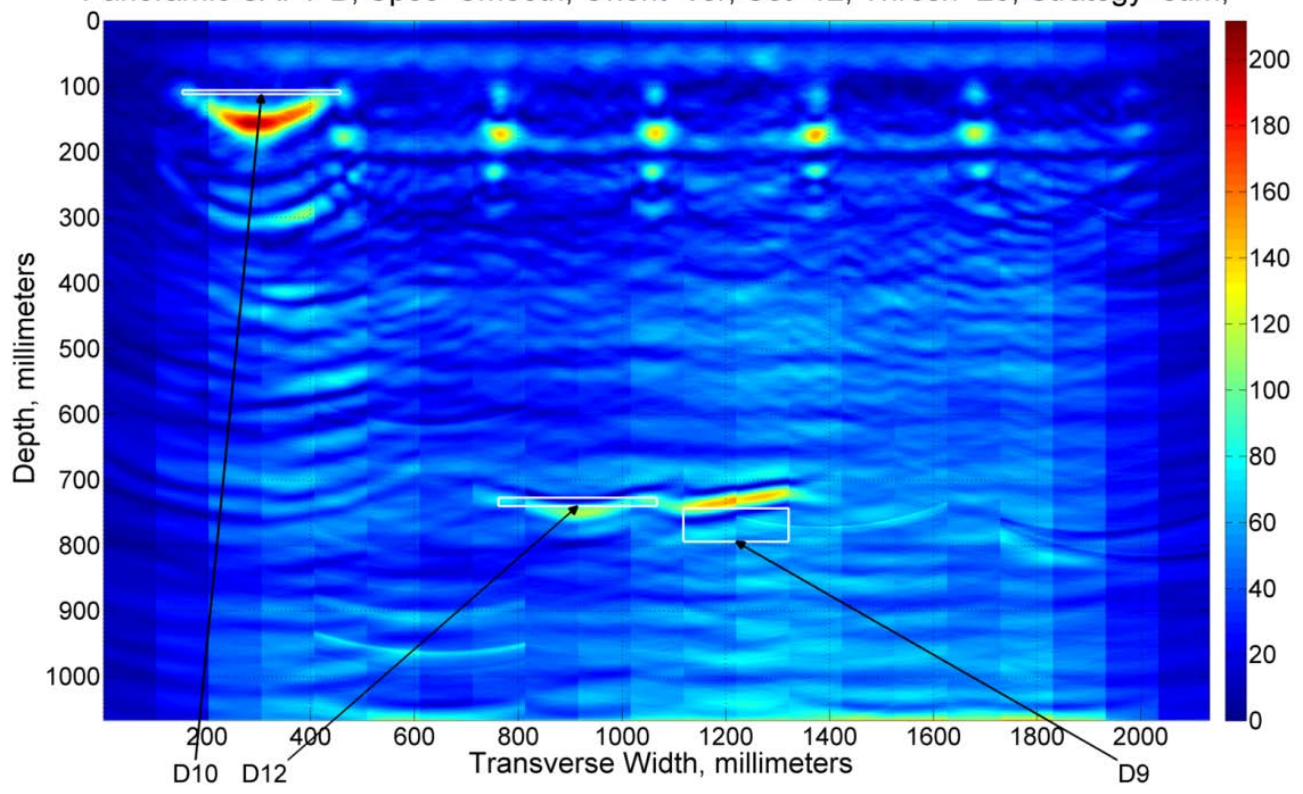

Fig. B. 151. Vertical Set 12, Node 0. 


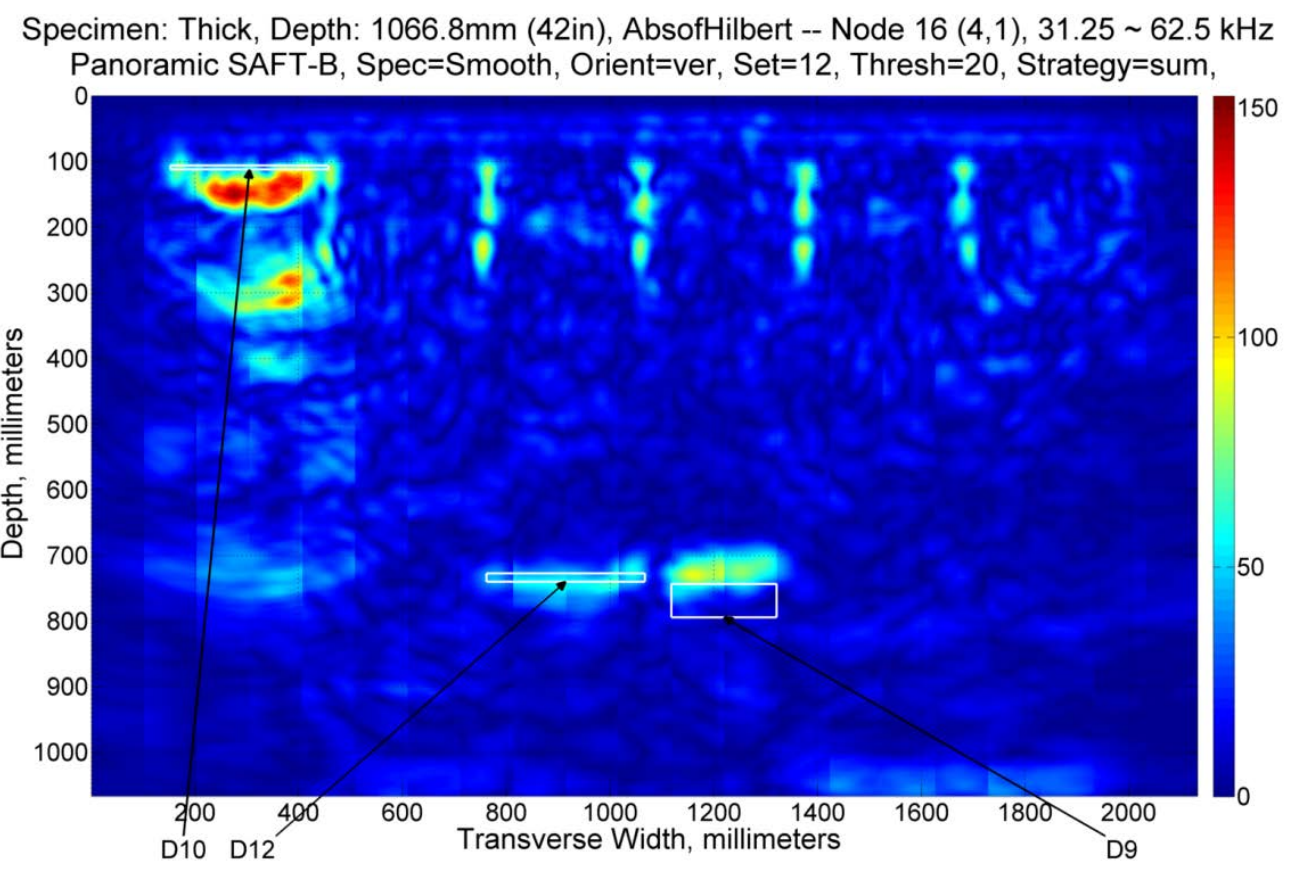

Fig. B. 152. Vertical Set 12, Node 16.

Specimen: Thick, Depth: 1066.8mm (42in), AbsofHilbert -- Node $18(4,3), 93.75 \sim 125 \mathrm{kHz}$ Panoramic SAFT-B, Spec $=$ Smooth, Orient $=v e r$, Set $=12$, Thresh $=20$, Strategy $=$ sum,

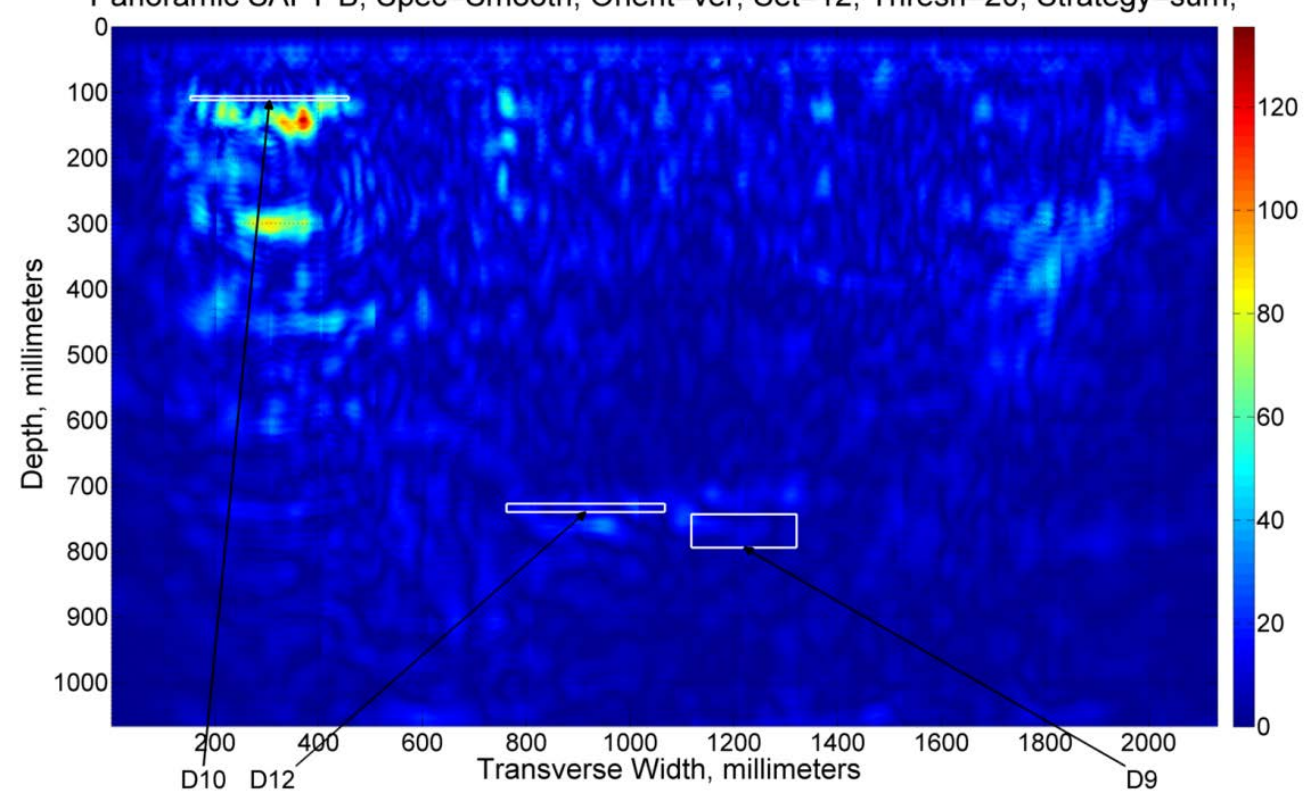

Fig. B. 153. Vertical Set 12, Node 18. 


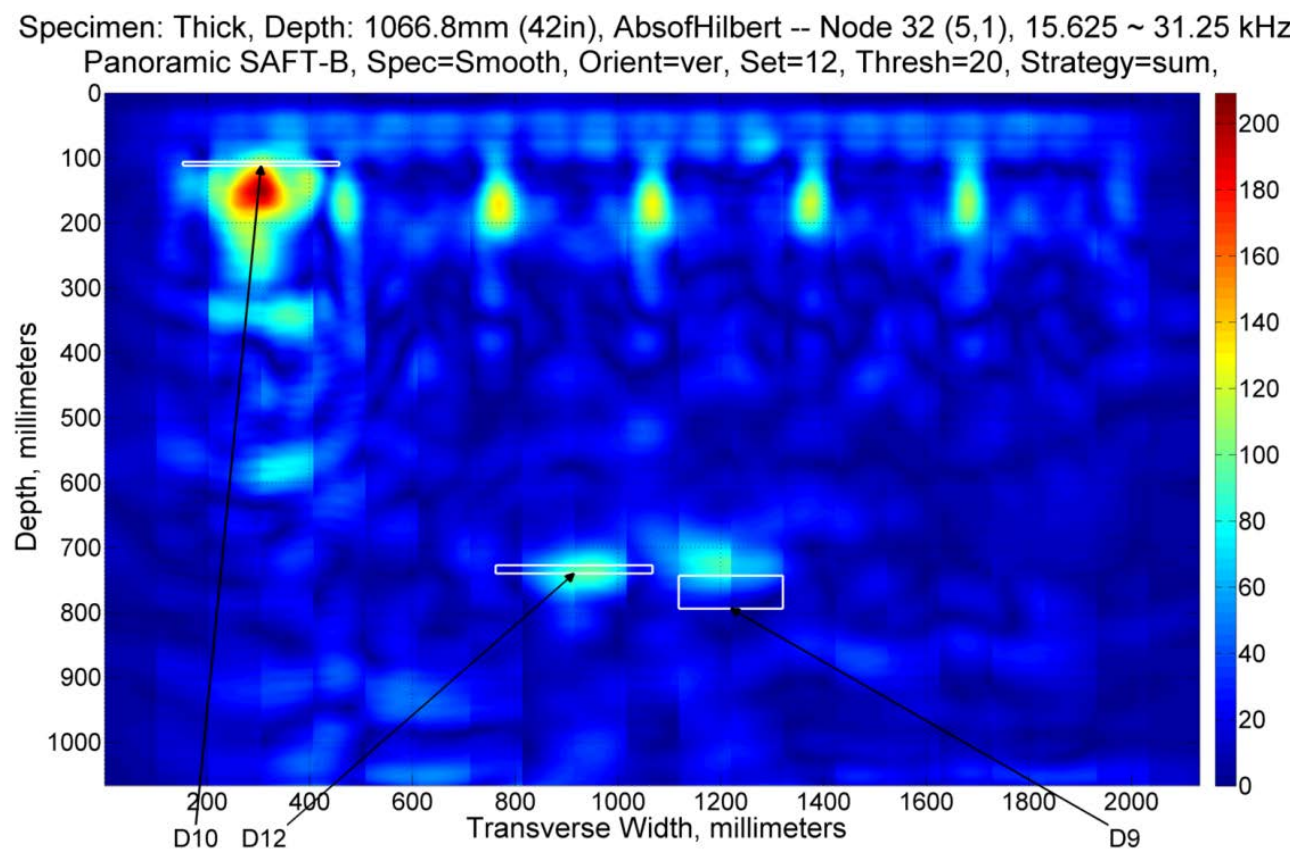

Fig. B. 154. Vertical Set 12, Node 32.

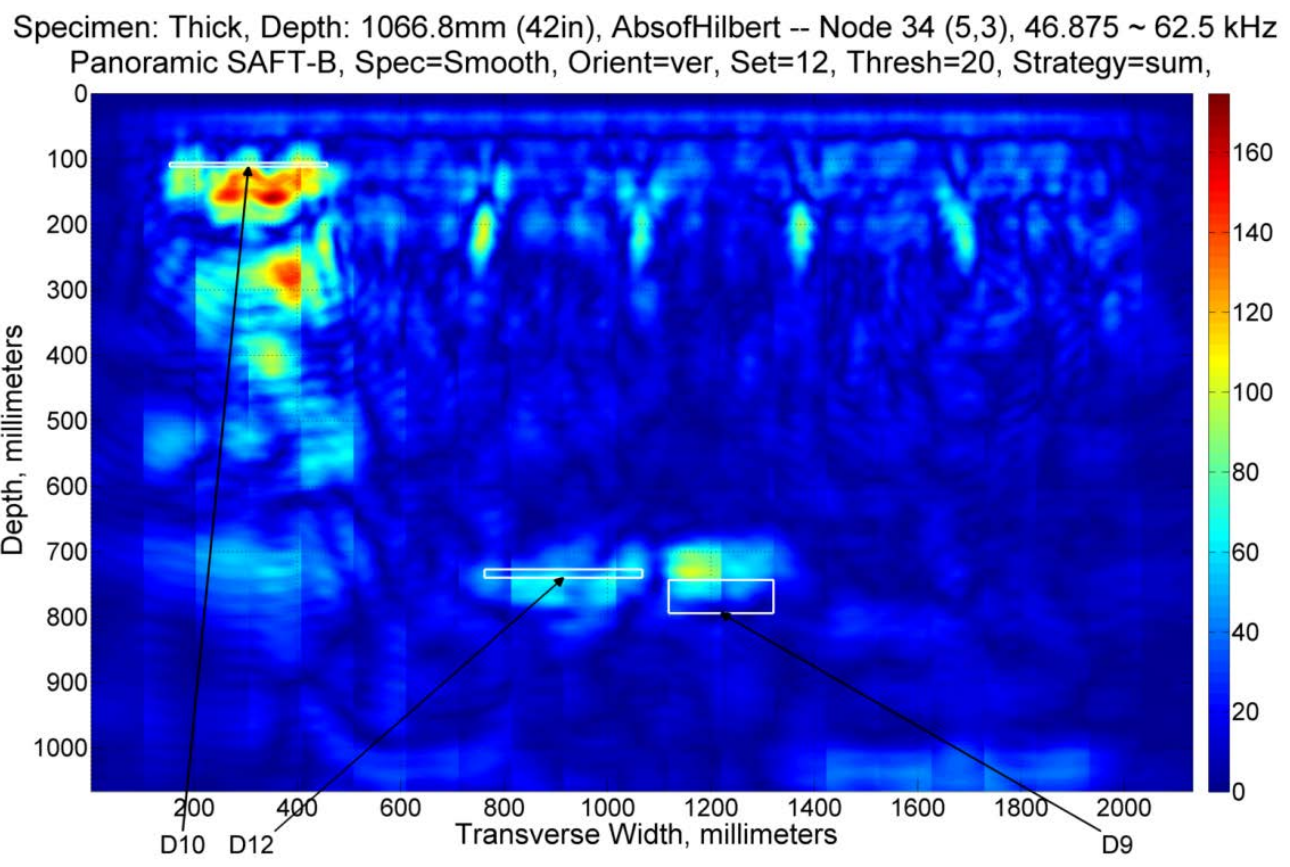

Fig. B. 155. Vertical Set 12, Node 34. 
Specimen: Thick, Depth: 1066.8mm (42in), AbsofHilbert -- Node $0(0,0), 0 \sim 500 \mathrm{kHz}$ Panoramic SAFT-B, Spec=Smooth, Orient=ver, Set=13, Thresh=20, Strategy=sum,

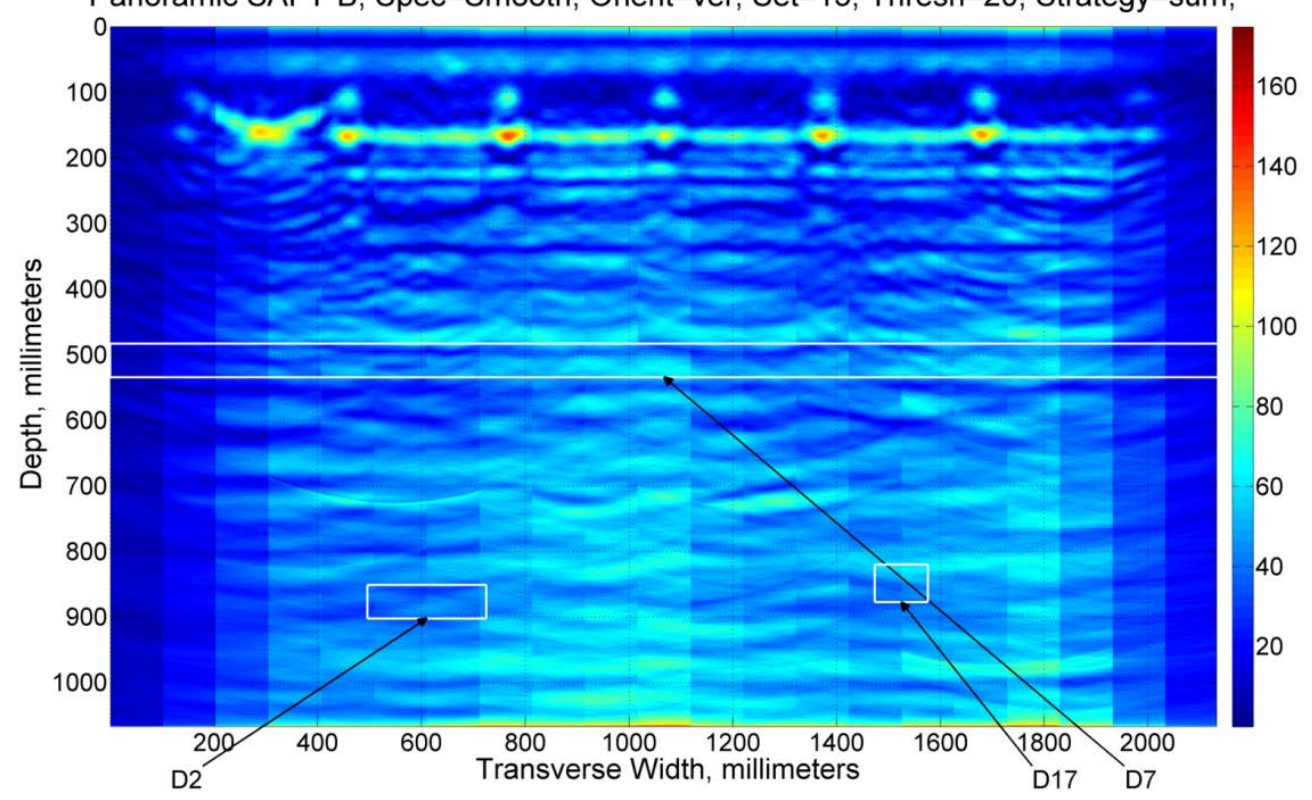

Fig. B. 156. Vertical Set 13, Node 0.

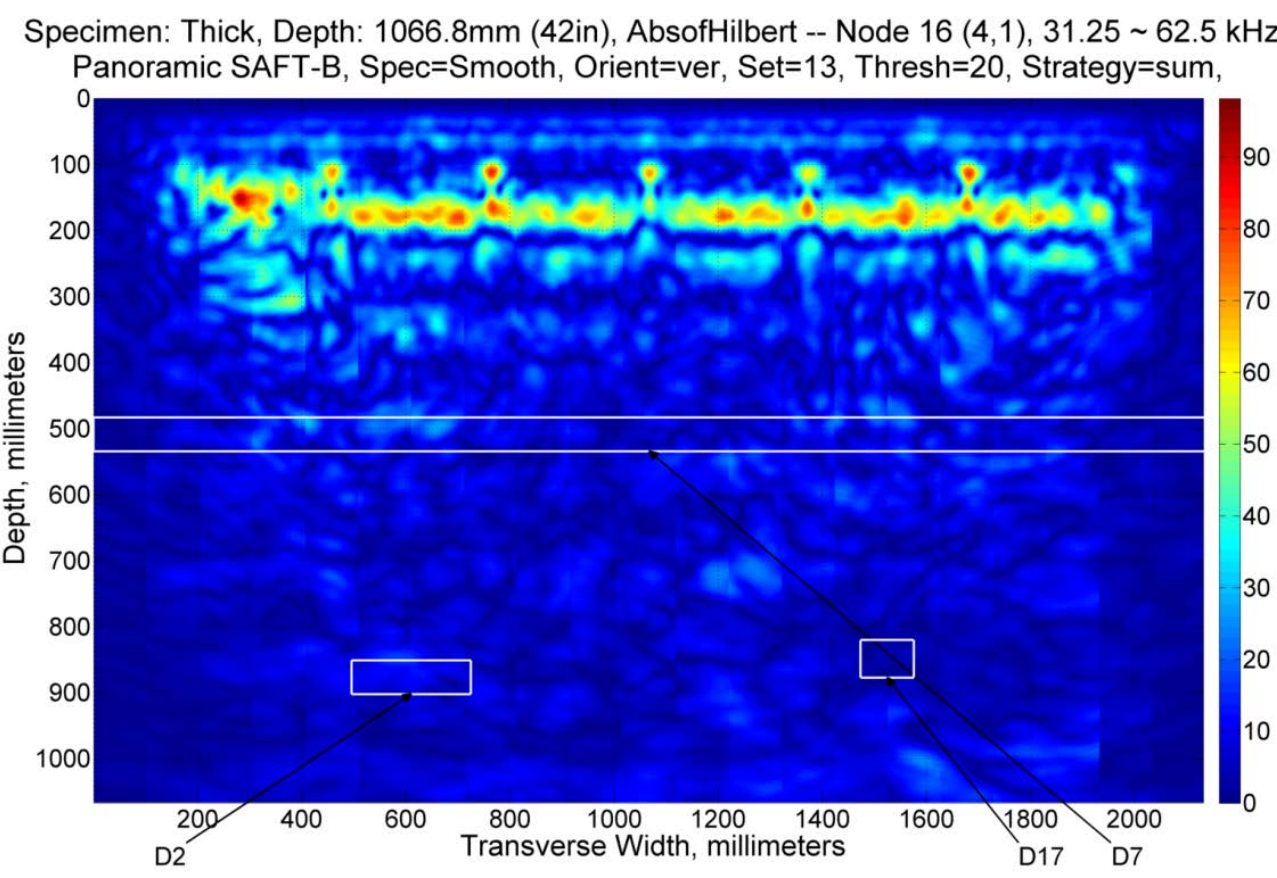

Fig. B. 157. Vertical Set 13, Node 16. 


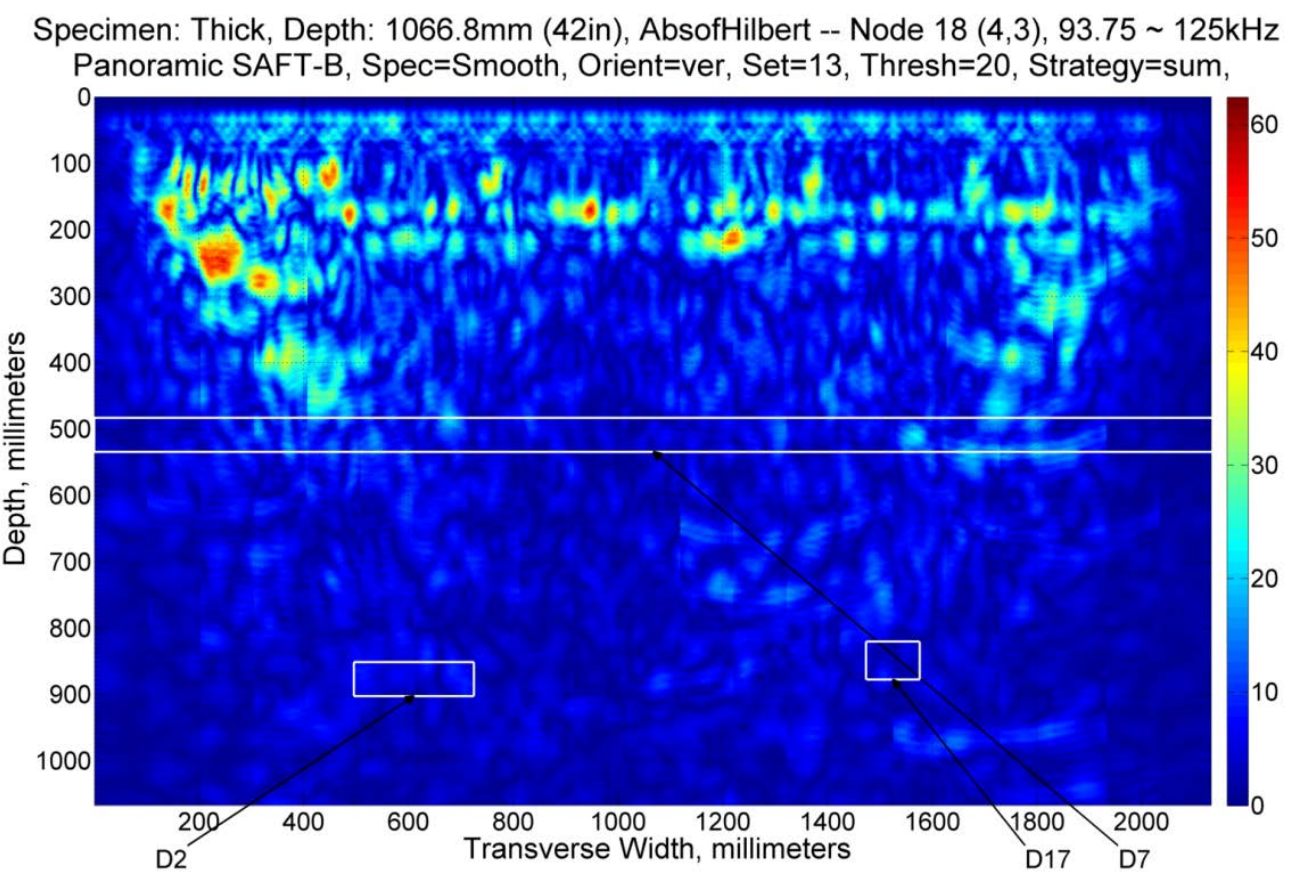

Fig. B. 158. Vertical Set 13, Node 18.

Specimen: Thick, Depth: 1066.8mm (42in), AbsofHilbert -- Node $32(5,1), 15.625 \sim 31.25 \mathrm{kHz}$ Panoramic SAFT-B, Spec=Smooth, Orient=ver, Set=13, Thresh=20, Strategy=sum,

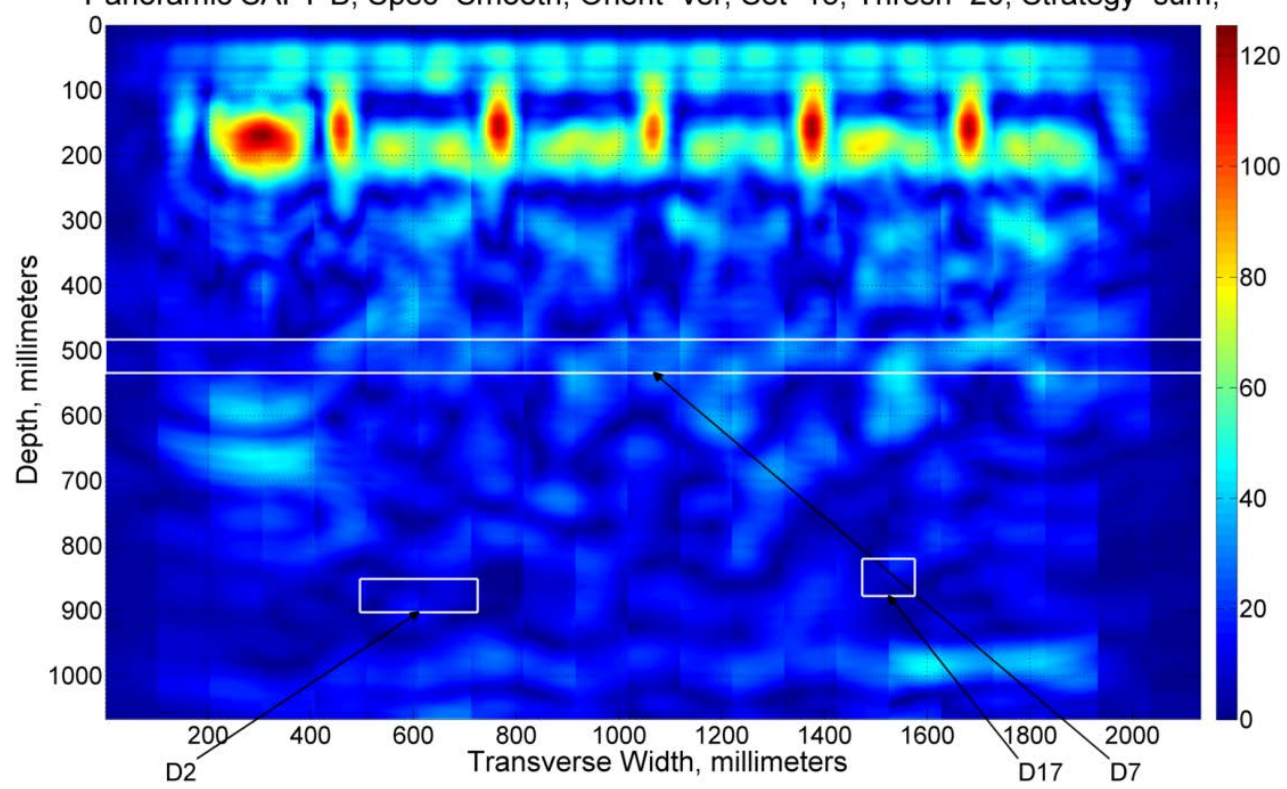

Fig. B. 159. Vertical Set 13, Node 32. 


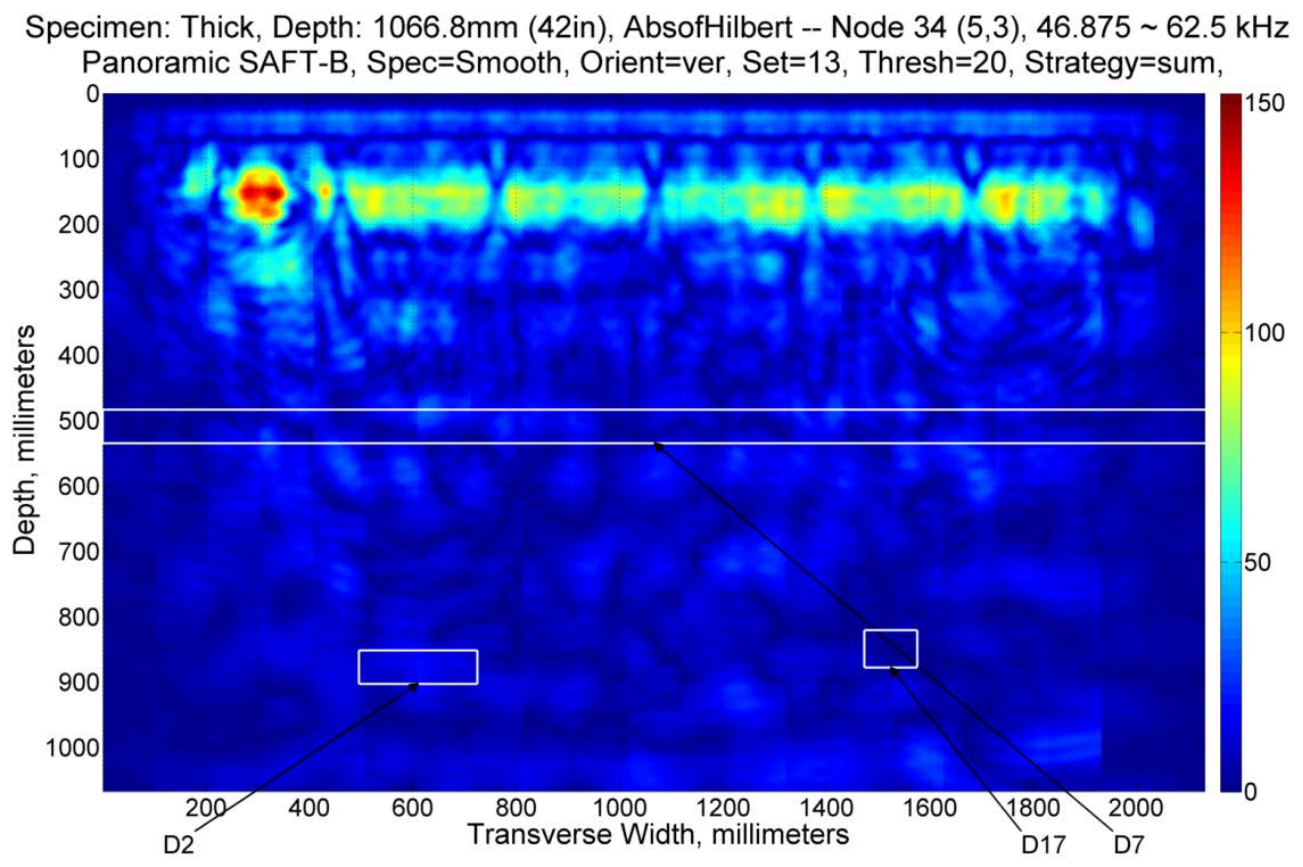

Fig. B. 160. Vertical Set 13, Node 34.

\section{SET FOURTEEN - DEFECT 4, DEFECT 7}

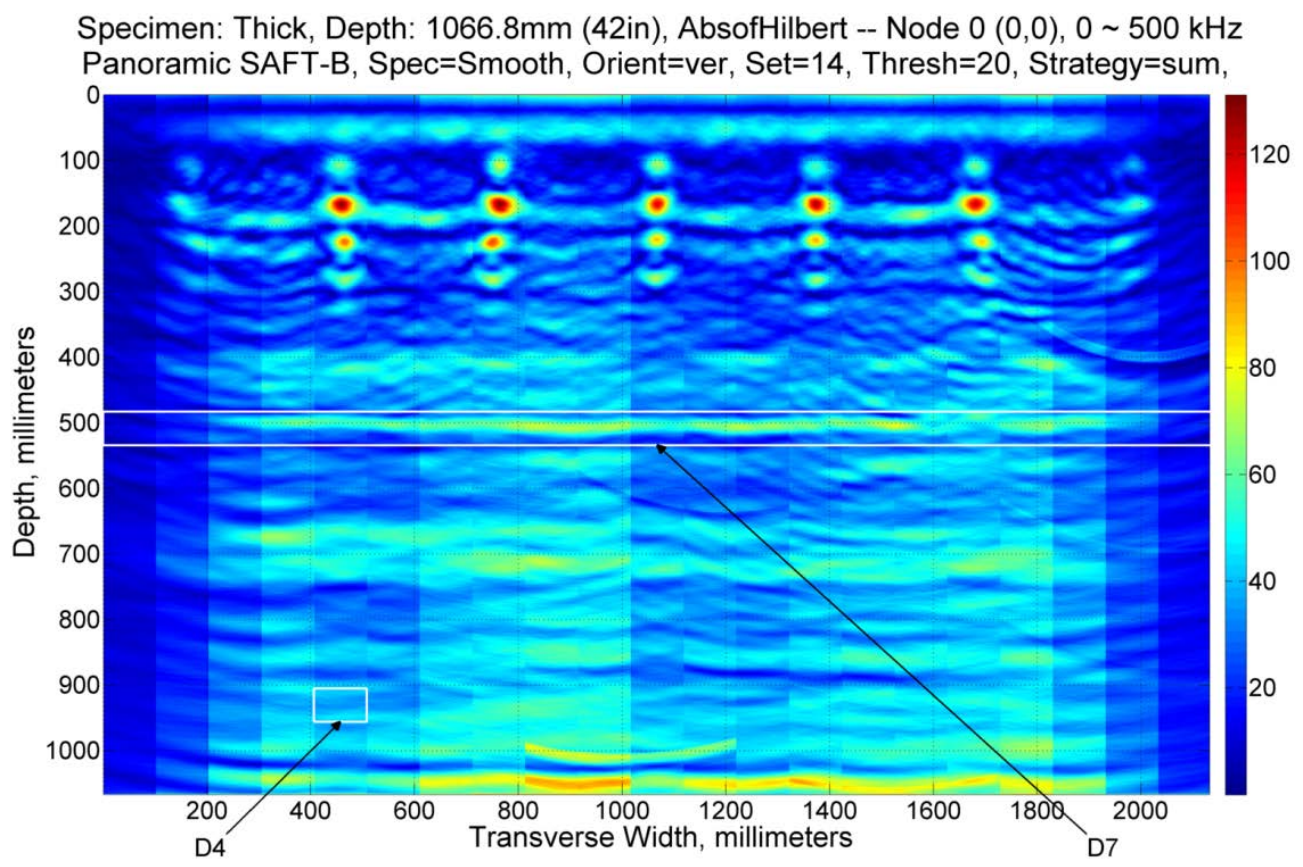

Fig. B. 161. Vertical Set 14, Node 0. 


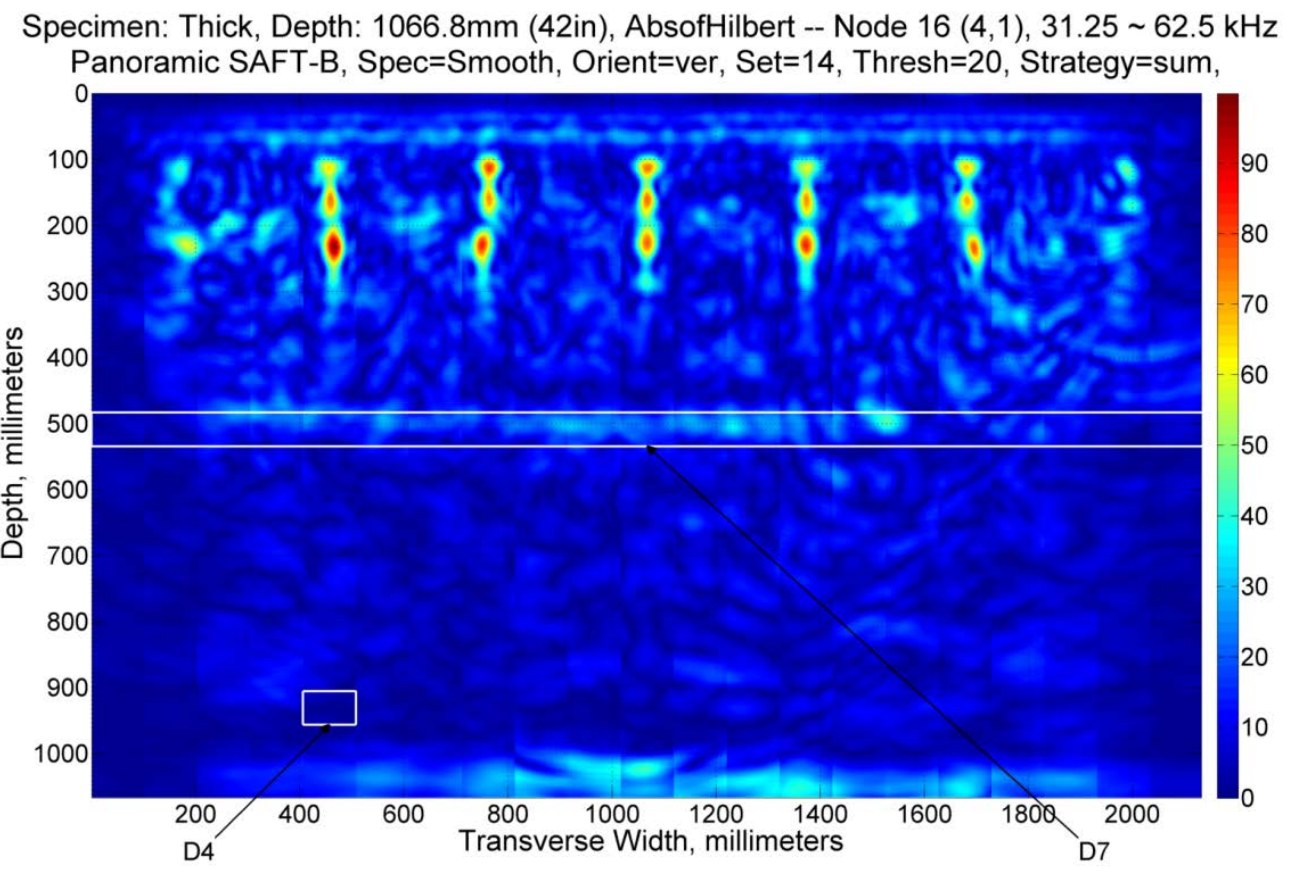

Fig. B. 162. Vertical Set 14, Node 16.

Specimen: Thick, Depth: 1066.8mm (42in), AbsofHilbert -- Node $18(4,3), 93.75 \sim 125 \mathrm{kHz}$ Panoramic SAFT-B, Spec=Smooth, Orient=ver, Set=14, Thresh=20, Strategy=sum,

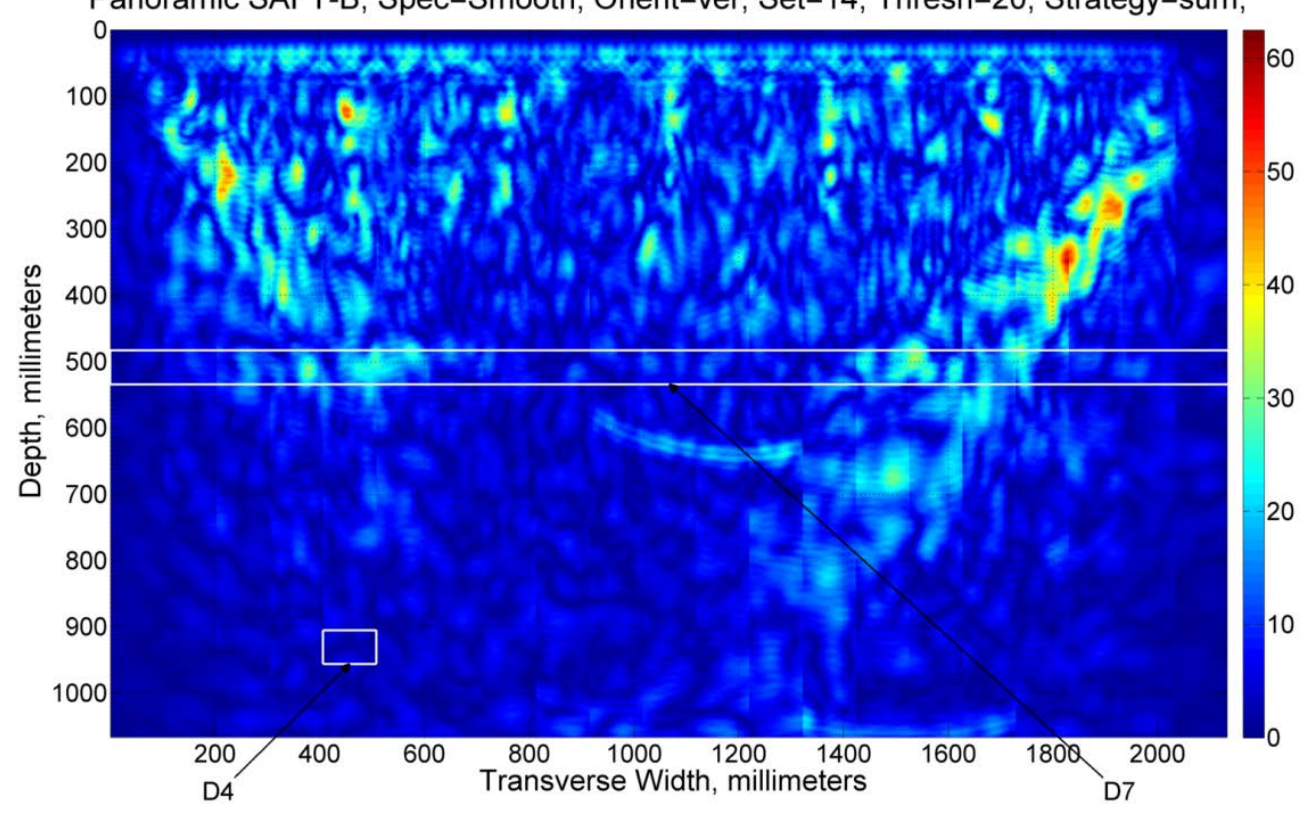

Fig. B. 163. Vertical Set 14, Node 18. 


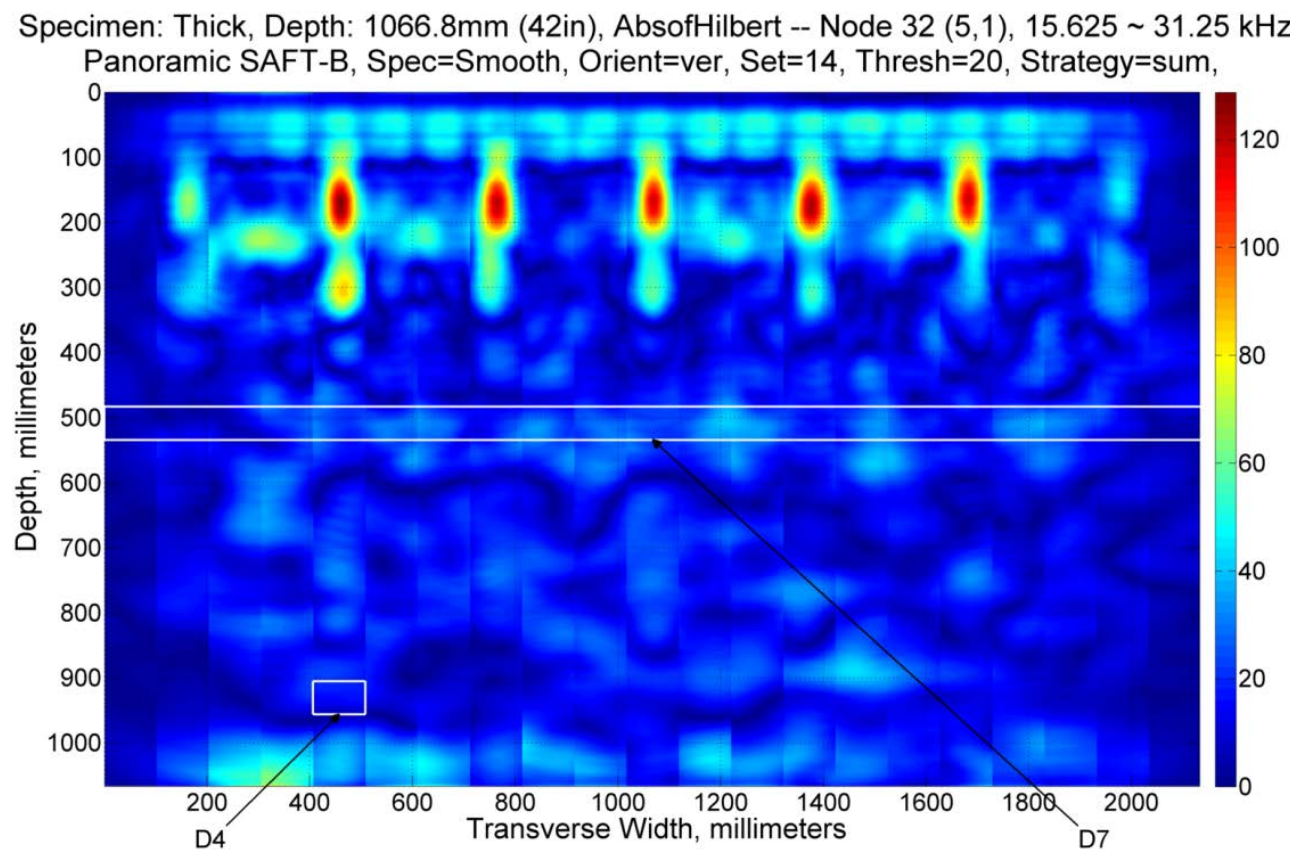

Fig. B. 164. Vertical Set 14, Node 32.

Specimen: Thick, Depth: 1066.8mm (42in), AbsofHilbert -- Node 34 (5,3), $46.875 \sim 62.5 \mathrm{kHz}$ Panoramic SAFT-B, Spec=Smooth, Orient=ver, Set=14, Thresh=20, Strategy=sum,

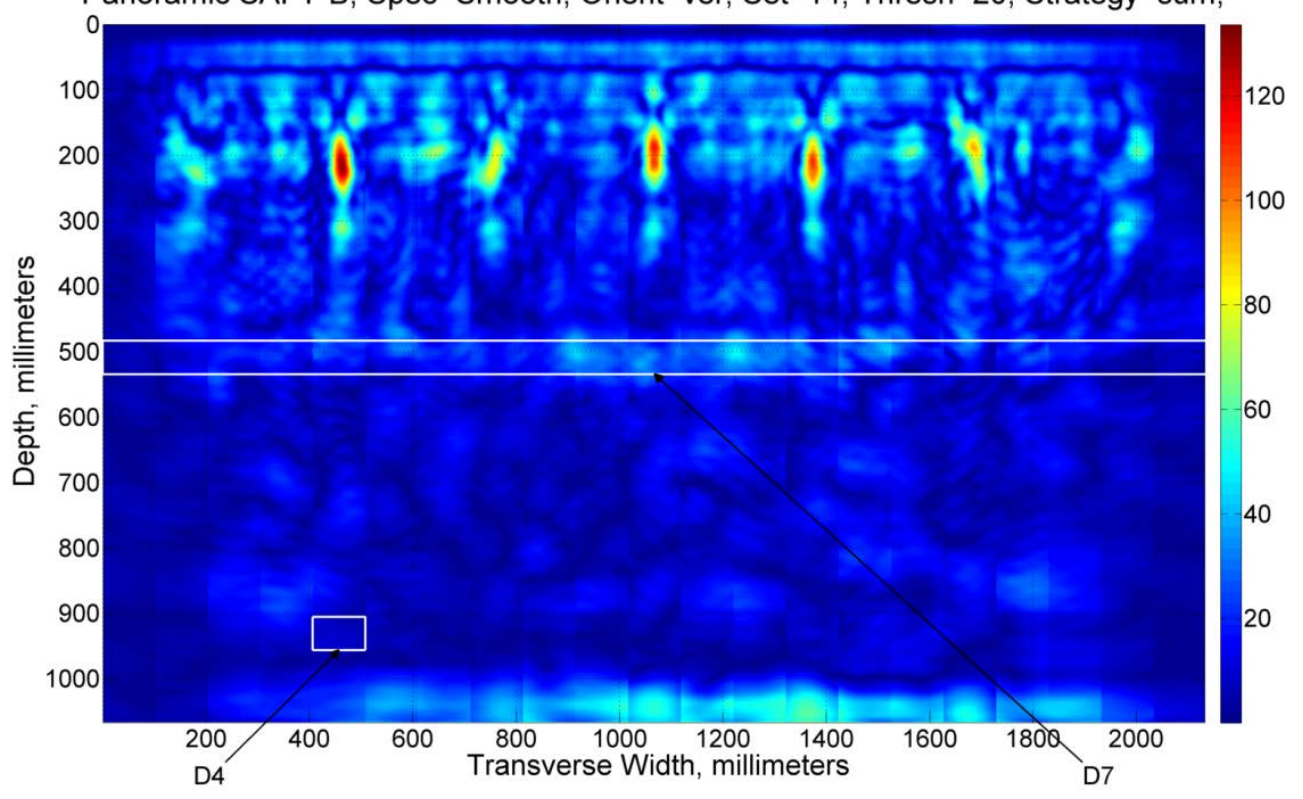

Fig. B. 165. Vertical Set 14, Node 34. 


\section{SET FIFTEEN - DEFECT 4, DEFECT 14}

Specimen: Thick, Depth: 1066.8mm (42in), AbsofHilbert -- Node $0(0,0), 0 \sim 500 \mathrm{kHz}$ Panoramic SAFT-B, Spec=Smooth, Orient=ver, Set=15, Thresh=20, Strategy=sum,

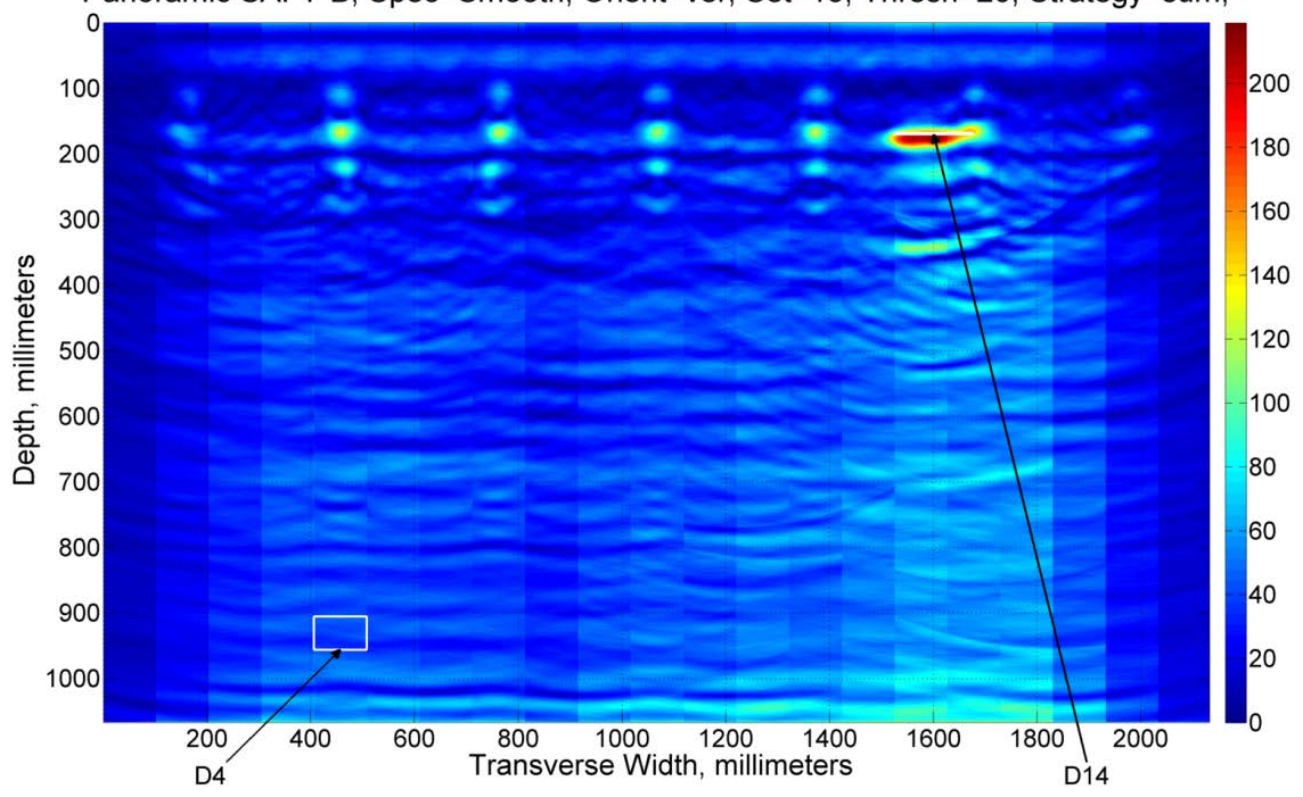

Fig. B. 166. Vertical Set 15, Node 0.

Specimen: Thick, Depth: 1066.8mm (42in), AbsofHilbert -- Node $16(4,1), 31.25 \sim 62.5 \mathrm{kHz}$ Panoramic SAFT-B, Spec $=$ Smooth, Orient $=v e r$, Set $=15$, Thresh $=20$, Strategy $=$ sum,

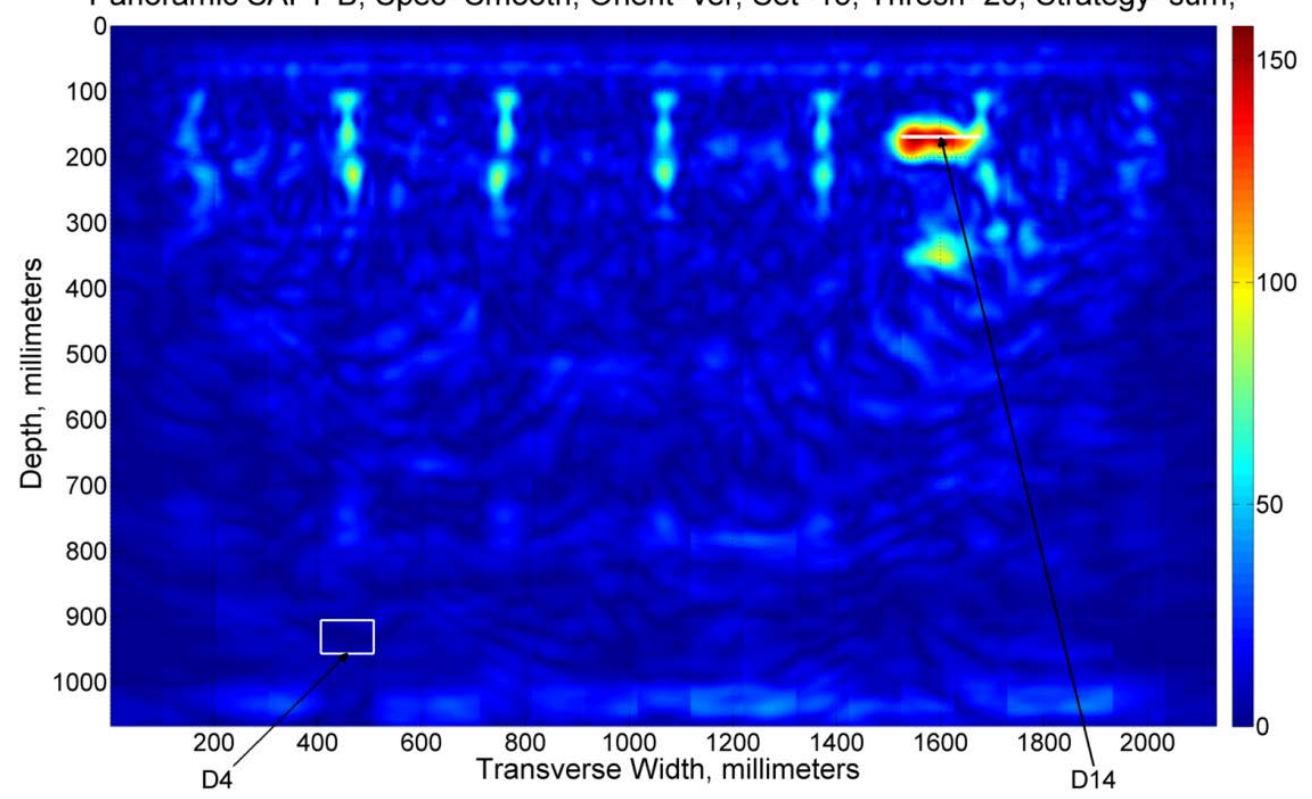

Fig. B. 167. Vertical Set 15, Node 16. 


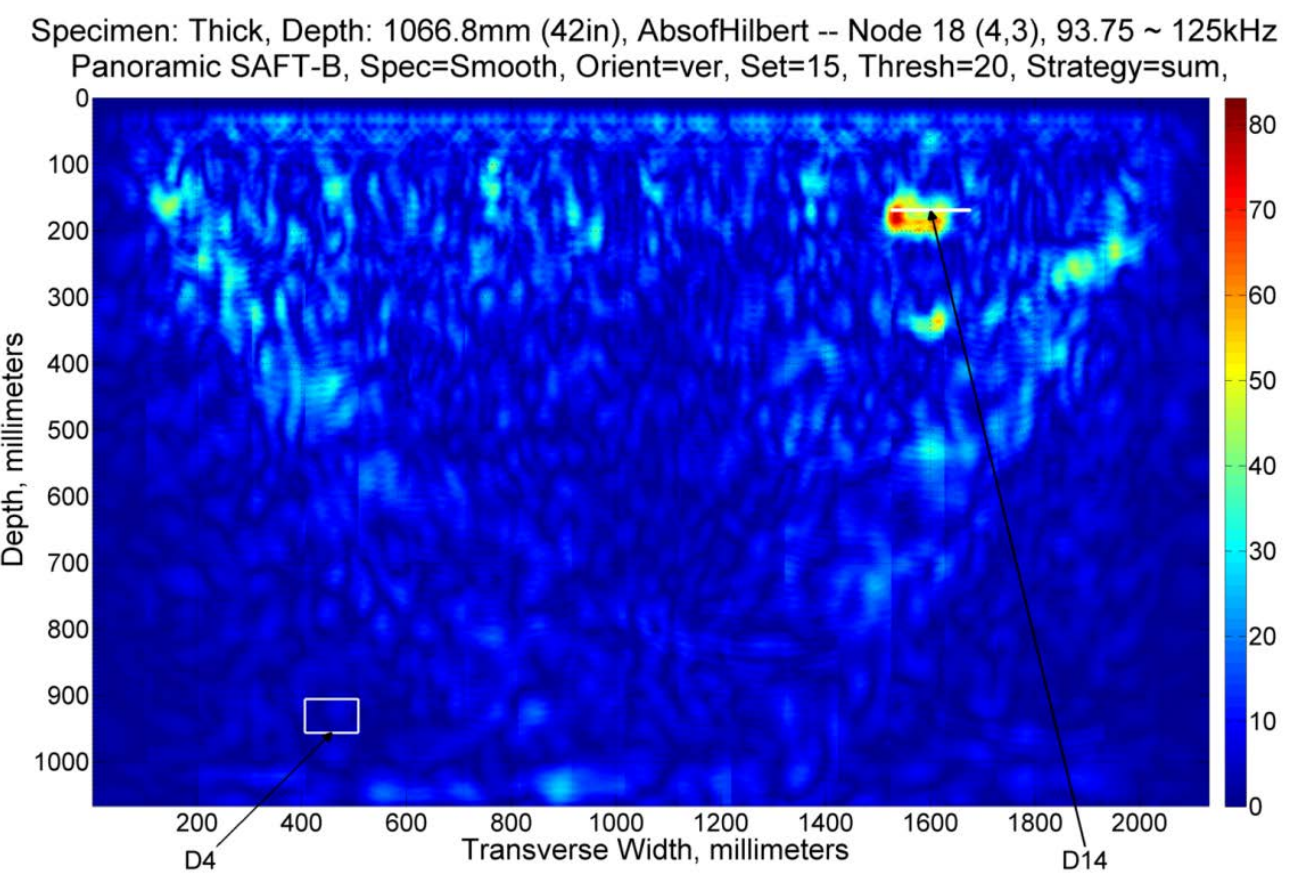

Fig. B. 168. Vertical Set 15, Node 18.

Specimen: Thick, Depth: 1066.8mm (42in), AbsofHilbert -- Node $32(5,1), 15.625 \sim 31.25 \mathrm{kHz}$ Panoramic SAFT-B, Spec=Smooth, Orient=ver, Set=15, Thresh=20, Strategy=sum,

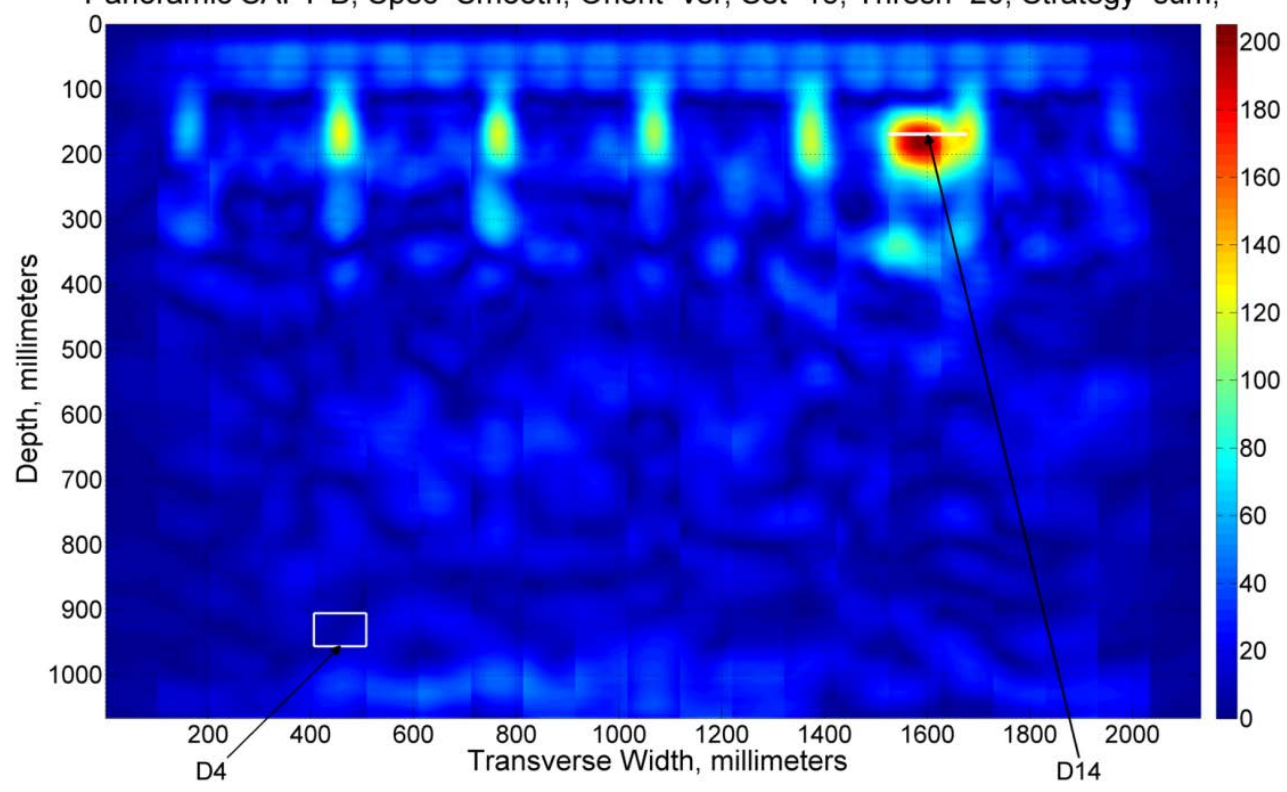

Fig. B. 169. Vertical Set 15, Node 32. 


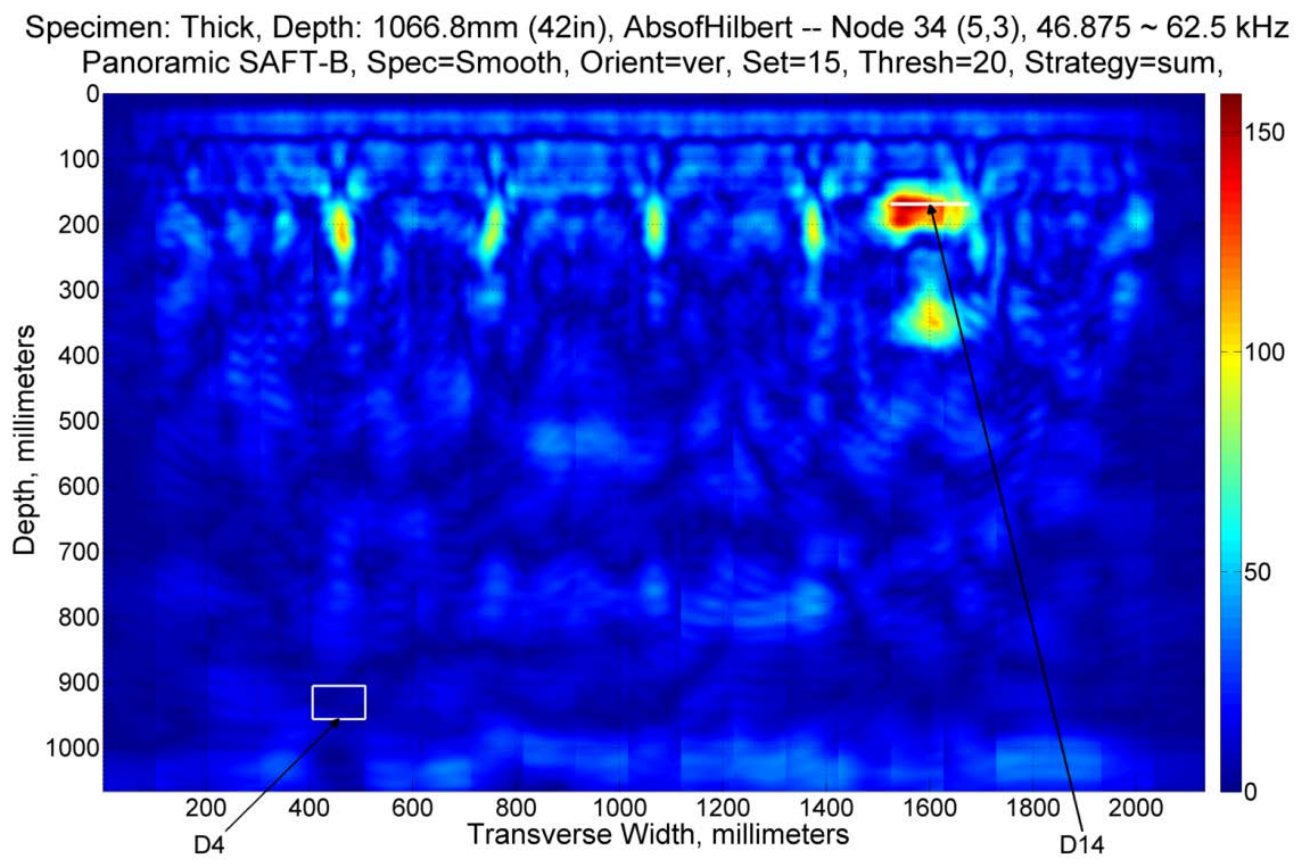

Fig. B. 170. Vertical Set 15, Node 34.

\section{SET SIXTEEN - NO DEFECT}

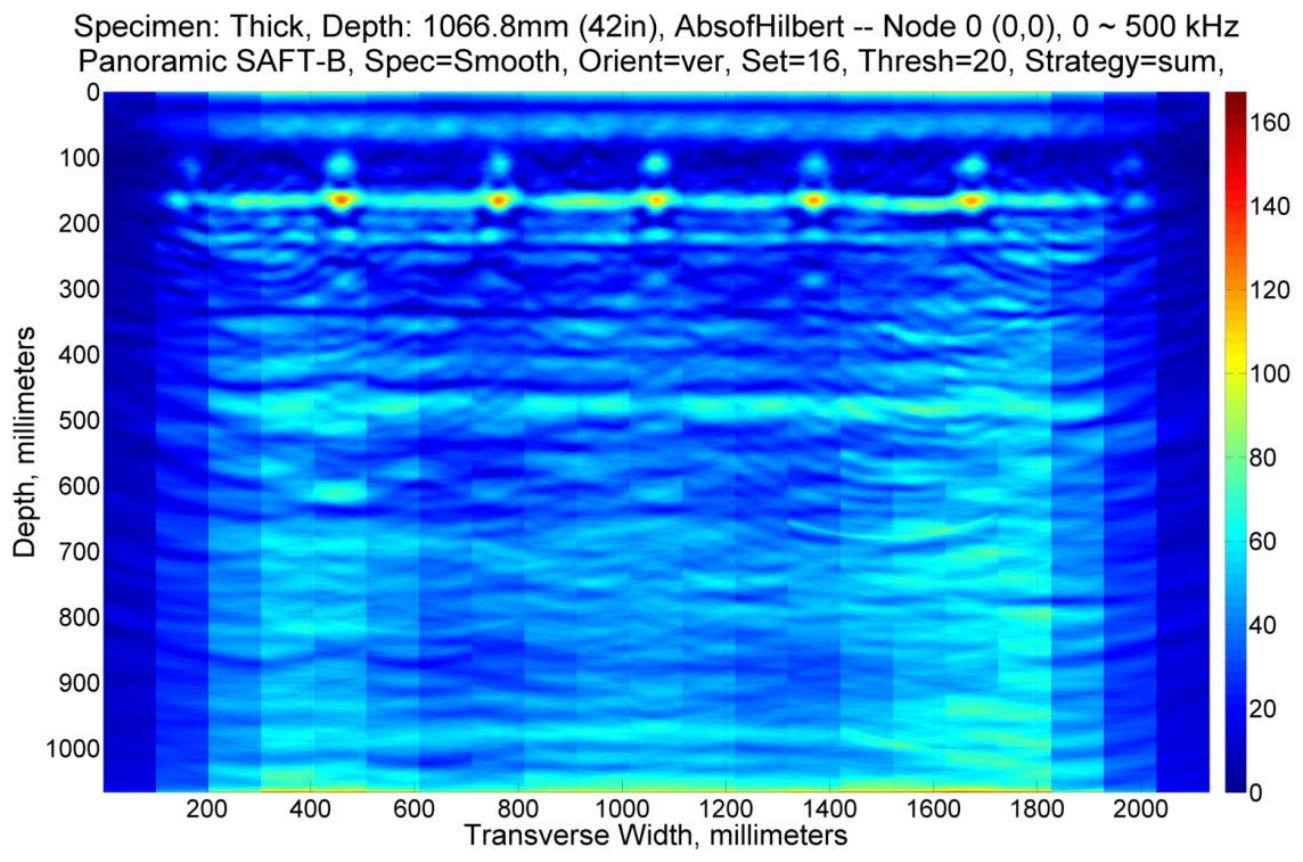

Fig. B. 171. Vertical Set 16, Node 0. 


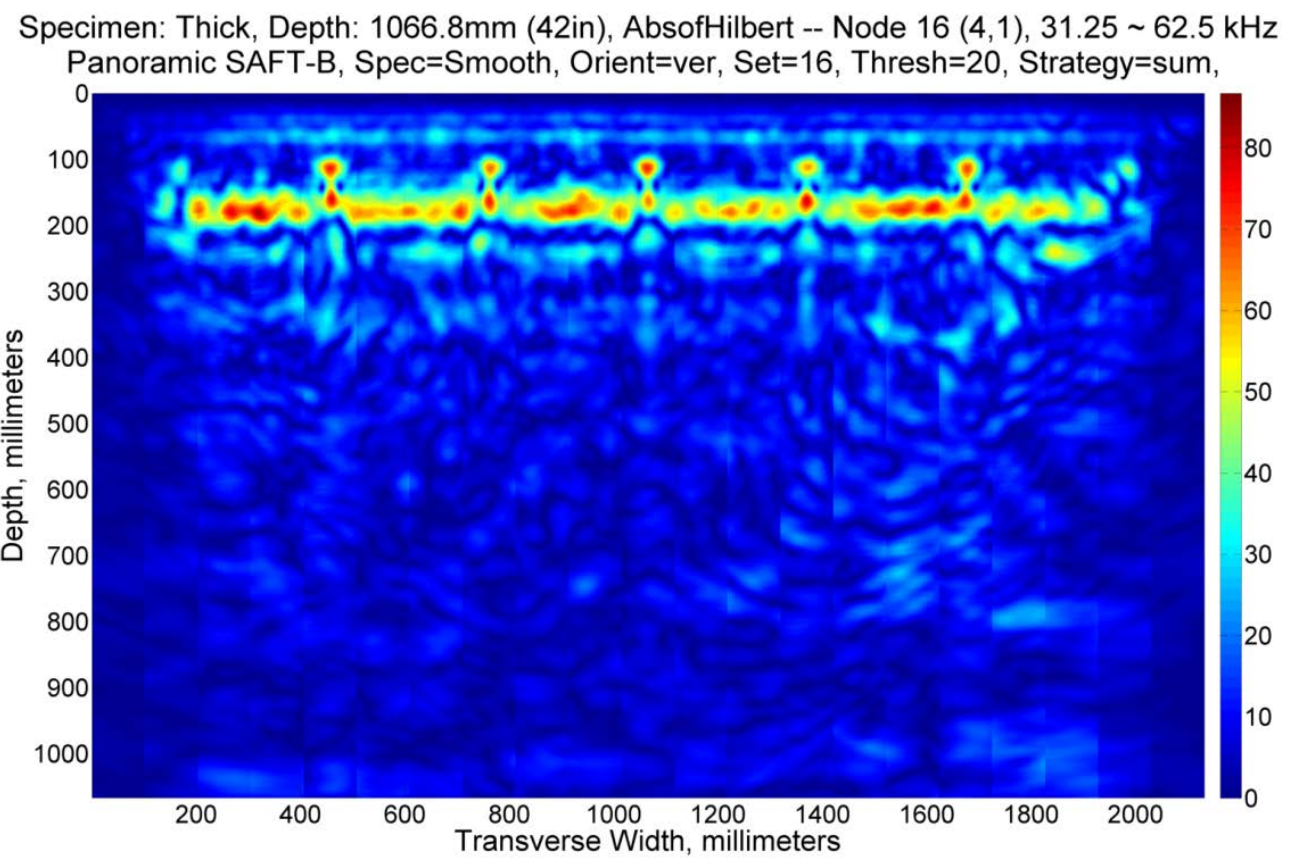

Fig. B. 172. Vertical Set 16, Node 16.

Specimen: Thick, Depth: 1066.8mm (42in), AbsofHilbert -- Node $18(4,3), 93.75 \sim 125 \mathrm{kHz}$ Panoramic SAFT-B, Spec=Smooth, Orient=ver, Set $=16$, Thresh=20, Strategy=sum,

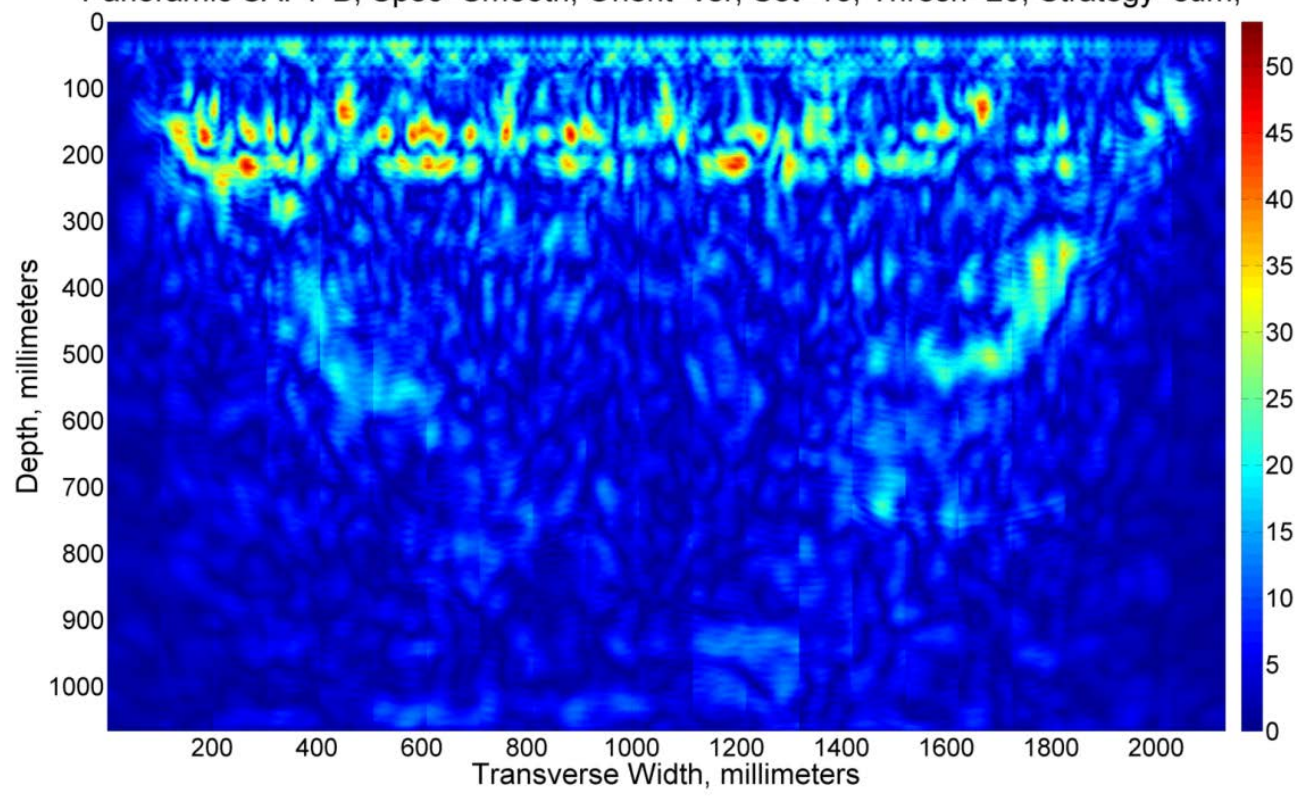

Fig. B. 173. Vertical Set 16, Node 18. 
Specimen: Thick, Depth: 1066.8mm (42in), AbsofHilbert -- Node $32(5,1), 15.625 \sim 31.25 \mathrm{kHz}$ Panoramic SAFT-B, Spec=Smooth, Orient=ver, Set=16, Thresh=20, Strategy=sum,

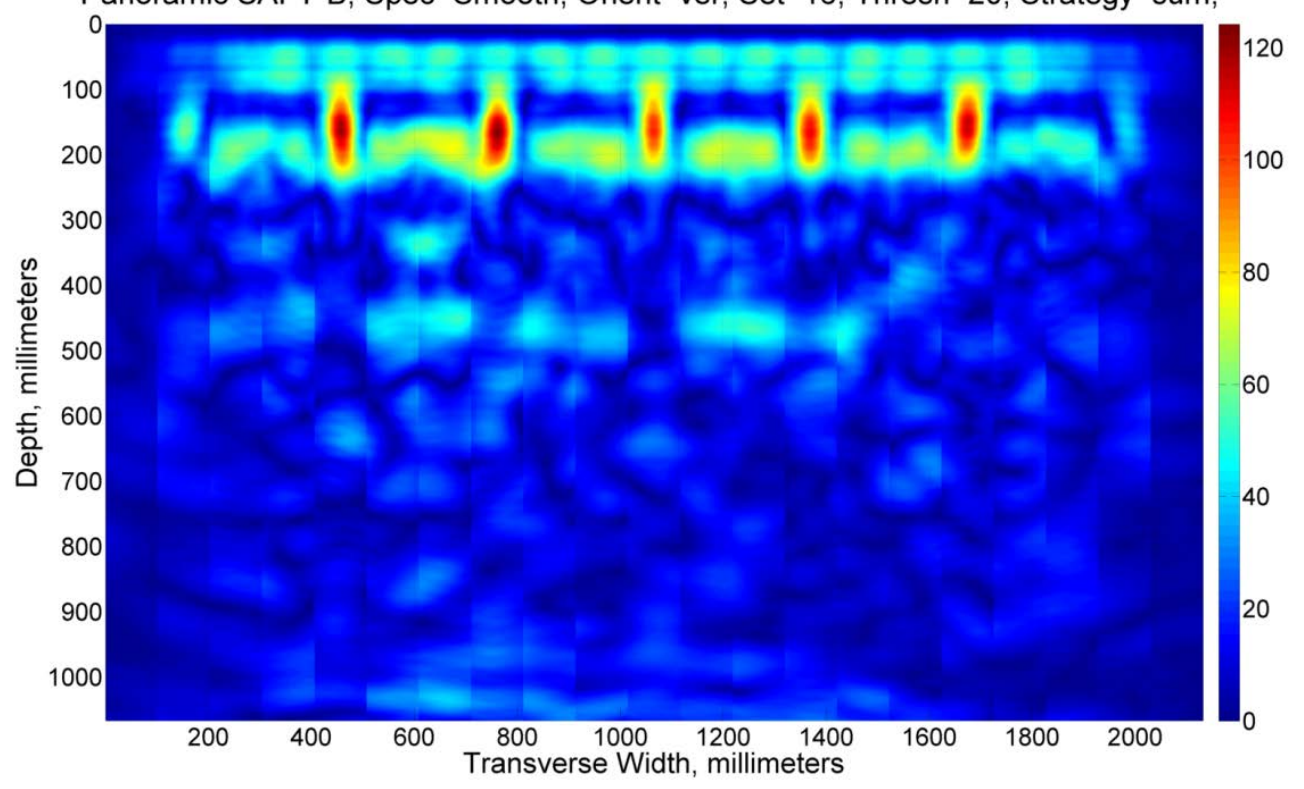

Fig. B. 174. Vertical Set 16, Node 32.

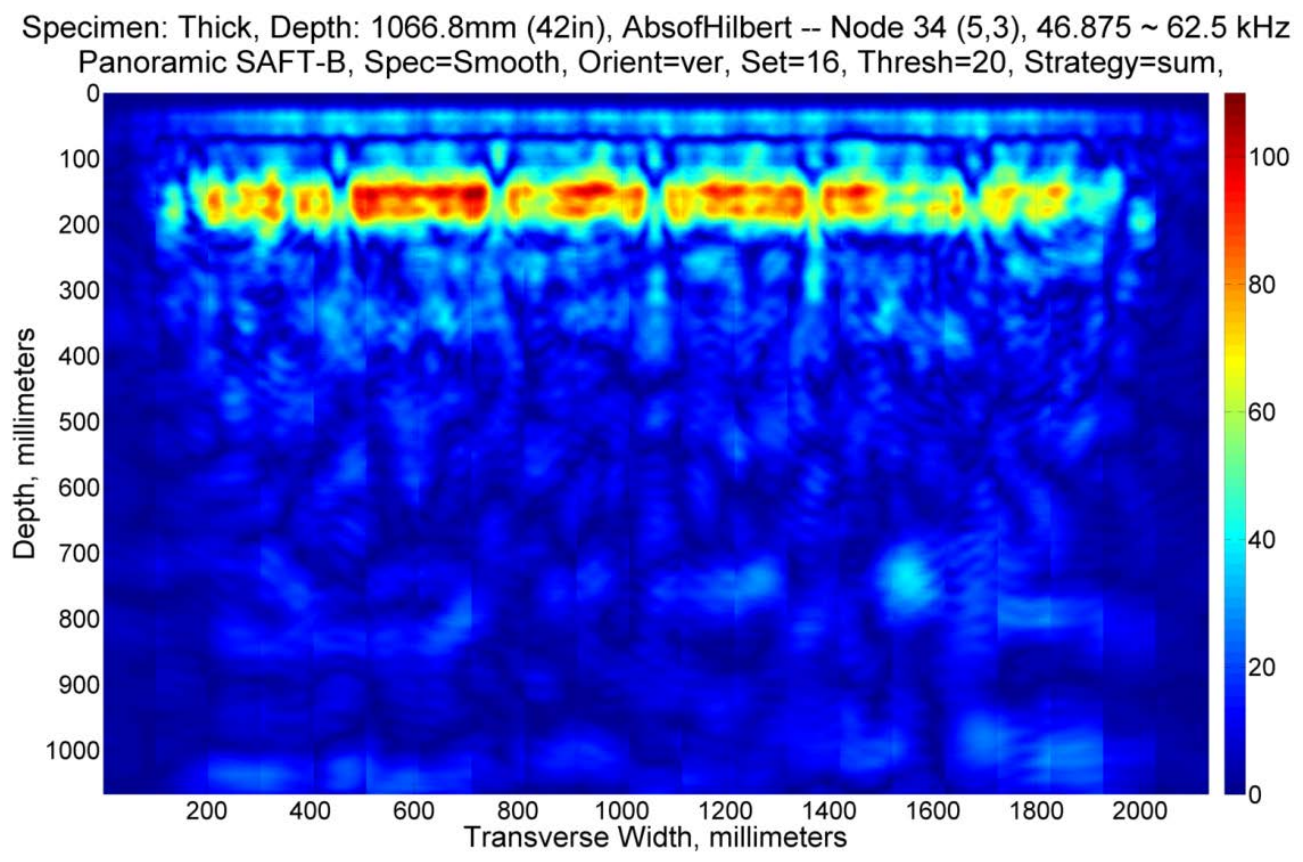

Fig. B. 175. Vertical Set 16, Node 34. 


\section{SET SEVENTEEN - DEFECT 16, DEFECT 19}

Specimen: Thick, Depth: 1066.8mm (42in), AbsofHilbert -- Node $0(0,0), 0 \sim 500 \mathrm{kHz}$ Panoramic SAFT-B, Spec=Smooth, Orient=ver, Set=17, Thresh=20, Strategy=sum,

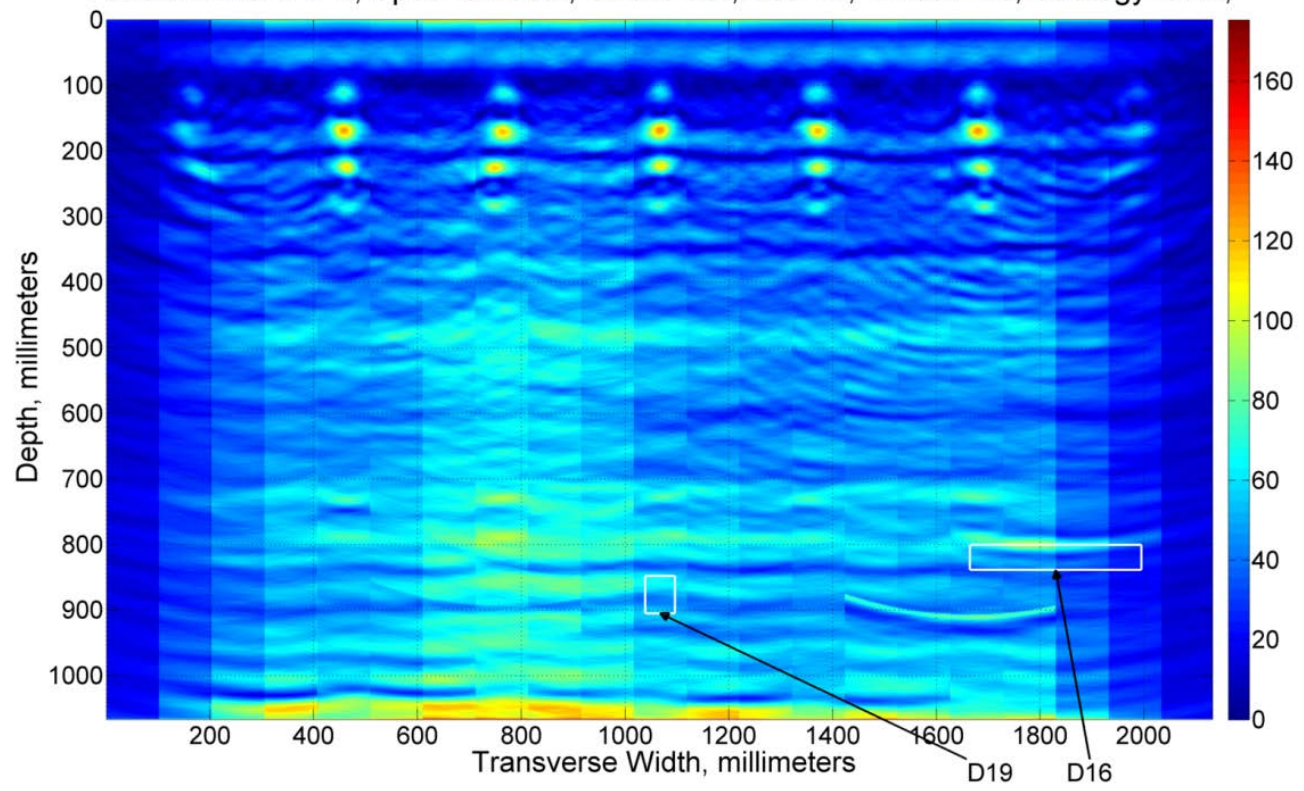

Fig. B. 176. Vertical Set 17, Node 0.

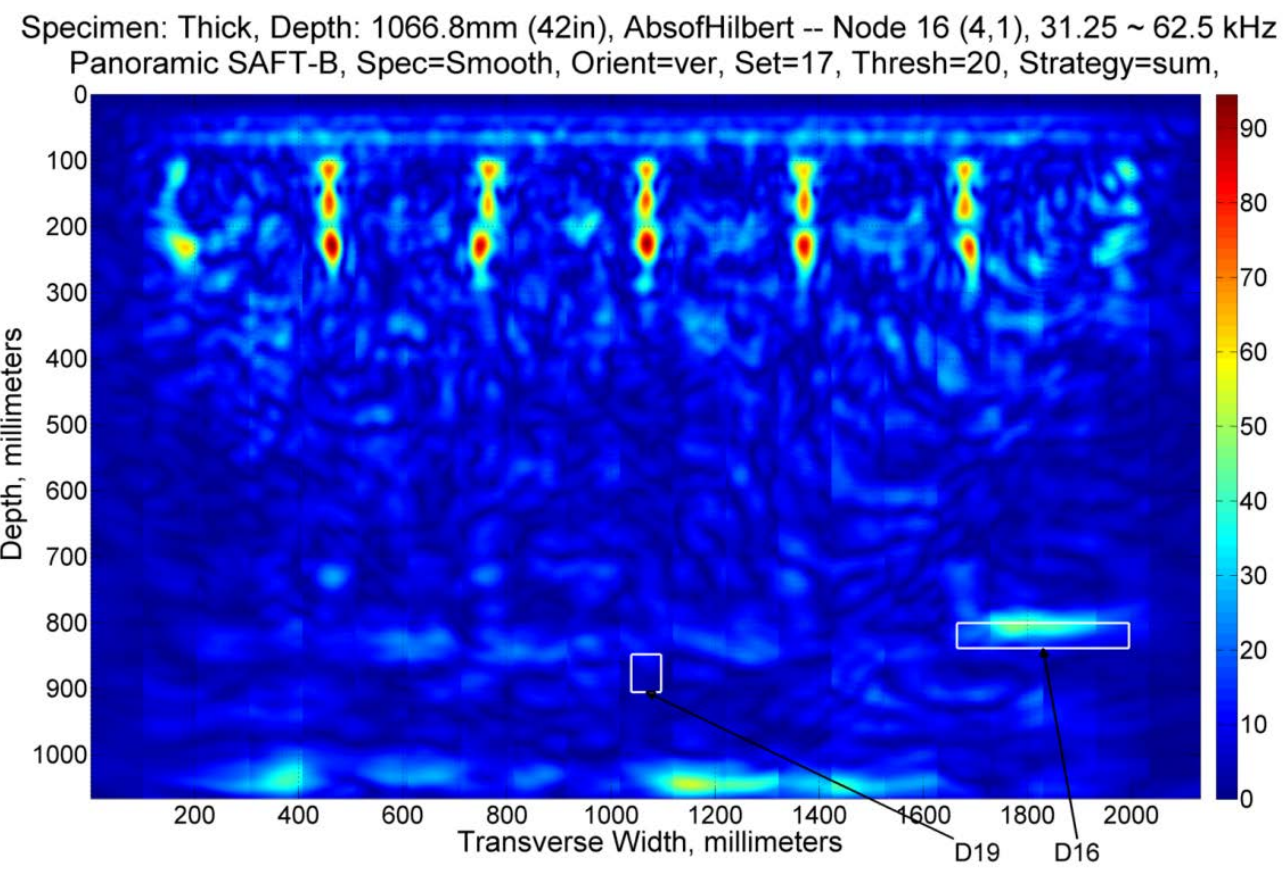

Fig. B. 177. Vertical Set 17, Node 16. 


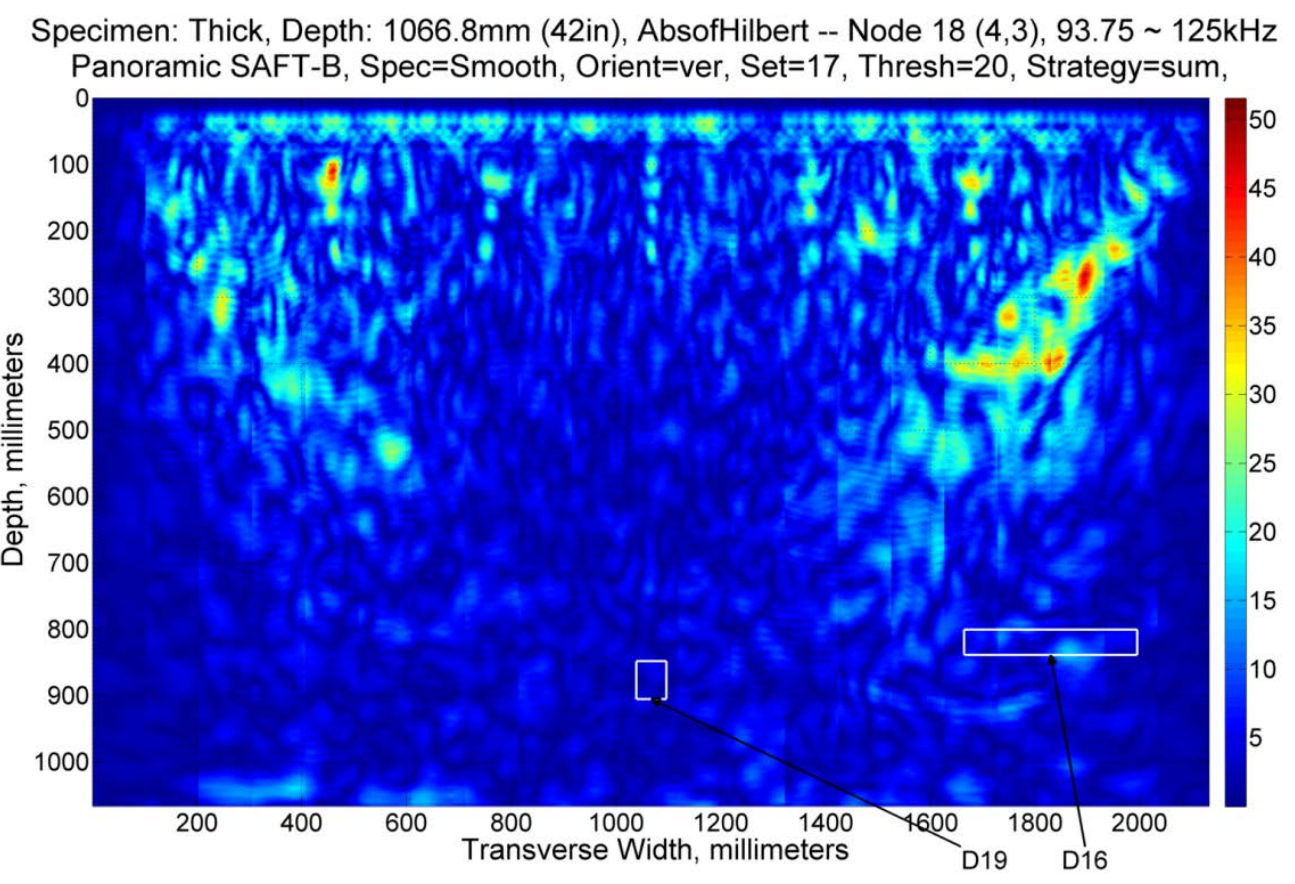

Fig. B. 178. Vertical Set 17, Node 18.

Specimen: Thick, Depth: 1066.8mm (42in), AbsofHilbert -- Node 32 (5,1), $15.625 \sim 31.25 \mathrm{kHz}$ Panoramic SAFT-B, Spec=Smooth, Orient=ver, Set=17, Thresh=20, Strategy=sum,

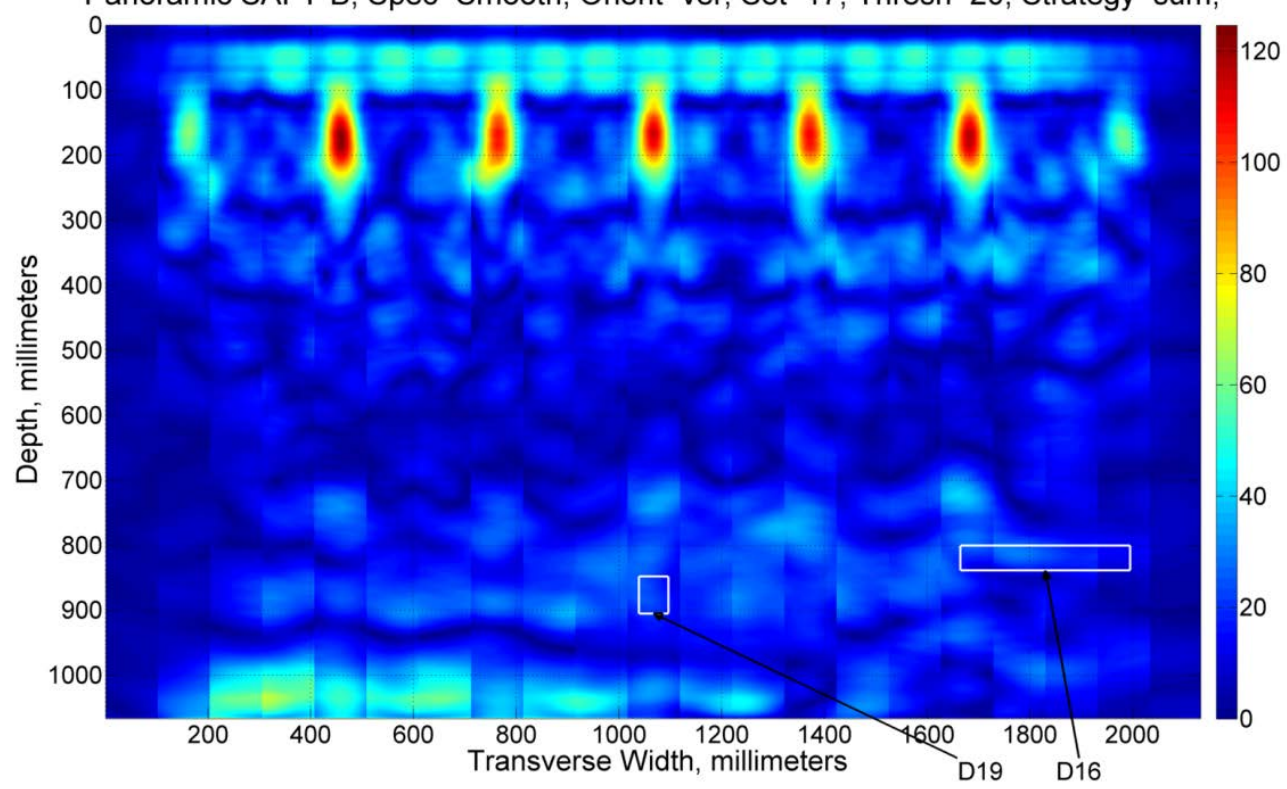

Fig. B. 179. Vertical Set 17, Node 32. 


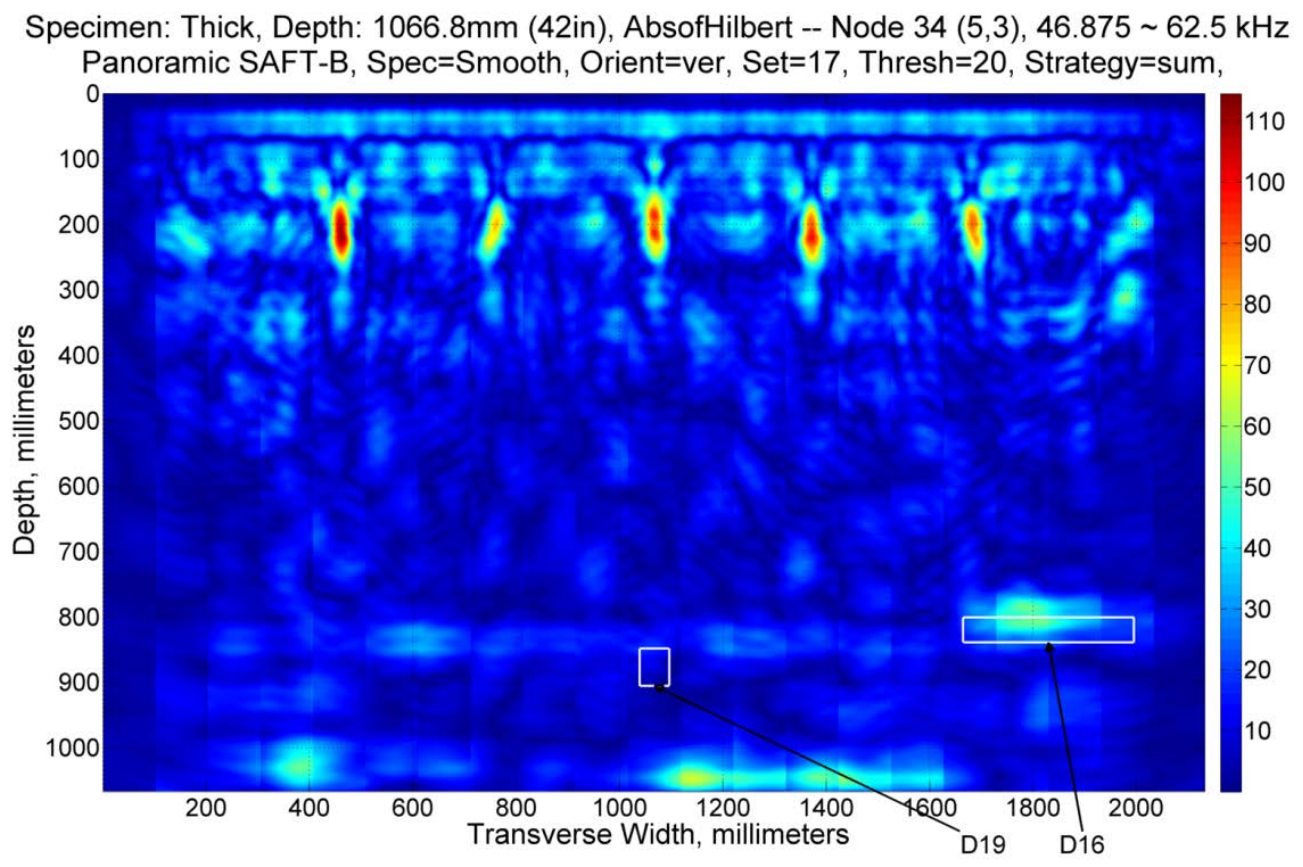

Fig. B. 180. Vertical Set 17, Node 34.

\section{SET EIGHTEEN - DEFECT 18}

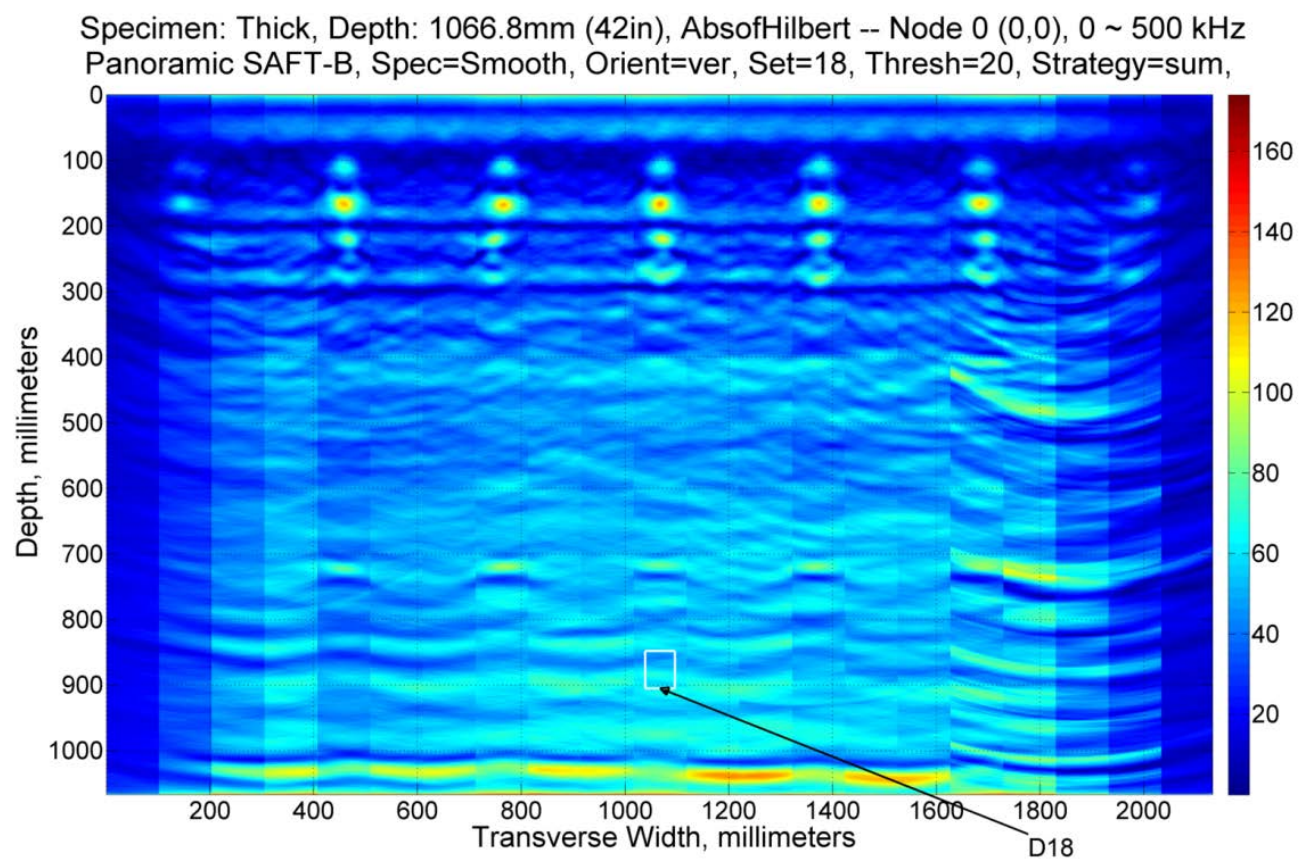

Fig. B. 181. Vertical Set 18, Node 0. 


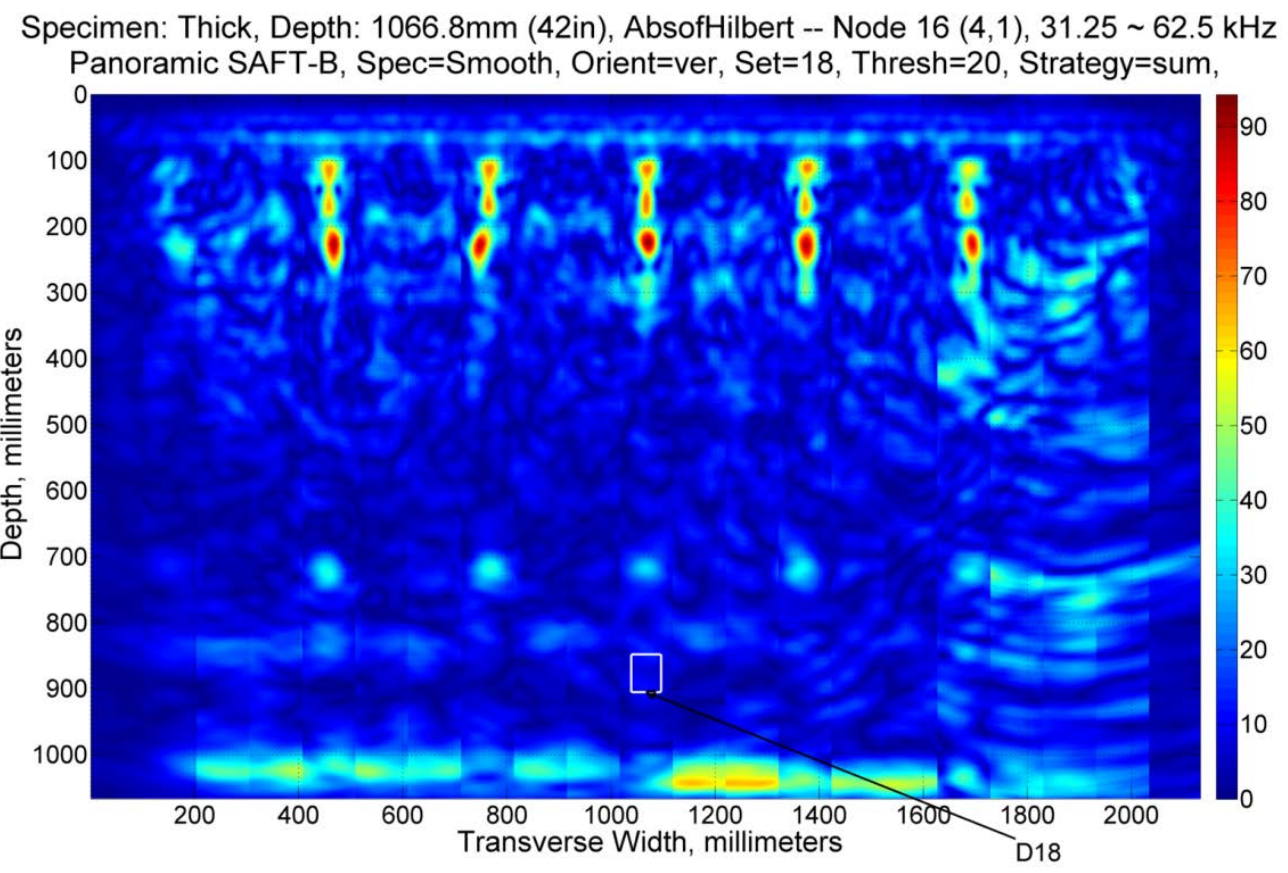

Fig. B. 182, Vertical Set 18, Node 16.

Specimen: Thick, Depth: 1066.8mm (42in), AbsofHilbert -- Node $18(4,3), 93.75 \sim 125 \mathrm{kHz}$ Panoramic SAFT-B, Spec=Smooth, Orient=ver, Set=18, Thresh=20, Strategy=sum,

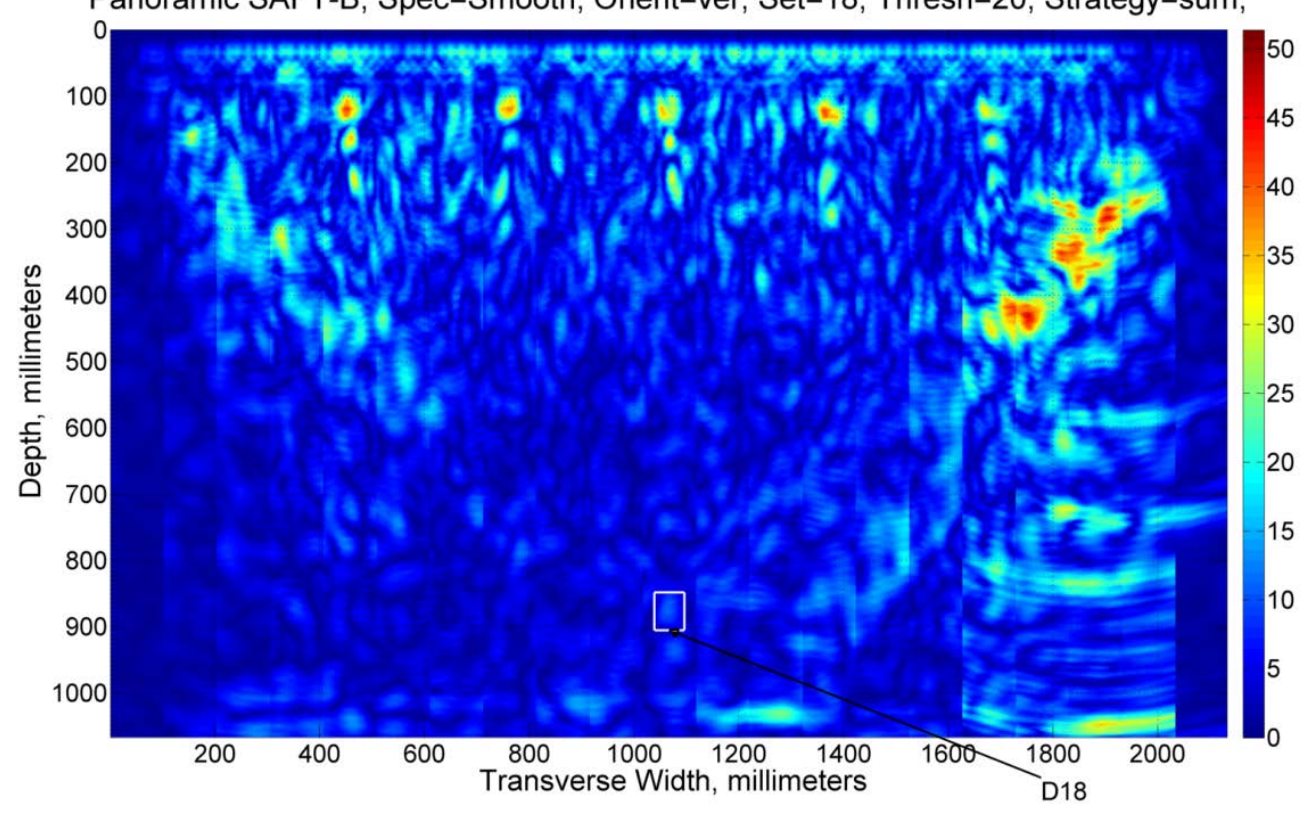

Fig. B. 183. Vertical Set 18, Node 18. 


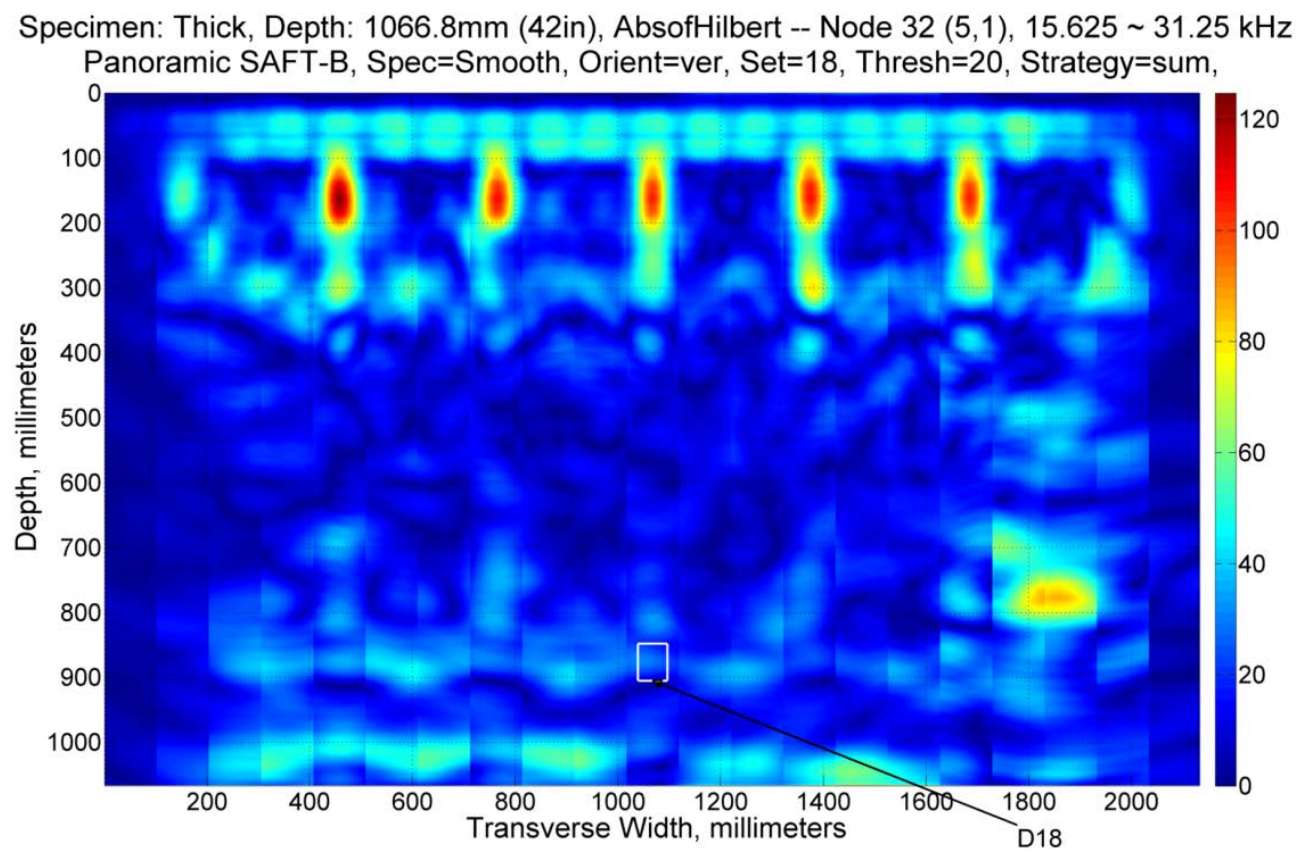

Fig. B. 184. Vertical Set 18, Node 32.

Specimen: Thick, Depth: 1066.8mm (42in), AbsofHilbert -- Node 34 (5,3), $46.875 \sim 62.5 \mathrm{kHz}$ Panoramic SAFT-B, Spec=Smooth, Orient=ver, Set=18, Thresh=20, Strategy=sum,

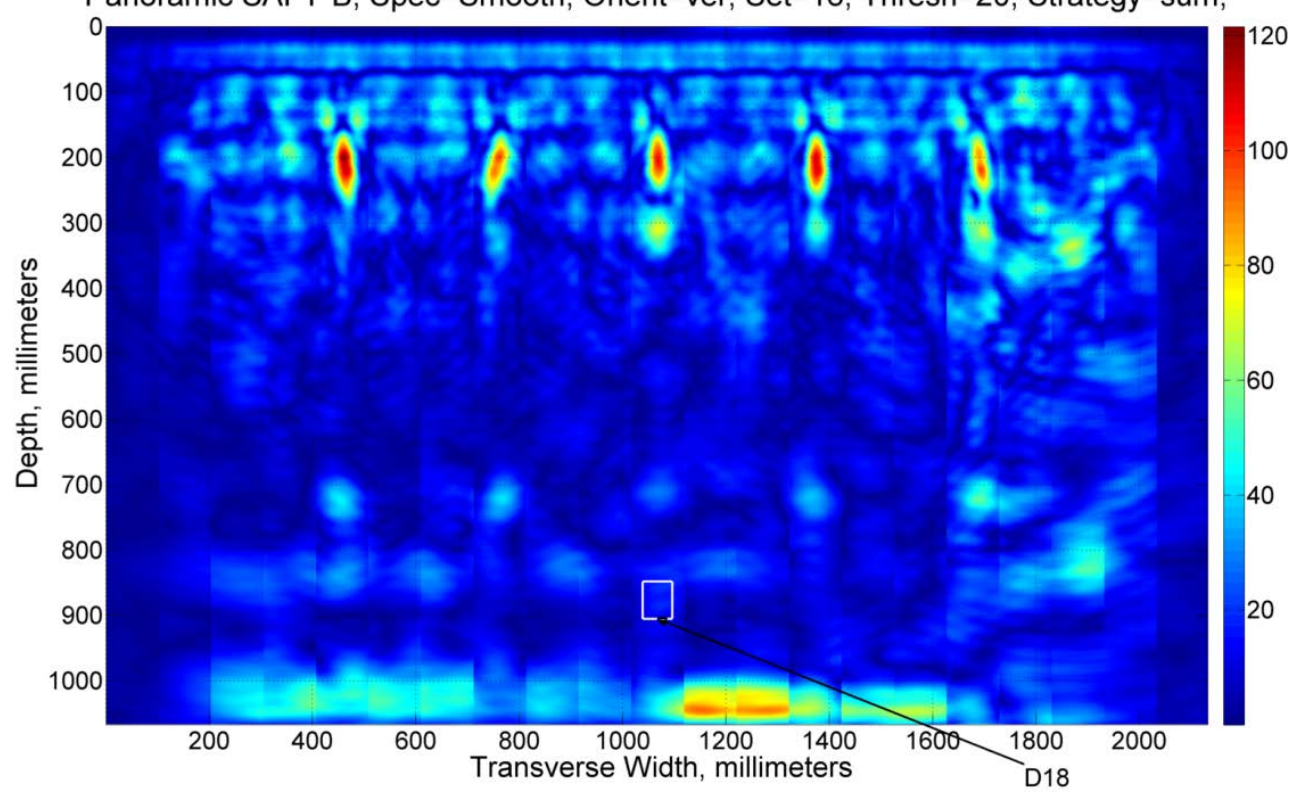

Fig. B. 185. Vertical Set 18, Node 34. 


\section{SET NINETEEN - NO DEFECT}

Specimen: Thick, Depth: 1066.8mm (42in), AbsofHilbert -- Node $0(0,0), 0 \sim 500 \mathrm{kHz}$ Panoramic SAFT-B, Spec=Smooth, Orient=ver, Set=19, Thresh=20, Strategy=sum,

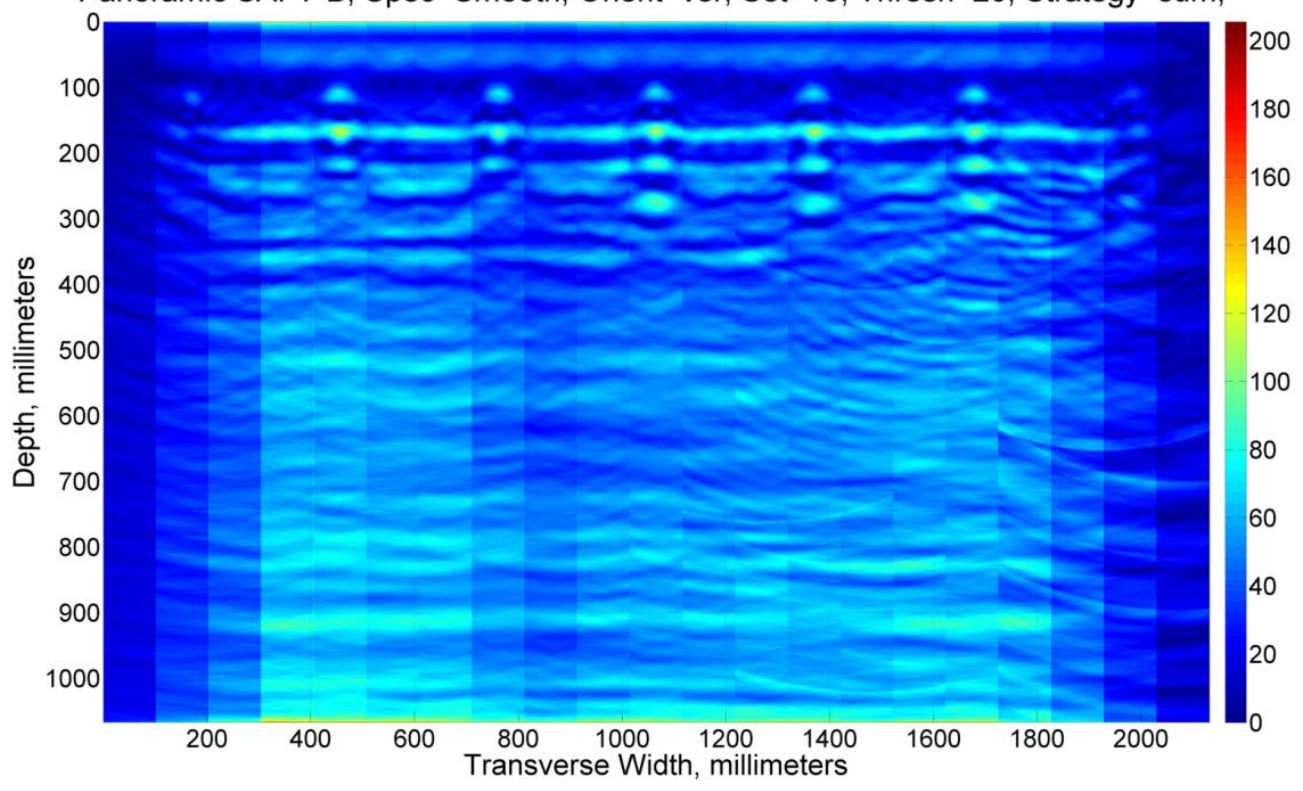

Fig. B. 186. Vertical Set 19, Node 0.

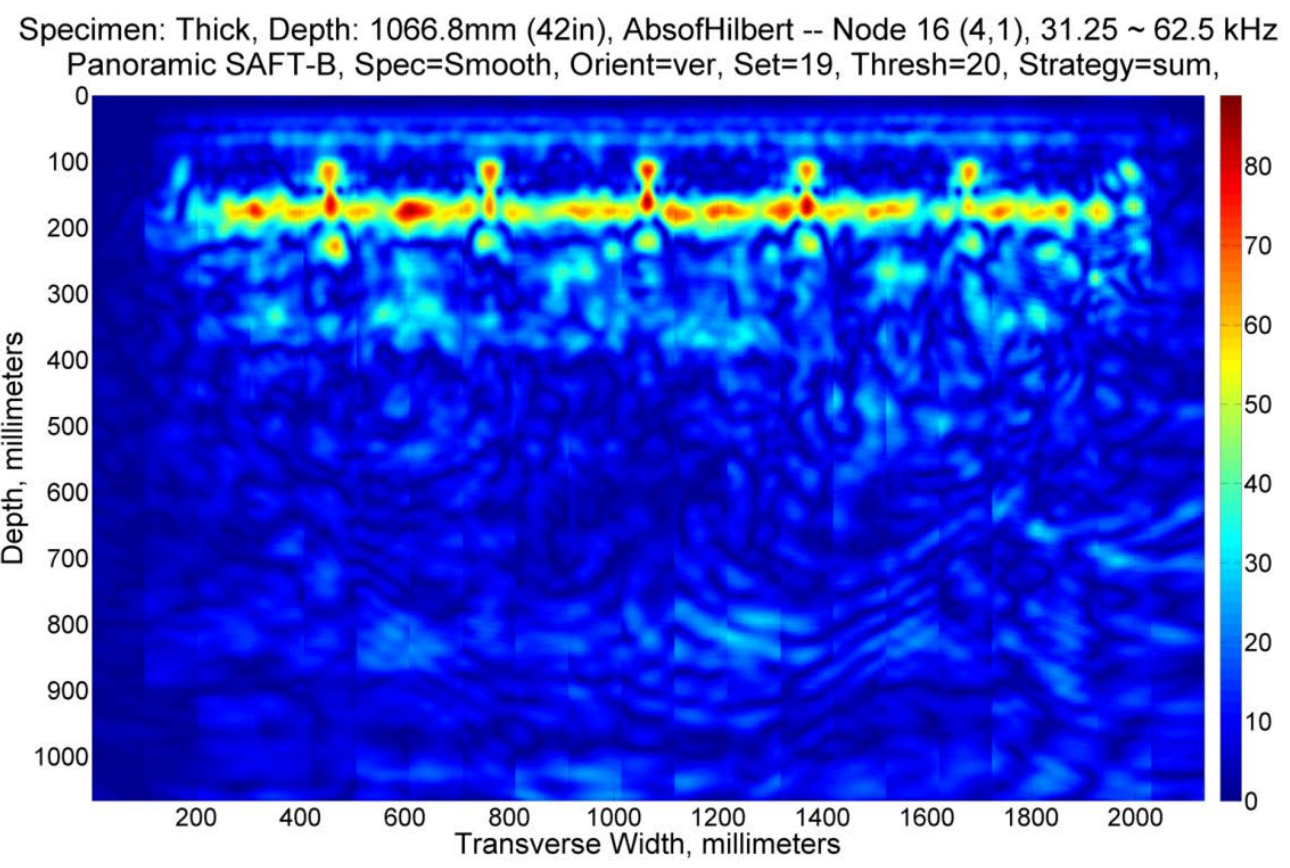

Fig. B. 187. Vertical Set 19, Node 16. 


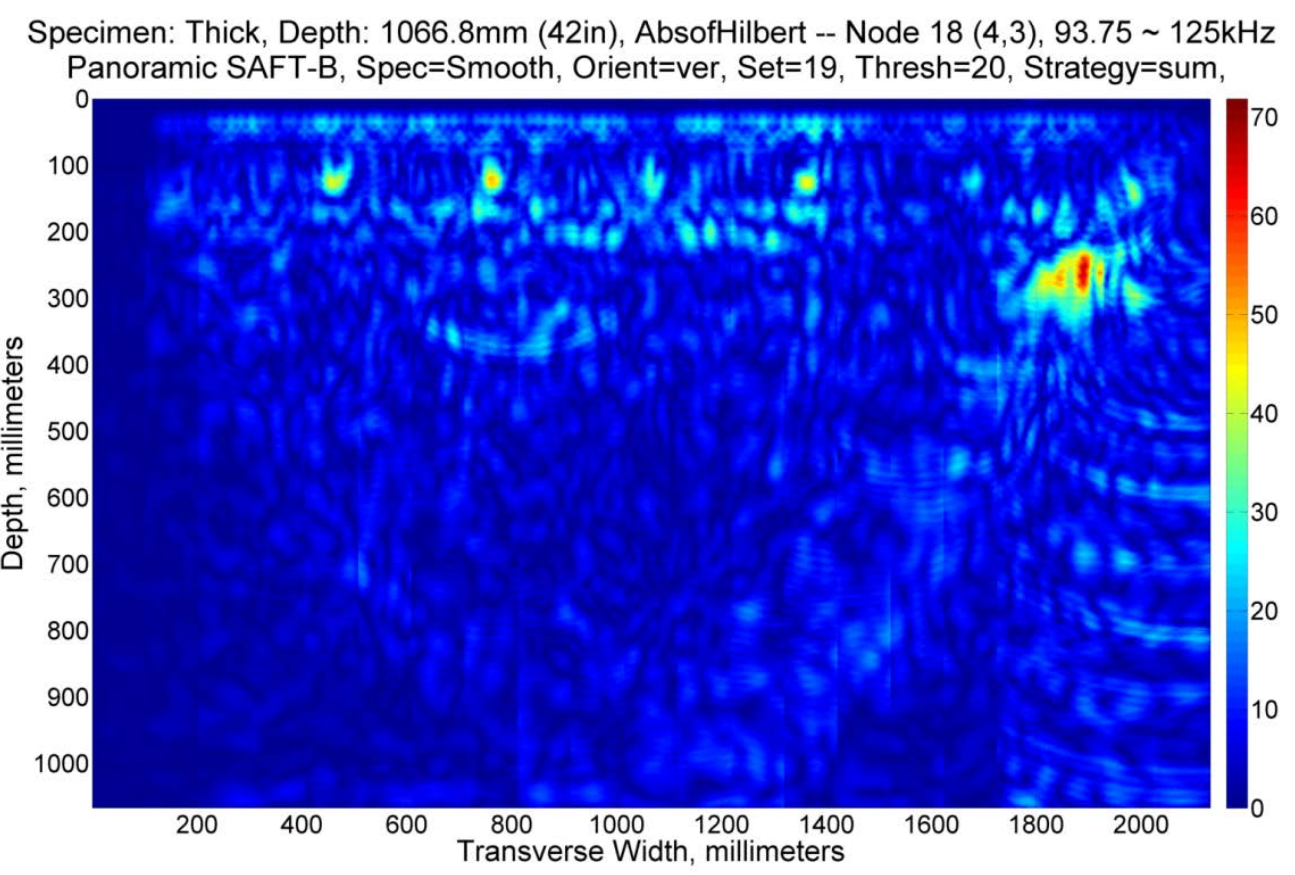

Fig. B. 188. Vertical Set 19, Node 18.

Specimen: Thick, Depth: 1066.8mm (42in), AbsofHilbert -- Node $32(5,1), 15.625 \sim 31.25 \mathrm{kHz}$ Panoramic SAFT-B, Spec=Smooth, Orient=ver, Set=19, Thresh=20, Strategy=sum,

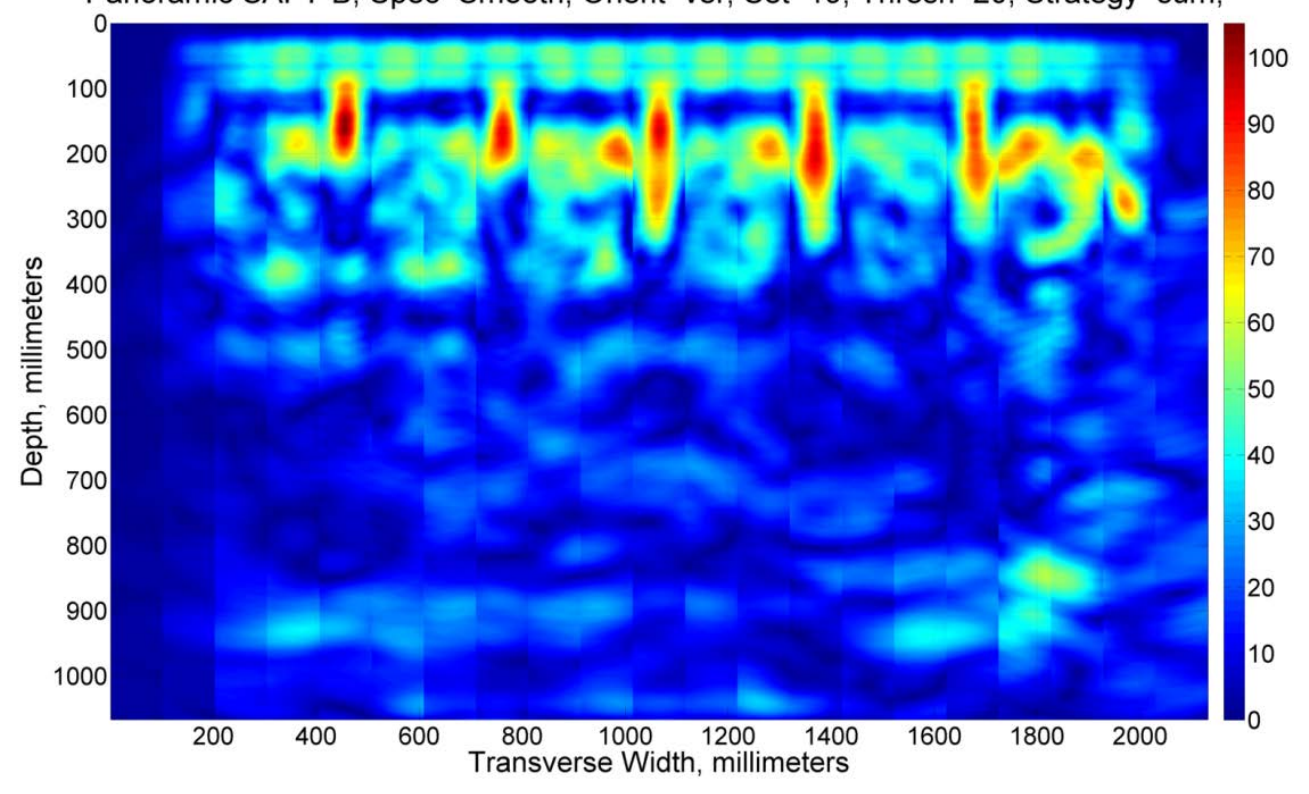

Fig. B. 189. Vertical Set 19, Node 32. 


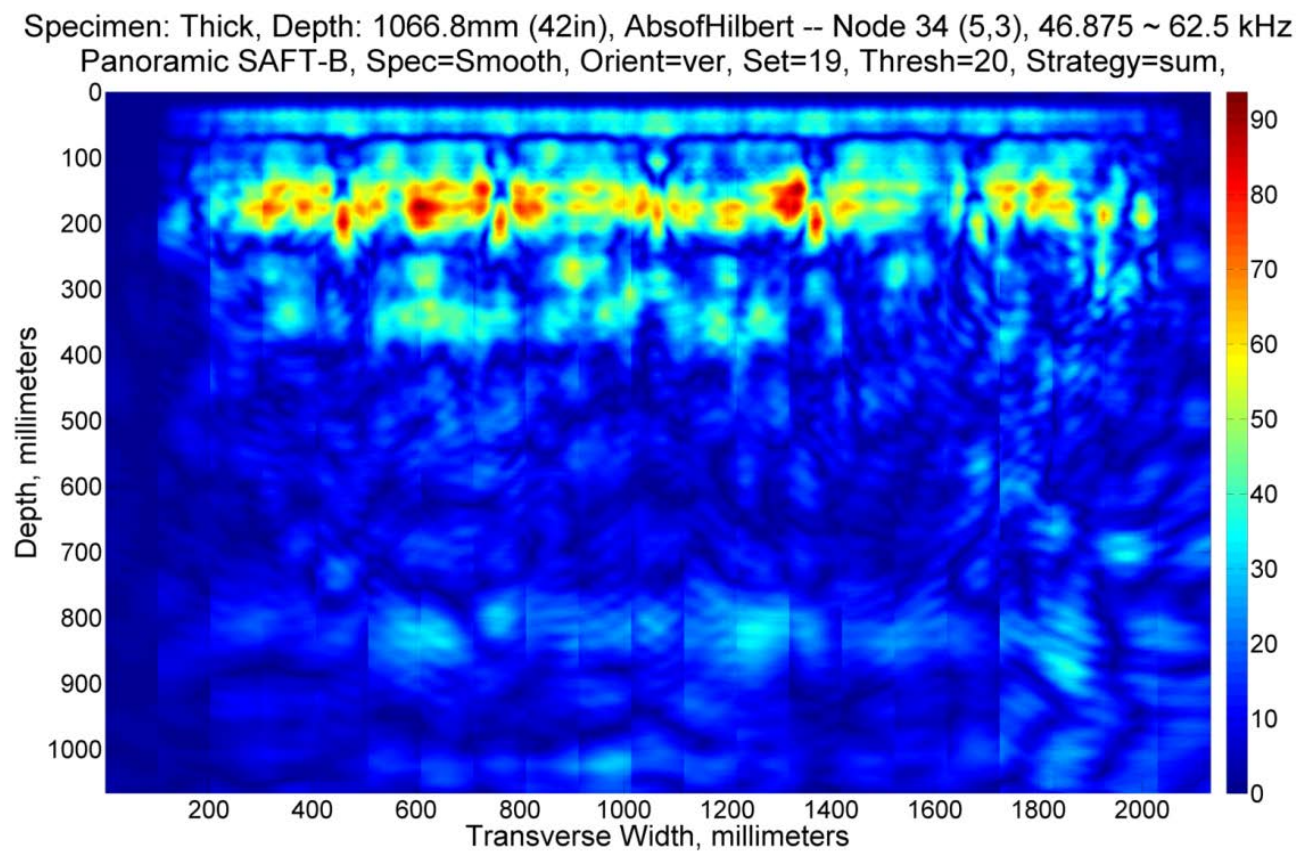

Fig. B. 190. Vertical Set 19, Node 34. 
